This document was too large to scan as a whole document, therefore it required breaking into smaller sections.

Document number: so- WH-D -238

Section_ 1 of 2

Title: Wast Compatibility, Safety swedes and anal Ruses for Tank $241-T-110$ Punt Mote samples

$-p_{g} \cdot \varepsilon 0 T-307$

Date: 5/15/92 Revision: A000

Originator: Nkuzum $\& \mathscr{A}$

Co: RESH

Recipient:

Co:

References: $\varepsilon \Delta T-620399$ 


\begin{tabular}{|c|c|}
\hline $\begin{array}{l}\text { 2. ID: (teriving argandzetian) } \\
\text { Bistribution }\end{array}$ & 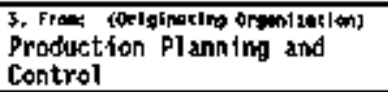 \\
\hline 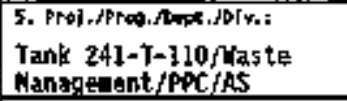 & 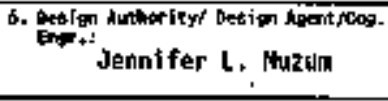 \\
\hline
\end{tabular}

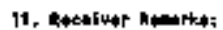
For release.

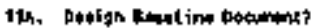

[]
4. Binted EPT MotT

NitA

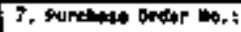

$$
\text { H/A }
$$

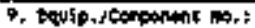
H/A

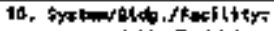
241-T-110

12. Najar Asan. Bm. Mo.r Nif

13. Darait/pand $k$ mpelleacten ito.t Hif

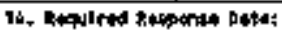
$05 / 15 / 97$

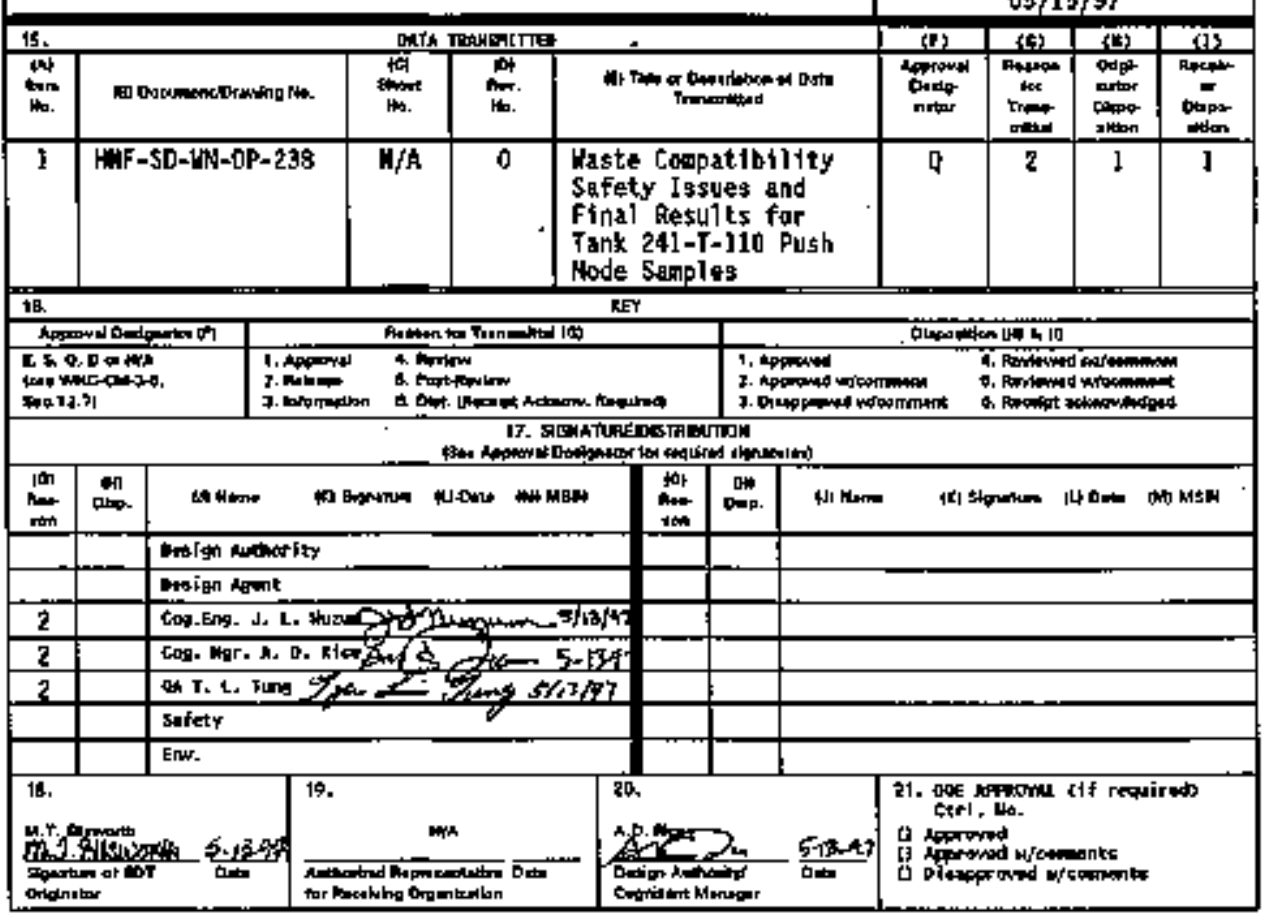




\section{Waste Compatibility Safety Issues and Final Results for Tank 241-T-110 Push Mode Samples}

Janntfer L. Huan

Rust Foderal Services of Hanford, Inc, Kichland, Wh 99352

U.S. Department of Energy Contract DE-ACO5-96RL13200

EOT/ECH: EDT-620399 UL: 2070

OTg Code: 75768 Charge Code: M037B

BAR Code: EN 3120074 Total Pages: $6 / 2$

Key Nords: Waste Conpatibility, Safety Issues, Flnal Results, Jank $241-$ T-110, 241-T-110, T-110, T Fara, Push Mods, Samples

Abstract: $\quad$ H/A

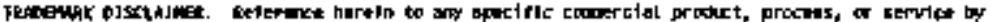

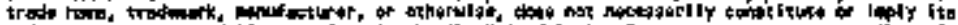

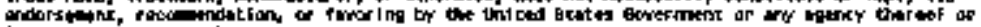

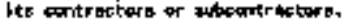

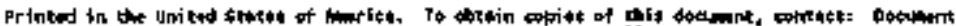

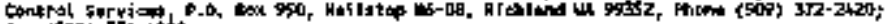
fex 400 ) $34-4969$.
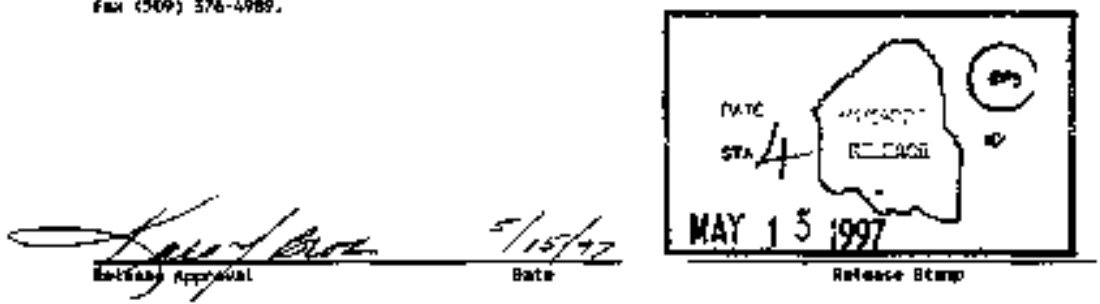

Approved for Public Reloase 
HAF-SD-WM-DP-238. REV. O

\author{
ANALYTICAL SERVICES
}

\title{
WASTE COMPATIBILITY SAFETY ISSUES AND FINAL RESULTS FOR TANK 241-T-110 PUSH MODE SAMPLES
}

PToject Coordinator: JEMitfer L. MRUW

Prepared for the U.S. Department of Energy office of Environeontal Restoration and Waste Nanagement.

by

222.5 Leboratory

Fust Federal Services of Hanford Inc. P.0. $600 \times 700$

Richland, Washinyton 
NAF-5D-WN-OP-238， REY, D

TH1S PAQY IMTERTIONALY LET BLAKK 
MAF-SD-MT-DP-238, REV. O

TABLE OF COMTETS

Warrative ........................ J

[-110 Sappla Breakdmm (Attackinant 1) $\ldots \ldots \ldots$

saplo Data 5 longary $\ldots \ldots \ldots+\ldots \ldots$

Chatn of Custody Foms . . . . . . . . . . . . 49

Photagraphs . . . . . . . . . . . . . . . .

Bulk Dens ity Morksheets $\ldots+\ldots \ldots+\ldots \ldots$

Data Yorification and Detiverable Sumary keport (Attachment 2) . . . . 104

sample kand1ting . . . . . . . . . . . . . . . . . 193

Extrusian Morkitst $16499(99)+\ldots \ldots \ldots$

Extrus ion Workj ist $16500(100) \ldots \ldots . . . \ldots 190$

Extrus ion Work7ist 16501 (101) ............... 197

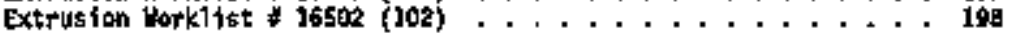

Extrusion Hork1 ist J6503 (J03) ............. 19g

Extrustion Hork115t 15504 (104) ............, 200

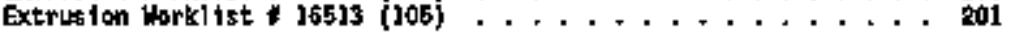

Extrus ion larkl ist \# 16514 (106) $\ldots \ldots \ldots+\ldots \ldots$

Extrus 1on Hark11st \# 16515 (107) $\ldots \ldots \ldots \ldots$

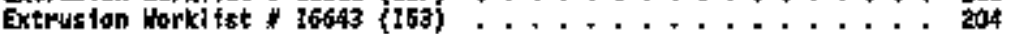

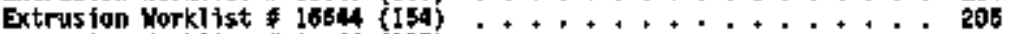

Extrus ian tork1 tst 16546 (155) $\ldots \ldots \ldots \ldots$

Ektruston Morkitst 16726 (187) . . . . . . . . 207

Extrusion Morklist 16726 (jos $\ldots \ldots \ldots$

Ext?us lon tork1 ist 16727 (199) .............. 209

Extrusion Workl ist 16728 (193) . . . . . . . . . 2J0

Extrusion Morkl15t $\neq 16889$ (102) $\ldots \ldots+\ldots+\ldots+\ldots \ldots$

Saple Praparations .................. 2J2

Fustan 0igest Horkl ist $16689(14 B, 149) \ldots \ldots \ldots$

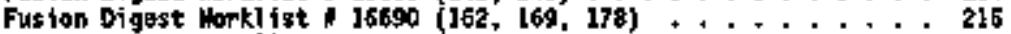

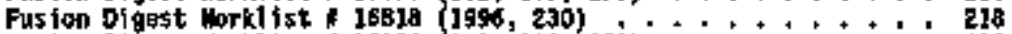

Fuston Digest Work' ist $16820(232,233,268) \ldots \ldots \ldots$

Fusion Digast Horklist / 18821 $(269,270) \ldots \ldots \ldots \ldots+\ldots \ldots$

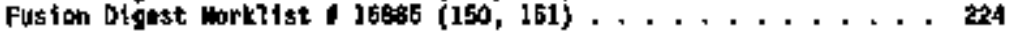

Jnorganic Unalyst $6 \ldots \ldots \ldots \ldots$

Differential scanning Carortwatry (DSC)

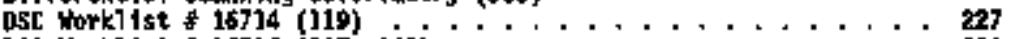

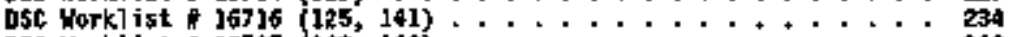

DSC Horkl1st of $15717(142,143) . \ldots \ldots 240$ 
ANE-SD-LIN-OP-23A, REY + 0

TABLE OF CONTEMTS (Cantinugd)

DSC Hork1 ist $16718(144,145) \ldots \ldots \ldots \ldots \ldots$. . . . . . 247

osC Morkl ist $16719(146,14) \ldots \ldots \ldots$

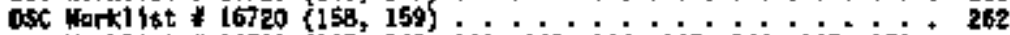

DSC Horklist $16796(125,141,142,143,144,145,146,147,158$.

159) $\ldots+\ldots+\ldots \ldots+\ldots+\ldots$

DSC Morkl 15t $+16025(292,193,214) \ldots \ldots \ldots \ldots$

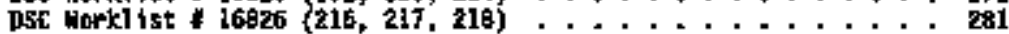

DSC Horkl 1st + $16827(219,220) \ldots \ldots \ldots 2$

osc Norkl ist 16828 $(221,222) \ldots \ldots$

DSC Markitst $16896(192,193,214,216,217,219,219,220,221$

222) $\ldots+\ldots \ldots$

osc Hark11st 1 16904 (11s) . . . . . . . . . . . . 3008

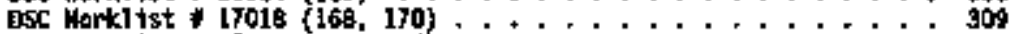

osc Horklist $117019(174,175) \ldots \ldots \ldots \ldots \ldots$

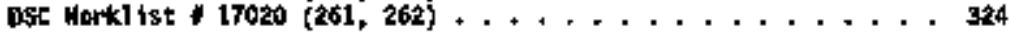

DSE Norkl15t * 1702] $(263,264)+\ldots+\ldots \ldots \ldots+\ldots \ldots$

DSC Hork115t *17022 $(255,266) \ldots \ldots \ldots$

D S W Workl 1st 17125 (368, 170, 174, 176, 261, 262, 263, 264, 265

266) .................. 346

Thermograviatric Analysis (TOA)

The Horklist 16707 (119) $\ldots+\ldots \ldots+\ldots+\ldots$

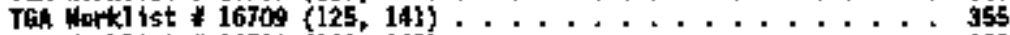

TGin Workl ist $16710(142,143) \ldots \ldots \ldots \ldots \ldots$

Teh Nork1 1st 1671$](144,145) \ldots \ldots \ldots \ldots$

TGh sorkl ist \#16712 (146. 147) ............ 374

Toh Yorkl f5t $16713(158,159) \ldots \ldots \ldots$

Teh work1 1st * $168300[192,193,214) \ldots \ldots \ldots$

Toh Norkl ist $26831(216,217,218) \ldots \ldots$

TGh Yorkl ist $16832(219,220) \ldots \ldots$. . . . . . . . . 409

Ted Norklist * $35833(221,222) \ldots \ldots \ldots$

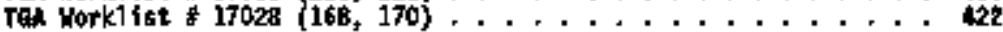

FGA Horki 1st $\neq 17029(174,175) \ldots \ldots+\ldots \ldots+\ldots \ldots$

T6h Horkl15t $17030(261,262) \ldots \ldots \ldots+\ldots \ldots$

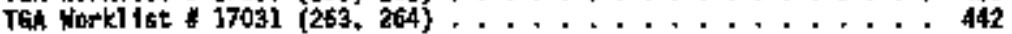

TGh Workl ist $17032(265,266) \ldots \ldots \ldots$

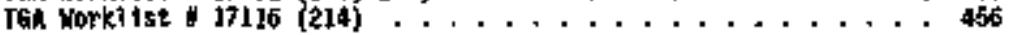

Specific Gravtty Analysis (Sps)

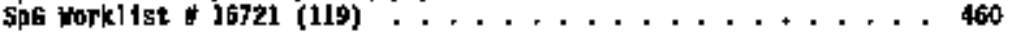

Sp6 Morkl ist $16749(204)+\ldots \ldots \ldots$

Ion Chrowotography (IC)

IC Norkl ist \# J6706 (119) . . . . . . . . . . 472

If Whark1 tst $\$ 17115$ (381) ................ 479

Induct ively Coupled p1asua spectrophotonter (ICO)

ICP tork1 lst $\#$ J6639 (J19) $\ldots \ldots \ldots+\ldots \ldots$ 
HMF-SD-Hit-DP-23g, REY. G

TABLE OF Co:rTETS (Continuad)

Total Jnorganic Carbon / Total Onganic Carbon (TJCTOC)

TuCrac Norkl ist $\$ 16990$ (381) , ................. 496

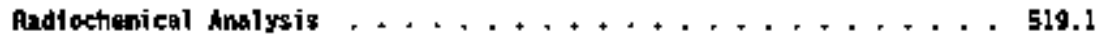

Total Alpha (AT)

AT Horktist f l6695 $\{119\}, \ldots \ldots \ldots \ldots$

AT Warki ist $\$ 16899(150$, isi $\ldots \ldots \ldots$

AT Worklyst $17065(232,233,269) \ldots \ldots 34$

AT Hork1 ist i 17066 $(162,169,178) \ldots \ldots \ldots 56$

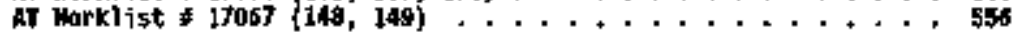

AT Morki ist $1706 \mathrm{~g}(269,270) \ldots \ldots 56$

AT Workt ist * 17072 $(106,230,231) \ldots 574$

Plutonitu 239/240 (Pu239)

Puz39 Worklists $*$ (3B1) $\ldots \ldots \ldots \ldots$. . . . . . . EBG

This Dacumit consists of pages 1 through 604. Pages $\mathrm{ji}, 2,13,32,50,69$, 898, 105, 104, 213, 226, 493, 494 and 519.2 woro intentionaily ioft thank.

TRMOEMAPKS:

Peirkin-Elmer Is a Rogistered Tradomirk of Rosearch and Manufacturing Company. Inc. Hetcler is a Rogistered Traderark of Mettler Eloctronics. 
HFF.\$O-WN-OP-23\$, REY, O

MURATIVE 


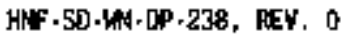

THIS PAFE INIENTIONGLIY LEFT ELANK 


\author{
HNF-SD-WM-DF-236, REV, 0 \\ 222-S ANALYTICAL SERVICES \\ TANK 2A1-T-110, CORES 1\$0 AND 181, \\ ANALYTHCAL RESULTS FOR THE FINAL REPORT
}

\title{
Summary
}

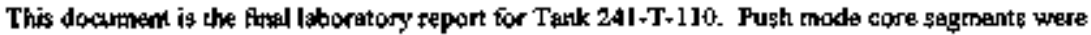
removed ffom risers 2 and 6 between Jathaty 29, 1997, and February 7. 1997. Sezmemts were received and extroded at 222-S Lahoralory. Analyses were performed in accondance with Tank

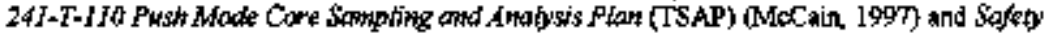
Soreening Deta Quality Objextive (DQO) (Duketow, et aL, 1995).

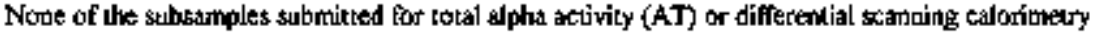
(OSC) analyses exceded the notification limits stated in DOQOO.

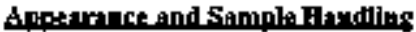

Tuo cores of eaght segments were expexted fom this tank. Complete coras were oblained.

Altachnaent I illustralea subaanples generated in the letoratory for analygis and identifies their sources. This reference also retates tank farm identification numbers to their corresponding 222-S Loboralory sample numbers.

\section{Core 180}

Segments 1-8 were removed from Tank 24 i-T-I lo Riser 6 batween Febratary 6, [997, and February 7, 1997. All sejments were received by 222-S Laboralocy betweten Febriary 10, 1997. and February 14, 1997. There was no somple tetrieved from Segineat 5. Table I stummarizer extresion information.

\section{Care 181}

Segments 1-8 were removed from Tank 241-T-110 Riser 2 between lanuary 29,199 , and February 3, 1997. All segments were received by 222-S Laboratory on February 3, F997. Table 2 summarizes extrusion information.

Fleds Blank

A field blank was provided to 222-S Laboratory with Cors 181 . This sample was treated ag a deajpable liquind in acoondance with TSAP.

\section{Hydrostatic Head Fluid Ftank}

There was ing indication of the use of hydrostatic bead fluid (HCFF) in sampling nor a HFF bignk provided to 222-S Laboratory. 


\section{INF-\$D-WM-DP-238, RCV, 0}

Table 1. Sample Retelpe and Exirasion Information for T-110, Core 180.

\begin{tabular}{|c|c|c|c|c|c|c|c|c|}
\hline \multicolumn{9}{|c|}{ 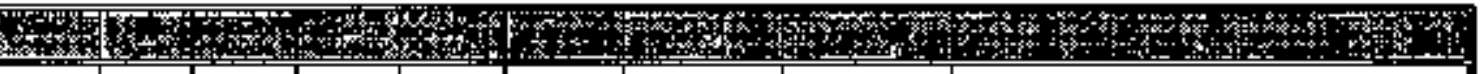 } \\
\hline श्र.1 & $\mathbf{I}$ & best & 33097 & sagen & 190 & ம* & 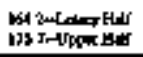 & 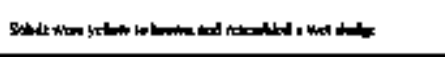 \\
\hline क्री2 & 2 & $249 \pi$ & 23097 & 22697 & 190 & $\omega$ & 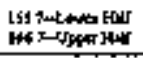 & 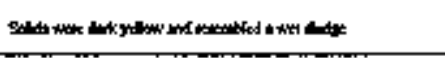 \\
\hline 92 & 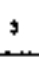 & 2iver & $\operatorname{sent}$ & aypy & 0 & $\bullet$ & 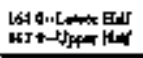 & 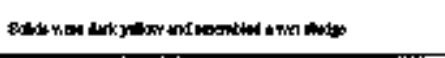 \\
\hline int-1 & 4 & vans & Dhort & jatirn & 190 & ** & r & 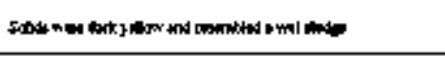 \\
\hline ons & s & $2 n$ & thort & inpt & 80 & 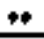 & $\leftrightarrow$ & 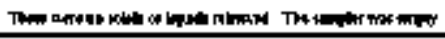 \\
\hline ris & 6 & vam & amis & nem & 19.9 & 44 & 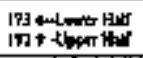 & 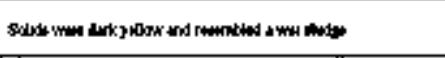 \\
\hline ns & 1 & 21ㅐㅐ & shent & 2מח & 180 & $\leftrightarrow 4$ & 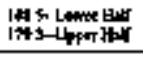 & 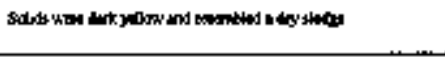 \\
\hline nn-1 & $\mathbf{\varepsilon}$ & inst & $1 \mathrm{nam}$ & indast & 100 & od & 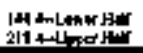 & 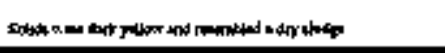 \\
\hline
\end{tabular}




\section{HNF-\$D-WM-DP-238, REV.*}

Table 2. Sample Recetpe and Extrustion loformation for T-110, Core 18L.

\begin{tabular}{|c|c|c|c|c|c|c|c|c|}
\hline$B=$ & Bhat: & 2097 & 2009 & נימדומ & $\varphi$ & 2aj JeDranikt & 00 & 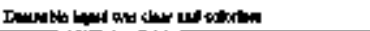 \\
\hline Yisog & 1 & $\log r$ & 2ym & bxis & נố & 00 & 195 & 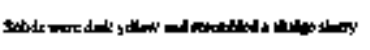 \\
\hline Prino & 3 & 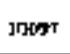 & 2397 & $26 m$ & 190 & Do & 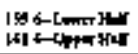 & 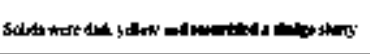 \\
\hline श्रस्थ] & $\mathbf{3}$ & 10\%वT & 259 & 2nn & 190 & pq & 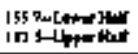 & Soldb were ded. y \\
\hline ขI-12 & 4 & atsers & कभग & $200 \pi$ & 190 & $0 \bullet$ & 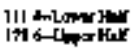 & 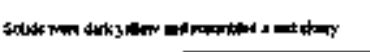 \\
\hline 4 4.-33 & 5 & ways & 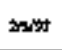 & 7axt & เค. & $\bullet \bullet$ & 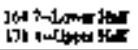 & 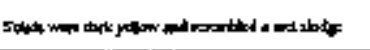 \\
\hline MT-14 & 6 & $\operatorname{lnom}$ & 2ANet & D地的 & ם סננ & 00 & 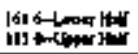 & 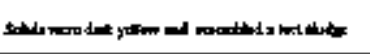 \\
\hline rit-15 & 7 & jarer & $2 A B P$ & bim & 1900 & 00 & 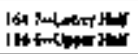 & 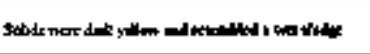 \\
\hline $9 \times 16$ & . & jovg & 2397 & mas & 1900 & $\Delta$ & 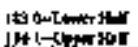 & 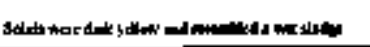 \\
\hline
\end{tabular}




\section{GNE-SD-WM-DP-238, REV.}

\section{Anahtical Respists:Summary}

The Date Summary Tsable (Table 3) Included in thls repon compiles molignical restlis assodatod with each subgemple gubmitted in accordance with $\mathrm{T} \$ \mathbf{A P}$ and $\mathrm{DQ}$.

Liquid aubgamples prepared for analybis by an acid adjugtruent of the ditect subagmple are

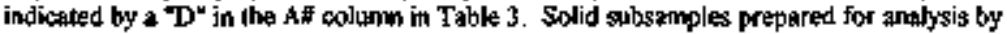
performing a fusion digest are indicated by a "F" in the A" columbr in Toble 3. Sotid sibsamptes prepared for anslysis by performing a water digest are indicented by a "W" in the A toolumn of Table 3.

Near infirmed spectroscopy and porce्mt waler by gravimexry analyses were performed on drainable liquid and half soginent semplea by Numulec Hanford Company to 222-S Laboratory. These results will be reported in a revision to this docountent.

\section{Inogranic-Annlytat}

\section{Differaulial Scanning Calurimetry (DSC) Anstysis}

The exothermic energy', babed on dy meight of unbsample, was calculated for all subiaroples. The average of the thermogravinetric antlysis (TGA) results for each subsample was used in the Ary weigtr correction for that subample. Statiatical evaluation of the resulta by calculatiog the $95 \%$ upper confisdence limit is not performed by 222-S Laboratory and, thas, not cousidered in this report.

Relatiwe percent difterence (RPD) between sample and duplicale for Upper Hall Segment 5 of Core 181 (S97T000170) was $200 \%$. Poor precision is dut to a very singl exotherm detected on

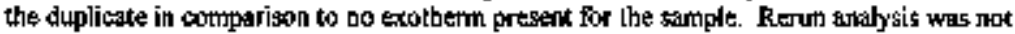
requested.

\section{Thermegrnvinetric Anglysis (TGA)}

TGA resulits were typicaly determined by summing woight loss steps below $200^{\circ} \mathrm{C}$. Weight loss steps above this were not used to determine the result. More information mady be obtained by examining the raw data.

A second analysis of L Dower Half Segment I of Core 180 (S97T0002 14) was parforned (denoted by a "J" in the replicate (R) columun of T Bble 3) due to difterenkes in appearance of the tbermograms between the sample and duphicate. These differences were not seen in the replicate analysis. The resulte for both andlyses are presented in Toble 3. 
BNF-Sb-WM-DP-238, REV. 0

\section{Density}

Bulk density was requealed only on lower half segmenls in accordance with TSAP. Regpils from

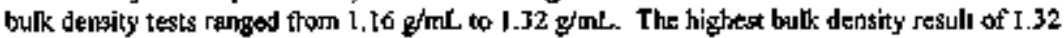
g/mL wota used to enkoulake the solid wotal alpha actinjity notificalion limit for this tank \{46.6 $\mu \mathrm{Cig}$.

\section{Spedife Gravity (SpG)}

There were no exceptiong to the quality control (QC) parameters staled in TSAP for thest subsomples.

\section{0w Chronatography (IC)}

There were no exceptions to the QC parametess starsd in TSAP for these subsamples.

\section{Inductively Coupled Plasma Spettrophodtneiry (ICP)}

There were no exceptions to the QC paranaters gtated in TSAP for these gubsamples.

\section{Totsl Orgunic Carbon (TOC)}

There were no exceptions to the QC paranteters stated in TSAP for these subsamples.

\section{Total Inorganic Carbon (TtC)}

For all TIC athalyses, a "Tctal Inorganic Carbon Analysis Repon" worksheet is indeded so raw date. Dus to programumigg limitations with the TIC instrument softwere the sample gize listed on this workstreet is incorrext. This velue is not used in the fintl caleulations (also included) and has no bossing on the results in Table 3. Ragults aro presented in Table 3 as opportunistic There wete no customer deflised TIC QC parametert. The restulis are not discorssed.

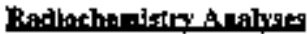

Attachmedt 2 contains the Data Verification and Deliverable (DVD) Sunmary Report for fadicchenistry malyses. This report summarizes tesults from radicchemistry analyzes and provites data qualifiers and total propagated uncertainty (TPU) values for results. The TPU values are based on the uncertainties inherent in each thep of the analysis process. They may be uard as an additional reference to doterming "reagonable" RPD values which may be usted to actepl valid date that do not meen the TSAP acceplatec criteria. A teport guide is provided with the repport to assist in understanding this gummary report. 


\section{Total Alpha (AT) Analysis}

AT malyses were requested for lower half segments only in accordance with TSAP. Liquid AT

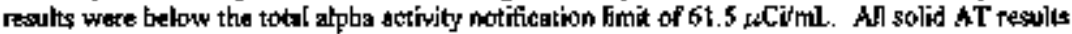

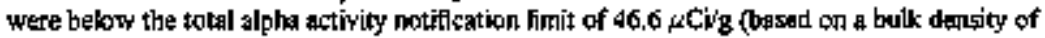
$1.32 \mathrm{~g} / \mathrm{mL}$ ).

RPD between arople and deplicate excoedied $20 \%$ for four subsamples. Segrants 3, 4, and o of Core 180 (597T000231, \$9TT000232, and S97T000233) and Segment 5 of Cora I81 (S97T000169) had RPDs of 20.8\%, 31.4\%, 39.5\%, and 21.4\%, nepectively. High RPD is due to low sample alpha aclivity. Rerun analyses were not requested. AT analysis of five subramples resulted In low spike tocoverles. For Segnaents I athd 7 of Core 180 (S93TT000230 and $\$ 97 T_{000269)}$ and Segmeat 8 of Core 181 (\$9TT000196), renun rasulta showed similar spike recoveries, aiggesting the cause to be matrix interference. Conkinuing rerruns were not requested. For Segpment 4 of Core 180 ( $89 / 2000232$ ) and Segment 7 of Core 181 (S9/TO00162), the apike recovepring were urithin control linits for the QC standard. Renun analyses were not requested.

Platonitan 259/240 (Pu)

There were no exceptions to the QC paranieters salted in TSAP for these subsarnples. 


\section{Erosedures}

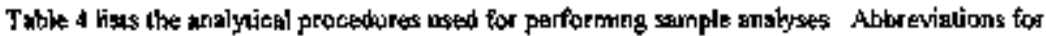
analyses are defined in the table notes

Toble 4. Anfigtical recediutes

\begin{tabular}{|c|c|c|c|}
\hline $\cos$ & $\begin{array}{l}\text { Sold } \\
\text { Lopid }\end{array}$ & Nith & 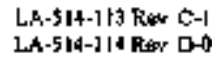 \\
\hline Ta4 & $\begin{array}{l}\text { Solved } \\
\text { Liquid }\end{array}$ & WW & $\begin{array}{l}{[A-514-] \mid 4 \text { Rew }} \\
{[A-560-1 \mid 2 \text { Rew } 5-0}\end{array}$ \\
\hline 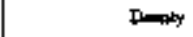 & Solvd & Wh & LO.160-103 Rer 10. \\
\hline AT & $\begin{array}{l}\text { Solpd } \\
\text { Lionind }\end{array}$ & 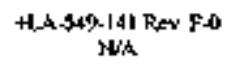 & LA-XKA-EOCI Rev E-D \\
\hline sp 0 & Lichd & NA & LA.510.]IZ Ker D.I \\
\hline IC & Ltopht & Wh. & LA-S33-10S Reu $[-1$ \\
\hline $\mathbb{1 0}$ & Laqued & ilta & [A.5D5.]6t Rer E.] \\
\hline $\mathbf{T} \times \mathbf{x}$ & Liqupd & Wus & $E-4-34\}-100$ Res E-0 \\
\hline TJC & Liqund & N/A & $\angle 4-34 z-160$ Ren E- 0 \\
\hline $\mathbf{P V}$ & Ligut & $N_{A}$ & LA-D4J-128 RE: B -0 \\
\hline
\end{tabular}


ETF-SD-WM-DP-238, REV. 0

\begin{tabular}{|c|c|}
\hline Nows: & \\
\hline 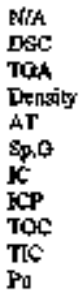 & 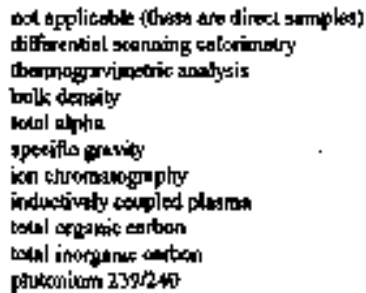 \\
\hline+ & Huion diged \\
\hline
\end{tabular}




\section{ENE-SD-WM-DP-23s, REV, 0}

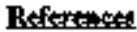

Dulcelow, G. T., J. W. Funt, H Babad, and J. E. Meachan, 1995, Tank Siffety Screening Darta Quabty Objectrus, WHC-SD-WM-SP-t04, Rev. 2, Weglingtouse Hanford Company, Richlaad, WA 99552.

MoCain, D.J., 1997, Tonk 24t-T-1 No Push Mads Cons lonpling ard Amalysis Plom, HNF-SD-WM-TSAP-I13. Rev, 1, Westinghouse Hatford Company. Richland, WA g9s52.

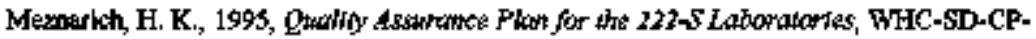
QAPP-016, Rev. 0, Wegtinghousa Hanford Company, Pichland, WA 99352. 


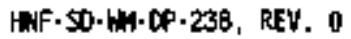

T-110 SAWPLE BREAKDCWN (ATTACHEENT 1) 
WWF-SD-WH-DP-23B, REY. O

TKIS PAEE IUTENTHOAHWY LFFT ELAKK 
$\mathrm{T}-110$

Core:181

Seg: Field Blank

\$97T000099
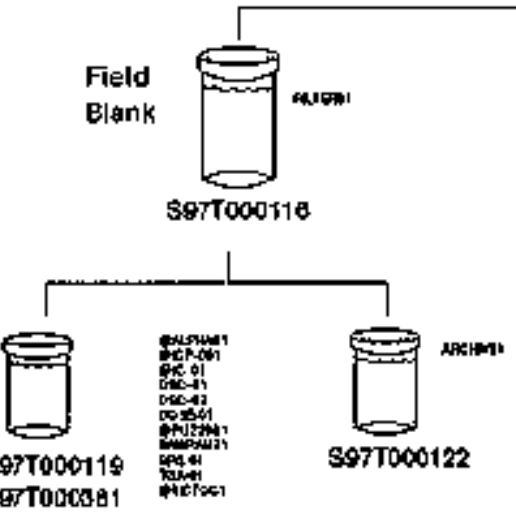
$\mathrm{T}-110$

Core: 181

Seg: 1 (97-09)

S97T000100

\section{Attachment 1}

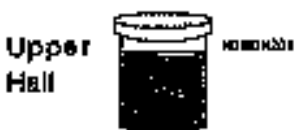

897T000127

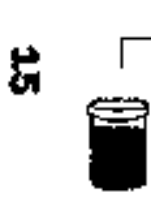

8977000141

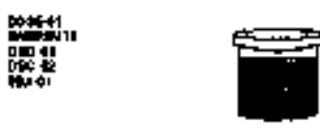

S97T000134

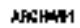

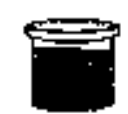

\$97T000126

4hatr

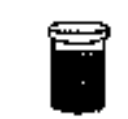

E97T000125
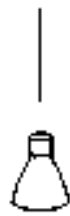

Alomin

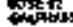

cosing

ort

G4해

25

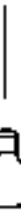

Lower

Hạll

B97T000124

S97T000140 
$\mathrm{T}-110$

Core:181

Seg: 2 (97-010)

S97T000101

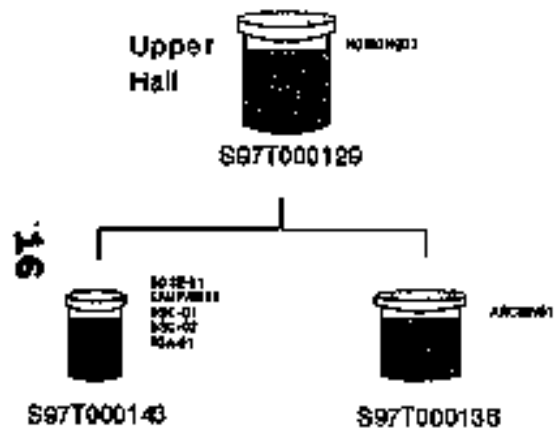

\section{Attachment 1}
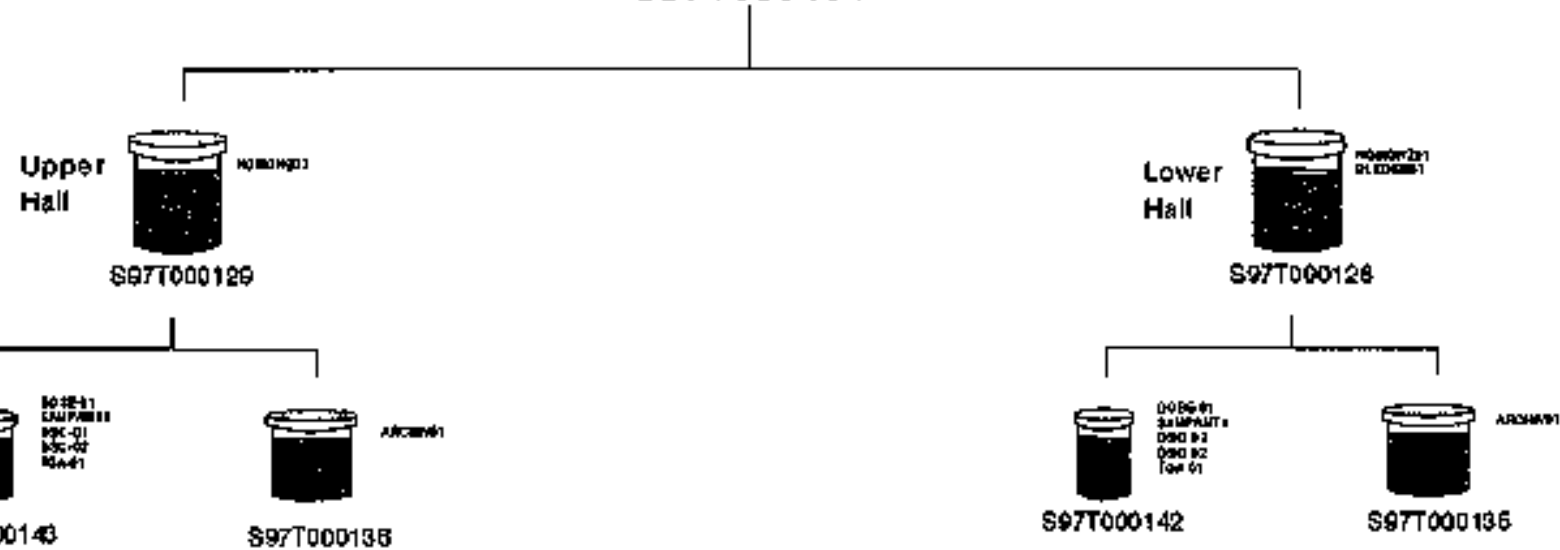

|

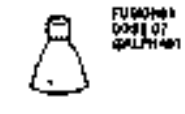

597T000149 
Core:181

Seg: 3 (97-01t)

S97T000102

\section{Attachment 1}

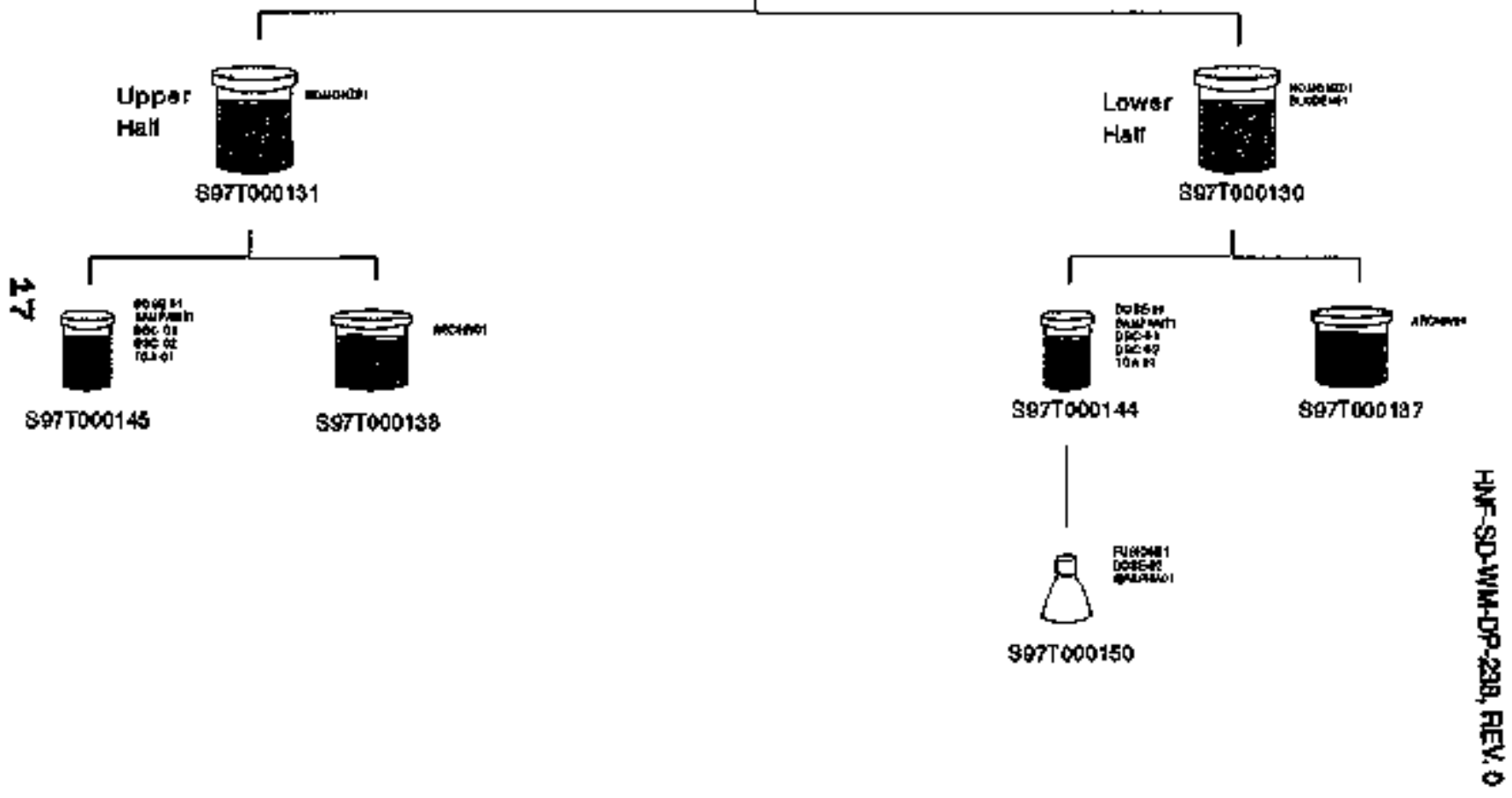


$T-110$

Core:181

Seg: 4 (97-12)

\$97T000103

\section{Attachment 1}

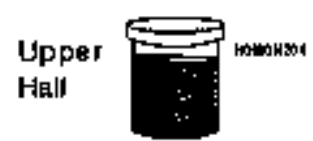

S97T000182

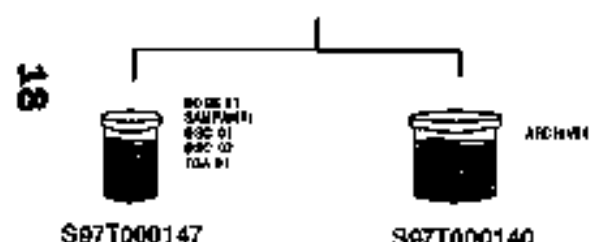

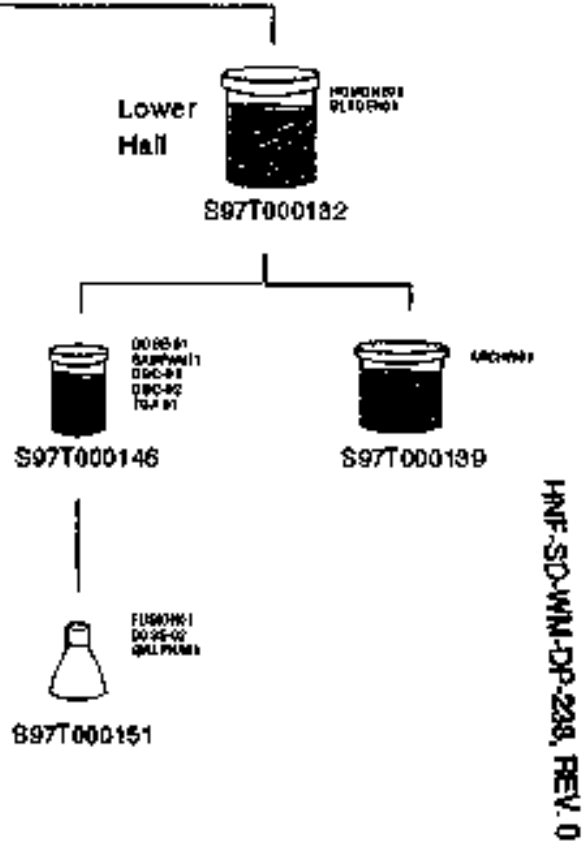




\section{$\mathrm{T}-110$}

Core:181

Seg: 5 (97-13)

S97T000104

\section{Attachment 1}
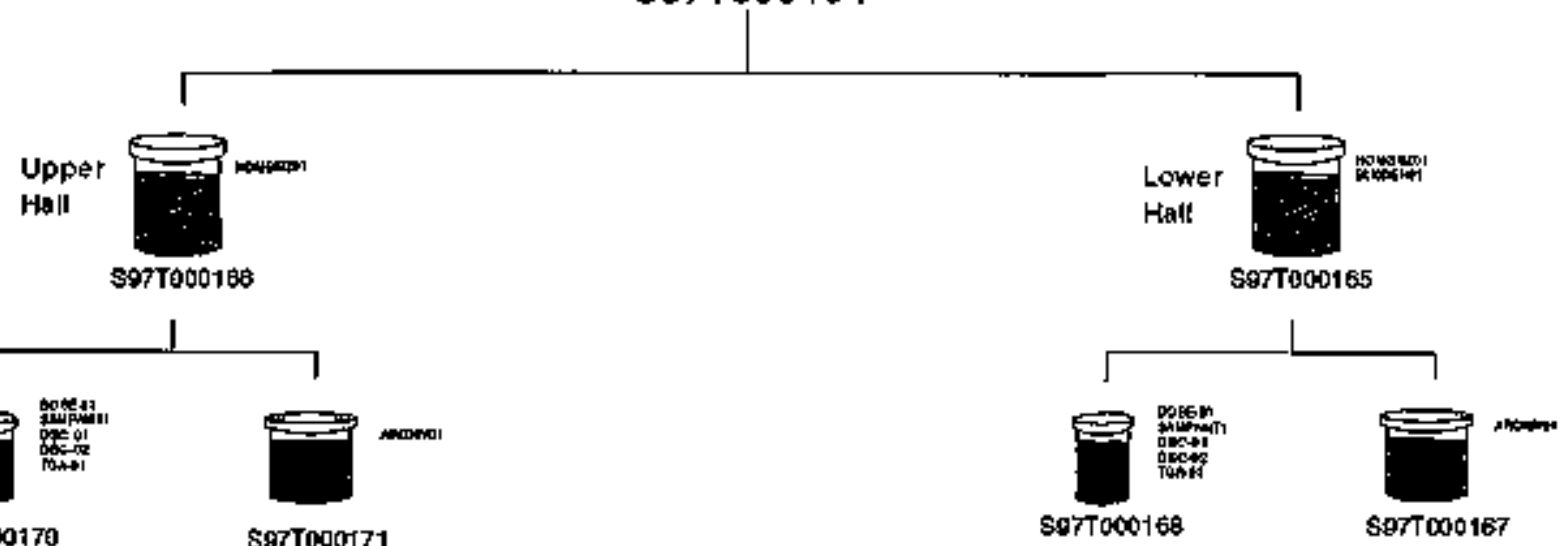

s97Tonor71 


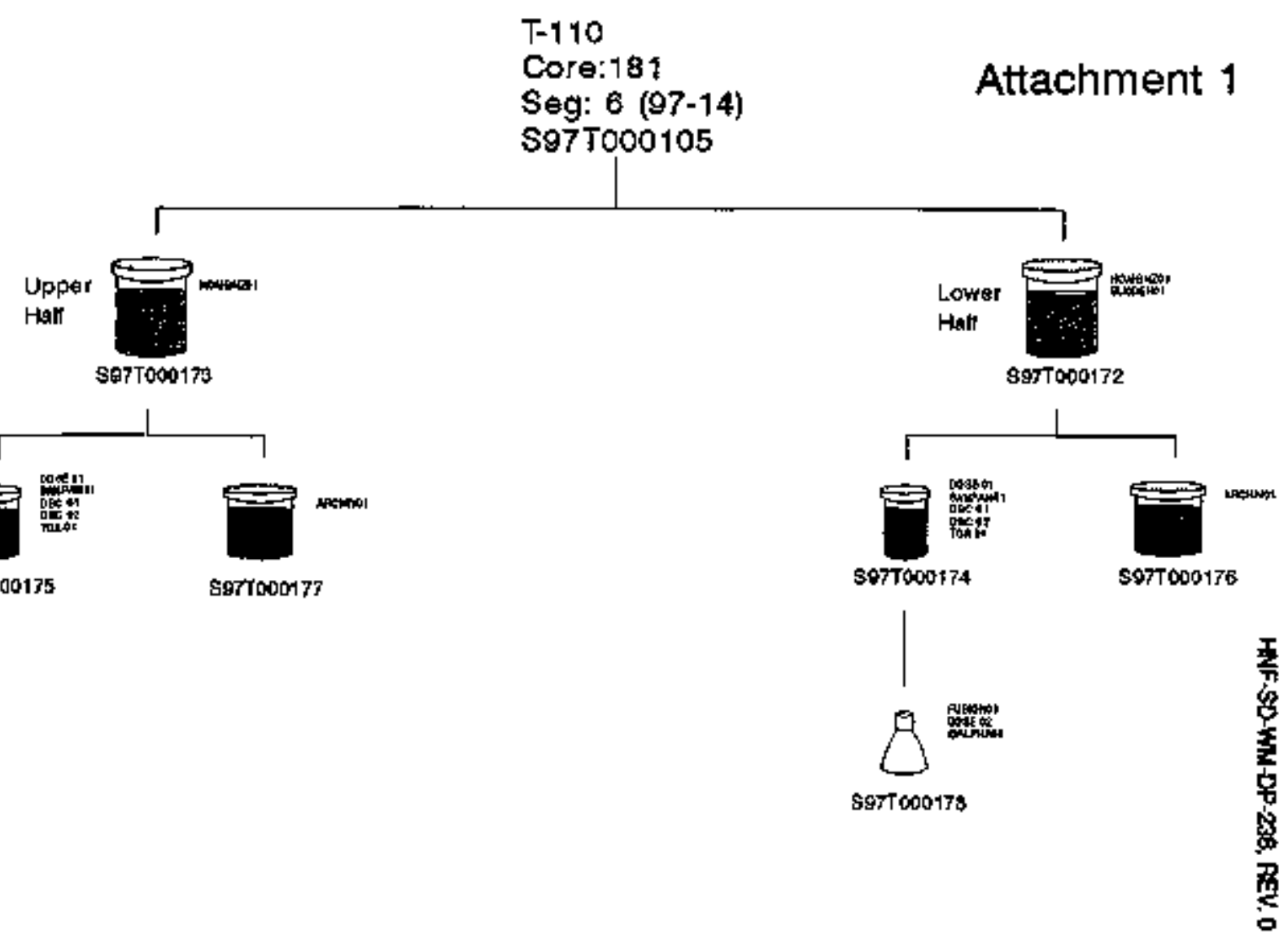




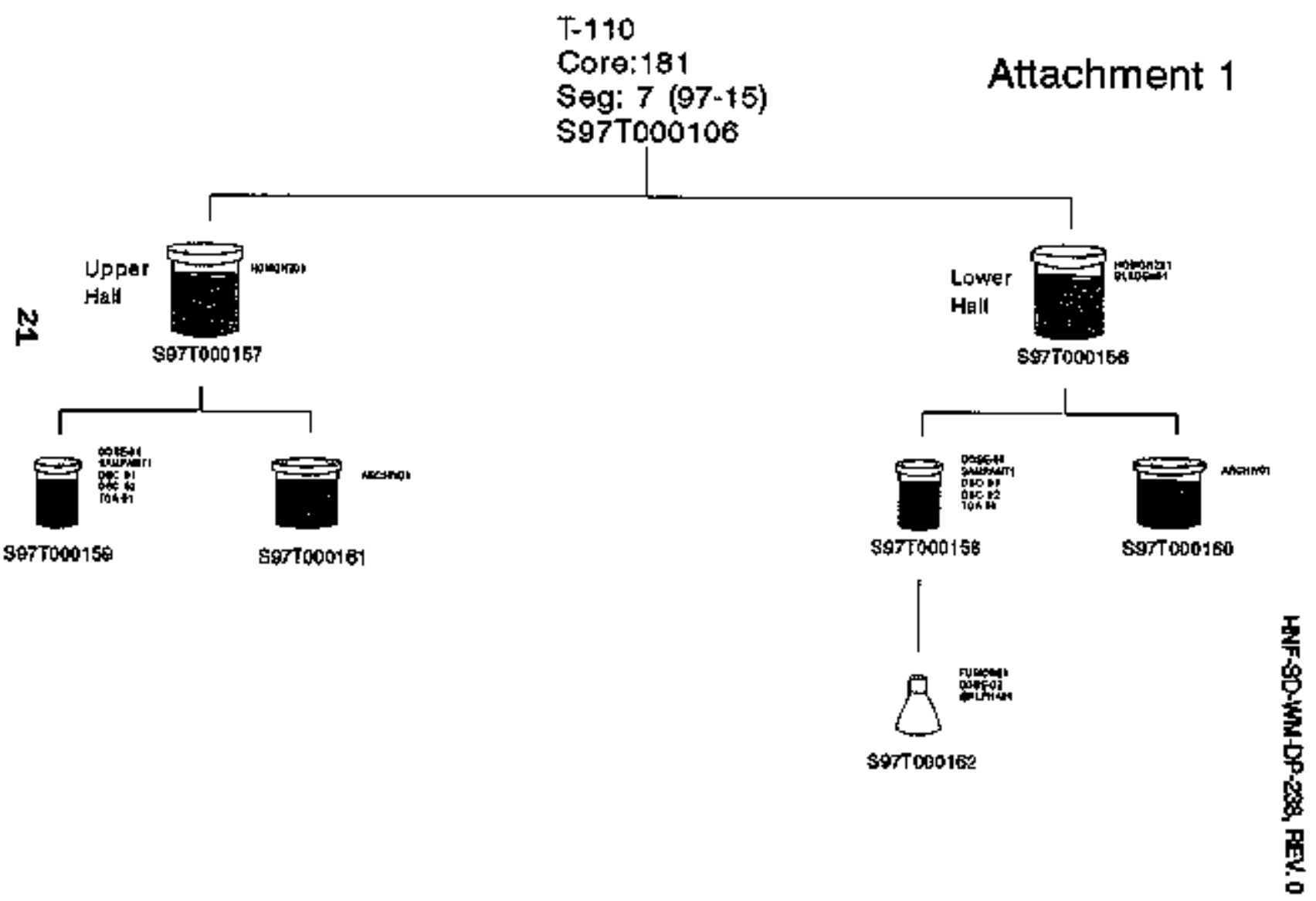




\section{$T-110$}

Core:181

Seg: 8 (97-16)

Attachment 1

\section{S97T000107}
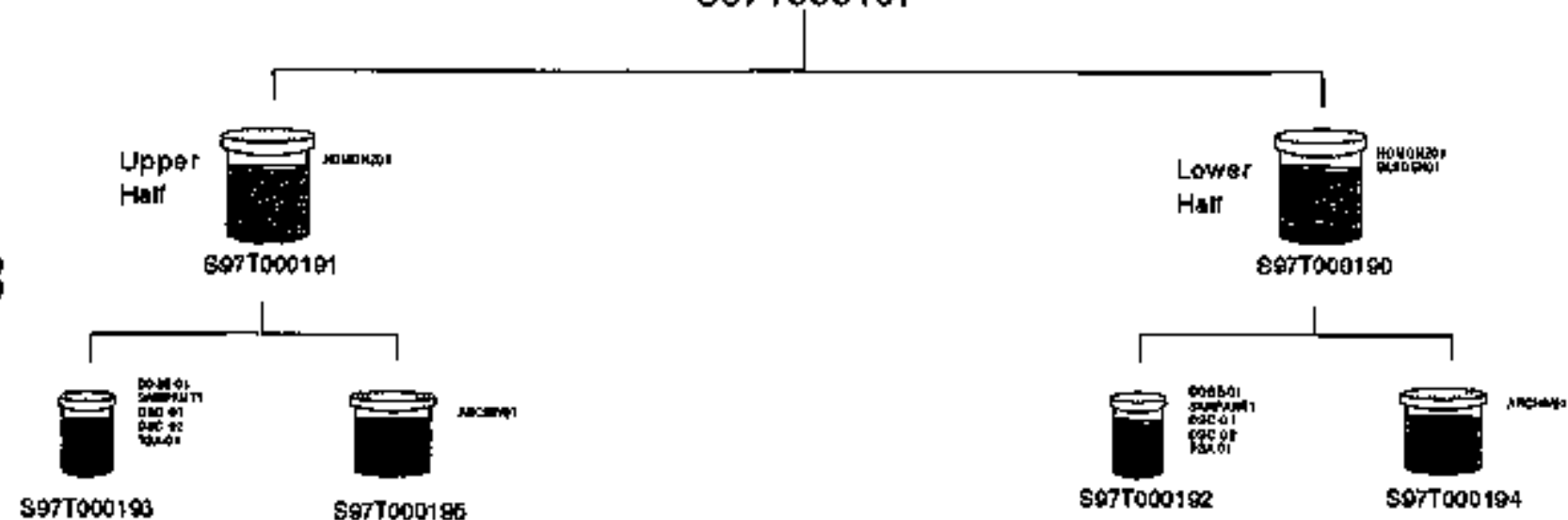

1

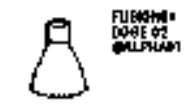

s971000 196 
HNF-SD-Wh-DP-236, REV. D

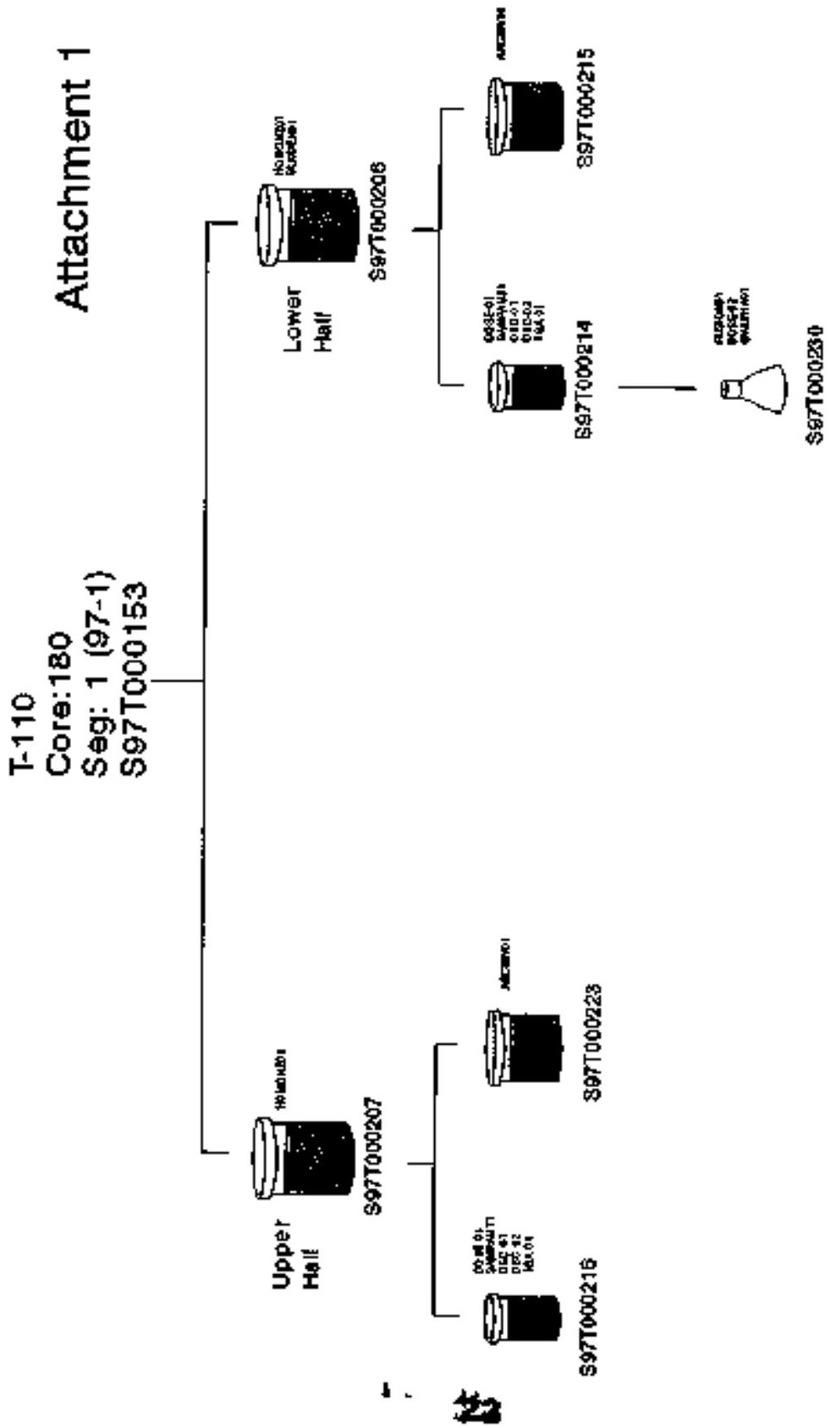


$T=110$

Core: 180

Seg: 2 (97-2)

S97T000154

\section{Attachment 1}

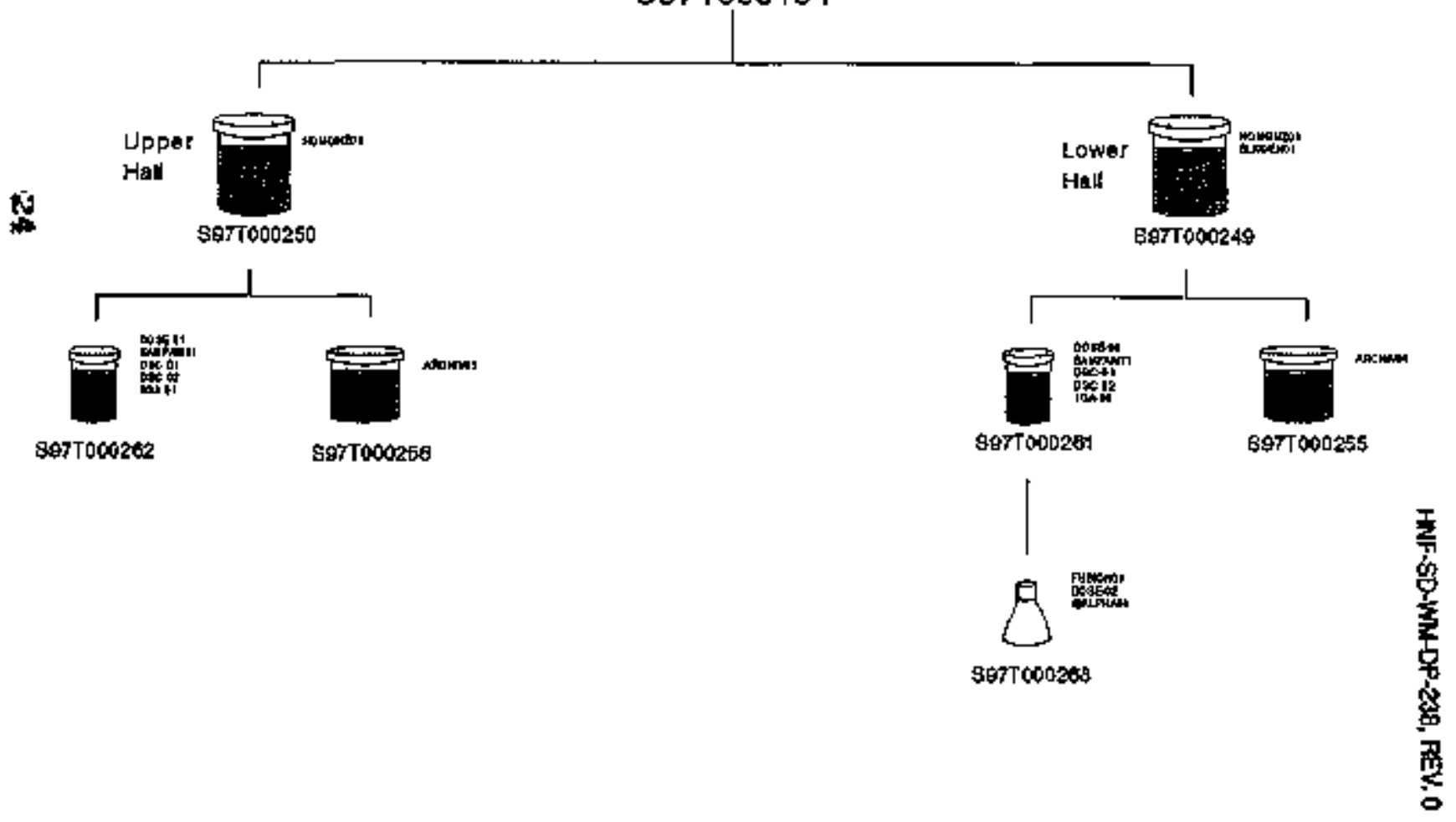




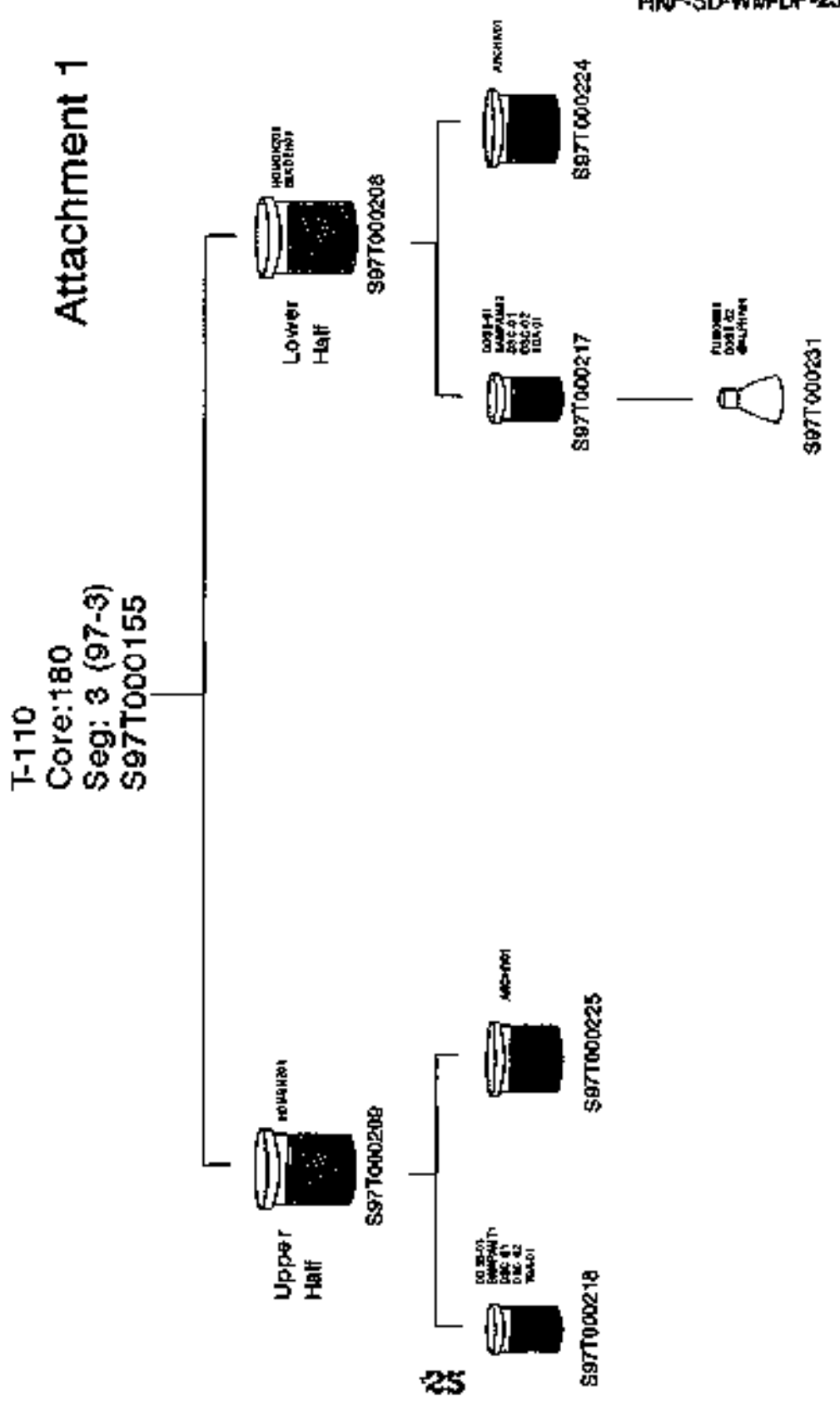


$\mathrm{T}-110$

Core: 180

Seg: 4 (97-4)

S97T000187

\section{Attachment 1}

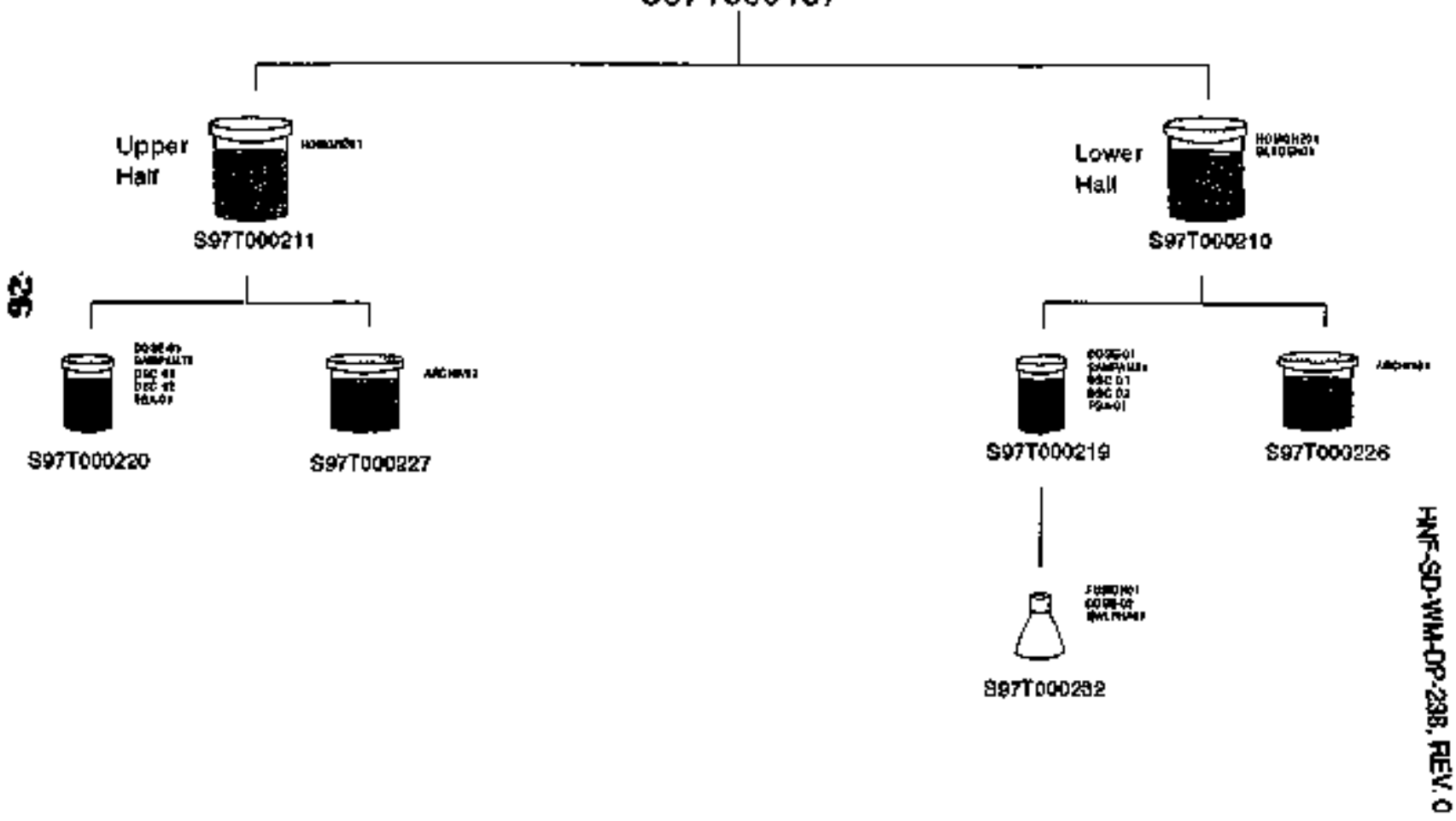


T-110

Core: 180

Seg: 6 (97-6)

S97T000189

\section{Attachment 1}

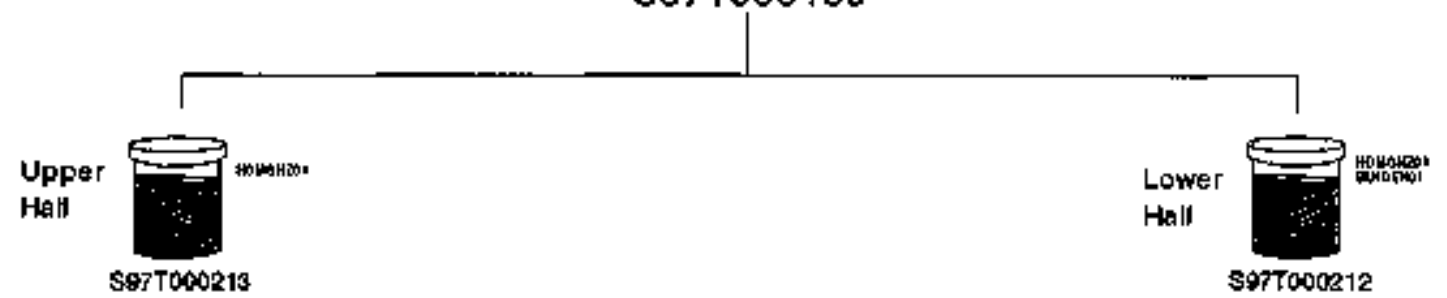

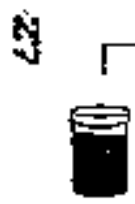

\$97T00020
S97TO00213

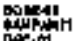

ofe먼

sict

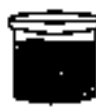

897000228

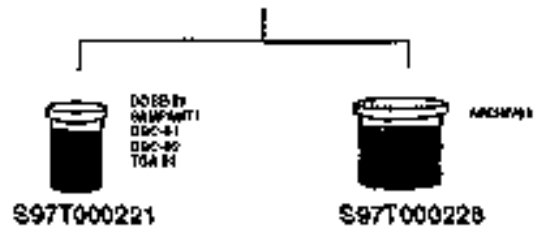

$\mid$

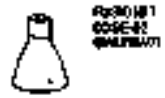

serT000293 


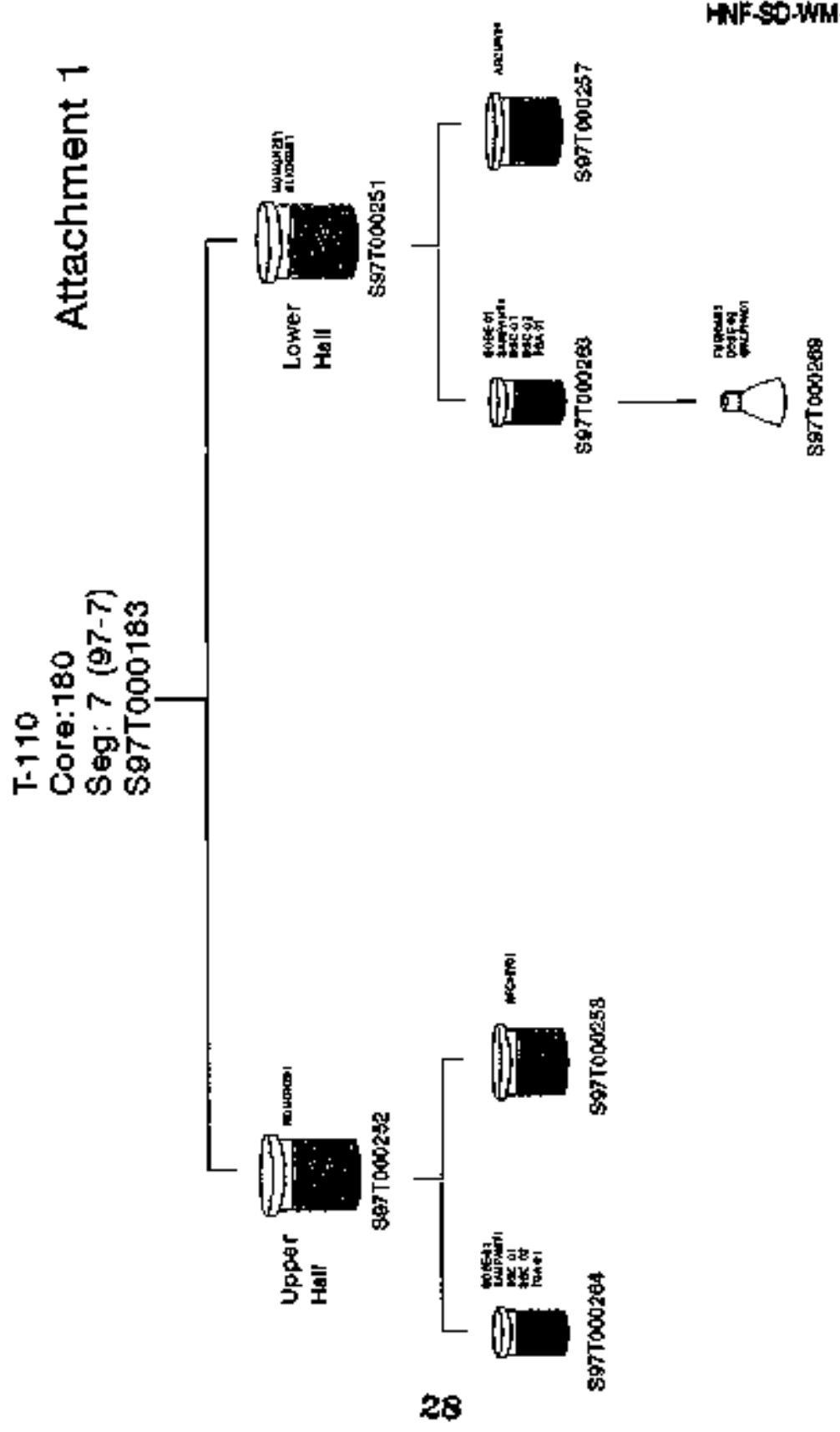


T-110

Core: 180

Seg: 8 (97-8)

S97T000182
Attachment 1
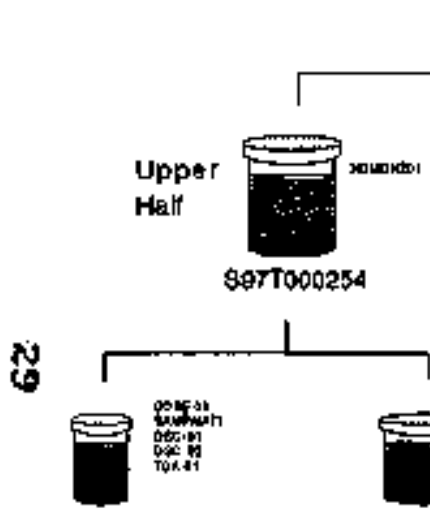

serTo00203

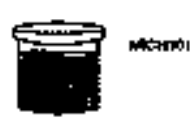

S97T000200

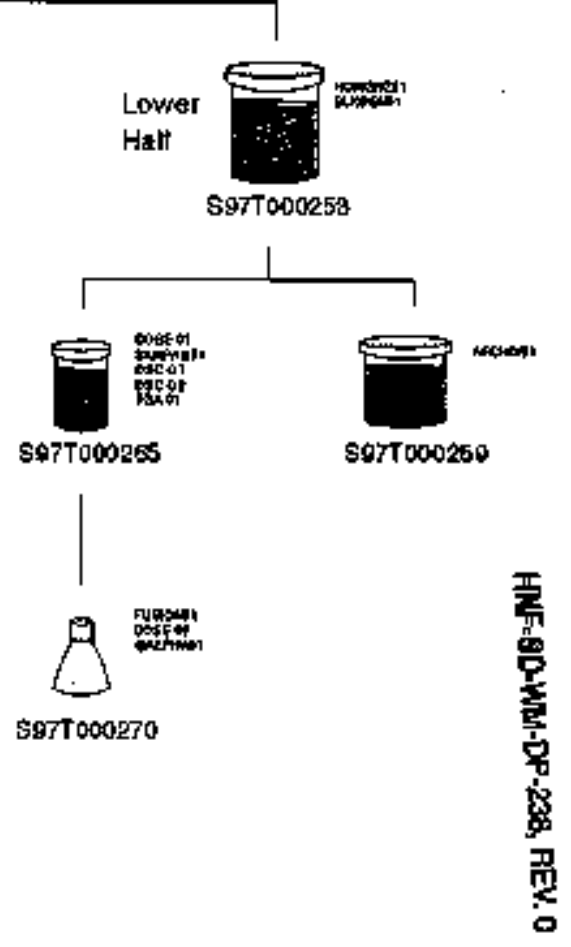




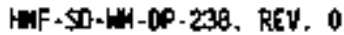


HWF-\$O-WN-OP-238, REY, O

THIS PAFE IHTENTIOAALLY LEFT GLANK

31 
foln 1. Buta samer 1abte

$1 \cdot 110$

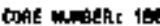

Beblewt k: 1

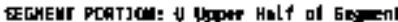

\begin{tabular}{|c|c|c|c|c|c|c|c|c|c|c|c|c|}
\hline Snotef $\mathrm{E}$ & hof & crulyte & terit & St:mand y & Bitol... & Beroult & 므멍 itext. & & RPO * * & Syle & Dot lin: & Eorrt Enth \\
\hline 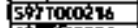 & & DSG Exothori Dry Coleulored & deresta Bry & nta & $\mathrm{n}^{\mathrm{ra}}$ & $0.0 .0=100$ & b.00t+0.0 & $0.00=0.0$ & 6. 西 & bing & $n$ & n's \\
\hline 987000216 & & os: Exethern of Parkin ficu & talledo & भiका & nre & $0.000+60$ & D.004t00 & 6_62:00 & & hith & nin & Ifte \\
\hline & & I bater by Tuh on gart in Eloup & & W.21 & nol & 36 & 7.60 & & D.56 & nita & & \\
\hline
\end{tabular}

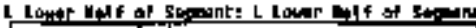

\begin{tabular}{|c|c|c|c|c|c|c|c|c|c|c|c|c|}
\hline Engles R & hel & enelyte & Lhoth & stantind $x$ & BLEt & Ansoult & auplicate & Anereget & $\cot x$ & tigk ten $y$ & Dest LElte & Conrk Emt \\
\hline Bg & & Bull Density of Enold & Int & $n$ & nat & 1.30 & nt & $\mathrm{n} / \mathrm{a}$ & ntu & nip & $5+0,0101$ & nt? \\
\hline Edomonth & & Desc Exntum Dry Calculatent & Joules/g Drr & nis & nst & 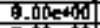 & Q.604+010 & D. & D. & nive & $\mathrm{n} \sqrt{\mathrm{a}}$ & 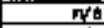 \\
\hline Bğtorats & & 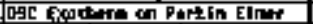 & Joredis & D. W & 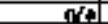 & Q. & $6.004+60$ & 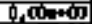 & क. 西 & nia & n厂r & ats \\
\hline 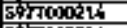 & & 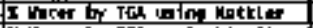 & $\mathbf{I}$ & 9.4. & nta & frt.람 & Fi. & 1,8 & 0. & nide & nर्ब & nth \\
\hline G 97000216 & & 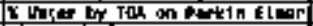 & $\underline{E}$ & D. & nirat & 9.5 .00 & 㣚.91 & 43,8 & 157 & nde & nis & pis \\
\hline 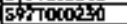 & F & 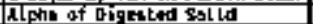 & ISt & B5.54 & a. $44+6)$ & 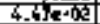 & $4.61=02$ & $4,350-102$ & 5.53 & 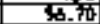 & 5.006-i3 & क्षा1 \\
\hline
\end{tabular}

s. 


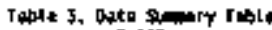

$$
T-110
$$

cDoe numa: IBO

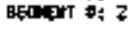

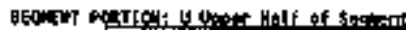

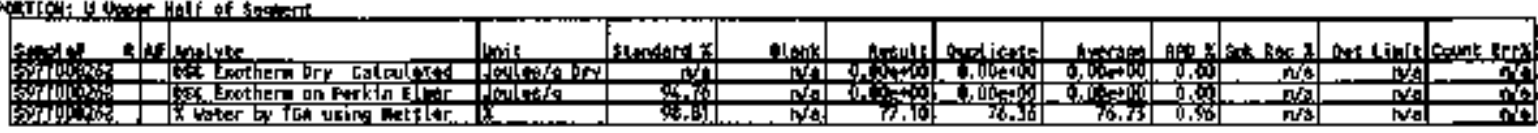

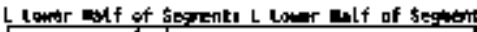

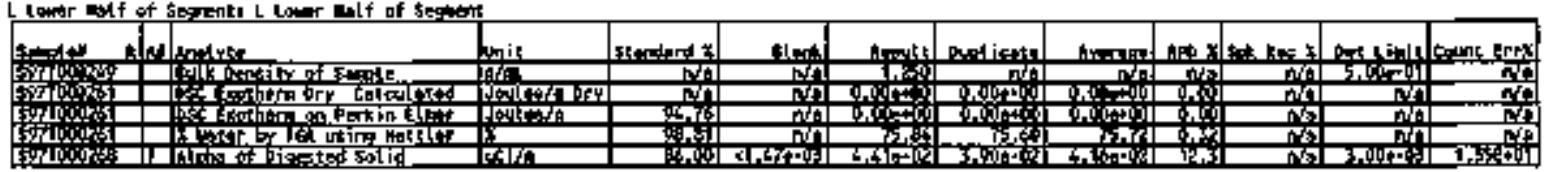

乡્ڤ 


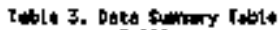

OPE UMCER: 1 QP

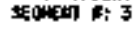

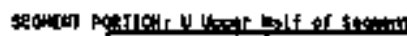

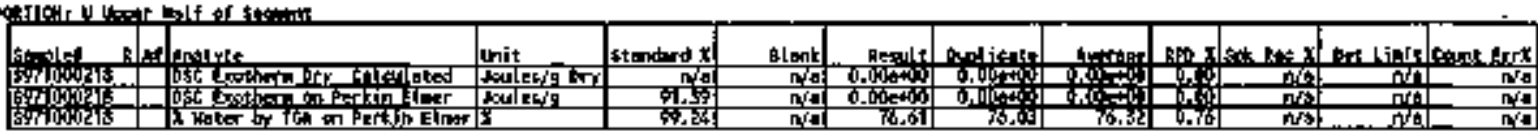

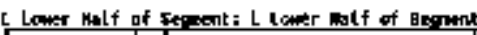

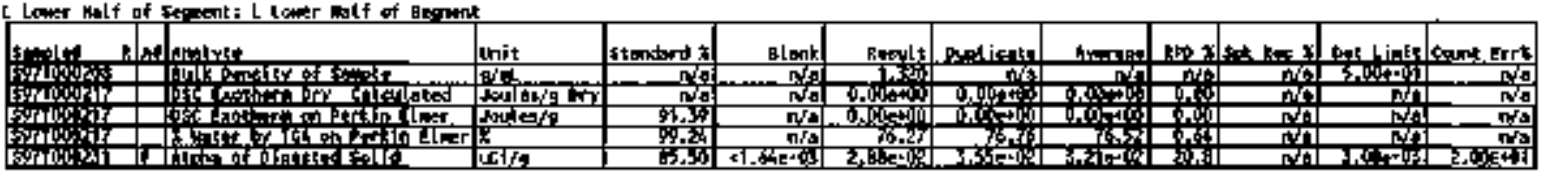




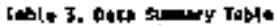

T=11

Coge unatik is

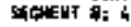

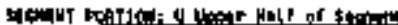

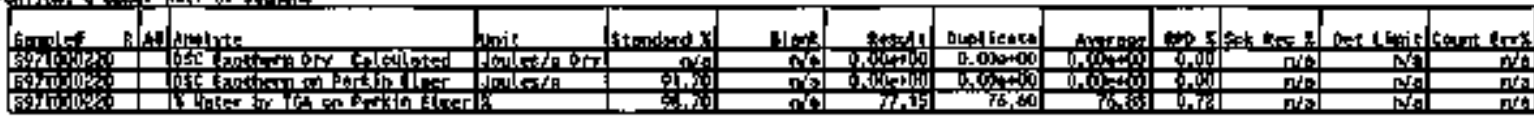

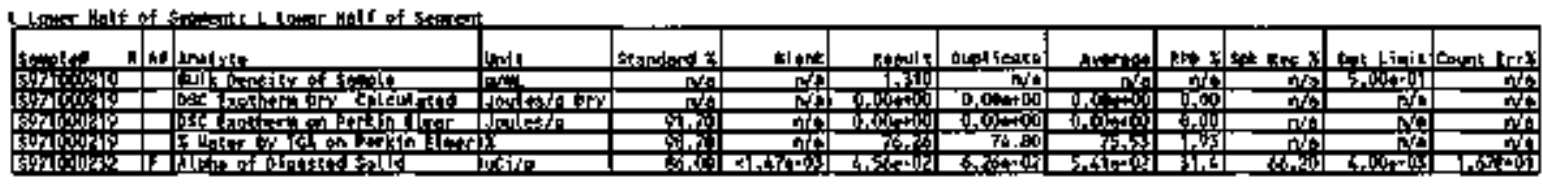


COAE MMges: 1\$0

औGuHT W: 6

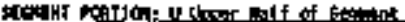

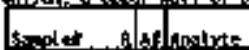

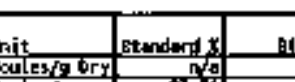

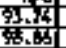

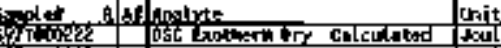

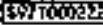

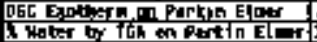

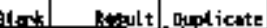

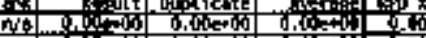

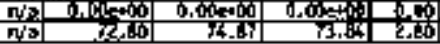

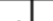

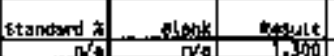

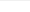

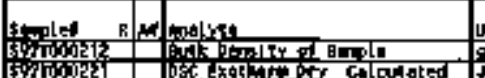

Init

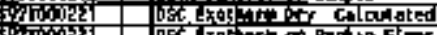

4.

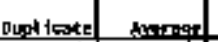
ar

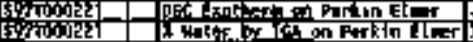

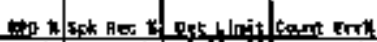

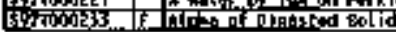

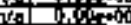

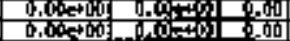

P.s.te




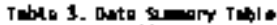

CDAE anger: 10h

Eavan है?

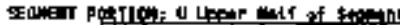

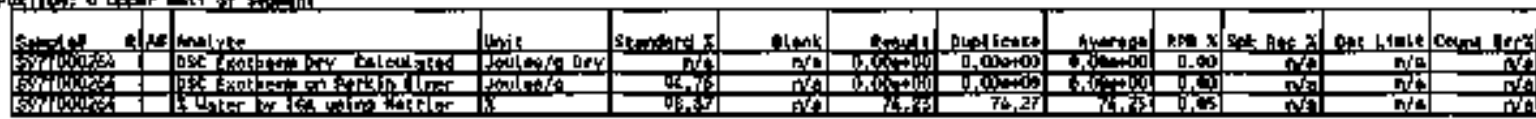

\begin{tabular}{|c|c|c|c|c|c|c|c|c|c|c|c|c|}
\hline 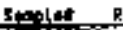 & st & Anatrit. & Lmic & Gert $x$ & 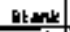 & trater & buplons. & Antrases & 由 & هpe & tol LIefi & Conmentert \\
\hline Ejy tovula & & 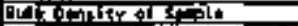 & मूल & nto & n't & & nto & nth & $\mathbf{n} / \mathrm{s}$ & resp & $5.000-1$ & Nit \\
\hline Eyotom & & 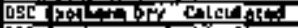 & 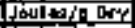 & & n's & & D.0.04ton & $8,60+00$ & D.0.0 & nis & n/a & nith \\
\hline 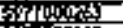 & & 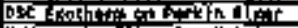 & 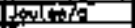 & & It & & i, $00+00$ & 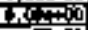 & 0,00 & npe & nis & nte \\
\hline 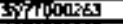 & & 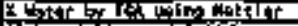 & & & 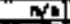 & & Pि. 표 & 7.91 & 0.多 & iva & nia & nto \\
\hline ats & 1 & 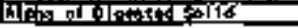 & trit]/2 & & 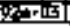 & & F, 표에 & & $3 .+5$ & & $5.000=03$ & \\
\hline
\end{tabular}

4 
CSAE UUBER: 10

EgGurer $\omega: \mathrm{B}$

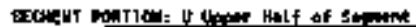

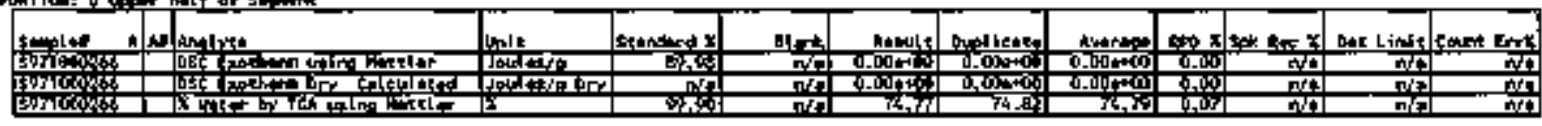

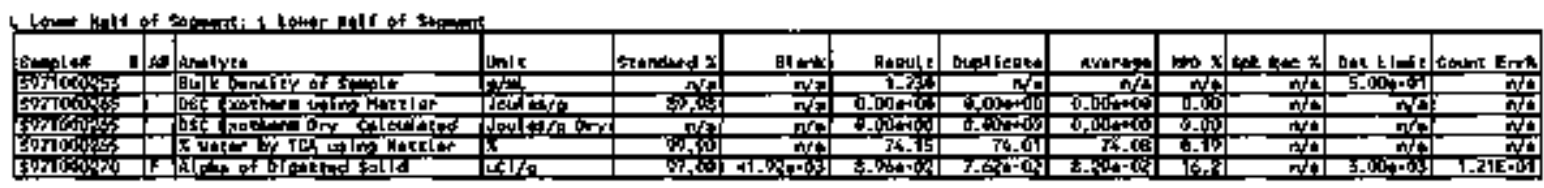

幽 
Table 5. Dats amoory rable

$T=110$

Cone nattit: 181

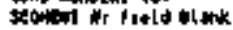

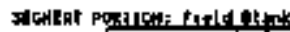

\begin{tabular}{|c|c|c|c|c|c|c|c|c|c|c|c|}
\hline & alinalyty & Lint & Ekrandind $x$ & 6tank & & & dirercet & 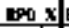 & Ept RED $\mathrm{r}$ & & $t$ [xt] \\
\hline 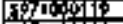 & 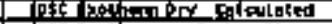 & doulesta Dery & 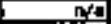 & n's & $+\infty$ & $=$ & $0,00 \mathrm{n}=0$ & 0.00 & n? & $n: 2$ & D小? \\
\hline Fis? & 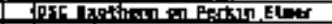 & 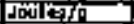 & 电地 & nis & 还 & Eक्षण & $0.00+\infty$ & (0.00 & nin & $n \pi$ & nis \\
\hline 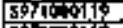 & Finififle rovely & 50.9. & 150.7 & nis & & 西a-b & 9.50-01 & 0.10 & nta & & n'b \\
\hline 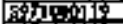 & 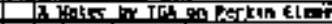 & $\sqrt{2}$ & Fis & nive & 6.6 & 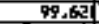 & (9.8.84 & 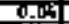 & ivo & $\dot{t}$ & Nisa \\
\hline उदिका & 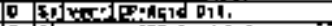 & 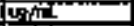 & 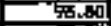 & $61,00=6$ & 4. $600=65$ & 28.00e-3 & nd & nita & nta & & $n \sqrt{3}$ \\
\hline 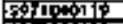 & A & קin & 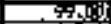 & $3.90=29$ & & & $\pi / 9$ & तis & 席司 & & $n \sqrt{2}$ \\
\hline Enotogs & trosties & पivin & कि. & & & & $\mathrm{Mr}$ & nito & rita & & का1 \\
\hline 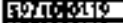 & 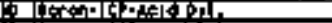 & ginta & 10.5 & - & [1] & D.t. & 1.63 & $2.0 \%$ & $2+3$ & & $-0 \sqrt{7}$ \\
\hline 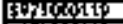 & [F]" & $4 h^{2}$ & 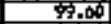 & -51 & & 20:-1 & n? & $\mathbf{n} \mathbf{u}^{\prime}$ & 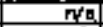 & & $\mathrm{NM}$ \\
\hline 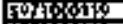 & 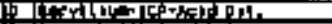 & $\ln 2$ & 原与 & 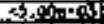 & & ही: & niv & nda & $5, \sqrt{19}$ & & $\sqrt{7}$ \\
\hline Eirtoang & 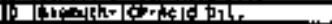 & $0,1 / 2$ & ब证. 0 & & & $6=$ & 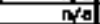 & not & nos & & M1 \\
\hline 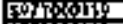 & 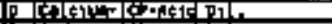 & Minich & 94.26 & 2009 & 35] & 10,10 & 18.10 & a, 6 & $m$ & & $\pi \sqrt{1}$ \\
\hline Eirtogan] & 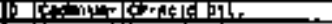 & Vilnt & 面. & $\Rightarrow, 40.03$ & & $36-2$ & $\mathrm{n}$ & $n / 3$ & nis & & ht? \\
\hline 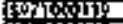 & 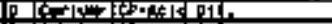 & loyfen & KQ. & 901 & II & 1 & $\mathrm{Nan}$ & 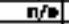 & N/t & & तif \\
\hline Hratoour: & 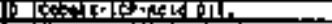 & wyin & 6.1.5 & 2 & & $=1$ & n'to & $n / b$ & nits & & $n h$ \\
\hline 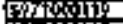 & 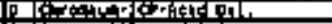 & yylat. & TQ.2 & & & $=\mathbf{z}$ & Na & $n \sqrt{2}$ & 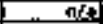 & & $n$ \\
\hline 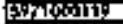 & 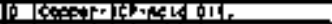 & vert. & Wht.2 & & & & $\mathrm{nos}$ & no & not & & int \\
\hline Fratos:y & 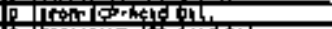 & nind.h & 70.0 & & & 7 & No & $n \sqrt{0}$ & ofs. & & It. \\
\hline Estrotulity & 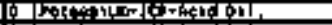 & xy & 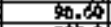 & & & 2000 & Euta & $n \sqrt{2}$ & nfe & & Ith \\
\hline 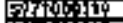 & 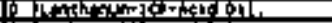 & IIstith & & & & $0=1$ & nda & $n \sqrt{n}$ & 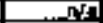 & & nth \\
\hline 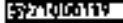 & 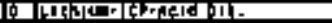 & Imsth. & 100.4 & & & $\frac{152}{452}$ & $n+2$ & $n \sqrt{a}$ & n. & & nto \\
\hline Extagosiry & 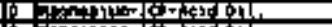 & Irat: & Tobs & रा। & tho & 360 & 3.35 & 7.15 & $\mathrm{n} \times \mathbf{n}$ & & nis \\
\hline 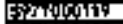 & 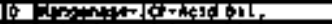 & LYT:4 & & & & $=4$ & 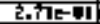 & 0.14 & no & & $n$ \\
\hline 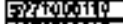 & | & M:A.h & & & & 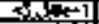 & $n / a$ & $\mathrm{nta}$ & 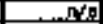 & & +2 \\
\hline 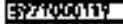 & 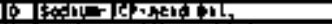 & GR:L & Tob. 2 & & & 11.50 & 11.80 & $7, \sqrt{2}$ & Nut & & nct \\
\hline 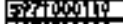 & 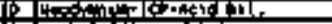 & tras.h & 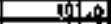 & रा & & रF,004-1 & $n / \mathrm{ra}$ & nre & 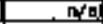 & & $n+2$ \\
\hline Fistuon & 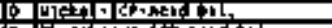 & $\cos \sqrt{2} 4$ & 100.2 & E. & & रा.60e-1 & nfa & nyt & 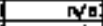 & & $n$ \\
\hline 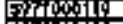 & 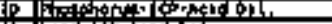 & tras: & 和, & 30,00 & ब606 & $5,4.47$ & nif & atu & nva & & $n^{2}$ \\
\hline Estivoulp & 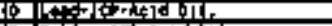 & 1906 & 50.20 & रा.00\%-01 & 6,ope-dit & 2f,00e-1. & nIs & $\mathbf{F}^{\prime}$ & rota & & $t^{\prime \prime}$ \\
\hline Fiדosin & 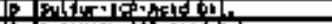 & Ind:- & 9.120. & $=1.00=0$ & d高赑 & $9,0.0$ & 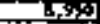 & in & nvㅁㅁ & & $\sqrt{2}$ \\
\hline Fritowath & 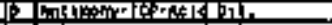 & $\sin 2$ & 7,0 & 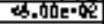 & de=b & 0:-b & ती & nyln & 자묘 & प्रi & {$[\sqrt{7}]$} \\
\hline Frरकonds & 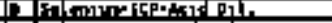 & whe & 90 & 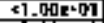 & 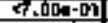 & 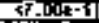 & nप & Fva & 과늘 & $7,004-01$ & ary \\
\hline 5 rTodata & 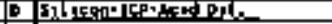 & ज्ञा & 9.20 & 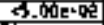 & 6.5 & Fisu & $4,1+4$ & 1.05 & infla & $=0$ & तथ \\
\hline 圈行6017\% & 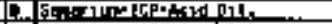 & Lor & 900,7 & $\div 1.001-01$ & [0A- & & NR] & ENt & nta & & nis \\
\hline ERT Toon & 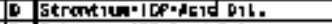 & win & $7,0,4$ & $41.00=-02$ & & & IC대닌 & पas. & nts & E. & Fra \\
\hline EFrobial15 & 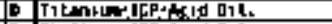 & G5 & Pria & 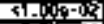 & & & का & $n / 2$ & art & $\mathbf{F a g}$ & EN? \\
\hline 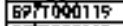 & 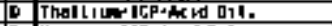 & Qㅅㄴㄴ & & & 5 & 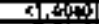 & का & 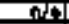 & ord & 400 & nfo \\
\hline EqरTण0115 & 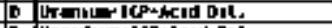 & t地它 & & 5 & 5 & स. & Nh & nta & $n$ & & 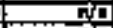 \\
\hline 昭下 & 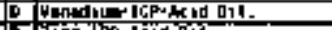 & ting & & & & & $\mathbf{N}$ & Not & 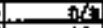 & 01 & n? \\
\hline 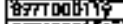 & 6) & than & & & & & 4.56e-6. & 它国 & $\pi / 2$ & & FIP \\
\hline Egrlogats & 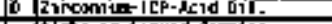 & 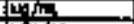 & & & 5 & $27.00=2$ & n'to & $n+3$ & nt. & & त्य \\
\hline 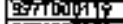 & 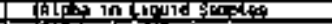 & [r.s] & & & & $5, \mathrm{BE}-\mathrm{Br}$ & $3,51-5 \mathrm{~N}$ & S. 3. & & & Ex: \\
\hline 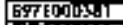 & 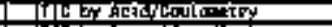 & min: & & W] & 4,10 & 14,80 & 14.45 & 4,27 & & ] & ND \\
\hline Girionisus & 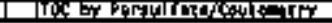 & [rat... & & & (1) & $4,6=1$ & $\mathrm{~m} / \mathrm{l}$ & $n / \mathbf{a}$ & & & nt \\
\hline
\end{tabular}




\begin{tabular}{|c|c|c|c|c|c|c|c|c|c|c|c|c|}
\hline bolet & 然 & Anslyte & thalt & 5tmatord 8 & & & Dupl lotks & Awr:- & 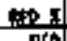 & A Rec $x$ & oet Linit & Cosik Erst \\
\hline 900 & & 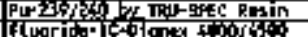 & 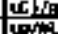 & क्र. & & & & & & $-n$ & $3.5 \mathrm{ep}$ & 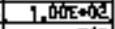 \\
\hline 64.0005 & & & $\log 2 x+1$ & 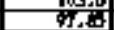 & 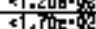 & 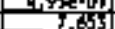 & & 750 & का & $\frac{180}{2 n}$ & $\frac{1.3 t 2 e-1}{10}$ & 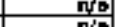 \\
\hline 而 & & 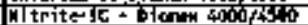 & $\cos 2$ & 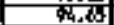 & 2.060 .04 & Tाएत & & $m$ & nto & nto & 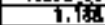 & $m_{0}$ \\
\hline 0065 & & 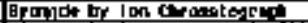 & oine & 10.2 & $-1,250$ & 2 & 4.360 & & & nta & i.3t+ & av \\
\hline & & 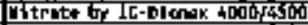 & 4 & 10.9 & $2,0 \mathrm{ke}=61$ & di1 & 7.090 & 7,5 ; & 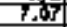 & n? & $1,1,0$ & nita \\
\hline 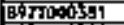 & & 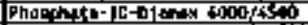 & wyile & क्रा-6? & $=1,20-6$ & 1.320 & जद्युज & 02 & nic & $n+4$ & 1.3 .00 & $n$ \\
\hline 60501 & & 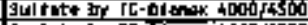 & $0 y^{-1}$ & Ints, 5 & $4,5,0$ & 18.56 & & 16.9\% & 2.34 & nin & $1,35 \mathrm{TE}$ & NA \\
\hline $897+0$ & & 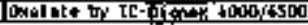 & $0 y$ & ज्ञ道 & 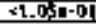 & 1.155 & 5.1600 & $\pi / a$ & nita & M & 1.1.53 & $\mathrm{nda}$ \\
\hline
\end{tabular}


tatala 1. Dorta Buncor Table

$1+110$

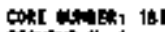 \\ stritut it 1}

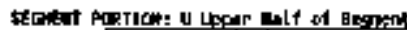

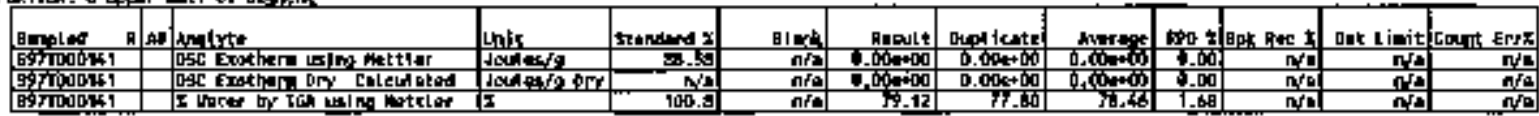

\begin{tabular}{|c|c|c|c|c|c|c|c|c|c|c|c|}
\hline 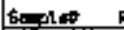 & Naf analyte & linite & Stondind $\mathrm{x}$ & Blan & Berier & aupllicnte & Averefo & Lept $y$ & Dft Rec $x$ & OEL LInit & Eougt Erri \\
\hline $80^{8}$ & 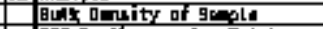 & $\pi$ & n & nis & 1.160 & & & & $\frac{\pi}{2}$ & $3.00-64$ & Nit \\
\hline Eqrowatz & 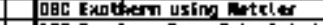 & Joules's & E.S.8 & nis & 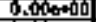 & $0.00+60$ & 0.000 & 0.000 & $\mathrm{ma}$ & $n / a$ & nt? \\
\hline 89000138 & 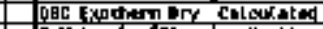 & 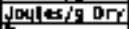 & 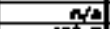 & nis & 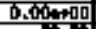 & $0.00+60$ & & 8.000 & M & n & Nis \\
\hline 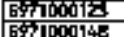 & 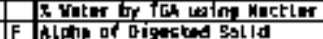 & & $\begin{array}{ll}106.8 \\
8.30\end{array}$ & 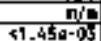 & $\frac{16.93}{704021}$ & & क. & $\frac{0.63}{61.6}$ & 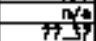 & $\frac{n+3}{5, \infty}$ & 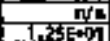 \\
\hline
\end{tabular}


Tablo 3. Date samaxy Iable

$1=110$

cose wurg: IBt

ocersirg $y_{4}$

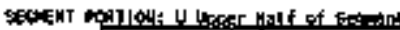

\begin{tabular}{|c|c|c|c|c|c|c|c|c|c|c|c|}
\hline 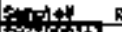 & Ax Inol hte: & Inasts & stordard $x$ & 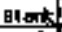 & $B=15$ & licnte & & & 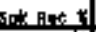 & bot & jant_Errk. \\
\hline Fintolosats & 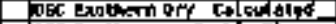 & DETLER OFY & $\mathrm{n} / \mathrm{a}$ & $n / a$ & & 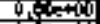 & 0.1000000 & $0,0]$ & 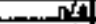 & $n+3$ & तथ \\
\hline EYTCOTSL & 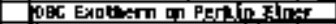 & 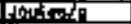 & 92, 국 & $n / 2$ & 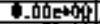 & 015000 & O. Donew & 6,0 & NSI & not & aing \\
\hline Erriogent & 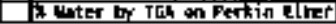 & Hen & क्रापर्भा & nis & 8.9.4. & 76.15 & 70.05 & Q.8.8 & 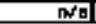 & $n[2]$ & nith \\
\hline
\end{tabular}

\begin{tabular}{|c|c|c|c|c|c|c|c|c|c|c|c|c|}
\hline 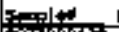 & R 1 at & Holytn, & undt: & Etenturd $x$ & Blont & REvilts & Prolitante & Avarasy & $8.2 !$ & क Ros t t & & Egentrat? \\
\hline - Sydutores. & & 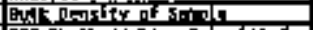 & AT: & $n / 4$ & n:? & & nit & & No & $\mathrm{n} \mathbf{p}$ & ival & ntis \\
\hline 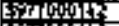 & & 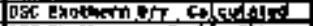 & Dorelata 0 Ty & $n / a$ & $n$ & 0.01 & $0 \pi \cos 20$ & $0.00 \mathrm{gan}$ & 7,00 & तi & 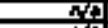 & Dus? \\
\hline Fydulort? & & 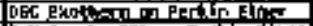 & Itक्ष.IP & \$2, 62 & $\mathrm{n} / \mathrm{n}$ & 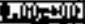 & in $100+00$ & 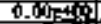 & & n?tit & n: & nth \\
\hline Sîtulutu & & 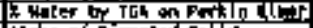 & & 67.91 & $n \mathrm{rg}$ & 7.51 & 6.09 & $70,7)$ & $1,60$. & त. & SR] & sid: \\
\hline Findoutis & $\mathbf{F}$ & 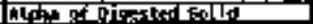 & I大 & $E 9,5$ & $=1.45 \mathrm{E}-103$ & $6,0,6=019$ & $12 \leq-0$ & 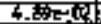 & ज्ञ & nt? & & 난다 \\
\hline
\end{tabular}

tis 


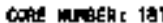

EOTEHT *: 3

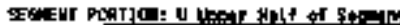

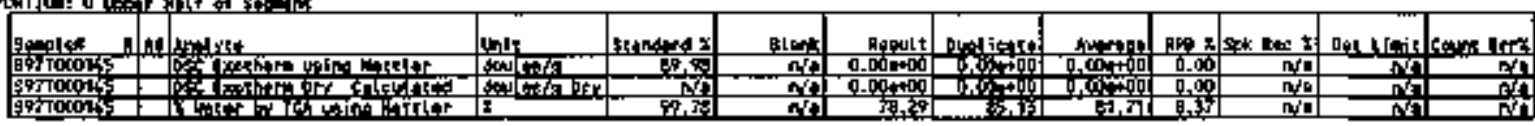

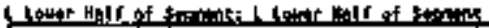

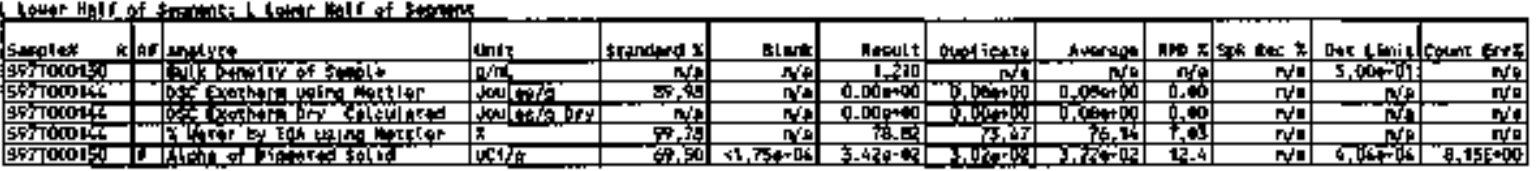

at. 
DAE UMges: 1Bt

gesomern is:

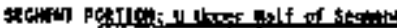

\begin{tabular}{|c|c|c|c|c|c|c|c|c|c|c|c|}
\hline 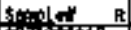 & 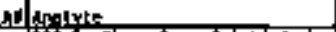 & Chjit & stan:tand $x$ & 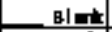 & & H.: & & & Spt. Rarc $\mathbf{t}$ & Det LInit & therk.Errst \\
\hline Eiralo & 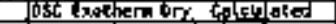 & 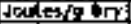 & & $n / 4$ & Q. 010040$]$ & & & 5.00 & $\mathrm{n} / \mathrm{t}$ & nia & n n \\
\hline 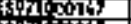 & 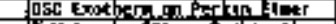 & Jorlesta & $\$ 5.63$ & n난 & $0.00 \operatorname{coth}^{2}$ & & [0] & 0.00 & ning & $n / 4$ & n'D \\
\hline 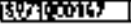 & 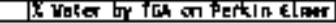 & $\mathbf{E}$ & 4.9.9 & $\mathrm{mat}$ & $2,0,0$ & 76,610 & (6.4. & 1.03 & n'ta & nat & n'D \\
\hline
\end{tabular}

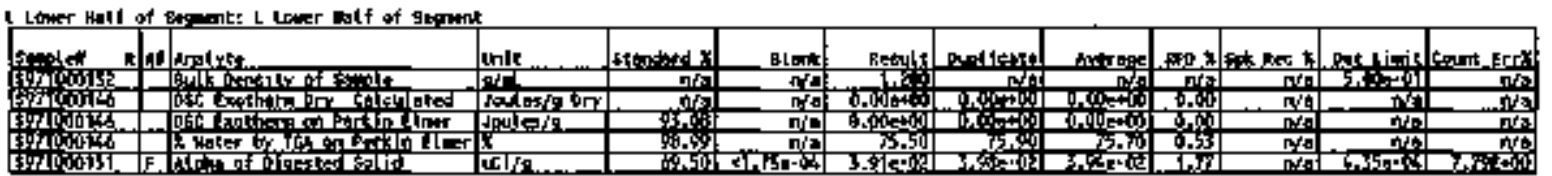




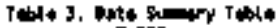

T-120

CORE UNokt 18

Hexim in: 5

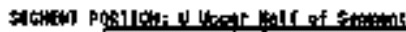

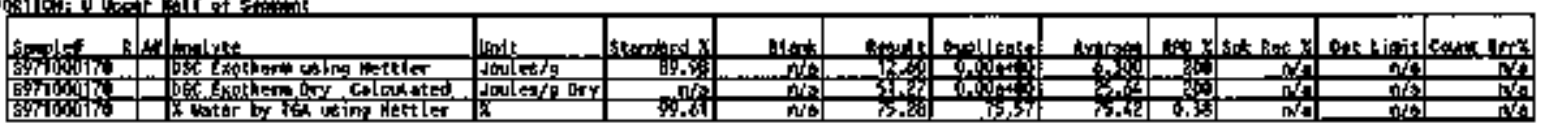

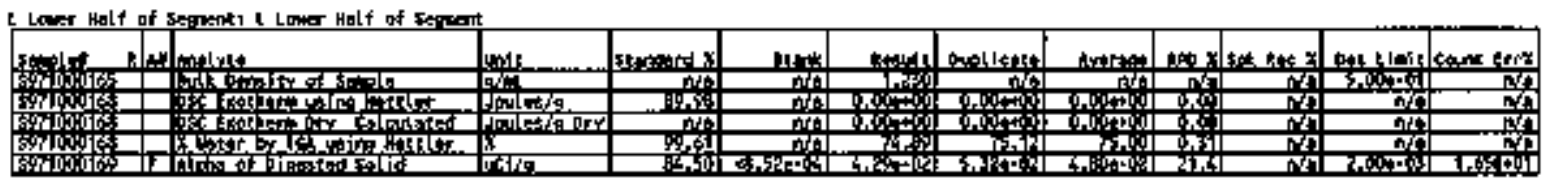


tots RLE: LA: 101

SEEDENT I 1

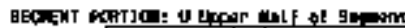

\begin{tabular}{|c|c|c|c|c|c|c|c|c|c|c|c|}
\hline 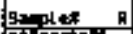 & he petilist & Unif & 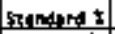 & Blent & Etsils & & & 明 $\mathrm{x}$ & gepher $\mathrm{R}$ & 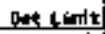 & Count ErrX \\
\hline Bका & & Bdata ory & Non & $\pi \sqrt{2}$ & 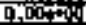 & D. Dototion & 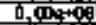 & $0 . \omega$ & mat & $\mathrm{n}$ & $n$ \\
\hline 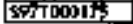 & 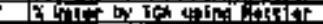 & E & 10\% & Nis & & & & H. & $n / 2$ & w & s \\
\hline
\end{tabular}

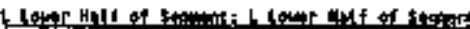

\begin{tabular}{|c|c|c|c|c|c|c|c|c|c|c|c|}
\hline Shoon? & 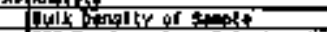 & N/21 & 늠 & 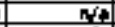 & & & $\mathrm{N}$ & $n / \bar{s}$ & nfta & 3,004-01 & $\mathrm{Na}$ \\
\hline 5कroming & 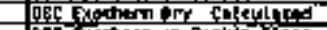 & 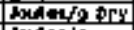 & & nta & $\overline{Q .00}$ & 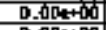 & $0,000+0,0$ & 0.0 & nifs & $\mathrm{N}^{2}$ & not \\
\hline 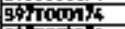 & 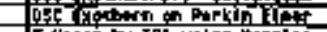 & bulcillts & & 尌 & & 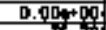 & & 0.00 & sip & nda & n;a \\
\hline 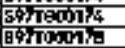 & 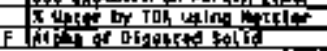 & $\frac{2}{2}$ & $\frac{1003}{8150}$ & 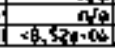 & 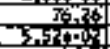 & 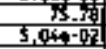 & 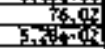 & $\frac{0.35}{9.4 .6}$ & nis & $\frac{20}{3,004-103}$ & 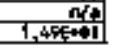 \\
\hline
\end{tabular}

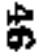


CheF What 18t

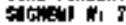

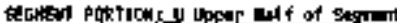

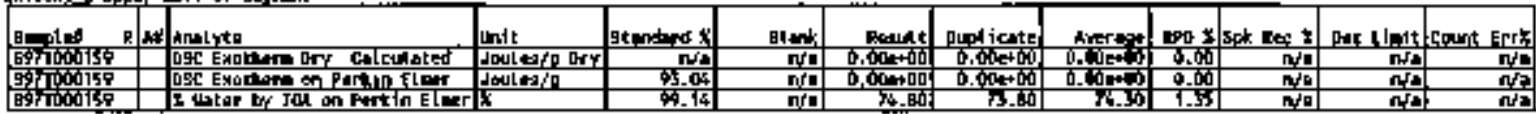

\begin{tabular}{|c|c|c|c|c|c|c|c|c|c|c|c|c|}
\hline tanglet 月 & net & Anelrt! & 血至 & Btandend $x$ & Blank & Resux & Deplacote & Awerage & $60 \pm$ & Sak $\operatorname{Rec} 2$ & ant Lingt & Coumit Err̈̈ \\
\hline 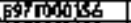 & & 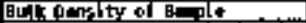 & git & $n$ & n'0 & 1.26 & $\mathrm{n} / \mathrm{a}$ & $\pi / 2$ & $n n^{\prime}=$ & N & $5,000-010$ & $n \sqrt{a}$ \\
\hline Bgrlonots. & & 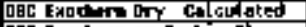 & Joulesta ory & na & nfol & 6.060.0. & D. bueton & 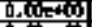 & 5.00 & rida & n/a & $\pi / 2$ \\
\hline 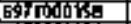 & & 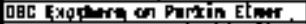 & doule/r & 5,64 & n'a & D. & D, 而血e & D. & क西页 & nin & $n$ & $\pi / \Delta$ \\
\hline 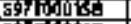 & & 5 water by The on Rertin Iner & $x$ & क, 14 & n'a & $\sqrt{1.27}$ & H.63 & 3,04 & 2.15 & riv' & $n \sqrt{9}$ & 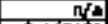 \\
\hline 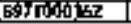 & $\mathbf{F}$ & Alperef of preted solid & ICI/ & EAs? & 4.5.5n-00 & G.TSO-DR & 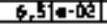 & 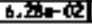 & Fe & 3.44 & F贯 & 1.44E+ \\
\hline
\end{tabular}


Toble 3. Data prangy toblo

$\uparrow=194$

cont mutata 18

tEoturis:

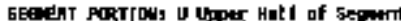

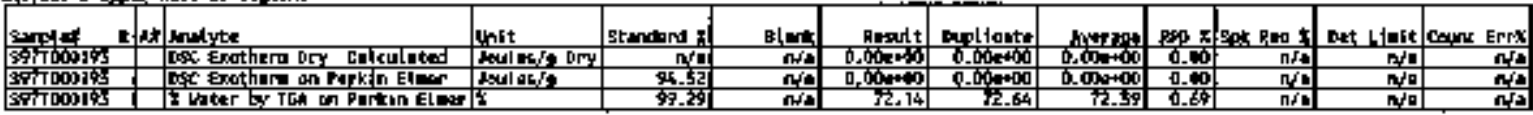

\begin{tabular}{|c|c|c|c|c|c|c|c|c|c|c|c|}
\hline gardef & Af Aralyte & Unit & Standind $t$ & Blm & Besoult & waterte & Anereot & $60 x$ & 月世 $\mathbf{I}$ & bet Linit & $\operatorname{Con}$ Errix \\
\hline 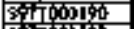 & Eulk Denstry of singlt & 9t5 & $\begin{array}{ll}\mathrm{N}^{\prime} \mathrm{g} \\
\end{array}$ & $n$ & 1.250 & & & $n f$ & $n \rightarrow$ & 3.006-01 & $\mathrm{n}$ \\
\hline 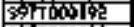 & Ex, Exothern ony calculnted & sulesto ory & nftu & $n / 2$ & $0.0,0.406$ & 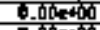 & $\frac{D}{10}=0$ & 0.00 & $n$ nes & n/प & صلى \\
\hline 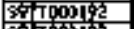 & 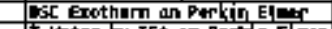 & 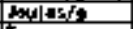 & & $n$ & $0.060^{3}+0$ & 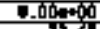 & $6.00=0$ & 0,06 & 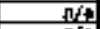 & $n \sqrt{n}$ & ה \\
\hline & 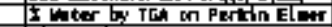 & & 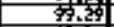 & $n / \sqrt{2}$ & 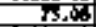 & 12,3 & 恼. & 6.59 & nIf & & n's \\
\hline Sक्र $0001 \%$ & 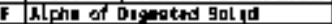 & usto & & -7.641-63) & $3,6,0,03$ & 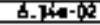 & E.TIn-(T) & & 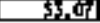 & 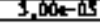 & LESEROA] \\
\hline
\end{tabular}

象 
14F-5C-14-09-239. REV, 0

CHAIN Of CUSTCOr FORMS 
HOAF-SD-LN-DP-239, REV, O

THIS PASE TNTEATIONALLY LEFT BLANK

58 


\section{COPY}

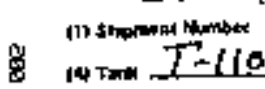

crots

CHAIN-OF-CUSTOOY AECOAD FOA COAE SAMAPEING

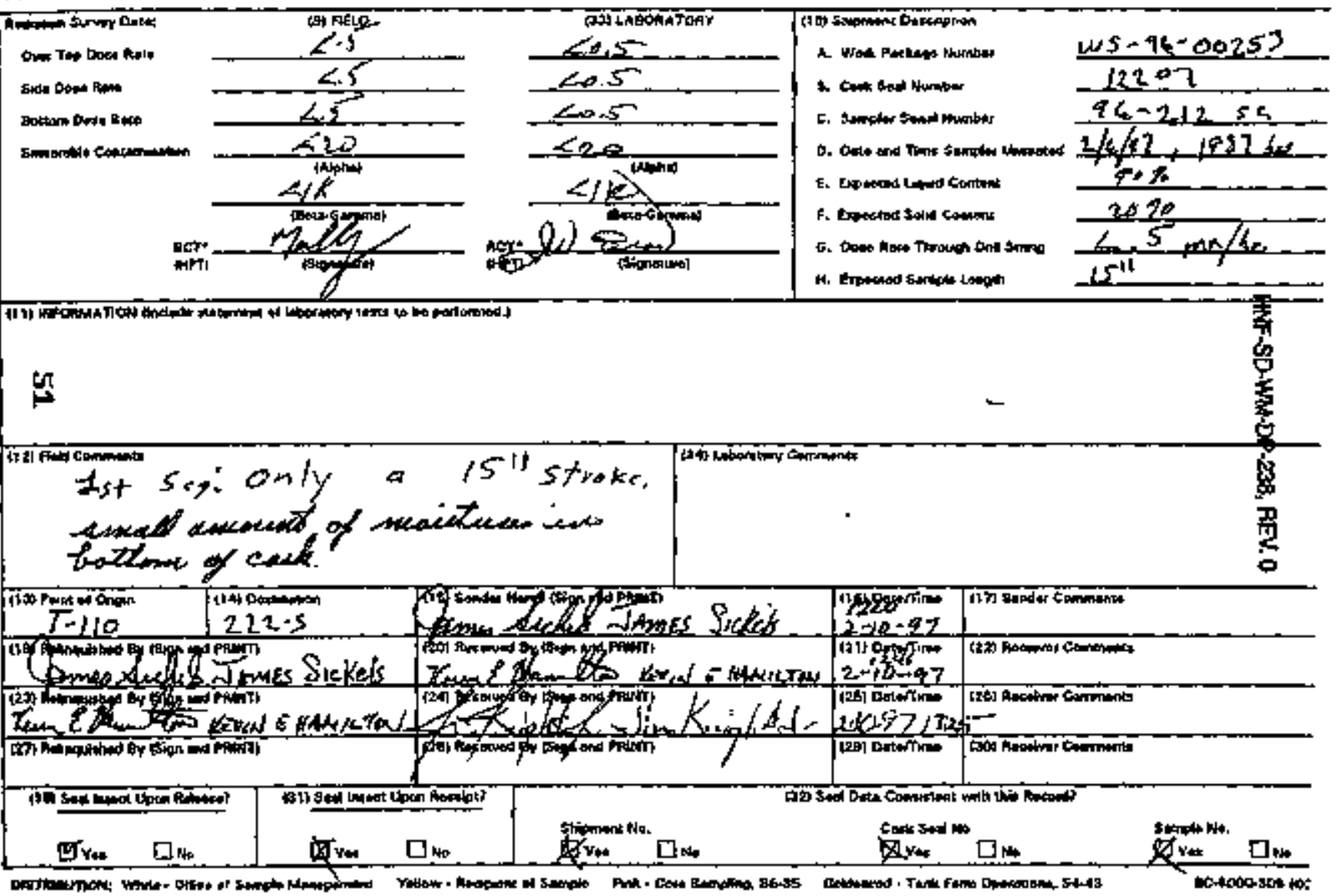




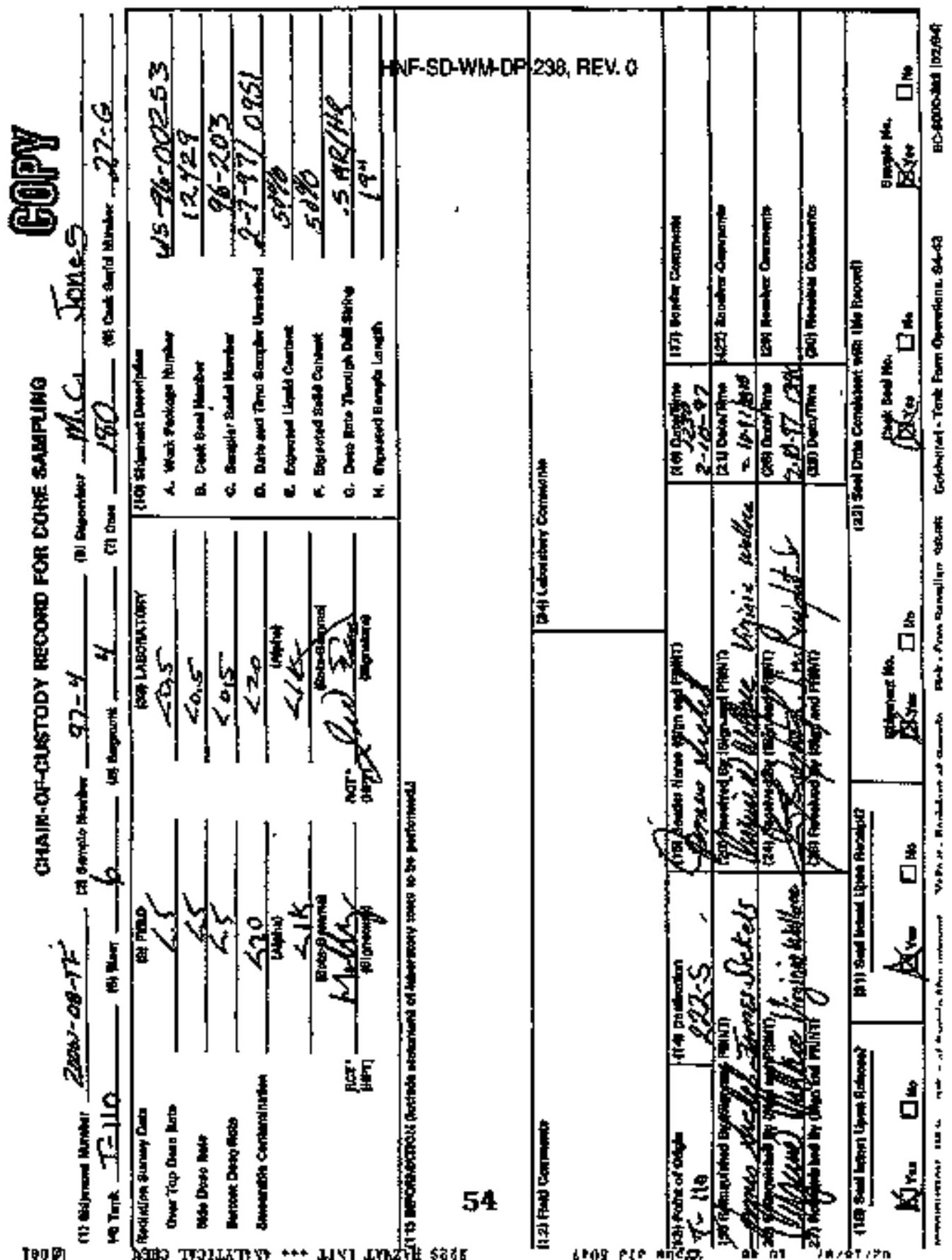




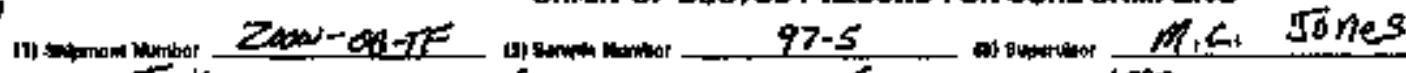

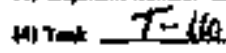

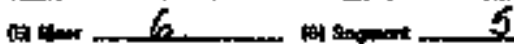

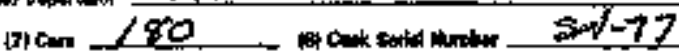

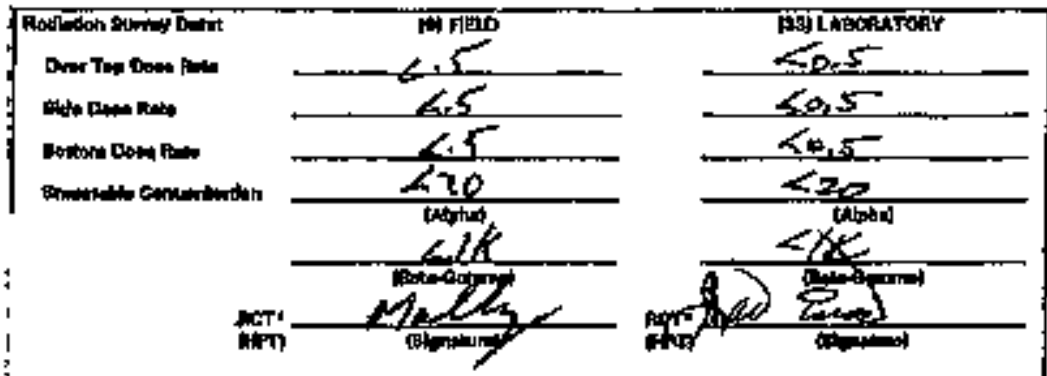

it 1)

t1

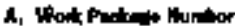

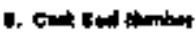

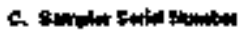

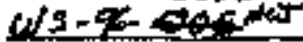

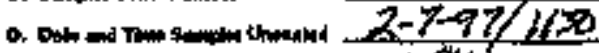

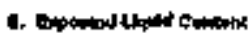

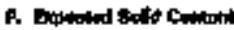

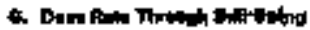

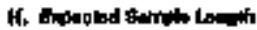

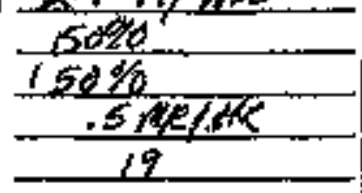

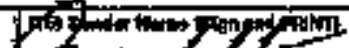

2 त 12425

$87-20$

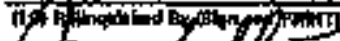

F, C L t

Vertiont giakes

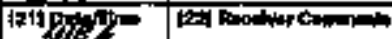

$\Rightarrow$ mbentals

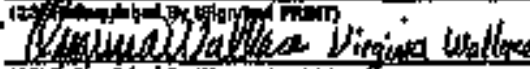
|zT) if

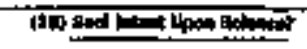

S

口品
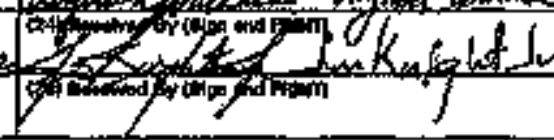

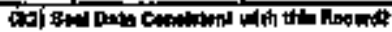




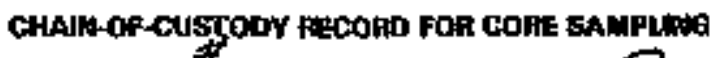

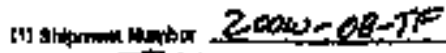

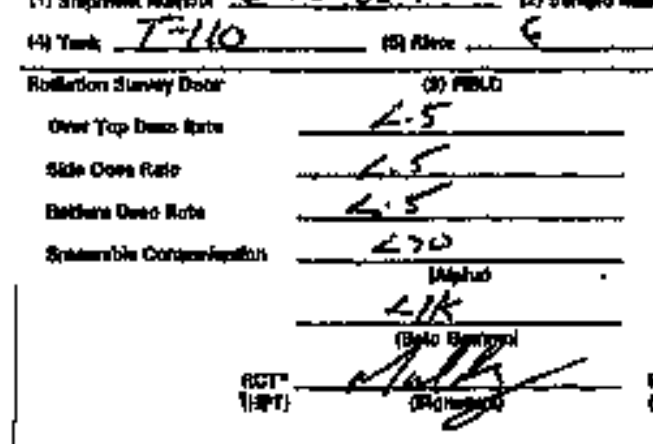

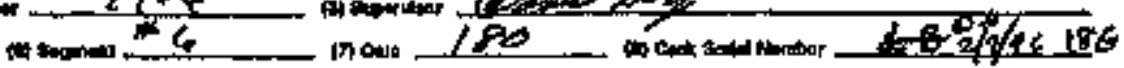

a

\section{ROPPY} a F

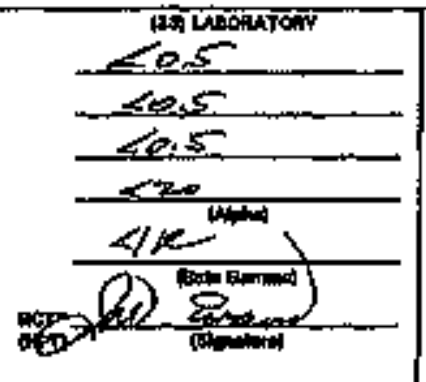

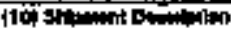

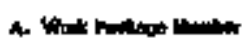

B. Cret gand humpor

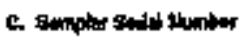

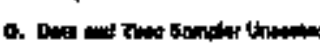

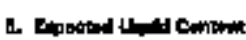

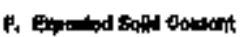

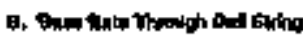

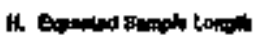

\section{$4 \sin 6,-00253$} 12283

$26-201$

$2 / 1 / \alpha^{2} / 2 \theta_{A+\infty}$

-.

$10 \%$

$\leqslant 5 \max$ $-12^{4}$

sto

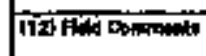

$$
\text { 3. }
$$

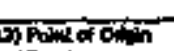

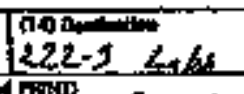

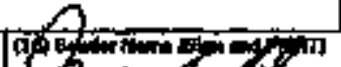

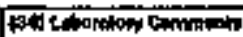

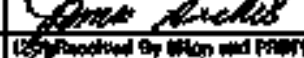

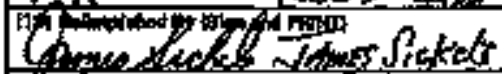
(1)

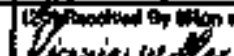
Finity

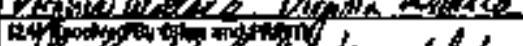

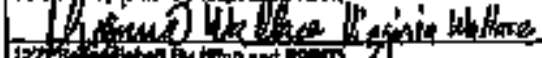
Fation

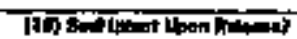

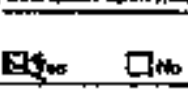
Fat P.

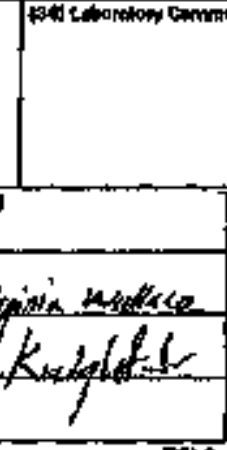

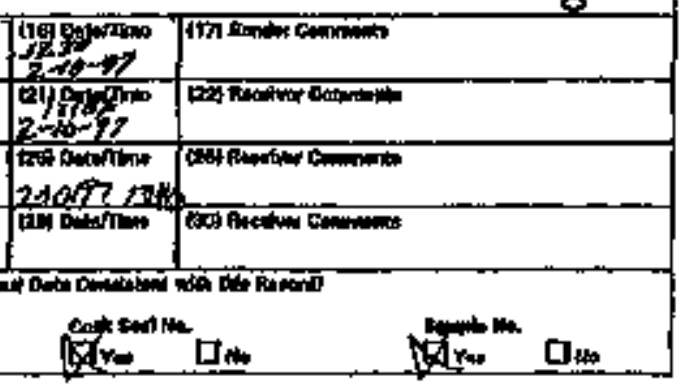

axe 

$T+110$

.
CHAIN-OF-CUSTODY RECOAO FOA CORE SAMUL, INO

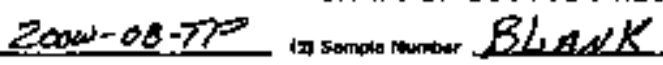

in sospent Blowik

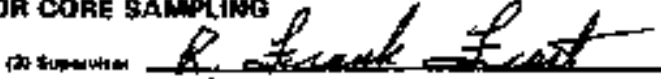

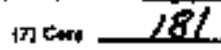

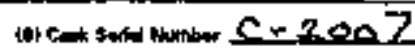

I

constand

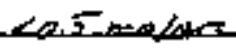

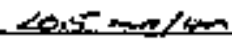

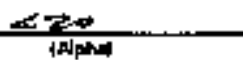

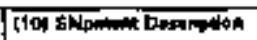

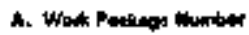

B. Gant sal Number

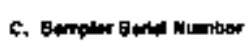

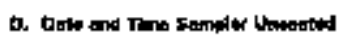

ws $-9 h-00252$

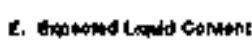

$\frac{4 / k}{C+1 / 2+2}$

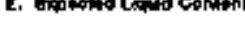

i 1179

F. Eppoted Bopd comint

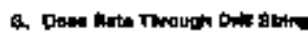

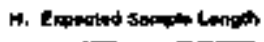

$96-213$

2-3-97 60190

$-100 \%$

$-0 \%$

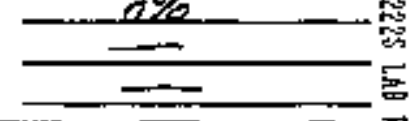

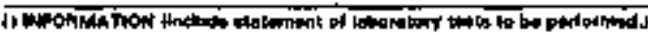

$$
\text { is }
$$

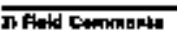

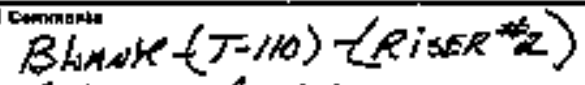

deioniged $\mathrm{H}_{2} \mathrm{~S}$

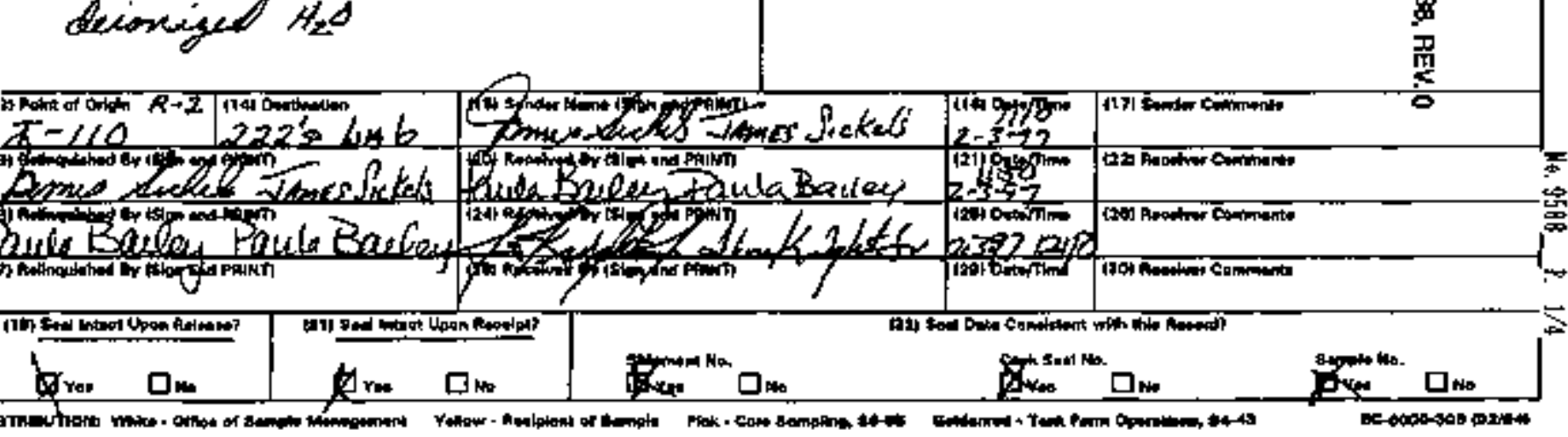




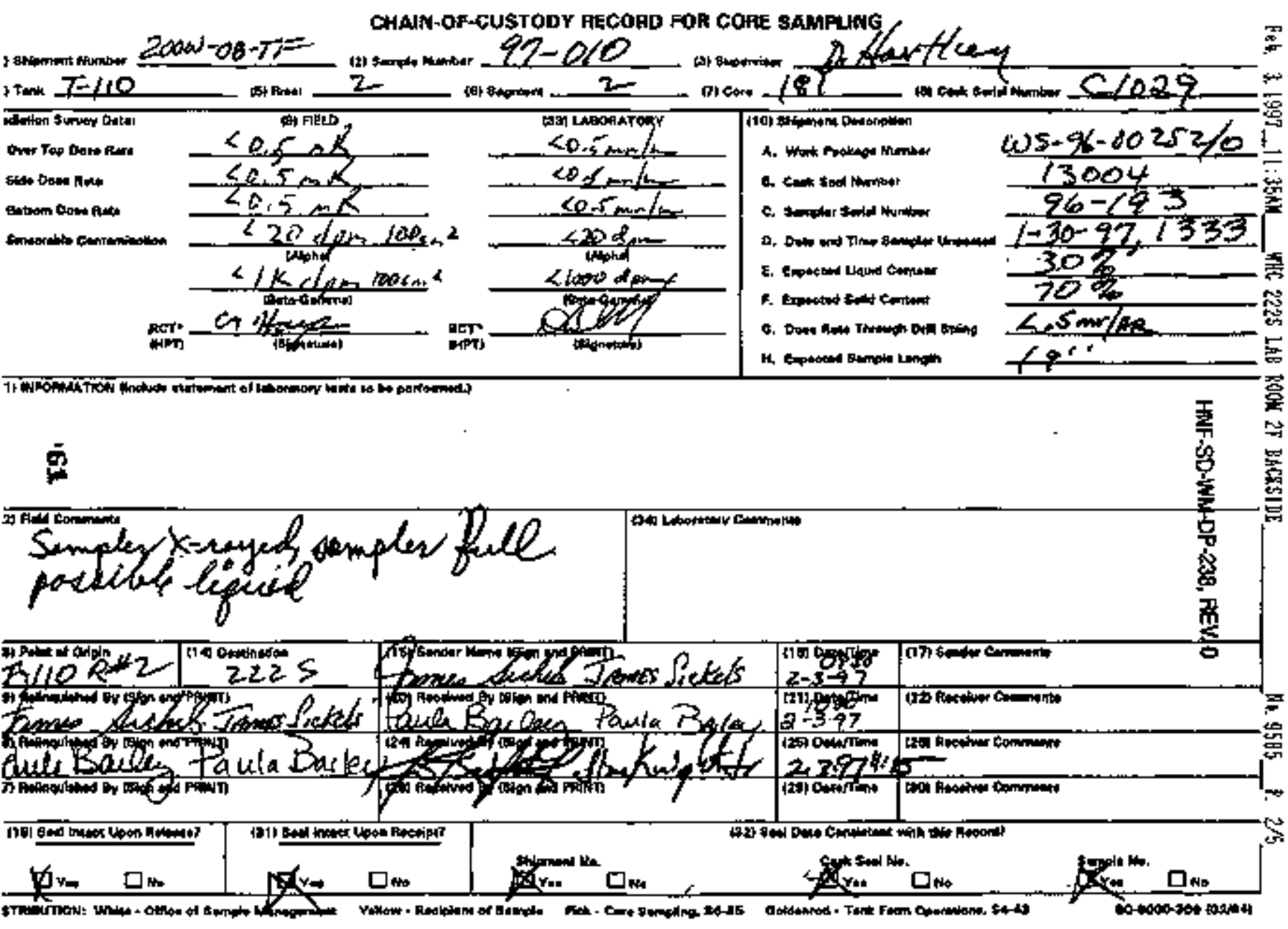




\section{CHAIM-OF-CUSTODY RECORD FOR COFE SAMPLEG}

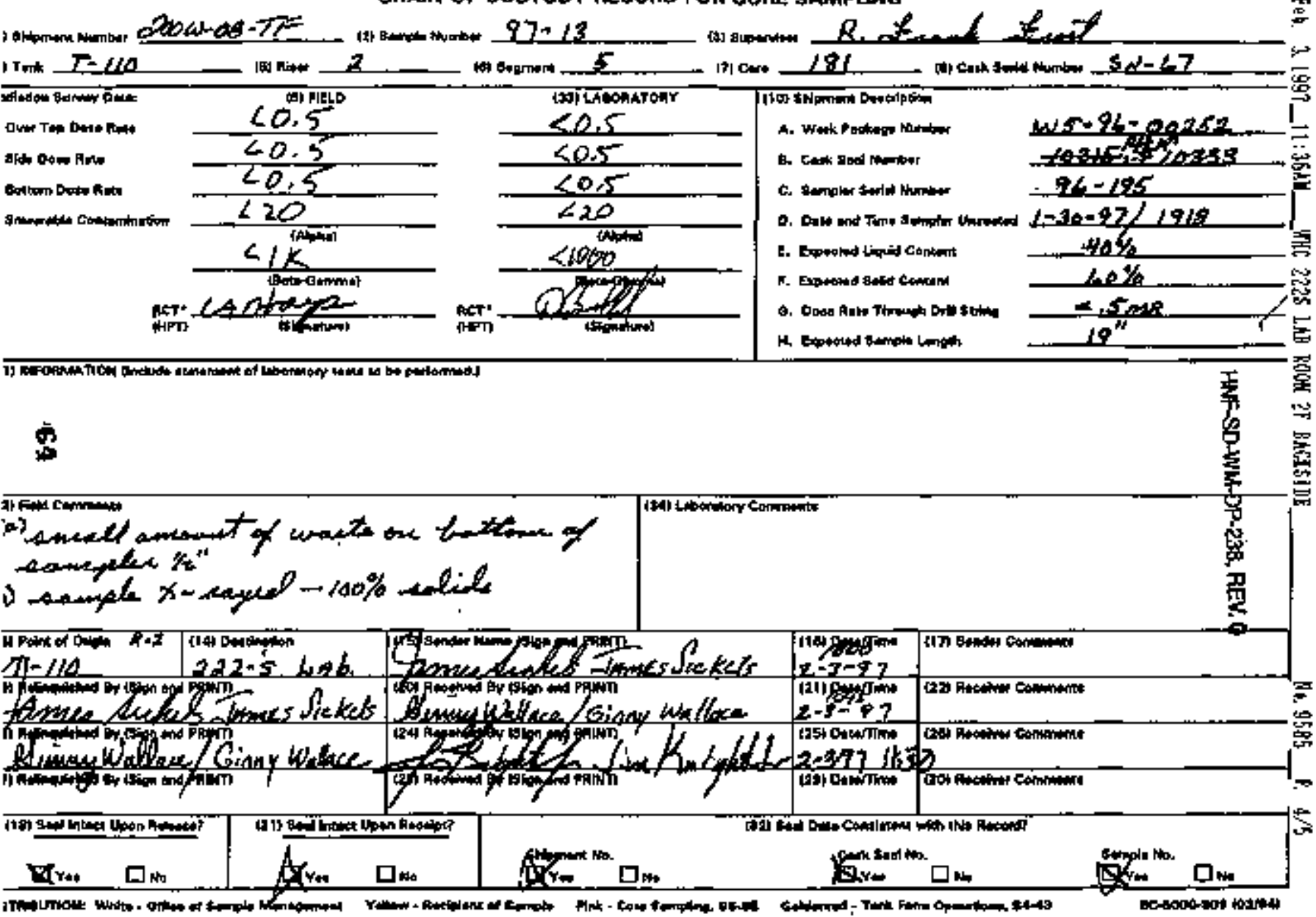




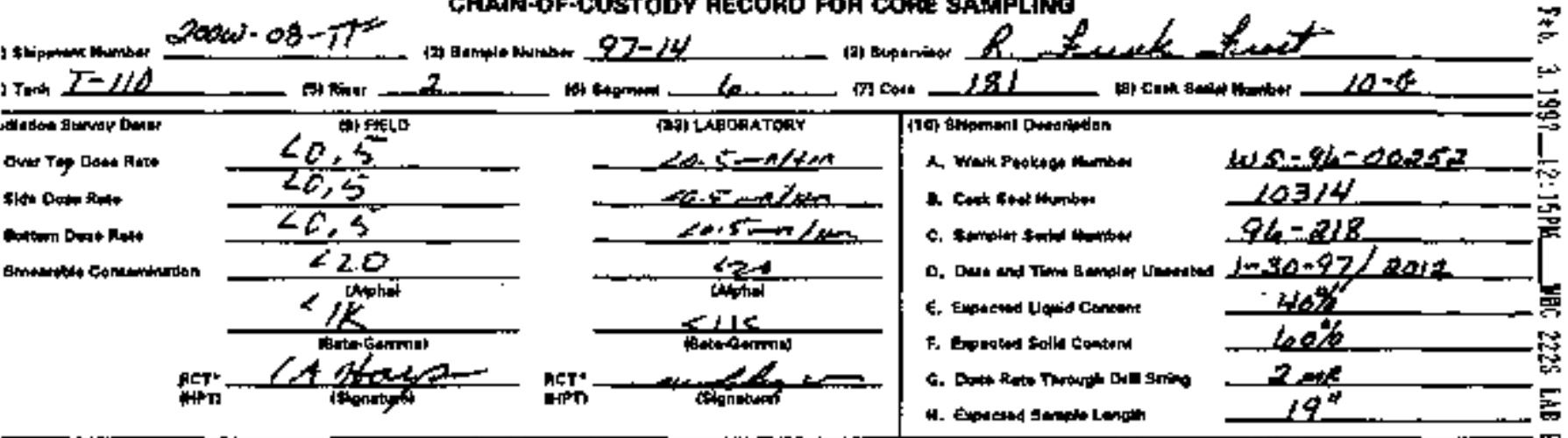

\begin{tabular}{|c|c|}
\hline 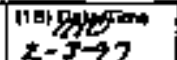 & 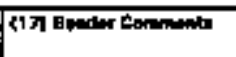 \\
\hline $\begin{array}{l}211 \text { Detwitim } \\
2-5 \rightarrow 7\end{array}$ & 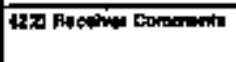 \\
\hline 2,32124$)$ & 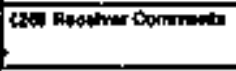 \\
\hline 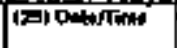 & 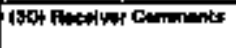 \\
\hline
\end{tabular}

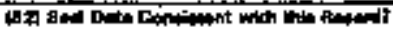

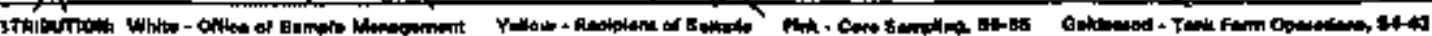

Boworon.

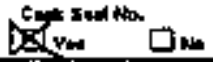




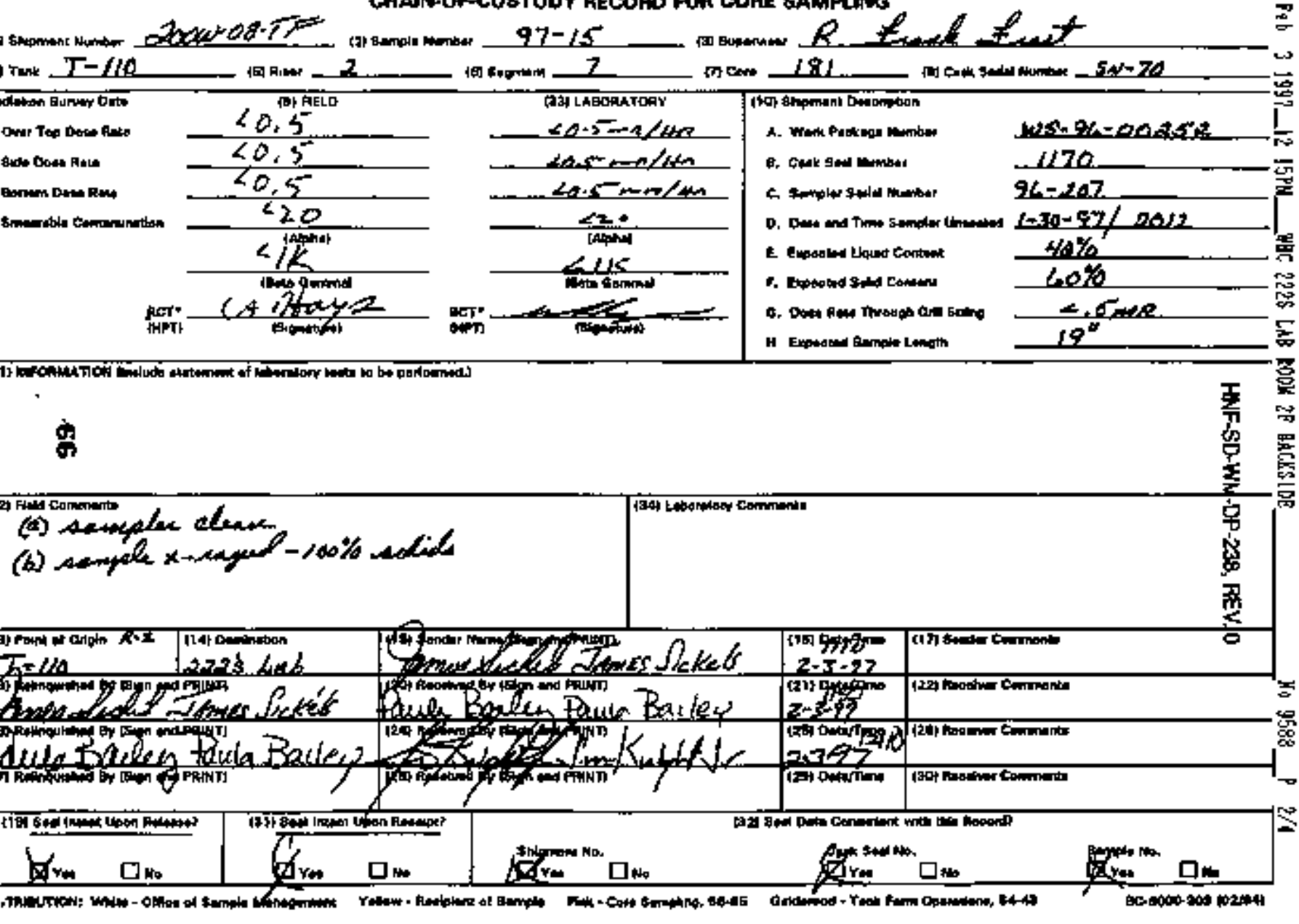


HWF - SD-LA-DP-238, REY, O

PHOTUERAPHS

IBS 
HEF.5D-1W.DP.239, REV. O

TAIS PAGE INTERTIONALLY LEFT BLNX 
HNF-SD-WW-DP-238, REV O

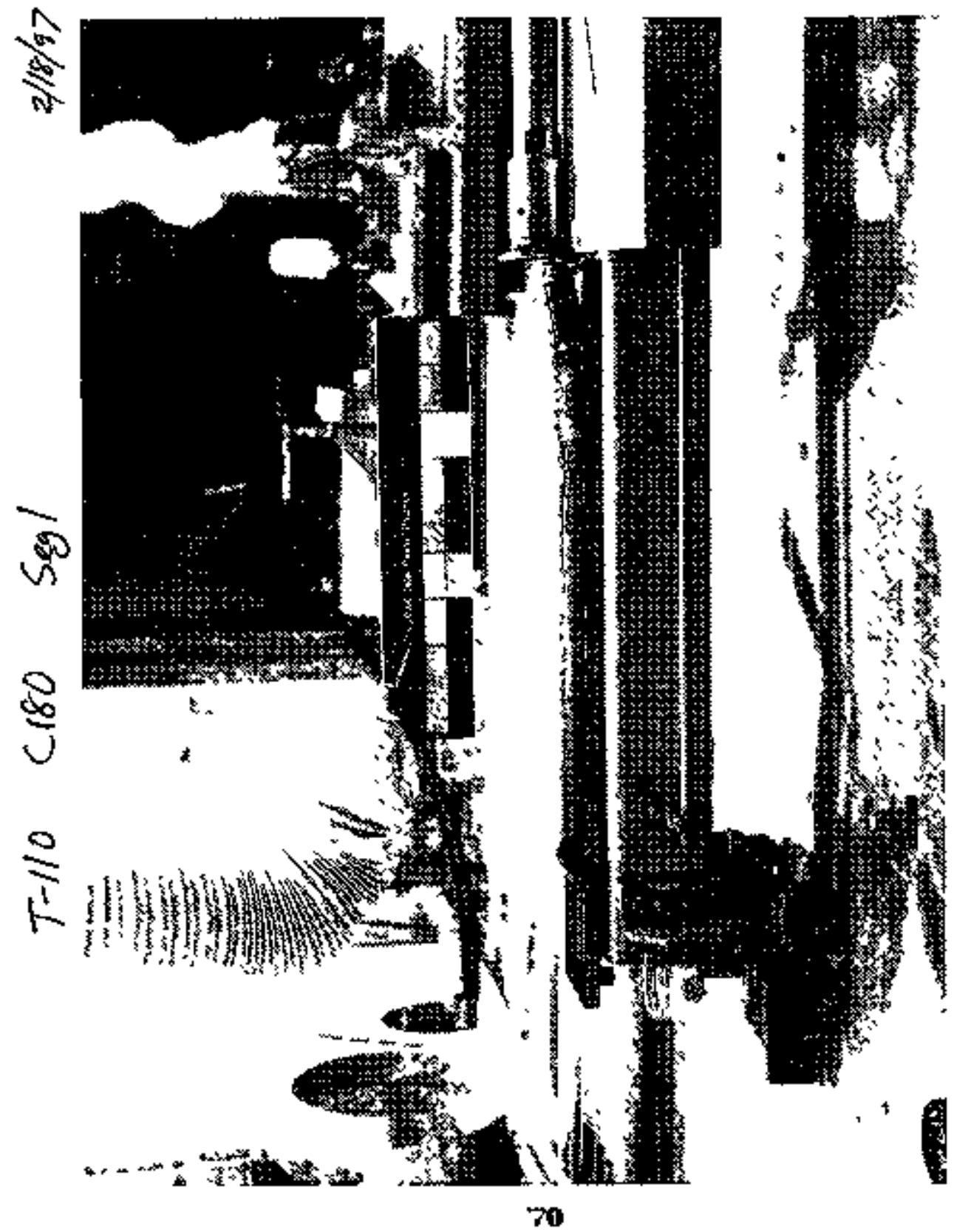




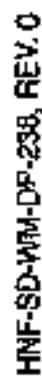

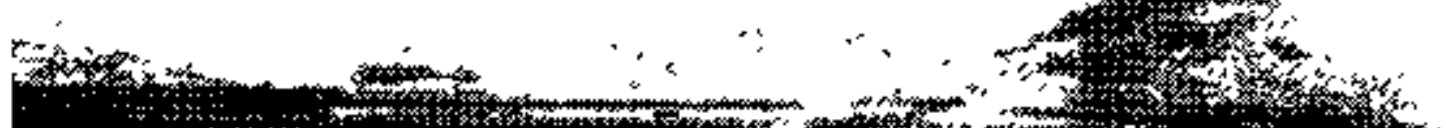

.
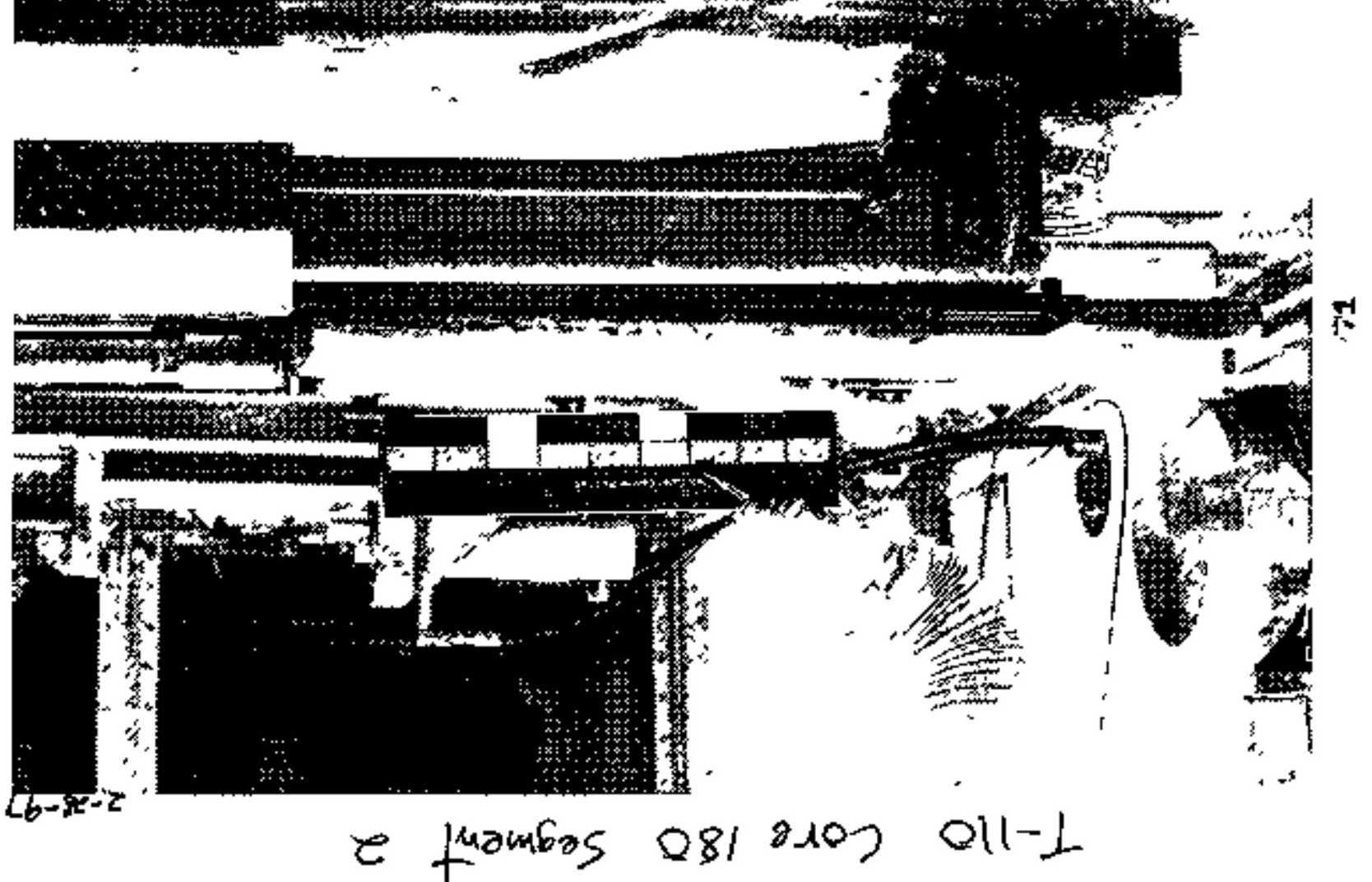


\section{$\frac{1}{2}$}
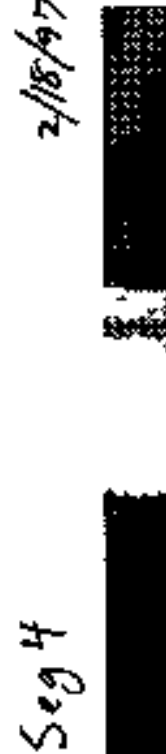

$\stackrel{8}{v}$

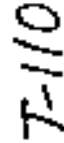

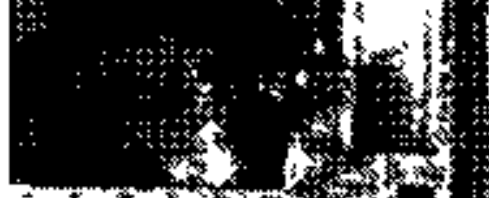

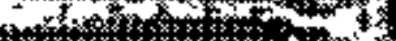

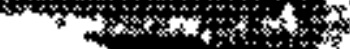

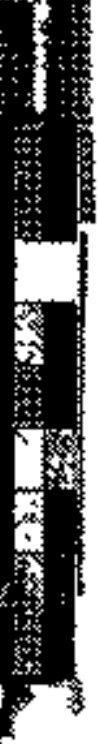

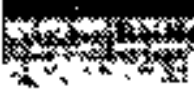

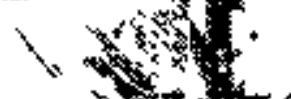

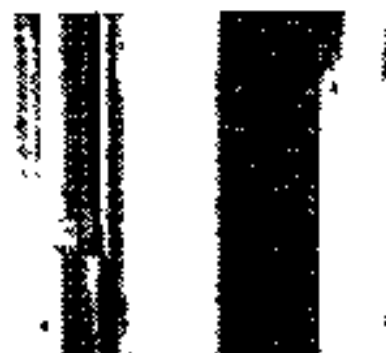

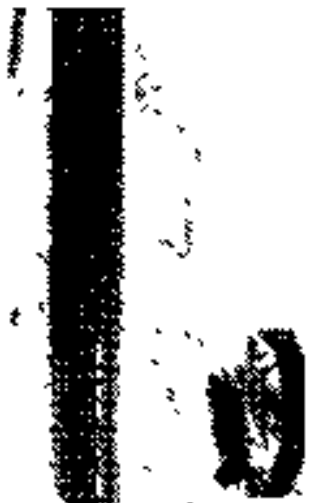

8
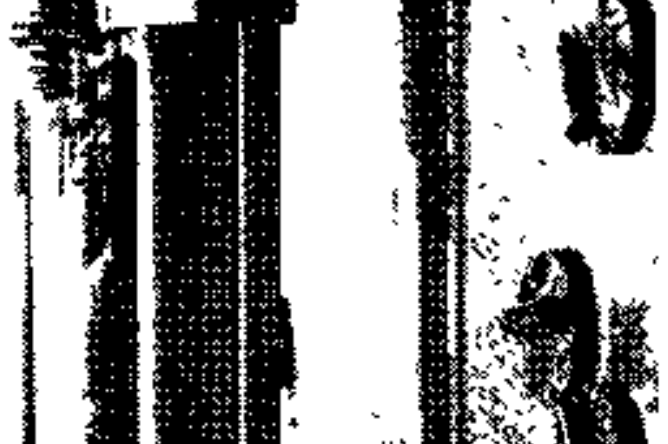

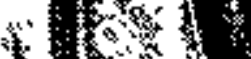
Ax 列 $3 \times 3=8+2$ 12\% ?. 3.x. $x$ istion

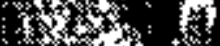

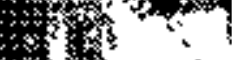

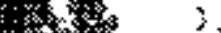

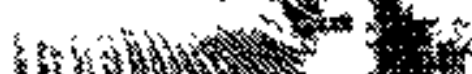

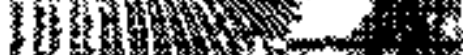

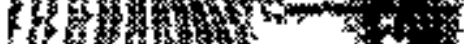
(3)

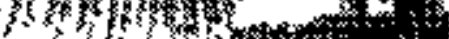

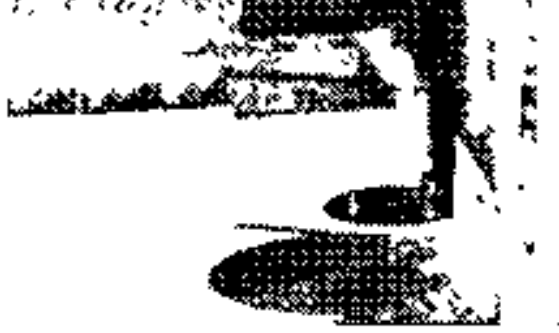


HNNF-ST-WM:-DD-239, REV. O

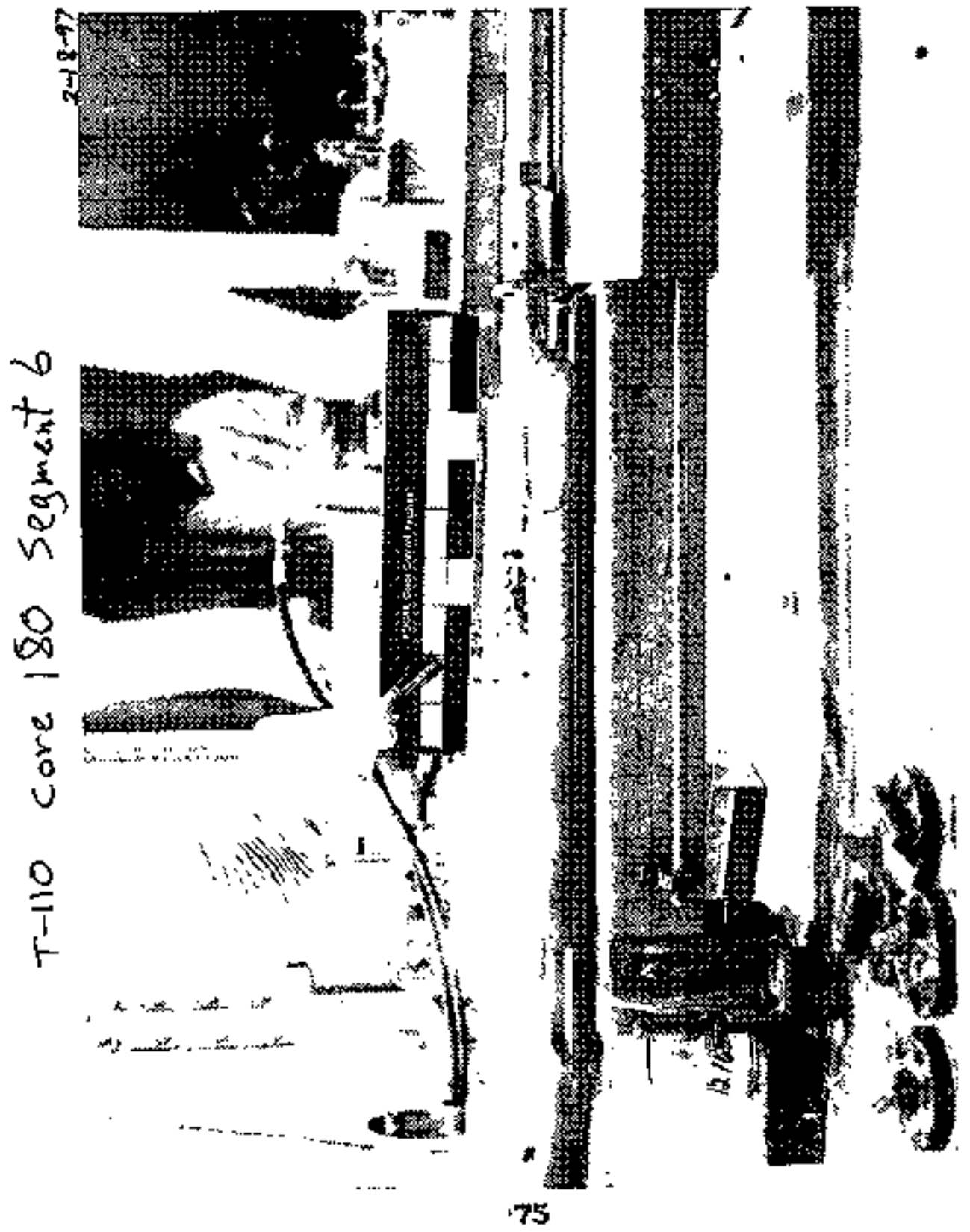


HNF-SD Wh-DP-23B, REV,O

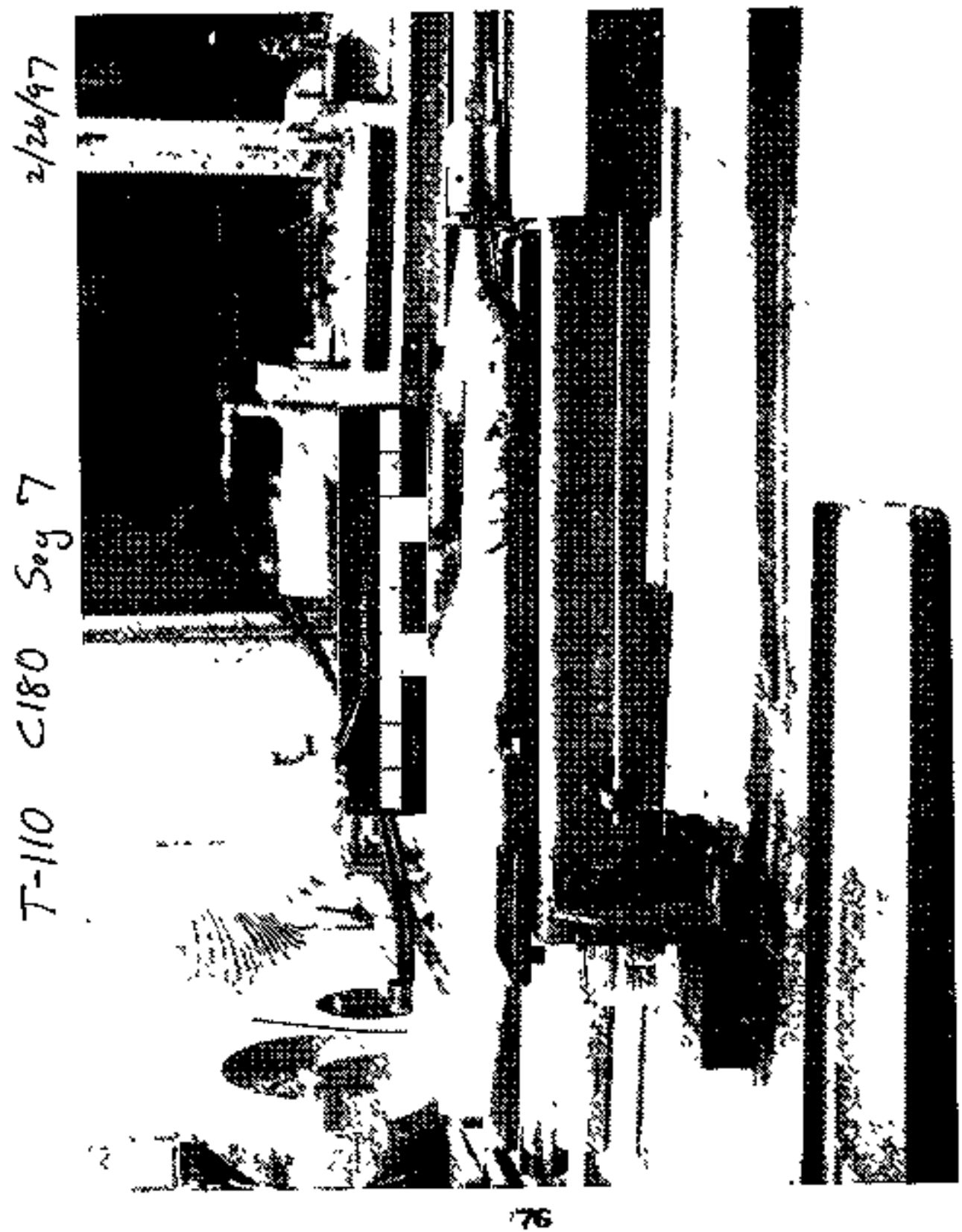




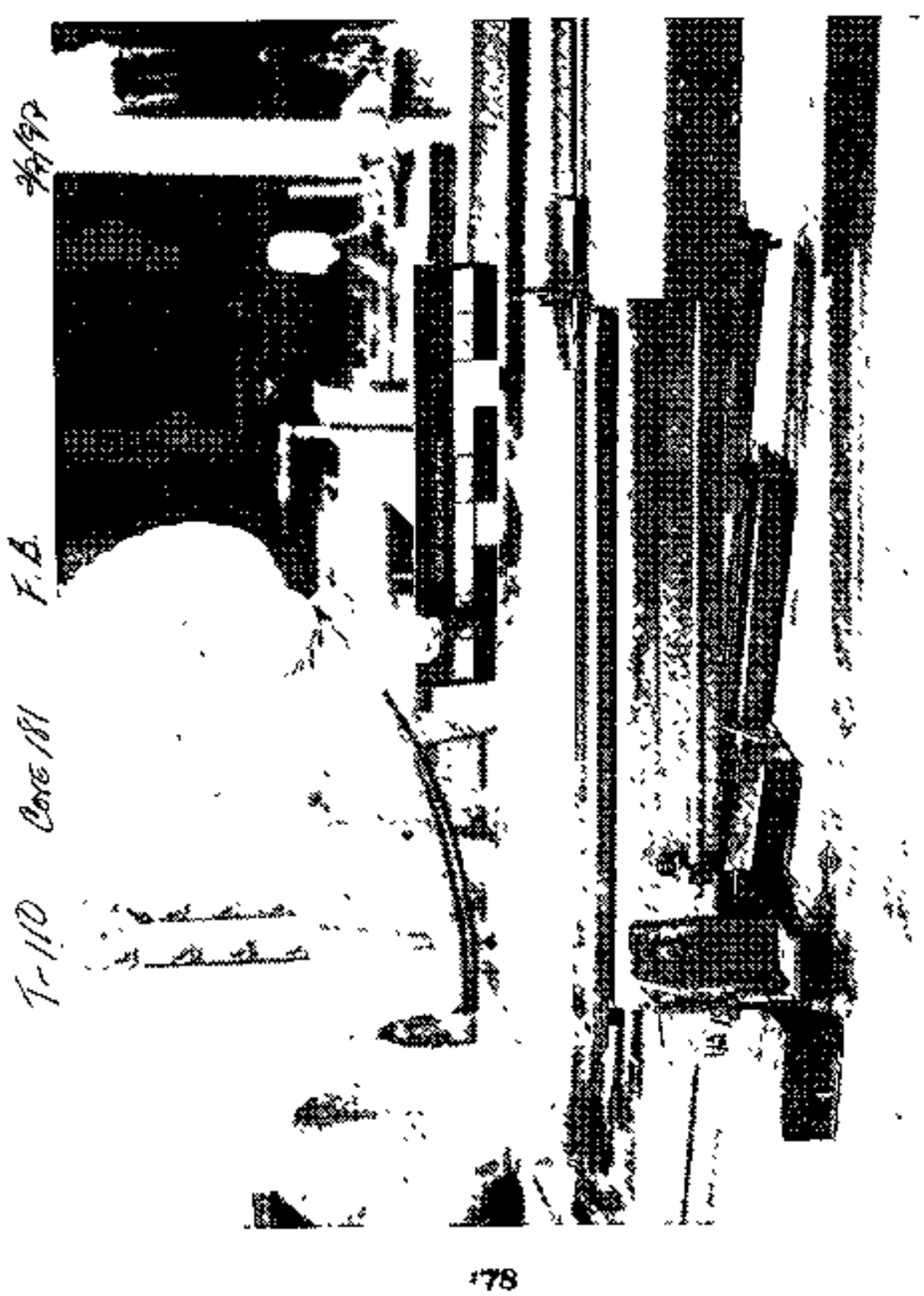


HNF-SD-WIM-DP-2S8, FEV. O

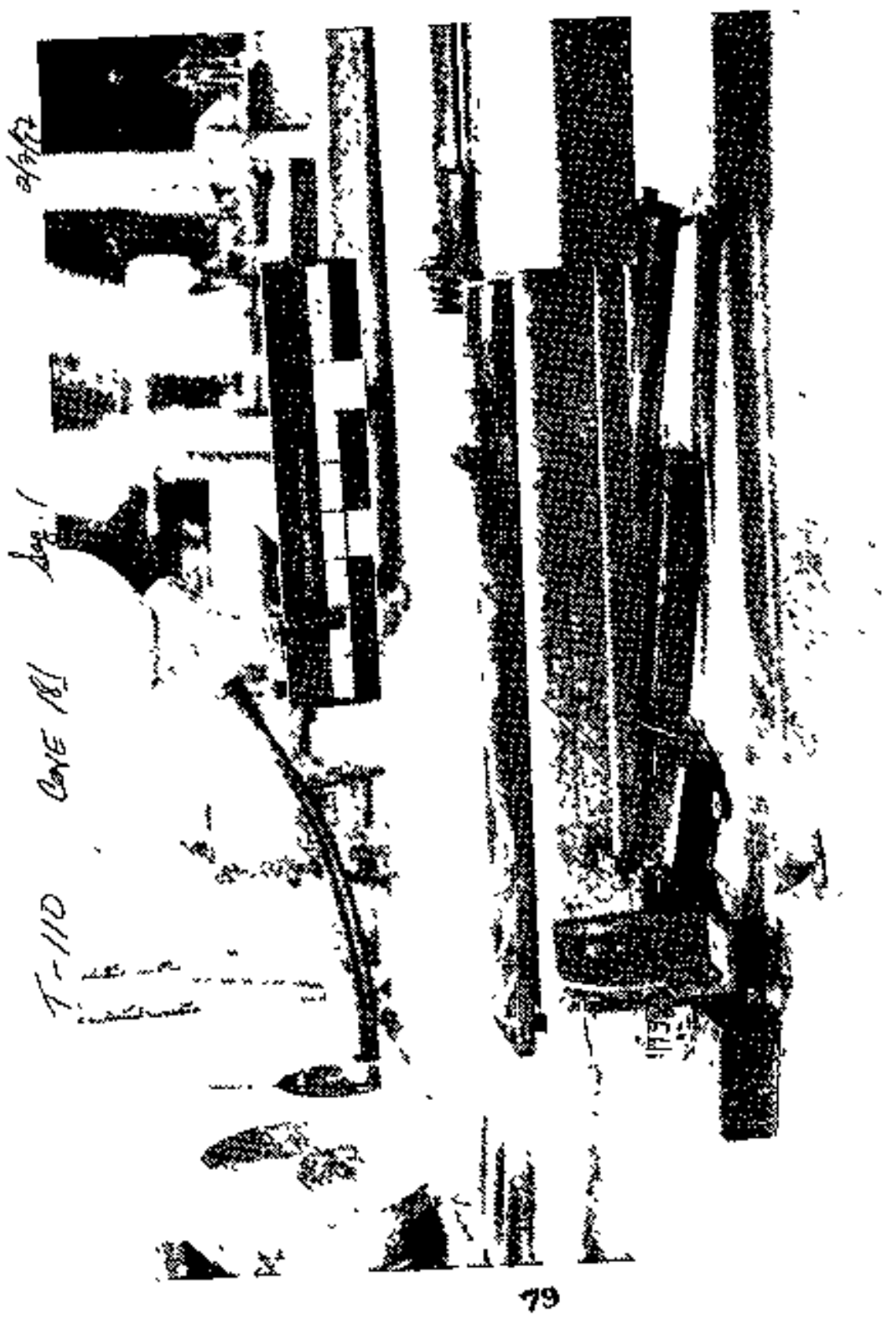


HNF SD-WH-OP-23日, REV.O

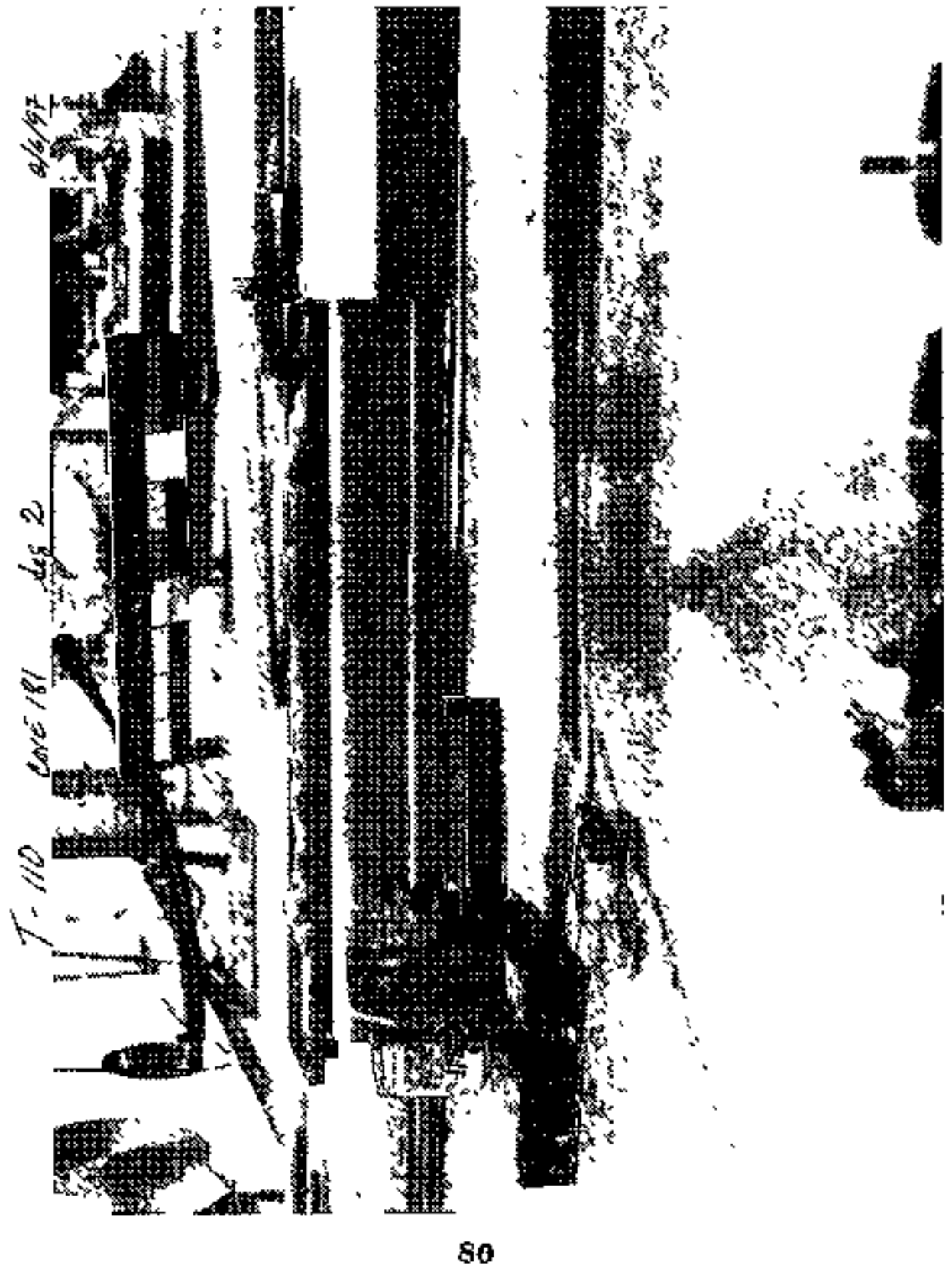


HNF-SD-WWA-DP-23B, REV. 0

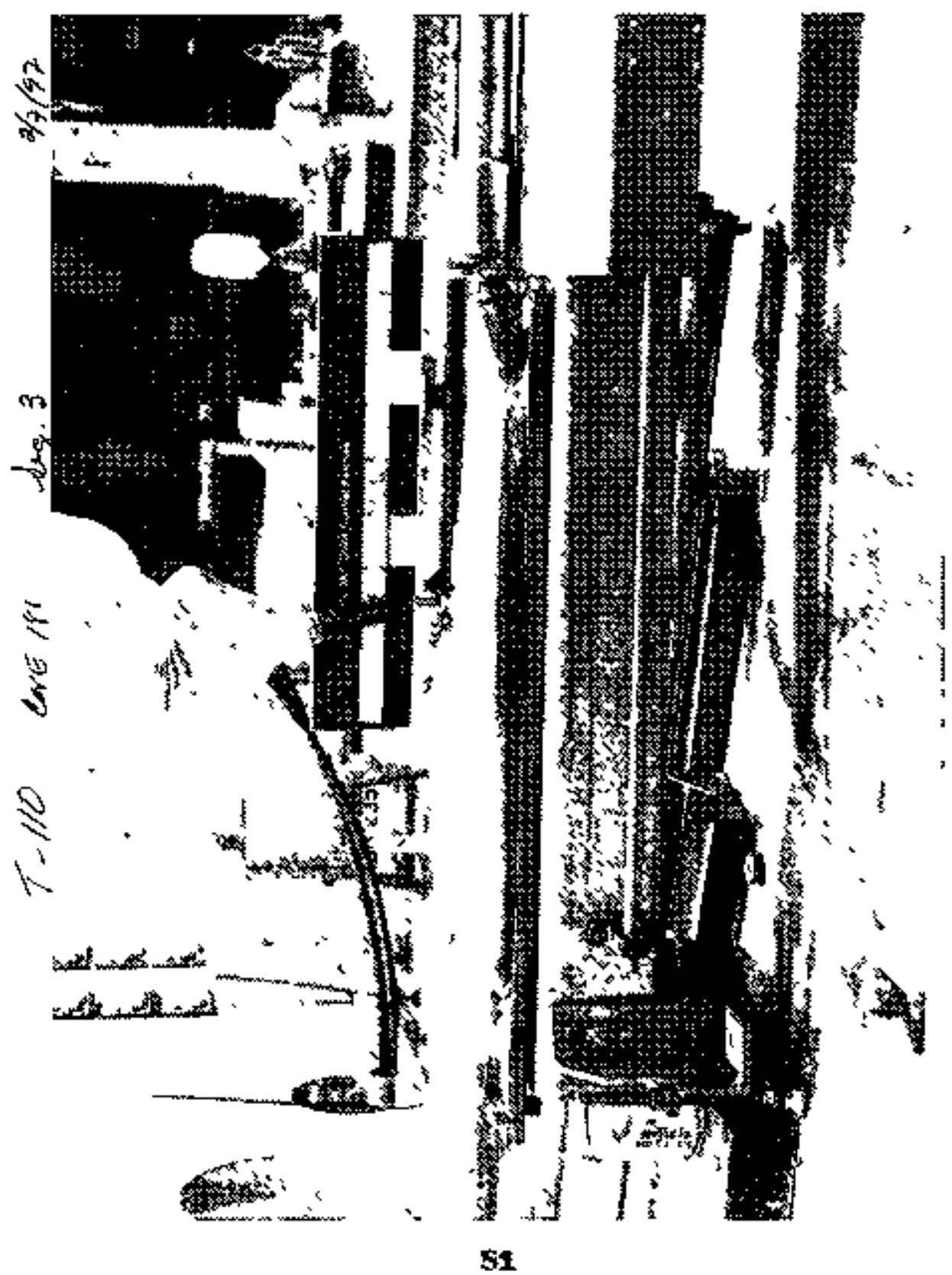


HNF-SR,WL DP-238, REV 0

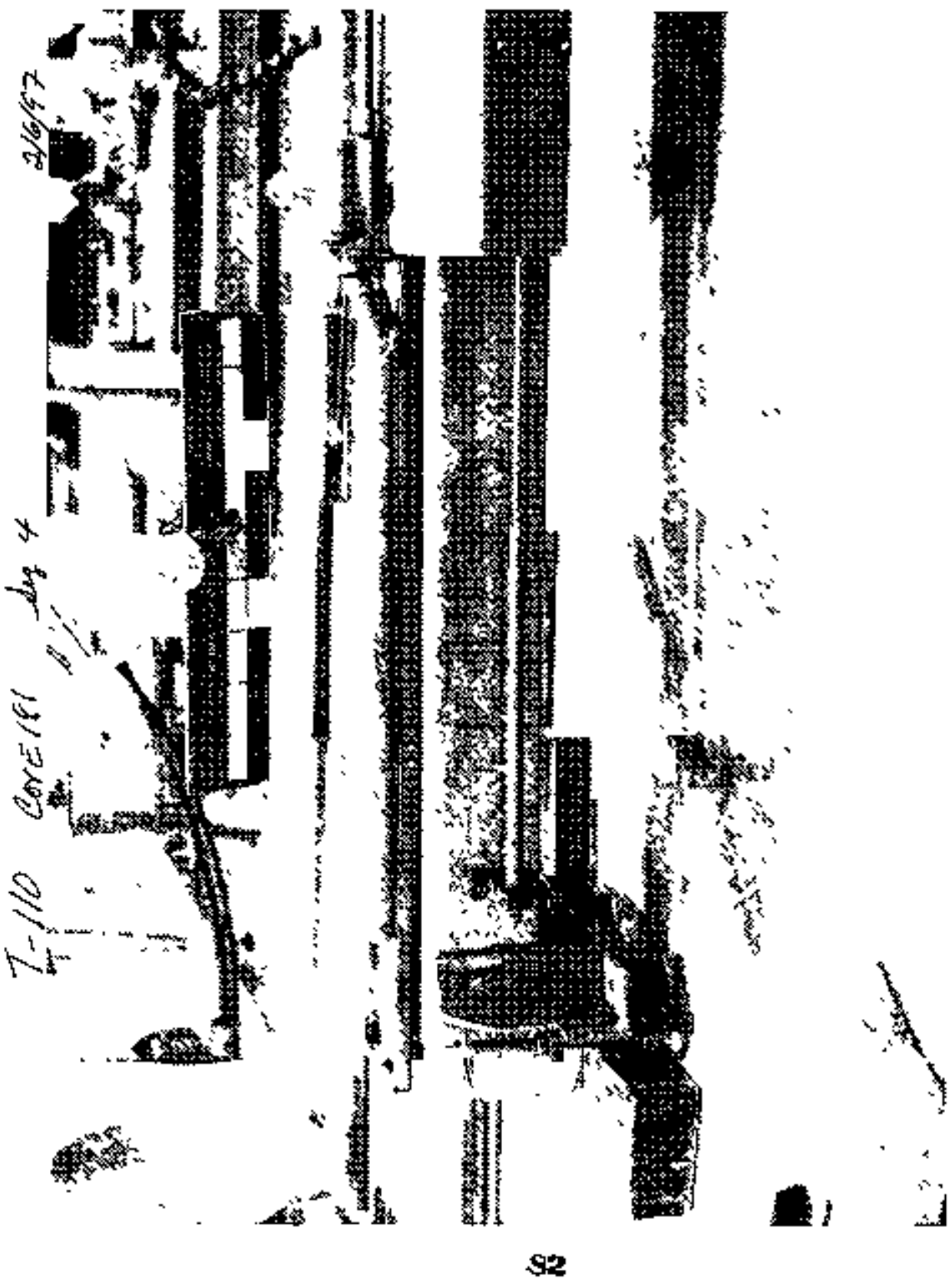




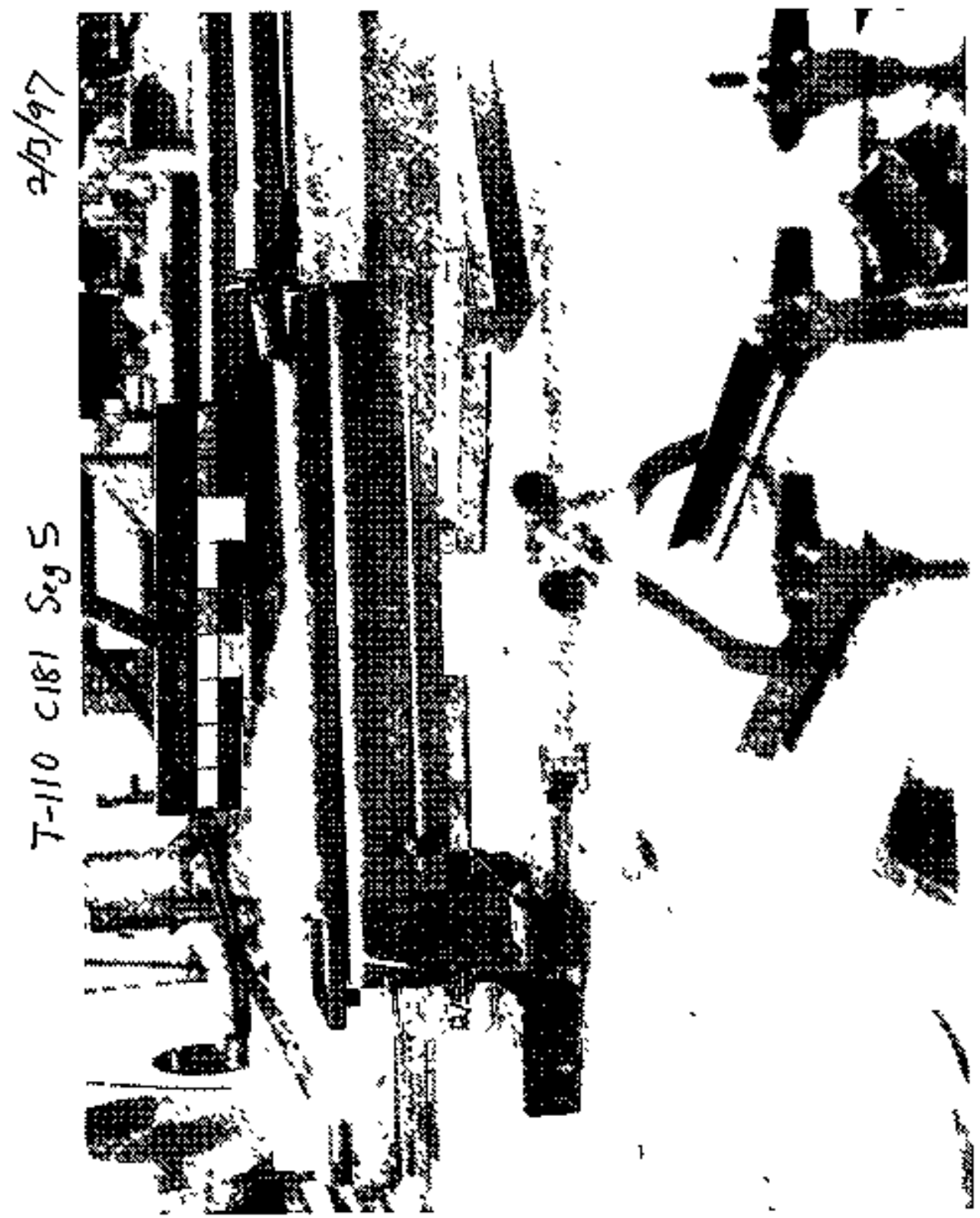



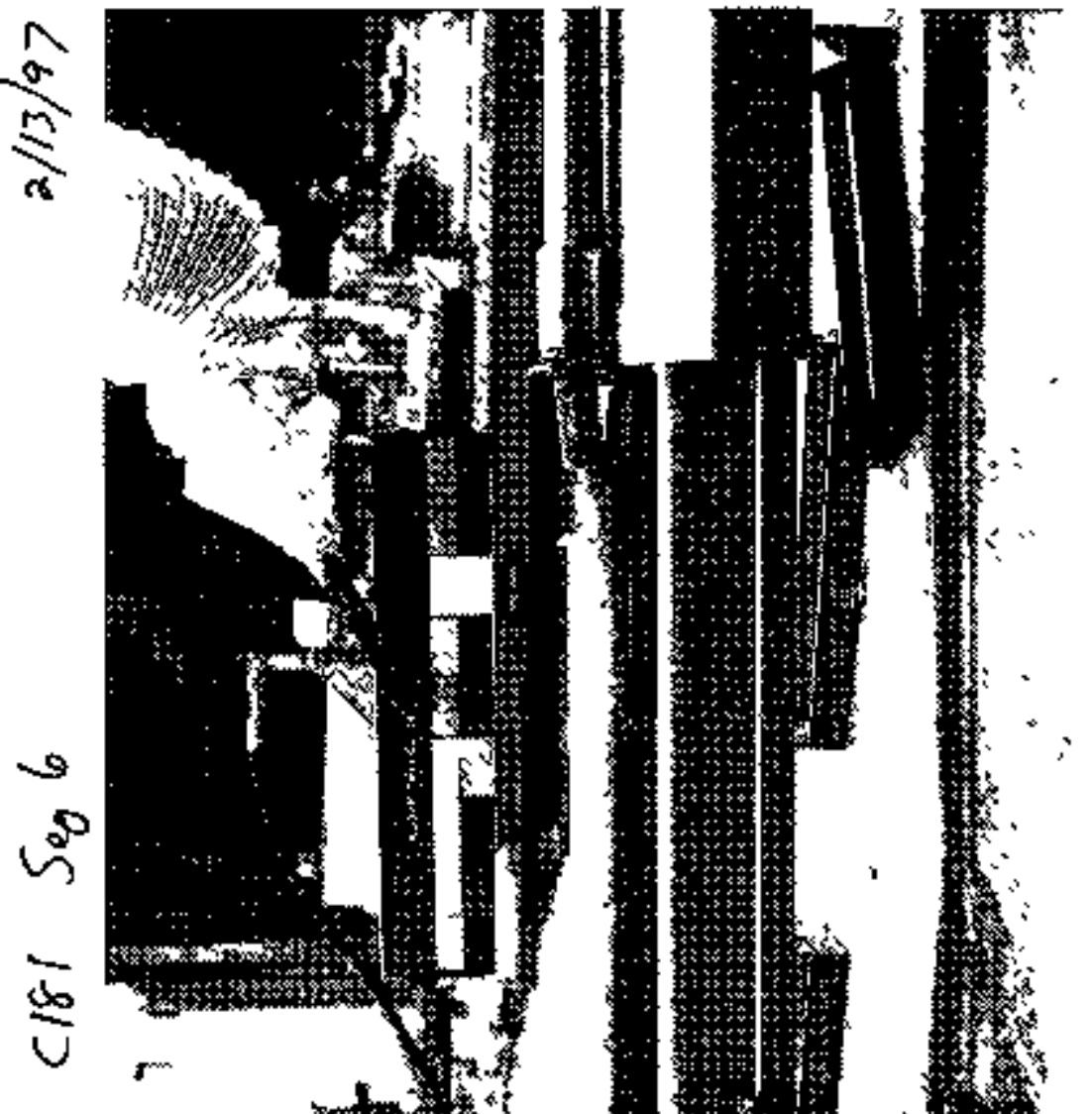

$\stackrel{9}{5}$
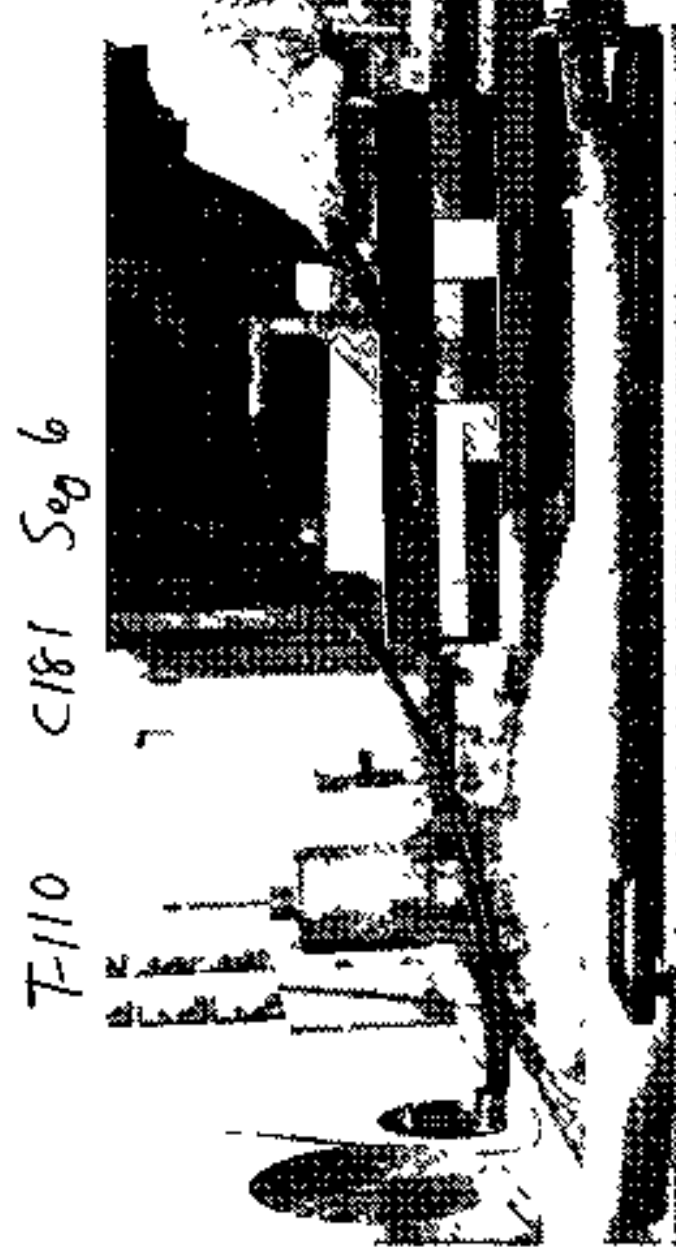
HWSF-SO-WHA-DP-23B, FEV. O

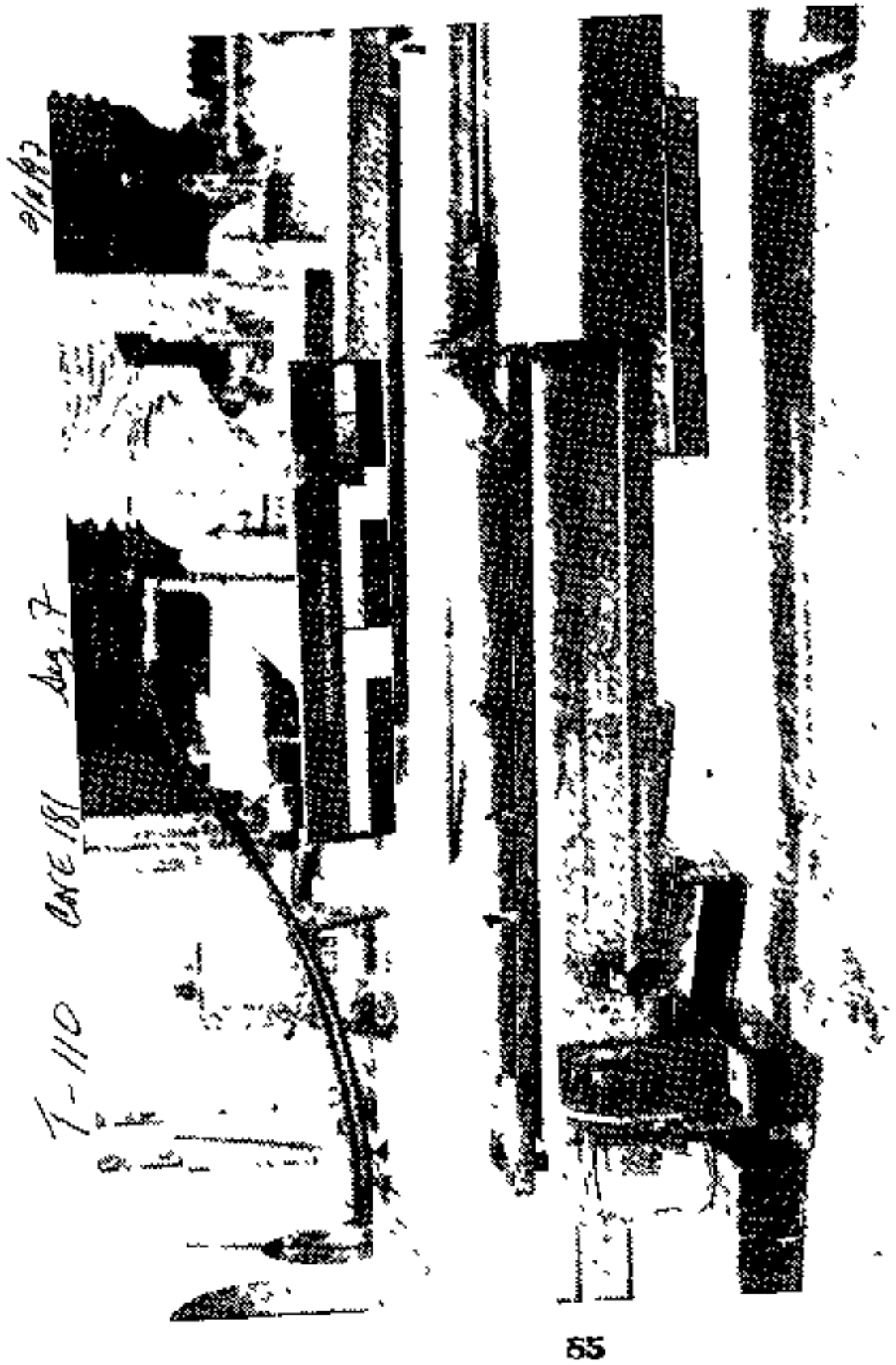




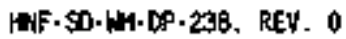

BULK OEASITY WORKSEETS 
HKF-SD-MA-OP-238, REE, O

THIS PNá IKTEMTOONLLY LEFT BLAN 
Requestor: Foteex

Tank: $T-110$

Core: 180

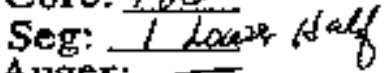

Augex:

Sample ID: 597 Tex0.206

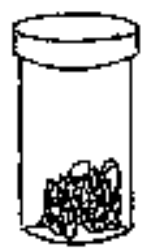

son time

ᄃA Tra

Homegendzation

Trme (Mín)

Jarf: $12 / 55$

Jar/Vial Size: 250 的 Initial Weignt: $g$

Final Weight:

Net Weight: g

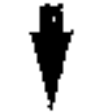

$$
\text { : }
$$

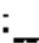

${ }^{8}$ $g$

Conet:

Final Vol: 100 mL Initial Weight 7.43 g Final Weight: 20.360 Net Weight: 1293 Sample ID:

Appearance/Narrative:

12.935 .29

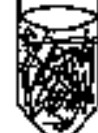

Tank:

Core:

Seg:

Ariger:

Sample ID:

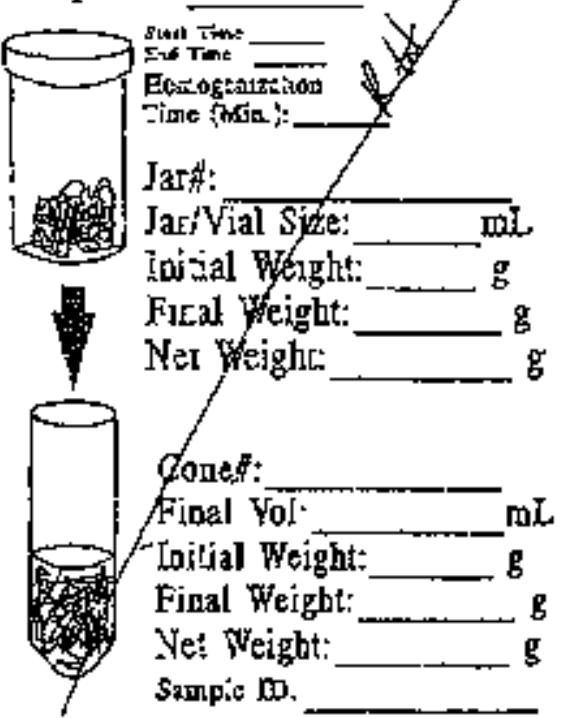

Appearanee/Narrative.
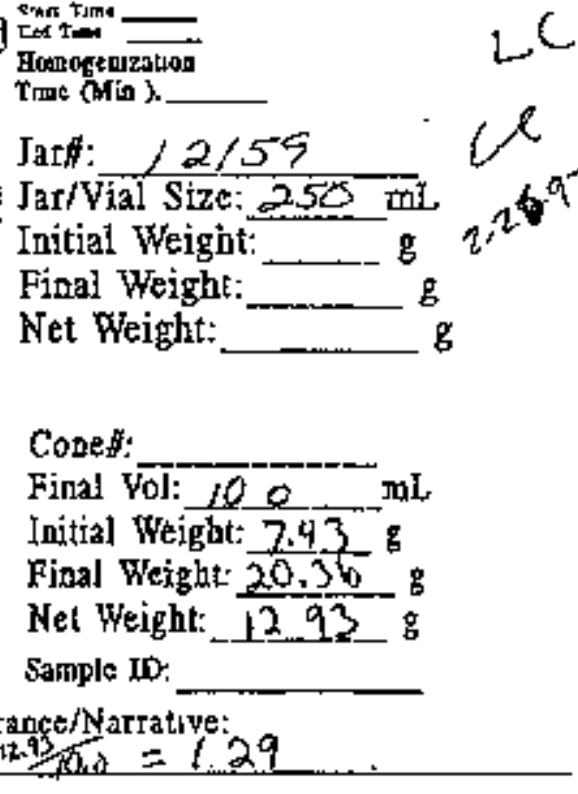
Requestor: 26 oturyutio

Tank: T-LP

Core: 180

Seg: $2 \leq 4$

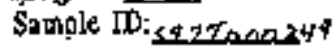

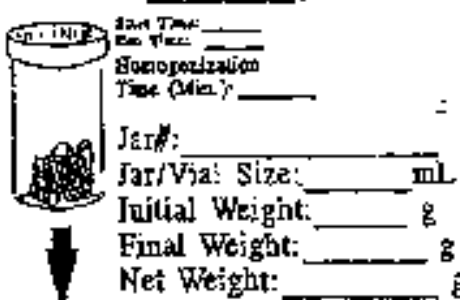

\&

\section{Bulk Density Worksheet}

Date:

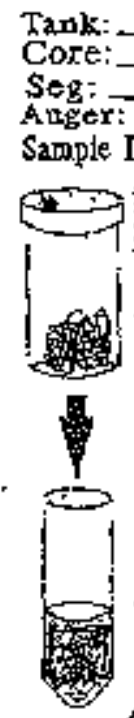

Copte: 60

Fian: Vol: 5

Ixilia! Weght: $y$ st- 5

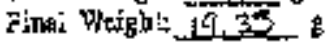

Net Weigit-1L58-

Somnk ID:

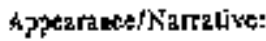
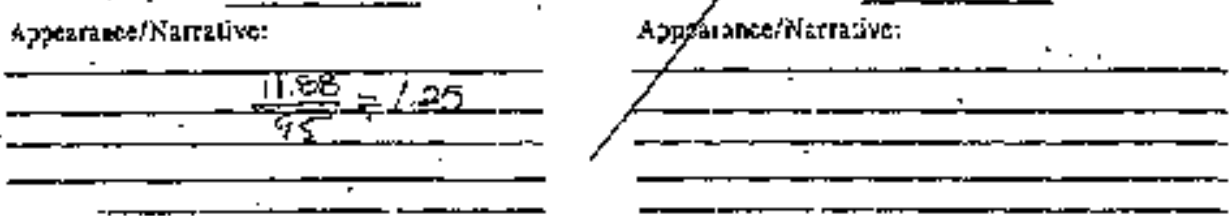
Requestor:

\section{Bulk Density Worksheet}

Tarik: $Z=110$

Core: 186

Seg: 3 ㄴt

Anger:

Sample DD:57t7000,208

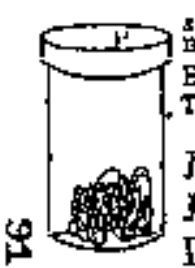

- Initial Weight

Appearance/Narralive:
Final Weight:

Net Weight:

Coneff:

Final Vol: $9.0-m L$ Initial Weigbt: $7.67 \mathrm{~g}$ Final Weight: $19.53 \mathrm{~g}$ Net Weight: 1.86 . Sample ID: $11.84=1.32$

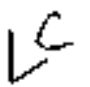

L g $\mathrm{g}$ g

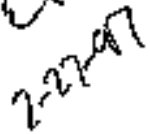
$\mathrm{g}$ 
Requestor: F Stes

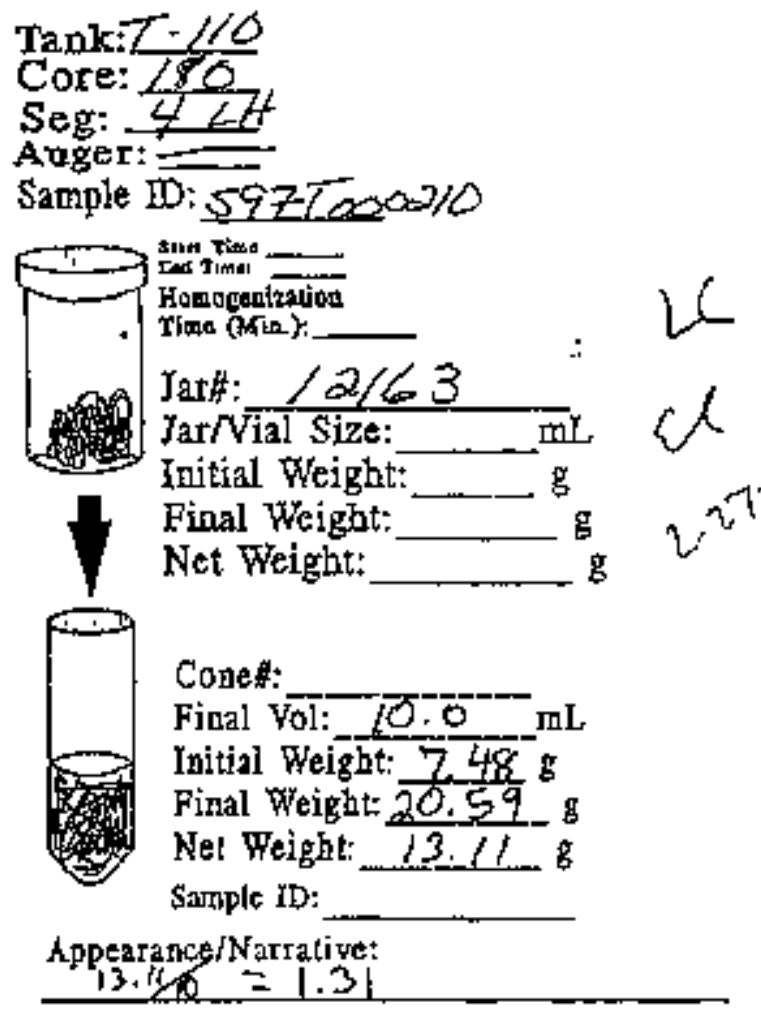

\section{Bulk Density Worksheet}

Date: $227-97$

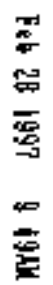

Tank:

Core:

Seg:

Auger:

Sample ID:
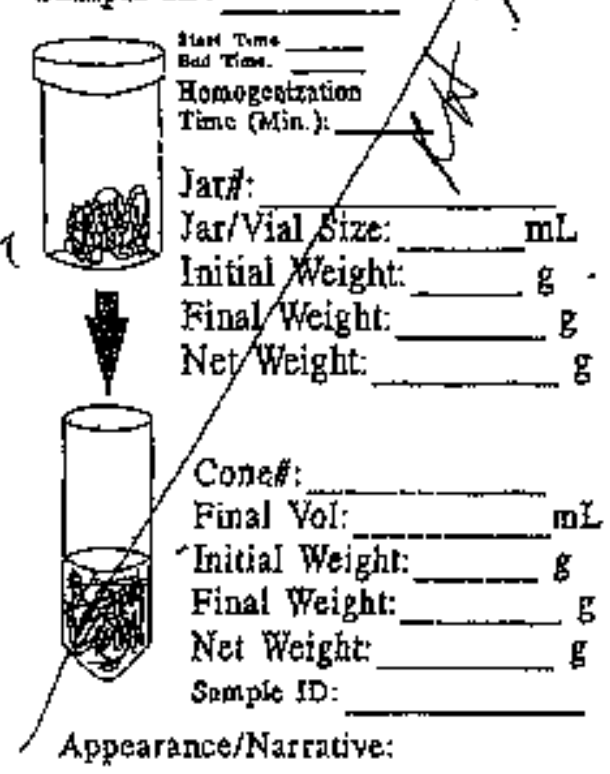


\section{Requestor: ESteer Bulk Density Worksheet}

Date: $2-26-97$

Tank T-11O

Core: 180

Seg: $6-74$

Augex:- -

Sample $\overline{D:} 59700001 / 2$

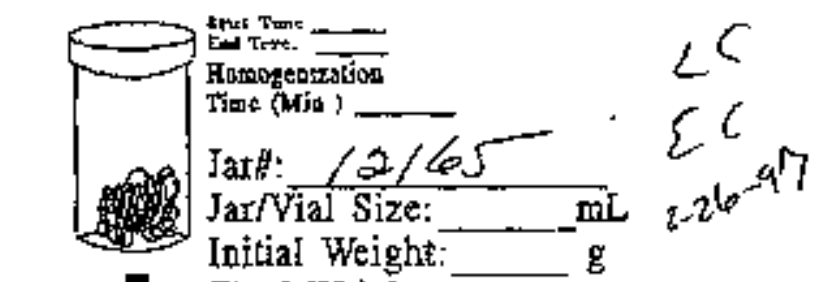

\& Final Weight: $-\mathrm{B}$

Net Weight:

$g$

Conef:

Final vol: $9.25 \mathrm{~mL}$

Initial Weight: $7.46 \mathrm{~g}$

Final Weight: $\frac{7.49}{19.49}$

Nef Weight: $12.03:$

Sarmplc ID:

Appearace/Narialue:

$1201 \times 25=130$

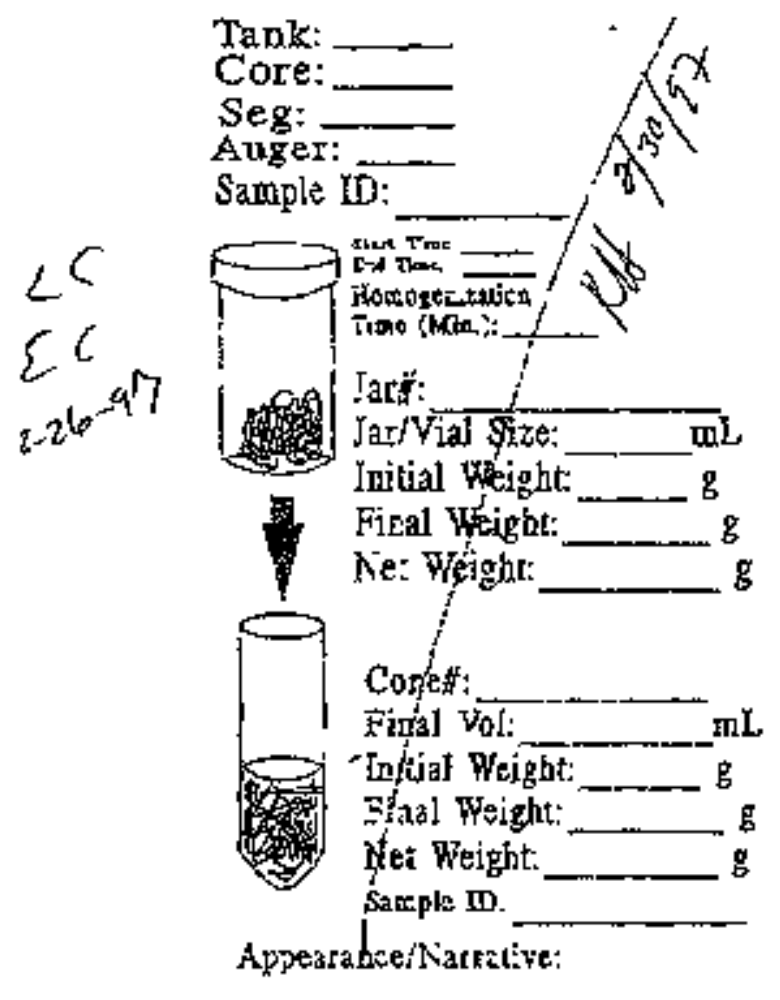

Tank:

Core:

Auger:

Sample ID:

Fical Wight:

8

g

8

产

돈

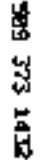

$\%$ 
Roquestor: Ych_nush

\section{Bulk Density Worksheet}

Date: $1-6-92$

Tank: THo

Core: 180

Seg: $\frac{1+4}{2+4}$

Atrger:

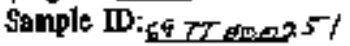

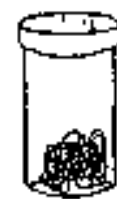

.

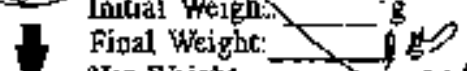

Tamosedistian

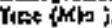

Jar : $\quad 12167$

Jat $\mathrm{Yial}$ \$ize:

Initial Weigh

Net hitight:

Canet:

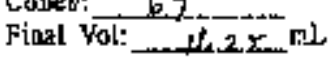

Inici하

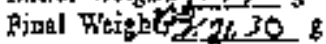

Net Weight /9.83 \&

Smpk to. 69770002 ist;

AppertancelNotratsy

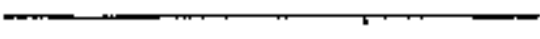

Than:

Cace:

Seg

Auge:

Sample ज:

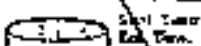

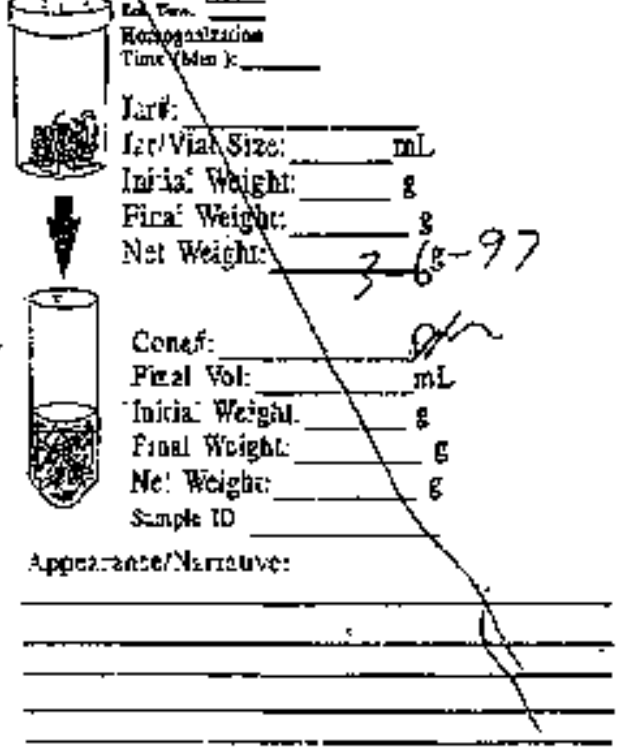


Requestor: IL Nuzem

\section{Bulk Density Worksheet}

Date: $3-6-97$

Taxk: 俩e

Core: $\angle 64$

Seg: ght .

Auger:

Sasiple ID: sayt atoes $x$

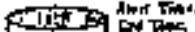

(3)

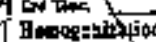

Time (Mins )

Jarit:

Jar

sy

Initial Weigt

Einal Weight:

Net Weight:

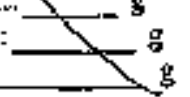

Conef:

Fina vol $\sqrt{10+5}$ :

Isinal Werght $2.45:$

Final Feight: 20.09

No: Weighc 12,61 C

Sunpht It.

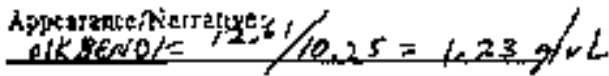

Tank:

Core:

Sect:

Anger :

Sampti ID:-

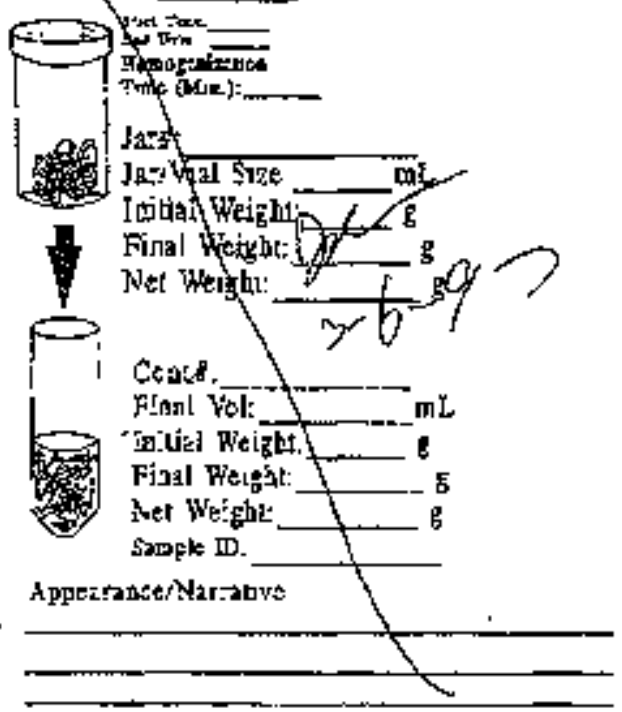


Requestor: $\mathcal{H}$ Nyzum

Tank: Ff10

Core: 181

Seg:

Auger:

Sample 10: 597 T600124

\section{Bulk Density Worksheet}

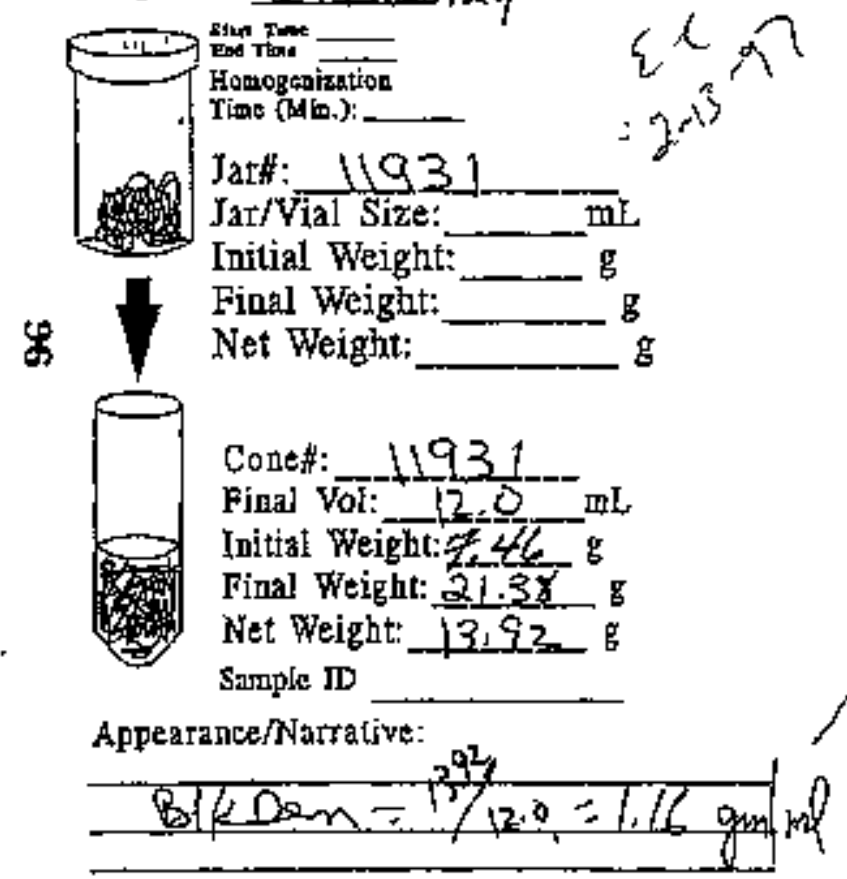

Tank:

Core:

Seg:

Auger:

Sampie ID:

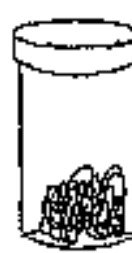

5NiTh Thm

Ind Time

Homogeristitide

Time (Min.):

Jarit:

Jar Viā́ Size:- $\mathrm{mL}$

Initial Feight:

Final Weight:
Net Weight:

7 Final Weight:

Date: $\frac{2 \sqrt{6} / 97}{7}$

Coneff:

Final vol: mL

-Initial Weight:

Final Weight:

Net Weight:

Sample ID

Appearance/Narsatìy: 
क्ष

MNA-SD-WM-OP-238, FEV 0

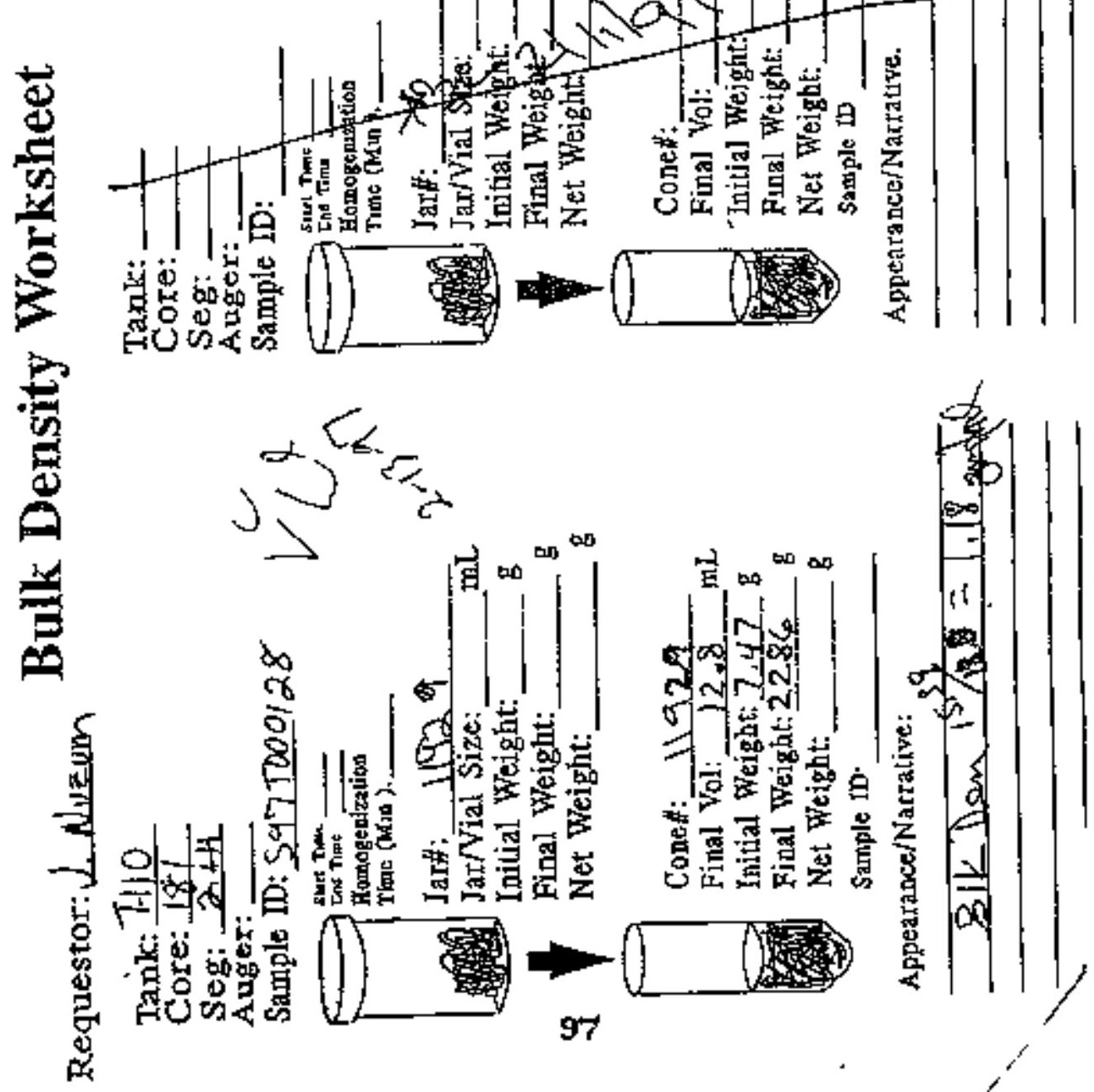


के

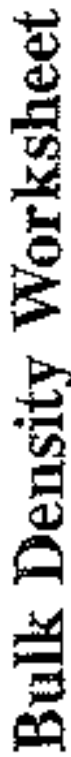
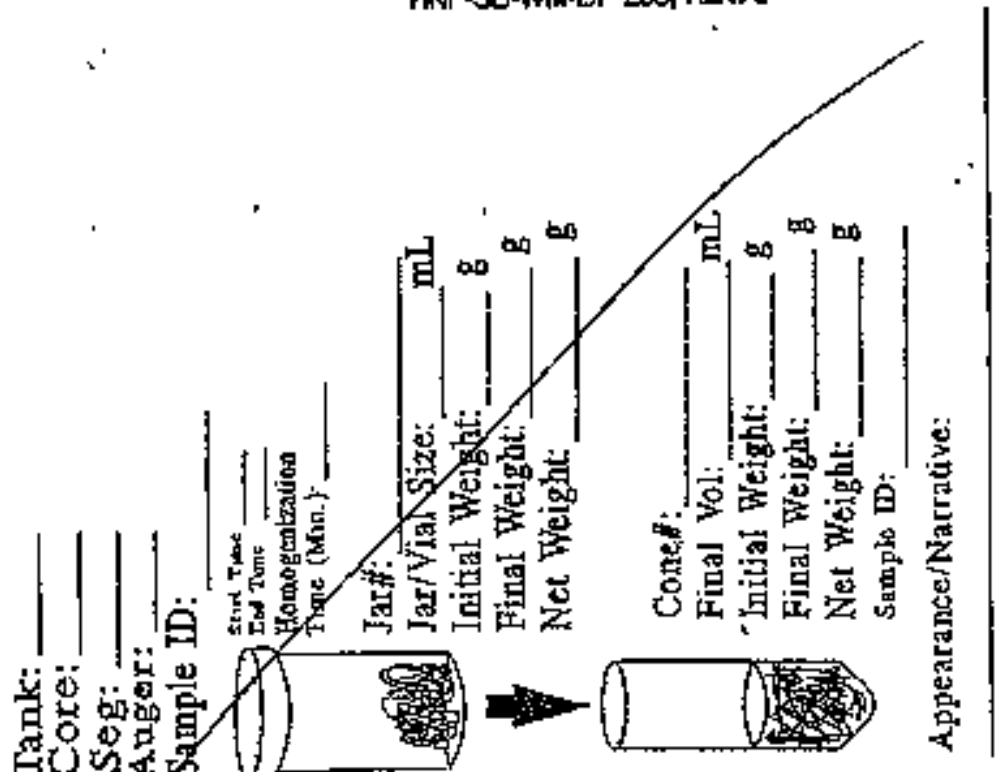

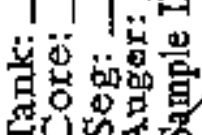

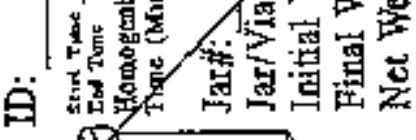
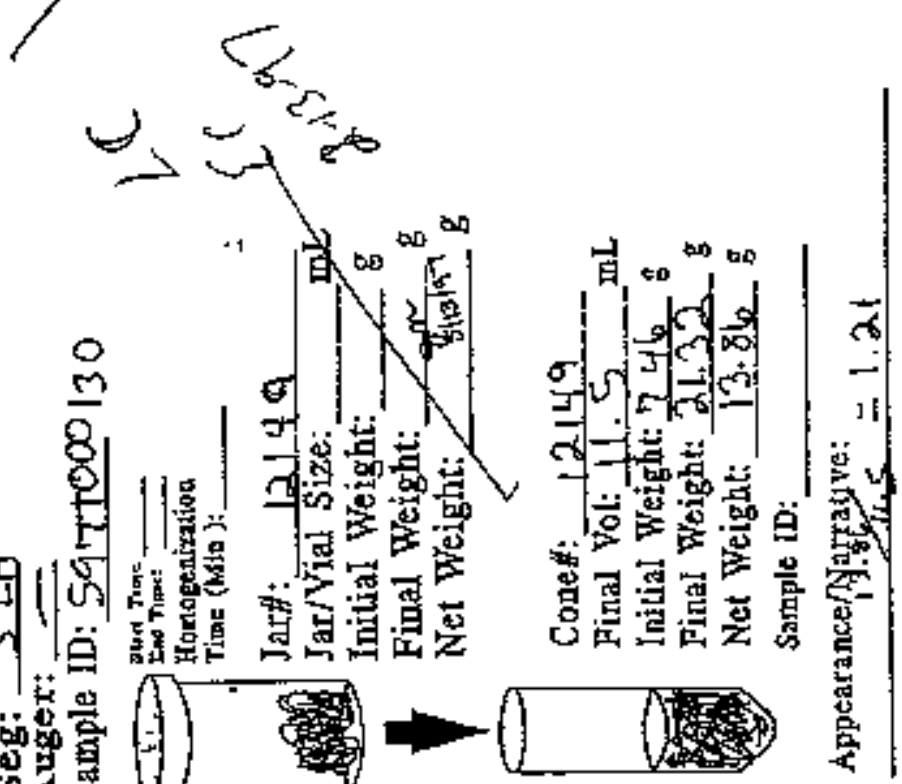

의 1 圆

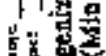

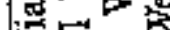

点

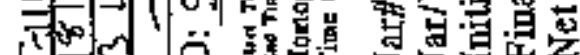

ir 并

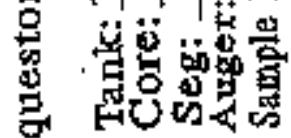
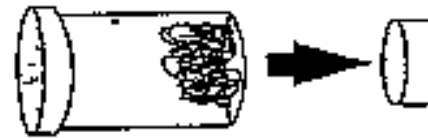
Requestor: J Nuzum

\section{Bulk Density Worksheet}

Tank: T-10

Core: $-\frac{181}{41}$, nisth"

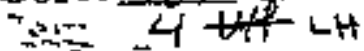

Anger:

Simox 1D: 5977000132

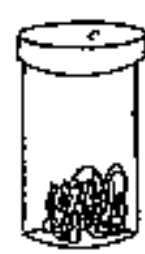
5्ञxत्र T=8

Homoranczention

Tranc (M:D)

Jart: 11934

Jar/vial Size:

Initial Weight:

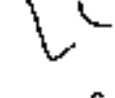

(1) Final Weight:

Net Weight: $g^{m} \leq[3[F] \mathrm{g}$

Coneff:

$+193$

Final vol: $12.2 \mathrm{~mL}$

Initial Weight: 745 g

Pinat Weight $22.14 \mathrm{~g}$

Net Weight: 14.69

Sampic ing

Apacarance/Natrat $\quad 1,20$

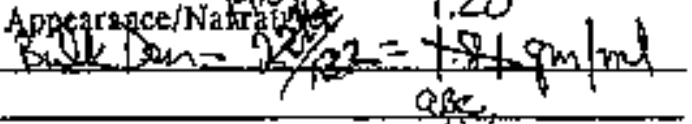
2 $119 / 97$

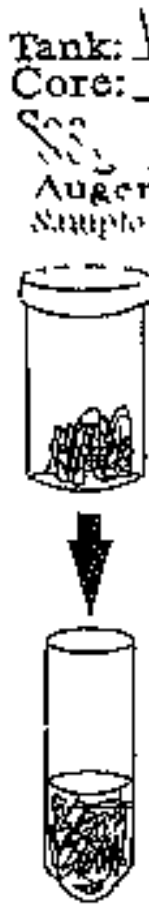

Appearance/Narfative:

Krimegentration

The (BNA)

Jat:

fand $\sqrt{\text { ial Size: }}$

Initial Weight

Fingl Weight:

Net Weight:

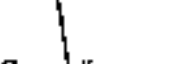

Cond :

Final Vol

'Intial Weight'

Final Feight:

Net Whight:

Sample D

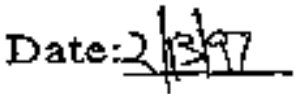
min

\section{$=$}

( $\mathrm{mL}$

丞 g

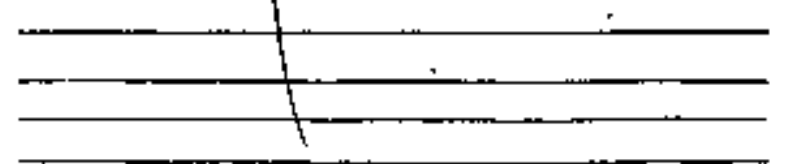

웅
号
$\rightarrow$
$\leftrightarrow$ 
Requestor: $3-2.4020$

\section{Bulk Density Worksheet}

Tank: Teto

Core: $\angle$ Ki

Seg: $\frac{S+4 t^{4}}{2}$

Augex:=

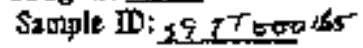

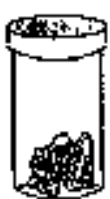

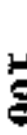

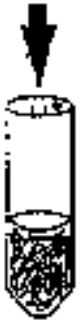

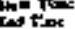

Eetated

Tin: $a \sqrt{1}$.

Jark: $\quad[2,5]$

Jat vial Size: - wal

Initial weighr:

Final Puight:

Nec Weighs:

Cosep: 12153

Fian: vil: th 35 an

Inital Weight: 252 :

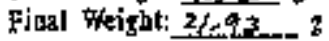

Net We:ght:L4t:

sampie [D.

Appeararce/Narzarizitity

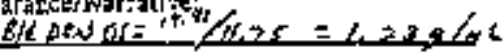

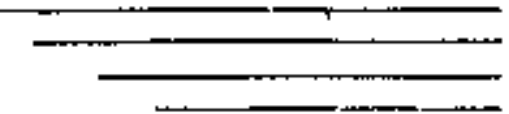

Tanks

Core:

Seg:

Aluter:

Sample IT:

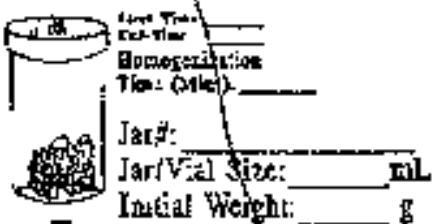

7 Final

Vet Wejphe

Vet pejric

$\cos n \sin ^{\circ}$

Eina! Wol:

'itutlal Weisht:

F:nal Werght:

Net Neight-

Sampio is:

Appenracecisozrasiz:

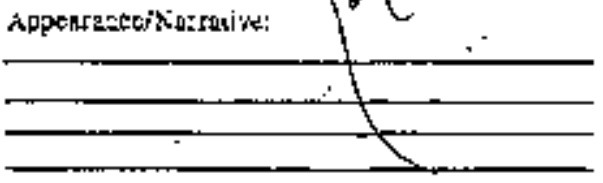


Requestor: 그션

\section{Bulk Density Worksheet}

Date: $3 \cdot 7-97$

Tank:T-200.

Core: 1 is

Seg: 644

Auger:

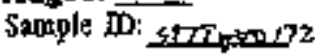

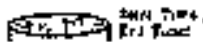

Afill

\& Final Wight: Net Weight:

$\underset{20}{20}$

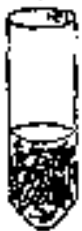

Conet 12155

Final vo: $f 0 . \bar{B}$ mL

farial Weighi: t.5.5. $\mathrm{g}$

Fisal Weight $2 \mathrm{LS}$ - 8

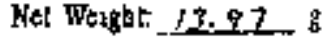

sample ID.

Appearastomatealip:

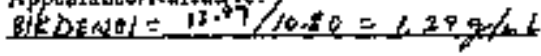

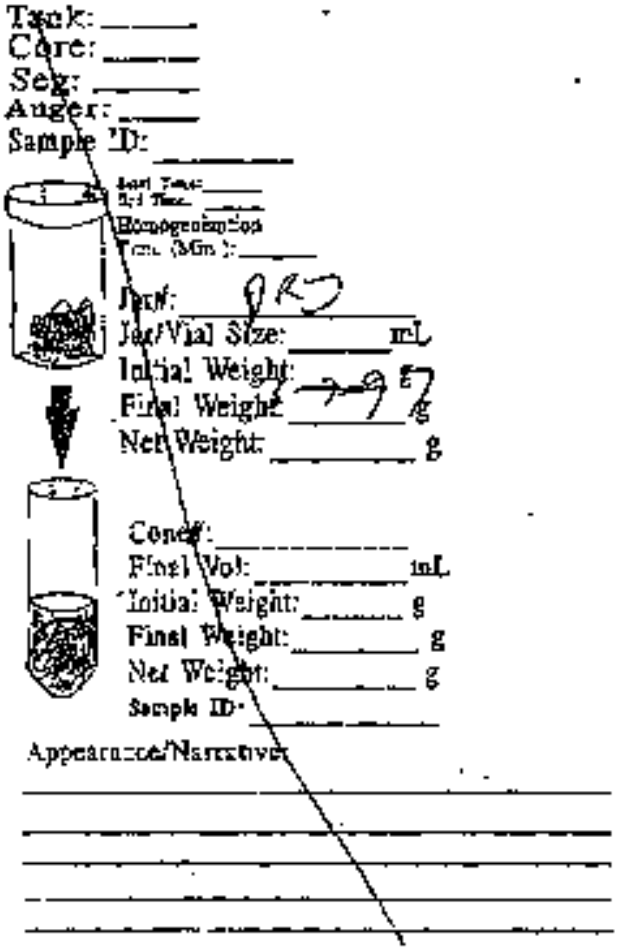




\section{Bulk Density Worksheet}

Requiestor: $\triangle M Z Z M A$

Tank: $T-110$

Core: $|\&|$

Seg: 4

Auger: =-

Sample ID:597/000 190

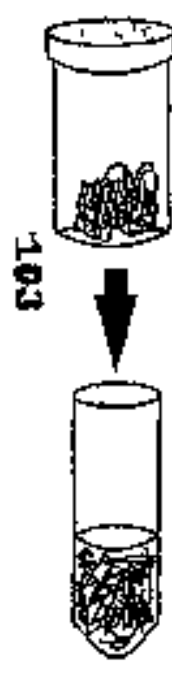

슬 Tilas

Homogenilyation

Tine (Brit.):

Jat\#: $12154=2 C$ Sat /Vial Size:__—

Initial Weight:

Final Weight:

Net Weight:__— $\mathrm{g}$

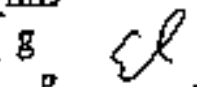

\section{Conet:}

Final Vol: $9.5-\mathrm{mL}$ Initial Weight: 7.50 : Final Weight: $9.66-1$

Net Weight: $12.16-g$

Sample ID: 597 T000/90

Appearance/Narrative:

$$
1.1 Y \% .5=1.28
$$

Tank:

Core:

Seg:

Anger:

Sample I:

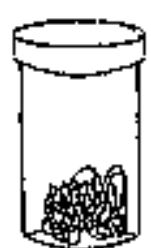

5 tind Tinc

Homogenization

Time (Minis):

Jast:

Jar/Vial Size:

Initial Weigh

Final Weigl,

Net Weigh

\section{g}

Cone

Fina Yol:

-Ini al Weight:

Date: $2-27-97$

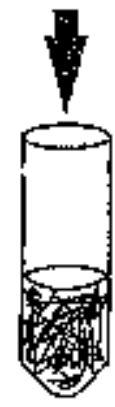

Fual Weight:

et Weight:

SEmplo ID:

Apper ance/Narrative:
胥

窎

薹

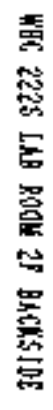

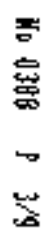


HF- 5 D-WH.EP-23B, REV. 0

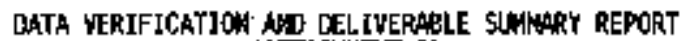
(ATRACHUYT 2) 


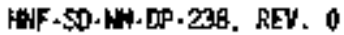

THIS PAGE IRTEHTIONALIY IEFT BLANK 


\section{CIleat Tins}

rank ratio

SUN

$$
\text { TAEIE OP CONTERTS }
$$

About this section $\quad$, ' , ' I

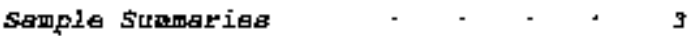

Frep Batch strmary , - , ' 5

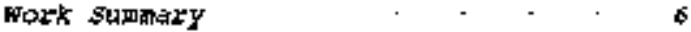

Method Blants $\quad$ - $\quad$, 9

Inb Contral samples . . . . 10

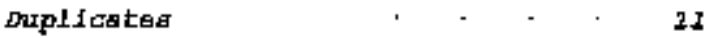

Matrik spikas , , , 19

Data Shests , . . . 21

Nethod sumaries , . - ' 26

Report Guides , . - . 3 I

End of Section . . . ' 46

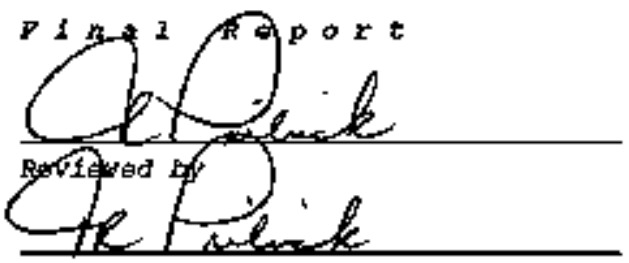

Iab $1 d 22 z-S$

Protocal s5T

Vorsion 1, Form $\mathrm{PVD} D \mathrm{TOC}$

Version 108

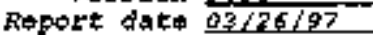




\section{ZZZ $=S$ HABORATORT}

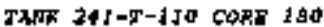

\$pe propit11 Contate I. I. Ifrtunt
REPORTTUIDE

\section{cItent mers}

Tank T्येo

A日OUT THE DATA

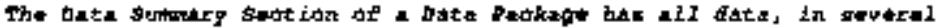

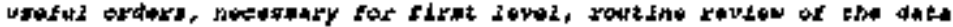

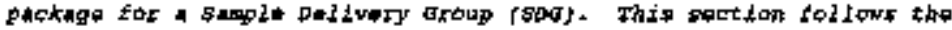

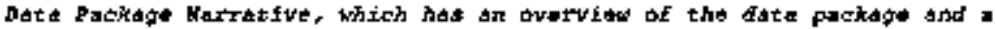

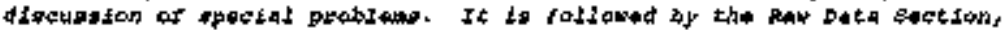
which has full ditalle.

The Data gumary section had several groupa of raporthd

\section{SAGPE SOMMARTES}

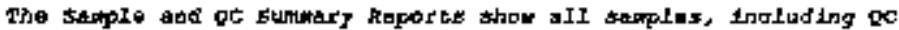

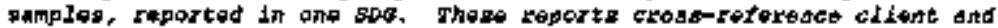
lab senple idontiflere.

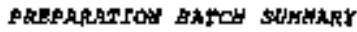

Hin Freparation batoh sumery lepart fhows al2 propazacton bachag iltb grouplage reflecting how work was argariad, zolevant to tha

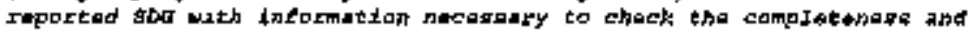
conlstethey of the sog.

FORX SUAMARY

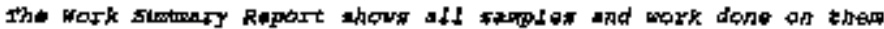
rolovent to ene reportod sow.

MeTron atians

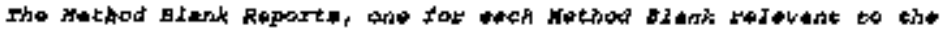

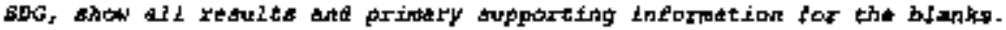

2AP CONTROL SHAPES

the tob coptrol sanpla Reporta, one tor exch Lab Control Sanpla relevant to the SDG, show all rosalta, rovoveriod and prtary sapparting

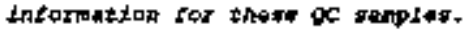

DUPLICNTE

Fina 1 ingoxt

\section{PEPORT GTIDES}

Onge I

SUMAART DAFA SECTION

yngeI

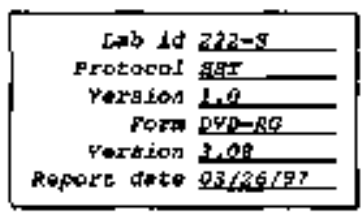


Z2 $2-S$ LABORATORY

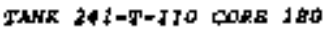

spo 9700 dit Cadtat I, I. Myzenil GUIDE, $\operatorname{con} t$.

Client Task

Tank Thare

A

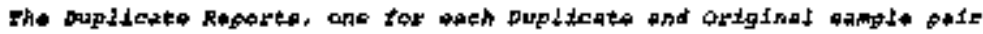
relevant to tha soG, ahow all rexalts, dfferennces asd primary etpporeting laforthation for thest of oamples,

MATAIX SPIRES

Tha Natrix spike Reporta, one for anth spiked and orifinal reppla gair

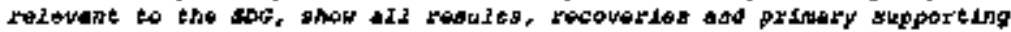
leformation for thate pe sapolas.

DATA SHEETS

The Data ghent Reporta, one far ach cliert sample is the spo, show all

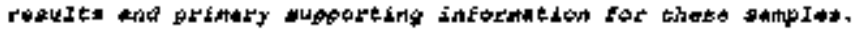

METPOD SUWHARIS

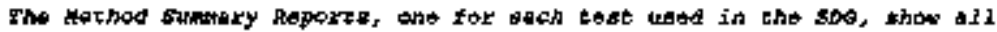

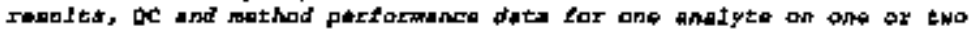

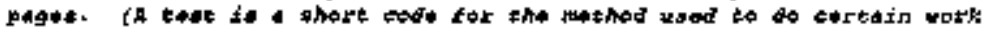
to the elfent's apmaifioation. )

REPORT GUYPES

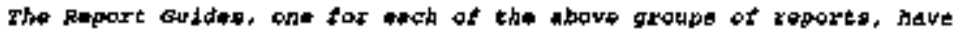

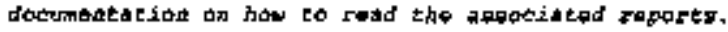

\section{Final R*port}

\section{IIFFORE GhIDES}

Pape $a$

SOHAARY DATA SECTIOW

Fage $?$
KAB iA 22\%-s

Protadol SSP

veredon 1.0

For OWN $=\mathrm{RO}$

$V$ ersion 3 , OB

peport 4afe 오/2/26/97 
HNF-SD-WhA-DP-2S8, REV. 0

\section{2-S LABORAPORY}

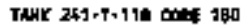

509 950, 11

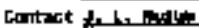

SAHPLE SUHSART

tet aent thes

$T+1+110$

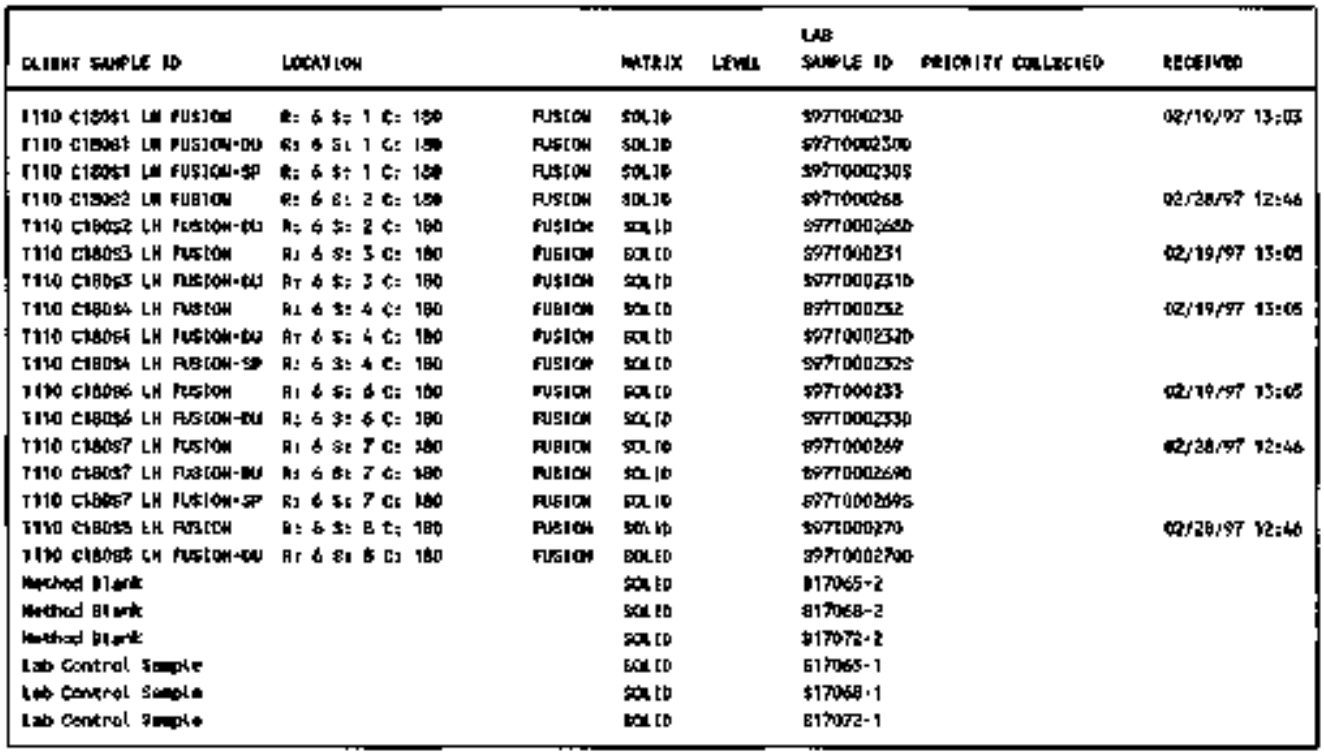

Final Report

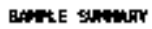

Papa 1

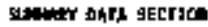

Pata 5
Leb Id 7275 .

protocel 코디

Viritan 10 Form Pror.5

Vitraien 5.05

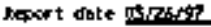


HWF-SD-WM-DP-238, REV. 0

\section{2́-S LABORATORY}

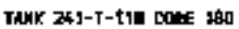

QC STMMARY tl iose nus

rark rol

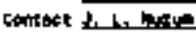

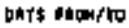

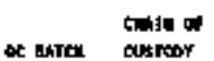

CIIDI ENGE IO

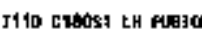

ग10 thace? LN TH\$

T1 chooss LH FUB30

7110 tharar LN Fatom

ร110 [18036 LH rUS]O

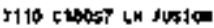

T110 ctods: [H FLE]O

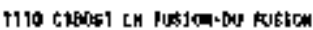

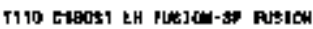

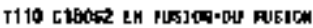

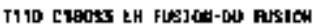

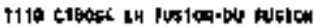

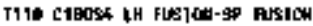

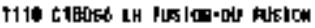

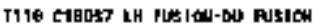

T110 c16057 in pusidw-s pusiom

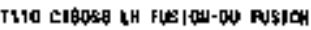

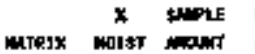

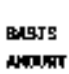

17\%

SWIPUE I0
ดESIRTMKT FHTE ID

\section{șan}

\section{mathod ink}

inthod Bient

nrthod ol pris

lab tontrol burple

lab compod sinte

lab Central surpl.

\section{L.}

5010

SDL. 15

sou.10

gDL]0

50.10

sCL.JD

ร. 16

sol.

म्र. I0

sta. 10

म्य. Ip

क्ष, 10

pr.

sac 16

म्व. 10

s.10 soptonieso

sớ Doozs

5 FTdotat

sFT To0k2

stotocisz

sistrocess

FPTt00azro

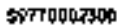

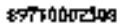

sp? 1000asto

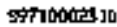

5propouzazh

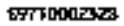

s971000aß

soit thockso

sprtoutors

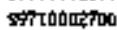

50410

50乌 10

D9 10

Bo으 Io

son 10

$\operatorname{sen}$ it
๑17055-2

g1706t-2

-170r8?

घ17000-1

$\$ 17058-1$

sirot2-1

\section{Fizal Report}

oc Brumer

Peg: 1

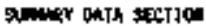

Pr日e 4
Lob la atas.

prosocol \$\$I

Veraton 10

Form P1P=06

Vocpton the

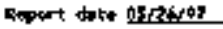


HNF-SD-WA-DP-238, REV. O

ZAJ 5 LABORATORY

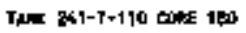

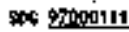

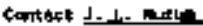

PREP BATCF StUMARY

Client Lette.

Tonk 1-110

\begin{tabular}{|c|c|c|c|c|c|c|c|c|c|c|c|c|}
\hline \multirow[b]{2}{*}{ TEST } & \multirow[b]{2}{*}{ matestx } & \multirow[b]{2}{*}{ kत्राn } & \multicolumn{2}{|c|}{ 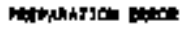 } & \multirow{2}{*}{ CLIEIT } & \multirow[b]{2}{*}{ mDEE } & \multicolumn{2}{|c|}{ HLdnç HETs } & \multicolumn{3}{|c|}{ auldraes } & \multirow{2}{*}{$\begin{array}{l}\text { antI } \\
\text { Fiess }\end{array}$} \\
\hline & & & wirt & Zn $x$ & & & Ret & ELAX & LES & OLPRTLO & hE/OAIG & \\
\hline \multicolumn{13}{|c|}{ 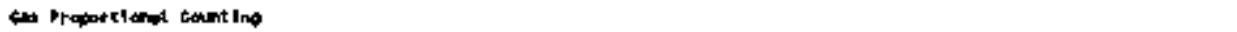 } \\
\hline \multirow[t]{3}{*}{$k T$} & stedo & Alphe Anatroits & 90000018 & 15.0 & 3 & & & 1 & 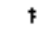 & $5 / 3$ & $1 / 1$ & \\
\hline & & & 9)=0\$1 & 13.0 & 2 & & & 1 & 1 & $2 r \boldsymbol{z}$ & $1 / 1$ & \\
\hline & & & oroposos & 15.0 & $\mathbf{1}$ & & & 1 & 1 & 2,2 & $1 / 1$ & \\
\hline
\end{tabular}

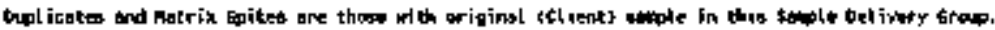

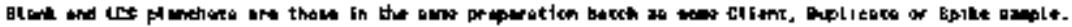

Final Report

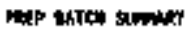

Poge 1

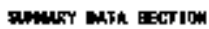

popt 5
Lib id 222-5

Pracecol 最

Whrtyon 10

Fare ous-pap

varsion 5 .

wivor date getzeror 


\section{ZZZ-S LABORATORY}

tank $244-T+110$ tone 180

WORK SUMARY cilent nuts

I*⿻ $\mathrm{I}=1 \mathrm{1th}$
La Eupat Jo

tLIEN suite it bocktya

danor

Prferby

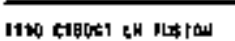

te \& st 1 CE Too

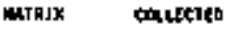

sox.IF

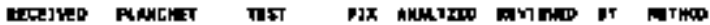

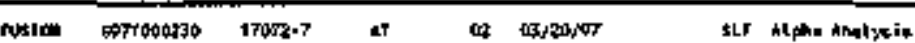

abt9/97

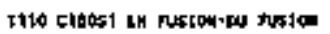

SOL.JP

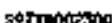

170rato AT

as oratit

\$LF Alphe arshyets

ค: क $3: 1$ c: 100

由19

T110 c18081 LH Rusion-6P Fuside 年|

Bgrtoooeros 1 1 porz-g

oz of $200 / 97$

SLF Alpha sratysis

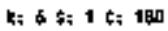

$02 \mathrm{rg}$ \%

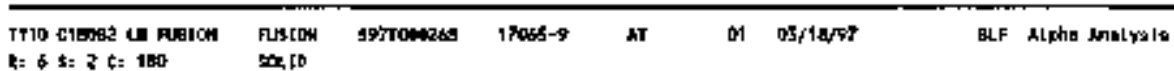

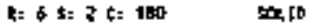

$02 \pi$ क्याप

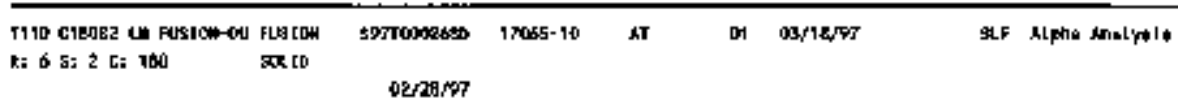

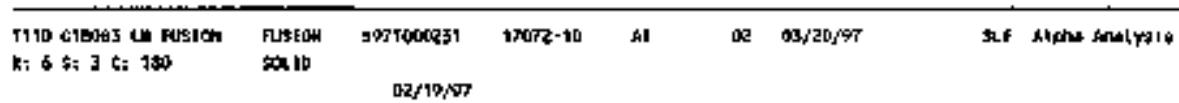

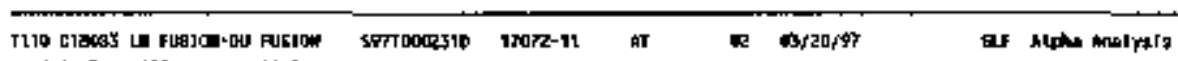

R1 6 5: 3 [? 1 bo Botlo

Dep19/s)

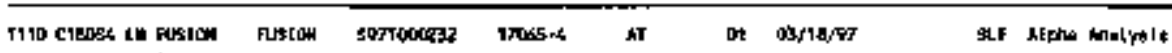

t: 6 B: \& C. aet $20 \pi$ EO

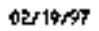

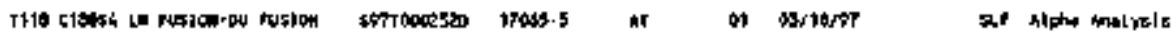

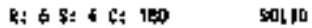

ater $19+97$

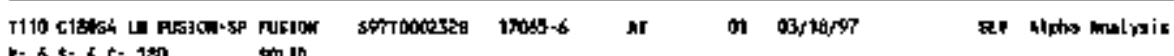

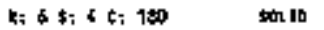

$02 / 19 / 97$

\section{Flnal Report}

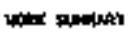

ragk 1

अ

tod 4
I A b 1d 227-s

Protocal s:5L

verion d, d

Fare $010-0 \times 5$

veraion $30 \%$

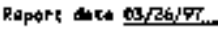




\section{2-S LABORATORY

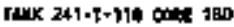

WORK SUMAARY, cont.
CIfint Ines

Iath 1.116

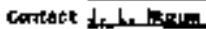

La stant it

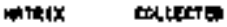

CLIENT aNPLE ID Lockt16

Dustour

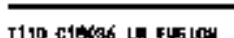

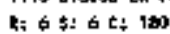

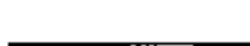

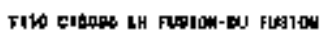

II: $65: 6$ C: 200

에

Fuelow Dotronots 17065-7 NT

$\$ 0,10$

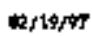

SLF Alpho Nolye1s

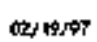

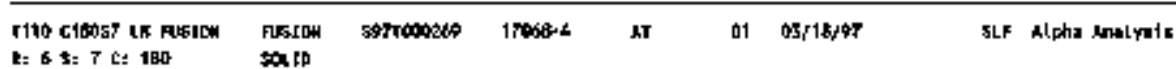

thitits

\begin{tabular}{|c|c|c|c|c|c|c|c|}
\hline 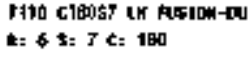 & 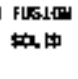 & 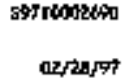 & $17068-5$ & ג1 & 01 & $03 r 16 / 67$ & SLF Alphe Noulysis \\
\hline
\end{tabular}

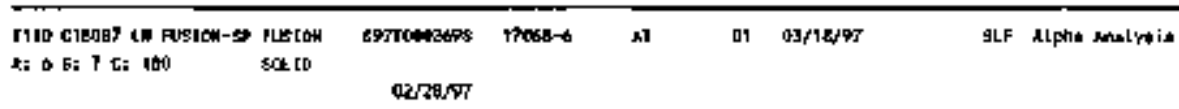

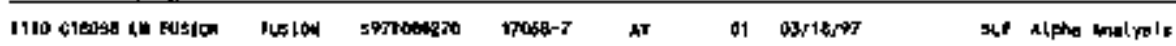

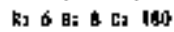

sal to

02/26가

\begin{tabular}{|c|c|c|c|c|c|c|c|}
\hline 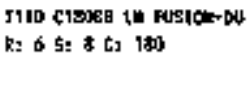 & $\begin{array}{l}\text { n:Fiow } \\
\operatorname{sen} 10\end{array}$ & 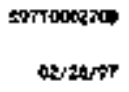 & Irose-B & O1 & $05 / 19 \% ?$ & 뽀, & Alphs molptit \\
\hline Method BI ont & Fallo & Evross -2 & $170065-2$ & .1 & 05,1987 & gLF & Alpht lnstrate \\
\hline Hothod Blanth & $\sin$ & genous-? & moss. 2 & ו1 & $03 / 1 \mathrm{k} P \mathrm{P} ?$ & she & Alpta valyels \\
\hline Weshod slank & $\operatorname{sen} 16$ & $41 \pi 0 \pi 2-2$ & TNR2: & $4 \pi$ & $03200 \%$ & s. & Alphs molpais \\
\hline 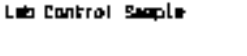 & BOR I0 & suthos-1 & Irocs-1 & Mr & 01/20j? & st.F & 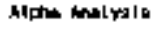 \\
\hline
\end{tabular}

\section{Final Report}

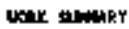

Pape eे

andikt bits sIstion

Dagh 7

ith id 227-1

Protocol sy

Nortion 10

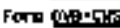

Vurtion

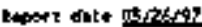




\section{2-S IABORATORY}

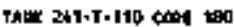

500 Troo0111

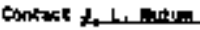

WORK SUNAARY， cOnt +

Cl Ient I.s.8 Tonk 1. 1 1 0

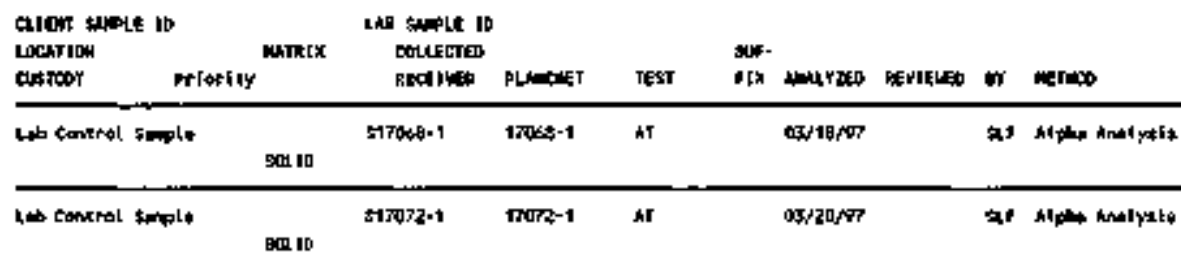

\begin{tabular}{|c|c|c|c|c|c|c|c|c|c|c|c|c|c|}
\hline TEN & Priorlity & COUWTS & $O F$ & $\begin{array}{l}\text { TESTS } \\
\text { HEEREF }\end{array}$ & BY & $\begin{array}{r}\text { SAMPLE } \\
\text { CLIEx }\end{array}$ & $\begin{array}{l}\text { TYPE } \\
\text { ח nonf }\end{array}$ & KE & B:AlW & $\operatorname{tcs}$ & DHP & Bike & IDTAL \\
\hline A1 & & Mphn Arolyrte & & $222-s$ cob $k$ & nalycteal & il precendure 7 & 7 & & 5 & 3 & $y$ & 3 & 로 \\
\hline Tणा 14s & & & , & & & & $\mathbf{T}$ & & 5 & 3 & T & 5 & $\pi$ \\
\hline
\end{tabular}

Pinal Report

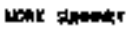

Pare 5

drellit buTd उECTIOW

Doge $B$ lab td $22-9$.

Protocod 5 sI

Nortal on 1,0

Fom ontors

Norteton 3.4.7.

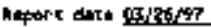


HNF-SD-WH-DP-23B, REV. O

\section{ZZZ-5 IABORATQRY}

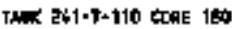

396 87000111

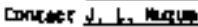

BIA $\boldsymbol{B}$ KS

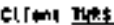

Tank I-LH?
Lot esmin id alysts-1

Dept and 1d

C

\begin{tabular}{|c|c|c|}
\hline IMYTF & crs "\# & RESAT \\
\hline & & \\
\hline
\end{tabular}

Total alpate

1247.441

5.7265

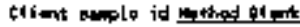
waber1 al Jkerrix

80.10

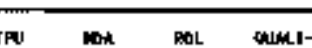

7 urlifg uclig rides Yesl pktp tuich

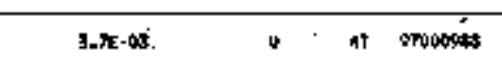

\begin{tabular}{|c|c|c|c|c|c|c|c|}
\hline \multicolumn{2}{|c|}{ 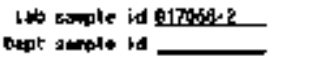 } & \multicolumn{6}{|c|}{ cllint ossple id bethos lint: } \\
\hline MKANE & Ch日 nO & $\begin{array}{l}\text { EEguLy } \\
\text { úidig }\end{array}$ & $\begin{array}{c}2 \sigma \mathrm{Tr} \\
\boldsymbol{x}\end{array}$ & 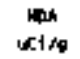 & $\underset{10 x}{\tan }$ & $\begin{array}{l}\text { OAnLI- } \\
\text { FEERS }\end{array}$ & 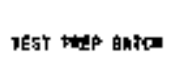 \\
\hline Itotet inpus & $12007.40-1$ & 45.2t-03. & & 3.2E+ & & $\mathbf{u}$ & 97000901 \\
\hline
\end{tabular}

\begin{tabular}{|c|c|c|c|c|c|c|c|c|}
\hline \multicolumn{2}{|c|}{ 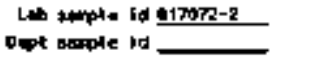 } & \multicolumn{5}{|c|}{ 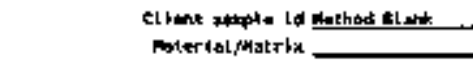 } & \multicolumn{2}{|r|}{ - 50.1P } \\
\hline AlenLTII & Chs ap & $\begin{array}{l}\text { HEBULT } \\
\text { WCI I }\end{array}$ & $\begin{array}{c}2 \sigma \text { Tल } \\
x\end{array}$ & ichos & $\underset{c t s p}{\tan }$ & $\begin{array}{l}\text { eumL1- } \\
\text { Pleks }\end{array}$ & 145T & HAd |nhen \\
\hline Toral Alpha & $72587-46-1$ & (3.06-13 & & $3.0 \mathrm{~d}-0 \mathrm{a}$ & & $v$ & st & 97001995 \\
\hline
\end{tabular}

Plnal kepart

mank

Pags 1

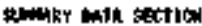

Drat 9
Lat ind 20225

Proted 结

Hersion 10

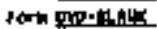

Uarzien 50 .

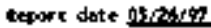




\section{$222-G$ IABORATORY}

sot oxpogu

contopt dis lutst
TMK ख1-1-110 QIPE IFo

LAB CONTROL SAMPLES ctlent Ty+9

Tark I. 110

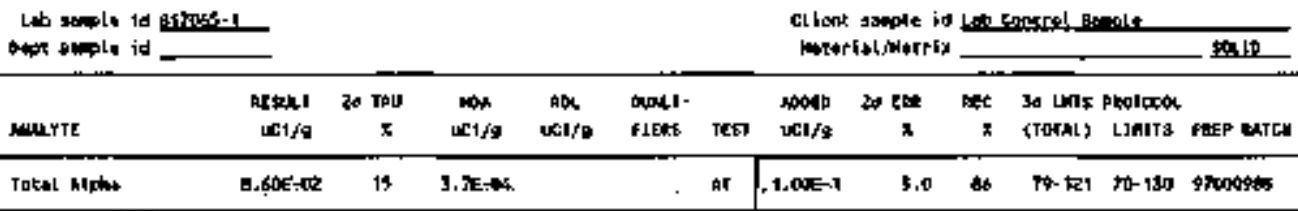

\begin{tabular}{|c|c|c|c|c|c|c|c|c|c|c|c|}
\hline \multicolumn{7}{|c|}{ 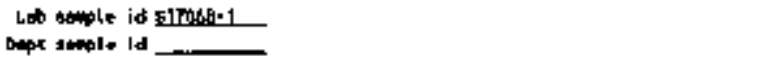 } & \multicolumn{2}{|c|}{ 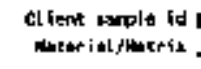 } & \multicolumn{2}{|c|}{ 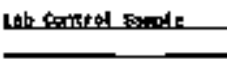 } & $-1 \times 1.10$ \\
\hline MHLLrTE & $\begin{array}{r}\text { IESSIT } \\
\text { LC1fo }\end{array}$ & $\begin{array}{c}\text { zo Tru } \\
x\end{array}$ & istis & $\begin{array}{l}\text { not } \\
\text { uct } f 0\end{array}$ & $\begin{array}{l}\text { oymL] - } \\
\text { F[ERS }\end{array}$ & TEST & $\begin{array}{l}10010 \\
1 \mathrm{CI} / \mathrm{s}\end{array}$ & $\begin{array}{c}30 \text { ERR } \\
x\end{array}$ & $\begin{array}{r}\text { REC } \\
x\end{array}$ & $\begin{array}{l}\text { \$a Lurs Phoroter } \\
\text { (TOTAL) LIMIIs }\end{array}$ & Resp BATCH \\
\hline Total Hipha & 9.टती $E-02$ & is & $3.1 \mathrm{E}-\mathrm{-0}$ & & & AT & $9.46=2$ & 5.0 & $\phi$ & 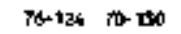 & 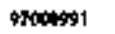 \\
\hline
\end{tabular}

Final Report

LAS DOmRL stretes

Pagt 1

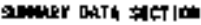

Pagn 10

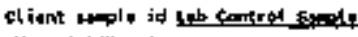
Mater1abilucr11 sath

dept sople ld

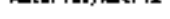

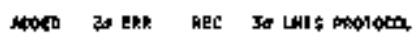

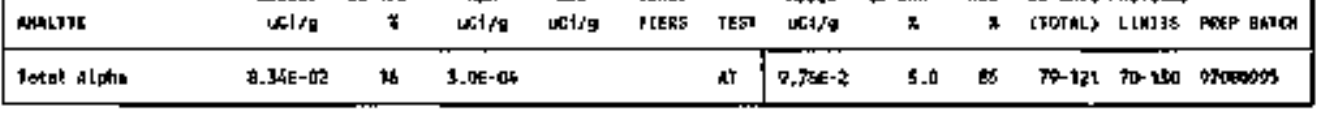

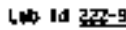

Proracel 腿

vertien 1,0 Fara 마이다.

Vertion 1 . nd

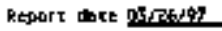




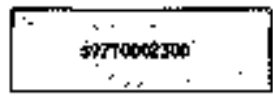

\section{ZZZ-S LAEORATORY}

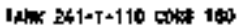

DUPLICATE

506 92000111

Sontact tr he vale ploLACATE

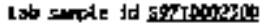

Dept id
WiGIIGL

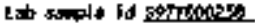

bupt acepde ld

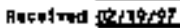

cilent Rises

rext $Y, 110$

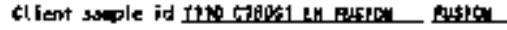

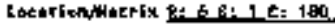
2004. collected Choln of castody lit

\begin{tabular}{|c|c|c|c|c|c|c|c|c|c|c|c|c|}
\hline АнитT. & $\begin{array}{c}\text { MPLtchre } \\
\text { uCFft }\end{array}$ & $\begin{array}{c}\boldsymbol{Z} \boldsymbol{T} \boldsymbol{T} \\
\mathbf{x}\end{array}$ & tes & 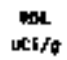 & $\begin{array}{l}\text { oumbl - } \\
\text { rpers }\end{array}$ & JEST & $\begin{array}{c}\text { oxjeimul } \\
\text { utiffs }\end{array}$ & $\begin{array}{c}\text { Zar TPU } \\
=\end{array}$ & $\operatorname{lom}_{\mathrm{UC1} / \mathrm{9}}$ & $\begin{array}{l}\text { oun T- } \\
\text { yeth\$ }\end{array}$ & 5 & 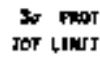 \\
\hline Totel stpht & $4.6,56-03$ & 22 & 3-1e-43 & & & AT & 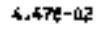 & 22 & 3. $14-0.3]$ & & 4 & 47 \\
\hline
\end{tabular}

\section{Final feport}

DLLICXIEB

Paga 1

swimer Ohrs sRerson

log. 11 las id 2050

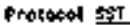

varsion 1,0 rom provory

Yexan 5.

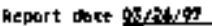




\section{ZZーS LAEORATORY}

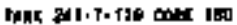

sकराpocessto.

DUPIICATE

\$46 900,01

Cantect Dipl|chII

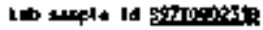

bept umpto id

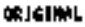

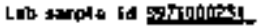

Dopt tanplot id

Pecplived 매/19/97
CItent IFR

Torth r+110

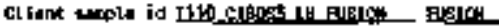

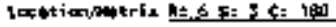
ando Col laceed

chain of autrods id

\begin{tabular}{|c|c|c|c|c|c|c|c|c|c|c|c|}
\hline NUMYYTE & $\begin{array}{c}\text { ocstichnt } \\
\text { teifs }\end{array}$ & $\begin{array}{c}201 \mathrm{x} \\
x\end{array}$ & $\cos$ & wci/s & $\begin{array}{l}\text { AIILLI- } \\
\text { F IERg }\end{array}$ & TEgt & 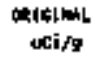 & $\begin{array}{c}\text { 20 TH } \\
\Downarrow\end{array}$ & 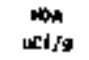 & $\begin{array}{l}\text { कus l- trot } \\
\text { FIEDE } x\end{array}$ & 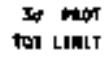 \\
\hline Totel Hichs & $3.55 \mathrm{E}-02$ & $\boldsymbol{B}$ & 5. $0 \mathrm{E}-0 \mathrm{~F}$ & & & MT & $2,8=02$ & है। & $y, 0 \leq-0 t$ & 1 & 31 \\
\hline
\end{tabular}

\section{FinaI Report}

Whicates

PopQ $Z$

butilat onTh section

1094 12
Lab id $292-5$

Protocol t5I

vortian I.to

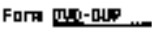

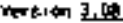

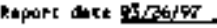




\section{2-S LAEORATORY}

ThWY 249-T-110 DOE: 180

sitootion

DUPLICATE

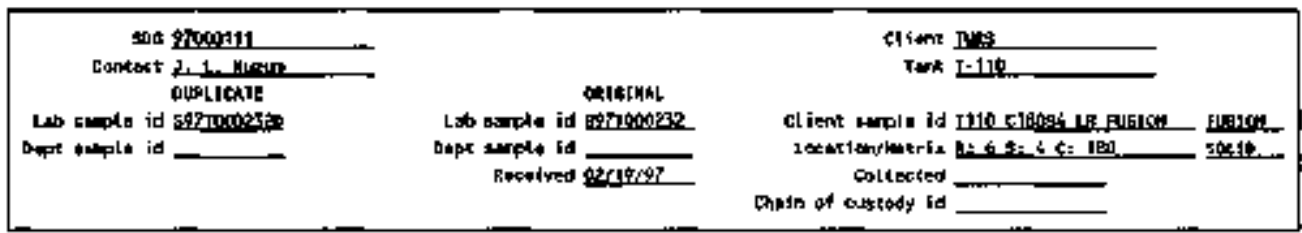

\begin{tabular}{|c|c|c|c|c|c|c|c|c|c|c|c|}
\hline מatrot & $\begin{array}{c}\text { Doptente } \\
\text { utifo }\end{array}$ & $\underset{x}{2 r}$ & utifo & $\begin{array}{l}\text { hit } \\
\text { uciff }\end{array}$ & 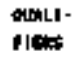 & TET & $\begin{array}{l}\text { aelatuat } \\
\text { ucifó }\end{array}$ & $\begin{array}{c}\text { A } \\
y\end{array}$ & $\begin{array}{c}\boldsymbol{\omega} \\
\mathrm{uti} / \mathrm{s}\end{array}$ & $\begin{array}{l}\text { aikl- tet } \\
\text { FIEss } x\end{array}$ & $\begin{array}{l}\text { उof Gtor } \\
\text { Tरा IIDIt }\end{array}$ \\
\hline totel Alphn & $0.265 \cdot 03$ & 21 & $4,00-05$ & & & hT & $s, 5 d-02$ & 螘 & $3 . \pi+\infty$ & 푀 & 46 \\
\hline
\end{tabular}

\section{FInal Report}

\section{aUPLICNEE}

Pape 5

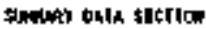

paste is
Lab id 27z-1

protocol 환

viriaten 10

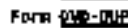

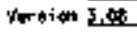

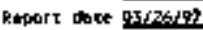




\section{2-S IABORAPORY}

TAN $241-T-T H O$ EDAE ID

DIPITEATE

sto or:311

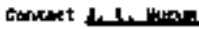

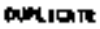

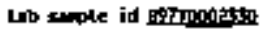

Itpe suaple ld

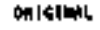

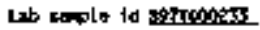

Dept aral 1 1d

Encolined gartiot? clime tritt:

Tark I-I10

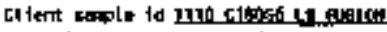

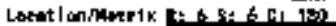
nathe sote colleted chinin of euptody id

\begin{tabular}{|c|c|c|c|c|c|c|c|c|c|c|c|c|}
\hline Nhurte & $\begin{array}{c}\text { DUwlokte } \\
\text { wise }\end{array}$ & $\begin{array}{c}\text { Ze T*U } \\
\boldsymbol{x}\end{array}$ & $\underset{\text { WERtg }}{\text { COR }}$ & wat & $\begin{array}{l}\text { culul- } \\
\text { rtaks }\end{array}$ & TEgr & $\begin{array}{c}\text { ory asmol } \\
\text { utifo }\end{array}$ & $\begin{array}{c}301+y \\
x\end{array}$ & whis & 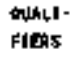 & nes & 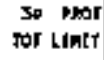 \\
\hline 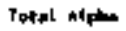 & 6, ex,-03 & 30 & 3:Be-13 & & & at & 4, bue-ol & E2 & $c_{+} 0 t-05$ & & 40 & 47 \\
\hline
\end{tabular}

\section{Iinal Roport}

\section{Aritekrex}

Fars 4

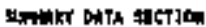

Vhat 16 un id 12005

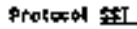
varalon 1.0 pore rmexts

rantion 3. 3 .

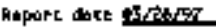




\section{$Z Z 2=S$ LABORATORY}

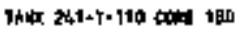

DUPLICATE

no groodid

contoct d. Whase mitchte

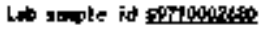

oapt ample ld otcoun

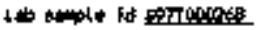

Dent anole id totelived estrosp?
cIIent 73:

tenk I. 110

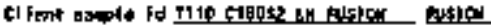

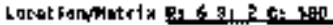

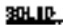
Euin of eatody ud

\begin{tabular}{|c|c|c|c|c|c|c|c|c|c|c|c|c|}
\hline mukyt & 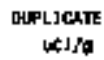 & $\begin{array}{c}\text { 20 } \\
\mathbf{x}\end{array}$ & 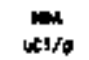 & 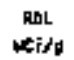 & $\begin{array}{l}\text { Muxl- } \\
\text { Adtint }\end{array}$ & Irti & $\begin{array}{c}\text { aitucul } \\
\text { ncisds }\end{array}$ & $\begin{array}{c}2 \pi \text { TFU } \\
y\end{array}$ & $\underset{4 E E}{\operatorname{MOA}}$ & 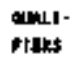 & $\begin{array}{r}\text { IPD } \\
\pi\end{array}$ & 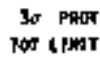 \\
\hline lotal Nlph. & $3.942-08$ & 86 & $3.51=00$ & & & A1 & f.d1 $-0 \%$ & $7 \%$ & 3.42-秌 & & 12 & 48 \\
\hline
\end{tabular}

\section{FinaI Report}

DURIEATEP

But 5

STEMAY BuTd EECTIOM

pope t5
Lot id 27305

Dratocel spr

version 1. Pore tho-pis

varaten this:

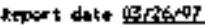




\section{2-S IABORATORY}

TANC 241-T-1 If Dote tho

DUPEICATE

sto gorpent1

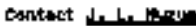
oftichte

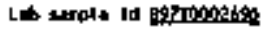

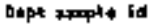

chitrink

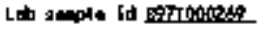

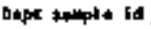
Recatred thidatif?
Cxtwing Tits

rank I-Tito

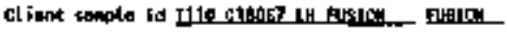

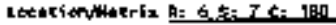

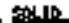
collected thain of etsto id

\begin{tabular}{|c|c|c|c|c|c|c|c|c|c|c|c|}
\hline MULTE & $\begin{array}{c}\text { Detichit } \\
\text { ctifs }\end{array}$ & $\begin{array}{c}201+4 \\
x\end{array}$ & undifs & ucifo & 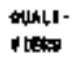 & TEST & 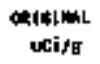 & $\begin{array}{c}2 . \mathrm{T} \\
1\end{array}$ & $\operatorname{mot}_{\mathbf{m}}$ & 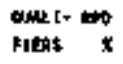 & $\begin{array}{l}\text { 3o mbi } \\
\text { rol Linti }\end{array}$ \\
\hline Total HIons & 7. $2 \mathrm{~A} E-0 z$ & 10 & $3: 51-05$ & & & 中T & $7,70-12$ & 19 & 3n- & 7 & 41 \\
\hline
\end{tabular}

Final Report

Het JeAter

Page 6

simint ohrd stersou

Pout 16 a 1d 272-s

orotocod 5\$1

versian an

Fom uㅏㅁㅐ

Yation Repert dect pitsuct 
HAN-SD-KN-DP-Z38, REV.O

ZZZ-S LABORATORY

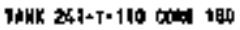

DUPLICATE

son 2ratit

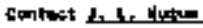
DTLIChTE

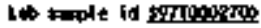

onpt anple id ctiorin

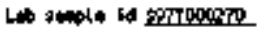

bept ample 1d

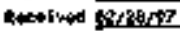

ctient thits

1mat r-110

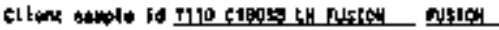

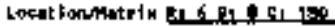

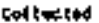

thin of euptody las

\begin{tabular}{|c|c|c|c|c|c|c|c|c|c|c|c|c|}
\hline AraLITE & $\begin{array}{c}\text { oupldch16 } \\
\text { uctfy }\end{array}$ & $\begin{array}{c}\text { 2. IPV } \\
x\end{array}$ & 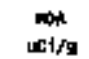 & $\begin{array}{c}\text { Mpl } \\
\text { ukiffo }\end{array}$ & $\begin{array}{l}\text { OHA B : } \\
\text { FJEs }\end{array}$ & IESI & 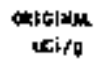 & $\begin{array}{c}\text { to TWU } \\
z\end{array}$ & $\begin{array}{c}\text { mas } \\
\text { uCisg }\end{array}$ & $\begin{array}{l}\text { and.]. } \\
\text { FIERg }\end{array}$ & $\begin{array}{r}m p D \\
x\end{array}$ & $\begin{array}{l}\text { 30 कीOT } \\
\text { TET LIMLT }\end{array}$ \\
\hline Toter ilpho & $7,6 \sigma^{-1}-\phi$ & 20 & ·.2h-18 & & & AT & sitto $\infty z$ & 19 & t.2E-tras & & 16 & 47 \\
\hline
\end{tabular}

\section{FInal Report}

DUPLICATEg

Pope $?$

BicengY DATh SECTKN

poy 17
Lob id Rz-s

Protocal FY

Yaraton 10

Poth therpens

Harzion 5.6

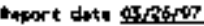




\section{2-S IABORATORY}

THWX 261-T-11 c00 160

MATRIX SPINE

Exo spotoll:

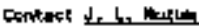
murix SP1दE

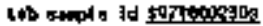
Dept mpta ta arenum.

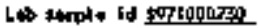

Depe sarpl* Id

hecrived 00rista? tol itrt D...X

I that T. 110

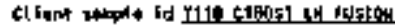

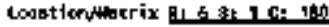
obltotid thin in of cutcosy did

\begin{tabular}{|c|c|c|c|c|c|c|c|c|c|c|c|c|}
\hline MULTtE & $\begin{array}{l}\text { SPIWE } \\
\text { uxifa }\end{array}$ & $\begin{array}{c}2 \times \text { IfU } \\
x\end{array}$ & netro & $\begin{array}{c}\text { BOL } \\
\text { stits }\end{array}$ & 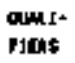 & ITSF & $\begin{array}{l}\text { ADOED } \\
\text { CAit/ }\end{array}$ & $\begin{array}{c}2 \pi \mathrm{et} \\
x\end{array}$ & $\begin{array}{c}\text { ORIEIMAL } \\
\text { utifra }\end{array}$ & $\begin{array}{c}20 \text { TPU } \\
x\end{array}$ & 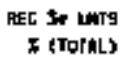 & $\begin{array}{c}\text { PROPOWL } \\
\text { L]MIT\$ }\end{array}$ \\
\hline fold Alphs & 1,050 d1 & & & & & M & - f.8. & 5,0 & $4,67]=-\phi 4$ & t; & 5 \$. 6.115 & $\bar{\gamma}=12 \pi$ \\
\hline
\end{tabular}

Final Report

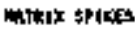

Pros 1

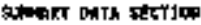

popt is ub id m2-8

stak따어 15T

version 1,0

Fore worte

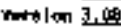
heport tore esizhich 
HWF-SD-WM-DP-238, REY, 0

\section{2-S LABORATORY}

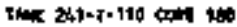

HATRIX SFIKE

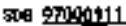

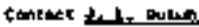
nhilerx EPIKT

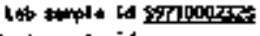

Dept aspot id

21

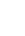


HNF-SD-WM-DP-239, REV. 0

\section{2-S IABORATORY}

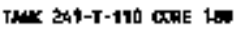

smatious:

内人tRIX SPITE

\$4 97000111

contpex tr tuare Whithr 由likt

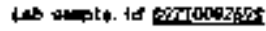

Bept Emale id ohtilnul

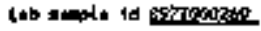

Dept entrof id

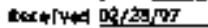

다 Eins twit

Tank I-11]

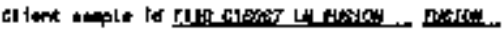

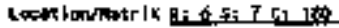
Colloctod Choin of cunkady id

\begin{tabular}{|c|c|c|c|c|c|c|c|c|c|c|c|c|}
\hline MMALTTE & $\begin{array}{l}\text { *FIrE } \\
\text { uctide }\end{array}$ & $\begin{array}{c}30 \mathrm{~W} \\
\mathrm{x}\end{array}$ & $\underset{\mathbf{m}}{\mathbf{m e d}}$ & $\begin{array}{c}\text { QDL } \\
\text { uCIJIG }\end{array}$ & $\begin{array}{l}\text { DU:4l- } \\
\text { Fteks }\end{array}$ & TEgT & $\begin{array}{l}\text { voso } \\
\text { wetis }\end{array}$ & $\begin{array}{c}\text { 五 likt } \\
x\end{array}$ & 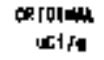 & $\begin{array}{c}2 \mathrm{IPH} \\
x\end{array}$ & 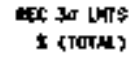 & $\begin{array}{l}\text { cortots } \\
\text { LINI TS }\end{array}$ \\
\hline Total Alphn & 申.tited on & & & & & dT & $1 . N a t$ & 5.0 & 7. $78 E-02$ & 10 & $53 \quad$ B. 114 & 75-1ES \\
\hline
\end{tabular}

\section{Finel Report}

\section{NAthan sptits}

Dist 3

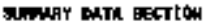

Pro 20

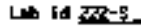

Prokesol हबा

yorsion 1.0 Fore $240=18$

Yertion 3.00 enpert antw 마. 


\section{HWF-SD-MN-DP-2S8, REV.O}

\section{2 $2-S$ LABORATOR}

TANR 241-T-3JO CORE d BO

s975000310

$D A F A$ SE E T

Tl10 cftost Let russol

sor gropous

cantagt r. T. Hasm

Lat sampla td sepropolatio

begt dample $x$ I

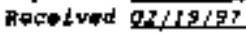

trfent TURS

Tank Thelp

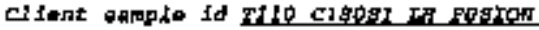

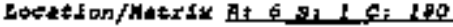
Collected

chain of antiofig id

\begin{tabular}{|c|c|c|c|c|c|c|c|}
\hline AYAITTE & CAS NO & $\begin{array}{r}\text { REStUtS } \\
\text { UCLI/g }\end{array}$ & $\begin{array}{c}\text { to } \\
4\end{array}$ & 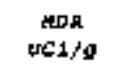 & $\begin{array}{c}R b B \\
w C 1 / g\end{array}$ & $\begin{array}{l}\text { QUREt- } \\
\text { FIRRg }\end{array}$ & TSR \\
\hline Toral Alpht & $22567-46-1$ & $+.47 E-ه 7$ & 27 & $3+15-03$ & & & $\boldsymbol{\lambda} \boldsymbol{z}$ \\
\hline
\end{tabular}

$F+1 \neq 1 \quad R \in p o r t$

DeTA SHEETS

Page 1

SUHNARY DXTA SERTION

Page 21
Lath if $222-5$

Protoent 곡

Yertion the

Foxm DYN-AS

Soraion 3.DS

separt date od $12 d / 2)$ 


\section{HNF-SD-WH-DP-23B, REV 0}

$2 Z 2-S$ LABORATORY

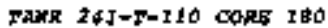

DITA SHEET

TII clsosa 라 FUtROA

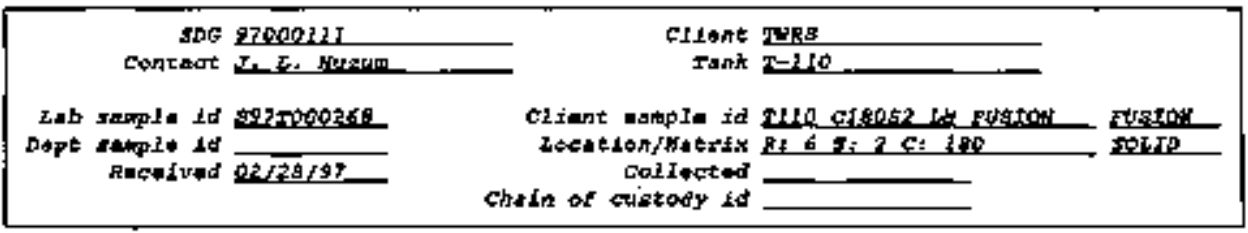

\begin{tabular}{|c|c|c|c|c|c|c|c|}
\hline AHAEMTE & ches wo & 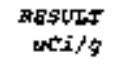 & $\begin{array}{c}20 \mathrm{TPQ} \\
t\end{array}$ & $\begin{array}{c}M D A \\
\mid N C i / g\end{array}$ & $\begin{array}{c}A D L \\
u c i f g\end{array}$ & $\begin{array}{l}\text { OUALI- } \\
\text { FIERs }\end{array}$ & rsst \\
\hline Potal Alpha & $12587-46-1$ & $1.41 t-03$ & 22 & s.fX-OS & & & $A T$ \\
\hline
\end{tabular}

FInat R日pot

DAIA SHEETS

Page 2

SUAMARY DATh SECTION

Rงgฺ 22

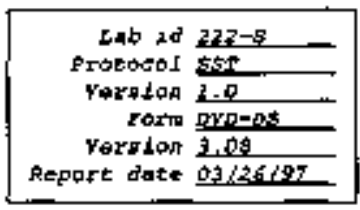




\section{HNF-SO-WM-DP-238, REV, 0}

$222-S$ LABORATORY Tath 24I-T-110 CORE 180

\section{DA TA SHEET}

TI20 c580s3 in roston
\$97Pa0023I

\section{CIIOHF TWRS}

$T$ ent $z=110$

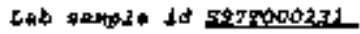

Dept tarple id

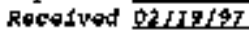

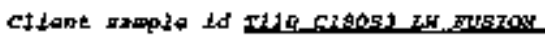

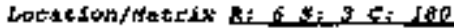
collactipd chari of chetody ita

\begin{tabular}{|c|c|c|c|c|c|c|c|}
\hline whtהerrs & CAAS No & $\begin{array}{r}\text { RESDET } \\
\mathrm{wCi} / \mathrm{P}\end{array}$ & $20 \mathrm{TPU}$ & $\begin{array}{c}\text { ADA } \\
\text { aCi } / \mathrm{g}\end{array}$ & $\underset{a C d / \theta}{R e r}$ & $\begin{array}{l}\text { QVAEI- } \\
\text { FIERS }\end{array}$ & FEst \\
\hline Total slphat & $12987-46-1$ & 2. $-5 A E-D 2$ & 25 & $9.05-09$ & & & $A T$ \\
\hline
\end{tabular}

FIn 1 RAport

\section{DATA ' SHESF'}

Paga 3

SUMAART DAPA SECTION

Pagा 23

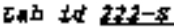
Protatol sist

vereson Lo Pars pUD=05

Vorpion $3.0 B$ deport datu attafty? 
232-S LABORATORY Ftadx 2dJ-F-110 cokt 180

DA TA S HEET
TIIO Clposf HA FUSTOH

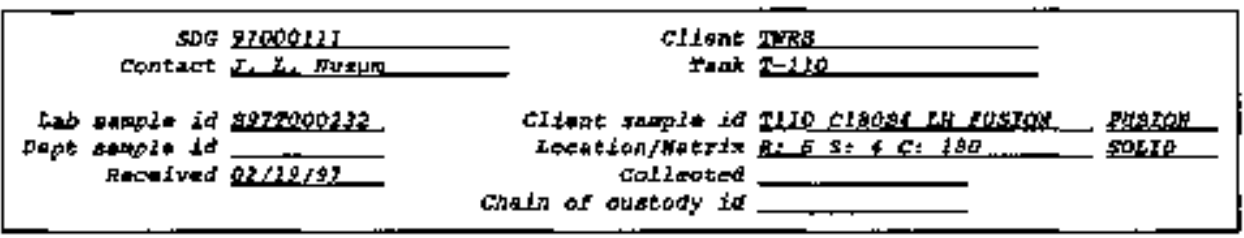

\begin{tabular}{|c|c|c|c|c|c|c|c|}
\hline ANAEYTE & ors aro & $\begin{array}{r}\text { RESULT } \\
\text { UCi/g }\end{array}$ & $\begin{array}{c}20 \operatorname{mov} \\
\mathrm{t}\end{array}$ & $\begin{array}{c}\operatorname{mox} \\
\omega C i / f g\end{array}$ & $\begin{array}{c}\text { RDZ } \\
D C i / \theta\end{array}$ & $\begin{array}{l}\text { OUALI- } \\
\text { FIERS }\end{array}$ & TEST \\
\hline Total Alpha & $12587-16-1$ & 4.58E-03 & 37 & $3.78-03$ & & & AF \\
\hline
\end{tabular}

FInal Report

\section{DATA SHEETS}

Pag* 1

SWHAHRY DATh SECTION

F*⿻丷: 24
Latb id 727-5 Prototol t55

Yargion 10 FODN DVP-RS

Vartion 2 as:

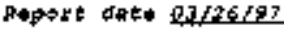


$222-5$ IABORATORY

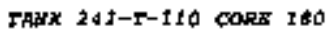

DA A SHEBT
Tifo clgost f th fustow

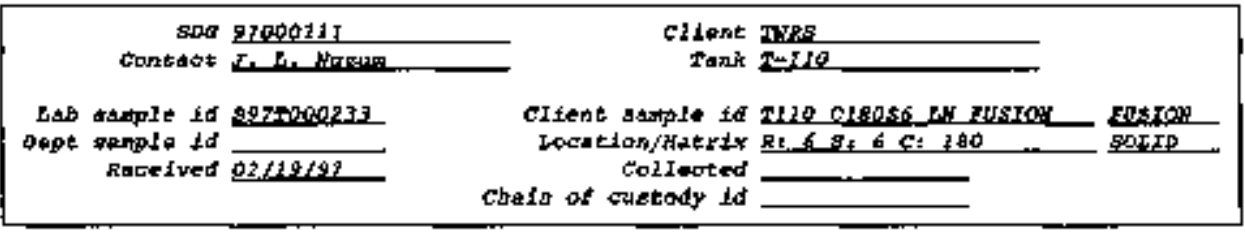

\begin{tabular}{|c|c|c|c|c|c|c|c|}
\hline ANAEYTE & cas to & $\begin{array}{r}\text { RESULT } \\
\text { UCI/g }\end{array}$ & $\begin{array}{c}20 \mathrm{TPO} \\
t\end{array}$ & $\begin{array}{c}\text { Mad } \\
\text { LCEfg }\end{array}$ & $\begin{array}{c}R p d \\
\mathrm{aCI} / \mathrm{g}\end{array}$ & $\begin{array}{l}\text { ONTI - } \\
\text { ZIBAS }\end{array}$ & FESP \\
\hline rotal hlpha & $12587-48-1$ & $4.04 t-03$ & 24 & $4 . a z-03$ & & & AT \\
\hline
\end{tabular}

FInat Repot

DAFA ANEST

Page 5

SUJMARY DATR SECTYOM

P*90 25

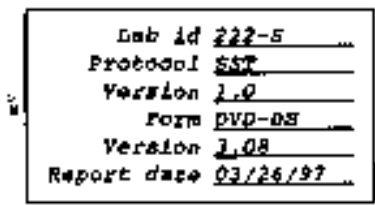


$222-B$ I $A B O R A T O R Y$ raxk 2dJ-F-I10 cors 180

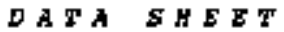

Tise clgos7 LN EUtsor

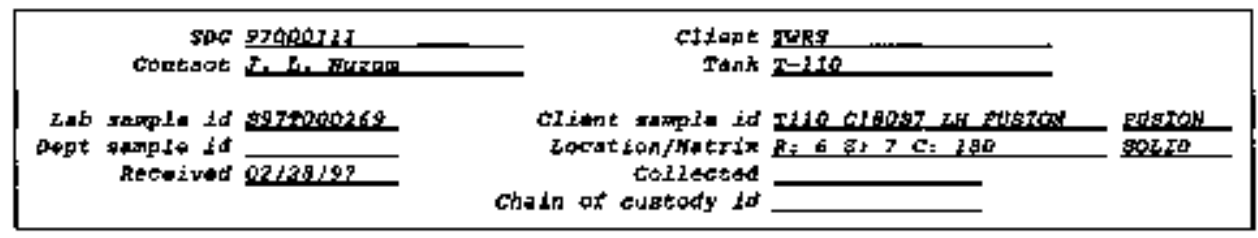

\begin{tabular}{|c|c|c|c|c|c|c|c|}
\hline MWAEYT & Eus wo & $\begin{array}{r}\text { pesstar } \\
\text { vel/s }\end{array}$ & $\begin{array}{c}70 \mathrm{TP} \\
8\end{array}$ & $\begin{array}{c}\operatorname{Mox} \\
\operatorname{tec}[\mathrm{f} / \boldsymbol{\sigma}\end{array}$ & 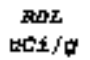 & $\begin{array}{l}\text { porari- } \\
\text { prefs }\end{array}$ & TEST \\
\hline Total Alpha & $1.187-46=I$ & $3.78 \mathrm{~B}=02$ & 19 & $3.28-03$ & & & $A r$ \\
\hline
\end{tabular}

FinaI R日port

DATA SHEETS

PAOt

SWNWARY DATA SECTIOM

pape 26
Lab id 22\}-s Protacol EST

Verradon B.Q. Form DrDas

verglas 胥.09 Raport date $0 \mathrm{~s} / 26 / 9 ?$ 


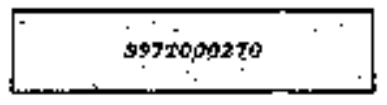

\section{Z2Z-S LA日OAATORY

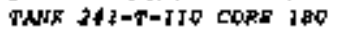 \\ DA TA SEE T}

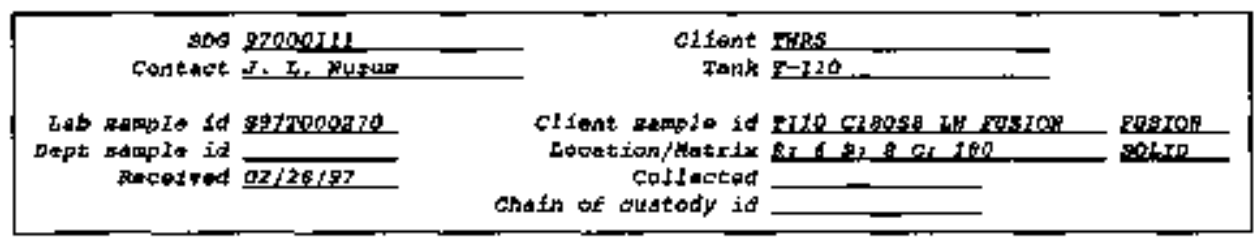

\begin{tabular}{|c|c|c|c|c|c|c|c|}
\hline Axhtras & CAS No & $\begin{array}{r}\text { REgJLT } \\
\square C 4 / g\end{array}$ & $\begin{array}{c}20 \mathrm{TPU} \\
\mathrm{t}\end{array}$ & $\begin{array}{c}M D A \\
v C i / g\end{array}$ & $\underset{\text { vet } i / g}{R p L}$ & $\begin{array}{l}\text { QWALI- } \\
\text { PERS }\end{array}$ & Thes \\
\hline Total repk & $125 * 2-16-1$ & $0.960-04$ & 19 & $\$ .28-a z$ & & & r \\
\hline
\end{tabular}

Inal Rqport

DATA SNEETS

Page 7

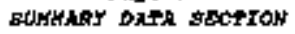

Fage 27
T120 treose La rotrol chath of dustody io 


\title{
222-5 LABQRATORY

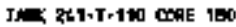 \\ NETHOD SWhHARY

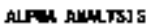

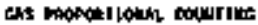

c11ent Mats

Twit I+11e

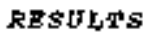

c Ims stipur in

-raporar lon bareh proposa.

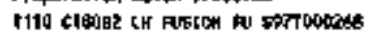

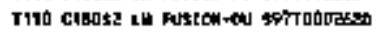

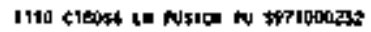

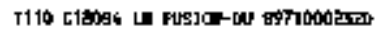

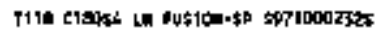

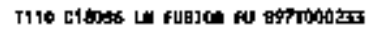

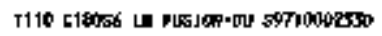

warhod Blutik

E17065.2

Lto fontrof esmpla

\$1705. 1
\end{abstract}

L) RW DUF.

1) Toted

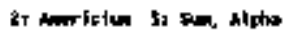

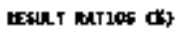

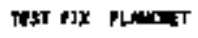

Mlphe

zit (CESA)

cantopis

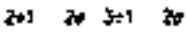

Prequation wateh shoosst

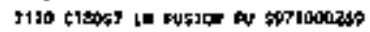

T110 [1703s L fubjo

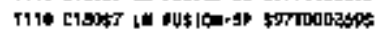

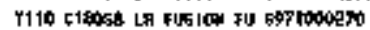

J190 c190;

Mrthod tienk

由17056?

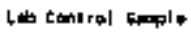

$\$ 17058-1$

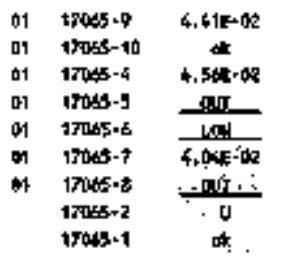

Preperation bureh groocios

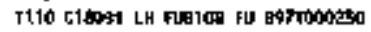

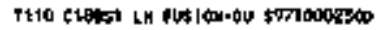

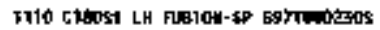

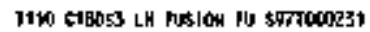

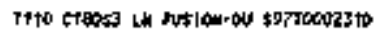

butiod Elant:

घ170र2-द

Lob tontrol Baple

s170re-1

\begin{tabular}{|c|c|c|}
\hline$\$ 1$ & 37068 - & $7.716=06$ \\
\hline$m$ & 1Ptod-5 & ar \\
\hline 어 & $17068-6$ & L此 \\
\hline or & $1700,7=7$ & 由. \\
\hline \multirow[t]{3}{*}{ or } & J7068-b & ak \\
\hline & 17tost-2 & $\mathbf{v}$ \\
\hline & 17068.1 & ak \\
\hline
\end{tabular}

weirad wollap and linets froe nethod

\begin{tabular}{|c|c|c|}
\hline od & $17012-7$ & 4:4TE-R \\
\hline 08 & $17072-4$ & ok \\
\hline oe & $130 h=\phi$ & سمبا. \\
\hline $\mathbf{\alpha}$ & $17072 \cdot 10$ & $2,28 t+12$ \\
\hline \multirow[t]{3}{*}{$\phi 8$} & $17078-11$ & arr \\
\hline & $180 r x-2$ & $v$ \\
\hline & 170?2=1 & ak \\
\hline
\end{tabular}

Final Report

VEThos sumurtes

Pag 1

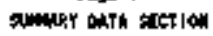

28

wa ld खियद्

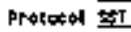

Yeoraton 10

rorm pyouctst

neratan 3.0.

Report ther os/2t/q7 


\section{己゙̇ず LABORAFORY}

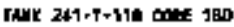

Test et motrik ardo

The Tस्W0111

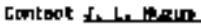

METHOD SUPHARY

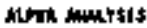

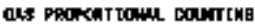

tlint tits

rark I-119

\section{HETHOD PERFORHANCE}

Wh the tif-

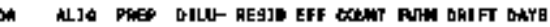

Nure-

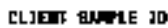

MUPLE IO

IET FIX ucifo

a the ritow

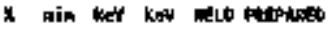

IIED Berreto

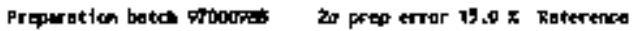

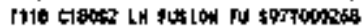

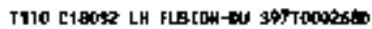

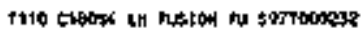

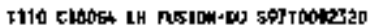

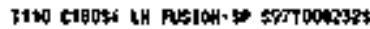

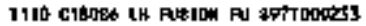

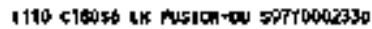

Mothod Blart

B170st-2

Lth tomtrod soped

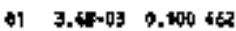

01 3.3E-05 0.100 करो

of 3.74-03 0.100500

01 4.tectos 0.100 566

व1 $0,100 \mathrm{s00}$

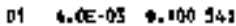

$01 \quad 3,84-03 \cdot 100 \operatorname{ses}$

$3, f_{1}=-05 \cdot 100 \mathrm{sm}$

i. $\pi 00 \times 1.00500$

$\begin{array}{lll}1,00 & 27 & 30 \\ 1,00 & 27 & 37 \\ 1,00 & 27 & 30 \\ 1,00 & 27 & 30 \\ 1.00 & 27 & 59 \\ 1,00 & 27 & 30 \\ 1,00 & 27 & 50 \\ 1.00 & 27 & 30 \\ 1.00 & 27 & 30\end{array}$

(65) $/ 13297$ 03/19

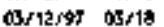

ब3/18/27 Do/19

$03 / 2,97$ 03/ 1 \%

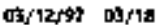

Qs:

$03 / 12 / 97 \quad 03 / 19$

(1)

$\mathbb{C O}_{j} / 10 / 97$
Lecteps

noritid

Lectorab

inertous

Lafizentos

inarate

Lactens.

vations

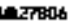

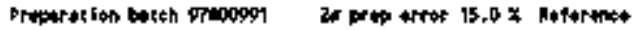

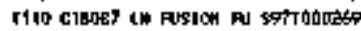

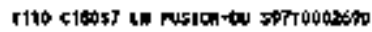

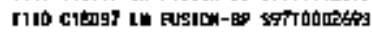

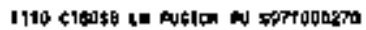

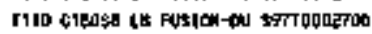

method Blart

D1706e-2

it toritrol saphe

$\pm 1706+1$

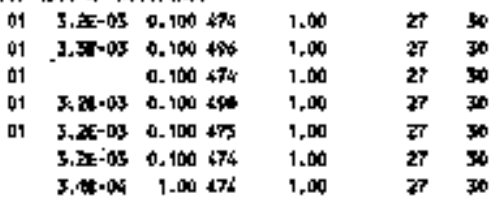

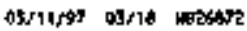

$03 / 1978$ 03/14

os 11 ist of

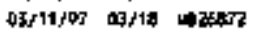

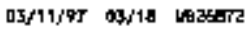

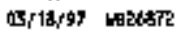

따/1940 LARSET?

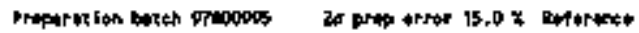

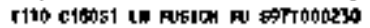

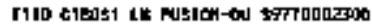

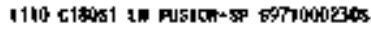

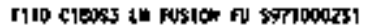

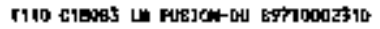

Whthod tolunk

Lub Control sompl.

$179 \% 2$

s170:-1

\begin{tabular}{|c|c|c|c|c|}
\hline o 2 & 3, w. & 0.100513 & 1.00 & 27 \\
\hline ㅁℶ & 3. 단-05 & $0.100 \$ 13$ & 1.00 & 27 \\
\hline 02 & & D. 100513 & 1.00 & 27 \\
\hline 02 & $3.00-05$ & 0,100400 & 1,00 & द7 \\
\hline 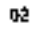 & 3.DE- & 0.100694 & 1.00 & 27 \\
\hline & $3,04-03$ & 0.100 & 1.00 & 27 \\
\hline & 3. 05.05 & $1.00 \mathrm{LBt}$ & 1.00 & 27 \\
\hline
\end{tabular}

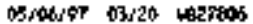

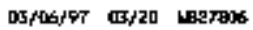

03sowis7 03/20 wazk06

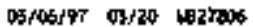

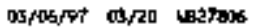

osfary wratos

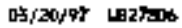

\section{Final Report}

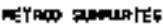

ㄴat $?$

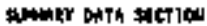

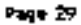

Lab 1d 2323-t

Protocol sitit

vertien 1.0

Forn 0.8-9:

repalan 300

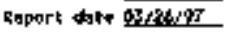




\section{$222-S$ LABORATORY}

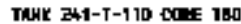

Tat ax watrdx

$\rightarrow 0$ intodt1

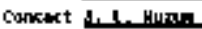

\section{NETHOD SWHART, cont.

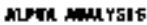

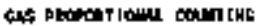

cI tert In:s

Int $T-110$

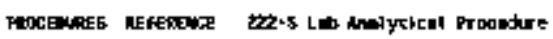

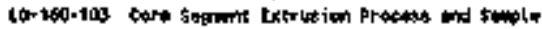
Prepacaxion, Rav it

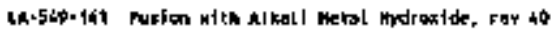

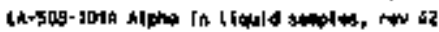

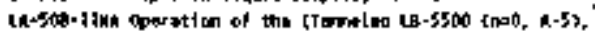

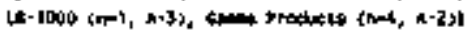

Alphar Bect Gounting brstors
AYERLGS 2 in

for 25 sules

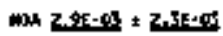

thictentr 23 -
FInal Repoxt

NETWO STEARIES

Page 3

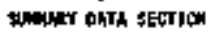

powt 30
L由 bat zaz-t

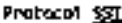

Version $1 . t$

Jore

varsion s.r.

Report date $63 t^{2} 6 \% T$ 
$22 \boldsymbol{2}-5 \quad$ I A BORATORY raNK 24J-T-150 Lase 190

stis g7000

confact T. L. Pantam
REPORTIIDE

SANPLE SUMHAY
CILent Gist

Tank $T=110$

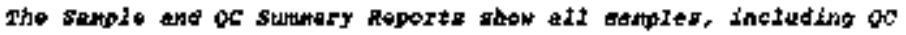

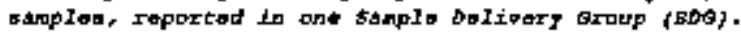

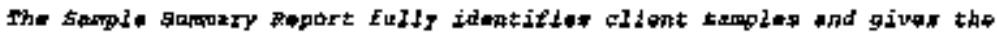

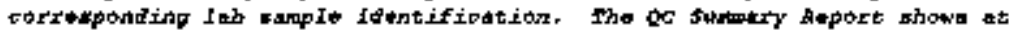

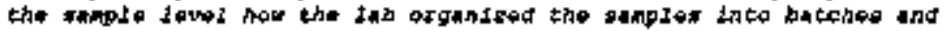

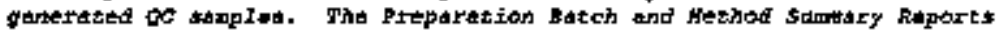
ghow thif at the anatyais levol.

The followith sotas apply to these reporte:

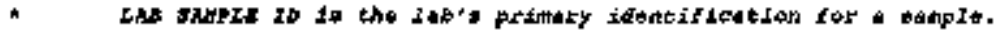

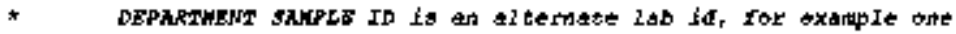
asalgned by * radianberiatry degaztmont in a lab.

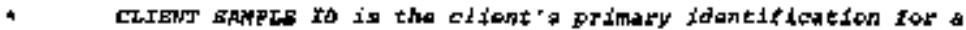

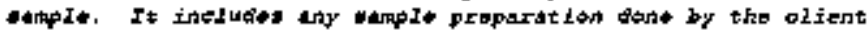
that is necessary to tdentily the sanple.

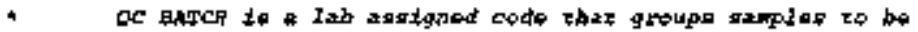

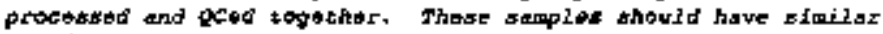
matricog.

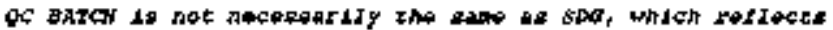

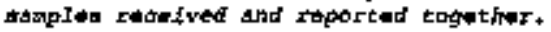

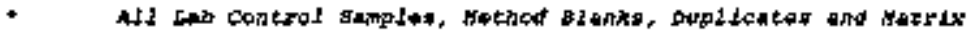

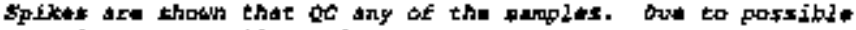

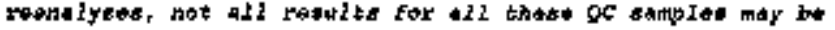

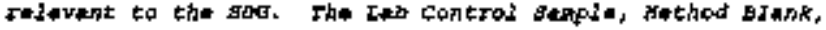

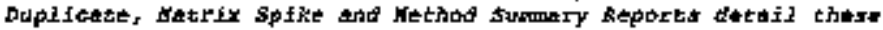
rel at torphipr -

FInt Ragot

\section{REPOAT Gorors}

Pay* 2

GLMHARI DATA GECTIOA

Pag* $3 I$

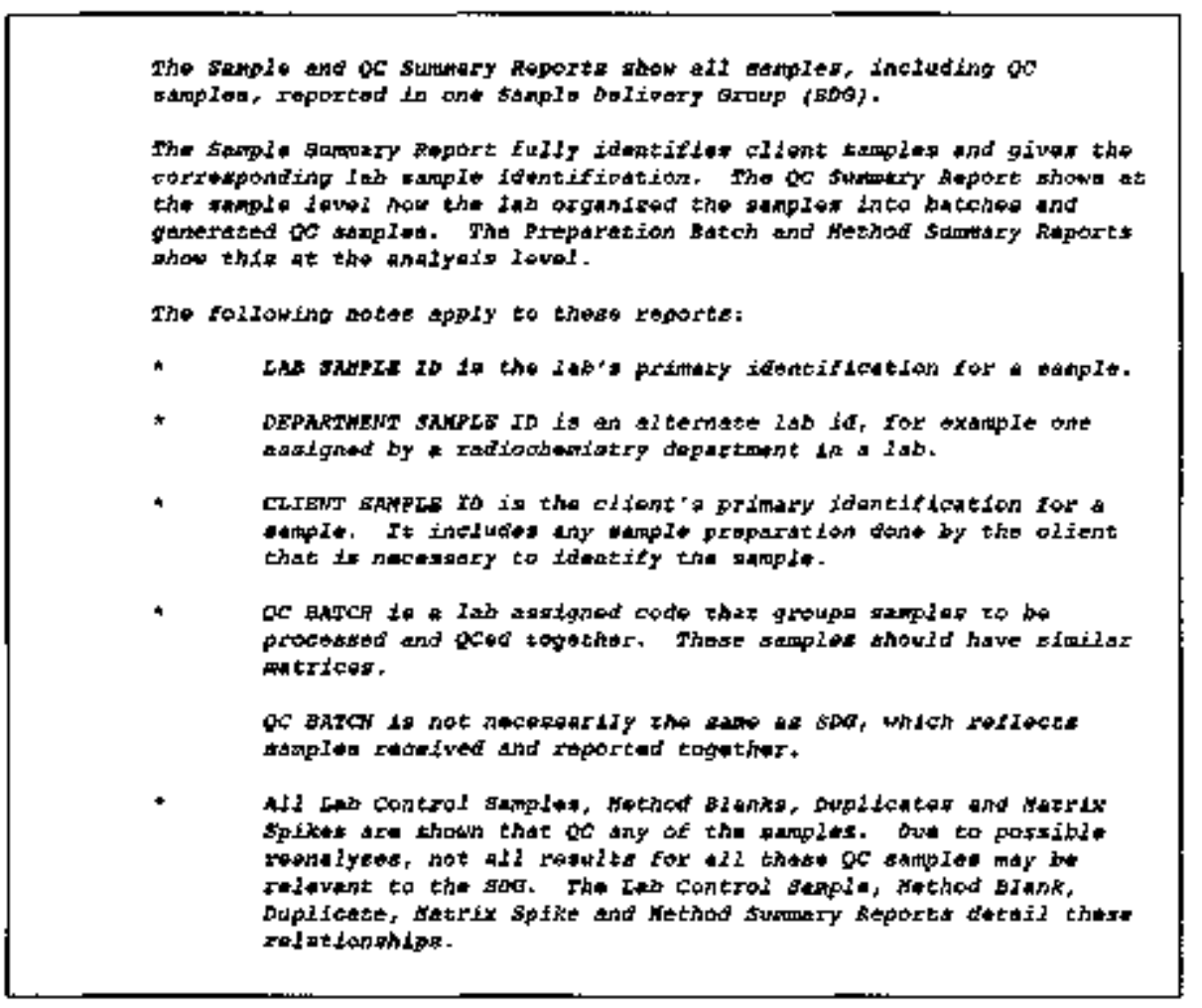


$222-5$ I ABOAATORY

spa g70003n.

contact I. Th Nareur
TANR 24I-T-IJO CORB I80

REPDRT GUIDE eltant تصRS -

rastk T-110

\section{FREPARTIOHARTH SUMNARY}

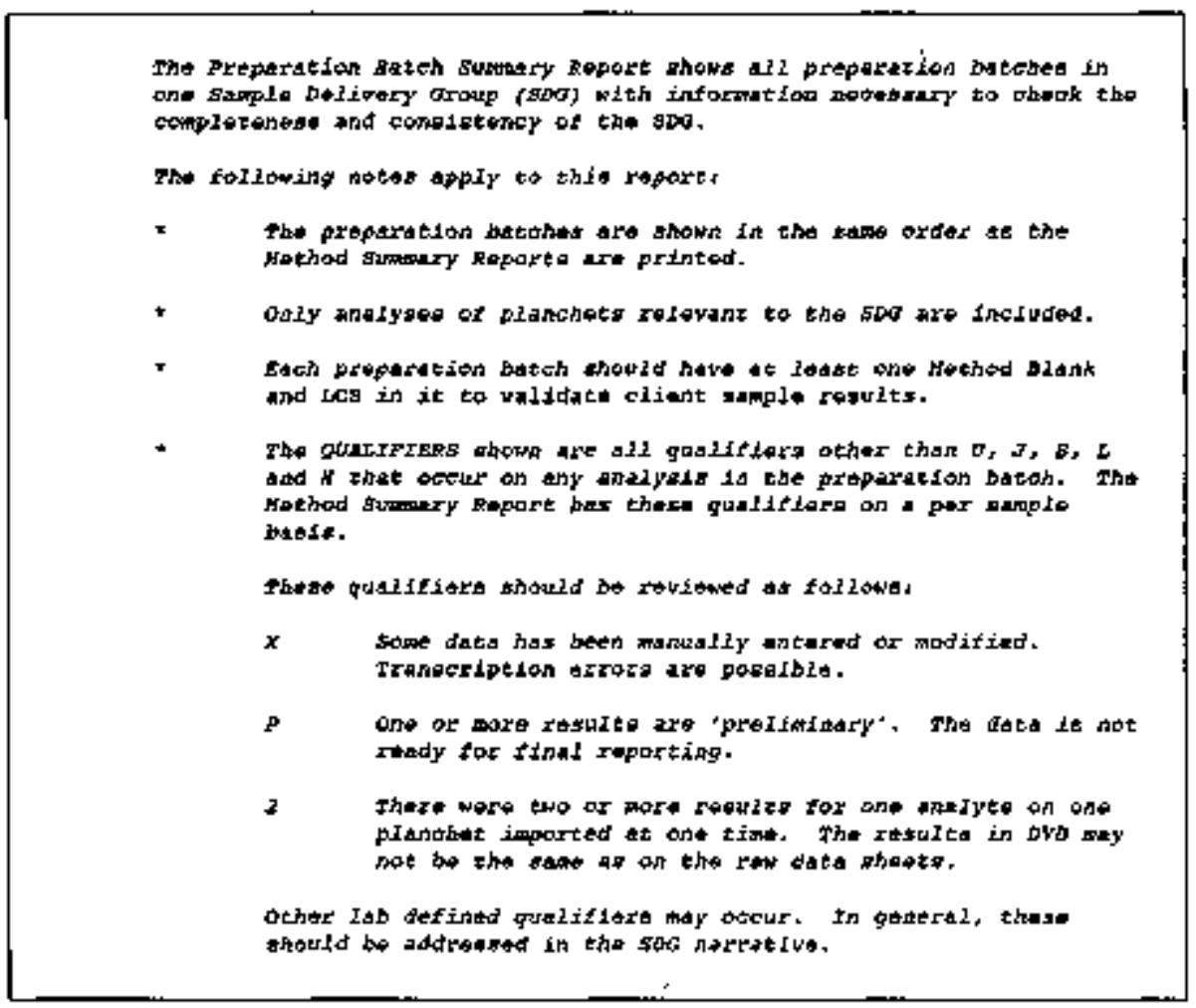

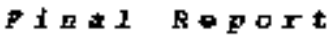

REPORT eUtoE'

page 2

STUNARRY DHTA SBRCTION

Eage 33

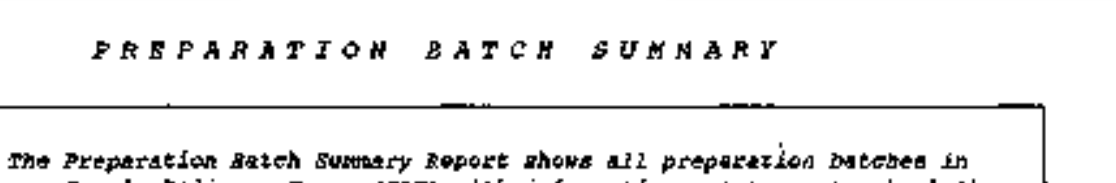

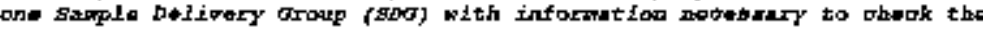

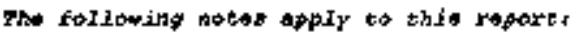

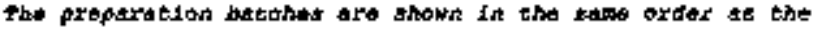

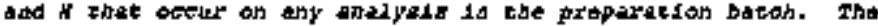
napiote

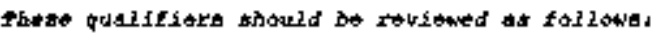


$22 z-s$ IBORATORY

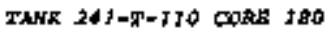

sot gropout

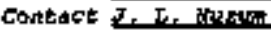

REPORT GUIDE

cliert gyas

Fark $\mathrm{I}-\mathrm{I} 20$

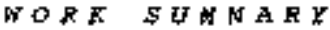

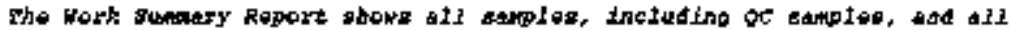

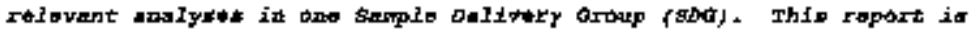

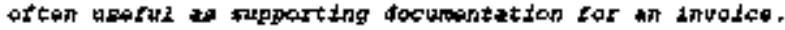

The following nater apply to this ropars:

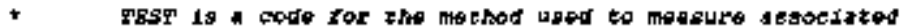
andytor. Results asd zaluted infozmution for each andyta

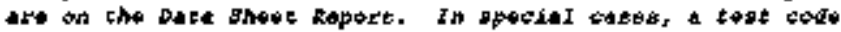
ared in the mumary data section it not the sam an in

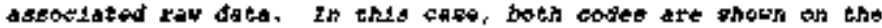
Tozk fumsary.

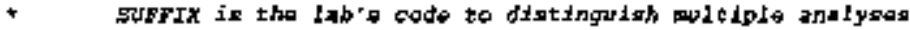
[reoounts, coworks, reanalyas, of a fraction of the osmplo.

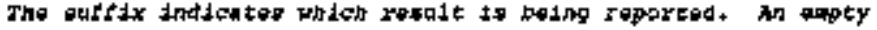

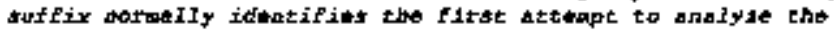
>apipas.

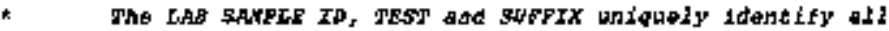

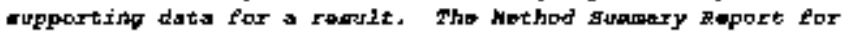

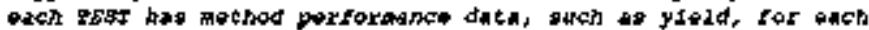

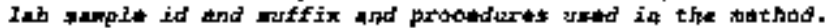

$\bullet$

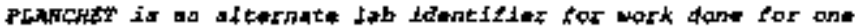
tott. It, combited with th TEST asd surFIX, way bo the best tefate to rau data,

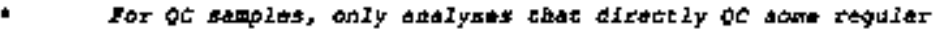

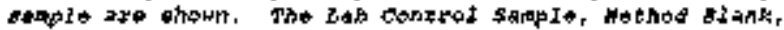
Duphlade, Natrix spike and pethod sumary kaportg datail these reIat fonshipg.

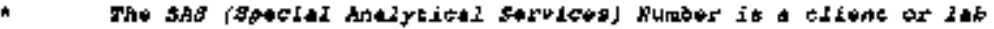

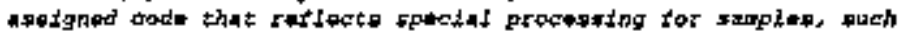

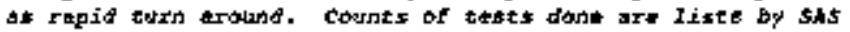

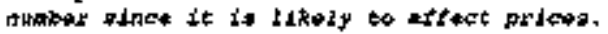

\section{$\forall 1 \Delta * 1 \quad R * p \circ r t$}

\section{REPDRT AUTDES}

Fage $\rightarrow$

SUMaRY DATA SECrYon

קage $\$ 3$
Int $20272=6$ Protacol gars

Vorsfarc L. D FOFI PVO-RG

Vereion 3.07 Aqport date a $93 / 26 / 97$ 
$222-S$ IA BORATORY

TALS $241+T-110$ CORE 180

spe g7oporid

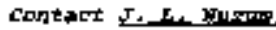

REPRT G

client TRs

sask toth

\section{DA TA SHE E T}

The data shet Aopart ahaws all rearlts and primary supporting

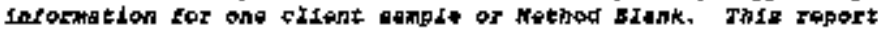
corrtaponds to both the CLP Iaorganiot and orvanics bath thate.

The following toter aply to this fapdoti

- TBSf to a aoda far the mathod used to meagure an analyte. If

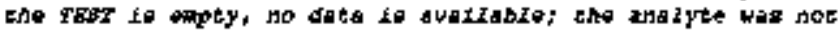
end hysed far.

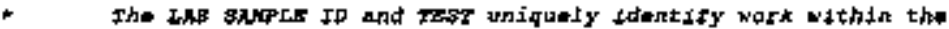

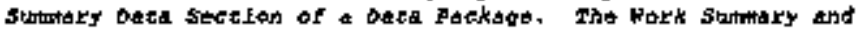

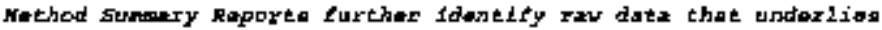
ente work.

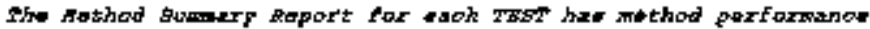

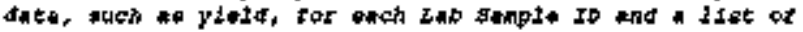
proutatures utead in the thethod.

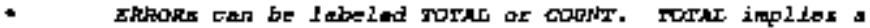
preperatiod (mon-countino mothod) orrer has boen soled, as

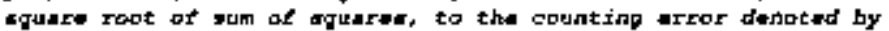

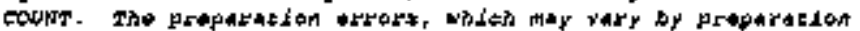
batch, art khowh on the Mathod surtming kepart.

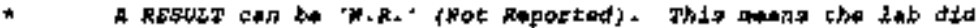

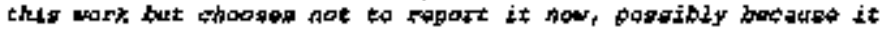
wal reported at anothor tinte.

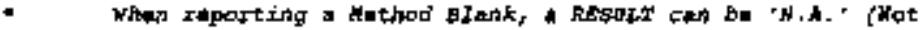

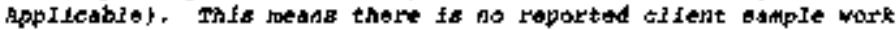
in tha ano preparation batoh as the Elank's rtault. This is

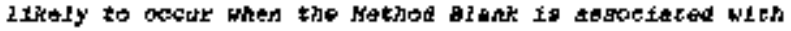

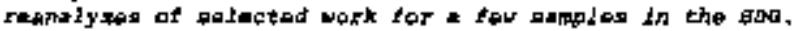

The coslowing quelifiers are detluad by the DVp aystem:

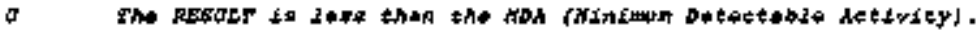

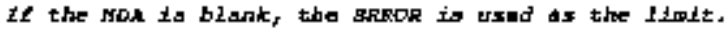

Final $R \in p$ at

REPORT LUTLES

Page 4

EUAGART DATA ARCTION

Page 34

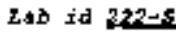

protocol ssist

Persian L.

Forat Rede-RE

Version 3,08 
$Z Z Z=S$ LABORATORY TANR 2+1-T-IJO GORE I80

aDo 2000111

Cordatet I, I, Myztrat
GUIB, CORt.

Cl7att Tres

Fank ThID

\section{DATA SHEET}

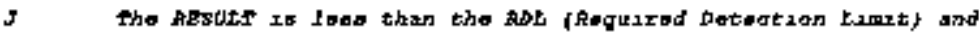
no U pulatiat is assigned.

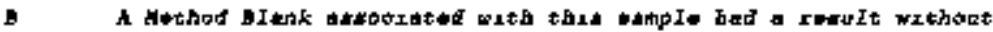

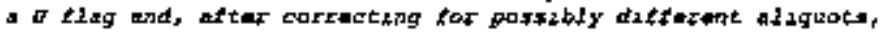

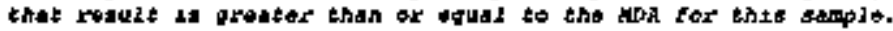

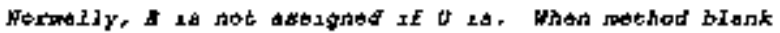

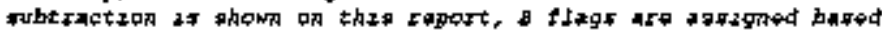

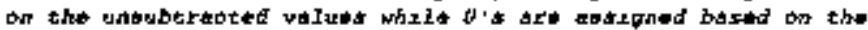

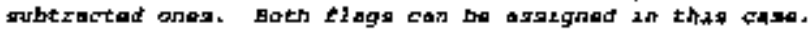

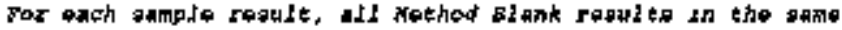
preparation batch are compared. The Hethod sumary Rapart focumonta thas ad other QC rolationabipa.

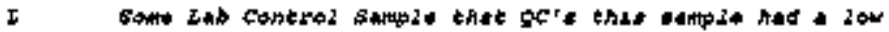

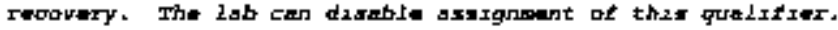

H gralar to ' $L$ ' arcapt tha zacovery was high.

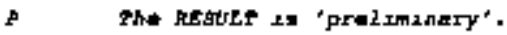

Y Same date nertmaxy to ongpute the RESOLT, BRROR or wDA mal manuzily gntered or modifref.

$\mathbf{2}$

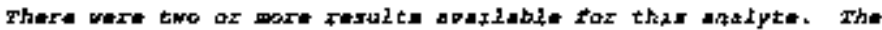

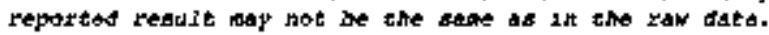

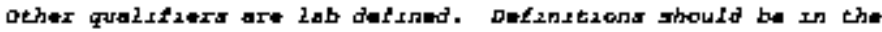
SDG harraceve.

Tho following ralues axe underlened to Imflicate porsible problassi

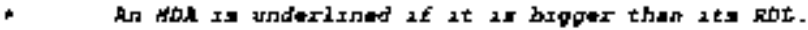

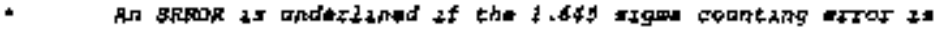

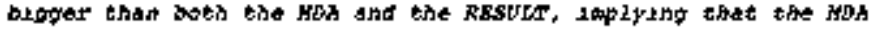

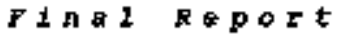

RAPURF OUIDS

Paga 5

SWriskry DגTh gtcrian

Page 3.5
Int b 하 $7777-8$

Pratacol s.94

Voroxin LID

Porr PYD-RB

Varason 3,0?

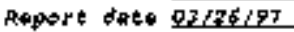


$z Z z-S$ LABORATORY

SOQ gT000HTh

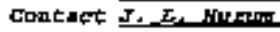

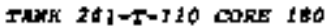

GUIDE, cont.
tIstort Tysf

rank Thlth

\section{$D$ T $A$ A HE}

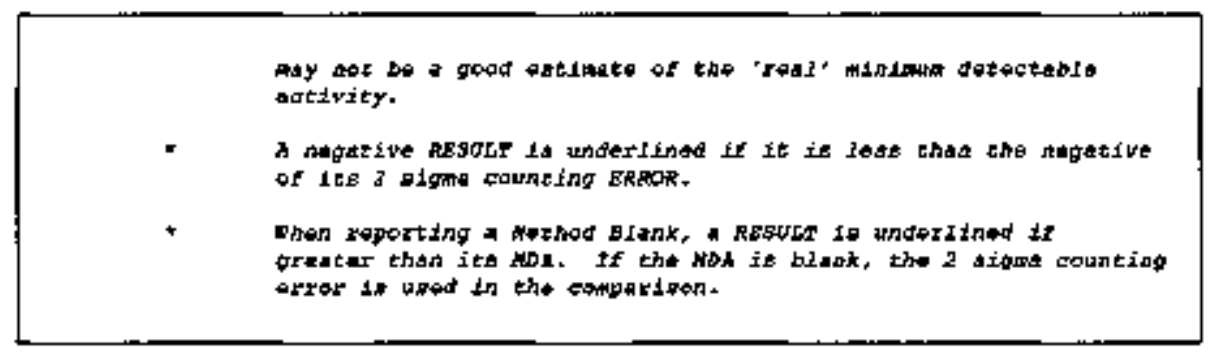

IntI R*Port

REPORT dUIOES

Fapt 6

STHAART DNTA SECTIOH

Page 36

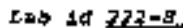

Protocol str

Fersion L.P

POLT PVP-Ro

Pararon Suth

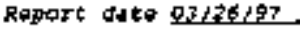


$Z Z Z-S \quad L A B O R A T O R Y$

soc gradow

Contact T. T. NyzuII
TAkK 24I-T-19A cole 190

REPORT GUIDE

\section{EIfort TrRg}

Tank T-zIO

\section{IAB CONTRDE SAHPIB}

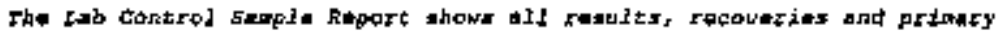
aupporzing litformation tar one Iab castzas ganpla.

The tollowing notas apply to thap rapart:

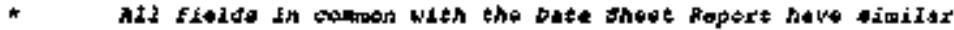

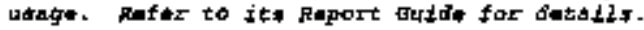

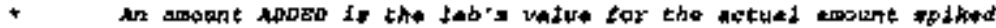

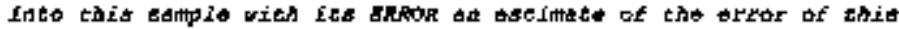
amount -

An amount added is unterlined it its ratio to the corrapponting

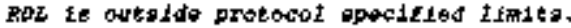

- REC (Rocovery) IS AEsULT Givided by ADbEO mpressed as a percont.

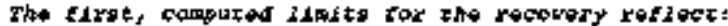

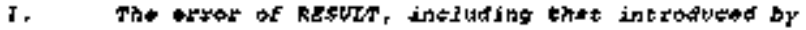
raending the rexult priar to priating.

If the lindtr are laboled fTorat, choy include

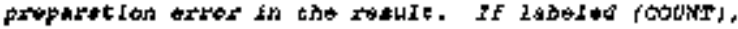
the g do not.

2. Tho Arxot of ADBEO.

3. $\lambda$ Iab specifieds per enalyte biad. The biad chenges the

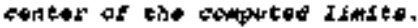

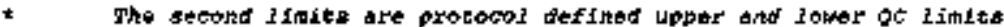
for the recovery.

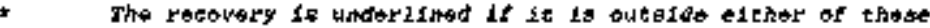
raspea.

- Labaratory control limita 2re definod in preceduro co-543-103.

\section{$P$ int $2 R \in p a x t$}

REPORT OUIDES

Page 7

SUGHARY DATत SECTYOM

page 3)
Lab id 2A2-s

Protocol 5.5T

$V=2010$ a th

FOIN OUD $=\mathrm{RB}$

vargion 3.0s

Aeport date axtapts? 
$222-5$ IA BORATORY

sod 27agonit

contact 士t tr Nyred
TANK 241-T-110 COAB 180

GUIDE, GOnt.
CLAOAE TyRs

rark $T-120$

IAB CONTROE SAMPIB

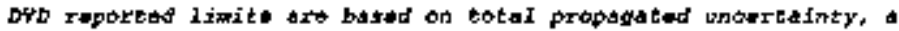

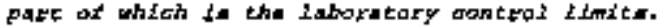

$F 1 \mathrm{~L}+\mathrm{R} * \mathrm{p} 0 \mathbf{r}$

\section{Rathr cutars}

Page 8

SOFMIRY DATA SACTIOR

Patre 3 t
L2b Lt" 272

Pratocal sEt

Varaion la For $\mathrm{BFD}=\mathrm{R}$ C

varion ich Reptrt dato 03/26/97 
$222=S \quad L A E O R A T O R Y$

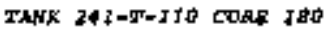

soo gropour

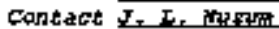

REPORT GIDE

CIfant TSBS

Tank Trise

DUPLICA T $E$

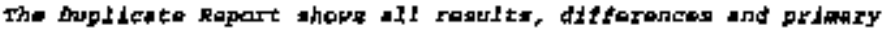

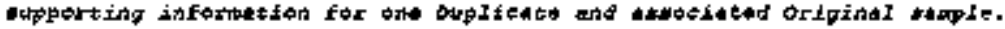

Tha tollowing notas apply to thio rapore:

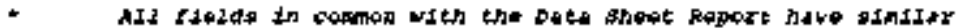

datape. This appliat bots to the Duplioate and driginal ataple

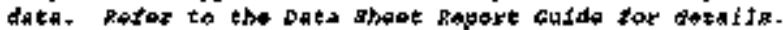

If the pupdtagte has data for a TEST and the 2ab gld hot do this

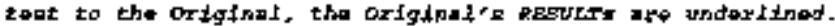

* The RPd fRelative Percent Difterencel lo the aboolute vadue of tha differtide of the hRgules divided by their average exprastad 4 a parcapt.

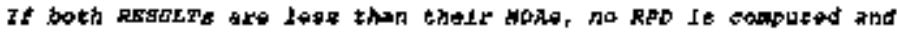
a 'Al it printed.

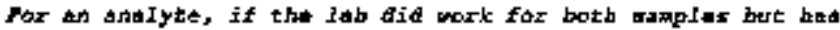

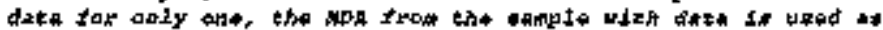
the othax's rexalt in the RPD.

- Tho firat, donpated linit id the mm, ad equare roat of arer of aglares, of the exrose of the readte divided by the average

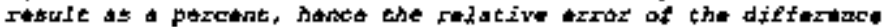

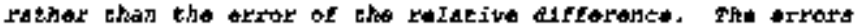

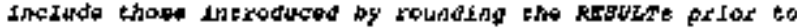
printing .

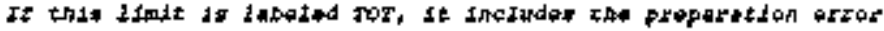

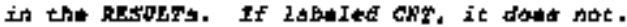

This value reported for thid ifrit in at most $g$ gh.

- The accond limit tor the Rph to the lazgoz of:

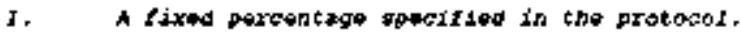

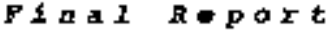

\section{REPORT GUtOEG}

Page $g$

STMARR DRTA BRCTION

Fage 39
$L A B$ if $2 x 2-8$ Protocel spre

varion to Pora DUD=RA

veraion 308

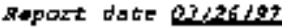


$222-5$ LABORATORY

TANK 24I-T-JJO COAS I80

$\operatorname{spos}$ grapot11.

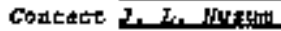

GUIDB, $=0 \pi t$.

ftint Twes

Thak $T=1$ ile

DUPLICATE

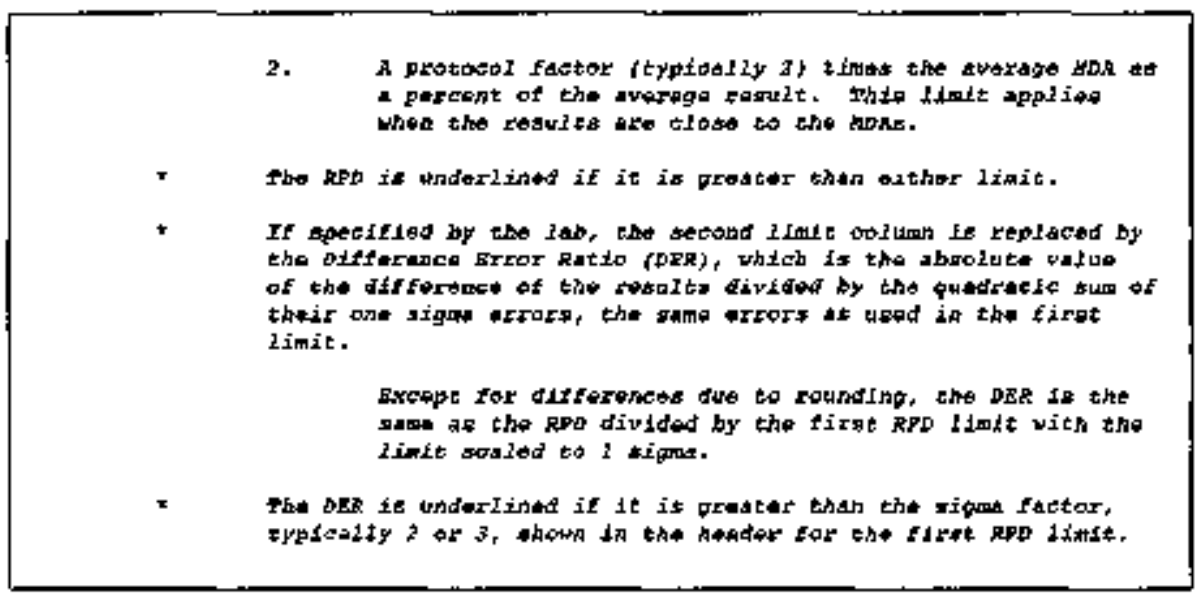

FIn $\approx 1 \quad R * p a r t$

SEPOAR AHTES

Page 10

SUMMARY DATA SECTION

eage to
Ineb Lt 2az=s

bratacal sat

Vorten La

Fornt QVD-BQ

Vurkion $3,0,9$

Rtport onto 03/26/97 
$2 Z 2=5$ LABORATORY TANK 24d-T=TJQ CORB IBQ

aDg $9+20001$ if

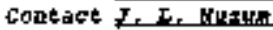

REPORT GOIDE

tLient TyAS

rank T-1RP

\section{HATRIX SPIXE}

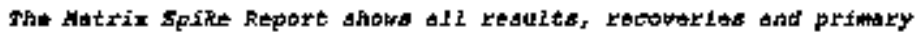

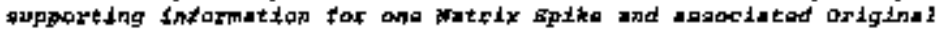
asminte.

Tha following notas apply to this zaports

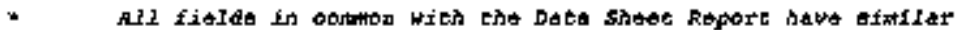

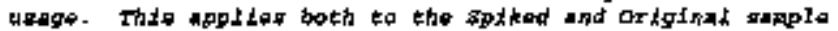
data. Aefer to tho bata shoet keport baido for details.

It the spike har data far a terat and the lab did rot do thix

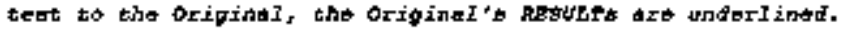

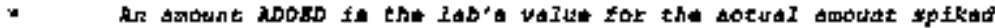

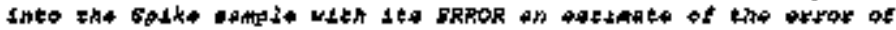
this anowat.

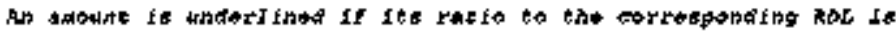
outside protacol mpecteted limitr.

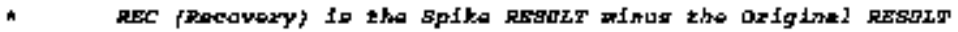

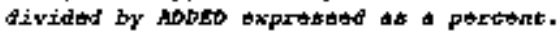

-

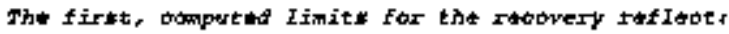

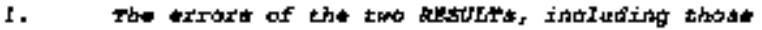
Introduced by rounding tham priar to priatine.

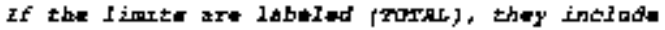
preparation arxor is the reture, If labeled fcowitys chay do not.

2. The extrox of ADDED.

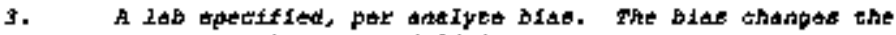
Gencer of the conputad isfitz.

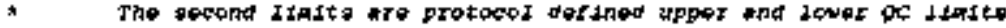
for the recovery.

Final Report

DEPORT GUIDES

Papt 11

SOUYARY DATA SBCTION

Dage at
Lab if $27 z-s$

Protocol 포다

Vartion de

rorT DYP-Ra

varston 3.00

Report date $03 / 26 / 97$ 


\section{HWF-SD-WM-DP-298, REV O}

\section{$22 Z-S$ LA SORATORY}

Thror 241-T-J10 COAB 580

sbo g700014.

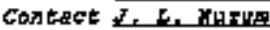

GIIEE, cont.

HATRIX GIRE cinte Thst

Iank r=IR

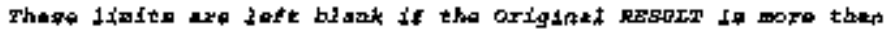

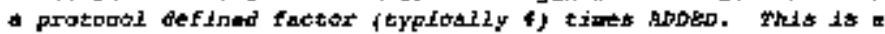

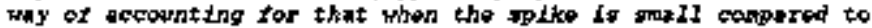

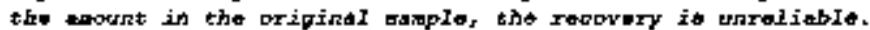

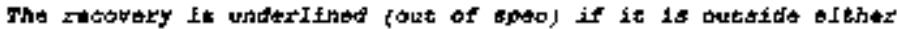
of thapo rengen.

Ftad Report

REPORT UUTOSB

Paste 12

gUt,

Pagd 12
Lab 1d $72 x=s$

Protocal 뽀난

Heraion 10

FOx DYP-RO

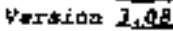

Rtpore det 오?/26/97 
$272-5$ I A B R H TAWR 24l-T-jJo CORE IBO

spa s7000다

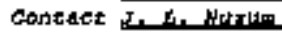

REPQR GUIDE

Cltant TFiㄹ

rank Trus

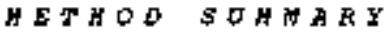

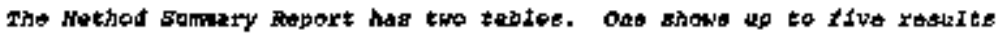

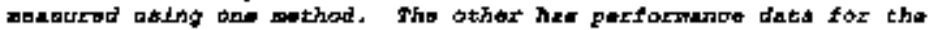

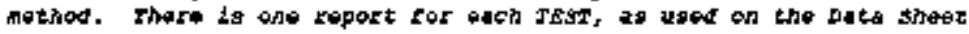
Reprot.

The toitowing noces mpply to this repart:

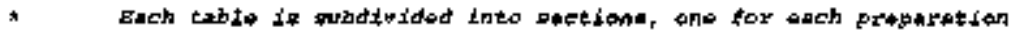

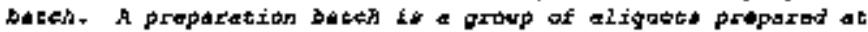

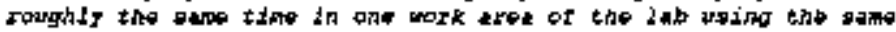
mothog.

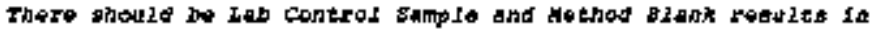

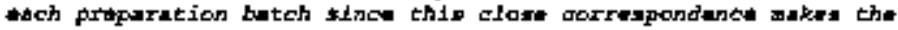

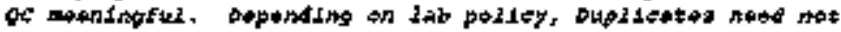

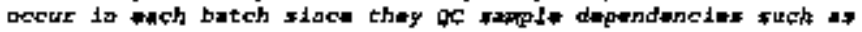
netrix effoces.

I The RAP TEST calatuh ahows the test gode ased th the raw data to

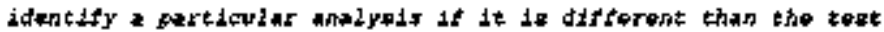

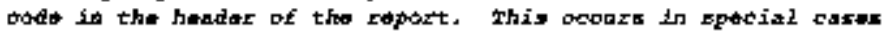

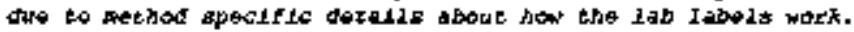

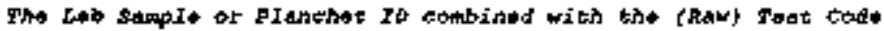

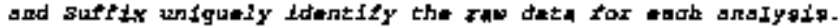

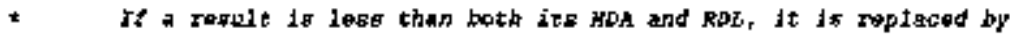

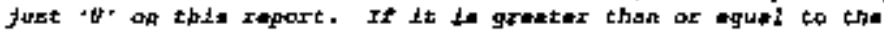

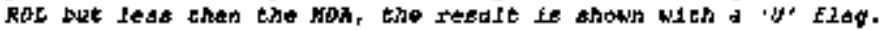

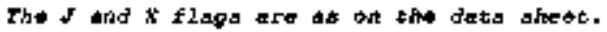

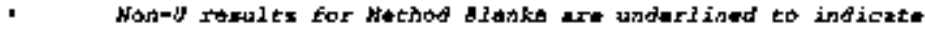

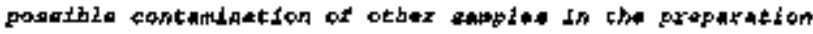
bazch. Tho Mathod gidak sepdzt has supportirg data.

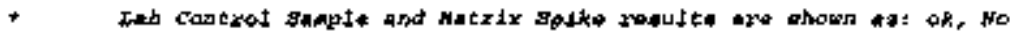

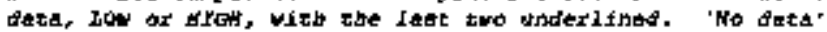

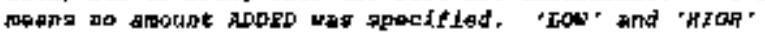

Finaz Report

\section{Rapor artors}

page 13

SUMadRY Dath stcriak

Page 43
Ind Id 272-5 Pratocol sis?.

varuion 2 . Form pupara

Vursion 2 .0. Report $A$ ate $03 / 76 / 97$ 


\section{$Z Z Z=S$ LABORATORY} JANS 24J-T-JIO CORE IBO

S00 \$70001f1

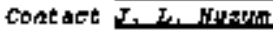

GUIDE, cont. there Thas

rank T-L2O

M $Z$ THOD SUMAR

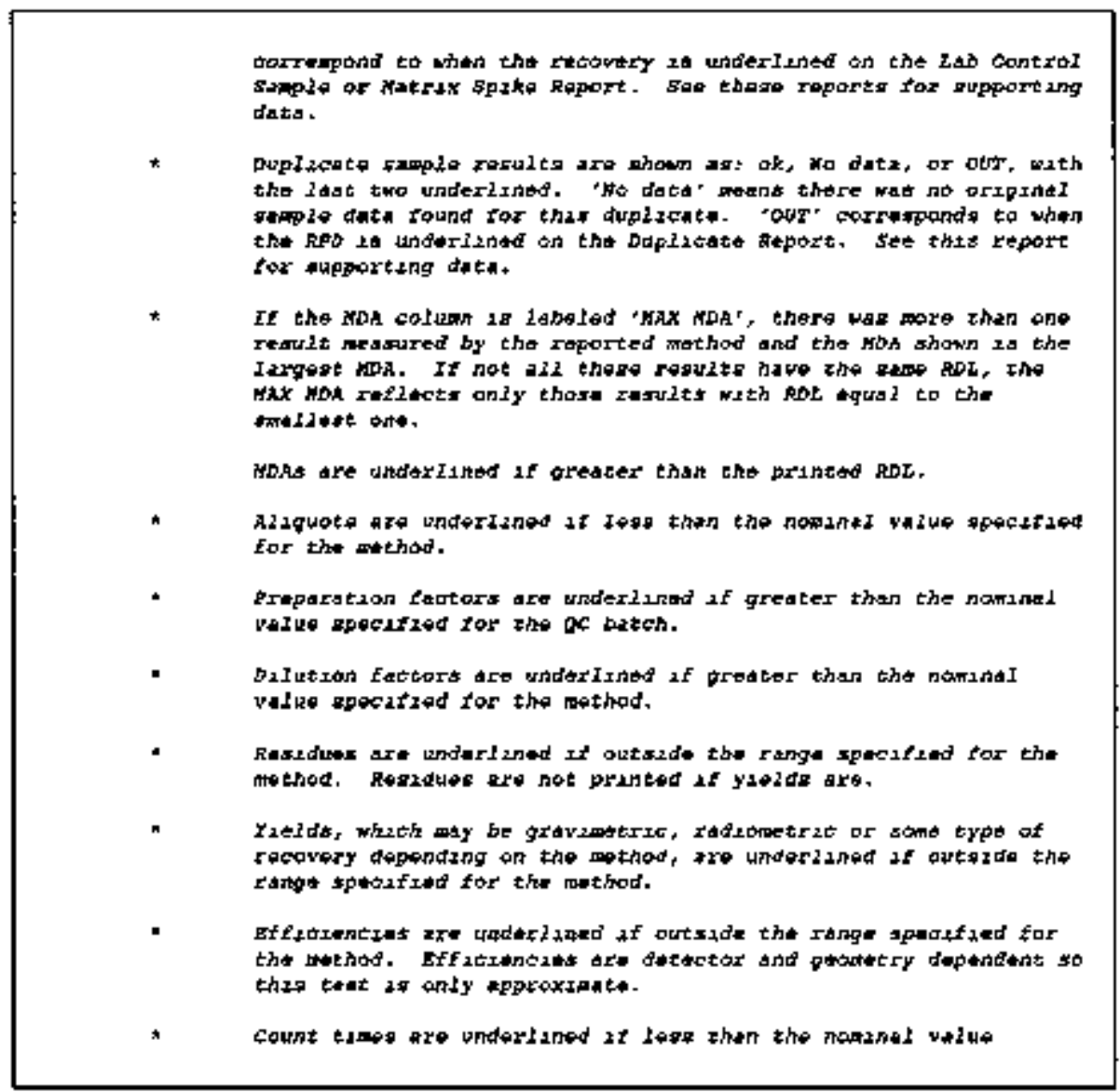

FInaI Report

Rappors gatass

$P * g * 74$

SOUWCRY GAFA SECTrOX

PAS* 14
Lab 24 t272-5

Protacol SST

vapton 3.0

Form pYrora

Verstor 3.09

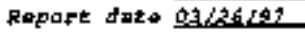


$2 Z Z=S \quad$ I B

spg s7000211

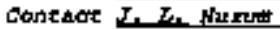

TANK 24I-T-נJO CORE 180

OUIDE,

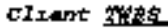

Tank Tolo

METHOD SUMHARY

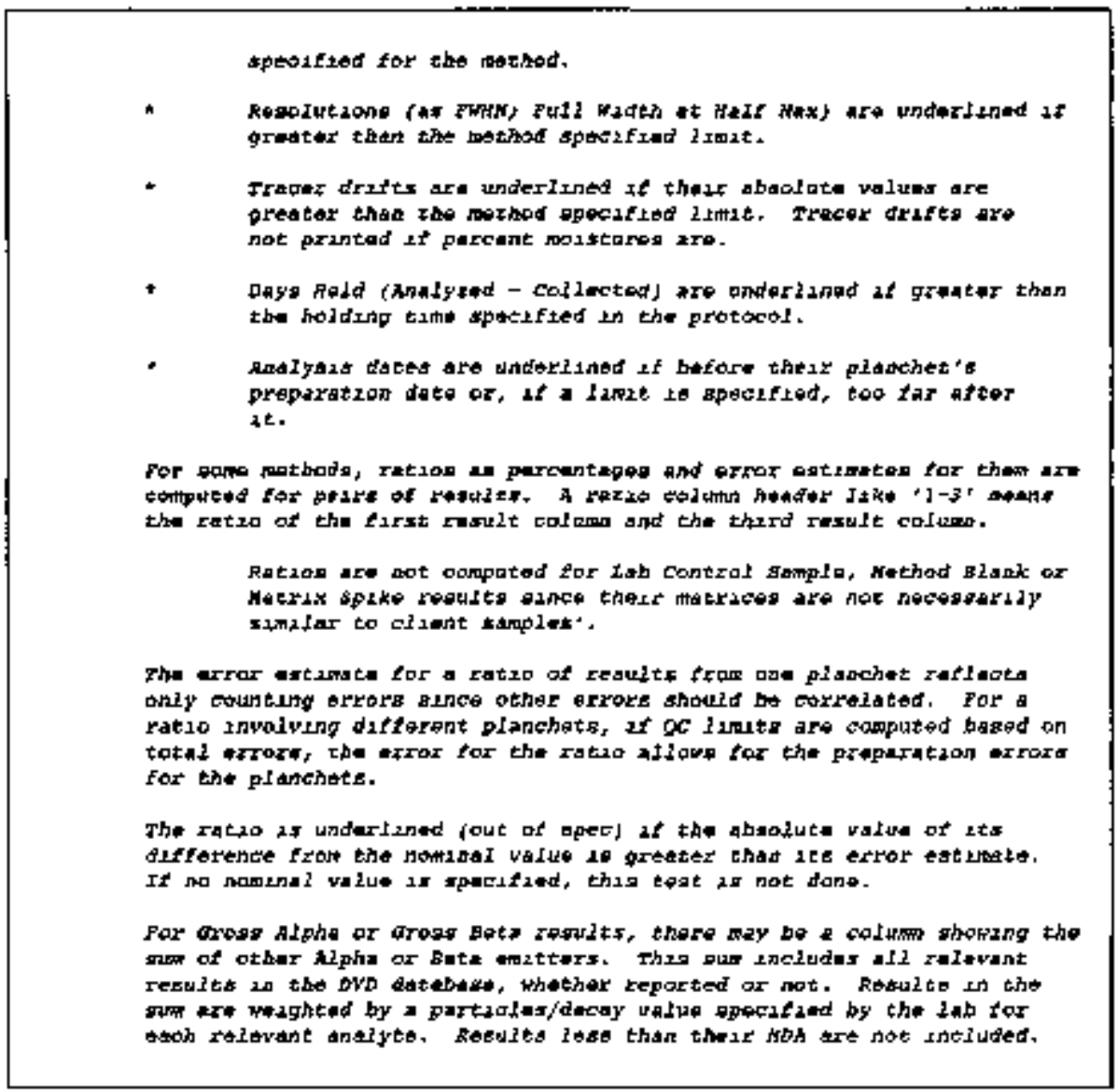

EIn十 2 a port

REPORT GUIPSS

Fage 13

SUHadRY DATA SECTID

Page 45
Iad IA 2:7-5

Pratocal sss

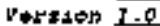
Form aym $\rightarrow$ R4

Veretor 3,08 Report date at:36197 
22 a - S IASORATORY

SOG gropdilt

contact 5 .
PINK 21d-P-110 CORE 190

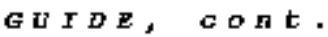

\section{C110ar Fras}

rank $T-100$

HETHODH H A R

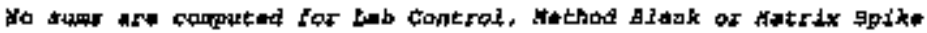

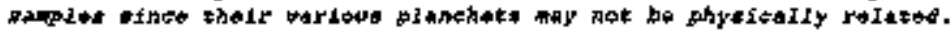

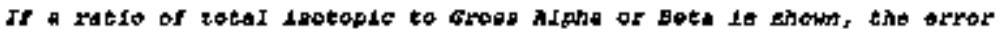
far the ratio reflecti both the arzor fa the trors ragrit and the serr,

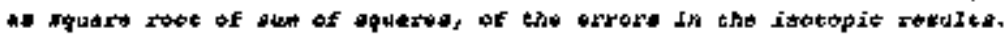

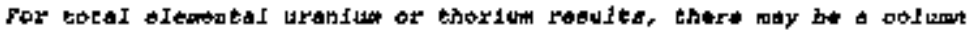

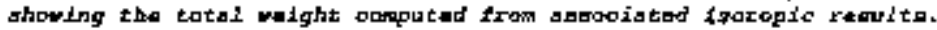

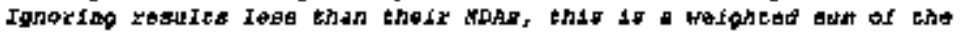

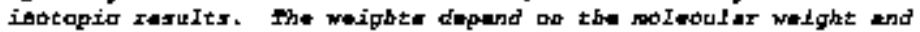

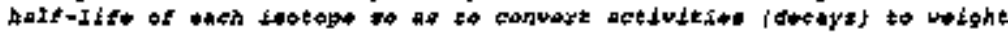
\{atars\}.

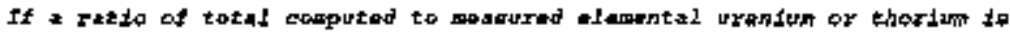
showh, the arrot for tho rat fo raflects the oriog in all the monguremants.

IInaI $R *$ port

REPORT ETHES

Page I6

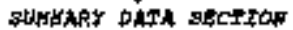

Papt 46 neth iff $27 x-5$

protocol SST

Yerman th

ForA blose $k E$

Peralon 3.08

Roport dato $03 / 36 / 27$ 


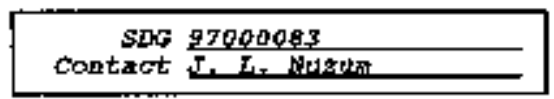

\section{SDA 97000083}

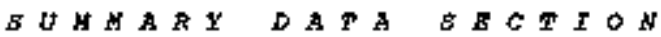

\section{Client TwRS}

Tank $x=110$

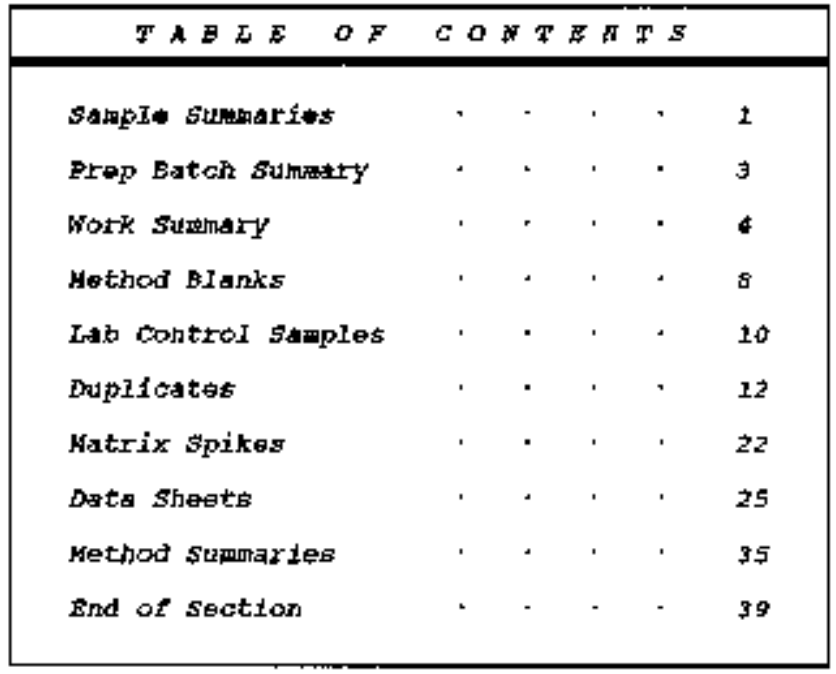

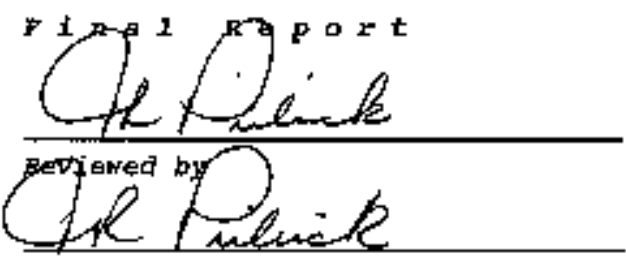

Approved by
Lab id 츤도 Protocol s5is

Version 1. Sor

vergion 준요 Report date b3/35/07 
HNF-SD-WH-DP-Z3B, REV. O

\section{2-S LABORATORY}

TH

906 으..00.0.5

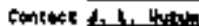

SAMPLE SOHWARY

cl1mt TH:F

Tari $T-110$

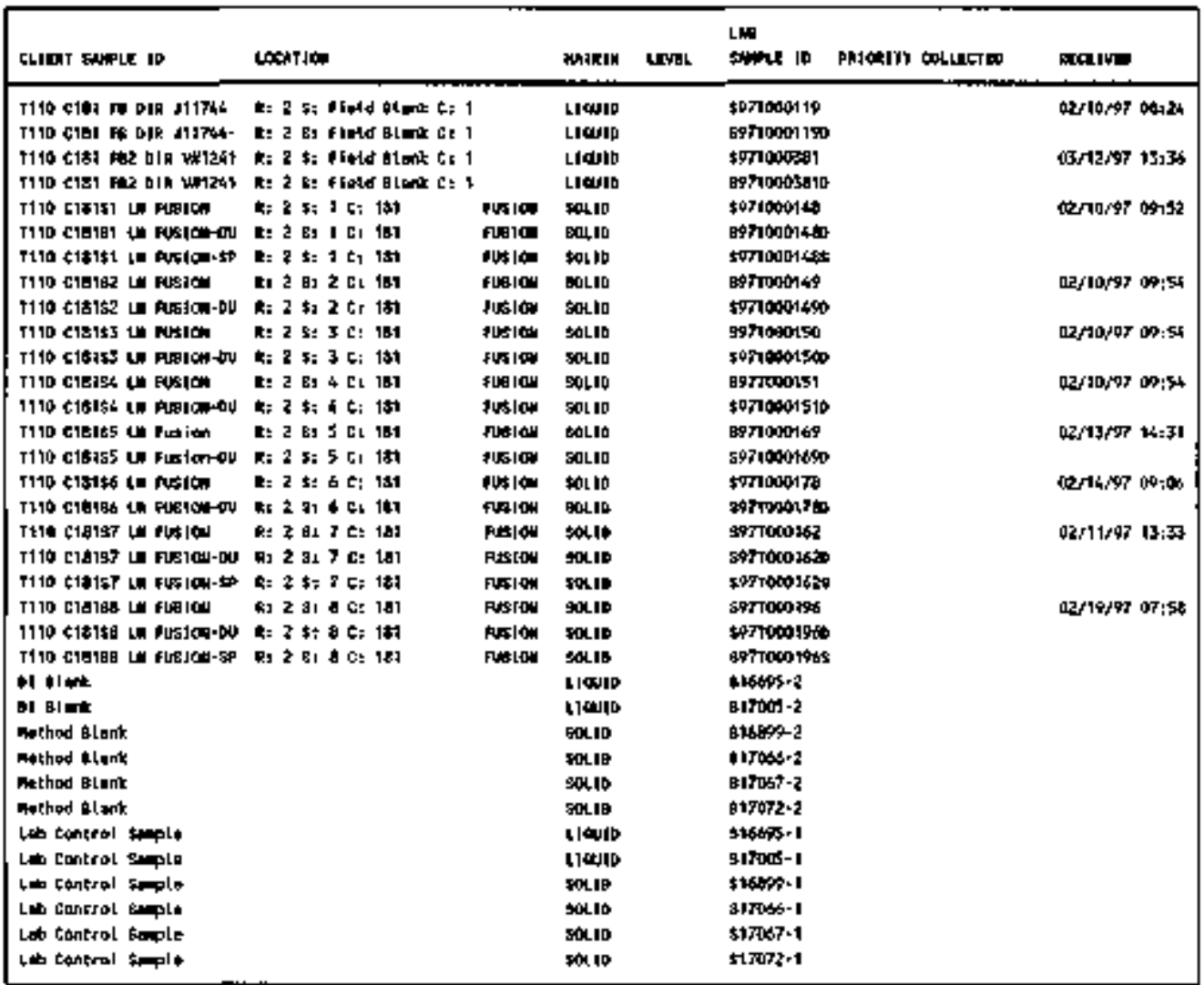

Final Report

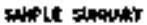

Dowe 1

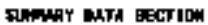

tor 1 in id $727-5$

protocol s5I

veraion 1.0

Fory 마.

veraion 3, th:

ipporl dint 03,26tor 


\section{2-S IABORATORY}

TMx $24 t-T-110$ CXPE 181

OC SUMMARY cl1tant Igest

a $\mathrm{i}$ + 110

\section{thistrof} e enteh Dustem
Q CEN SWULE ID

(E)

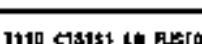

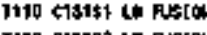

TIN c1818z in Rascow

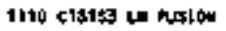

Tlto c1btat IE Rescow

Thto cattos ur furion

Ttro cIstso in Risipu

Ttw cIdts in Astow

T1\% C181t: ut festov

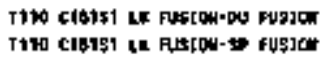

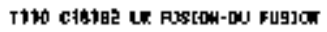

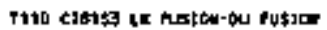

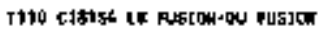

1110 c131ss it Fuianod pusien:

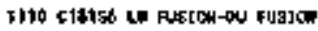

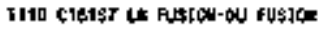

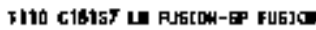

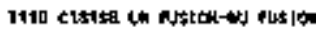

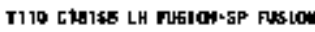

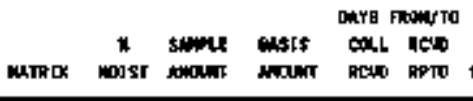

DAYG Fomito

to 10

bot 10

如以

श्रा ए

क्व 10

stio

serito

는 10

anco

皮的

sor co

如比

so.ro

站10

ancto

510

Barco

$\sin 5$

socis
LIN PHALE ID

\begin{tabular}{|c|c|c|c|c|}
\hline $970000 d 3-\mathrm{c}$ & 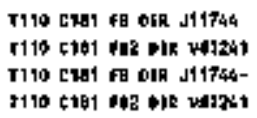 & 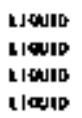 & $\begin{array}{l}46 \\
16 \\
16\end{array}$ & 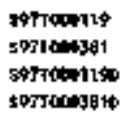 \\
\hline I Itous & 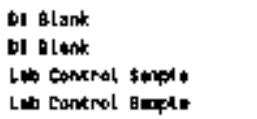 & 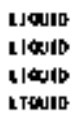 & & 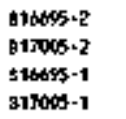 \\
\hline tats & 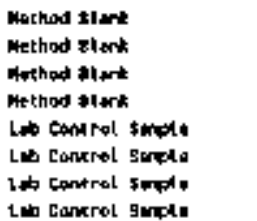 & $\begin{array}{l}50418 \\
50 L 18 \\
50110 \\
50 L 18 \\
50110 \\
90 L 10 \\
50410 \\
90410\end{array}$ & & 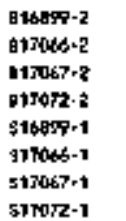 \\
\hline
\end{tabular}

\section{PInal Report}

e Busmar

Ptoe 1

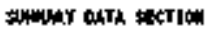

Powe 2
DIBH अare If
(⿻ 1d 2m-s

Pratocal 5st

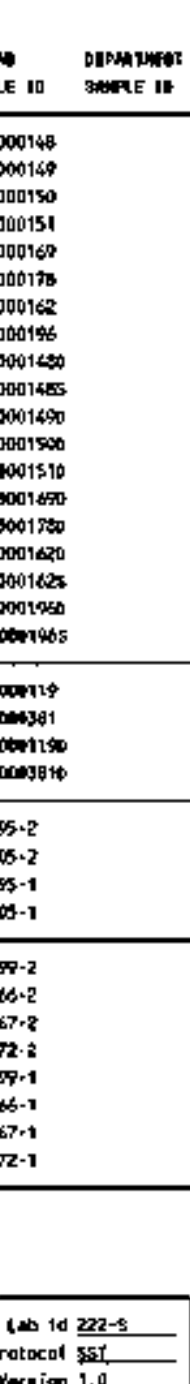

46 SनिT000146

di

44 STrTanatso

d4 Ertoún15।

d1 क्गरण00160

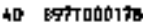

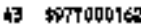

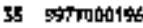

ts 507000140

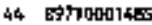

44 5975001490

44 barlonalses

th 5071001510

di s9rto001450

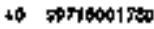

43 s9nooniszo

43 s9ritoplda

35 toropotos

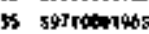


HNF-SD-WHA-DP-236, REV. 0

\section{2-S IABORATORY}

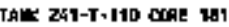

bot thogugt

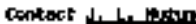

PREP ARJCH SUNAARY

Cotafic $\mathrm{xas}$.

rart $I=11$ e

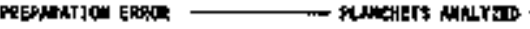

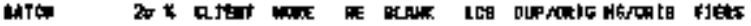

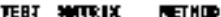

Alpin Bepectoroacupy

by boutt plutonica.z30

9000007

15.0

11 1 1 t

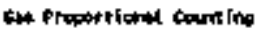

Later

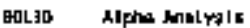

$11 \boldsymbol{I}$

\begin{tabular}{|c|c|}
\hline 9700ass & 15.0 \\
\hline \$robolats & 15.0 \\
\hline Prowopes & 15.0 \\
\hline \$7000 990 & 15.0 \\
\hline 9 roposes & 15.0 \\
\hline
\end{tabular}

1
3
3
1

$\begin{array}{llll}1 & 1 & 3 / 2 & \\ 1 & 1 & 3 / 3 & 1 / 1 \\ 1 & 1 & 2 / 2 & 1 / 2 \\ 1 & 1 & 1 / 1 & 1 / 1\end{array}$

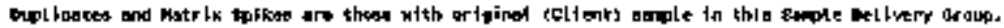

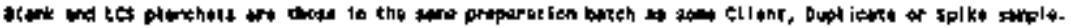

PinaI Report

PRE BATCH GUNunr

Page 1

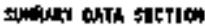

Poter 1
$\$ 156$
Lob td ita.5

Drotocal s

Vecrion 9,0

fara bub-eat

rarsian Ith

Raport tate $03 / 24 / 67$ 


\section{$Z Z Z-S$ IABORATORY}

sto grogon:t

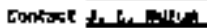

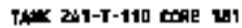

RORK SUMAARY
Cllane Twa

Yost 1110

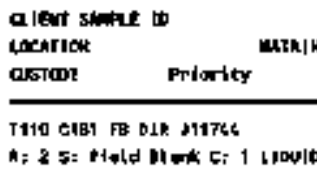

IN clbi fB o[R Jitha-

t: 2 s: F1atd glant c; 1 bIould

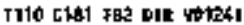

ค; 2 5; field elenk ta I L loulo

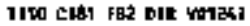

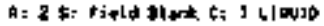

LtB trole 中

ल्मसकाए

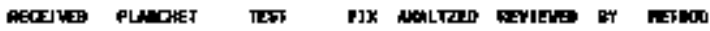

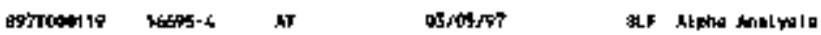

atriar?

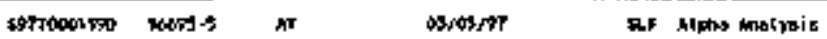

abritor

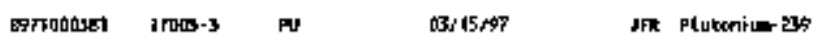

$09 / 12197$

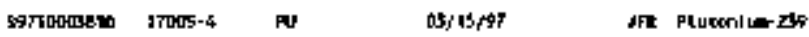

$03 / 12 y 9$

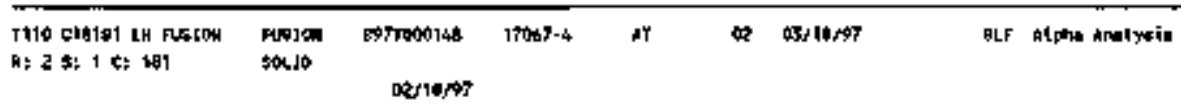

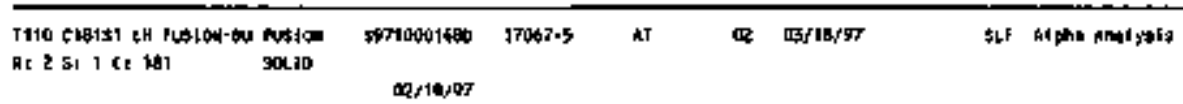

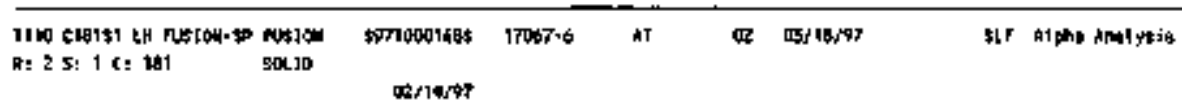

\begin{tabular}{|c|c|c|c|c|c|c|c|c|}
\hline 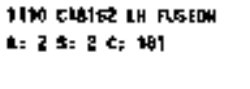 & $\begin{array}{l}\text { Projan } \\
\text { \$obsto }\end{array}$ & $\begin{array}{l}\text { Bd700014\% } \\
\text { B/20/\% }\end{array}$ & 170d & IT & it2 & 03 16 167 & ELF & alphe inalyein \\
\hline 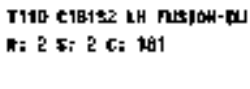 & $\begin{array}{l}16518= \\
50 L .19\end{array}$ & $\begin{array}{l}\text { topre001490 } \\
02+10,87\end{array}$ & 170 & $\boldsymbol{\lambda T}$ & 四 & 따18/97 & sLF & Alphe dintall \\
\hline 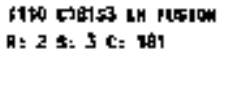 & $\begin{array}{l}\text { prasta } \\
\text { sale }\end{array}$ & 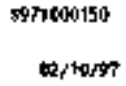 & 1660904 & $A T$ & & 1031407 & $\$ \mathbf{1}$ & alphe ardityoib \\
\hline
\end{tabular}

\section{FInal Report}

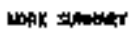

Pant 1

sumat Buth EEctom

bew 4

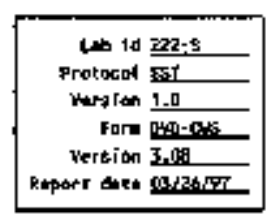




\section{ZZ2-S LABORATORY}

Tarr 241-7+110 00: 181

Exe gonocer torlsct thentut
- WORR sUNHARY, cont.

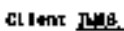

Ionk $1=110$

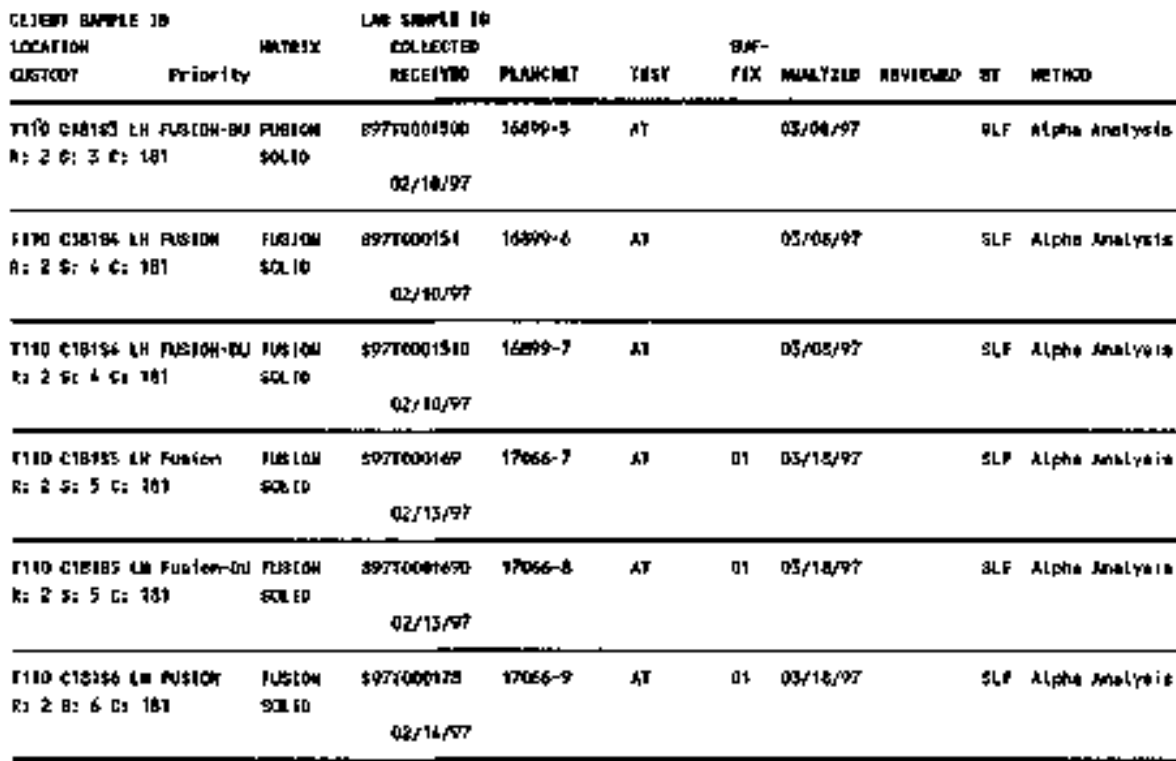

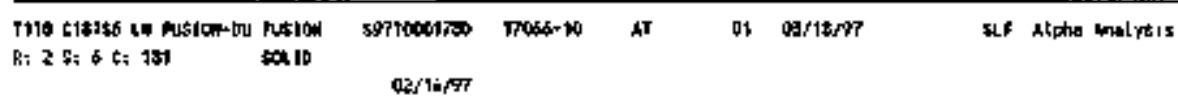

\begin{tabular}{|c|c|c|c|c|c|c|c|c|}
\hline 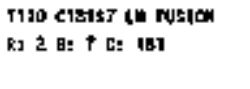 & $\begin{array}{l}\text { nut lon } \\
\text { sincto }\end{array}$ & 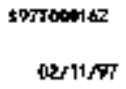 & $77056-6$ & hT & ㅁำ & $03 / 1 E / D T$ & 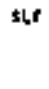 & Nlpht Mnstrtis \\
\hline 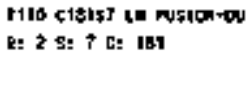 & $\begin{array}{l}\text { pus row } \\
\text { tencto }\end{array}$ & 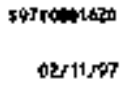 & tTosh-5 & Sl & 01 & $03 / 1</ \%$ & $\mathbf{s t r}$ & Alphe molrete \\
\hline 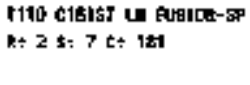 & $\begin{array}{l}\text { Fustow } \\
\operatorname{tallo}\end{array}$ & $\begin{array}{l}\text { soltiolded } \\
\text { Def } 11 / 97\end{array}$ & $17056-6$ & at & 01 & 03/18/4? & SLF & Alpha enalyste \\
\hline
\end{tabular}

\section{FIna2 Report}

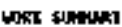

난 2

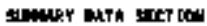

tage 5

lab id $372-5$

Protocel ghI

receton 10

Fom BYp-ctex

Teraten 3 .h.

Ropert takt osidert 


\section{ZZZ-5 ISBORATORY}

tht Prounces

Contract t. T. Thו
Tam ed-t-1to crae bol

WORK SUHAARY, cont.
CII Int Thts

Tork $\mathrm{I}+11 \mathrm{~d}$

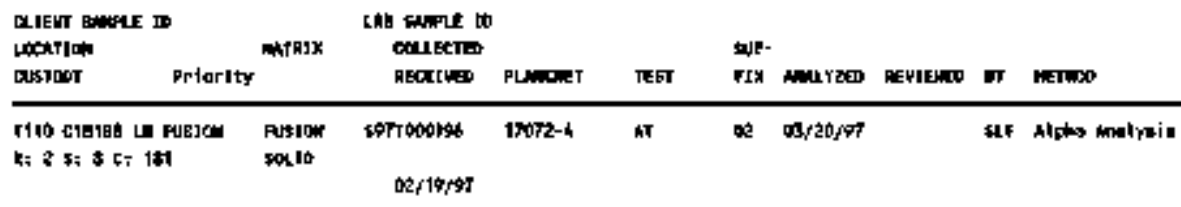

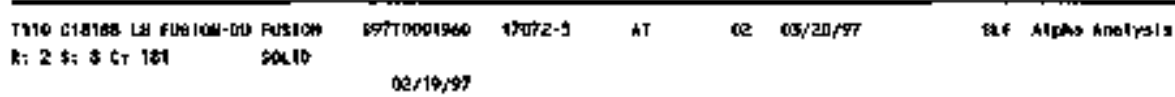

\begin{tabular}{|c|c|c|c|c|c|c|c|c|}
\hline 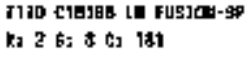 & $\begin{array}{l}\text { Rusiow } \\
\text { BCR ID }\end{array}$ & $\begin{array}{l}\text { softodolsos } \\
\text { 02/19/9) }\end{array}$ & 1 intz-6 & ผT & $\mathbf{w}$ & $03 / 20 / 97$ & Ex & Hpat malpia \\
\hline
\end{tabular}

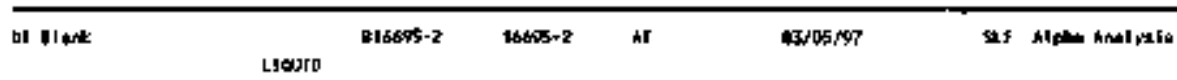

\begin{tabular}{|c|c|c|c|c|c|c|c|}
\hline Harlod telank & $\operatorname{son} 10$ & B16.499-2 & 16497 & คT & $05 / 100 / 37$ & st.f & 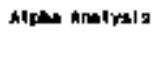 \\
\hline D) Pland & Liouto & |ltoos-z & 1rocs-? & PN & AW15AV7 & JfR & Plusond a 230 \\
\hline Wethod Bh ril & sodud & G170hod? & 1 rods-2 & AT & tevistgr & a.t & nipho irstersis \\
\hline nrthod vlow. & 때 & $\operatorname{lng} 7 \cdot 3$ & $17067 \cdot 2$ & $\alpha T$ & 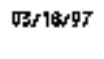 & $\$$ \$LF & Alphe irtulyt is \\
\hline nethod BLal & $\$ 0$.JD & 白17072 2 & $17072 \cdot 2$ & $\Delta t$ & arzoply & a. & hlpha Arstrsis \\
\hline Lab tantrol somple & L tould & $\$ 16605 \cdot 1$ & $16805 \cdot 1$ & AT & $03,06+97$ & al & Hiphs inal rsis \\
\hline lab canerol \$taple & goL.JD & 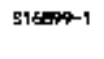 & $16899-1$ & NT & 따 $106 / 97$ & $\mathbf{s . F}$ & Atphl Anslyols \\
\hline teb contral Beple & LIQJID & a17oos-1 & 1Ytot-1 & nu & [15/2, & JFR & Plutonhlu بط \\
\hline
\end{tabular}

\footnotetext{
Flat Report

whax dromiti

Pot 3

घnowet nard secrug

Pupv 6
}

L 1d $222-9$ Protocod 5.

Veraloh 1.0

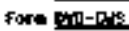
varsion 3.69

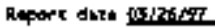


HNE-SD-WL-DF-238, FEV O

\section{2-G LABORATORY}

300 groshos

conter 2. 1, mine
รมس 241-1-110 1010

WORK SUAHARY, cONt. catenc n:

Tone I.110

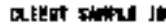
Lestriour Dastor

lab cantrol \$onpla

$$
\text { mitegx }
$$
Prlorty
Liv stin 10 crutecter

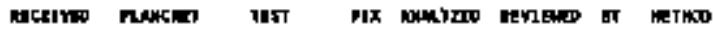

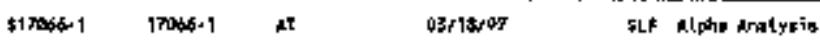

sot.

lab cencreal suple

$517067+1 \quad 17067+1 \quad 11$

a.stitity

sto alpht Irolytix

sx. To

Lat rontrod somple

strorz-1 1707e-1 ג

ofrastof

SLF Alphe Molycte

\begin{tabular}{|c|c|c|c|c|c|c|c|c|c|c|c|c|}
\hline 1It\$r & mipedtr & counts & $O P$ & TESTS BY & $\begin{array}{r}\text { SAMPLE T } \\
\text { AIFT }\end{array}$ & $\begin{array}{l}Y P E \\
\text { חAC }\end{array}$ & $\mathbf{n s}$ & MUT. & Les & $\mathbf{p r}$ & PIXX & Tिम \\
\hline AT & & Mpand Anotvis & & e22-s Lob Anatyticnt & A Procedure & & & 4 & 4 & 由 & 3 & 27 \\
\hline hi & & 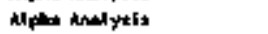 & & 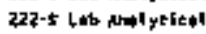 & 1 brocture 1 & & & $\mathbf{I}$ & 1 & 1 & & 6 \\
\hline PU & & Pluterniger 240 & & $22 z=\leqslant$ Lob inslytical & I Erocodure ? & & & 1 & 1 & 1 & & 6 \\
\hline jOTALX & & & & & 10 & & & 6 & 4 & 10 & 5 & 35 \\
\hline
\end{tabular}

Final Report

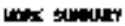

Pog $=4$

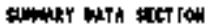

Pay 7
Lab 1d 222:s Protocal 55I

vation 1.0

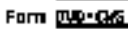
Vut i ,

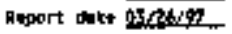




\section{ZZZ-S HABORATORY}

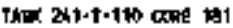

to $970000 \mathrm{~g}$

Dantact J. Thents.

BLABRS

ct fork 19g

Tatk pettio

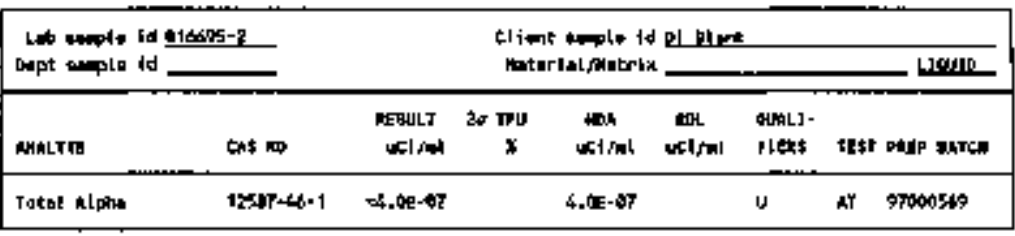

\begin{tabular}{|c|c|c|c|c|c|c|c|}
\hline \multicolumn{2}{|c|}{ 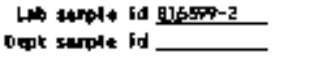 } & \multicolumn{3}{|c|}{$\begin{array}{l}\text { cl hanx 14xpl\& ba } \\
\text { moterial matrix }\end{array}$} & \multicolumn{2}{|c|}{ Mofhos tit:k } & 50410 \\
\hline akMtrne & LAS MD & 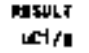 & $\begin{array}{c}2 \boldsymbol{m} \mathbf{T} \\
\mathbf{x}\end{array}$ & $\begin{array}{l}\tan \\
\text { ictita }\end{array}$ & $\begin{array}{l}\text { ene } \\
\text { atito }\end{array}$ & $\begin{array}{l}\text { BunLIJ } \\
\text { PtERS }\end{array}$ & IEgJ FAEP BATC' \\
\hline Told Nlpht & $17547-66-1$ & $\alpha, D \in=0 \mathrm{~S}$ & & $5,00.04$ & & cht & DN00818 \\
\hline
\end{tabular}

\begin{tabular}{|c|c|c|c|c|c|c|c|}
\hline 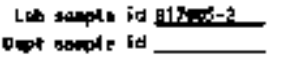 & \multicolumn{3}{|c|}{$\begin{array}{l}\text { Cl bonk sanple bd } \\
\text { maturlal matr bx }\end{array}$} & of misk & \multirow[b]{2}{*}{$\begin{array}{l}\text { Q4k, } \\
\text { PIERA }\end{array}$} & \multirow[b]{2}{*}{ ГEST } & \multirow[b]{2}{*}{ PAP BATE } \\
\hline NHALYIE & tofker & $\begin{array}{c}2+x \\
x\end{array}$ & $\begin{array}{c}\text { Whi } \\
\text { verijed }\end{array}$ & 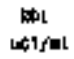 & & & \\
\hline 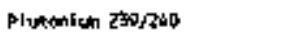 & 3.4. 40.06 & & 3.4Eung & & $\mathbf{v}$ & w & Wrapos6 \\
\hline
\end{tabular}

\begin{tabular}{|c|c|c|c|c|c|c|c|c|}
\hline \multirow{2}{*}{ 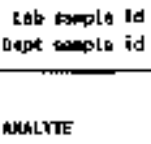 } & \multirow{2}{*}{ 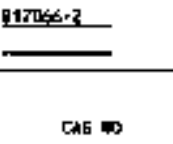 } & \multicolumn{3}{|c|}{ 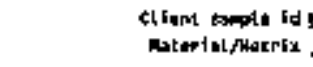 } & \multicolumn{2}{|c|}{ Finthod Blate } & \multicolumn{2}{|r|}{$=80.40$} \\
\hline & & $\begin{array}{l}\text { EFsint } \\
\text { webto }\end{array}$ & 20 Tru & 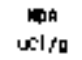 & $\begin{array}{l}\text { tex } \\
\text { Intfis }\end{array}$ & $\begin{array}{l}\text { CLLL [- } \\
\text { FIERG }\end{array}$ & TEST & PEIP BATR \\
\hline Total Alphn & 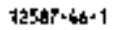 & 它.DE-伎 & & 2.DE-0B & & $\mathbf{u}$ & AT & sronotsts \\
\hline
\end{tabular}

Final Report

BUNKF:

pore 1

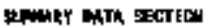

Paye

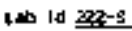

Pratocod seg

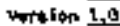

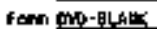

version 3.t.

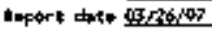




\section{2-S IAEORATQRY}

ERG BHot:

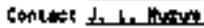

TANK 2\$1-T-110 COEE 16

매재

t)

$T$ The $T+110$

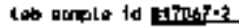

14\% 10

-

\begin{tabular}{|c|c|c|c|c|c|c|c|c|}
\hline MUNYTE & $C A 5$ & 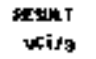 & $\begin{array}{c}\text { Pa TEU } \\
*\end{array}$ & $\begin{array}{c}\text { math } \\
\text { uch }\end{array}$ & $\begin{array}{c}\omega L \\
\omega b^{\prime}\end{array}$ & 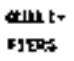 & TEST & PREP RATCH \\
\hline Tatal hlpos & $1245-46-1$ & $-242-05$ & & 2. 新-05 & & u & AT & कारण00996 \\
\hline
\end{tabular}

\begin{tabular}{|c|c|c|c|c|c|c|c|c|}
\hline \multicolumn{2}{|c|}{ 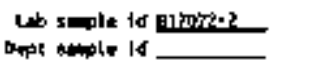 } & \multicolumn{3}{|c|}{$\begin{array}{l}\text { clfort soople td } \\
\text { MaEar fal have I }\end{array}$} & \multicolumn{2}{|c|}{ Bethod_Rlant } & \multicolumn{2}{|r|}{ 媪帅 } \\
\hline MHYTE & tas wo & $\begin{array}{l}\text { RESUYT } \\
\text { utifg }\end{array}$ & $\underset{x}{T}$ & $\cosh _{\text {uciso }}$ & $\begin{array}{l}\text { apl } \\
\text { ucifos }\end{array}$ & $\begin{array}{l}\text { minl. } \\
\text { Fugs }\end{array}$ & TEST & PЯEP ЕגTCH \\
\hline rotar Alplo & 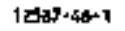 & $<5.0=05$ & & $3.0=0 x$ & & u & คT & proposs \\
\hline
\end{tabular}

Final Report

\section{oune:}

Page $z$

Sorvak tuth SEtTIO

5 tod 7

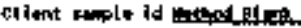

metertab/nerla 童旧

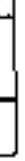




\section{Z-S LABOAATORY}

Thin 241-r-110 cote wat

mo gToos:

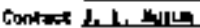

IAB COHPROD SAMPLES
Client 1Lats

Tork Tr1tp

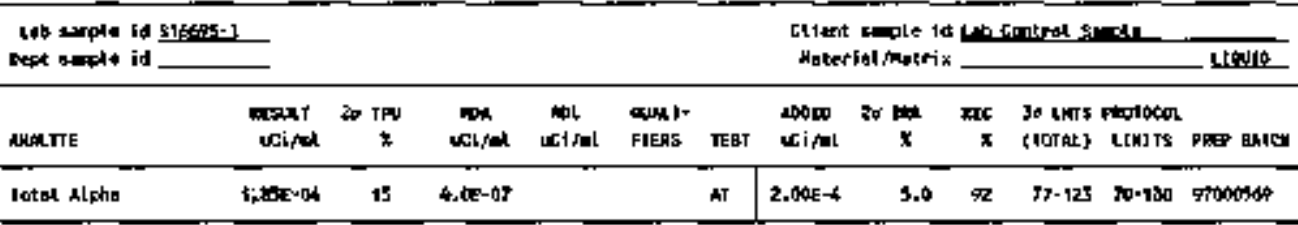

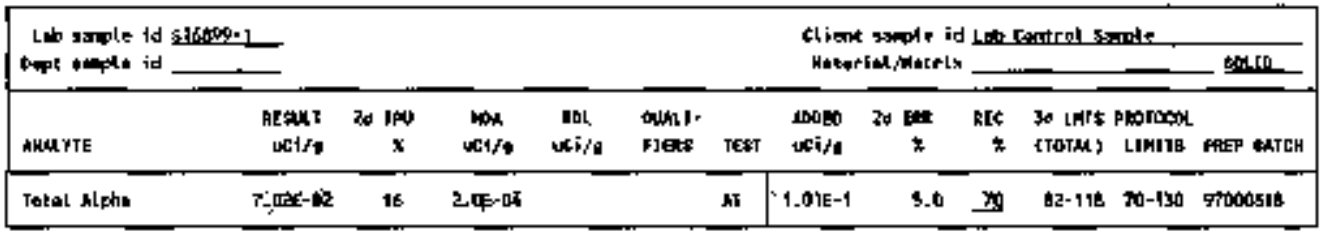

\begin{tabular}{|c|c|c|c|c|c|c|c|c|c|c|c|}
\hline \multirow{2}{*}{ 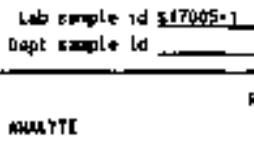 } & \multirow{2}{*}{ 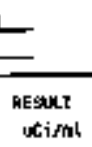 } & \multirow[b]{2}{*}{$\underset{x}{x}$} & \multirow[b]{2}{*}{$\cos _{\text {stiffal }}$} & \multirow[b]{2}{*}{$\begin{array}{l}\text { noL } \\
\text { ctition }\end{array}$} & \multirow[b]{2}{*}{$\begin{array}{l}\text { onliti- } \\
\text { piets: }\end{array}$} & \multirow[b]{2}{*}{ Tा } & \multicolumn{2}{|c|}{ 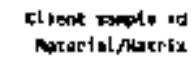 } & \multicolumn{2}{|c|}{ 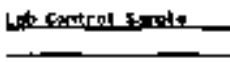 } & \multirow[t]{2}{*}{ - } \\
\hline & & & & & & & $\begin{array}{l}\text { AHOEb } \\
\text { uclited }\end{array}$ & $\begin{array}{c}\text { Dr Ext } \\
?\end{array}$ & REC & $\begin{array}{l}30 \text { unls prorocol } \\
\text { (foted Lintis }\end{array}$ & \\
\hline Dlurenter 2487340 & 1 âne-04 & 13 & b.Je-d & & & ต & 1:06-6 & 9.0 & 91 & ralz & groboses \\
\hline
\end{tabular}

\section{Final Report}

LB CCHTRLL SUPUES

bat 1

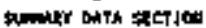

Page ID

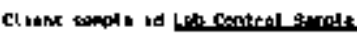

maturtaluntrix

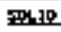

Lapt anople bo

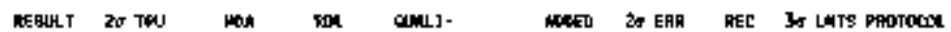

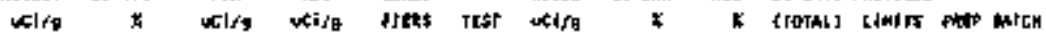

AMurte

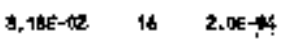

คT $\$, 6 e^{2}-2$

5.0 
HWF-SD-WA-DP-238, REV, 0

222-B IABORAPORY

Tax 241.1-110 Chet 1et

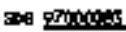

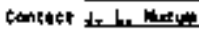

ctimt shes

Timk. I-110

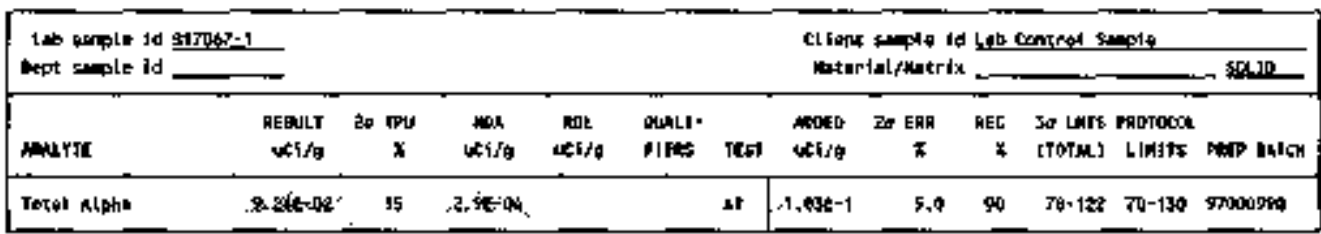

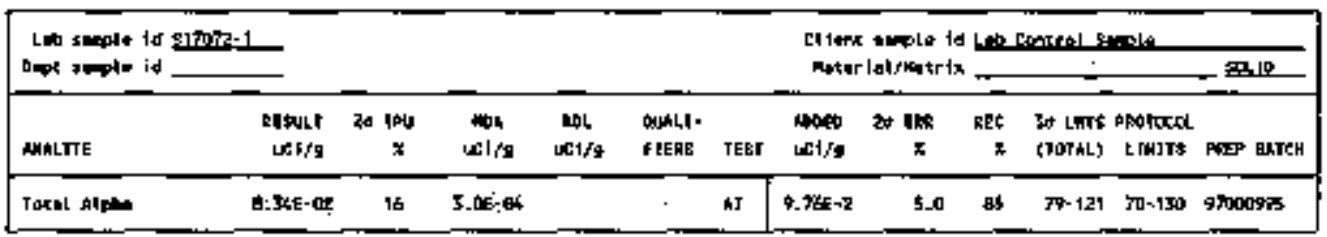

Final Repozt

LN cointhok shiples

Pupd $\mathrm{Z}$

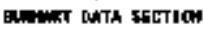

P的 11
La id post

prococol th:

burtion 12: Farn wh-lcs

toreion 5,00

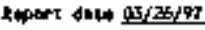




\section{Z-S HABORATORY}

Uh 201-5.110 की

DUPLICATE

506 97000모

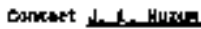
grictite

Lab end id sorrouthes

Dept mpple Id contarilu

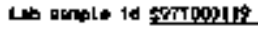

pot omple 1d seceliugd oftrors cl Im⿻ In: int

Tank I-110

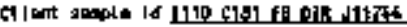

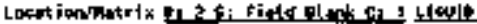
Codltctod

thain of tutbody id

\begin{tabular}{|c|c|c|c|c|c|c|c|c|c|c|c|}
\hline ANHLrTE & $\begin{array}{r}\text { pulchit } \\
\text { uclifed }\end{array}$ & $\begin{array}{c}20 \\
7\end{array}$ & $\begin{array}{c}\text { Ind } \\
\text { cifled }\end{array}$ & 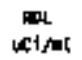 & $\begin{array}{l}\text { qux t- } \\
\text { FIExs }\end{array}$ & IEST & $\begin{array}{r}\text { क्prgimu } \\
\text { wither }\end{array}$ & $\begin{array}{c}20 \text { IPU } \\
\text { I }\end{array}$ & 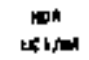 & $\begin{array}{l}\text { Dhul RF } \\
\text { f]ESS }\end{array}$ & $\begin{array}{l}3 \text { Plol } \\
\text { Tor } 1 \text { TwIT }\end{array}$ \\
\hline Tatal nlghe & \$.E5E-पT & 49 & 4.De-UP & & & NT & 5 37E- $0 T$ & 120 & 4.0F-0? & 5 & $200 \quad 145$ \\
\hline
\end{tabular}

Final Report

tolntohts $\$$

Pust I

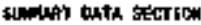

Vion 12

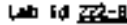

Pratocal 5xI

uproipn 1, ․

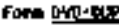

version 5 so

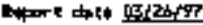




\section{2-S IAEORATORY}

TdN ASI-T-110 Dose 181

DUPAICATE

rito cibiat ir pusron

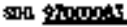

Contect 1.1. Hithe DUFictite

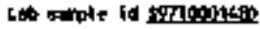

Dope sepple Id citat but

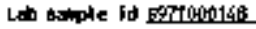
Dapk sample id

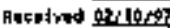

CIItent Thes:

t*at $I+120$

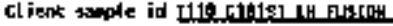

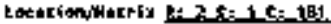
Eis.5 collacted phoin of euptody lid

\begin{tabular}{|c|c|c|c|c|c|c|c|c|c|c|c|c|}
\hline мичте & $\begin{array}{c}\text { DetschTt } \\
\text { LC1f\$ }\end{array}$ & $\begin{array}{c}\mathrm{yr} x \\
x\end{array}$ & ntifs & $\begin{array}{l}\text { not } \\
\text { ecs/o }\end{array}$ & $\begin{array}{l}\text { owhl. } \\
\text { flets }\end{array}$ & IEsI & $\begin{array}{l}\text { anicinm } \\
\text { utcita }\end{array}$ & $\begin{array}{c}\lambda 0 \text { त्य } \\
2\end{array}$ & $\begin{array}{c}\cos \\
\text { wClist }\end{array}$ & 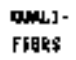 & $\begin{array}{r}\text { RPD } \\
7\end{array}$ & $\begin{array}{l}\mathbf{y} \\
\mathbf{1 0 T}\end{array}$ \\
\hline Iatel slphu & 6.680 .63 & 21 & $2.9 \%-03$ & & & st & $7,7004 \cdot 42$ & 20 & 2.60 .08 & & 12 & 12 \\
\hline
\end{tabular}

PInAI R*port

OWPLICATE:

lope $z$

SAMUSR WhT BECTICW

Pिp 13
Lob id that

Prototal \$\$I

Yercion 10

Porn prepup

Verpien 5,09.

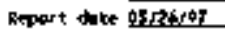


222-B IABORATORY

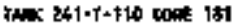

DUFLICATE
Bas 9xhothes.

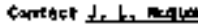
DUPLICATE

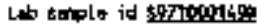

topr atrola 1d
DRIECINGL

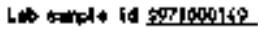
anpe sarche Id

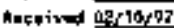

더른 Thes

$r a t+116$

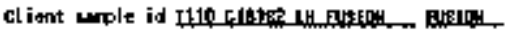

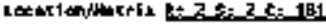
20sito

Chuin of euctody id

\begin{tabular}{|c|c|c|c|c|c|c|c|c|c|c|c|}
\hline HUITE & $\begin{array}{c}\text { MAPLICATE } \\
\text { urtof }\end{array}$ & $\begin{array}{c}x+5 \\
y\end{array}$ & ictids & 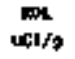 & $\begin{array}{l}\text { Punk: } \\
\text { flets }\end{array}$ & TEST & 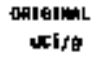 & $\begin{array}{c}Z * \text { TPU } \\
x\end{array}$ & $\begin{array}{c}104 \\
\text { MC5/89 }\end{array}$ & $\begin{array}{l}\text { ourl- tor } \\
\text { rinfs } x\end{array}$ & 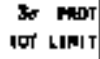 \\
\hline Totral Alphe & 5.12E- & $\$ 1$ & $2.6 t-05$ & & & h1 & $4+46-02$ & 21 & $2, x-\infty$ & $\boldsymbol{t}$ & $d t$ \\
\hline
\end{tabular}

Pingl R*port

IrALATEG

Pand 3

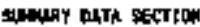

Pant is
Lot in $202+5$

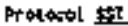

Uaraion je Forn therte

Yurtion 3.08 Repore duts 05/3thor? 
322-5 LABORATORY

TALC 241-T-IIt DOEE 101

DDPITATE

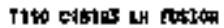

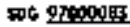

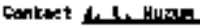
orfichte

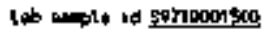
perpt tole id

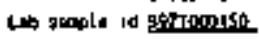

popt rmple id

becteived pingr? cl hing Ius

Fank r-91h

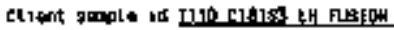
토.110..

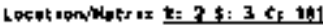
Collected

phain of abtody id

\begin{tabular}{|c|c|c|c|c|c|c|c|c|c|c|c|c|}
\hline AMLITII & $\begin{array}{c}\text { pusictie } \\
\text { uchit }\end{array}$ & $\begin{array}{c}20 \pi \\
7\end{array}$ & $\begin{array}{c}\text { Who } \\
\text { uciof }\end{array}$ & 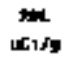 & $\begin{array}{l}\text { culas - } \\
\text { FIERs }\end{array}$ & 115T & 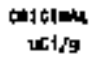 & $\begin{array}{c}30 \text { Th } \\
x\end{array}$ & $\begin{array}{l}\text { when } \\
\text { cotra }\end{array}$ & $\begin{array}{l}\text { tollel. } \\
\text { Fjexx }\end{array}$ & $x$ & 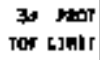 \\
\hline Total ilpho & 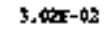 & 19 & 4.DE-06 & & $\mathbf{k}$ & AT & 3.4XE-02 & 17 & 4.05- 60 & $\mathbf{L}$ & the & 57 \\
\hline
\end{tabular}

Final Report

DUPLICHTEG

orge i

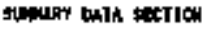

Pane is
Lat od $m+\frac{1}{4}$

propteof tyt

varuson 10

طب

Vurtian ti.06

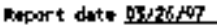




\section{Z-S LABORATORY}

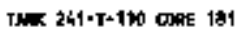

DUPEICATE

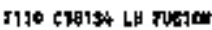

Sto omoons

Contack pustcat:

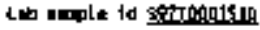

papt topla lat andsinnt

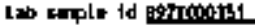

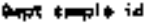
cectrued gatsorg

\section{Clinte lents}

Tark E=Ito

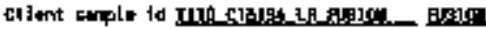

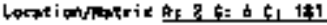
Dollexid

thes in of suttody id

\begin{tabular}{|c|c|c|c|c|c|c|c|c|c|c|c|c|}
\hline NuMtite & $\begin{array}{c}\text { tultents } \\
\text { uxifg }\end{array}$ & $\begin{array}{c}3 \\
2\end{array}$ & voh & $\underset{401 / 9}{4}$ & $\begin{array}{l}\text { Donil - } \\
\text { FIERG }\end{array}$ & TEBT & $\begin{array}{c}\text { onlofers } \\
\text { ucitis }\end{array}$ & $\begin{array}{c}200 \\
x\end{array}$ & $\begin{array}{l}\text { shith } \\
\text { uct if }\end{array}$ & 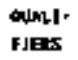 & 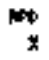 & $\begin{array}{l}3 \text { phal } \\
\text { Tor umit }\end{array}$ \\
\hline Total Mlposo & 5. PUE-DR & 17 & 4.로-06, & & 2 & AT & $3.916-12$ & 17 & 4.4E-04 & $\mathbf{L}$ & $z$ & 36 \\
\hline
\end{tabular}

Final keport

cumlchites

fare 5

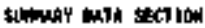

Pute 16
Lut $1 \mathrm{~d} m-\mathrm{m}$

Prokcol 회

Varzion 1.

pore pasure.

Hersion $3.0 \%$

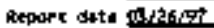




\section{ZZZ-S LABORATORY}

ThNK $24 \mid+T \cdot 19$ DONE Ho1

\$rThoptop

DUPLICATE

sto propolps

contect t, h. Herse artonfe

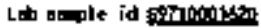

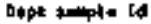

Serta]ul.

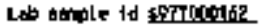

tope ample 1d Recelined Fitles?
Cl fent J...P

tonk t-110

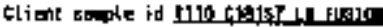

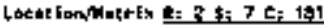

Coditrsters

Dain of cantody ho

\begin{tabular}{|c|c|c|c|c|c|c|c|c|c|c|c|c|}
\hline MUYTE & $\begin{array}{c}\text { buptidnt } \\
\text { |c1st }\end{array}$ & $\begin{array}{c}2 \pi y \\
x\end{array}$ & uct/s & uclifa & $\begin{array}{l}\text { SULII- } \\
\text { FJE:S }\end{array}$ & IEXT & $\begin{array}{l}\text { cistothnt } \\
\text { uch, }\end{array}$ & $\begin{array}{c}3+160 \\
I\end{array}$ & 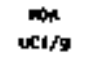 & $\begin{array}{l}\text { antd, } \\
\text { FIERg }\end{array}$ & $\begin{array}{r}\text { IPD } \\
I\end{array}$ & $\begin{array}{l}\text { 3o phor } \\
\text { moT LIICT }\end{array}$ \\
\hline 1otकl Allphe & 6.510 .03 & 20 & $2,00-03$ & & & $\mathbf{A r}$ & 6.05e-47 & 31 & 2.c4- 08 & & 7 & 43 \\
\hline
\end{tabular}

Final Report

OUPLJCAIEB

Ptgat 6

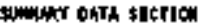

Pope 17
Lab id $M+5$

Propocol \$\$T

version 19

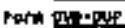

viraien 5.08

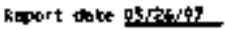




\section{ZZZ-S EABORATORY}

astiondsen,

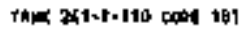

DUPII CAPE

r1to castes th Fopion

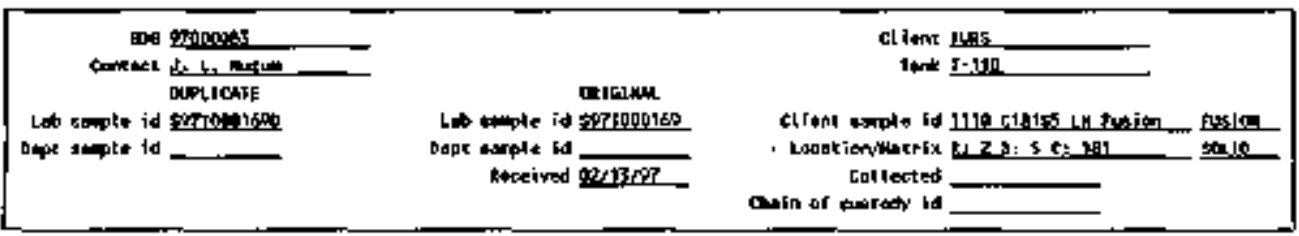

\begin{tabular}{|c|c|c|c|c|c|c|c|c|c|c|c|c|}
\hline 4hu, YTE & $\begin{array}{c}\text { оLPLICAT" } \\
\text { UC1/S }\end{array}$ & $\begin{array}{c}20 \text { TFU } \\
x\end{array}$ & $\begin{array}{c}\operatorname{mox} \\
\mathrm{uct} / \mathrm{g}\end{array}$ & $\begin{array}{c}\text { hol } \\
\text { cethos }\end{array}$ & $\begin{array}{l}\text { कum [- } \\
\text { FIERE }\end{array}$ & ТЕВт & 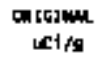 & $\begin{array}{c}\text { IPU } \\
x\end{array}$ & vetso & $\begin{array}{l}\text { onimi: } \\
\text { ffEes }\end{array}$ & RF & $\begin{array}{l}\text { 5. Pमा } \\
\text { TOT ITIT }\end{array}$ \\
\hline Tetal Alph. & 5_5x-02 & 21 & Z.1E-0క. & & & AR & $4.29 E-02$ & $\not Z$ & $2,1 \mathrm{~B}-\mathrm{DS}$ & & at & 46 \\
\hline
\end{tabular}

Final Report

OUPLICATS

Ptopt 7

tyenuer ahIR SECTICM

Page to lab id 292-5

Proveses \$\$1

vareion 10

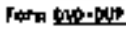

Varsion 3 .

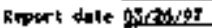


ZZ2-S LABORATORY

ThHK X1-T-114 towt 181

DOPIICARE
T11 क्षअत् in finton

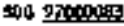

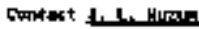
bunichte

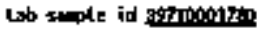

pipt tallold id ctotoine

ca sople id roptototh.

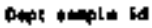

Reoelived Pintrgt
CI $\operatorname{canx}$ n⿴囗十:

Ionk r-116

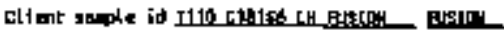

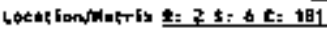
Gollected

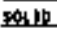

\begin{tabular}{|c|c|c|c|c|c|c|c|c|c|c|c|c|}
\hline matre & $\begin{array}{c}\text { DLICATS } \\
\text { undfs }\end{array}$ & $\begin{array}{c}21 \mathrm{Iy} \\
\mathrm{x}\end{array}$ & 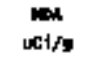 & $\begin{array}{c}\text { not } \\
\text { ucifo }\end{array}$ & $\begin{array}{l}\text { thlal. } \\
\text { Fless }\end{array}$ & IEST & $\begin{array}{c}\text { ofistiblint } \\
\text { uchion }\end{array}$ & 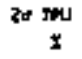 & $\begin{array}{c}\cos \\
\text { uci, } / 0\end{array}$ & $\begin{array}{l}\text { culds } \\
\text { FIERs }\end{array}$ & I & 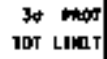 \\
\hline rottol Alphos & Gionside. & 茂 & 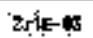 & & $\dot{i}$ & AT & $5.528-\mathrm{LC}$ & 22 & 2.25-0 & & 9 & 45 \\
\hline
\end{tabular}

FinaI Report

Dosiserins:

Page :

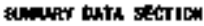

Pote 19
Lab ja $322-5$

Protocal stit

vertion 1,0 Fera mature

Version 3.06

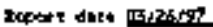




\section{2-S LABORATORY}

THX ZूT-T-IIL COAE toI

Bspotothos

DUP ICATE

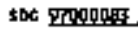

Contact fl hyru "in ECRTE

lab anple id sylabolyt

best phapld 1d

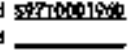

cartaimen.

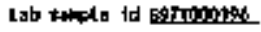
lopt acedt id

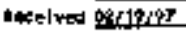

cl farx fl.its

Inonk 1.110

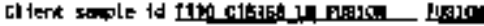

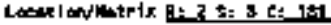
其1

col lected

chiln ol custody id

\begin{tabular}{|c|c|c|c|c|c|c|c|c|c|c|c|c|}
\hline musctit & 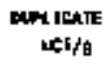 & $\begin{array}{c}20 \mathrm{rey} \\
\overline{7}\end{array}$ & tist & $\begin{array}{c}\text { cat } \\
\text { wito }\end{array}$ & $\begin{array}{l}\text { cund- } \\
\text { FInt\$ }\end{array}$ & IE\$T & $\begin{array}{c}\text { 0910Imi } \\
\times 5 i / 0\end{array}$ & $\begin{array}{c}\text { 2. TPU } \\
\boldsymbol{x}\end{array}$ & whts & $\begin{array}{l}\text { thul- } \\
\text { Ptas }\end{array}$ & $\begin{array}{r}1000 \\
7\end{array}$ & 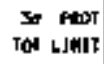 \\
\hline Tpkpl algin & 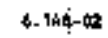 & 20 & 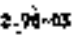 & & & AT & 5.40 .04 & $\mathcal{Z}$ & $3.05-05$ & & 5 & 4 \\
\hline
\end{tabular}

\section{FlibaI Report}

\section{acterts}

10 9

Buntry Darh strelto"

Vare do
Ith 19 X2.5 9rotecal 35I ve्wiक I.0 ford ondo version 3.09

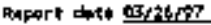


222-S IAEORATORY

TAMK $341-T-110$ BOCE It

DUPLICATE
DS q7owed

Conteck tesir. Duplica11

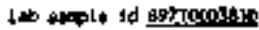

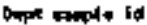

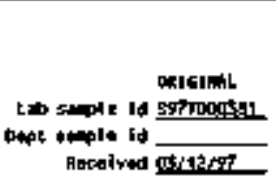

c1ithe Inits

Tore I-116

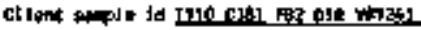

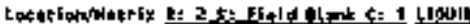
collected

phain of uapoty id

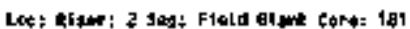

Final Roport

DLPLICRTEB

Paga 10

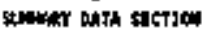

Pagu Z।

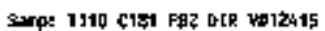

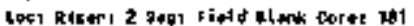

130 id tate

Protot sूT

Voraion a.t

Form RP=0MP

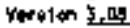

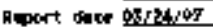




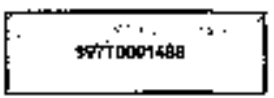

\section{2-S IABORATORY} thin 26!-T=110 talt 181

MATRIX SPIRE

sol grocting

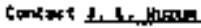
UAIII) झाt从E

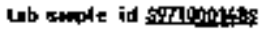
bepc suple Id

\begin{tabular}{|c|c|c|c|c|c|c|c|c|c|c|c|c|}
\hline Murte & $\begin{array}{l}\text { BPite } \\
\text { wet to }\end{array}$ & 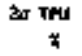 & untida & $\underset{1}{m L L}$ & $\begin{array}{l}\text { कox [- } \\
\text { FIns? }\end{array}$ & गत्रT & $\begin{array}{l}\text { Anes } \\
\text { ucista }\end{array}$ & $\begin{array}{c}2 \pi \text { Eat } \\
\text { मैं }\end{array}$ & $\begin{array}{c}\text { OxIeIIMAL } \\
\text { uclide }\end{array}$ & $\begin{array}{c}20 \text { Tru } \\
4\end{array}$ & 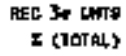 & 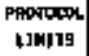 \\
\hline Iфtњl slpht & 1. & & & & & x. & $1 . \pm 5$ & 5.0 & T.50․ ㅁ? & 30 & $\overline{3 Z}$ s-118 & $\pi 5-125$ \\
\hline
\end{tabular}

\section{FInat Report}

\section{Whatky काxes}

raye 1

SIMAgr Dath काTा0

Pog* 22

Citent tros

10nk $r=110$

itialun

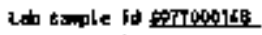
ongt ample to

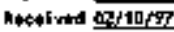

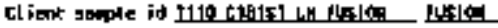

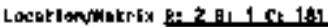
colli inctand Chain of artody id Ex下 


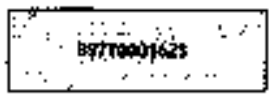

\section{2-S IAEORATORY}

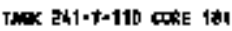

MATRIX SPIRE

\$t Troonst

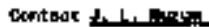

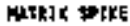

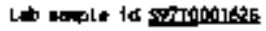

oept anlo id
Dalolimil,

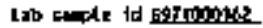

berer id herel red orf11,gr
Clint Thos

Tort. $\mathrm{I}-110$

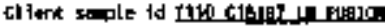

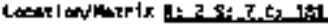
Elsto.

coll lectid soure

Galn of aubost td

\begin{tabular}{|c|c|c|c|c|c|c|c|c|c|c|c|c|}
\hline MuLyt: & $\begin{array}{l}\text { silke } \\
\text { utifo }\end{array}$ & $\begin{array}{c}2 y \text { TF } \\
7\end{array}$ & $\operatorname{mom}_{\text {stide }}$ & 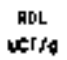 & musi- & TEST & $\begin{array}{l}\text { Notir } \\
\text { Whisto }\end{array}$ & $\begin{array}{c}20 \text { Ex } \\
\pi\end{array}$ & 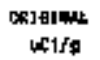 & $\begin{array}{c}\text { 2. IPU } \\
x\end{array}$ & 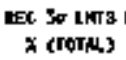 & $\begin{array}{l}\text { Fotora } \\
\text { t pul1s }\end{array}$ \\
\hline Sold alphe & $1,19 \%$ on & & & & & ดT & $1,7 \leq 100$ & 5,0 & $6,054-02$ & 21 & 호 혹-117 & $\pi$ \\
\hline
\end{tabular}

Final Report

MItix FIKEs

fot 2

STEAY BL SEet CON

toges 23 lab id atas

prazetal 391

Verolon ach

fond on-ths

verstion 3.06

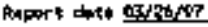




\section{$222-5$ LABORATORY}

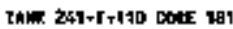

WAFRIX SPIXE
T110-carte in Fution
Sya trogpo:t

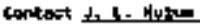
wirtix apjes

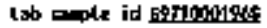
DApe toplo If ctroiul

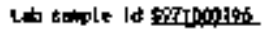

pope paple la

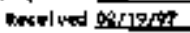

Cl banx 199.

Jonk 1.ith

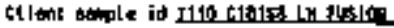

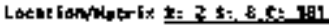

2us:10

collected

chatn of suabuty 14

\begin{tabular}{|c|c|c|c|c|c|c|c|c|c|c|c|c|}
\hline NuthIII & $\begin{array}{l}\text { BRIKE } \\
\text { WEIJa }\end{array}$ & $\begin{array}{c}\text { 20 IPU } \\
\text { I }\end{array}$ & $\lim _{x \mid 10}$ & $\begin{array}{c}\operatorname{noth} \\
4+5 / t\end{array}$ & $\begin{array}{l}\text { comL- } \\
\text { fritat }\end{array}$ & $B \| \$ T$ & $\begin{array}{l}\text { wem } \\
\text { we1to }\end{array}$ & $\begin{array}{c}\text { ट० घम } \\
x\end{array}$ & 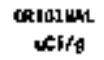 & $\begin{array}{c}\text { ê TrU } \\
x\end{array}$ & $\begin{array}{l}\text { PEC Jo uns } \\
\mathbf{z} \text { (TofmLt }\end{array}$ & $\begin{array}{l}\text { PUDTDCOX } \\
\text { LINIT\$ }\end{array}$ \\
\hline Iotal Alghe & $0,3 P C .00$ & & & & & $a t$ & 1.75e01 & 5.0 & 3.t46.05 & $\mathscr{2}$ & 포. 애에 116 & $\pi+1 \% 5$ \\
\hline
\end{tabular}

\section{FlnaI Report}

HTRIX अPIEE:

Pts 3

gumugy bath betcon

Page 24 lab 讨 $M 2+5$

Protetod t:I

ranton 10

forth owh th

Uersian tot.

topoct det 보/276 


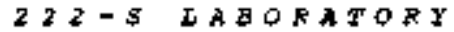
FAMK $3+I=F-110$ CORE $19 \mathrm{I}$

DATA SHEBT

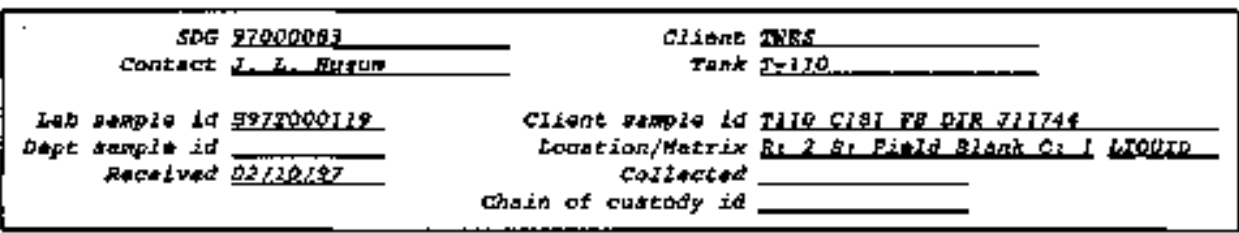

\begin{tabular}{|c|c|c|c|c|c|c|c|}
\hline 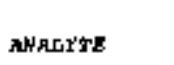 & ens wo & $\begin{array}{l}\text { ReswLT } \\
\text { ret//nd }\end{array}$ & $\begin{array}{c}\mathrm{ga} F \\
\mathrm{z}\end{array}$ & 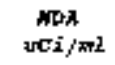 & $\underset{u C 1 / m I}{R D L}$ & $\begin{array}{l}\text { OUALI- } \\
\text { FrERS }\end{array}$ & TESTT \\
\hline Total Alphs & $12597-86-1$ & $5.37 x+02$ & 120 & $4.02-07$ & & & $\mathbf{A P}$ \\
\hline
\end{tabular}

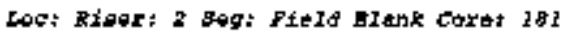

Fina R R port

DATA SHESTS

Page 1

SUMKARY DATA SECTIOM

Pngo is
Lab id 2280t5

Protocol gs57

varsion 10 Form DYR-bS

Vargion j.혀 keport date of/20/4t 


\section{$222-s$ LABORATORY TANR 141-T-190 CORB 181}

DTA SHEET
FILO C1\&1 PAR DIR Y*124I

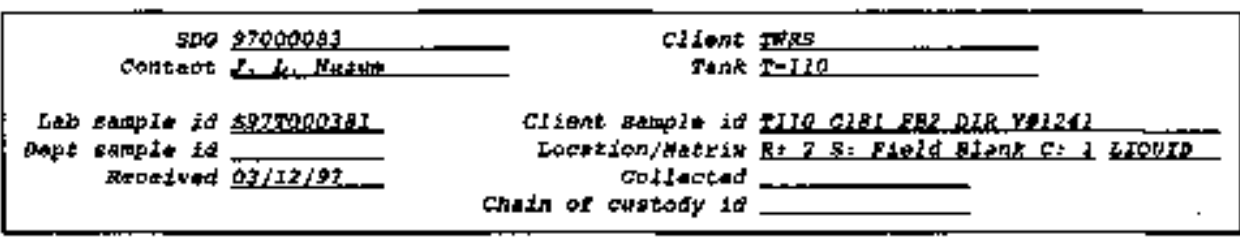

\begin{tabular}{|c|c|c|c|c|c|c|c|}
\hline AXALYKE & CAS NO & $\begin{array}{l}\text { Retsoth } \\
\text { uct/al }\end{array}$ & 20 TPU & 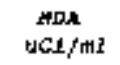 & $\begin{array}{c}R D L \\
U C L / \mathbb{R} I\end{array}$ & $\begin{array}{l}\text { QthAEI- } \\
\text { FIERS }\end{array}$ & TEST \\
\hline P3dtonilen $259 / 210$ & & $<3.45=06$ & & $9,18-06$ & & v, & $P V$ \\
\hline
\end{tabular}

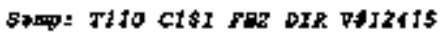

boy, Risar, 2 beg, Field Blatik corte IBd

Final Report

DATA begatP

Pajब 2

FUHMART DATA SECTION

Page 26
Lab id $2 \geqslant 2=9$

prococos ss

varmion Li

Fort avo $=05$

veraion j-숭

taport dato 03/25/97 
HNF-SD-WH-DP-238, FEV.O

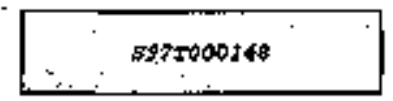
$2 Z 2-S$ LABORATORY TANK 24]-T-JIO COORE fक]

$D A T A \quad S H E T$

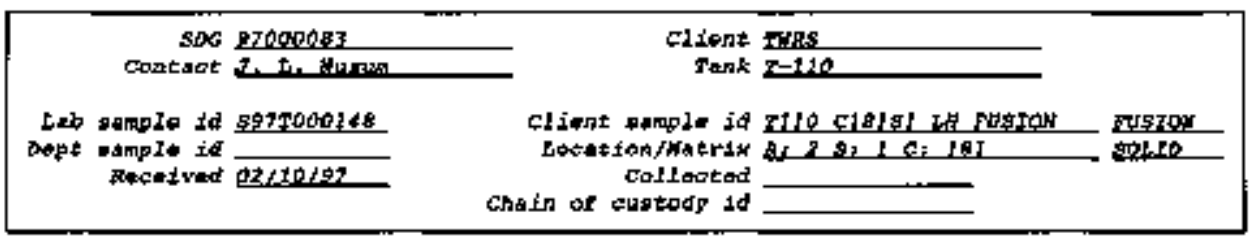

\begin{tabular}{|c|c|c|c|c|c|c|c|}
\hline ANAUYME & CAS MO & $\begin{array}{l}\text { RESULT } \\
\text { LELf } / 9\end{array}$ & $20 \mathrm{TPU}$ & $\begin{array}{l}\text { APA } \\
\text { UCA } / 9\end{array}$ & ues/o & $\begin{array}{l}\text { OUALY: } \\
\text { FISRE }\end{array}$ & Test \\
\hline Total alphn & $1258 t-16-I$ & $7.50 E-02$ & 20 & $7,98-03$ & & & $A F$ \\
\hline
\end{tabular}

Final Report

DATA STEETH

Page ?

gUHEARY DATA SECTTOH

Dage 27
$5 a b$ id $27 \vec{z}-s$

Frotocol s.5.

vargion $I-0$

Ford OYNDS

varasen 308

Report dat a 0/25/92 
$22 z-5$ LABORATORY ThXK 24]-T-I10 CORE IEI

DATA SHEET
WNF-SD-WN-DP-2SB, REV. 0

\section{$59700002+9$}

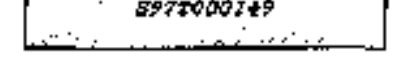

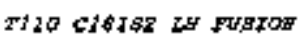

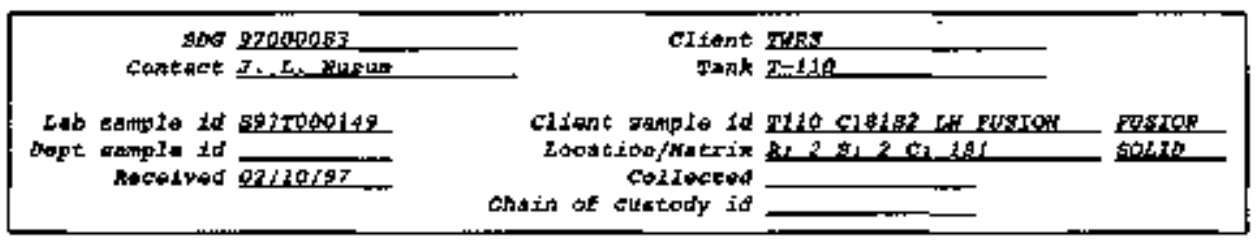

\begin{tabular}{|c|c|c|c|c|c|c|c|}
\hline NAIITB & CAS MO & $\begin{array}{r}\text { Rstopt } \\
\text { aci/g }\end{array}$ & $\begin{array}{c}2=58 \\
k\end{array}$ & $\underset{\omega C i / g}{\operatorname{mox}}$ & $\begin{array}{l}\text { Rot } \\
\text { uCijg }\end{array}$ & $\begin{array}{l}\text { OUALI- } \\
\text { FIBRE }\end{array}$ & TEtrt \\
\hline Iotal Xlphe & $12597-46-1$ & $+.665-02$ & 72 & 7. & & & $\mathbf{A r}$ \\
\hline
\end{tabular}

FInal Raport

\section{DATA \$HFETS}

Faga d

SUAHARY DAFA SECTION

Patge 79

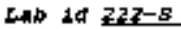

Protacol 5xP

Dorsion 1.0 Facp DYATOS

Vardion 3.af

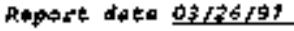




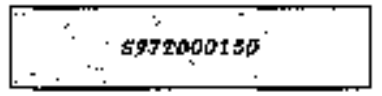

$222=S$ IABORATOAY

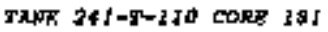

$D A T A S E E T$
HEV-SD-WM-OP-2SB, REV.O

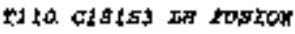

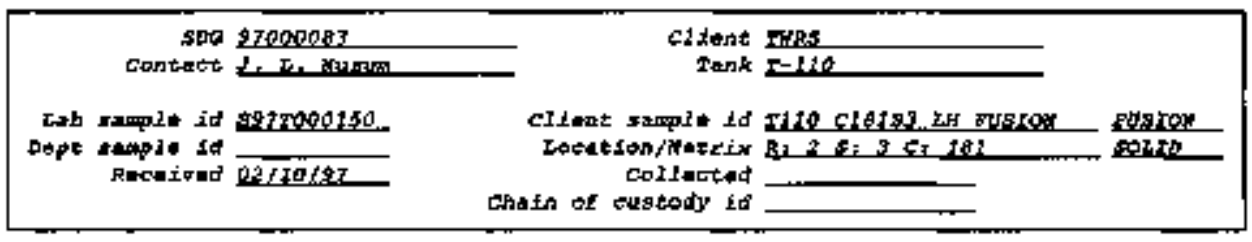

\begin{tabular}{|c|c|c|c|c|c|c|c|}
\hline ANALPTS & ans 10 & 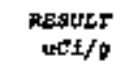 & 20780 & 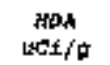 & $\begin{array}{c}R D L \\
\text { bai/g }\end{array}$ & $\begin{array}{l}\text { QPALI- } \\
\text { FrERS }\end{array}$ & Pest \\
\hline total derpha & $1258 t-4 \alpha-1$ & 20128 & t) & 4.OB-OA & & I & $N T$ \\
\hline
\end{tabular}

FIn 1 Report

\section{PATA BgESTH}

Fage 5

SUMAAFY DATA SECPION

$P \geq g * 29$ यab id 222-s Protacol 8sT

varafor th Fortt $0 U 0=05$

Veresan 3,0R Atport date 03/24/97. 
$222-S$ IAEORATORY

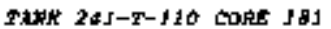

DATA SHEET

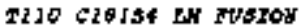

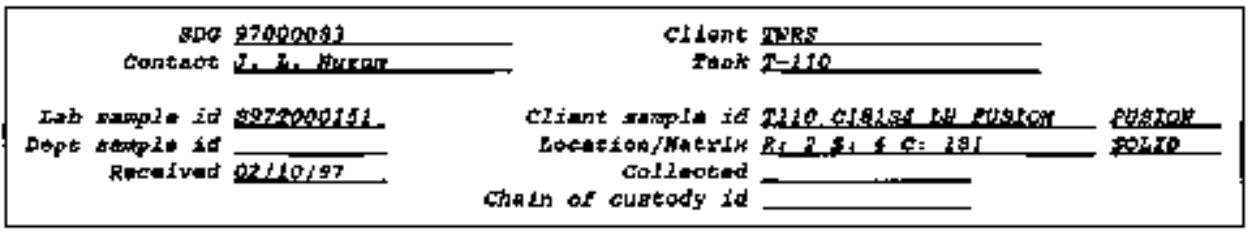

\begin{tabular}{|c|c|c|c|c|c|c|c|}
\hline AHALYTS & cas mo & 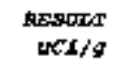 & 20 3 & $\begin{array}{c}A \operatorname{R} \lambda \\
\operatorname{uci} / \Omega\end{array}$ & $\begin{array}{c}A b t \\
H C D / g\end{array}$ & $\begin{array}{l}\text { QUanI- } \\
\text { FISRSS }\end{array}$ & $T S B T$ \\
\hline Totel dipha & $12597=46=2$ & $3 \div 915=02$ & 27 & $4,45-04$ & & $\mathbf{z}$ & $A T$ \\
\hline
\end{tabular}

FInI Repot

DNTA FHETS

Pagat 6

FUWHART OATA STCFIDN

Paga 30
Latb id 232-5. ProtocoI STS

Parision t.o parat aybohs

Vorefor 3. 0 B

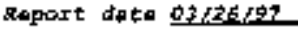


HNF-SD-WH-DP-238, REV, 0

222 - S LABORA TORY TXNK 24J-F-110 CORE 101

DATA SHET
Fio cjgigs LH Fuan

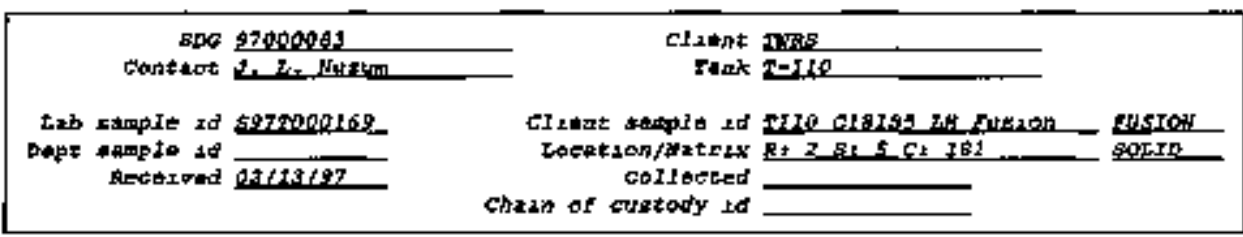

-

\begin{tabular}{|c|c|c|c|c|c|c|c|}
\hline NAXYTE & CAS No & $\begin{array}{r}\text { RESULI } \\
\text { uEtII/g }\end{array}$ & $\begin{array}{c}20 \text { TFV } \\
\text { t }\end{array}$ & $\begin{array}{l}\operatorname{man} \\
\forall C \perp / g\end{array}$ & $\begin{array}{c}R D L \\
d C_{2} / 9\end{array}$ & $\begin{array}{l}\text { OUAEI- } \\
\text { FIERS }\end{array}$ & TESF \\
\hline roEAI AIgha & $I 2587-46-J$ & $4.299 \mathrm{mpz}$ & $2 \overrightarrow{2}$ & $2.58-03$ & & & $A T$ \\
\hline
\end{tabular}

FIn 1 a

DATA 㕷EgTY

Paģo 7

SUNAART DATA SECTIOW

Eage it
Zab at 2a2-s

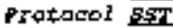

Vortion 10

Forn 0 L $=0 \mathrm{~S}$

varsion tos

Aeport fat as a 26107 
222 - 2 LABORATORY ratr 2A1-T-110 CORE IBd

DATA SHEET
TIO CASTS WN FUSO

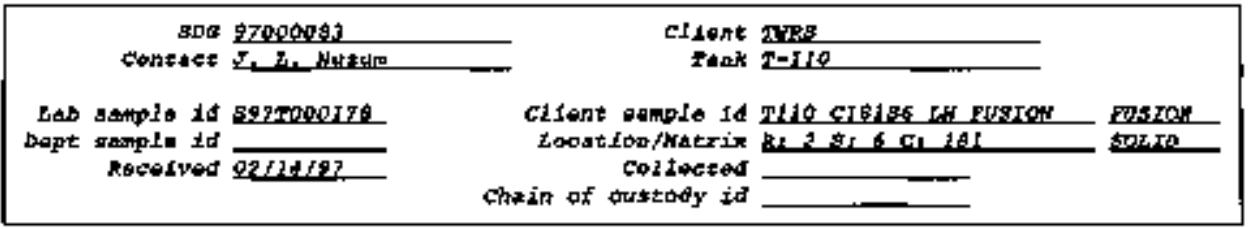

\begin{tabular}{|c|c|c|c|c|c|c|c|}
\hline Marrts & cas no & $\begin{array}{l}\text { RESULT } \\
\text { UC1/s }\end{array}$ & $\begin{array}{c}\text { Zo IPV } \\
t\end{array}$ & $\begin{array}{c}\operatorname{sind} \\
\sec 1 / 0\end{array}$ & 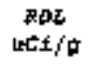 & $\begin{array}{l}\text { OOALI= } \\
\text { FIERS }\end{array}$ & TEST \\
\hline Toted $\mathrm{AL}_{\phi} \mathrm{hH}$ & $12597=46=2$ & $5.525-02$ & 32 & $2.25-09$ & & & $A T$ \\
\hline
\end{tabular}

FInaI Report

ONeB shriprs

Page?

Germari bath ERCTIOH

Papt 32

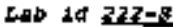

Protocol t5t

rextion LQ⿻

Forp DVD-p오

Yersion 308 Report sat od $036 / 27$ 
HNF-SD-WMADP-238, REV. 0

\section{$222-S$ IABORATORY} TAHK 241-T-1 to COAB 191

PATASHEE

897T000162

s92000162

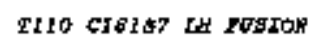

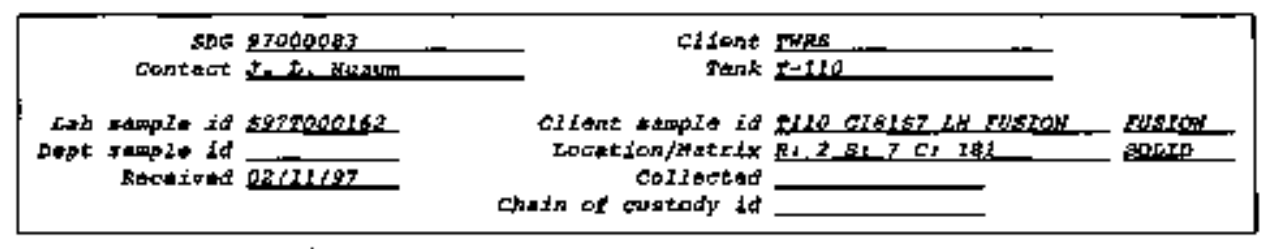

\begin{tabular}{|c|c|c|c|c|c|c|c|}
\hline MAIYTE & CAS NOO & $\begin{array}{r}\text { RESUET } \\
\text { UCDI/O }\end{array}$ & $20 \mathrm{TPU}$ & $\begin{array}{c}A B A \\
u C d / 9\end{array}$ & $\begin{array}{c}A b L \\
\omega C S / Q\end{array}$ & $\begin{array}{l}\text { QUAEI- } \\
\text { FIRRS }\end{array}$ & TEST' \\
\hline Totdr Alphn & $125.67-46-1$ & $6.052-02$ & $2 I$ & $2.05-03$ & & & $A T$ \\
\hline
\end{tabular}

Final Report

PATA SARETS

Pagt 9

SUMHART DAFA SECTION

eage is
Lab id 222-s

Protocol sim

$v e r g i a n$ t.o

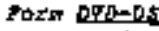

Dexsion $3.0 \mathrm{~B}$ tegart date ardafidet 
Z2 $2-s$ IABORATORY

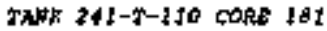

DATA $B$ FE E T
FIO CT8158 EH FOSTOF

\begin{abstract}
$\operatorname{sag}$ gropopos?
contact ith Hown

Recolvod at/16/0?
\end{abstract}

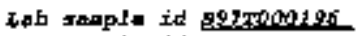

Dopt senplo id

\section{Client Tras \\ Fabe 7110}

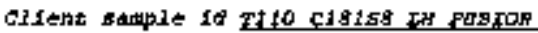

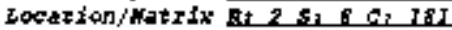
collooted

chath of custody sot

\begin{tabular}{|c|c|c|c|c|c|c|c|}
\hline MAATTE & CAS FO & $\begin{array}{r}\text { ResoLT } \\
\text { WCI/g }\end{array}$ & $\begin{array}{c}20 \text { Tptr } \\
4\end{array}$ & $\begin{array}{c}W D A \\
\operatorname{CO} 2 / \phi\end{array}$ & 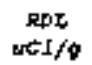 & $\begin{array}{l}\text { QUACI- } \\
\text { PYERS }\end{array}$ & $2 E \$ T$ \\
\hline Toted A Apho & $12567-16=3$ & $5.888-02$ & 27 & $3,0 x-03$ & & & ته \\
\hline
\end{tabular}

FinaI neport

OdPR grteEs

Papo 10

STMHARY DATX SECTTOY

bage is
LAD 10 $77 F=8$

Protacal is th

veraion 1.0

EOrm oVD-DG

Versidan 30 a

Rogert date 0.3/76/97 


\section{$222-5$ I ABORATORY}

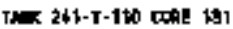

lest ar Matrix Hough

tot 97woons

contect L1, thith
METHOD SUMWARY

NOHW NGLTSIB

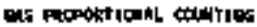

ctlent Ins:

$T$ ond $T+110$

\begin{tabular}{|c|c|c|c|c|}
\hline QJeT qNPE ID & Lit & $\begin{array}{l}\text { RMN QUNF- } \\
\text { TEET FנX }\end{array}$ & PLtectit & $\begin{array}{l}\text { 1) Iote } \\
\text { ilfint }\end{array}$ \\
\hline \multicolumn{5}{|c|}{ 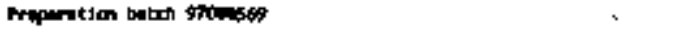 } \\
\hline $\begin{array}{l}\text { of Blat } \\
\text { rilot cits re och J11744 }\end{array}$ & $\begin{array}{l}=10065 \cdot 2 \\
\text { sprtonod } 19\end{array}$ & & $\begin{array}{l}16605 \cdot 2 \\
16695-4\end{array}$ & $\frac{v}{3.3 \pi=07}$ \\
\hline 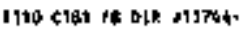 & spr100011\% & & $16655-5$ & ak \\
\hline Leantrod Bapla & s1tefs-1 & & $16685 \cdot 1$ & $\mathbf{n k}$ \\
\hline
\end{tabular}

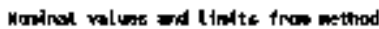

mar \{uxtsink?

$\Leftrightarrow$

Artrag:

\begin{abstract}
METHOD PERFORUANCE

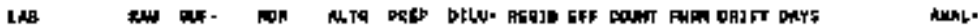

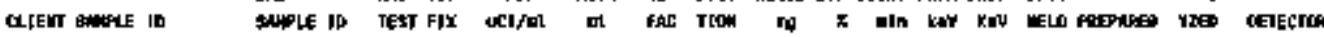

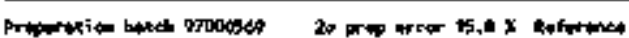

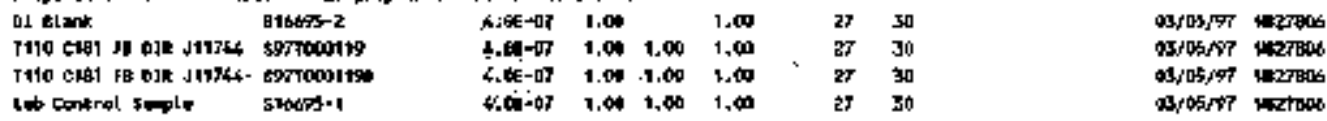

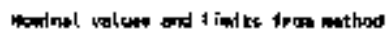

0.100

3.

30-55

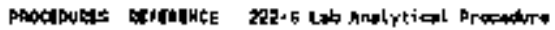

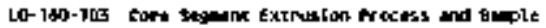

Preparation rer 17

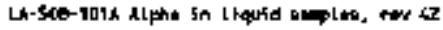

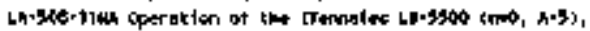

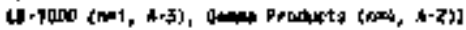
Alparberta counting Byntion
MYTIGEE \pm 2 to

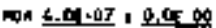

Foit 4 avm-15t
FIneI Roport

ratos zomates

Pot 2

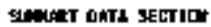

Poge 14

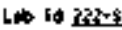

Procoeol 5 ST

Yareion 1it

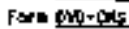

Verrion 3.46

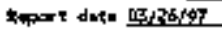




\section{Z-S LABORATORY}

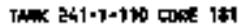

Teat pu_ Matr ik Llodulp spo spopoces

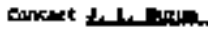

METHOD GUUMAR

PLUTCMUM- בM

ALPHA BPELTIDECOPT citart I!:

$r=t+110$

\section{RESU゙TS \\ aJEW BMPLE ID \\ LA Rour suF- \\ Pluconile Plubenlu

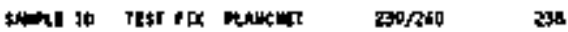

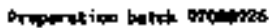

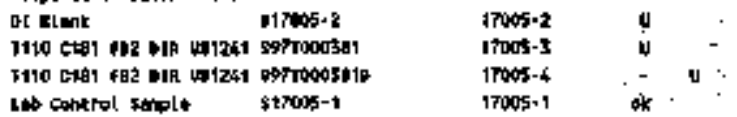

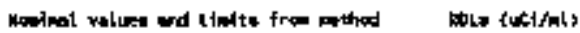

METHOD PERFORNAMCE

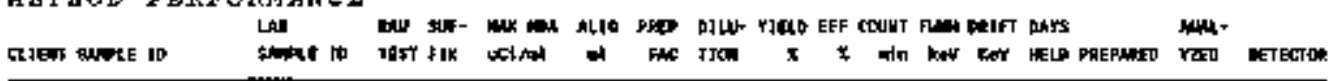

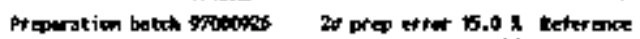

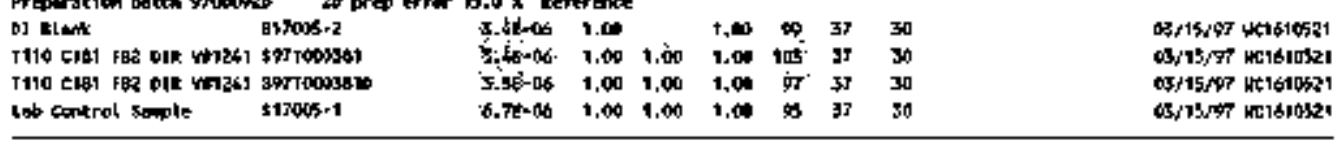

Nowiral values ond lintts fros method

0.200

30-005 3t

20-55

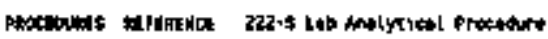

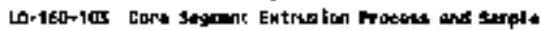

Propetion, ray 17

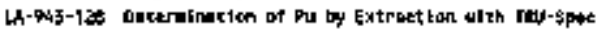

Rasin, rer $\mathbf{0}$ -

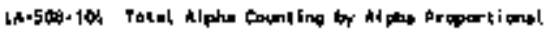

count bne, res 16

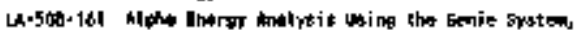
vor 12

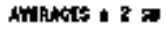

Fen 4 supups

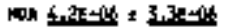

YIEL $\frac{92}{37} * \frac{7}{0}$

\section{Fin4l Report}

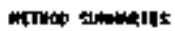

Pegt 1

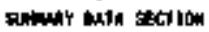

Degon 35

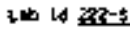

Pratocol बूy

veroran 1.0

ford ondo-rits

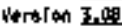

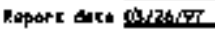


HNF-SDWH-DP-238, REV. 0

\section{2-S LABORATORY \\ Thur 241-T-110 DotE It}

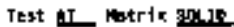
\$4 9700083 Contact de levt:
NETHOD SUWAARY

Mank murs]s

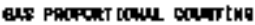

Cl fom 뇨도

Twक $\mathrm{I}=110$

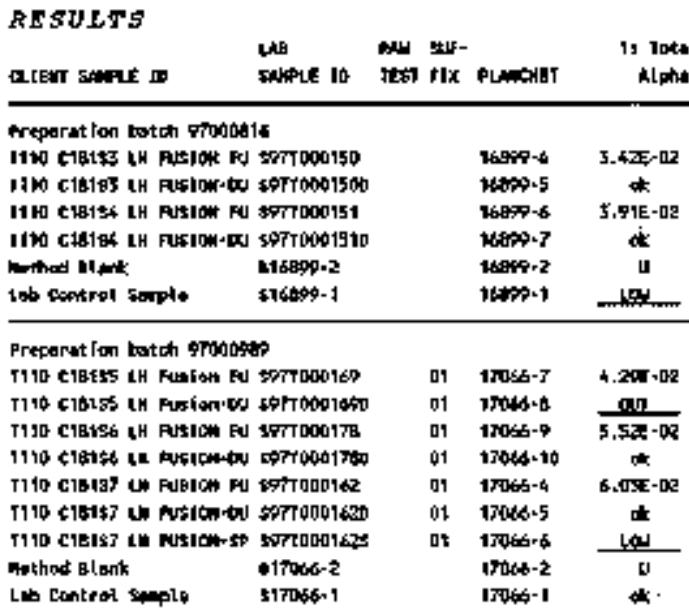

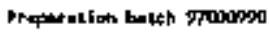

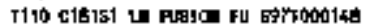

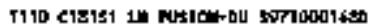

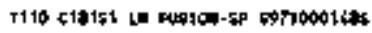

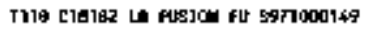

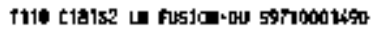
notiod Elonk B1 rost-2 Lob control sople 817067-1

\begin{tabular}{|c|c|c|}
\hline 02 & Itobs-4 & $7.50 \mathrm{E}-0 \mathrm{Q}$ \\
\hline 02 & 13067.5 & 些 \\
\hline 婄 & $17067-4$ & 10. \\
\hline or & 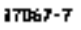 & $4.64 c-08$ \\
\hline de & JPodr-ts & ok \\
\hline & artots-z & v \\
\hline & 1704ti-1 & t+ \\
\hline
\end{tabular}

Prowation botsh shasiss

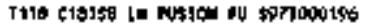

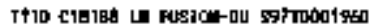

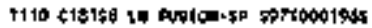
Hethoo Blent b1707z-2 Lo tomtrod sapla 5170re-1

\begin{tabular}{|c|c|c|}
\hline 02 & 17072=d & 5. Bac- 02 \\
\hline व2 & $13 t 2-5$ & or \\
\hline QR & $17072-6$ & لم. \\
\hline & Irtra-2 & U \\
\hline & $17072-1$ & ut \\
\hline
\end{tabular}

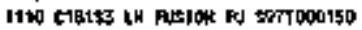

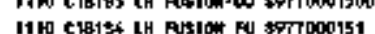




\section{ZZZ-S IABORATORY

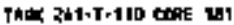

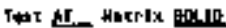

Boc 97 :0015s

concet d. Prmn

\section{METHOD SUNHARY MPAth motrists

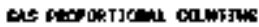

cliont tost

Tunt

\section{NETHOD PERFARHANCE}

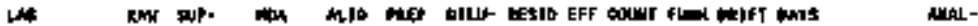

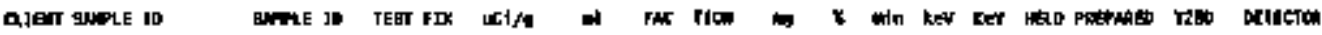

\begin{tabular}{|c|c|c|c|c|c|c|c|c|c|}
\hline T11日 & 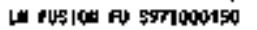 & 4. & 0,500505 & 1.00 & 27 & 50 & $05 / 05 / 9 T$ & 世rat & aztond \\
\hline T+10 c1a15s & L & 4ige-04 & 0.500 d96 & 1.0 & 27 & 30 & $03,05+97$ & प5/48 & 4hasens \\
\hline T110 대내 & الثا & $4.45-05$ & $0+500543$ & 1.00 & a) & 30 & $03 / 05 / 97$ & a) & veateat \\
\hline T110 cนtss & LH FLBIOW-DU ESTTOmOn5D & - 4 左-40 & 0.300 gez & 1.0 & 27 & 90 & $03, \tan 57$ & $03 \sqrt{66}$ & 1820606 \\
\hline Wethod bland & 日utat? 2 & 4.0E-104 & $0.500505^{\circ}$ & $1, \boldsymbol{\omega}$. & 27 & 30 & & $H 08 \% ?$ & 48rtas \\
\hline |t b tanktal & 1660\%-1 & $8.9=-04$ & $1.00505-$ & 1,00 & $\boldsymbol{r}$ 、 & 0 & & 408,99 & Lazans \\
\hline
\end{tabular}

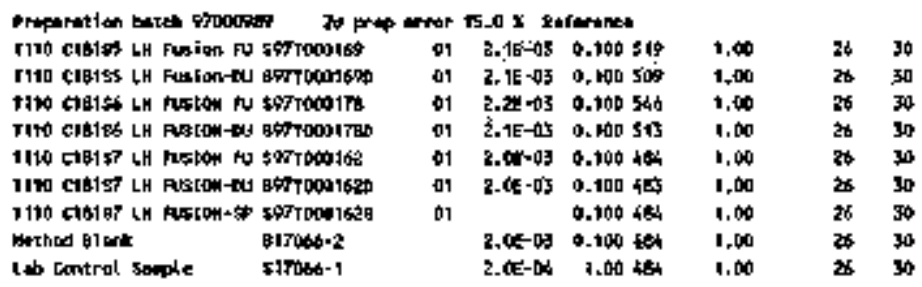

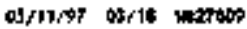

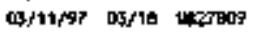

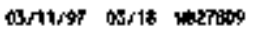

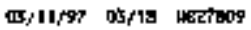

03л1s97 osite mertion

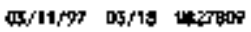

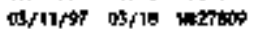

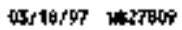

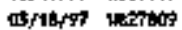

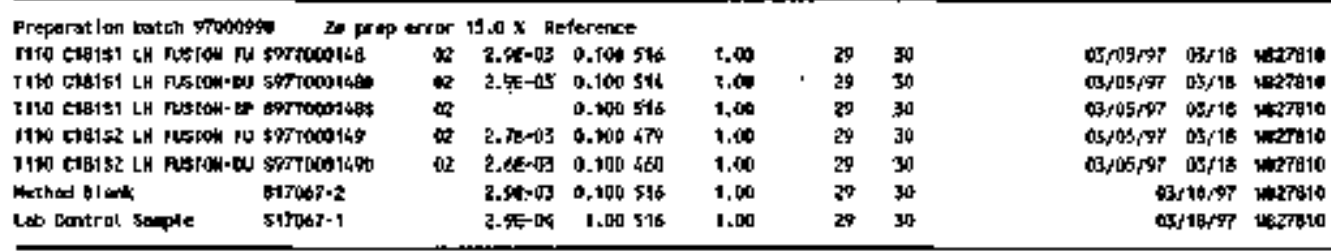

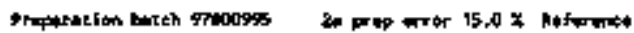

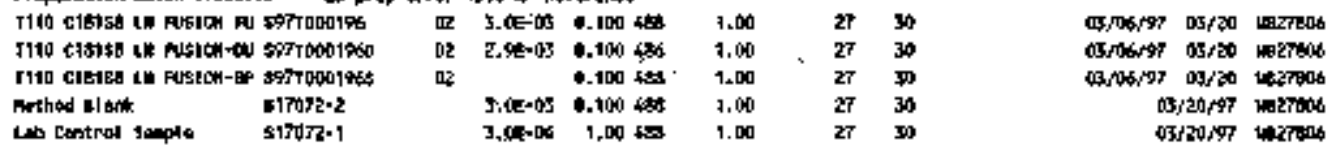

0.100

$\mathbf{3 0}$

$20-55$

FinaI Report

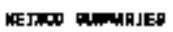

Popa 4

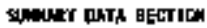

Page 3 .
Lsto id 27205

Propक巾 \$\$三丨

Varsion 10

Sore tuth-thts

Uarsion 3.64 Recort dete 03126007 


\section{$2 Z Z=5$ LABCRATORY}

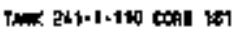

Iat 시 Whurix

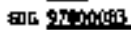

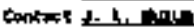

\section{METHOD SUNHARY, CONE. ALWh NULLYIs:}

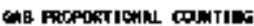

HNF-SD-WN-DP-238, FEV, 0

cilent T.s.

$T$ Ton $T+110$

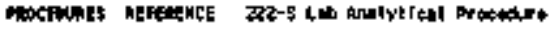

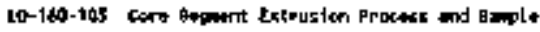
- reparaclon, rer 17

Lh-560-14t pusion with hlkall wetol Hodrowide, rev 40

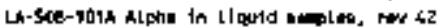

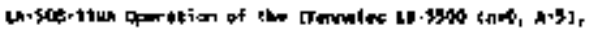

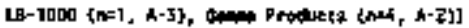
Alphortests thingling sycters
Final heport

EThor andurce日

Pogn 5

SMmak DATh SECTIC

page 50
Lab 1a 272-5

Protocel 战

voraton 1.0

Fom Therass.

Vequan 5.

Ragort dore ofristid? 
HPF-50-MN-DP-238, REY, D

SAHPLE HABLLI\$S 
HW.5D-WW-DP-238, REV, O

THIS PAGE INTENTIOARLLY LEFT BLAK 


\section{LABCORE Data Entry Template for Worklist\# 16499}

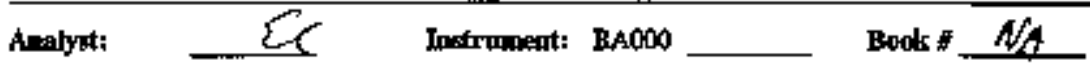

Method: 10-160-103 ReviMod $03 \partial$

Work's Conment: T-110 CIBI FIELD BLANK RISER 2 EXTRUSLON

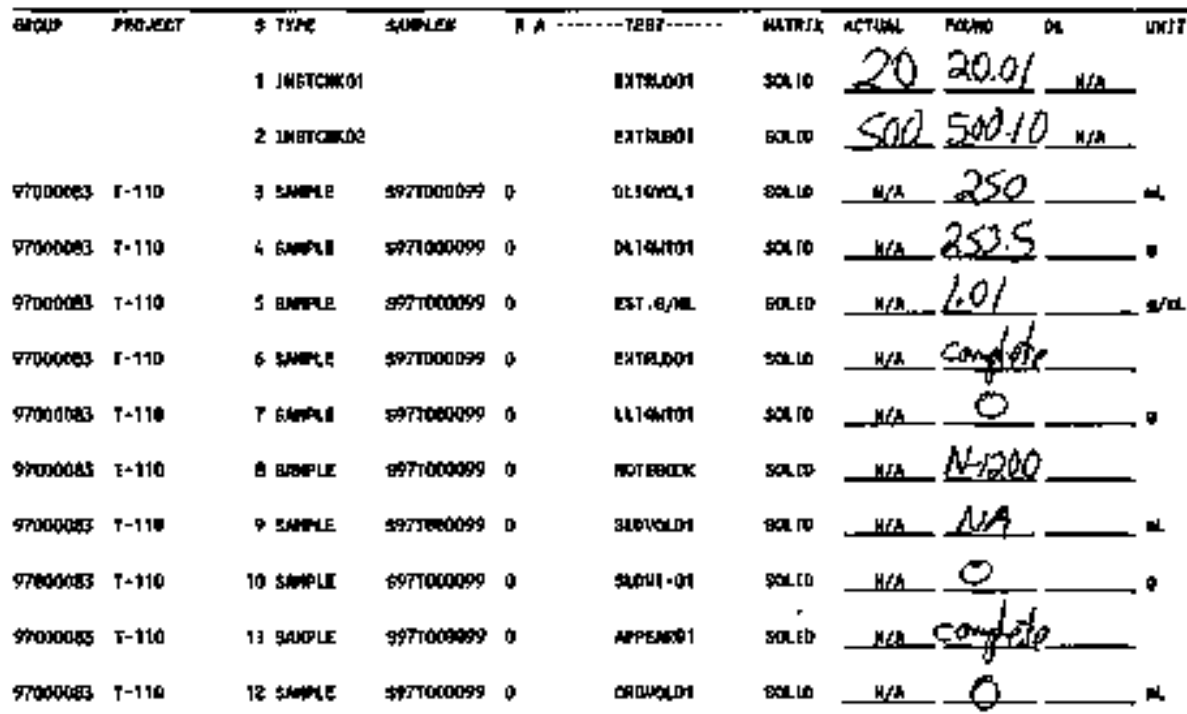

Final page for worklist \# 16499

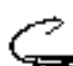

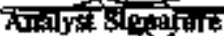

(1)

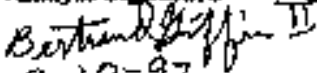

$2-10-97$

\section{$2+2.77$}

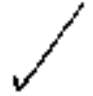

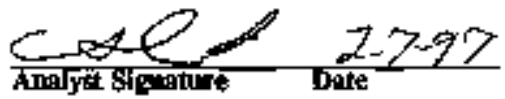

Dara Btoy Comuners:

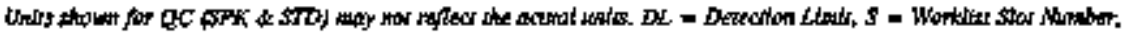

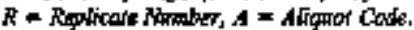




\section{LABCORE Data Entry Template for Worklist 16500}

Analyst:

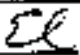

Instrument: BAOOO

Boots :

Method: LO-160-103 RewMod

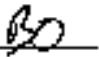

\section{Wortalst Comment; T-110 C181 SES 1 RISER 2 EXTRUSION}

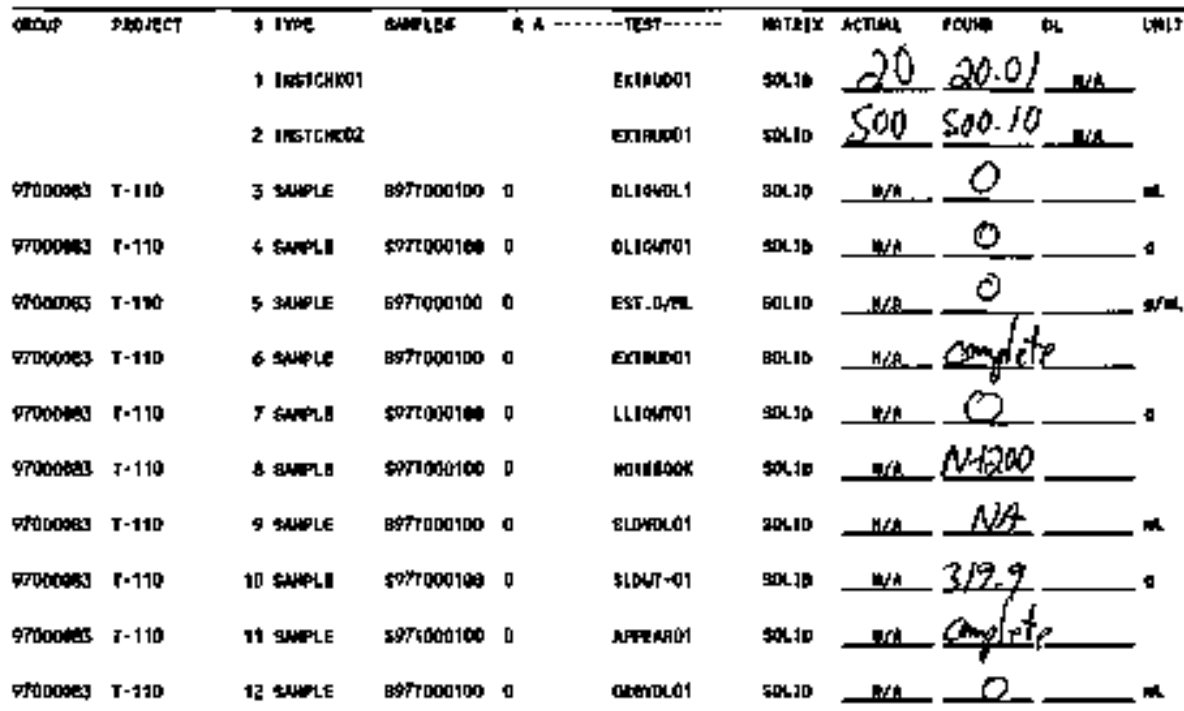

\section{Final page for worklist \# 16500}

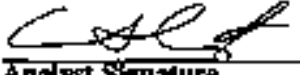

Anegat Sije-1ure

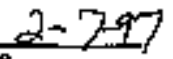

$2+29$

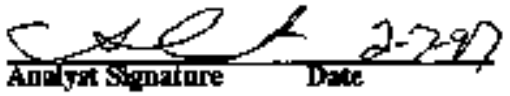

Dare Enity Cownonts:

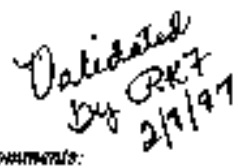

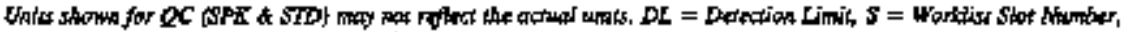

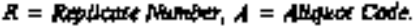




\section{LABCORE Data Entry Template for Worklist 16501}

\section{Anedgit:

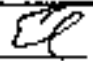

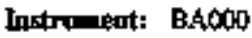 \\ Method: LO-160-103 RerMod 130 \\ Wartlist Cosment: T-110 C181 SEG \# 2 RISER 2 EXTRUSION}

Book $\# \perp / A$

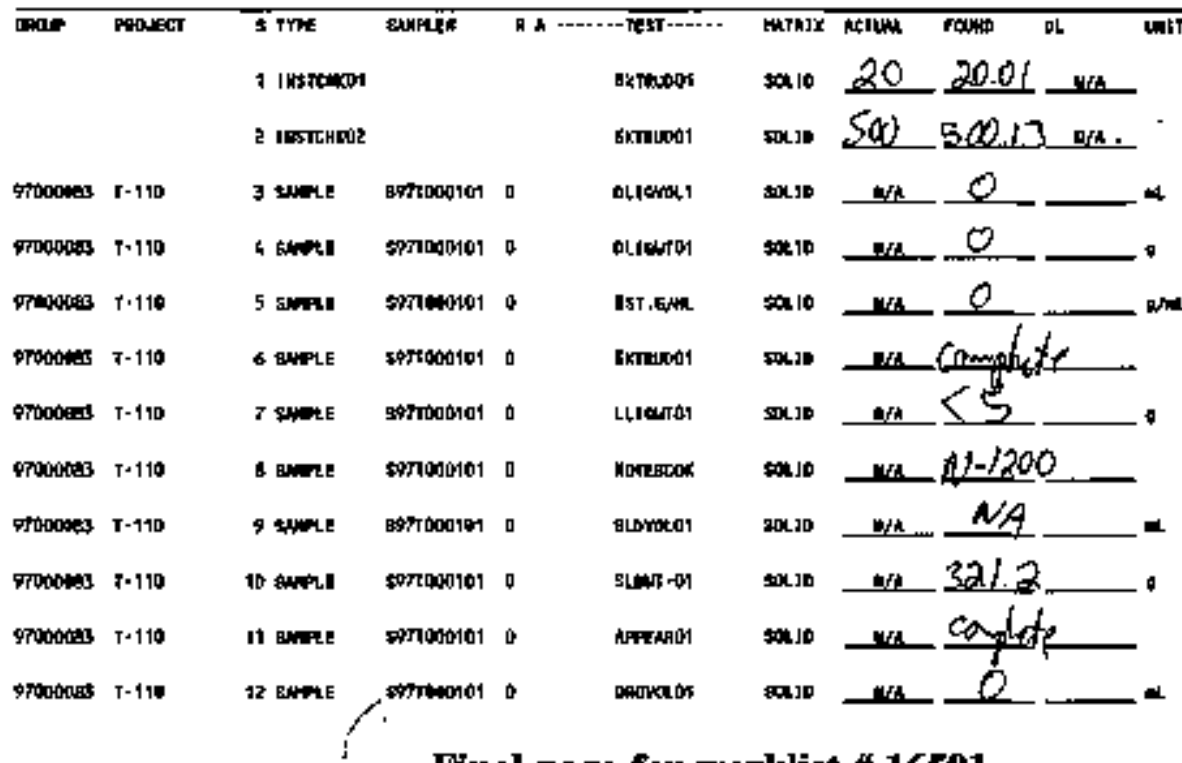

\section{Fingl page for worklist \# 16501}

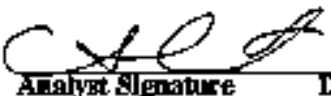

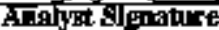
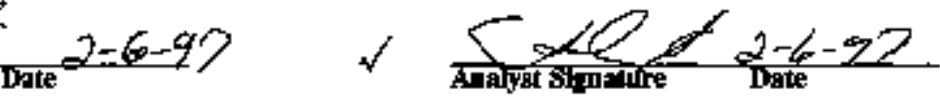

Data Entry Cominnts:

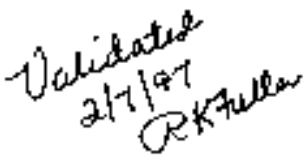

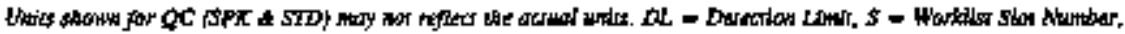

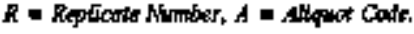




\section{LABCORE Data Entry Template for Worklist 16502}

Arallyx:

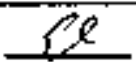

Instrument: BA000

Book : $N A B$

Methudt LO-160-103 Rew/Mod BO

Workllet Comment; T-110 C181 SEG \# 3 RISER 2 EXTRUSION

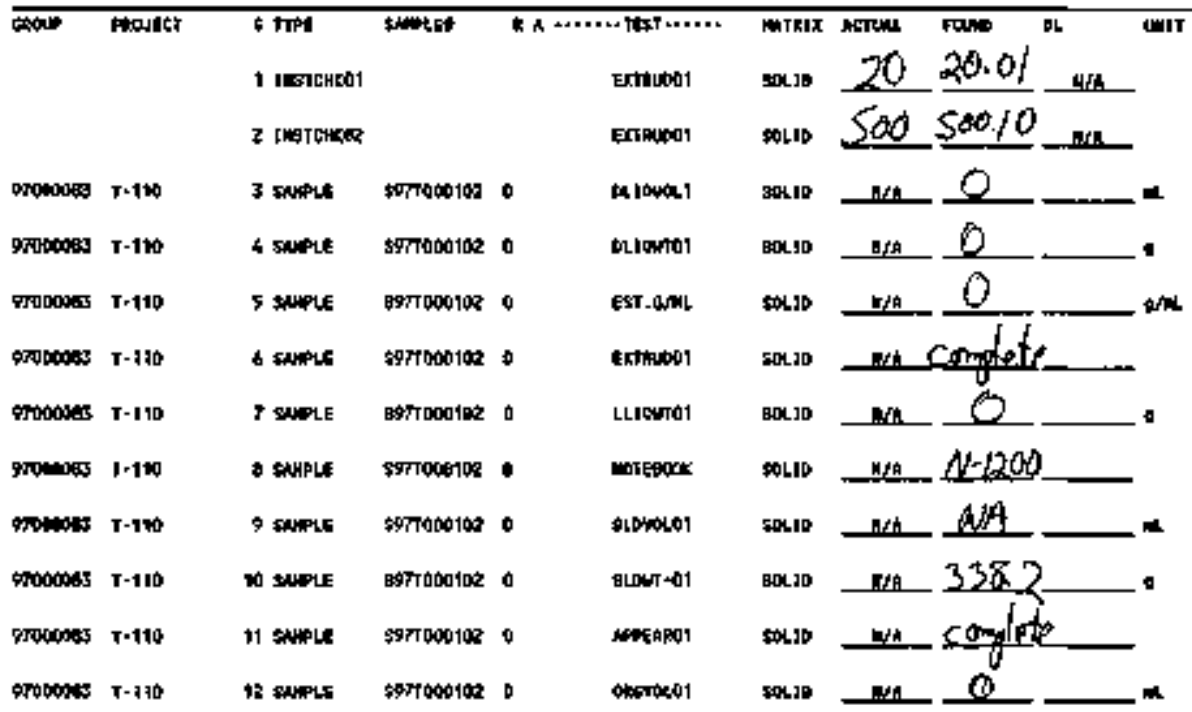

\section{Final page for worklist \# 16502}

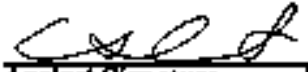

Ans For Siqueture

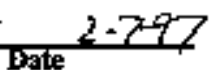

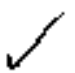

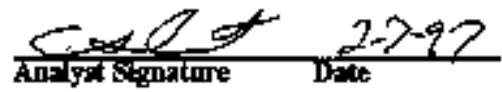

Dafa Emy Comuthins:

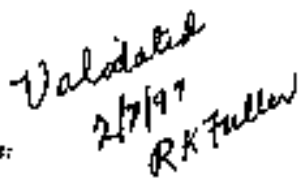

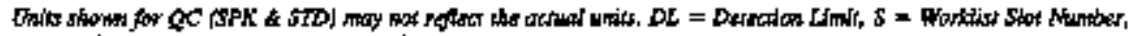

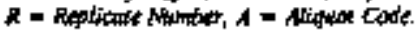




\section{LABCORE Data Entry Template for Workist 16503}

Analyat:

Indument: BAOOO

Book

Method: LO-160-103 RevMod , G-

Worllist Comenent: Tw10 Cli1 \$FG \# 4 PJSER 2 EXTRUSION

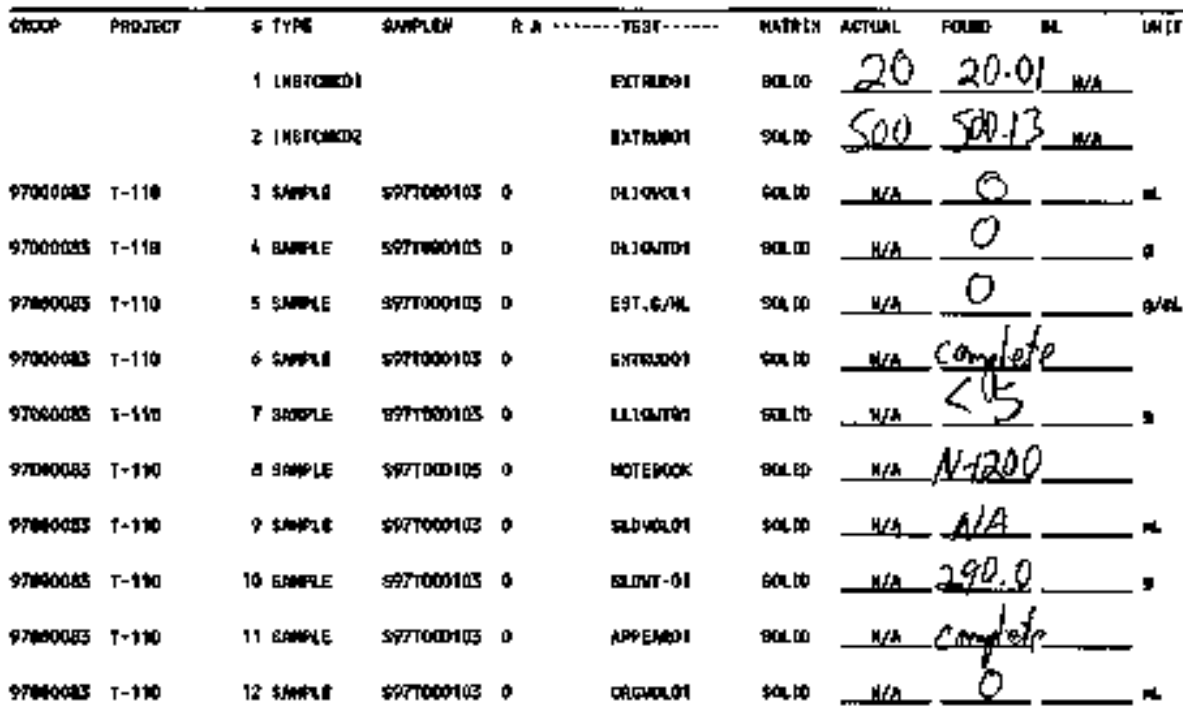

\section{Final page for worklist \# 16503}

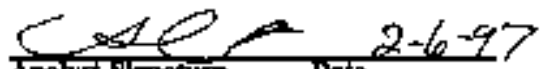

Analys sporivie Date

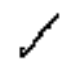

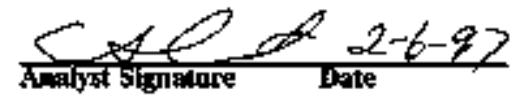

Dalp Eotry Cpongmt:

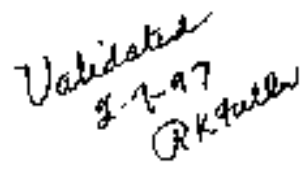

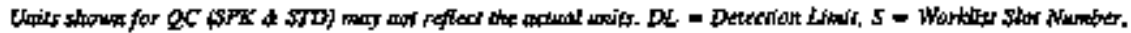

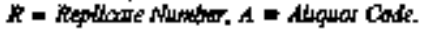




\section{LABCORE Data Entry Template for Worklist\# 16504}

Andyit: J(F

Instrinent: BADOO

Book * 새 .

Metliod: LO-160-103 Rev/Med B-C

Wort lst Corant: T-110 CIII SEG \# S RISER 2 EXTRUSHON

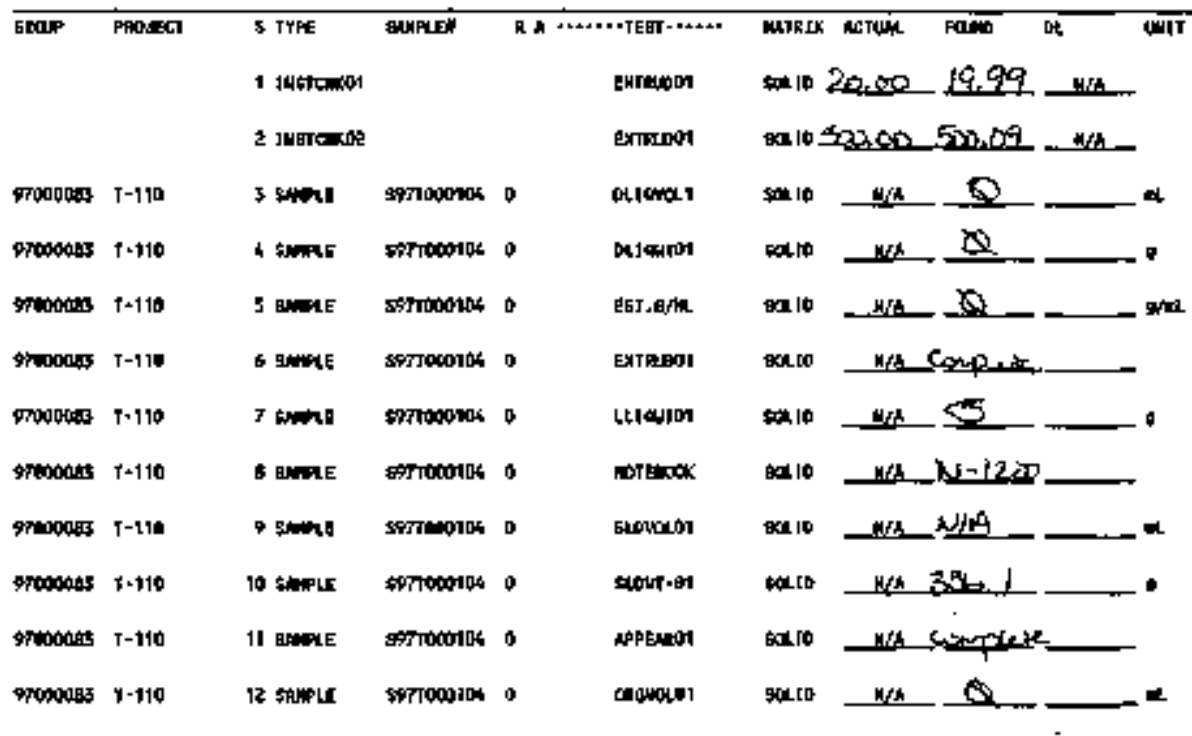

Final page for worklist \# 16504

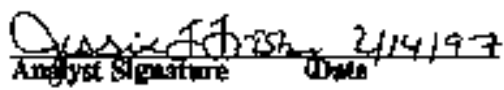

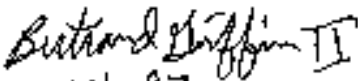

$2-14-97$

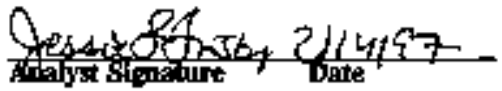

Dava Daty Comeners:

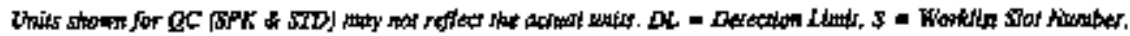

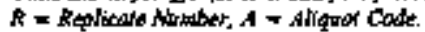




\section{LABCORE Data Entry Template for Worklist\# 16513}

fase: $I$

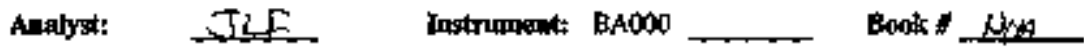

Method: Lo-160-103 Rewhthod ß-1-0

Wortalks Comment: T-110 C 181 SEG \#6 RISER 2 EXTRUSTON

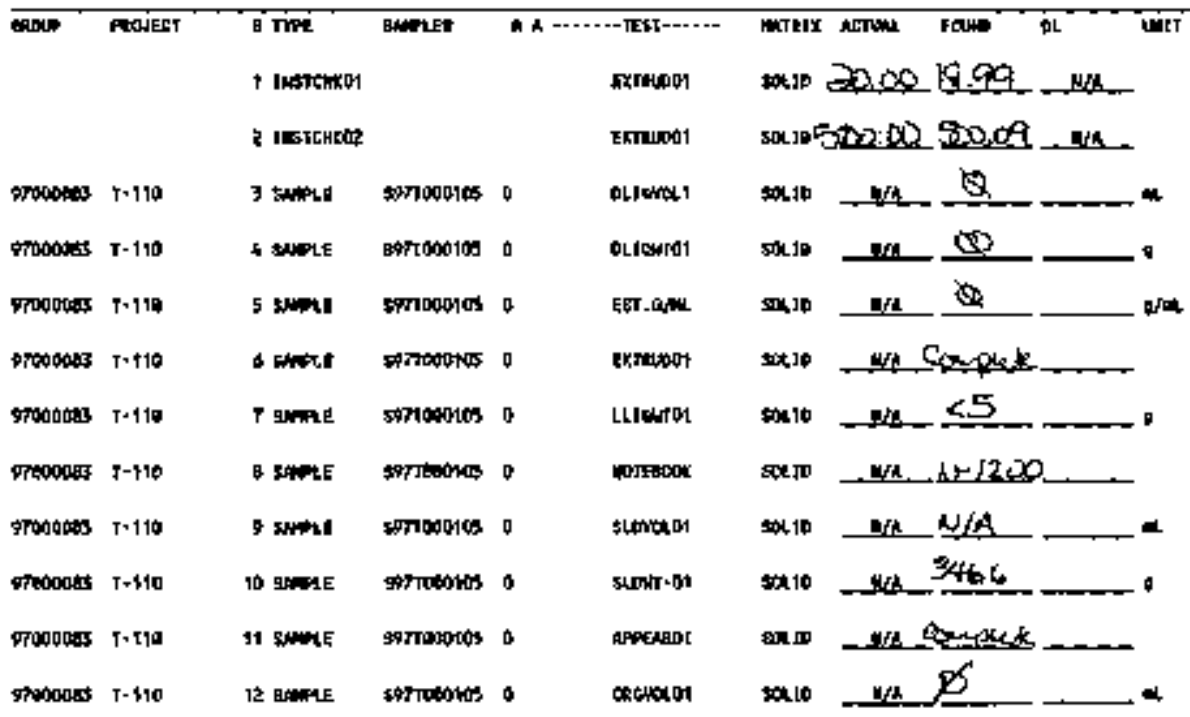

\section{Finat page for workllst \# 16513}

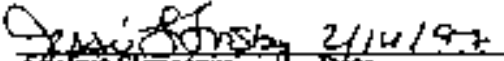

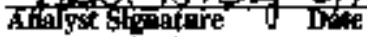

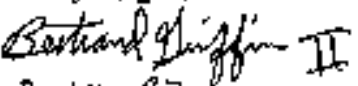

$2-14-97$

Data Dotry Compiats:
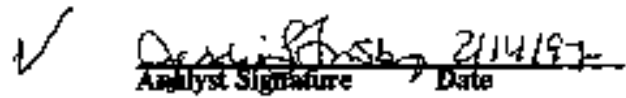

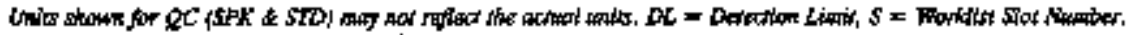

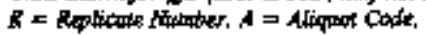




\section{LABCORE Completed Worklist Report for Worklist\# 16514}

Analyst: jlf

Method: lnstrabent: BAs000

RewiMod $B, D$
Boakf

Workllst Comment: T-110 C 181 SEO \#7 RISER 2 EXTRUSION

\begin{tabular}{|c|c|c|c|c|c|c|c|c|}
\hline Seq Type & Sampled & $\mathbf{R A}$ & Trest & Metifx & Actual & Fontutd & DL of Fiteld & Unit \\
\hline 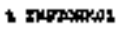 & & a & 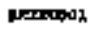 & JokT & $\mathbf{2 v}$ & 20.6 & 76.900 & 4 Bacerwhy \\
\hline 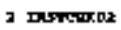 & & 0 & 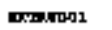 & $\operatorname{apc} m$ & 100 & do, & $14+, 01=$ & e leoertecy \\
\hline 1 mintex & xtrsponal & $\nabla$ & 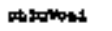 & Notrots & 뜨는 & $\bullet$ & & $m$ \\
\hline 1 sкани: & iptropgk & a & ритентау & I0kTR & $\mathbf{p} / \mathrm{A}$ & - & & 9 \\
\hline 3 vme & 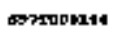 & 0 & arsobar & arantb & . & 4 & & tothe \\
\hline 1 Aluprest & 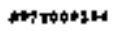 & a & wreopal & Itents & $m / x$ & ocmplate. & & \\
\hline$z \operatorname{cocos} x$ & 5Trvadue & 0 & Exismen & Fonth & $x=$ & 5 & & $=$ \\
\hline botatrs & sMmondt & 0 & Harlonot & 바타무 & 바. & Dratert & & \\
\hline sinose & myTaatix & $a$ & atruceal & 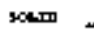 & mia & of & 0.100 & $-x$ \\
\hline 10 antrax & $s+\pi, 001 \mathrm{NS}$ & 0 & achanal & $\operatorname{soc} a$ & L & M1.J & 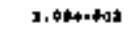 & 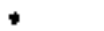 \\
\hline & ANmbobsh & 0 & Arperats & Harto & $E / 2$ & Anatrot & & \\
\hline 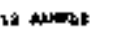 & Ayphálox & $b$ & otorratis & 1010 & 20 & b & & $=$ \\
\hline
\end{tabular}

\section{Conments Section:}

Commenis for sample: S9TT000106 and tesl APPEAROI.

"Exuruded 19 inches of solids which werc dark yellow in color and the texlure yats a wet sludge (D3). Sibsampled in half sepments.

\section{Final page for worklist $\mathbb{1 6 5 1 4}$}

Py

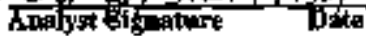

Analyst Signatura Dake

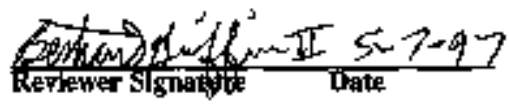

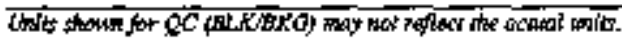




\section{LABCORE Data Entry Template for Worklist\# 16515}

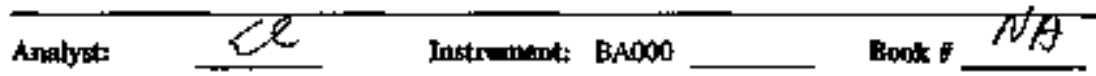

Method: LO-160-103 Rev/hod $B \mathrm{BO}$

Warkes Conment: T-110 C 181 SEO $\#$ R RSER 2 EXTRUSION

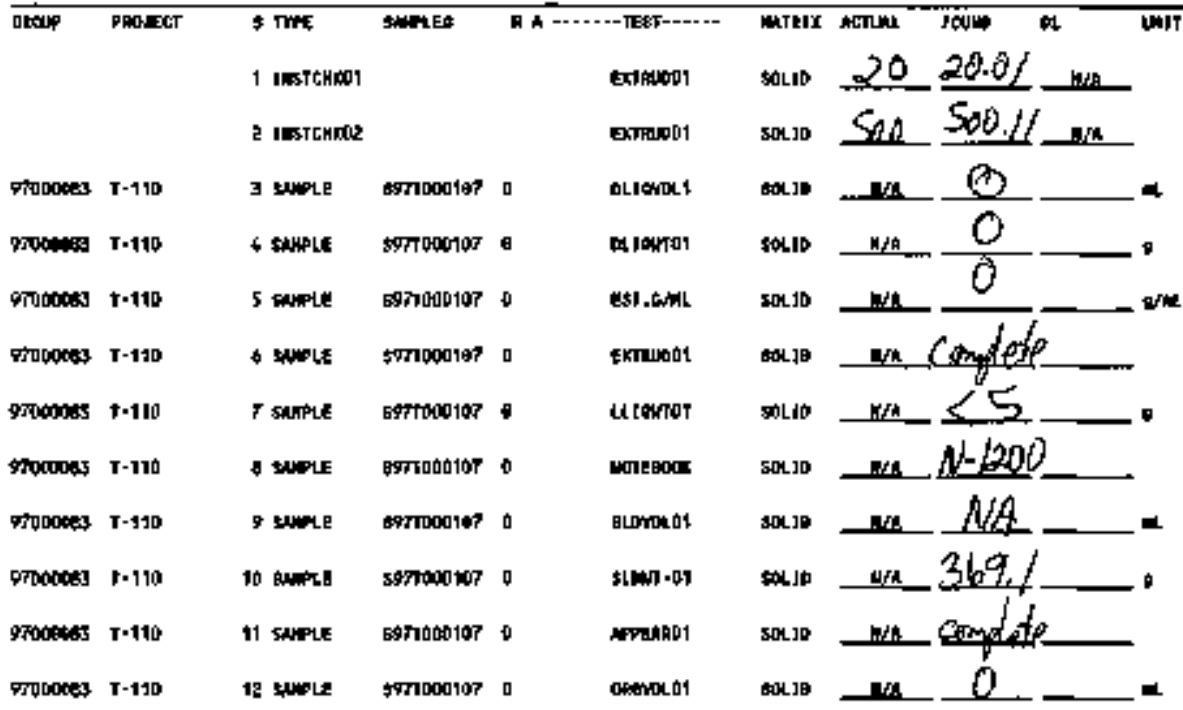

Final page for worklist \#16515
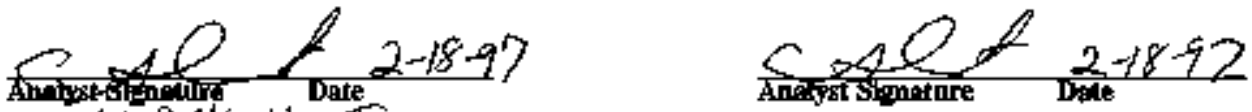

$2-27-6-7$

Dara Emry controm:

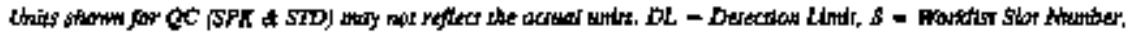

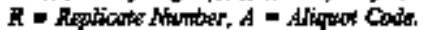




\section{LABCORE Data Entry Template for Worklist \# 16643}

\section{Alinilyst:

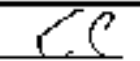 \\ Instrumen: BAO0O \\ Methed: L0.160-103 Rev/Mod BC \\ Wort" at Commend: T-110 C180 SEOHI R6 EXIRUSION}

Book $A /$ A

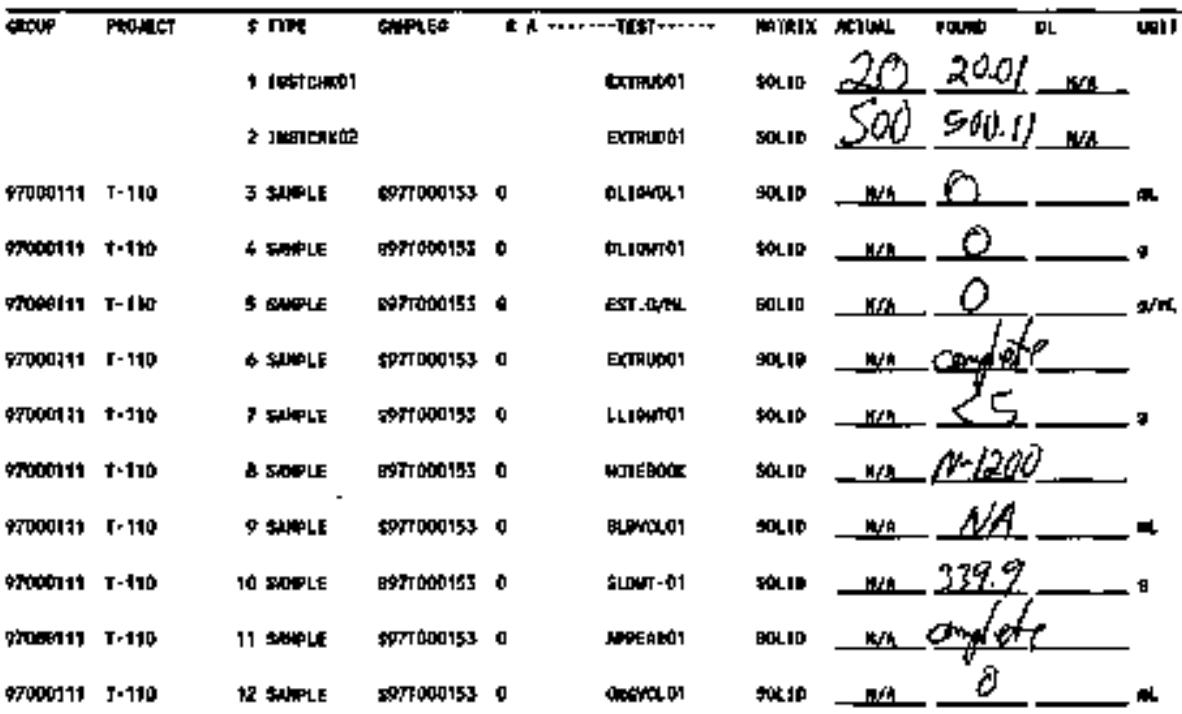

Final page for worklist \# 16643

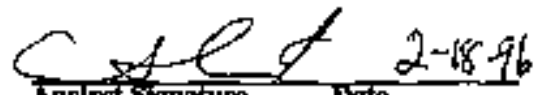

Anajot signiore sontand on

$$
2-27-97
$$

Dale Erry Common:

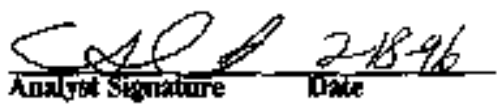

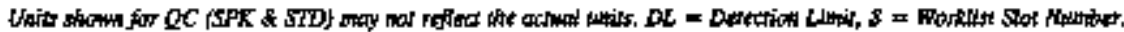

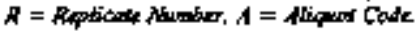




\section{LABCORE Data Eintry Template for Worklist\# 16644}

Amalys: $\quad 027$ Instroment: BA000

Broik of $D / A$

Method: LO-160-I03 Rev/Mad $R-\not C$

Wotk'st Corment: T-1LD C1B0 SEG\#2 R6 EXTRUSION

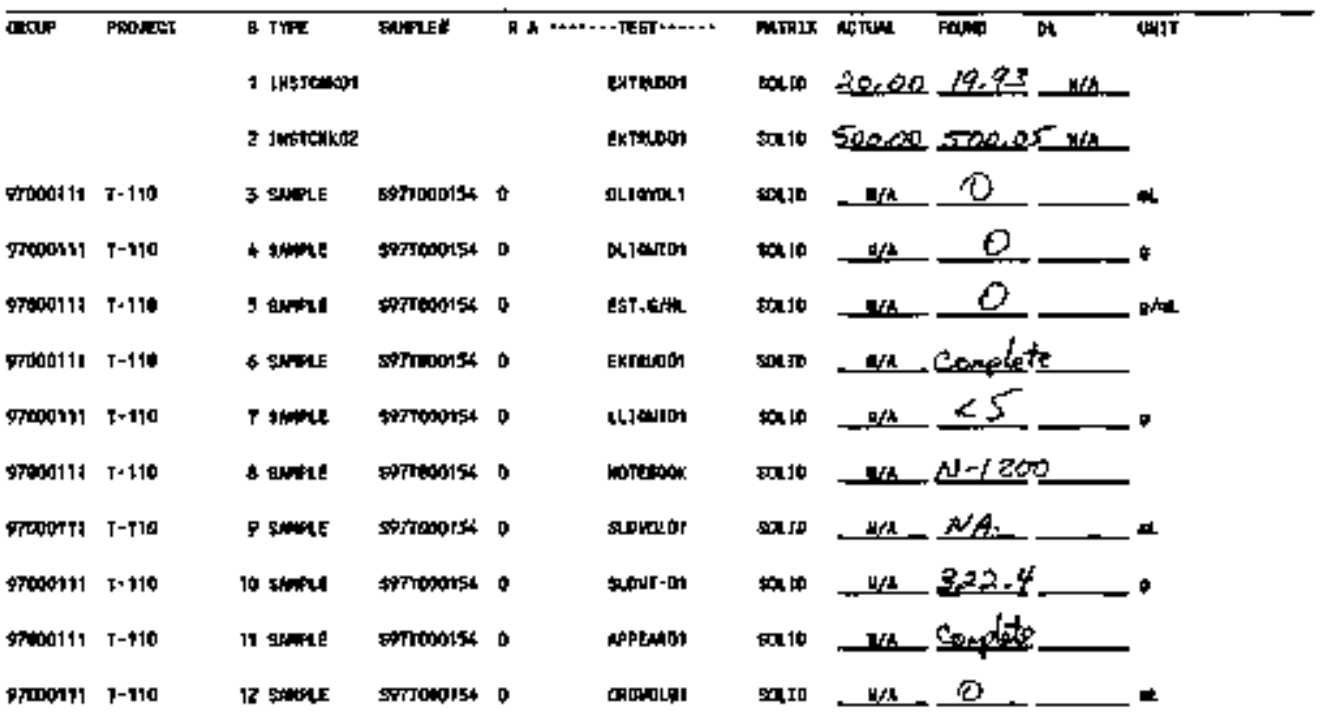

\section{Final page for worklist \# 16644}

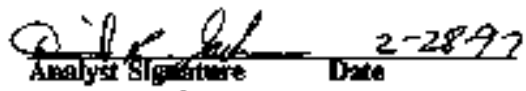

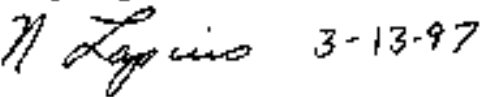

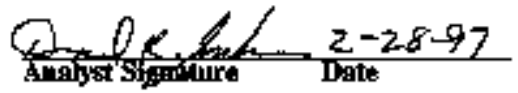

Derd Eny Campantr:

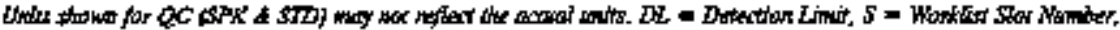

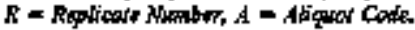

205 


\section{LABCORE Data Entry Template for Worklist 16646}

\section{Analyst: TIS Instrument: BA00O \\ Book $\underline{x}) A$ \\ Melliad: LO-160-103 Rewhod $\mathrm{PrC}$ \\ Worklist Comment: T-1 10 CL \$O SEG R RO EXTRUSION}

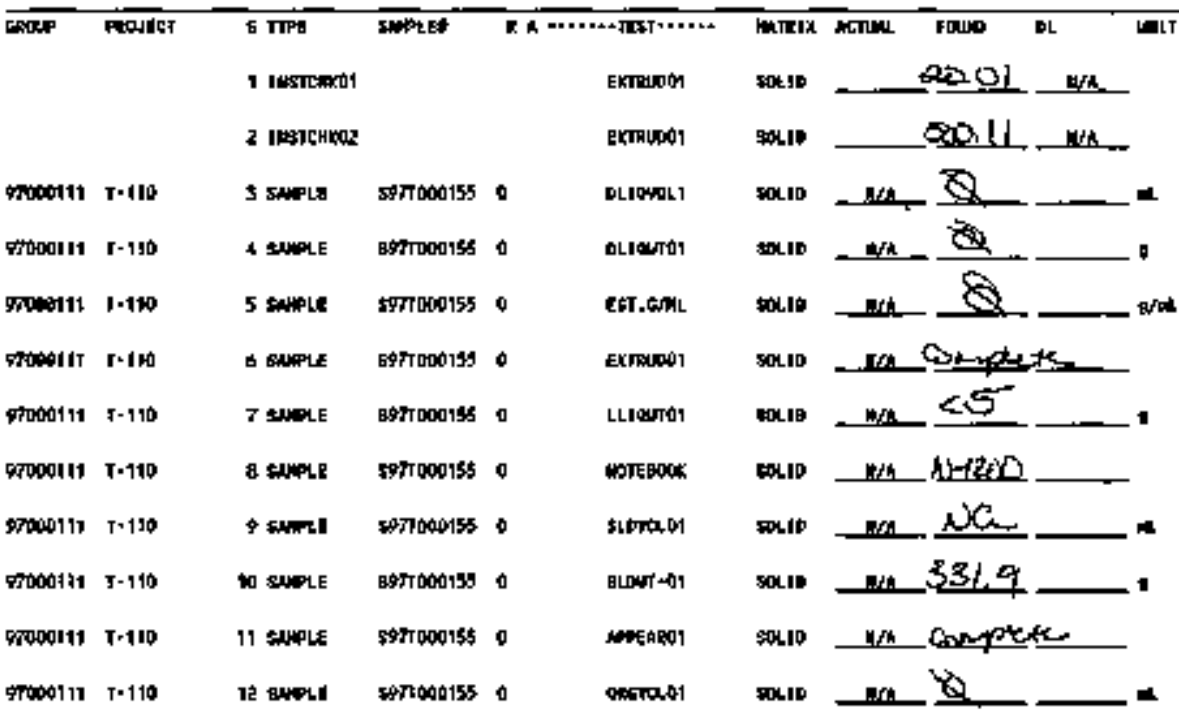

\section{Final page for worklist \# 16646}

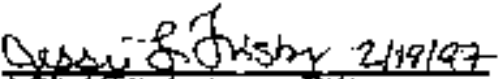

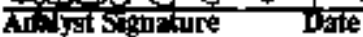

20thand sindim

Dpto Erry Commets:

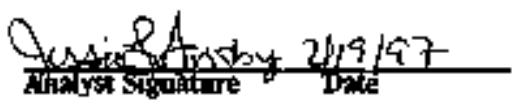




\section{LABCÓ́RE Data Entry Template for Worklist 16725}

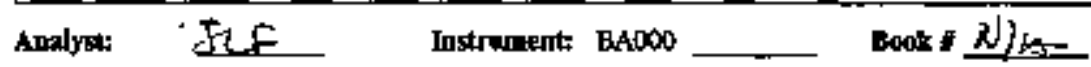

Method: LO-160-103 Rev/Mod $3-2$

Worklly Comment: T-110 C180 SEG \& 4 RISER 6 EXTRUSION

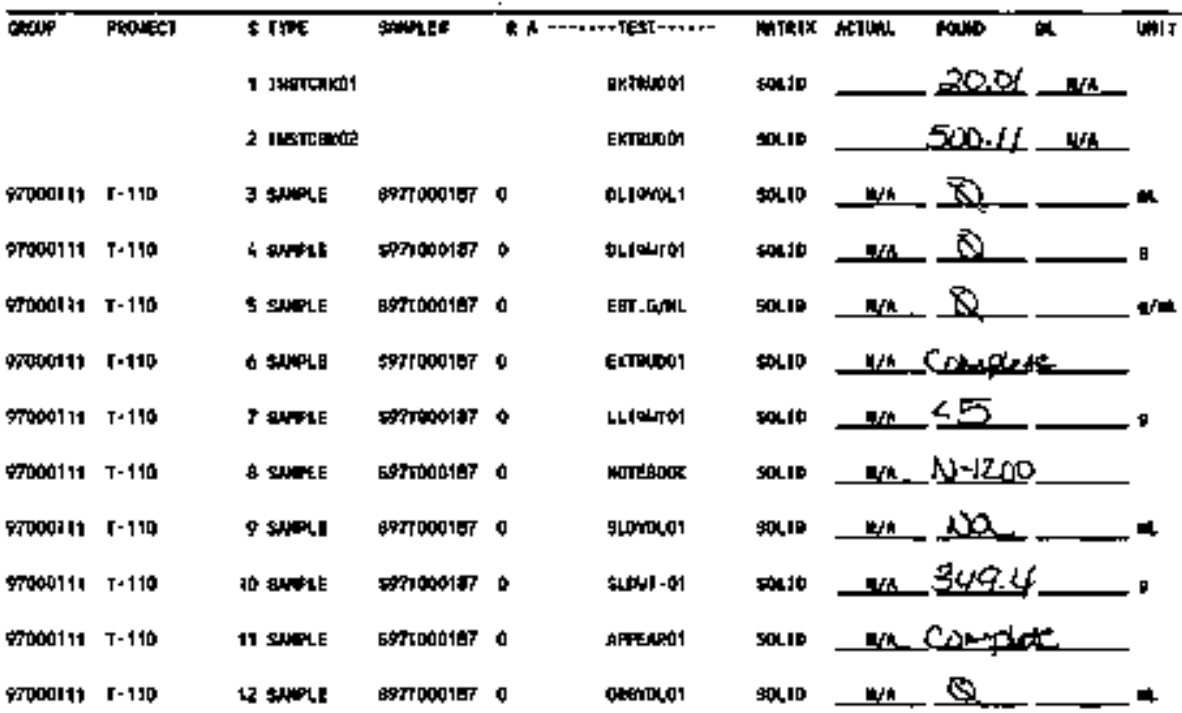

Final page for worklist \# 16725

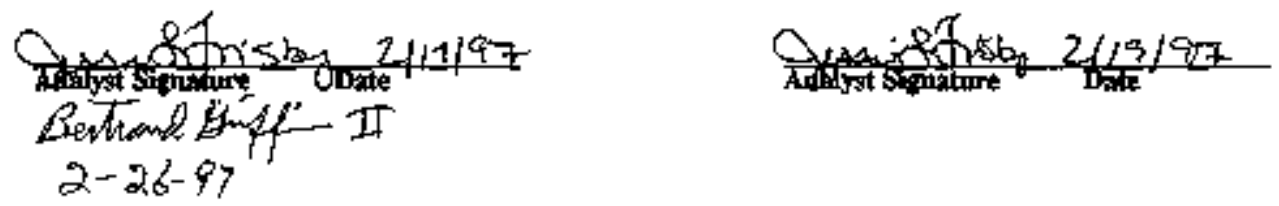

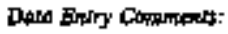

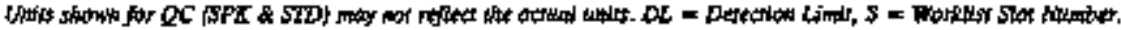

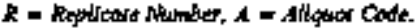




\section{LABCORE Data Entry Template for Worklistf 16726}

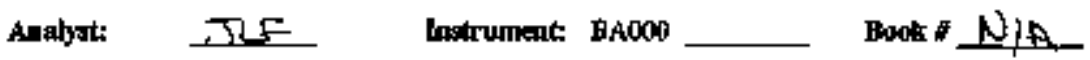

Method: L.160-103 Rev/Mod B.

Worklist Comment: T-1 10 Cl\$O SEG 5 RISER 6 EXTRUSLON

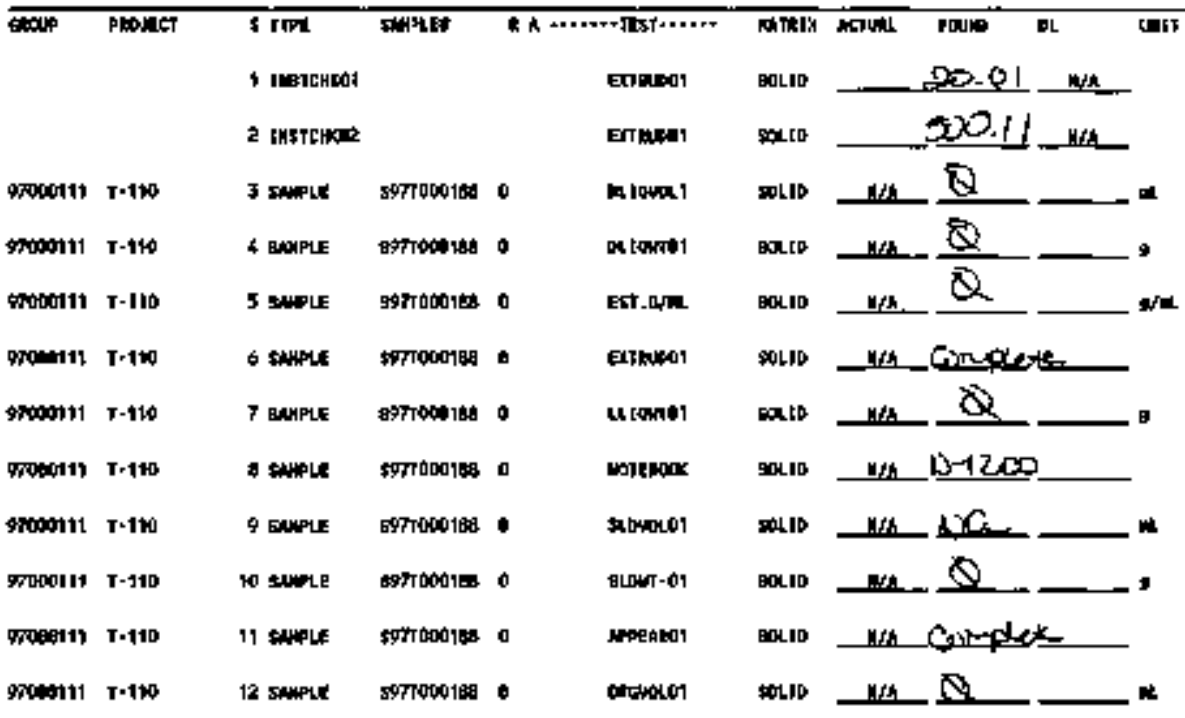

Final page for worklist \& 16726
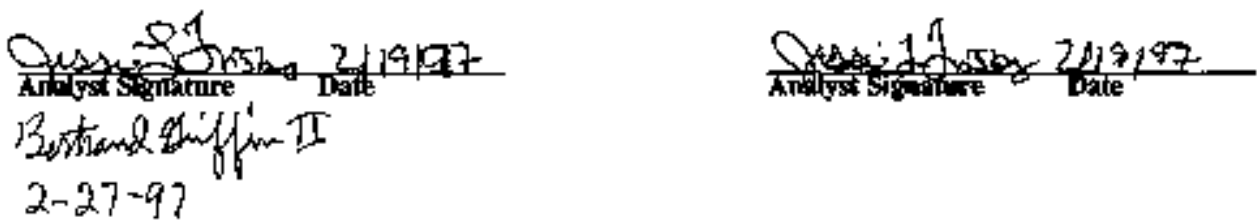

Dard Etry Comatsia:

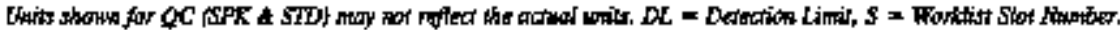

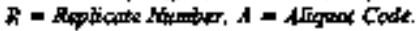




\section{LABCORE Data Entry Template for Worklist\# 16727}

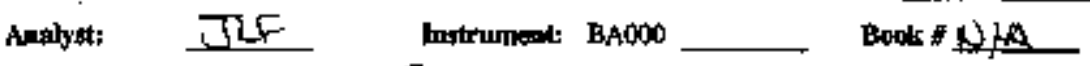

Method: LO-160-103 Rov/Mod 旦-2

Wortills Corment: T-110 C190 SES \# 6 RISER 6 EXTRUSION

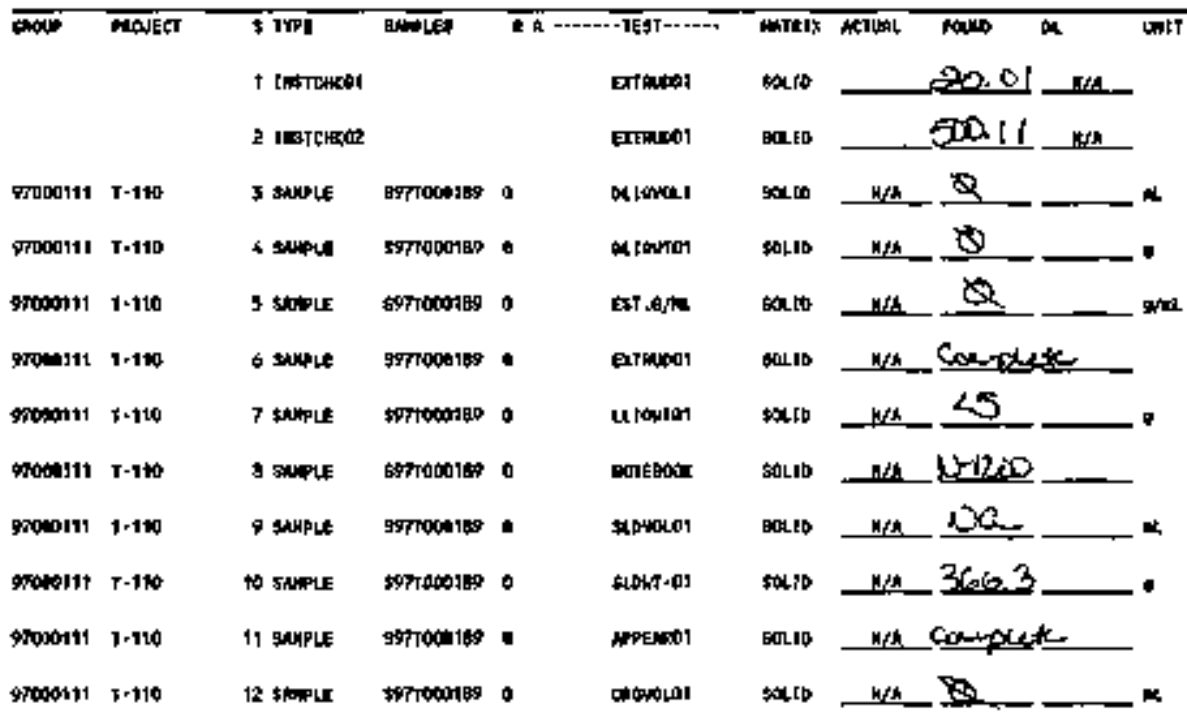

Final page for worklist \# 16727

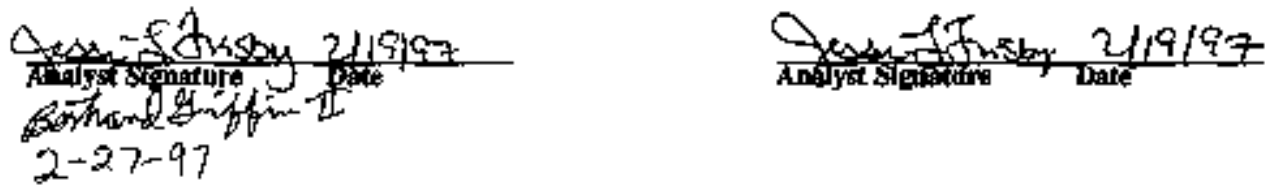

Dana Etrry Comatteras:

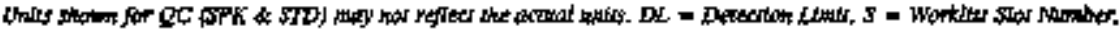

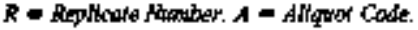




\section{LABCORE Data Entry Template for Worklist\# 16728}

Antalyst: exs Instrumend: BAOOO Dook \#

Method: 10-160-10\% Rew/Mod $\ell=0$

Werkltst Cominent: T-1 10 C180 SEG \& 7 RISER 6 EXTRUSION

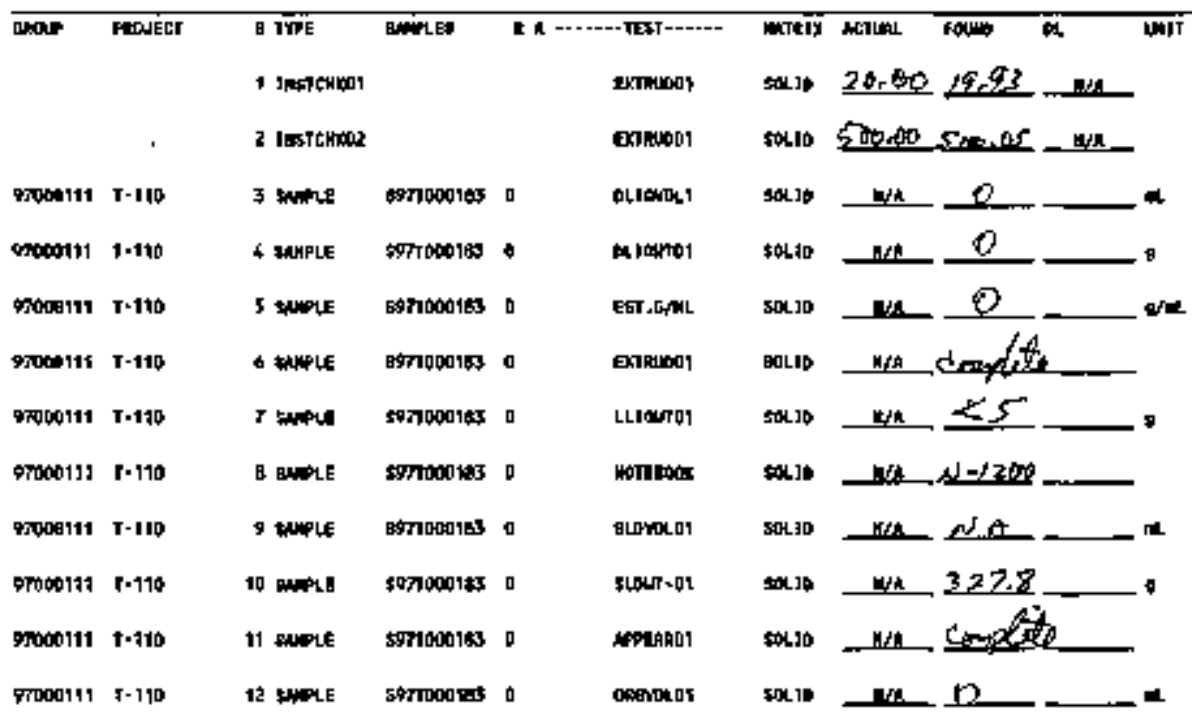

\section{Final page for worklist \# 16728}
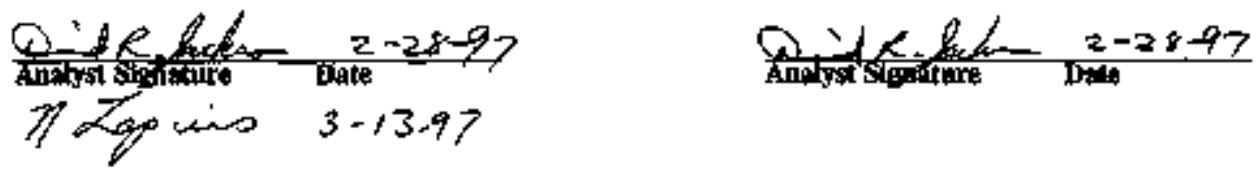

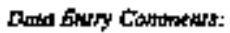

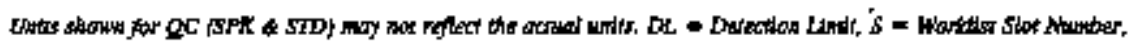

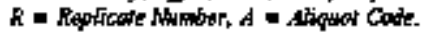




\section{LABCORE Data Entry Template for Worklist\# 16889}

inabyst:

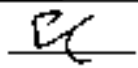

Indtrument: BA000

Book $* A / A$

Method: L0-160-L03 Rew/Mod

10

Wotkllst Commeta: T-110 CI80 SEG * \& RISER 6 EXTRUSION

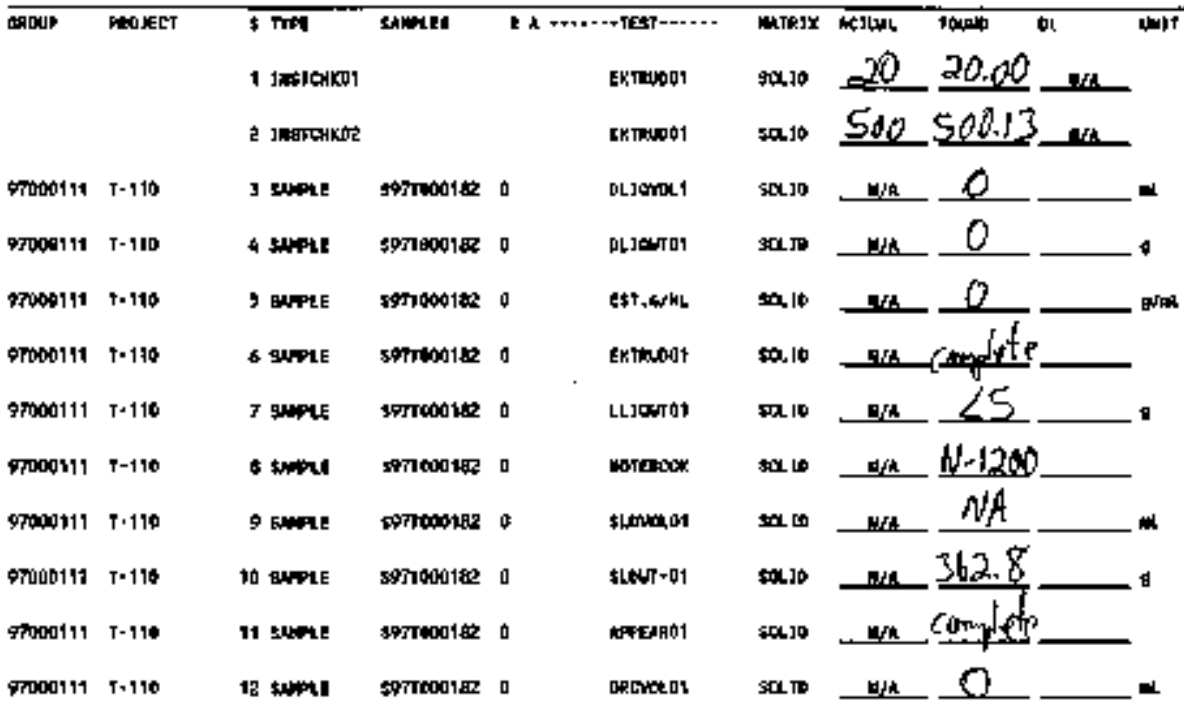

\section{Final page for worklist \# 16889}
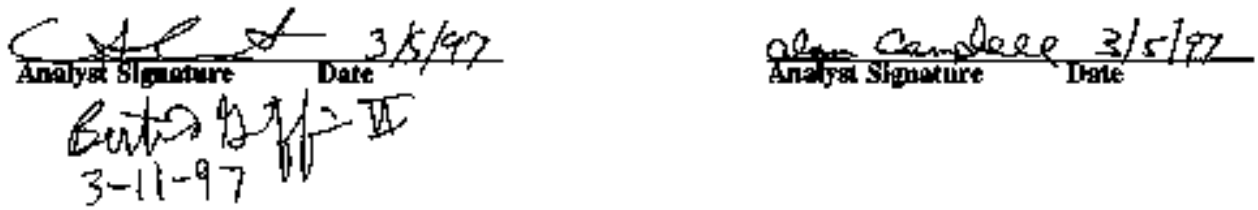

Dare Eatry Gormests:

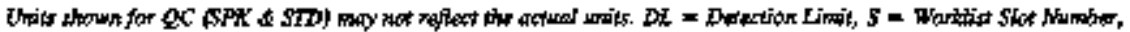

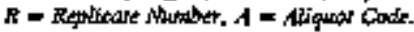


HNF-SD-WH-DP-23B， REV. 0

SAMPLE PAEPARATIOHS 
HE-SD-WN-DP-23E，REY. 0

THIS PAGE INTENTIOHALLY LEFT BLAK

213 


\section{LABCORE Data Entry Template for Worklitt" 16689}

Pिए: I

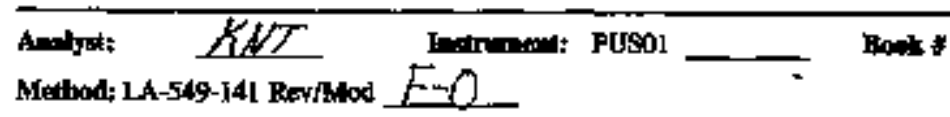

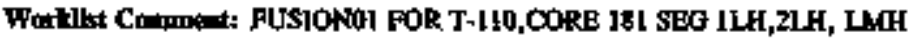

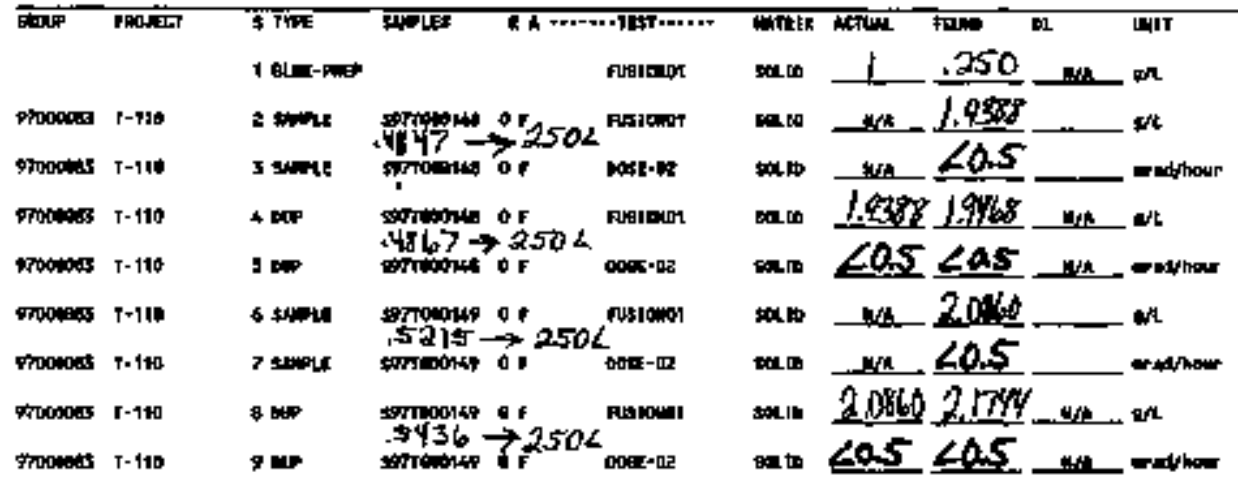

\section{Flmal page for worklist \#16689}

$7 \operatorname{2tan} 3-5=97$

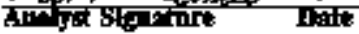
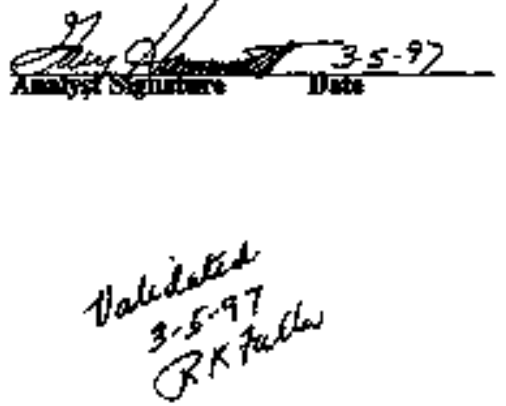

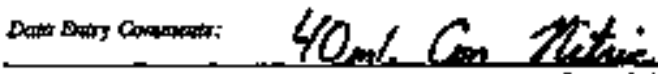
HPT wan seott Zandibut

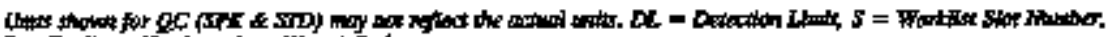

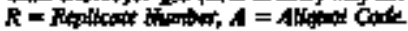




\section{LABCORE Completed Worklist Report for Worklist\# 16690}

Anolyst: $k$ mi

Instriment: FUSO1

Books:

Method:

Req/Mod

Workt'st Comment: FUSIONO1 FOR(T-110,CORE 181,SEG 7LH,SLH,GLA,FOR JEN

RTS

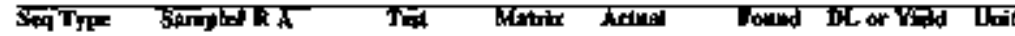

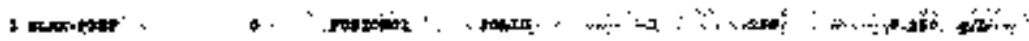

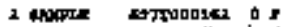

3 anory abridaich in

1 ote

5 फs

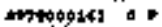
morem boin-tht pangeway

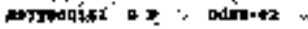

4 avoris moxim

7 ances arnopits 5

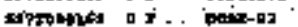
sprovers a $x$ pameral

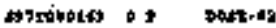

ind

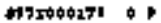

It unctil 1. Mopphín. 1 피

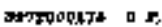

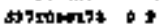
ropgeve? pongeat: restiont

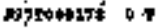

Dap-4:

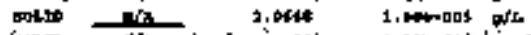

. "

2006. - Mas res.

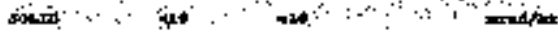

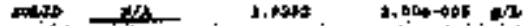

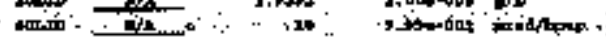

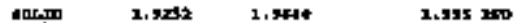

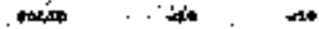

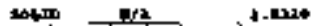

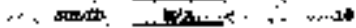

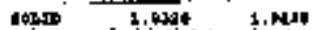
$\therefore \quad: \because \quad$ mianto

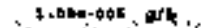

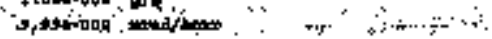

\section{Final page for worklist\# 16690}

\section{L六的}

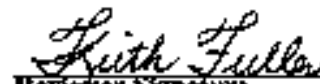

Revitur Signofire

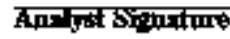

D尔

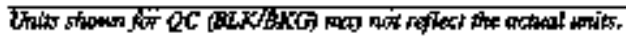

\section{Date}




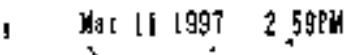

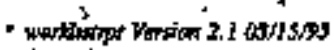
DIIIAT IASA

\section{LABCORE Data Entry Template for Worklist/ 16690}

\begin{tabular}{|c|}
\hline Annlpt: $\quad K N T$ \\
\hline
\end{tabular}

Eoot :

Methed: LA-s4g-141 ReviMod F.O

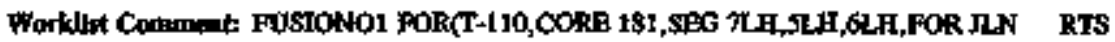

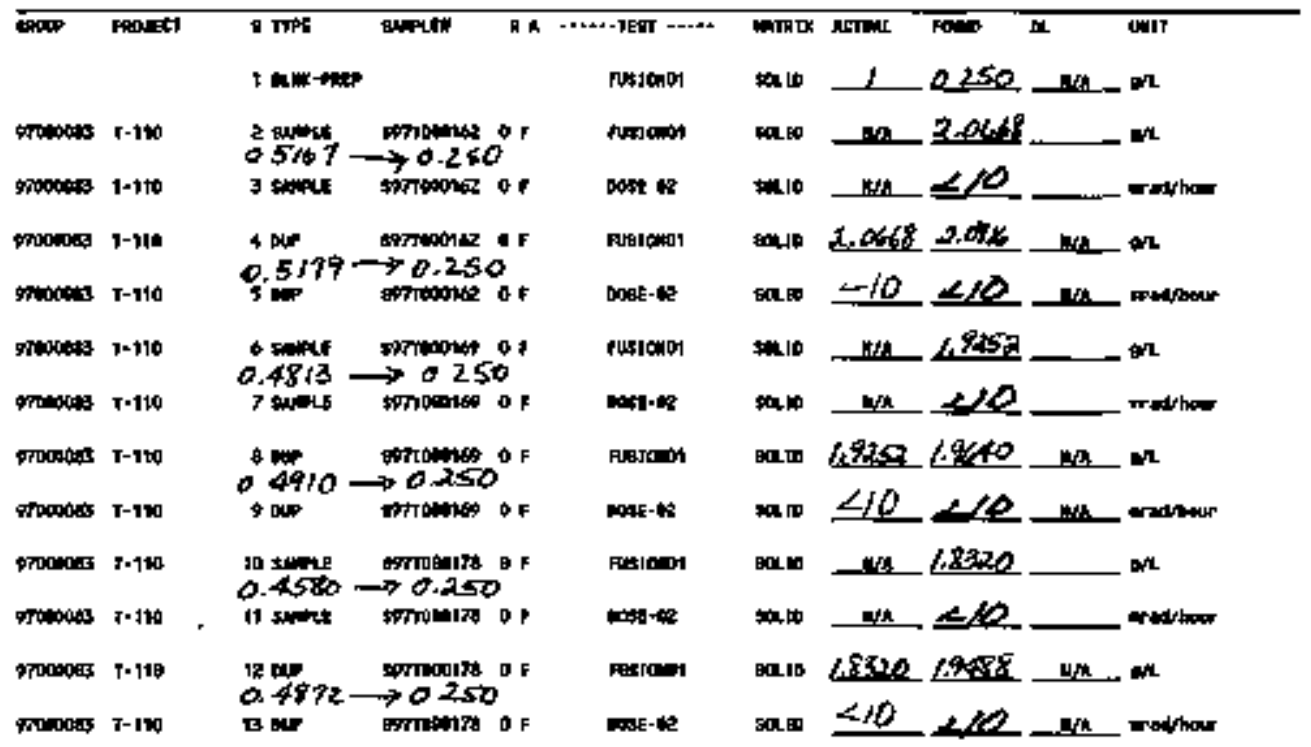

\section{Finsl page for wortlist \& 16694}
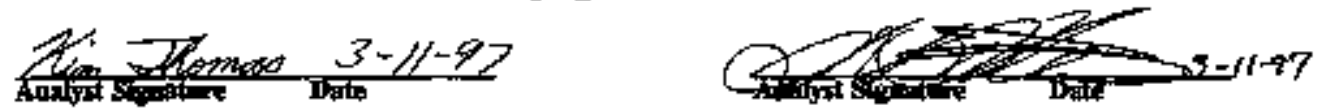

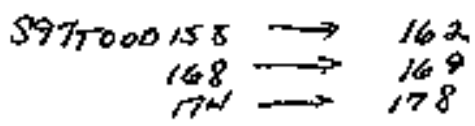

Dam Bary Commers.

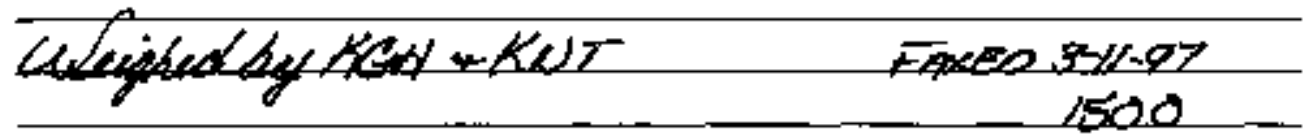

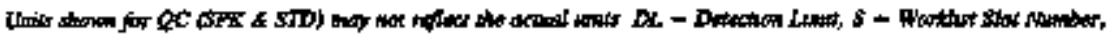

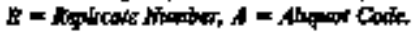




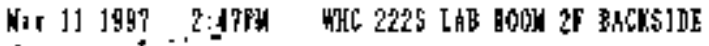

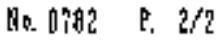

HWF-SD-WHU-DP-238, REY.O

Page: $]$

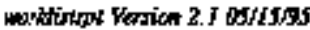

ootshy ro:32

LABCORE Data Entry Template for Worklistł 16690

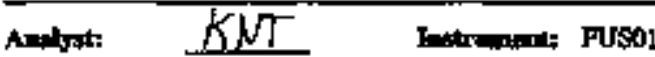

Deot F

Wethed: LA-S49-141 Rew/Mod F.O

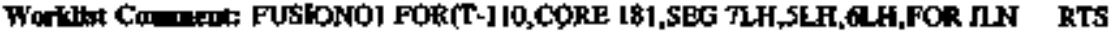

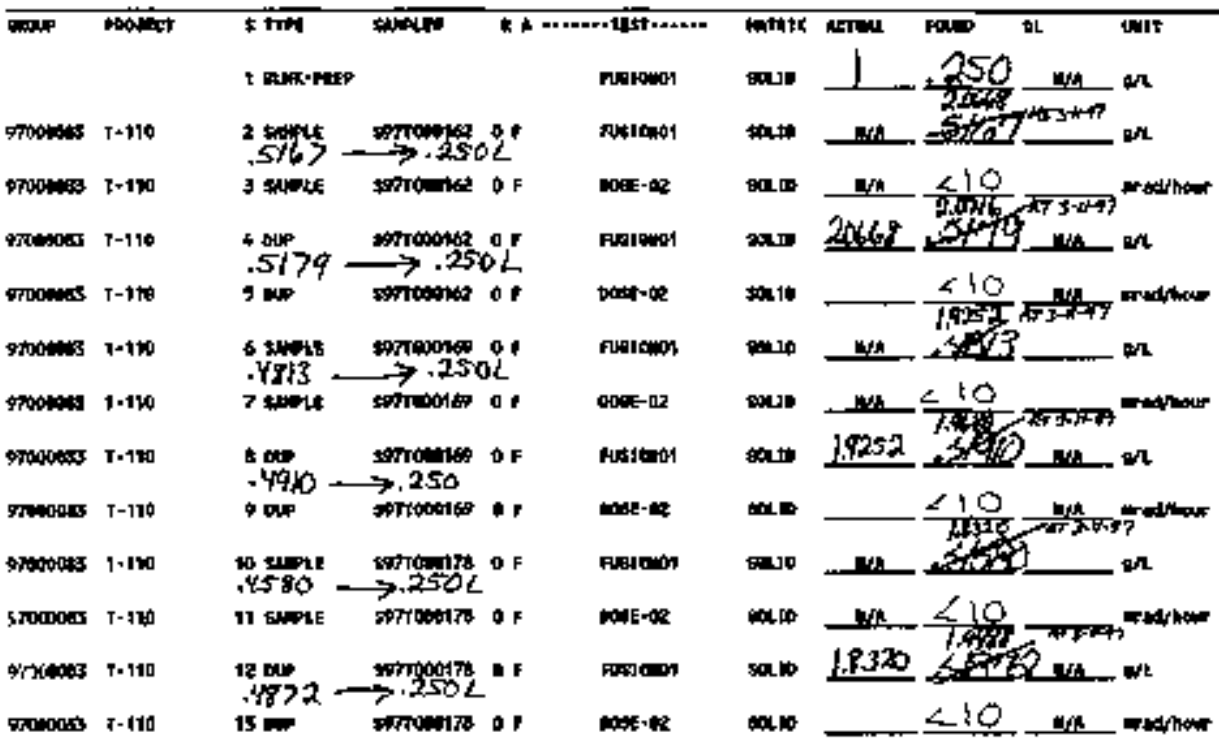

\section{Fingl page for worklist \#16690}

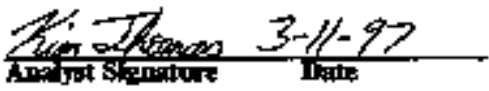

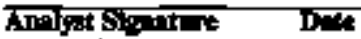

$59772000168-759770 \times 00162$ $397700016 \%-59 \pi 000 \%$

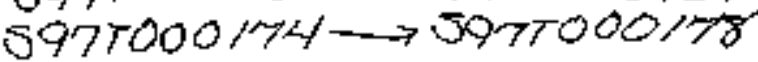

Dater Bury Coinmin:

Ulinged by kethes ther

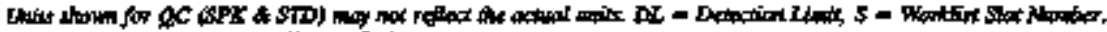

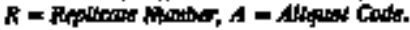




\section{LABCORE Completed Worklist Report for Worklist\# 16818}

Antlyst: knt

Instrumet: FUSOI

Bot:

Method: Revladod

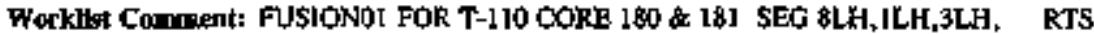

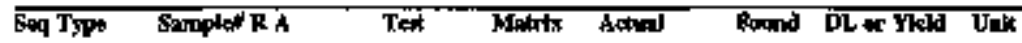

\begin{tabular}{|c|c|c|c|c|c|c|c|c|}
\hline 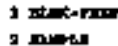 & 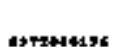 & $\bullet$ & triflodilt & 15 & 1 & -156 & 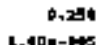 & $A$ \\
\hline xam & gorpeded 6 & $+\mathbf{r}$ & cotot & porm & 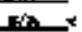 & 1.5 & +.014atra & med/fbutr \\
\hline & 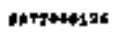 & $\bullet$ & 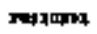 & P4क & 2.4612 & 2.45E & b.2H & mo \\
\hline & 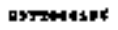 & $\bullet r$ & wax & जorm & $44 \mathrm{x}$ & 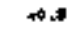 & & maldhen \\
\hline 4 ancex & 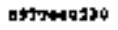 & $\bullet$ & Fo4304k4 & ecthls & 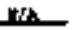 & $2-3404$ & $1.00+1465$ & $9 / \mathbf{h}$ \\
\hline$\nabla$ mater & $A V N=197=0$ & & noar-12 & Dorim & wh & 0.5 & $3.13=-607$ & 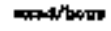 \\
\hline & 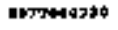 & $* \mathbf{F}$ & thedzonin & mat IP & 2.1406 & 1.450巾 & $0-144$ & $\boldsymbol{\omega r}$ \\
\hline & 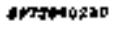 & $\bullet \mathbf{r}$ & Dode- 10 & mon & $20 . x$ & 80,3 & & maditim \\
\hline $\mathbf{n}$ & "YT2*10331 & +1 & tadsolen & eatatb & $T^{\prime / A}$ & 2.4302 & $1.004=406$ & $s / 1 /$ \\
\hline & 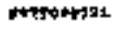 & & betiz-as & 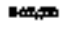 & 보로 - & $\bullet-x$ & D.1llabot & \\
\hline & dotedonas & & nosinter & 묲 & a, ess & 2.6040 & D.Te4 & ner \\
\hline $\mathbf{1}$ & sproped & 8 & 5apad & who & at. 5 & at & & redex \\
\hline
\end{tabular}

\section{Comments Section:}

Cemments for sauplet $\$ 97$ T000196 and test FUSIONo].

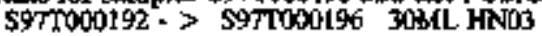

Comments tor samplat S97T000230 and test FUS[ON\%0]. S9T1000214 - ? $59 \mathrm{TTOO0230} 30 \mathrm{ML} \mathrm{HNO3}$

Comoments for samplat S97TOOO231 and test FUSIONFOH. S97T000217 ; S9TTOOO231 30ML HNO3

\section{Final page for worklist: 16818}

Antyst Signwire Drte
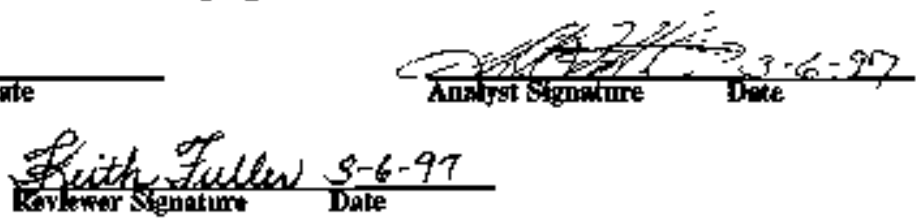


\section{LABCORE Data Entry Template for Worklist/ 16818}

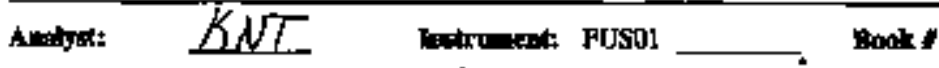 \\ Melbod: LA-549-141 Rowhad $F-O$ \\ Wortilit Comment: FUSFONO1 FOR T-110 CORE I\% \& 181 SpG \&LH,LL,3LH, KTS}

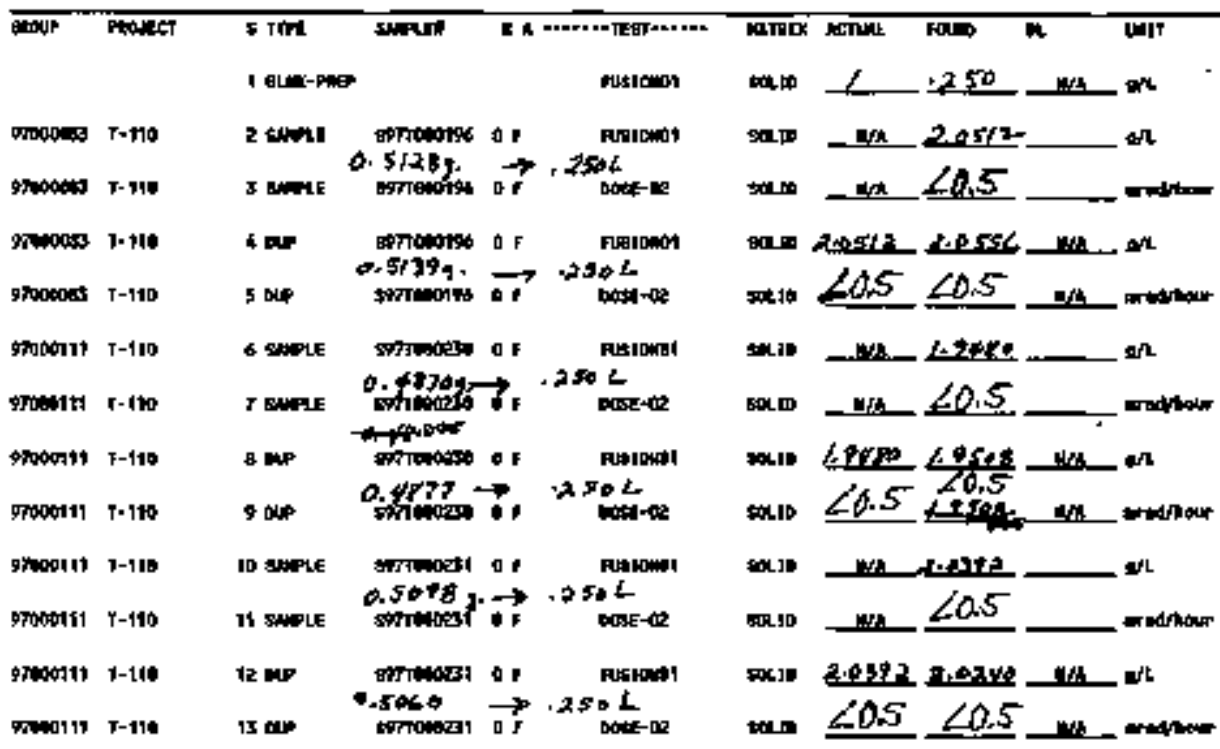

\section{Fingl page for worklist \# 16818}

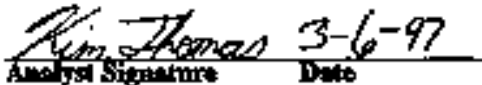

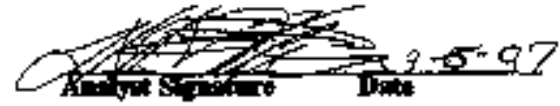

$647000182 \longrightarrow 5075000156$

547 roodity $\rightarrow 547$ rat 230

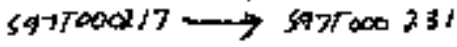

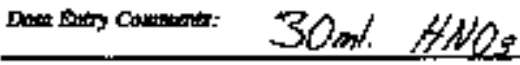

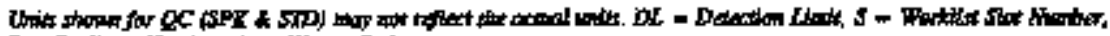

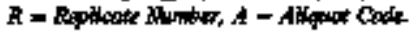

$$
219
$$




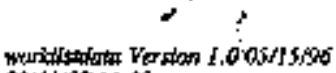

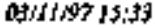

HNF-SD-WHAP-238, REV 0

Page: $I$

LABCORE Completed Worklist Report for Worklist\# 16821

An:alpst: kgh

Instrment: PUSOI

Boets"

Method: ReyiMod

Workiltat Commant: FUSIONOt FOR TANK T-J10 CORE 1808181 SEG, 7LH,8LH, RTS

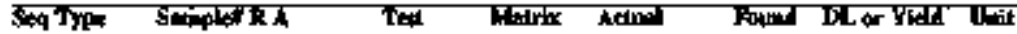

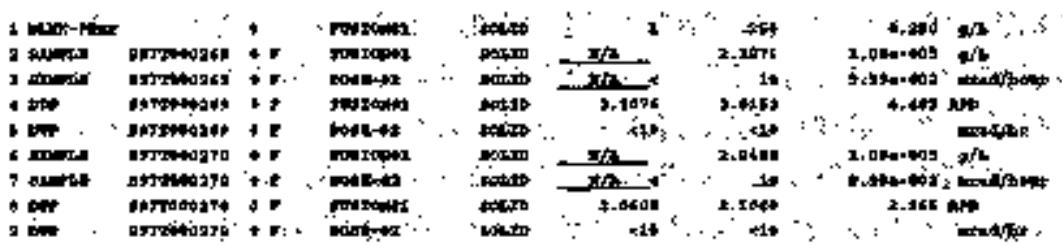

\section{Final page for worklist 16821}

7. 10

$\operatorname{Lrex}$

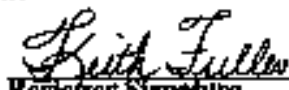

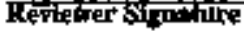

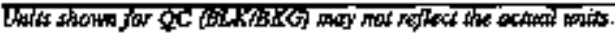




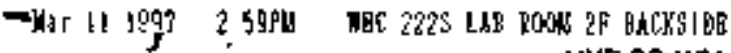

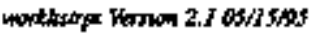

HNF-SD-WIA-DF-298, REV. 0

Wo $0787 \& 2 / 2$

Q2olsy is:si

\section{LABCORE Data Eutry Tenplate for Warklist" 16821}

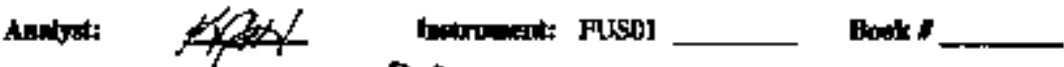

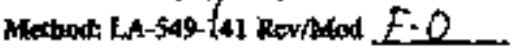

Warthist Compm.t: FUSIONO1 FOR TANT T-110 CORE 1804181 SEG, 7LH,8LH, RTS

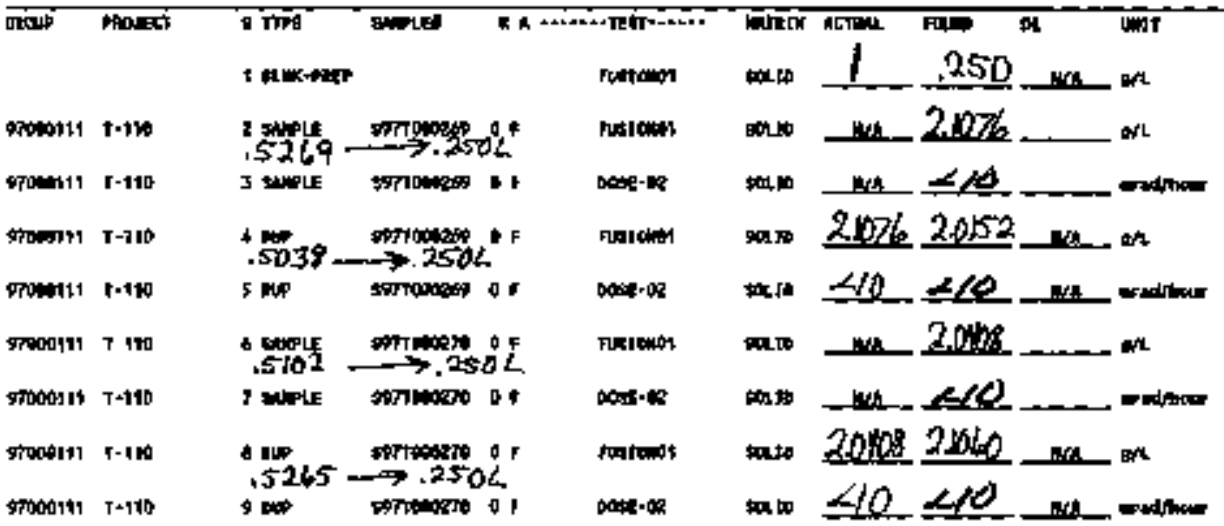

Final page for worklist 16821

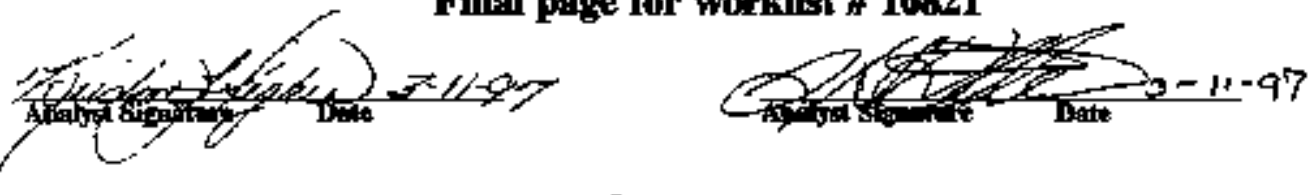

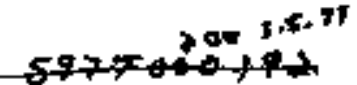

$$
\begin{aligned}
& 57770000269 \rightarrow 59770400269 \\
& 265 \rightarrow 59770040+70
\end{aligned}
$$

Duso Entry Comorosti:

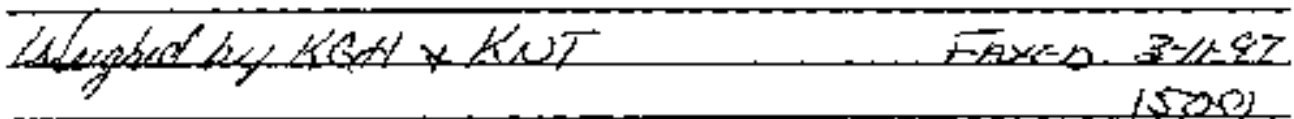

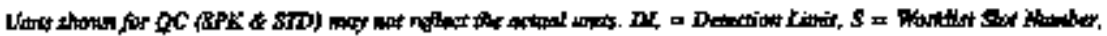

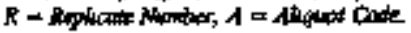




\title{
LABCORE Data Emtry Template for Worklist/ 16821
}

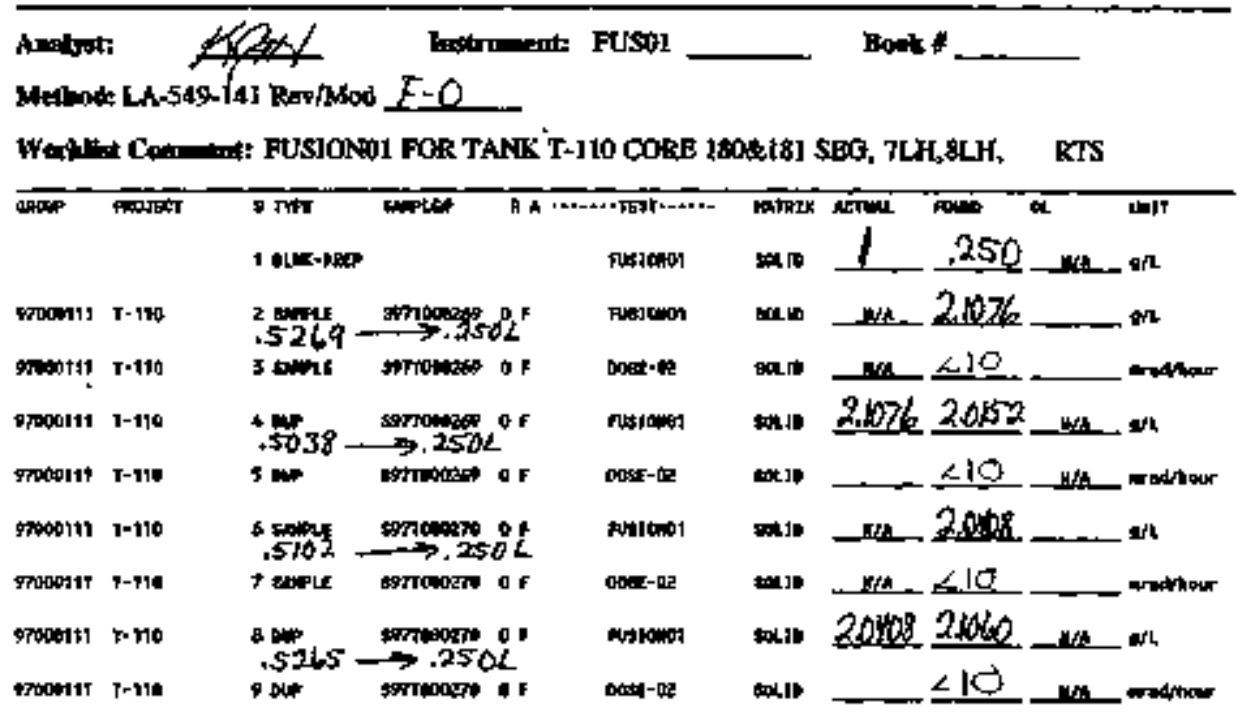

\section{Final page for worklist \# 16821}

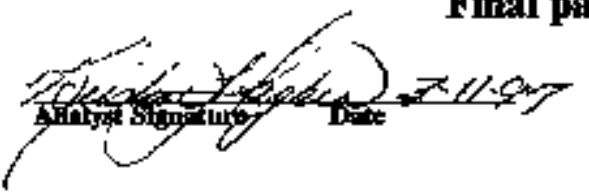

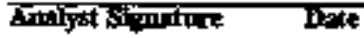

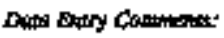

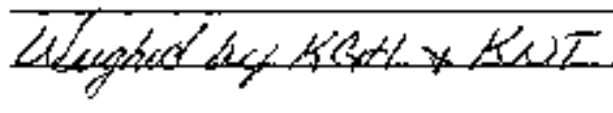

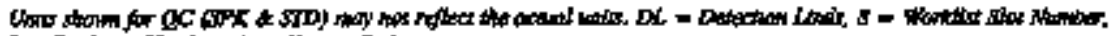

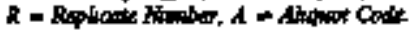




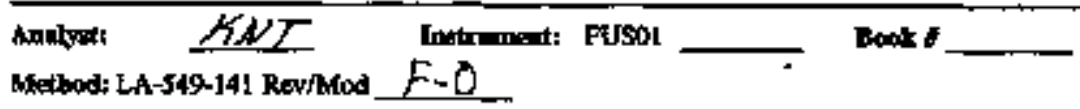

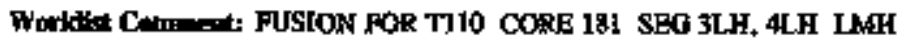

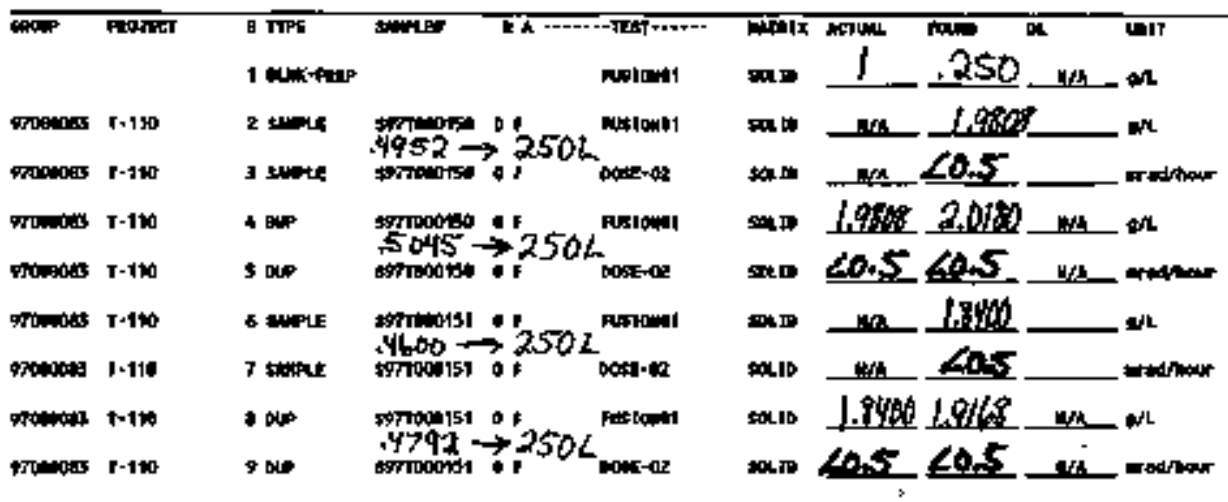

Fingal page for worklist \# 16885
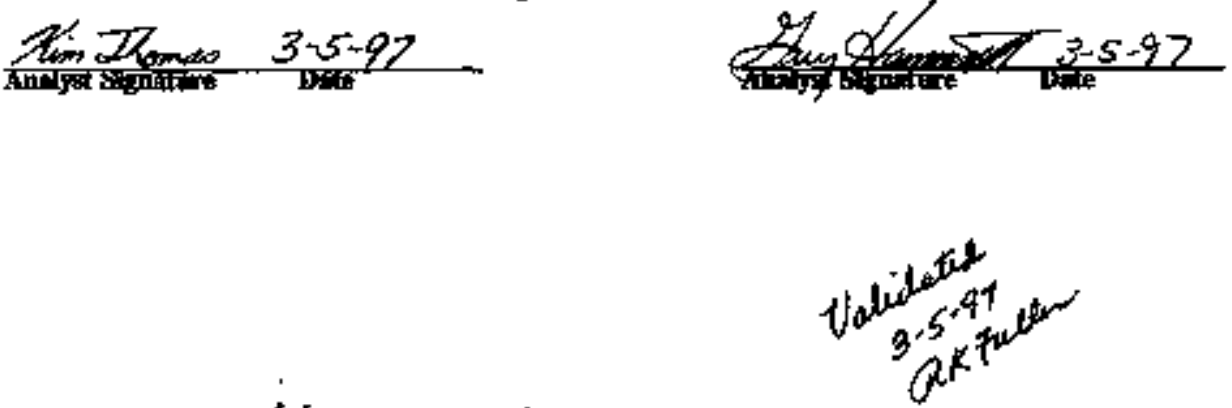

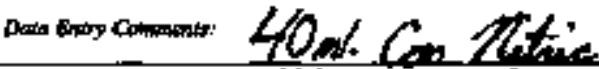

HPT ane sout handuland

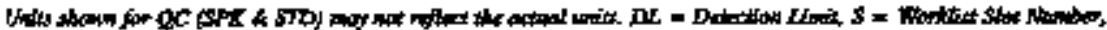

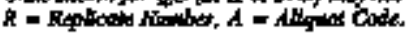


HWF-SD-MN-DP-238, REW. O

IHCEGAMIC ANALYSIS

.225 
HWF-SD-1W-DP-238, REV. 0

THIS PAGE INTENTIONALLY LEFT BLAKK

$+226$ 


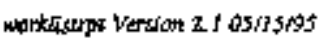

HAF-3D-WN-DF-238, REV. 0

Pasee I

at200\% 00:30

LABCORE Data Entry Template for Worklist\# 16714

Analyst: SMLF Instrment: DSCO 3.

Methodt LA-S14-114 Res/Mod D-O

Workllst Comment: DSC-0I FOA T-110 GRAB (T-1 (19)RUN UNDER NITROGEN) RTS

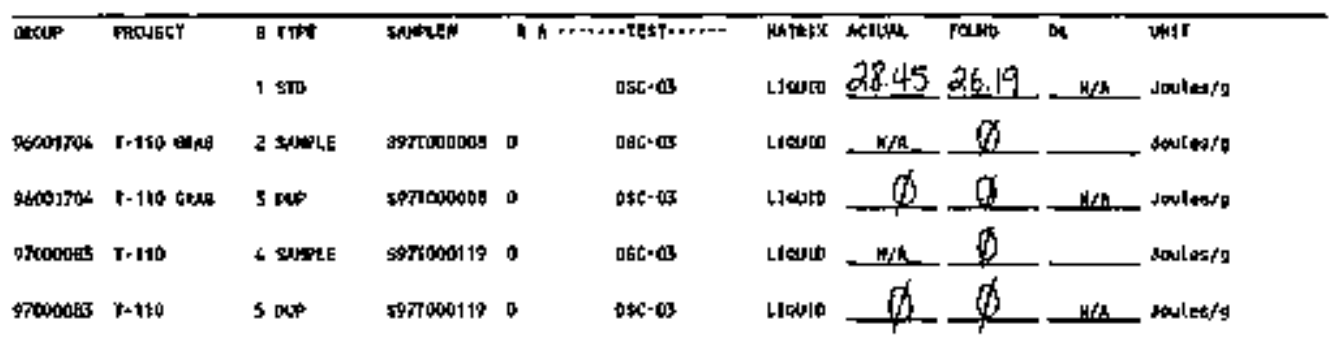

Final page for worklisfy 16714

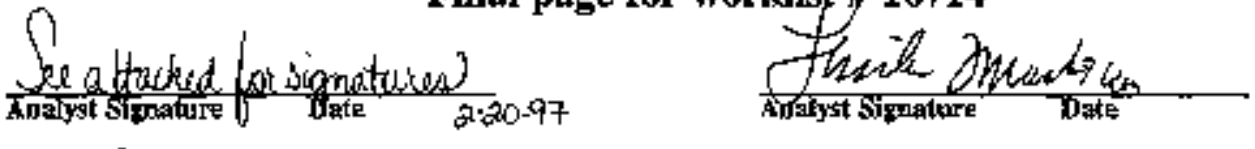

Verifid/ralidated iy

Blandina

Valensinela?

Drwo Estry Commenta.

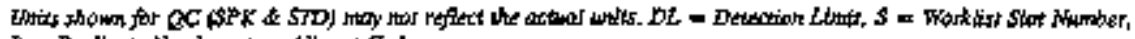
$K=$ Replicate Numiber, $A-A$ ilowat Code.

227 


\section{LABCORE Data Entry Template for Worklist"\# 16714}

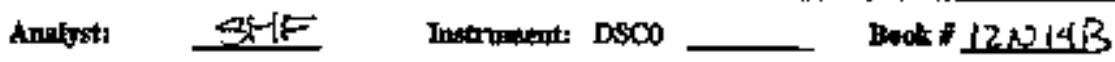

Method: LA-514-113 Rew/Mod

Worktist Compents DSC-0\} FOR T-LIO GRAB \& T-110)RUN UNDER NITROGEN) RTS

\begin{tabular}{|c|c|c|c|c|c|c|c|c|c|c|}
\hline \multirow[t]{2}{*}{ Ar } & \multirow[t]{2}{*}{ Btolla } & 3 TYE & Beoutes & \multicolumn{2}{|c|}{ 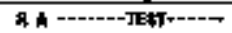 } & \multirow{2}{*}{ 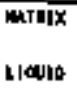 } & \multirow[t]{2}{*}{ 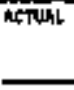 } & \multirow[t]{2}{*}{ Fas } & \multirow[t]{2}{*}{$\mathbf{m}$} & \multirow[t]{2}{*}{ UIT } \\
\hline & & $1 \$ 7$ & & & 1 & & & & & \\
\hline 9ootinet & F-110 exp & I artit & Bormpooplat: & 0 & $\ln x-01$ & Itais & Wh & & & saulnet \\
\hline 96001704 & $T-\tau$ end & 30 & stroosus & 0 & $0<0=01$ & Lاله & & & & dochop \\
\hline 9 topotass & $r-120$ & 4 土уири & काropit1s & $\diamond$ & $000-01$ & เ10ho & mas & & & Jachind \\
\hline Tropous & $T=110$ & $5 \mathrm{DP}$ & פ9T00:19 & 0 & astan & IJEla & & & & reotent \\
\hline
\end{tabular}

Final page for worklist \# 16714

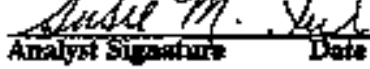

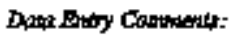

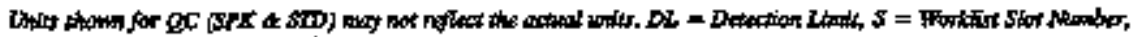

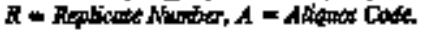




\section{wewe 1: das}

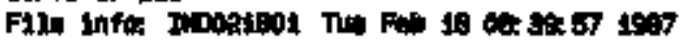

giplis inditit: 10,80

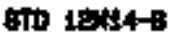

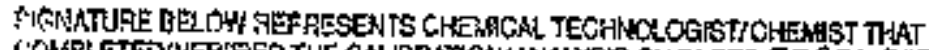

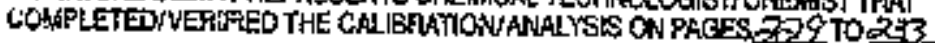

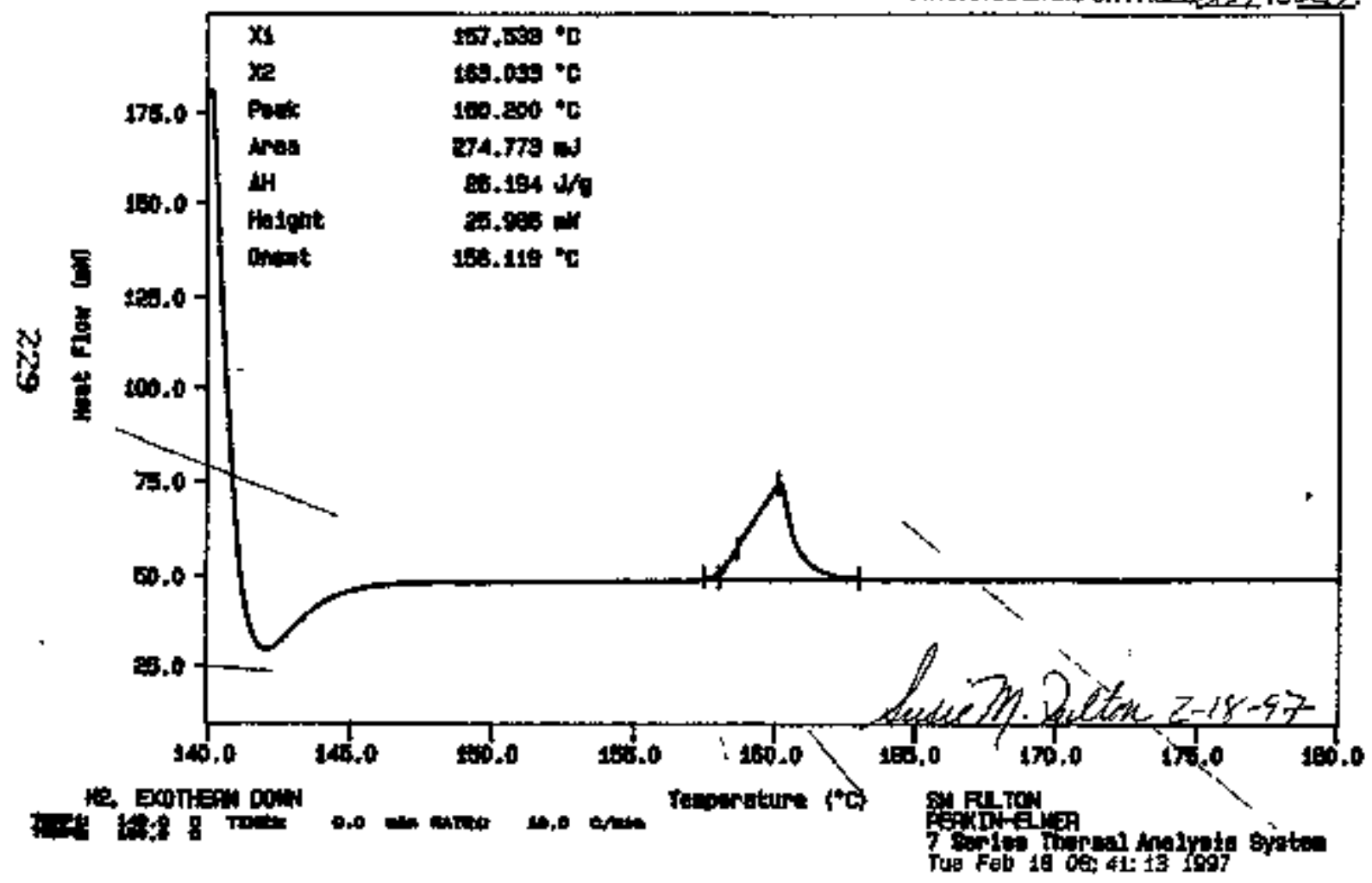


Grva : Dac

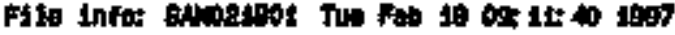

Oomplo wologes 0.790

E977000008

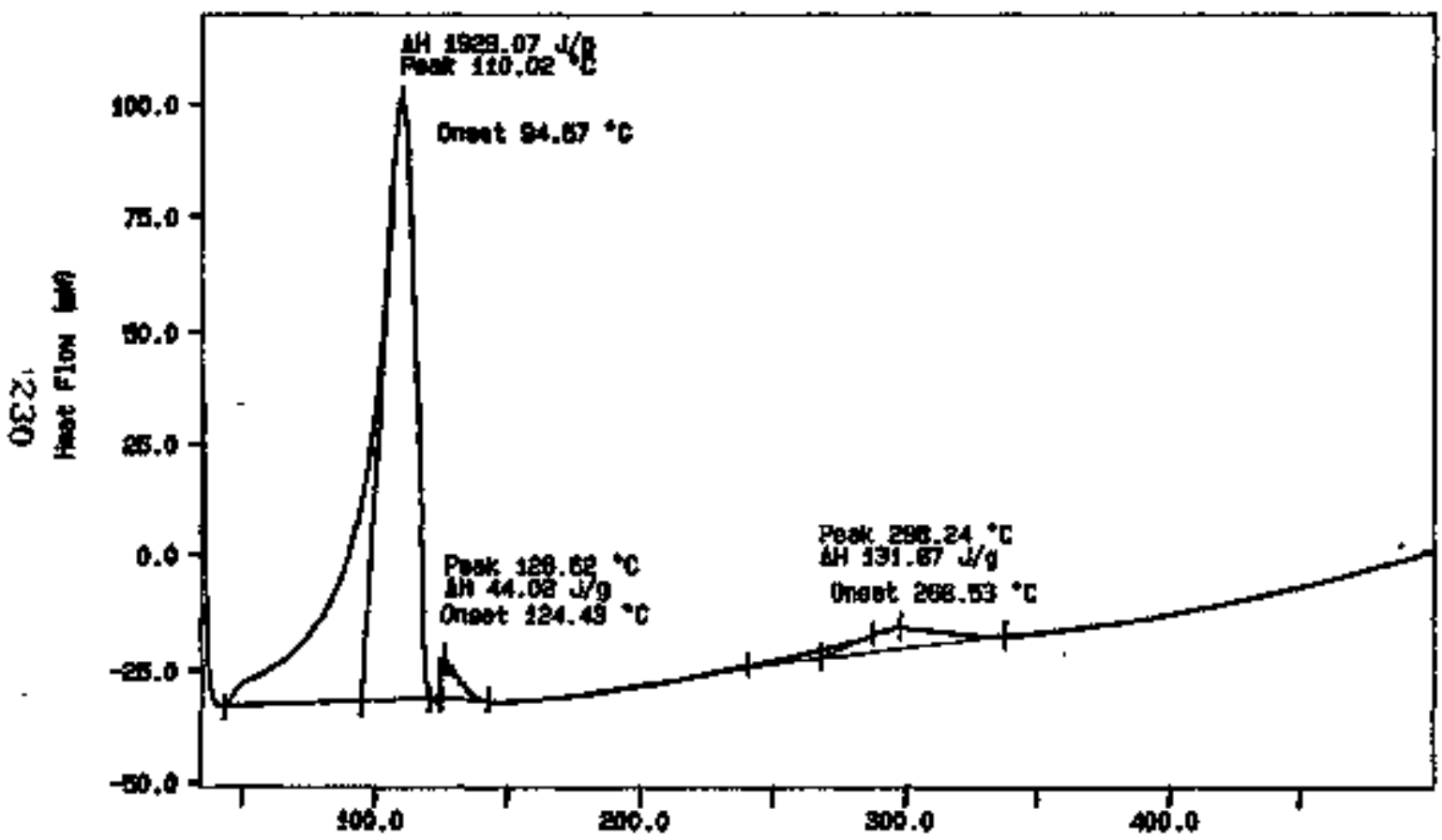

ine 100/min

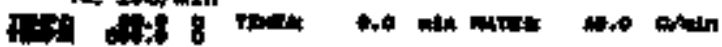

Teipersture 1+다

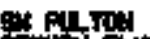

Einiting

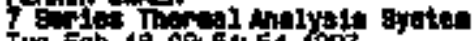


Grve is $\cos$

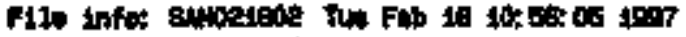

gaplo inicht 10.70

esproosose dop

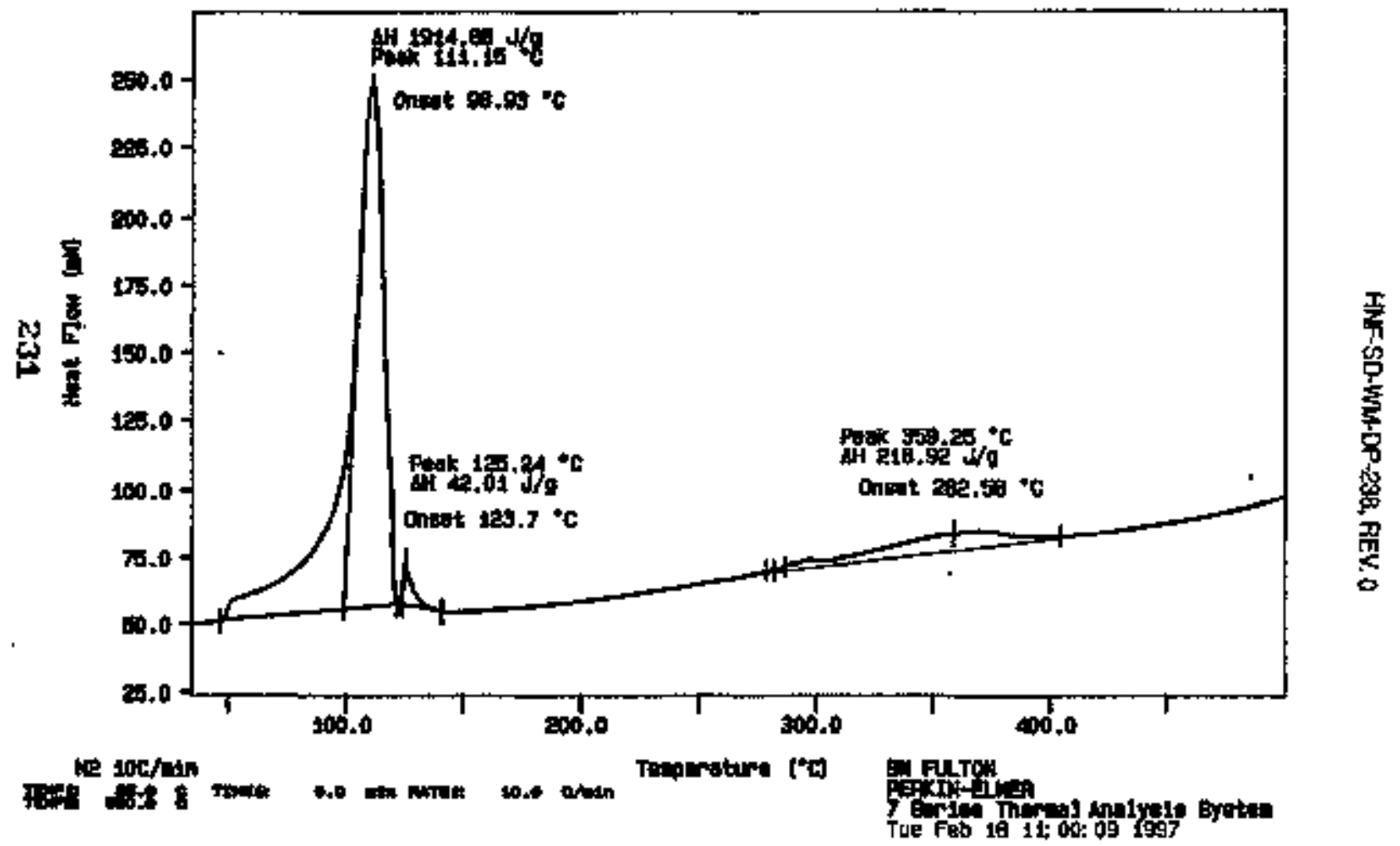


Arve :5 Dex

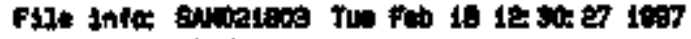

sopla wight a.atp $\boldsymbol{x}$

smooosts

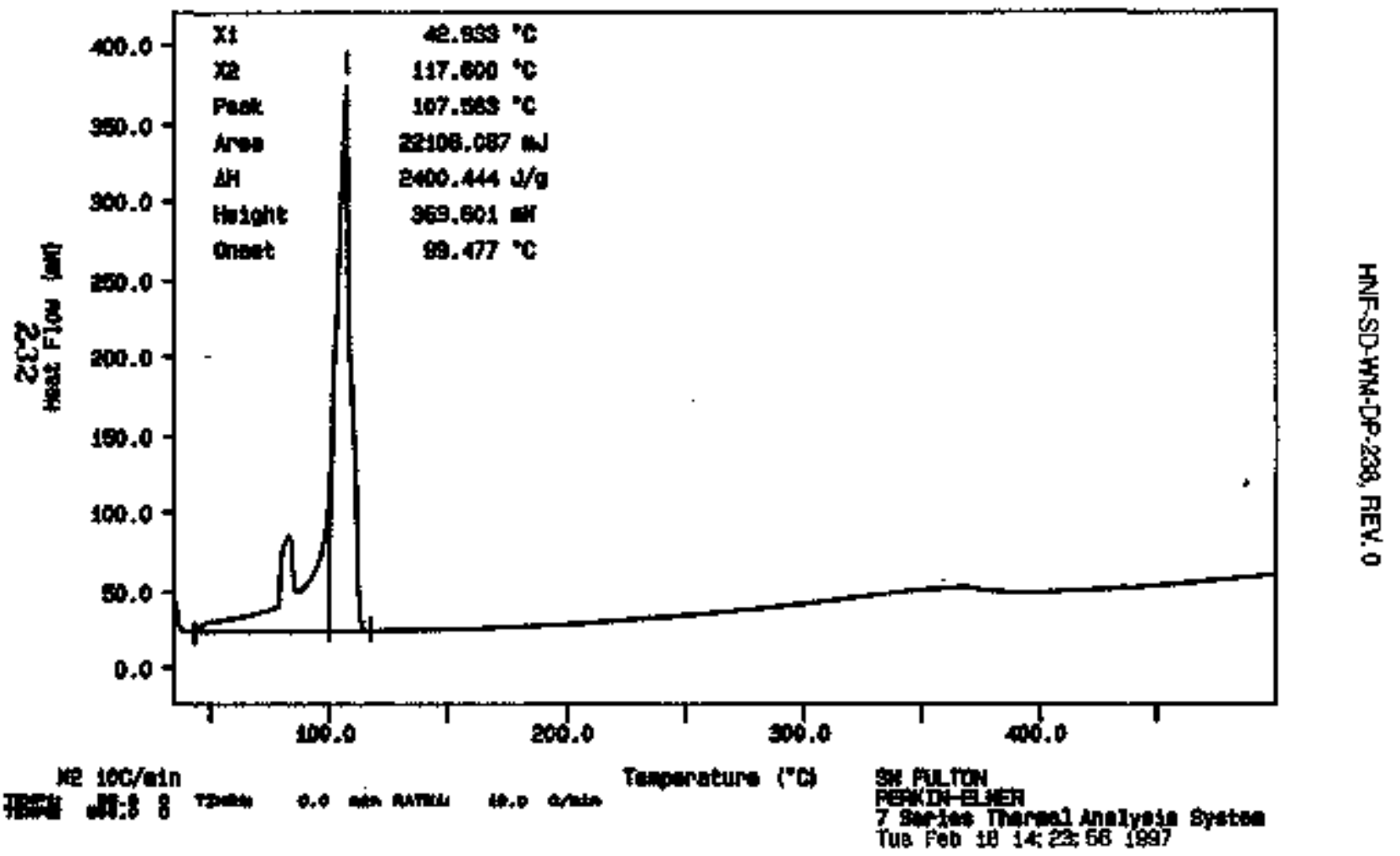


Drve it De:

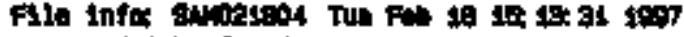
saple injotitio 8.300

Somiootis DuP

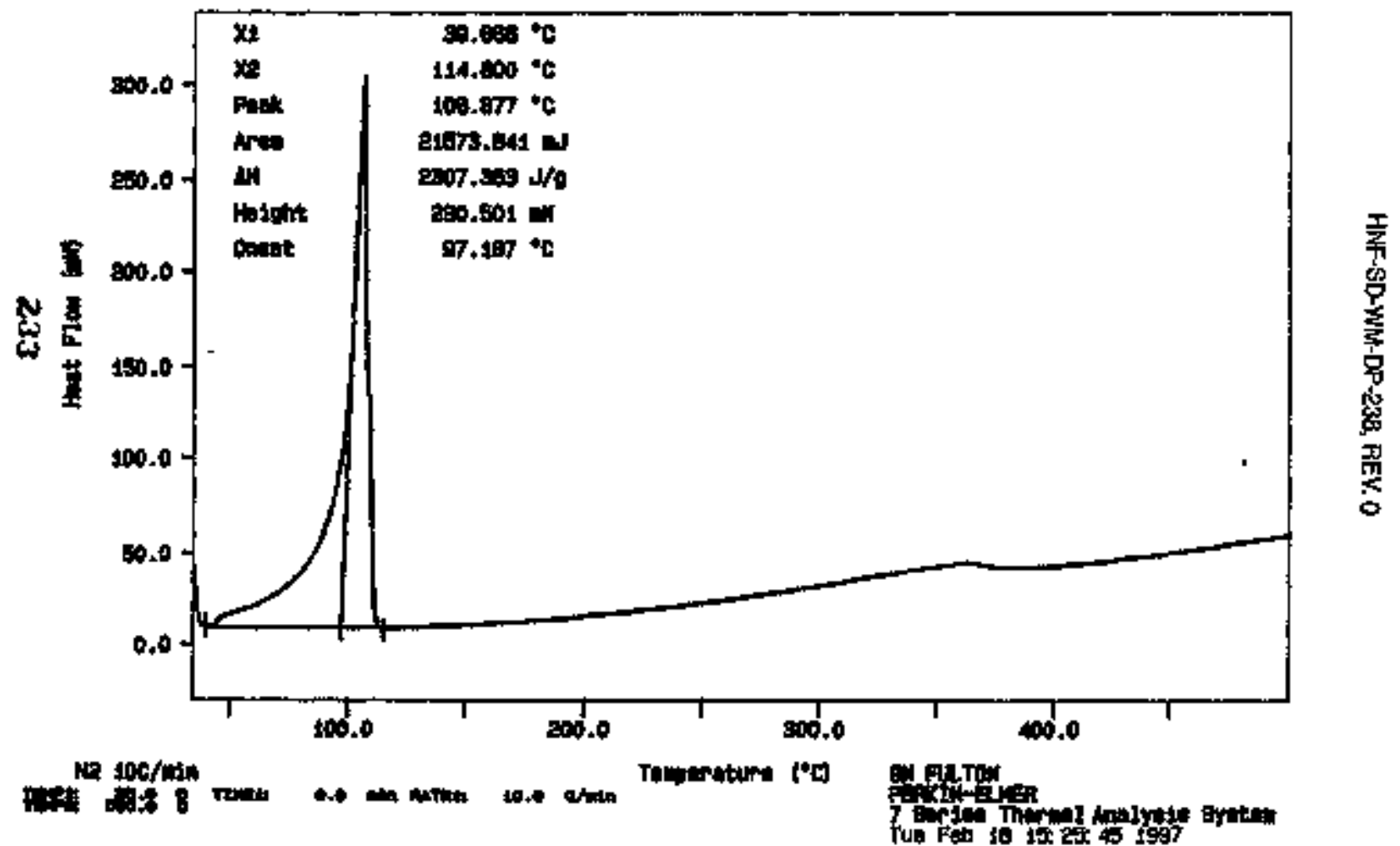




\section{LABCORE Data Entry Template for Worklist/ 16716}

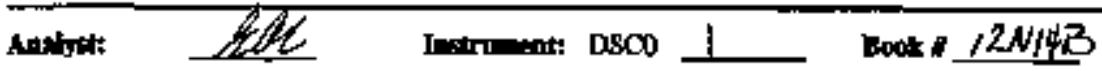

Method: LA-514-113 Rev/Mod C-1

Wachilst Conn:mint: DSC-01 FOR T-110 (RIFN UNDER NITROCEEN) INDLUM RTS

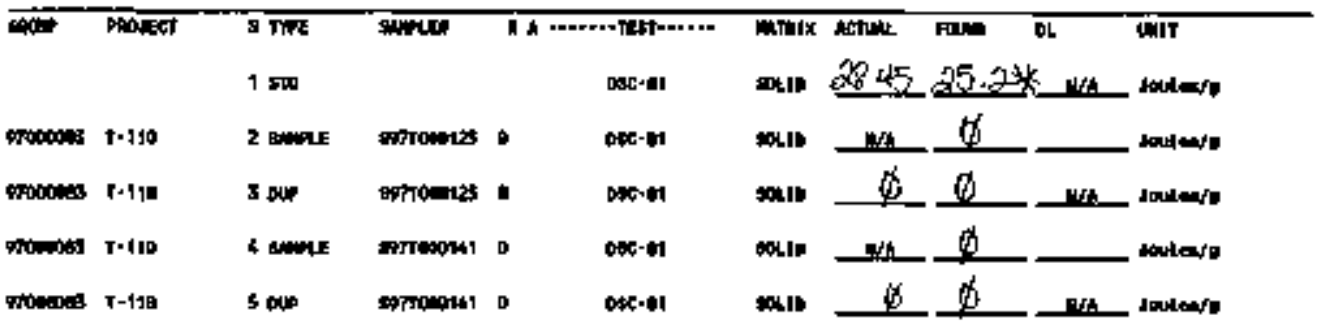
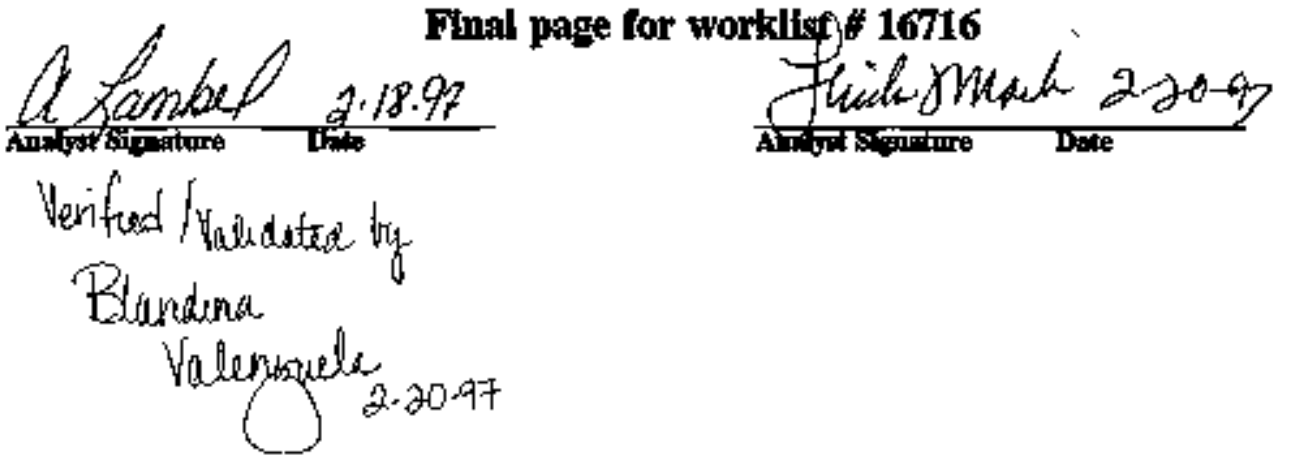

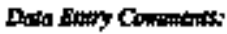

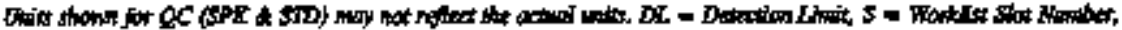

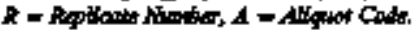


A 1 intal 0.1797 .

DSC STD I2N14-B

$22.700 \mathrm{mg}$

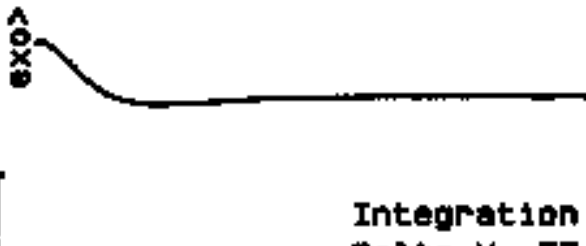

Delta $H \quad 574$ md

Ritex $10.0^{*}$ c/atn

$$
25.25 / 9
$$

Peak 158.5"C

-29. 1 ris

1
3
8
8

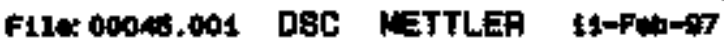

Ionts 0.0

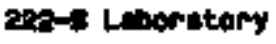

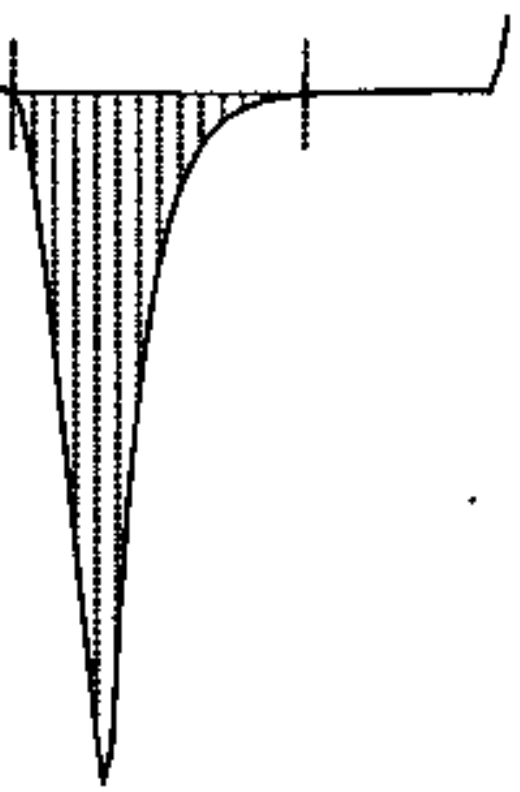

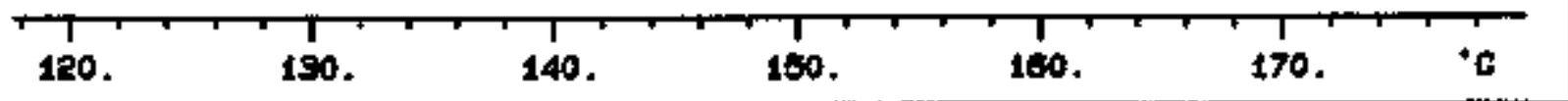




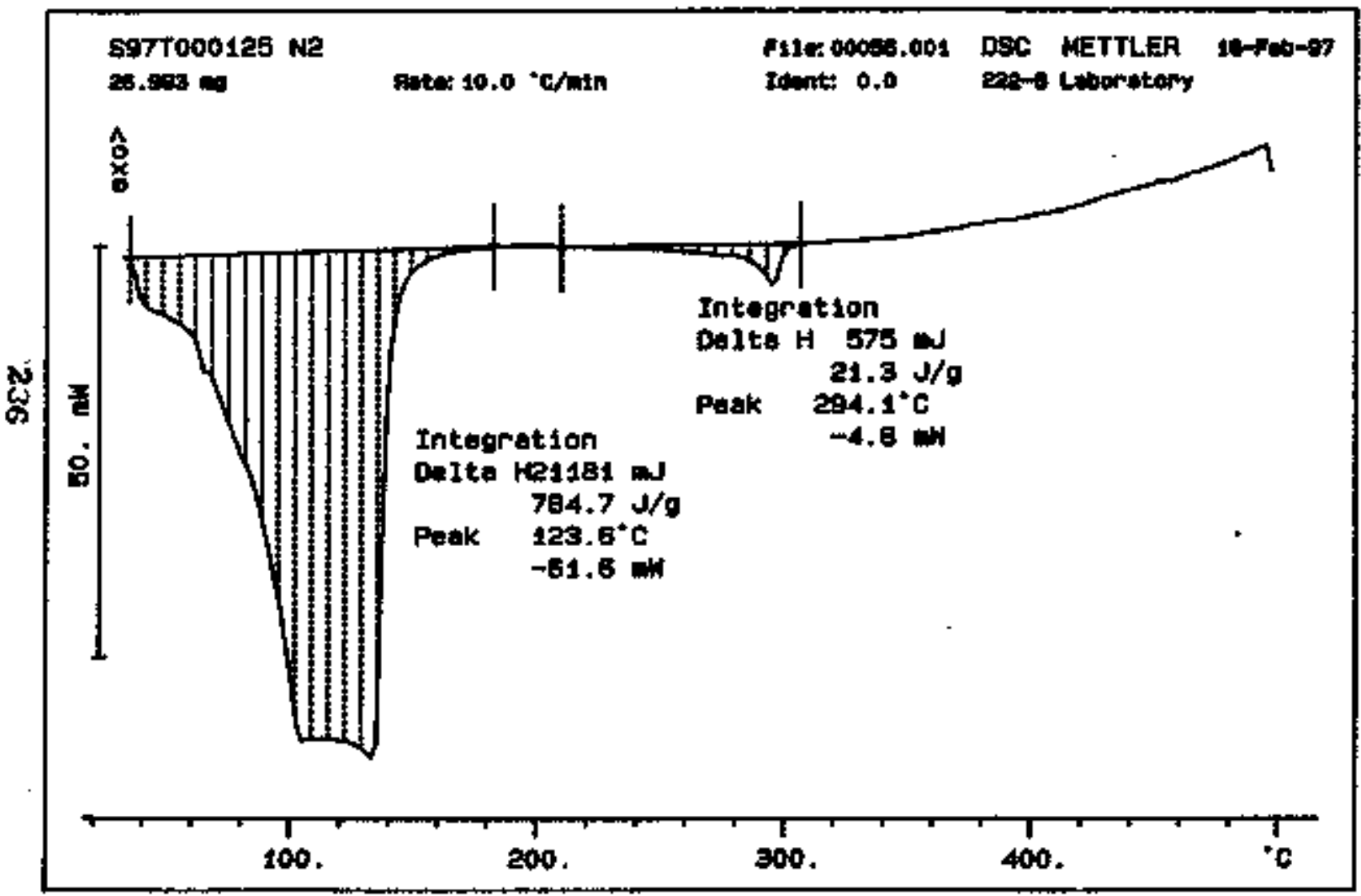




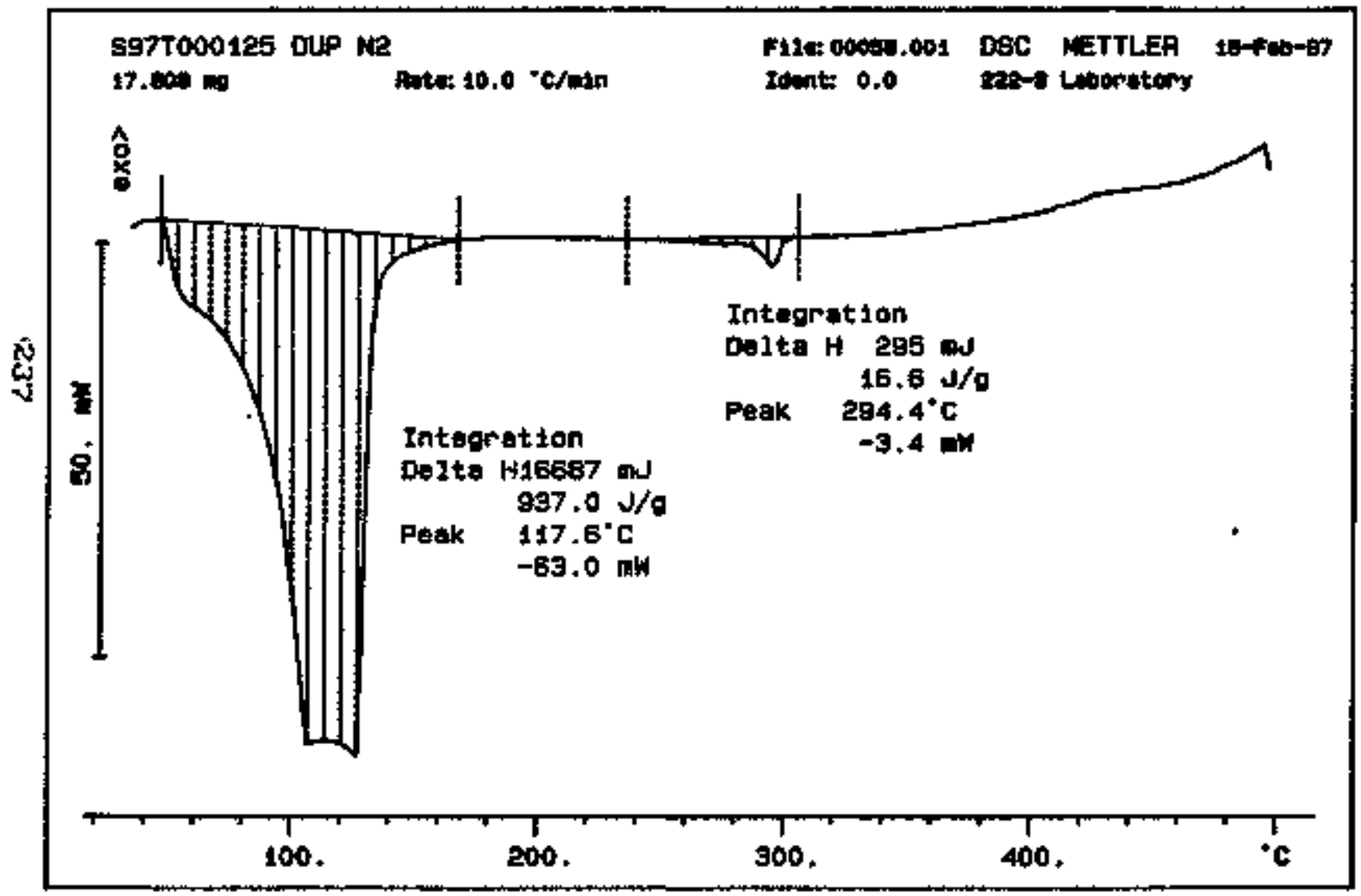

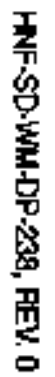




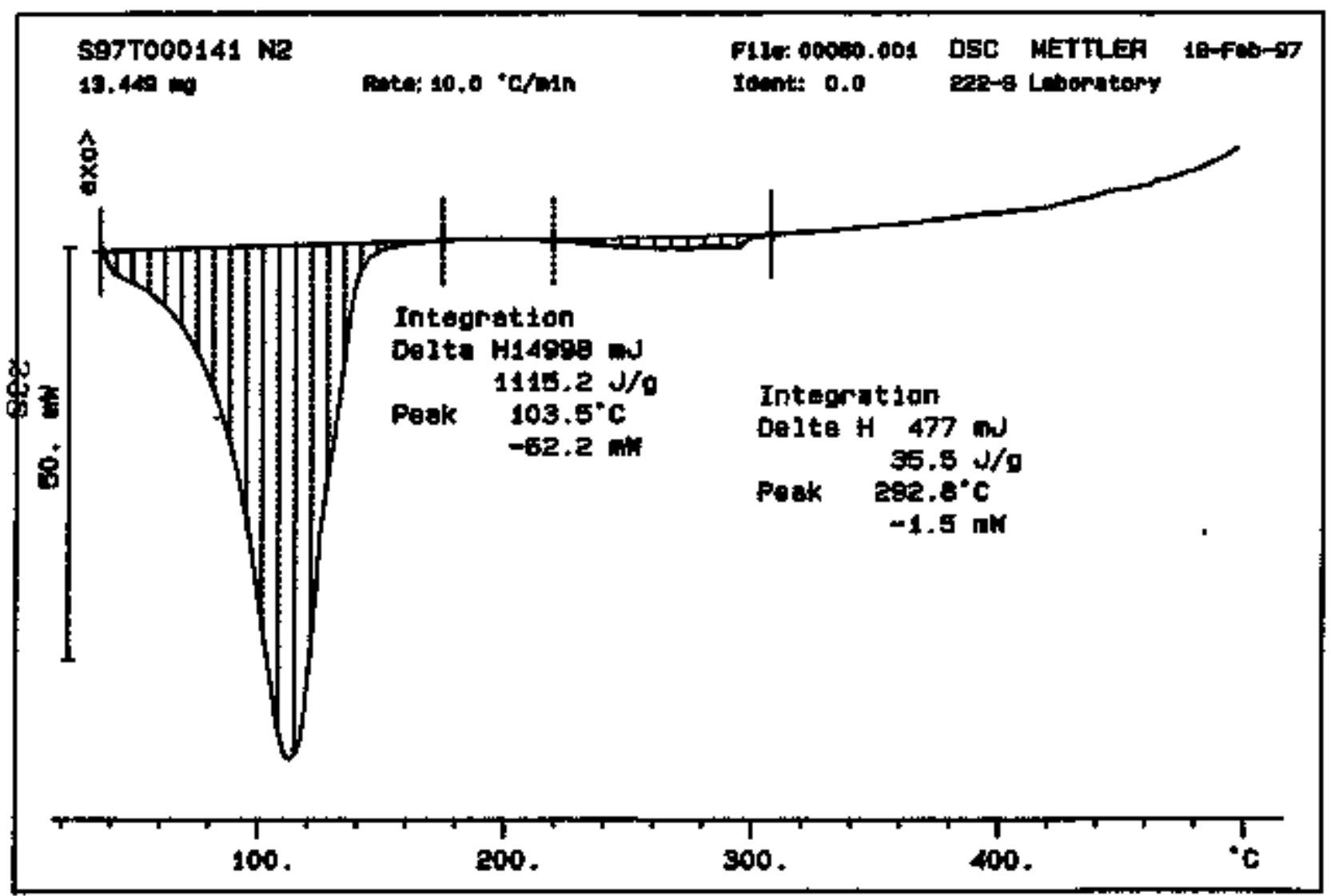

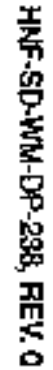




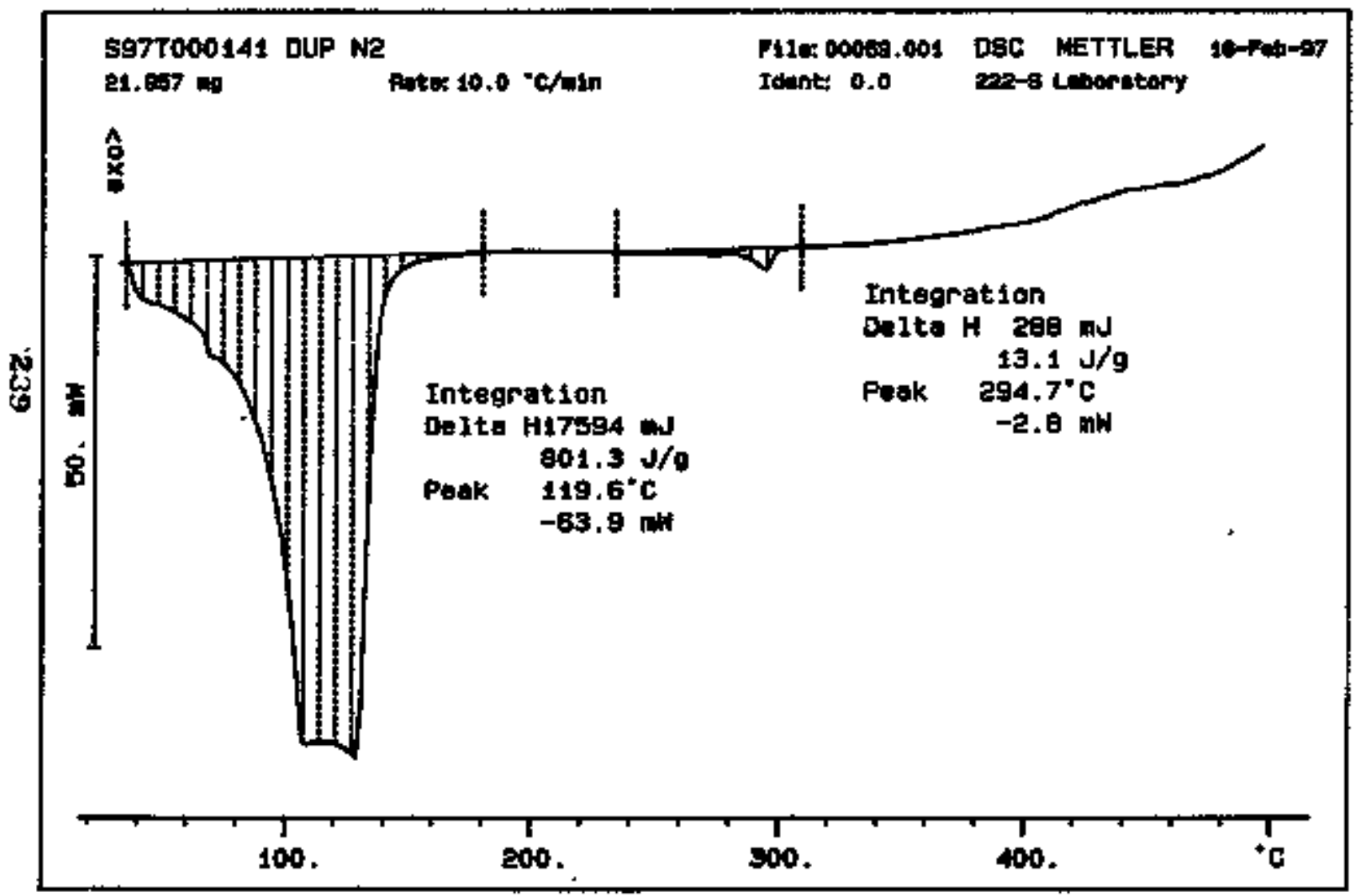

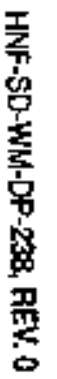




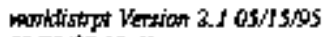

HNF-SD-WHA-DP-238, REV. 0

Pogt: I

(2)/2n's $3(2-4)$

LABCORE Data Entry Template for Worklist\# 16717

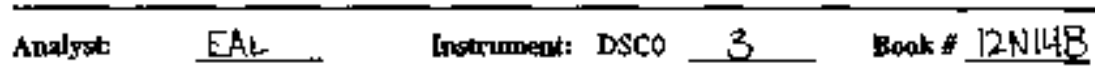

Methodi LA-514. I I4 Rew/Mind D-O

Watt'st Cowment: DSC-01 FOR T-1 10 (RUN UNDER NITROGEN) INDIUMA RTS

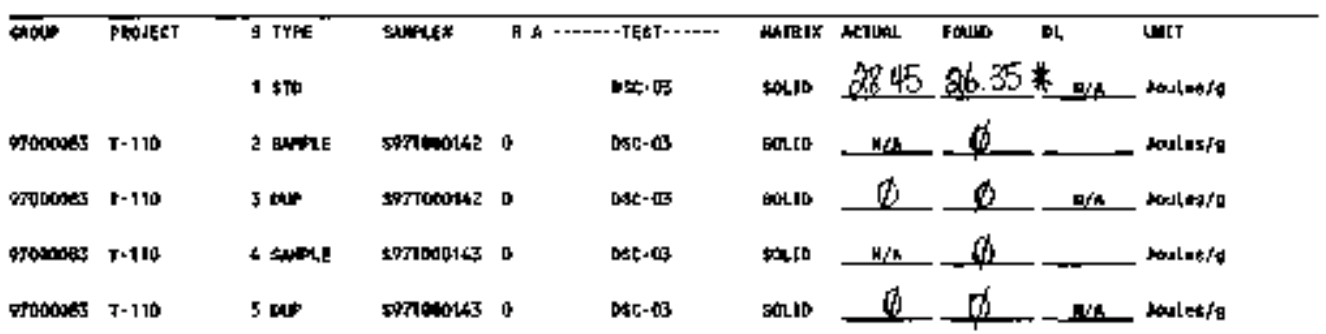

Final page for worklist \# 16717

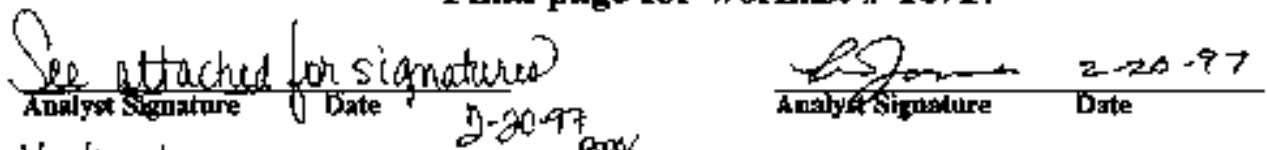

Verifued Nalidated

ory Bardinas.

Vatenguele

$3-547$

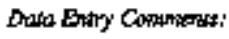

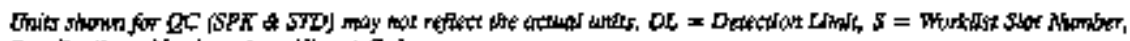

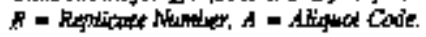

240 


\section{LABCORE Data Entry Tempłate for Worklist\# 16717}

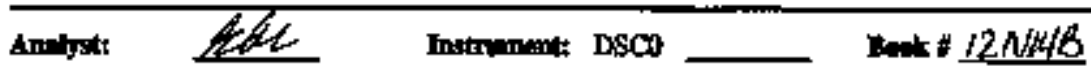

Mothod: LA-514-113 Rovphod

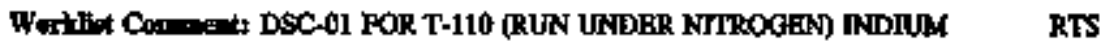

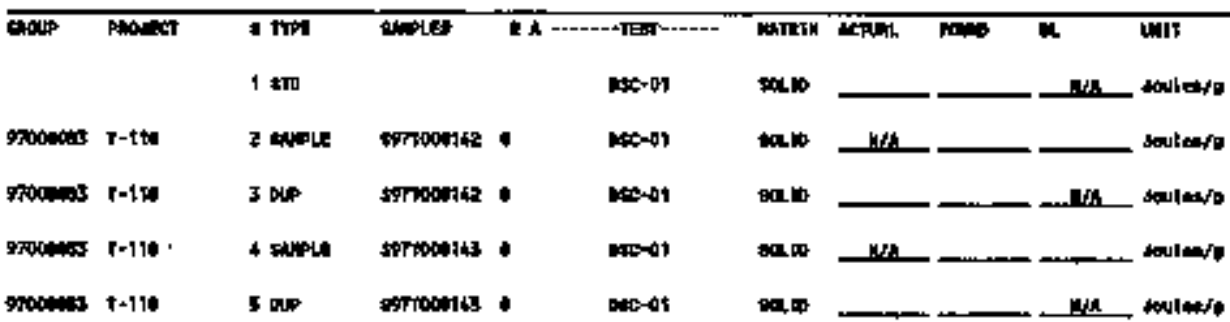

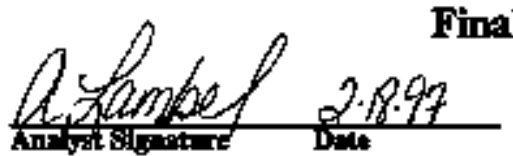

Final page for worklist \# 16717

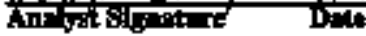

Doc-ol instrumint

was noed

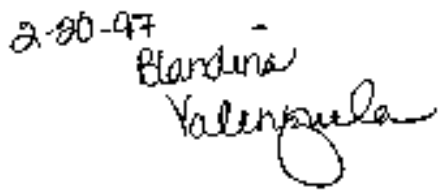

Data bany Candis:

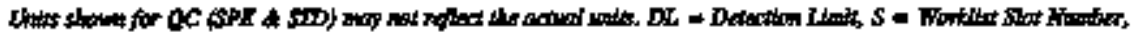

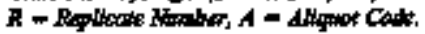


Curve it DaC

F11: info: INDOedool Tue Fob 19 0B ax 57 1997 sangle velont 10.450

5TU 12N14-9 mis
SIGNATUAE BELOW REPRESENTS CHEUICML TECHNOLOOST/CHEHIST THAT

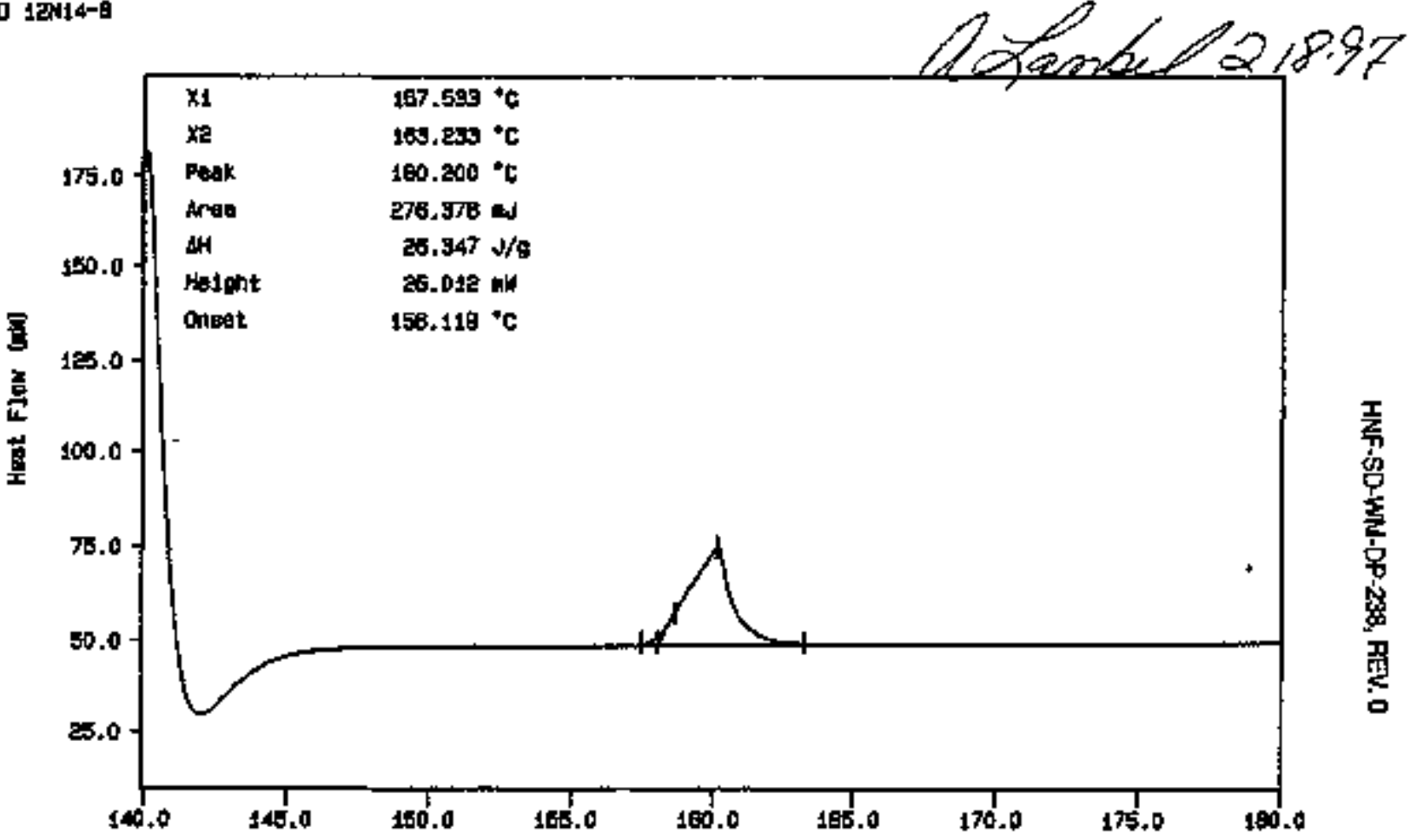

Ne. EXOTHUN DON

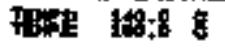

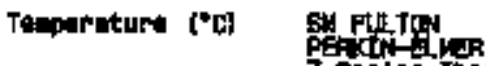

7 sopise Tharad hnolytio sraten 
Durw t

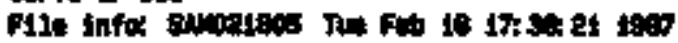

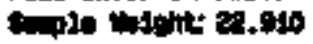

groponete

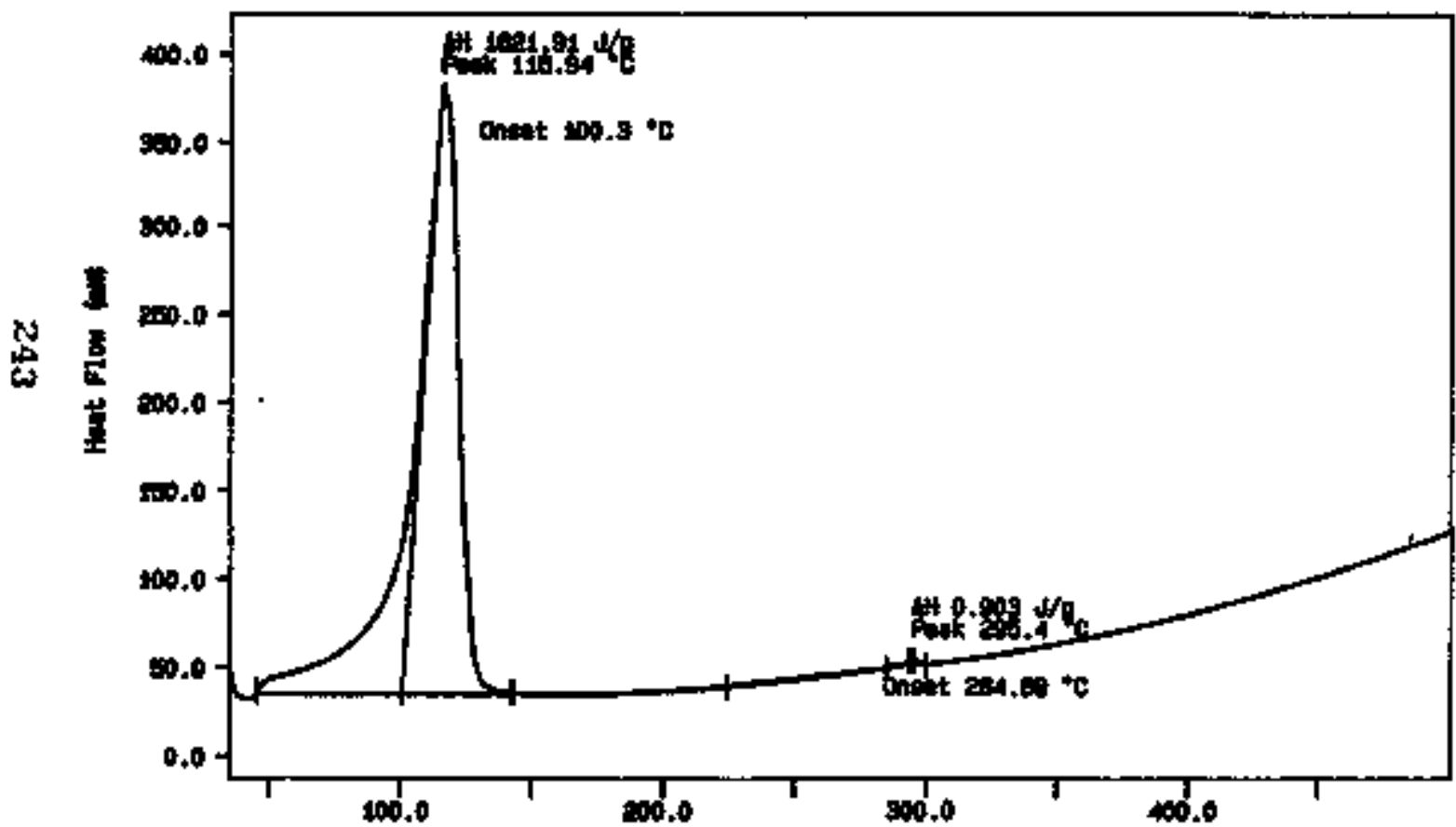

ne racking

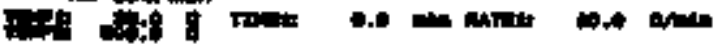

Temontare

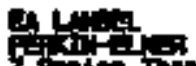

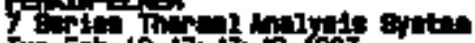
Tue F⿱⺈ 18 17: 47 6 is 
Corre 4: Dac

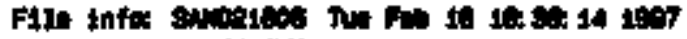

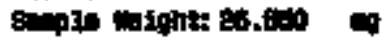

cortoposerse

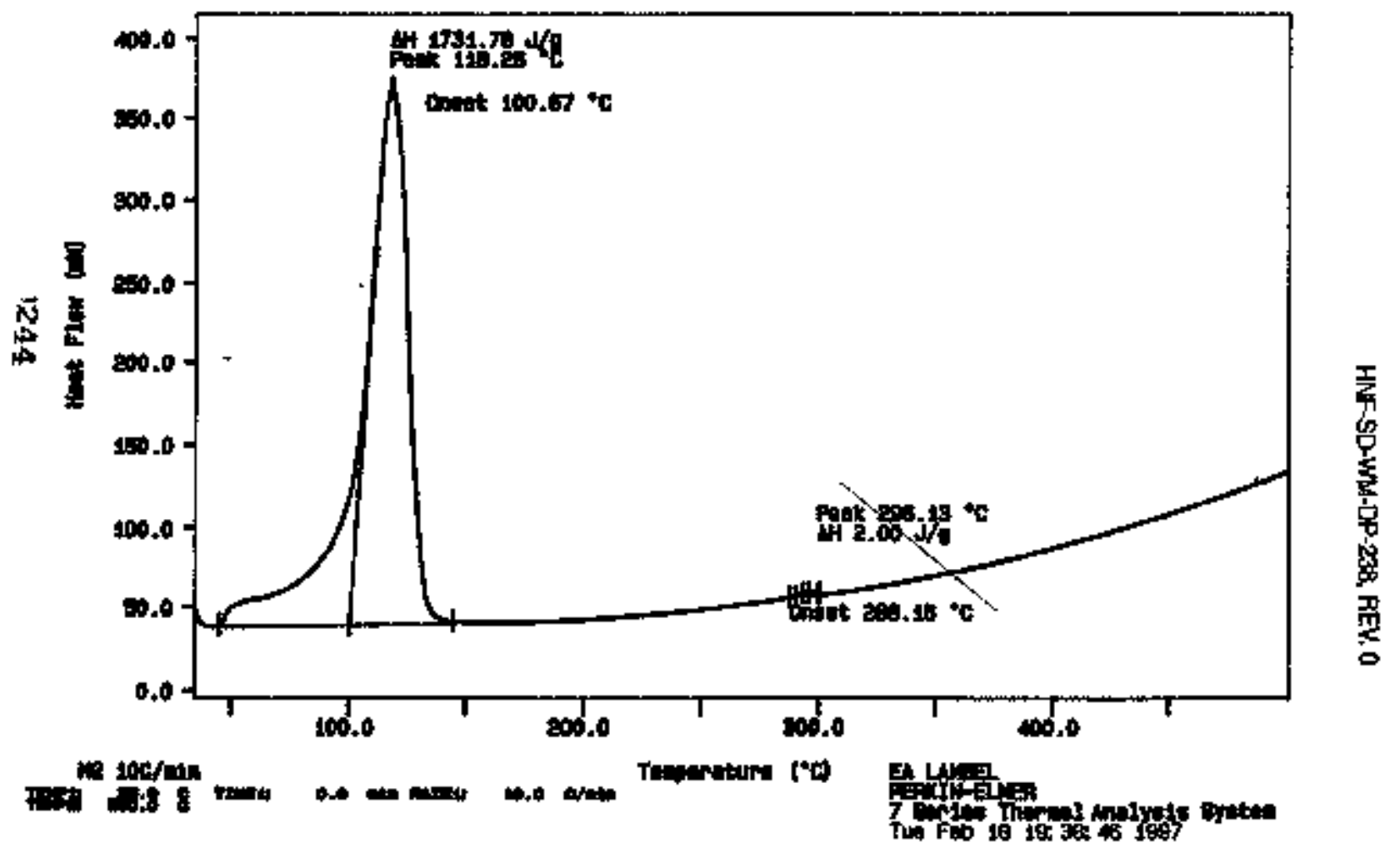


Curve I: ISt

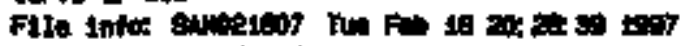

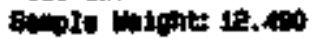

HTo00145

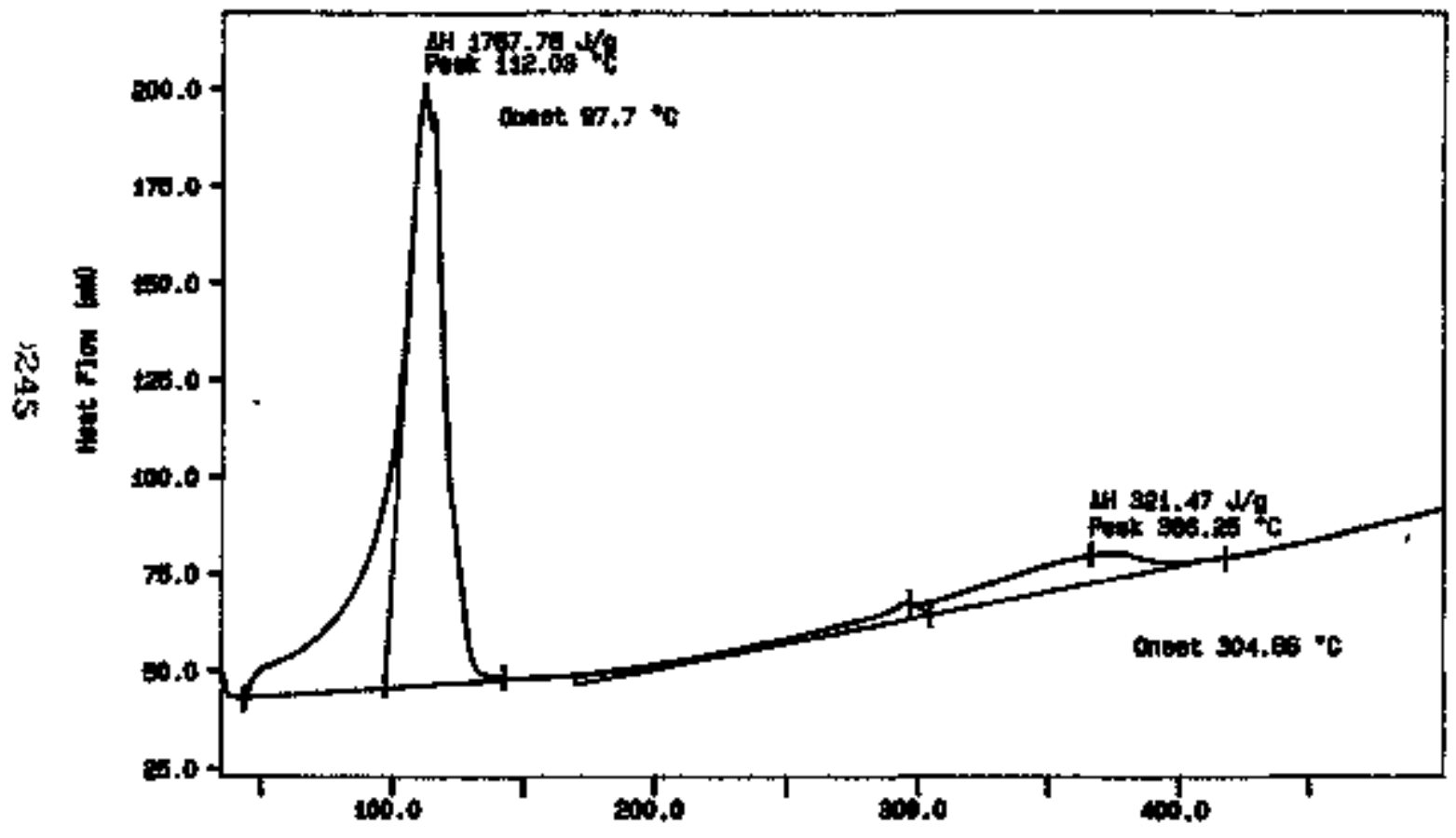

and J06/ain

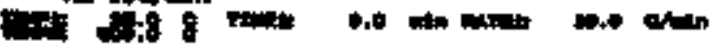

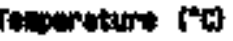


arve is ats

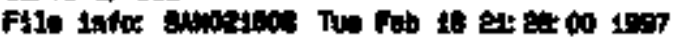

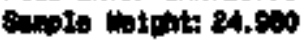

momolasp

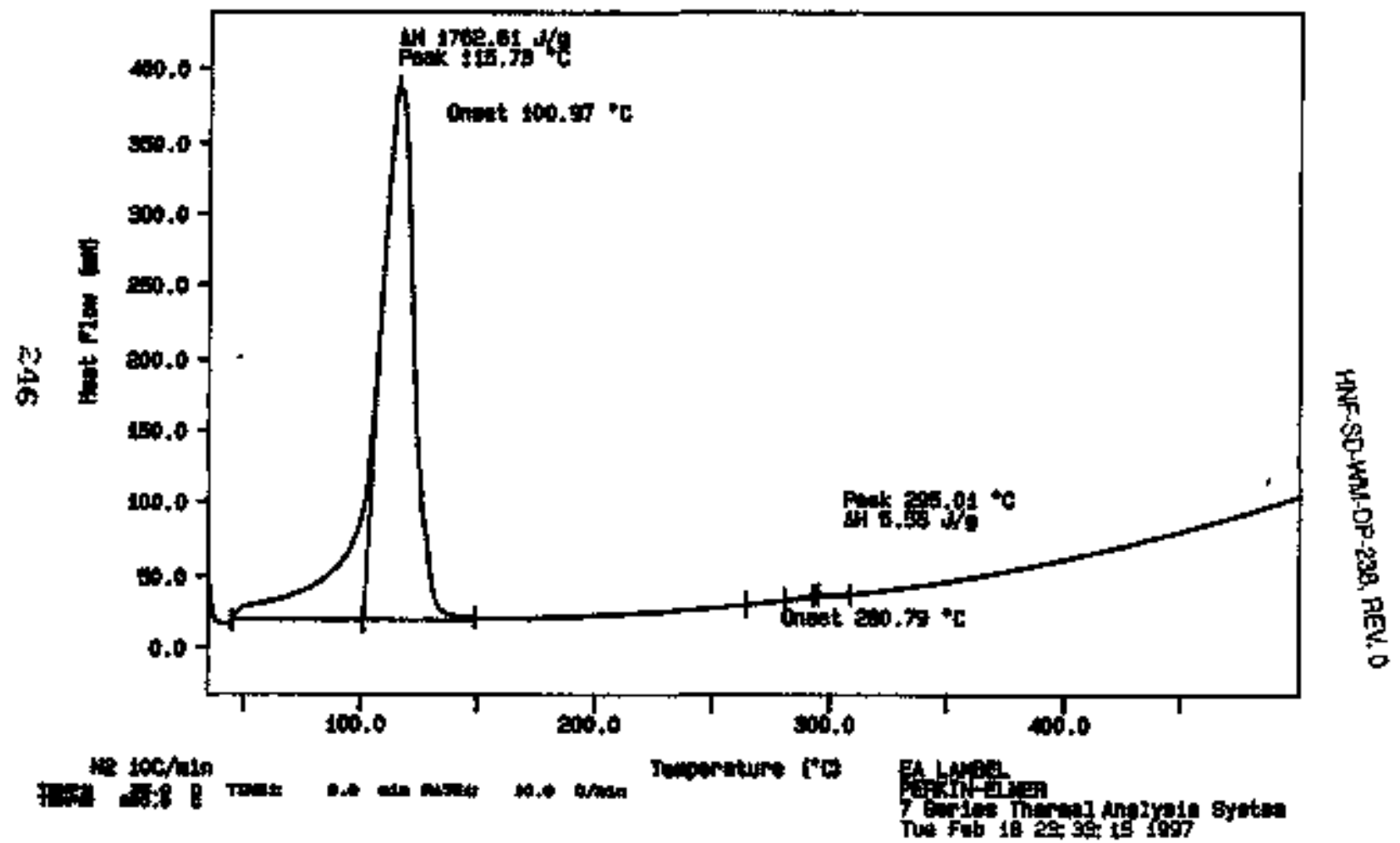


norktisme Persian 2.J 05tJ5\%s

HNE-SD-WH-DP-238, REV, O

Fage: $f$

$02 / 26 / 97$ 14:15

LABCORE Data Entry Template for Worklist: 16718

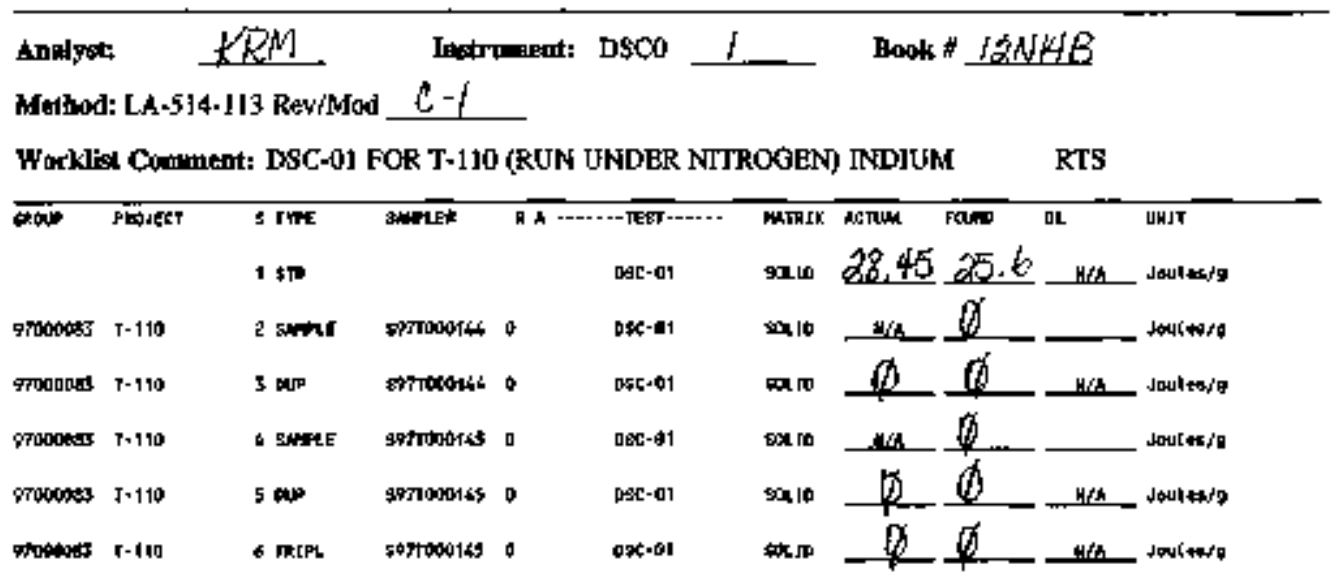

Final page for worklist \# 16718

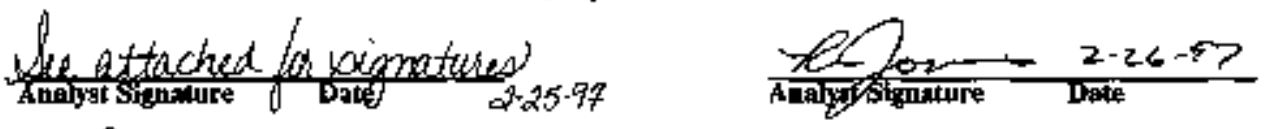

Verified/Nalidated by

Blandina

Valepondela $3-27-97$

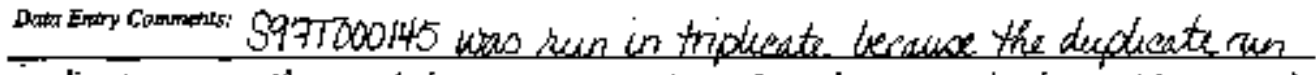
indicated another outotance pleoent witfin the sample (possiblyorganic) whth pery little water. the triplicate ran was aimilar to oniginal

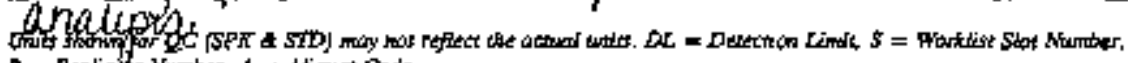

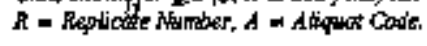

i:247 


\section{LABCORE Data Entry Template for Worklist" 16718}

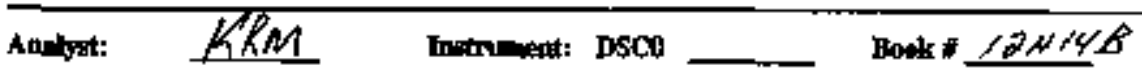

Methedt 1A-514-113 Row/Mod

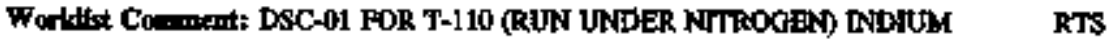

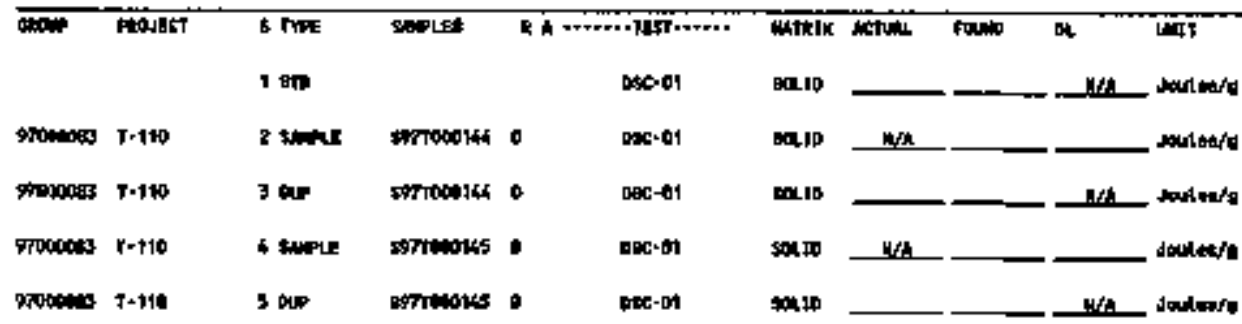

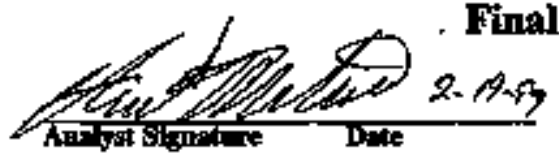

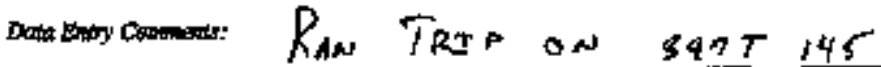

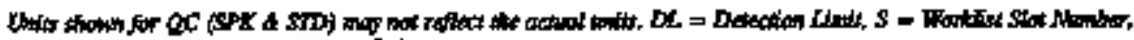

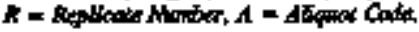




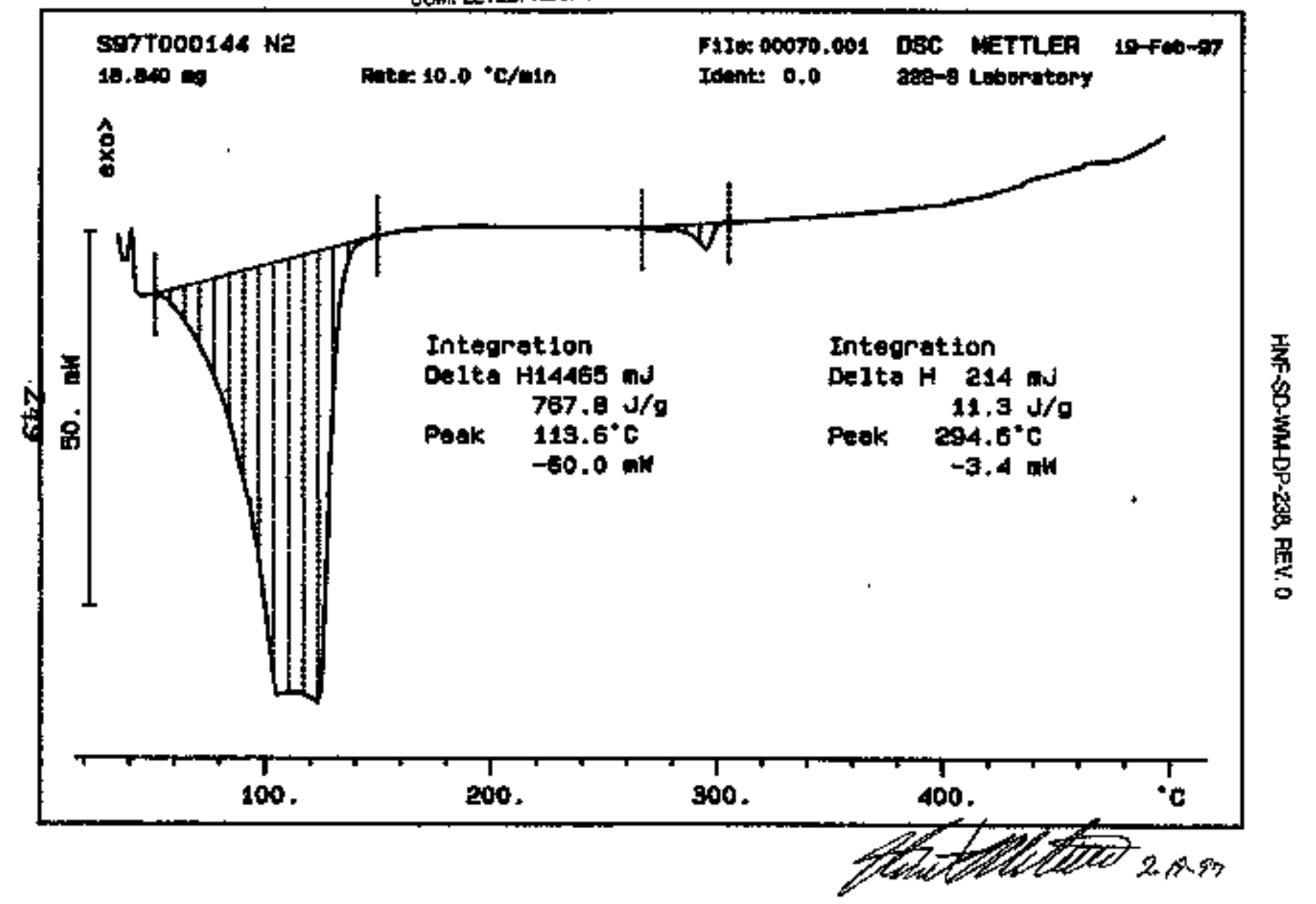




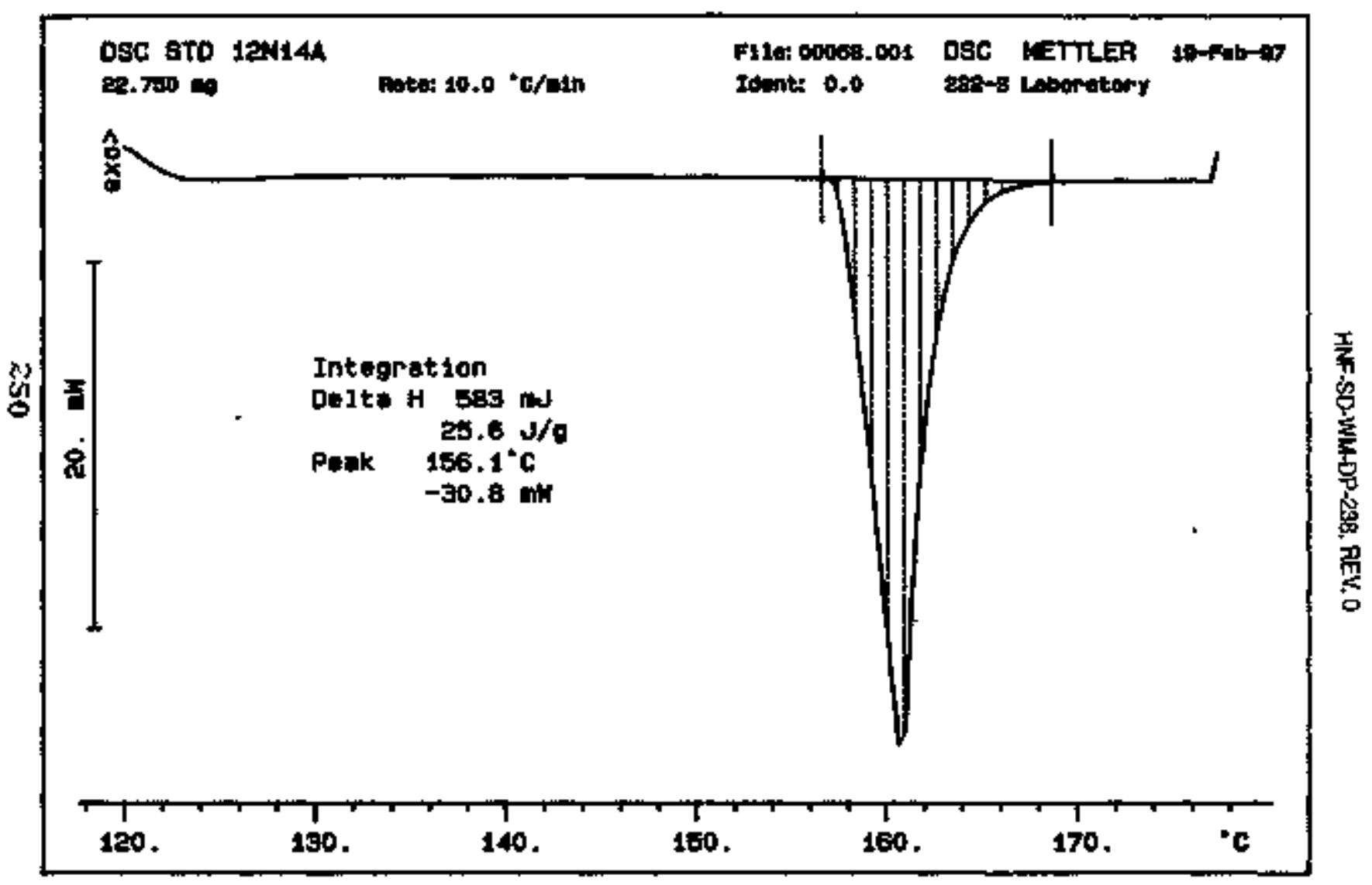




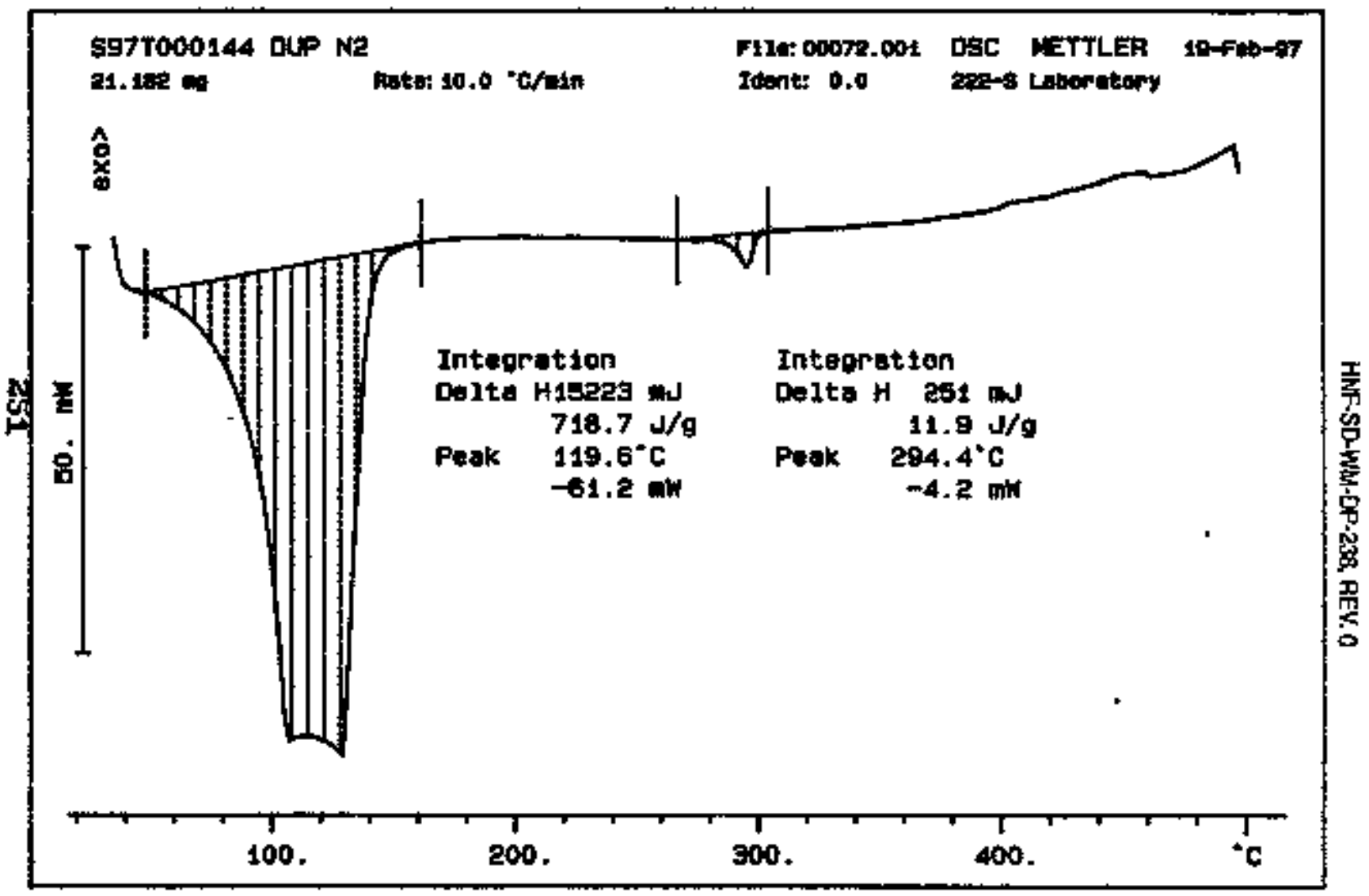




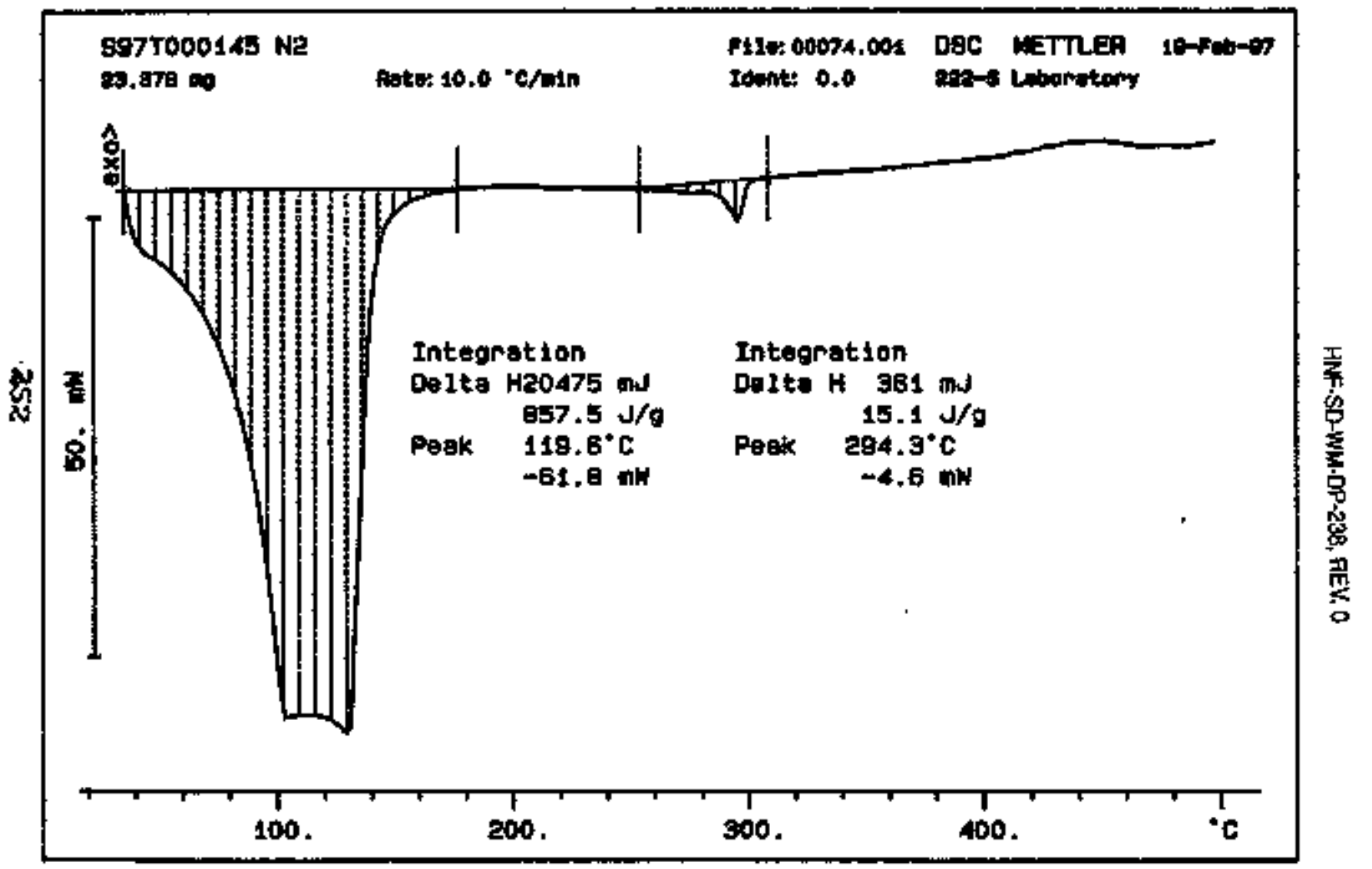




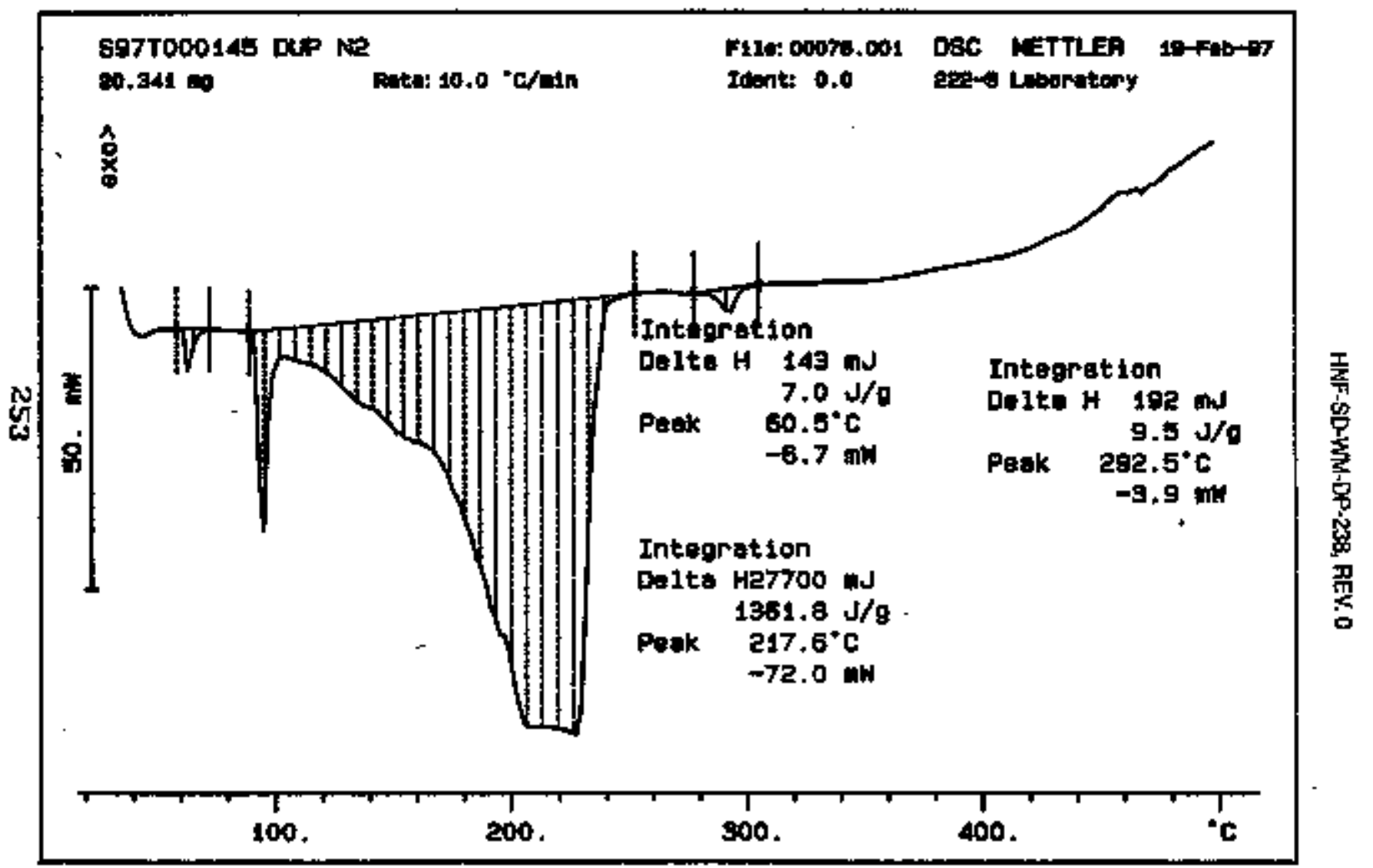




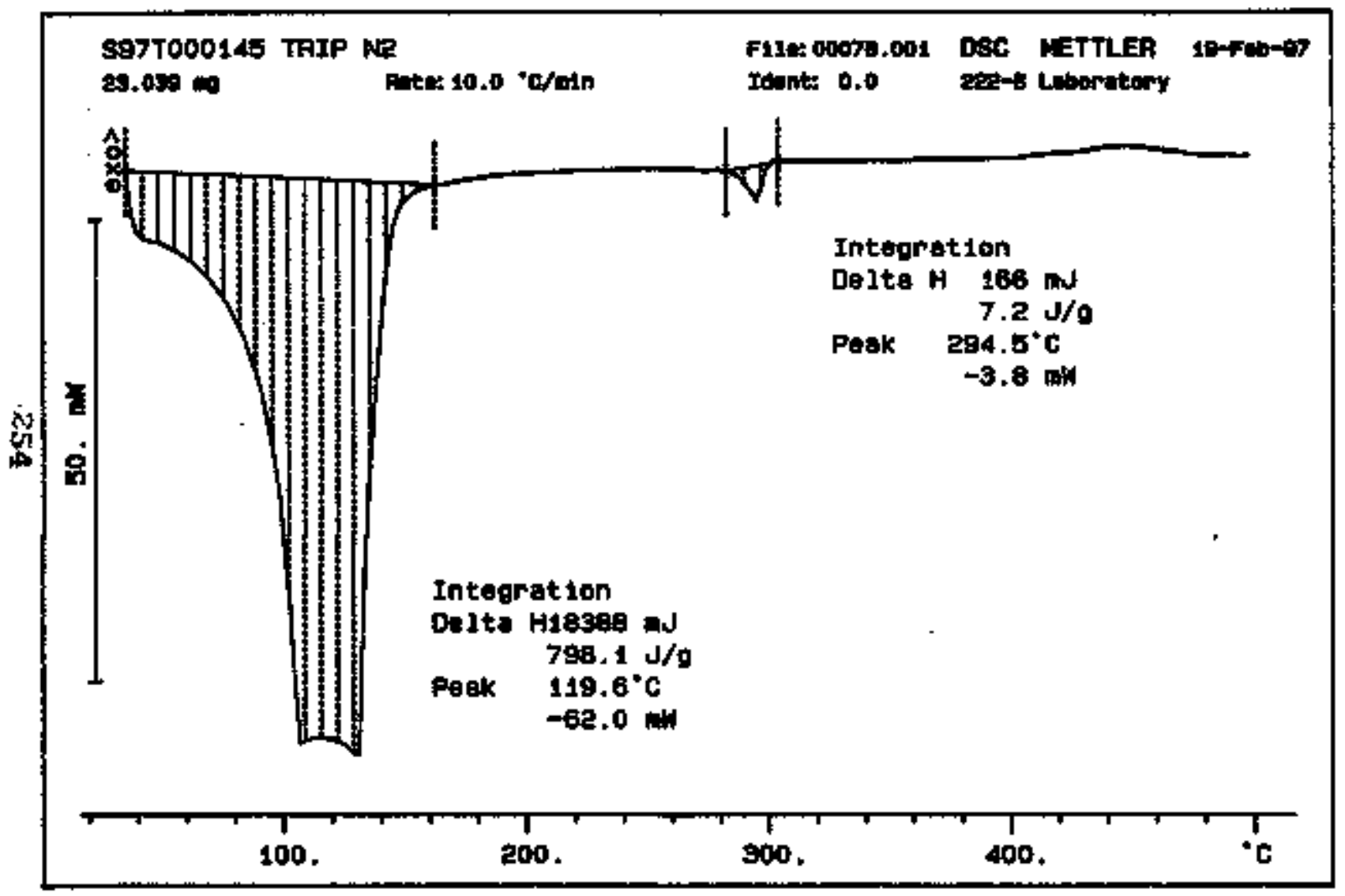

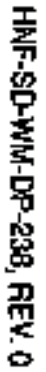




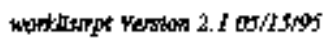

HNF-SD-WM-DP-238, REV. 0

Papz; I

$02 \pi, 29700 ; 04$

LABCORE Data Entry Template for Worklist\# 16719

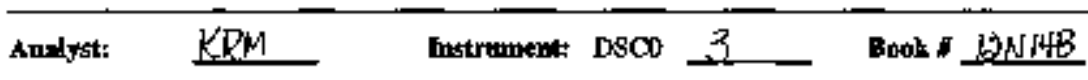

Metbod: LA-5id-1I4 Revihod $D-0$

Wortlks Comment: DSC-01 FOR T-110 (RUN UNDER NITROGEN) INDIUM RTS

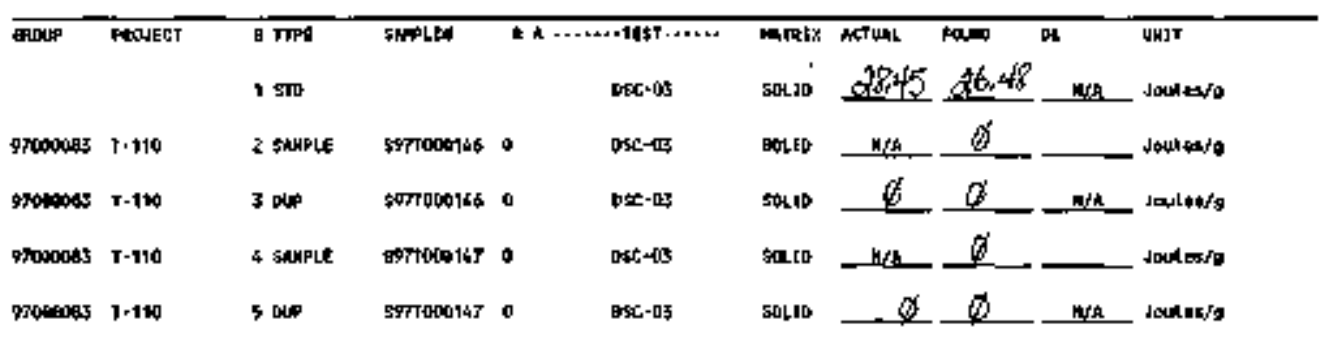

Final page for workligt \#16719

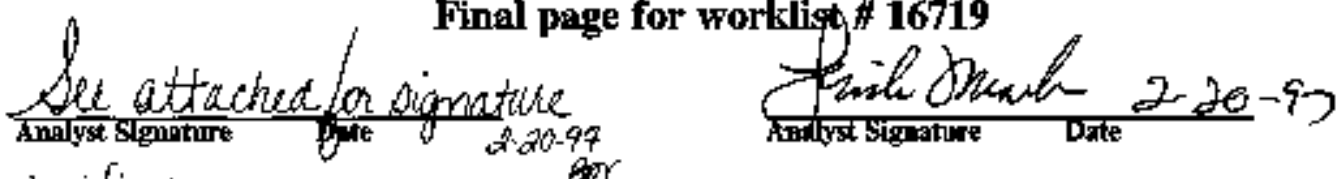

Verifiud Nolidated by

Blandena

Valenzopula

$$
2 \cdot 30-97
$$

Data Extry Compremit:

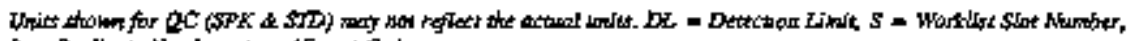

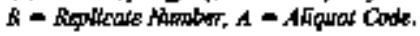

1255 


\section{LABCORE Data Entry Template for Woxkist\# 16719}

\begin{tabular}{|c|c|c|}
\hline Analyot: & $K R_{m}$ & Instrimenty DSCD \\
\hline
\end{tabular}

Mroknod: LA-514-1!3 Revilhod

Wort't CuI DSC-01 FORT-110 (RUN UNDER NITROGEN) RDIULS RTS

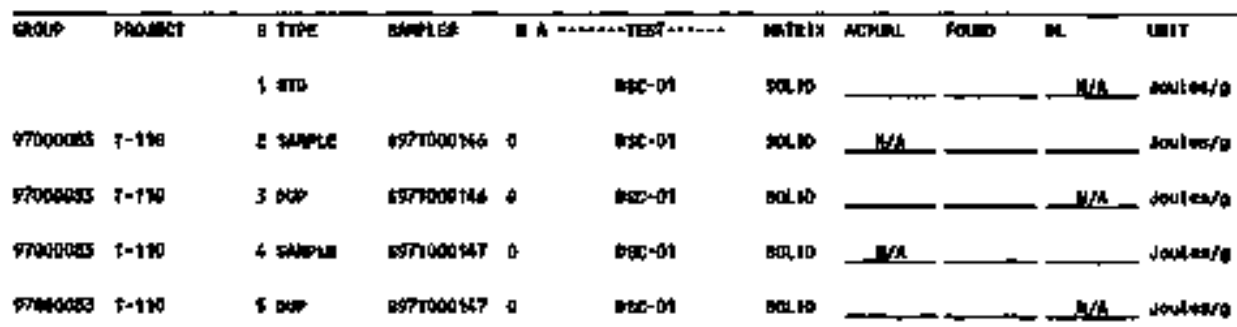

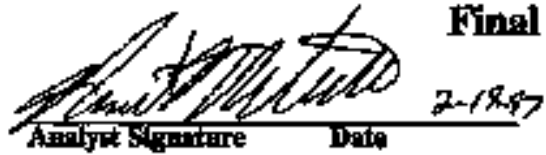

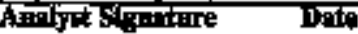

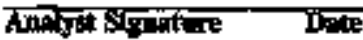

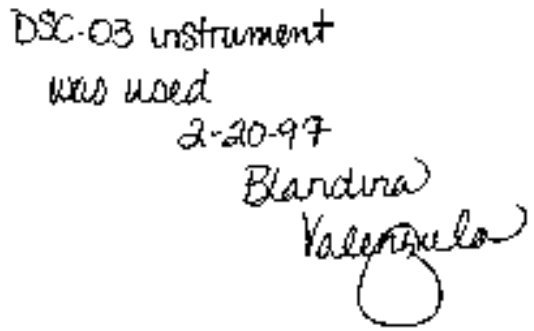

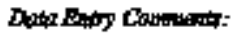

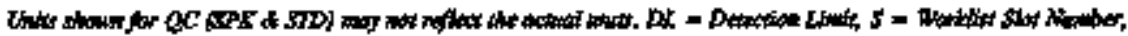

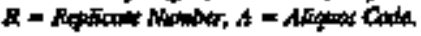


Dumre t: DEt

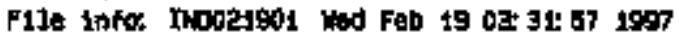

senple wiont $10,4^{\circ} 0$

m

8 I0 1814-9

अGMTLFE BELOW REPRESENTS GHENHWL TECHNOLCASTOHEMST THAT

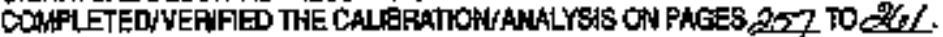

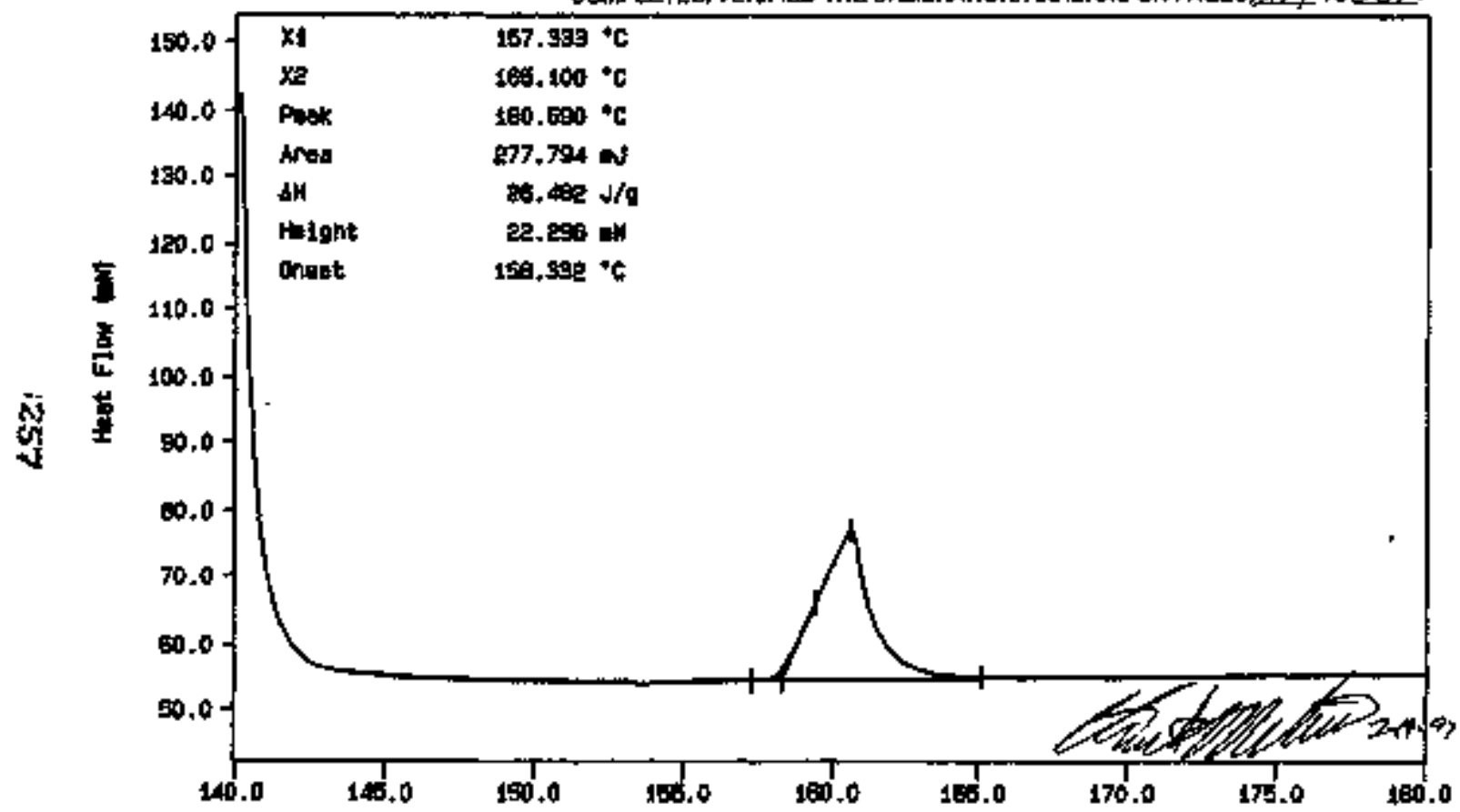

Ne. EXOTHEPA DOFN

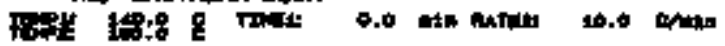

Tomperature (*c)

KS WOITIH

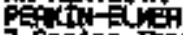

7 orion thoreal Ansiygte Syaten led Fot 19 is: 40:33 iog 
Cunve It ISG

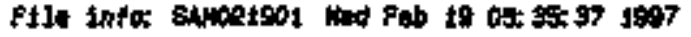

sumplo thitht: 23.420 4

BorTo00145

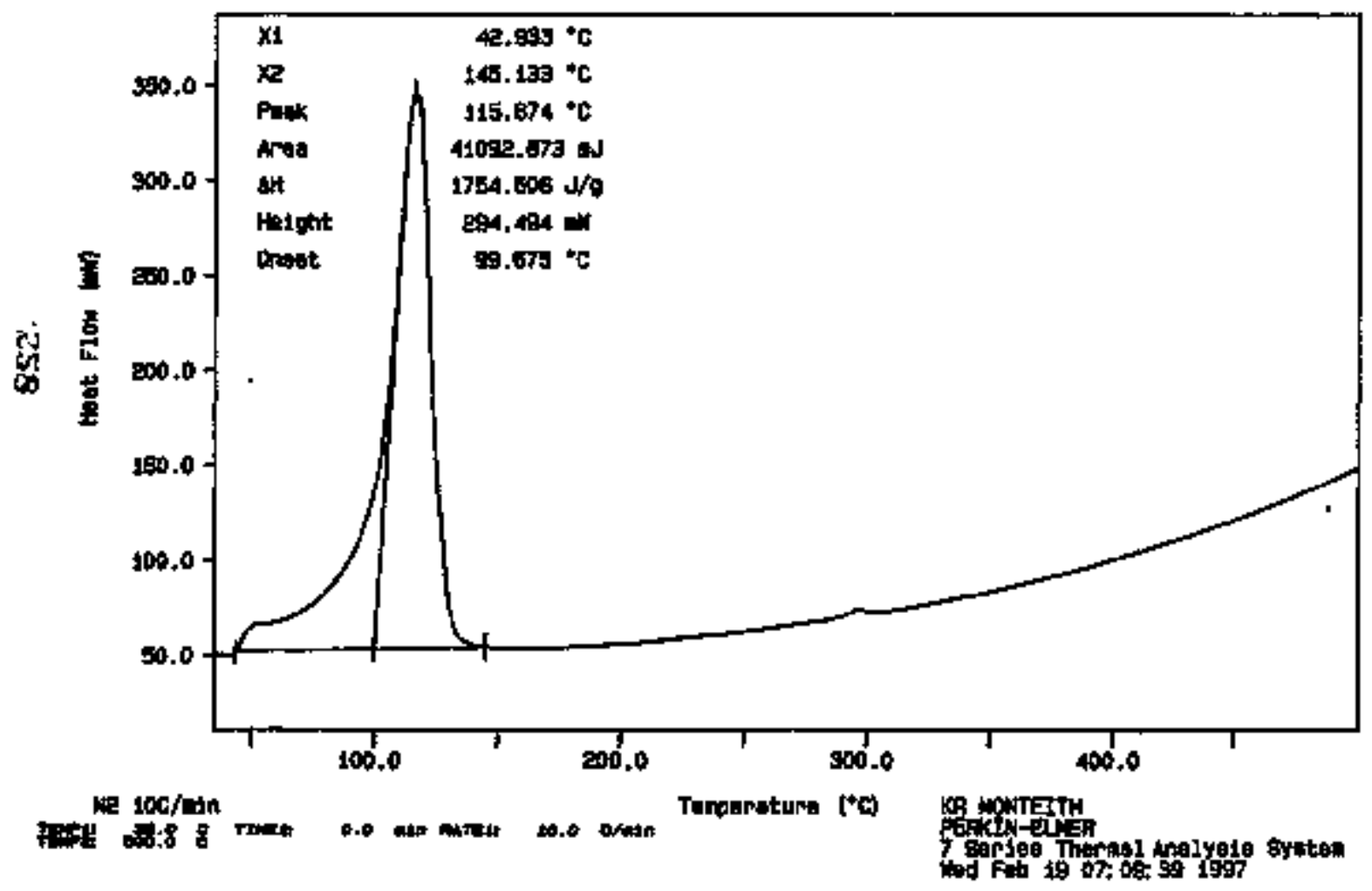


Crove 12 Dot

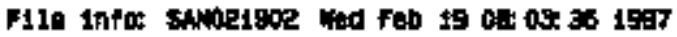

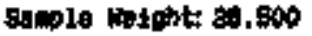
is

sortopolas Dis?

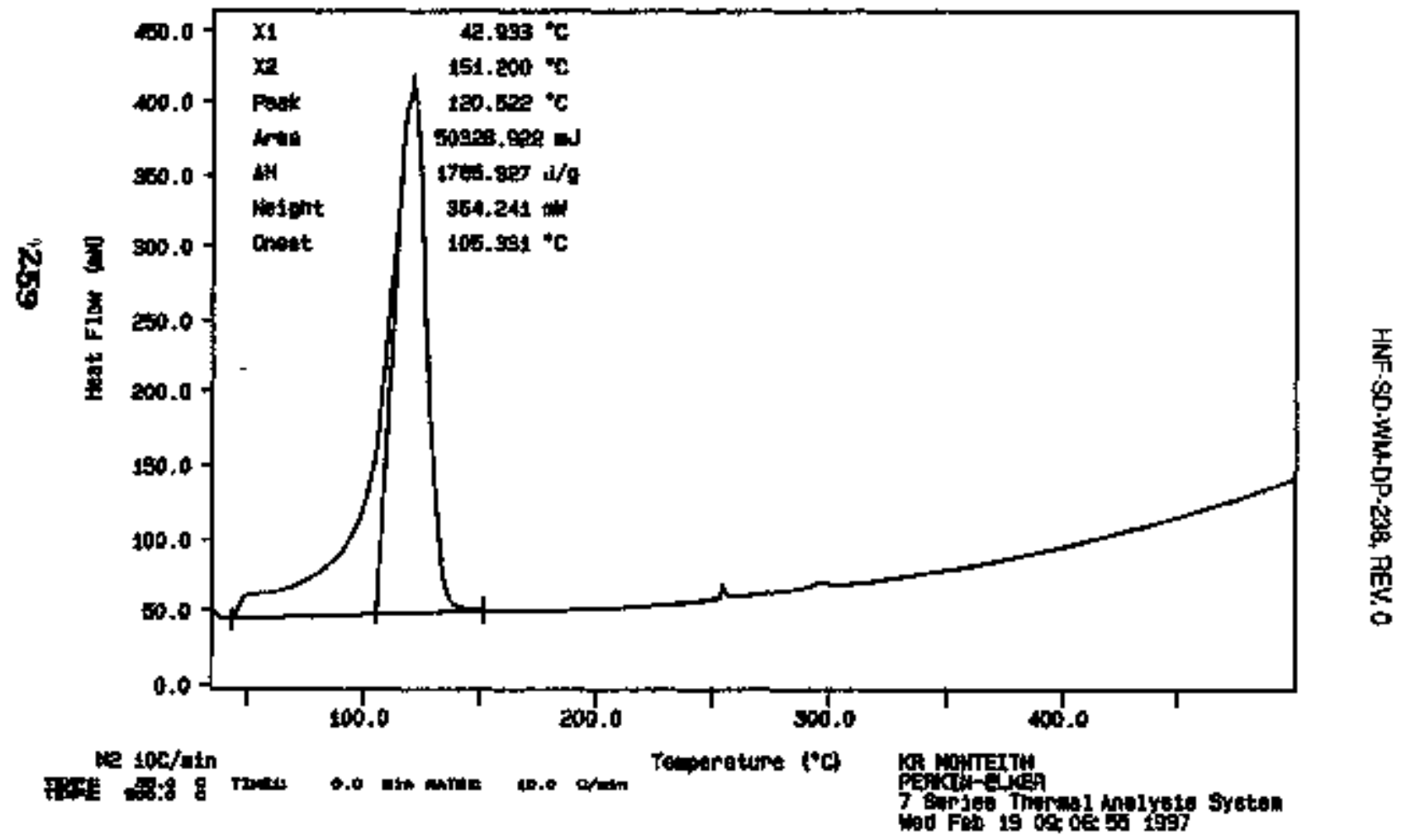




\section{Curve I}

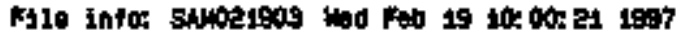

somplo winint: $97+600$

0077000147

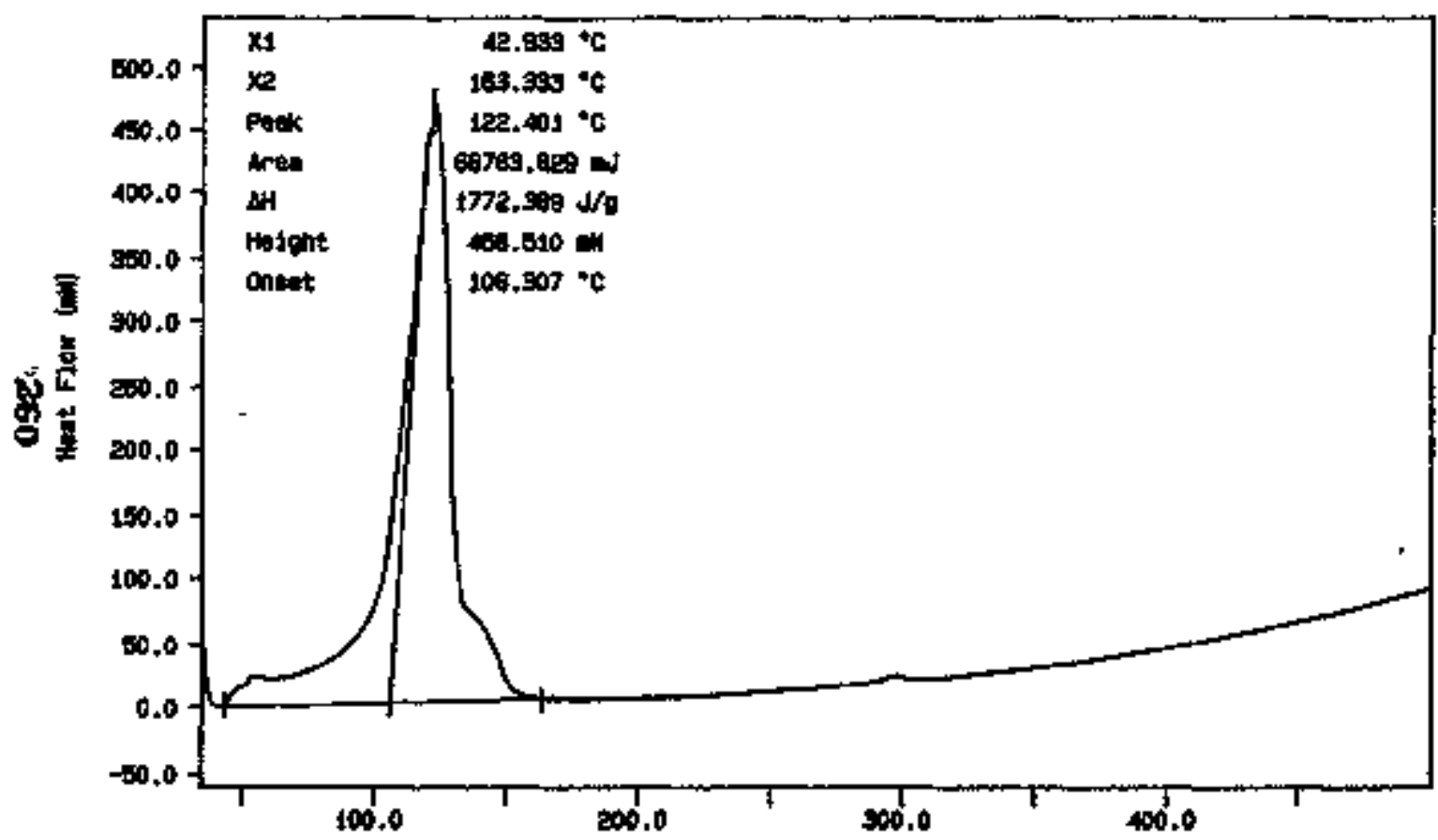

Ne 100/ain

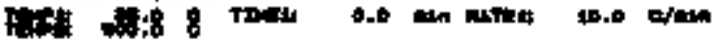

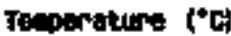

Fin

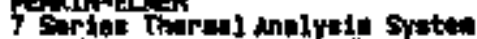

Ned Fob 19 11:3: 17 1097 
Curve 1 Detc

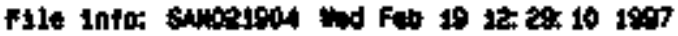

temis lotght: 98,400

Do

sim7000147 gip

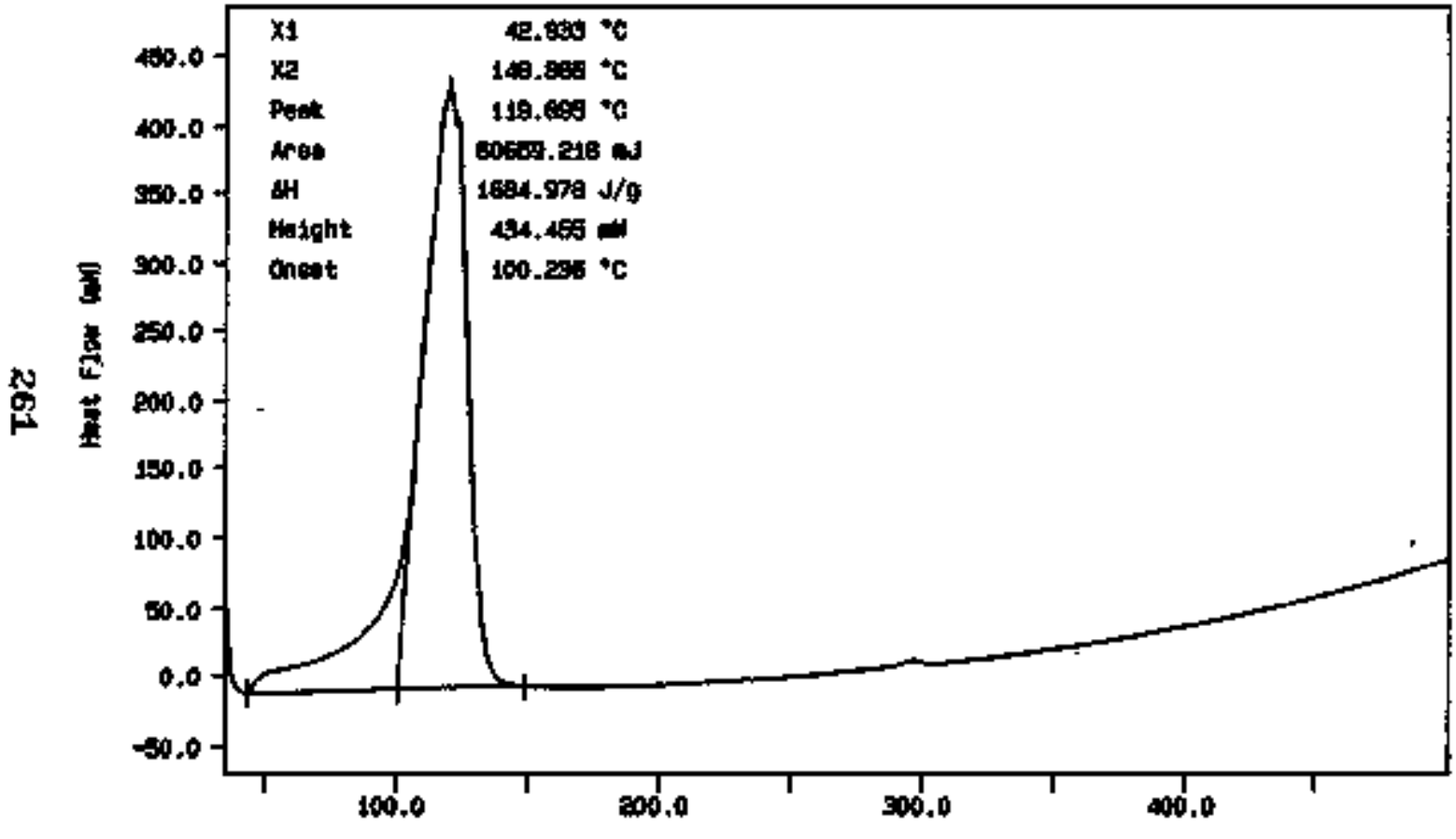


morklievpt Verten 2.J osis 5 iss

HANF-SD-WHA-DP-238, REV O

Page: I

$02 / 26977+26$

LABCORE Data Entry Template for Worklist"\# 16720

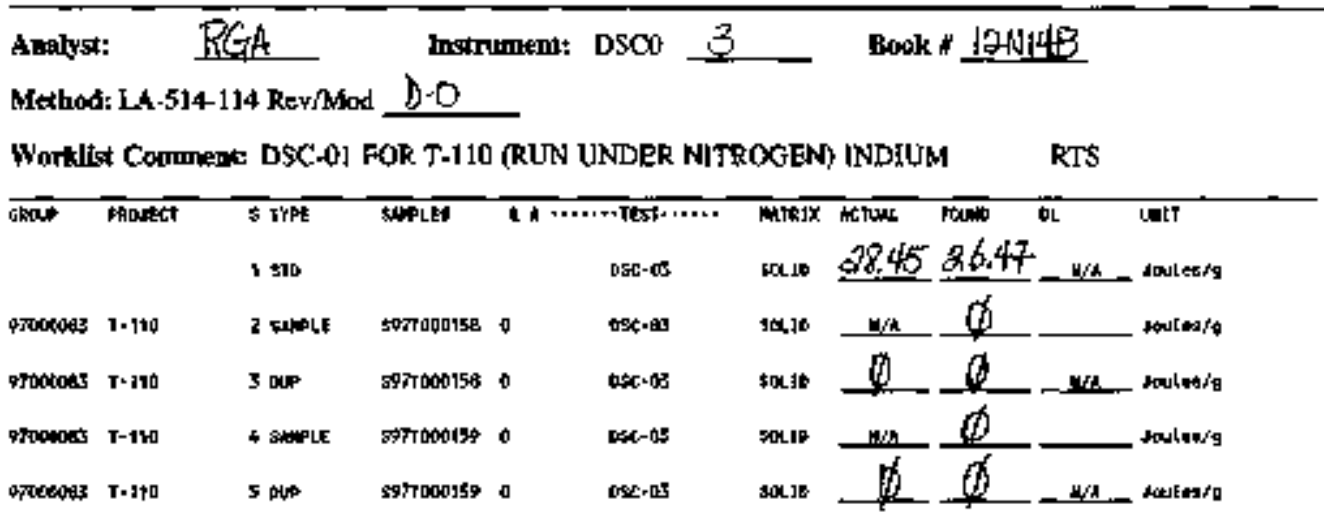

Final page for worklist \# 16720

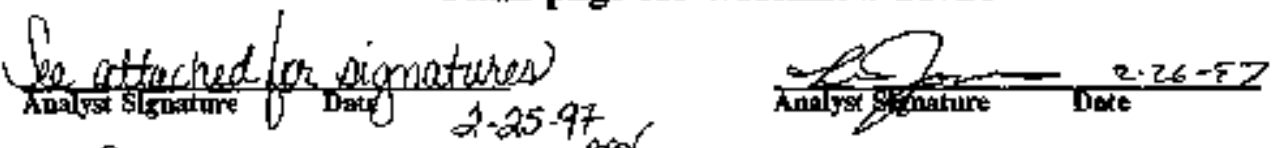

Verified/ralidabed by

00

Valentale 2-27.97

Dont Entry Comperts:

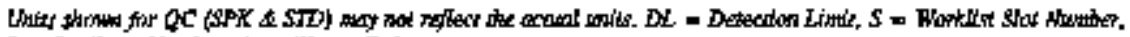

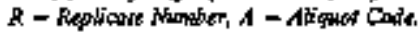

262 


\section{LABCORE Data Entry Template for Worklistt 16720}

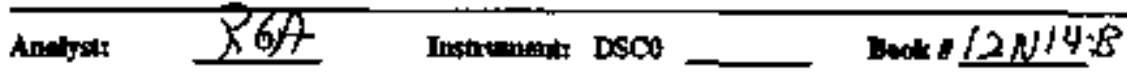

Malmit LA-514-113 Rewhiod

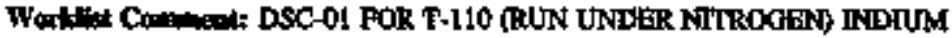

RTS

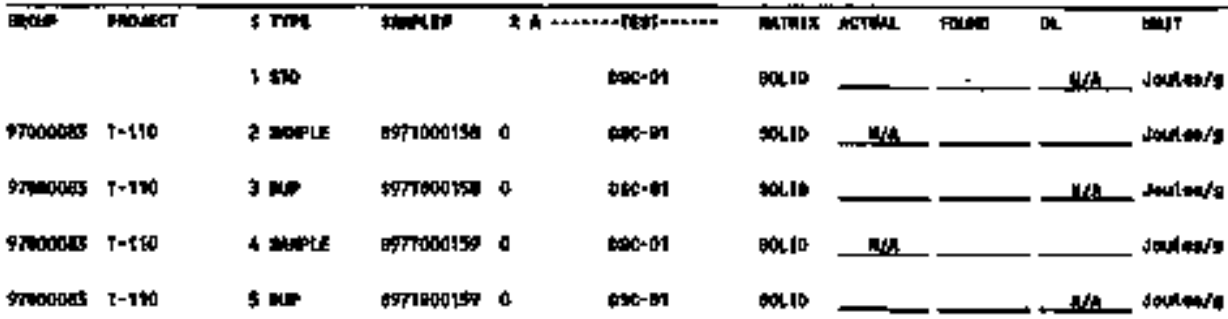

\section{Fthal page for worklist * 16720}

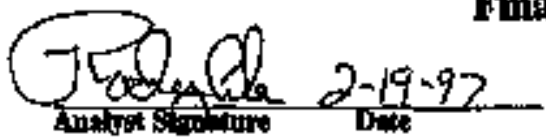

$\triangle S C .03$ instrument

woo uod.

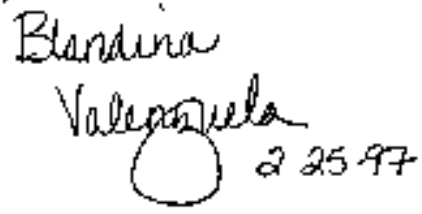

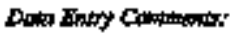

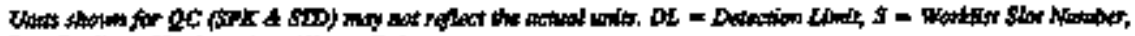

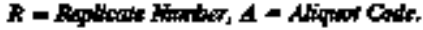


Drrve it Das

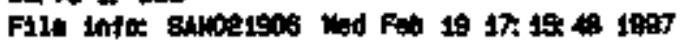

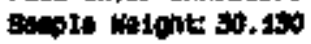

agroxolso sin

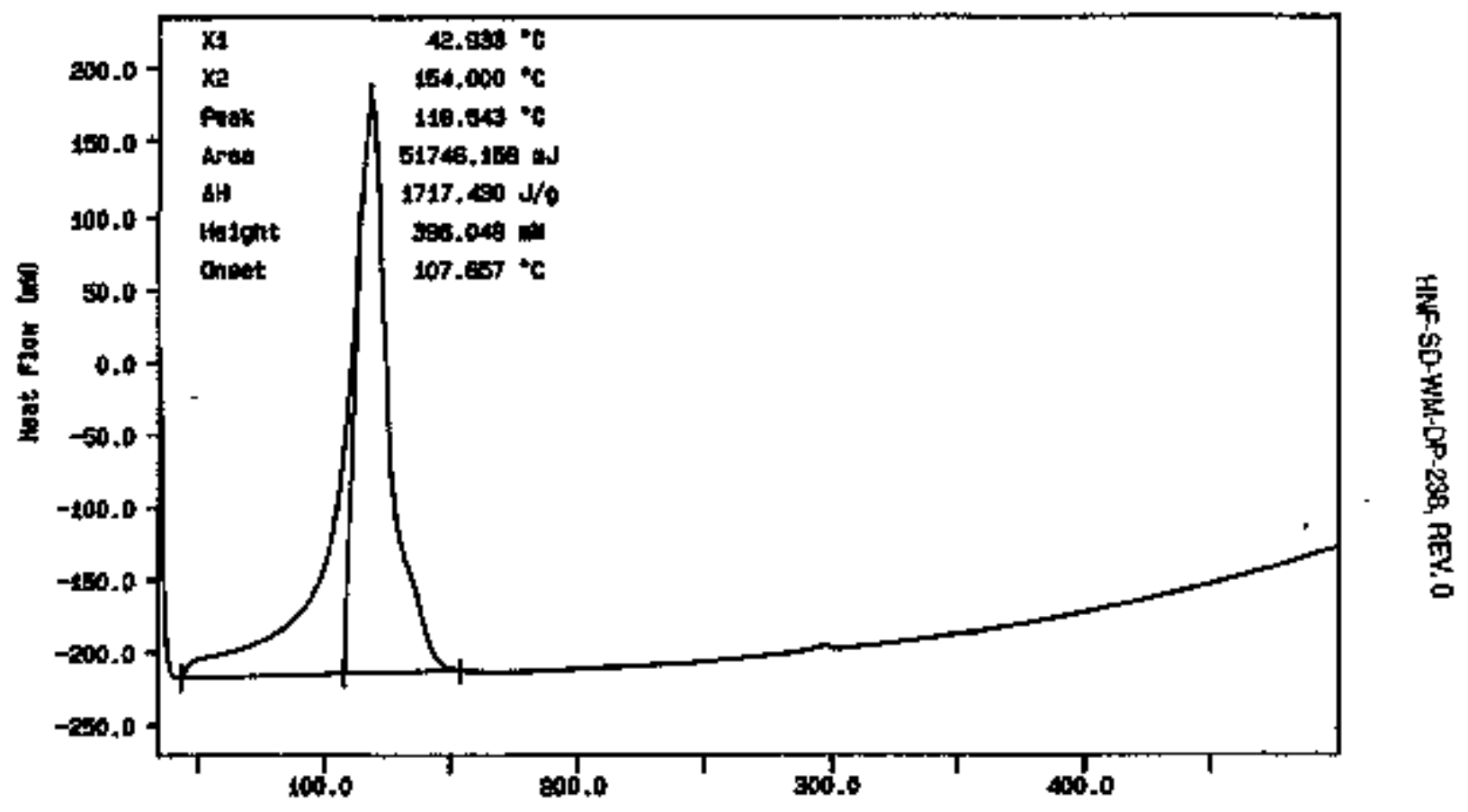

in ipc/ain

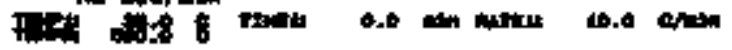

Temoreture (tch 
Curve 1: DSt

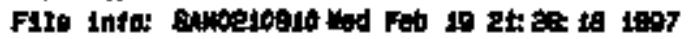

gaple inight: 4.730

Bg7700059 nup

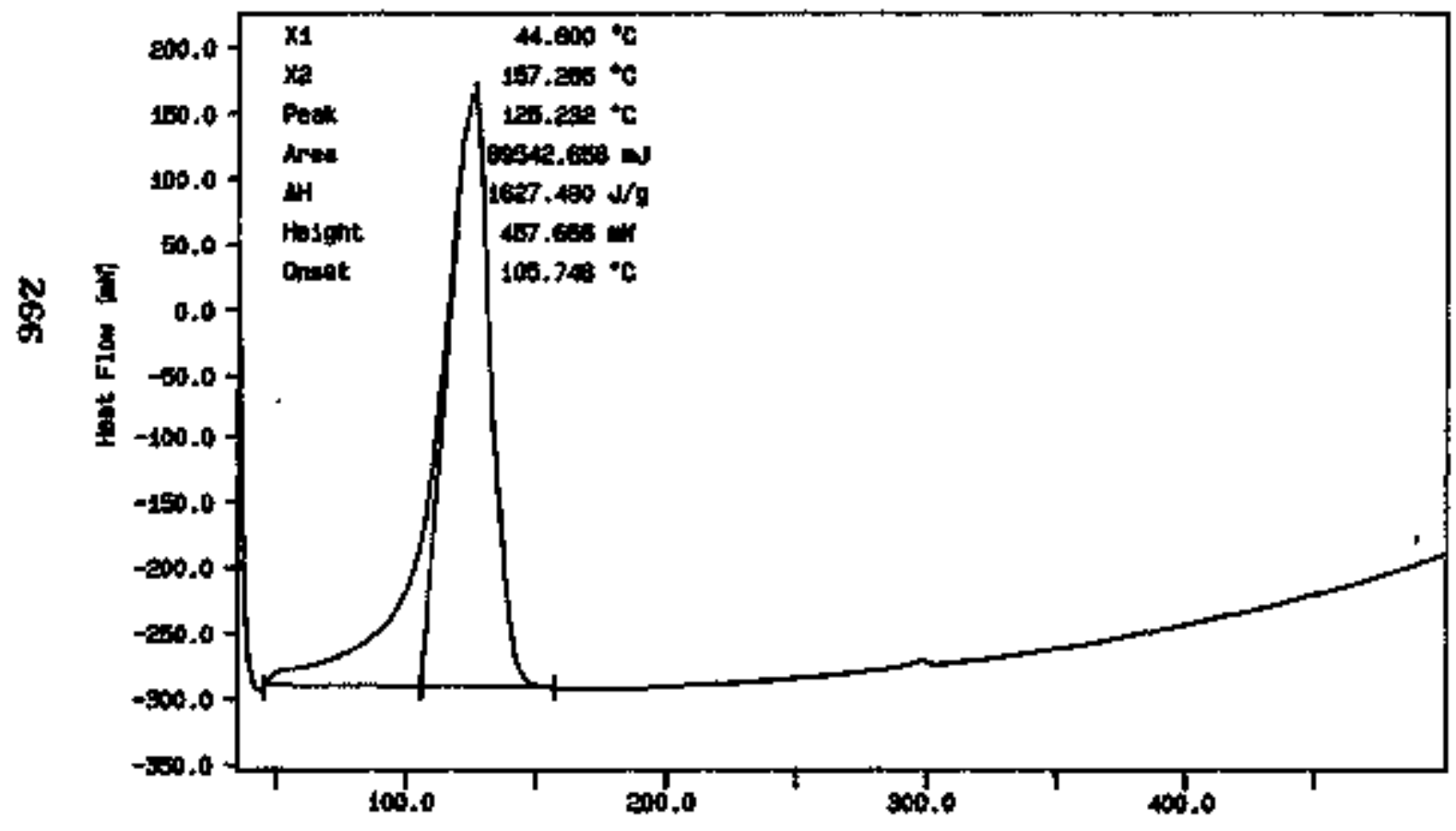

\section{wa 100 inn}

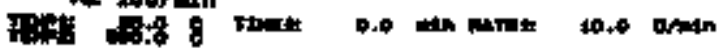

Tumarotare $f^{\circ} \mathrm{t}$ 
Arve if Des

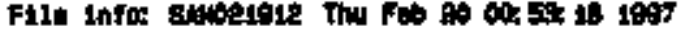

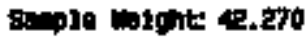

sertotoles sut

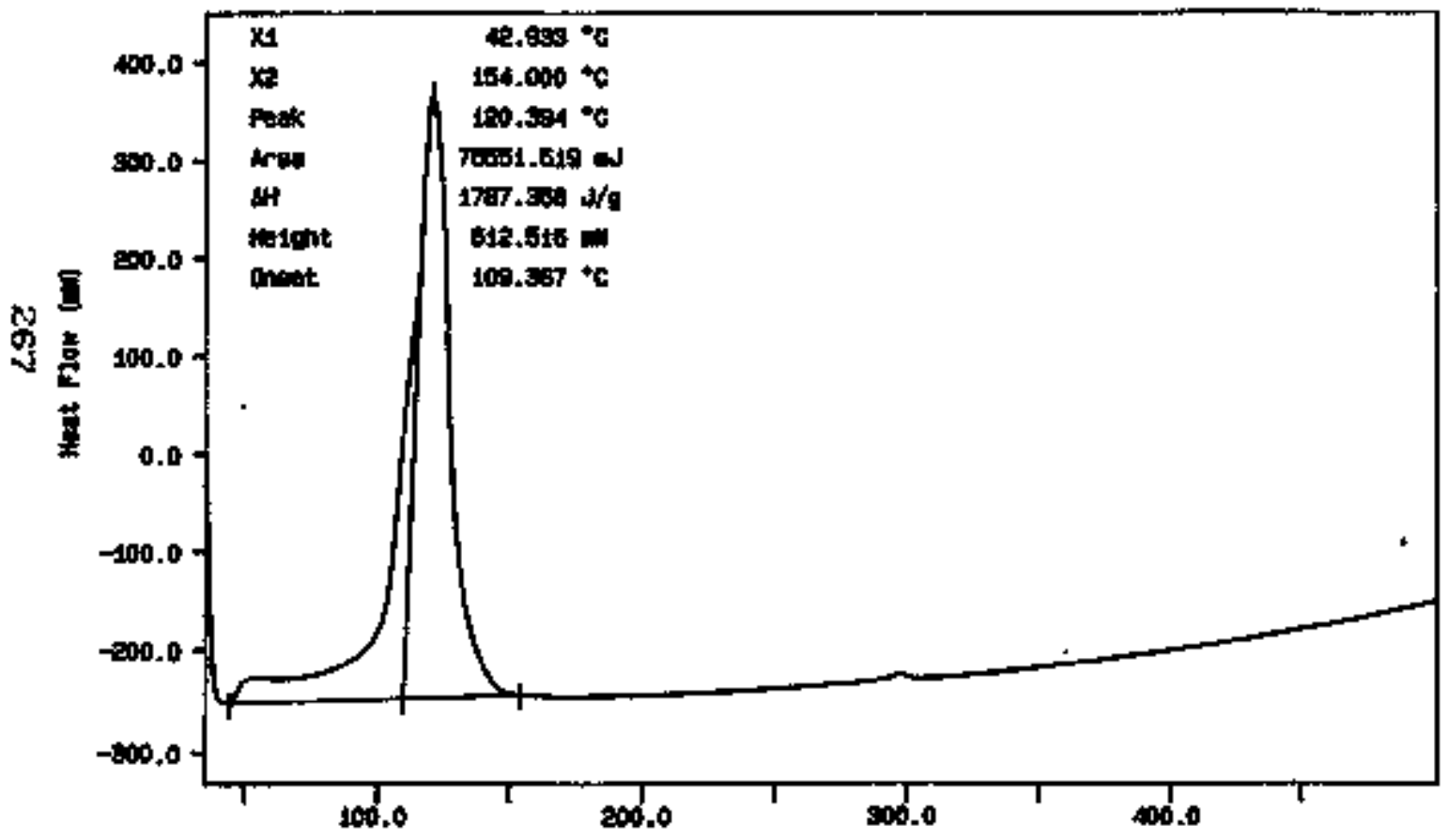

蝶 100/ain

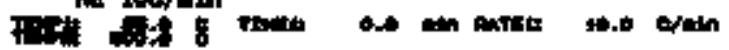

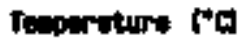

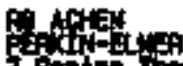

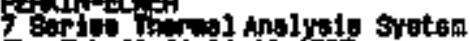

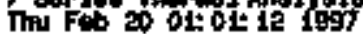


Curve it tosc

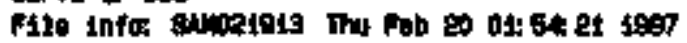

beple wight: 40.690

897700010 UP

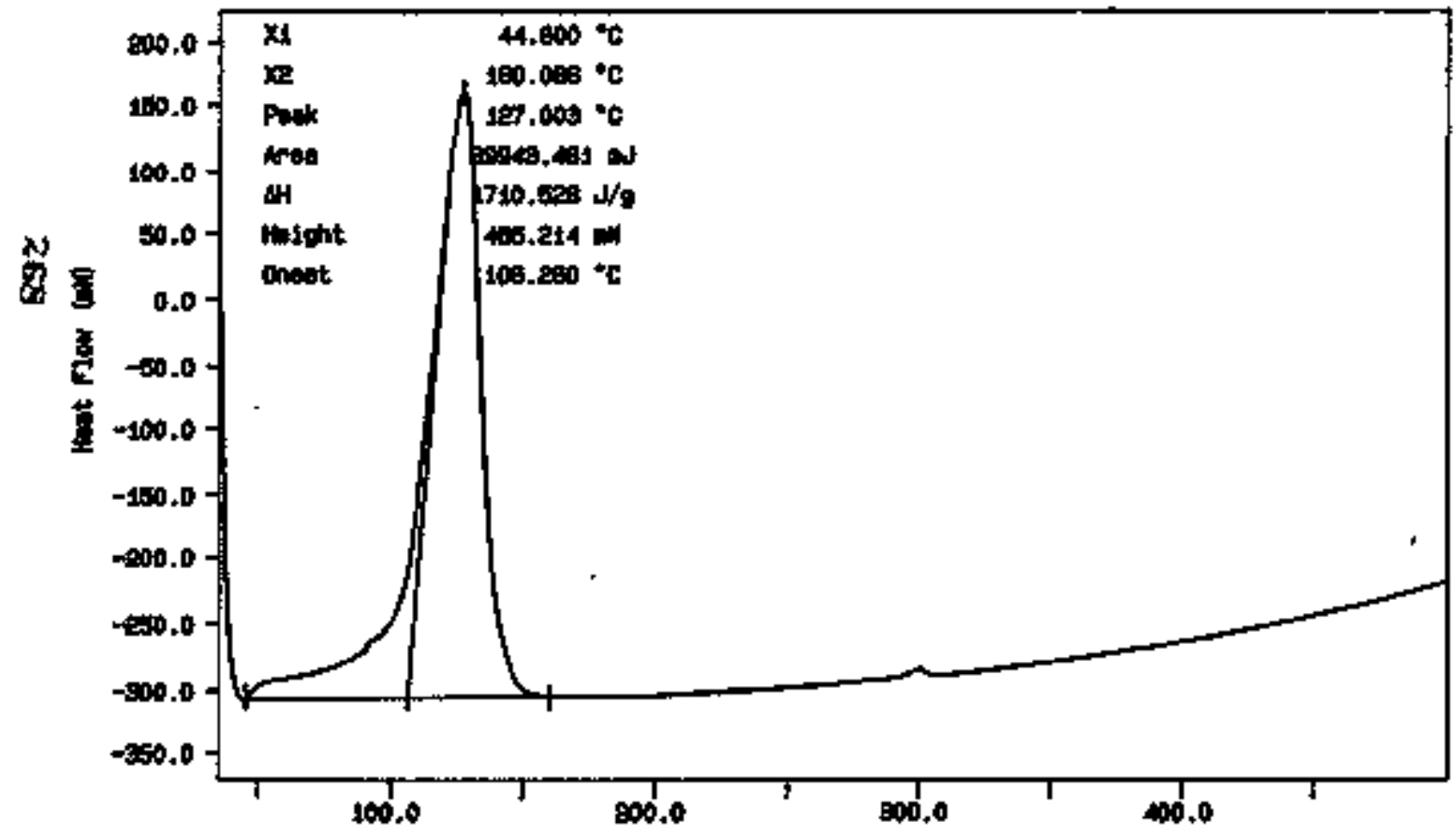

106/ain

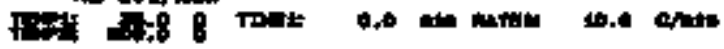

Temparatere (아

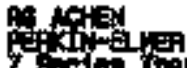

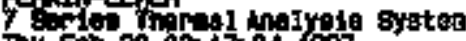

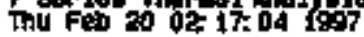




\section{LABCORE Data Entry Template for Worklist\# 16798}

Amalyst: TOS Instrument; DSCO]

Book *

Mrthod: LA-514-113 Rev/Mod

Wocklist Conoment: T-110 dry DSC., bdy

\begin{tabular}{|c|c|c|c|c|c|c|c|c|c|c|}
\hline ERONP & PFon:CT & 5 The & sapusdiln & k & - ATE\$T- - & WHIR [R & IETU冈่ & potren & M & Lإl|T \\
\hline groboths & $T-110$ & 1 3нพr| & क्रTtopotz5 & 0 & एक्ट & $\operatorname{son} 10$ & wth & & & Jalporg Dry \\
\hline 970000Rs & $T=110$ & 2 bue & ş7troovids & b & Dac-位 & BOLIO & & & oth. & soules/9 Dry \\
\hline 9700001 & $T=110$ & 3 swhth & 59r1600161 & D & $0 \$ C-\mathbb{C}$ & 50 & 배 & & & Jouk nota pry \\
\hline 97500085 & $T-110$ & 4 tro & धनाT000141 & D & 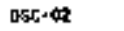 & 30160 & & & Ith & Joul pars bry \\
\hline sroconst & $T=110$ & 5 SNDEE & s971000167 & D & QSE-直 & sert to & & & & Joudear's Dry \\
\hline procoudes & $T-110$ & \& DUP & $59 \pi 1000142$ & 1 & DSc-Q? & $\sin 10$ & & & WKA & Joulst fo pry \\
\hline proocoses & $T-110$ & 7 ENTE & sotropol 43 & 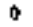 & $0 \div 5 \cdot 62$ & 品占 & & & & Joulays'p pry \\
\hline 93200063 & 1.110 & \& DEP & Bstroou163 & 0 & asc-ed & scoud & & & ./A & Jouley to try \\
\hline stouotis & $x=110$ & 9 saldeE & $59 \mathrm{MPOOH}+4$ & 0 & DSE- $D$ & $50 \mathrm{~d} 10$ & & & & Joultes gr or \\
\hline 9roocoes & $r-110$ & VD DEP & $\operatorname{son} T 0 \phi 16 t$ & 0 & $0 \neq c \cdot a 3$ & $\operatorname{san} 16$ & & & Noth & Joulloty Dry \\
\hline परफ006s & T.11t & 11 sUPLE & B9fтtodo145 & 0 & DBC.02 & $\operatorname{soc} 10$ & & & & Jodlet's bry \\
\hline 97000003 & $y=110$ & 12 bo & 9971000145 & D & agc-ce & $\sin 10$ & & & $\mathrm{M} / \mathrm{A}$ & Joulextg orr \\
\hline pracocose & $r-110$ & 13 ENPLE & s97nodo146 & 0 & $0 ; c \cdot 4$ & $\operatorname{sat} 1 \mathrm{t}$ & & & & Joull 4 to bry \\
\hline T7000063 & T-110 & 16 oup & Bg.7000146 & $\mathbf{0}$ & DBC- 02 & sot.jo & & & $m_{A}$ & doulg \\
\hline protitus & $x \cdot 110$ & 15 巨مبнt| & $597101016 ?$ & 0 & $D \$ C \cdot \mathbb{Q}$ & 10 & U & & & Joul 4 W' Dry \\
\hline 9xococos & T-310 & 16 DUP & 397T000147 & 0 & $0 B C-0$ & $\cos 10$ & & & mith & Jowlestr $\mathrm{Dr}$ \\
\hline प7woposs & 1.110 & 17 ث4:LLE & s97700015s & 0 & $\Delta s c-02$ & 50210 & 느 & & & doulate Dry \\
\hline 9700003 & $t=110$ & 16 ow & $\$ W 0015$ & 0 & DSC-ם & 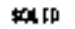 & & & Wra & Jouk bata Gry \\
\hline
\end{tabular}

Dut Evry Comporsts:

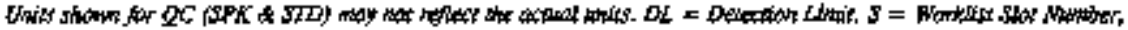

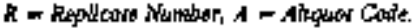




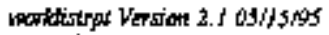

HNF-SD-WH-OP-23B, GEV. 0

$02 / 27 / 47 / 14,44$

LABCORE Data Entry Tenplate for Worklist/ 16798

\begin{tabular}{|c|c|c|c|c|c|c|c|c|c|c|}
\hline कीa & PROACKT & TIPE & S4P E E & & 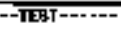 & MATEIX & fRTLIIL & polat & bi & int? \\
\hline stopoulas & $\tau-110$ & 19 в닫 & 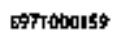 & 0 & $\cos x-b e$ & 200.10 & with & $(1)$ & & sculeters Dry \\
\hline 9700000 & T-1T0 & Do p pos & SFro:al59 & 0 & $\phi c t-d z$ & $=0610$ & th & $A$ & & Joukeso bry \\
\hline
\end{tabular}

\title{
Final page for worklist \# 16798
}

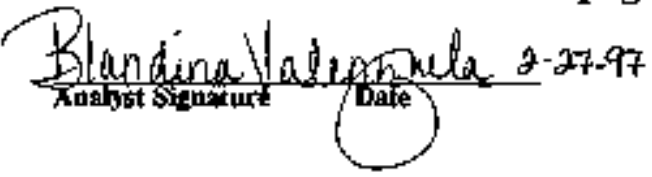

\author{
Antys Scatare Date
}

Date Enry Canments:

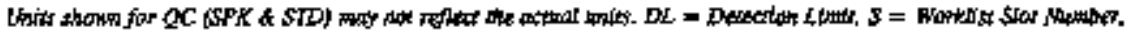

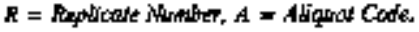


HNF-SD-WMFDP-238, REV. 0

\section{T-110 dM DSC \\ CALCINLTED ORY OSC.}

\begin{tabular}{|c|c|c|c|}
\hline SAMPLE, Hr. & DSC RESNLT $(\mathrm{v} / r)$ & TEh RESULT ( $\mathbf{A}$ mater) & ORY DSC RESULT \\
\hline 5977000125 & 18 & & \\
\hline 1250 & 6 & & \\
\hline 141 & $\Leftrightarrow$ & & \\
\hline 1410 & of & & \\
\hline$\mu \mathrm{a}$ & (1) & & \\
\hline 1420 & $\phi$ & & \\
\hline 143 & $\phi$ & & \\
\hline 1430 & 6 & & \\
\hline |4 4 & 6 & & \\
\hline$M 4 D$ & is & & \\
\hline 145 & $\phi$ & & \\
\hline 1450 & 0 & & \\
\hline 14 & 6 & & \\
\hline 1460 & 0 & & \\
\hline 幽标 & $y$ & & \\
\hline$M F D$ & 4 & & \\
\hline $15 \%$ & (f) & & \\
\hline $158 D$ & (b) & & \\
\hline & & & \\
\hline & & & \\
\hline & & & \\
\hline & & & \\
\hline
\end{tabular}




\section{LABCORE Data Entry Template for Worklist\# 16825}

Page: I

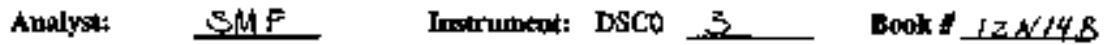

Method: LA-SL-114 ReviMod $I 2-O$

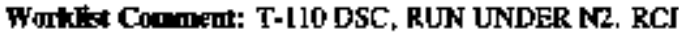

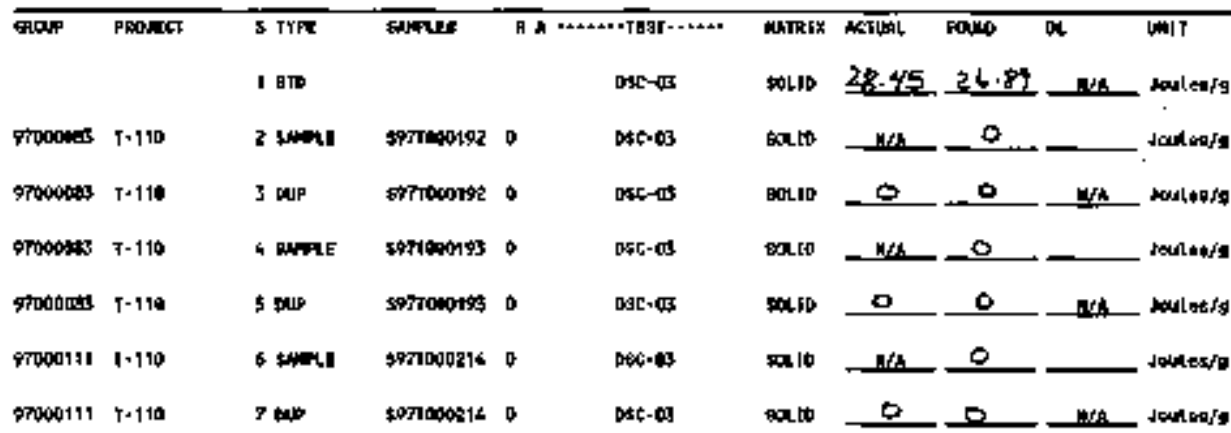

\section{Final page for worklist \# 16825}

See Attached for Signature Ans:yst signisture Da falidatud (3)tachetor $3 / 5 / 77$

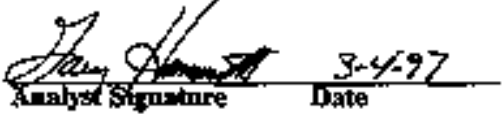

Dater Edry Coxhmints:

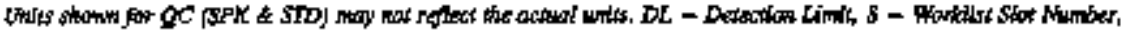

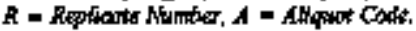




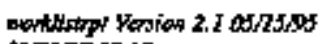

HNF.SD-WH-DP-238, REV. 0

Pror 1

A2RASPI IS, 15

LABCORE Data Entry Template for Worklist\# 16825

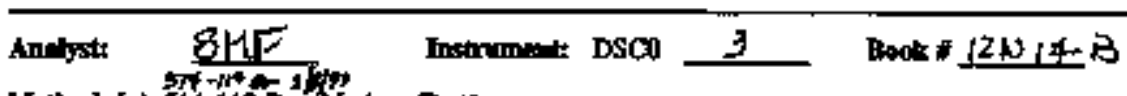

Mechod: LA

Works Connet T-1 10 DSC, RUN UHDER N2, RCF

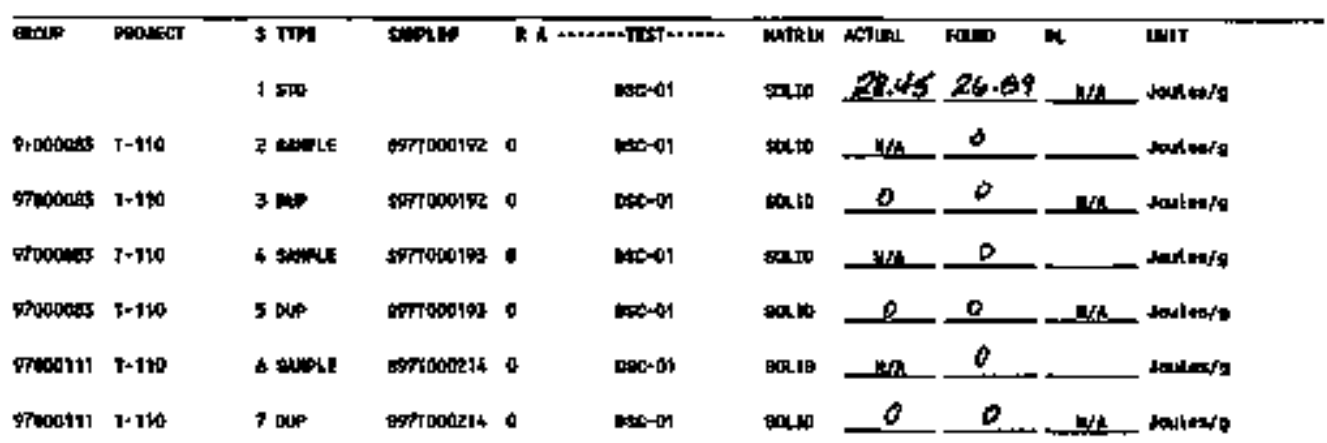

Final page Ror wortalist \# 16825

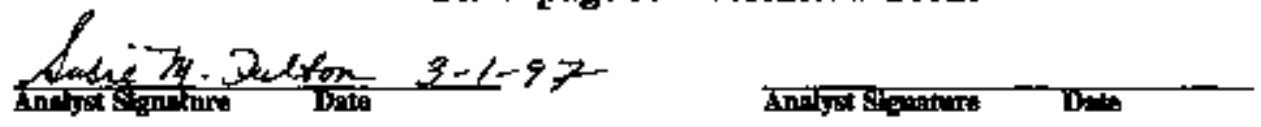

Faxes Both Dso And TGA to 3B

16 pages total

a) $0640 \quad 3-2.47$

505

A- Paxted on the

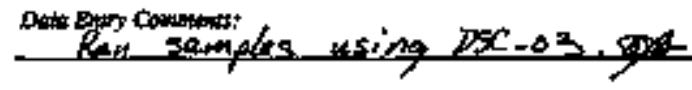

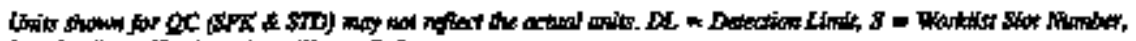

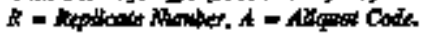

273 
Aorve is DSC

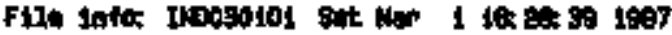

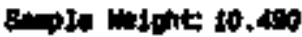

잉

BTt 1EN14-8

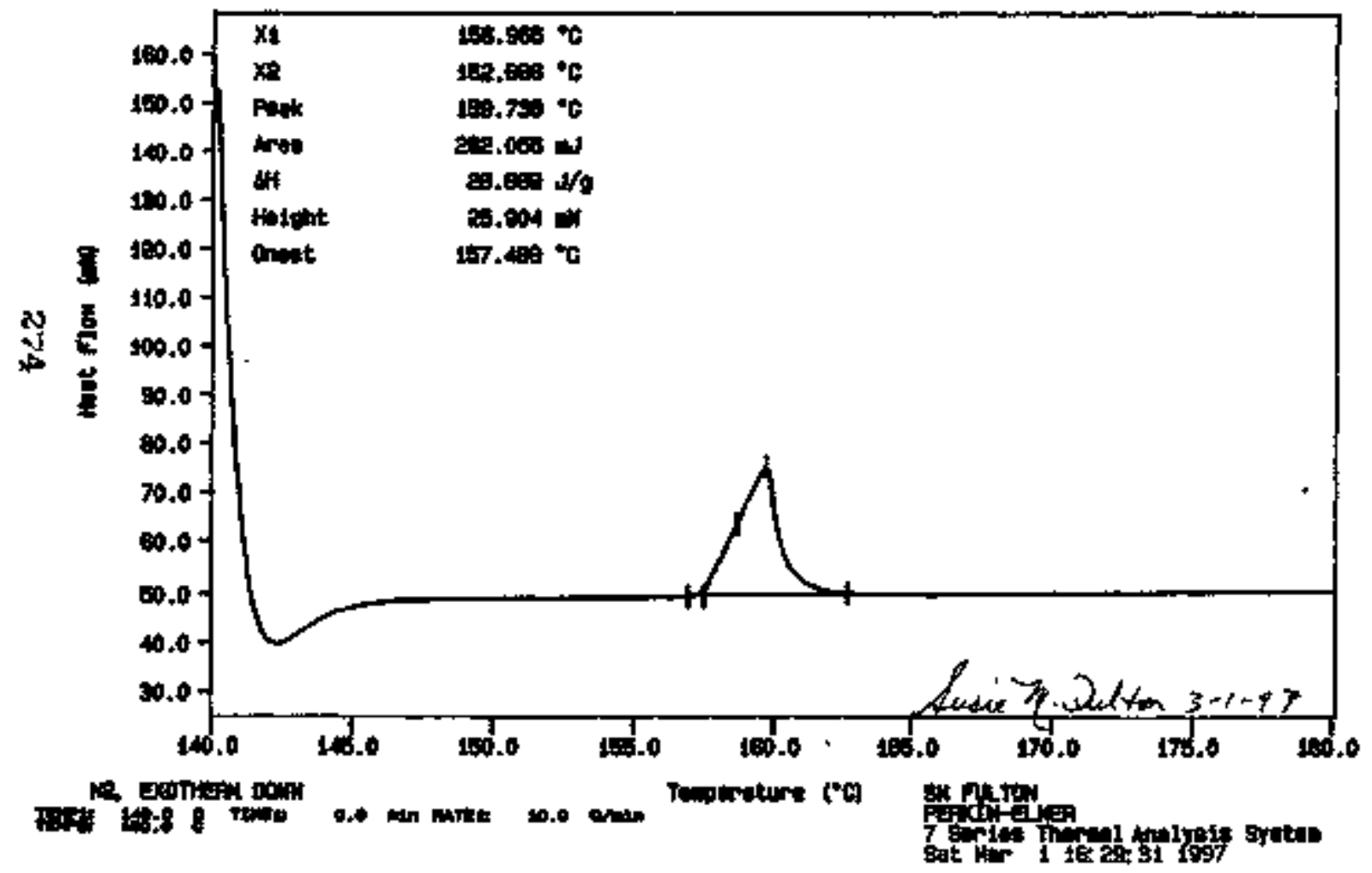

贾 
Dirit it toc

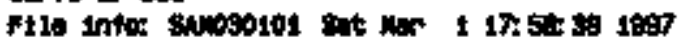

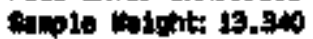

sortoosin?

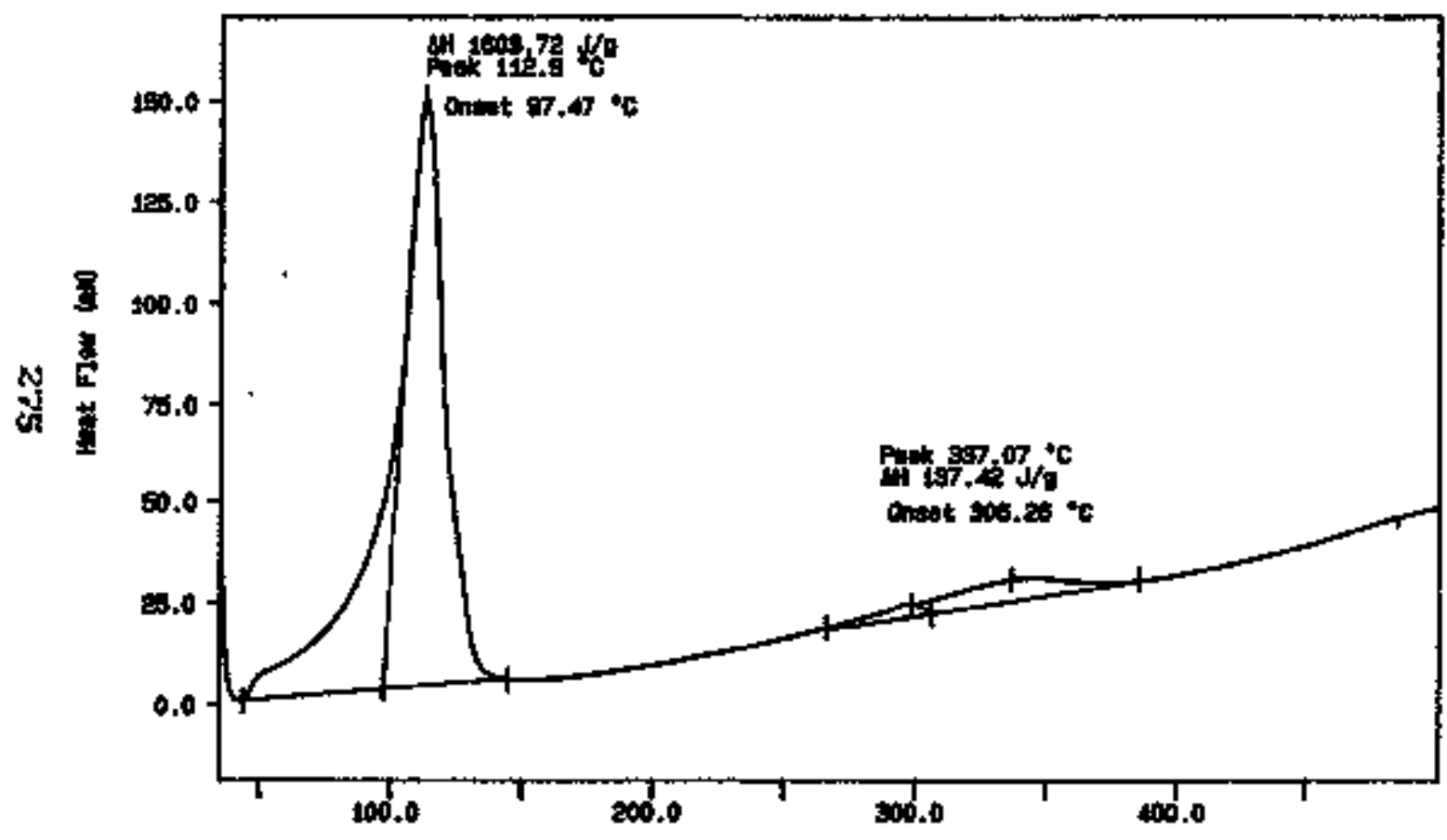

va sechoin

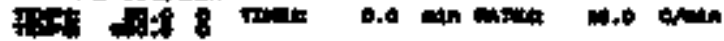

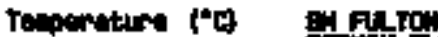

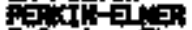

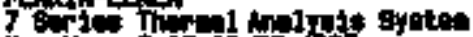

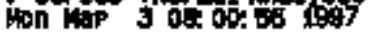


tonve it 000

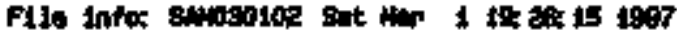

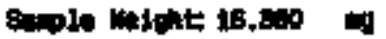

serToowse do

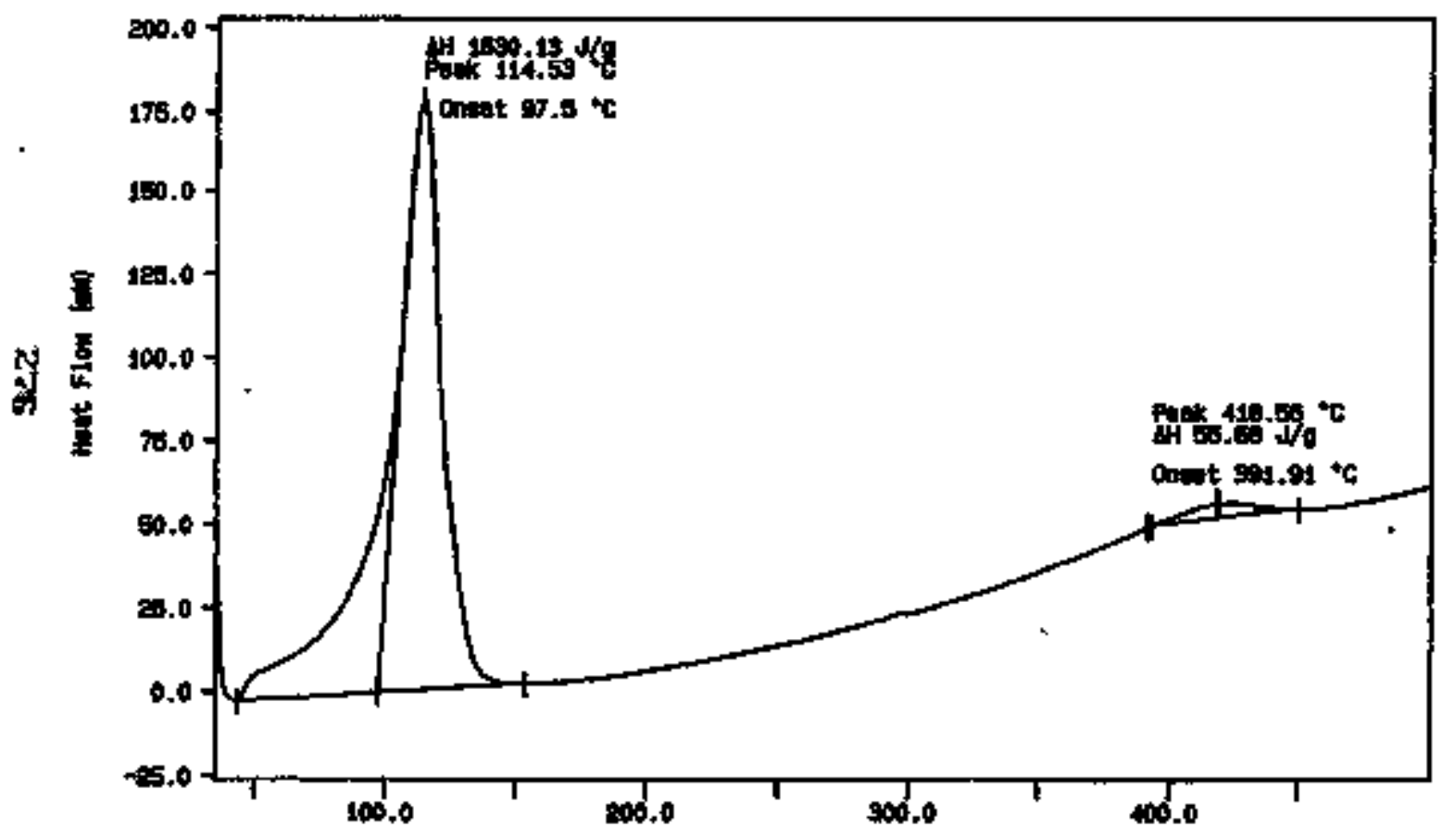

in 100/in

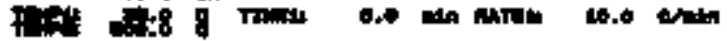

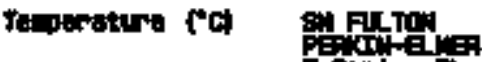

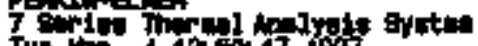

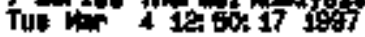


Punve is Dac

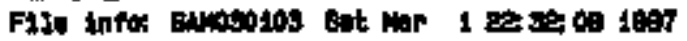

8uple mights t9.20.

8:HTooutge

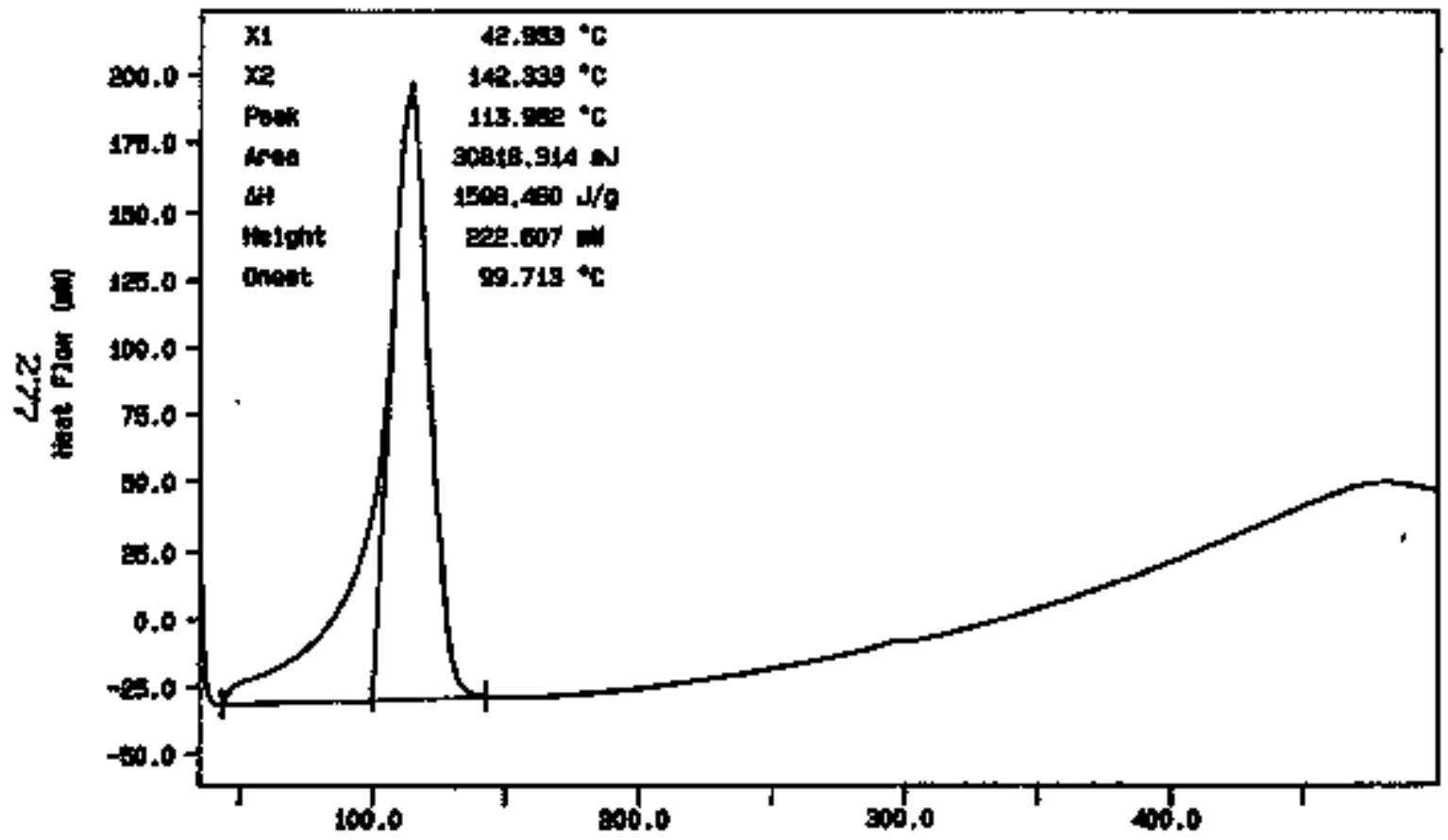

No wackin

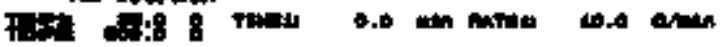

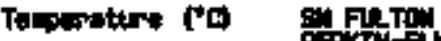




\section{Cumpe to Dec}

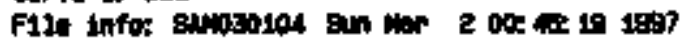

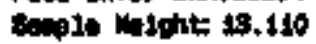

entroptos Dur

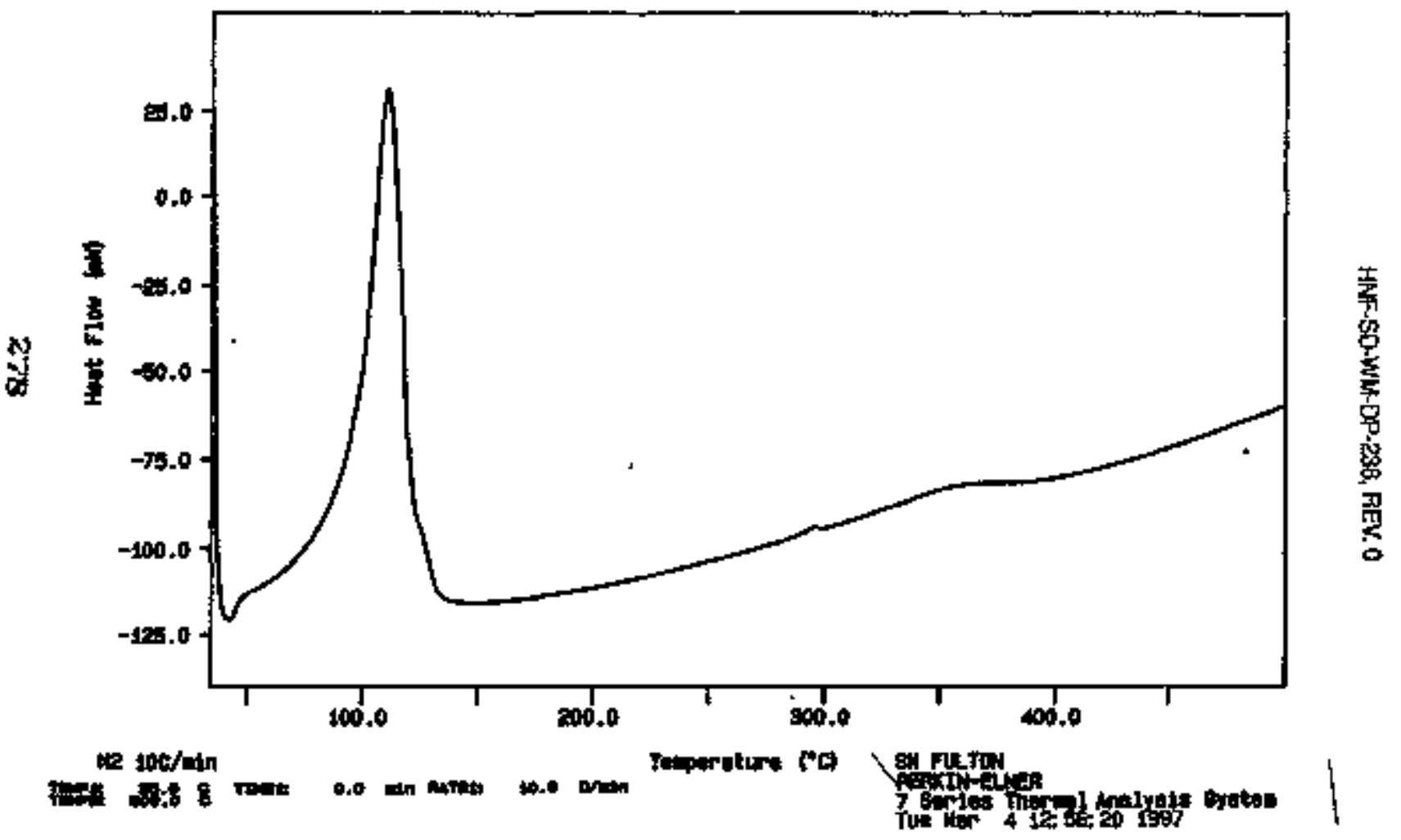




\section{Curva t: Dos}

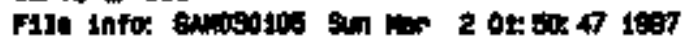

somple wipht: 10, dep

expoust4

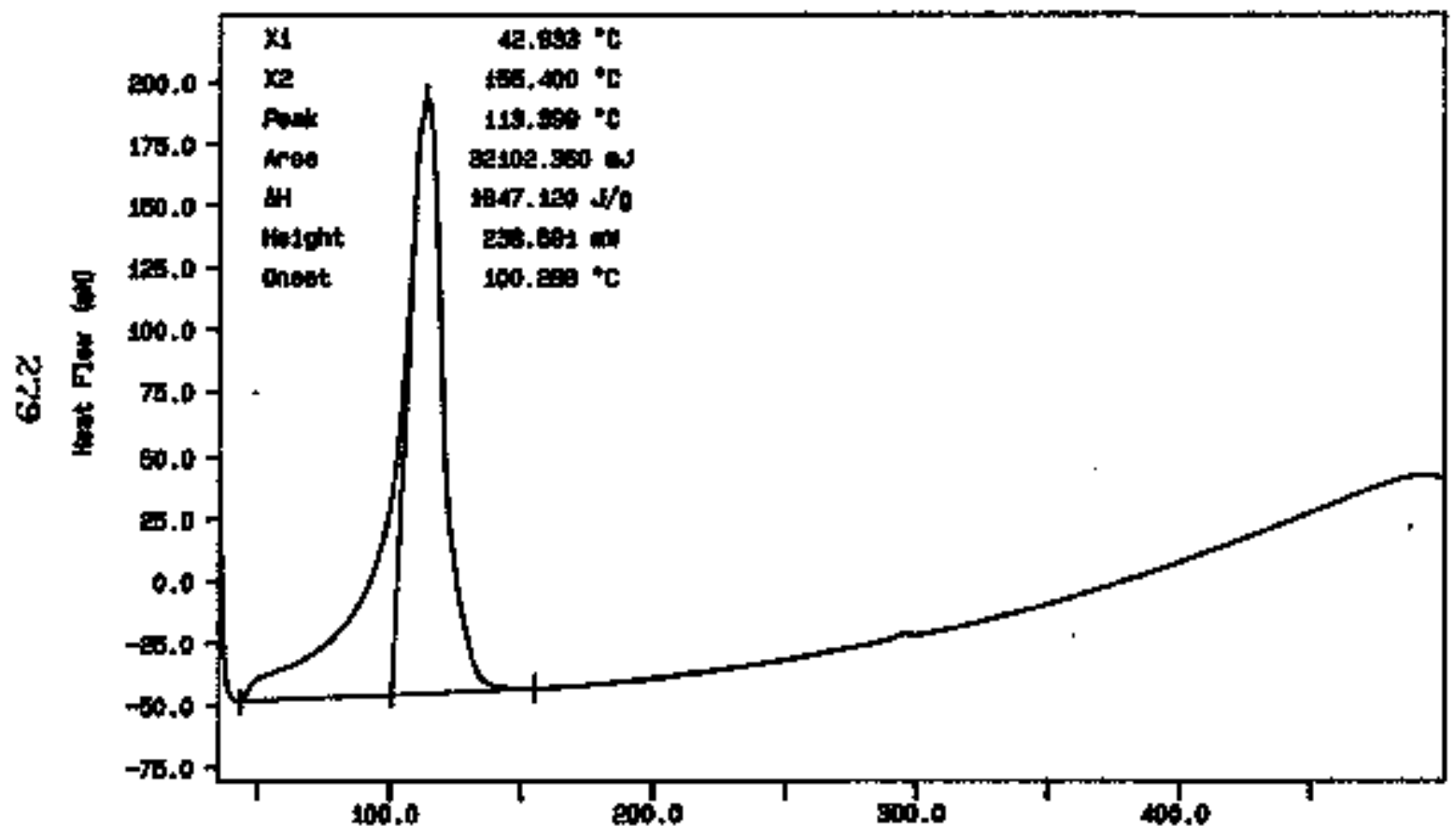

$10100 /$ ain

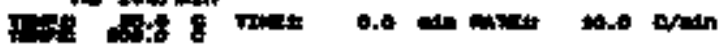

Tenourvarn ('ch

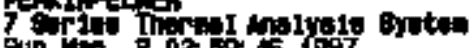

sun la ed ot 


\section{Drve to to:}

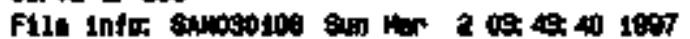

Bmple wint; 49.96

Erpropoest oup

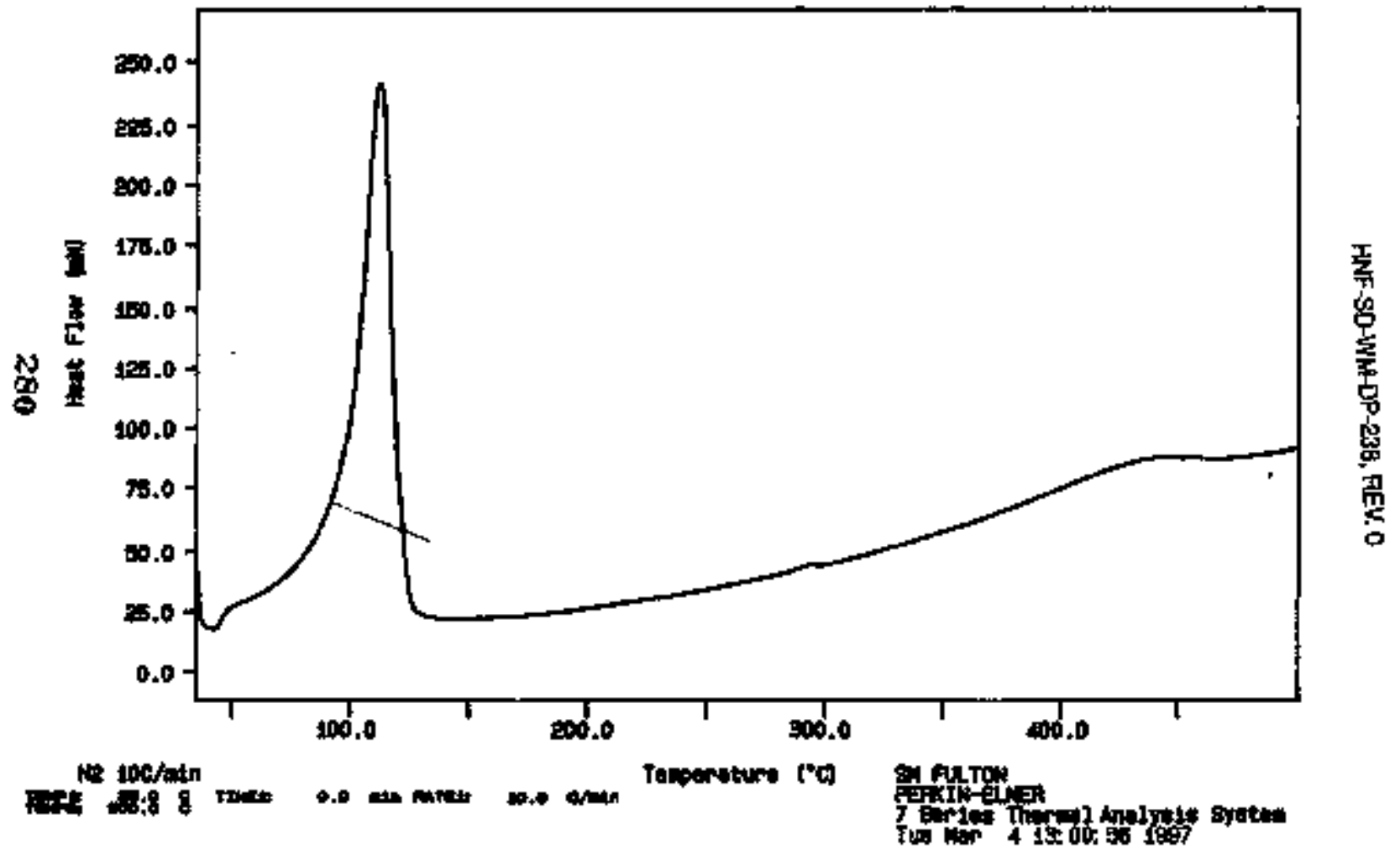


Writs

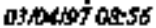

\section{LABCORE Data Entry Template for Worklist\# 16826}

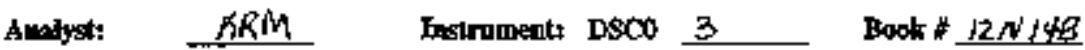

Method: LA-514-114 RewAMd _D-D

HNF-SD-WU-DP-298, REV. 0

Forkllat Comnent: T-110 DSC, kUN UNDER N2. RCI

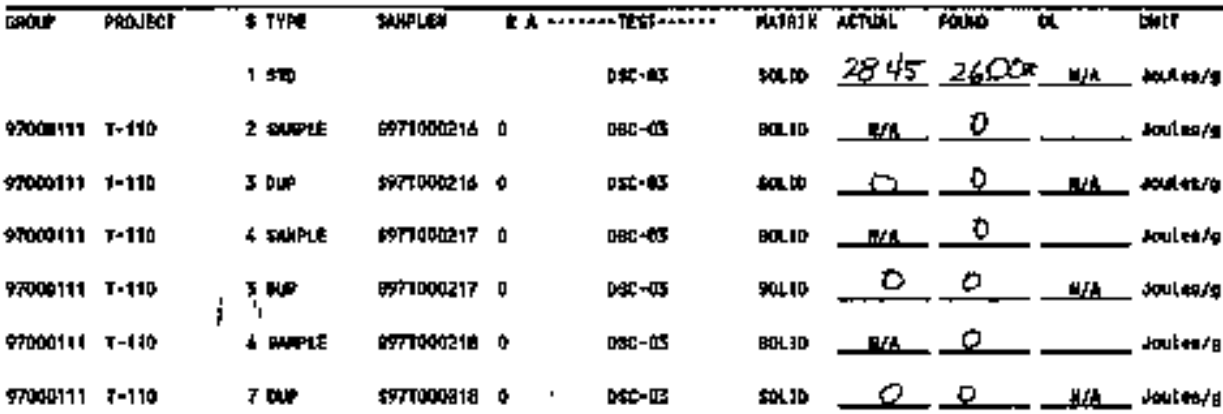

\section{Final page for worklist \# 16826}

See Alachad for Signatixe

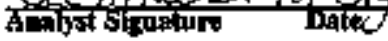

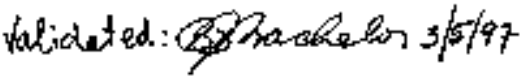

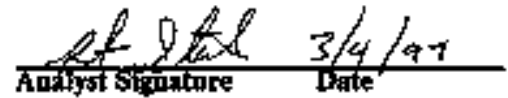

Dem Enty Comants:

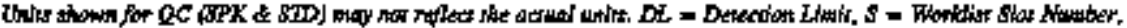

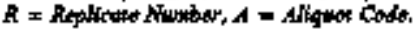




\section{LABCORE Data Entry Template for Worklist 16826}

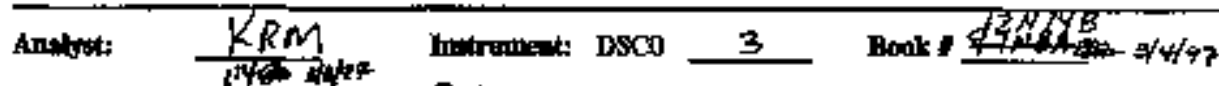
Mintbodi LA-514-iYg Reviod D-b

Forkilet Cimmatit T-110 DSC, RUN UNDER N2. RC

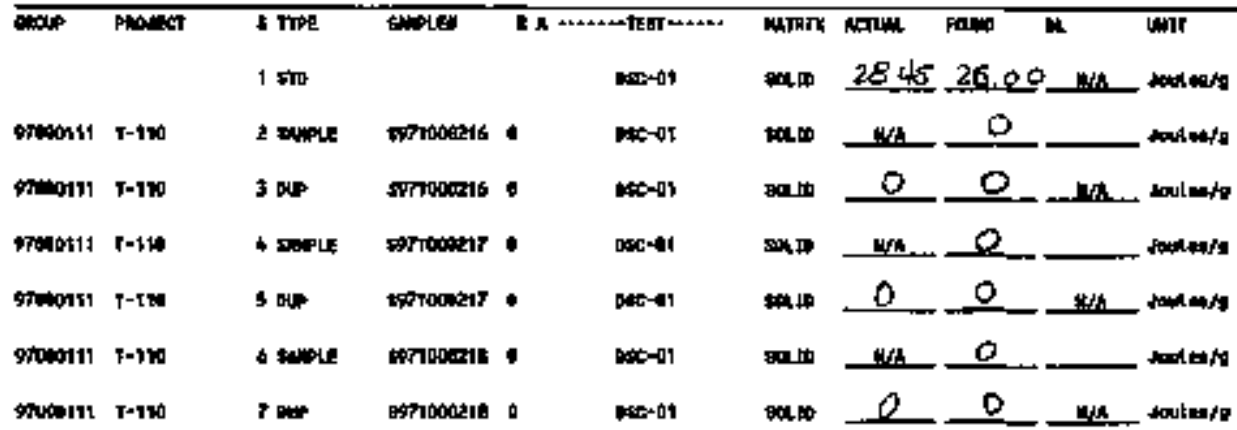

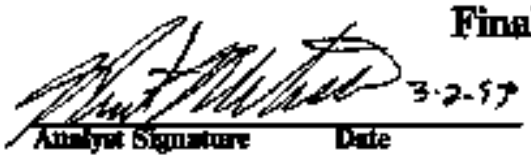

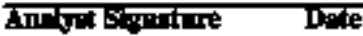

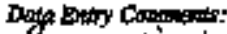

fun

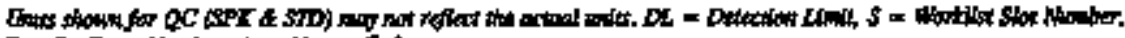

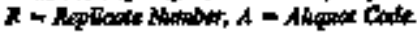


HWF-SD-WH-DP-238, REV. O

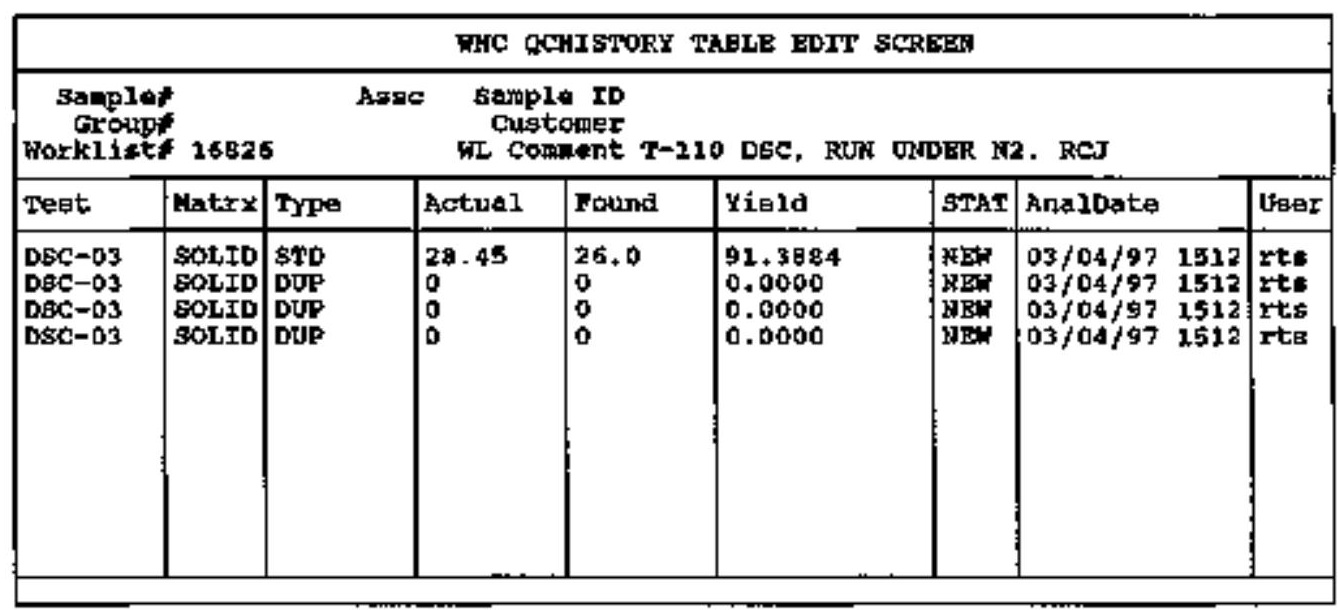

Saý(F12) EnA(F3) 
HNF-SD-Wh-DP-2SB, REV. 0

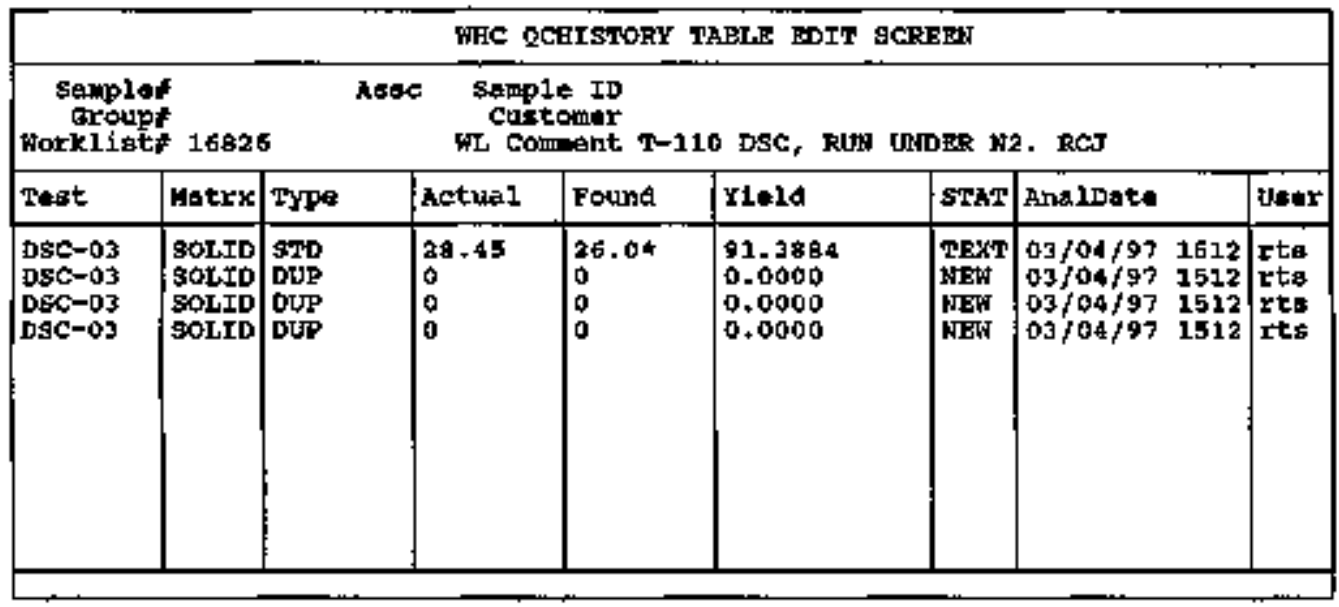

Gave (F12) End (F3) 
arve it ner

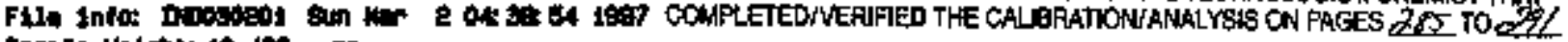
8tople Wotght: 10.490 ing

810 IEnt4-B

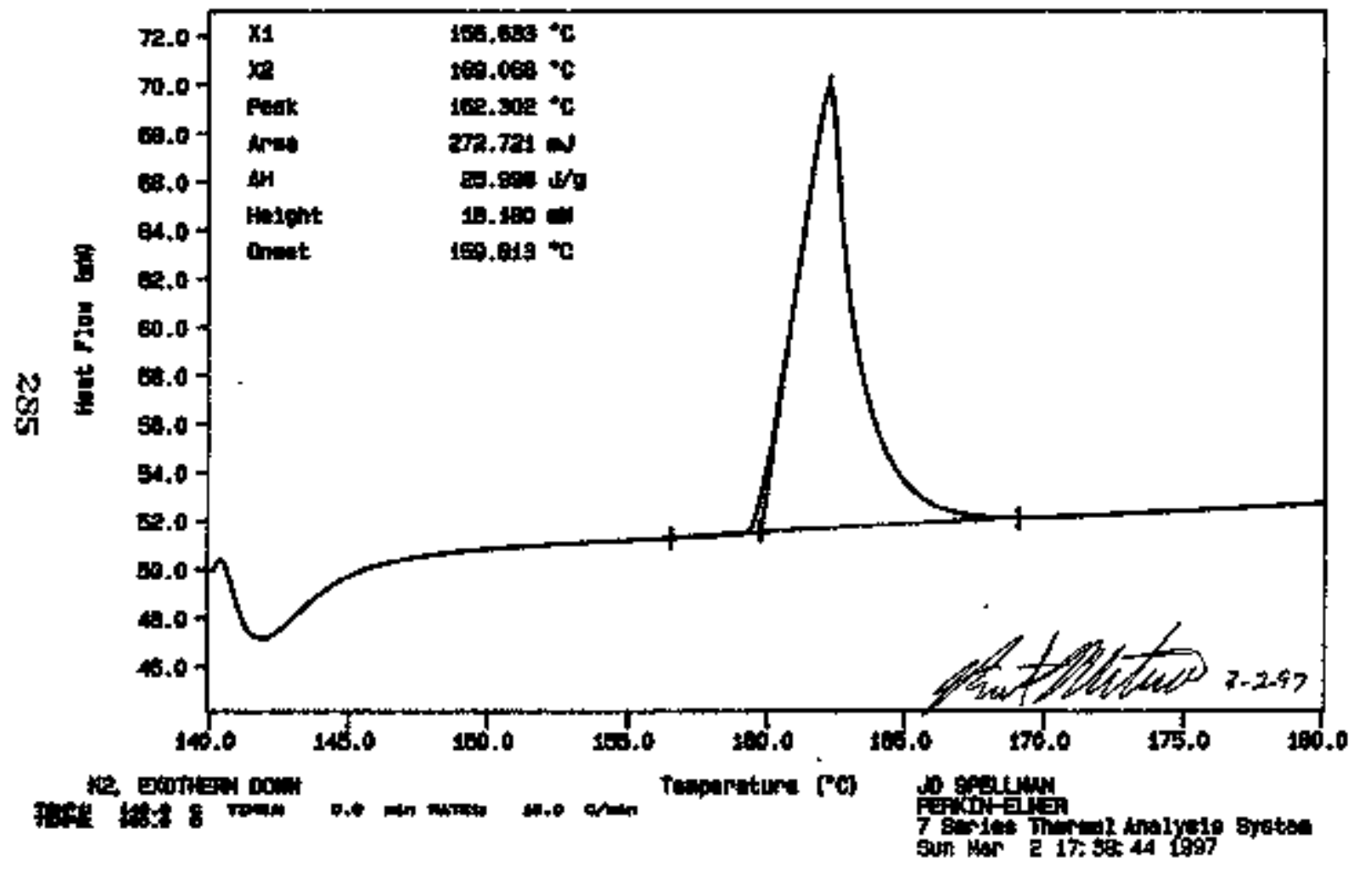

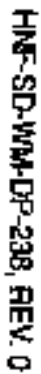


furite it DE:

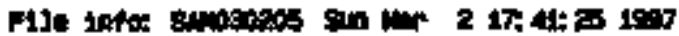

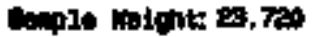

EPTTOARAB

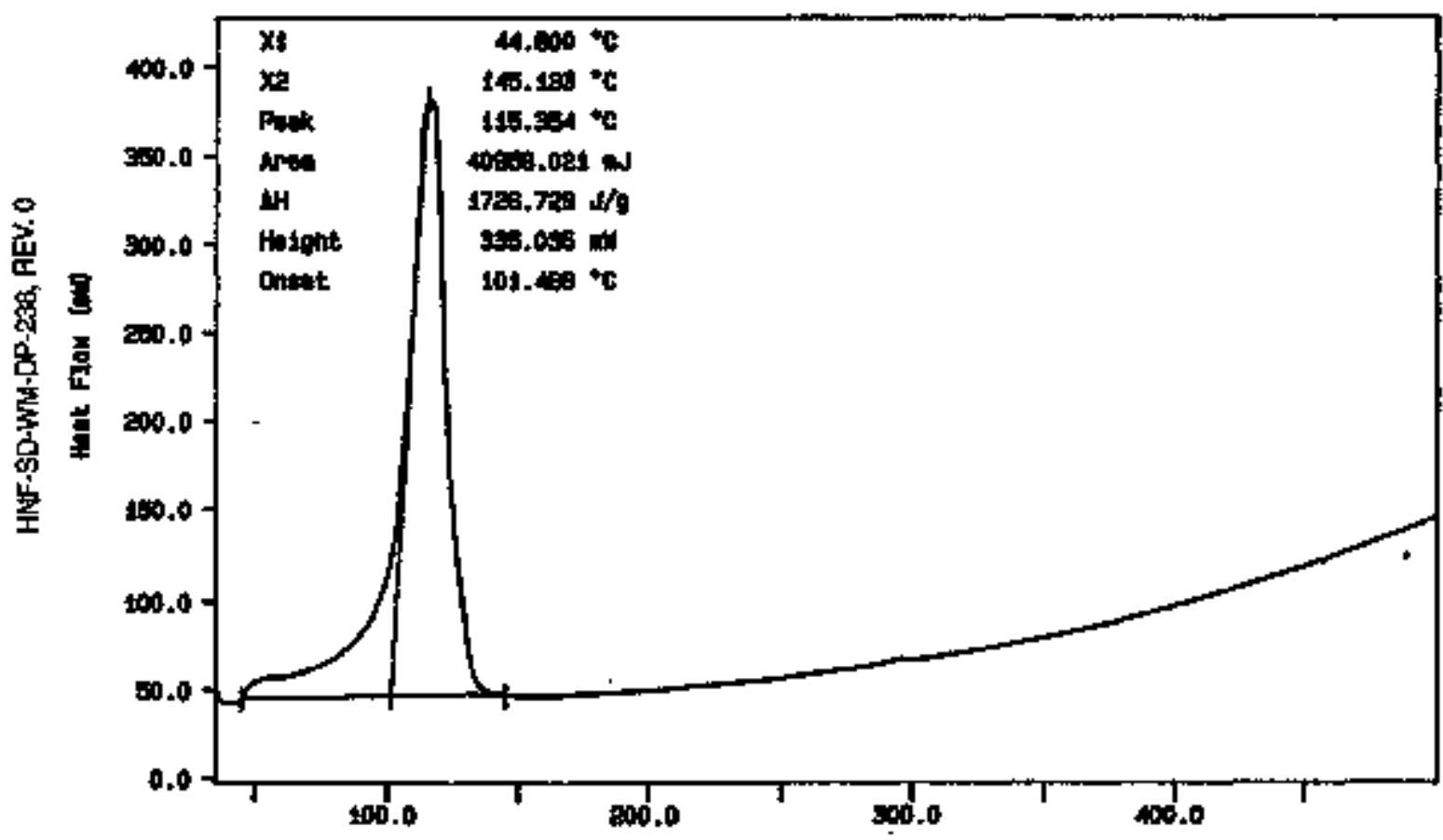

10 100/min

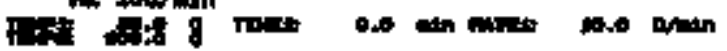

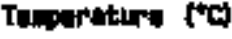


Durre it Dex

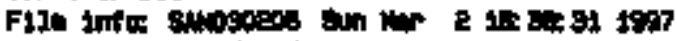

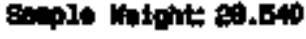

897Twoes: DVP

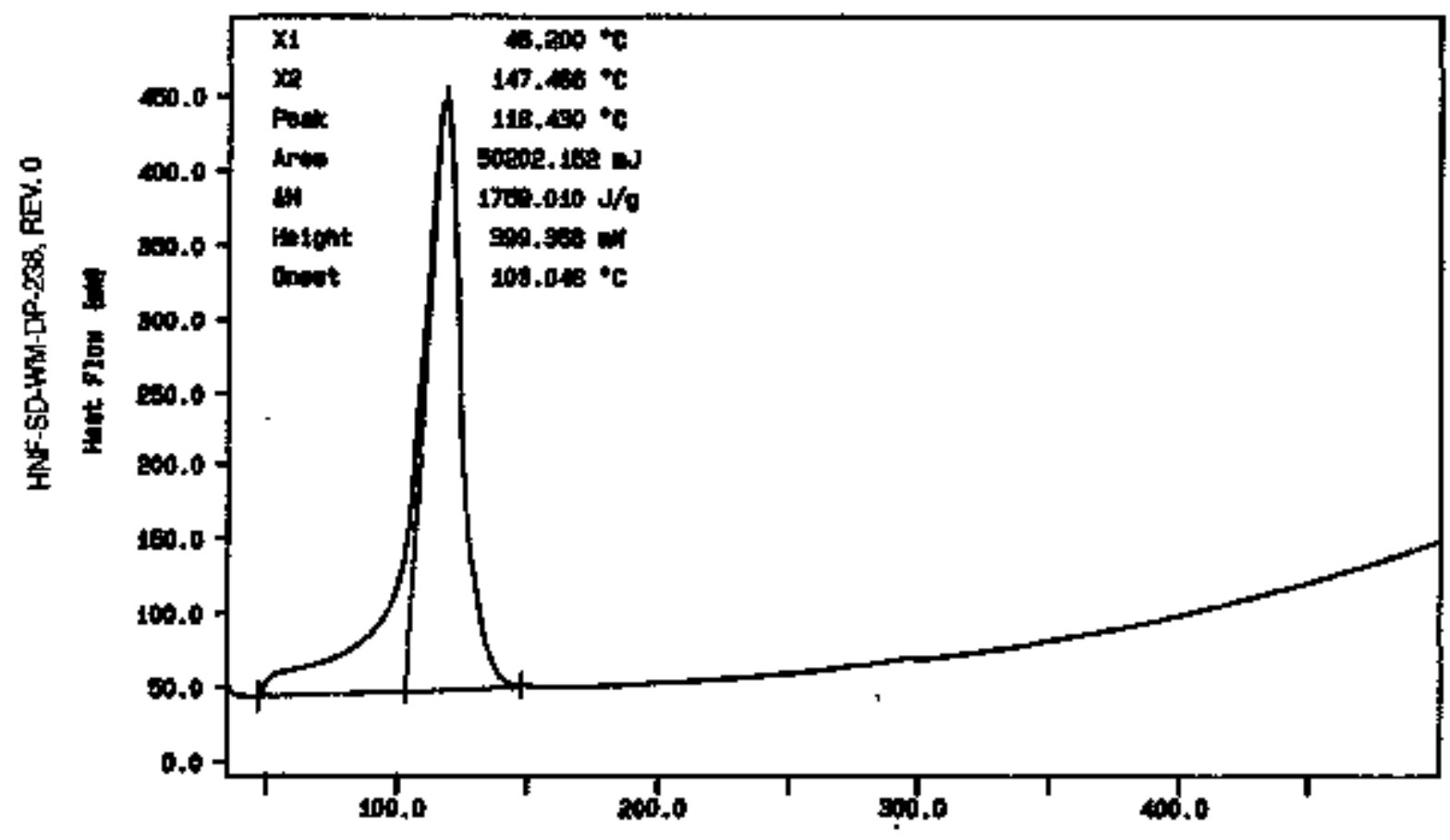

102 100/adn

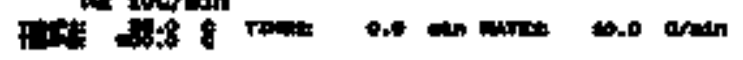

Touprotere t*o

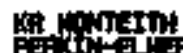

Fistinis

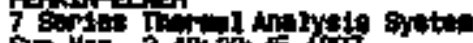

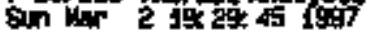


Darve A: gec

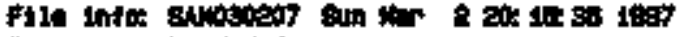

Bapla Wolphte to

entoverit

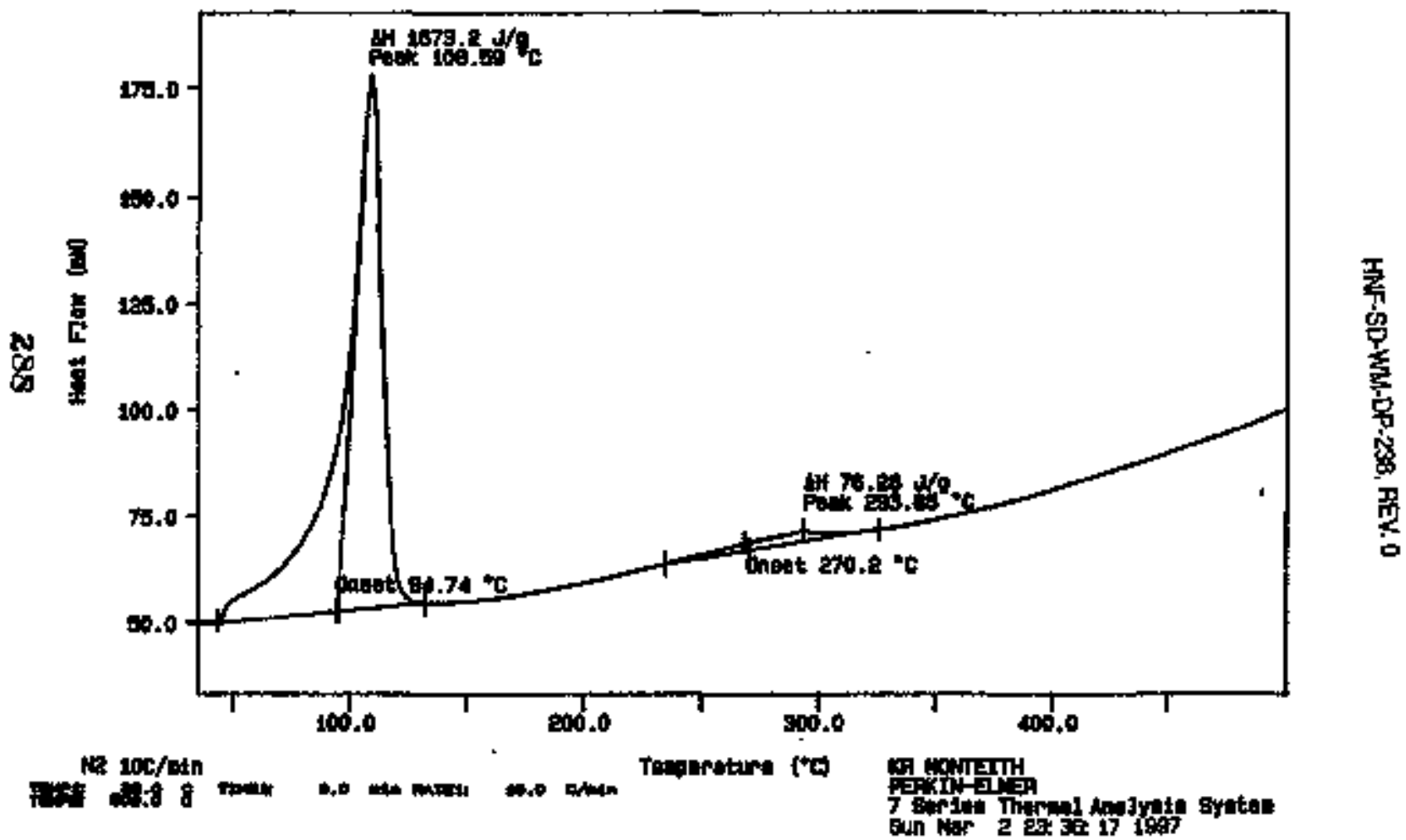


Cump is Dst

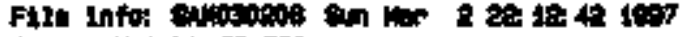

geple wisht: sa.

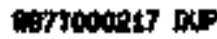

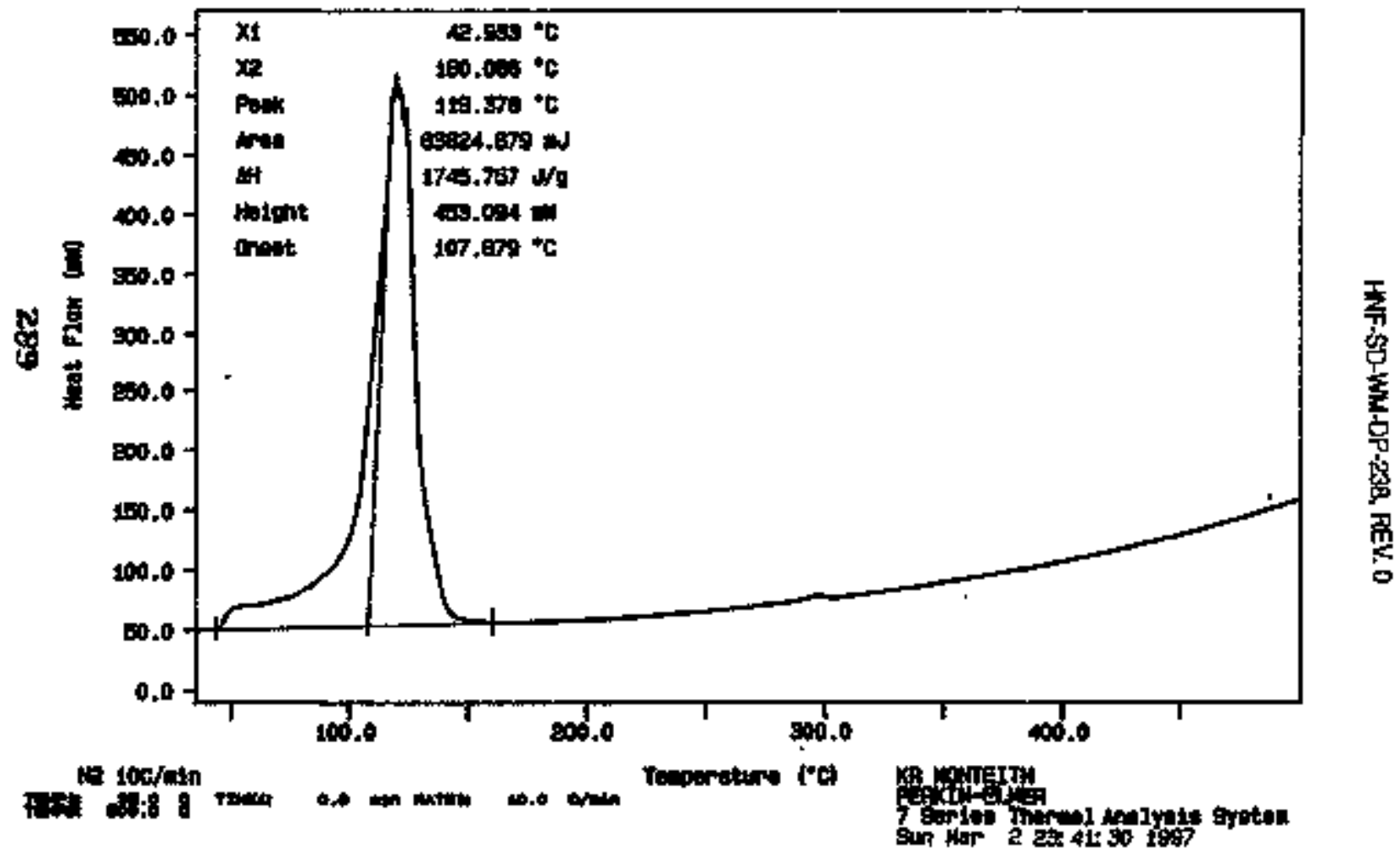




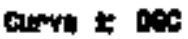

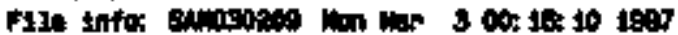

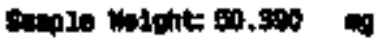

serTutats

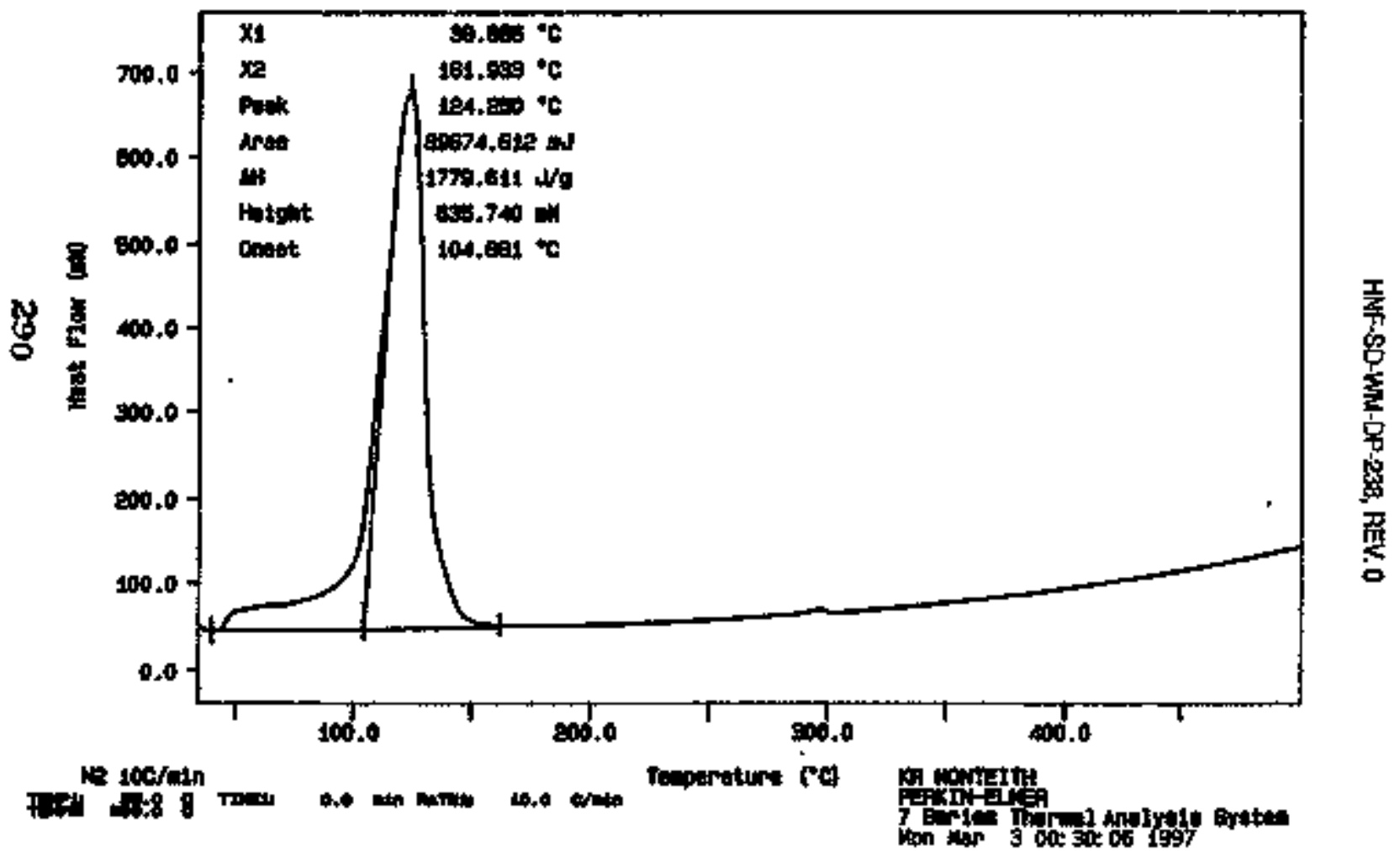


Cum 1t Dec

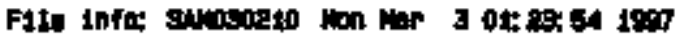

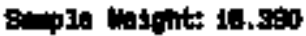

sortopoese ar

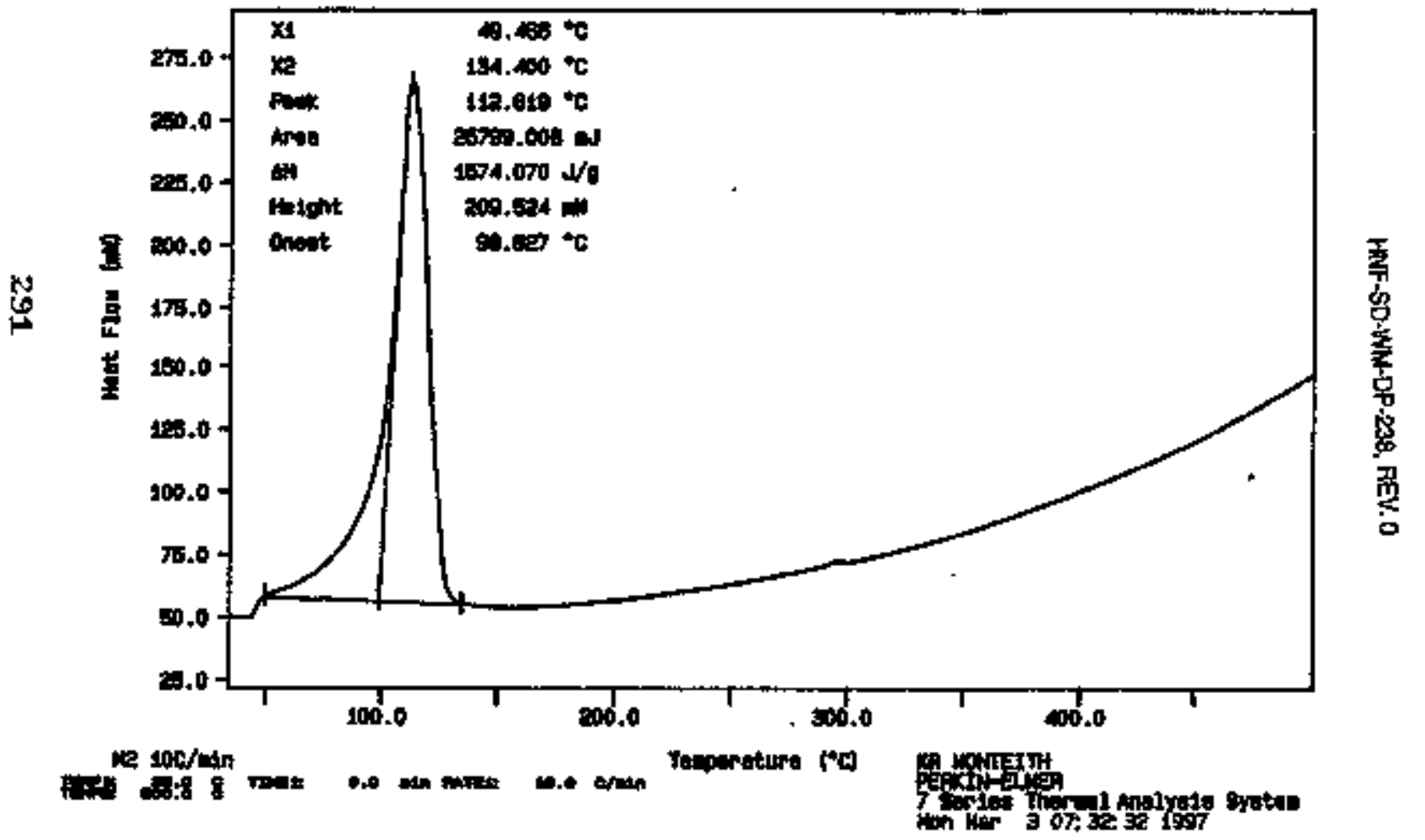




\section{LABCORE Data Entry Template for Worklist\# 16827}

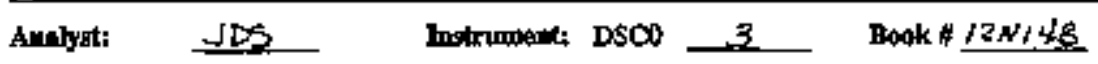

Metiod: LA-514-114 Rew/Mod D-O

Workllst Con ent: T-I10 DSSC, RUNN UNDERR N2. RCI

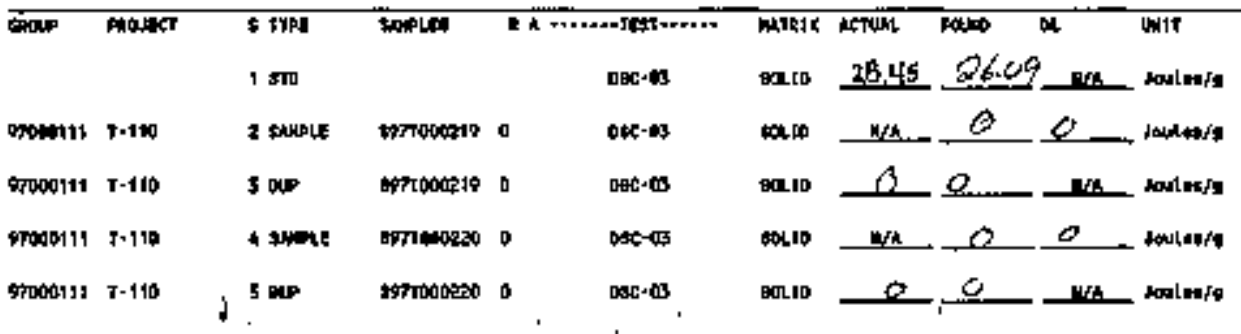

\section{Final page for worklist \# 16827}

Seg Atrached. for Signathere

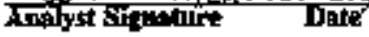

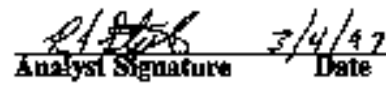

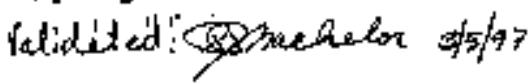


moritheng Vasion 2.1 osisess

HWF-SD-WhHP.Z3B, REV, O

Dargen 18.16

\section{LABCORE Data Entry Template for Worklist\# 16827}

Pages I

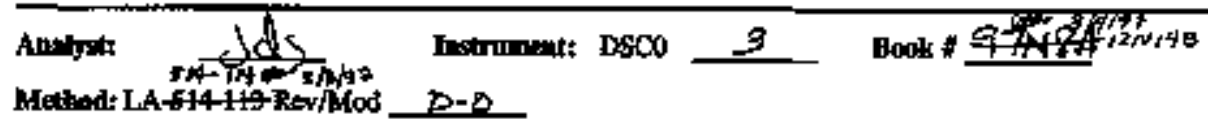

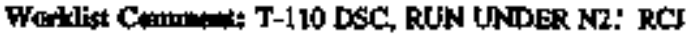

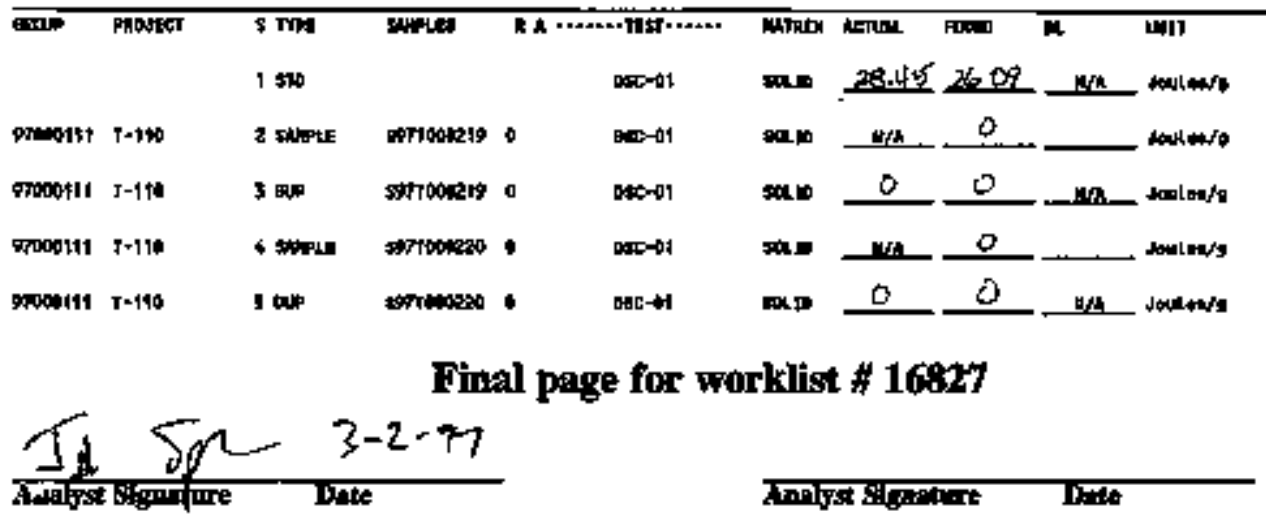

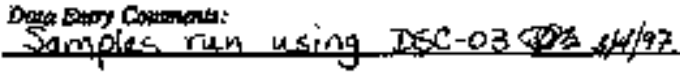

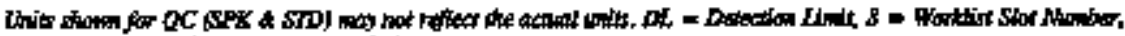

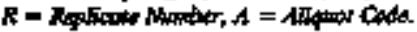


Drite if Dec

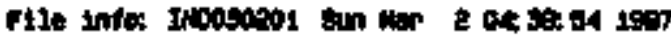

single wifhts 10.40

SID taitite

ㅁ.

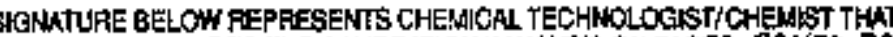

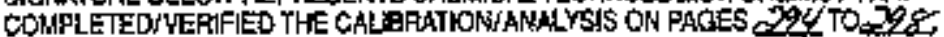

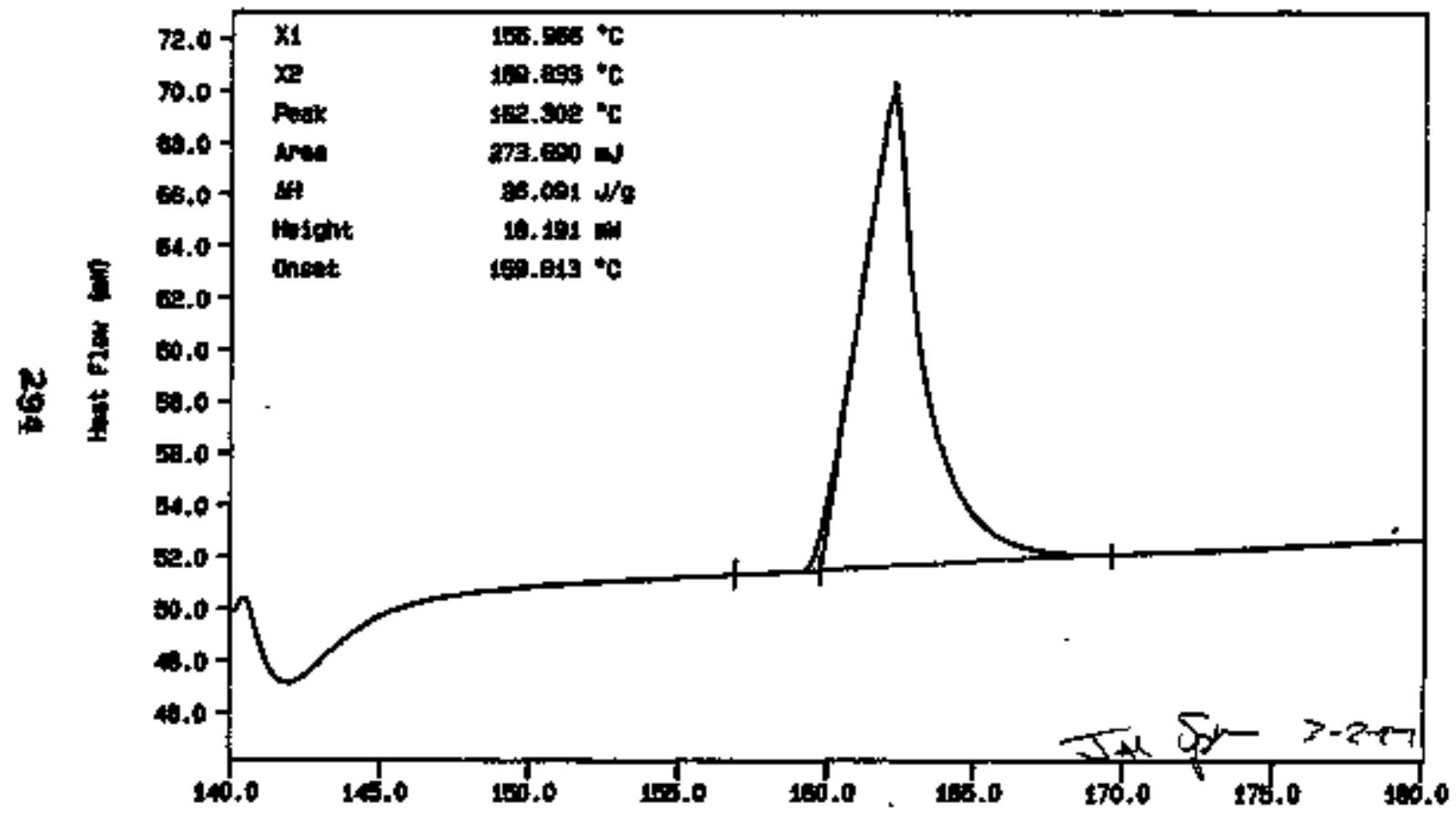

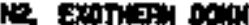

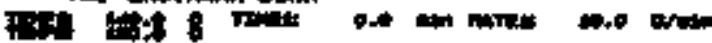

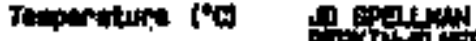


Gure I: oets

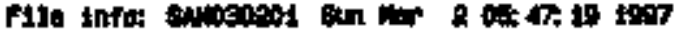

bonole wight 27,270 os

B9700045

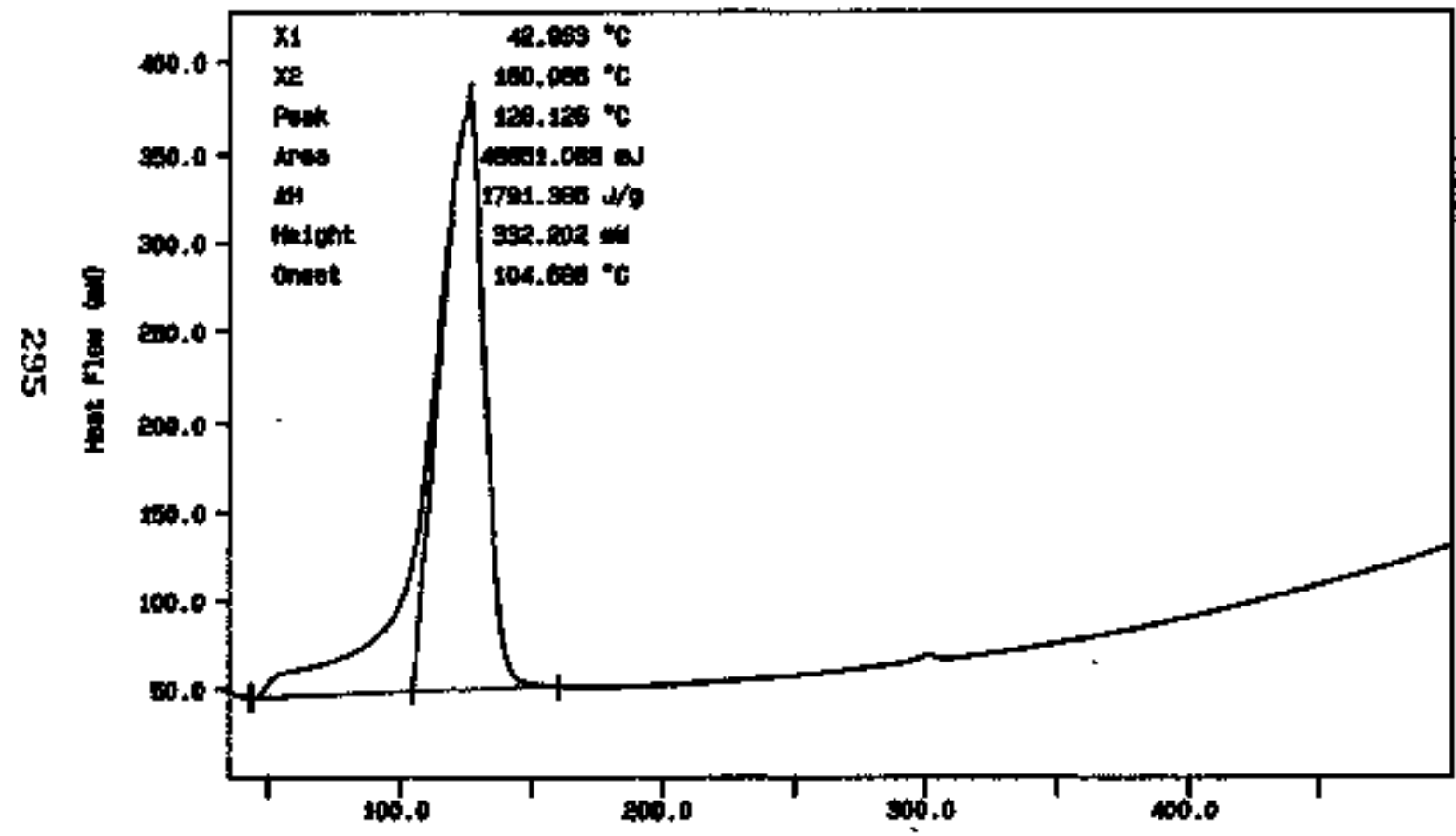

10.2 100rotn

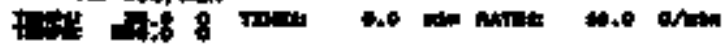

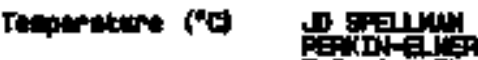

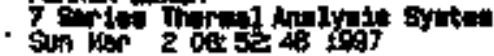


Cune t: DOS

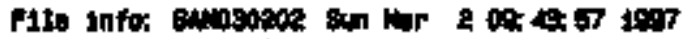

Geale mishte 11.700

Eortoon:tious

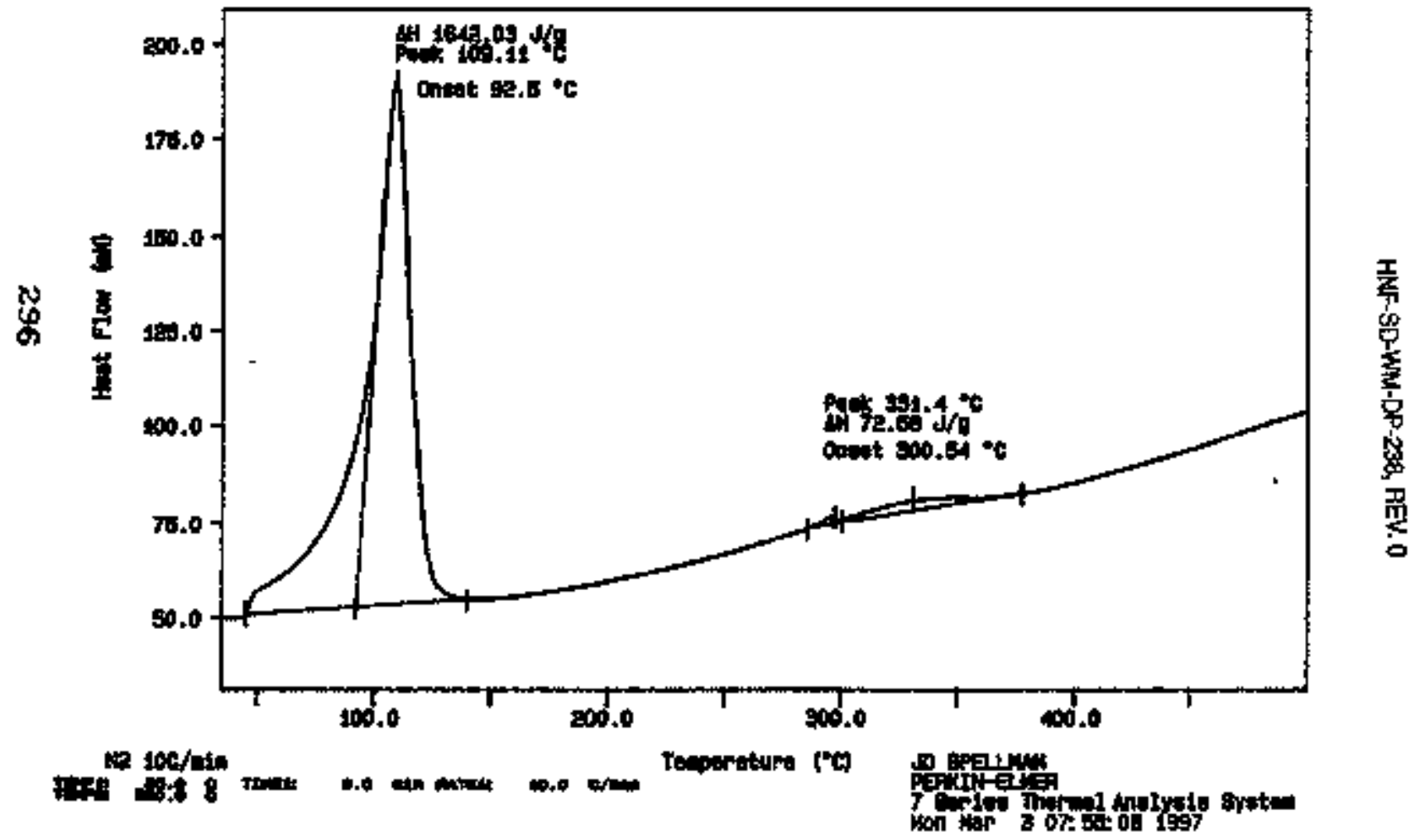


Curve is $0 \mathrm{Ac}$

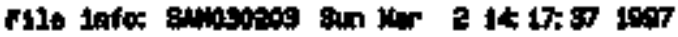

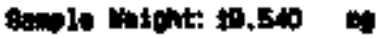

entoperso

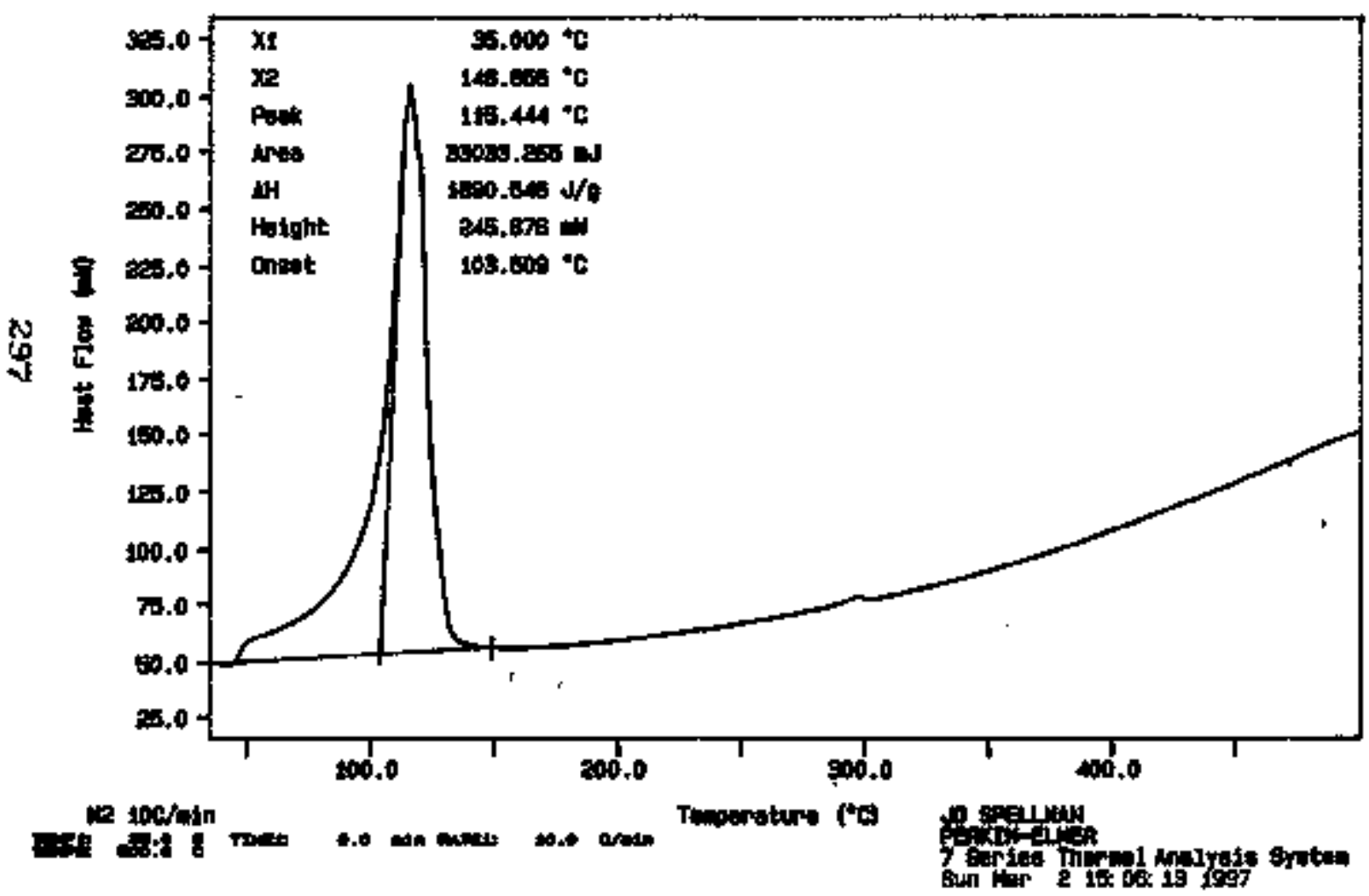


Dima it 0:8

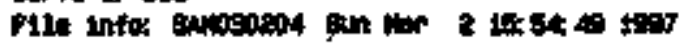

encle wiphts 94,40

sirtuponacop

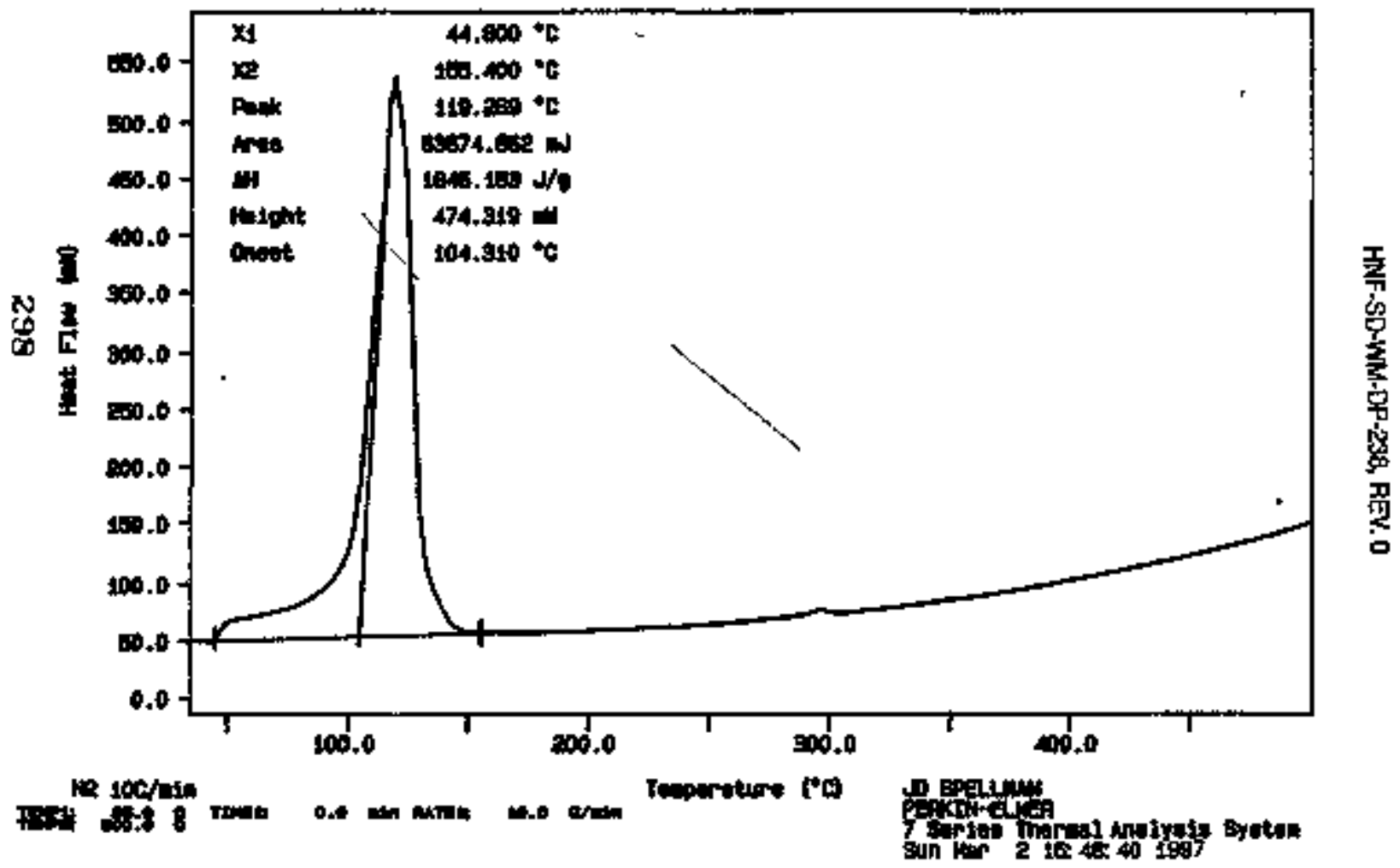




\section{LABCORE Data Entry Template for Worklist" 16828}

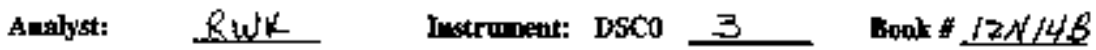

Method: LA-S14-114 Rev/Mod DD-O

Workllst Comment: T-1 10 DSC, KUN UNDER N2. RCI

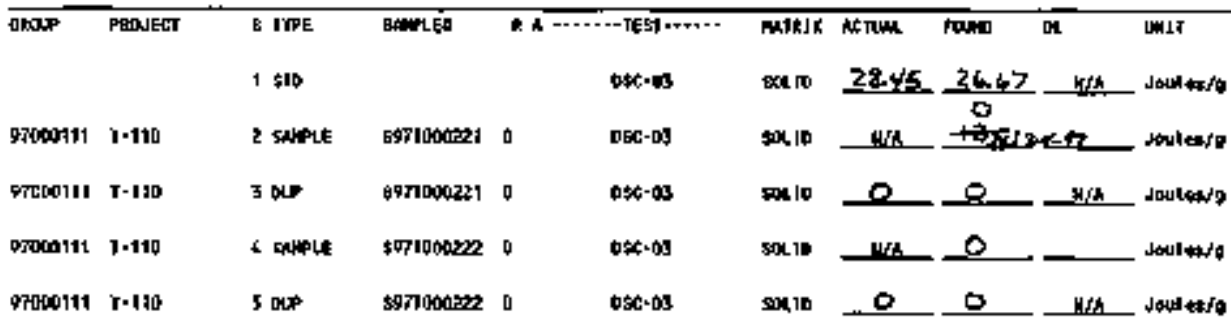

\section{Final page for worklist \# 16828}

Sep Affacled for Signature

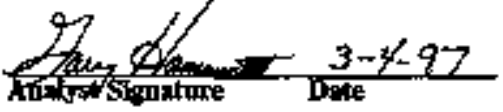
Waldated: Soptachalo $8 / 9 \%$

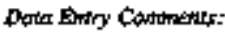

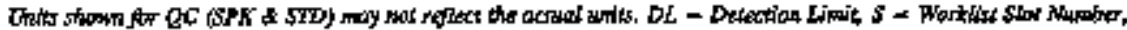

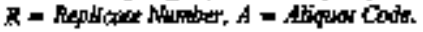




\section{LABCORE Data Entry Template for Worklist\# 16828 .}

\begin{tabular}{|c|c|c|c|c|c|}
\hline Analyst: & $72 k$ & Estrune:at: & DSCO & 3 & Dook $12 A / 4-B$ \\
\hline
\end{tabular}

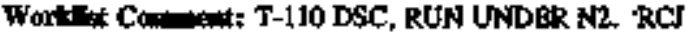

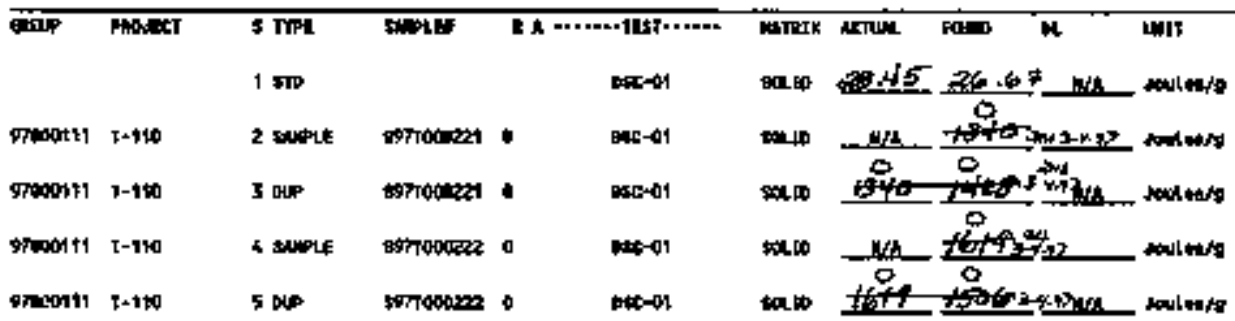

\section{Final page for worklist \# 16828}

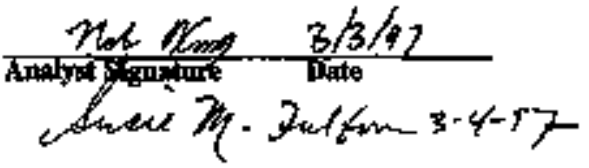

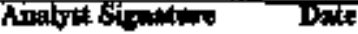

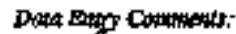

han semples asing $b s c-0 y$.

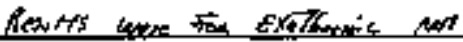

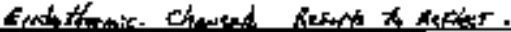

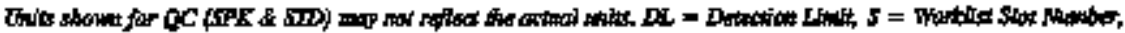

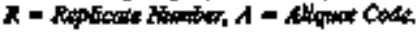


Curve I: DaC

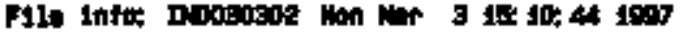

expin wight: 10,40 a

8ID 19N44-6

SIGNATUARE BELOW REPRESENTS CHEIACAL TECANOLOGLST/CHEMST THAT

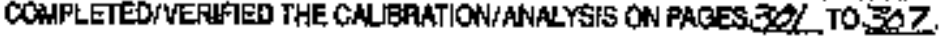

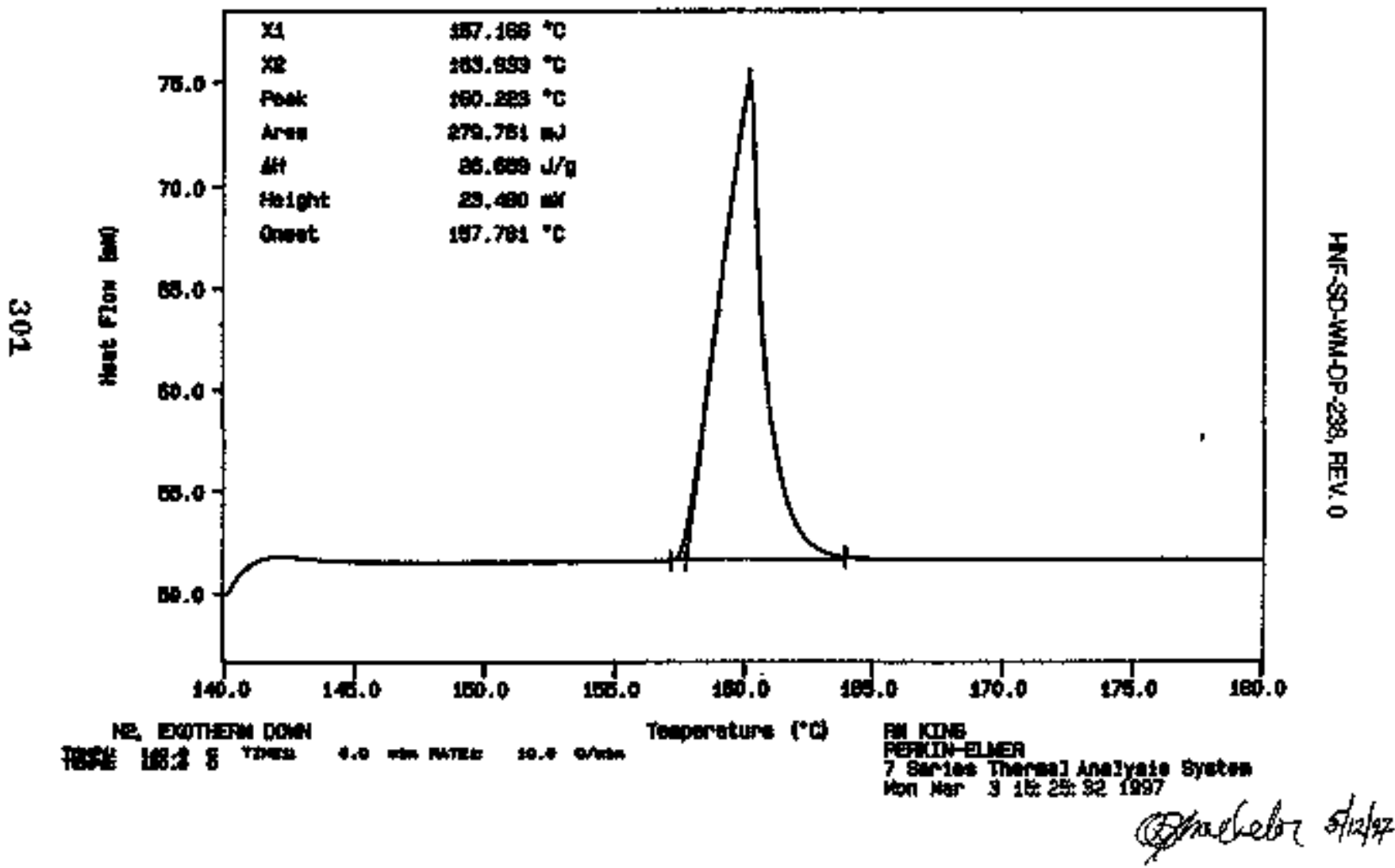


Cumit 1 DEC

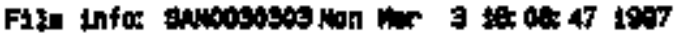

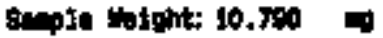

eqrotorat

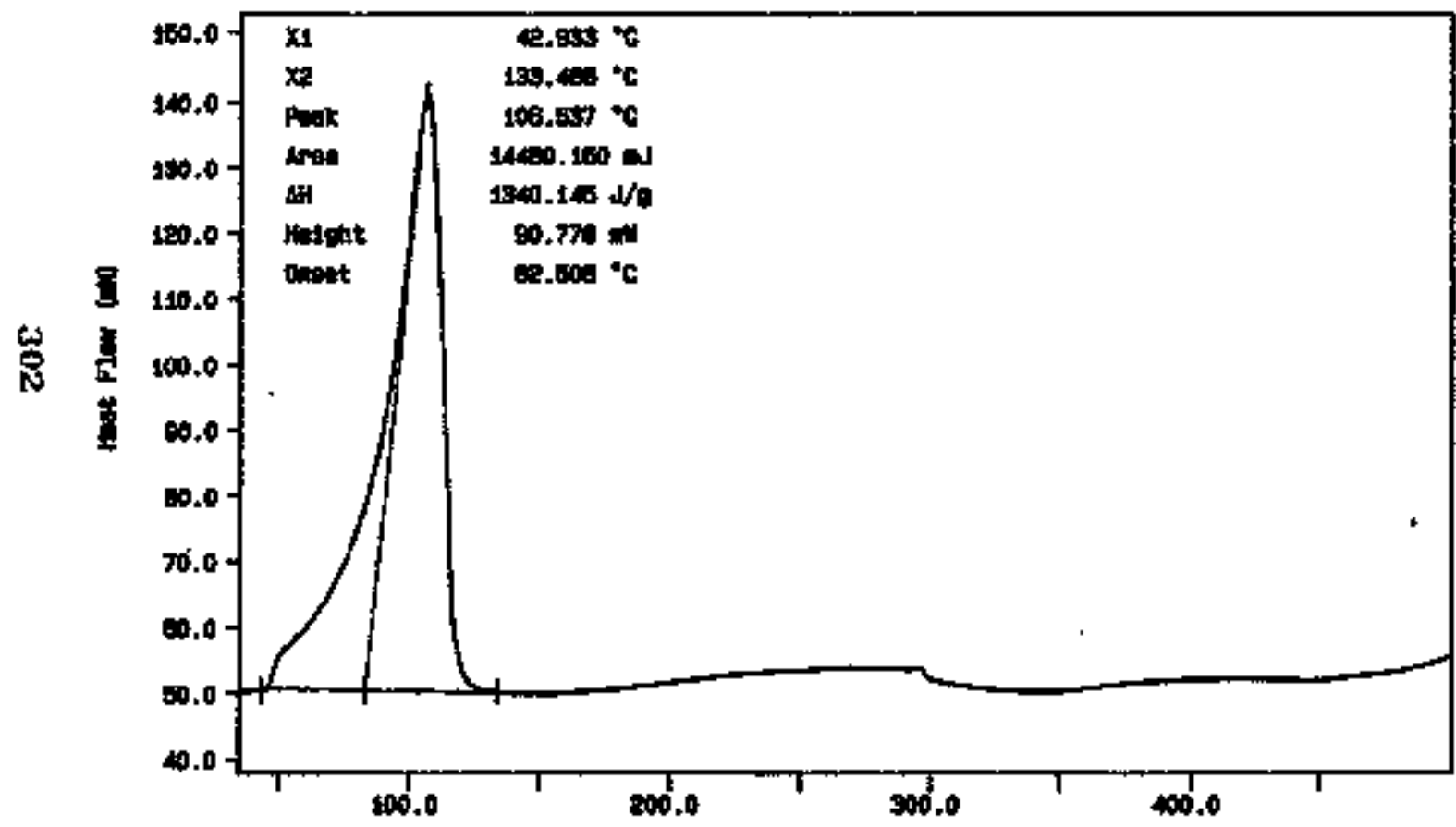

42 503/4s 푸년 3.8 8

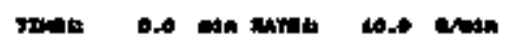


Crmit 1: a:t

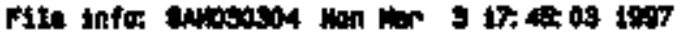

ocole Ningt: 40.740

somoopar top

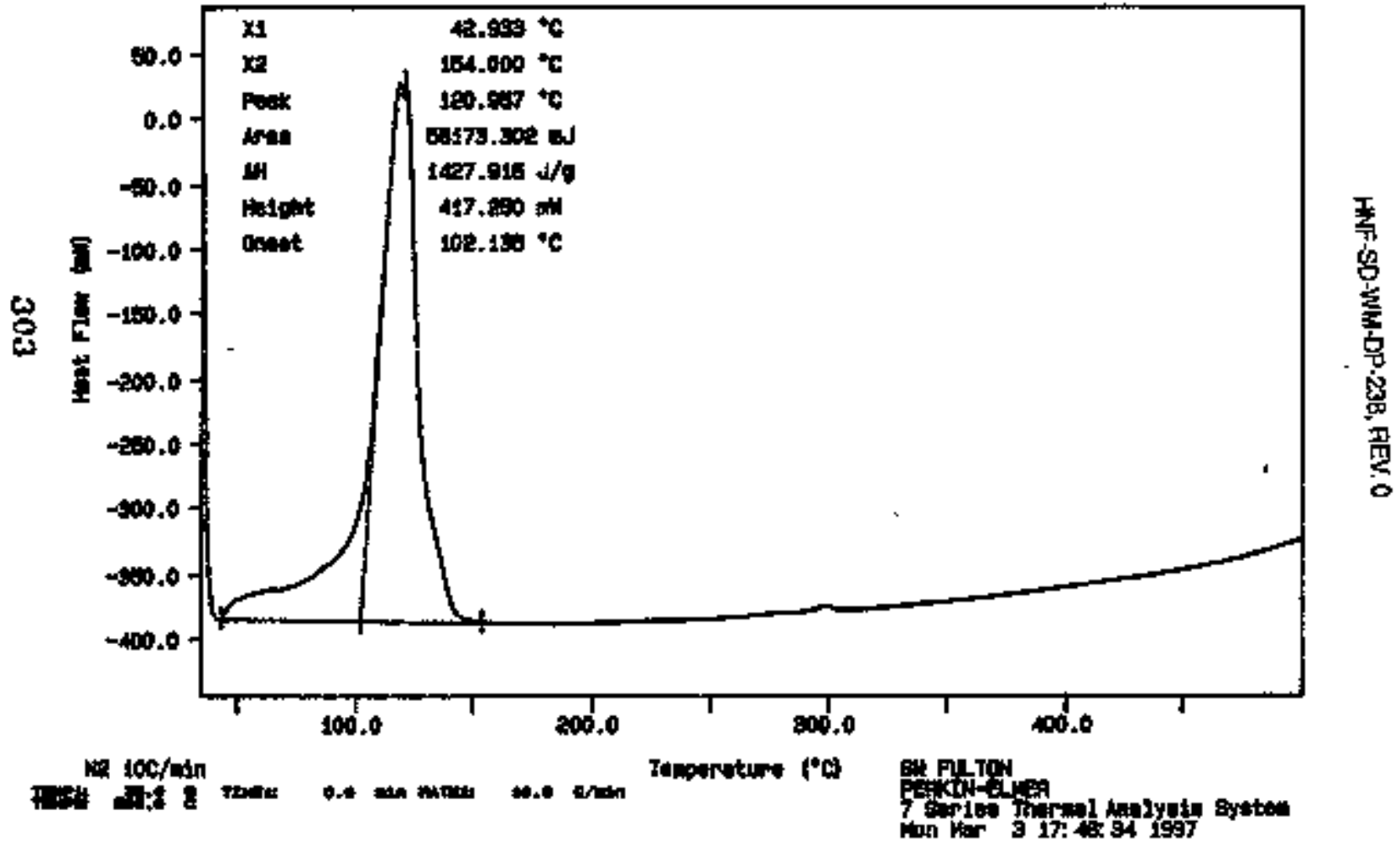


Arret it Dat

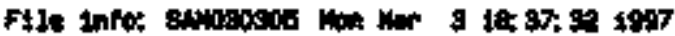

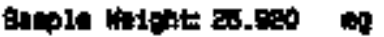

wrowoes

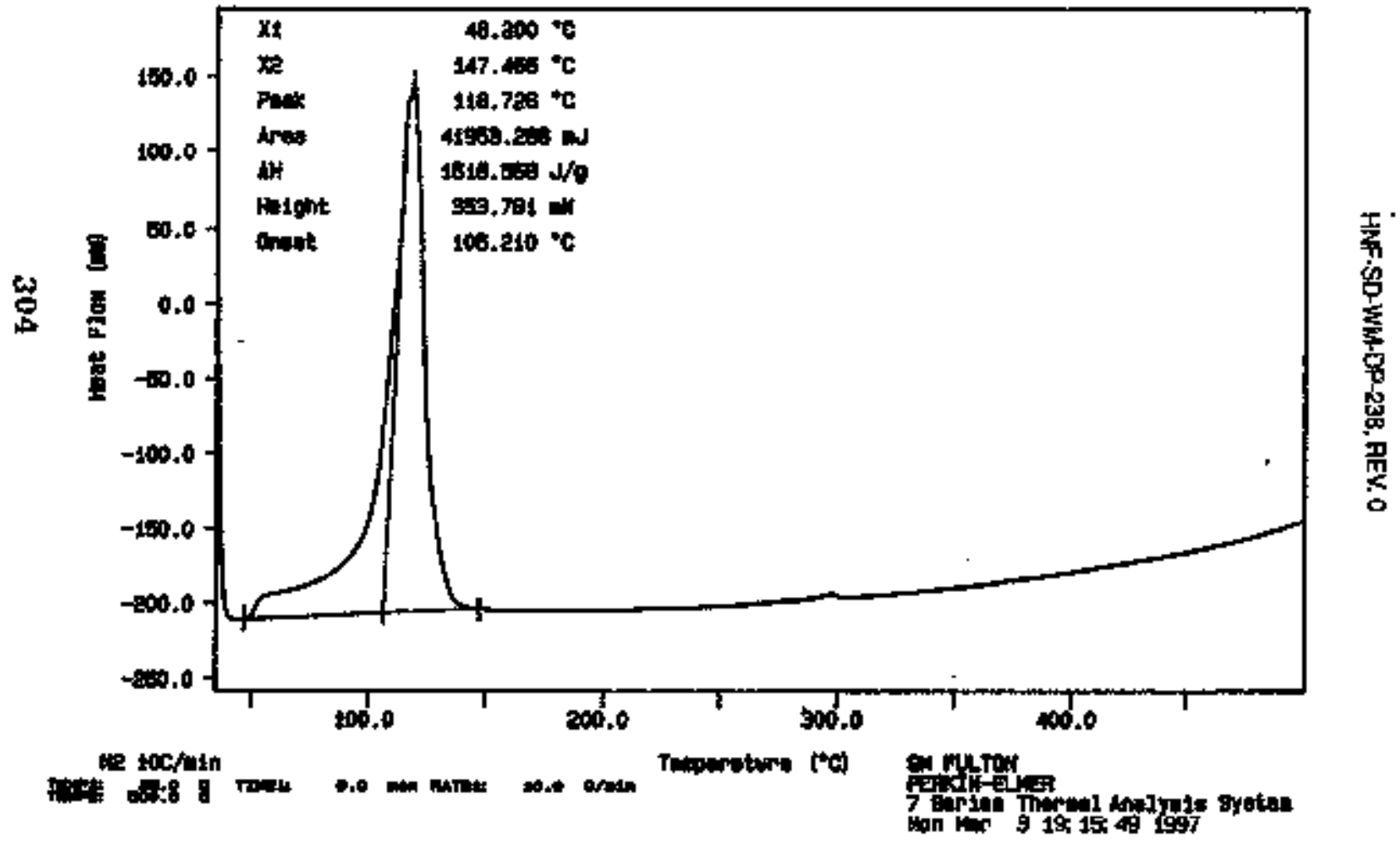


Curre $t$ Dac

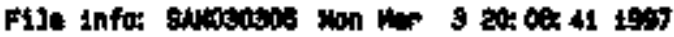

soople Woight: 21.010

geptootion DP

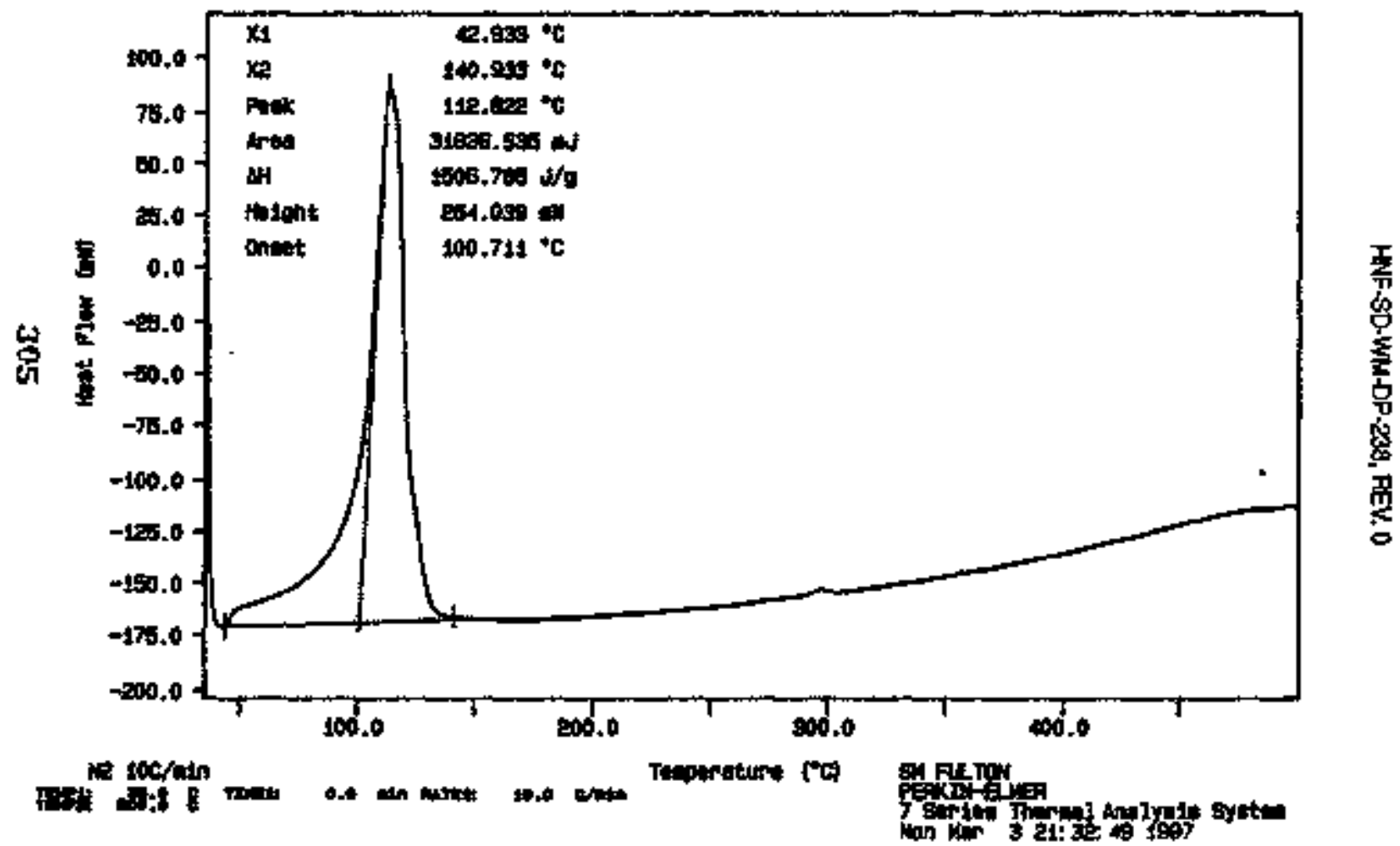




\section{MNF-SD-WH-DP-236, REV 0}

werditivet Yersion 2.I 05/15/95

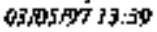

\section{LABCORE Data Entry Template for Worklist" 16896}

Pogt: $\quad$

\section{Analys: $\quad 0, b$ instrumand: DSO01 \\ Dook $A / A$

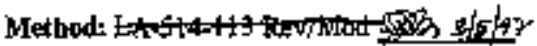

Worklst Comineat: DSC-02 T-110. -PFB

\begin{tabular}{|c|c|c|c|c|c|c|c|c|c|c|}
\hline thits & FADNECT & 日 ITPE & aNoLE & & 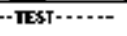 & Imtrlax & AETML & fouls & OL & เEt \\
\hline srowoods & $t=110$ & 1 salpher & sortoanipg & $a$ & $\operatorname{cosc}=02$ & SOL 10 & $N / h$ & $\theta$ & & Jad tota ory \\
\hline gropoods & T-110 & 2 ONT & 697T000192 & 0 & 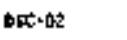 & SOLL IO & 2 & $D$ & N/4 & roules/s bry \\
\hline Dropowas & $\mathbf{r}-110$ & 3 tele & B9r 「D00193 & 0 & DBC-DX & SOL.10 & $m a$ & 2 & & Nodlen/g bry \\
\hline s7obotas & $t=110$ & 6 oup & काT0m10 & 0 & Dstende & 50LID & $\mathrm{D}$ & $\theta$ & Wh & 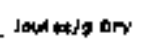 \\
\hline 9700011 & 1-110 & 3 ваNPIE & 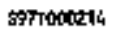 & $\bullet$ & 0 - 05 & $\Rightarrow 10$ & Ith. & $Q$ & & 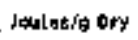 \\
\hline 9700111 & $T-110$ & 6 & ExT1000214 & o & Doss-az & solcto & $\phi$ & $\dot{\varphi}$ & wt. & Joun essig OTr \\
\hline srosont1 & 1.110 & 7 \$NWASE & SDTtocing16 & $\bullet$ & $83 x-02$ & BOLIO & Mth & $D$ & & Jannera Ory \\
\hline Drapol11 & $T-110$ & $B$ avp & shtatoven & 0 & $D \& C .02$ & $\$ 010$ & 2 & 0 & Heth & 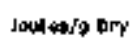 \\
\hline $970001 t 1$ & 7.110 & P\$FLUE & Sprtoc0R17 & o & Desc- 127 & SOHLIt & Mth & $a$ & & Jadestg tor \\
\hline groondt & $T-110$ & 10 머 & \$97004017 & o & 10202 & SoLIS & $Q$ & 0 & Whe & Joungars on \\
\hline 97900111 & $T-110$ & 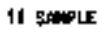 & 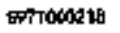 & 0 & $0 \leqslant c \cdot \Delta 2$ & toto & llts & 0 & & Joud \&t/o Dry \\
\hline srapott1 & 1.110 & $17 b$ & 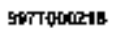 & 0 & $050-02$ & SOLLIO & $\dot{0}$ & 0 & nest & 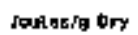 \\
\hline 07000111 & $T-110$ & 13 Ëomple & potocoses & 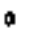 & $0 x t-02$ & 50 to & ㅂas & $D$ & & 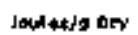 \\
\hline प्रra0til & 1.110 & 14 DUP & 907т00யद & 0 & $\cos 2 \cdot 0 t$ & SoLIO & 0 & 0 & nos & Jarlesig ory \\
\hline 97000111 & $T \cdot 110$ & 15 smptr & StTotorato & 0 & asc-az & Bento & Wth & $\theta$ & & Joud ts/g ory \\
\hline shoont1 & $1 \times 110$ & 16 ofP & कPToders? & - & -101402 & Sollo & $a$ & $D$ & With & dadikfo ory \\
\hline 9hosed1' & שונה-ד & 17 SUه!E & seftonos? & 0 & $n \dot{x} \cdot 0 z$ & $\operatorname{soc} 10$ & nth. & 0 & & Napled/s bey \\
\hline 97009111 & T-140 & To & sqTithocel & 0 & $0 x-10$ en & FOL.10 & 0 & 0 & . is & Pallow/g Dry \\
\hline
\end{tabular}

\section{Dasa Droy Comethent:}

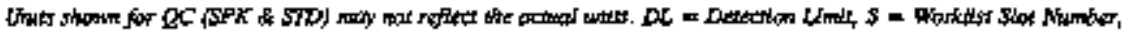
$k=$ Reptlone hunter, $A$ = Alipeos Code. 
warklismpt Vaskon 2.1 Dotrsigs

HIF-SD-WH-DP-296, REV. 0

Fage: $\quad 3$

O3/55/97/3:399

\section{LABCORE Data Entry Template for Worklist\# 16896}

\begin{tabular}{|c|c|c|c|c|c|c|c|c|c|c|}
\hline क्स००ण & FDVNECT & \$ टभम & \$NDL" & & rreqs & mathix & ACIIIIL & Folm & $\overline{r a}$ & LEtT \\
\hline 970011 & $\uparrow+11$ & th sway & Frtingazs & $\bullet$ & Desfor & Ba.10 & $N_{1} / A$ & 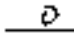 & & Jorid \&ato to \\
\hline $97000+11$ & $T-119$ & 20 ous & 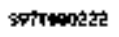 & 0 & ofec- 12 & sat it & 0 & 12 & $4 / 4$ & Jouldes/月 \\
\hline
\end{tabular}

\section{Final page for worklist \#16896}

Q82) 2010

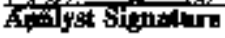

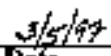

Date

Analyst Sigasture


This document was too large to scan as a whole document, therefore it required breaking into smaller sections.

Document number: $50-W M-D P=238$

\section{Section 2 of 2}

Title: Woste Compatibieity, Safety Desues and Thal Runtes for Tank 241-T-vo pust Hote saneus

Pge. $308-604$

Date: $5 / 15 / 92$ Revision: A000

Originator: Yugum gos

Co:_._ESSH

Recipiertit:

Co:

References: $\varepsilon O T-420399$ 


\section{LABCORE Data Entry Template for Worklistf 16904}

Anolyot:

$$
\rho \text { p }
$$

listrumenat: DSCO1

Bopts $\#$

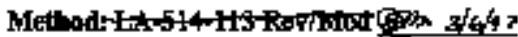

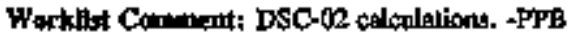

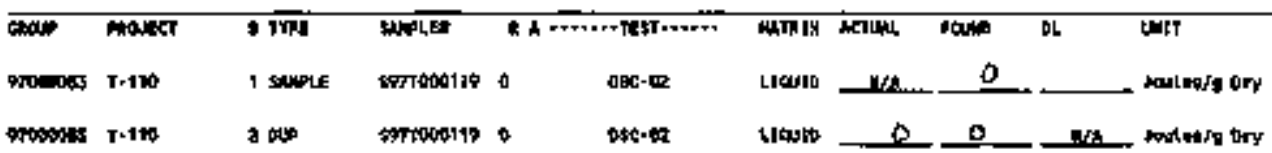

\section{Final page for worklist \# 16994}

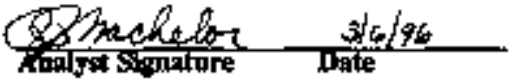

Anelyst Stguature Date

Dera Faty Comorantar

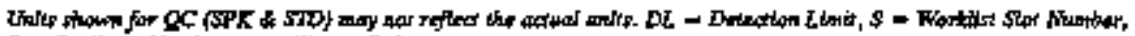

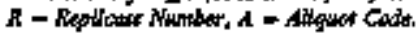




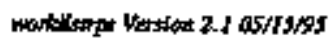

HNF-5D-WW-OP-2S\$, REV, 0

mage: 1

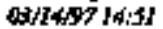

LABCORE Data Entry Template for Worklist 17018

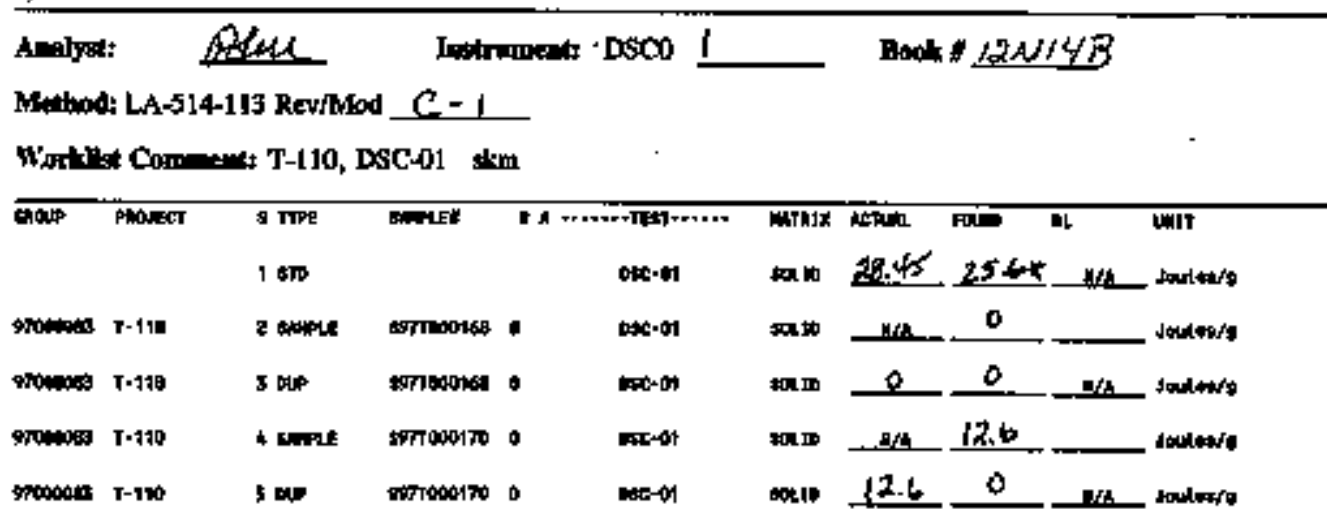

Final page for worklist 17018

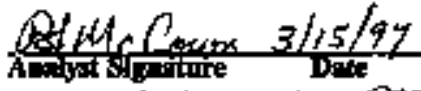

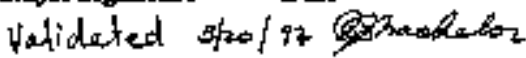
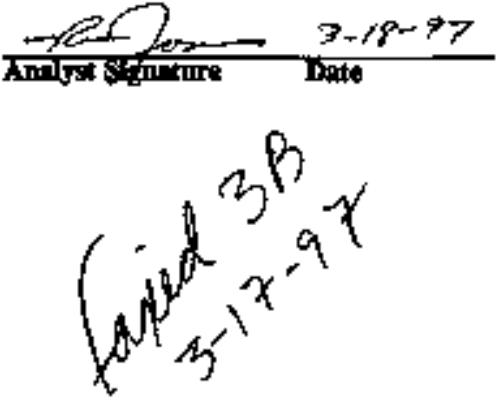

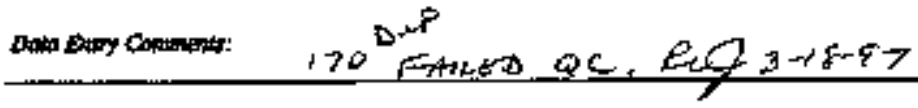

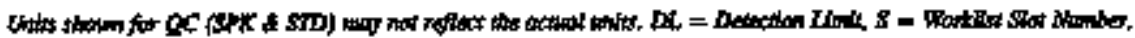

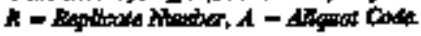

309 
WhC OCFISTORY TRBLE EDIT \$CREMAN

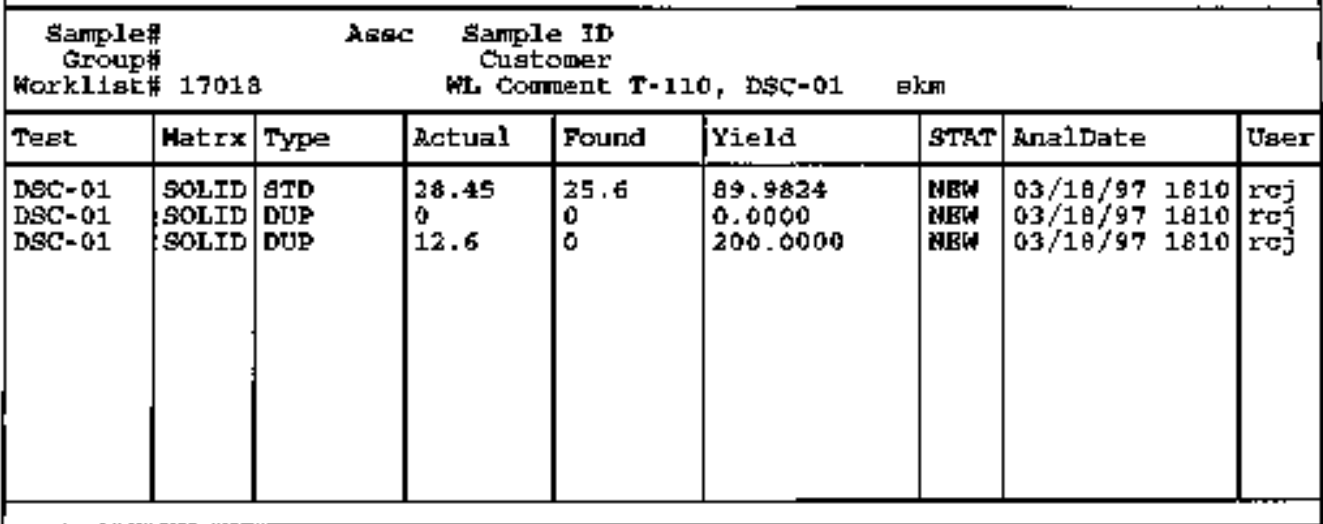

Save (F12) Bnd (F3) 
HNF-SD-WMHPP-298, REV O

\begin{tabular}{|c|c|c|c|c|c|c|c|c|}
\hline \multicolumn{9}{|c|}{ WHC QCHISTORY TABLE EDIT SCRERN } \\
\hline \multicolumn{3}{|c|}{$\begin{array}{l}\text { Sandis\# } \\
\text { Oroup\# } \\
\text { Morkilot\# } 17018\end{array}$} & \multicolumn{3}{|c|}{ 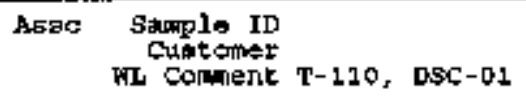 } & oknat & \multirow[b]{2}{*}{ AtnalDats } & \multirow[b]{2}{*}{ Deter } \\
\hline T\&ąt & Materx & Typ: & Actual & Found & Y1410 & 9TAT & & \\
\hline 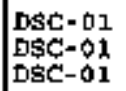 & $\begin{array}{l}\text { SoLtD } \\
\text { solid } \\
\text { soLid }\end{array}$ & $\begin{array}{l}\text { STD } \\
\text { DUP } \\
\text { DUTP }\end{array}$ & $\begin{array}{l}28.45 \\
0 \\
12.6\end{array}$ & $\begin{array}{l}25.6 * \\
0 \\
0\end{array}$ & $\begin{array}{l}99.9284 \\
0.0000 \\
200.0000\end{array}$ & $\begin{array}{l}\text { TEXT } \\
\text { PEN } \\
\text { WEW }\end{array}$ & $\begin{array}{l}03 / 28 / 971810 \\
03 / 18 / 97 \text { 1810 } \\
03 / 18 / 97 \text { 1910 }\end{array}$ & $\begin{array}{l}\text { rej } \\
\text { rej } \\
\text { rej }\end{array}$ \\
\hline
\end{tabular}

Save (F12) End (F3) 


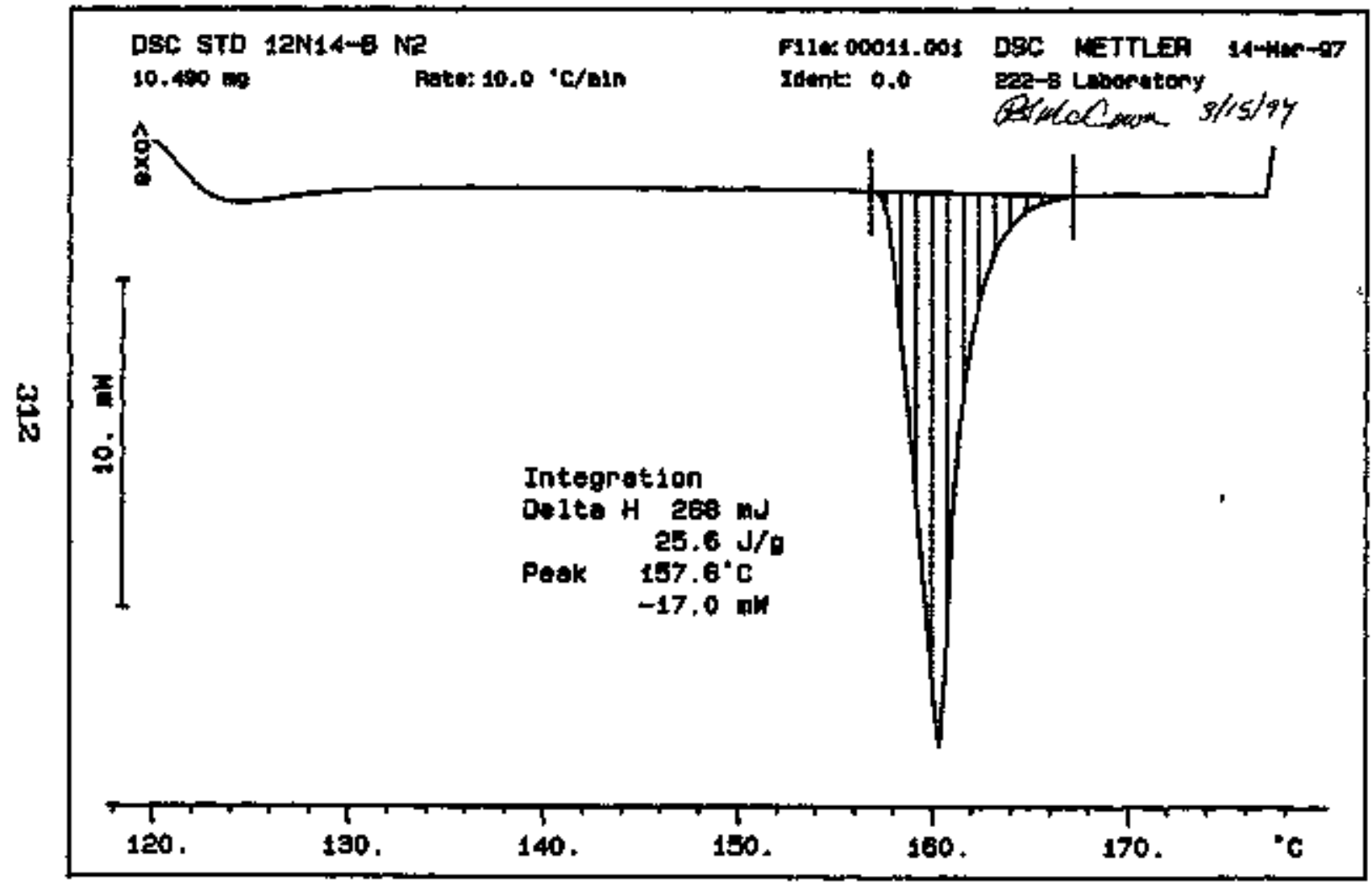




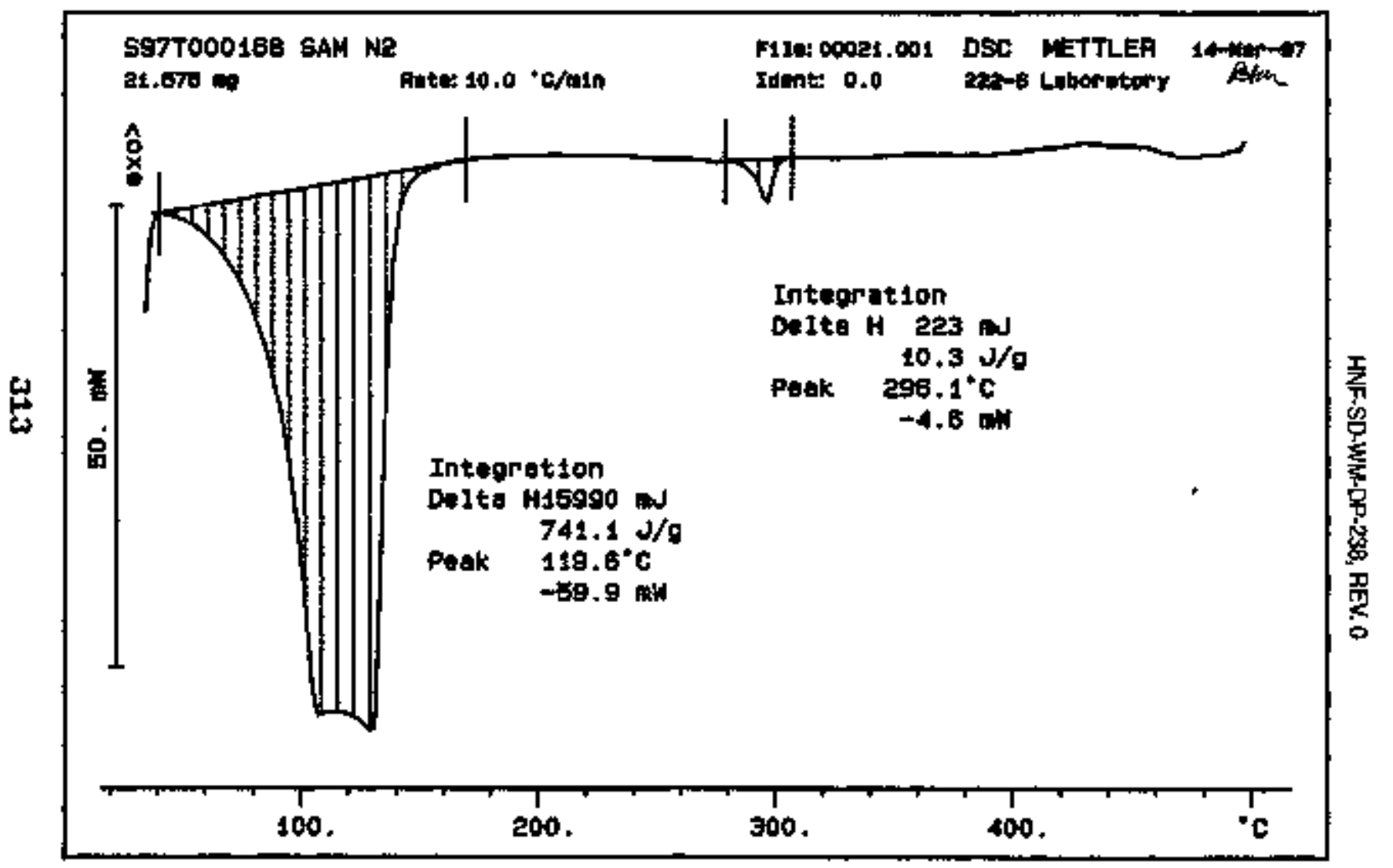




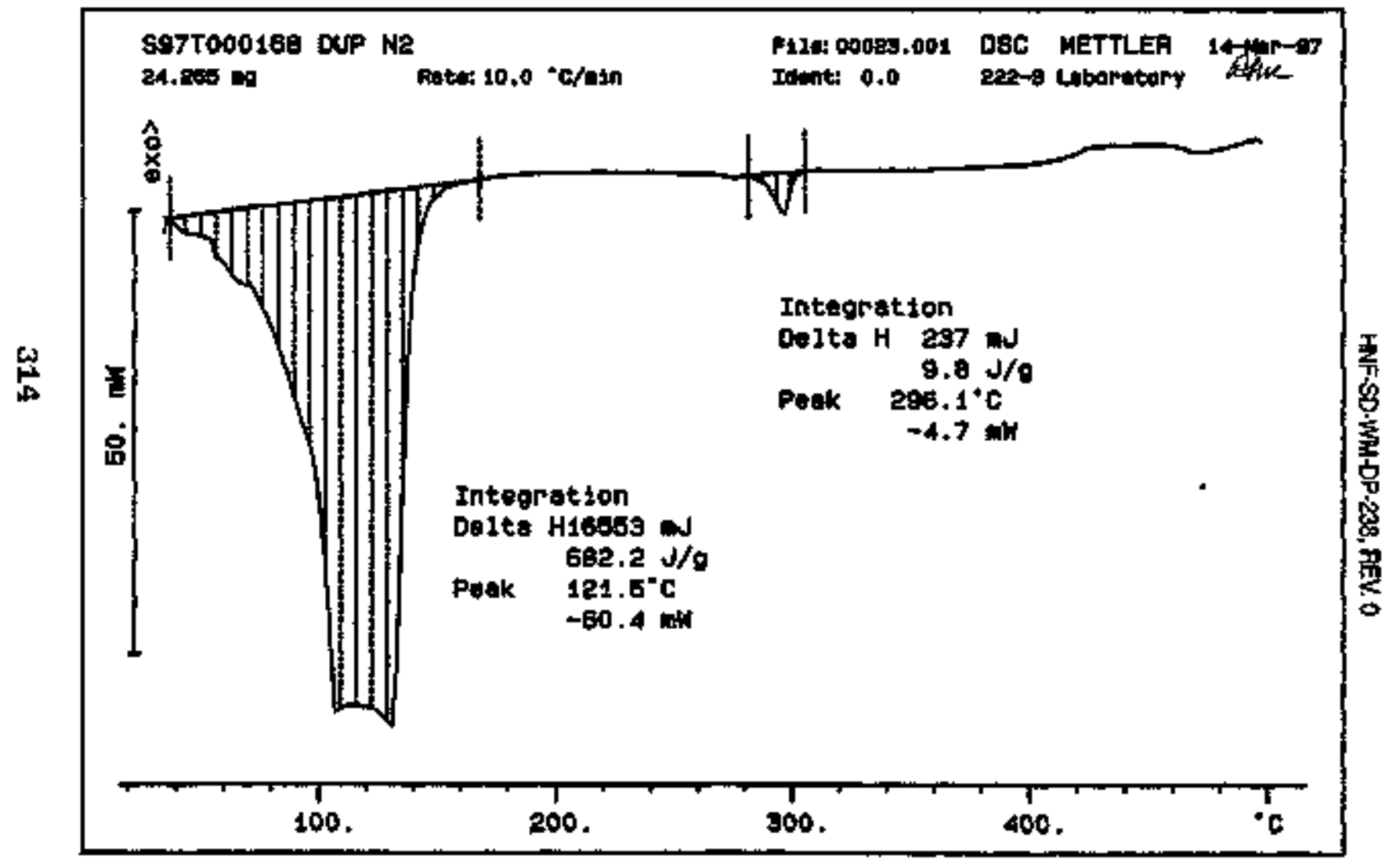




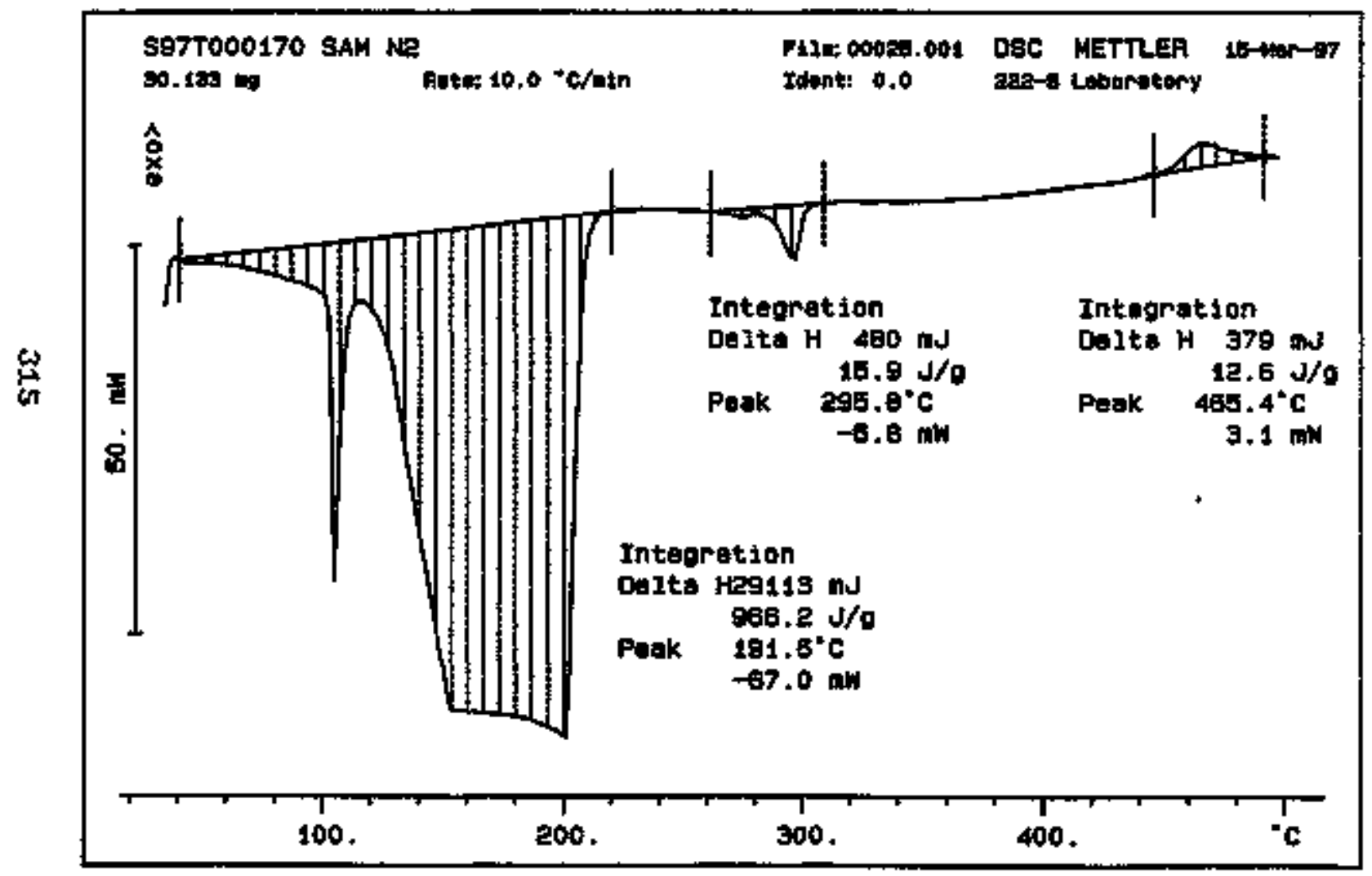




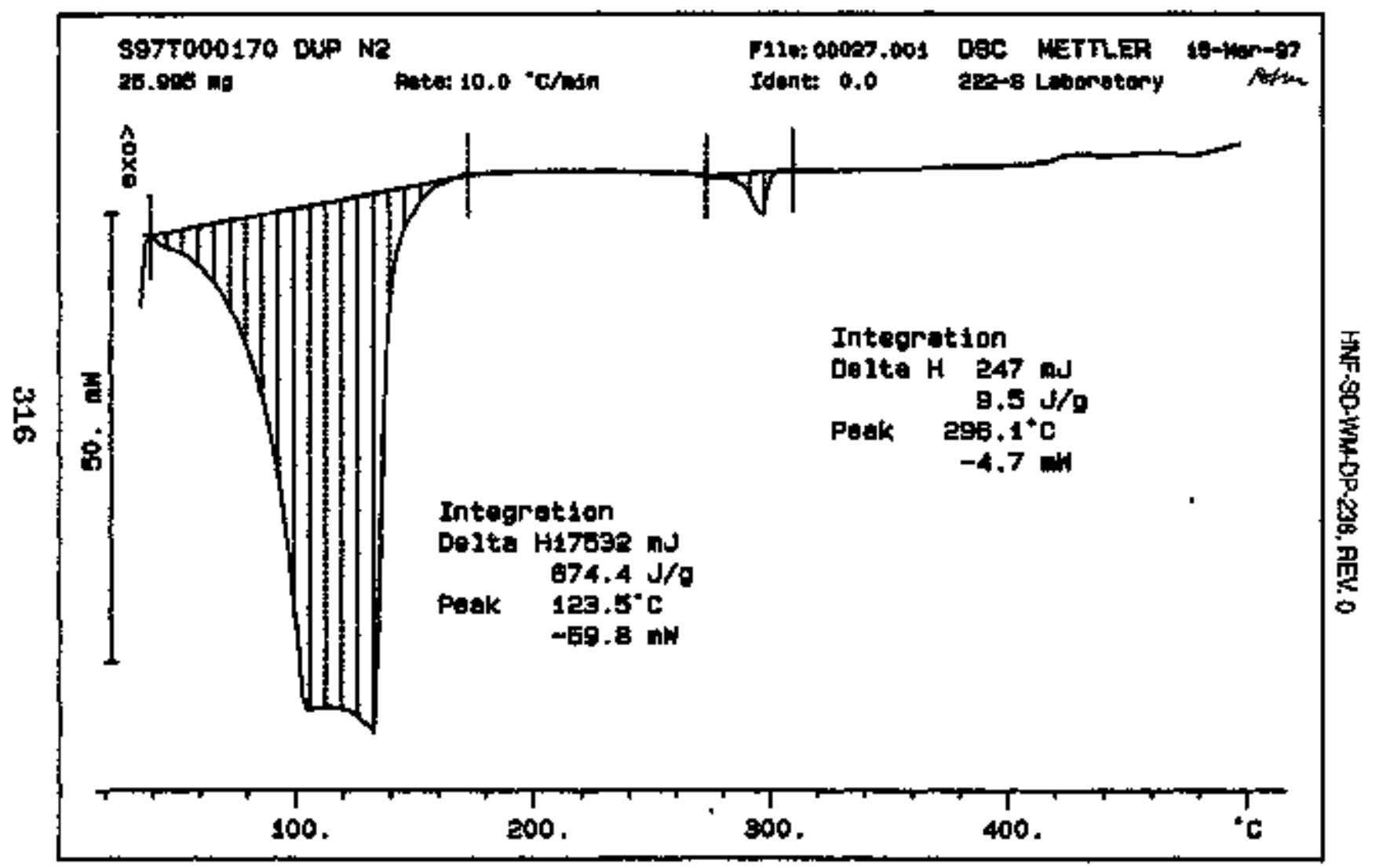




\section{LABCORE Data Entry Tenplate for Worklisł 17019}

\section{Anabilt \\ Instrumen: DSCO 3 \\ Bpak $* \angle 2 N / 4 / 3$}

Method: LA-514-1 14 Rew/Mkd _D-D

Worklist Comentint: T-110, DSC-01 atm

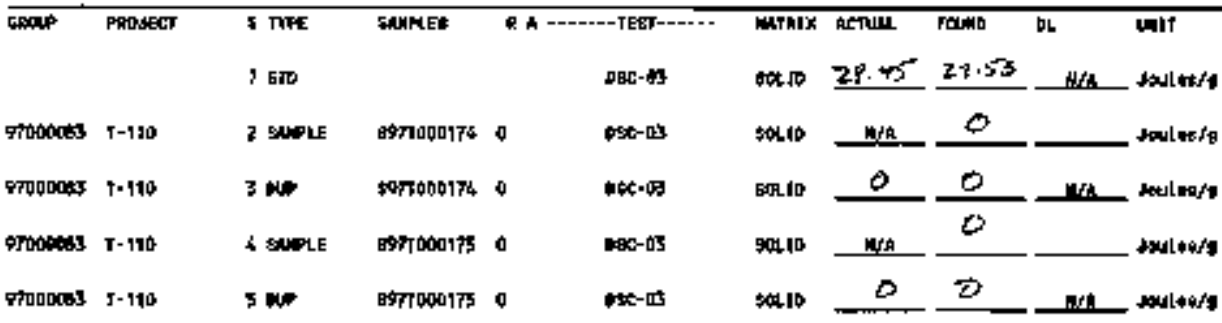

\section{Final page for worklist \# 17019}

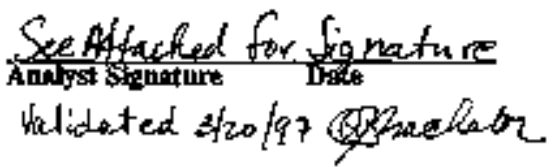

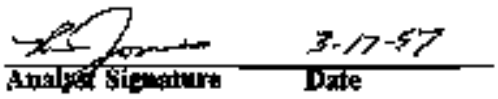

Dan Extry Conmant:

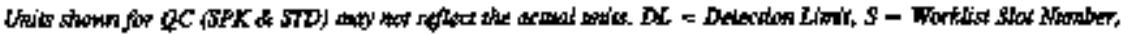

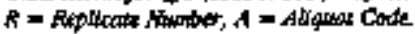




\section{LABCORE Data Entry Template for Worklist 17019}

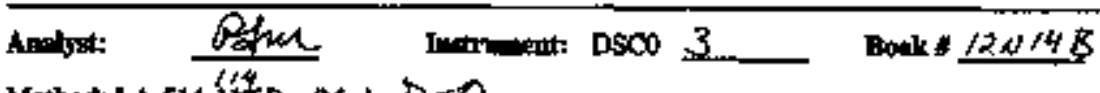

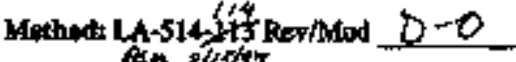

Ben s/4st

Worlist Crmmet: T-110, DSC-01 stm

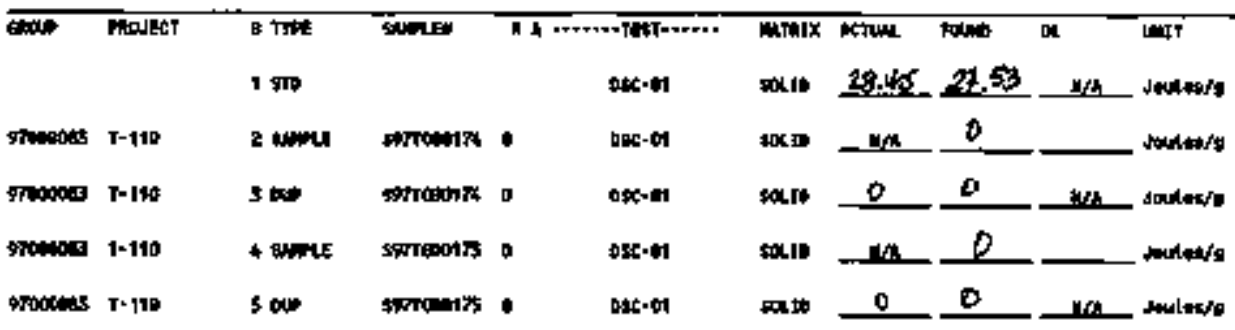

\section{Final page for worklist \# 17019}

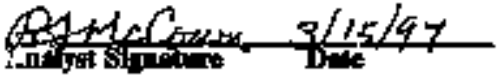

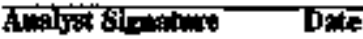

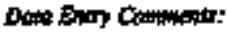

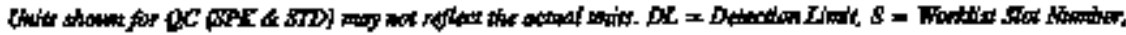


cume I: $\mathbf{t}$

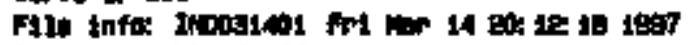
goplo waght 10.4:0

IID 1214:SHENATURE BELOW FEPAEOENTS CHEHCN TECHNOLOGST/CHEYAST TUAT

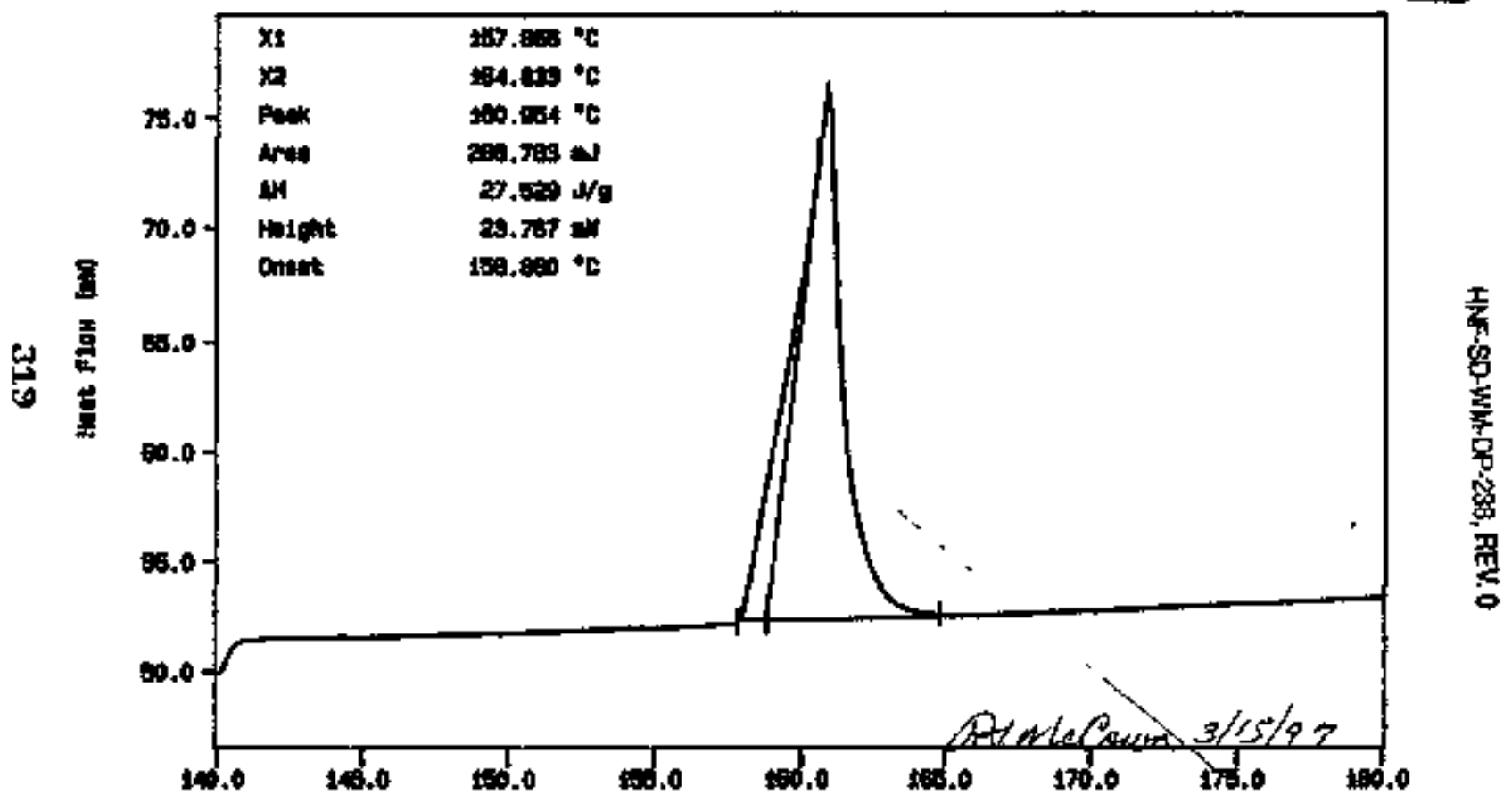

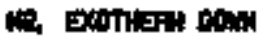

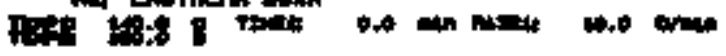

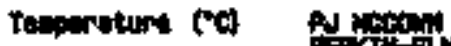


Pupe is Das

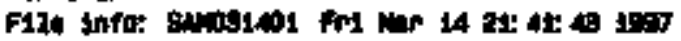

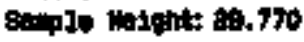

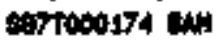

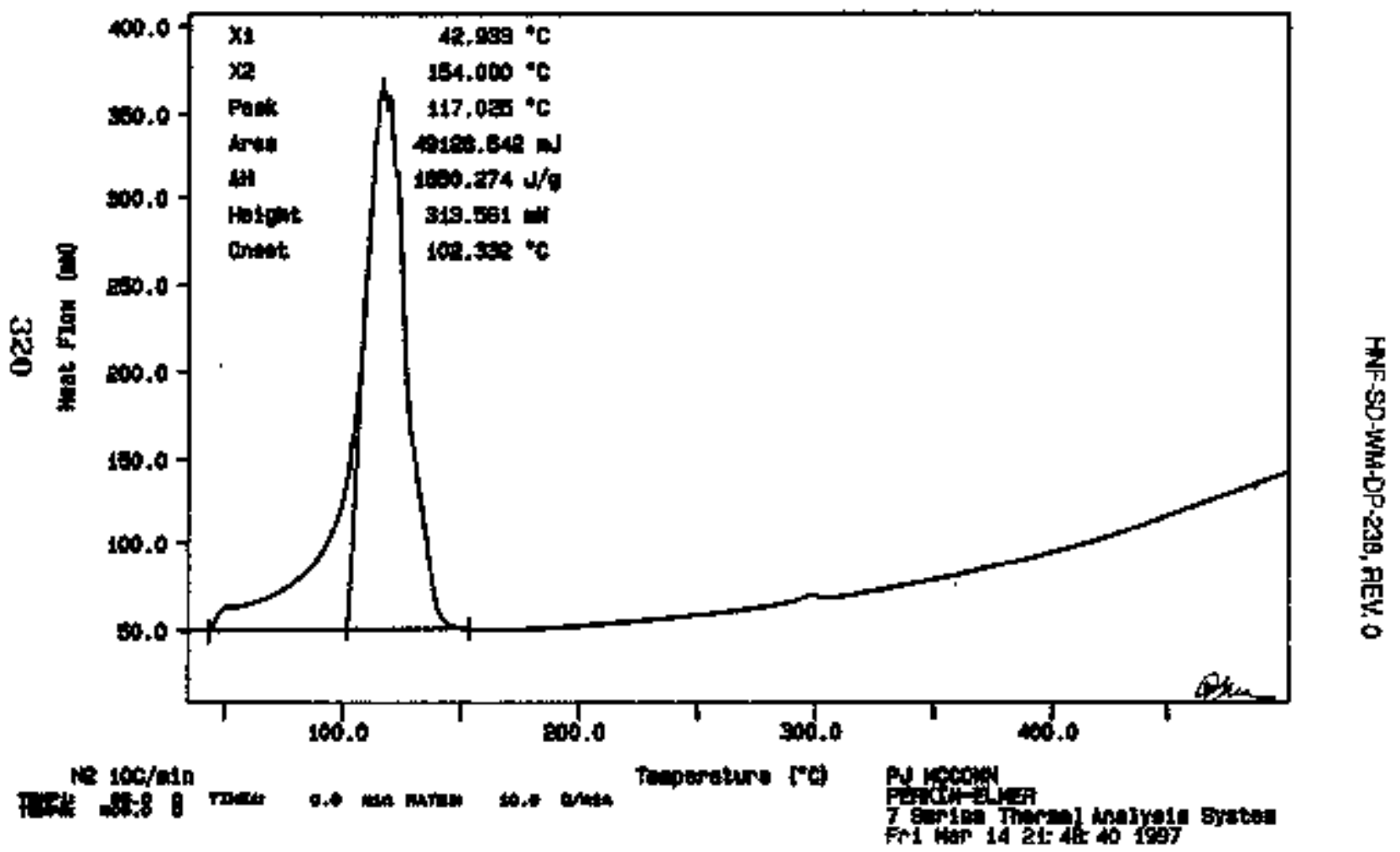


Curve if Dec

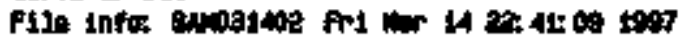

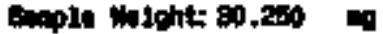

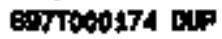

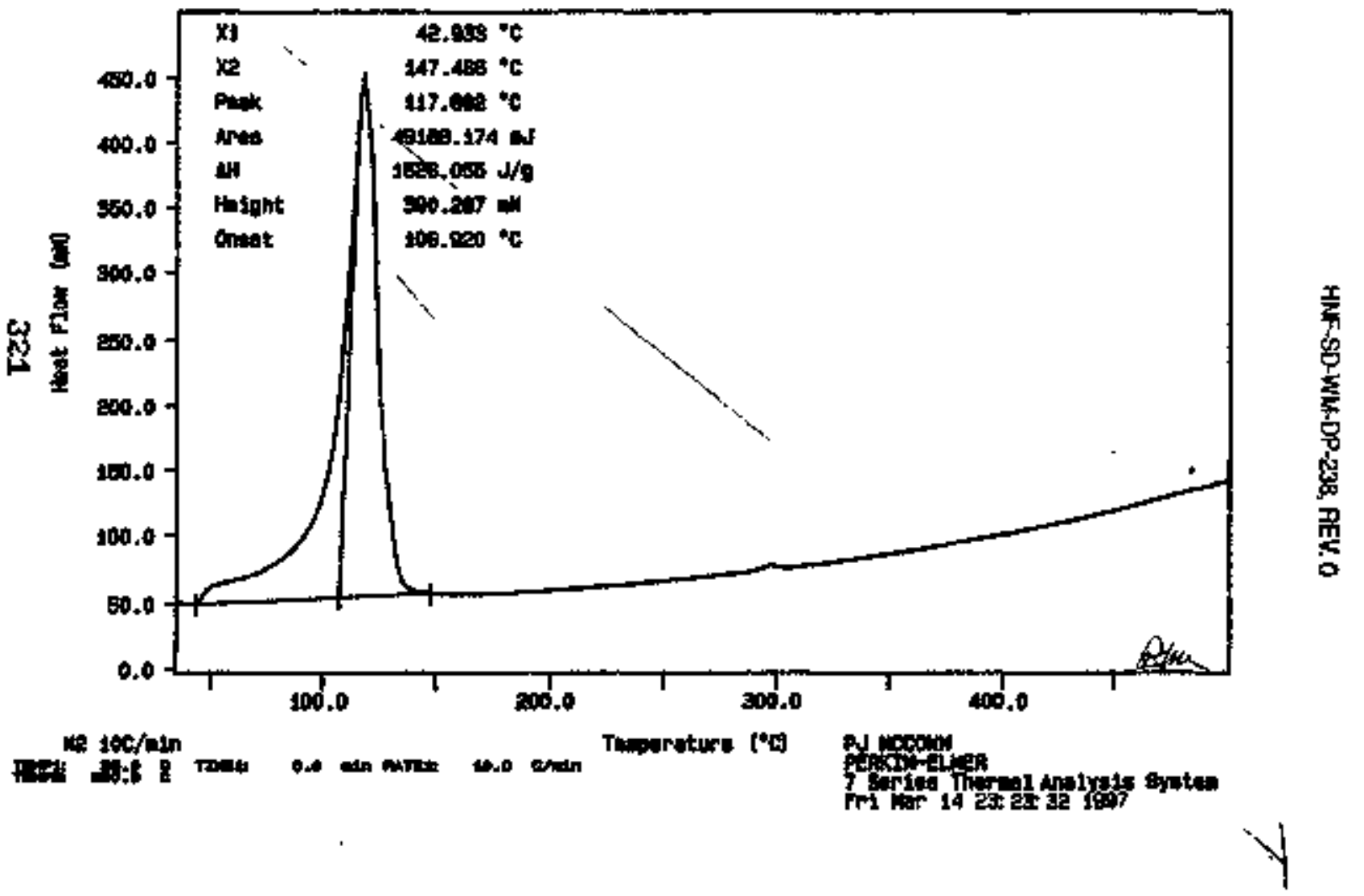


Derve it net

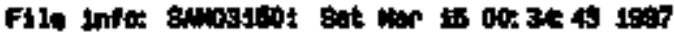

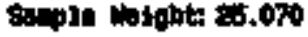

a.

oprovosirs sint

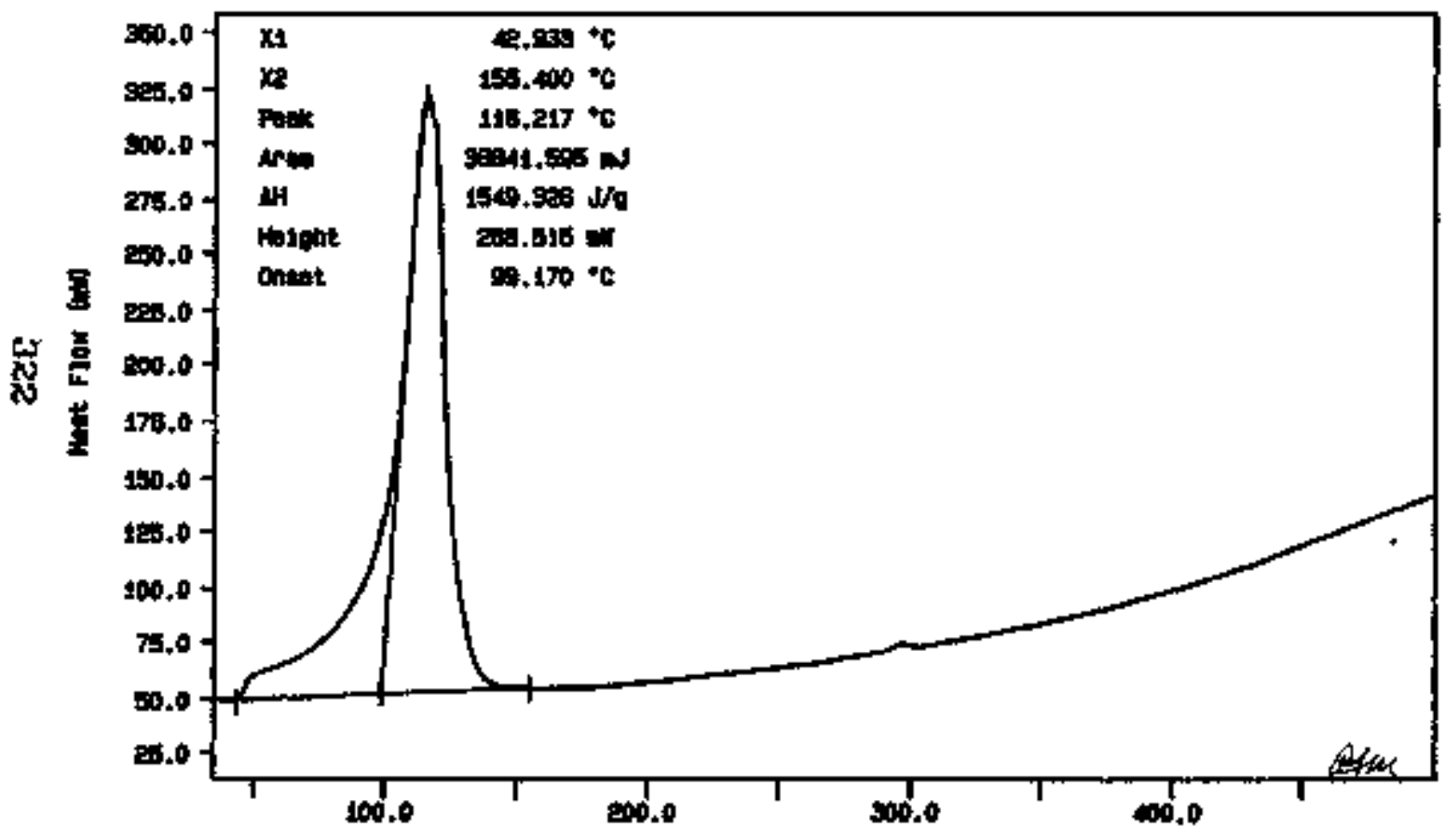

ne tockaln

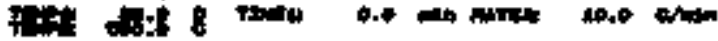

Tolparoture 10ㅇ

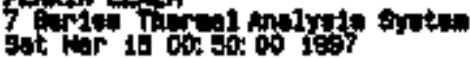


Corne it Dot

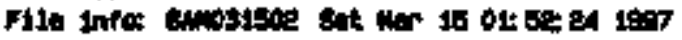

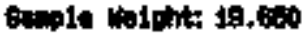

EvT000174 alp

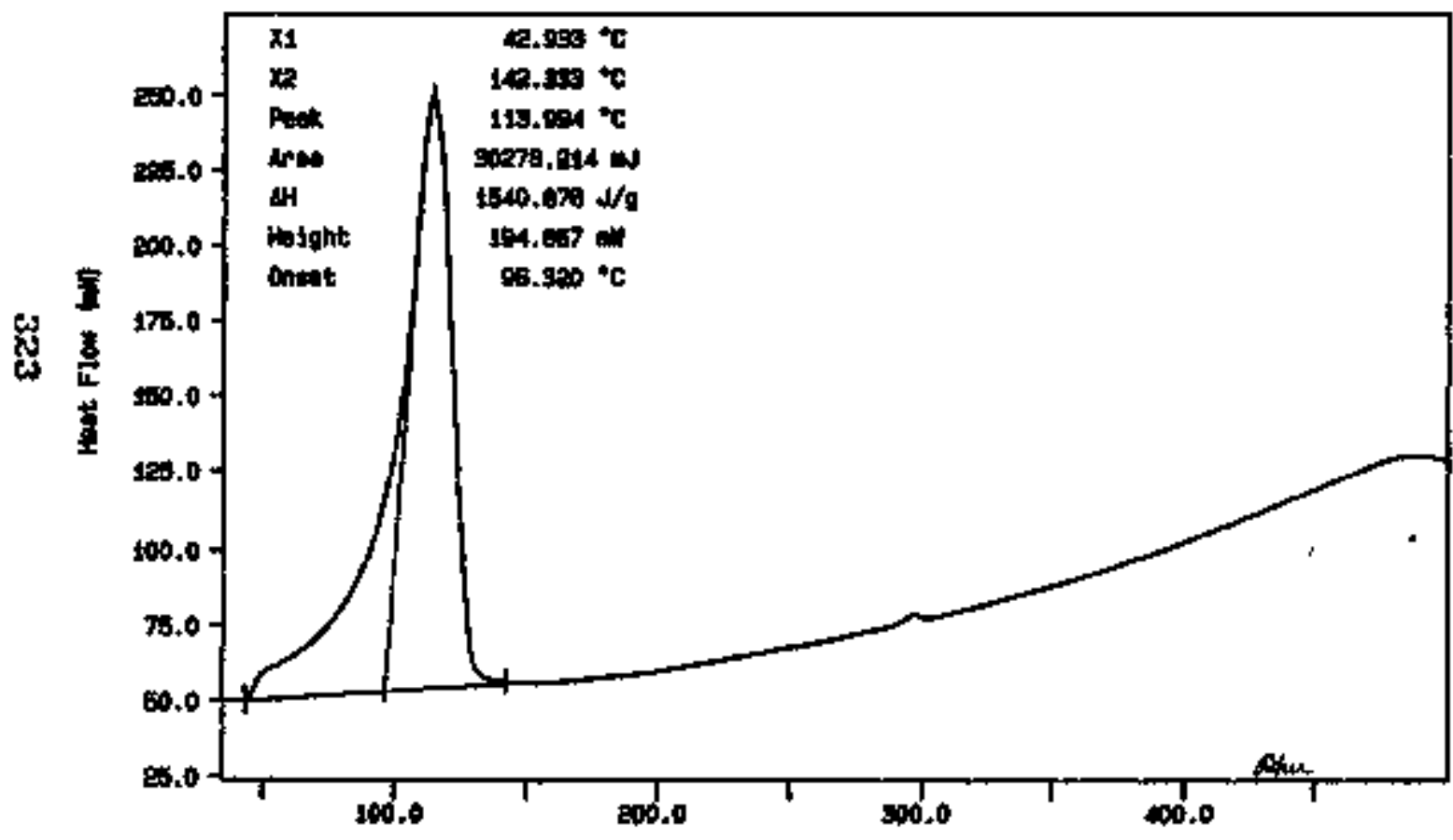

10 $100 / 0$ on

Fidi

tempotste

Pil

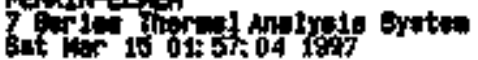




\section{LABCORE Data Entry Template for Worklist 17020}

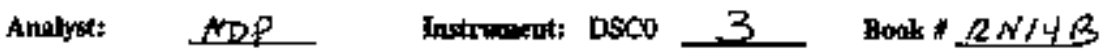

Methed; [A-S]4-114 Rev/Mod D-D

Wpeklist Compous: DSC-01, T-110 skm

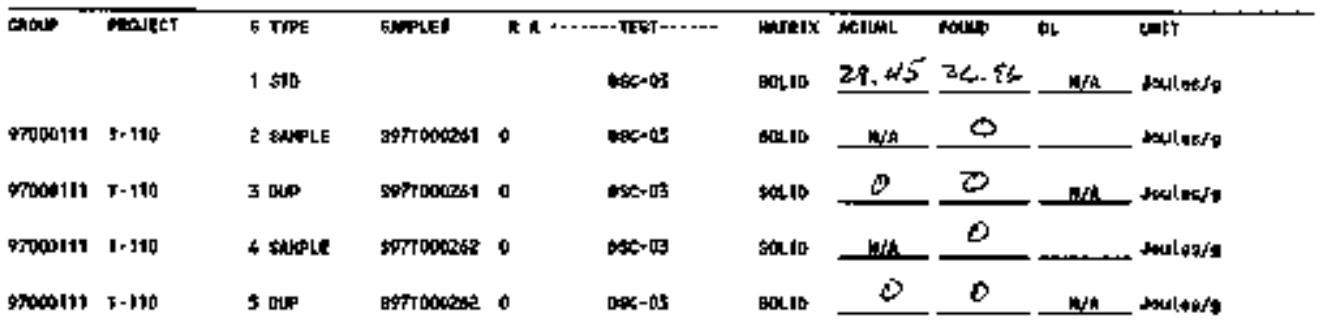

\section{Füal page for worklist \# 17020}

Sec Affuched for Signature Analyt somiture ivato

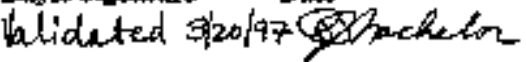

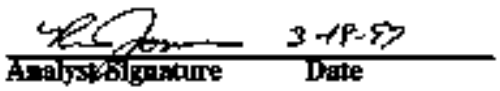

Daw Butry Combent:

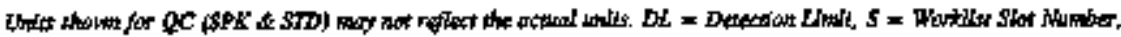

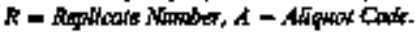




\section{LABCORE Data Entry Templete for Worklist\#̈ 17020}

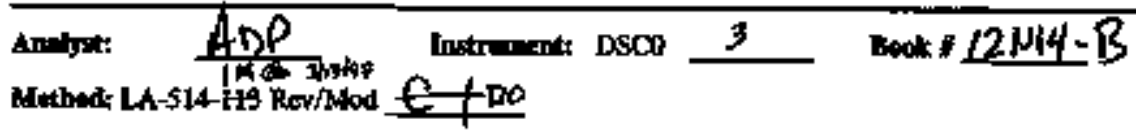

Werkliat Commedr DSC-0l, T-110 akm

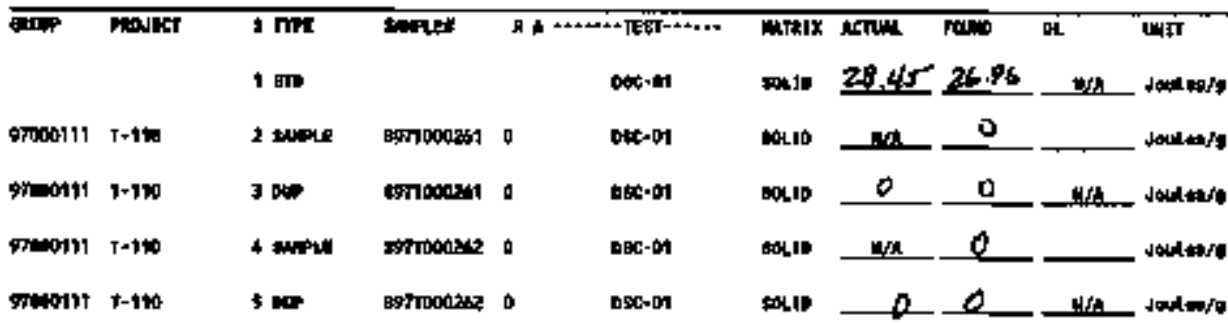

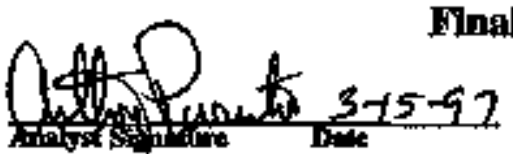

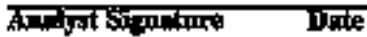

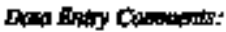

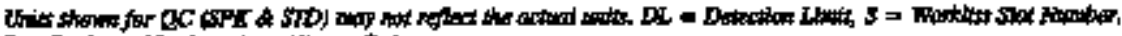

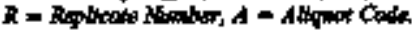


carve it ort

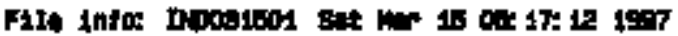

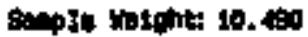

c.

oT0 satsde

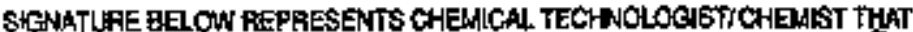

COUPLETEDNEFIFIED THE CALIBRATIONIANALYSIS ON PAGES $3 X_{6}$ TO 320 .

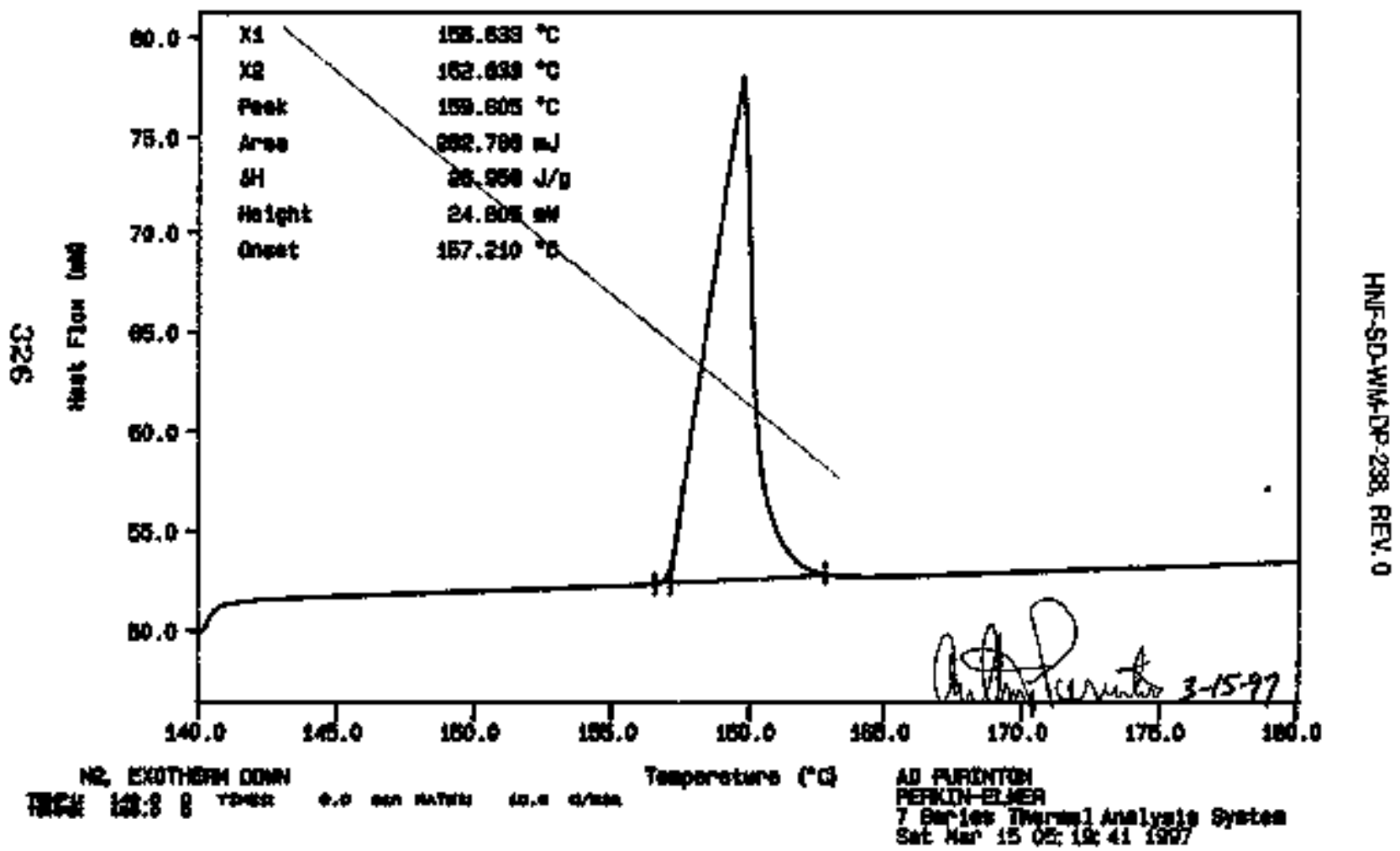


Durva 1 눌

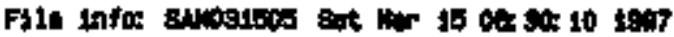

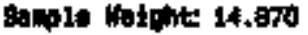

partototert but

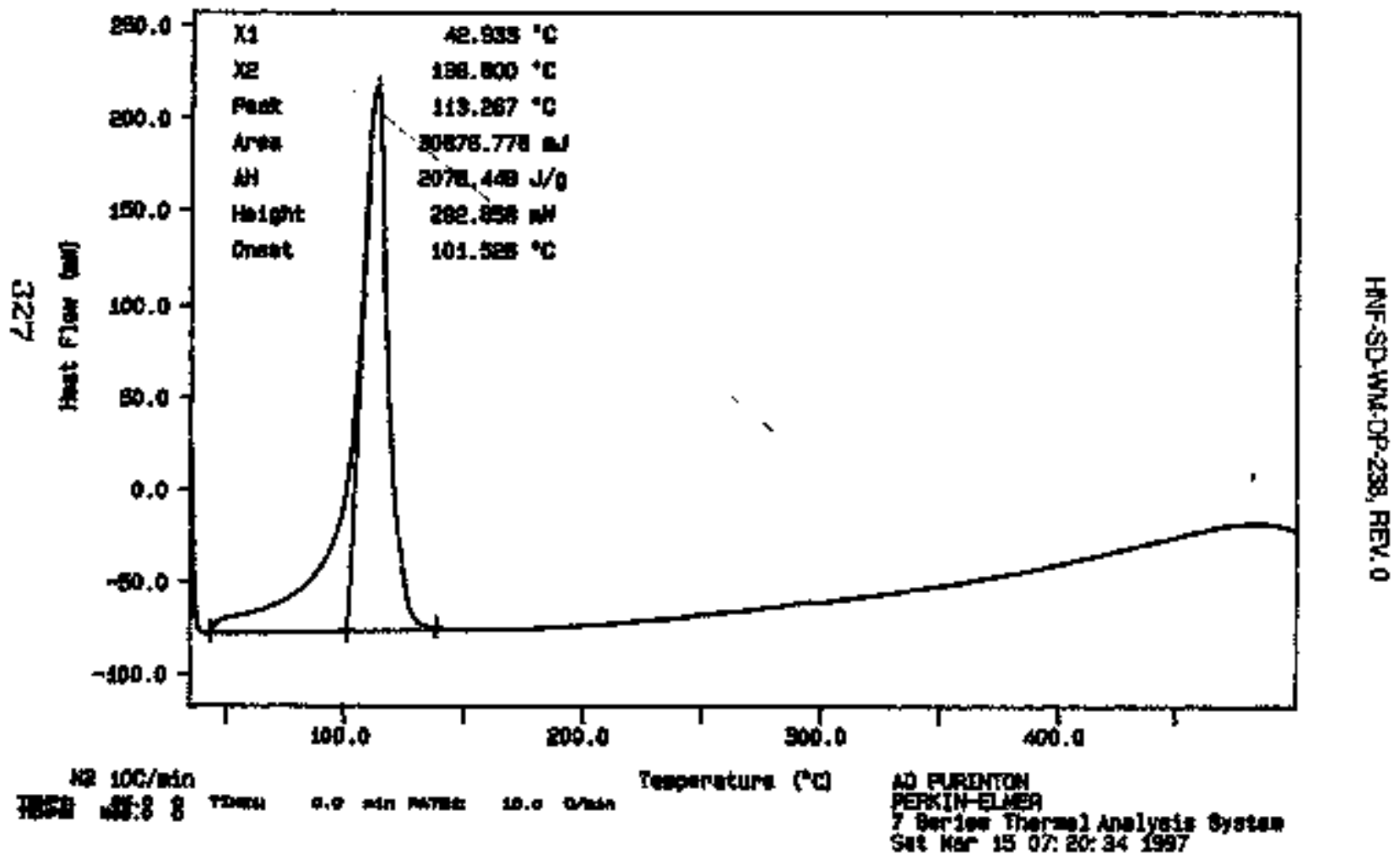


Dimb is bete

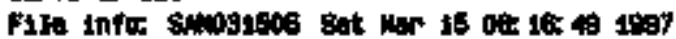

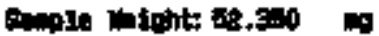

Eorpopect pur

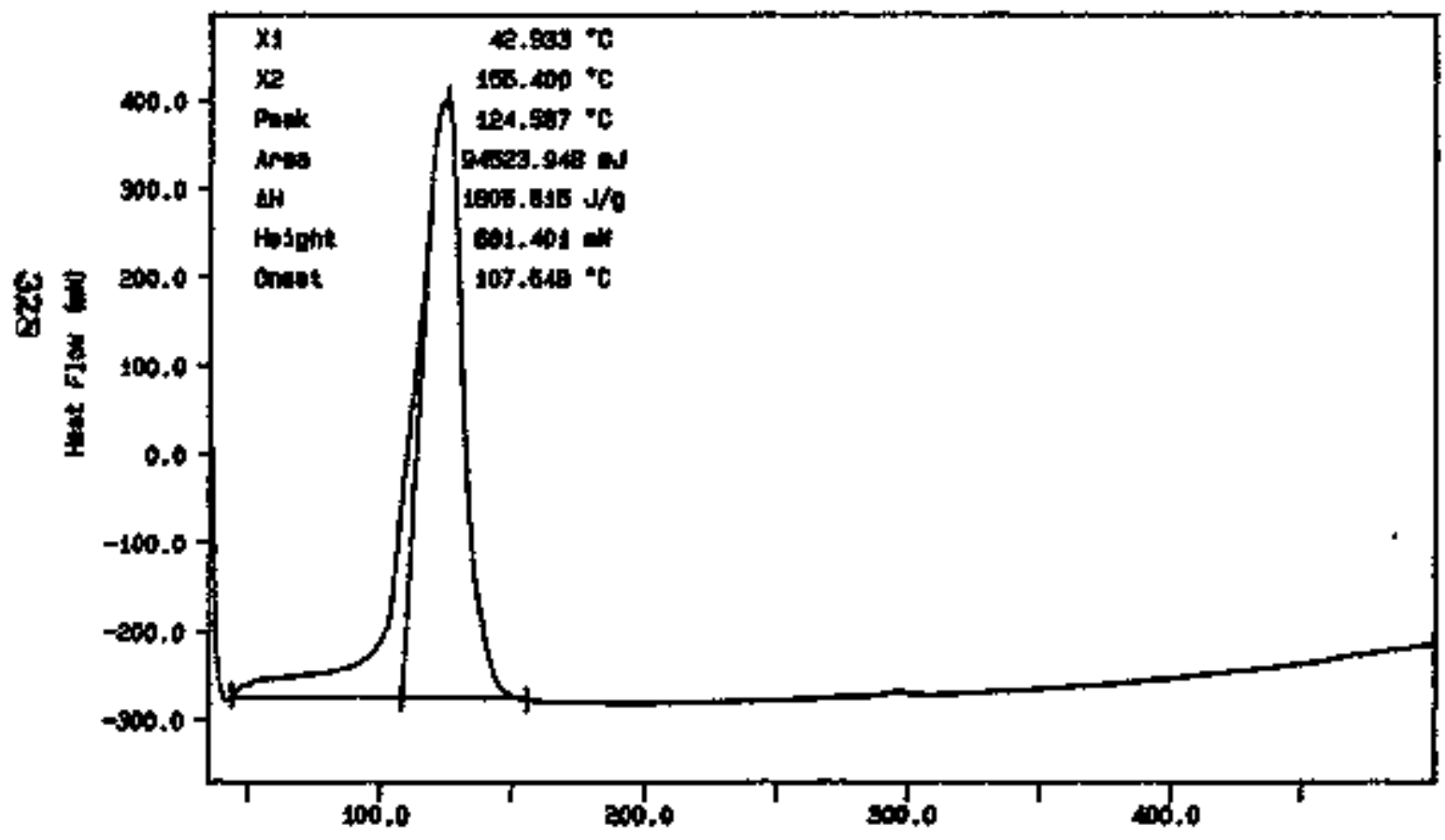

in toc/an

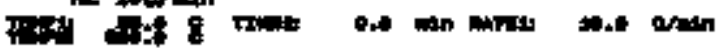

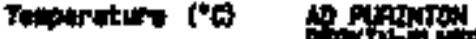


Corret to Dac

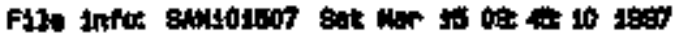

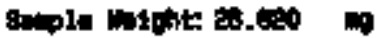

sarousece shi

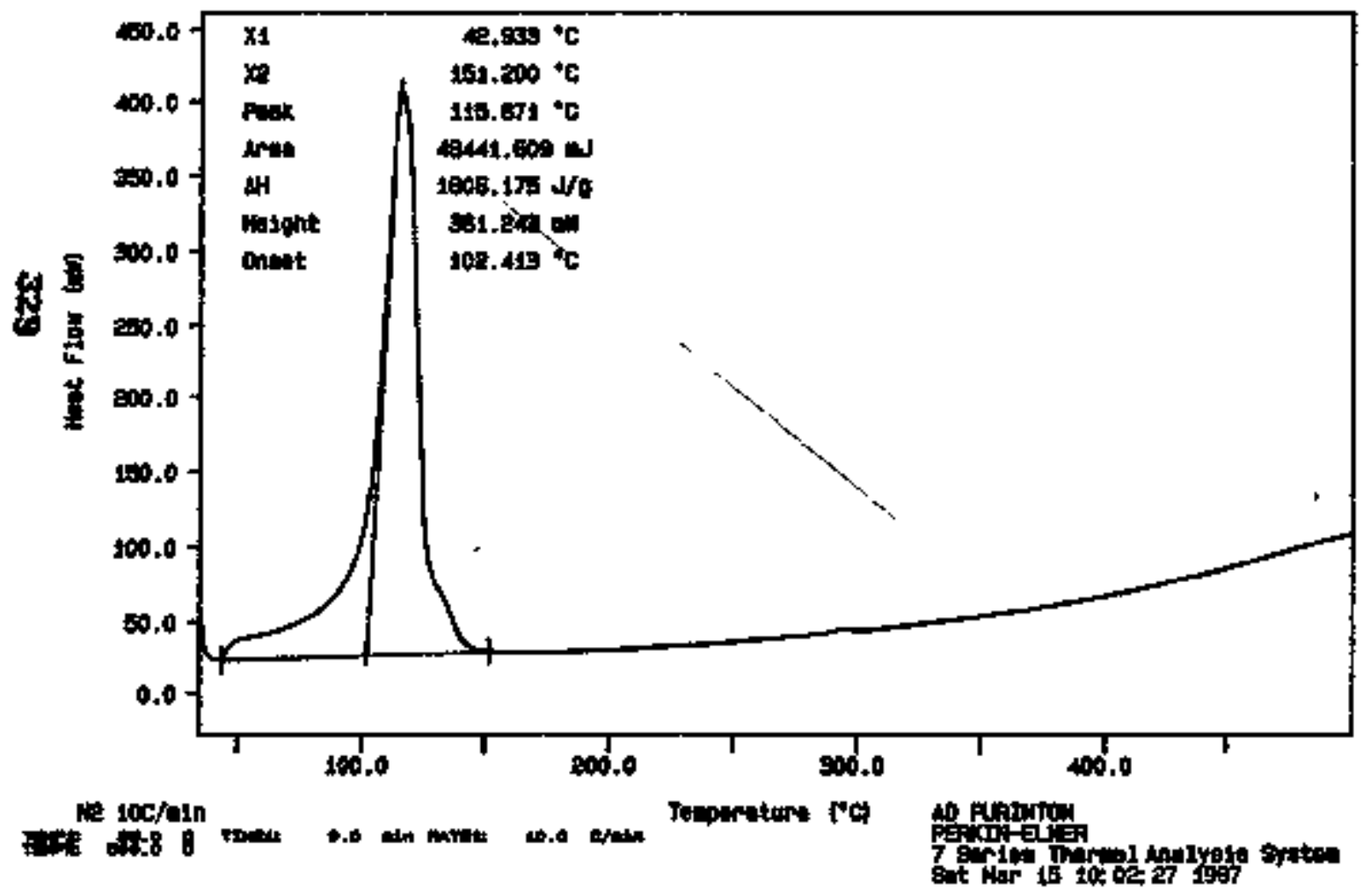


Dave it p:c

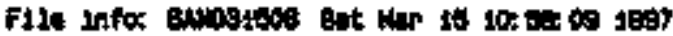

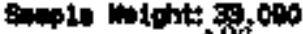

8mongar ent

$$
\text { DUP }
$$

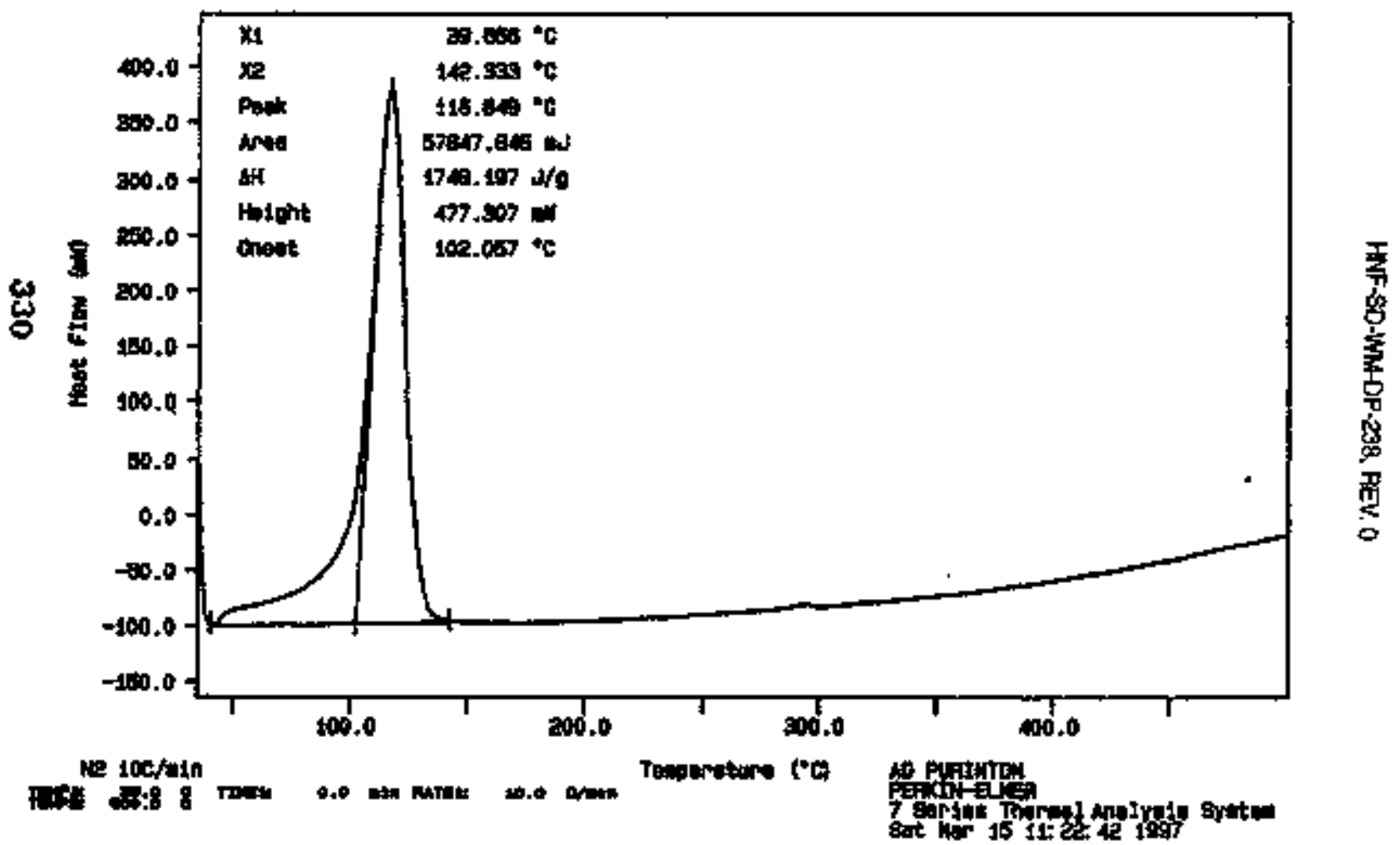




\section{LABCORE Data Entry Template for Worklist\# 17021}

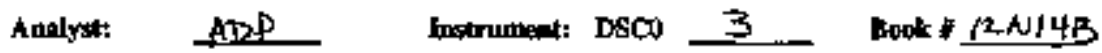

Nethod: LA-514-114 Rev/Mod, D $\sim$ D

Worklks Comment: T-110, DSC-01 skm

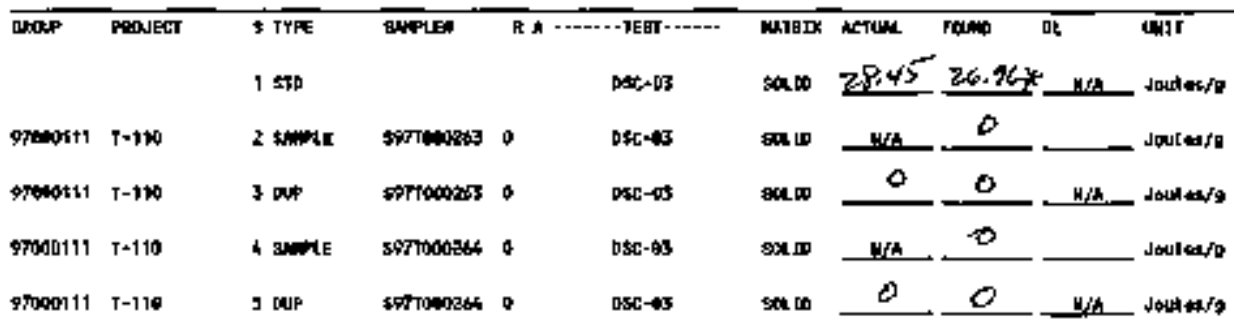

Final page for worklist \# 17021

Seefftacted for Sonature

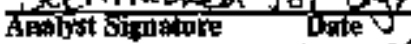

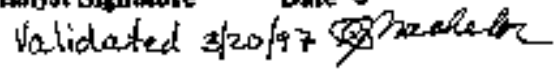

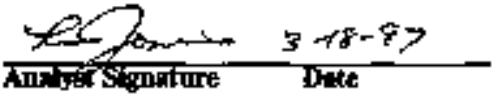

Daw Evity Conthers:

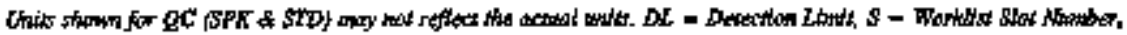

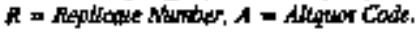


HNF-SD-WWDP-238, REV. 0

\section{WHC QCHISTORY TABLE EDIT SCREEV}

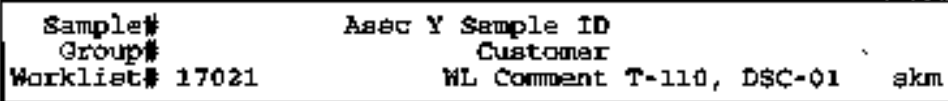

\begin{tabular}{|c|c|c|c|c|c|c|c|c|}
\hline Test & Matrx & Typ由 & Actual & Found & Yield & STAT & AnalDate & Deer \\
\hline $\begin{array}{l}0 s c-03 \\
08 C-03 \\
D \leq c-03\end{array}$ & $\begin{array}{l}\text { SOLID } \\
\text { SOLID } \\
\text { SOLID }\end{array}$ & $\begin{array}{l}\text { 6TD } \\
\text { DUP } \\
\text { DOP }\end{array}$ & $\begin{array}{l}38.45 \\
0 \\
0\end{array}$ & $\begin{array}{l}26.96 \\
0 \\
0\end{array}$ & $\begin{array}{l}94.7627 \\
0.0000 \\
0.0000\end{array}$ & $\begin{array}{l}\text { NEN } \\
\text { NEW } \\
\text { NEW }\end{array}$ & 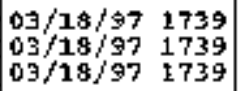 & $\begin{array}{l}r c j \\
r e j \\
\text { rej }\end{array}$ \\
\hline
\end{tabular}

SAve $\{F 12\}$ End $\{F\}\}$ 
HNF-SD-WM-DP-238, RAEV 0

WHC QCHISTORY TABLB EDIT SCREEN

\begin{tabular}{|c|c|c|c|c|c|c|c|c|}
\hline $\begin{array}{r}\text { Sann } \\
\text { Grol } \\
\text { Norkli }\end{array}$ & 1702 & & $\begin{array}{l}\text { Y } \mathrm{Y} \text { 9an } \\
\text { cht }\end{array}$ & $\begin{array}{l}\text { ID } \\
\text { omex } \\
\text { neint } T-\end{array}$ & 0. $\cos 5-01$ & kn & & \\
\hline Teqt & Matcrx & Туре & Actual & Found & Yield & \$rat & Analdate & tiser \\
\hline $\begin{array}{l}D S c-03 \\
D S c-03 \\
D S c-03\end{array}$ & $\begin{array}{l}\text { \$OLYo } \\
\text { \$OLY } \\
\text { sotro }\end{array}$ & $\begin{array}{l}\$ \mathrm{TT} \\
\text { aup } \\
\text { Dutp }\end{array}$ & $\begin{array}{l}23.45 \\
0 \\
0\end{array}$ & $\begin{array}{l}26.96 \hbar \\
0 \\
0\end{array}$ & $\begin{array}{l}94.7627 \\
0.0000 \\
0.0000\end{array}$ & $\begin{array}{l}\text { TEXT } \\
\text { MEW } \\
\text { WEW }\end{array}$ & 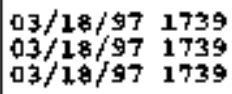 & $\begin{array}{l}r e j \\
r \in j \\
r q j\end{array}$ \\
\hline
\end{tabular}

Save (F12) End (F3) 


\section{Gurve Is Dec}

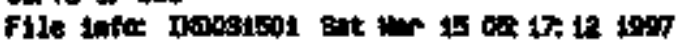

\section{Bundo lakpht 10.400}

$8 T 0$ fais4-9.

- 18 8TI IEA14-E TDOFIEOE

Hot blow tin

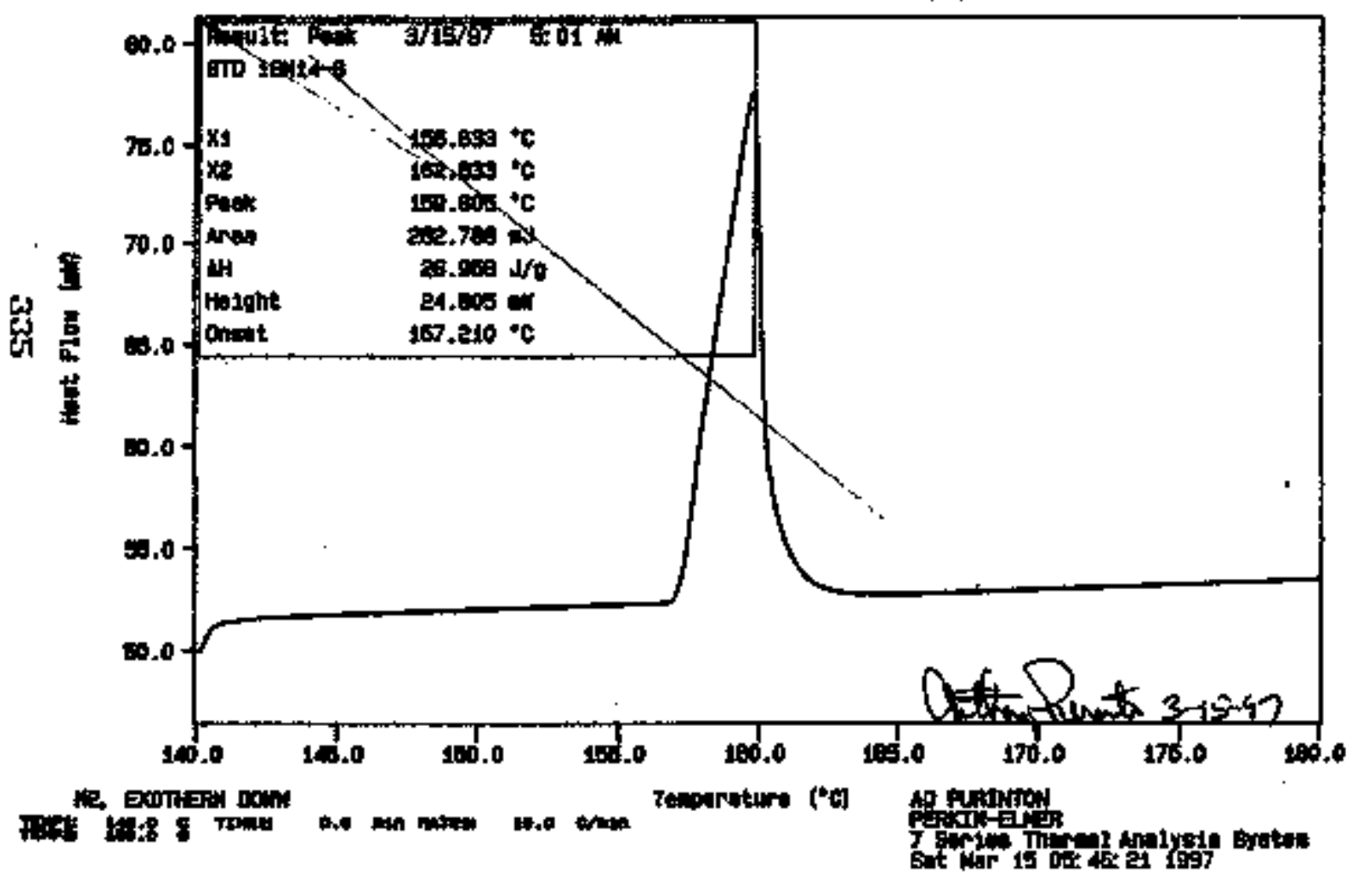




\section{Carve it Dex}

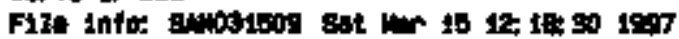

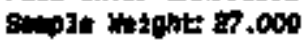

mprocosos sil

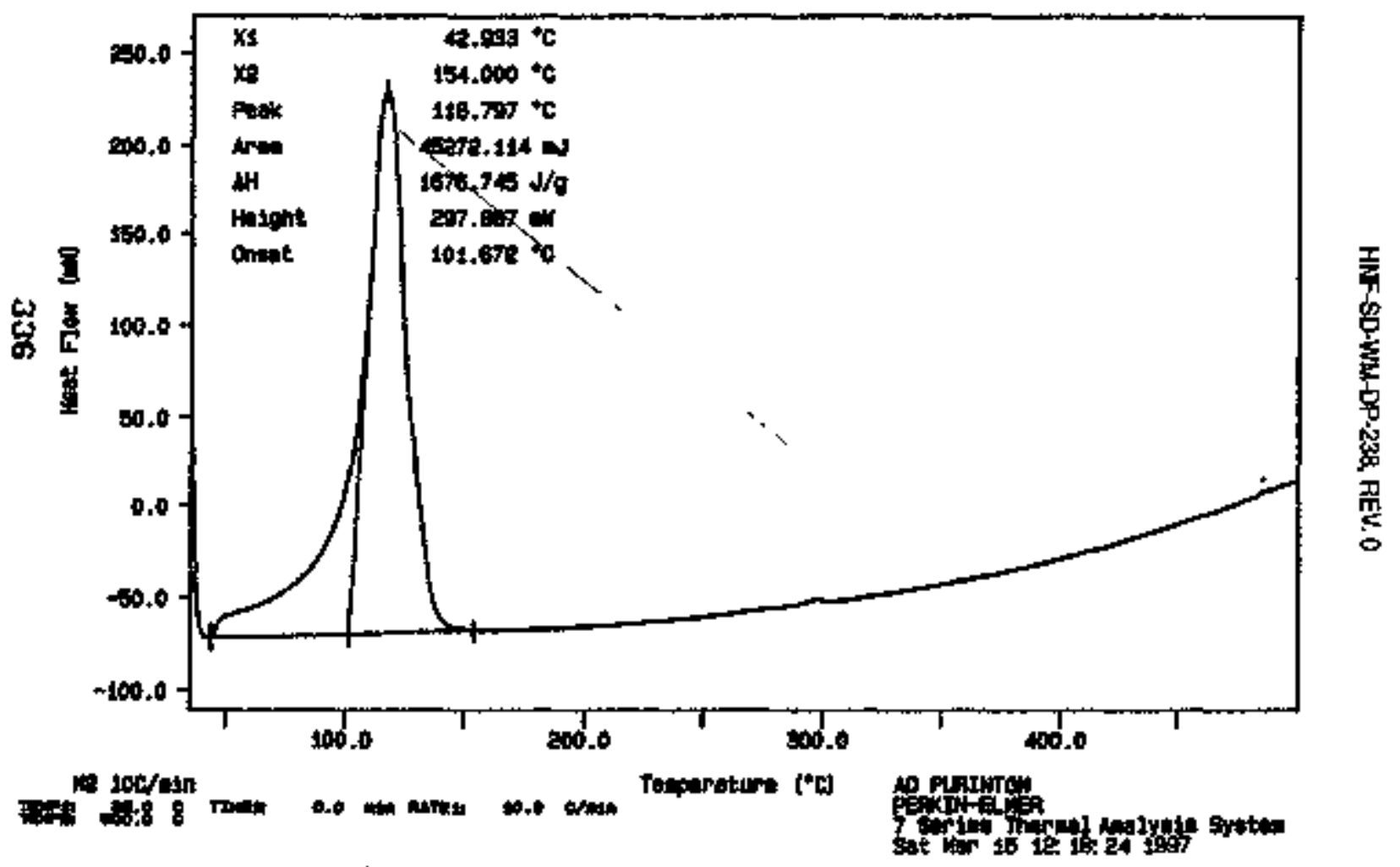


Erive it Dob

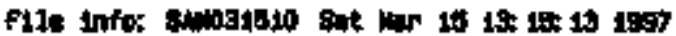

siple indoht 18.040

sorodots ET

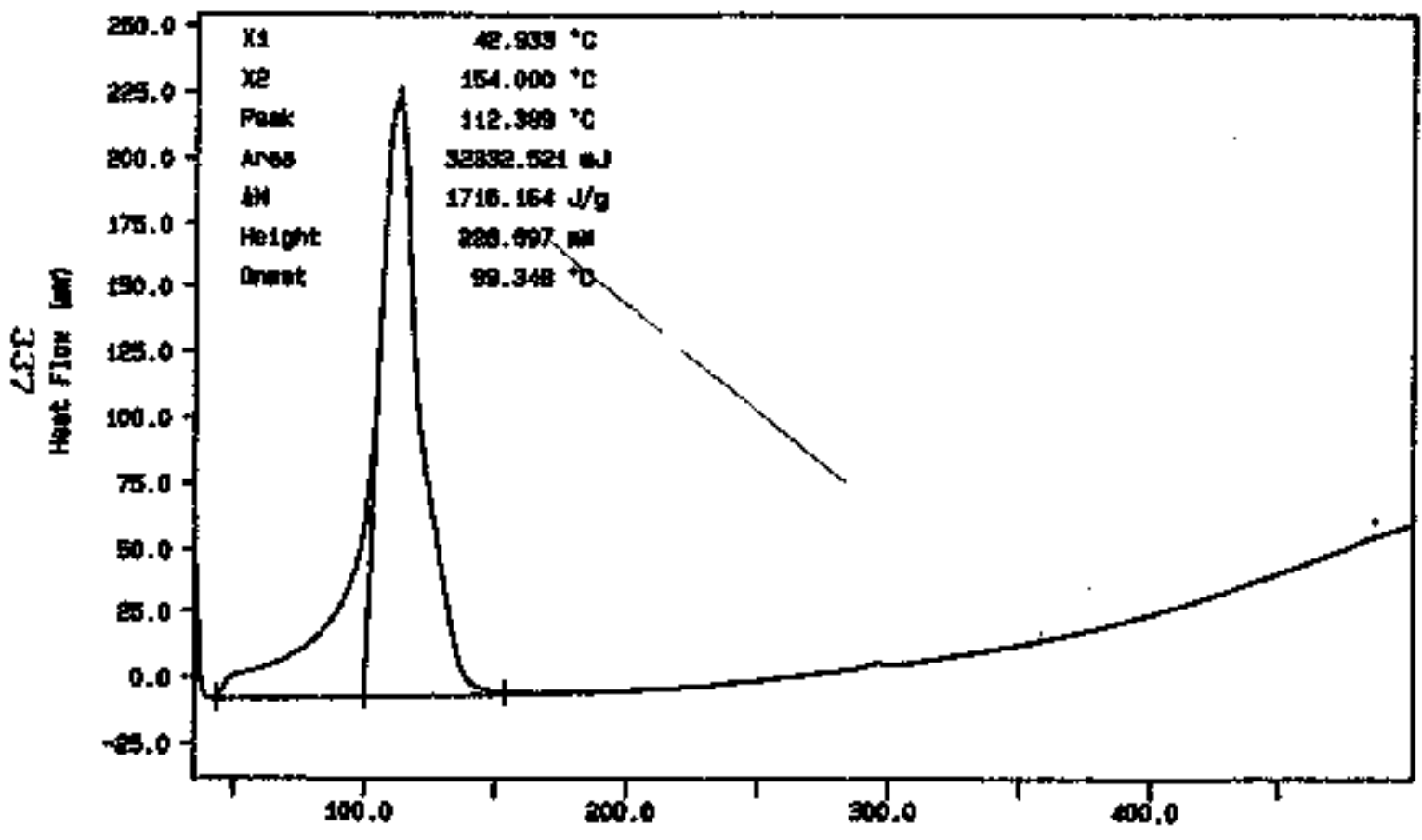

102 105/4a

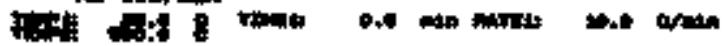

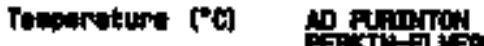


CrNo it net

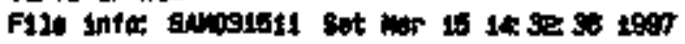

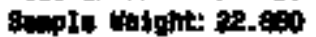

sertoonates tas

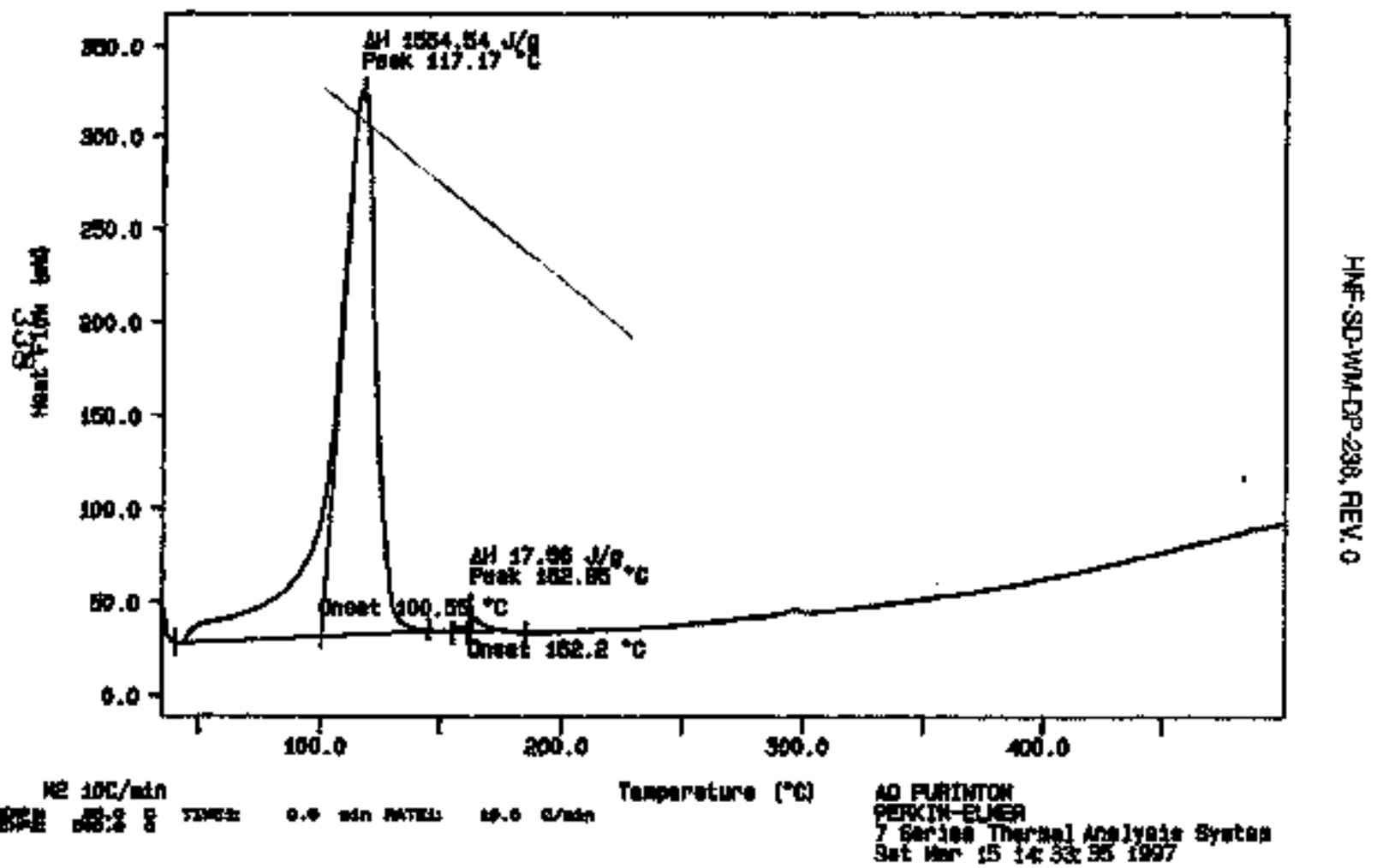


Curve it ose

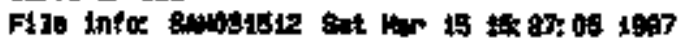

sapin rospht 3s.eso

onroodest dup

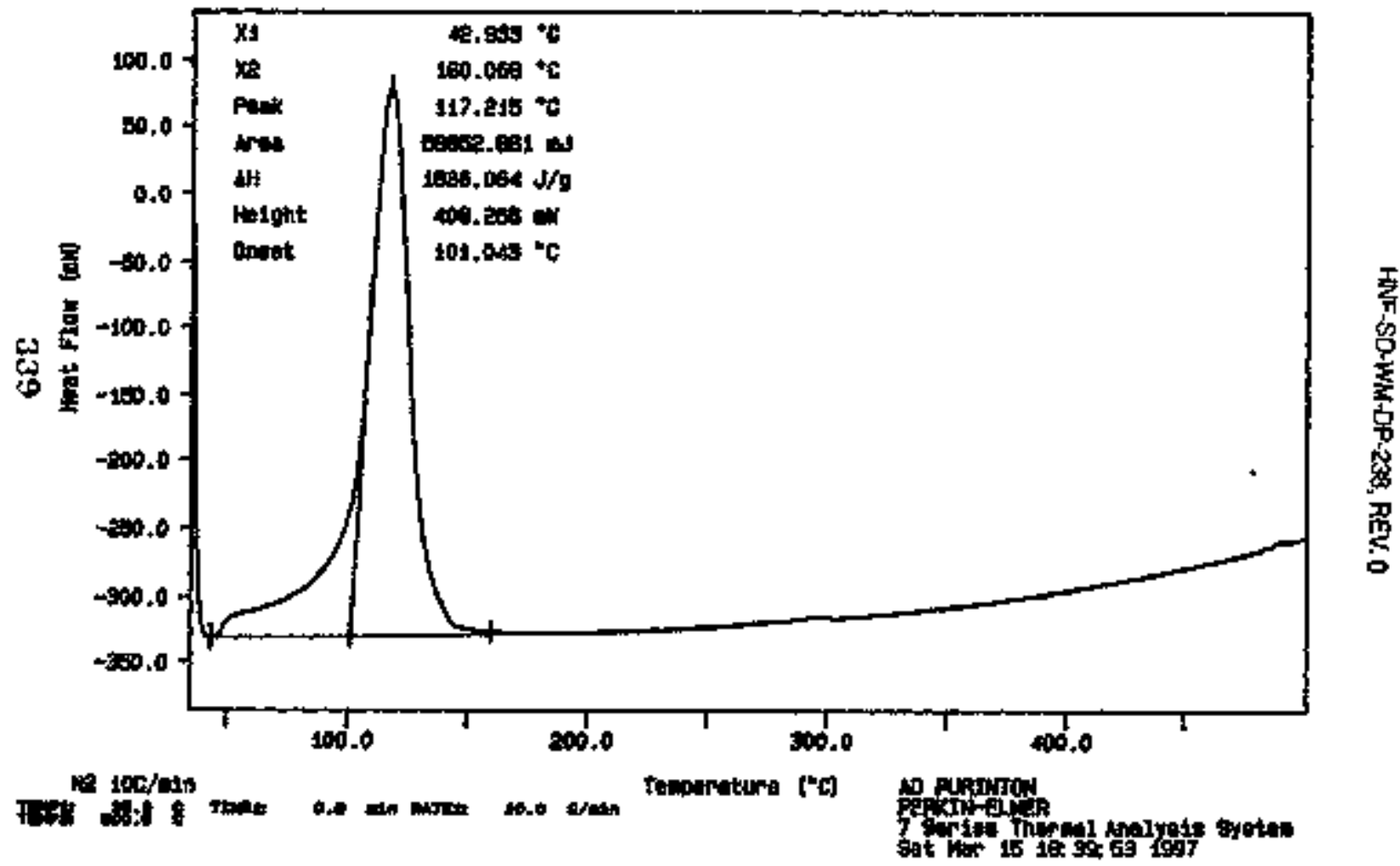




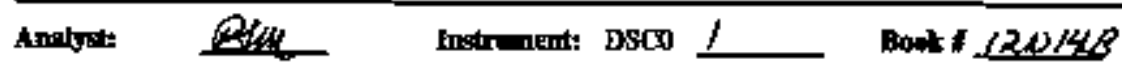

Metbod: LA-S14-113 Rew/Mod S.

Wortist Conmonte T-110, DSC-01 stom

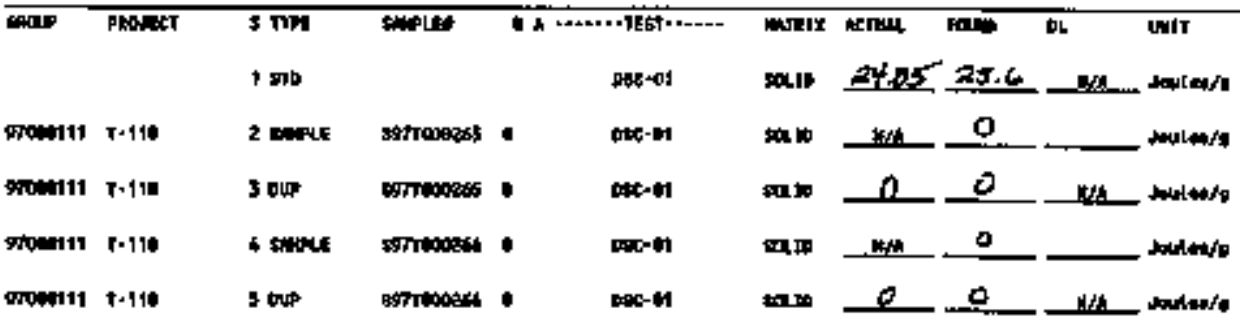

Final page for worklist 17022

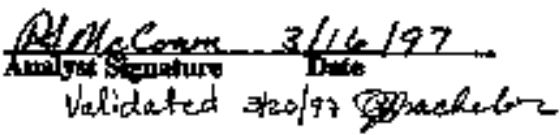

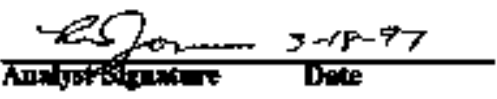

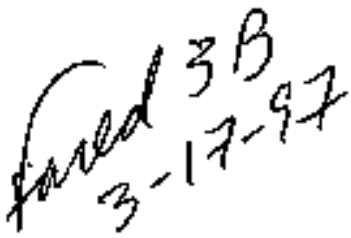

Deta Botry Chingis:

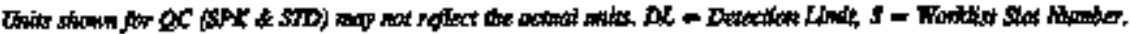

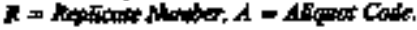




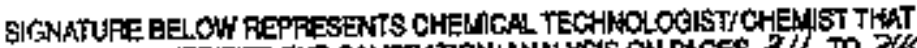

CONPIFTEDNVRAFEO THE CALBRATON/ANALYSAS ON PAGES XI/ TO 345

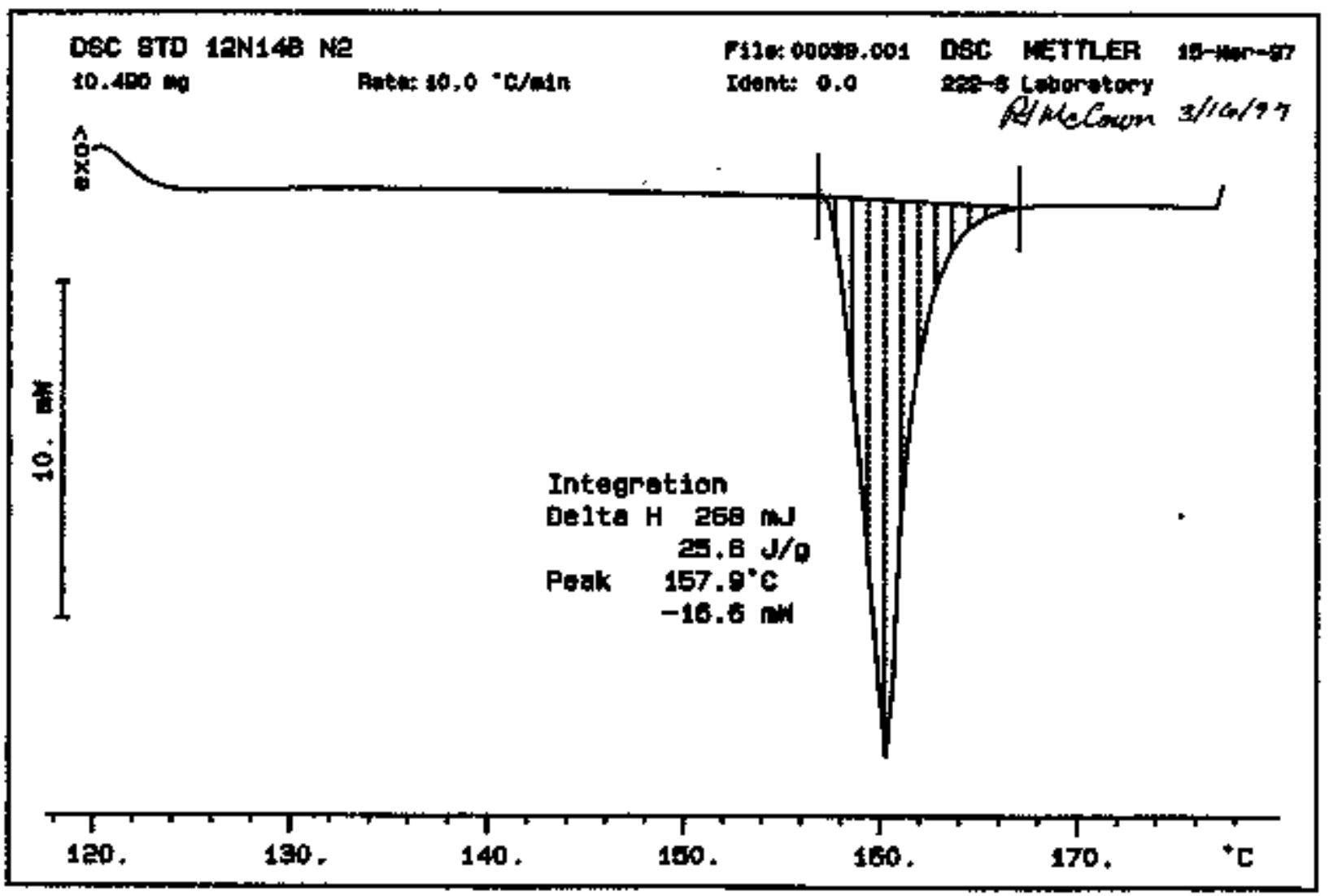




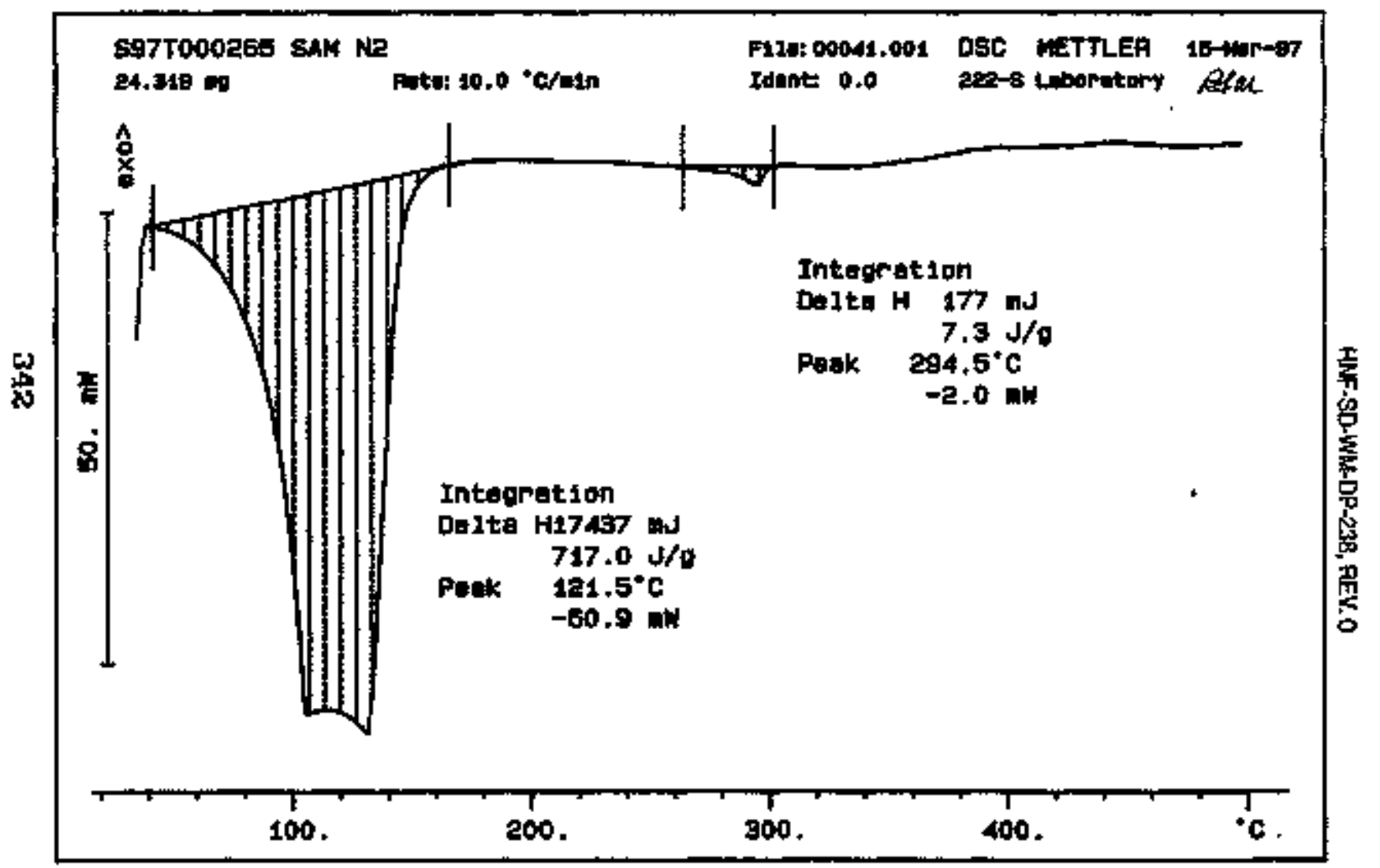




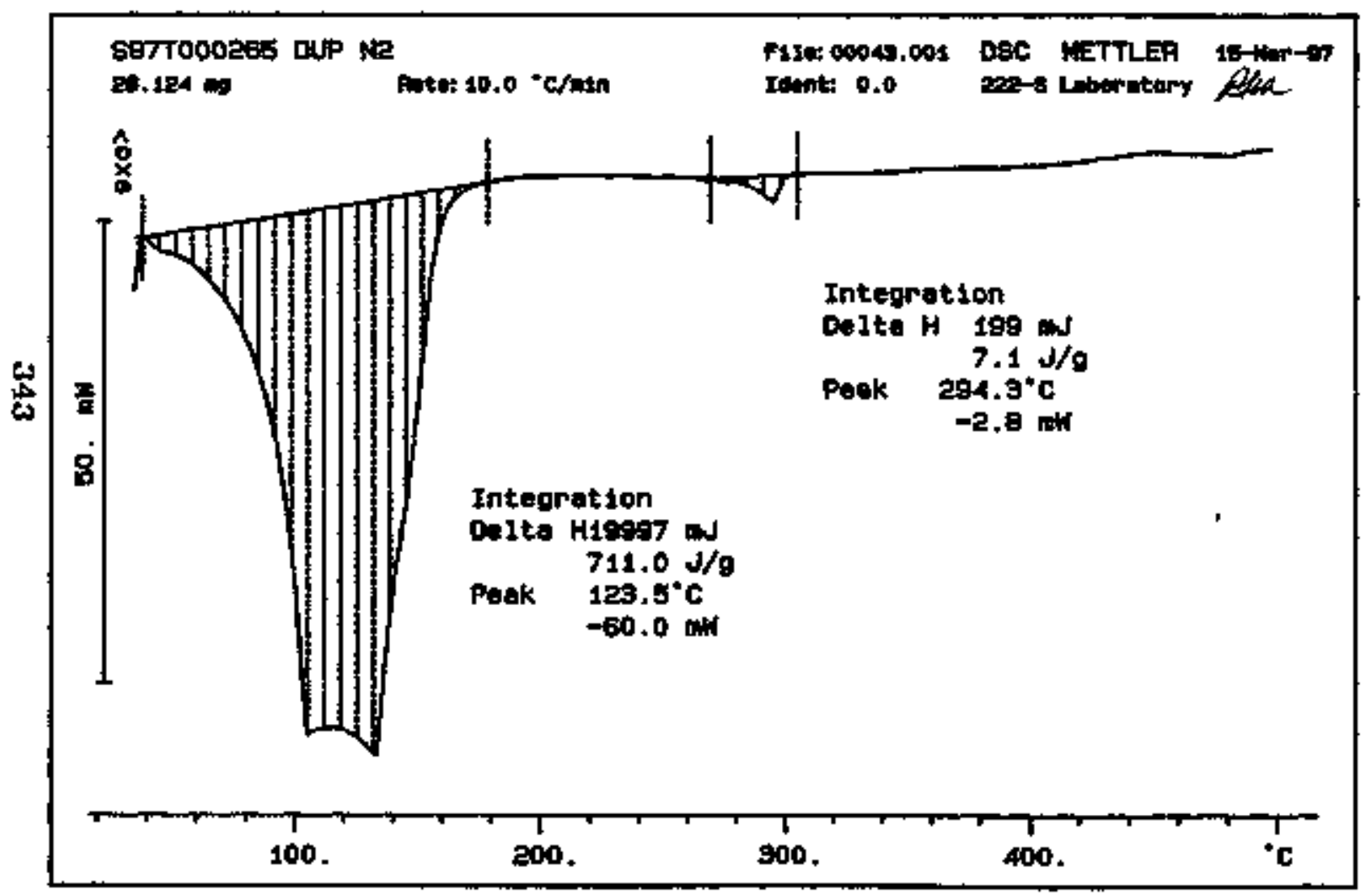




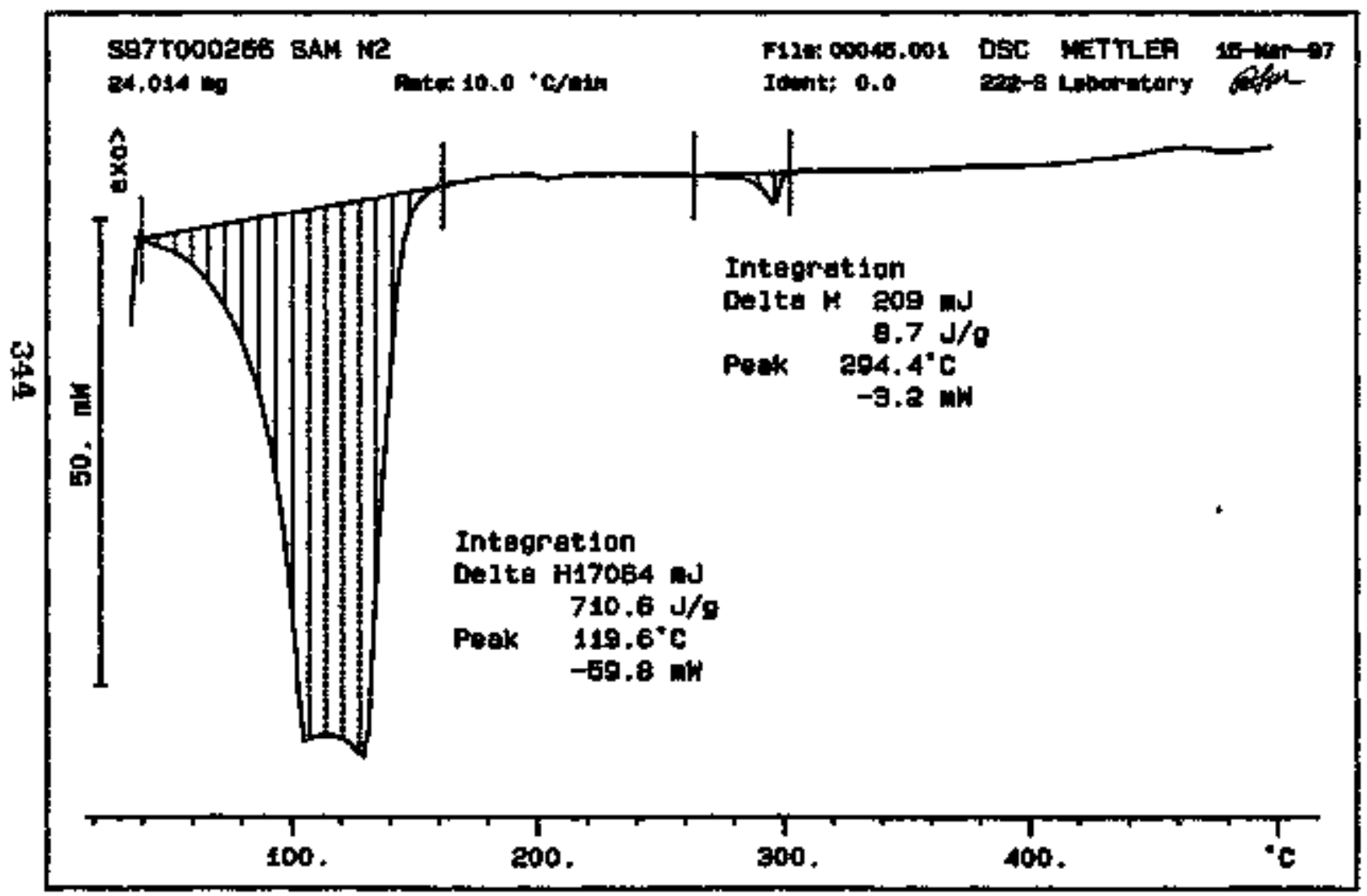




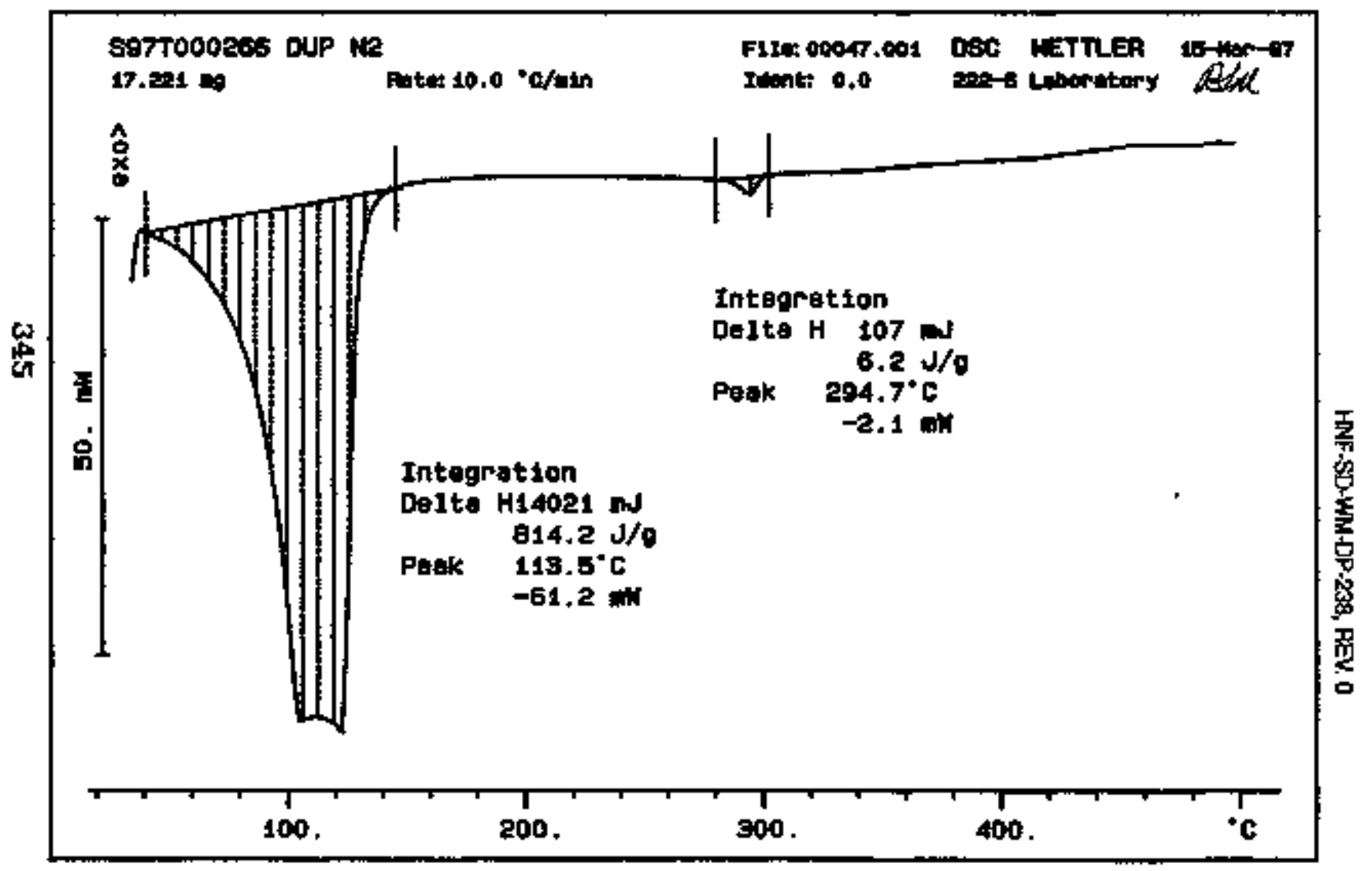




\section{LABCORE Data Entry Template for Worklist\# 17125}

Amplyat:

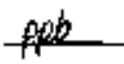

Instrumint: DSSOPI

Book * NA

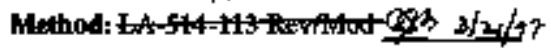

Werkltst CoIf-ent: DSC-02 T-110. ^PPB

\begin{tabular}{|c|c|c|c|c|c|c|c|c|c|c|}
\hline $\operatorname{tap}$ & PAOt:ct & 5 TtPE & ENATE & & 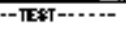 & WhTtIX & 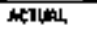 & Nound & $\mathrm{K}$ & Lktr \\
\hline 970000s & $T+110$ & 1 shine & sqrodoutd & D & $b s c=02$ & soc.10 & 다새 & ○ & & latingsing Dry \\
\hline 9700006s & $5+130$ & 2 tep & $\$ 9 / 1000+4$ & 0 & $00 x-\infty$ & $\sin 18$ & $D$ & $\phi$ & 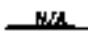 & recteas's Dry \\
\hline Troopes & 5,110 & 3 HWALt & 597000170 & 0 & bet:Des & stho & & & & Noultet 19 DH \\
\hline 97000003 & $T \cdot 110$ & t wh & $\$ W 1000170$ & 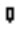 & $0 x c=\alpha$ & mol.10 & & & . & doul weds Dry \\
\hline Dropoces & $T-110$ & 5 supte & 9971000134 & 0 & $p+t-p R$ & SWID & H/h & 0 & & Lathers Dry \\
\hline 9roooces & $3-110$ & $6 D$ & Es7mopols4 & 0 & $D$ 技-DR & $\operatorname{sos} 10$ & $\varphi$ & 0 & $\underline{\Delta} / \boldsymbol{h}$ & Haty bry \\
\hline 9rooocel & $T-110$ & 7 BNept,E & Eqroboutrs & 0 & pac-oc & $50 \mathrm{C} . \mathrm{B}$ & sh & 0 & & foulenfe Dry \\
\hline Droooces & T- & 自 D大: & Bs7htopurs & a & bte-tat & Cotid & $\phi$ & 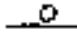 & N & douteds Dry \\
\hline \$ropo191 & T-110 & 9 aNex & Bghooctid & 0 & poc-0d & 800,10 & Eta & 0 & & fouledry ory \\
\hline 97000111 & $r-110$ & 10 난 & Bgrtotonas1 & $\theta$ & Gre-az & BOL.ID & 0 & D & 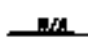 & Joukl befo DrV \\
\hline גנה & $r-110$ & 11 FAPUE & Bstroptates? & 0 & Bes.물 & BOLID & Dif & 0 & & Joutests Dry \\
\hline कroodi11 & $T-110$ & 12 pus & BAno00dat & 0 & $\cos -\phi$ & BDL.JD & 0 & te & m.ts & louteds dey \\
\hline Dropol11 & $r=110$ & IJ SNPLE & sortactosi & $\bullet$ & 1000 & 加ID & $M$ & 0 & & doulata pry \\
\hline crobouta & $r+110$ & 16 Dop & tortototess & 0 & Bम.tQ & 50L.L10 & 0 & $\theta$ & . & Joed $* t / \phi$ pry \\
\hline 97000111 & $T=110$ & 15 EMPLE & 59710001044 & 0 & DEx-QR & sol.10 & Itta & 6 & & Maleots ory \\
\hline 90000111 & $t \cdot 110$ & $t$ & sqriodoes4 & 0 & 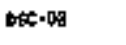 & Sol.10 & 0 & v & $\mathbf{n} / \mathbf{k}$ & Jarltwita bry \\
\hline 9rood111 & $t=110$ & 17 HPla & 5971000265 & 0 & $0 x+02$ & SLID & .....th & 0 & & 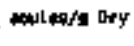 \\
\hline PTobol11 & t-110 & 18 DP & sortopos: 5 & 0 & 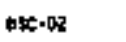 & $\$ 0 L 10$ & 0 & $\vartheta$ & NA & dechogle Dry \\
\hline
\end{tabular}

Darta Fitrify Combenss:

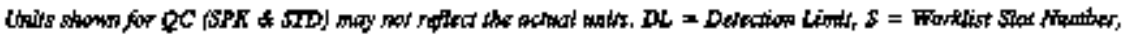

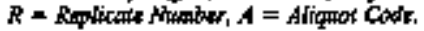




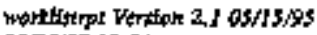

HANF-SD-WH-DF-238, FEV 0

as/20197 10-54

LABCORE Data Entry Template for Worklistä 17125

\begin{tabular}{|c|c|c|c|c|c|c|c|c|c|}
\hline WROCF & PADNACT & 5 Tine & 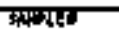 & 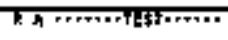 & Mhrerx & 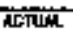 & FOMO & $\overline{D E}$ & WणII \\
\hline 9 soot11 & $T-110$ & 17 sact & 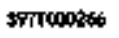 & $D S C-62$ & 娥比 & 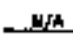 & 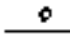 & & Joukesta ba \\
\hline 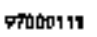 & $T-110$ & 20 ar & 5971000364 & 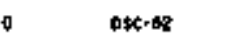 & 20.18 & 0 & $p$ & & towlez/g Dry \\
\hline
\end{tabular}

\section{Final page for worklist \#17125}




\section{LABCORE Data Entry Template for Worklist 16707}

Anolyst SMF Instruent: TGA0 $3 \quad$ Book \# 9 7ho-

Method: LA-514-114 REW/MOd D-O

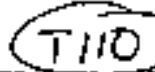

Worklbt Comment: TGA-01 POR T-110 GRAB (RUN UNDER FTTROFEN)TERLIQSTD RTS

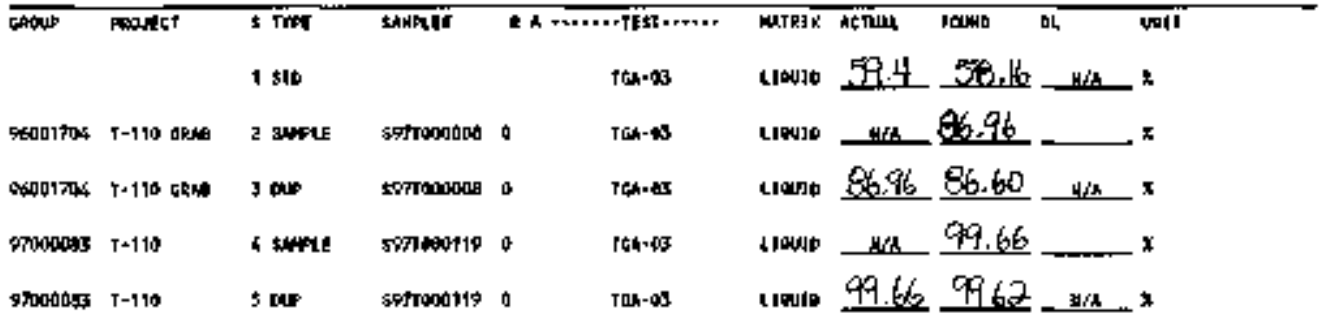

Final page for worklistif 16707

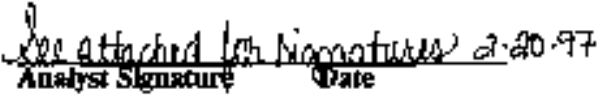

Verified Nalidatad by

Blandinta

Valengacele $\bigcup^{2 \cdot 20} \cdot 97$

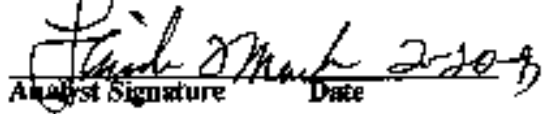




\section{LABCORE Data Entry Template for Worklist\# 16707}

Pogat $\quad t$

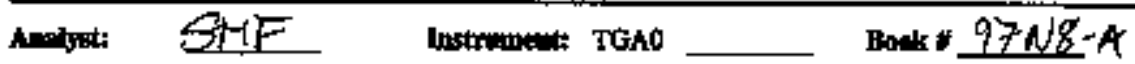

Methads LA-S60-112 RevaAod

Woklist Comm:at TCA-0I FOR T-110 GRAB (RUN UNDER NITROGENJTERLYOSTP RTS

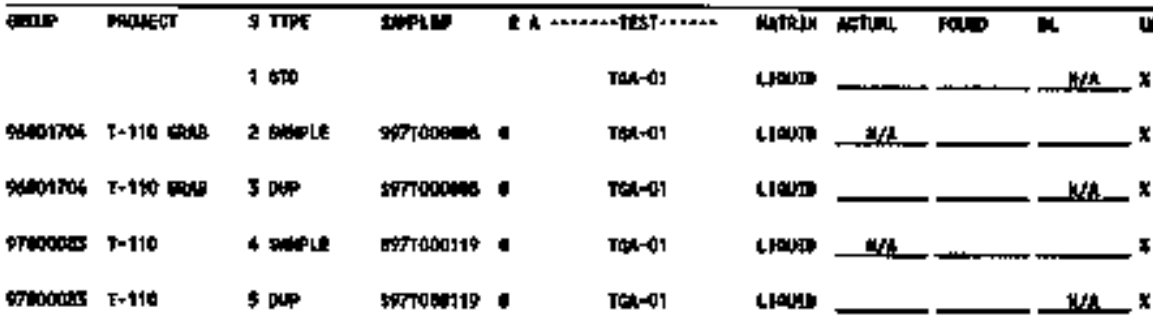

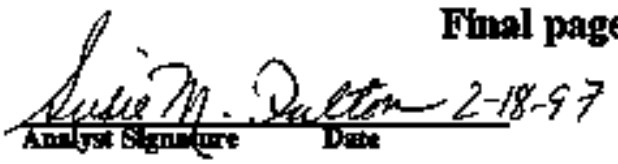

TEA 03 instruments

lith wod

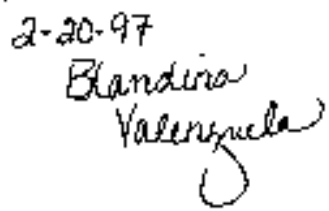

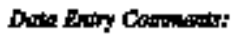

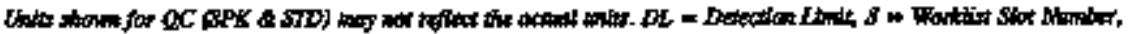

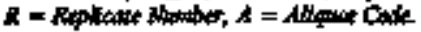


Durye it Th

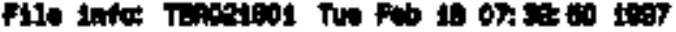

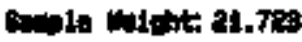

Tex 5 sto stiva-d

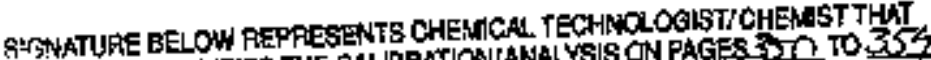
SHSTATUNE BELOW RET THE CNIBRATIONIANALYSIS ON PAGES STO TO 354

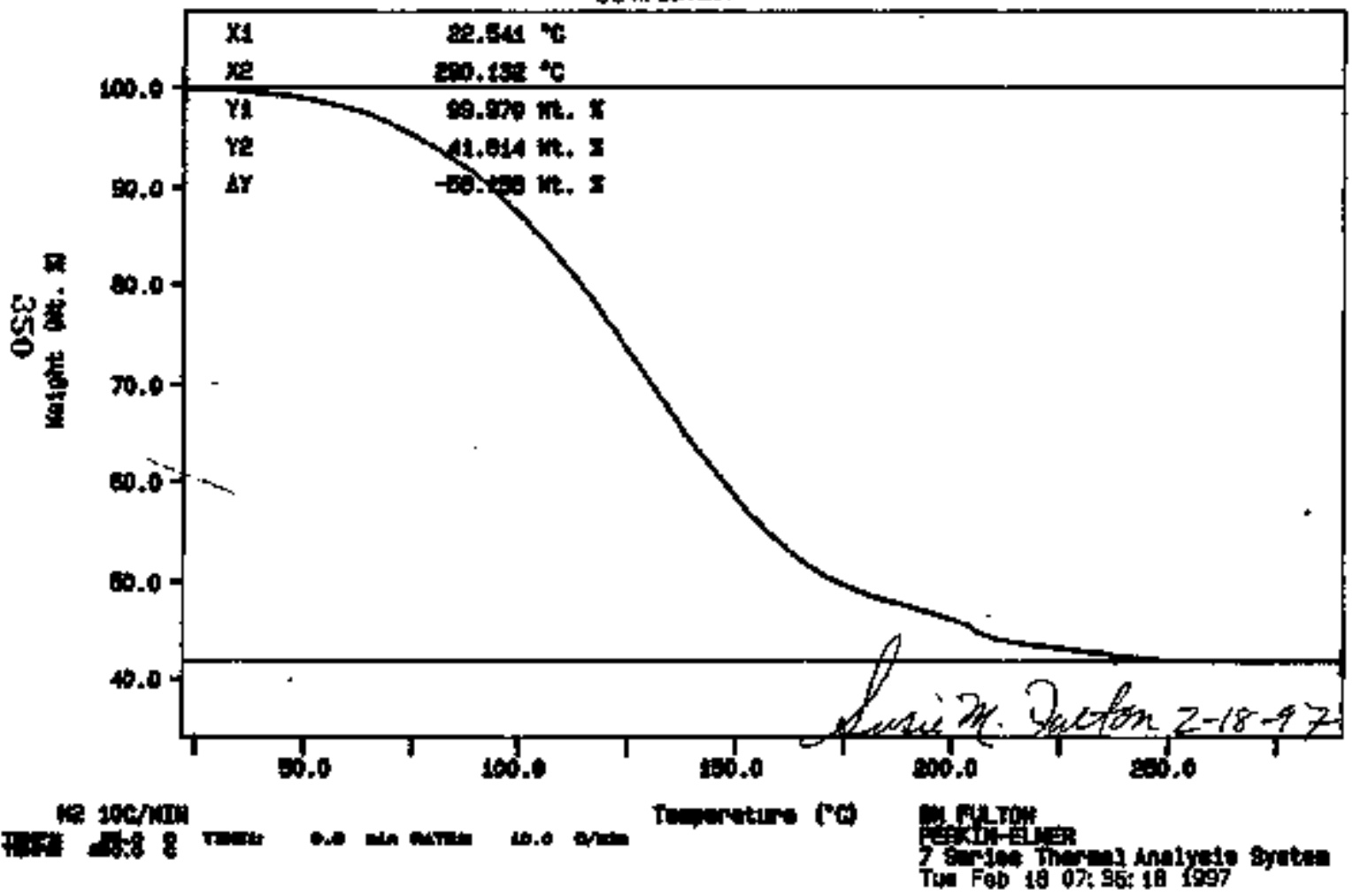




\section{Crive \& $T$ Th}

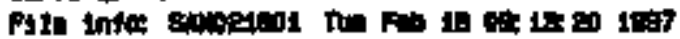

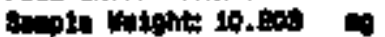

gropoopo.

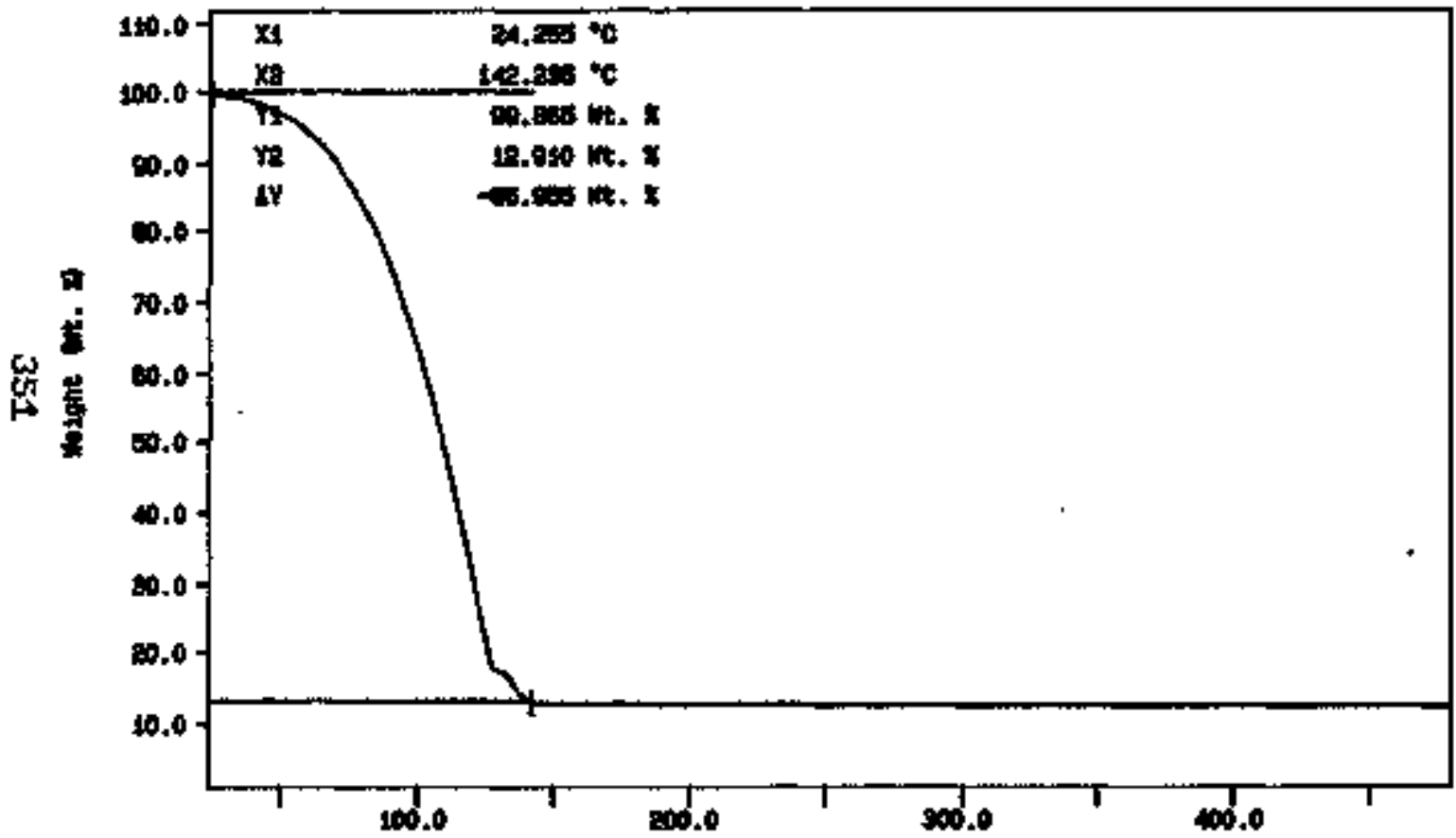


Dory is Th

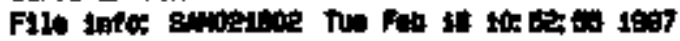

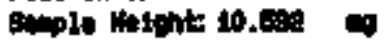

artoseres olp

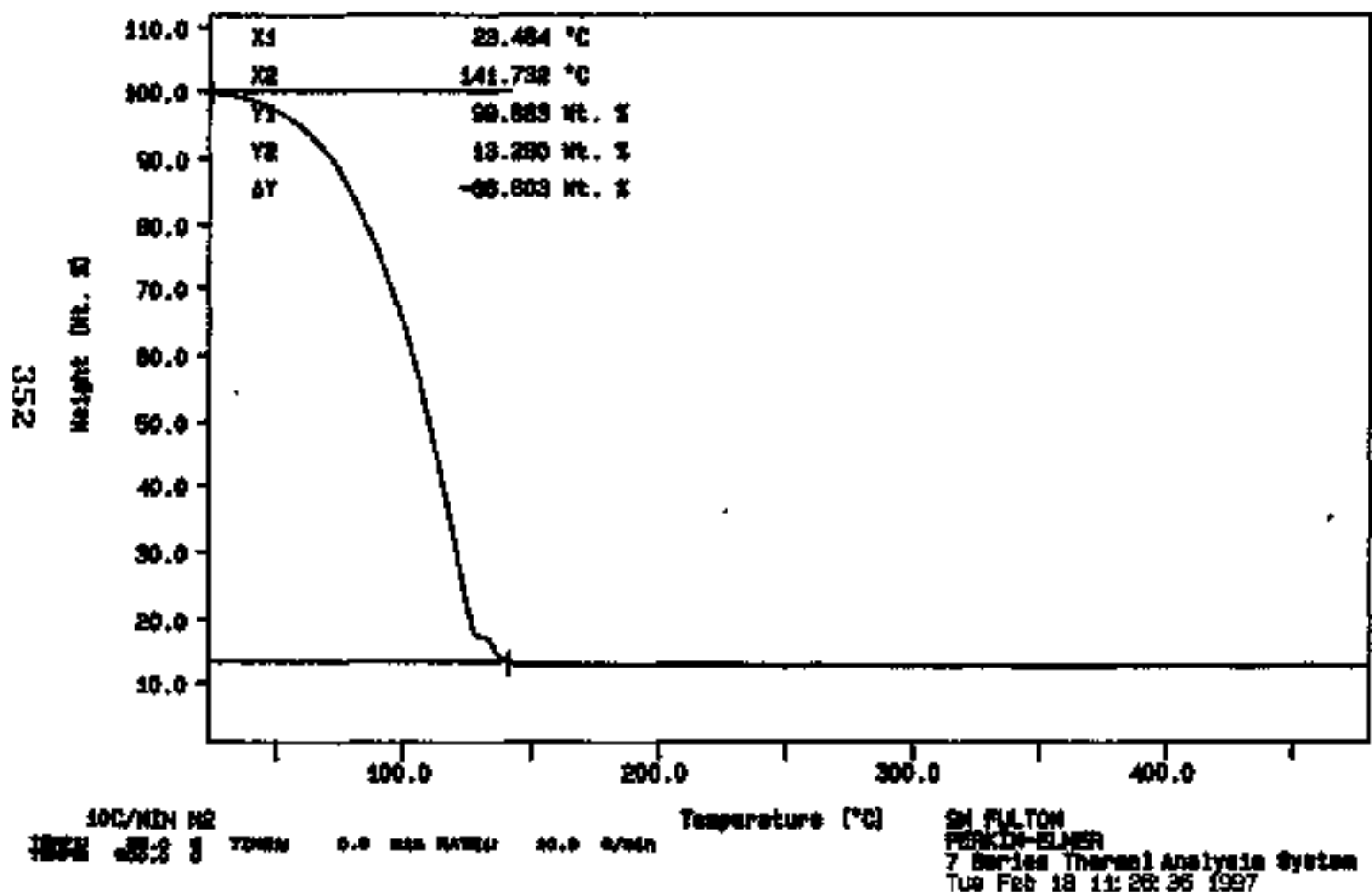


Cume to Tad

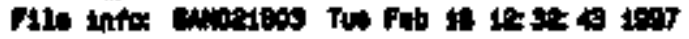

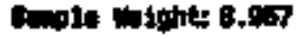

ExTo0044

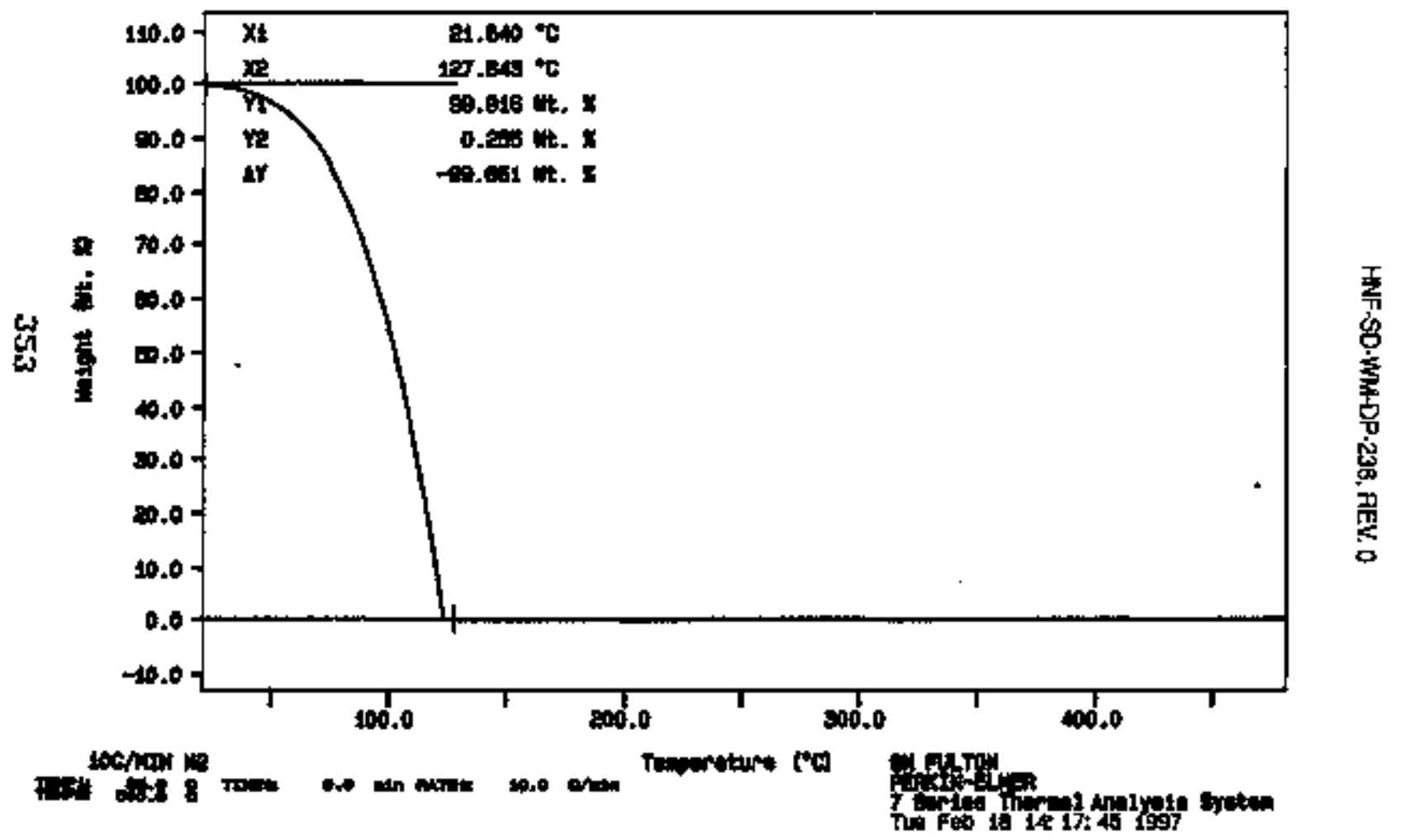


Gurve tt ras

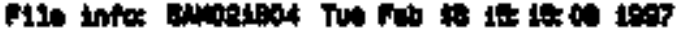

foplo lingents 9.000

S9700018 Dip

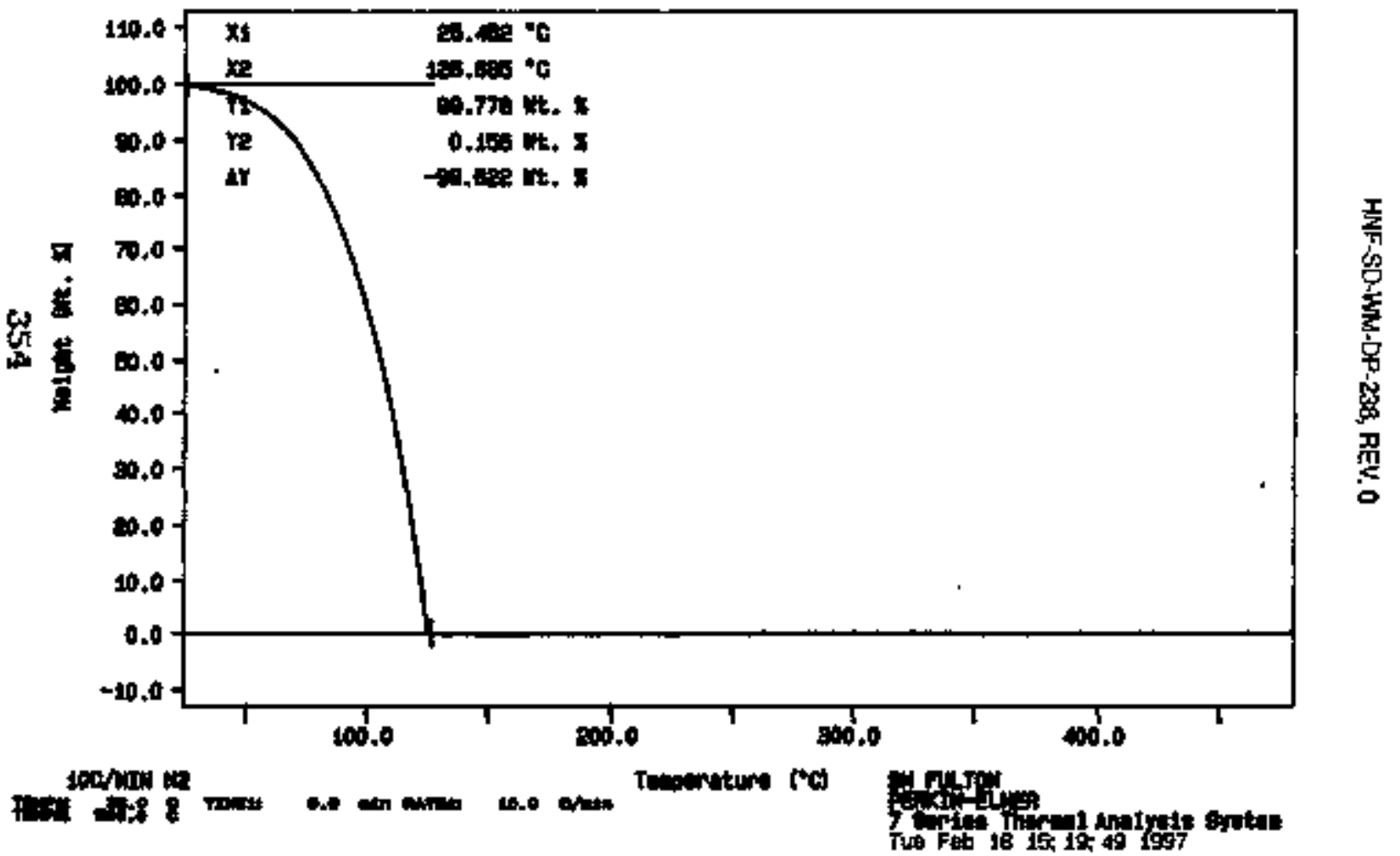




\section{LABCORR Data Entry Template for Worklist" 16709}

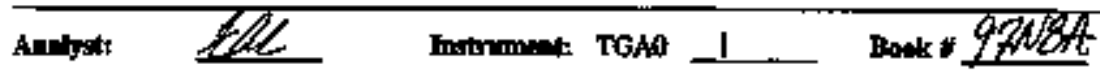

Methad: LA-560-112 Rev/Mad D-O

Wodklist Commont: TGA-01 FOR T-110(RUN UNDER NTTROGEN)TERLIQ RTS

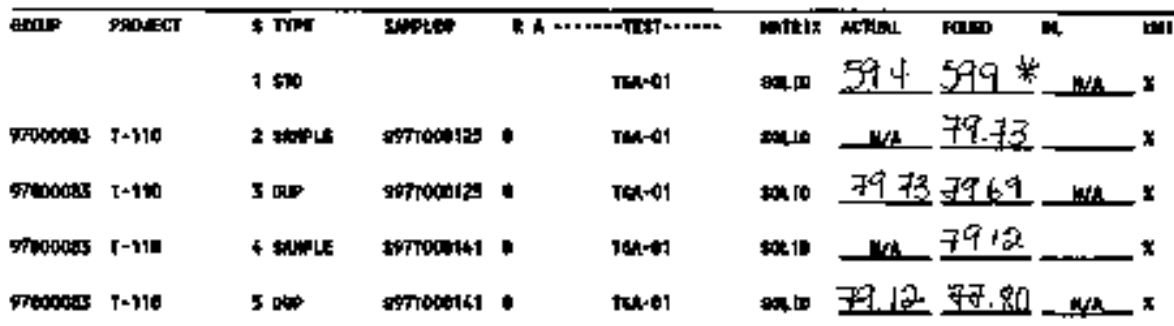

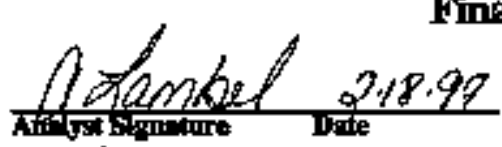

Verifud saleded bo

Suldurdere

Whetroces 20097

Final page for worklist \# 16709

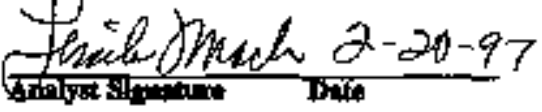

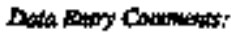

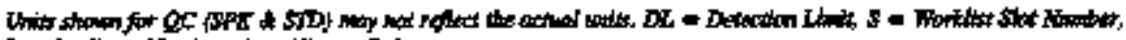

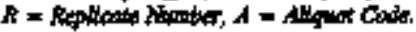




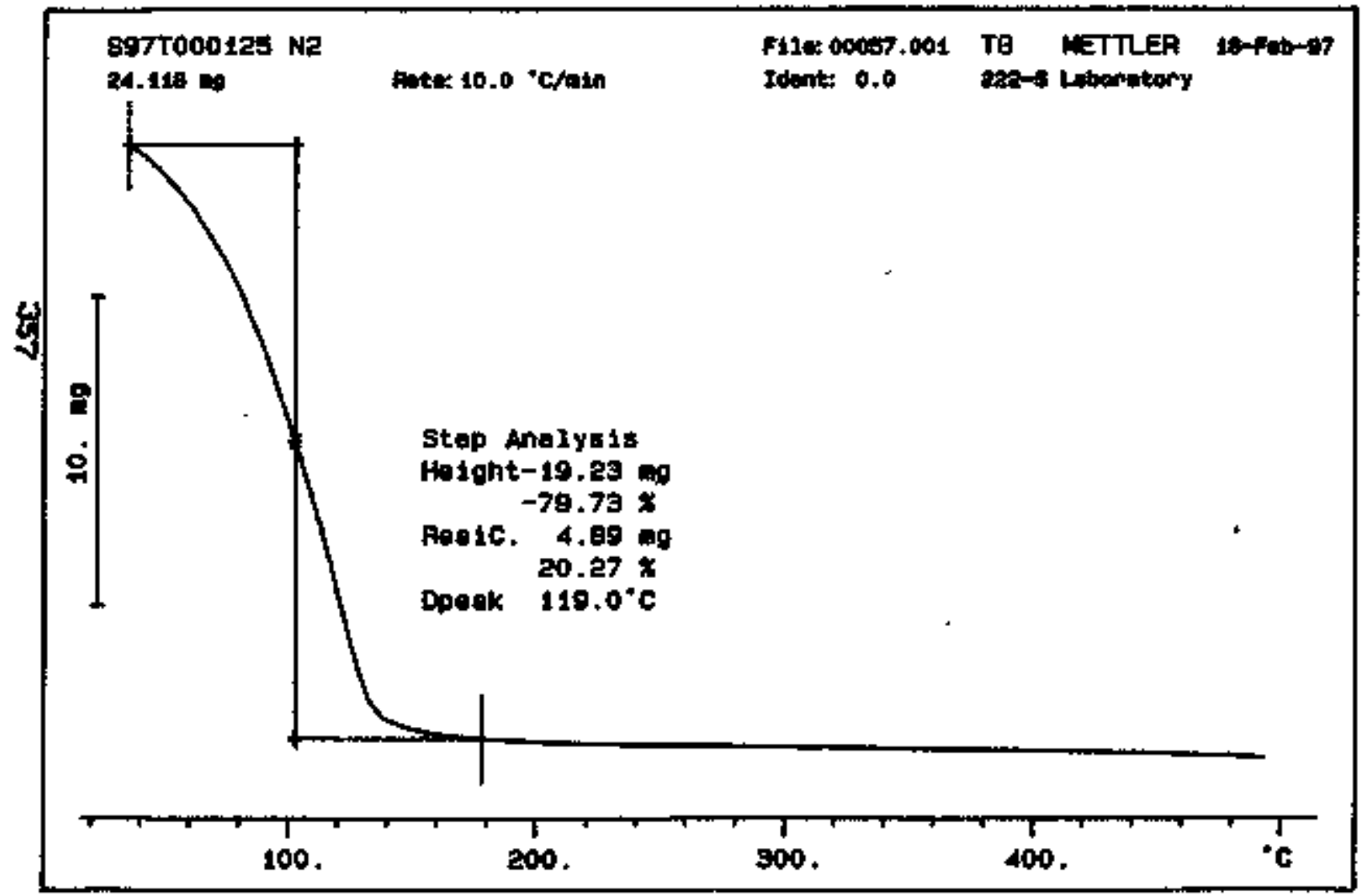

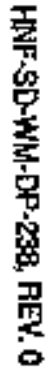




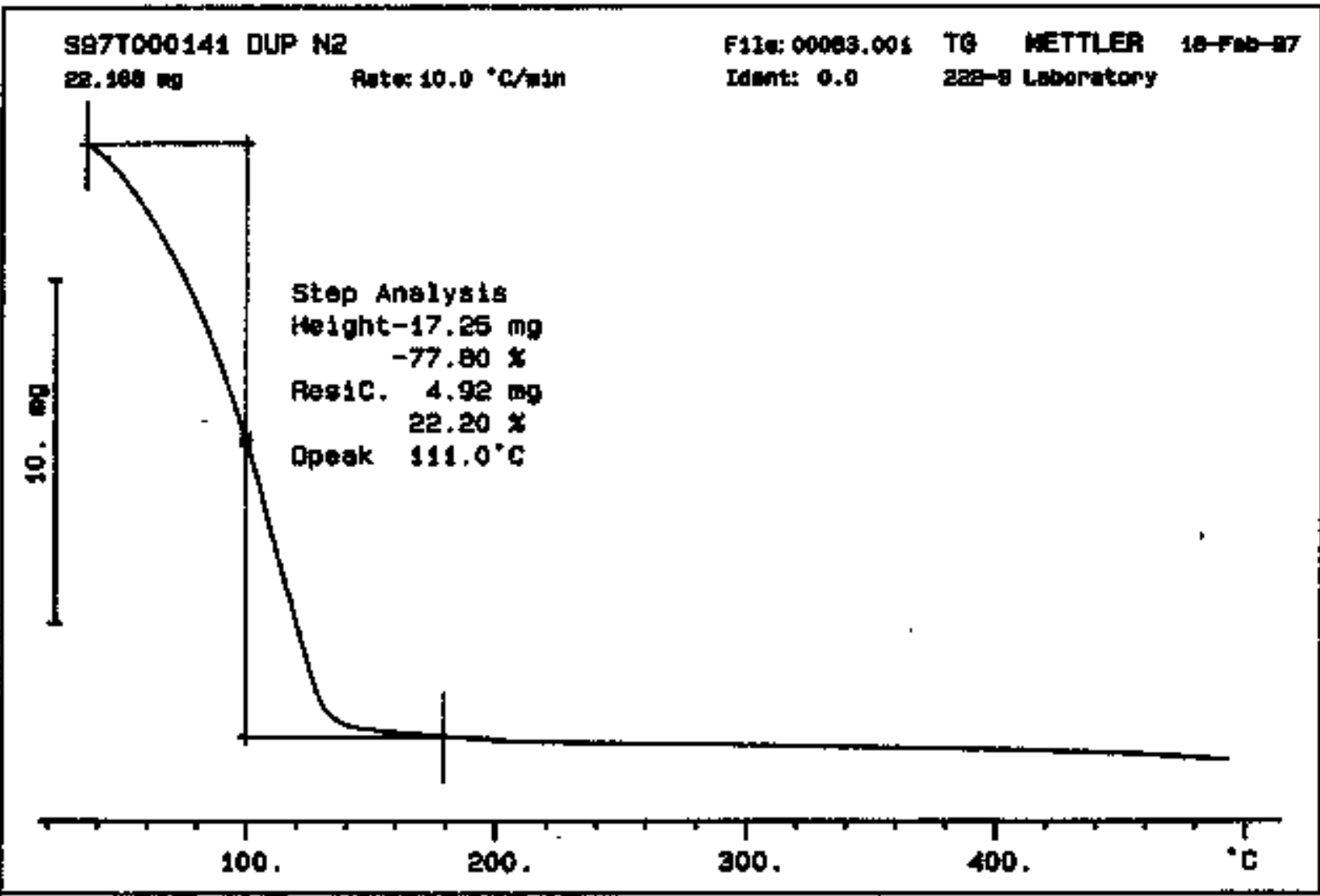




\section{LABCORE Completed Worklist Report for Worklist\# 16710}

Analyst: eal

Instrument: TYGAOS

Book GINBA

Method: LA-514- 114 Rev/Mod D D-O

Workllst Comment TGA-01 FOR T-119(RUN UNDER NITROGENYTERLIO

RTS

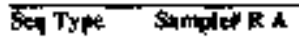

\begin{tabular}{|c|c|c|c|c|c|c|c|}
\hline 20 & & $\phi$ & 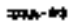 & Iothp & $x-9406$ & 07,100 & F-74t $t=1$ mers \\
\hline 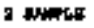 & 1977t40149 & 0 & कार- 6 & JOATP & $\mathbf{A} \mathbf{2}$ & 27.51 & $\mathbf{z}$ \\
\hline $\mathbf{D W}$ & IxTromen & 口 & IDR=d & 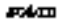 & 77.41 & 1dat & 1. سم \\
\hline 4 Punt & |rtye-4‡4] & $\phi$ & 200-向 & |णी & 보. & 76,0 & 4 \\
\hline 5 Fיק & 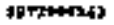 & 0 & $204-a x$ & $\operatorname{soc}$ & $70-74$ & 36.12 & •.750 7Ma \\
\hline
\end{tabular}

\section{Final page for worklist 16710}

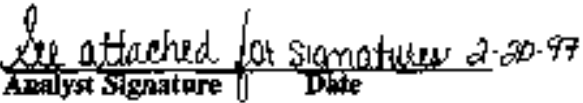

Ansibyt Slepature Date

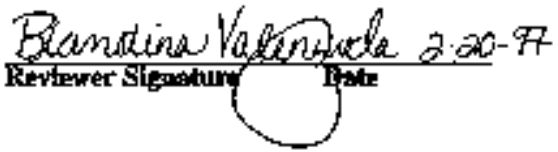




\section{LABCORE Data Entry Template for Worklist: 16710}

\section{Anolyst:}

Mellind: LA-S60112 Rethad

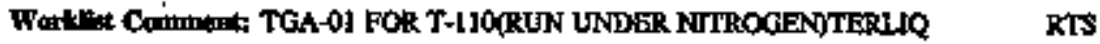

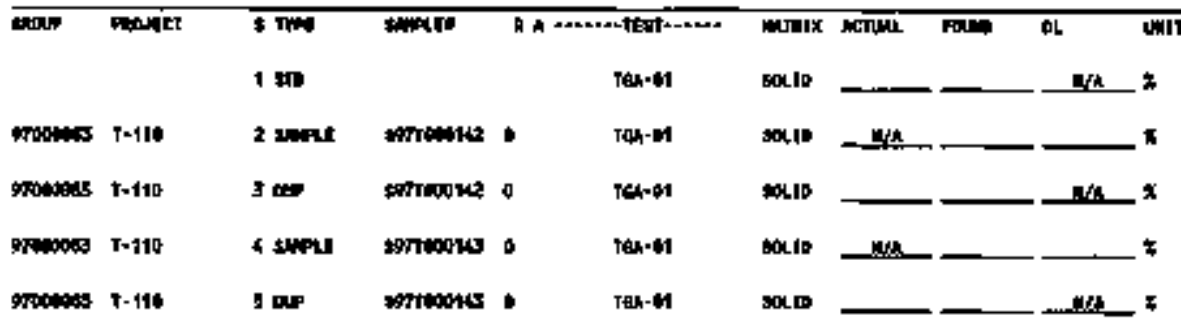

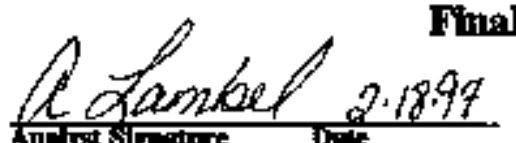

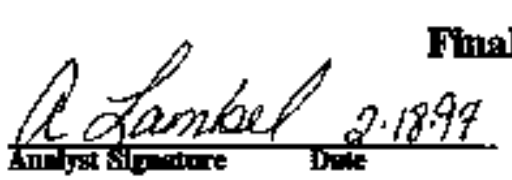

\section{page for worklist \# 16710}

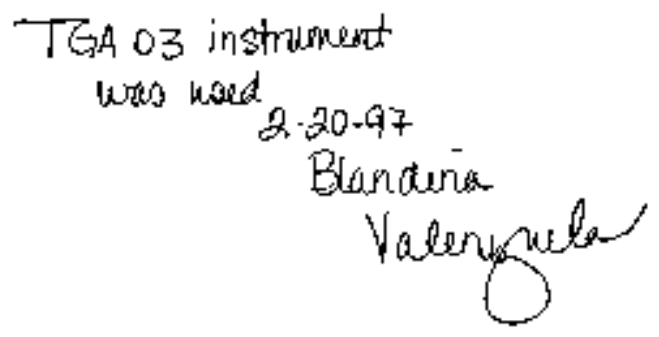

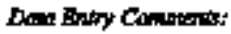

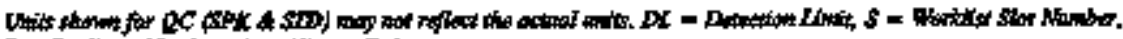

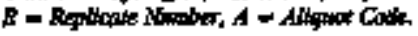


Berve 1s Tak

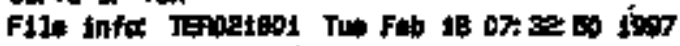

somis watght at.72.

Tak STT Son:-

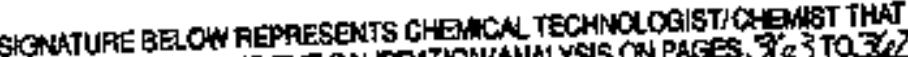

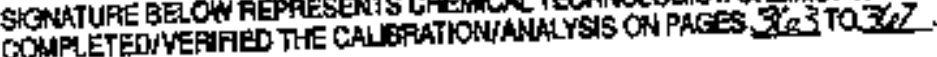

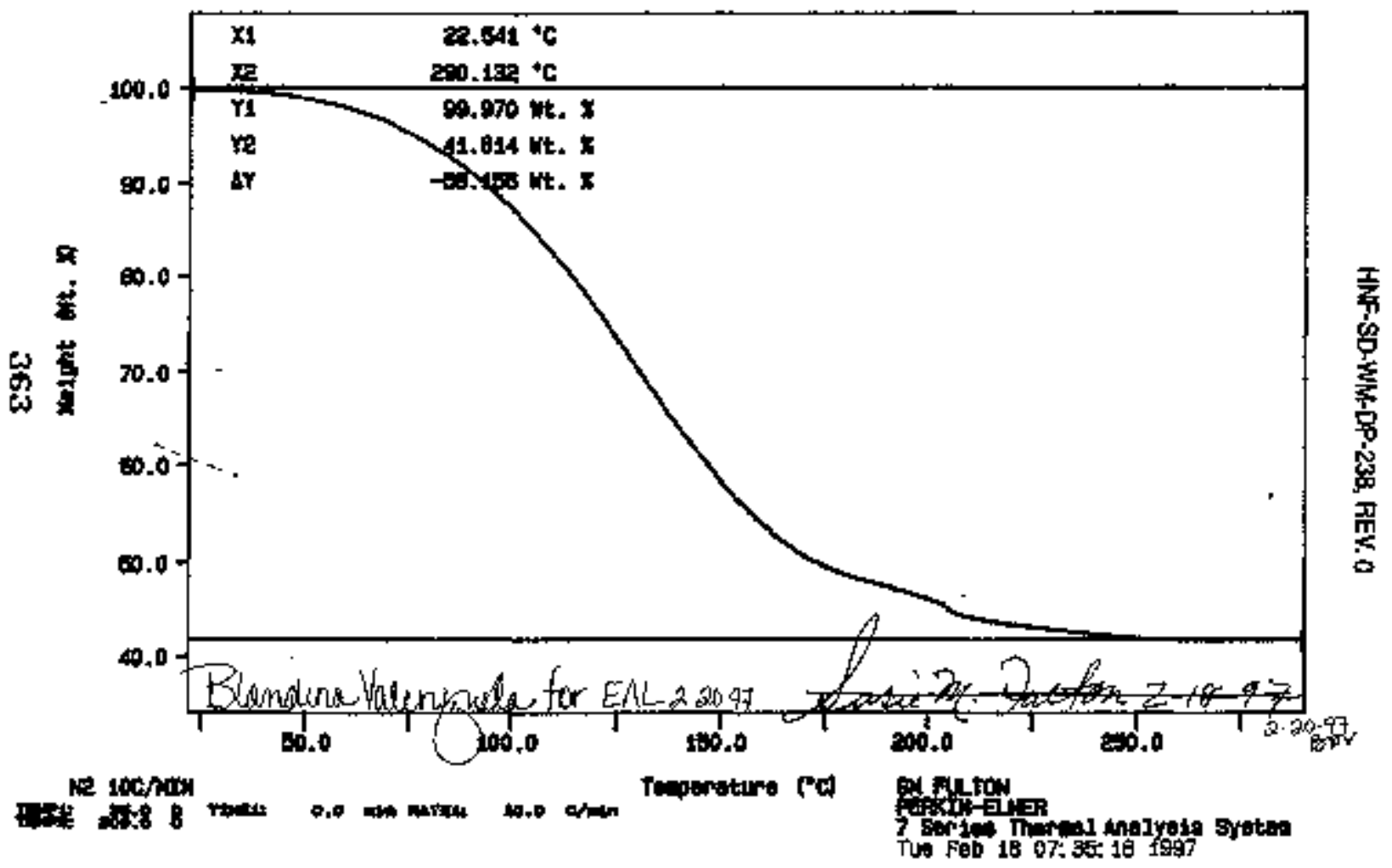


Arve t5 IBA

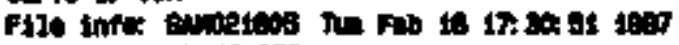

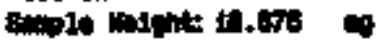

saptoose

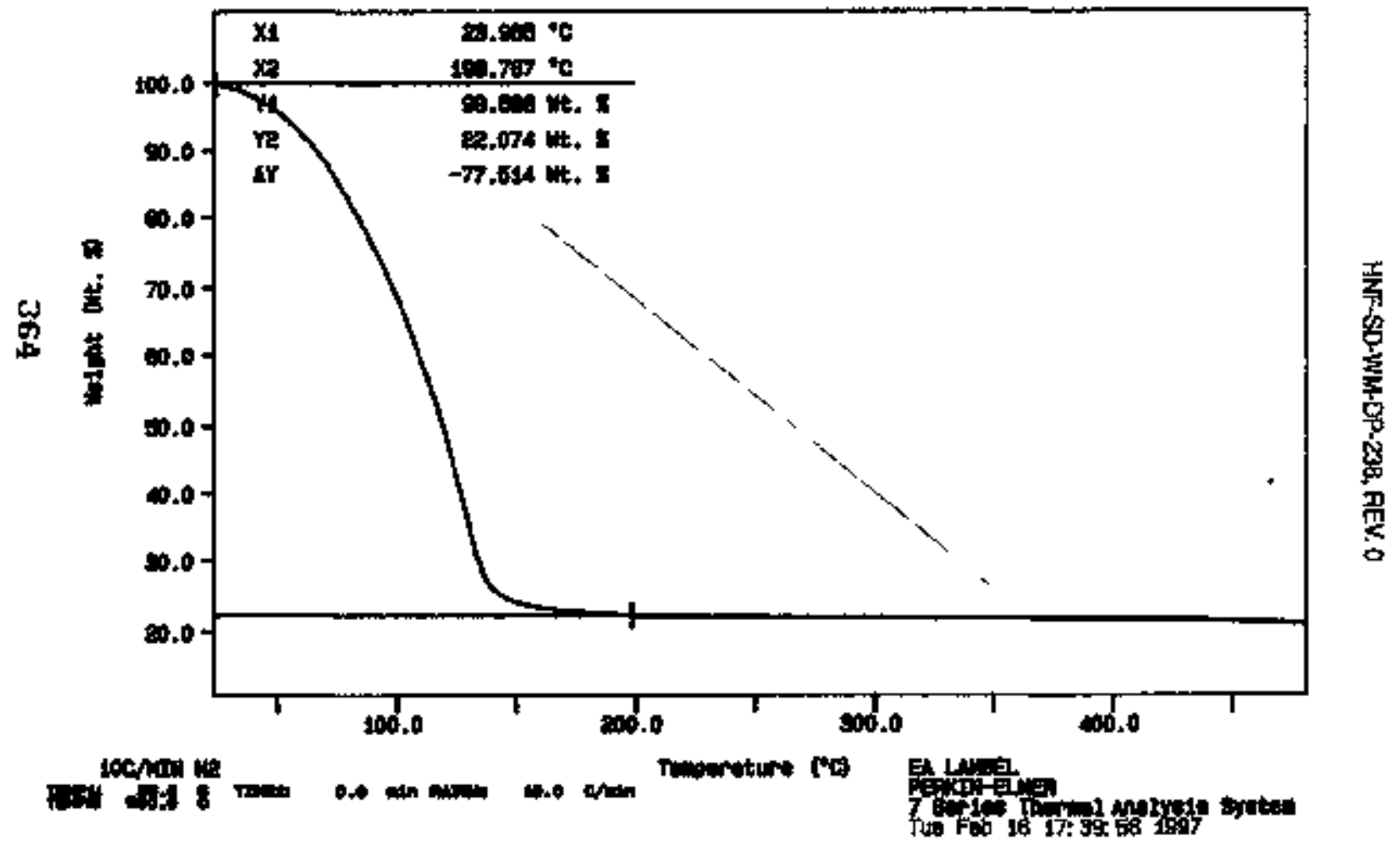


DUTr| 12 TEA

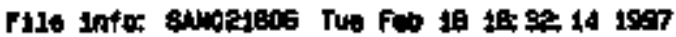

Auplo teight: 18.331

empopostant

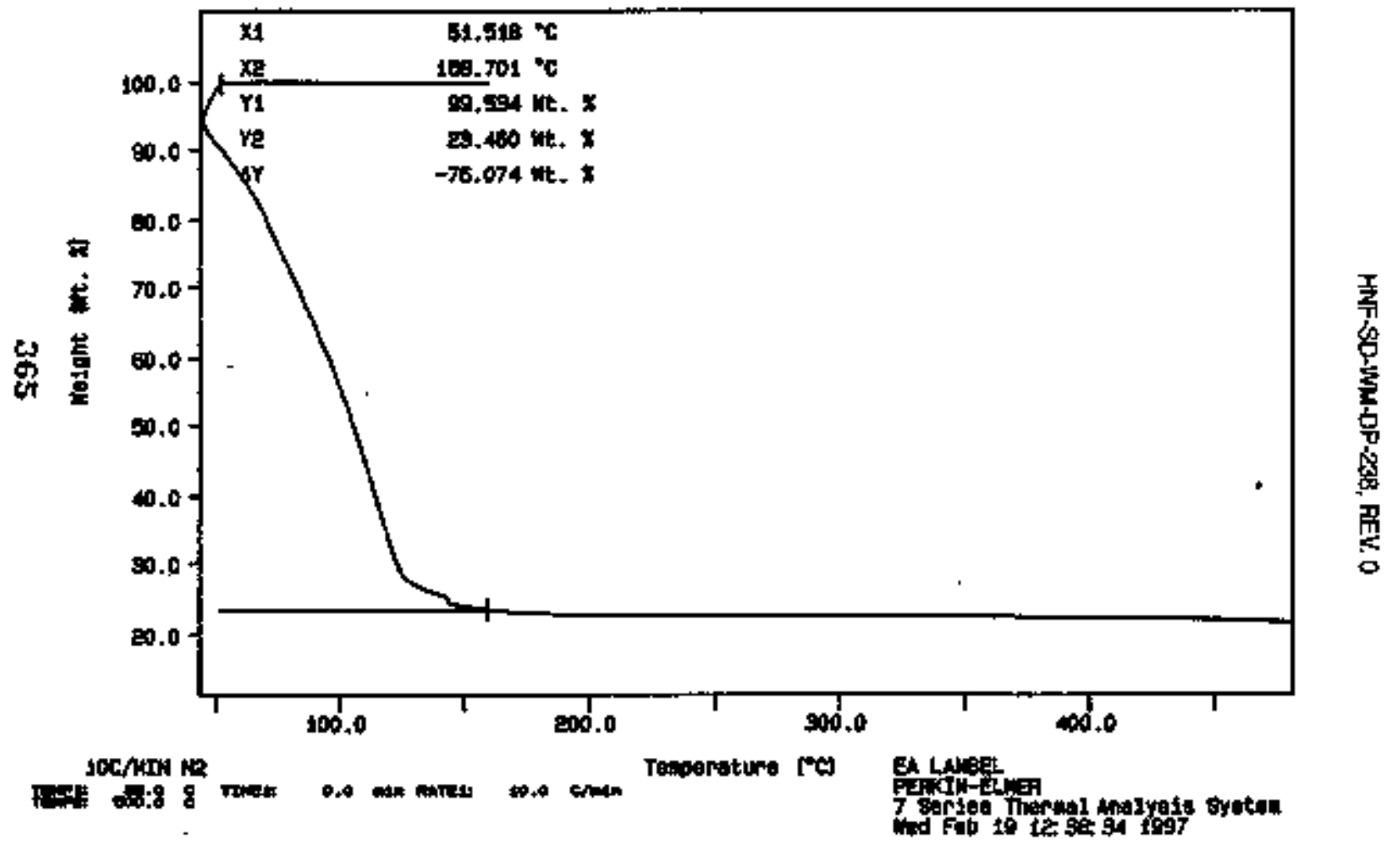


Bary is tin

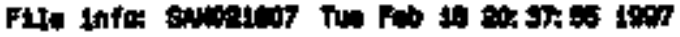

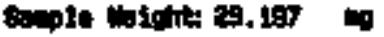

optotota

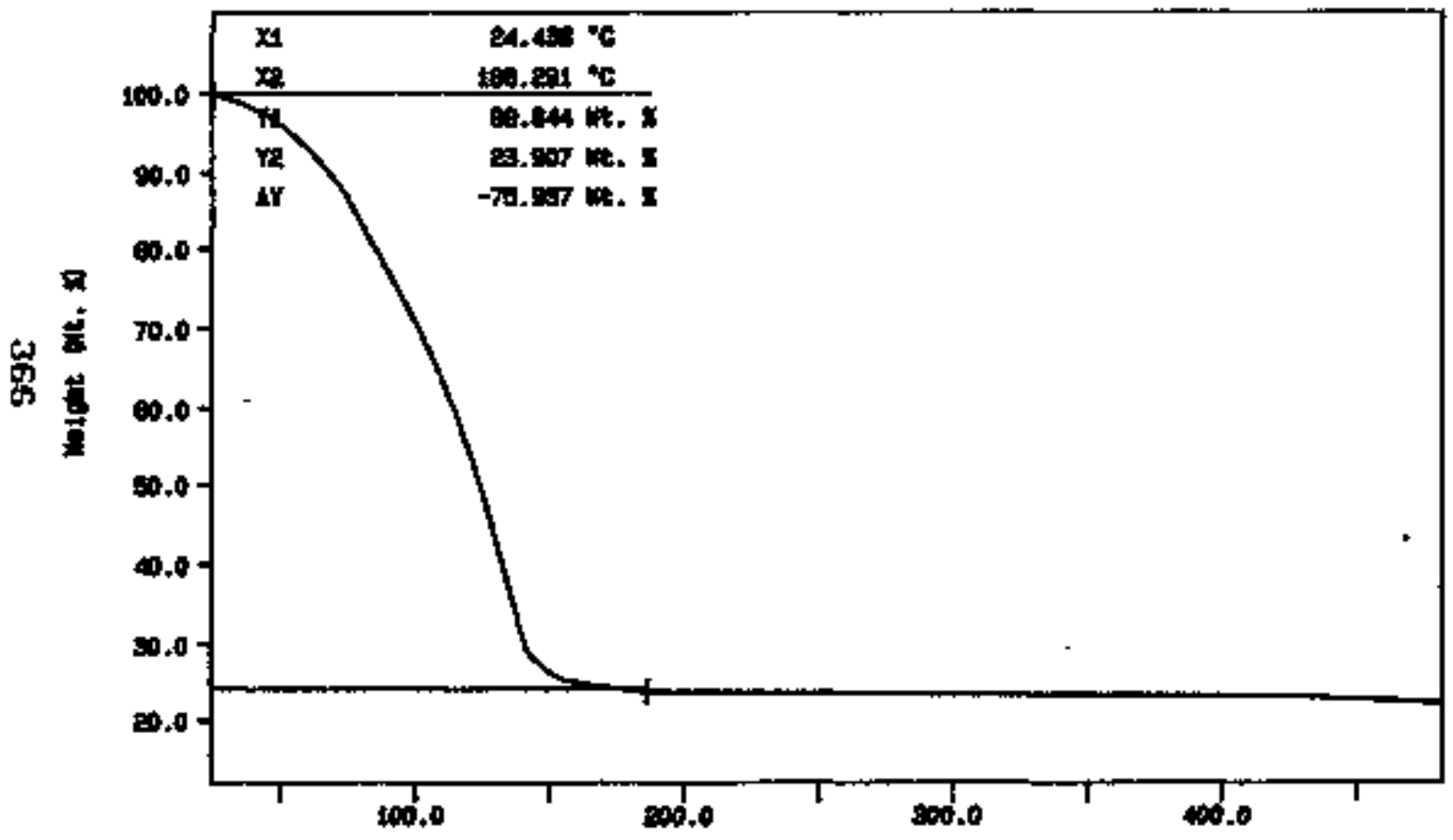

10CAn ke

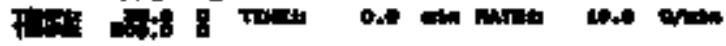

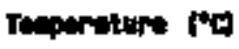


Com it ton

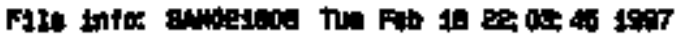

Benplo wight 27.40

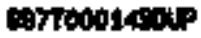

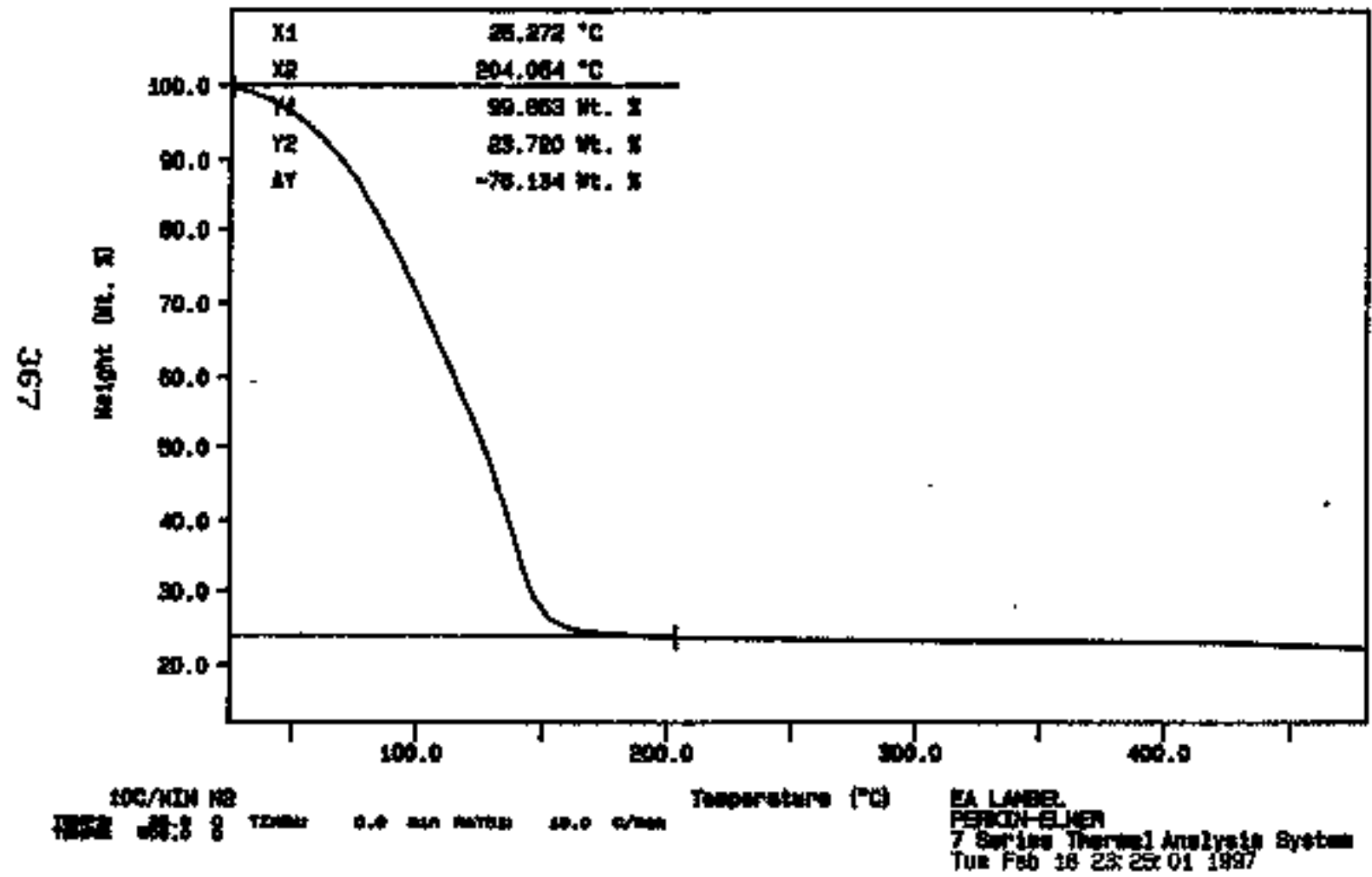




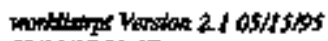

HNF-SDAWHAD-238, REV. O

Pasei $t$ 02/4.957 0.57

LABCORE Daka Entry Tempłate for Worklist\# 16711

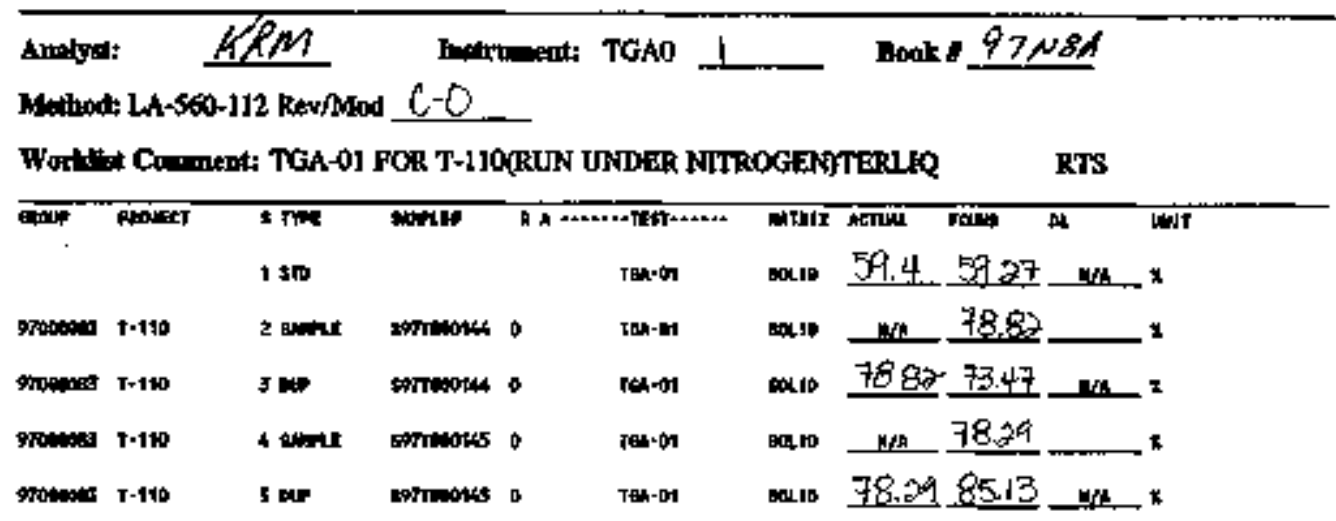

Final page for workligth 16711

2. $(x \rightarrow 1)$

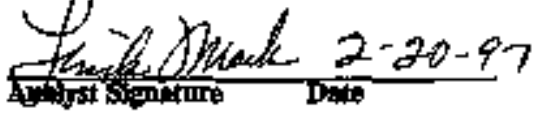

Verifid/Aalidatia by

Blandani

Valeryoula.

$2 \cdot 2047$

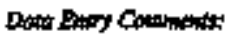

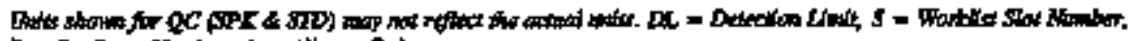

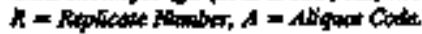

368 


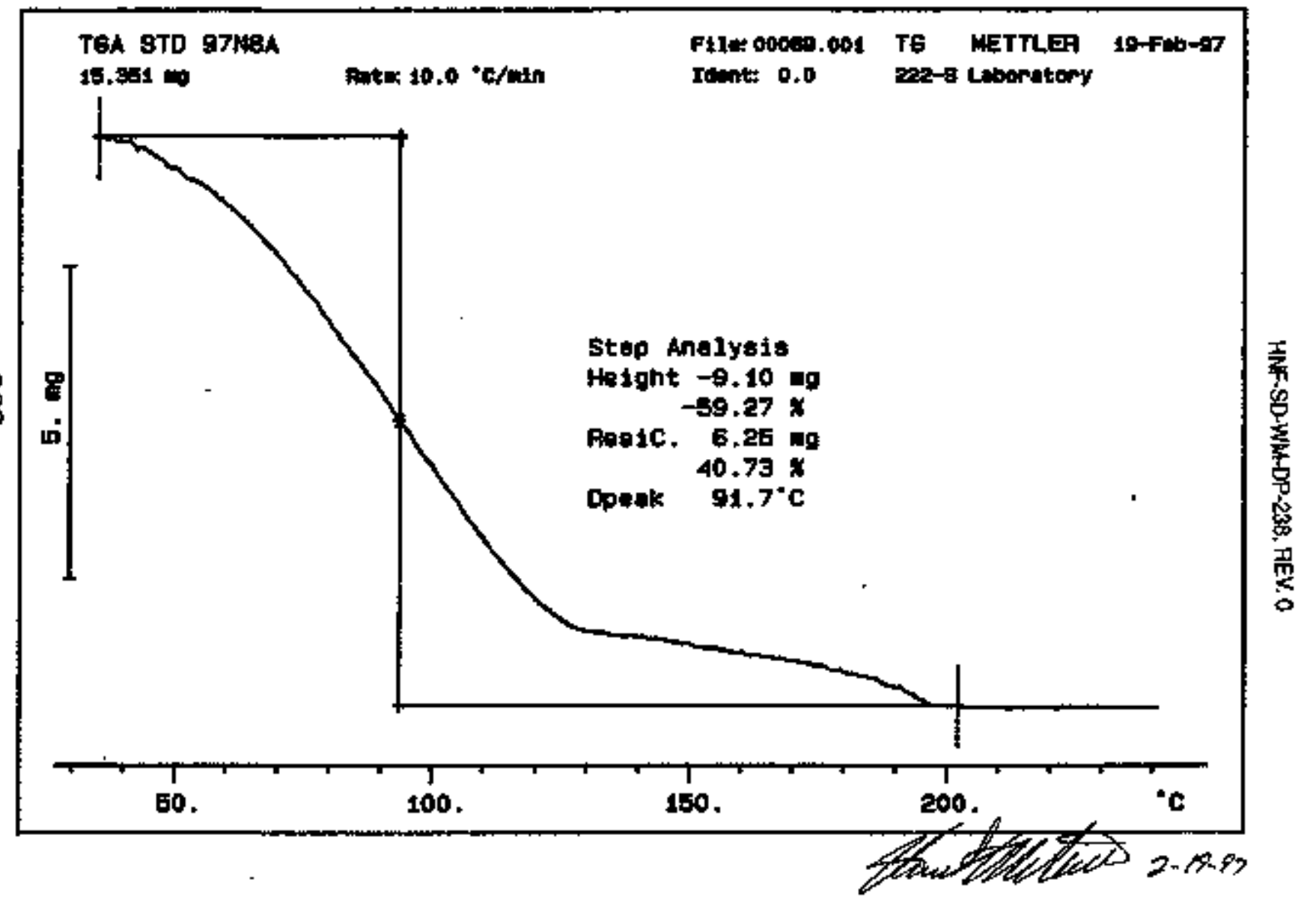




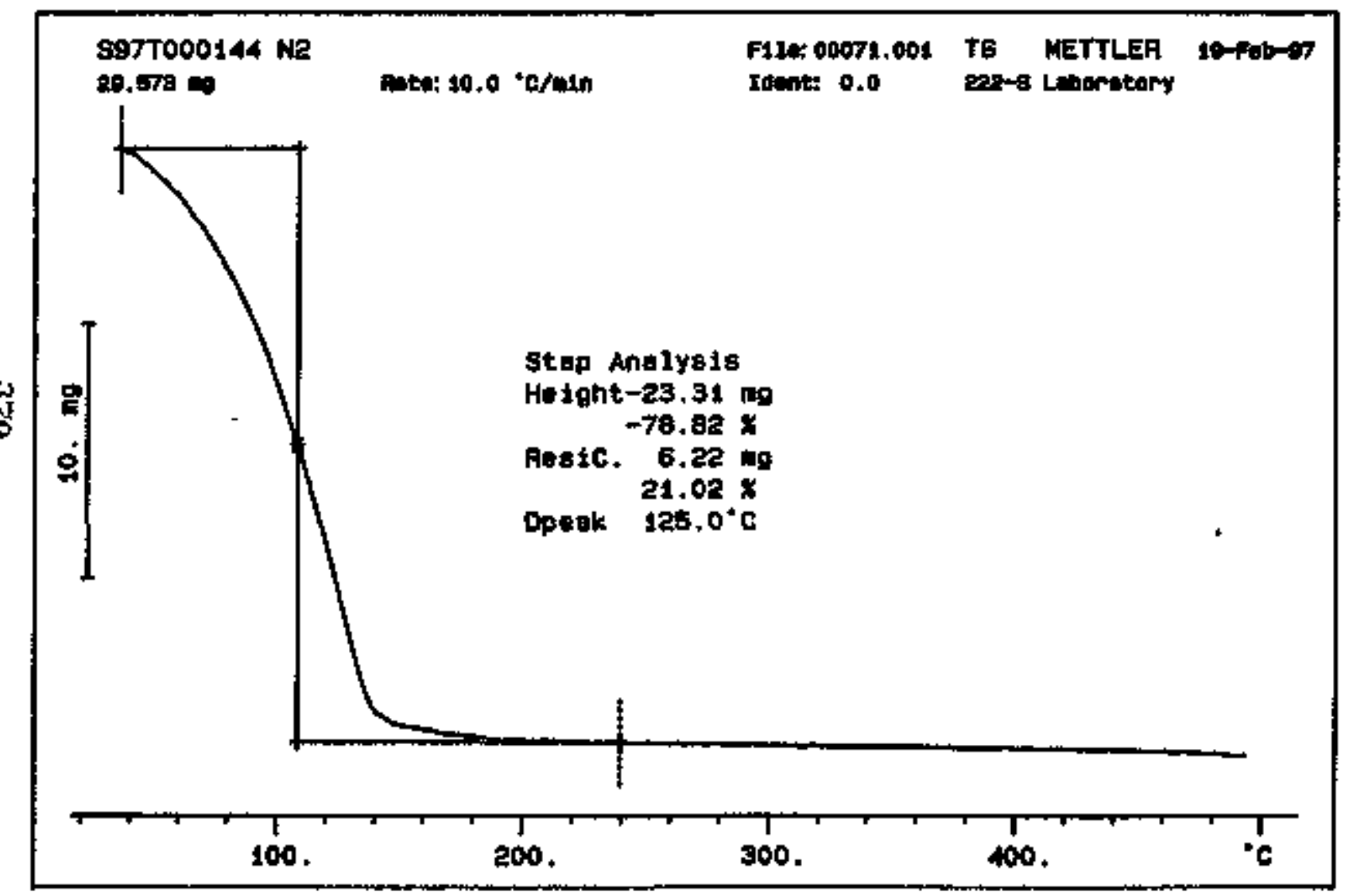




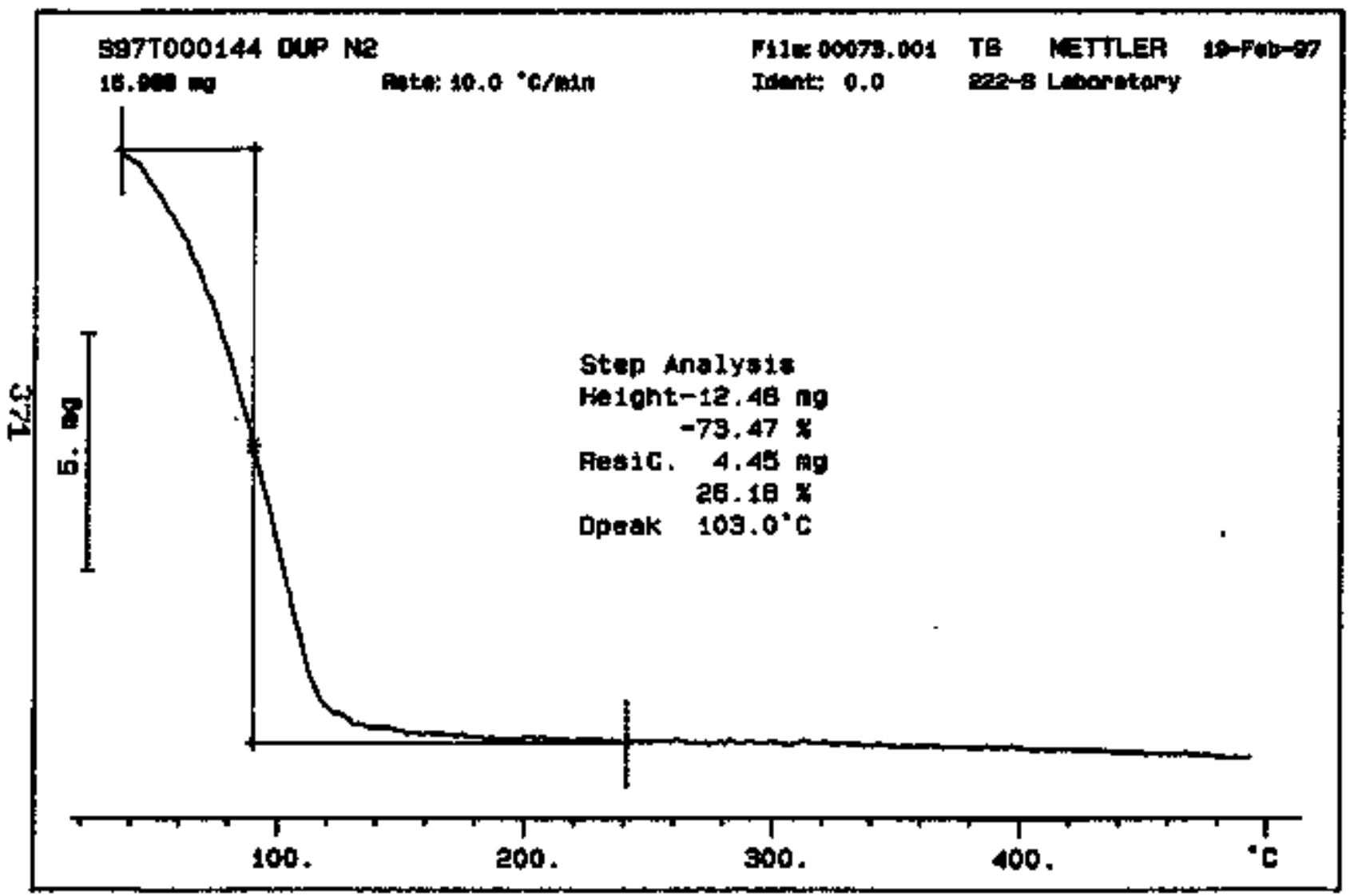




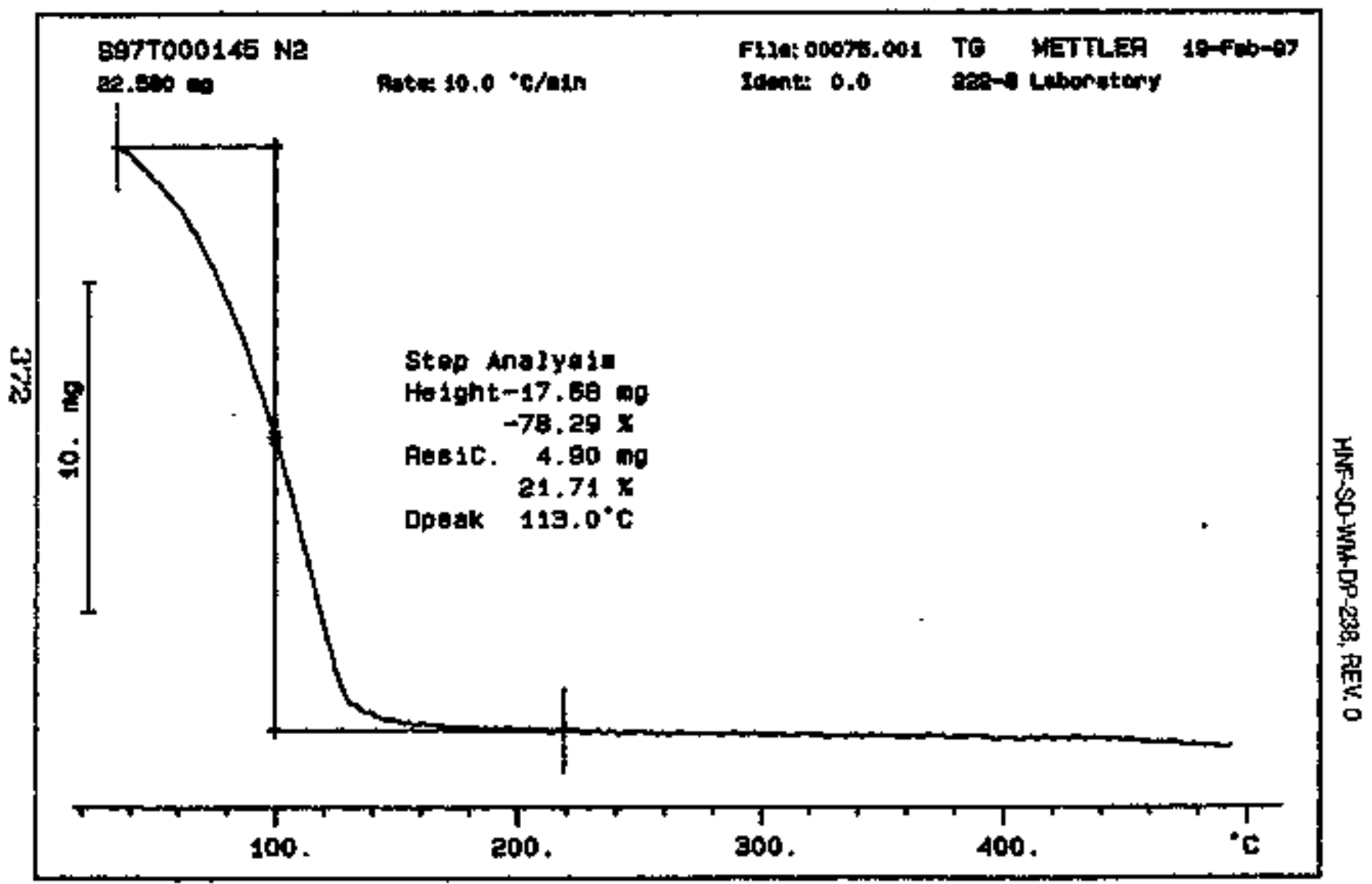




\section{LABCORE Data Entry Template for Worklist* 16712}

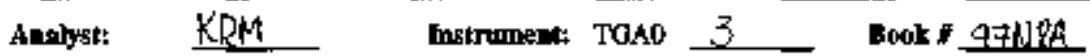

Methout LA-514-i14 Rev/MTod D-O

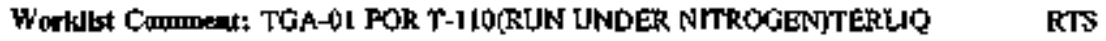

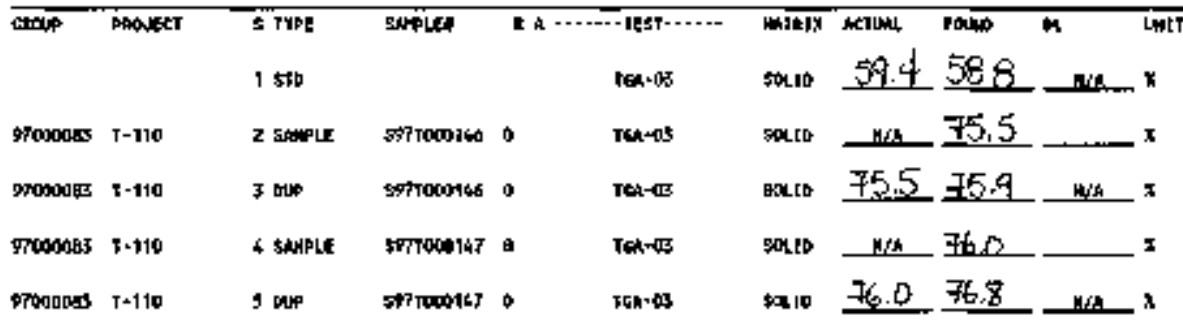

\section{Final page for workligt \# 16712}
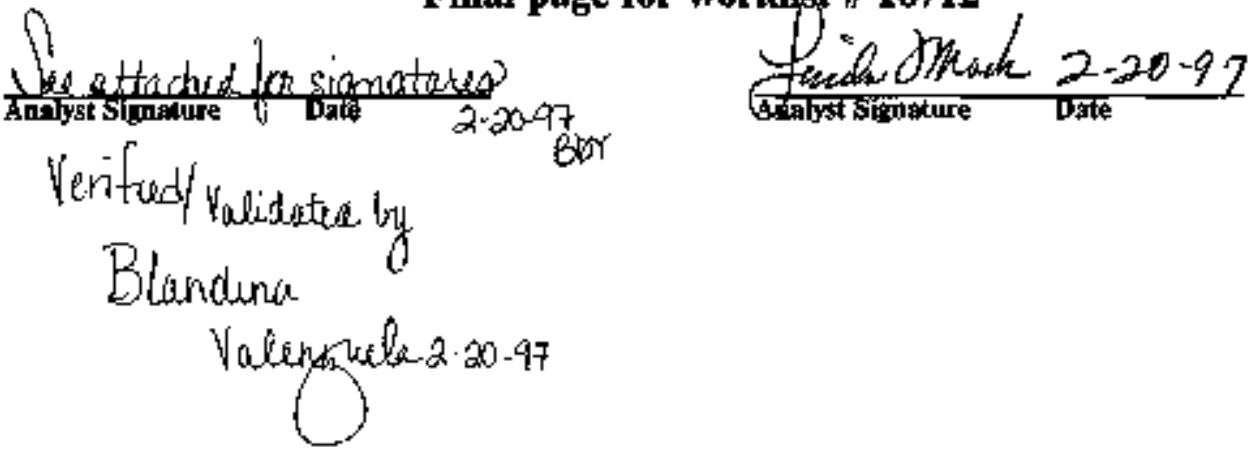


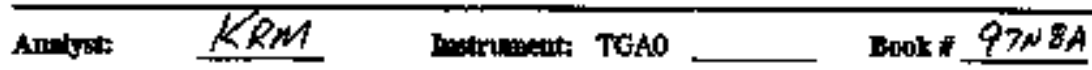

Method: LA-560-112 RowiMod

W-iklt Cun

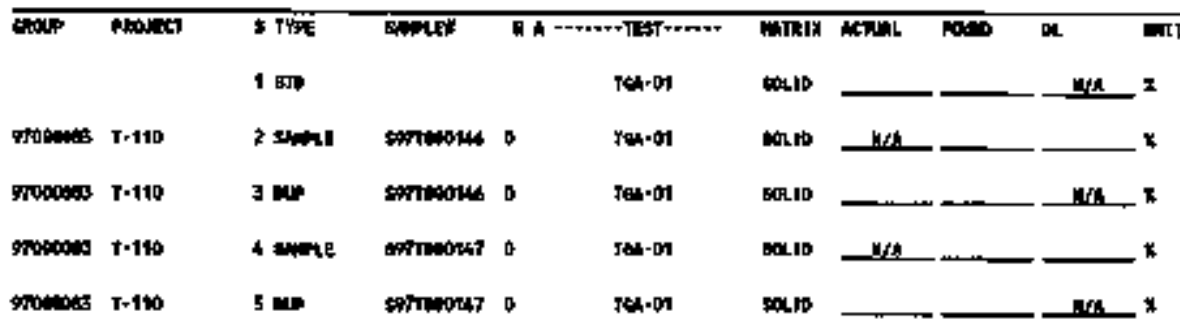

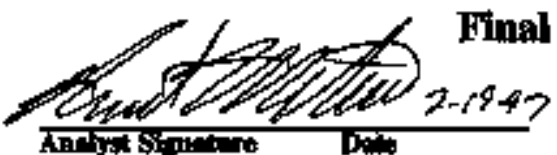

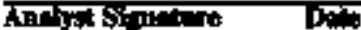

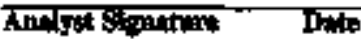

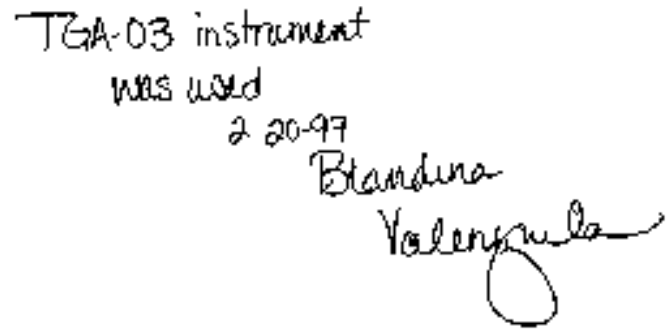

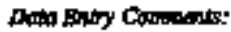

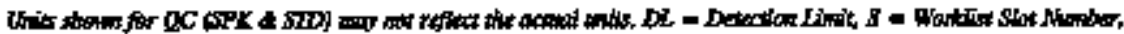

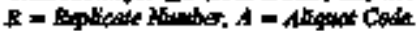


Gurv 1: T3h

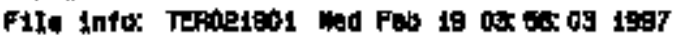

salpie Hasont: 21.311

Toh sto 97ing-k

eq

SIGMATUFE BFLOW REPRESENTS CHEACAL TECHNHLOCISTCHFMHT THAT

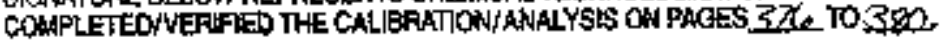

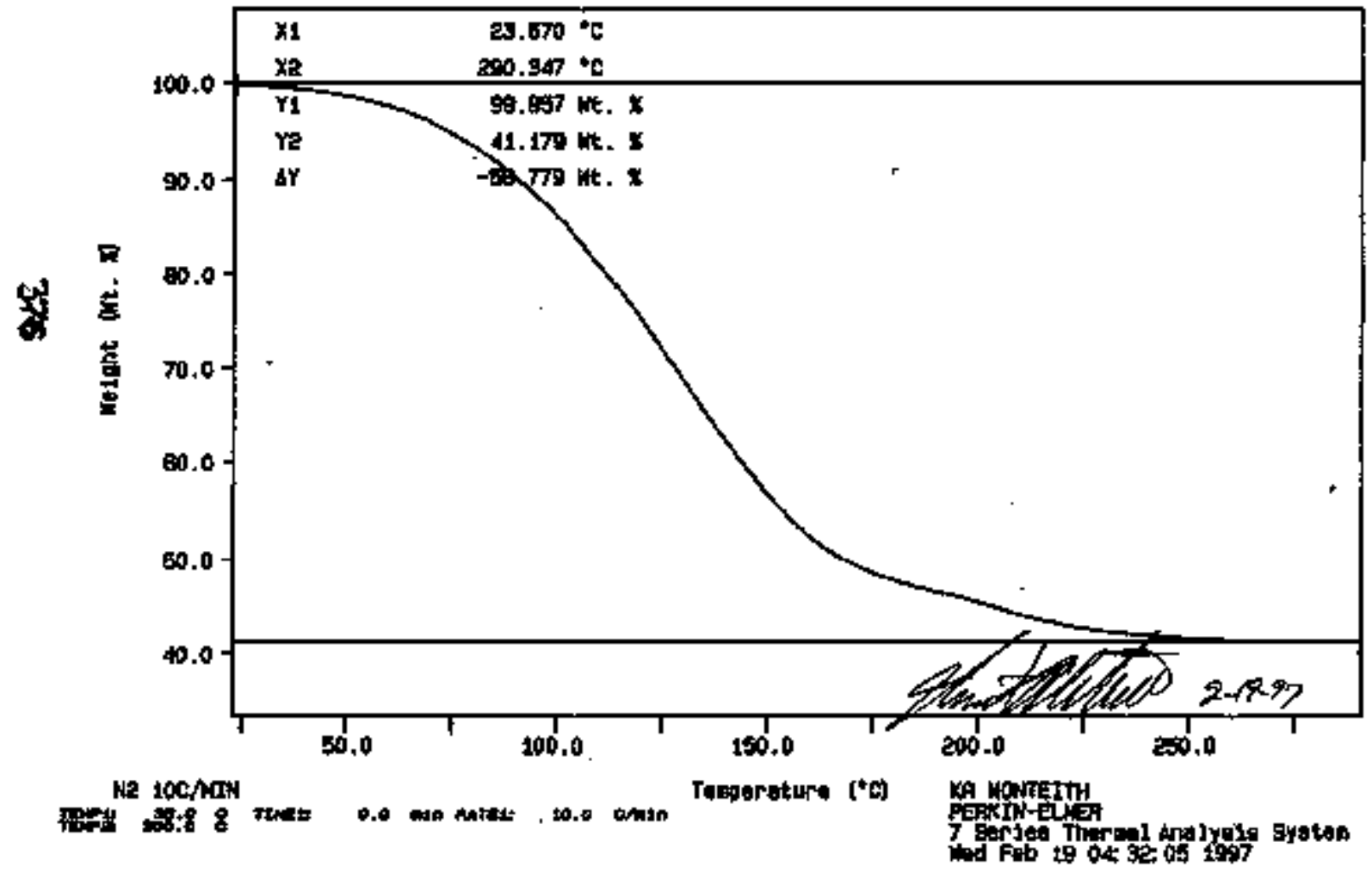


Curve it Tes

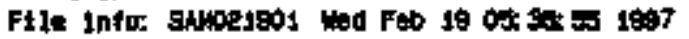

Sacpla Wadonts 16.959

9a7tooos4s

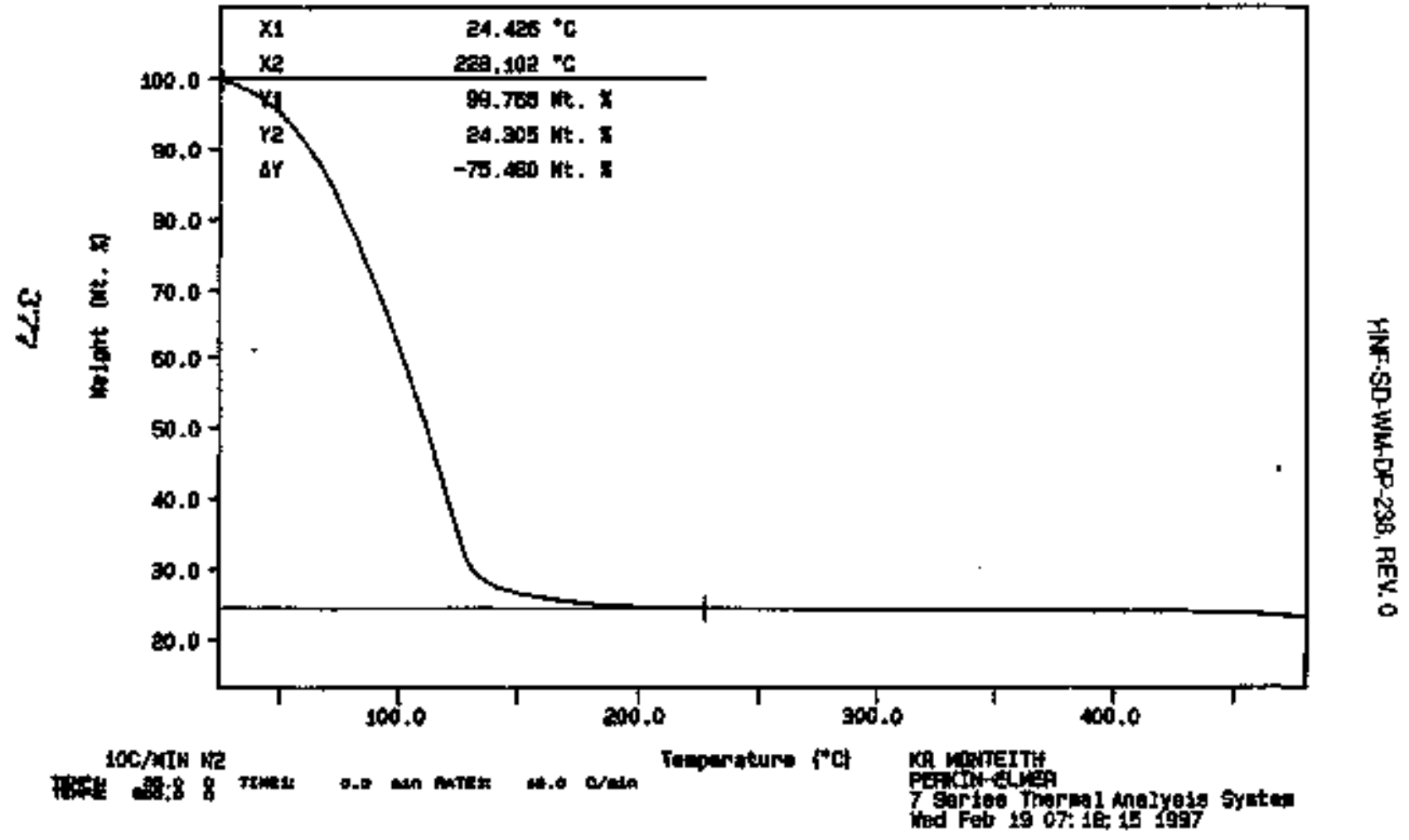


Curve d: TeA

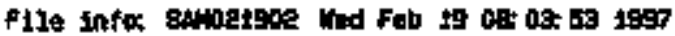

saplo wight: $12.697 \quad \omega^{*}$

sorTo0o1e dp

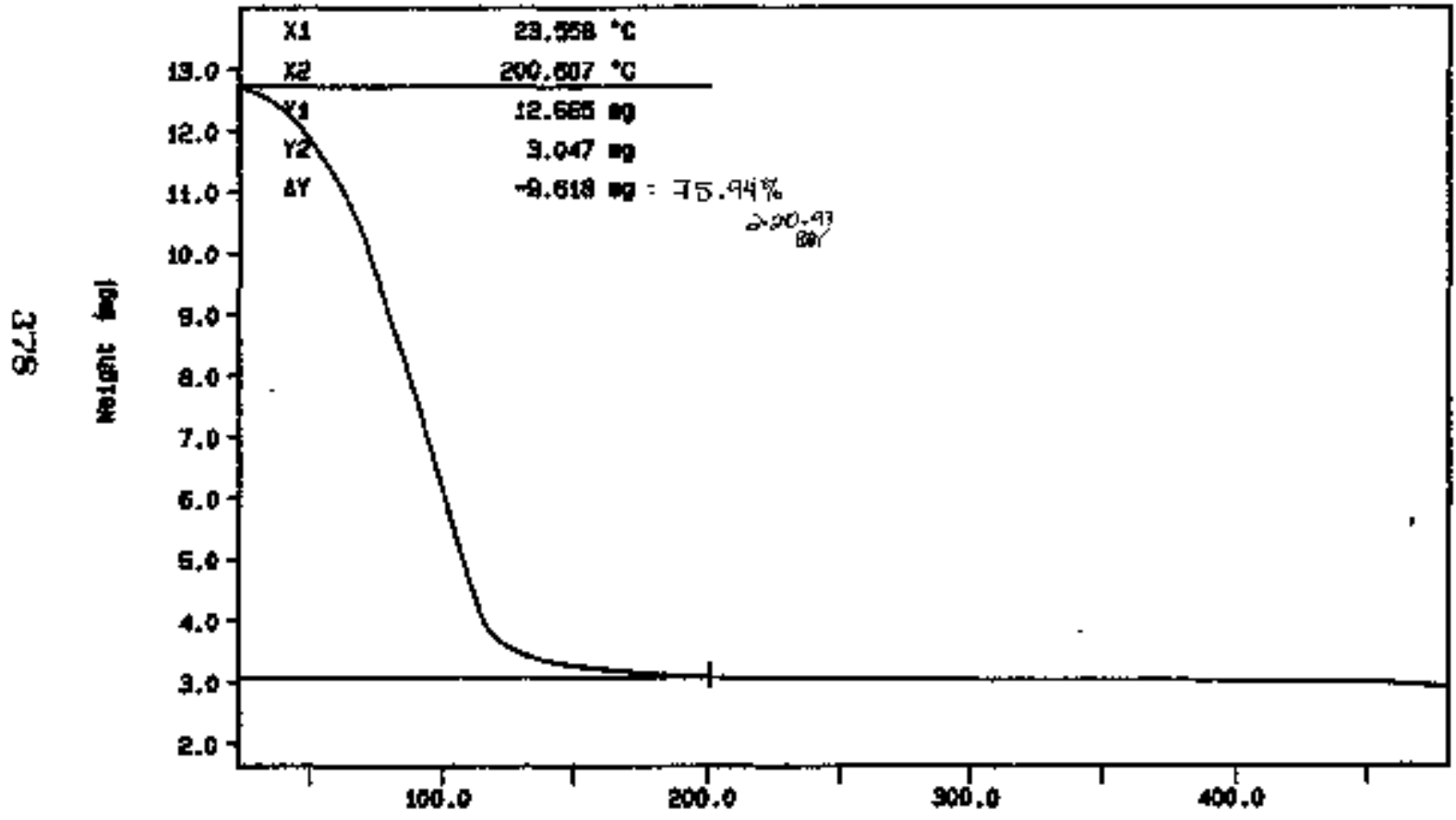

$10 \mathrm{C} / \mathrm{MN} \mathrm{Ne}$

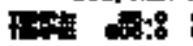

9.to namit

16.9 antint

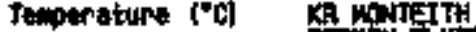

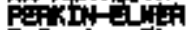

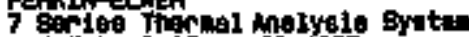
wist fot 19 ox 14; 231997 
Curva 1: Tak

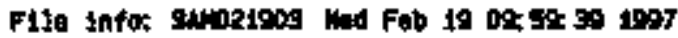

Sagle intoht: 29.317

enTo00147

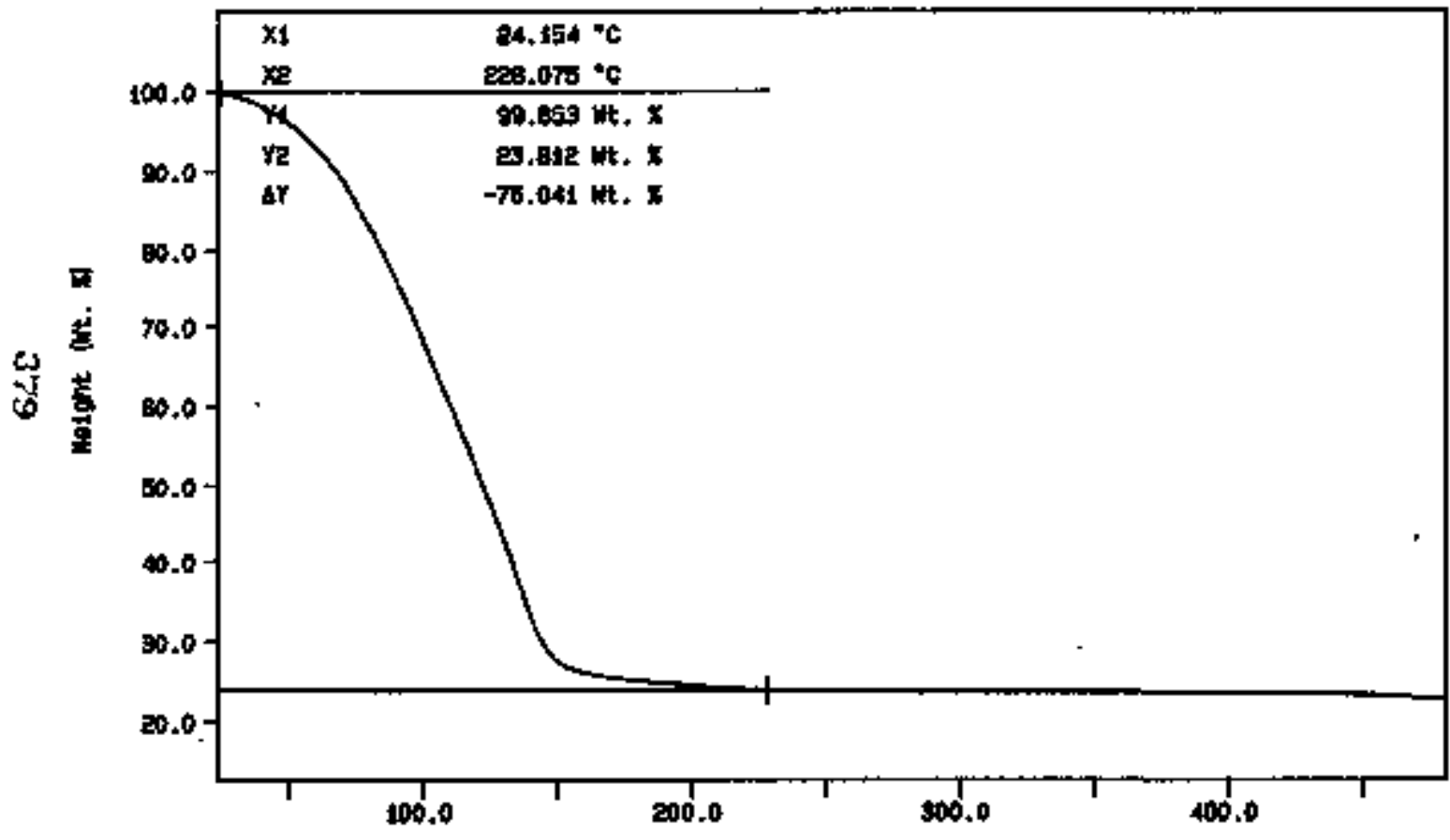

106/NEN HP

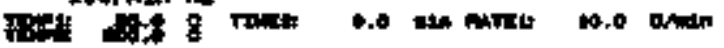

Treporiture ('C)

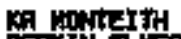

PinIthing

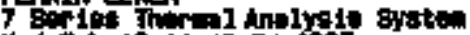

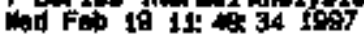




\section{LABCORE Data Entry Template for Worklist\# 16713}

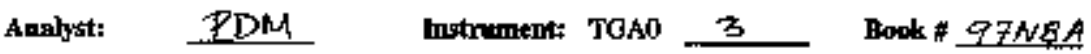

Method [A-514-114 Rev/Mod D D-O

Worklks Commeat TGA-0I FOR T-I10(RUN UNDER NITROGEN)TERLUQ RTS

\begin{tabular}{|c|c|c|c|c|c|c|c|c|c|}
\hline $\operatorname{sex}$ & DWOAECT & $\begin{array}{l}\text { B TIPE } \\
1 \text { STp }\end{array}$ & 3athet & & $\begin{array}{l}\text { "-TEET--.... } \\
\text { TSH.05 }\end{array}$ & $\begin{array}{l}\text { М1ค1K } \\
\text { क人. }\end{array}$ & $\begin{array}{l}\text { Nerin } \\
\text { sqy }\end{array}$ & 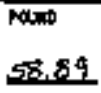 & $\begin{array}{l}\text { ol } \\
\text { Mrs } \\
\end{array}$ \\
\hline 97000008 & $5-110$ & 2 swhple & s9700015s & 0 & 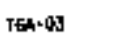 & sxus & $\mathbf{M}$ & 콰. 27 & \\
\hline 97000000 & $T-110$ & 3 Lur & 87T0015. & 0 & Ter- 05 & BOL.JD & 71.27 & 7.72.82 & rirt. \\
\hline shoovots & $5-110$ & 4 SAOPLE & sq7inooe 159 & 0 & ros-0s & 90L.10 & 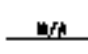 & 74.00 & \\
\hline 9700008s & $T+110$ & 5 bo & कातocolsp & 0 & Thandis & solib & 고노.마이 & 73.00 & $\mathbf{D}$ \\
\hline
\end{tabular}

Final page for worklist \# $\mathbf{1 6 7 1 3}$

Sep Attaciod for Sigereture

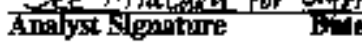
Whidated : granchedor

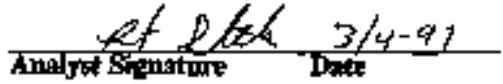

Data Entry Corimats:

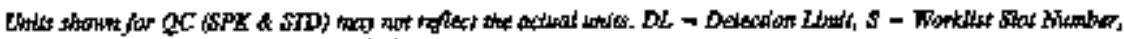
R $=$ Repdiste Minuber, $A=$ Allower Cade. 


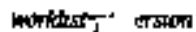

$02 / 26197,55$

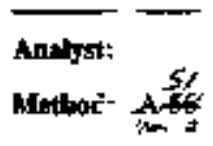

Werklk: Jomin

कीat

grookits $\cdot 110$

moroost $\quad 10$

woonats two

10000003110

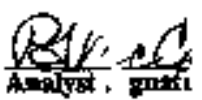

Anivist. piniti

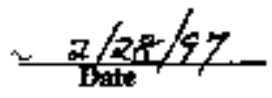

\section{Fina}

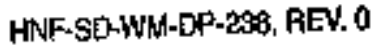

\section{- CORE Jata Entry Template for Worklist\# 167t3}

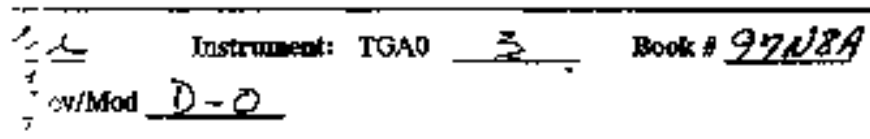

一它A-中1 FOR 'T-11G(RUN UNDER NITROGENYTERLIQ RTS

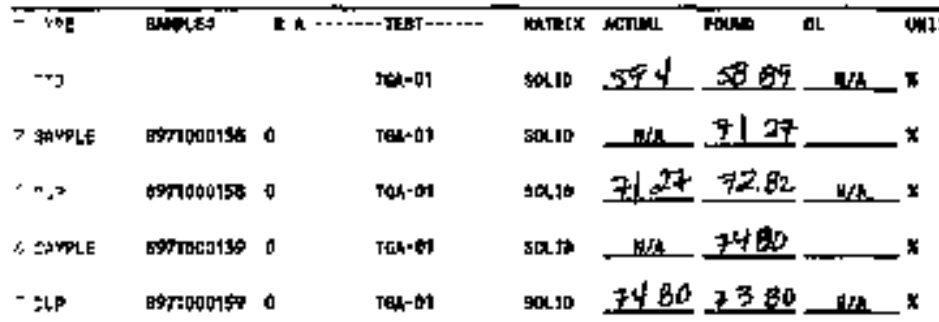

page for worklist \# 16713

\footnotetext{
F्यित क 3

$2 / 2\} 174$

C FI? tot
}

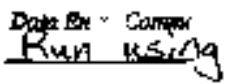

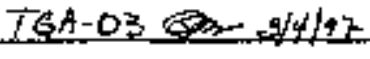




\section{Crive it Tek}

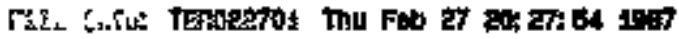

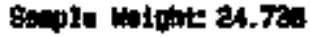

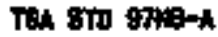

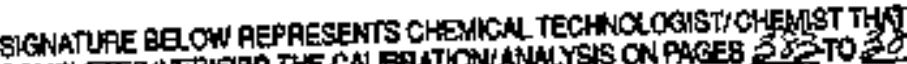

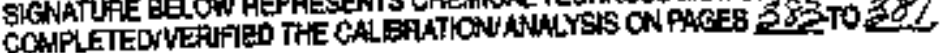

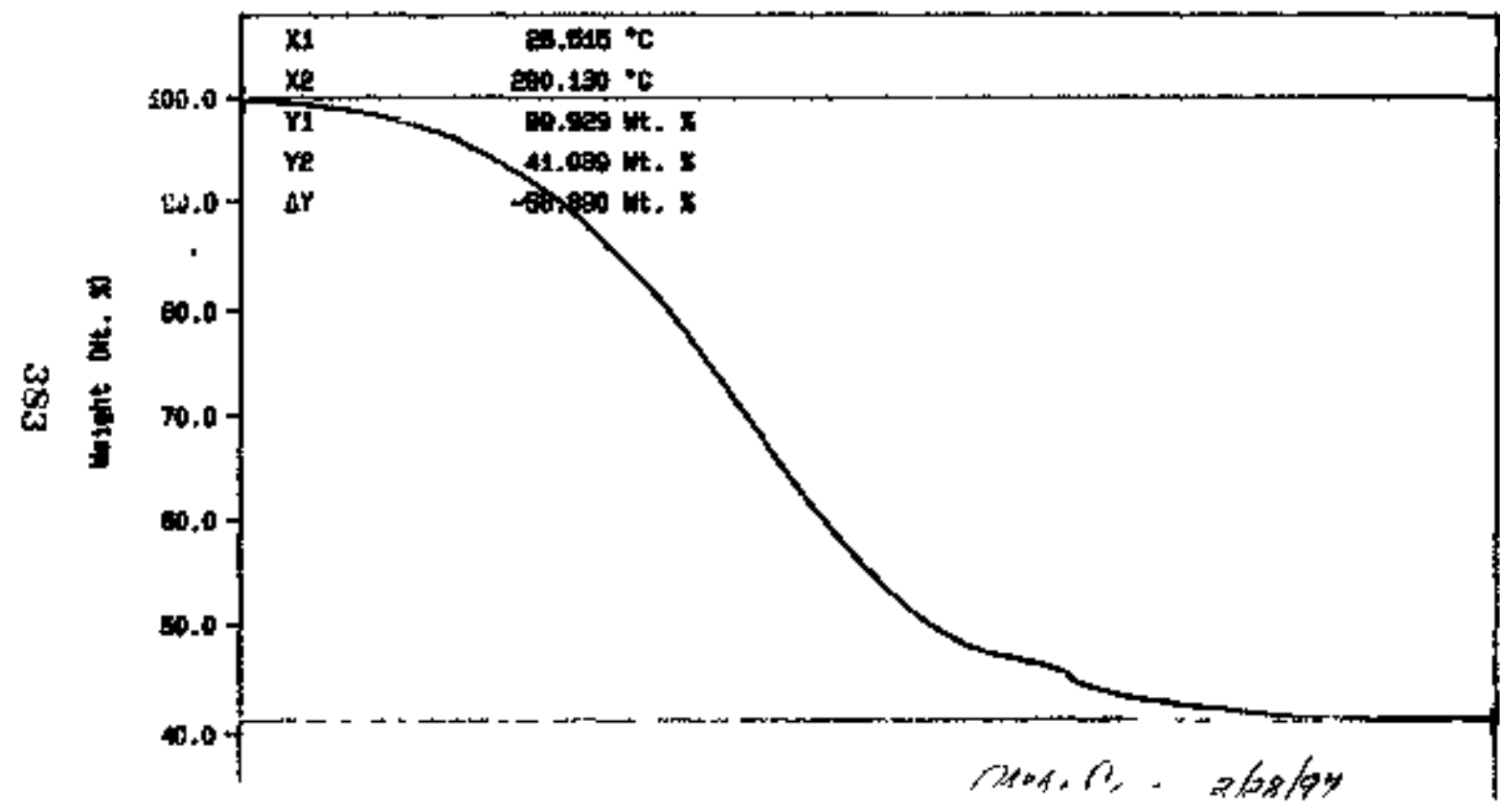


Gume I tax

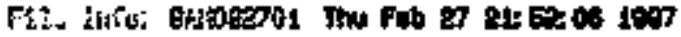

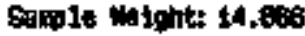

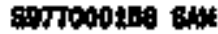

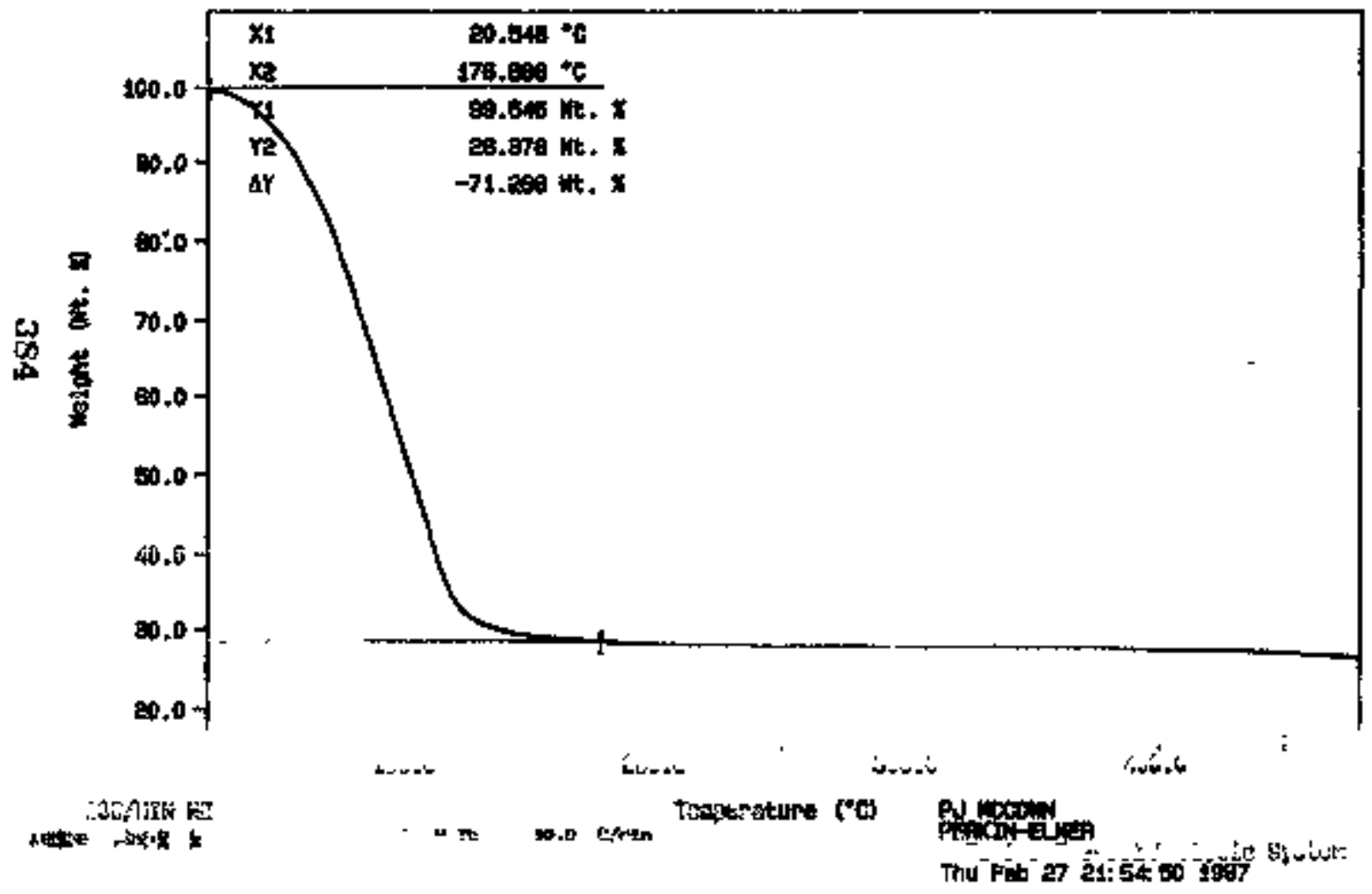




\section{Cume \& tex}

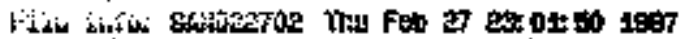
Banplo indolsta 16.570

\section{Eoprocoseg. orp}

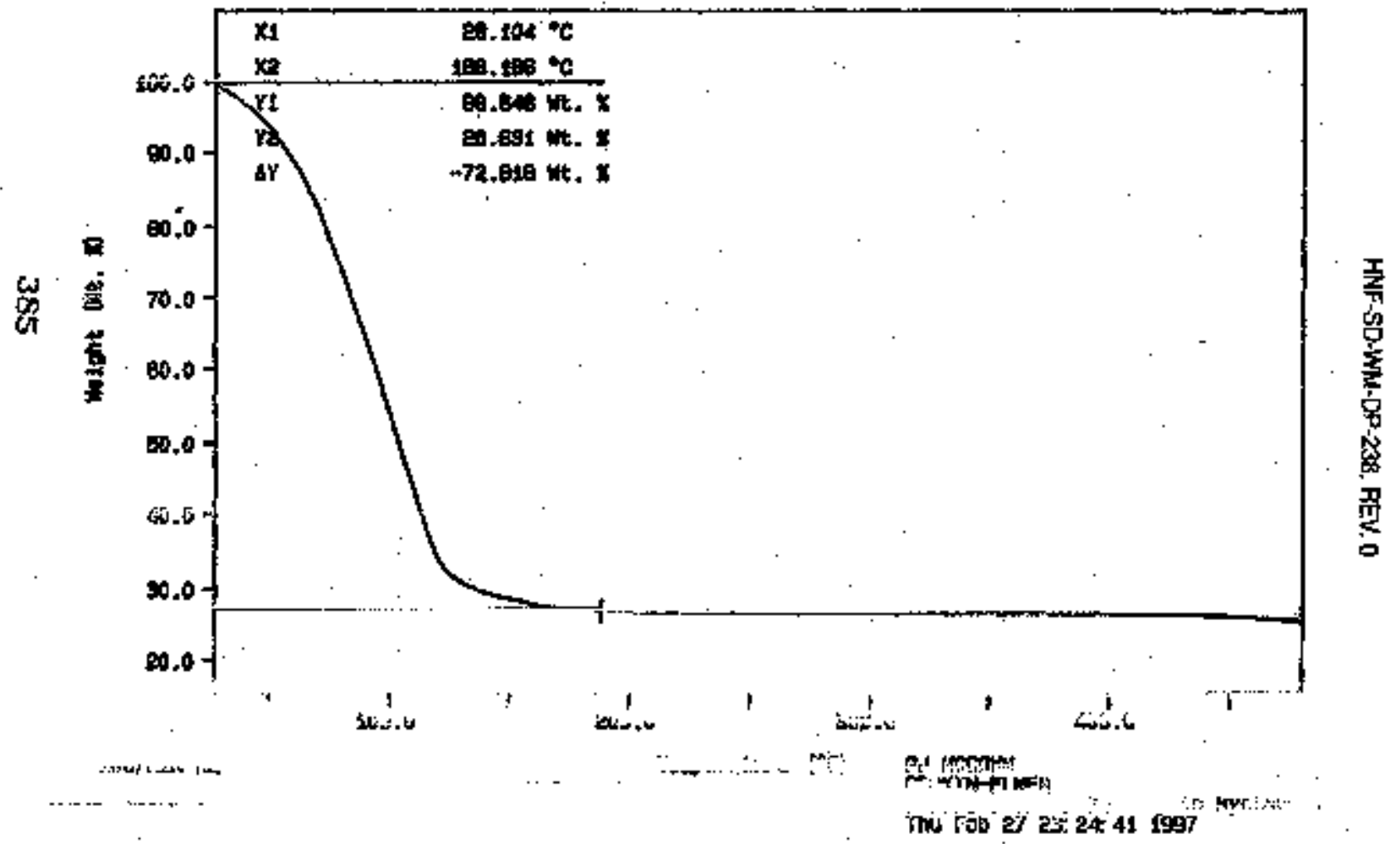


tuma is tet

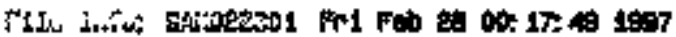

somple lotohts at.es1

somposta stit
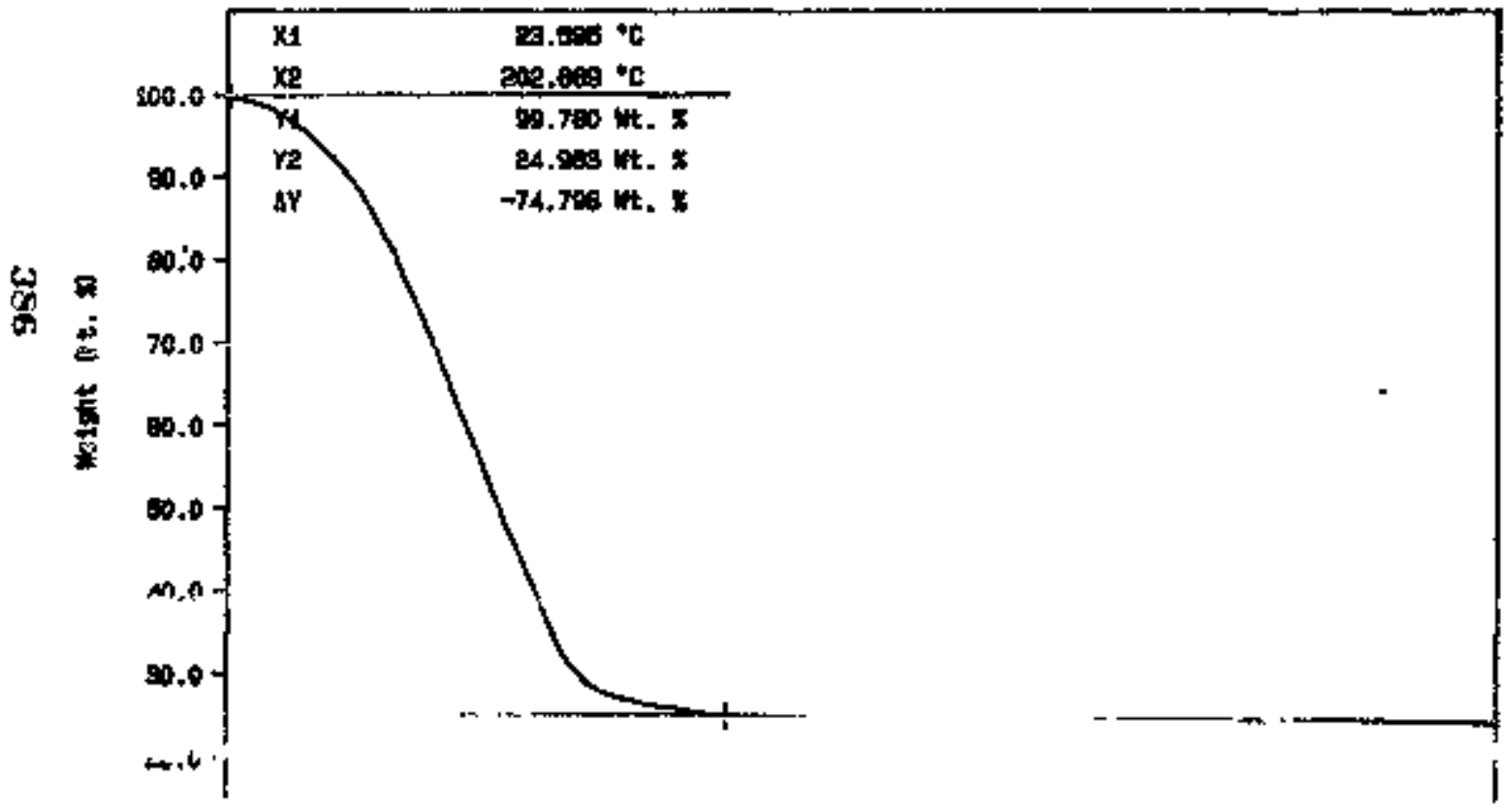

it․ㄴ - +-

itr. - v

Sant

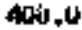

女。

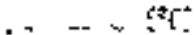

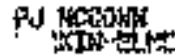

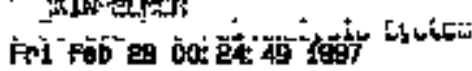




\section{Curve t: Th}

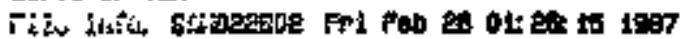
8aple mient 10, to ch

gefitoosis ap

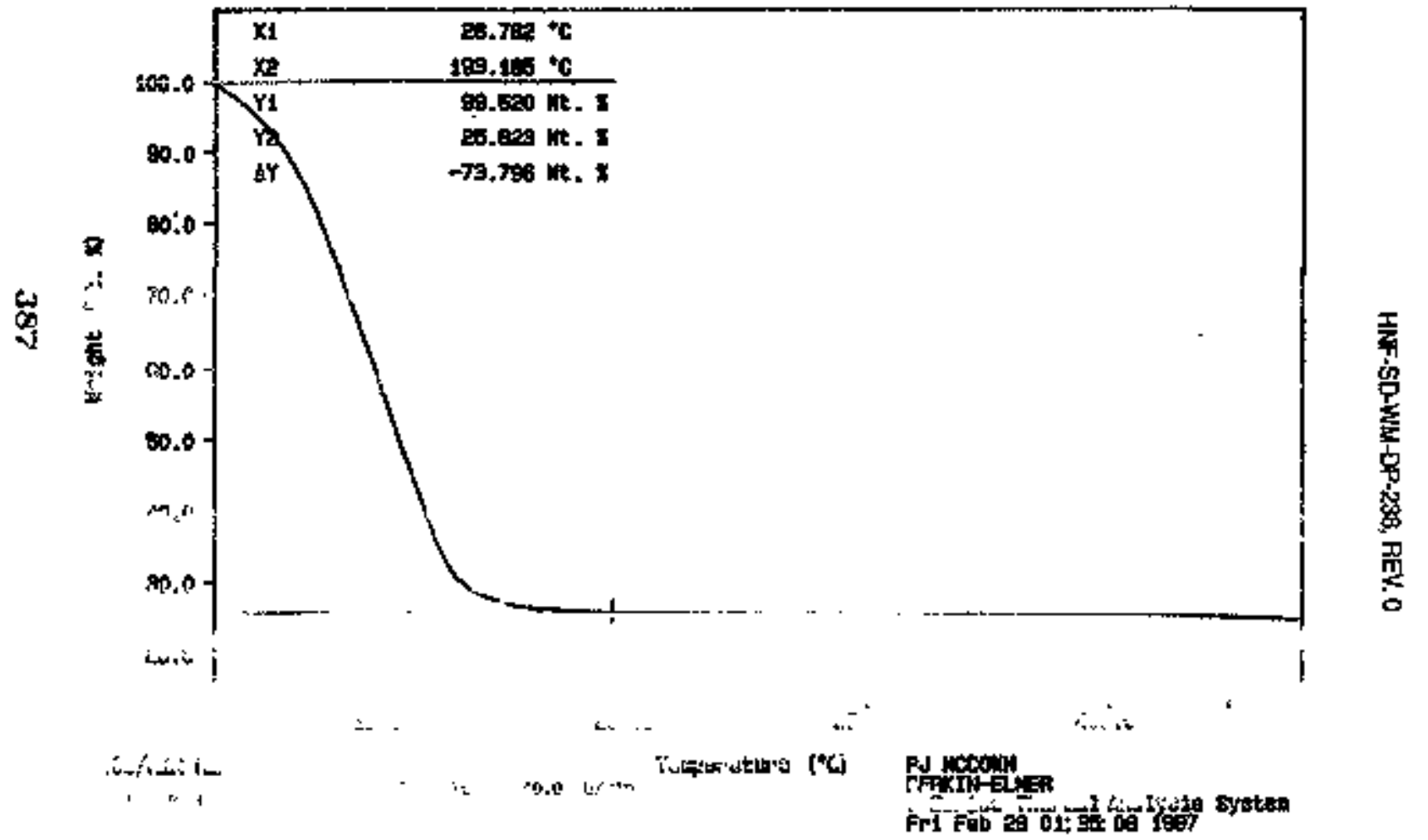




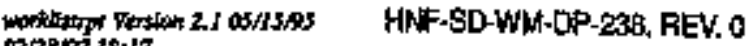

Fente 1 o2/28:5 18:17

LABCORE Data Entry Template for Worklist\# 16830

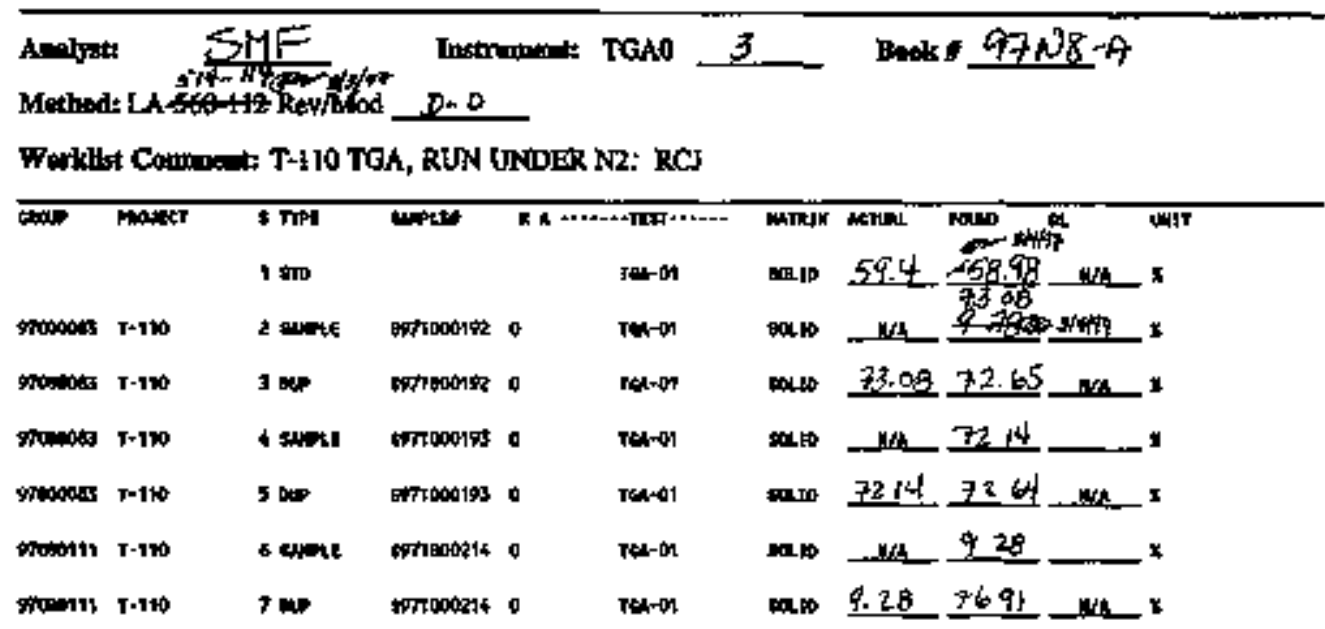

Final page for worklist $\# \mathbf{1 6 8 3 0}$
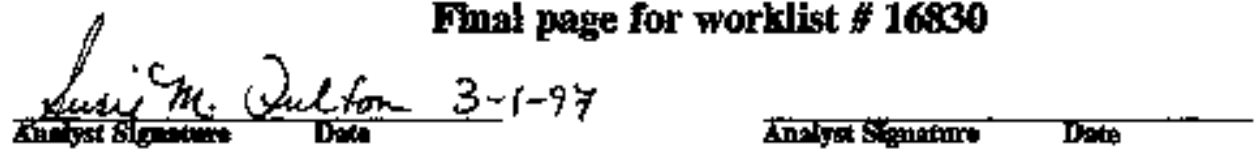

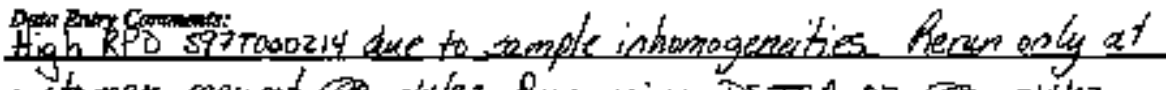

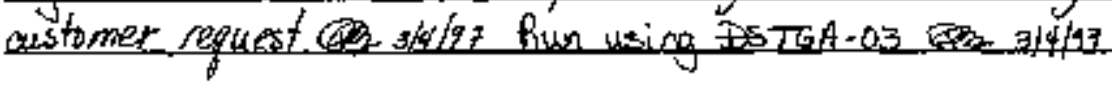

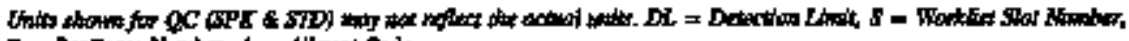

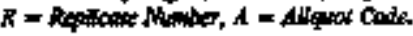

388 
HNF-SO-WH-DP-238, REV. 0

\section{LABCORE Data Entry Template for Worklist\$ 16830}

Analyt: SMF Instrmment: TGA0 3._ Bookf 97NSA

Method: LA-514-114 Rew/Mod D-O .

Worklist Comment: T-110 TOA, RUN UNDER N2. RCJ

\begin{tabular}{|c|c|c|c|c|c|}
\hline B ITP & 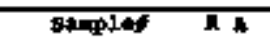 & 704 & Batrix & arotiph & Projte \\
\hline $1 \operatorname{sen}$ & & $50 x=03$ & BOCID & & \\
\hline 2 6NATr: & $\begin{array}{c}9977000192 \text { d } \\
\text { Amelyten Bequeited }\end{array}$ & $=0$ & sor.ts & 57000083 & $T-120$ \\
\hline 3 DUP & s977000192 & $\operatorname{Teth}-03$ & sacro & & \\
\hline 4 SAMPLS & 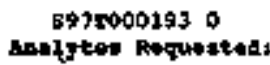 & ${ }_{1}^{2} \operatorname{Ten}-03$ & BOLID & 97000083 & $z-110$ \\
\hline 5 Dop & s97Tdoorigso & $\mathrm{T}$ (a)-03 & scorrb & & \\
\hline SWTPE & 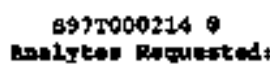 & $\begin{array}{l}\operatorname{Tax}-03 \\
\text { Thx- }\end{array}$ & ODLID & 93000111 & $7-110$ \\
\hline $7 \mathrm{DHB}$ & \$97พ0002140 & Fak-03 & sct.to & & \\
\hline
\end{tabular}

\section{Final page for worklist $\# \mathbf{1 6 8 3 0}$}

zee Attached for Signatues Results

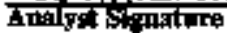

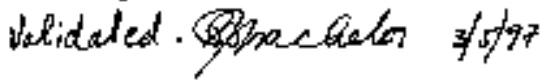

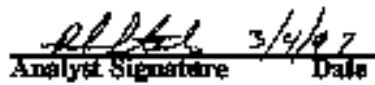

Duta Entry Coormants:

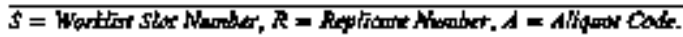


Ormin to Th

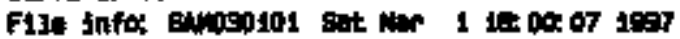

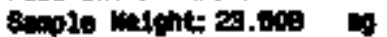

sortopotse

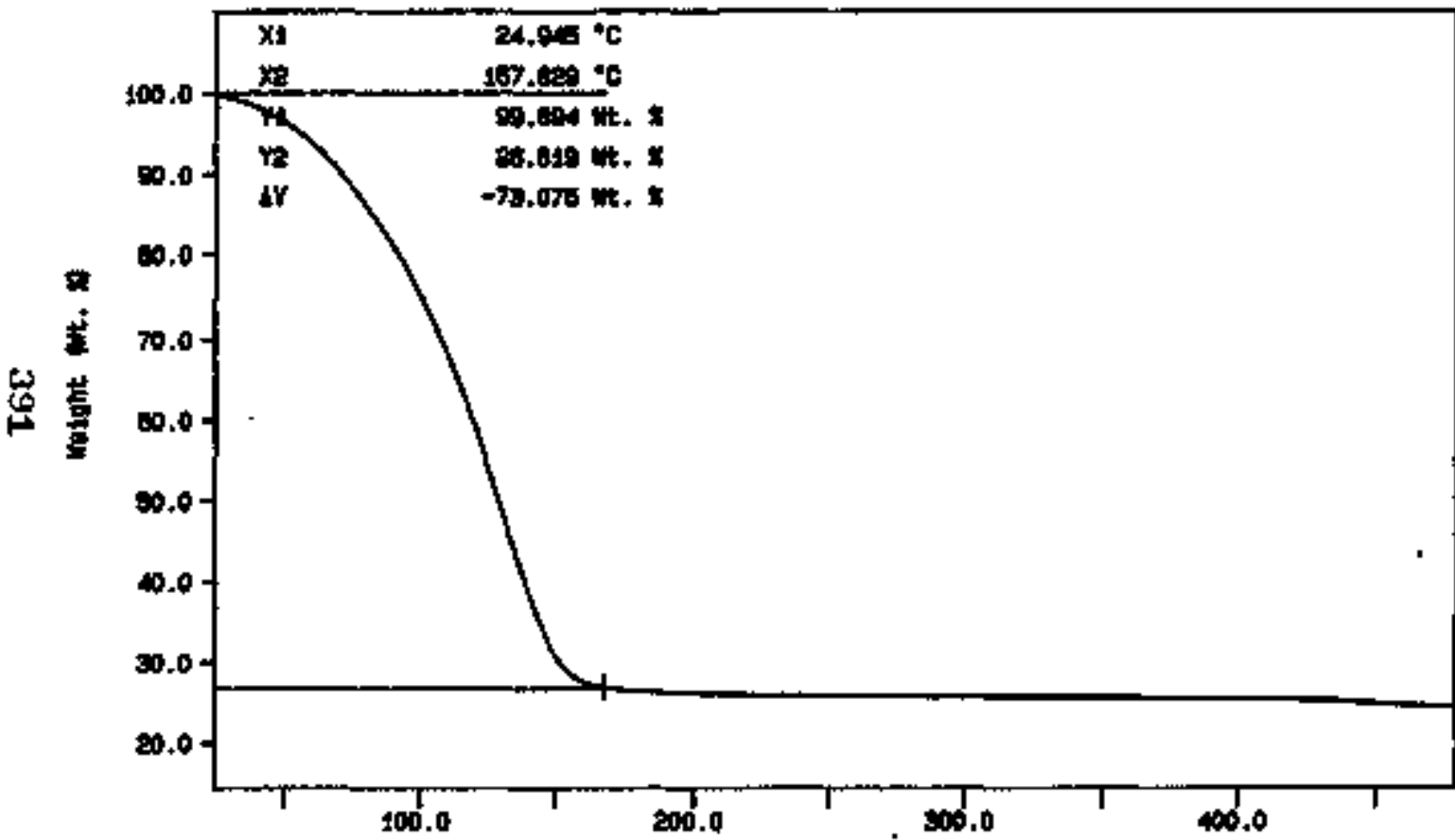

$100 / 1$ w $\mathrm{kP}$

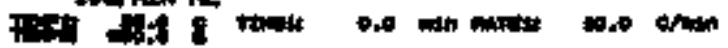

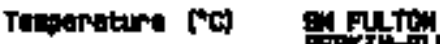

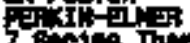

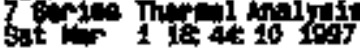


Drve is TR

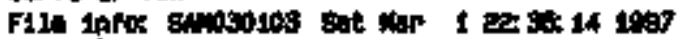

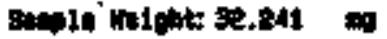

eorrooseg

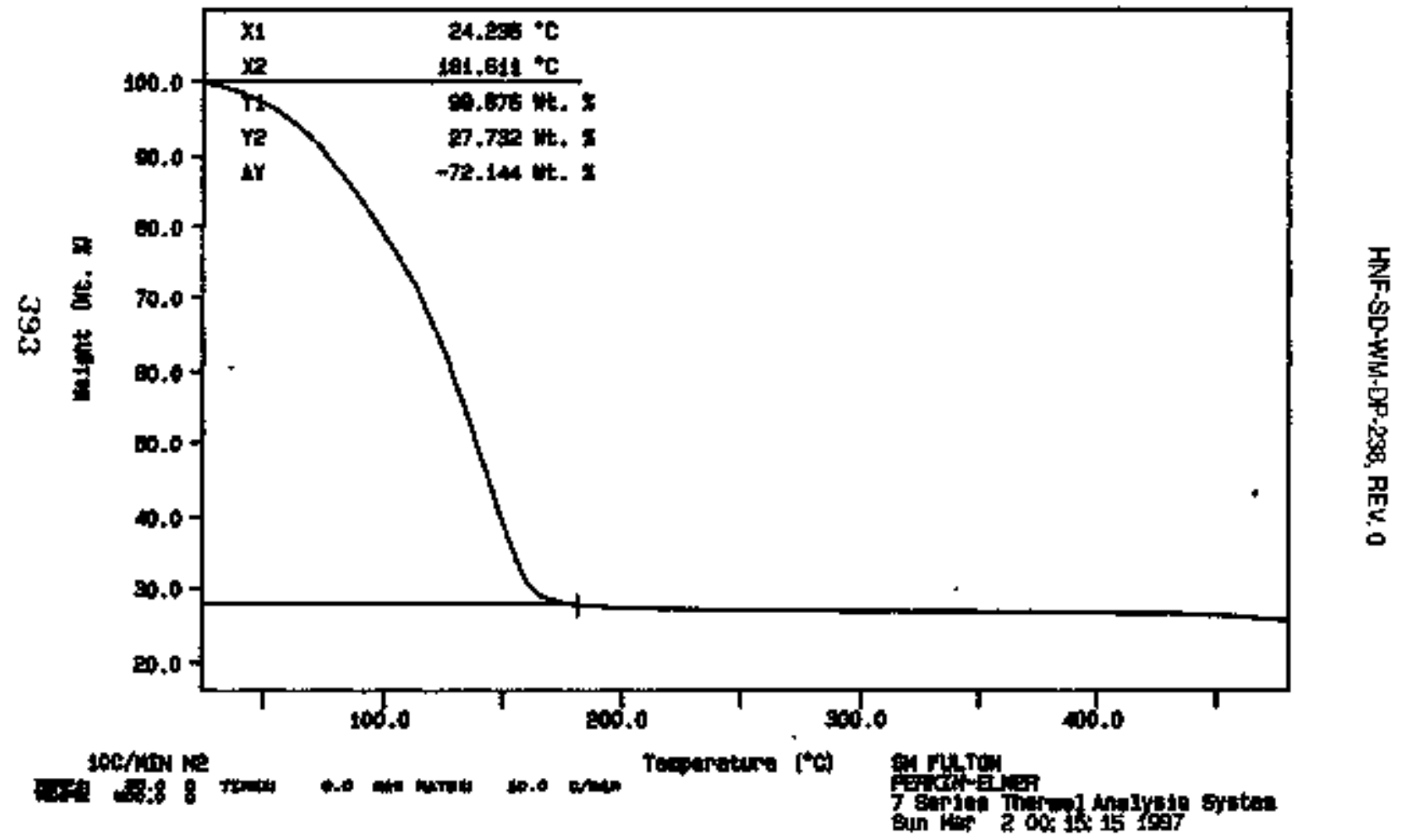




\section{Parret is Th}

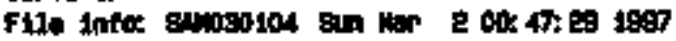

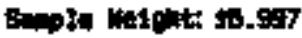

8grToostes do

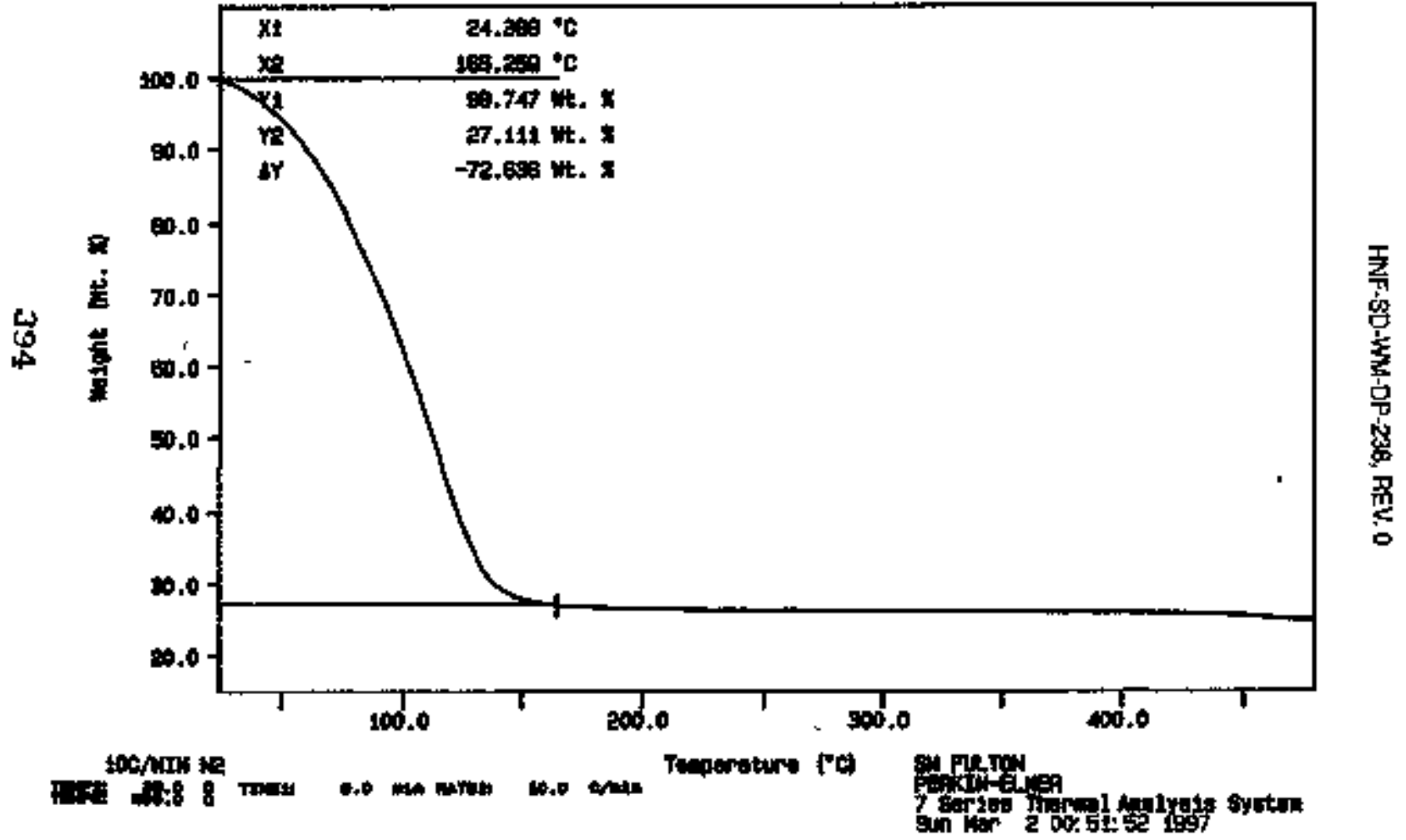


Crre II Tat

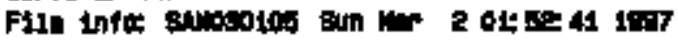
ompra wight: 12,097

:artoraedA

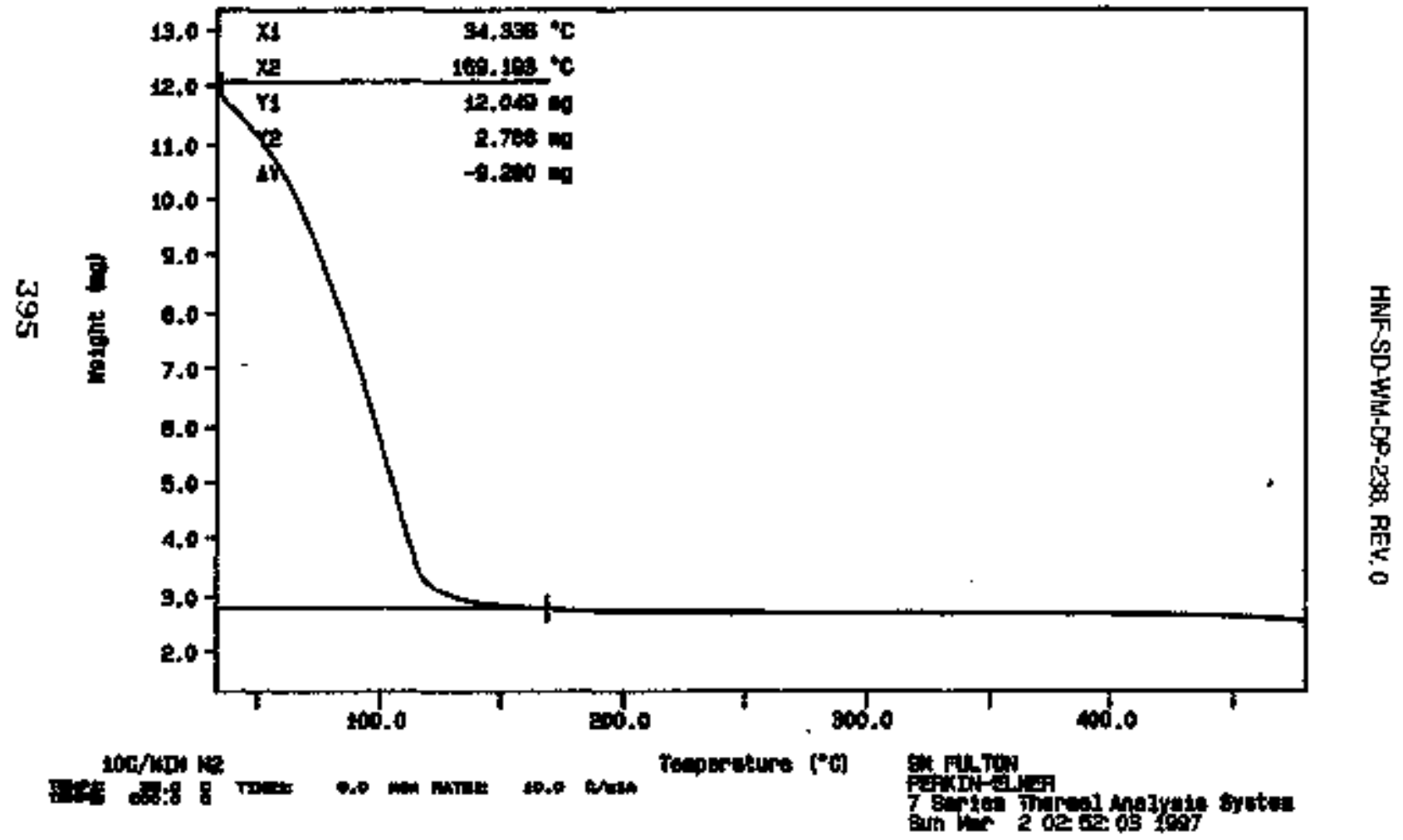


tarne 는

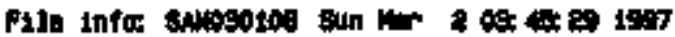

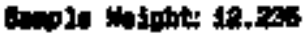

BorToperd do

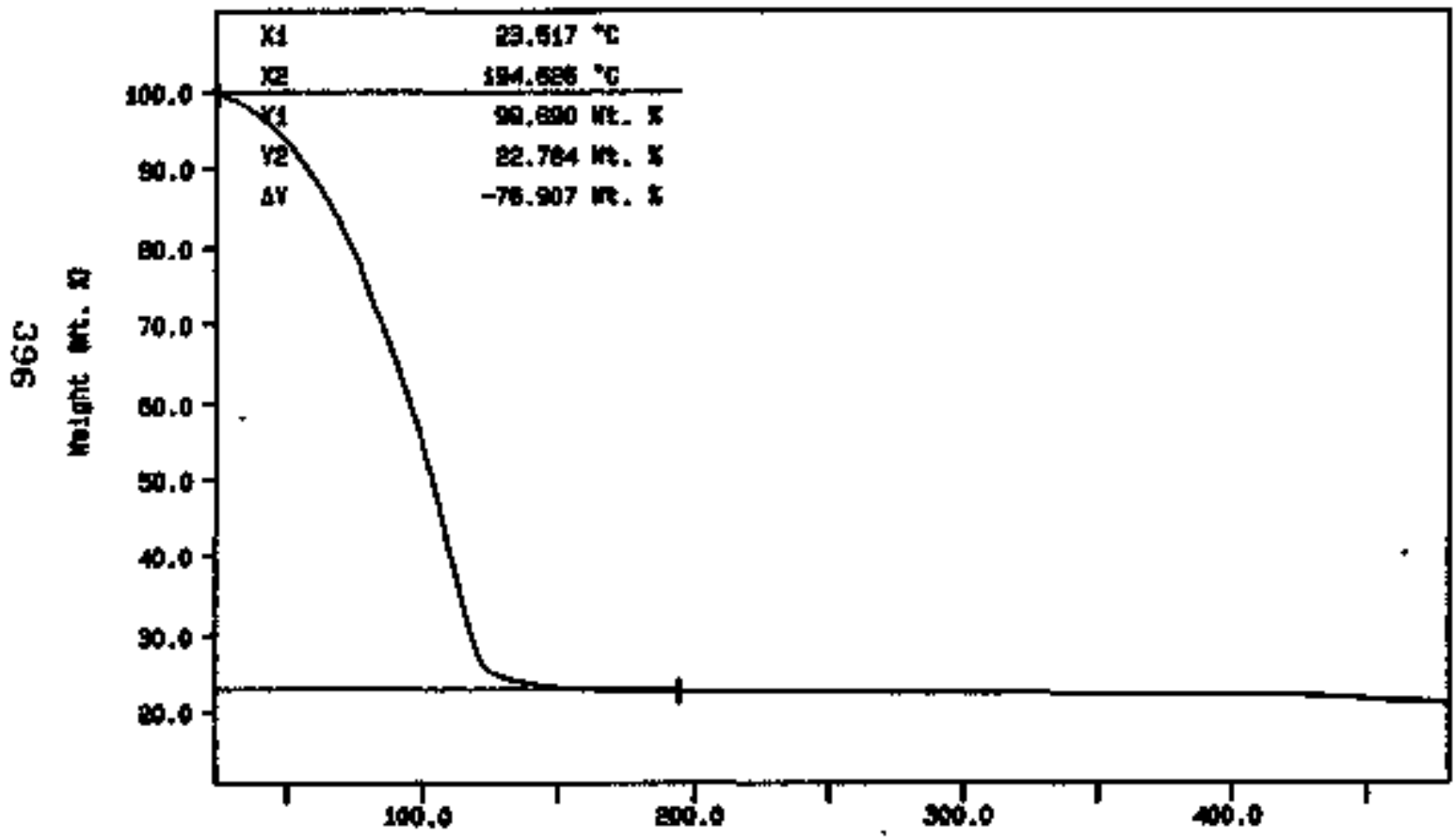




\section{LABCORE Data Entry Template for Worklist\# 16831}

Amalyst: HRM Instrument: TGAO 3 Bonk P7N8A

Mefhod: LA-S]4-114 Rev/Mod D-O

Warkid Commens: T-110 TGA, RUN UNDER N2. RCJ

\begin{tabular}{|c|c|c|c|c|c|c|c|c|c|}
\hline 0 & DA0द्यन्धा & $\begin{array}{l}\text { B TIVE } \\
1 \text { \$TP }\end{array}$ & ANPEt & D & 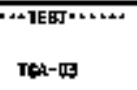 & $\begin{array}{l}\text { martix } \\
\text { क्भh } 16\end{array}$ & 59.4 & $\begin{array}{l}\text { का } \\
58.95 x\end{array}$ & is/h \\
\hline 9hwh11 & $t+110$ & I thal & $597+0016$ & 4 & ten-OE & solite & $n / 4$ & 76.98 & \\
\hline movonil & $T+1 น$ & 3 ave & 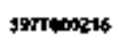 & 0 & $r e x-5 s$ & set It & 76.98 & 77.40 & 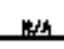 \\
\hline 9robiti & $t+100$ & 4 thent & 197tiosec1? & 0 & ten-4s & stete to & . & 76.27 & \\
\hline $9 x 000111$ & $1=110$ & 5 WP & SFTtDowat? & 0 & TGh-03 & 50 & Ho. 17 & 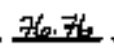 & Inth \\
\hline 9xation & T-110 & † HUPLE & 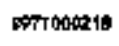 & 0 & Tan-03 & exto & . & 36.61 & \\
\hline 9xo0*111 & $T=110$ & $7 \mathrm{MP}$ & 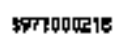 & 0 & $T \mathrm{Th}_{1}, \mathrm{As}$ & $\sin x$ & 710.6 & $7 f_{0}, 03$ & Wh \\
\hline
\end{tabular}

\section{Final page for worklist \#16831}

See Atached for sion atwe

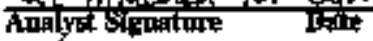

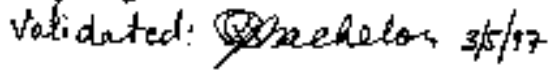

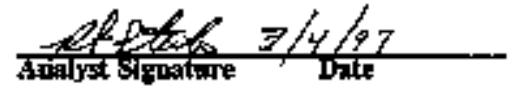

Dara Euny Cammours:

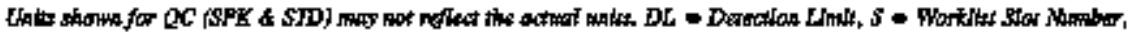

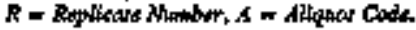




\section{LABCORE Data Entry Template for Worldist" 16831}

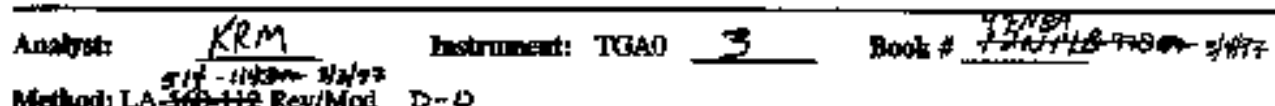

Method LASA the Rew/hod

D-b

Warift Connest T-110 TOA, RUN UNDER N2: RCJ

\begin{tabular}{|c|c|c|c|c|c|c|c|c|c|}
\hline ctore & pablect & $\begin{array}{l}\text { 自 trite } \\
1 \text { IIt }\end{array}$ & EHAte: & & 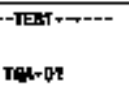 & $\begin{array}{l}\text { WhInIt } \\
\text { thels }\end{array}$ & 1CT0. & N58.95 & ma. \\
\hline soltotsys & $T-170$ & 2 surme & 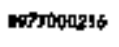 & a & $\mathrm{red}=\mathrm{pr}$ & tab & wh & 7488 & \\
\hline stopoti1 & $T-110$ & 3 owp & smotocets & $\bullet$ & $t+1-1$ & m & $\exists 6,98$ & 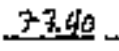 & m \\
\hline Troosts & $T=11$ & 4 somet & 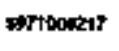 & $\bullet$ & TEx-4A & sing & 반 & 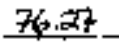 & \\
\hline moot11 & T-หม & $5 \mathrm{DW}$ & whoopal? & 0 & $n d-01$ & $\operatorname{sen}$ & 76,27 & 76.76 & . \\
\hline Prtallit & $t+1+0$ & \& subste & tropoesta & 0 & Tex-61 & gand & uㅡㄴ & 7606 & \\
\hline 970111 & T-1พฤ & $t \mathrm{wr}$ & 由) & 0 & 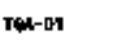 & setio & 76,61 & 76,03 & $m$ \\
\hline
\end{tabular}

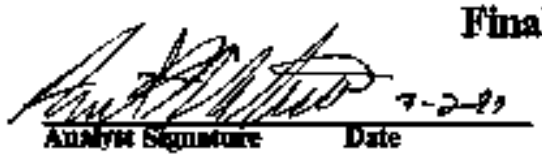

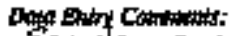

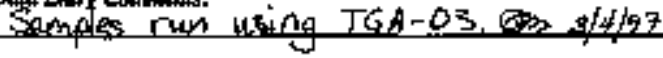

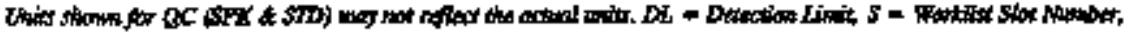

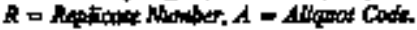


HNF-SD-WM-DP-238, REV. 0

WAC OCHIETORY TARLE EDIY SCRERA

\begin{tabular}{|c|c|c|c|c|c|c|c|c|}
\hline \multicolumn{3}{|c|}{$\begin{array}{r}\text { Sanplef } \\
\text { Grouxp } \\
\text { Worklist } 16831 \\
\end{array}$} & \multicolumn{2}{|c|}{$\begin{array}{l}\text { sample ID } \\
\text { Customer } \\
\text { WL Comment T }\end{array}$} & 0 Trah ROM & IDBR $\mathrm{N2}$ & ECI & \multirow[b]{2}{*}{ Ueer } \\
\hline Tert. & Watrx & TYPo & fotual & Found & tifeld & STAT & Analdate & \\
\hline $\begin{array}{l}\text { TGA-03 } \\
\text { TGA=03 } \\
\text { THA-03 } \\
\text { TGA=03 }\end{array}$ & $\left|\begin{array}{l}\text { SOLID } \\
\text { Spt.ID } \\
\text { SOLID } \\
\text { BOLID }\end{array}\right|$ & $\begin{array}{l}\text { STD } \\
\text { DUP } \\
\text { DUP } \\
\text { DUP }\end{array}$ & $\begin{array}{l}5.94001 \\
76.98 \\
76.27 \\
76.61\end{array}$ & $\begin{array}{l}98.95 \\
77.40 \\
76.76 \\
76.03\end{array}$ & $\begin{array}{l}99.2421 \\
0.3441 \\
0+6404 \\
0.7600\end{array}$ & $\begin{array}{l}\text { NEN } \\
\text { NEE } \\
\text { WEEN } \\
\text { NEW }\end{array}$ & $\mid \begin{array}{ll}03 / 04 / 97 & 1506 \\
03 / 04 / 97 & 1506 \\
03 / 04 / 97 & 1506 \\
03 / 04 / 97 & 1506\end{array}$ & $\begin{array}{l}r t s \\
r t s \\
r t s \\
r t g\end{array}$ \\
\hline
\end{tabular}

Save(F12) Find (F3) 
HNF-SD-WH-DP-239, REV. O

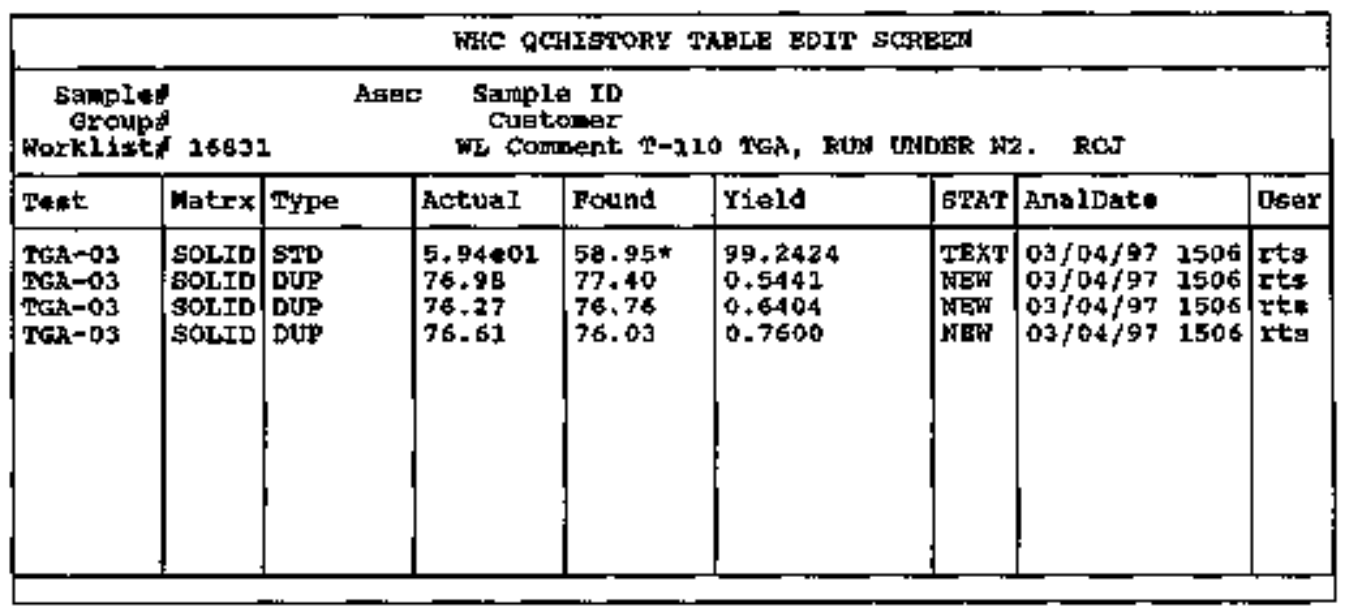

Save(F12) End (FI) 
Curve if ten

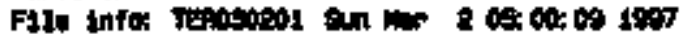

secple Wiqhte 85.691 ad

TEג घा० 97in-k

SAGNATURE BELOW AEPFESENTS CHEWHCA TECHNOCGIST/CHEHST THLT

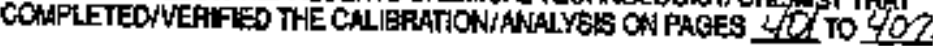

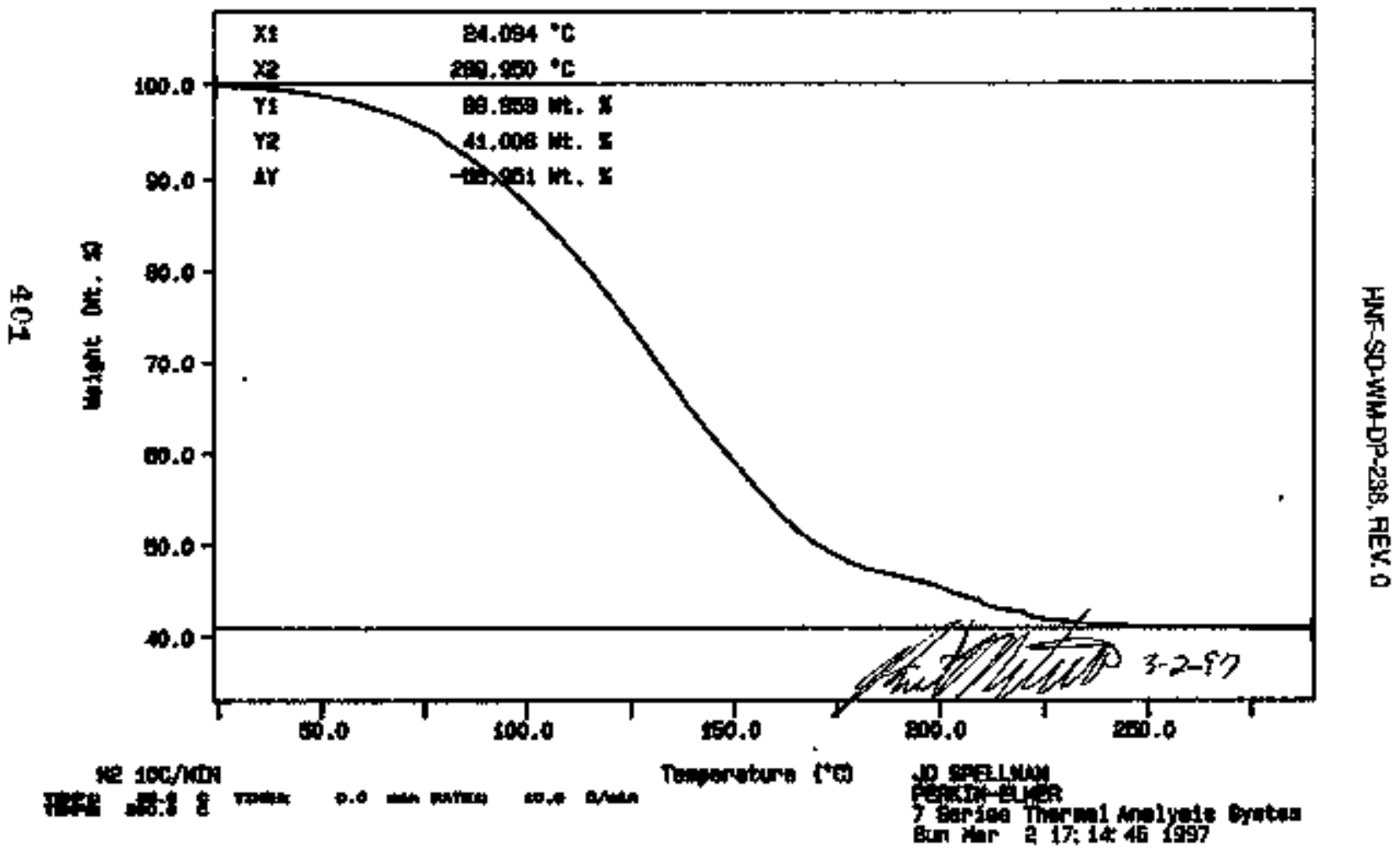


Curve :: TeA

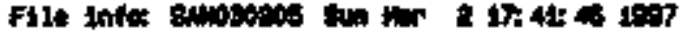

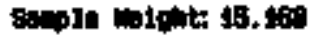

sertociess

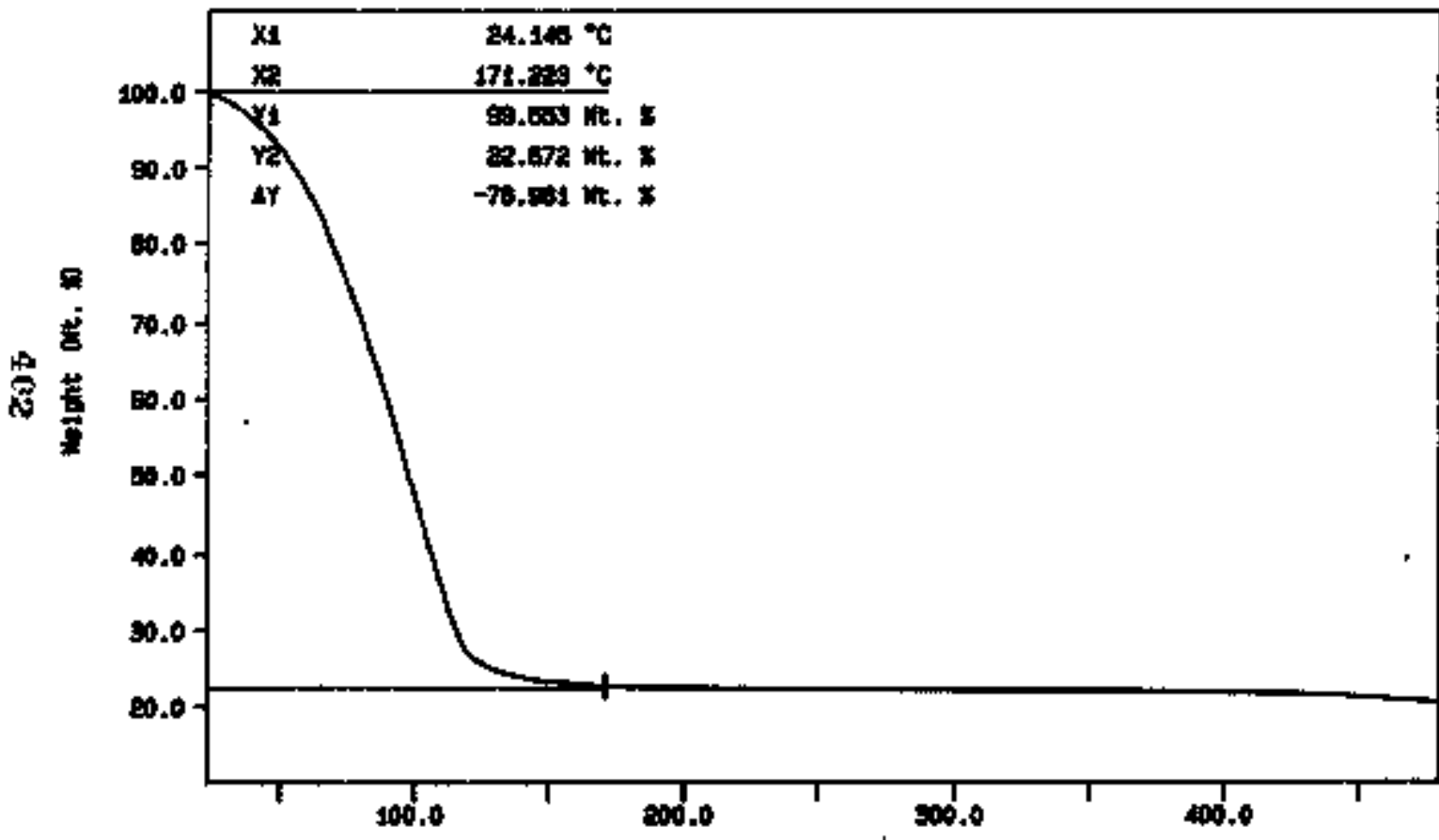

106/10 to

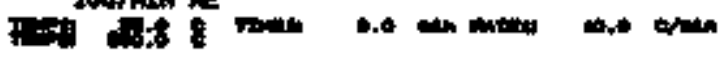

Tenporatare $f *$ 이

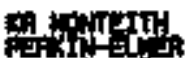

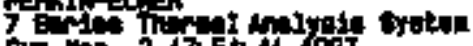

oun war 2 17, 5 t 41 igs 
Enve \&t ted

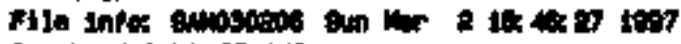

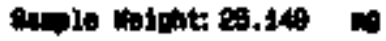

sortonsts or

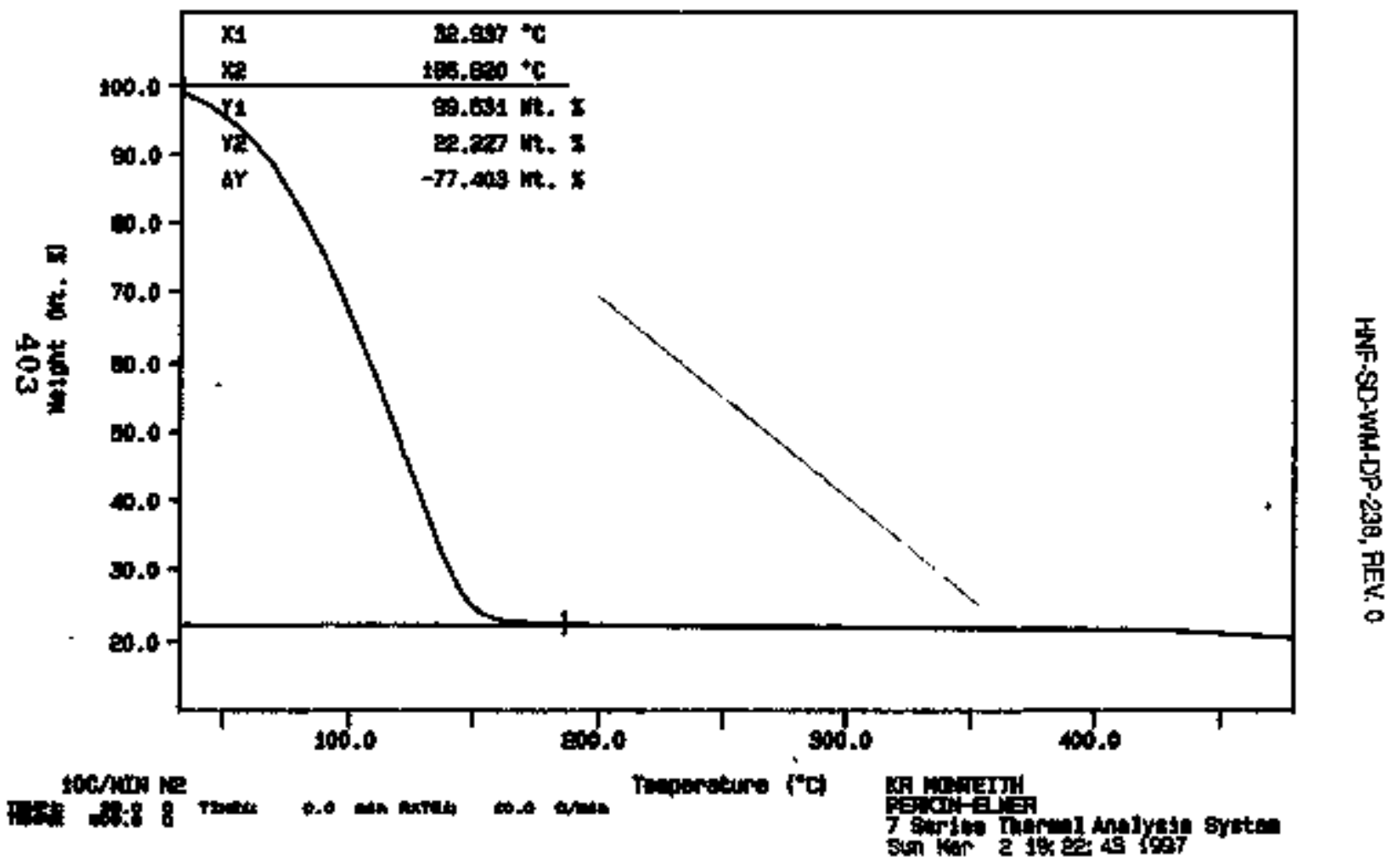


mint it Th

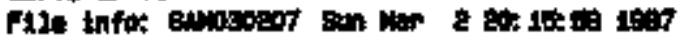

Piple might: 15.470

ETrapalt

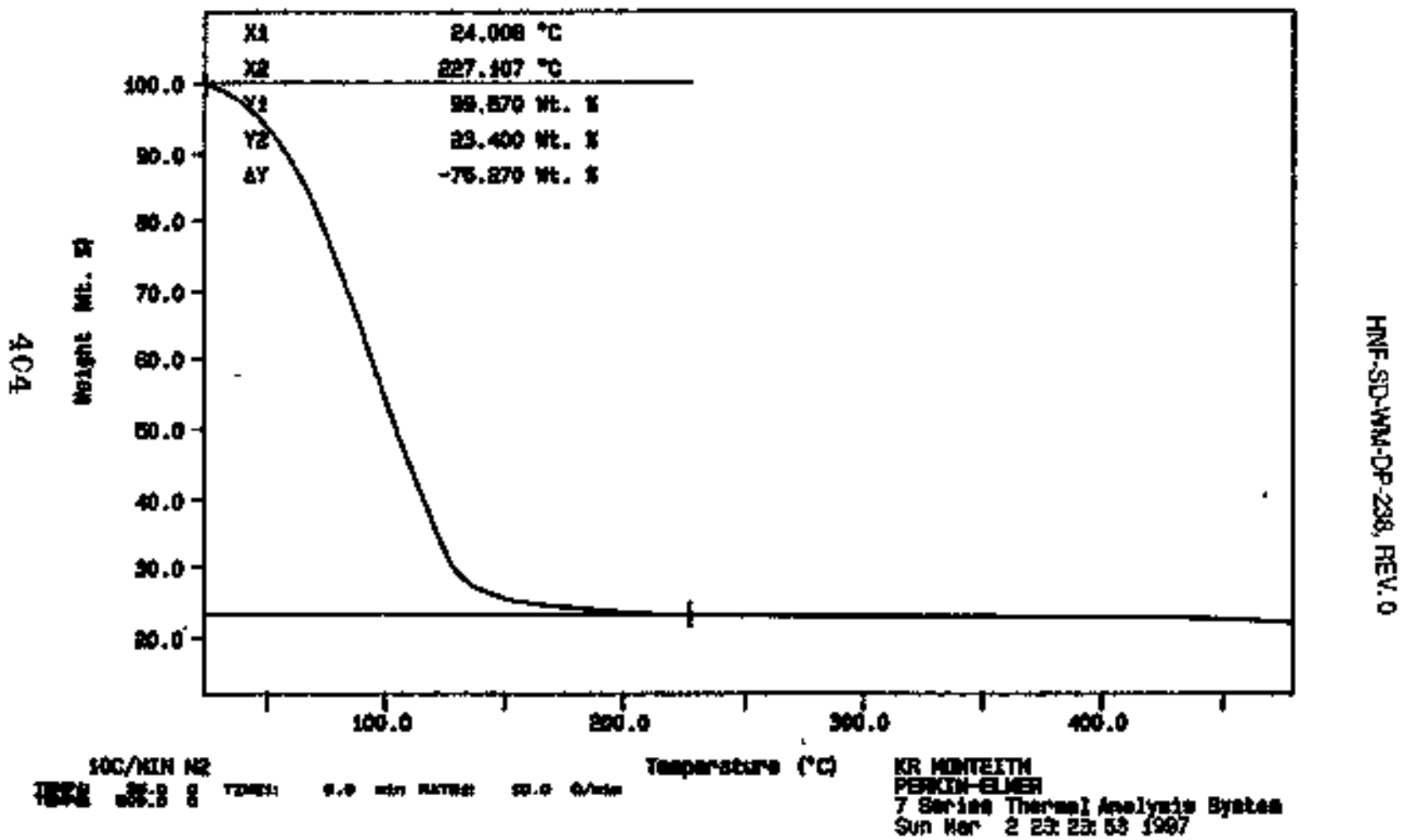


crave is Tê

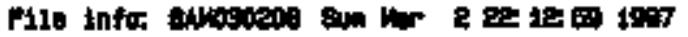
timple whohtt 18.98

BorToboat7 ITP

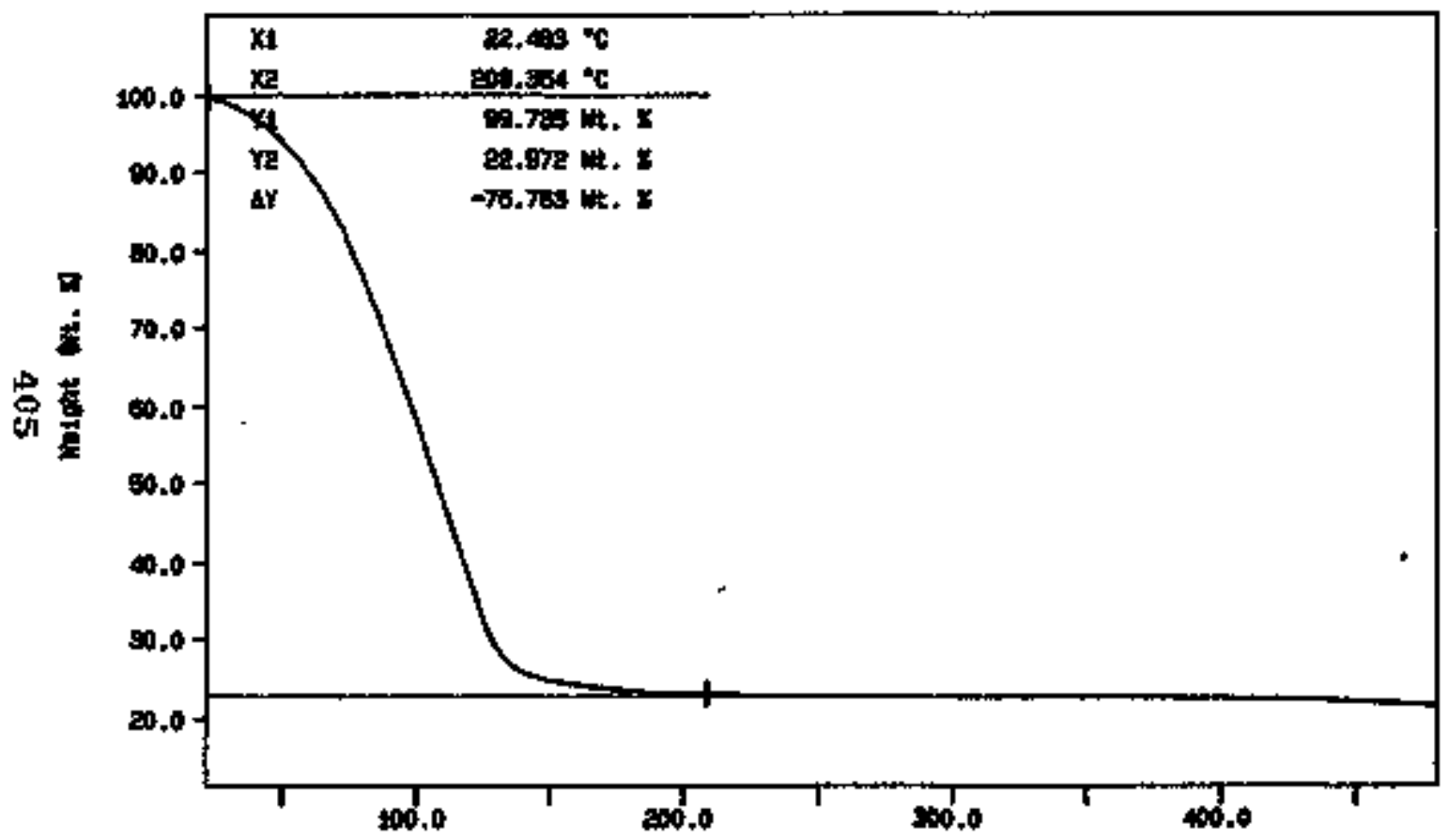

10FAitas ro:

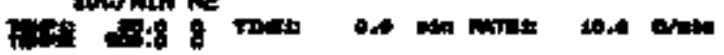

Theprature rot

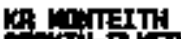

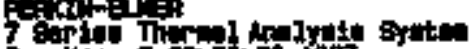


Corwe 1: Tak

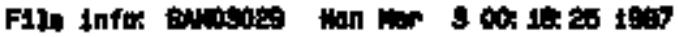

saple intght 13.007 ad

stroouest

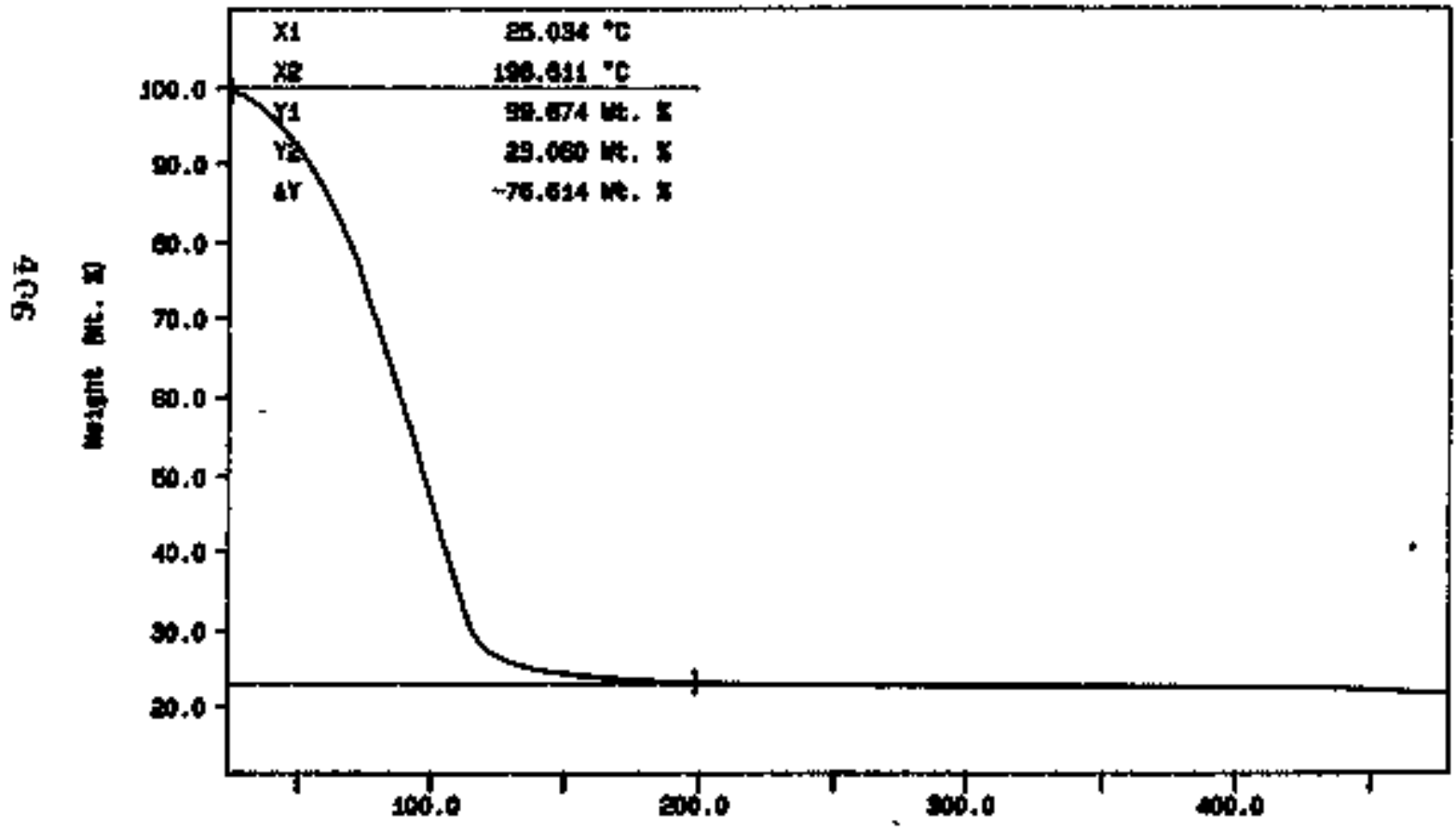

100/NIt in

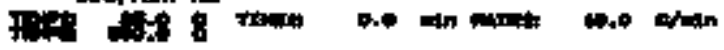

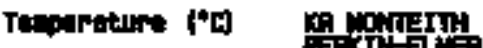

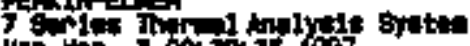

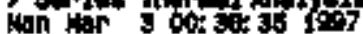


CINve it ER

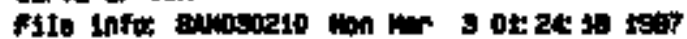

Ample wighte ig.tos es

Exroutita bep

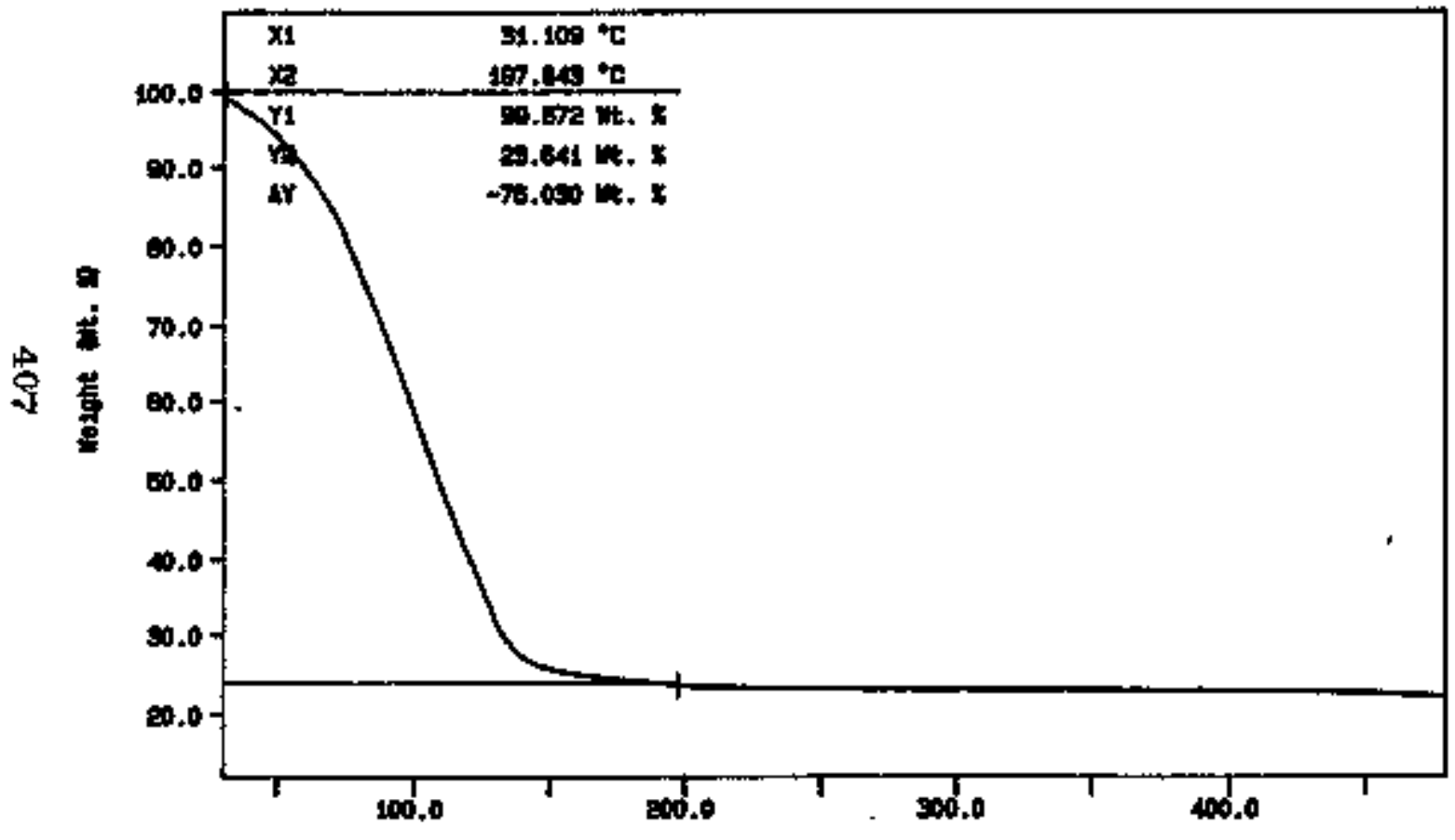

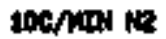

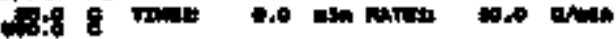

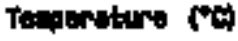

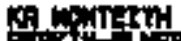




\section{LABCORE Data Entry Template for Worklist\# 16832}

Anolyst: Instriment: TGAO 3 _ Book 97XOA

Method: LA-514-114 Rev/Mod D-D

Workitist Cod gent: T-110 TGA, RUN UNDER N2. RCI

\begin{tabular}{|c|c|c|c|c|c|c|c|c|c|}
\hline GNOP & PRarect & $\begin{array}{l}0 \text { TYNe } \\
1 \text { เ }\end{array}$ & swipled & & 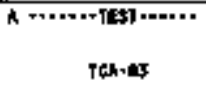 & $\begin{array}{l}\operatorname{melary} \\
\text { getcr }\end{array}$ & $\begin{array}{l}\text { Acrubl } \\
5.9 .4 \\
\end{array}$ & $\begin{array}{l}\text { nolp } \\
58.63\end{array}$ & was \\
\hline moconts & T-10 & 2 sure & mototoris & a & $201-6$ & Borlo & Noth & 77. 26 & \\
\hline Shoditt & T-110 & 3 dp & Sprionsto & D & tan.es & \%ald & $\overrightarrow{7} \mathbf{1}-2 b_{0}$ & 74 然 & mots \\
\hline snowitt & $T-110$ & 4 SULPL & किranoses & 0 & $\tan \cdot 03$ & $50 \times 18$ & aill & 77.15 & \\
\hline grocolit: & $t-110$ & rs bous & 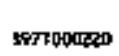 & 0 & Tthih & oxilet & 77.15 & 76.60 & 보 \\
\hline
\end{tabular}

\section{Final page for worklist \# 16832}

See Aftacked for Sinnature.

\section{直}

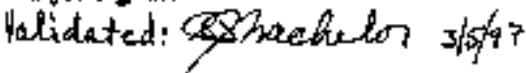

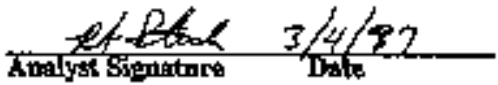

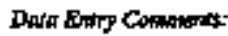

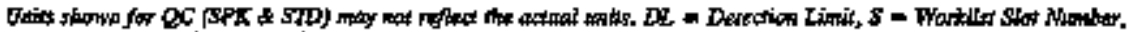

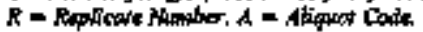




\section{LABCORE Data Entry Template for Worklist\# 16832}

Mage: I

Amalyity

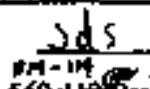

Intruent: TGAO 3

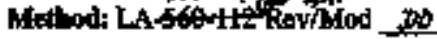

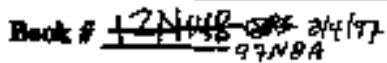

Wollit Coment: T-1FO TOA, RUN UNDER Ne: RCJ

\begin{tabular}{|c|c|c|c|c|c|c|c|c|c|c|}
\hline Exprar & Pharct & 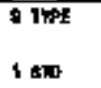 & IOPIE" & 11 & 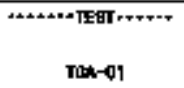 & mix & 5 sich & Paw & ma & $x$ \\
\hline Prototis 1 & ราเที & 2 entiry & 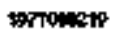 & - & $T+k-01$ & 踏15 & Ma & 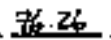 & & $\mathbf{x}$ \\
\hline Prooth & $T-110$ & 3 atp & Protime19 & $\bullet$ & 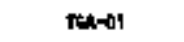 & סוnה & 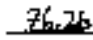 & 74.80 & H.th & $x$ \\
\hline probal1 & $t .110$ & 6 awrix & 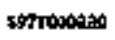 & 0 & TER:AI & sokit & ㄴ.S. & 홀/5 & & $x$ \\
\hline 97006111 & $T=t 10$ & B m中 & 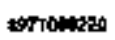 & $\bullet$ & Then & P国 & 77.5 & 7660 & $m$ & $x$ \\
\hline
\end{tabular}

Final page for worklist " 16832

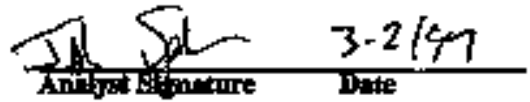

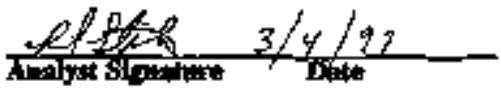

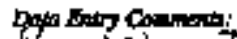

Bun using T6A-03.63 34k7

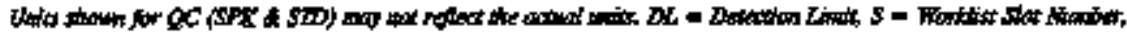

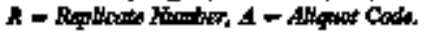


Qrve it The

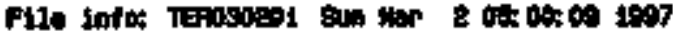

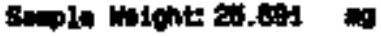

Ten 870 bhish

SIGNKTUFE GE OW REPRESENTS CHENCN TECHWCLOGISTHCHEMIST THAT

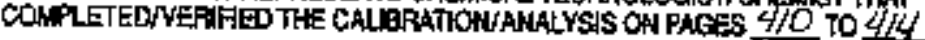

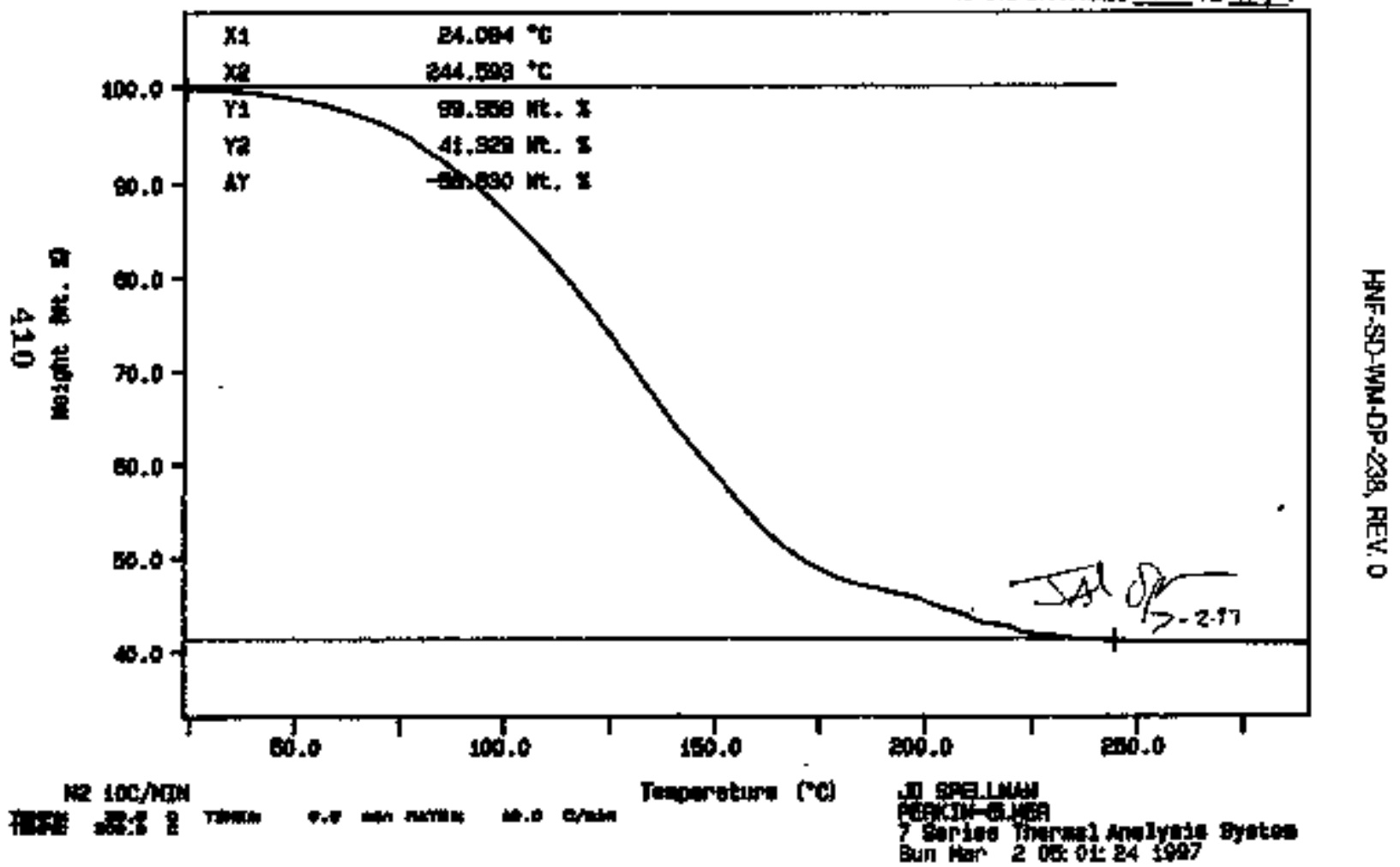




\section{Curva to Tex}

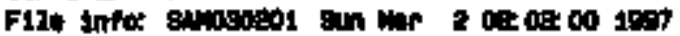

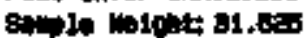

stroosts

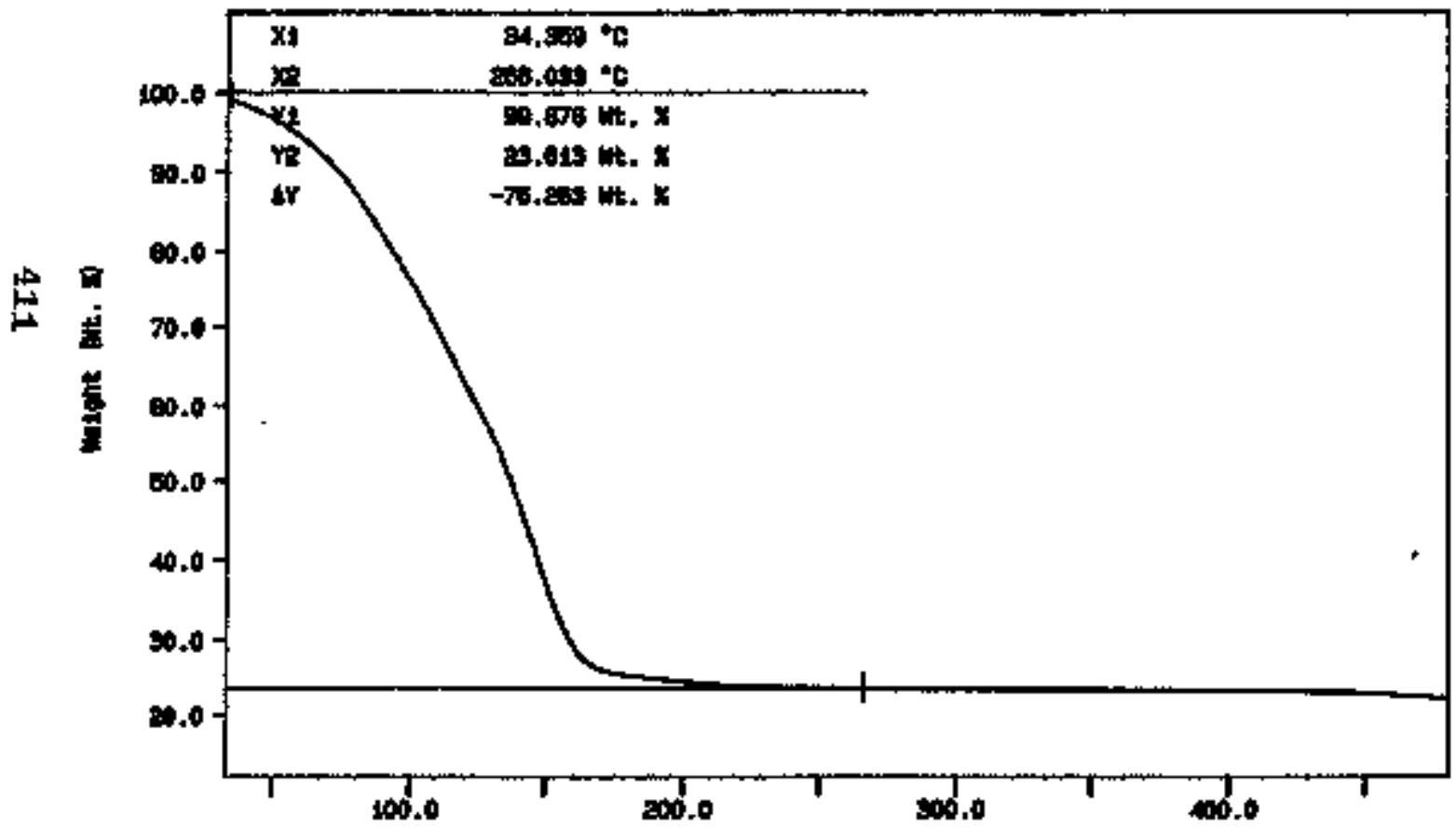

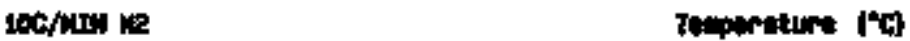

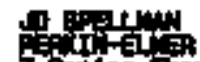

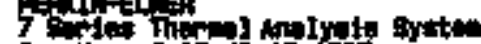

sum har a of 
Armor to Th

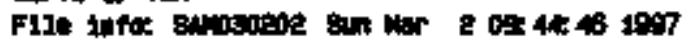

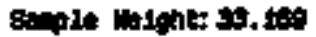

ensoutetious

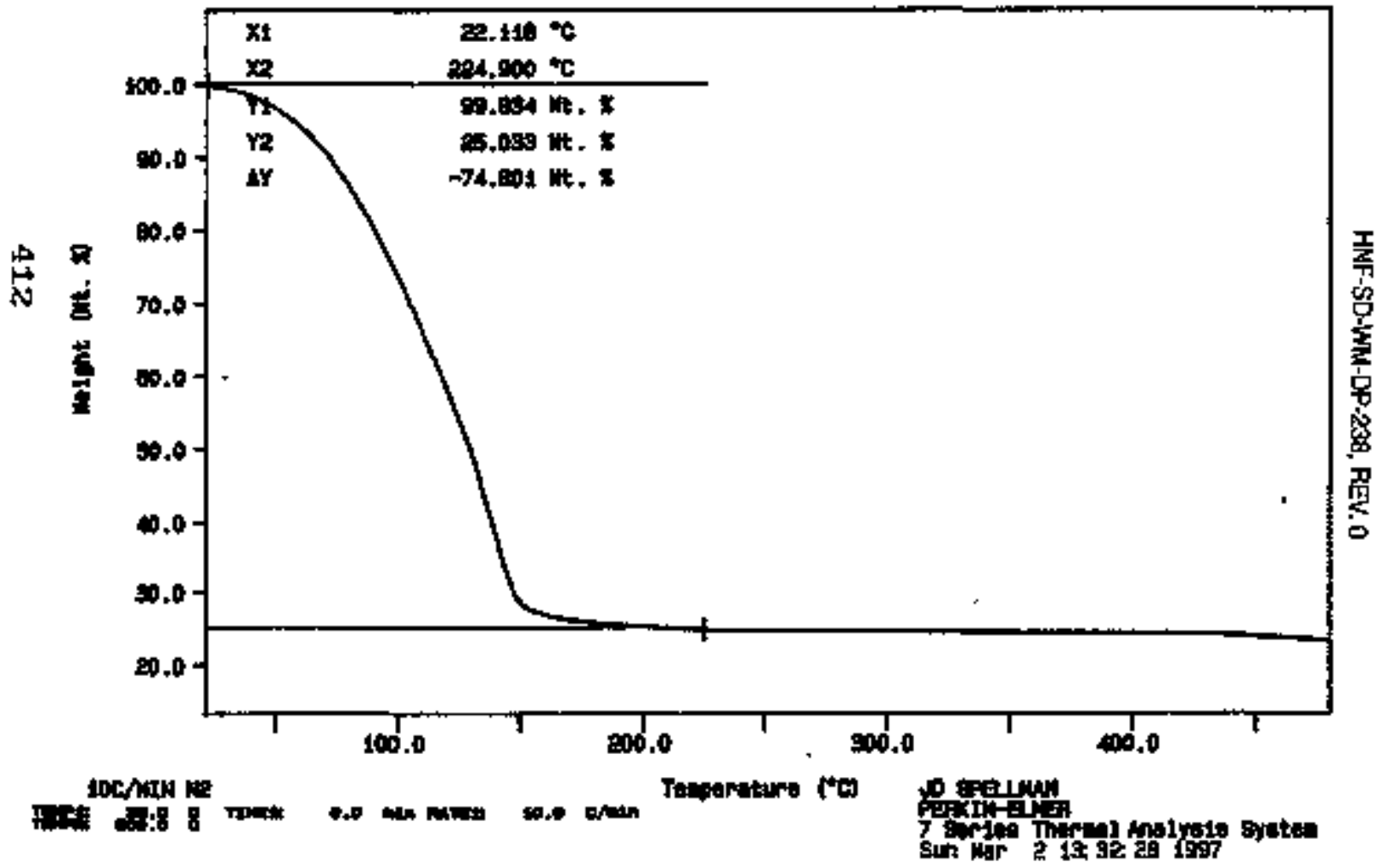


Drys it Try

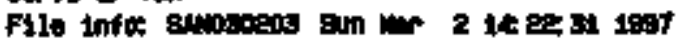

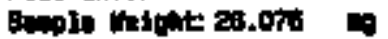

EgrToonest

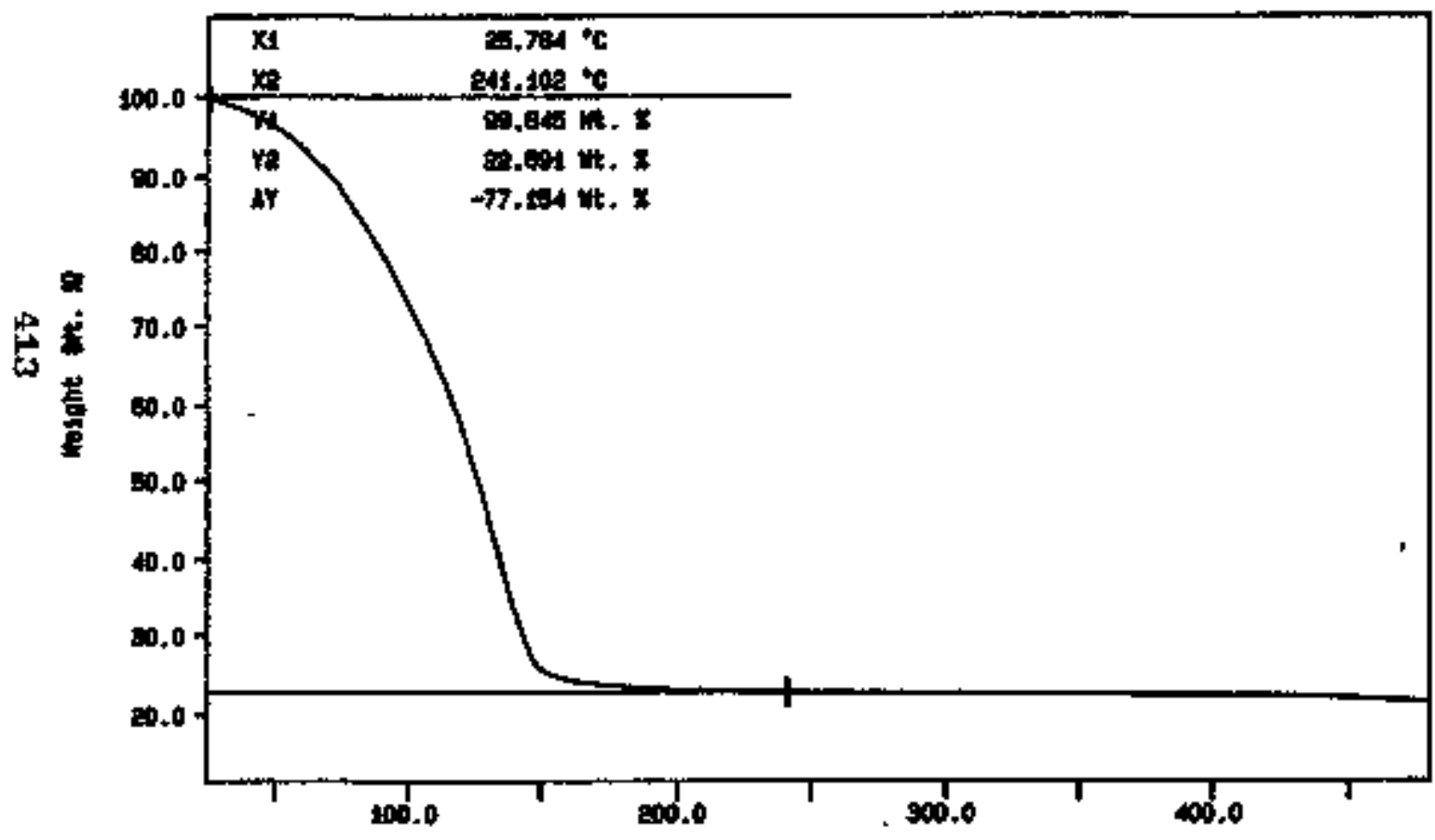

I00nath in

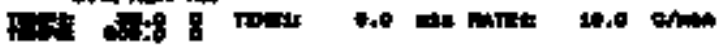
Teneretury ("여 
thre is Tet

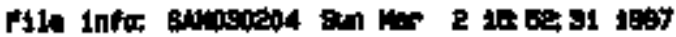
onple wigite 15.804

8.7Tsomitip

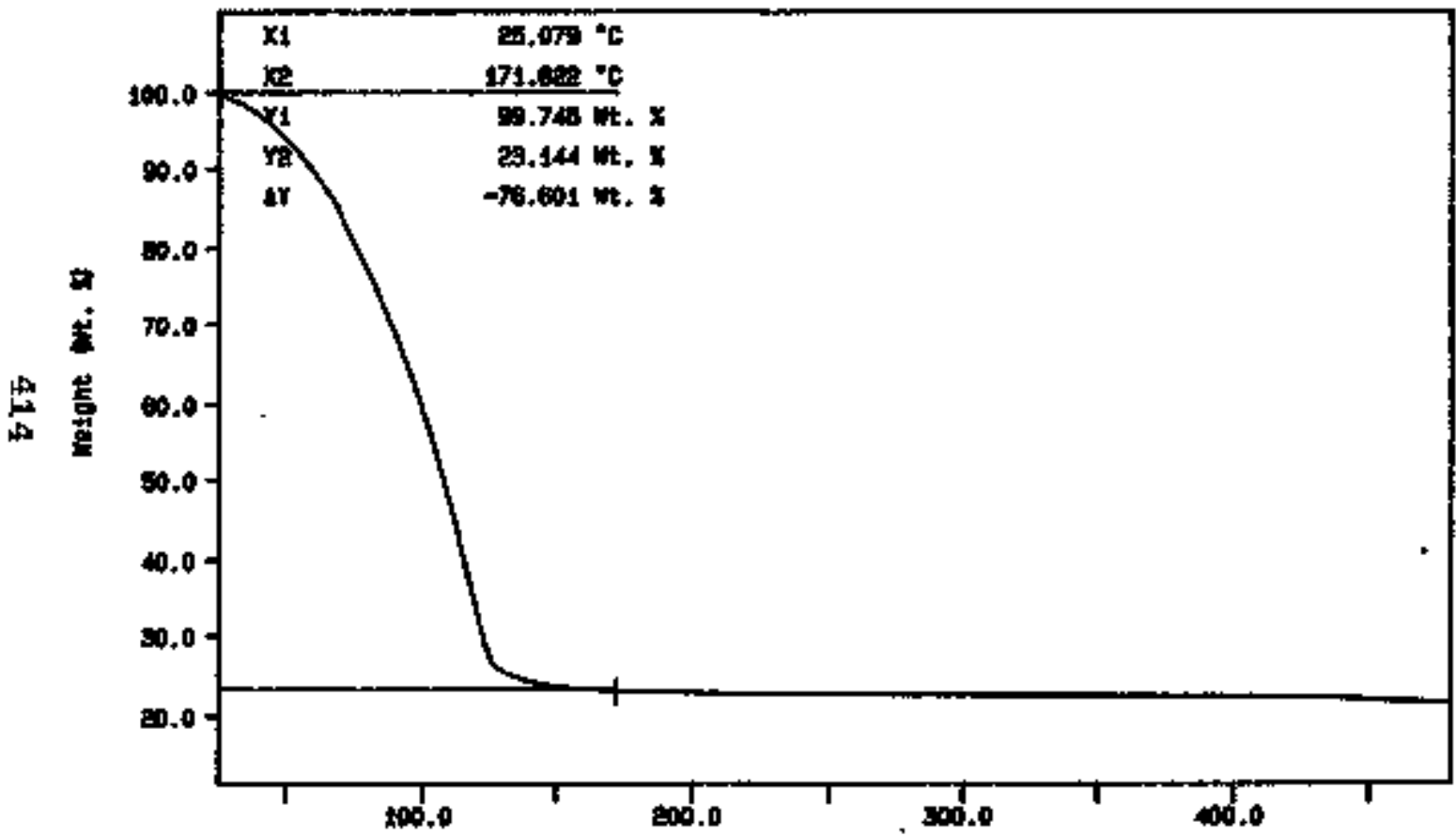

nochiti ka

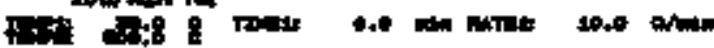

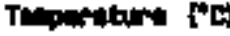




\section{LABCORE Data Entry Template for Worklist 16833}

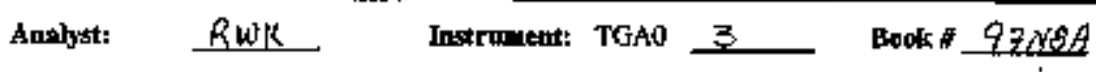

Metbod; LA-514-114 Rev/Mod D-O

Workllst Commen: T-110 TOA, RUN UNDER NR. RCJ

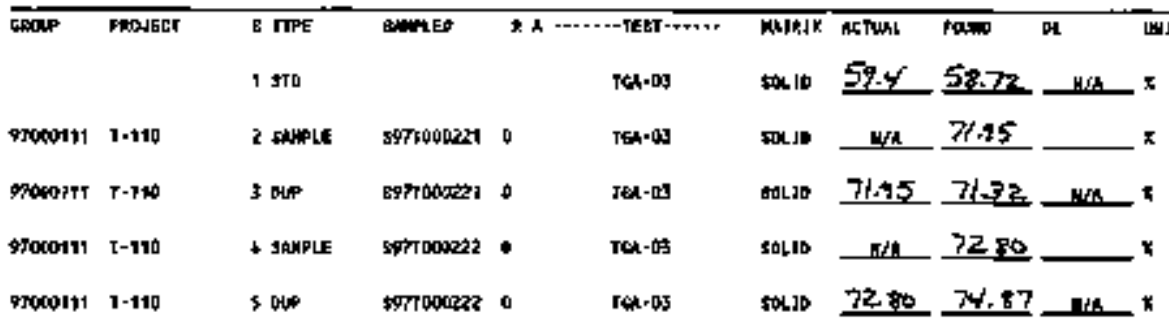

\section{Final page for worklist \# 16833}

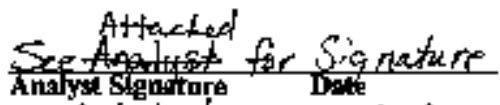

validated: Gopachelo $3 / 5 / 27$

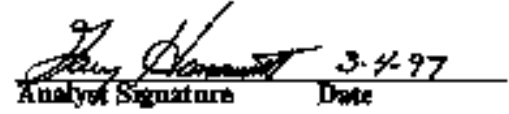

Dum Erry Canoustrs:

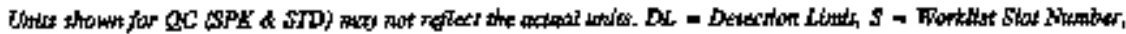

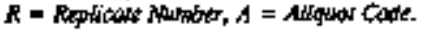




\section{LABCORE Dato Entry Template for Worklist" 16833}

\begin{tabular}{|c|c|c|c|c|}
\hline Angly & $\not R /$ & hotrimant: & TGAO & 3 \\
\hline
\end{tabular}

Method: LA Aly-ly

Q-O

Wortidis Commant: T-110 TGA, RUN LNDER N2. 'RCI

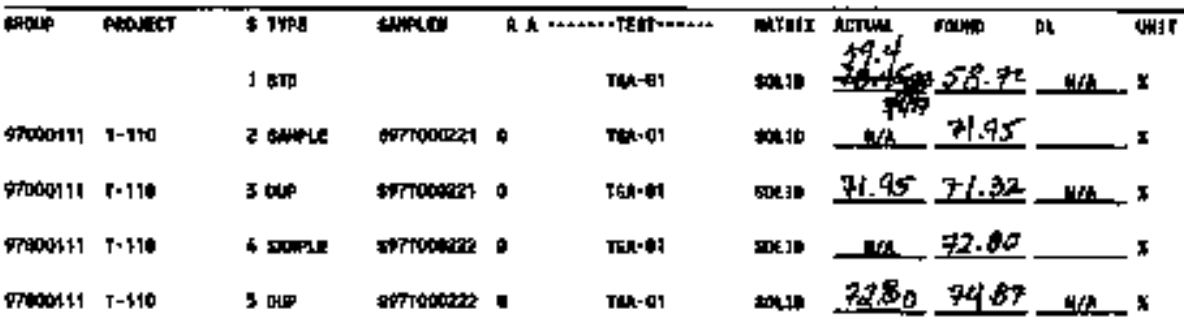

\section{Fingl page for warklist \#16833}
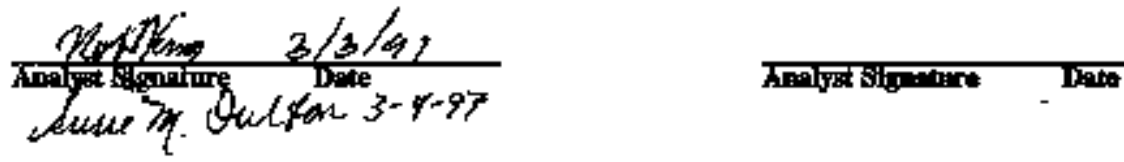

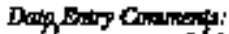

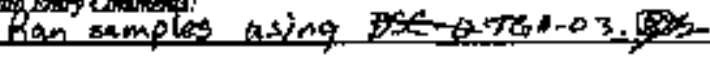

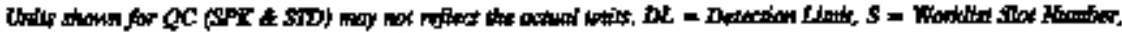

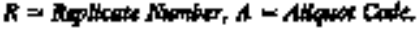


Cumil i: TEM

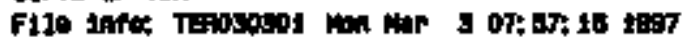

8teple intente co.7ag

Tax GTD orimat

a.

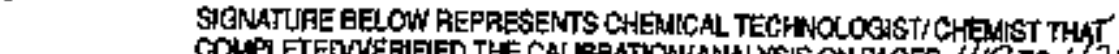
COMPLETEWVEFIED THE CALBAATONANALSIS ON PAGES 4 T TO 421 .

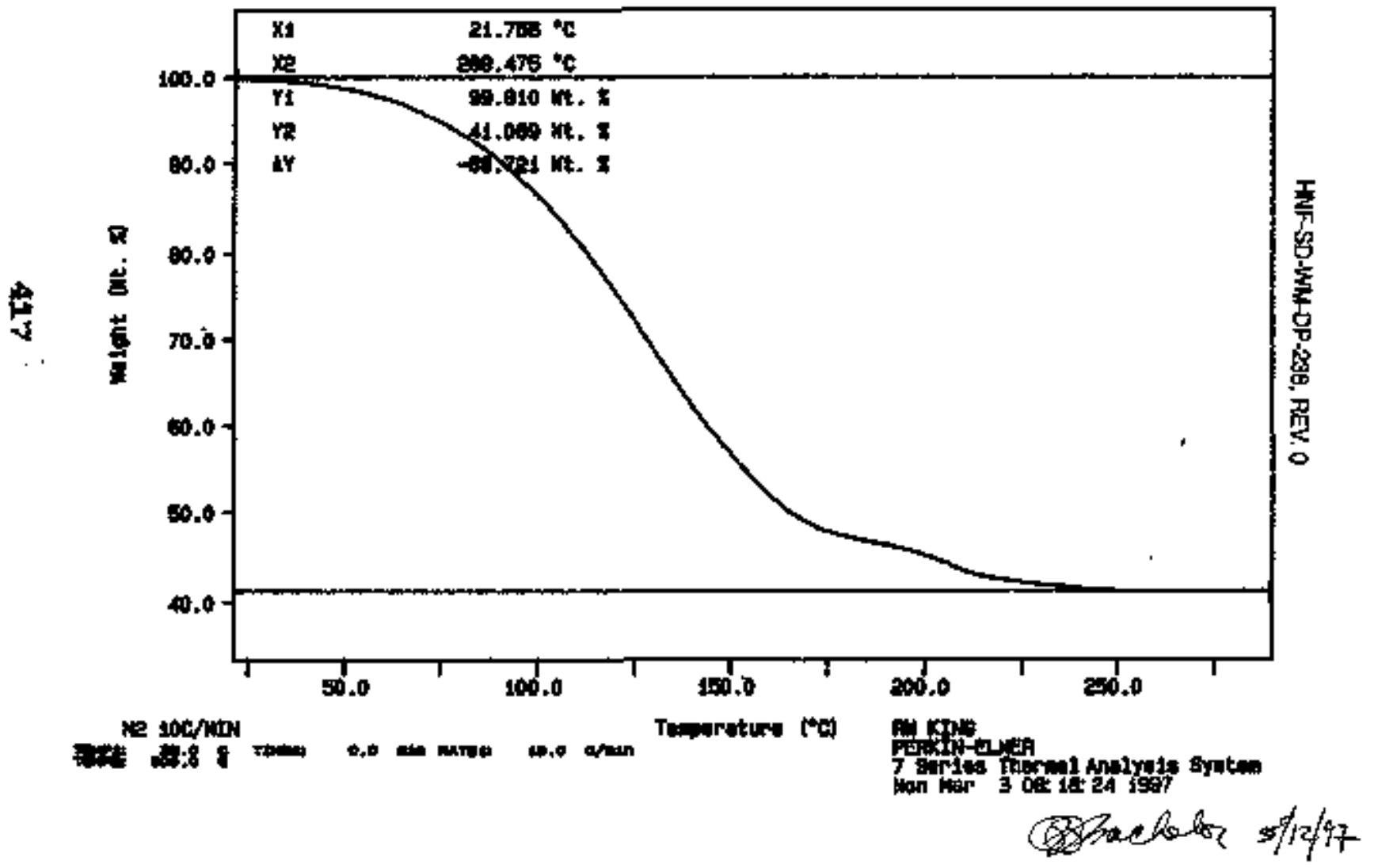


curnt 1: Tet

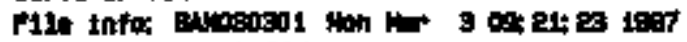

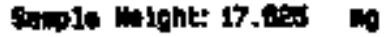

8ryooses

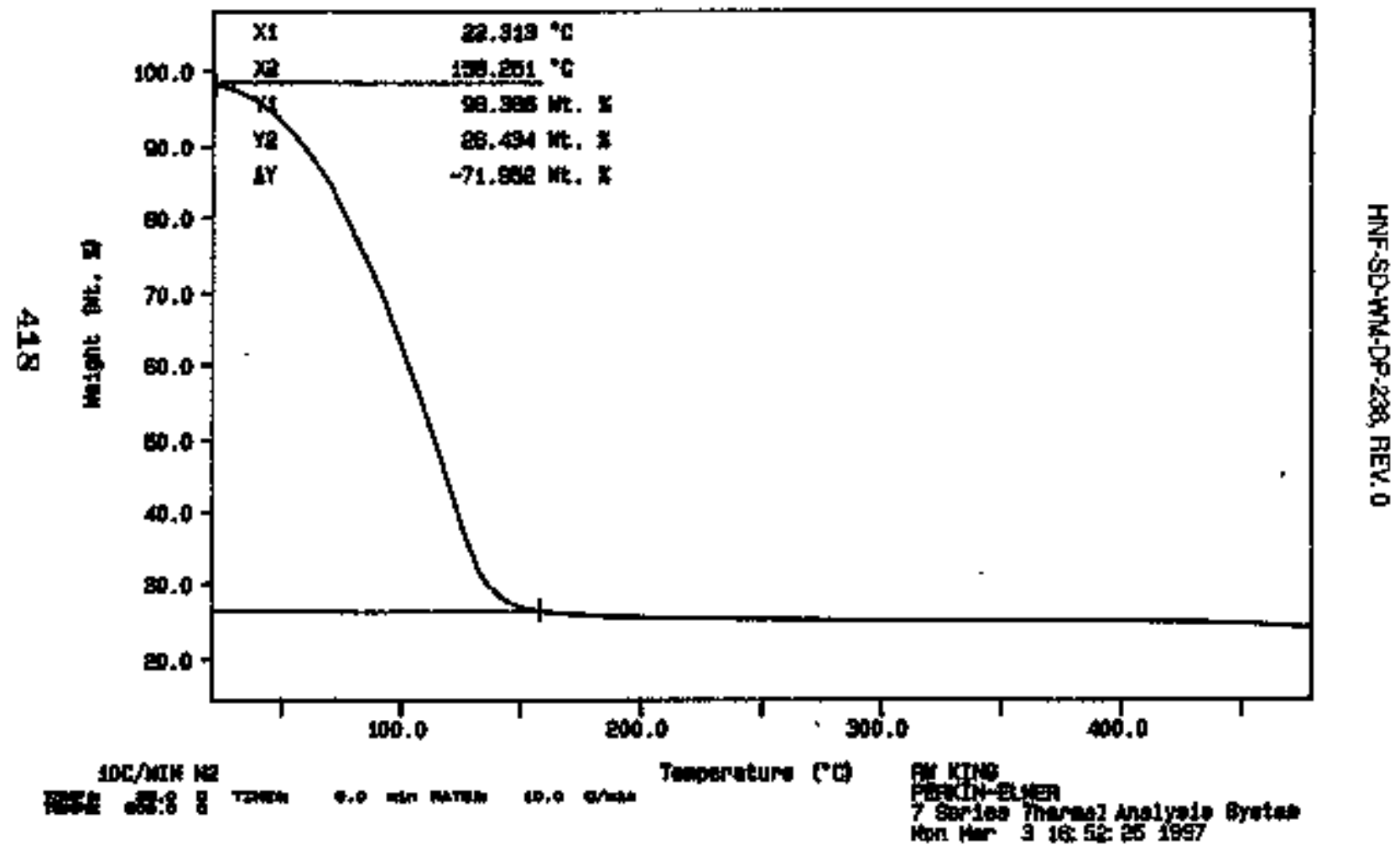




\section{Curve is Tex}

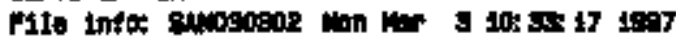

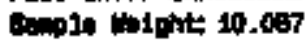

8070000es WMP

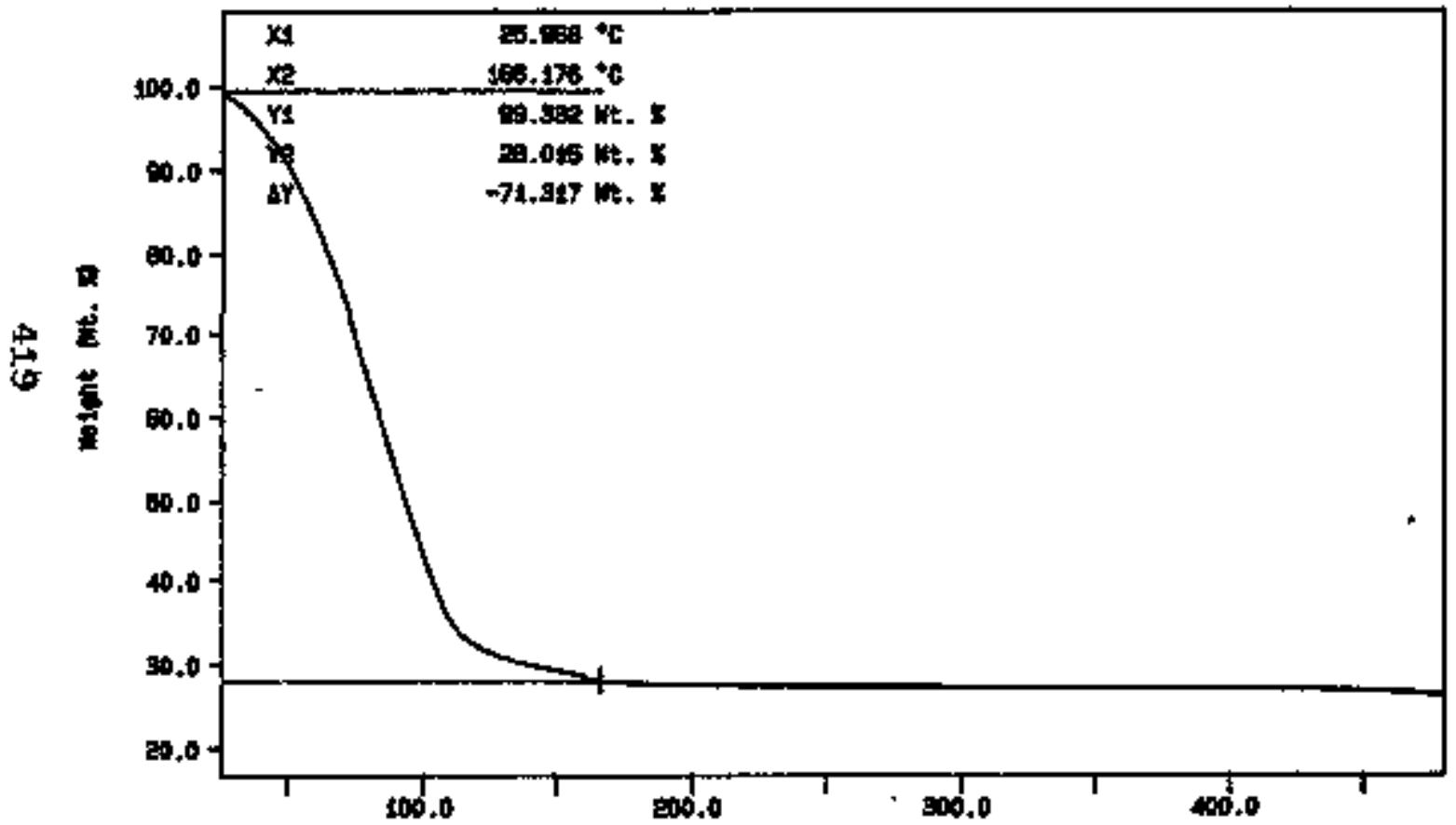

socortin in

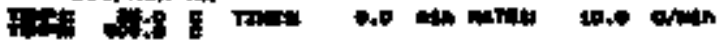




\section{Curw $\mathrm{t}$ TEA}

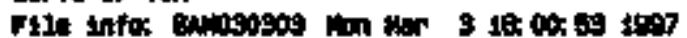

Sumplo instoht: 9.018

expoupose

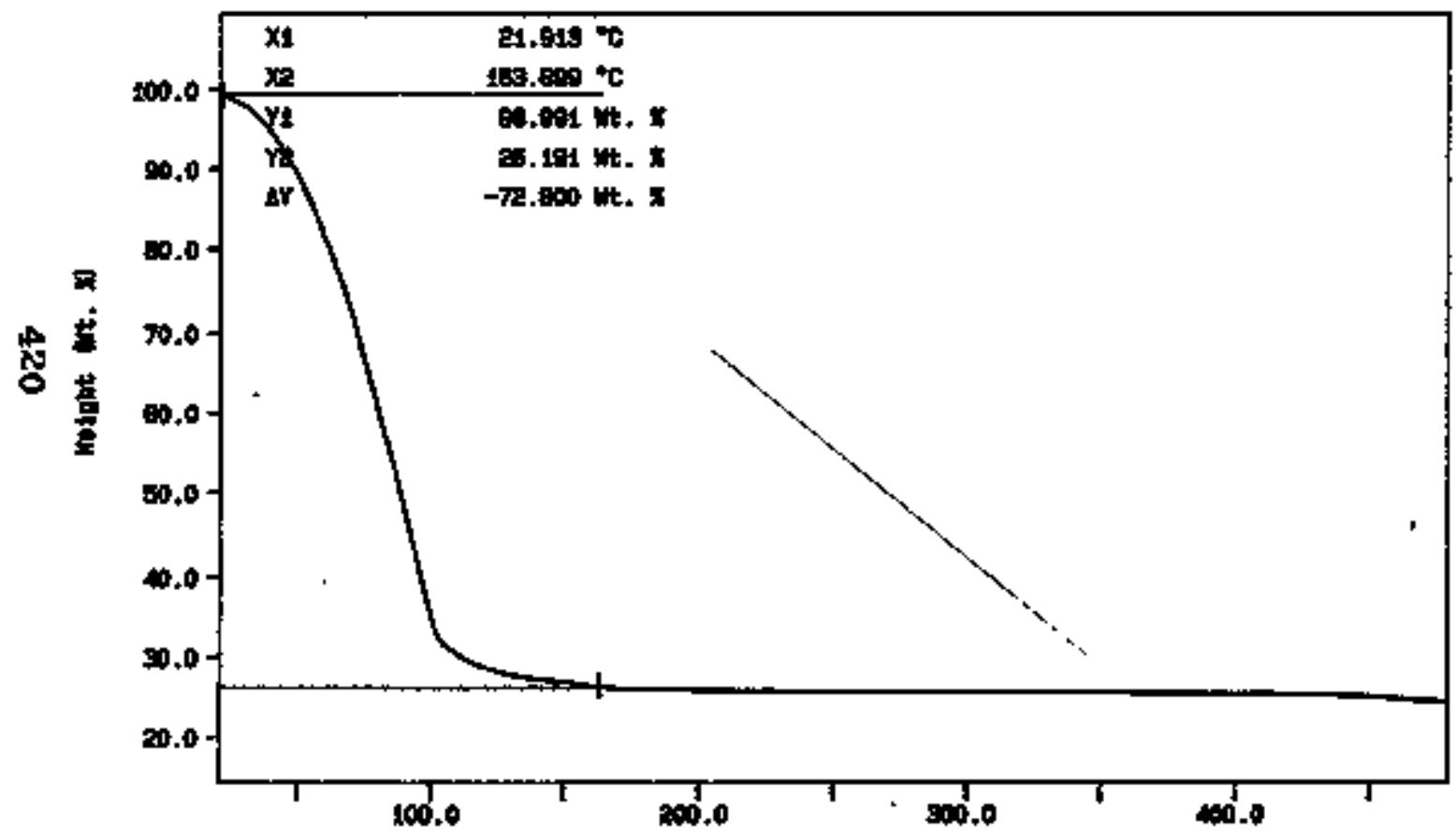

I06) in: is

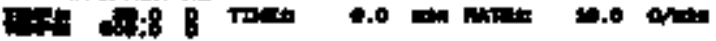

Tomperatare (10) 
Drpt it Tax

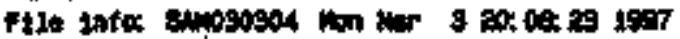

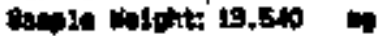

sirtoweper DiP

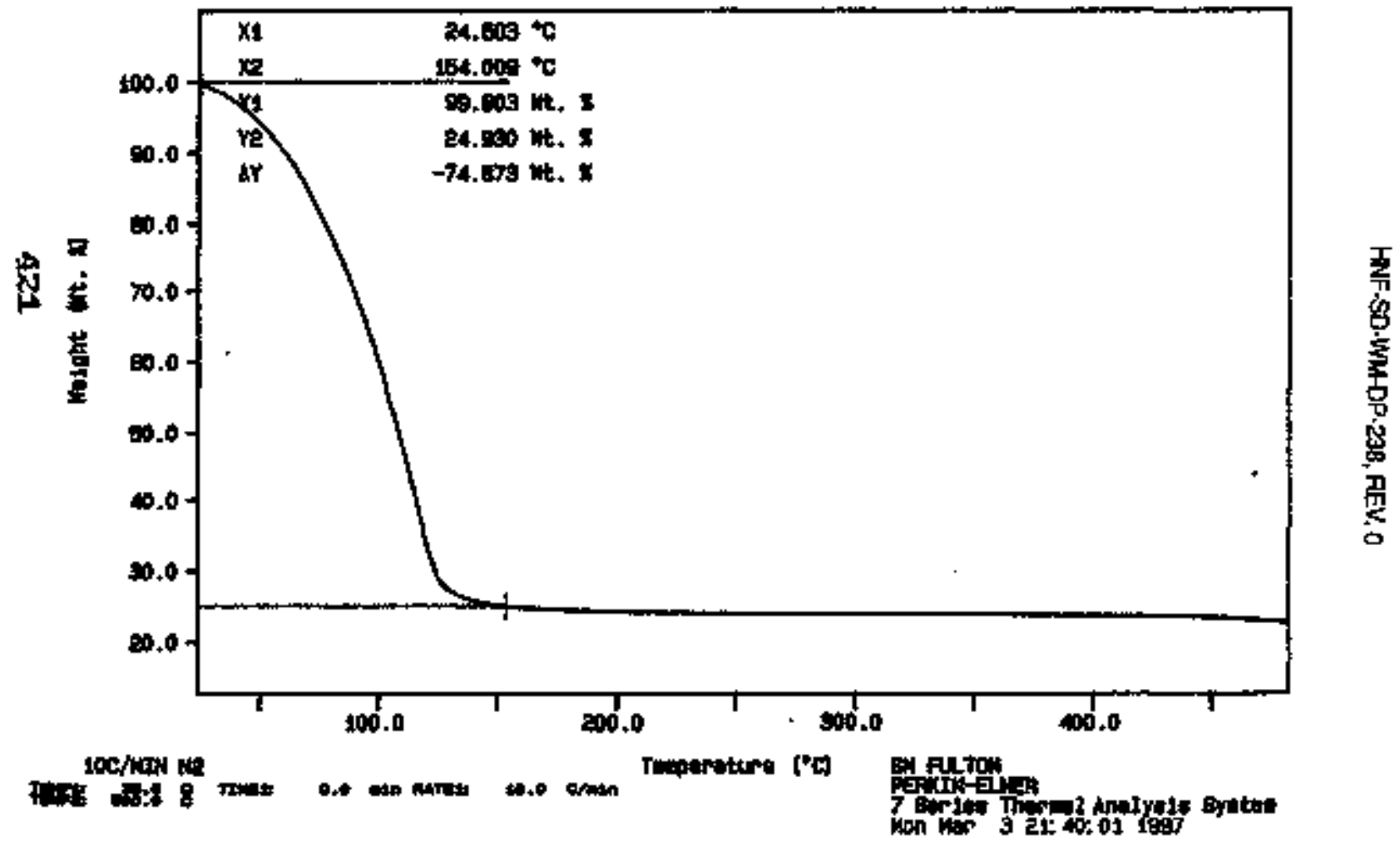




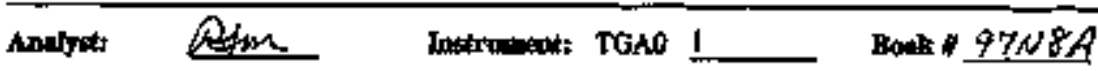

Method: LA-S60-112 Revined $(-D)$

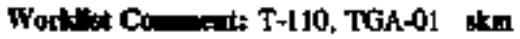

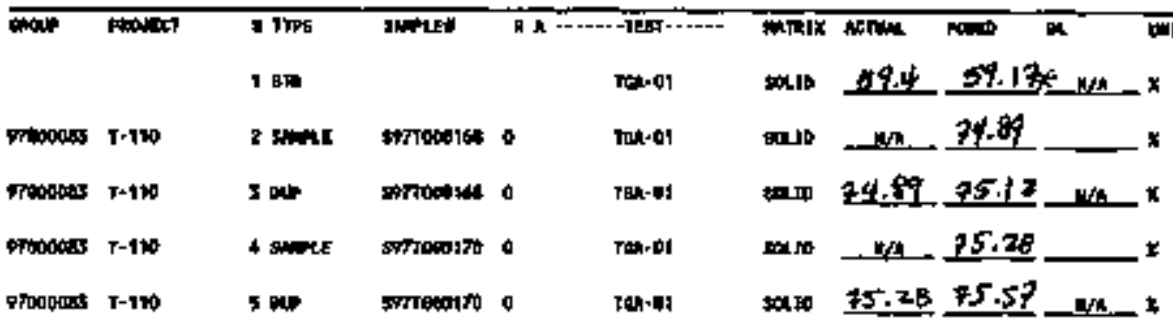

\section{Final page for worklist \# 17028}

Austofoum $3 / 15 / 17$

Walidatce 3/zol: 7 gracher

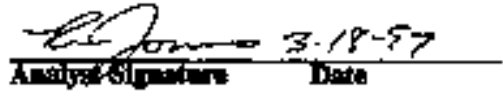

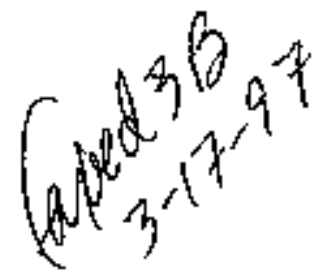

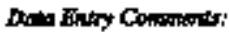

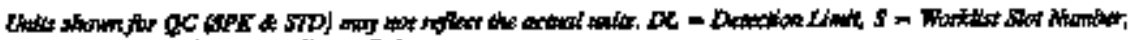

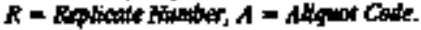


HNF-SO-WM-DP-23B, REV.O

\begin{tabular}{|c|c|c|c|c|c|c|c|c|}
\hline \multicolumn{9}{|c|}{ WHC QCHISTORY TAELB RDIT SCREBN } \\
\hline \multicolumn{3}{|c|}{$\begin{array}{l}\text { Eadiple\# } \\
\text { Group\# } \\
\text { worklidt\# } 17028\end{array}$} & \multicolumn{2}{|c|}{$\begin{array}{l}\text { Aser Eamplo ID } \\
\text { Cugtomet } \\
\text { W Comment } T\end{array}$} & TOA -01 & $\mathbf{s k n}$ & \multirow[b]{2}{*}{ Antidaks } & \multirow[b]{2}{*}{ 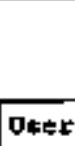 } \\
\hline$T=t$ & Metrx & Type & Actupl & Found & Yield & ФTAT & & \\
\hline $\begin{array}{l}\operatorname{ThR}-01 \\
\operatorname{TGR}-01 \\
\operatorname{TaA}-01\end{array}$ & $\begin{array}{l}\text { SoLIp } \\
\text { 90LID } \\
\text { sOLID }\end{array}$ & $\begin{array}{l}\$ T \mathrm{~T} \\
\mathrm{QOp} \\
\text { DOp }\end{array}$ & $\begin{array}{l}5.94=01 \\
74.69 \\
75.28\end{array}$ & $\begin{array}{l}59.17 \\
75.12 \\
75.57\end{array}$ & $\begin{array}{l}99.6126 \\
0.3066 \\
0.3845\end{array}$ & $\begin{array}{l}\text { NEF } \\
\text { NEW } \\
\text { DEW }\end{array}$ & 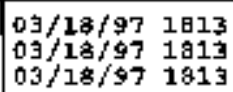 & $\begin{array}{l}r c j \\
\mathbf{r} x j j \\
\mathbf{r} \subset j\end{array}$ \\
\hline
\end{tabular}

Save (F12) End $\{\mathrm{F}\}$ 
HNF-SD-WM-OP-236, REV O

WHC OCHISTORY TABLB BDIT GCRBEN

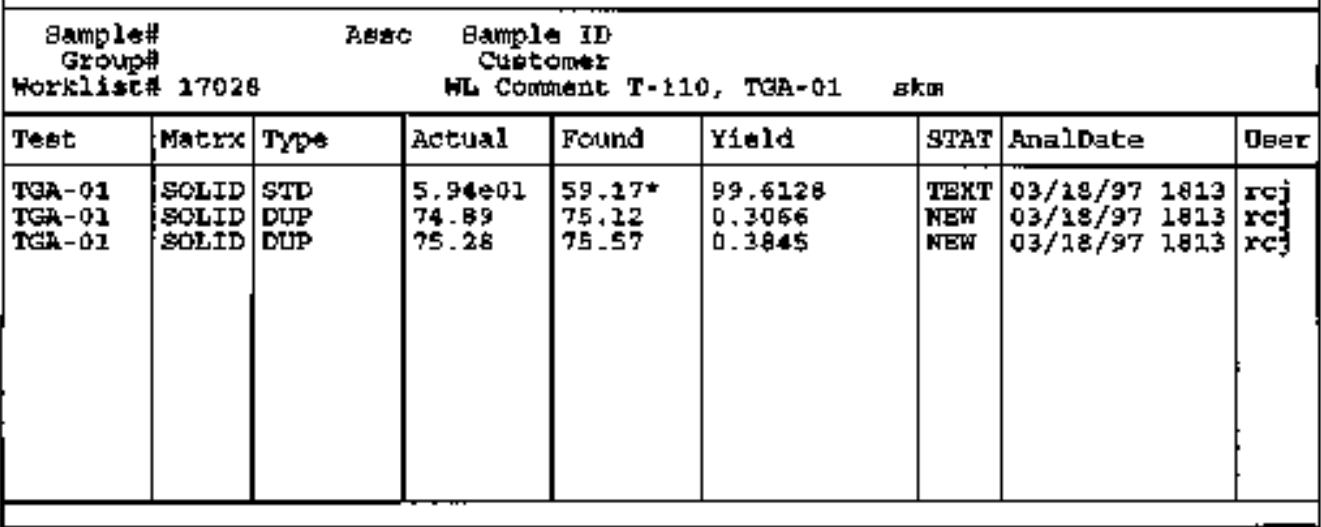

Bxue (F12) Bnd (F3) 
HATE BELOW REPRESENTS CHEMICAL TECHMOOOSIST CHESTST THAT

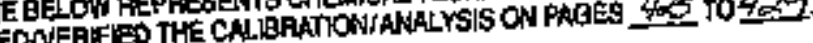

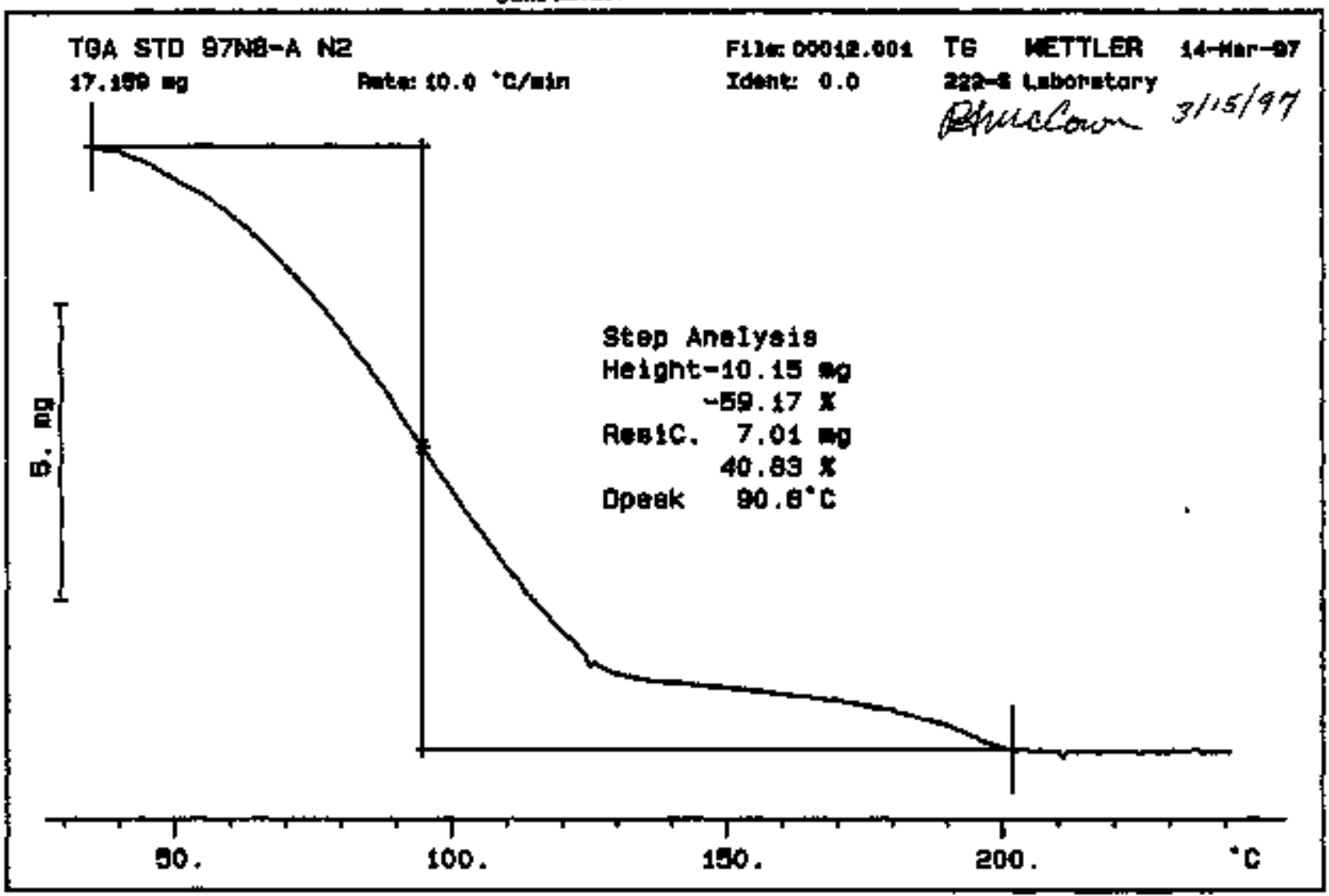




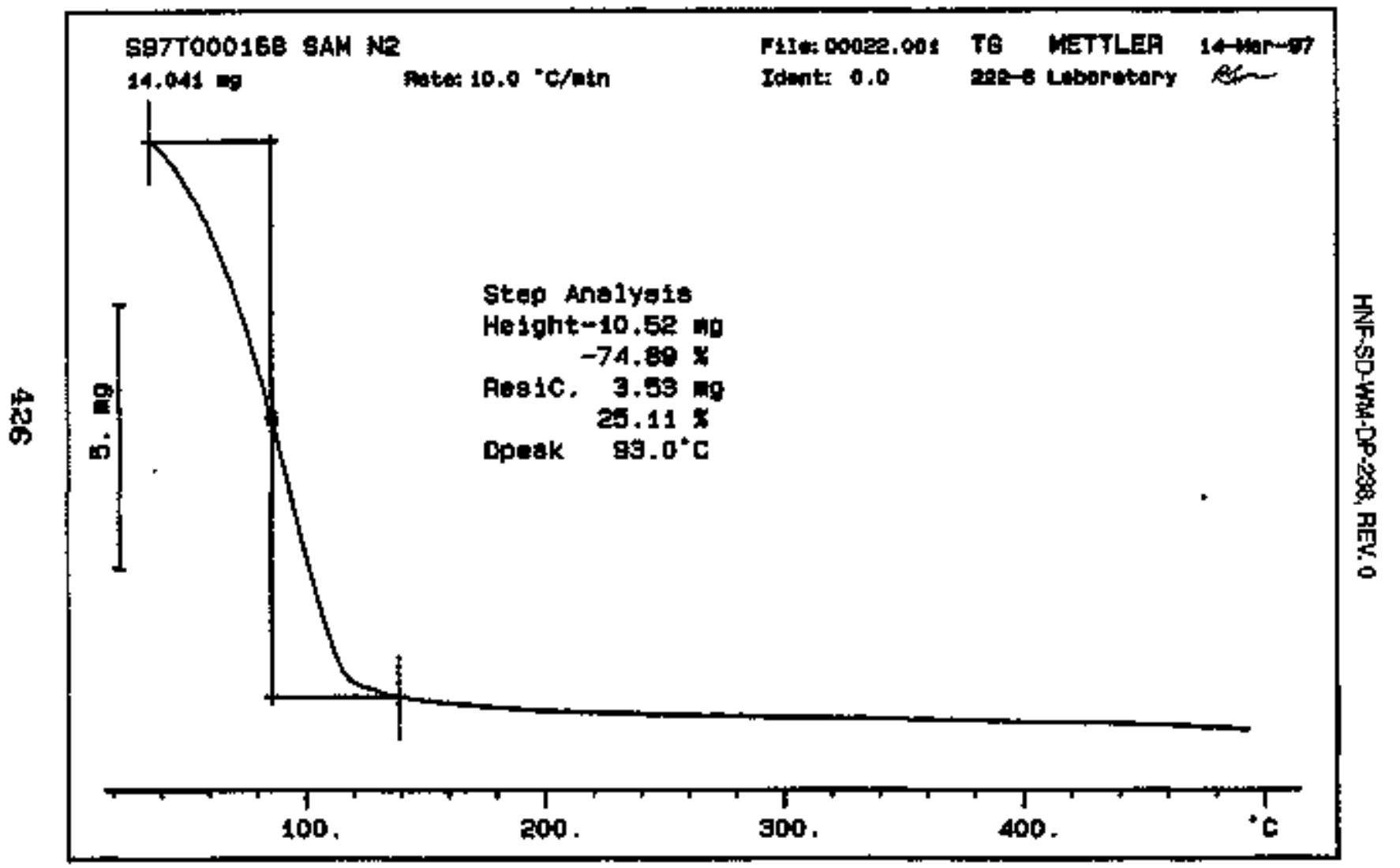




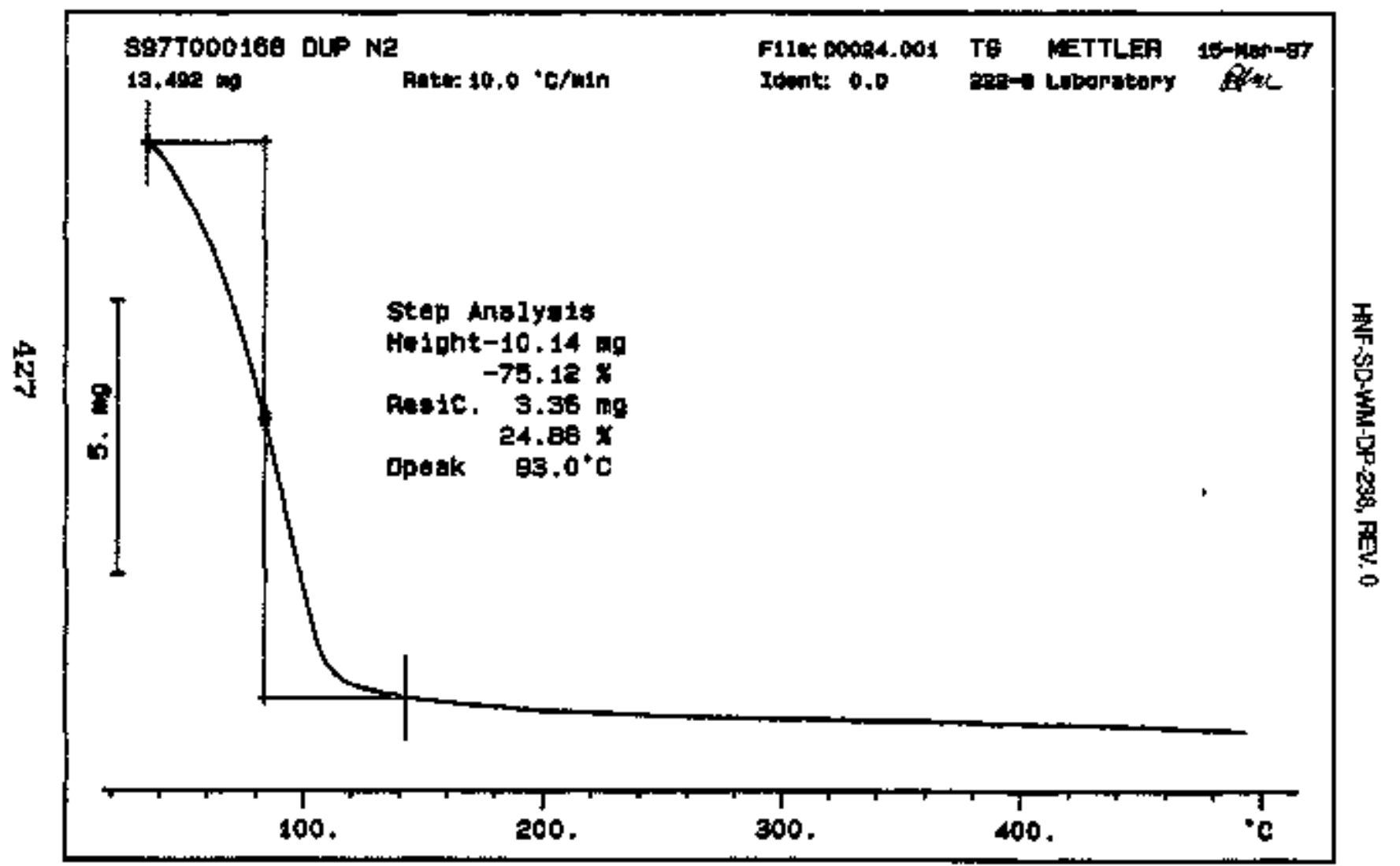




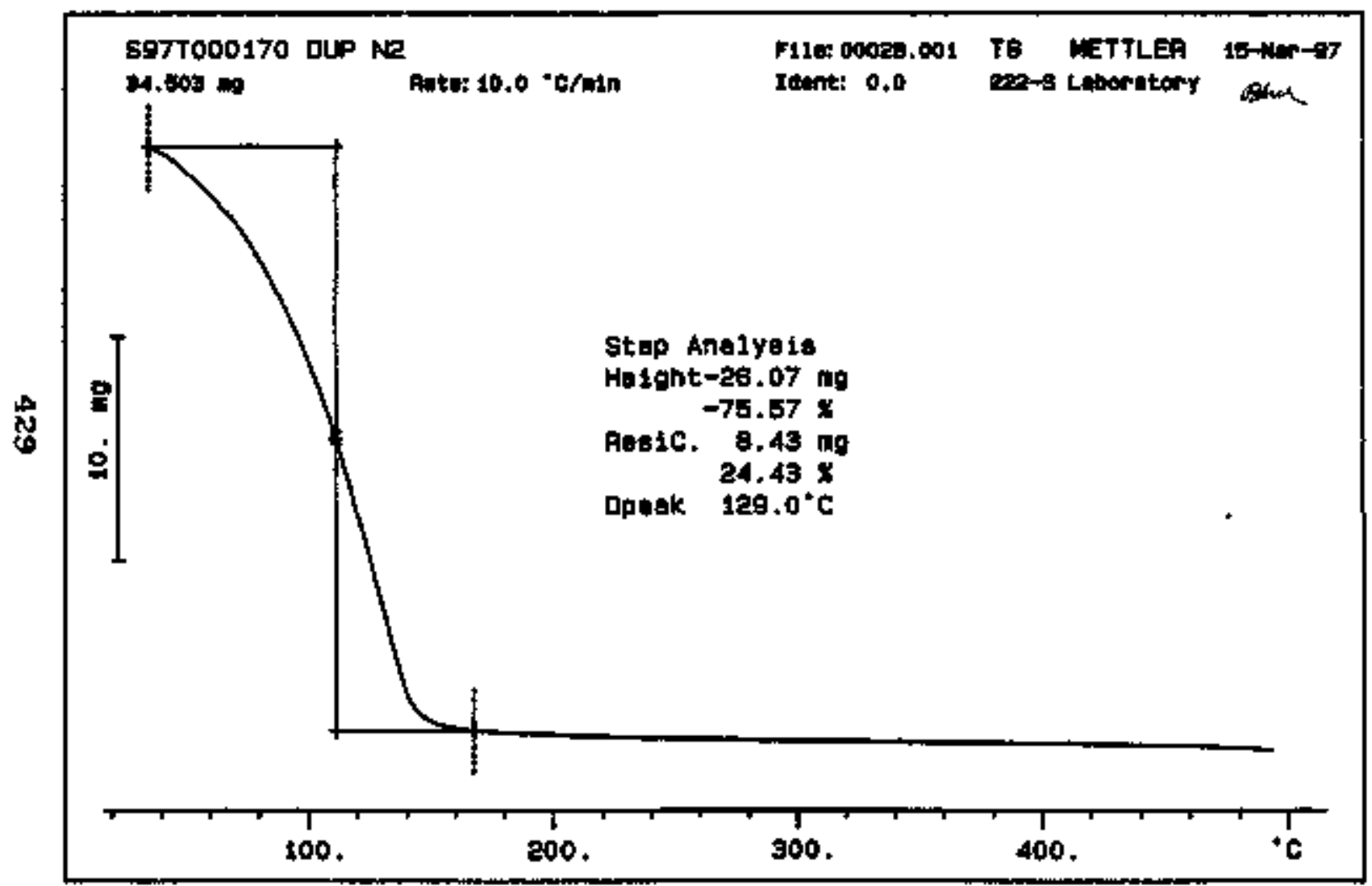




\section{LABCORE Data Entry Template for Worklist" 17029}

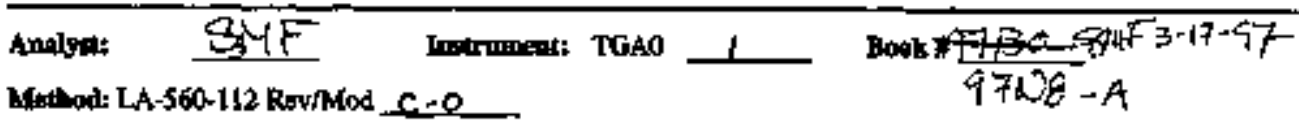

Wrat Coment: T-110, TGA-91 stm

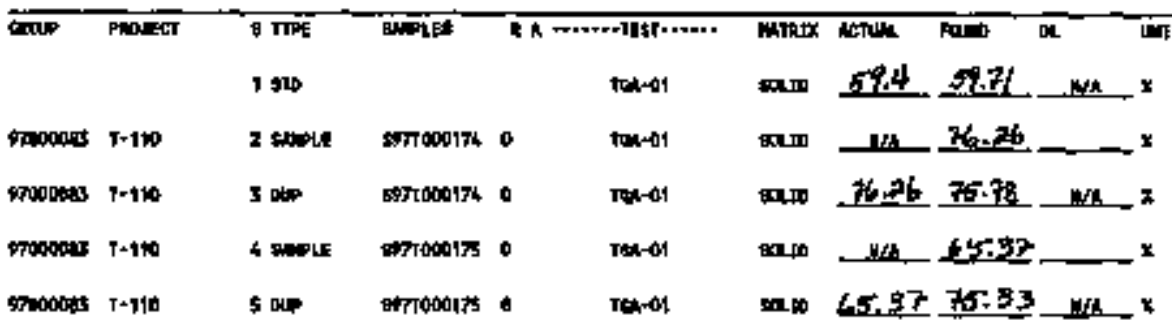

\section{Final page for worklist $\# 17029$}

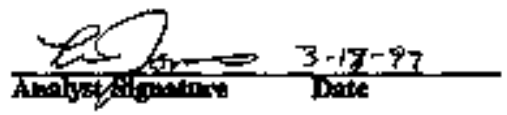

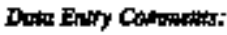

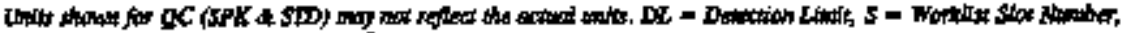

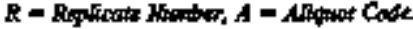




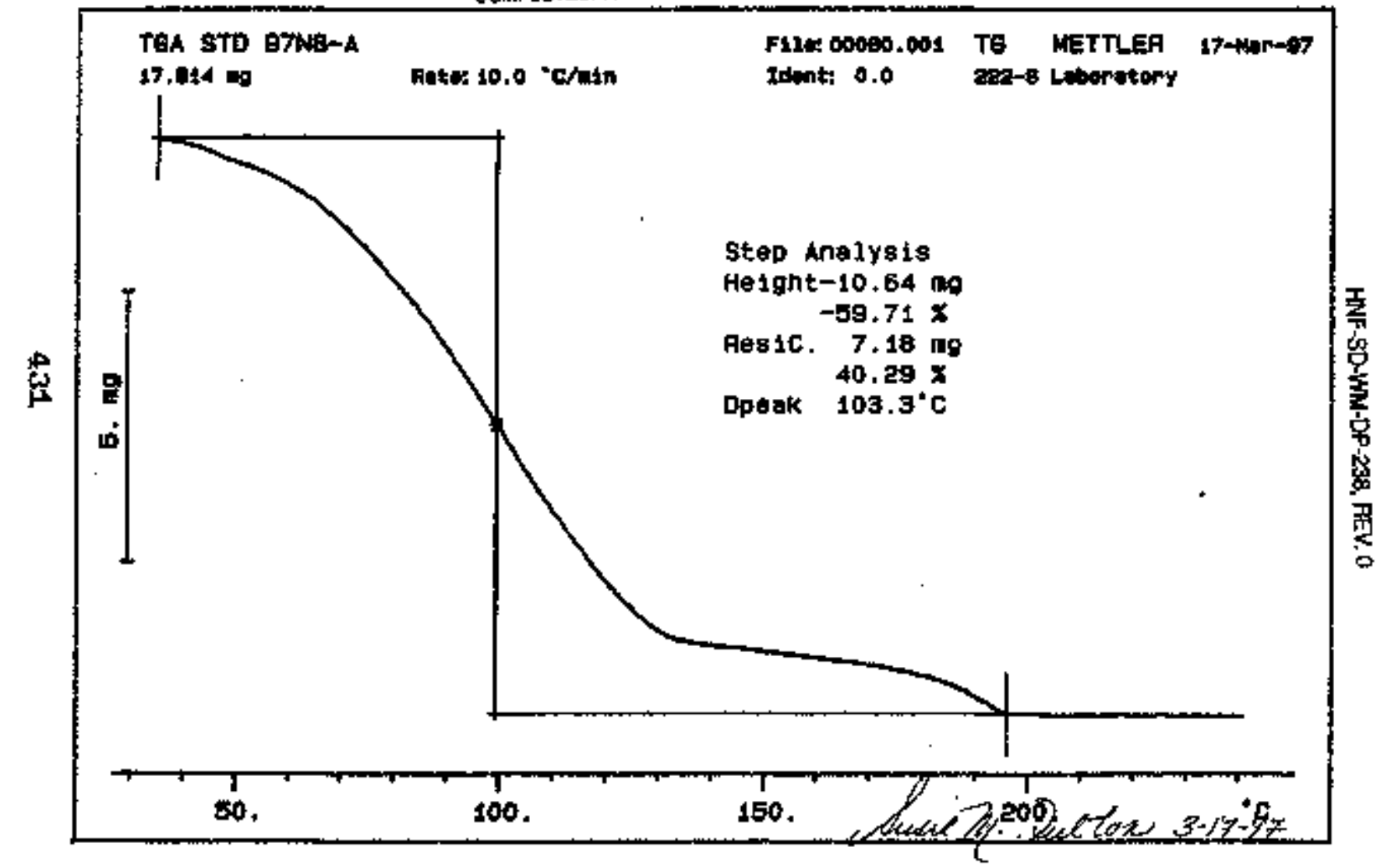




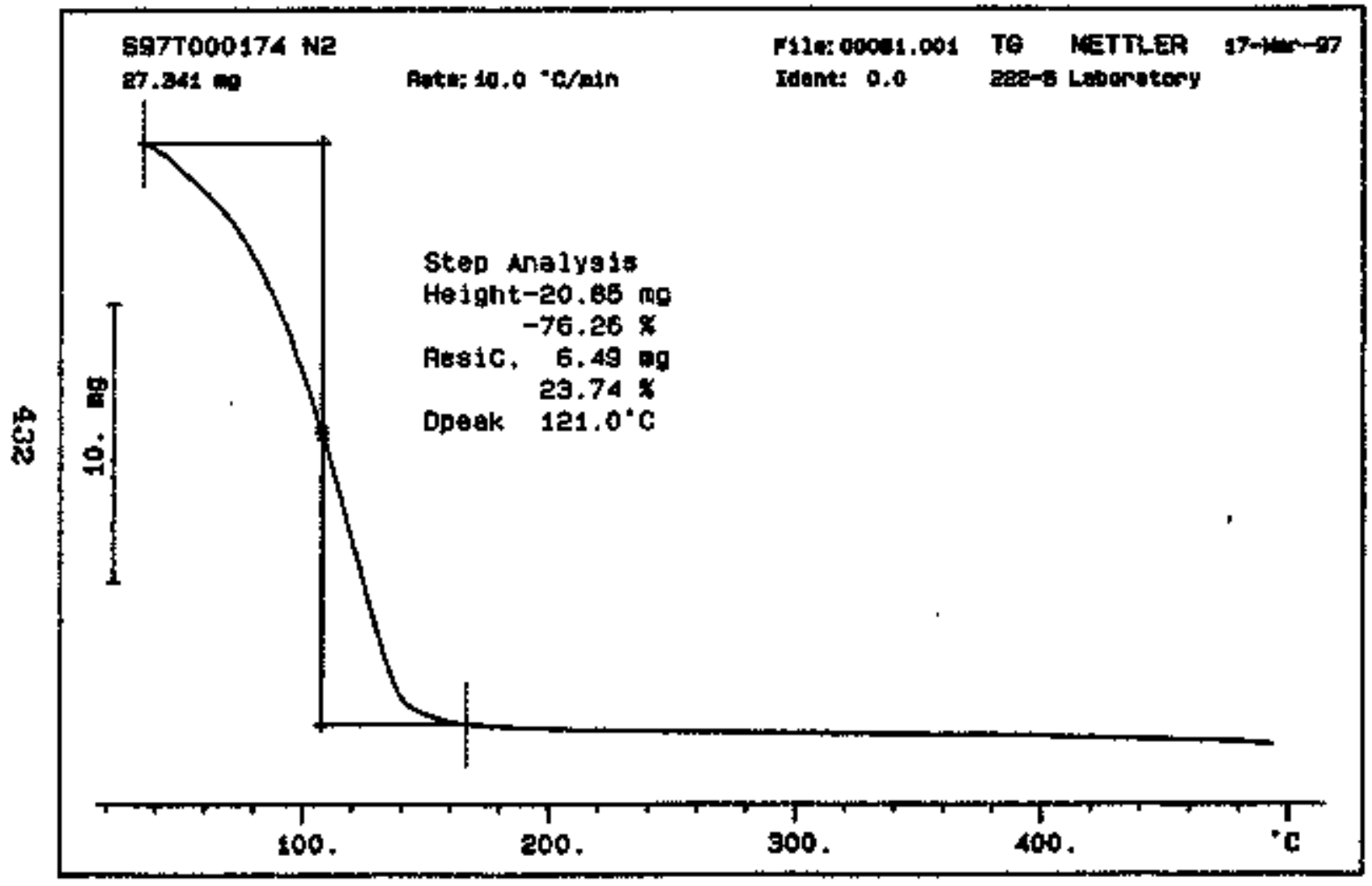




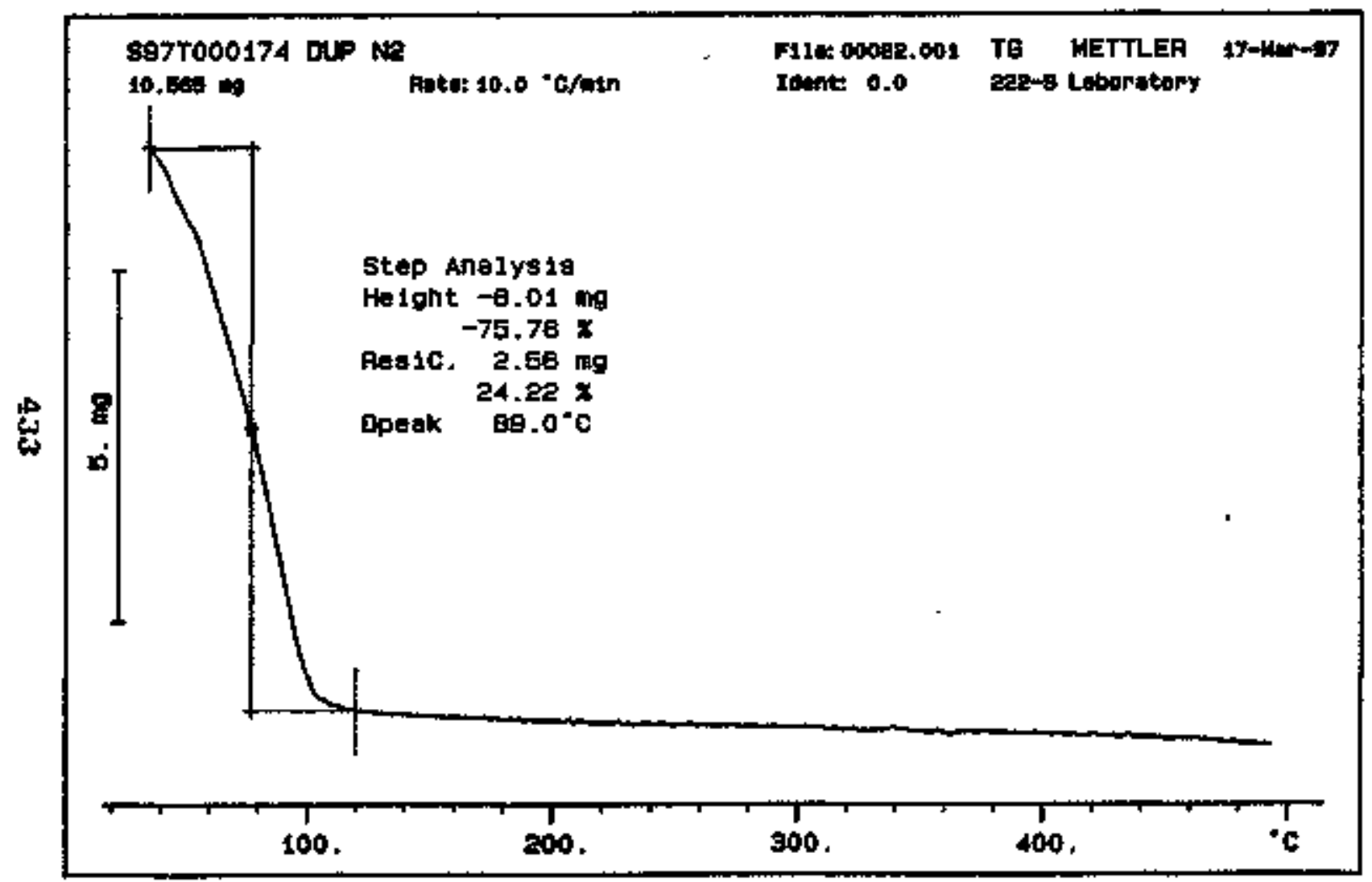

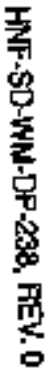




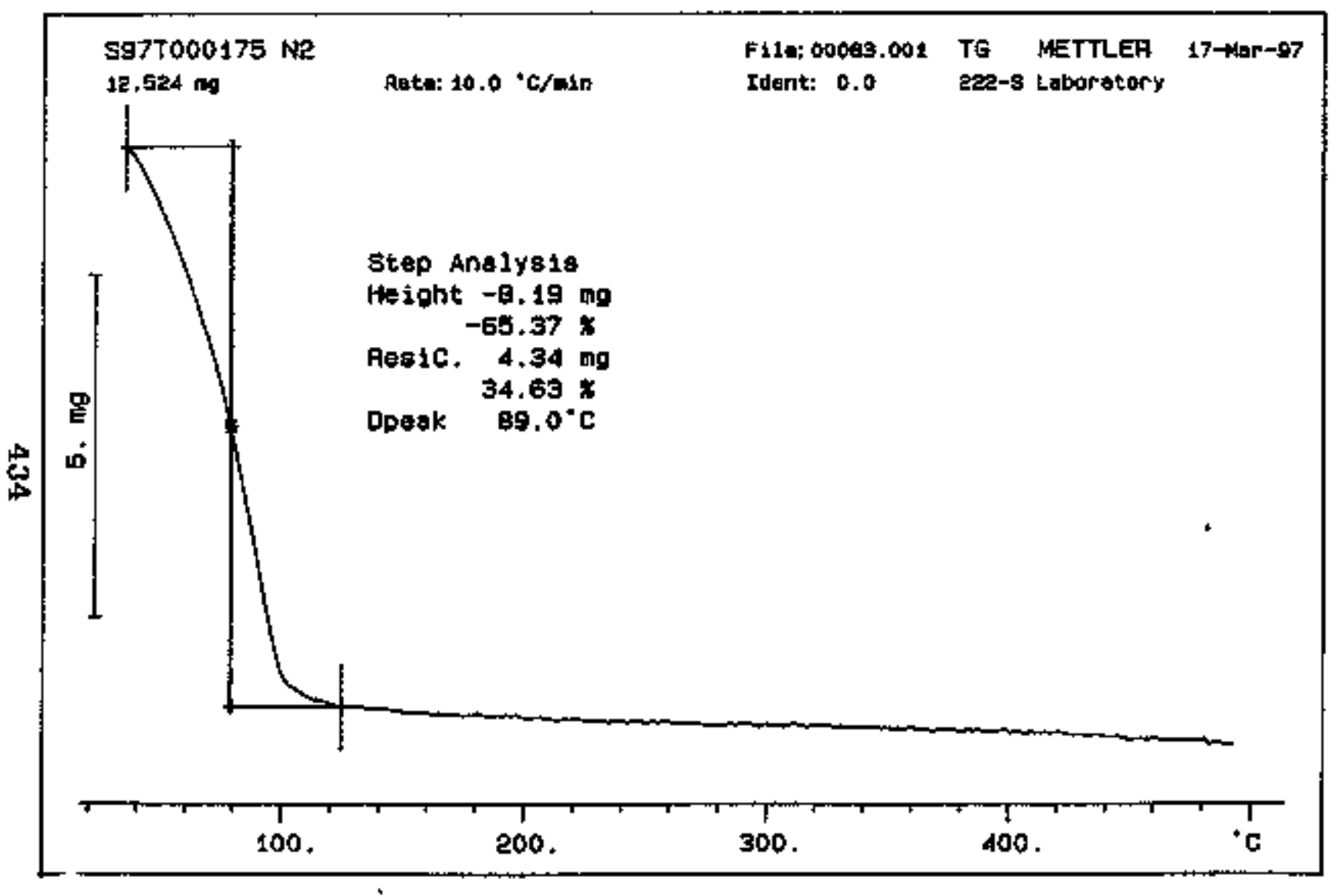

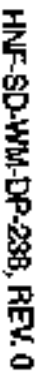




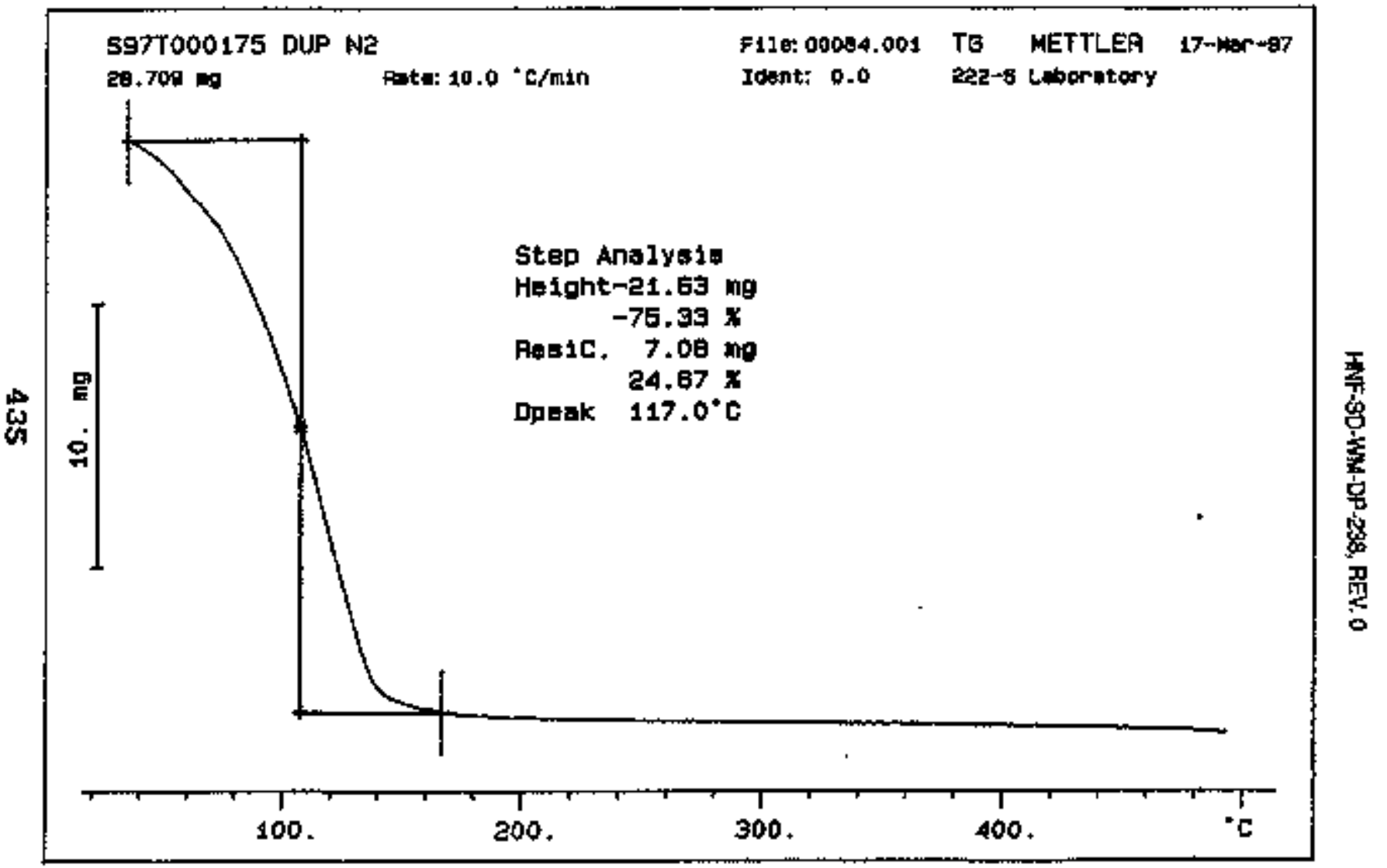




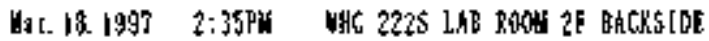

$10,1043 \quad 3, \pi / 2 d$

sportisury Versica 2, I as/15,9s

HNF-SD-WM-DP-2SE, REV. 0

Paga: I

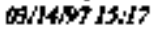

\section{LABCORE Data Entry Template for Worklist/\# 17030}

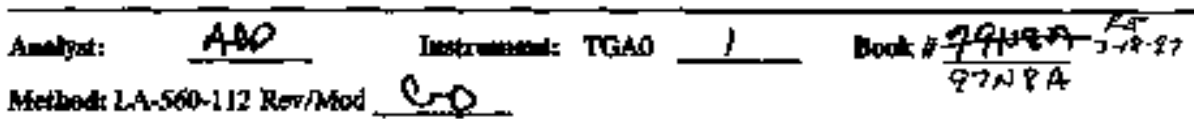

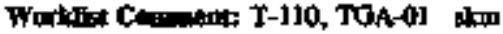

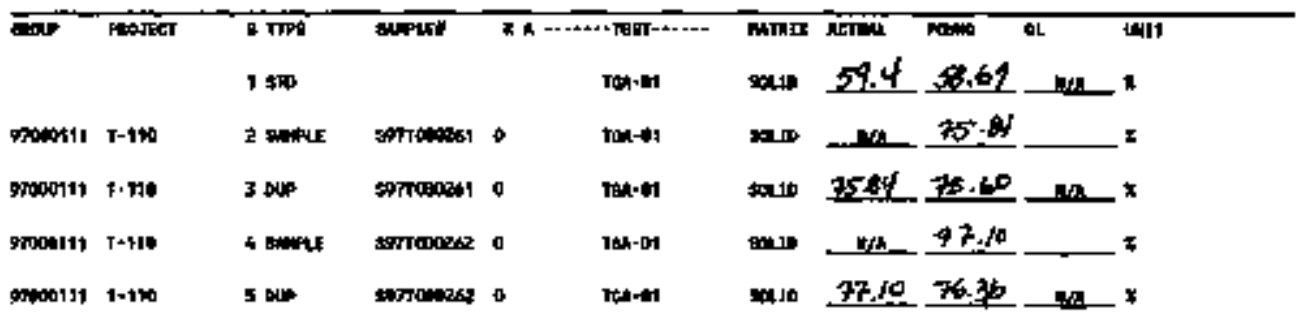

\section{Feal page for worklist a 17030}
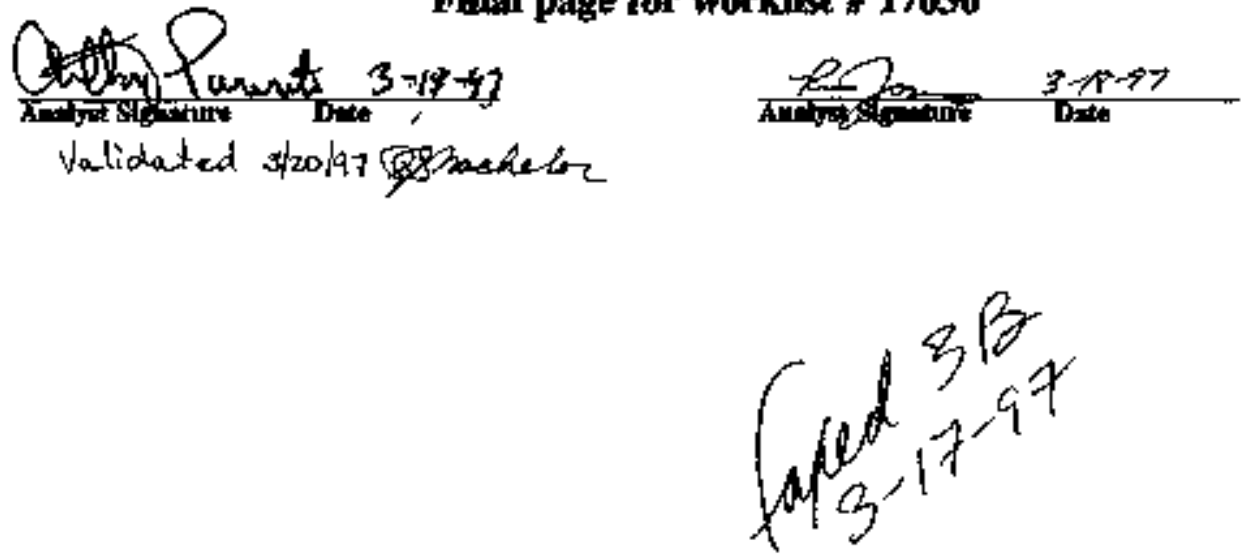

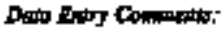

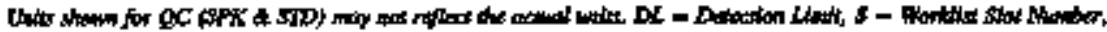

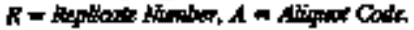




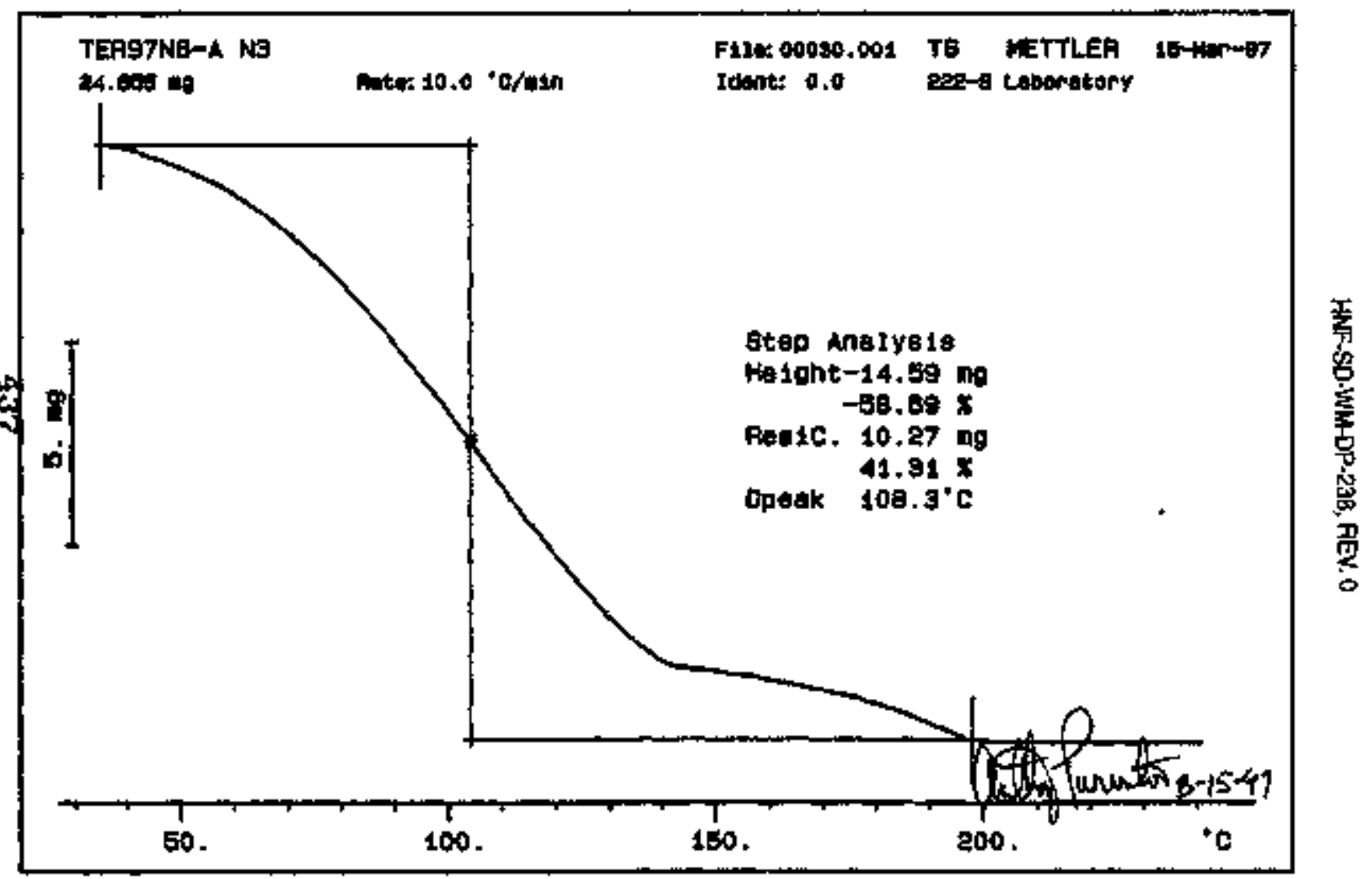




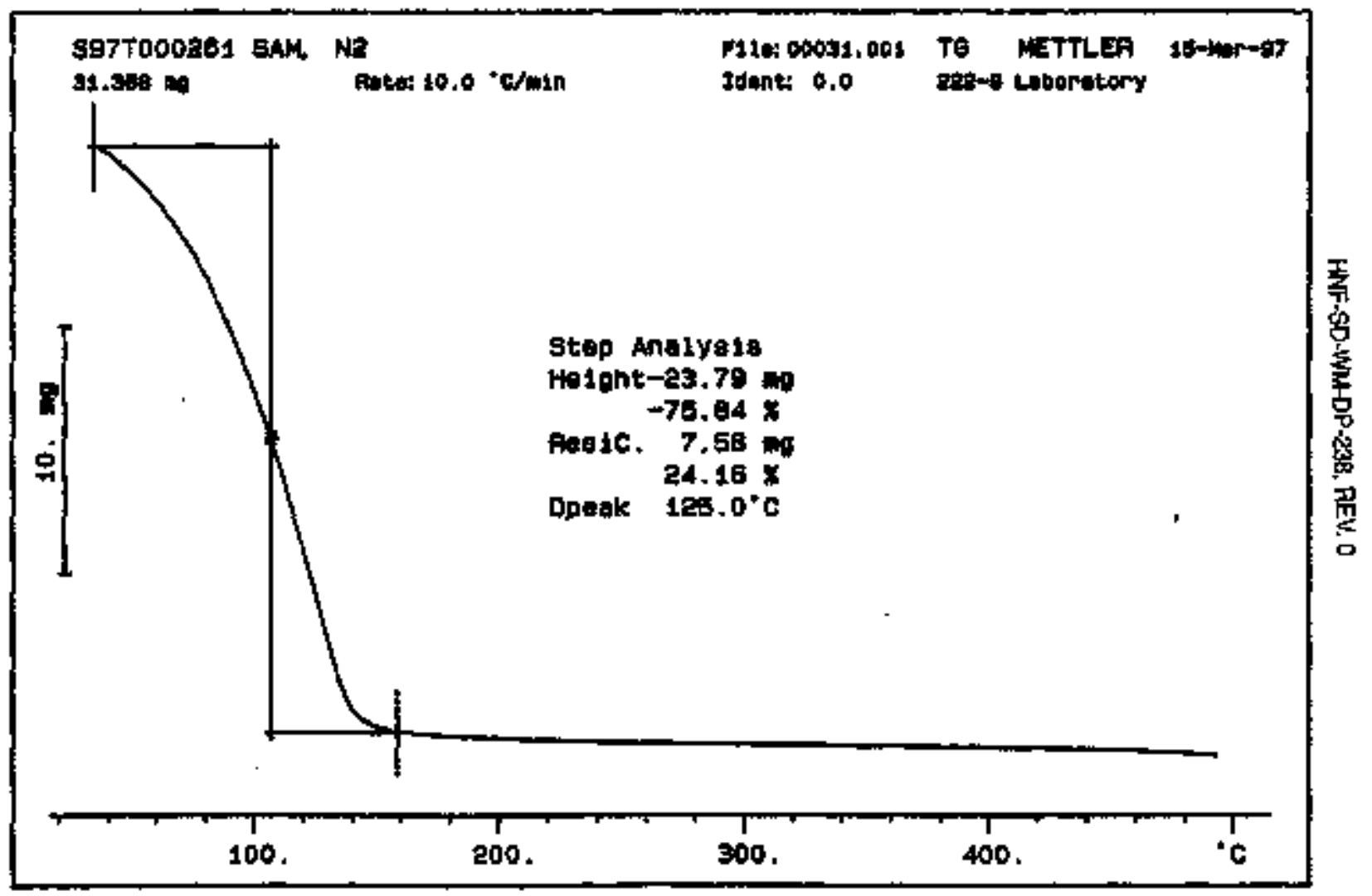




\section{setrooors oup Ne} 31.

8

fieto: $10.0 \cdot 0 /$ mbla

\section{File 0003a,c01 TE KETTLEA 1E-Ha-07}

Idwot: 0.0

\section{apen Laputany}

Stop hnolysto

Hight-29,90 wo

$-75.60 \times$

Res1C. 7.71 no

$24.40 \times$

Dposk $128.0^{\circ} \mathrm{C}$

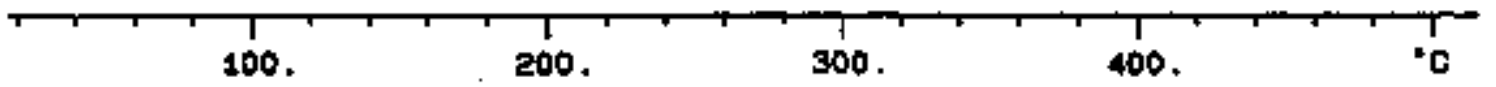




\section{T000282 SAN NR} $4.711 \mathrm{mo}$

fatte: 10,0 $0 / a$ in

\section{FH1es 00043.001 TQ METTLEA 19-Man-97} Ident: 0.0 loted Laborutory
Step Anelyate

Hent-31.39 $\mathrm{mg}$

$-77.10 \times$

Rentc, $9.32 \mathrm{mg}$

$22,90 \times$

Dpoek $137.0^{\circ} \mathrm{C}$

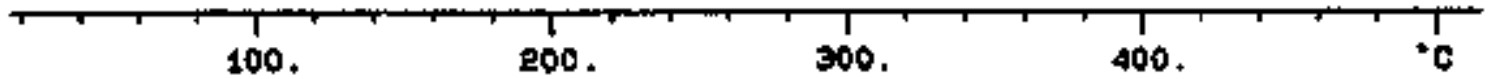




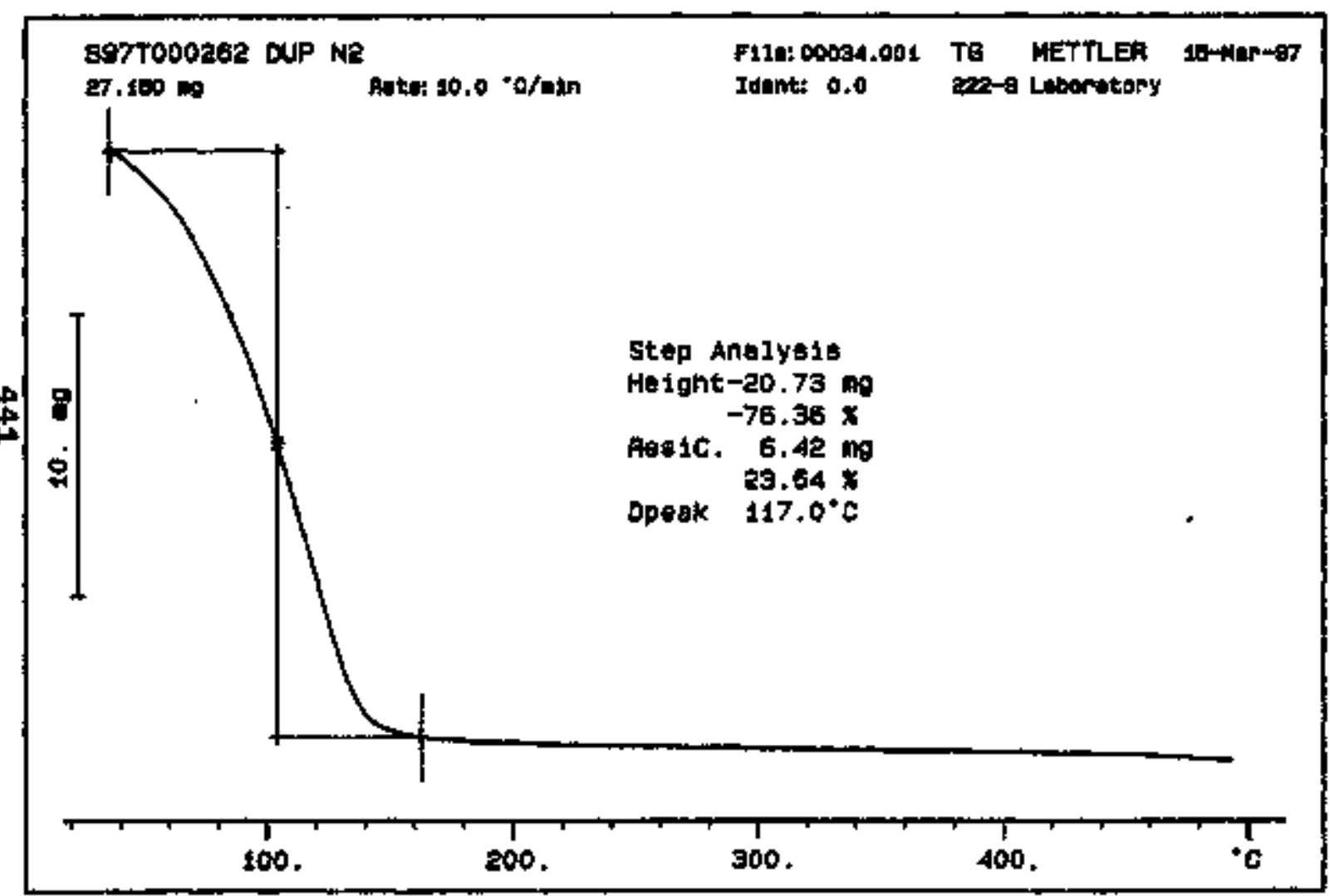




\section{LABCORE Data Entry Template for Worklist\# 17031}

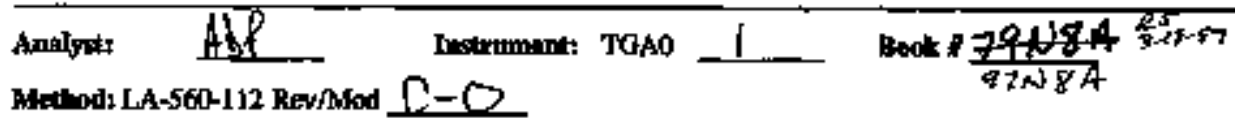

Wortitst Com: atiof T-110, TGA-01 skm

\begin{tabular}{|c|c|c|c|c|c|c|c|c|c|}
\hline car & Pedict & 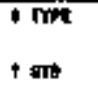 & $\sin (10)$ & & 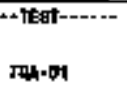 & $\begin{array}{l}\text { MाT } \\
\min x\end{array}$ & $\begin{array}{l}\text { NeThL } \\
5 y, 4 \\
\end{array}$ & 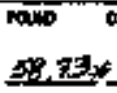 & MAL \\
\hline procotils & $T+1 \mathbf{w}$ & 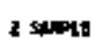 & $5 m 1-083$ & $\bullet$ & รQ4.91 & 略 & Mu. & 7 조‥59 & \\
\hline shogh11 & $r-110$ & 3 ber & termoopess & 0 & Tox-d & mll & 2.154 & 75.22 & Exs. \\
\hline trablis & $T-1: 4$ & if staper & squmorat & $\theta$ & Tenet & bot.t. & Ma & 19. & \\
\hline 9xo0hal11 & 5.190 & 5 ton & 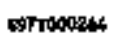 & 0 & ten-pot & stist & 푀쇼 & $94, * t$ & Difl. \\
\hline
\end{tabular}

\section{Final page for wortlist \# 17031}

1)

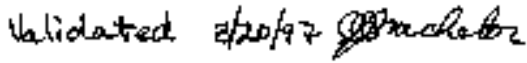

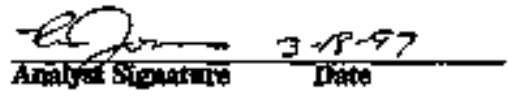

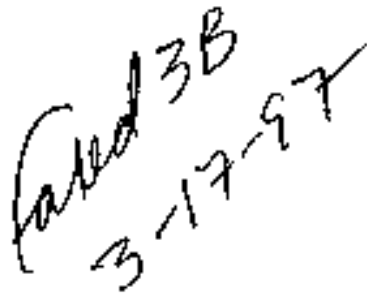

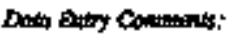

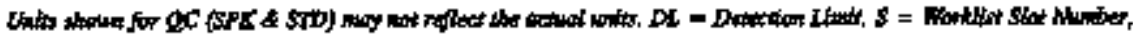

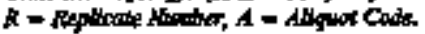


HNF-SD-WM-DP-238, REV. 0

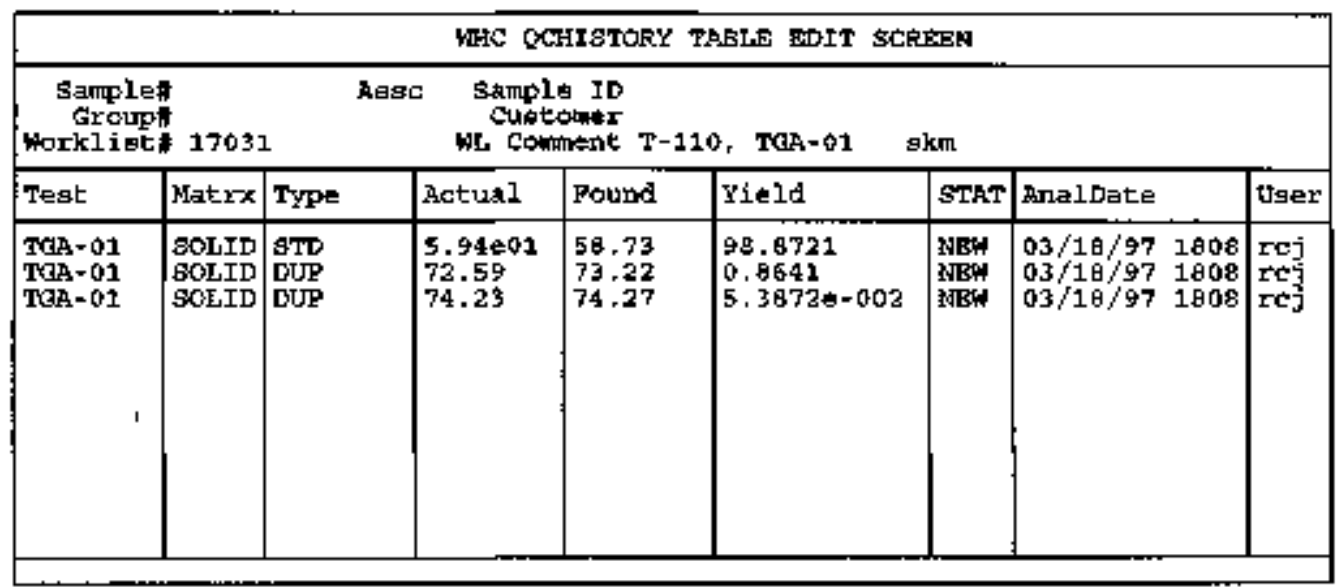

Save $\{F \perp 2\} \quad E n d(F\})$ 
HNF-SD-WH-DP-298, REV. 0

\begin{tabular}{|c|c|c|c|c|c|c|c|c|}
\hline \multicolumn{9}{|c|}{ WHC QOKISTORY TABLE EDIT SCREEN } \\
\hline \multicolumn{3}{|c|}{$\begin{array}{l}\text { Stwple\# } \\
\text { Group" } \\
\text { Horkl ist" } 17032\end{array}$} & \multicolumn{2}{|c|}{ 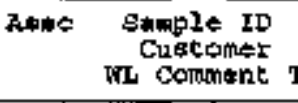 } & T-110. TTAB- 01 & kno & \multirow[b]{2}{*}{ Analdate } & \multirow[b]{2}{*}{ Joser } \\
\hline Tetet & Matrx & Type & natual & Found & Yield & GTAT & & \\
\hline $\begin{array}{l}T G B-01 \\
T G A-01 \\
T A B-01\end{array}$ & $\begin{array}{l}\text { SOLI } \\
\text { SOEID } \\
\text { SOLID }\end{array}$ & $\begin{array}{l}\text { sTip } \\
\text { oup } \\
\text { DUP }\end{array}$ & $\begin{array}{l}5.94903 \\
72.59 \\
74.33\end{array}$ & $\begin{array}{l}58.73 \pm \\
73.22 \\
74.27\end{array}$ & $\begin{array}{l}96.8721 \\
0.8641 \\
5.3672 E-002\end{array}$ & $\begin{array}{l}\text { TEXT } \\
\text { NEW } \\
\text { NEW }\end{array}$ & $\begin{cases}03 / 18 / 97 & 1808 \\
03 / 18 / 97 & 2800 \\
03 / 18 / 97 & 1808\end{cases}$ & $\begin{array}{l}x \in j \\
r \subset j \\
r \in j\end{array}$ \\
\hline
\end{tabular}

Save $\{F 12\}$ End $\{$ 3 $\}$ 


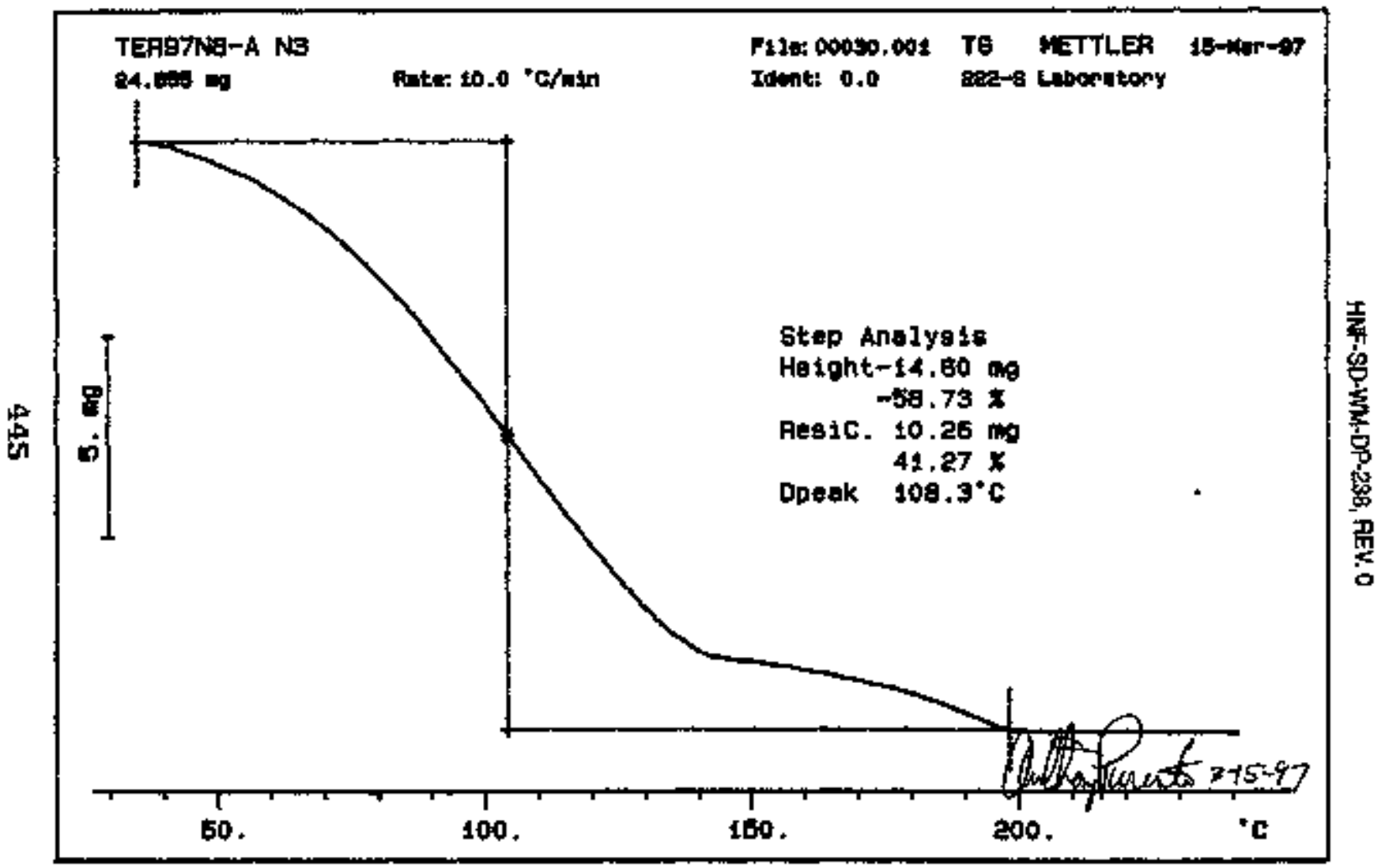




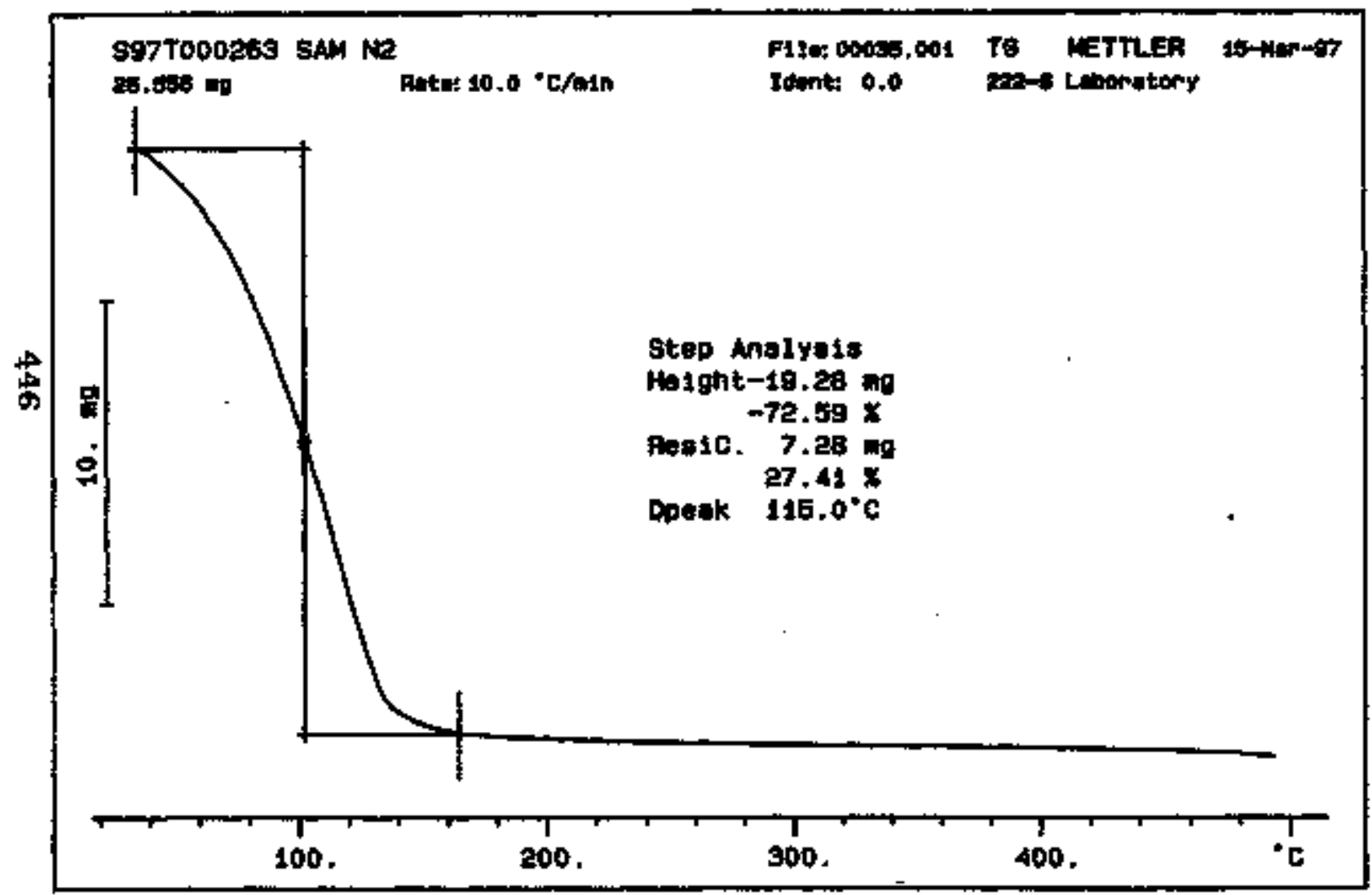




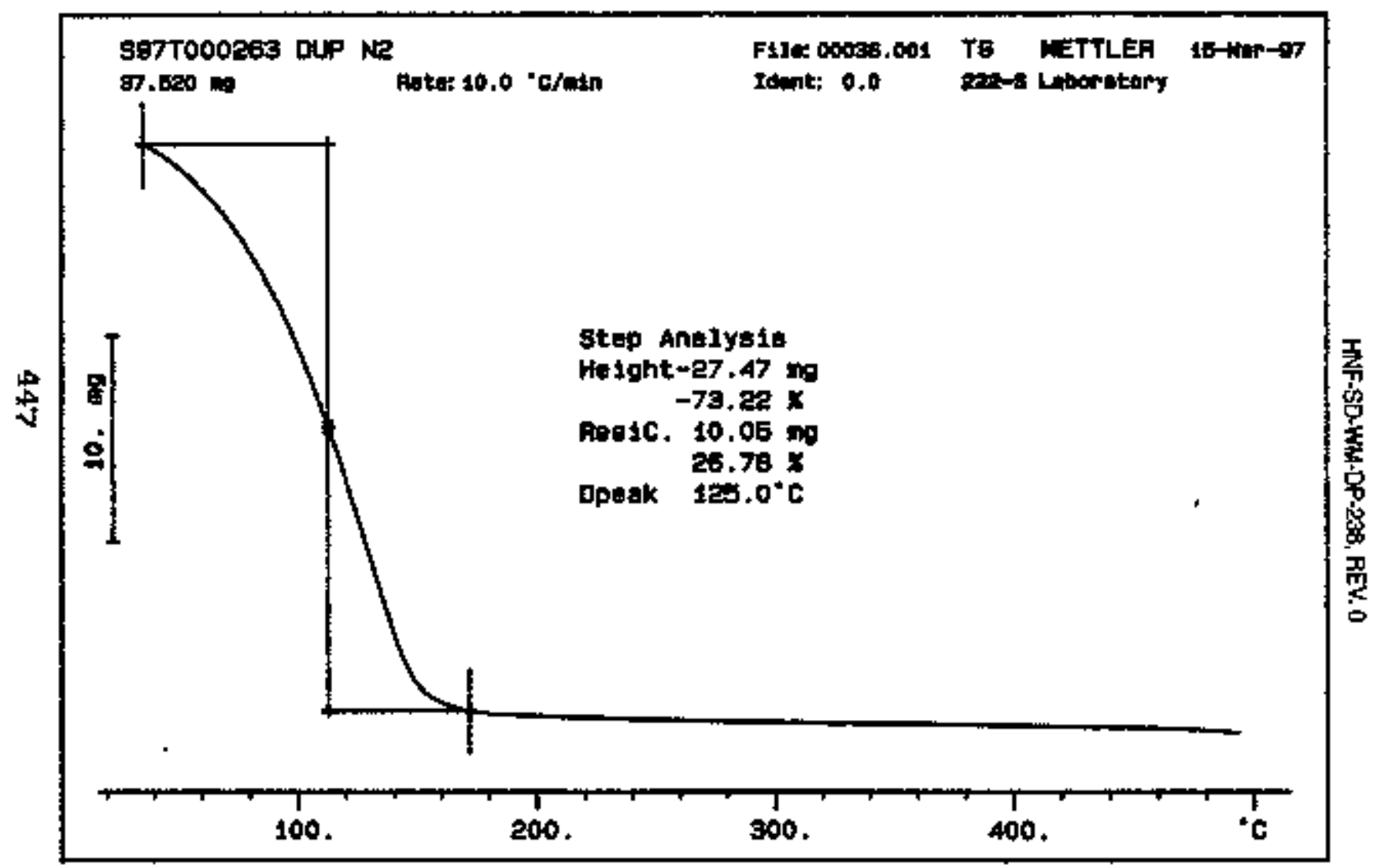




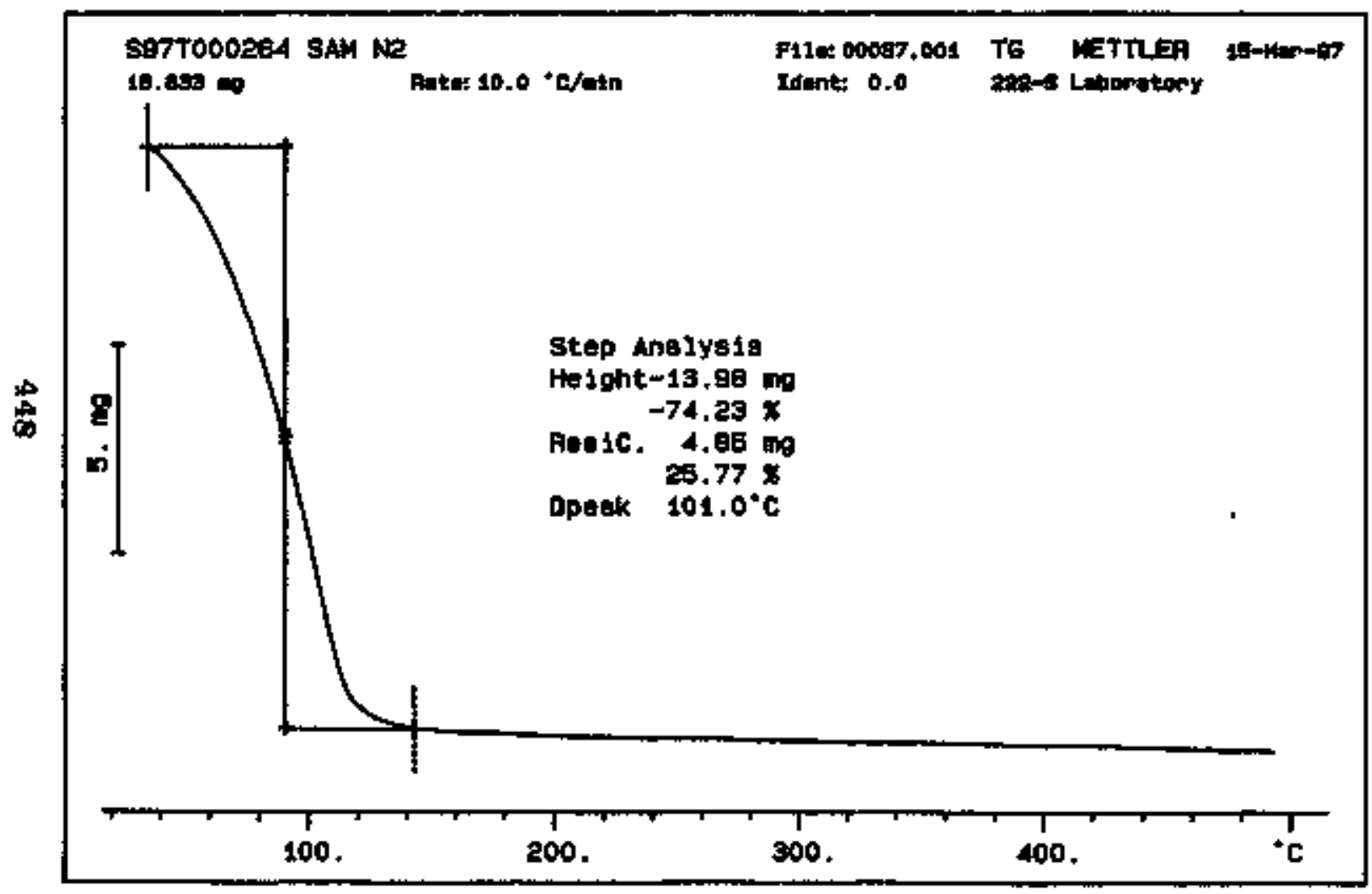




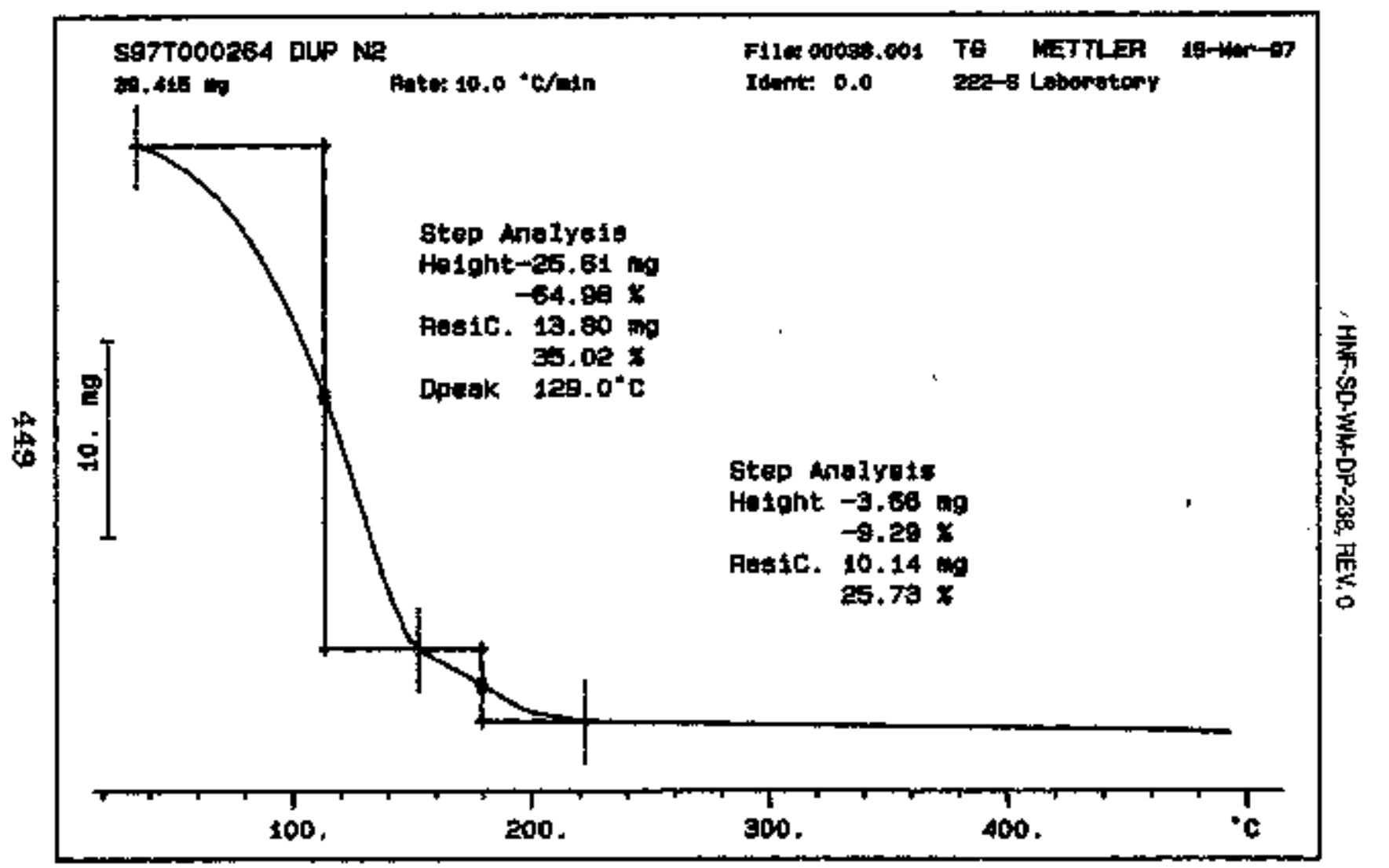




\section{LABCORE Data Entry Template for Worklistf 17032}

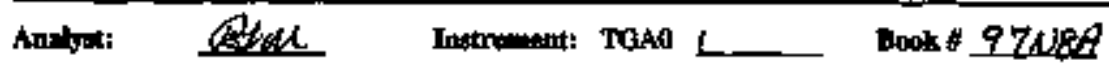

Methed: LA-561-12 RevilMod $\mathrm{CO}$

Worklist Commentit T-1 LO, TGA-01 skm

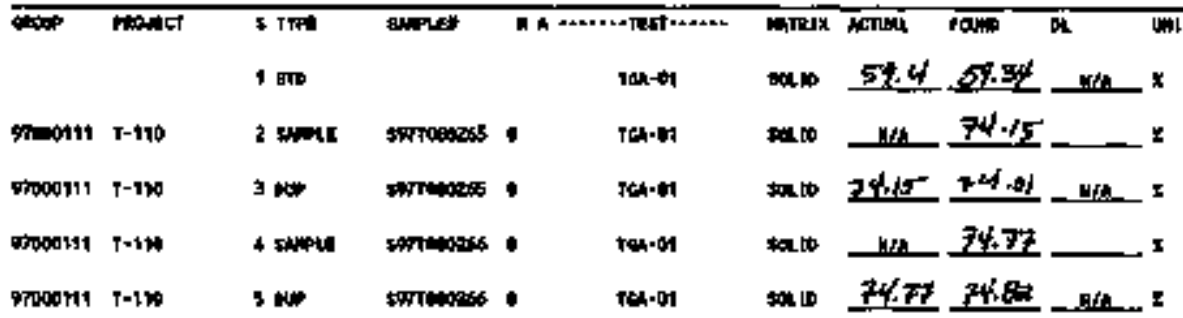

\section{Final page for worklist \# 17032}

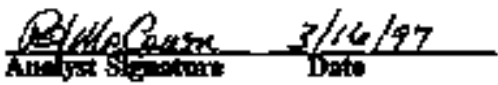

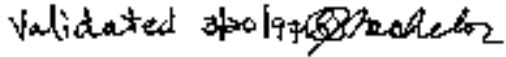
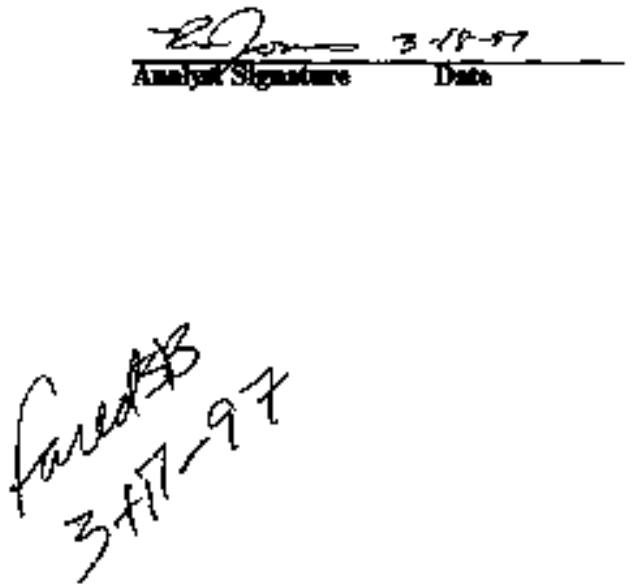

Data Bty Comites;

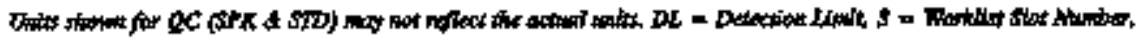

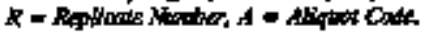


COWFHETEONERIFTED THE CALBBRATIONIANALYSIS ON FAGES HST TO LS5

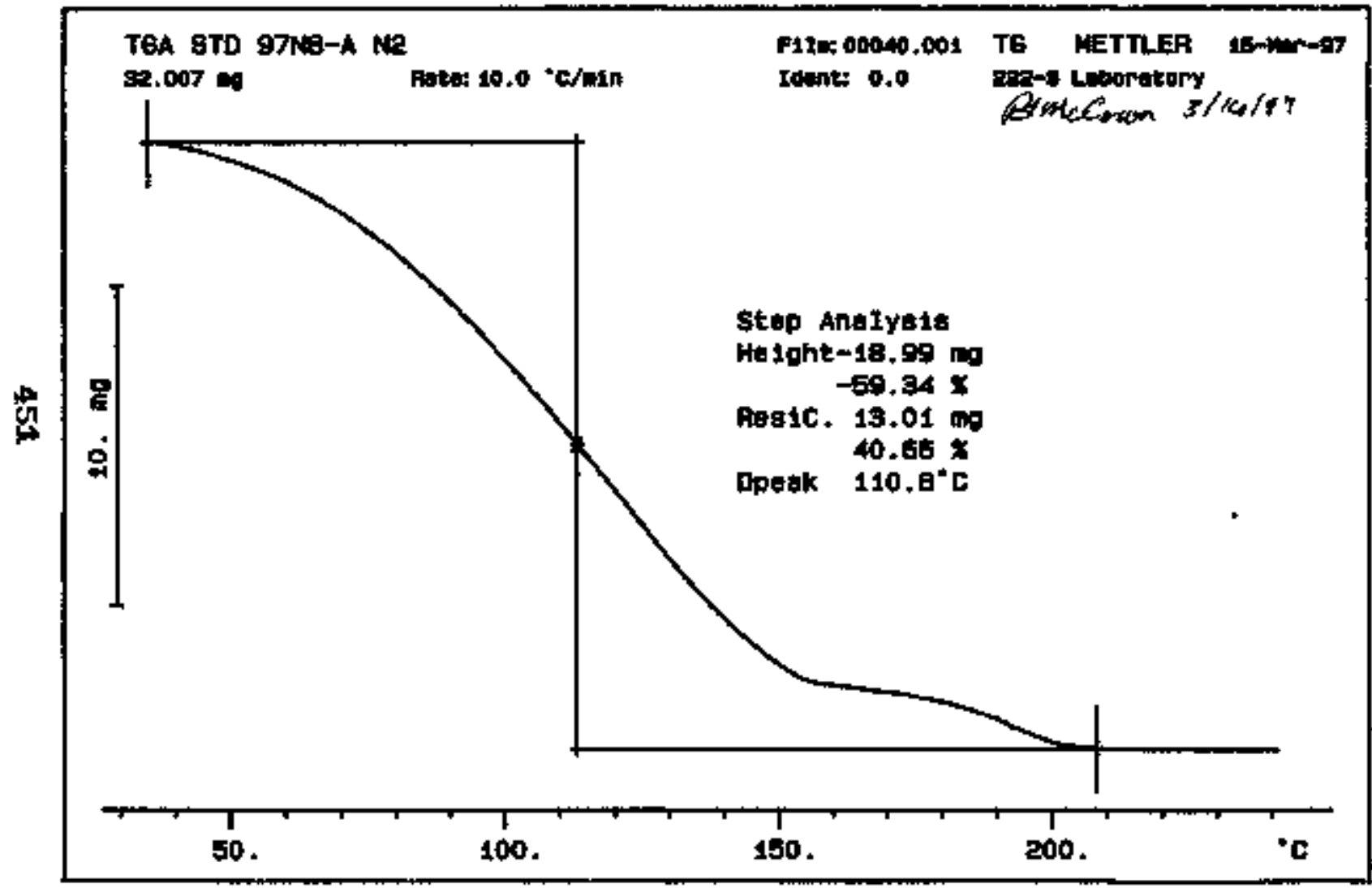




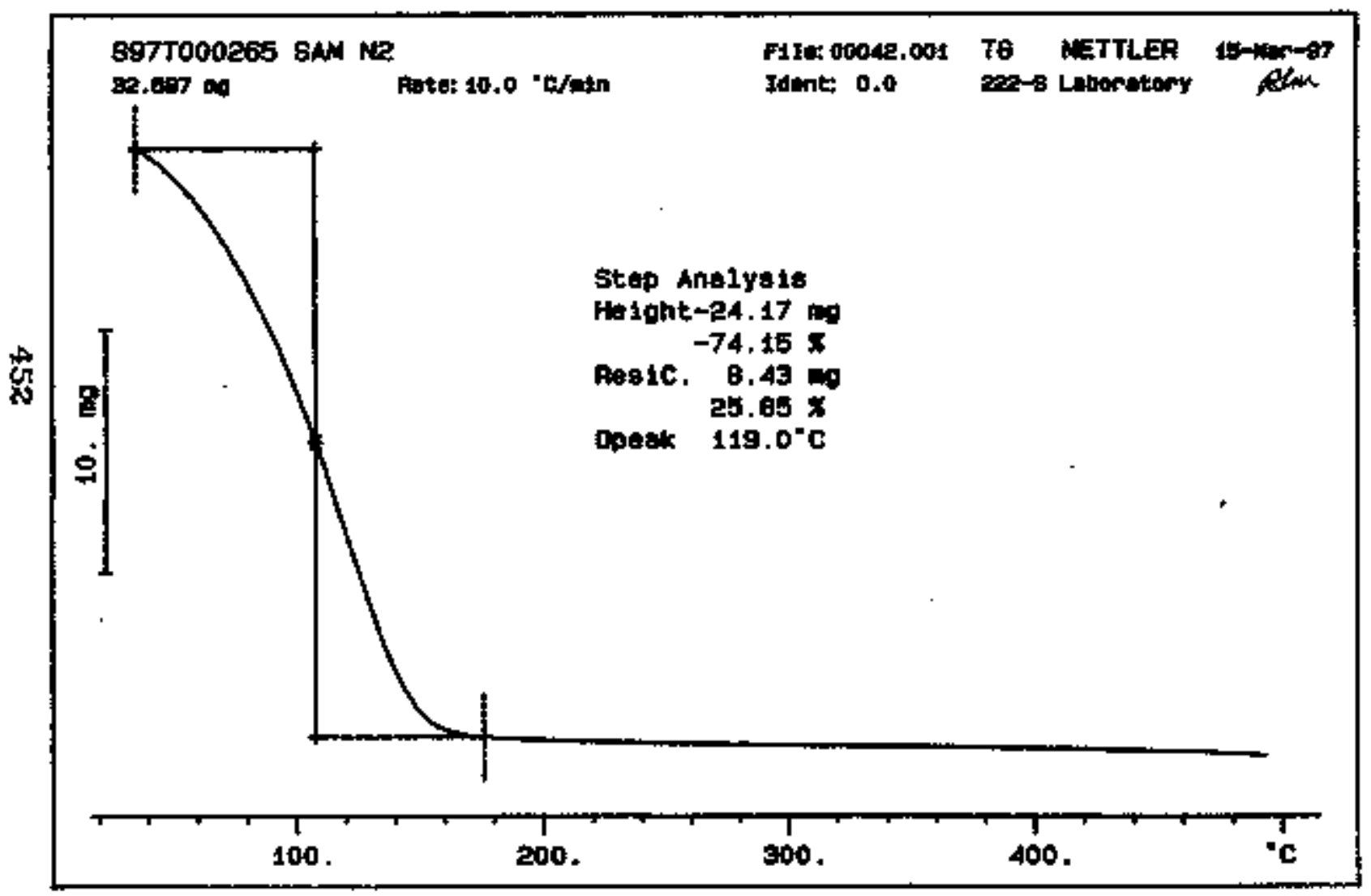

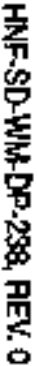




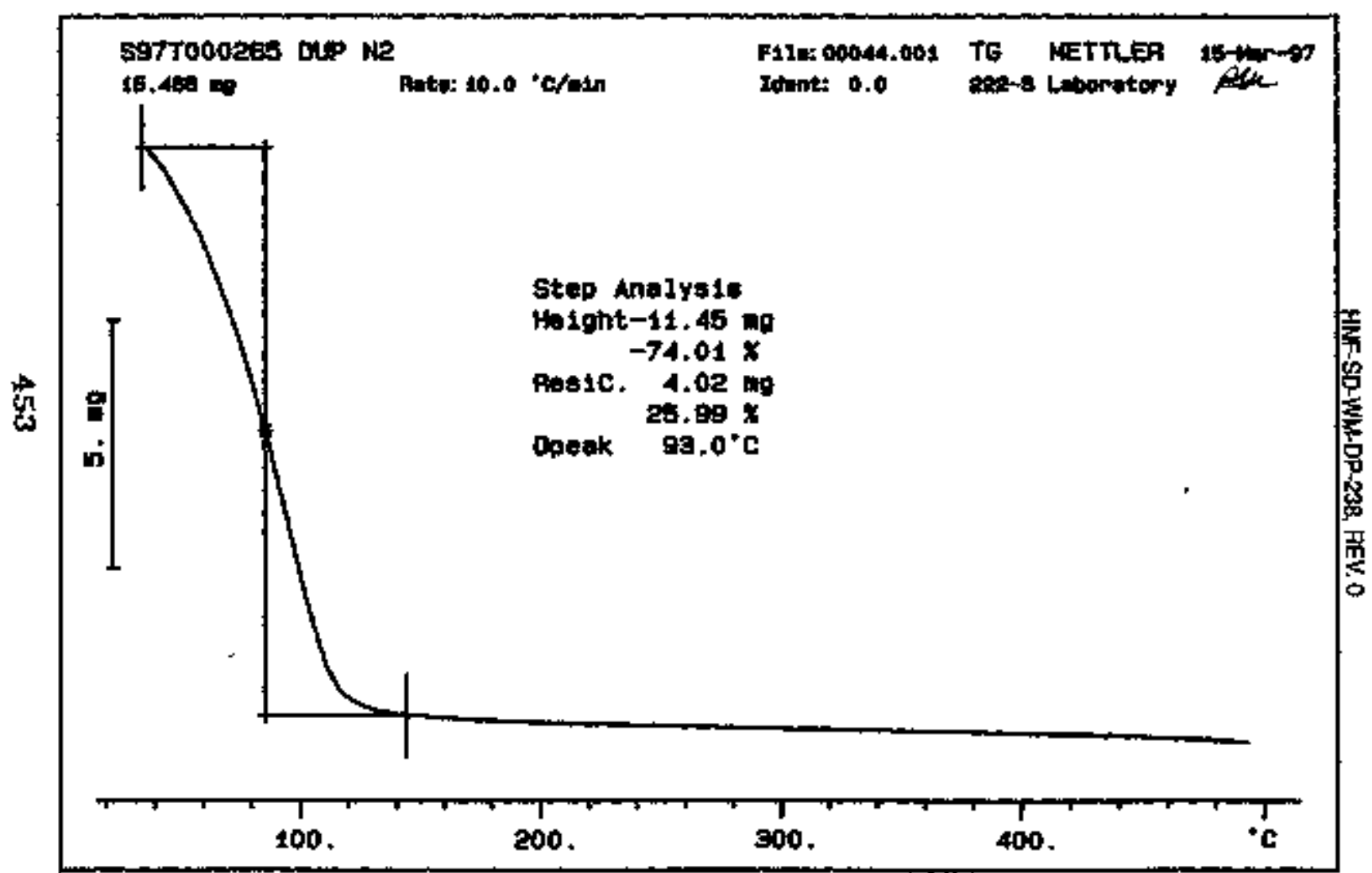




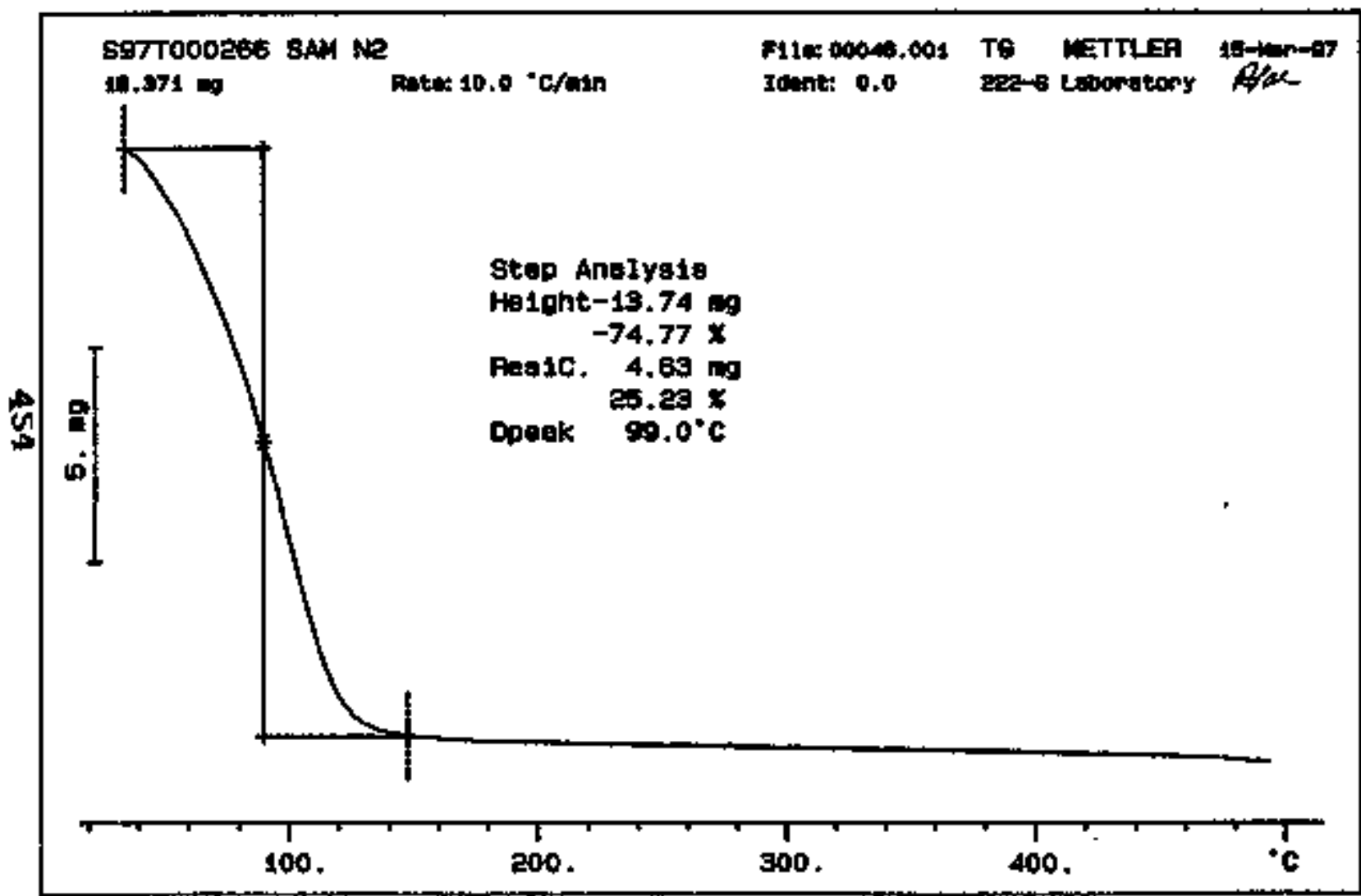

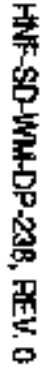




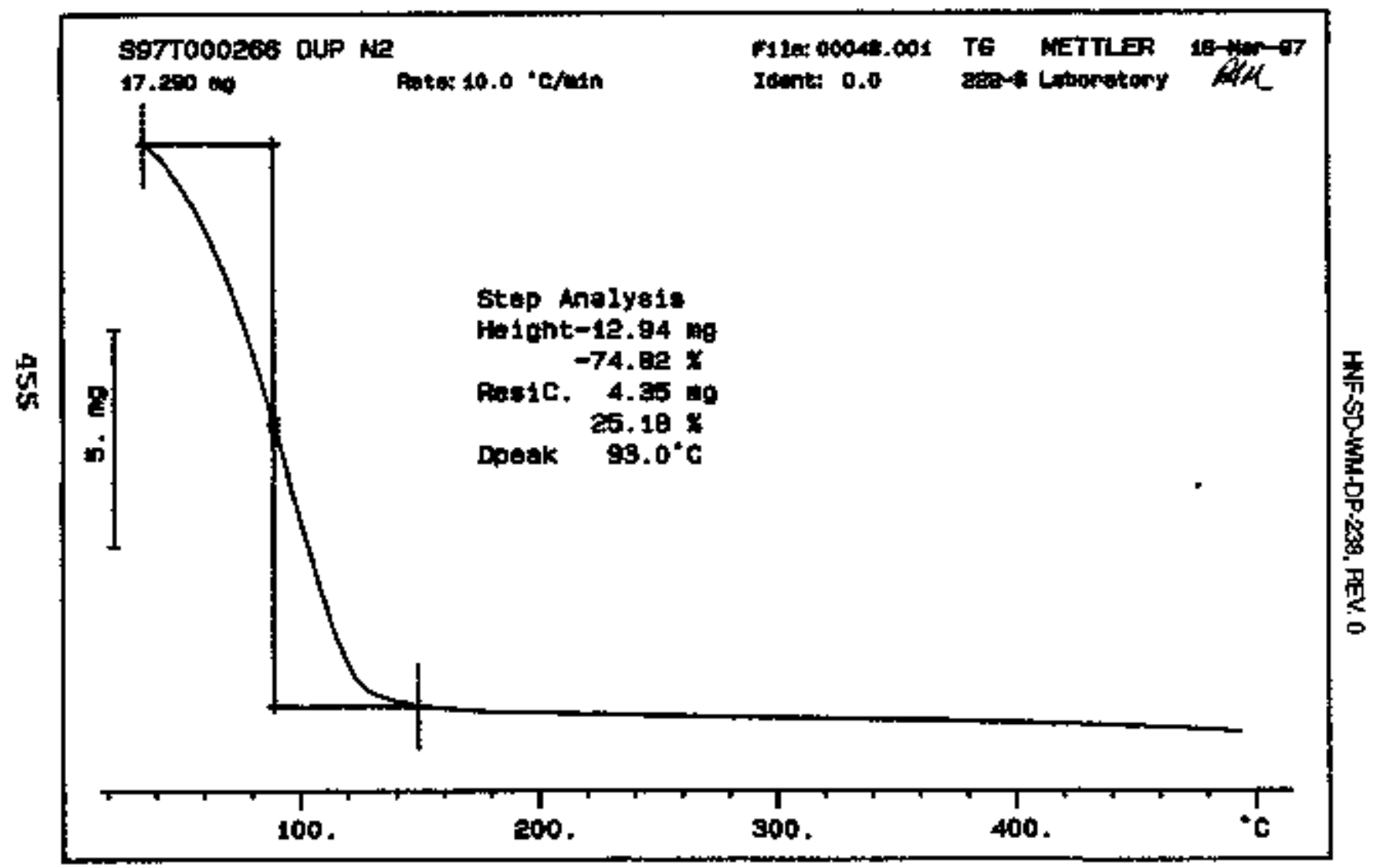


nowhingl Korion 2.1 cosilstes

HINF-SD-WH-DP-238, AEV. 0

fate: 1

Astapyst 0,22

LABCORE Data Entry Template for Worklista 17116

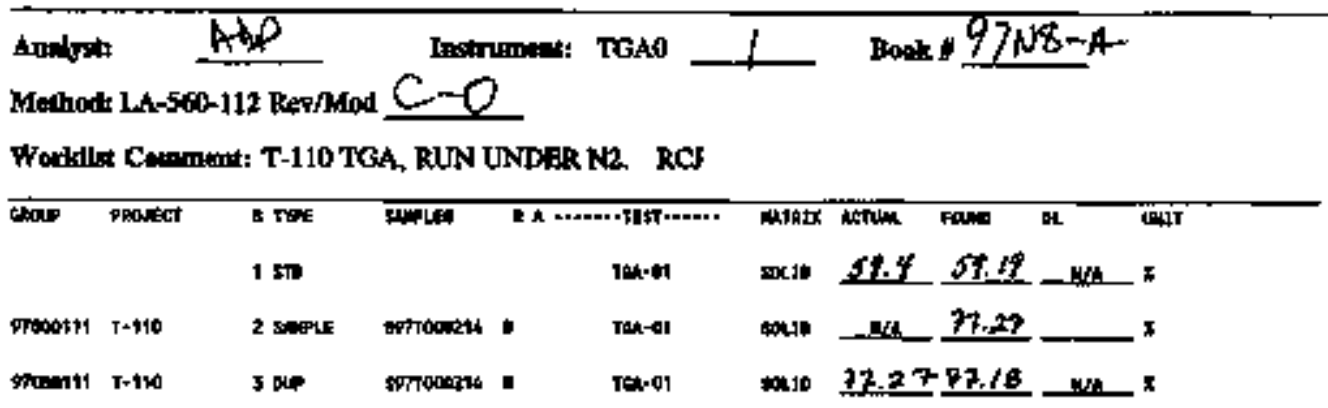

Fingl page for worklist \# 17116

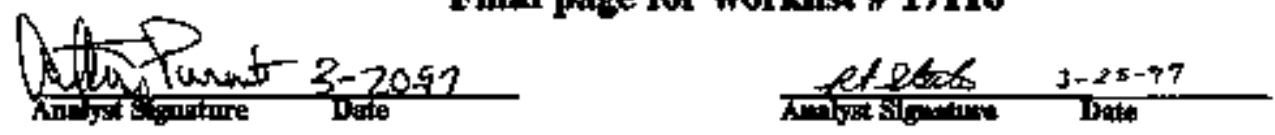

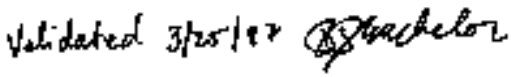

Dow bisy Consom

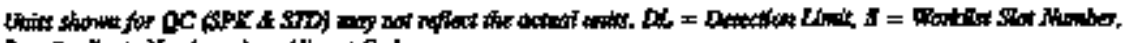

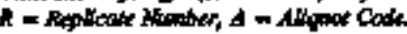

456 
TEA STL 97NB-A N2

20.870 a fotet $10.00 / \mathrm{mon}$
Frle: 00014.001

Idant 0.0
TG METTLER Bo-kar-ot ate-8 Leborotary

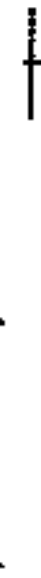

Step Anelyato

Height-15. $3 \mathrm{gg}$

$5.19 x$

Fot 1C. $10.72 \mathrm{ng}$

$40.81 \%$

Dpeak $101.7^{\circ} \mathrm{C}$

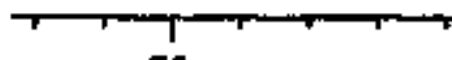

50.

100.

150. 


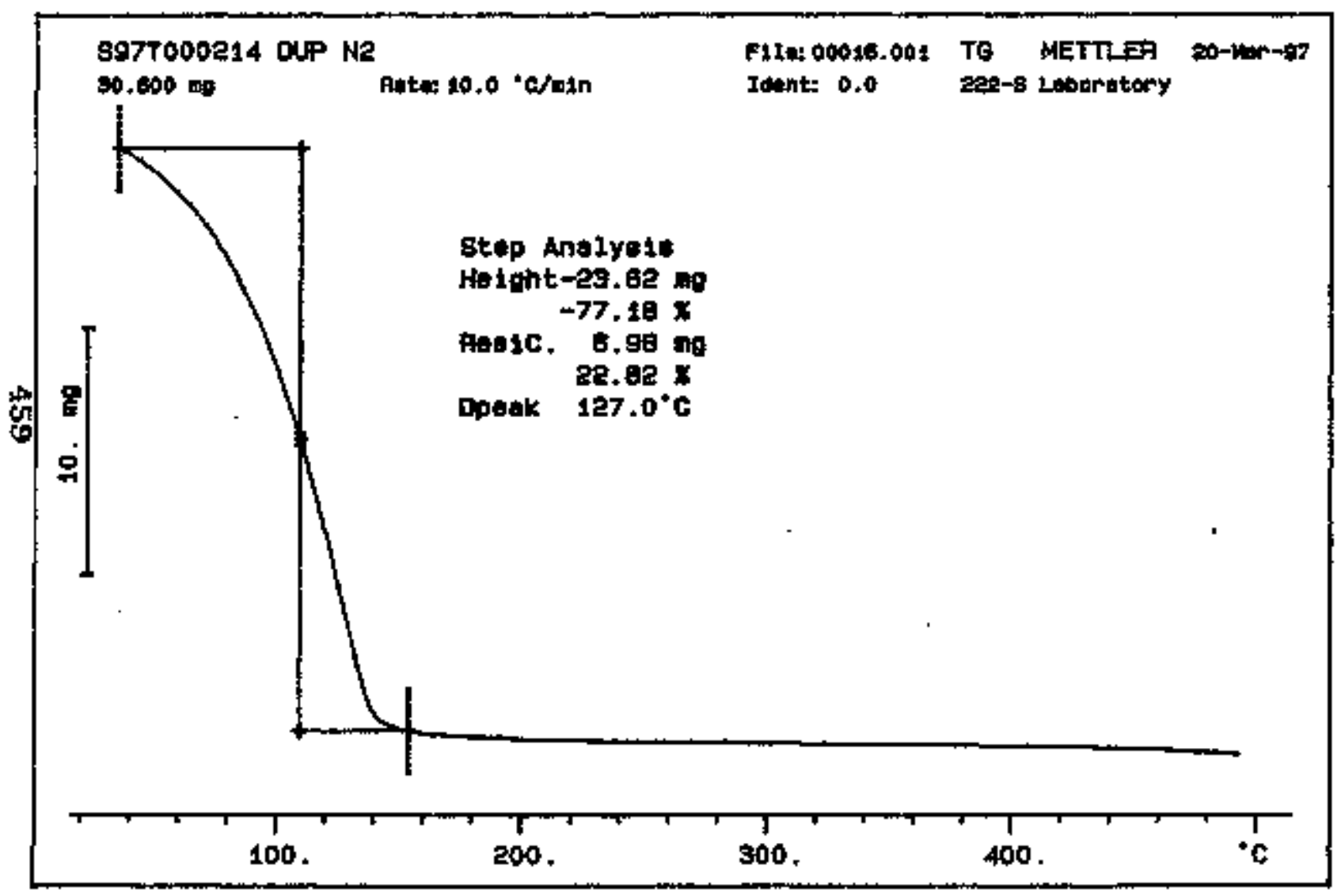

高 


\section{LABCORE Completed Worklist Report for Worklist\# 16721}

Anatist: 3dp

Indirwient: BAOOL

Bookf 133 indir-A

Method: $\angle A^{*}$ STo- 112 Rev Mad $D-1$

Workllst Comment: SPG-01 FOR T-110 (ZNCLZZ CAL PIPETT)

RTS

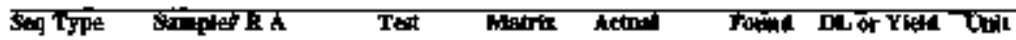

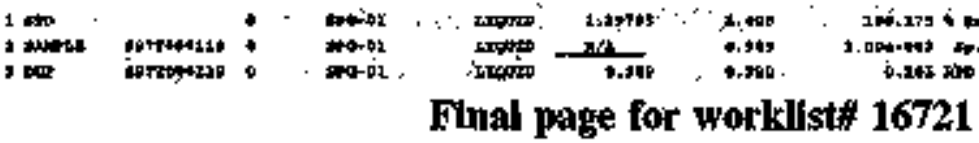

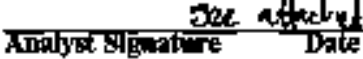
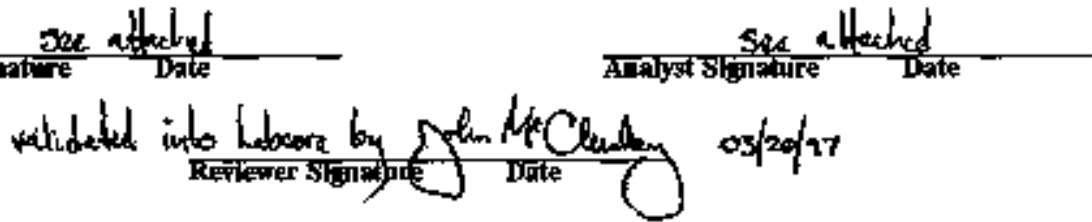

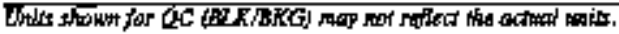




\section{LABCORE Data Entry Template for Worklist: 16721}

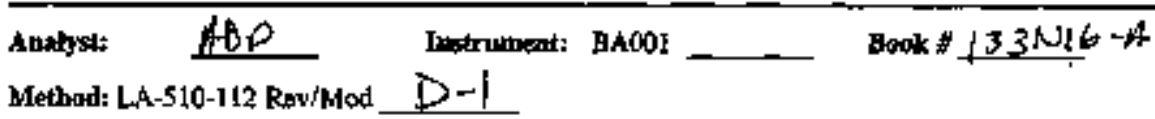

Worklist Comment: SPG-01 FOR T-110 (ZNCL2 CAL PLPETT) RTS

\begin{tabular}{|c|c|c|c|c|c|c|c|c|c|}
\hline $\sin$ & FAONECT & $\begin{array}{l}8 \text { IHPE } \\
1 \$ \pi 0\end{array}$ & supiek" & ค A & S46-01 & Lith & 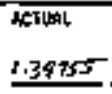 & $\begin{array}{l}\text { Nones } \\
1.400 \\
\end{array}$ & HoL \\
\hline 97\%000085 & T.110 & 2 泾田1 & sprepol 19 & 0 & 501 & Llarib & . Is & $\$ .984$ & \\
\hline 97000063 & $r \cdot+10$ & 3 axp & sshopotts & 4 & अ76-91 & Liourso & 4.484 & 0.790 & $\mathrm{~K} / \mathrm{th}$ \\
\hline
\end{tabular}

\section{Final page for worklist \# 16721}
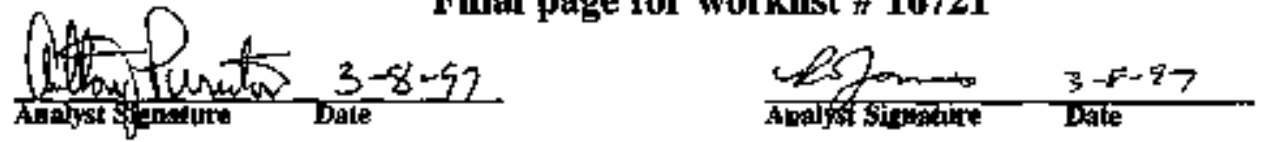

Dota Bntry Comorop:

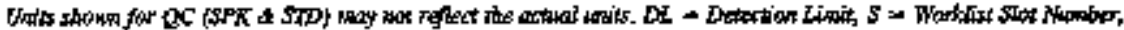

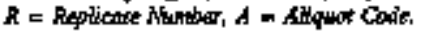



HNF-SD-WW-DP-Z38, REV. 0

PLACE ANALYTKCAL CARD IN BOX EELOWN OR ATTACH TRAVELER

\section{SPECIFIC GRAVITY : LA-510-112 (C-3)}

\begin{tabular}{|c|c|c|c|}
\hline 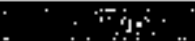 & & \$TAMDARD & STANDAFD \\
\hline STAROARD & 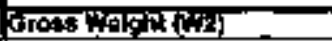 & 220193 & $1998 \mathrm{r}$ \\
\hline 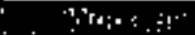 & Tort Wusth (W-1) & 1.9138 & 1.844I \\
\hline (67) & Mediht of Eolulien (W2-WM) & 0.10\% & 0.1048 \\
\hline & Voturne of solution pe & 76.2400 & 75.1400 \\
\hline 9P्E-a1 & Spacifte Gravky & 1.4040 & 1.391 \\
\hline i. & 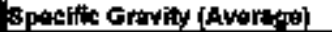 & 1,4001 & \\
\hline
\end{tabular}

प्राप्राए

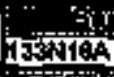

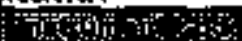

SAOST

$\because:$ : :

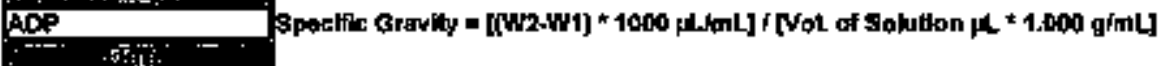

v RGSULT V

a

2:30 AM

Spuellic Groulty Averege :

1,400

Eross Wotyhl (Wz) $=$ thl of vial + gap + cotton + solullon

Tere Woigh $(W 1)=$ wh. of $v$ tal + cap + colton

ili: :

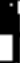

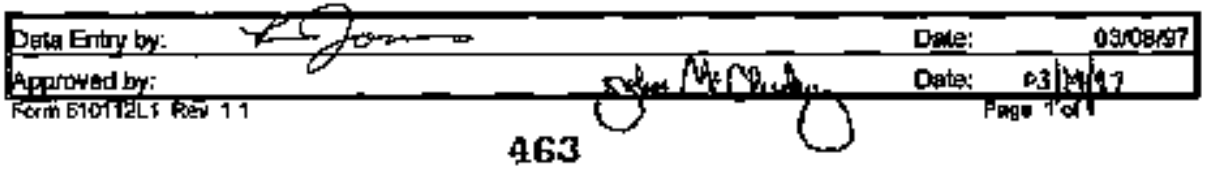


HAF-SD-WM-DP-238, REV. O

PLAGE ANALYTKCAL CARD IN BOX EELOW OR ATTACH TRALELER

SPECIFIC GRAVITY : LA-510-112 (C-3)

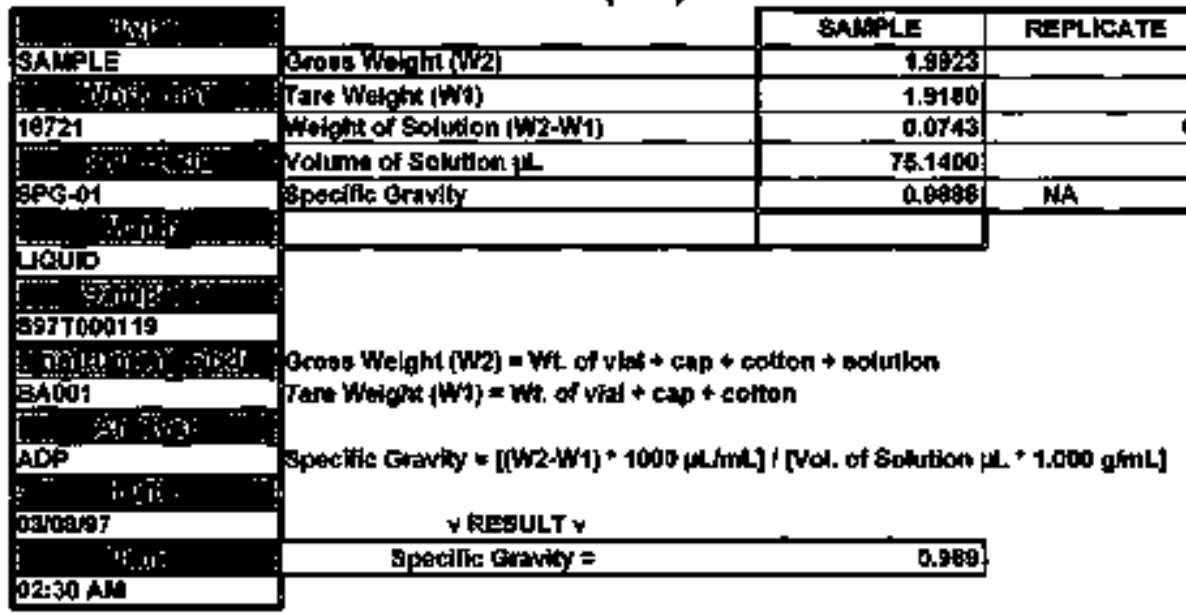

Data Entry by:




\section{SPECIFIC GRAVITY : LA-510-112 (C-3)}

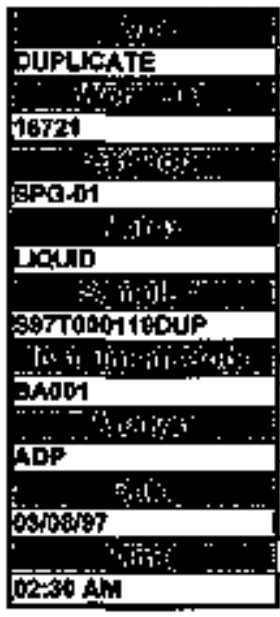

\begin{tabular}{|c|c|c|}
\hline & DUPLKATE & REPUCATE \\
\hline 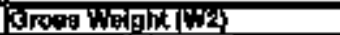 & $1.83 \mathrm{~s}$ & \\
\hline Tare Welght (W1) & 7.8649 & \\
\hline 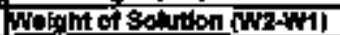 & 0.074 & $\mathbf{0}$ \\
\hline Yolun of solution pL & TS.1400 & \\
\hline Spoctilic Orartly & s.10002 & MA \\
\hline
\end{tabular}

Gross Wolgtk awa = Wh of val + cap + eotton + golution

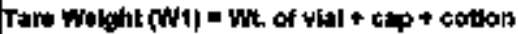

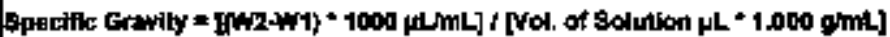
Y RESULT V Specific Oravily a 0,90

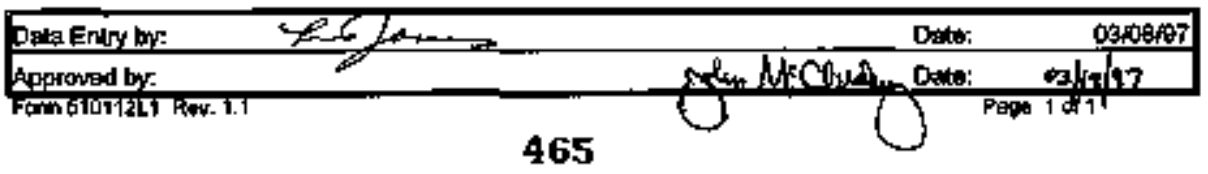




\section{LABCORE Completed Worklist Report for Worklist\# 16749}

Anelyst adp

Instrument: BA001

Bookff [33신..A

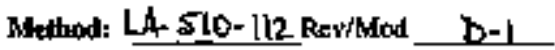

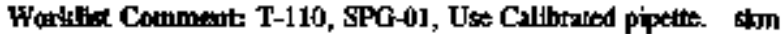

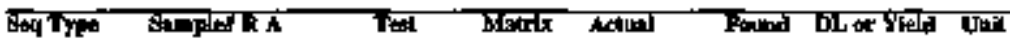

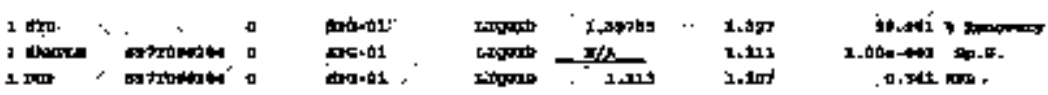

Final page for worklist: 16749

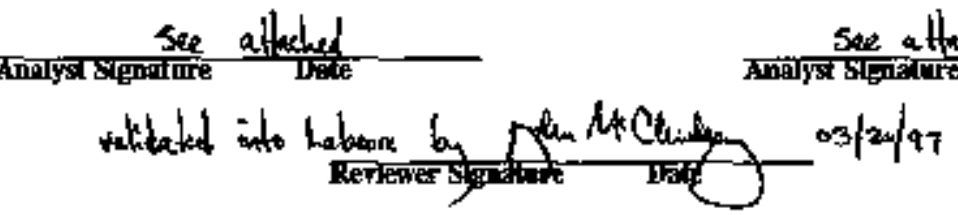




\section{LABCORE Data Entry Template for Worklist" 16749}

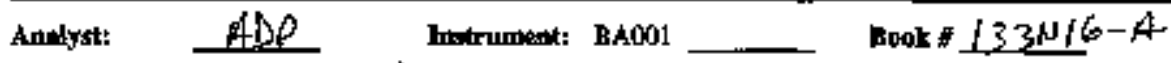

Method: LA-510-112 Rev/Mod $\mid \mathrm{D}-1$

Forklist Comment: T-1 10, SPG-01, Use Calibrated pipette. shm

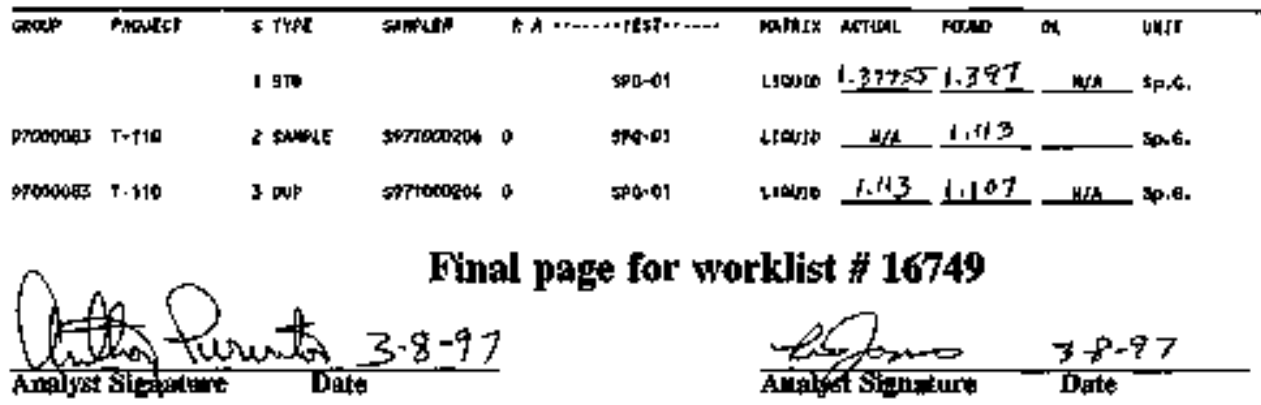

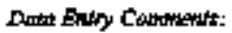

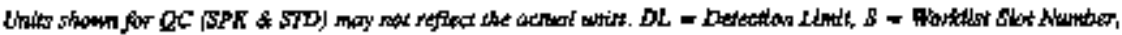

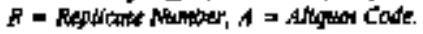




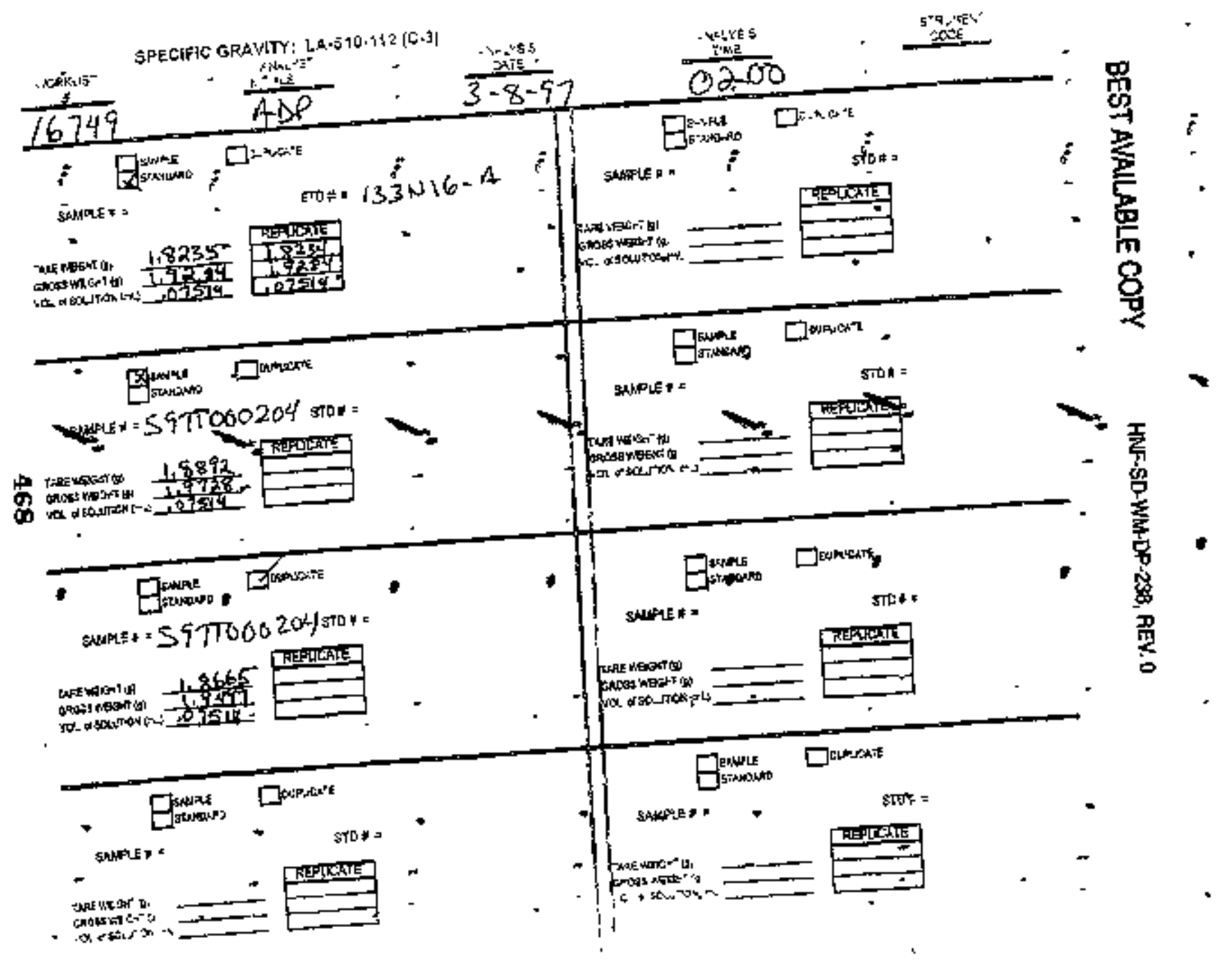




\section{SPECIFIC GRAVITY : LA-S10-112 (C-3)}

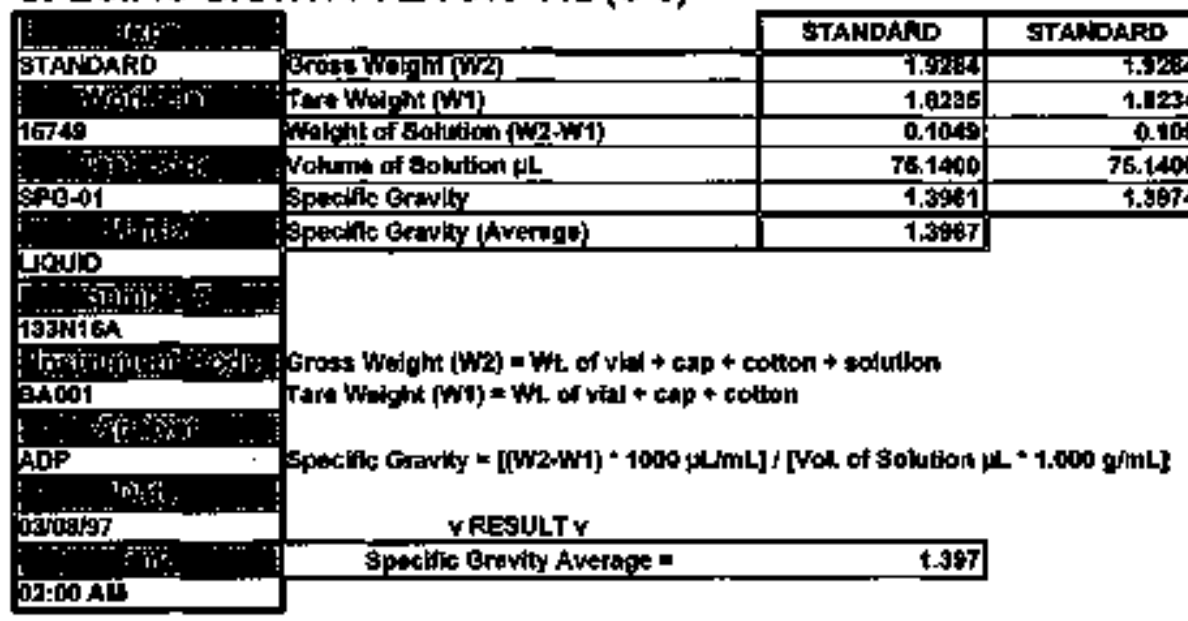

\begin{tabular}{|c|c|}
\hline Deta Emby by: - Er & Dosnoseng \\
\hline $\begin{array}{l}\text { Approved by: } \\
\text { Forp S101 }\end{array}$ & 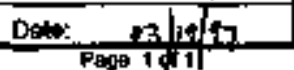 \\
\hline
\end{tabular}




\section{SPECIFIC GRAVTY : LA-510-112 (C-3)}

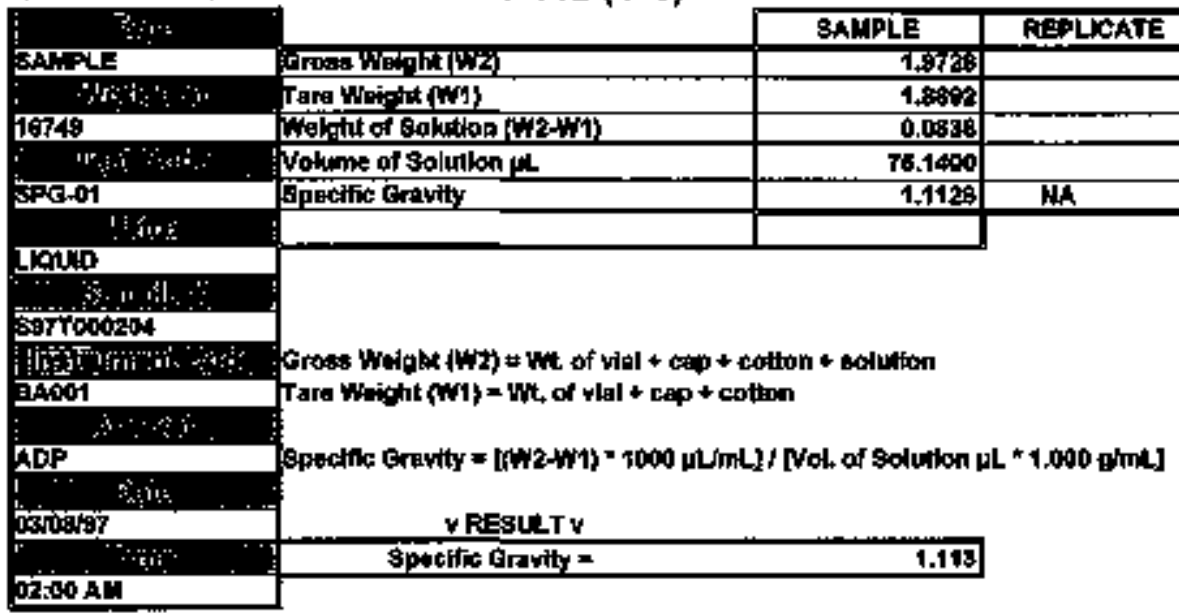

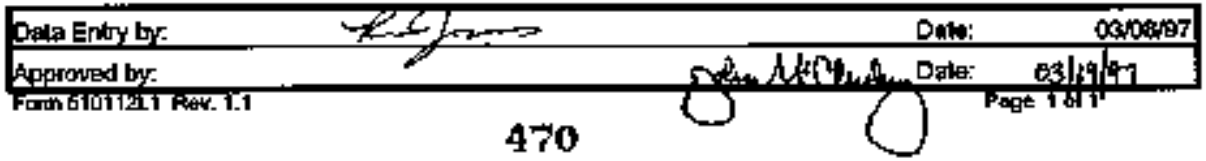




\section{SPECIFIC GRAVITY : LA-510-112 (C-3)}

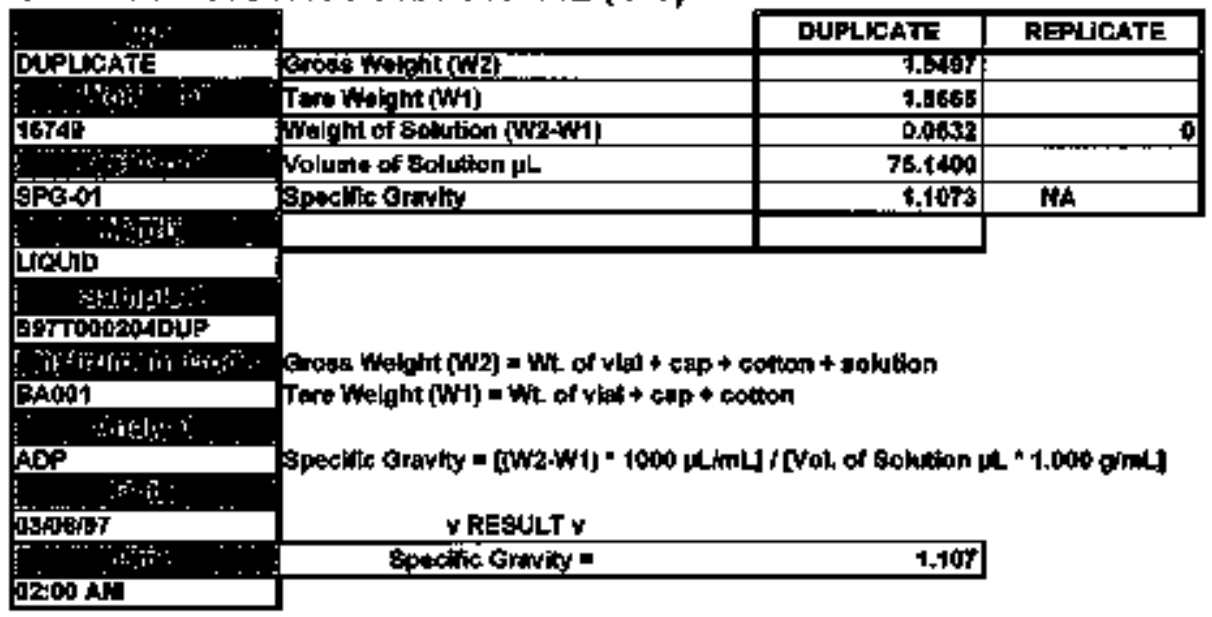

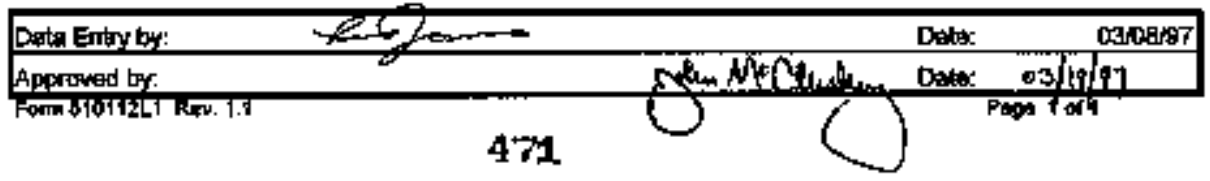




\section{LABCORE Completed Worklist Repart for Worklist/f 16706}

Autlyst: Igh

Inthourent; JCO2

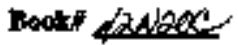

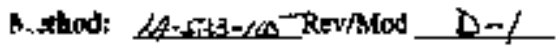

Morkist Comment G]C-01 FOR T-110

RTS

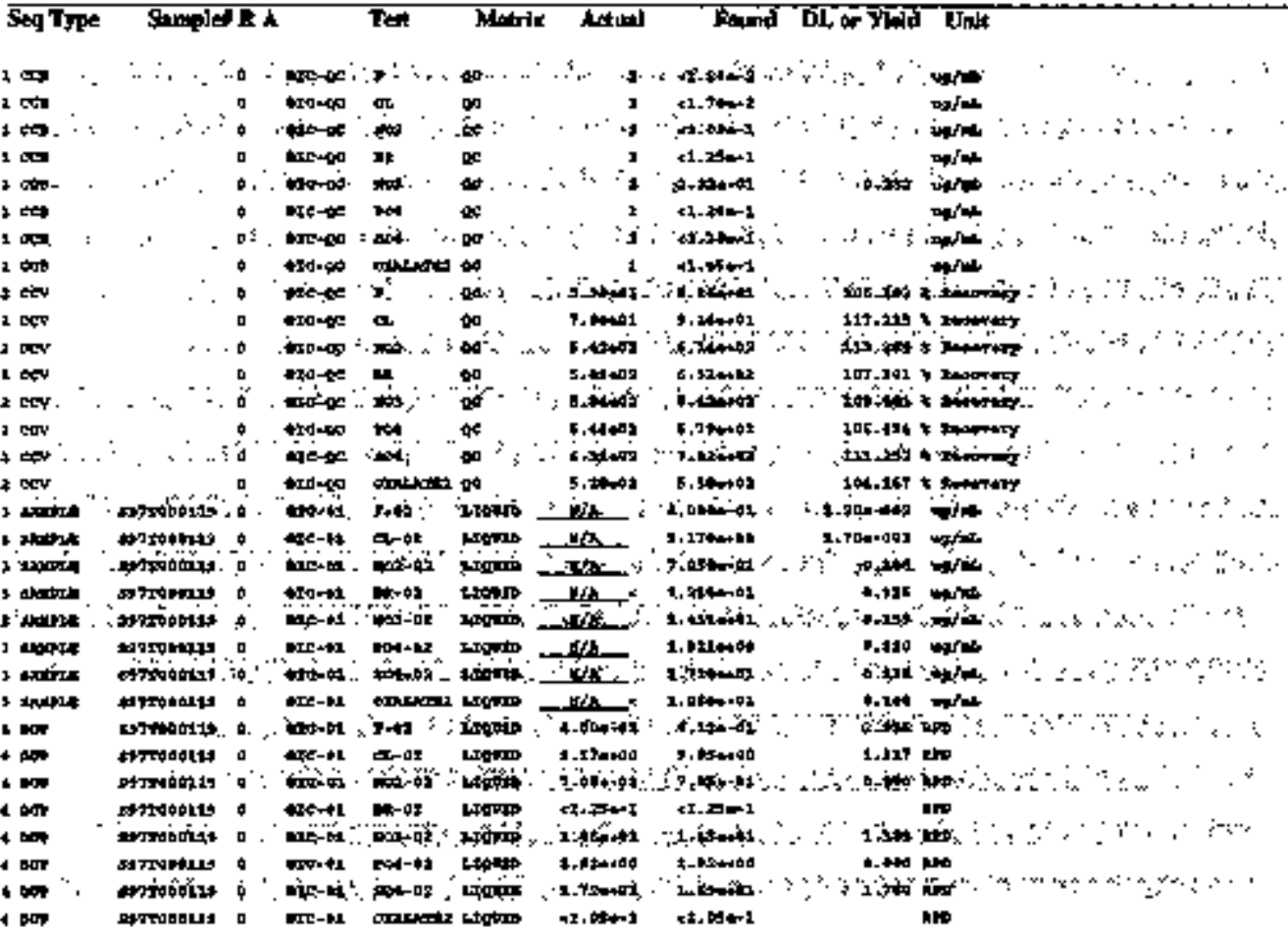

\section{Final page for worklist 16706}


atros 9714070

HNF-SD-WH-DP-Z38, FEV,

Page I

statist

LABCORE Data Entry Template for Worklist" 16706

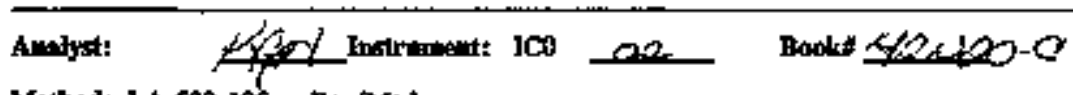

Meland: LA-S93-10S Rew/Mod

Wially Cimment IC-01 FORT-11日

RTS

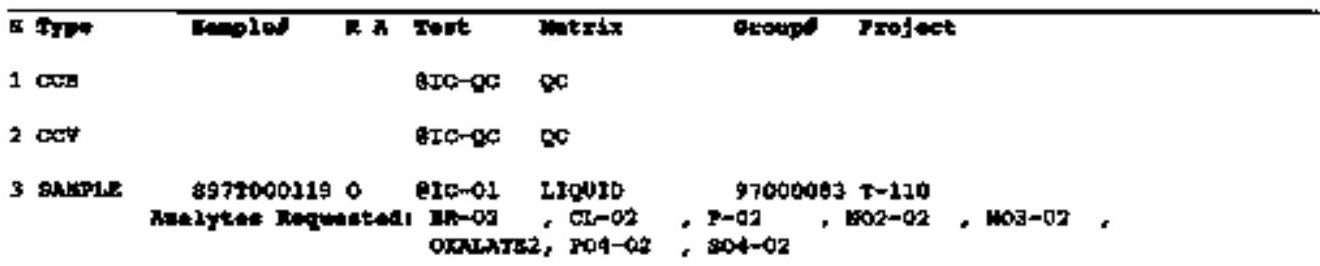

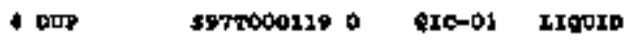

Final page for worklist f 16706

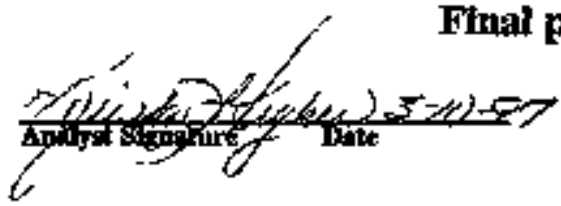

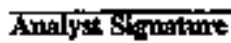

Date

Daka Buby Companives

$16706 \mathrm{~m}$ the, sct

IGZOLMAR.CSW

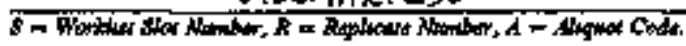

473 
HNF-SD-WM-DP-238, REY. O

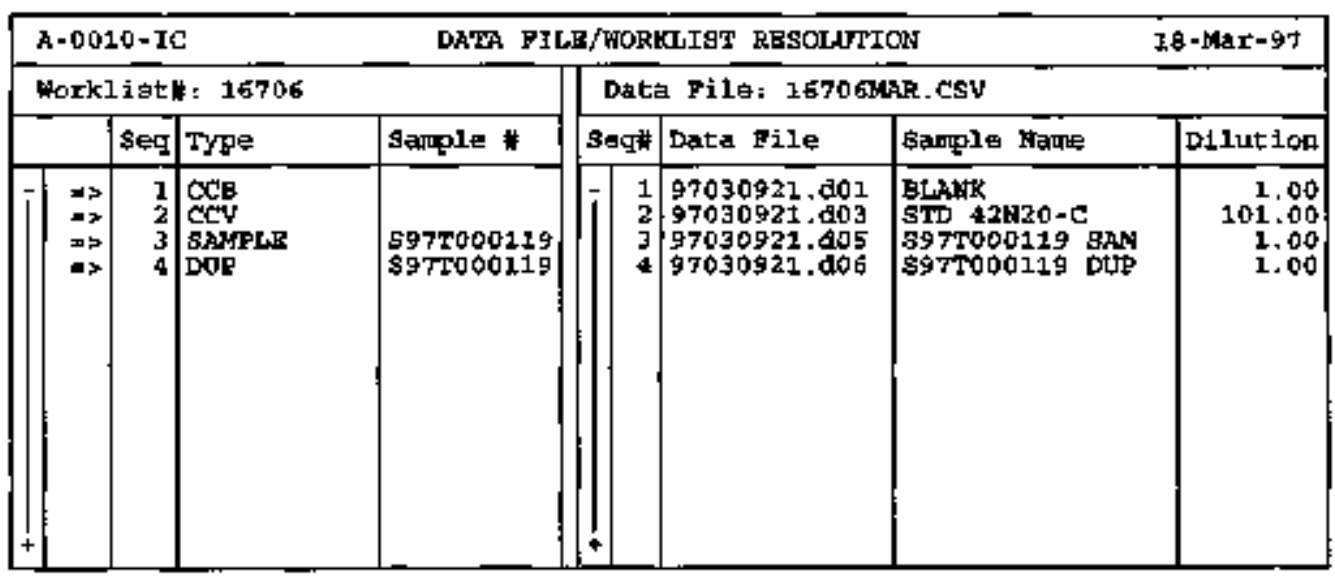

Save (F4) Abort (ShAt-F\}) LiakPiles (Ghift-F1) Uploadfile(FB) 


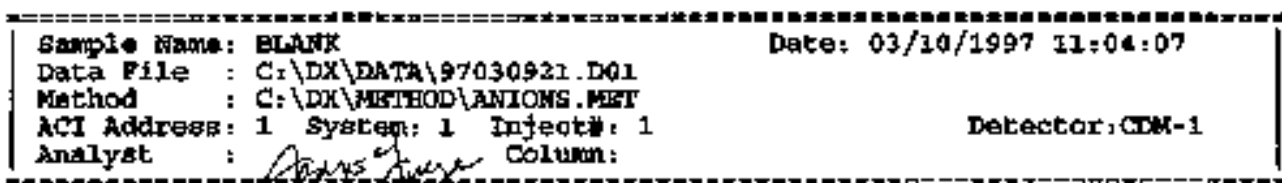

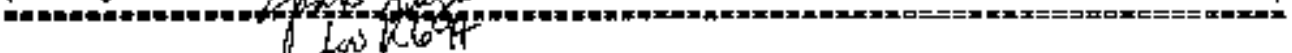
Caibration Volunef Dilution points pate start stup Area Reject. Exterinal 1 13000 5Hz $0.00 \quad 10.00$ 50

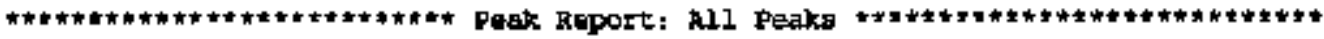

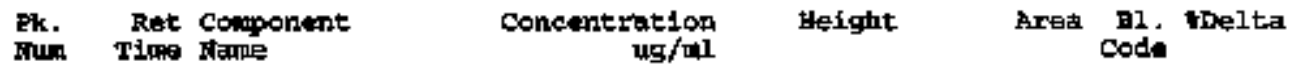

$21+39$

$3 \quad 1.55$ CELORIDR

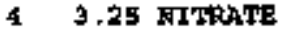

$5 \quad 5+92$ StTrFhB

6. 7.45

$\begin{array}{ccccc}0.000 & 55 & 319 & 1 \\ 0.000 & 20 & 68 & 1 \\ 0.006 & 55 & 213 & 1 \\ 0.332 & 67 & 503 & 1 \\ 0.124 & 45 & 504 & 1 \\ & 0.000 & 41 & 201 & 1 \\ \text { Iotalg } & 0.362 & 284 & 1827 & \end{array}$

.352 1 $1 \quad-0.22$ $1 \quad 1.67$ $1-2,79$ 1

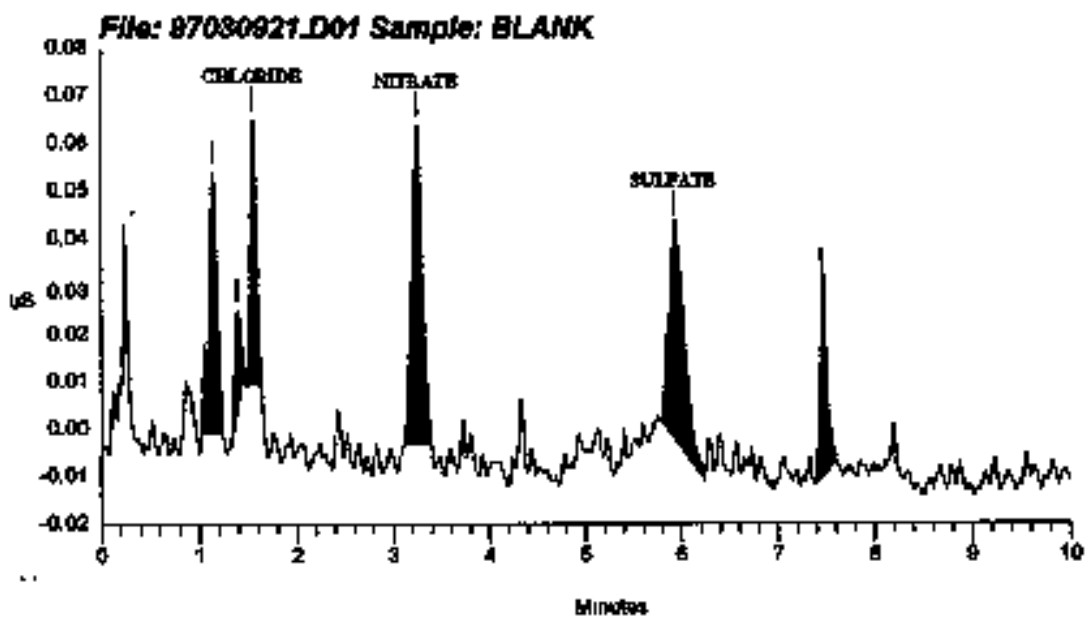

SCNUTURE ABOVE REPRESENIS CHEMRAL TECINOLOGISTCHFHST JJ

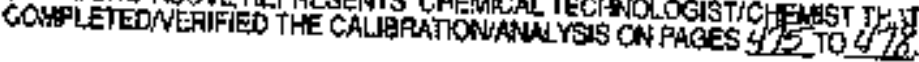




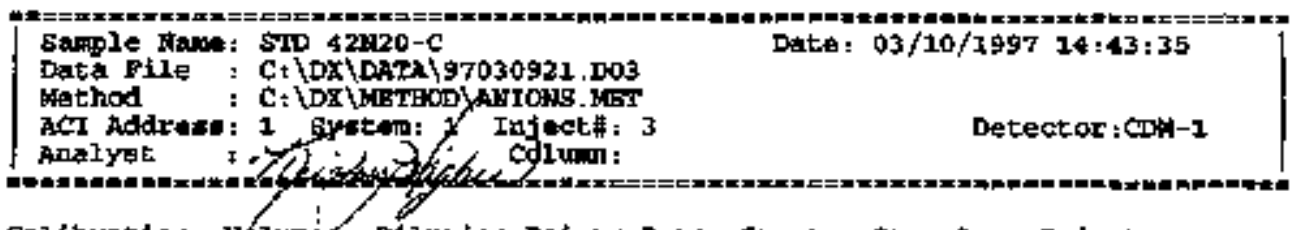

calibration volum Dilutiom Points Rata start \$tpp Area Reject

$\begin{array}{lllllll}\text { Extertal } & 1 & 3000 & 5 \mathrm{H} & 0.00 & 10.00 & 50\end{array}$

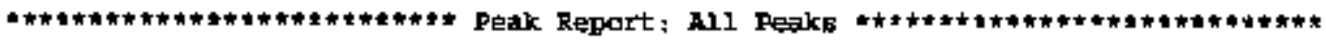

\begin{tabular}{|c|c|c|c|c|c|c|c|}
\hline $\begin{array}{l}\text { Pli. } \\
\text { Inum }\end{array}$ & $\begin{array}{l}\text { Rat } \\
\text { Timat }\end{array}$ & $\begin{array}{l}\text { Compothent } \\
\text { Jane }\end{array}$ & $\begin{array}{r}\text { Concentration } \\
\text { ug/on }\end{array}$ & 비믹 & $A x \in a$ & ide & HDelca \\
\hline $\begin{array}{r}1 \\
2 \\
3 \\
4 \\
5 \\
6 \\
7 \\
6 \\
10 \\
11 \\
13\end{array}$ & $\begin{array}{l}0.30 \\
0.87 \\
1.02 \\
1.41 \\
1.53 \\
1.87 \\
2.83 \\
3.21 \\
4.62 \\
5.91 \\
7.35\end{array}$ & 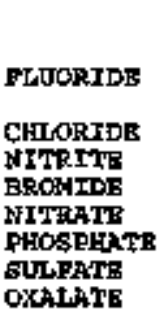 & $\begin{array}{r}0.000 \\
0.000 \\
62.600 \\
0.000 \\
93.597 \\
613.718 \\
631.625 \\
642.455 \\
579.217 \\
702.356 \\
550.272\end{array}$ & $\begin{array}{r}52 \\
87 \\
2486 \\
34 \\
2548 \\
9683 \\
5959 \\
6537 \\
1720 \\
5252 \\
2311\end{array}$ & $\begin{array}{r}337 \\
372 \\
72207 \\
292 \\
73146 \\
53155 \\
39693 \\
52248 \\
21534 \\
72644 \\
43606\end{array}$ & $\begin{array}{l}1 \\
2 \\
2 \\
2 \\
2 \\
1 \\
2 \\
2 \\
1 \\
1 \\
1\end{array}$ & $\begin{array}{r}0.00 \\
0.22 \\
0.54 \\
-0.12 \\
0.21 \\
-0.43 \\
-3.01 \\
-3.28\end{array}$ \\
\hline
\end{tabular}

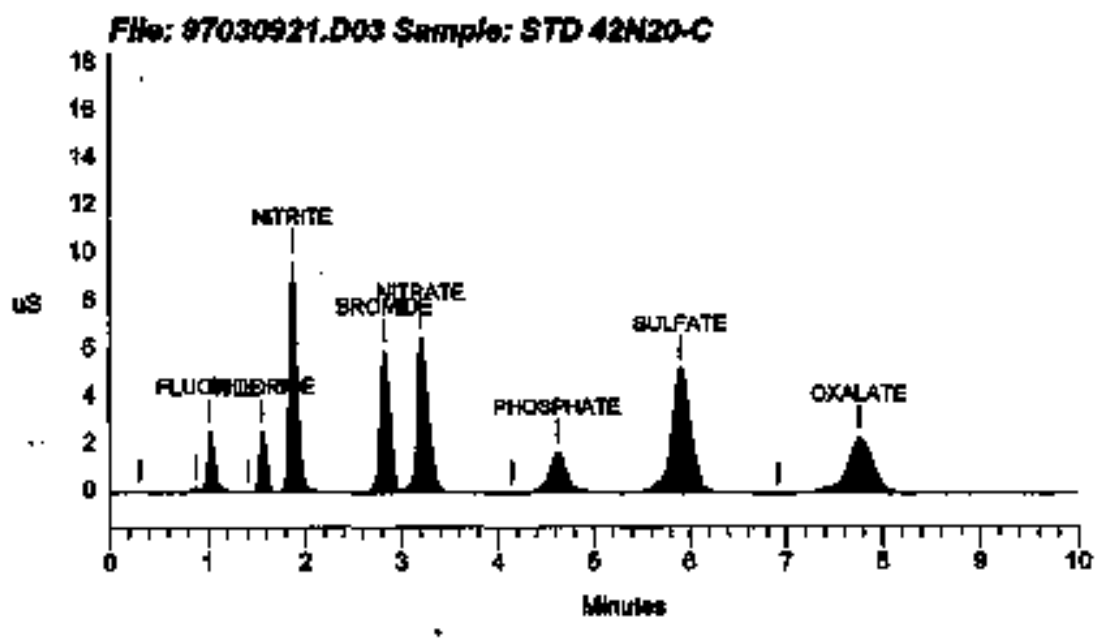




\begin{tabular}{|c|c|c|}
\hline 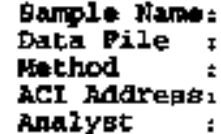 & 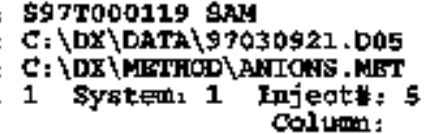 & Date, 03/10/1997 15+15:41 \\
\hline
\end{tabular}

Calbatiog Volume Dilntion Points Rate start Btop Artö Reject

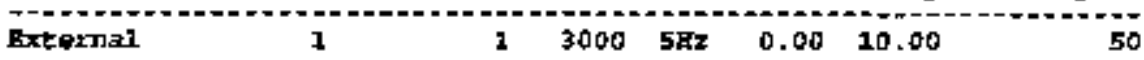

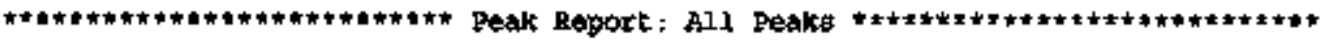

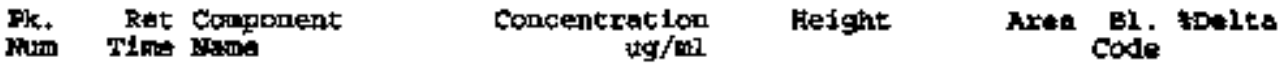

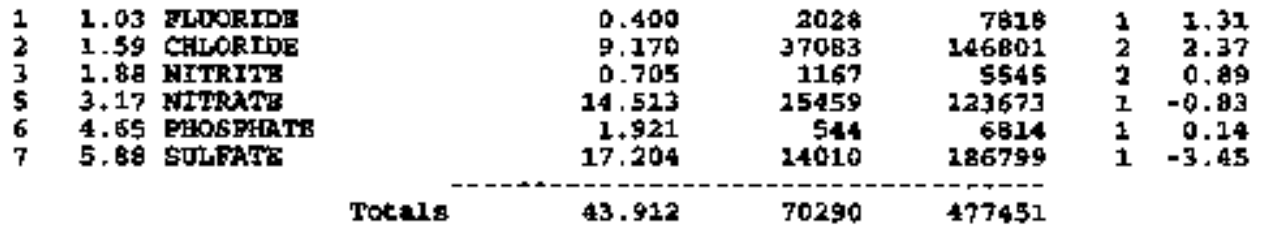

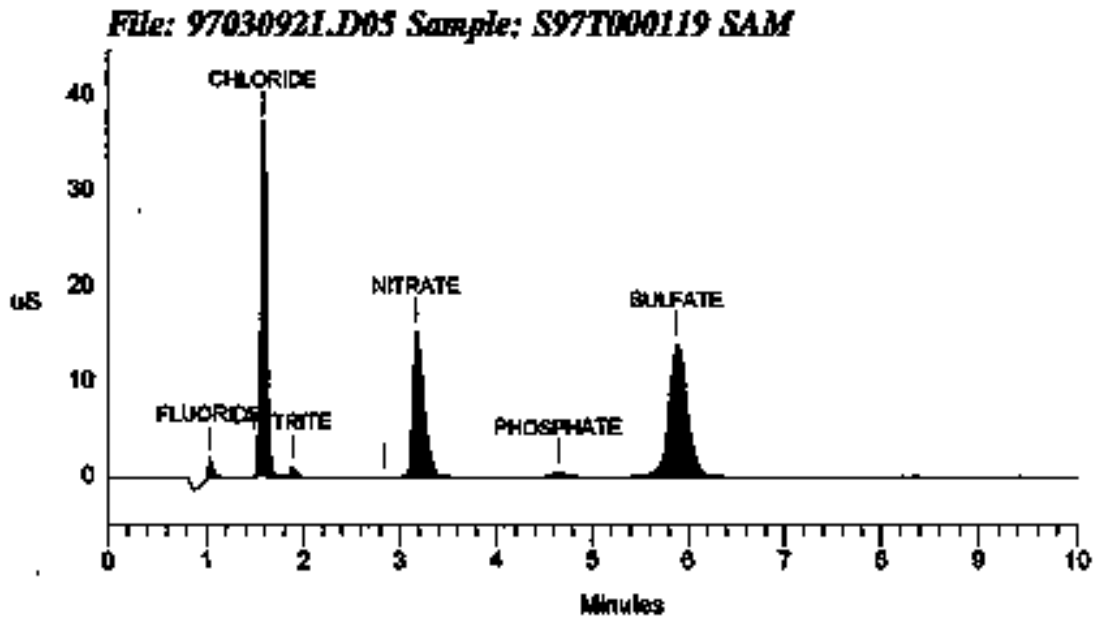


HNF-SD-WWH-DP-233, REV. D

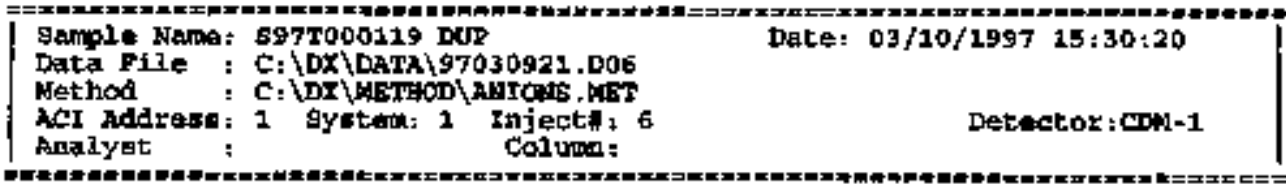

Fibration Volume Dilucion Pointe Rate \$tart stop Aret Reject

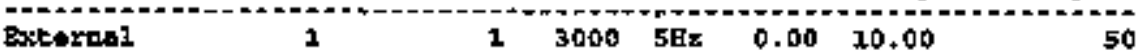

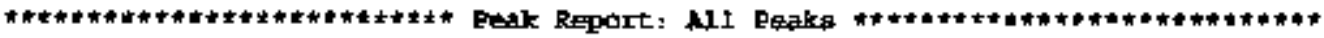

Pk. Ret Componint Concentration Height Ares Bl. tDelta

Num Time Hame $\operatorname{lig} / \mathrm{ml}$

Code

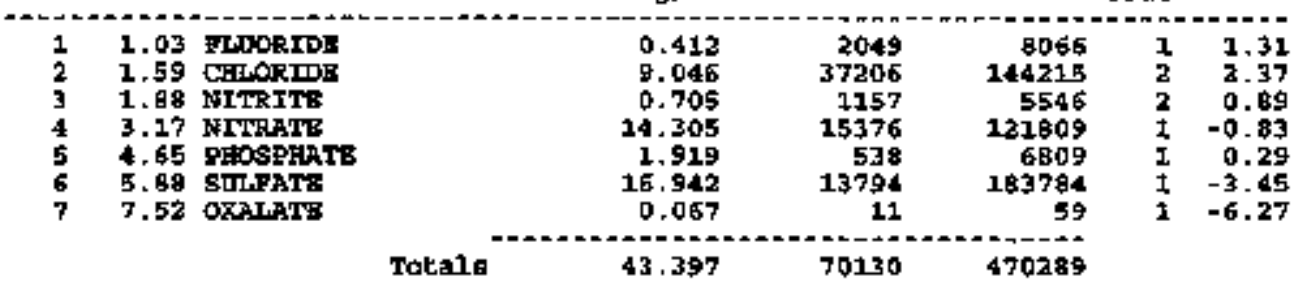

\section{Fle: 97030921.DO6 Sanples S97T00119 DUP}

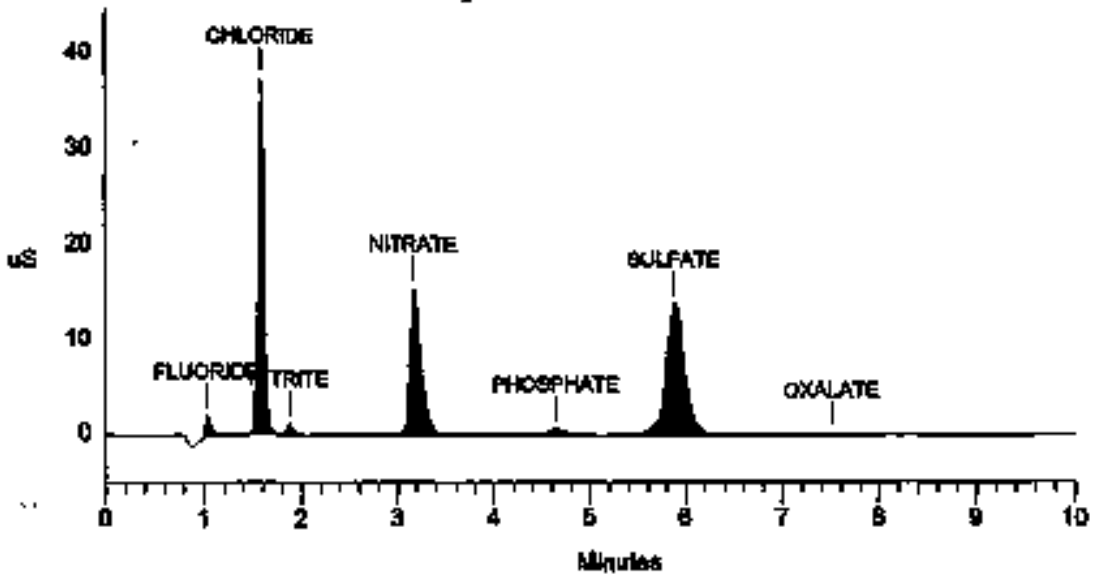




\section{LABCORE Completed Worklist Report for Worklist\# 17115}

Anglyst: eal

Instrunent: $\mathrm{ICO1}$

Book" fatad

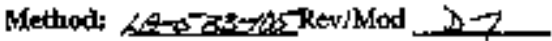

Workfist Comonent: T-I10 IC. RCJ

\begin{tabular}{|c|c|c|c|c|c|c|c|c|c|}
\hline Seq Ty山 & Serpple. & $\overline{\mathbf{R A}}$ & & Tets & Matrbx & Adod & Trones & Mer Ytad & Chis \\
\hline 1 प्त & & - & 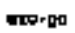 & $\boldsymbol{r}$ & क & $z$ & $-1.304-x$ & & na/m \\
\hline 1 的 & & $\downarrow$ & ere-the & a & $\approx$ & 2 & $-1-700-2$ & & 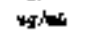 \\
\hline 10 & & 4 & are-acte & Don & מי & 1 & 1.4. & & xab \\
\hline c c다 & & v & Tx=pa & $\mathbf{m}$ & so & 2 & a rabt-z & & $2 g / x$ \\
\hline z & & 中 & wre-06 & 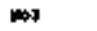 & $\approx$ & 7 & 8-40-4) & 4. gat & 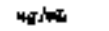 \\
\hline 20 & & 4 & anz-oc & nou & $\infty$ & 1 & $<1.20=-1$ & & a \\
\hline 1 따 & & $\square$ & torma & Ip4 & 68 & $\mathbf{x}$ & $-4-20+-1$ & & estiven \\
\hline 2 से & & $\bullet$ & $\varphi x=-\phi c$ & onvasites: & $\infty$ & 2 & $<-4-4=-9$ & & 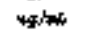 \\
\hline ger & & 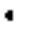 & 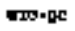 & r & $\phi$ & $5.10=01$ & t.120t+1 & 100.969 & 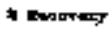 \\
\hline 200 & & 0 & ตคำ & 中 & s\% & 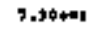 & $1.7+4+1+1$ & n. 14 & 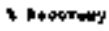 \\
\hline a & & b & 口II-ه & $\tan$ & $\approx$ & $5.40 \mathrm{mbz}$ & x.120+4g & M. cos & t Baporaty \\
\hline $2 \mathrm{ov}$ & & 4 & 由Ix-pc & $\mathbf{m}$ & $\phi$ & 3.69462 & $4.944+12$ & $1 \%, 170$ & * Hasemery \\
\hline 2005 & & v & 벼엉 & 車 & $s$ & D,tinea & 1-8ेteth & $149-104$ & 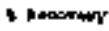 \\
\hline$\neq \mathrm{ev}$ & & 4 & 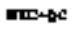 & 1006 & $8 c$ & $5.44 m b a$ & $+.130+4 a$ & 94. ESt & t Inoortary \\
\hline $2 \mathrm{ECV}$ & & 4 & 由खिम & $\operatorname{lon}$ & ه\$ & $C . \$ 1=\phi 2$ & $+.524+6 a$ & Ans, tso & $4 \mathrm{Mn} 4 \mathrm{~m}$ \\
\hline 1 त्का & & $\square$ & |c⿴囗十) & boshotets & He & 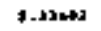 & $5.20=+44$ & 17.564 & e moootery \\
\hline 7 маяров & Deרraporn & 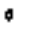 & m-at & $-d a$ & Ixम0س & $D / x$ & $+.900=40$ & 4.107 & ug \\
\hline 3 Ixomax & 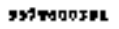 & 4 & $=00 \times 1$ & $x+2$ & Iकणेणn & 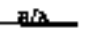 & T.1994441 & $4.14 t$ & Nationt \\
\hline I Inetat & 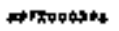 & $\nabla$ & 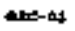 & ma-dt & кта丿) & wh & $1.1040+14$ & 2.1Ek & ugrax \\
\hline + Axacore & sorranase & a & 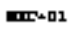 & -10.42 & हापुणा & $0 / x-5$ & $1.9754+41$ & 1. 3ts & $\log t=\pi$ \\
\hline 3 morrax & estougos & 4 & $\operatorname{tron} 01$ & $60 t=42$ & tAlq0 & Aba & t.13atomet & t. 5 ats & What \\
\hline 1 Anvilaty & 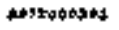 & 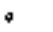 & 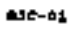 & $\omega t-a t$ & Lrogta & ㅂt & 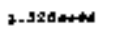 & \pm .280 & U. \\
\hline 7 нwpre & 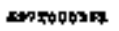 & 0 & 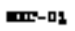 & -ad-az & याप्रणम & wh & $1.6760+44$ & 1.51u & up/at \\
\hline 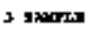 & 5ytwodret & 4 & ax-01 & cothraxis & IAtqur & $a$ & 1.J9s444 & 1.113 & Iים \\
\hline 4 mot & M & $\bullet$ & 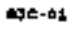 & 7-bo & 1Ateap & 4.x4-61 & $5-1+4-1$ & A. 93 & $\rightarrow$ \\
\hline p por & 5v7ragosm & a & גם-אח & (12 & Ixणमा & T.65 $60+44$ & $0.150+41$ & 4.320 & סמט \\
\hline - odr & Ertwonyor. & 4 & 4to.01 & 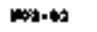 & 1Aldt & $0.1 \% 4$ & $42-100+$ & & $\omega$ \\
\hline+10 & 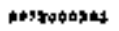 & $\bullet$ & 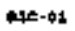 & n-w & Iт⿺辶⿻ & ct. 3tub & ct.sent & & 10 \\
\hline+ odr & rstranasen & - & m-01 & $00-42$ & Iryणn & T.41=aten & T.090140 & 1.018 & $=0$ \\
\hline - Deा & 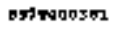 & 4 & |tr:01 & 106-4at & tInणDb & ax, tat+b & -1, T2A-4 & & $a+b$ \\
\hline 4 par & 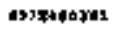 & + & $=14$ & act- & Ingam & 1.4tenthes & $1.220+101$ & 1. Is & $\mathbf{x}$ \\
\hline Dop & mytronown & 4 & 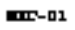 & Dxhlatin & घาपण्ण & A1,15er & $<2,16=$ & & war \\
\hline
\end{tabular}

Final page for worklist\# 17115

Analyst sisnsture Date

Analyst signature Daite

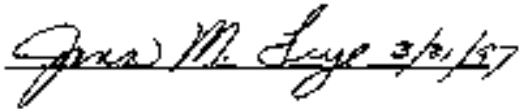

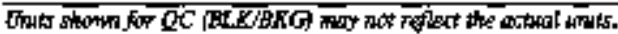




\section{LABCORE Data Entry Template for Worklistf 17115}

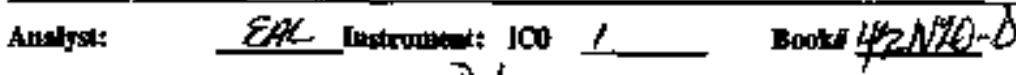

Mlathed: 1-A-533-50S Bew/biod

Warklist Com onet T-110 1C. RCI

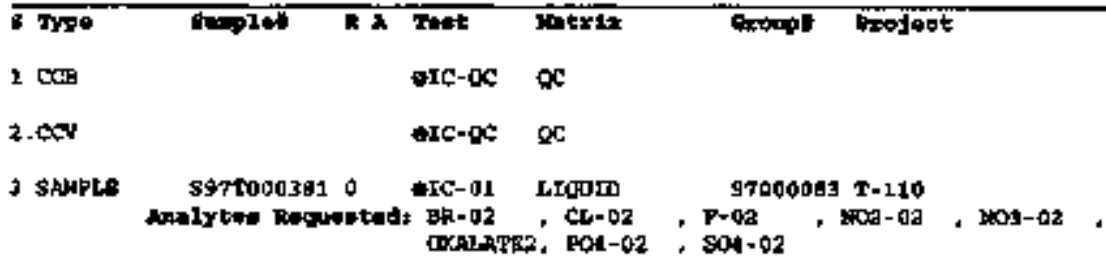

- DOF sajtoopjar o erc-ol irourit

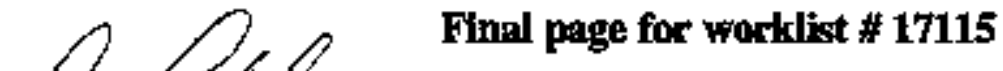

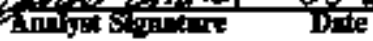

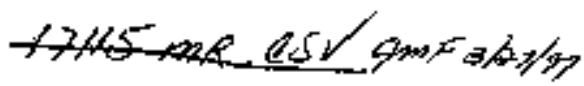

WPLCDOA $\overrightarrow{3}-27-\overrightarrow{7} \rightarrow$

Qte howate

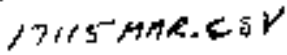

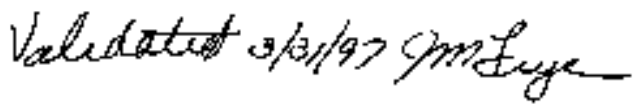

Dofo En Comant:

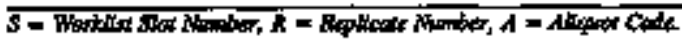

480 
HNF-SD-WM-DF-ZSB, REV 0

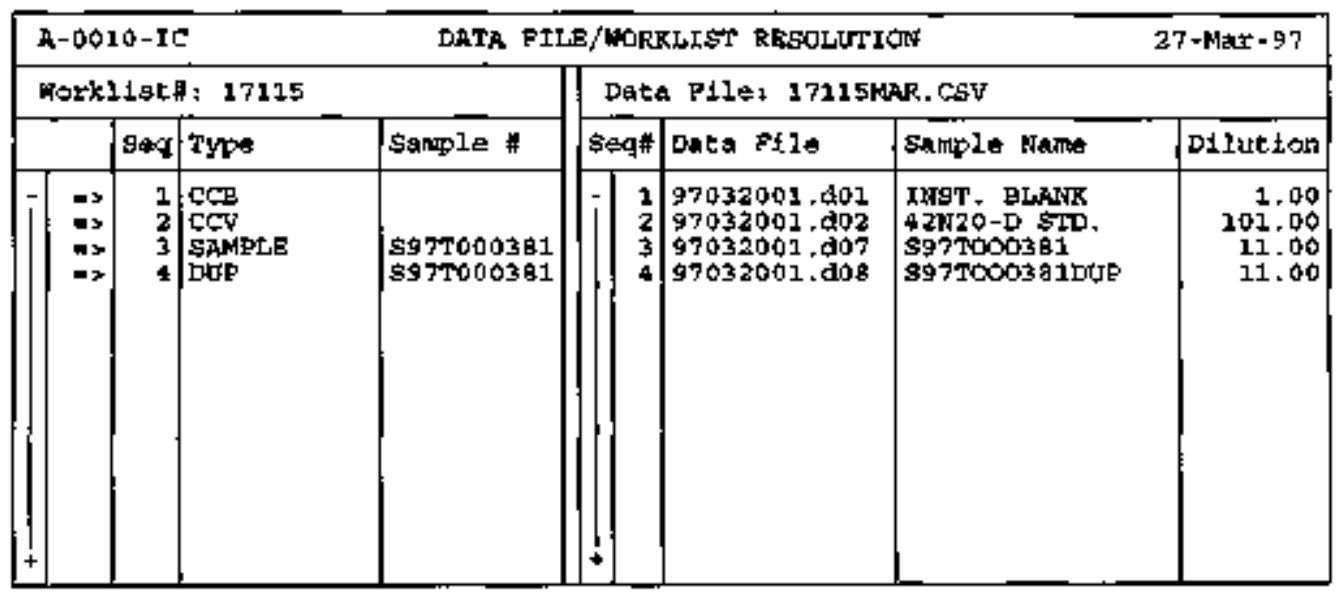

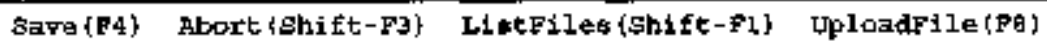

481 
Data Reproceaped On 03/24/1997 15153;17

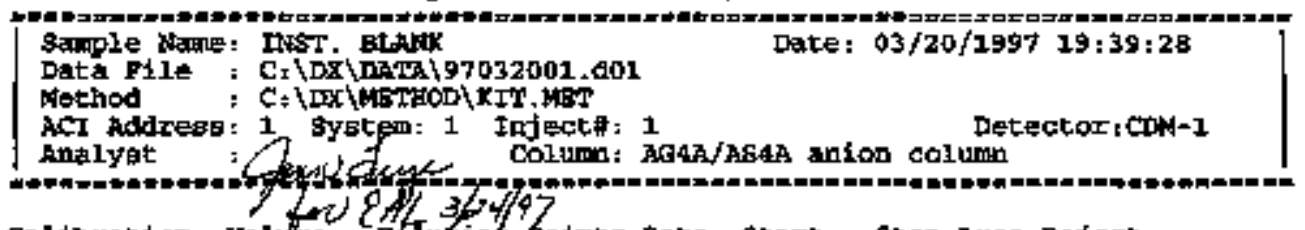
Calibration voline DITutiof foint kate start stop Area Reject

becterinal

1

13000 5Ez

0.0010 .00

50.

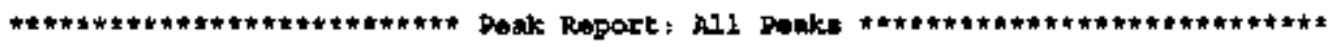

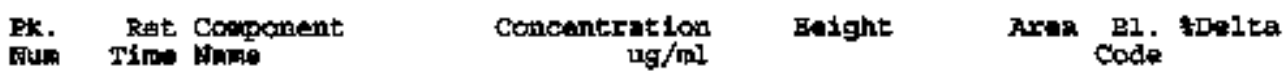

Sum Time Meno

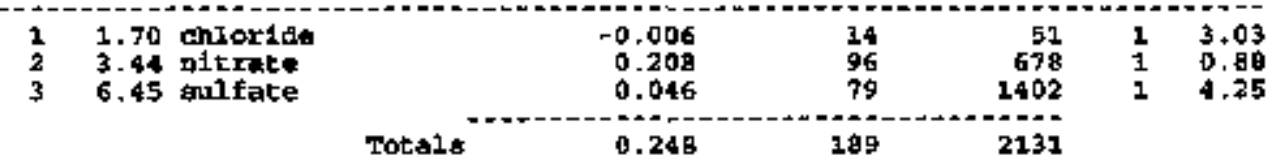

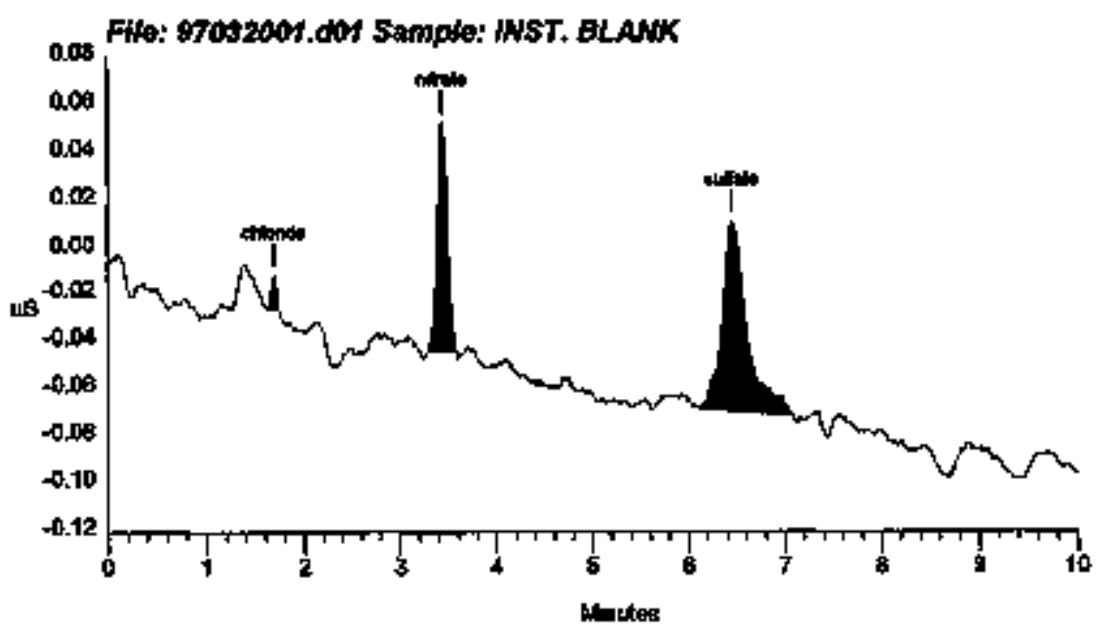

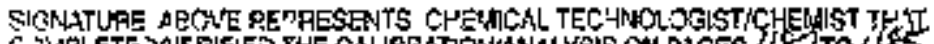

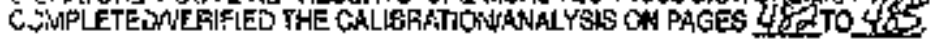


Data Reprocesued on 03/24/1997 15:53:18

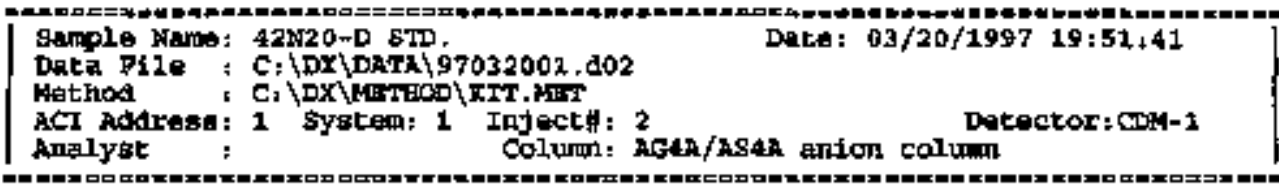

Calibration Volumb Dilution Points Rate gtart Stop Area Raject

Bxtermal $\quad 1 \quad 2013000 \mathrm{sHz} 0.00 \quad 30.00 \quad 50$

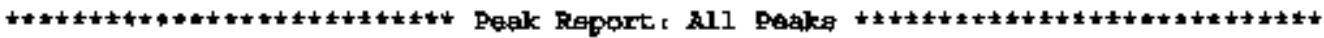

Ph. Rat Cowgorent Coocentration Feight Area Bl. tDelta

Hom Timp Naro

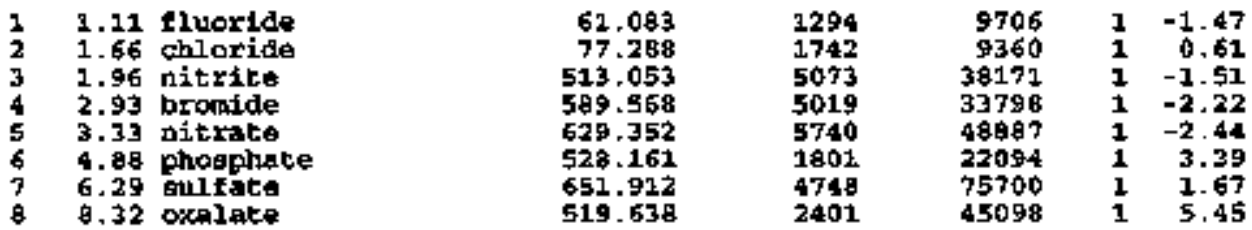

Total: $3570.055 \quad 27827 \quad 282815$

File: 9703200L.d02 Sample: 42N20-D STD.

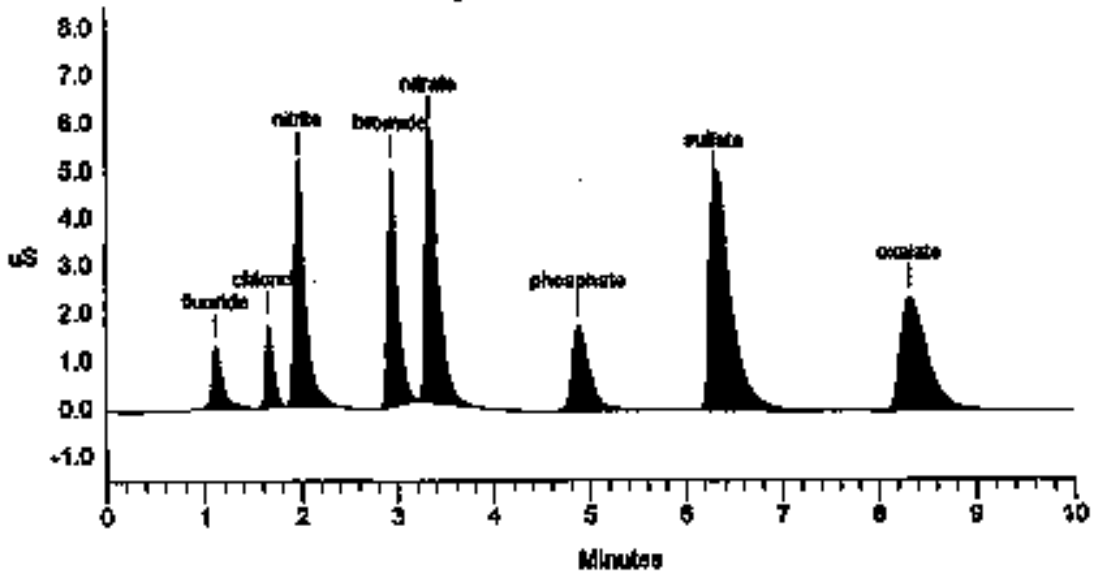


Data Reproctesed on 03/24/1997 15:53:21

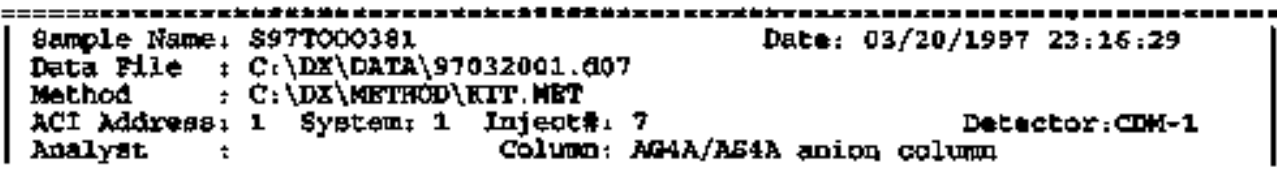

B日e

Calibration Volum Dilution points Rate start stop Area Reject

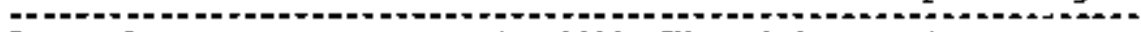

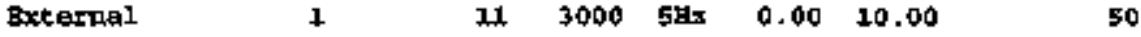

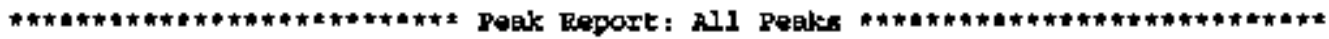

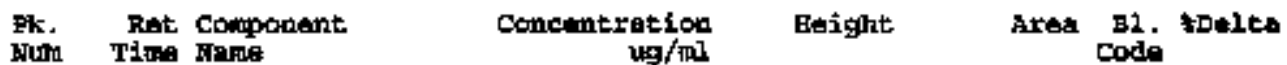

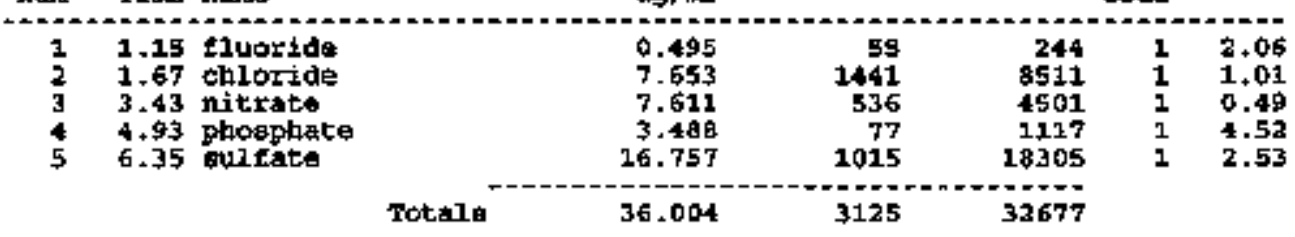

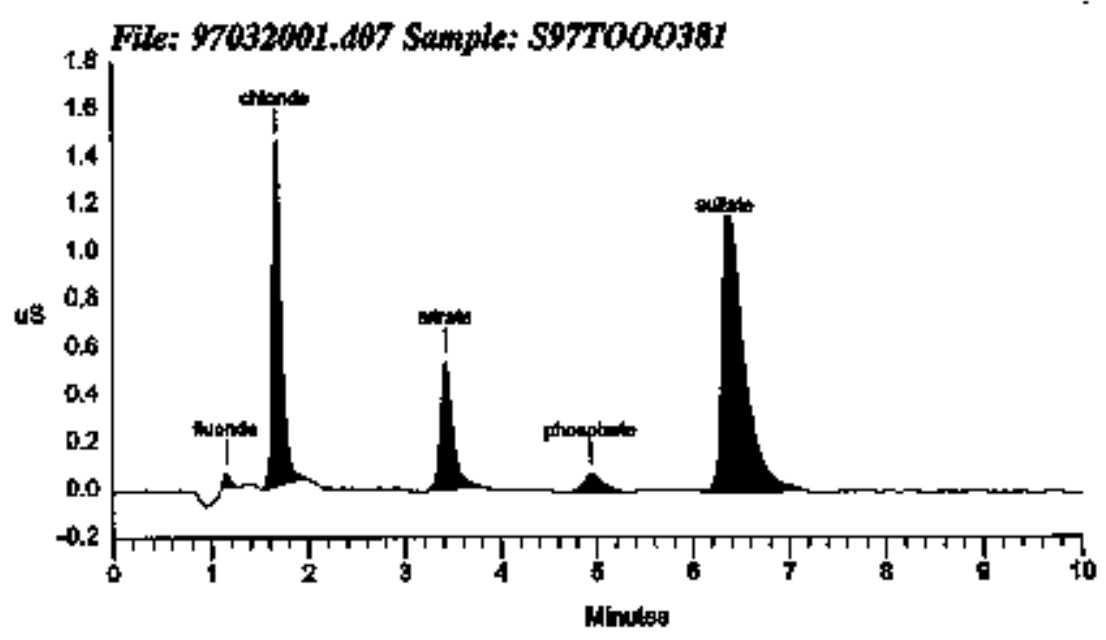


Datal Reprocersed on 03/24/1997 15:53:22

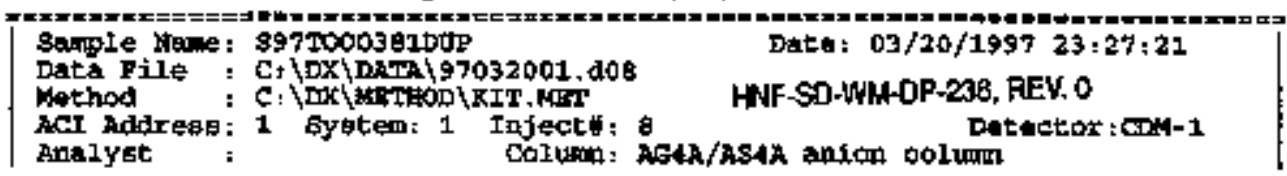

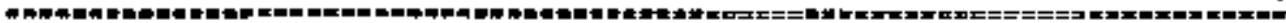

Calibration volume Dilution Points Rate start stop Area Reject

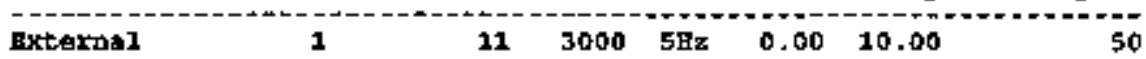

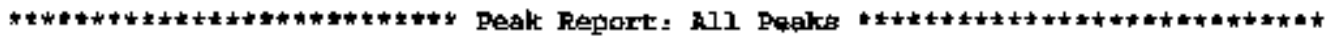

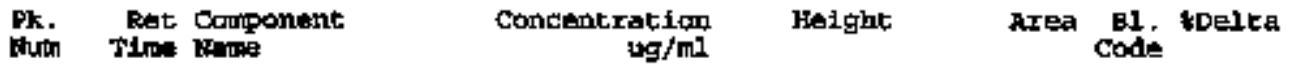
$\mathrm{Mg} / \mathrm{ml}$

$\begin{array}{ll}1 & 2.15 \text { fluoride } \\ 2 & 1.67 \\ 3 & 3.42 \text { hitrate } \\ 4 & 4.93 \text { phogphate } \\ 5 & 6.35 \text { sulfate }\end{array}$

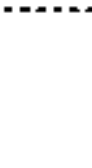

0.519

B, 147

7.094

50

1437

3.309

513

17.179

$7 \pi$

1008

36.248

3089

279

9060

1

2. 06

4129

1

1.01

Tot꾸요

$8 \quad 36.248$

1049

1

0.29

14.52

1

2. 53

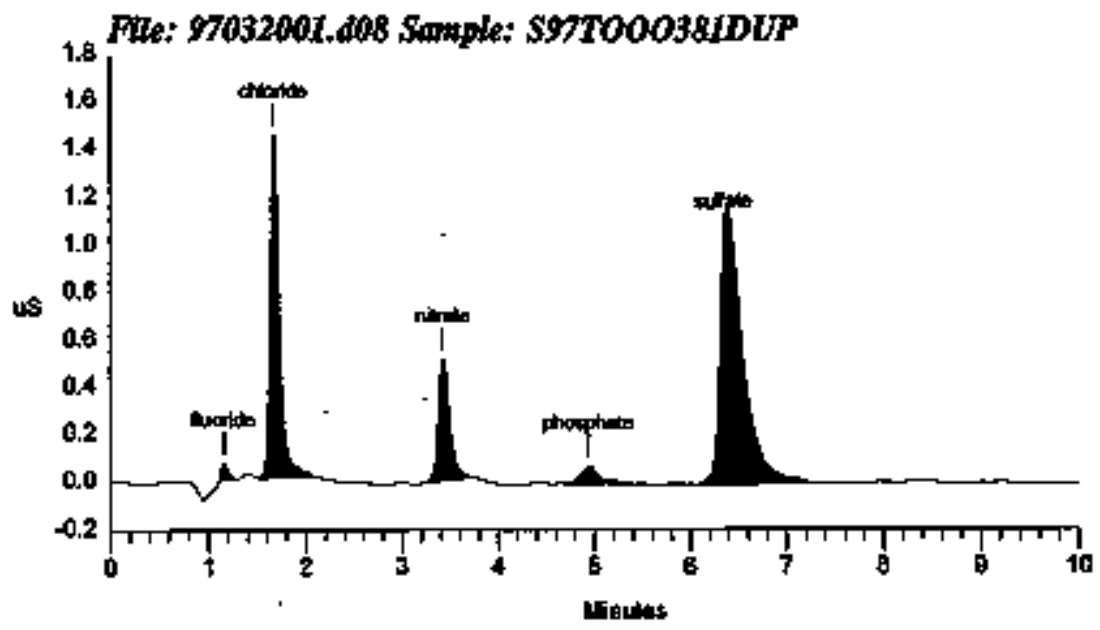




\section{LABCORE Data Eatry Template for Worklist 16639}

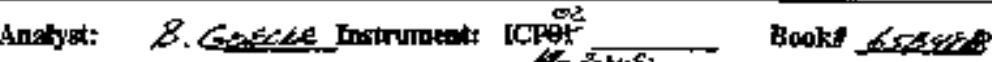

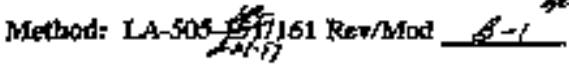

Workhst Cour dent ICP T-110 (DIRECT)

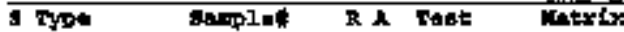

1 ter

2 ICS.

3 ITS

4 Itess

5 ICta.

6 BERDIL

7 SHAPLS

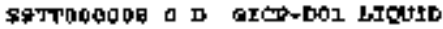

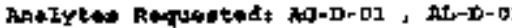

BE-D-07, BI-D-0]

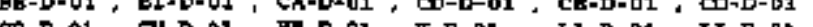

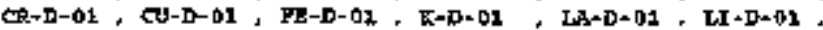

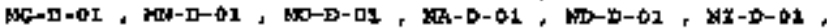

P-D-QI, PB-D-01, \$-D-01, sk-Du01, \$t-D-01, s1-D-01. SK-D-01, ER-D-01, TI-D-01, TL-D-01, U-D-dב, V-D-Q1. 20ㅇ-D-01,

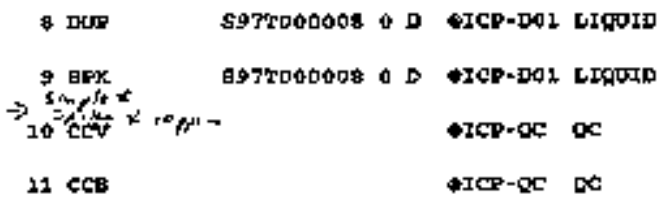

Dara Eary Conuneras:

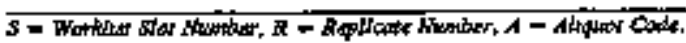


thosistis

HWF-SD-WHAD-238, REV. O

Page: 7

1.0004-1 LABCORE Data Entry Template for Worklist/ 16639

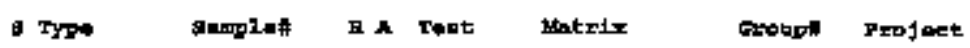

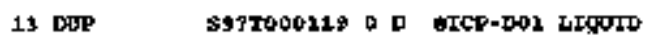

14 I멱

$15 \operatorname{ICs} A B$

16 ocv

17 대요 arep-oc a

eзcP-g; $\mathrm{CC}$

arce- $\alpha$ ac

$\operatorname{licpinaC}$ de

Final page for worklist \#16639

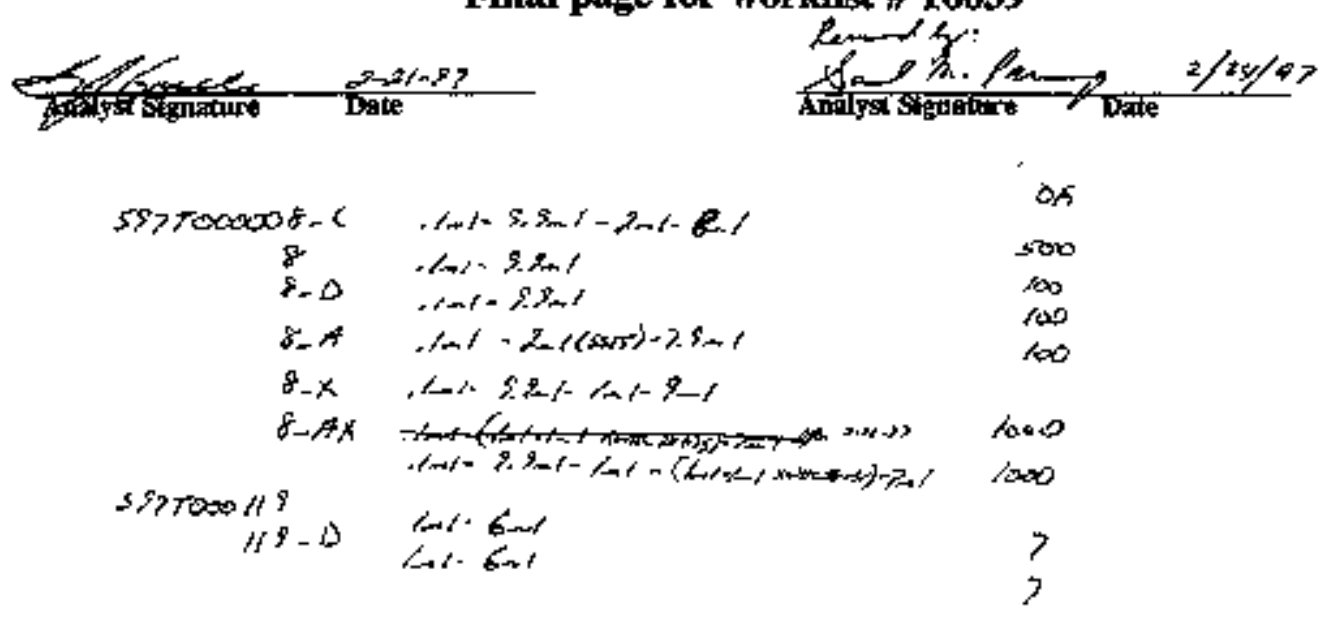

Dara Empl Conimears:

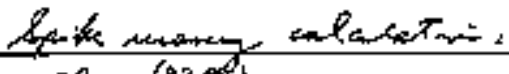

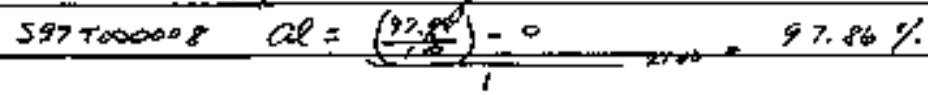

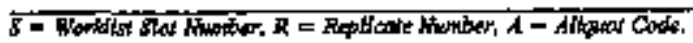

487 
HNHF-SD-WH-DP-23B, FEY. 0

forod opt apto.

$$
\begin{aligned}
& N_{a_{0}}=\frac{\left(\frac{97260}{1000}\right)-\left(\frac{34960}{1000}\right)}{10} \times 10+102.8 \% \\
& P=\frac{\left(\frac{15300}{1000}\right)-\left(\frac{5052}{1000}\right)}{10} \times 10=97.8 \%
\end{aligned}
$$

488 


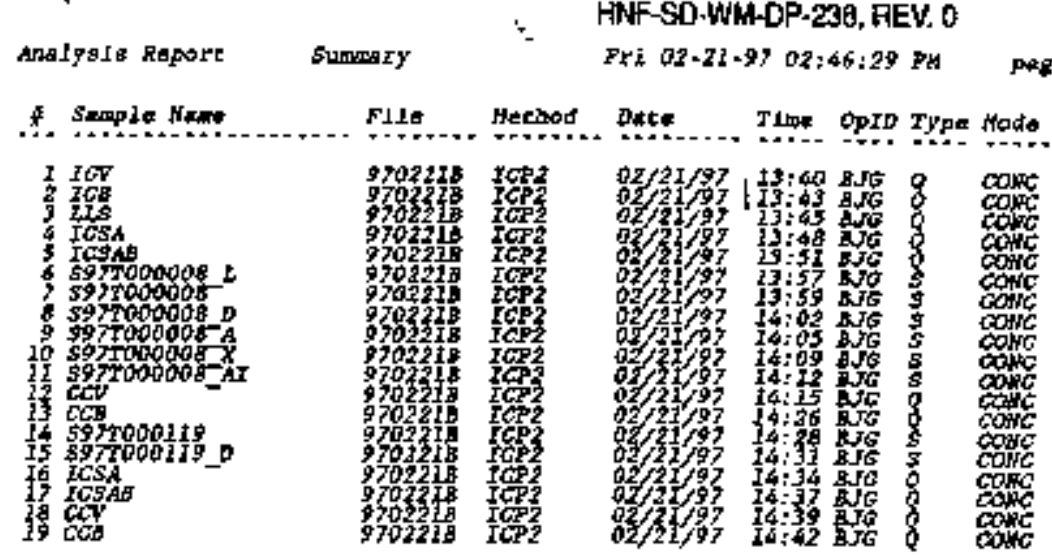

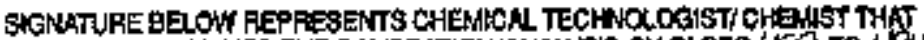

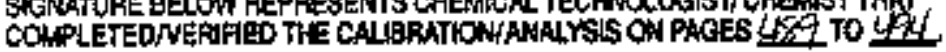

$$
\begin{aligned}
& \text { - } \\
& 7-1 N \\
& \text {, to stst }
\end{aligned}
$$

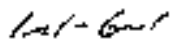

$$
\begin{aligned}
& 55>700500+119 \\
& \text { E-4R O" }
\end{aligned}
$$


Andysts Reporc

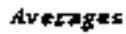

FrI $02 \cdot 21-9702: 46: 29 P W$

proge 2

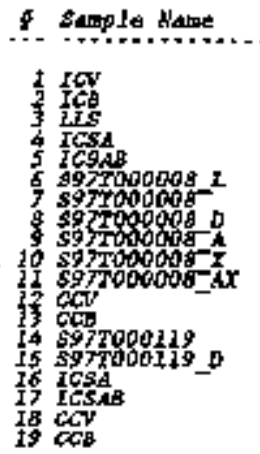

As

Al As......

B.

Es

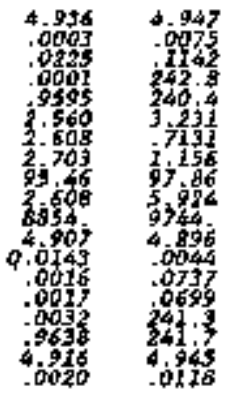

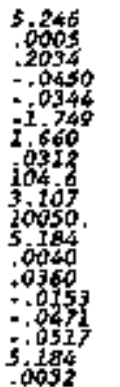

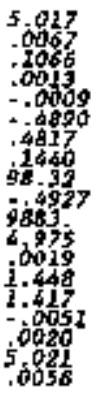

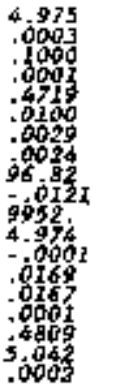

9.119

a1

ca

ad

ca.

Co

Cx

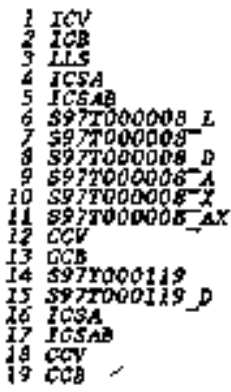

5.096

.0035

$.218 \%$

.0065

, 0035

6. 219

4.71

i. 088

1045

11, i2

3.085.

0287

: 1731

.0075

0023

5. 150

6.906

.0007

$54+2$

240.0

2.142

1.546

1.260

9404

3.039

g9.

.0016

I6. I2

If. 07

243.0

2455

5.017

.0010

- 미도

.0012

- 1350

$-0 \leq 30$

$-0213$

5.003

idora

5.062

$-.0062$

$-0022$

$-.4876$

- aoga

ogi 7

97.92

9681.

-9.523

4.970

10750

- odol

$\because 00 \%$

.0015

.9543

- dol

- 0405

4.998

5040

os

Eu

Fa

. 000

.0027

- 440

$-4692$

1 1693

و' 27

1298

9962

5.020

.000

.0081

- 128

4673

5.059

opos

cIor

apos

$-4774$

.1255

ols 8

o. 04

2096

990 .

5.047

.0002

.0018

- 0003

.472

,oy

\section{* Senpla Nama}

की

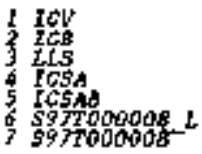

$\begin{array}{ll}5.359 & -.0089 \\ .0006 & : .0017 \\ .0213 & -.0014 \\ -.0051 & -.0298 \\ .008 & \therefore .0287 \\ .0076, & =.4591 \\ .0941 & -.0706\end{array}$

5.276
-.0003
9053
93.16
92.28
1.061
1.036

$\mathrm{r}$ . DQI3

5.009 , odo .0274 .0076

4607

34.25

34.58

133.0

35,53

9948.

4.965

$-.0029$

$-0029$

- aps?

4801

.0014

LE

4.834
0.0999
0.969
0.092
30227
316.4

5. I09

$-.0004$

. 04 ?

$\therefore 0055$

$-.0652$

$\therefore$ apgs

5.037

.0010

.0198

- 0020

1.026

-.2217
-.022 
HNF-SO-WH-EP-238, REV.O

\begin{tabular}{|c|c|c|c|c|c|c|}
\hline AnaLysig Rrgort & Averages & & Fri $02+21$ & $9702: 46$ & $29 \mathrm{PH}$ & Dage 3 \\
\hline * Samplo Mame & $C e$ & Eu & $F$ & 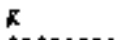 & 27 & LI \\
\hline 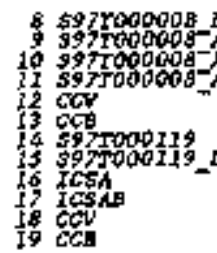 & 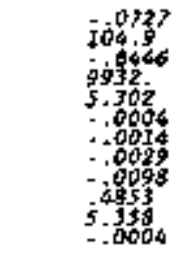 & 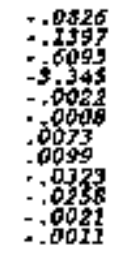 & 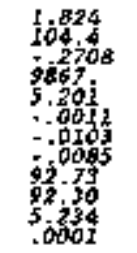 & 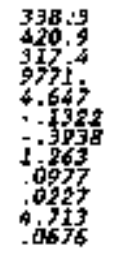 & $\begin{array}{l}0425 \\
100.8 \\
-6090 \\
10120 . \\
5.053 \\
-.0012 \\
-.0059 \\
-.0014 \\
=.0049 \\
5.128 \\
.0002\end{array}$ & 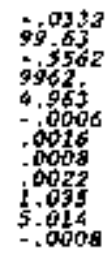 \\
\hline
\end{tabular}

\begin{tabular}{|c|c|c|c|c|c|c|c|}
\hline 5 & Sample Nas & $\mathrm{Hg}$ & An & 50 & $\boldsymbol{H a}$ & Nd & $\boldsymbol{N I}$ \\
\hline $\begin{array}{l}\frac{1}{2} \\
\frac{2}{3} \\
4 \\
5 \\
6 \\
7 \\
8 \\
5 \\
10 \\
12 \\
12 \\
13 \\
14 \\
15 \\
16 \\
17 \\
19 \\
19\end{array}$ & 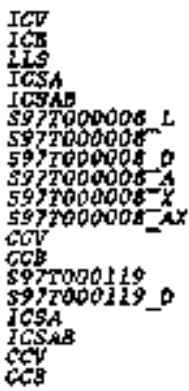 & 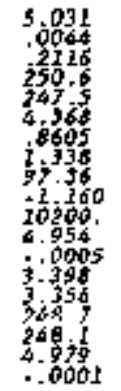 & 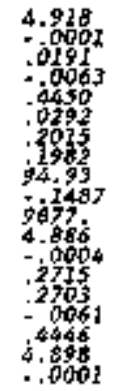 & 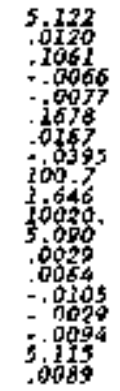 & 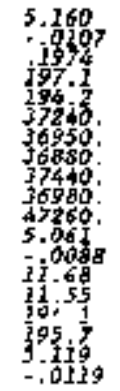 & 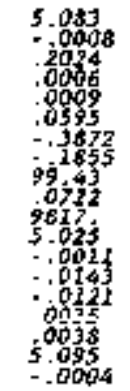 & 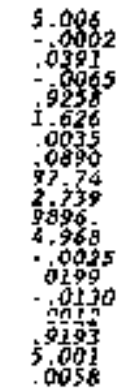 \\
\hline$\Delta$ & 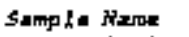 & P & Pb & $\boldsymbol{s}$ & $s b$ & $S d$ & $s_{2}$ \\
\hline $\begin{array}{r}1 \\
2 \\
3 \\
4 \\
5 \\
6 \\
7 \\
8 \\
9 \\
20 \\
12 \\
12 \\
13 \\
12 \\
15\end{array}$ & 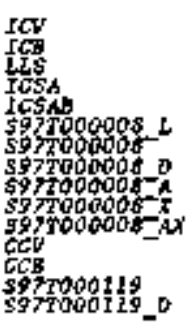 & 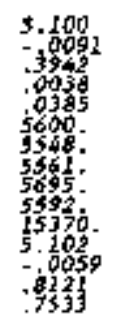 & 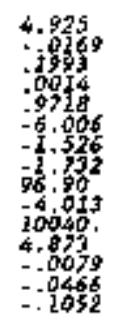 & 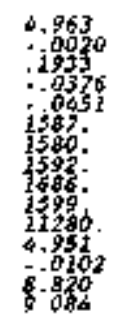 & 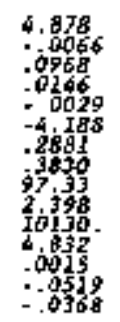 & $\begin{array}{r}4.825 \\
.0956 \\
0.2692 \\
.0090 \\
20.027 \\
6.001 \\
4.271 \\
102.0 \\
55.53 \\
9742 \\
4.753 \\
.0511 \\
3657 \\
.3787\end{array}$ & 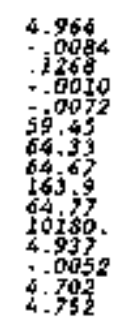 \\
\hline
\end{tabular}


HNF-SD-WMA-DP-236, REV, 0

Apalysis Regoze

Avartgats

F+1 02-21-97 02:46:29 PH

page 4

i 5omple Name

$P \quad$ PO

5b

S.

$s \downarrow$

\section{ICSA \\ 17 ICSAs \\ f ct?}

.0064
0.042
.00024

- 9052

sta

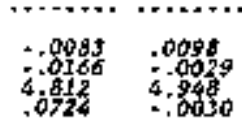

f Sanpita Name

Stro

sx

wh

$\pi 1$

.0044
-0245
.0002

TI

fr

$\frac{10 y}{2}$

$4 \mathrm{ICSA}$

5 ICSAD

squjo0000s L

7 sgivoodoos

9 sg7udoogs-

10 sq700000

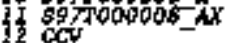

I CC

24597000129

If 597000119

IT ICSAB

to

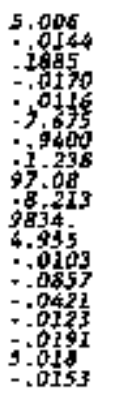

5.020

- dros

.0020

. ooro

.0027

- 0405

9.15

$-0965$

ؤे 1

4.952

-oóa

.

. o7t

.0020

5.024

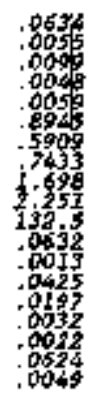

4.092

4.899

- odor

- 0305

.0200

.0003

ond

$-0072$

. 0045

$-2313$

- - 0 憵

9.1s

5206

4.993

$\therefore .0007$

$-.0049$

.0065

.0008

4. 9005

-. door

.14 .05

$-3.155$

$-7.541$

93.84

. 343

g⿺⿻一𠃋十

4.843

- .0062

.0276

ista

. 058

4027

1. otmpla Nana

$\boldsymbol{v}$

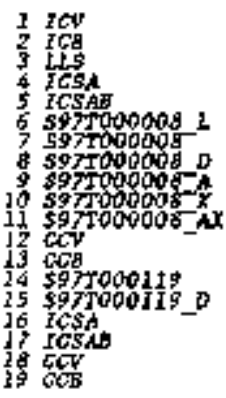

5. 115

-.0027

ogt

- 0034

.4671

$: 1.439$

$-2358$

in 198

100.2

$-2,354$

9928

5. 057

$Y$

Iת

Zr

$-.021$

- ajus

- odza

4665

5.097

\section{.0053}

- oong

- oods

.0063

.006s

$\therefore 783$

$-.0763$

$-0605$

.0306

76866

Ii 4 I

cojs

- oges

- 0043

- .00j2

. 065

.0062

- 055

5. 121

opo

do 50

.950

.3979

.1371

it68

IPI:

984 2

5. 073

- Dopl

. 452

- 4475

- Dón

,9495

5.044

. odjo

\subsection{5}

- .0025

OII7

- 0050

$=0050$

- 21 自

$-2658$

98. 57

1.524

99.2.

- 0or

$-.0104$

.0133

- 0047

$\therefore$. 0र5

5. 27

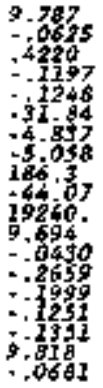


IMF-SO-WH-DP.238, REY， D

\section{THIS PAGE IETERTIONALEY LFTT BLANK}

\section{3}




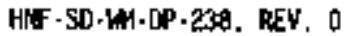

THIS PAFE IKTEMTIONALY LEF BLNK 


\section{LABCORE Completed Worklist Report for Worklist\# 16990}

Amalyst: slh

Ingtranant: CARB

Method:

Boole\# $\frac{25 N 12 A}{2+N 52 A}$

Workilst Comment: T-110 \& 2706T (S9TTPOA015) ETICTOC1

Seg TJpe Serpat

Test

1. Bax

1 IIX

2 क्षात

2 sका

3 arde

3 aupl

i wo

4 Bo

5.

5 t)

6 swpt:

(1) vovte

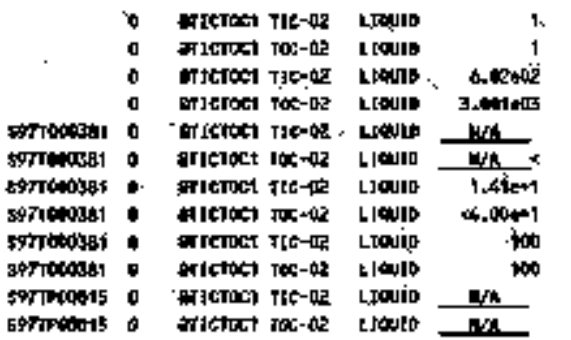

Final page for worklist/ 16900

\subsection{0 \\ s,o6it with}

1. 1. 1

$6,1+1,2$

te.exta

tikte-1

1240in 1 ,

$\infty .+1$

100. 1

4.3.3

z,

6. $69+1$
$5.00 \%+1$

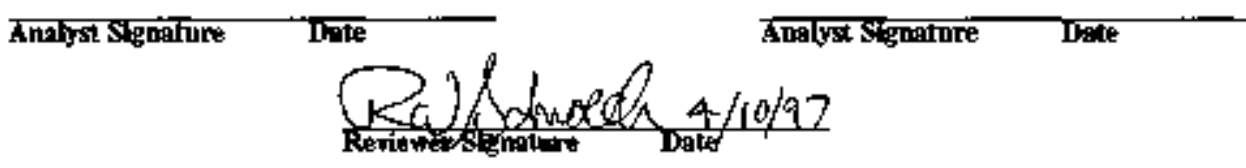

0.100 rovic

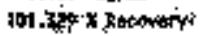

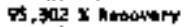

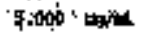

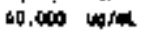

trost ap

NPQ

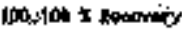

R.50A $x$ Reconery

5009 vist.

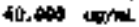




\section{LABCORE Data Entry Template for Worklist 16990}

Anilyg:

Instrumeit: CARB2

Boolk:

Methat: LA-342-100 Reyrkod

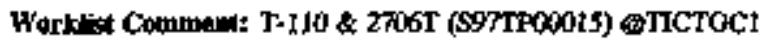

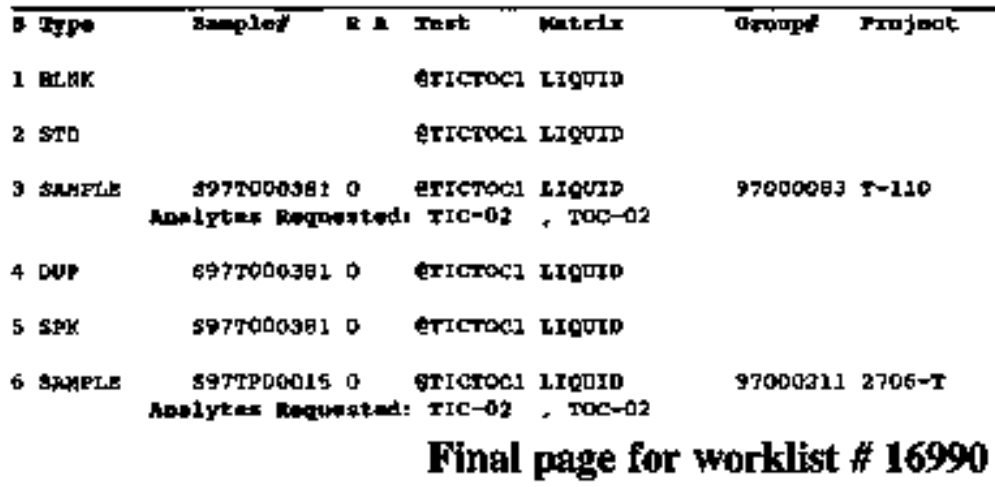

Anglyfis Signature

Date

Anthot 8anahre

Late

Dam Eniry Cammonts:

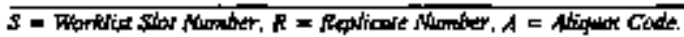




\section{LABCORE Data Entry Template for Worklist\# 1699}

Andint:

Method: LA-342-100 Row/Mod E-O

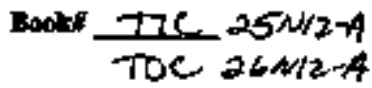

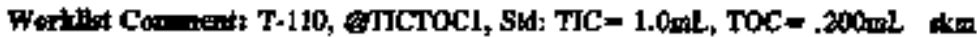

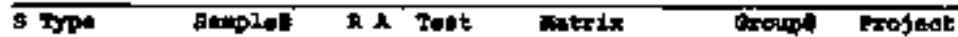

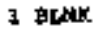

ArICTOC2 LETrig

2 䄱

MICTOC1 LrOUID

3 Shista

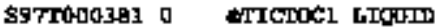

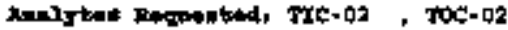

97000093 T-110

1 DUP

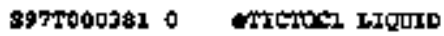

\section{Final page for worklist 16990}

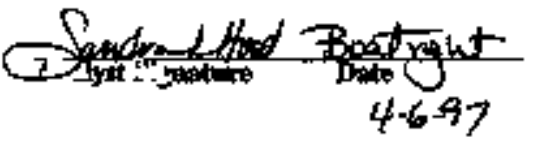

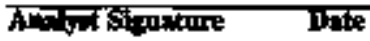

$4-6.97$

$\boldsymbol{1}$

;

Date Eutry Cannests:
Basts $4 / b / a 19$
*
1.2L 4
$\rightarrow \geq 017$
acted tis the $\omega L$

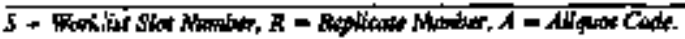


HNF-SD-WM-DP-238, FEV D

TIC- TOTAL INORCANIC CARBON ANALYSIS REPORT TICTOC REP 2.0

gamplet ETD 2

Sample size = 1000 th

Dil Factor $=1$

Blank ID *

Blank Valut = $=+28$ ug $/$ minute C
Date: $04 / 05 / 97$

+ Re

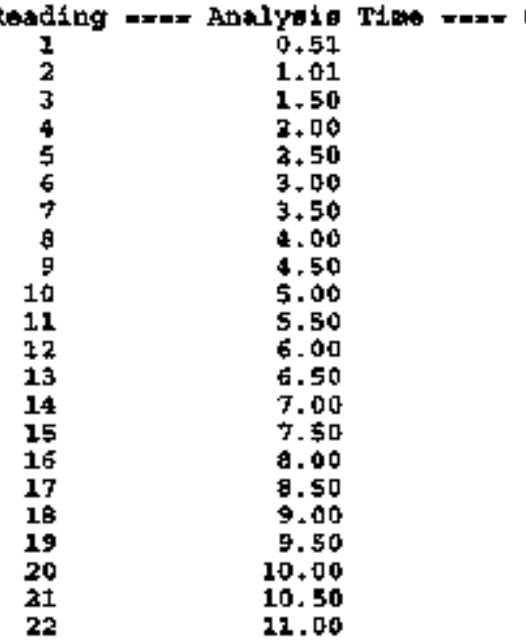

Coulatater =cxx *

$$
\begin{array}{r}
0.20 \\
0.50
\end{array}
$$

35,90

$195+10$

36.70

499,30

$552+70$

$582+30$

598.30

604.30

607.60

$\$ 0 \mathrm{~B} .90$

609.60

610.20

610.70

611.00

611.40

611.90

612.10

512,30

612.70

613.00
Timet 22,18,16

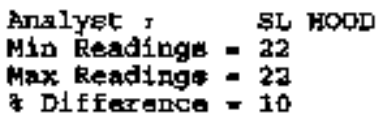

USBk IAPUT Btark VATS

BLAlKK VALUB = 3.079863 micrograms carbon

BLA19R FACTOR - 3, $379863 / 10.99951=+3.8 B-01$ ug/min Carton

BAMLLR RESULTS:

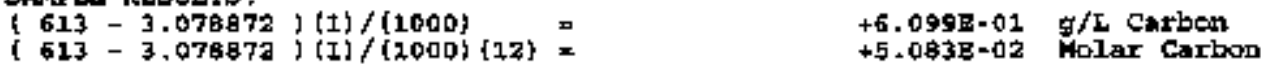

0.00

50.00

98.51

01.60

47,08

24.65

11.47

5,08

2.67

0.99

0.54

0.21

$0+11$

1. .10

0.00

0.05

0.07

0. 0.08

0.03

0.03

0.07

0.05

SGGNATURE BELOW REPRESENTS CHZMCNL TECHNCLOGHT/CHENUST THAT

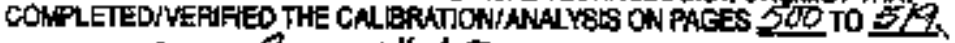
Sample Run By;

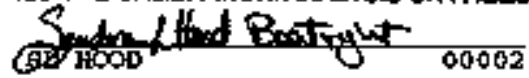


HNF-SD-WH-DP-238, REV. 0

TOC- TOTAL OROANIC CARBCO ATALYSIS RBPORT

TICTOC REV 2.0

Sanple: Sxt 2

Sample size $=200 \mathrm{uL}$

Dil 1 Factor $=1$

Blank ID \# =

Blank Value $=3,57 \mathrm{ug} /$ mimute $c$
Date : $04 / 05 / 97$

Time: $22 \div 33: 36$

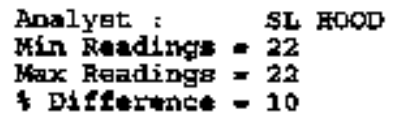

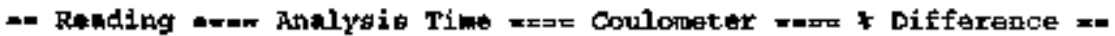

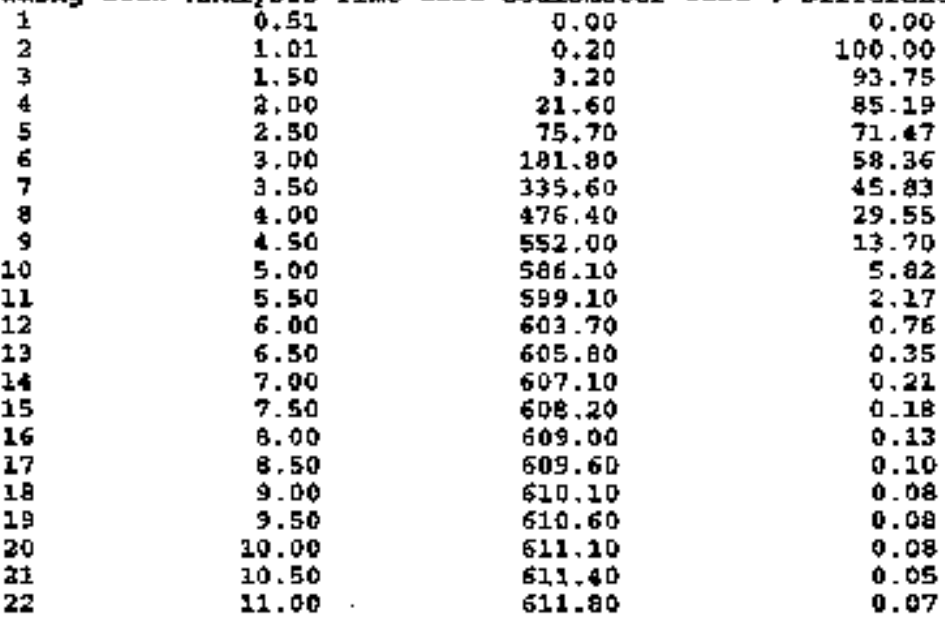

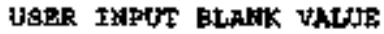

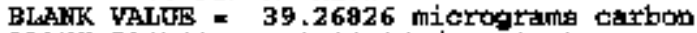

BIANK EACIOR = 39,26826/70+99951 =

+3.6E+00 ug/min cartorn

SFMPLE RESUTI' :

$\left\{\begin{array}{l}611.8-39.25562\}\{1\} /(200\}, 0)= \\ 611.0=39.25552\}(1) /(200)(12)=\end{array}\right.$

$+2.853 \mathrm{E}+00$

$+3+386 \mathrm{~B}-0 \mathrm{~d}$

gid/L Carbon

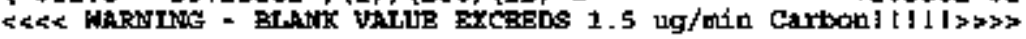

Nolar Carbon

$2 \cos$

Sample Run By

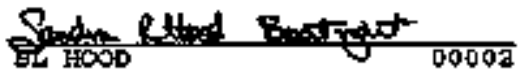




\section{HWF-SD-NAN-DP-238, REV, O}

TIC- TOYAL IFORGANIC CARBON ANALYBIS REPORT

TICTOC REV 3.0

<द BILANR AKALYBIS 3 s?

Bample: AsSE 2

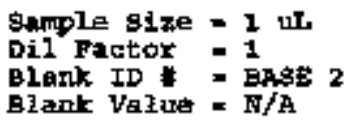

Timet $27,11,43$

$$
\begin{aligned}
& \text { Analyet, su Hoto } \\
& \text { Min Readingé a } 22 \\
& \text { Max Readiags }=22 \\
& \text { \& Differtance }=10
\end{aligned}
$$

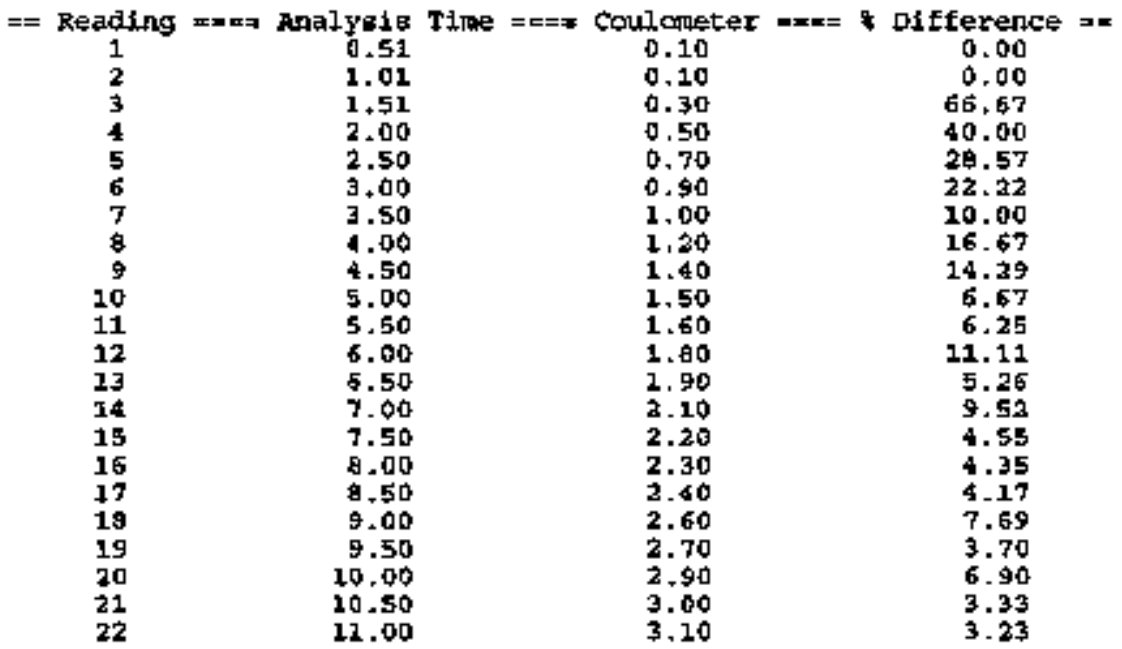

BLAKT VALUIE - 3,1 mieYograme cartoin

BLABTK FACTOR = $3.1 / 10+99609$ a

+2. 8R-01 ug/wan Carbon

gample Run BY:

$\overline{\mathbf{S E} 5000-70002}$




\section{HNF-SD-WH-DP-238, REV. 0}

TOC- TOTAI ORGRNIC CARBON NMALYAIE REPORT

TICTOC REP 2.0

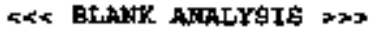

Samplex RAsE 2

tample size = 7 uL

Di1 Bactor = 1

Blank ID " DAsE 2

Blatk valug $=\mathrm{N} / \mathrm{s}$
Date: $04 / 05 / 97$
Tine: $21: 58 \div 50$

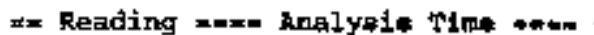

$\begin{array}{rr}1 & 0.51 \\ 2 & 1.01 \\ 3 & 1.50 \\ 4 & 2.00 \\ 5 & 2.50 \\ 5 & 3.01 \\ 7 & 3.50 \\ 8 & 4.00 \\ 9 & 4.50 \\ 10 & 5.00 \\ 11 & 5.50 \\ 12 & 5.00 \\ 13 & 5.50 \\ 14 & 7.00 \\ 15 & 7.50 \\ 16 & 8.00 \\ 17 & 8.50 \\ 18 & 9.00 \\ 19 & 9.50 \\ 30 & 10.00 \\ 21 & 10.50 \\ 23 & 11.00\end{array}$

coulometer 0.00

0.20

2.50

7.60

15.50

23.30

as, 50

31.40

33.10

34.70

35.060

Anulytt : st thoob

Min Readinge = 22

Max Roadinge = A2

- Difference - 10

35,50

36.00

36.50

37.00

37,30

37.70

38,10

38.40

38.70

38.90

39.30

- Difference =a

$100+00$

92.00

67.21

50.97

33,48

$18+53$

8.93

5,14

$2+\$ 3$

2.57

1. 69

$1+11$

$1+37$

1,35

0.80

1.06

1. .05

0.76

0.78

0. 51

1.02

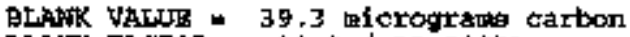

BLABTK FACTOR = $39.3 / 10+99951=$

$+3.57 \mathrm{E}+00 \mathrm{ug} / \mathrm{mL}$ ( Carkon

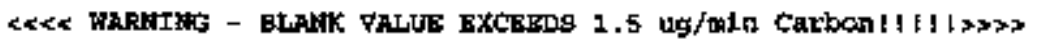

Saupl. Run By:

占 
HNE-SD-WH-DP-29R, REV. O

TIC- TOEAL IHORGARIS CARBOM ARALYGIS FRPORT

TICLDC REV 3.0

Gample: BIR $\mathbf{2}$

Sampla size = 1 ut

Dil Factor $=1$

BIank TD $\$=$

Blable Value - .2A ugfoinute c
Time; $22: 47: 25$

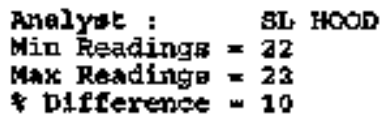

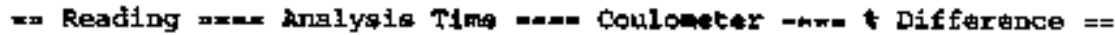

$\begin{array}{rrrr}1 & 0.51 & 0.20 & 0.00 \\ 2 & 1.00 & 0.60 & 66.67 \\ 3 & 1.50 & 1.70 & 54.71 \\ 4 & 2.00 & 2.60 & 34.62 \\ 5 & 2.50 & 3.20 & 18.75 \\ 6 & 3.00 & 3.60 & 13.11 \\ 7 & 3.50 & 3.90 & 7.69 \\ 3 & 4.00 & 4.20 & 7.14 \\ 9 & 4.50 & 1.70 & 10.64 \\ 10 & 5.00 & 5.00 & 6.00 \\ 11 & 5.50 & 5.20 & 3.85 \\ 12 & 6.00 & 5.40 & 3.70 \\ 13 & 6.50 & 5.70 & 5.26 \\ 14 & 7.00 & 5.00 & 5.00 \\ 15 & 7.50 & 6.30 & 4.76 \\ 15 & 9.00 & 6.50 & 3.08 \\ 17 & 1.50 & 6.80 & 4.41 \\ 18 & 9.00 & 7.10 & 4.23 \\ 19 & 9.50 & 7.40 & 4.05 \\ 20 & 10.00 & 7.60 & 2.63 \\ 21 & 10.50 & 7.90 & 3.60 \\ 22 & 11.00 & 8.10 & 2.47\end{array}$

DGER INPGT BLAMK YALUE

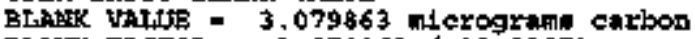

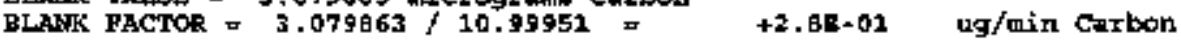

GARPLE RBSULTS:

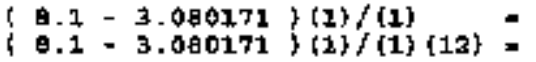

$+5.08+\square 0$

g/t Carbon

44.35-01 Molar Carbon

Sanple Rum Ey I

BL HOOD $\quad 00002$


Bantole: BLR 2

Dete: $04 / 05 / 97$

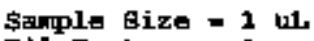

Dil Factor $=1$

Blank ID =

Blank value $=3.57 \mathrm{ug} /$ niduut: C
Time: $23 \div 01: 35$

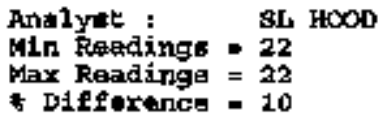

-

Read
1
2
3
4
5
6
7
8
9
10
11
12
13
14
15
16
17
18
19
20
11
22

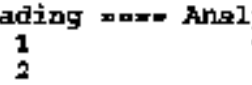

3

4

5

7

8

9

10

11

12

13

14

15

16

17

18

19

20

21

\subsection{1}

1,01

1.50

2.00

2. 50

3.00

3.50

4.00

4.50

5.00

5.50

5.00

6.50

7.00

7.50

a. 00

B. 50

9,00

$\$ .50$

10.00

1. 5.50

11. 00
Conzlamater

$$
0.20
$$

D. 70

5.20

14.20

22.50

27.50

31.00

32.60

37.80

34.40

35.00

35.50

35.90

36.40

36.90

37.20

37.60

30.10

30.20

38.60

38.90

39.20
Difference 0.00

71,43

86.54

$63+38$

34.69

18.18

11.29

4.91

3.55

1.74

1.71

1.11

1.11

1.37

t.35

0,81

$1+06$

1.31

0.26

1.04

0.77

0.77

\section{USER IAPUT BLAUKK VALUES}

BLAKK VALUB - $39.26826 \mathrm{mi}$ exograms carbon

BLAJKR FACTOR $=39.26826 / 10.99951=$

$+3.6 \mathrm{E}+00$ ug/tain sarton

BATPLB RESULTS:

$\left.\begin{array}{l}(39 . a-39.26564 \\ (39.2-39.26564\end{array}\right\}(1) /(1)(1)\{12\}=$

\& $5.00 \mathrm{E}-3 \mathrm{~g} / \mathrm{L}$ Carkon

(39.2 - 39.26564$\}\{1\} /\{1\}\{22\}$.

4.17 But Molar Carbon

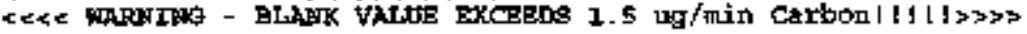


HLئA-SD-WW-DP-2S3, REV. 0

TIC- TOTAL IWORGAMTC CAREON AMALYGIS REPORT THCTOC REV 2.0

Sample: s9tTodzal

Sample slze - 1 uL

Dil Factar $=1$

Blank ID \# -

Blank value $=.28$ ug/minute 5
Date: 04/05/97
Tint: $23: 17: 48$

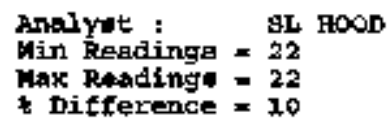

Aralyut : \$L FOOD

Max Roading $=22$

bifference $=10$

= Reading man Analysil Time man Conloneter

$\begin{array}{rr}1 & 0.51 \\ 2 & 1.01 \\ 3 & 1.51 \\ 4 & 2.00 \\ 5 & 2.50 \\ 6 & 7.00 \\ 7 & 3.50 \\ 8 & 4.00 \\ 9 & 4.50 \\ 10 & 5.00 \\ 11 & 5.50 \\ 12 & 6.00 \\ 13 & 6.50 \\ 14 & 7.00 \\ 15 & 7.50 \\ 16 & 8.00 \\ 17 & 5.50 \\ 18 & 9.00 \\ 19 & 9.50 \\ 20 & 10.00 \\ 21 & 10.50 \\ 22 & 11.00\end{array}$

0.30

0.80

2.50

5.60

9.10

10.90

12,30

13.20

13.10

14.00

14.40

$14+70$

15.00

15.30

15.50

15.80

16.10

16.10

26.40

16.60

16.80

17.20

\section{Differense -}

क. 0 D

62,50

68,00

55.35

3月. 46

16.51

11. 3 후

6.82

$4 .+5$

1.43

2.78

2. 04

2. 00

1.96

1. 29

1.90

1.86

0.00

1.83

2.20

1.19

2, 33

USEER IFATUT BLRWTK VALTE

BEALIR VALUB - 3.079963 microgramé caction BLAYH BACTOR = 3.079B63/10,99951 a +2, \$E-01 ug/oin Carton SAMPLB RRSULTS:

$\left\{\begin{array}{l}17.2-3.079419 \\ 17.2=3.079419\end{array}\{1\} /\{1\}\right.$ (1) (12) $=$

$+1.41 \mathrm{~B}+01$

$+1.18 B+00$

g/t Carton Molar carbon

SArQle Run "By: 
Barmit: $597 T 0381$

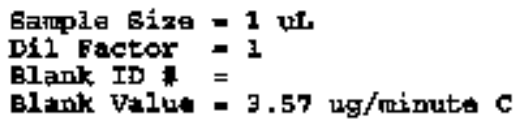

iginata $C$

Dated : $04 / 05 / 97$
Time: $23: 30: 35$

$$
\text { Analyat : BL HCOD }
$$

Min Readinge $=22$

Max Reading = $\mathbf{2 2}$

- Diffuronc* $=10$

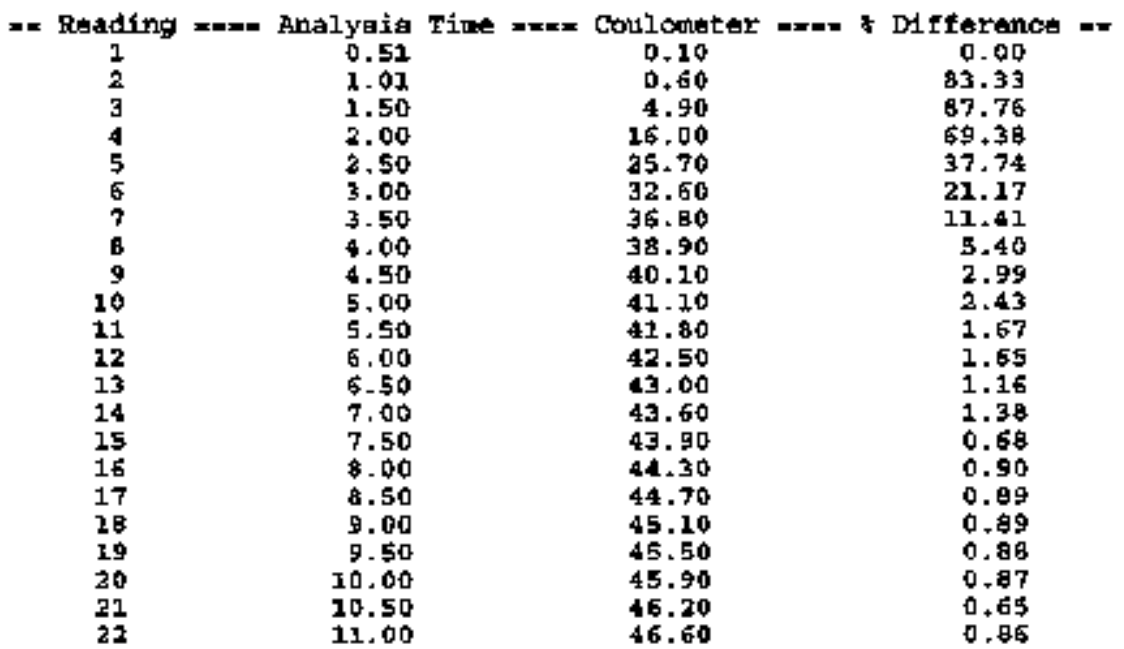

DGER IHPUT BLAKK VAETR

BIJNR VALUE * 39,26B26 mLerograng cartbon

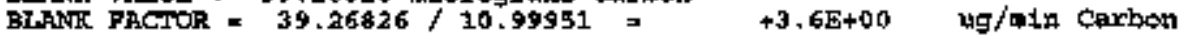

SAMPER RESTLTS:

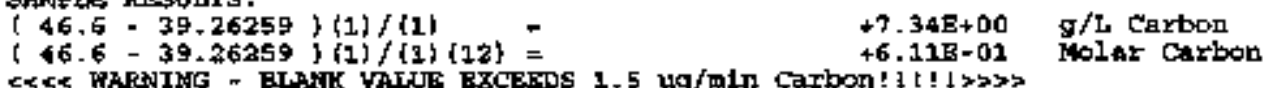

St

Sangle kun By:

SL HOOD 


\section{HNG,SD-WHAP-238, REV, O}

TIC- TOTAL IHORGANIC CARBON ARTAYYIS REPORT

TICTDC EEV $2+0$

gample: S97T0381 DJP

g*tul* giz* - 1 uL

Dil Factor $=1$

Blank ID $\approx$

Blonk Valut $m, 28$ ugforinute $C$
Date: 04/05/97

Time: $23: 43: 27$
Analyst : st 1000

Min Rendings : 22

Max Repdings = 22

t Diffarence $=10$

UȘER TWPLT BLABK VALUR

BLANK VALUK - 3.079863 nilerograms exrben

BANNK PACTOR - 3.079863/10.99951 = +2, 6E-01 ug/min Carbon

SAMpL Resulas:

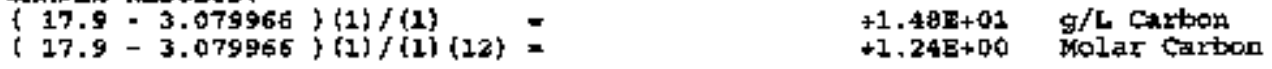

Sanple Run By:

SE $\$ 0 O D$ 
TOC- TUTAL ORGAMIC CARBCO AHALYSIS REPORT TICTOC RFV 2.0

Sample: s97T0381 DUP

Sanple size $=7$ ur

Dil Pactor $=1$

Blank ID \# =

Blank Value $=3.57 \mathrm{ug} / \mathrm{qu}$ nute $\mathrm{C}$
Datet $04 / 05 / 97$

$=\mathbf{n}$

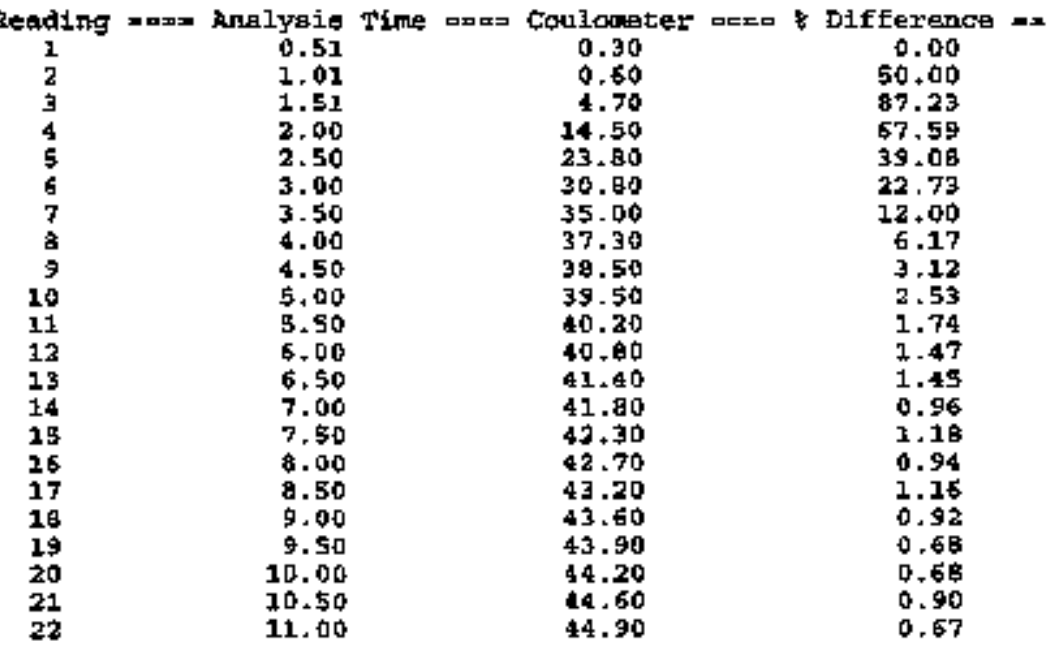

USER IRPOT BLANK VNUIE

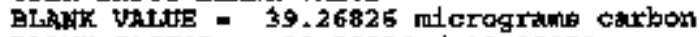

BLALEK FACTOR - $39.26826 / 10.99951 \times$

+3, 6E+0d ug/min Carton

SAMPIL RESULTS t

$\{44.9=39.25911\}\{1\} /(1)=$

$+5.64 E+00$

$+4,70 B^{4} 01$

g/L Carbora

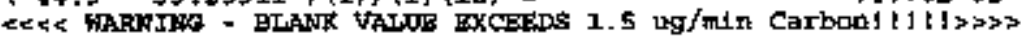

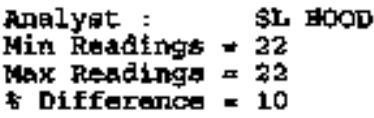


WHFSDWH-DP-2OB, REV D

TOC- TOTAL ORHANIC CARBON NIREYSIS REPOET

TICTOC REV 2.0

Sapple: 597T0381 SPK

getpl* sizt = $1 \mathrm{w}$

Dil Factor $=1$

Blarik ID * $x$

Blank valub $=3.57 \mathrm{ug} / \mathrm{minat}=\mathrm{C}$
Dace: $04 / 06 / 97$

Timet $01: 19+39$

\begin{tabular}{|c|c|c|c|c|}
\hline $\begin{array}{c}\text { Redifig } \\
1 \\
2 \\
3 \\
4 \\
5 \\
6 \\
7 \\
8 \\
9 \\
10 \\
11 \\
12 \\
13 \\
14 \\
15 \\
16 \\
17 \\
19 \\
19 \\
20 \\
21 \\
22\end{array}$ & $\begin{array}{r}\text { x=malysi } \\
0.51 \\
1.01 \\
1.51 \\
2.01 \\
2.50 \\
3.00 \\
3.50 \\
4.00 \\
4.50 \\
5.00 \\
5.50 \\
5.00 \\
5.50 \\
7.00 \\
7.50 \\
5.00 \\
8.50 \\
9.00 \\
9.50 \\
10.00 \\
10.50 \\
17.00\end{array}$ & Tife & $\begin{array}{c}=c \text { coulcueter } \\
0.00 \\
0.50 \\
5.10 \\
45.10 \\
129.60 \\
218.10 \\
275.80 \\
304.60 \\
319.90 \\
325.00 \\
337.70 \\
329.50 \\
330.70 \\
331.40 \\
332.10 \\
333.60 \\
333.30 \\
333.60 \\
334.00 \\
334.60 \\
335.10 \\
335.40\end{array}$ & $\begin{array}{c}\text { Deference } \\
0.00 \\
100.00 \\
93.65 \\
84.92 \\
64.93 \\
41.04 \\
20.92 \\
9.51 \\
4.72 \\
1.57 \\
0.82 \\
0.55 \\
0.36 \\
0.21 \\
0.21 \\
0.15 \\
0.21 \\
0.09 \\
0.12 \\
0.18 \\
0.15 \\
0.09\end{array}$ \\
\hline
\end{tabular}

USER IAPUT ELFNK YALDE

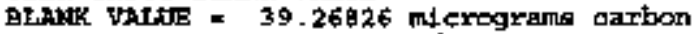

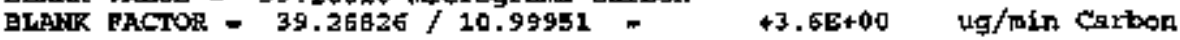

SHMLE FROULTS

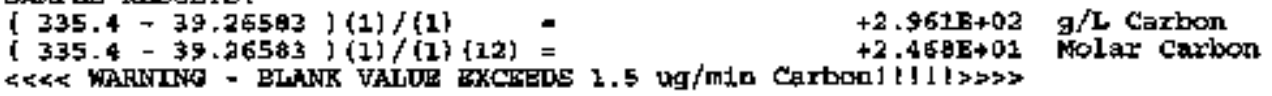

Analyat : SL Hopp

Reatinga - 22

Max keadinga - 22

a Difference = 10

Coulcometer man of toference =-

0.50

45.10

220.60

304.80

325.00

327.70

330.70

331.40

332.10

$33 a+60$

333.30

333,60

$334+00$

335.10

0.09 
TIC- TUTAL IWORGANYC CRRBOA ARALYSIS REPORT TICTOC REP 2.0

Banple: 697T0381 BPK Date: 04/06/97

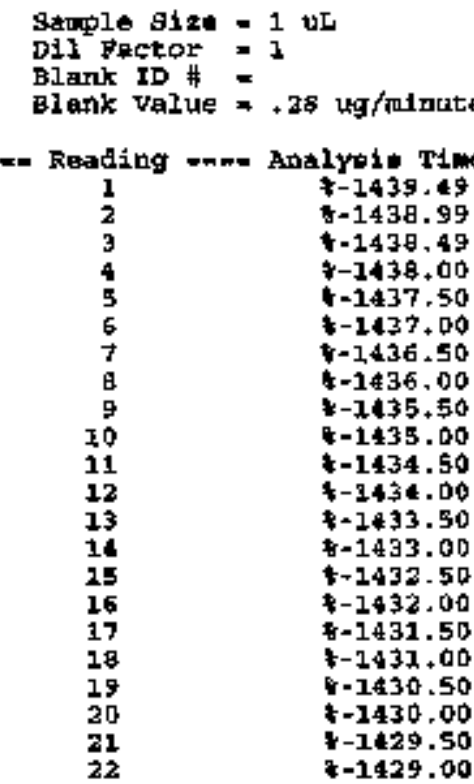

\author{
Analyot : $\quad$ GL HOOD \\ Min Readings $=22$ \\ Hax Rodilingt - 22 \\ Differepince $=10$
}

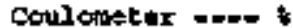

$$
0+\infty
$$

0,40

14.20

$90 \%+90$

181.10

244.20

280.20

298.40

307.50

311.70

313.60

$\$ 14.60$

315.30

315.70

316.70

$\$ 16.40$

316,00

$\$ 13.10$

313.40

317.80

$31 \mathrm{a} .10$

328.40

\section{Difterenct --}

0.00

100.00

97.18

6. 38

49.91

25,84

13. B5

6.10

2. 96

1.35

0.61

$0 . \$ 32$

0.22

0.13

0.23

0.09

0.13

0.09

0.09

0.13

0.09

0.09

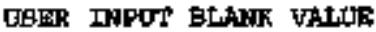

BIARR VALUE = 3.479663 micrograms cartbon

BLAMR PACTOR = 3.079863/10.99951 =

+2. $\mathrm{AB}-0 \mathrm{~L}$ ug/nin Carbon

SAMPLE RBELETS,

$\left\{\begin{array}{l}318.4-400.1200 \\ 318.4--400.1208\end{array}\right\}$ (1) $/(x)(1)(12)=$

$+7.195 \mathrm{E}+02$ g/t Carbon

+5.988E+01 Molar Caxpon

fo: Sample Run By,

s. $10=$

I ocosig

$5+2$

. 20 25/2-4

511 


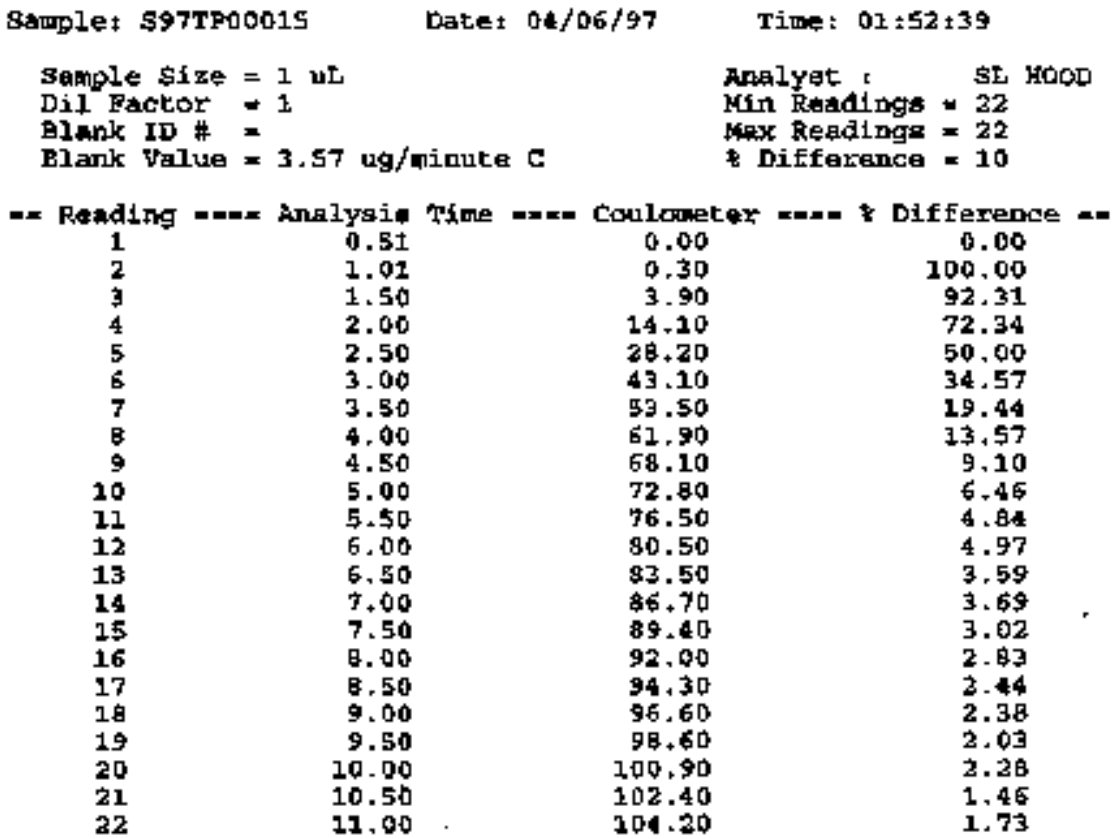

USER INPUT BLANK VALUIE

BIAMK VALUE $\approx 39.26 B 26$ Gicrograne carbon

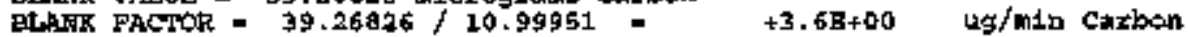

GAMPLE RESULT:

$(104.2-39.26286 /(1) /(1) \quad-\quad+6.494 \mathrm{~B}+01$

+6.494 a +01 gic Carbon

$\{104.2-39.26286\}(1\} /(1)\{12\}=$

$+5,4113+00$

Molar Carbon

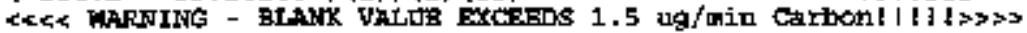

Sample Run By,

os the -

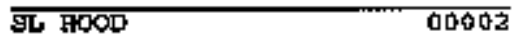

$0.987 / 3$ 


\section{TIC- TOUTAL INORGANLC CARBON ARTALYSIS REPORT} TICTOC REY 2.0

Samplet S97TP0es15

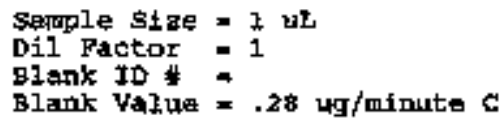

Timet $01: 38131$

$$
\begin{aligned}
& \text { AnRlyat } x \text { SL ROOD } \\
& \text { Min Readingm = } 22 \\
& \text { Max Reading* = } 22 \\
& \text { * Difference }=10
\end{aligned}
$$

\begin{tabular}{|c|c|c|c|c|c|}
\hline $\begin{array}{l}\text { tepding } \\
1 \\
2 \\
3 \\
4 \\
5 \\
6 \\
7 \\
8 \\
9 \\
20 \\
11 \\
12 \\
13 \\
14 \\
15 \\
15 \\
17 \\
18 \\
19 \\
20 \\
21 \\
22\end{array}$ & $\begin{array}{r}\text { Anelyeig } \\
0.51 \\
7.00 \\
1.50 \\
2.00 \\
2.50 \\
3.00 \\
3.50 \\
4.00 \\
4.50 \\
5.00 \\
5.50 \\
6.00 \\
6.50 \\
7.00 \\
7.50 \\
8.00 \\
1.50 \\
9.00 \\
9.50 \\
10.00 \\
10.50 \\
11.00\end{array}$ & then & $\begin{array}{c}=\text { Couloter } \\
0.00 \\
0.30 \\
2.40 \\
9.20 \\
27.50 \\
22.80 \\
35.60 \\
27.20 \\
26.10 \\
28.60 \\
29.00 \\
29.30 \\
29.50 \\
39.90 \\
30.20 \\
30.50 \\
30.70 \\
30.90 \\
31.10 \\
31.40 \\
31.70 \\
32.00\end{array}$ & $n a n=$ & 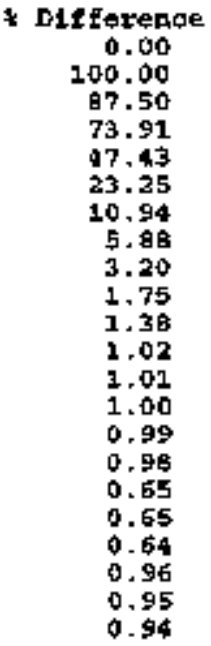 \\
\hline
\end{tabular}

UKSR INPUT BLANR YALUB

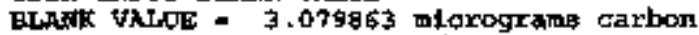

BIANK PACTOR - 3.079063/10.99951 - +2.8B-01 ug/min caxbon

SAMPLE RESTHTS:

$\{32=3.076925\}\{1\} /\{1\}$

$+2.89 E+01$

g/L tarban

$\{32-3.078925\}\{1\} /\{1\}\{12\}=$

$+2 .+1 \mathrm{~B}+0 \mathrm{O}$ Molar Carbon

Gantplo Rut But:

ss $|w|=0.977$ 


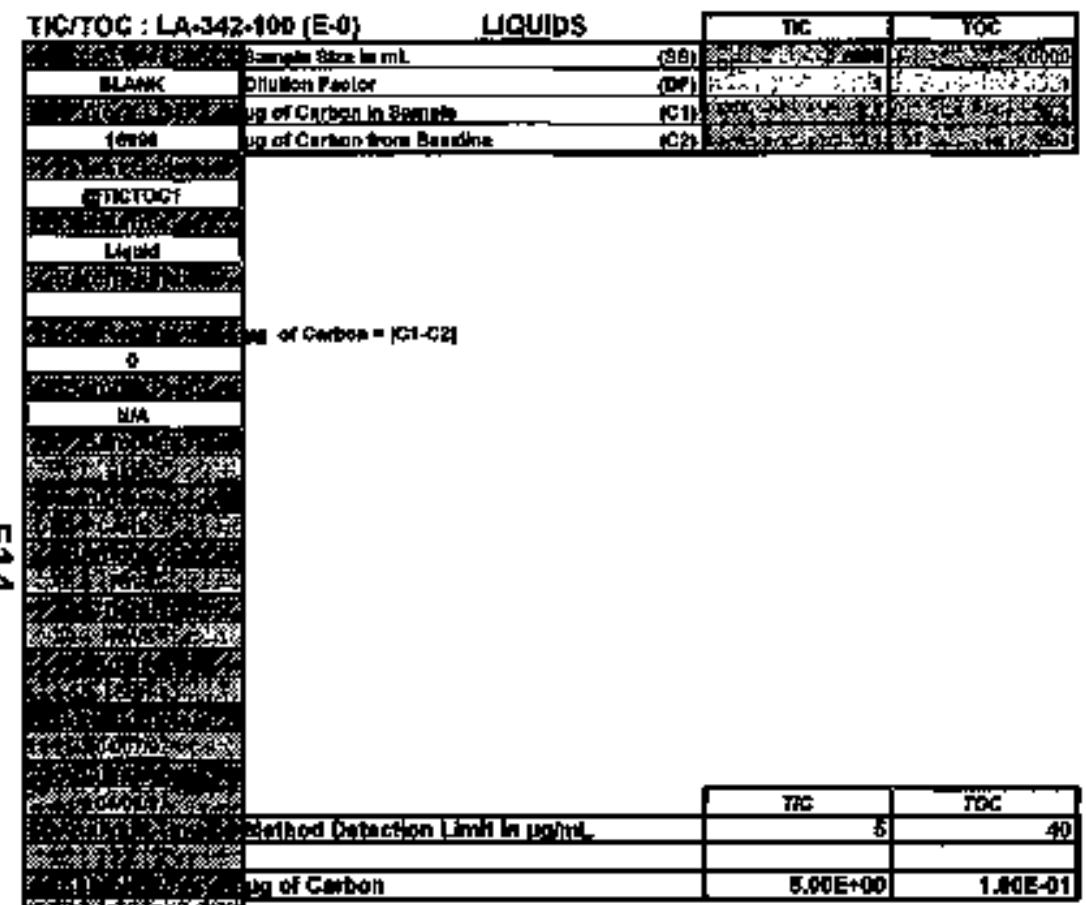

要

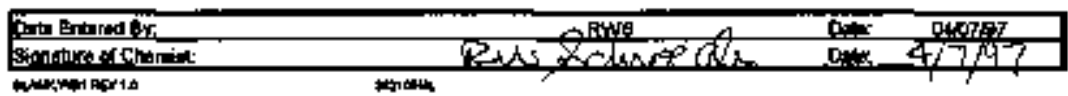




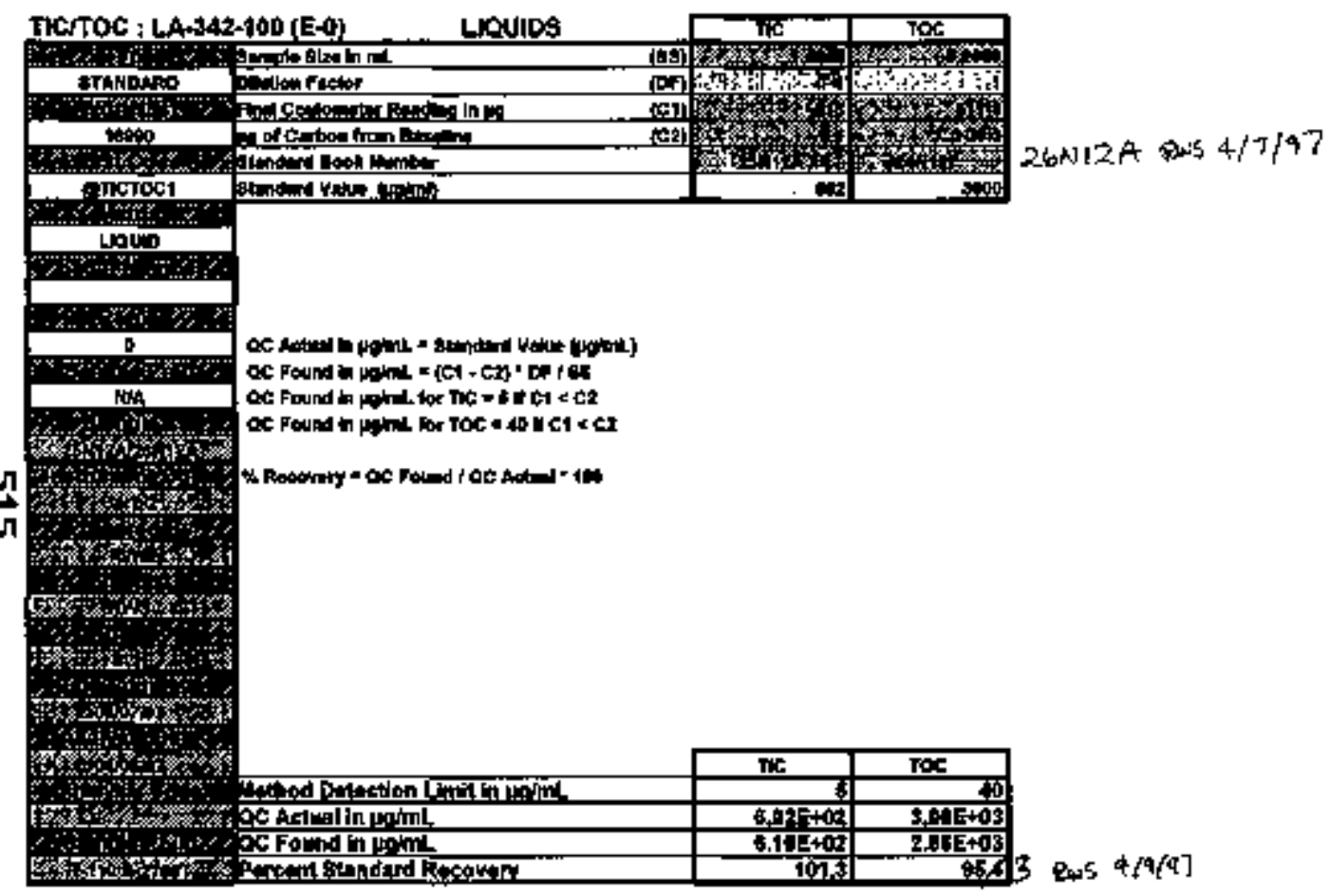

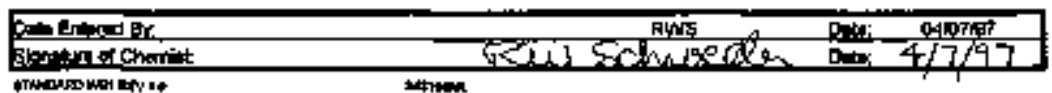




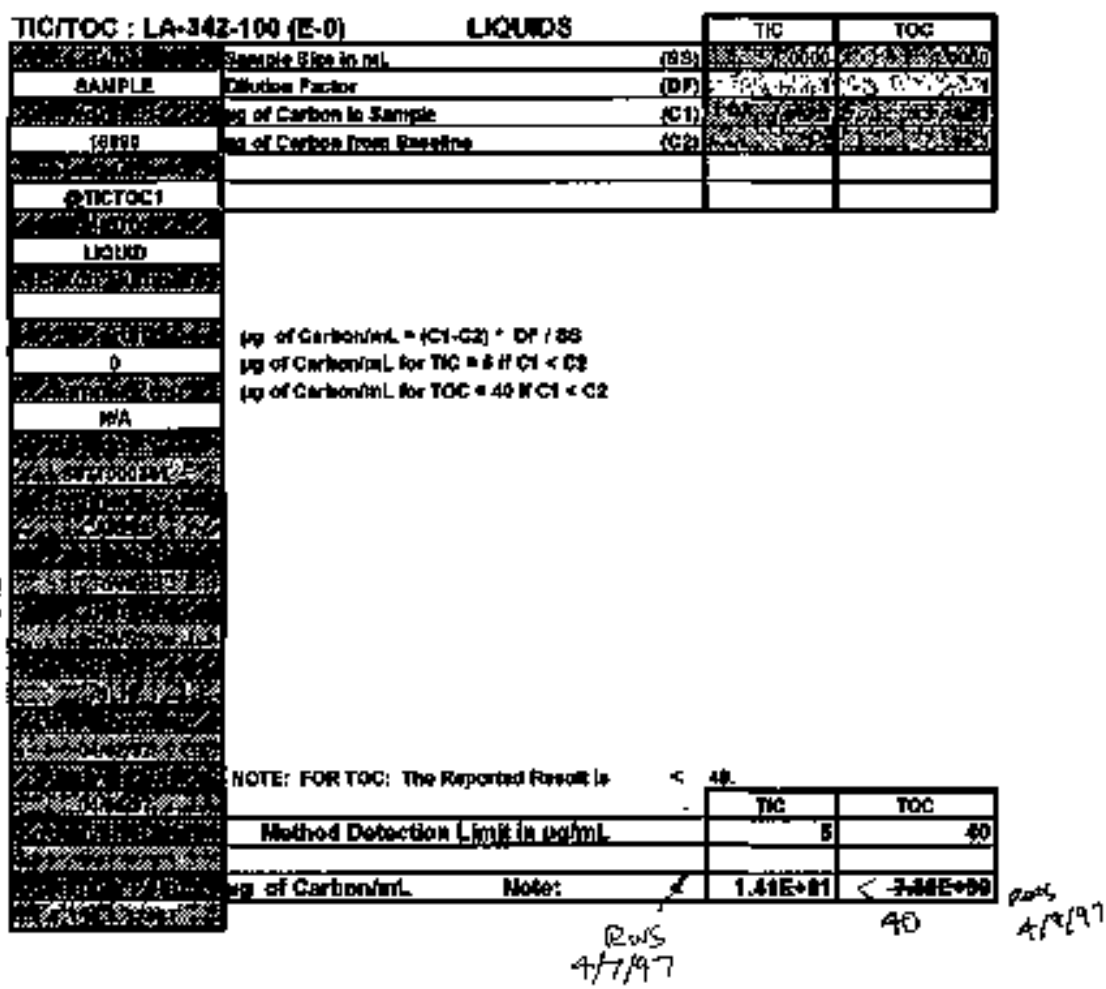

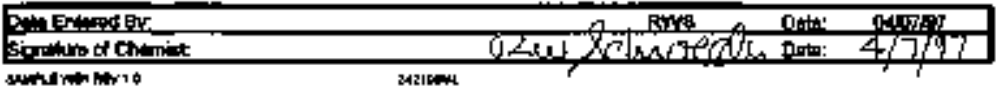




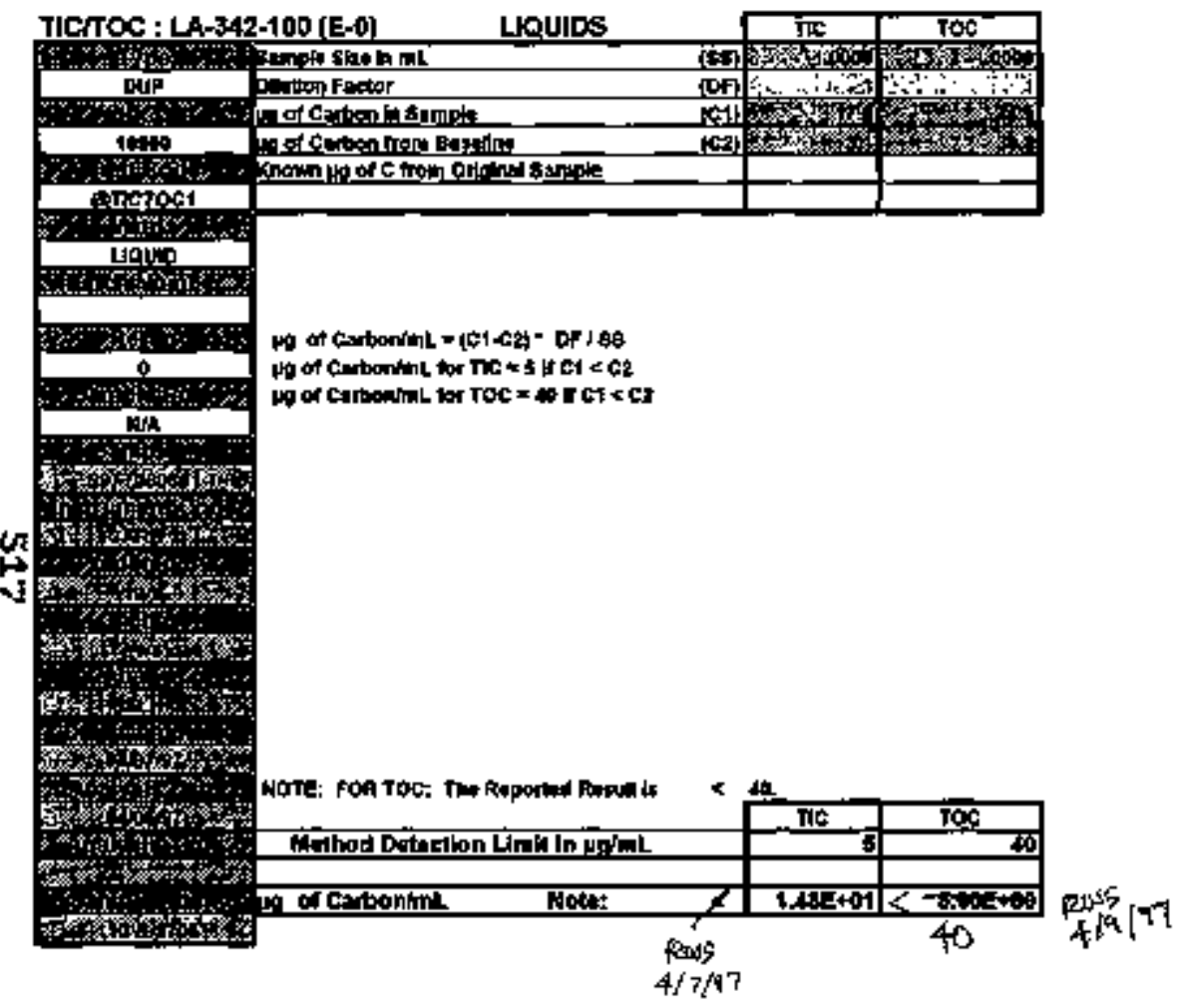

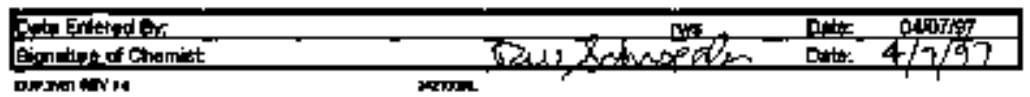




\section{HNF.SDWW-DP-239, REV. 0}

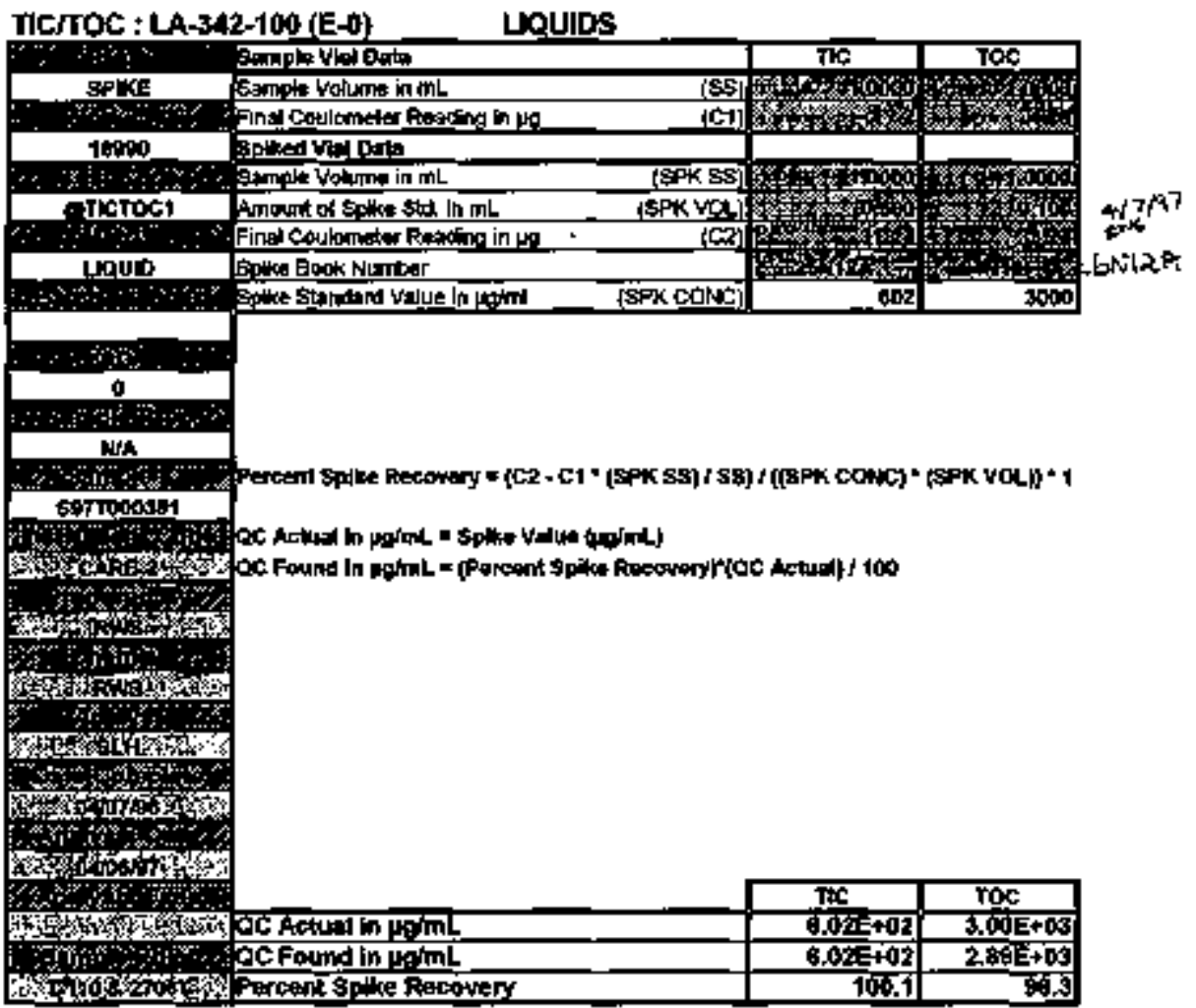

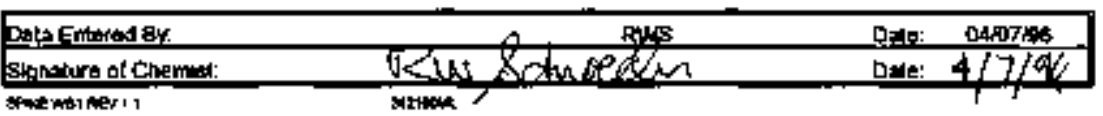

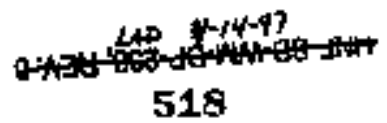




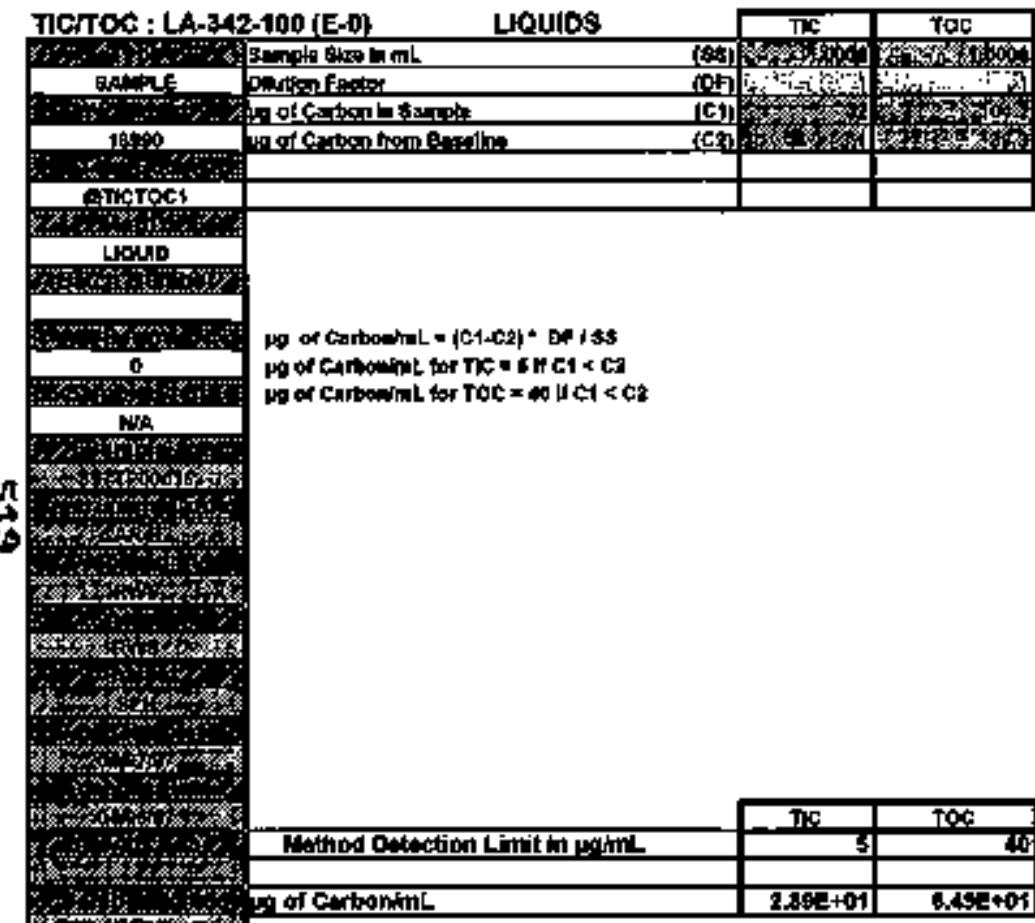

管

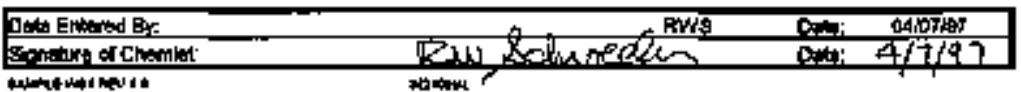


HAF-5D-161-OP-239，REV+Q

RADIOCHENICAL ANALYSIS 


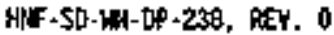

THIS PAEE INAENIONALLY LEET BLANK

519.2 


\section{LABCORE Completed RadChem Report for Worklist"\#: 16695}

Analyst: jus

Instrument: ABI6

Book

Method: Revhorod

Workliot Conment: T-110, BALPHAOL, Deter. s.s. using Lndum. Jkm

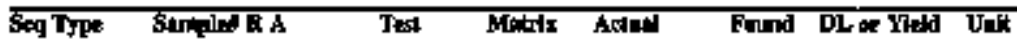

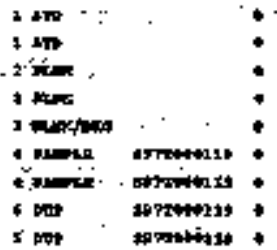

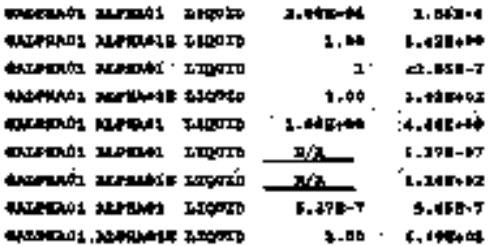

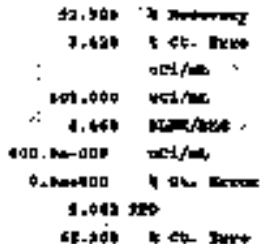

Final page for worklist\# 16695

Annost Signature

Date 


\section{LABCORE Data Entry Template for Worklist 16695}

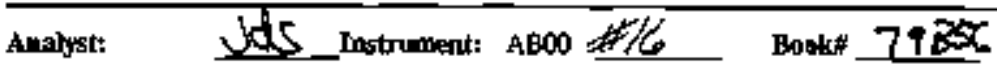

Methad: LA-50S-10] Rev/Mod_F-O

Wocklist Comanent: T-] 10, BALPHA01, Deter, 8.s, using Ludlum, stem

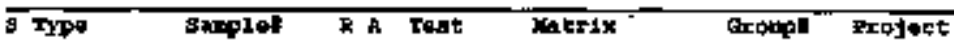

1 STR GRPHAOI LIOLID

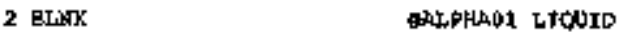

3 BLATC/bN3 AALPHAOZ LIQUIO

4 SAKLE 5PT000119 0 AALPEBOL LIQU10 970000e3 T-110

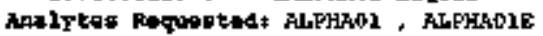

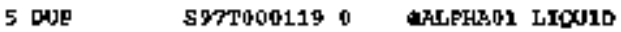

\section{Final page for worklist \# 16695}
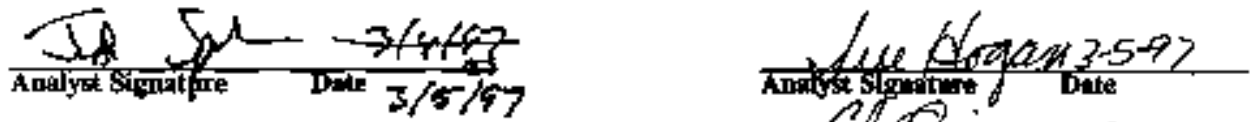

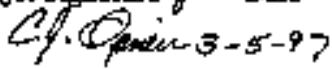

Data Bany Comporst.

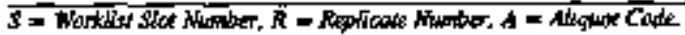


HWF-SD-WH-DP-29日, REV O

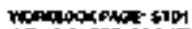

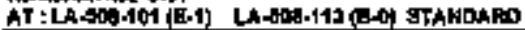

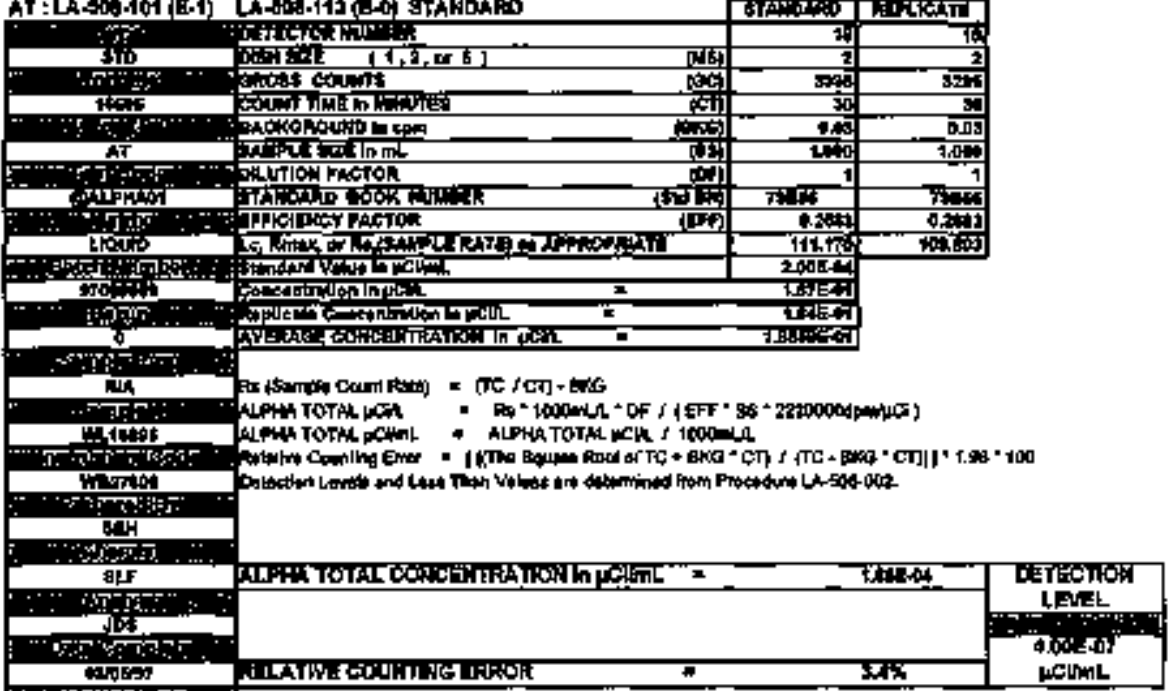

: antis

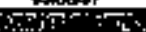

4ETTIM

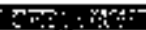

TH:

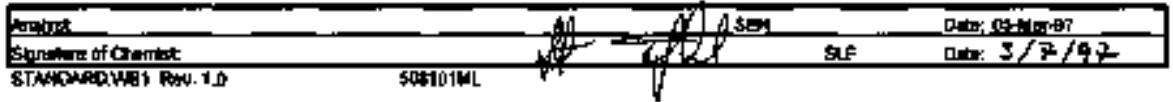


HAYF-SD-WW-DP-Z36, REV. 0

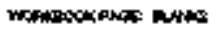

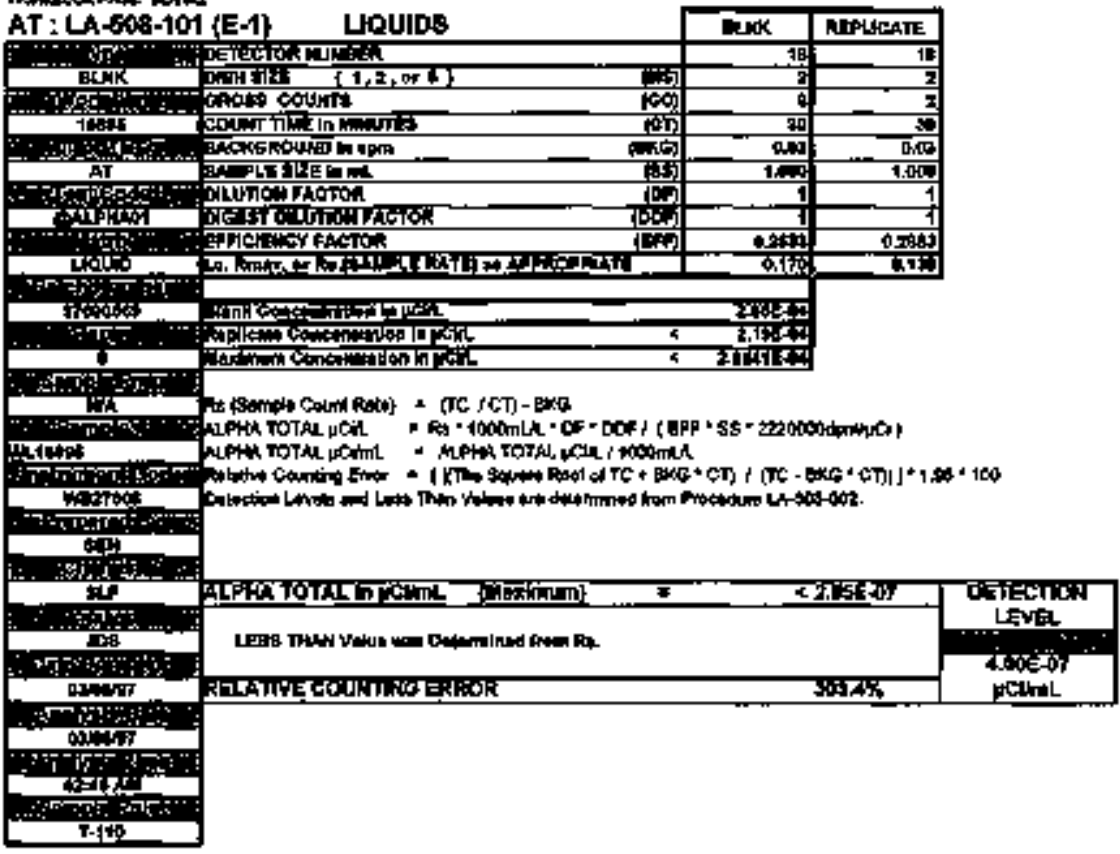

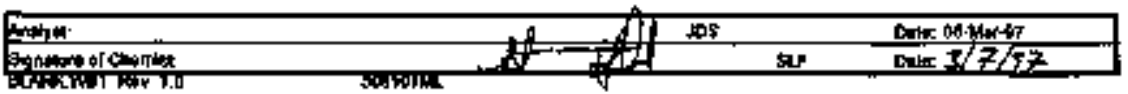


WOfurboox moR' 3lum

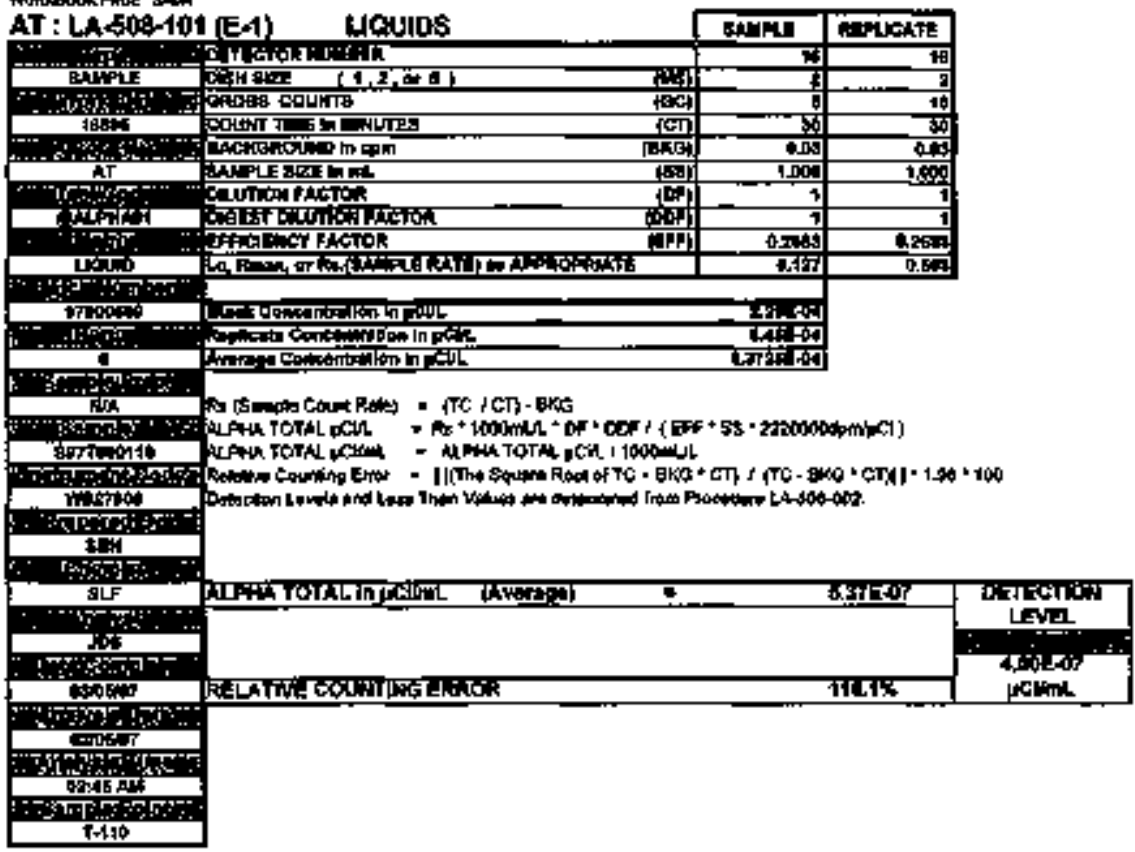

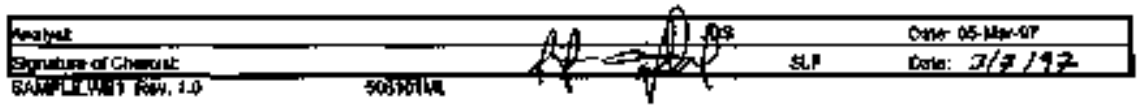


HEसF-SD-WHA-DP-238, REV. 0

mprroox Ruct: DUPs

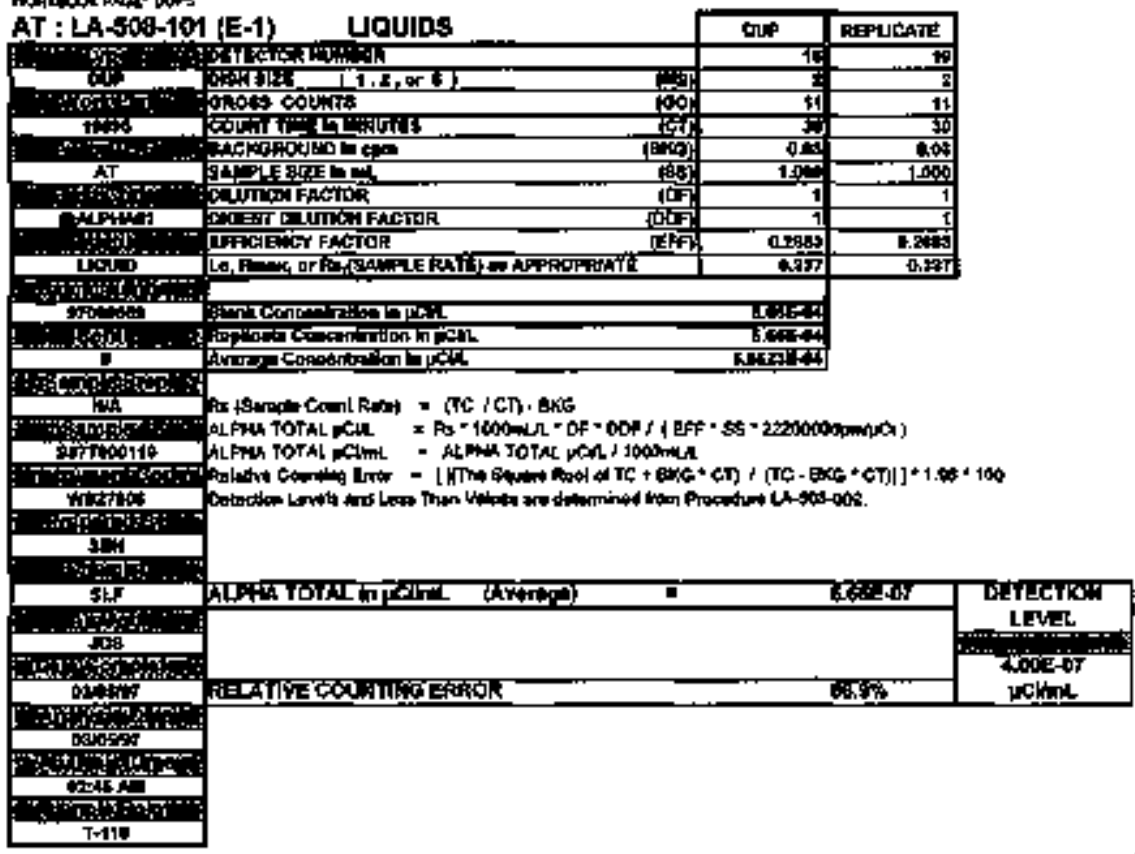

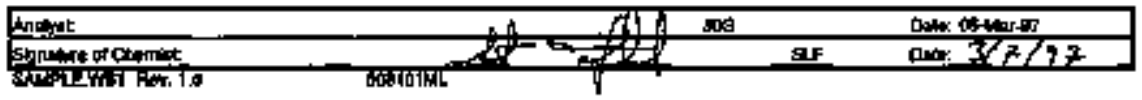




\section{LABCORE Completed RadChem Report for Worklist: 16899}

Analptet glh

Instrament: AAl6

Bookt

Metbod: Revillod

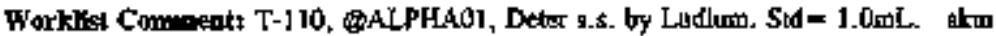

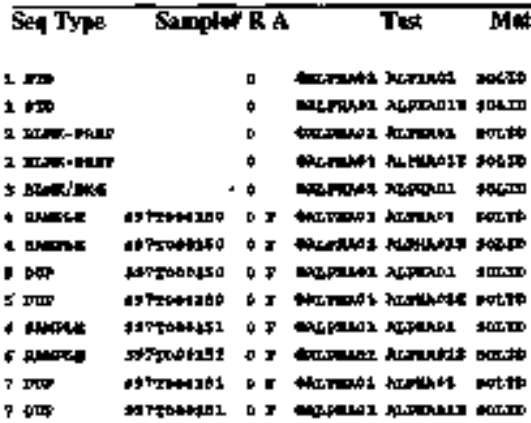

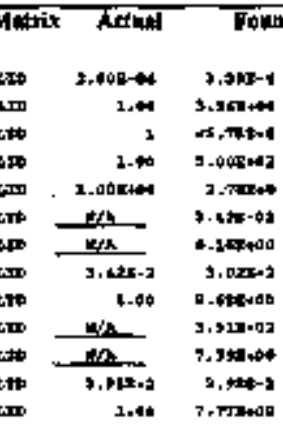

DLor Fisd Tris

Final page for worklist 16899

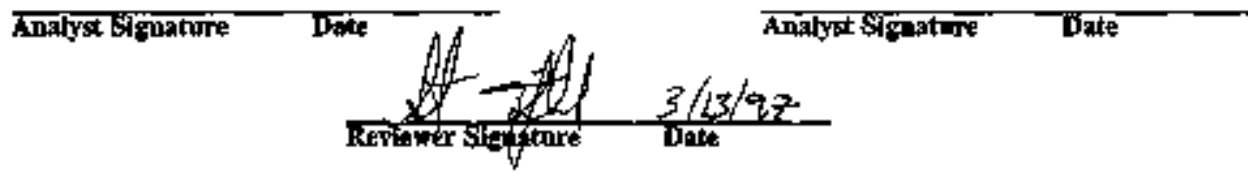


$03,05 \% 7+3: 50$

HISF-3D-WhA-DP-238, REV, 0

Page: 1

A. 0 and .s.

LABCORE Data Entry Template for Worklist 16899

Analyst:

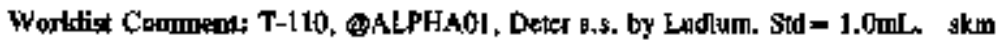

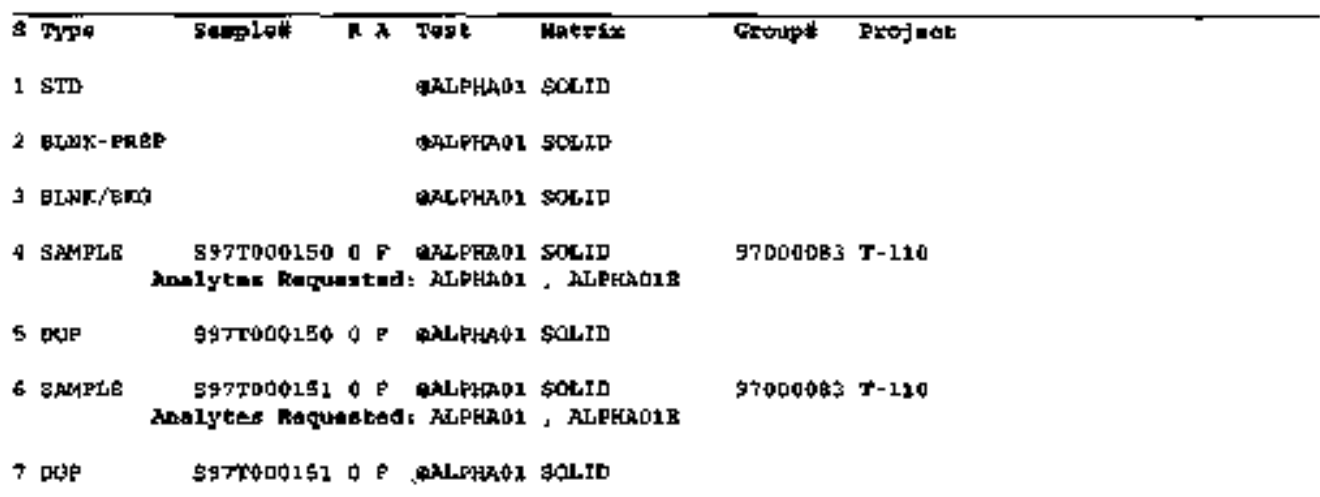

Final page for worklist \# 16899
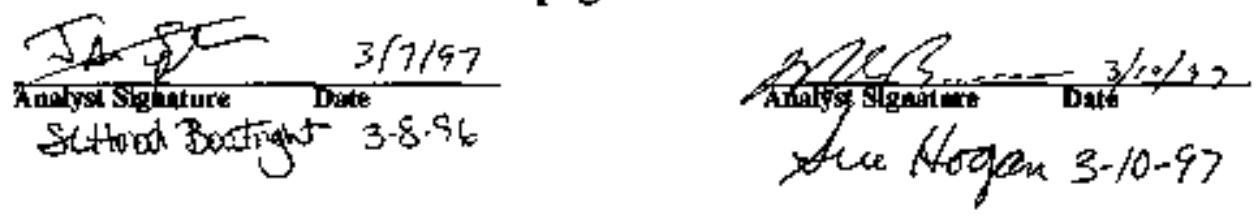

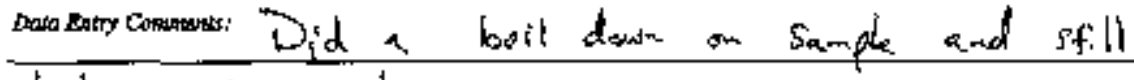
hind crusty dishes

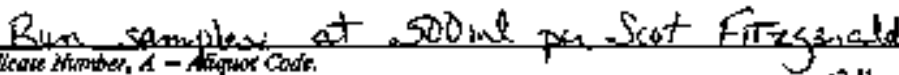

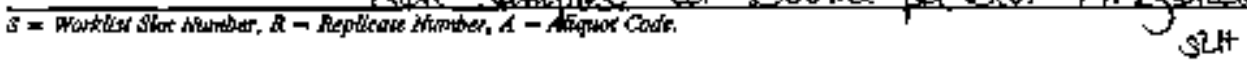
527 
HFF-SD-WM-DP-238, REV. O

monkook 1401

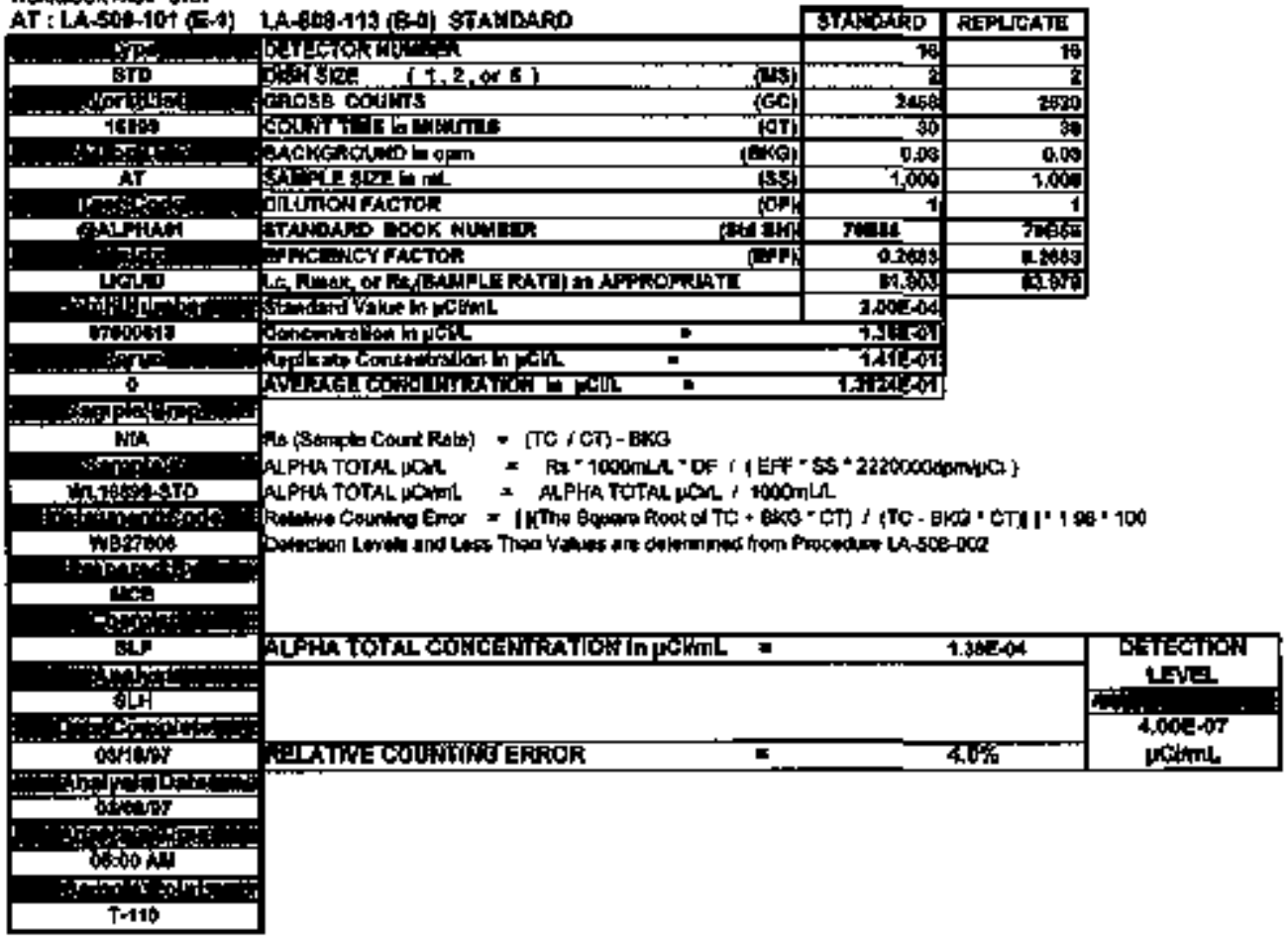

\begin{tabular}{|c|c|c|}
\hline Annlat & MCe & 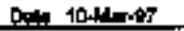 \\
\hline 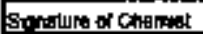 & & Date $3 / 12 / 17$ \\
\hline
\end{tabular}


HNF-SD-WW-DP-Z3G, FEV O

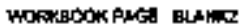

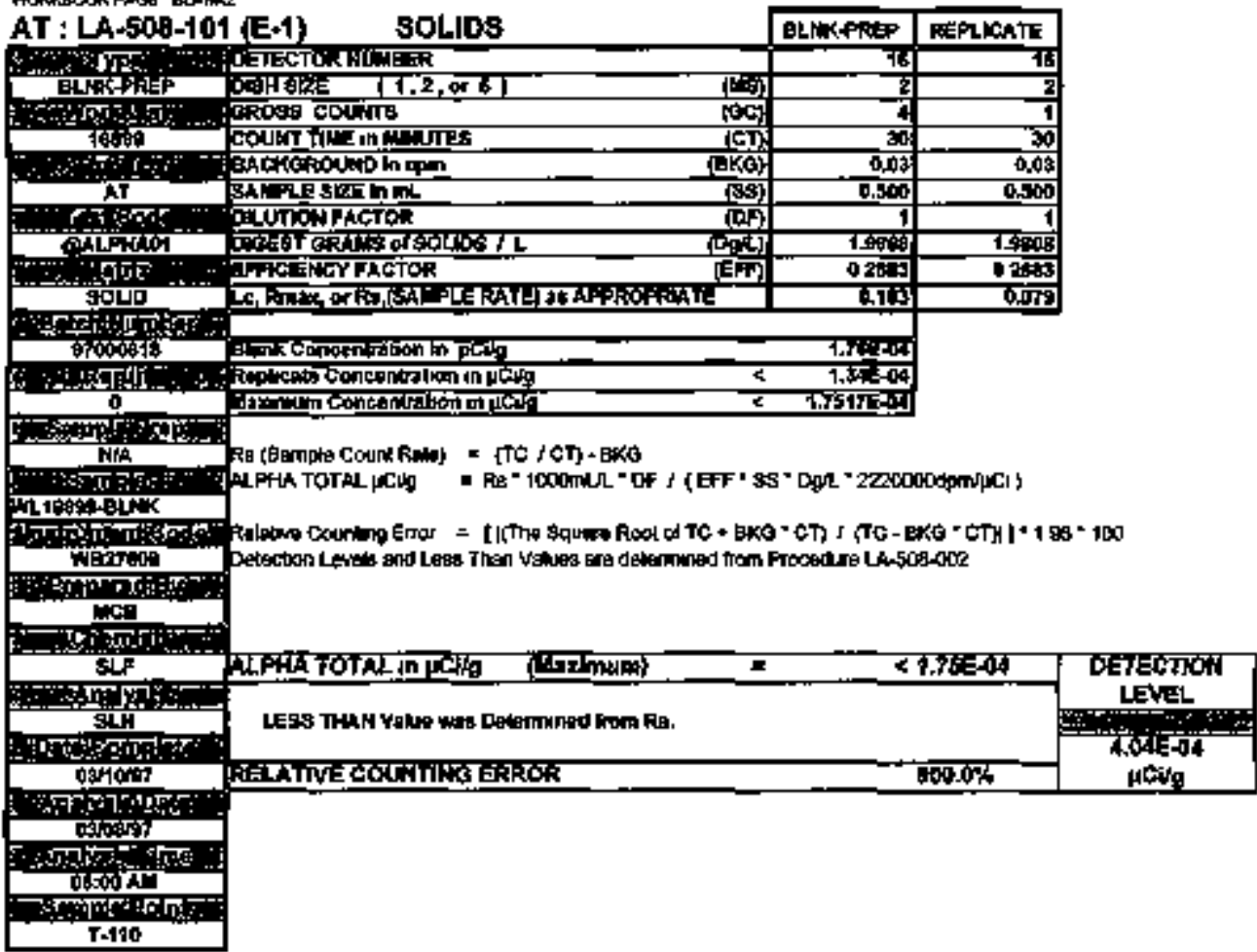

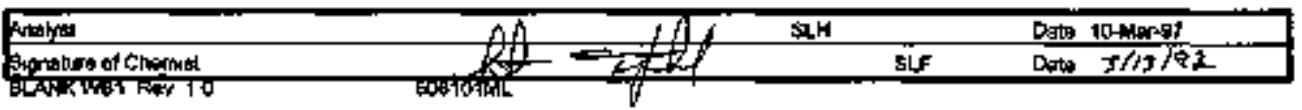

\section{9}


HI:-SD-WH-DP-238, REV. 0

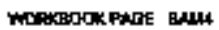

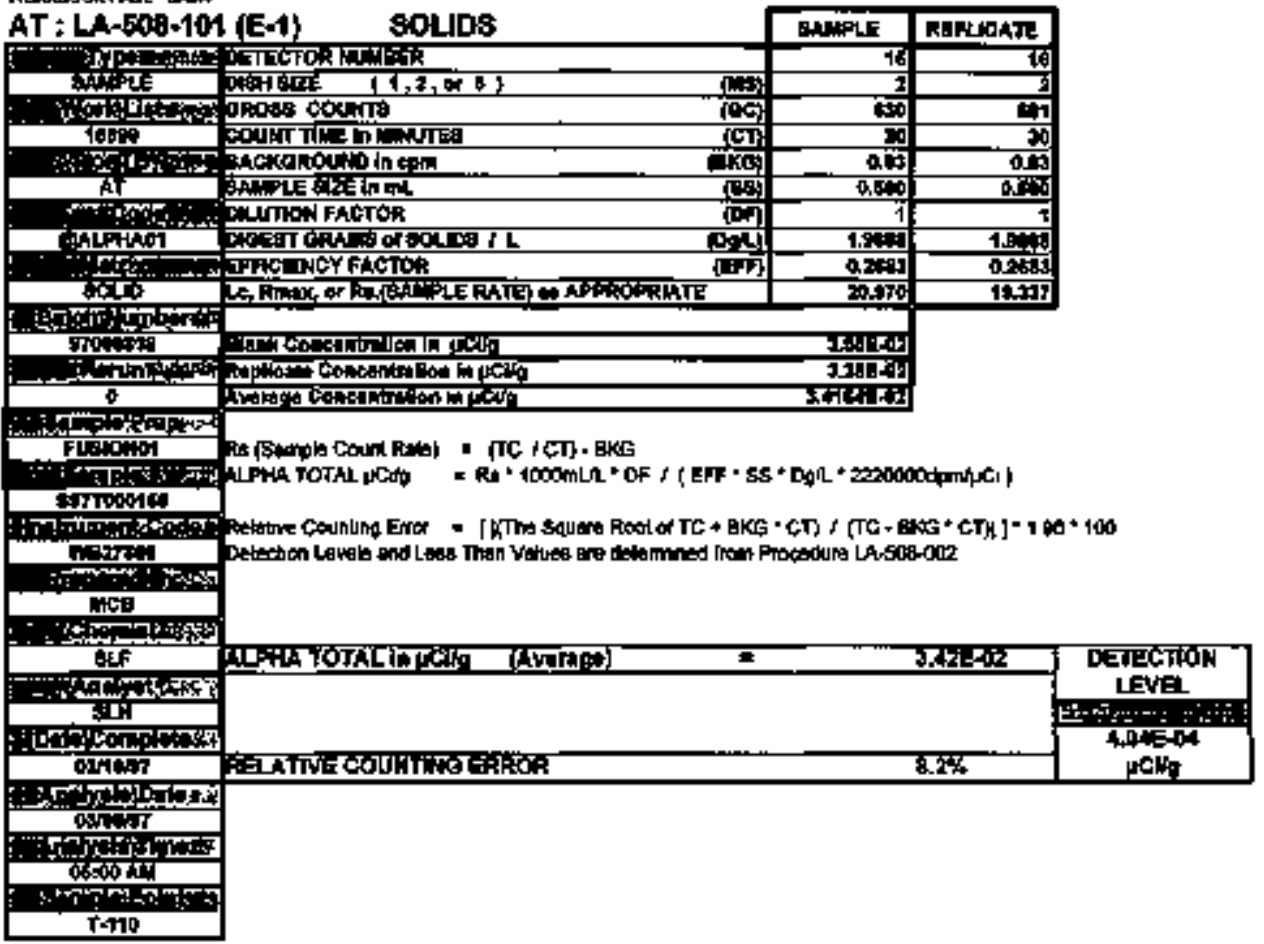

\begin{tabular}{|c|c|c|}
\hline 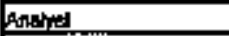 & S내 & Dele 104tor-s? \\
\hline sproturid af Chemet & & Doin $3 / f=$ \\
\hline
\end{tabular}


WOFHaON PHaF oups

HWF-SD-WM-DP-239, REV. O

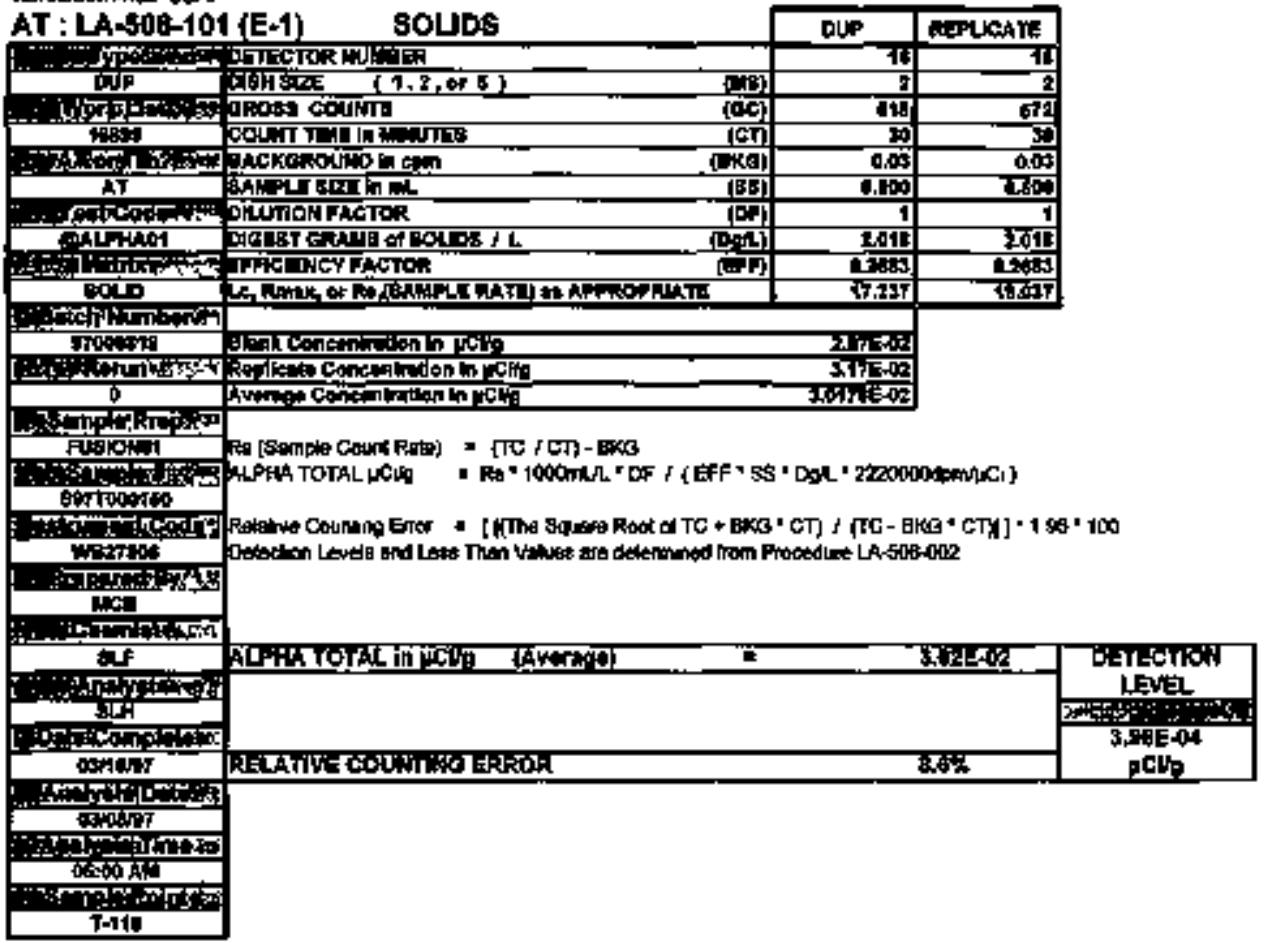

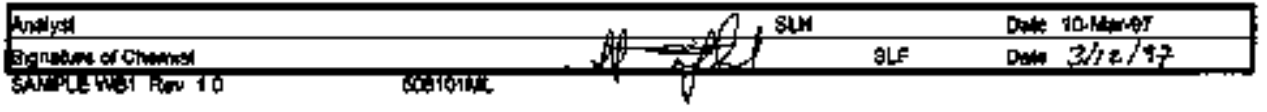


HAF-SD-WH-DP-238, REV. 0

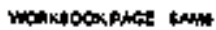

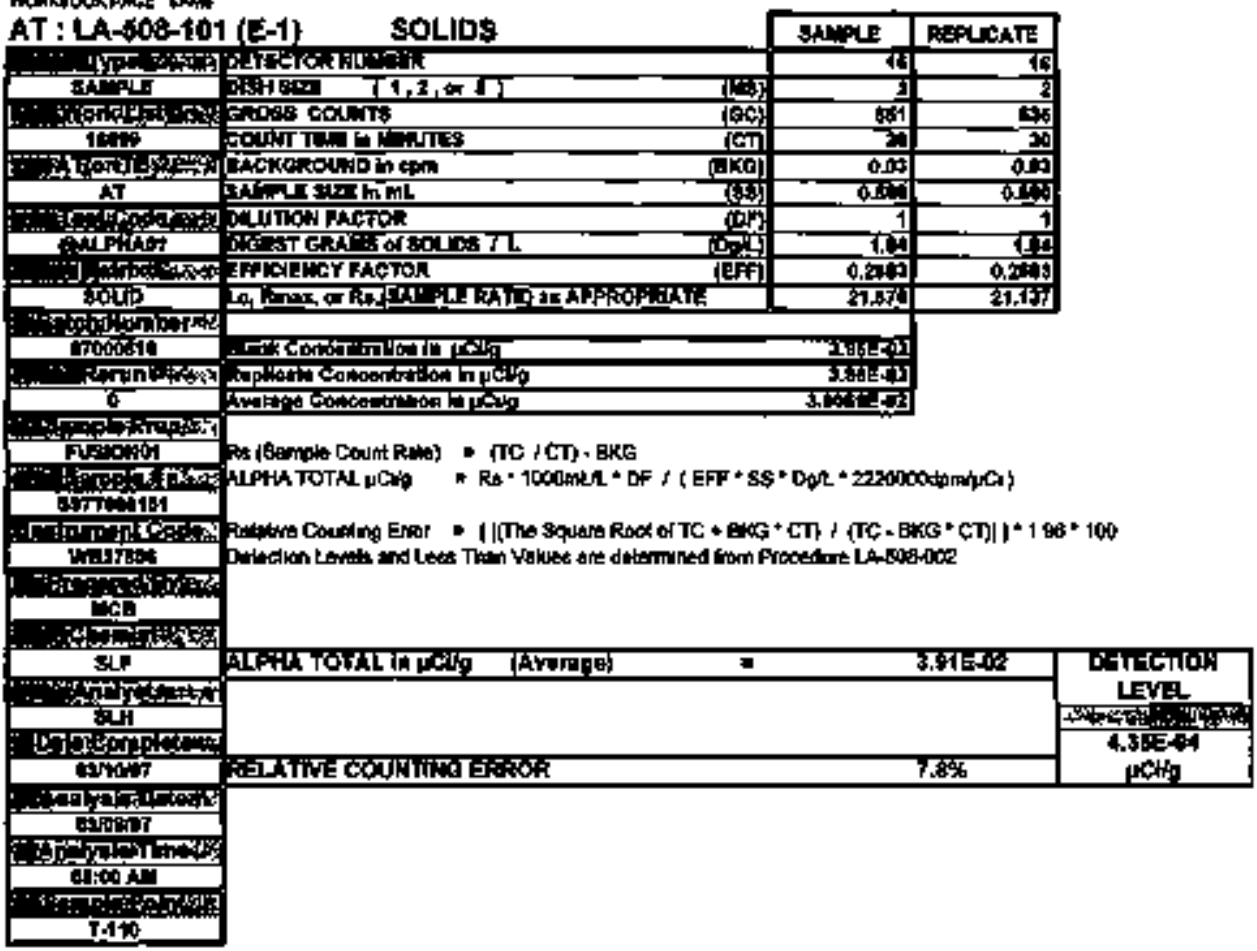

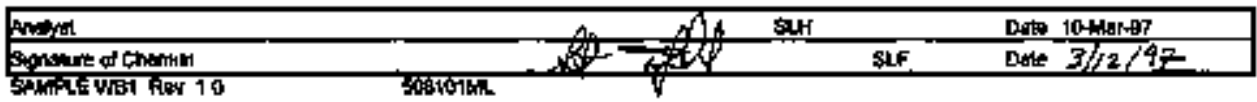




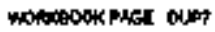

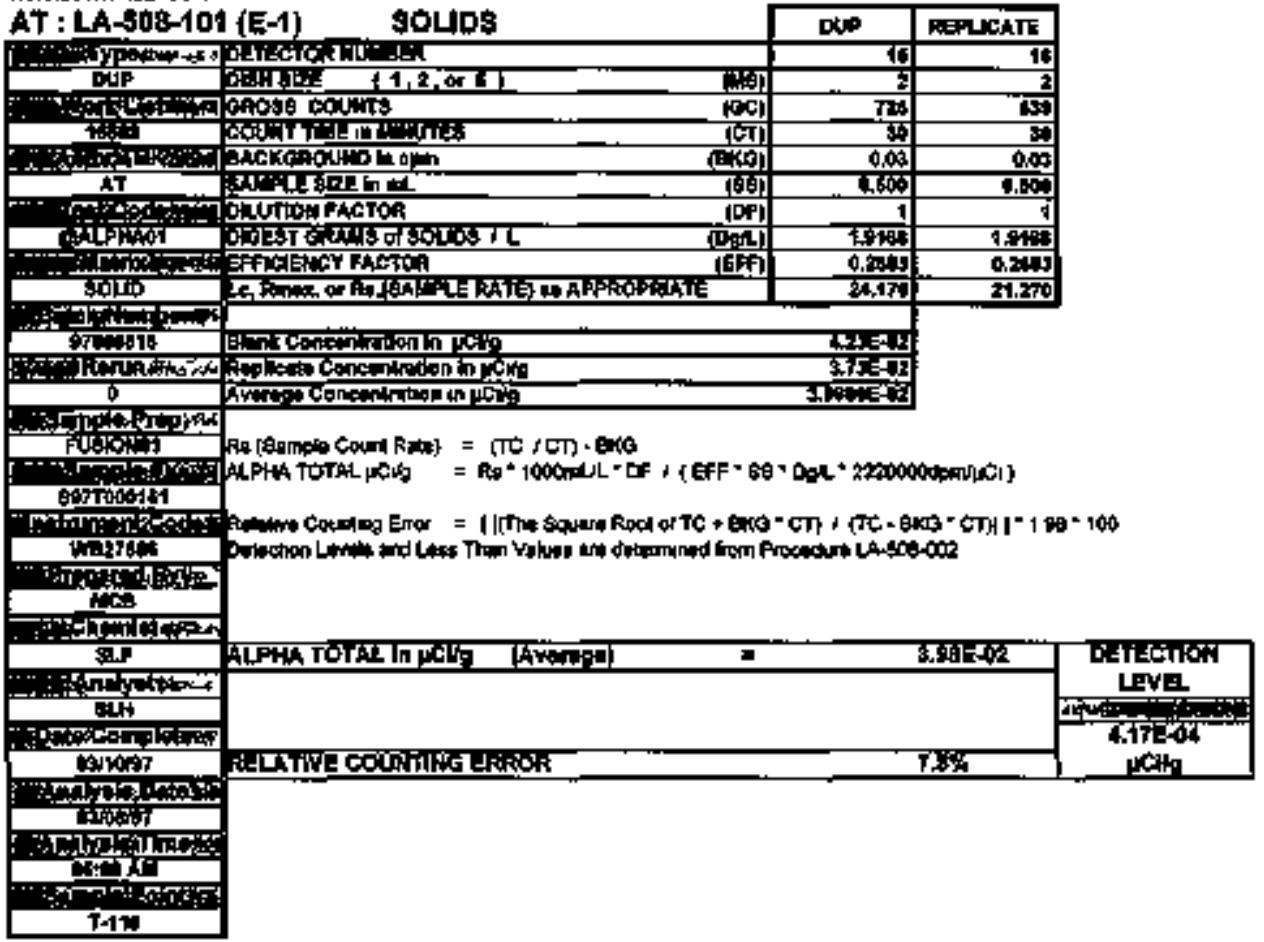

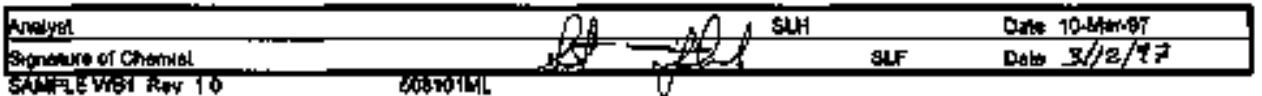




\section{LABCORE Completed RadChem Report for Worklist:" 17065}

Analyst anf

Instroment: AB26

Booky

Method: Rewhod

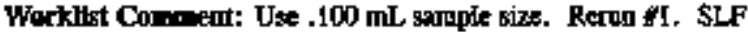

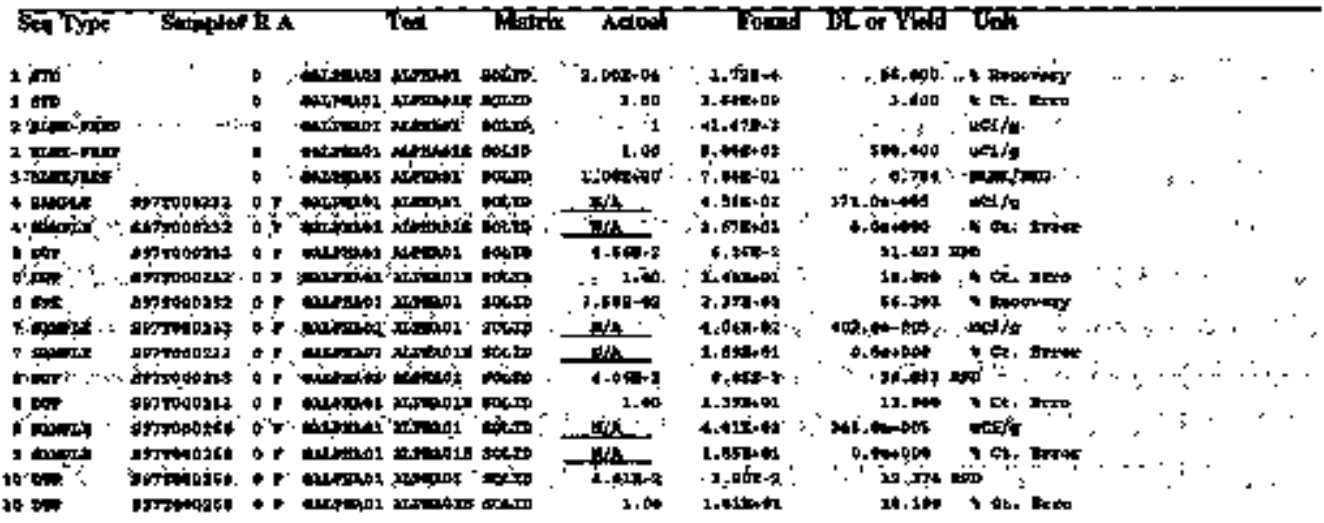

\section{Final page for worklist 17065}

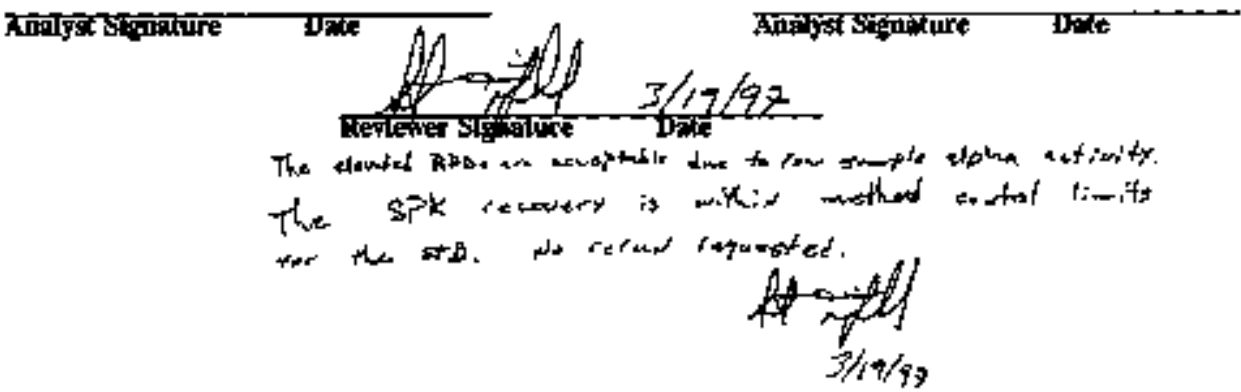

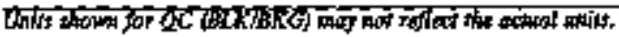




\section{LABCORE Data Entry Template for Worklistf 17065}

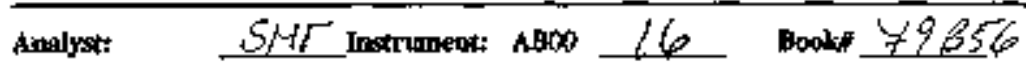

Method: LA-508-101 Rewhod F-O

Worklist Commeare Use .100 mL sample size. Rerun \#I. SLF

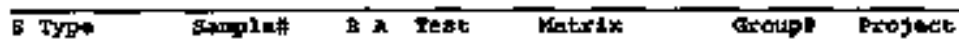

1 sTD

extpeator schin

2 BLAX-PEEP

AALPERBI SOLTD

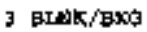

AHLERAB SOLID

1 SAMPLE

$\operatorname{sit} \pi 00232$ a $\theta$

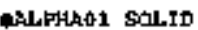

$97000111 \mathrm{~T}-110$

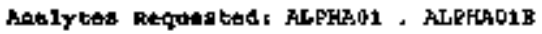

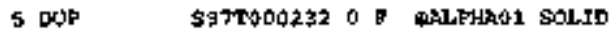

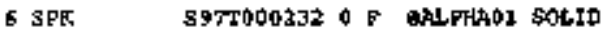

+ Solvpse

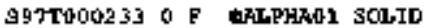

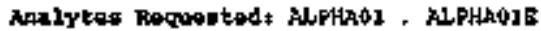

$97000111 \mathrm{~T} \cdot 110$

9 LUP S97TDDO233 O F DALPARDI SOLID

9 GMMPEE G9TT000268 O F ARPHAO SOLID

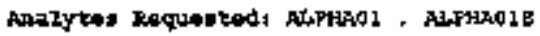

11 DUP

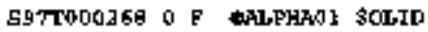

Final page for worklist \# 17065

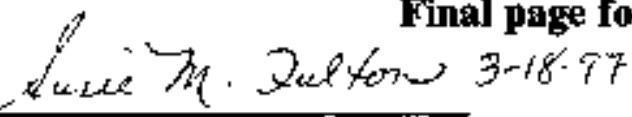

Anatgra

nualys inating bate

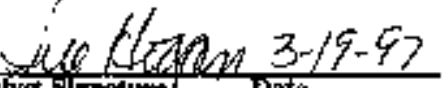

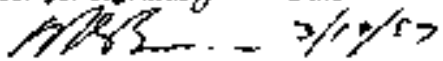

Doxa Eutry Cannewts:

ASR 423843 . $100 \mathrm{AL}$

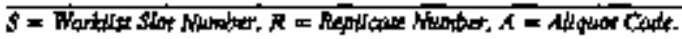




\section{HAF-SD-WM-DP-238, REV. O}

Whoporare 8TD1

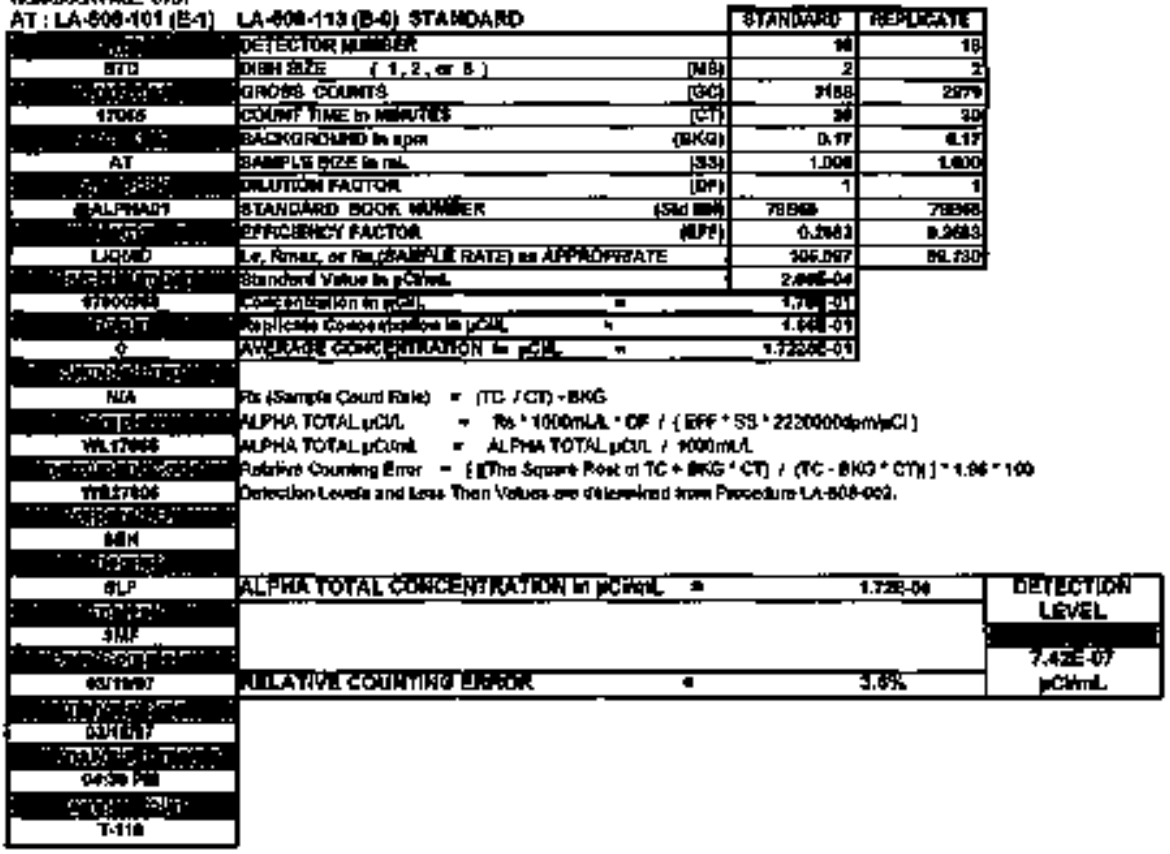

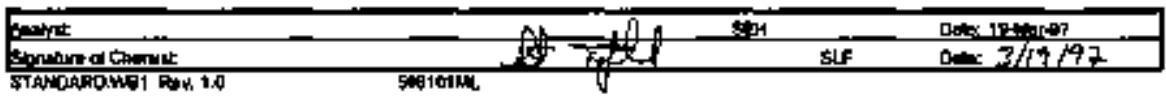


HNF-SD-WH-DP-233, REV. O

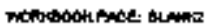

\begin{tabular}{|c|c|c|c|}
\hline 4 & $([2]-1)$ & BLNK-PQtgP & 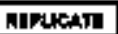 \\
\hline & 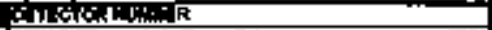 & Tा। & T] \\
\hline & 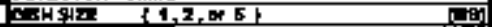 & 2 & J \\
\hline$\because 7$ & 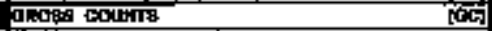 & 3 & $\overrightarrow{4}$ \\
\hline Trite & 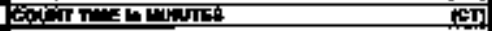 & 过 & F \\
\hline$\therefore$ & 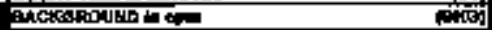 & 217 & प117 \\
\hline hi & 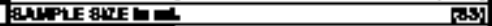 & 0.100 & D.KS \\
\hline & 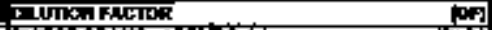 & 1 & 1 \\
\hline & 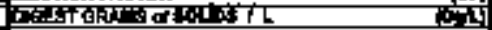 & 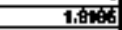 & 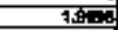 \\
\hline & 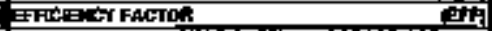 & ㅁ.2683 & 2.5 \\
\hline अणं0 & 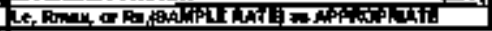 & Q17 & (1.126 \\
\hline
\end{tabular}

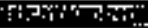

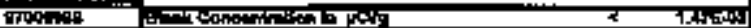

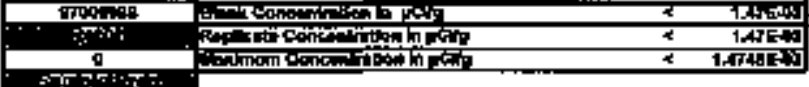

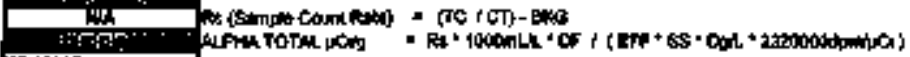

introts

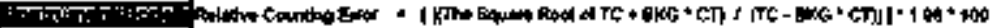

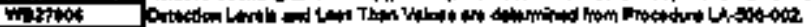

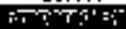

IN4

:

8.

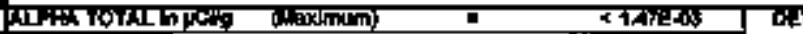

!ריה:

(1)

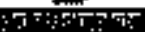

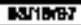

(1)

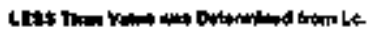

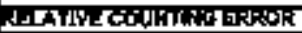

wordo

brictro บ는
$\therefore$ ग

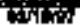
$\because 7$
Aritive
r.:
$\mathbf{T - 1 0}$

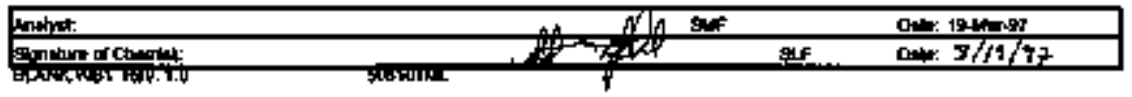


HNF-SD-WH-DP-2GB, REV O

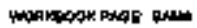

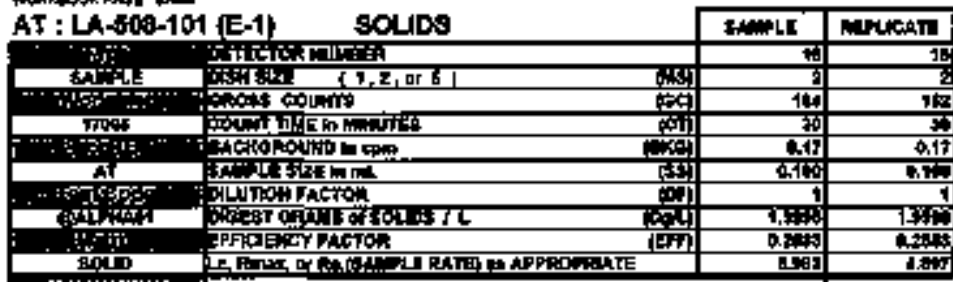

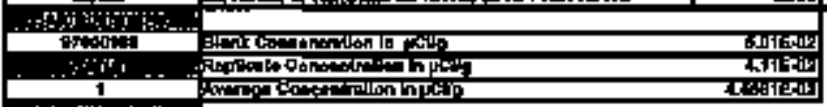

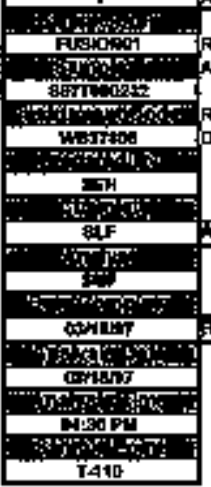

fa (S

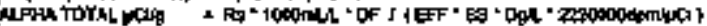

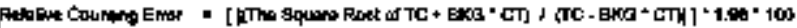

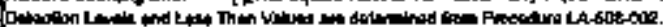

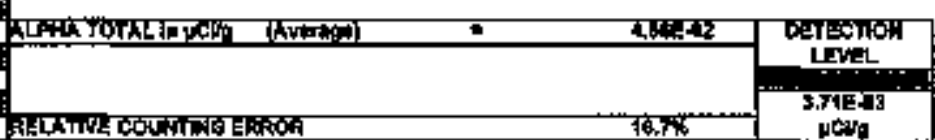
1 ptwa

\begin{tabular}{|c|c|c|}
\hline ansart & $8 y$ & D. 19:-D? \\
\hline 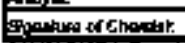 & & a-x. $7 / / 4 /$ \\
\hline
\end{tabular}




\section{HNF-SD-WH-DP-29日, FEV O}

4apospros oups

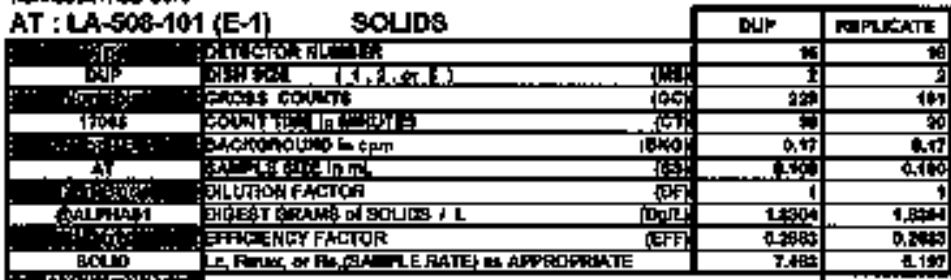

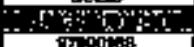

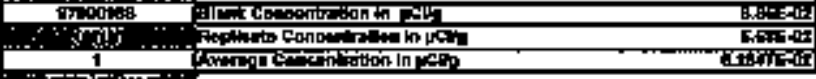

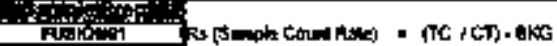

$\because$ "rip.

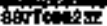

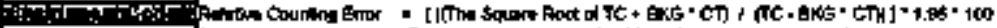

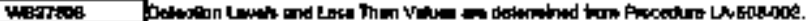

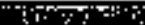

족

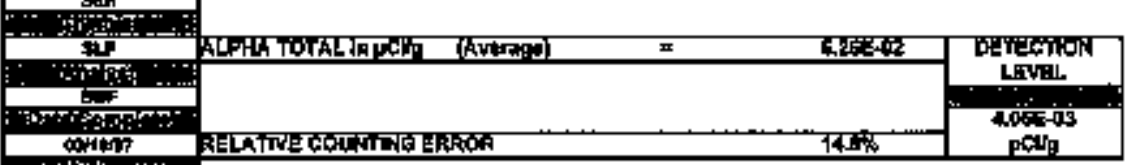

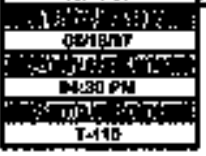


HNF-SD-WHA-DP-238, REV. 0

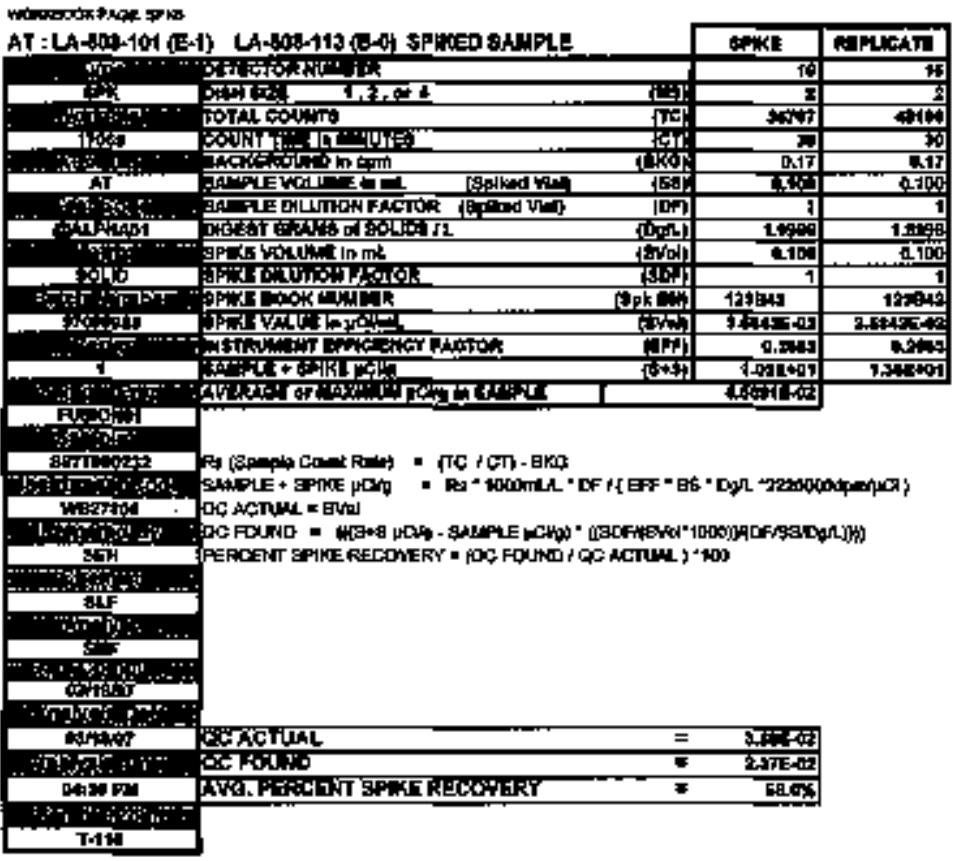

\begin{tabular}{|c|c|c|}
\hline 的出 & $\mathrm{EDH}$ & 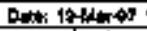 \\
\hline 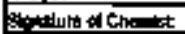 & & datidits \\
\hline
\end{tabular}

$$
\begin{aligned}
& \text { The thented RPB for the SPH + SPK/REP is the }
\end{aligned}
$$

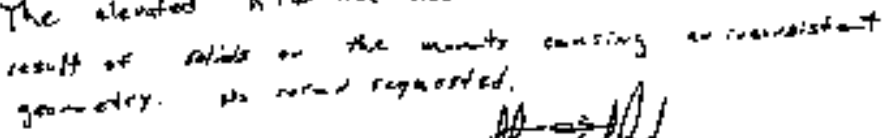

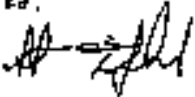

$$
\begin{aligned}
& \text { s/ } / 1 / 7
\end{aligned}
$$


IFNF-SD-WM-DP-238, REV. 0

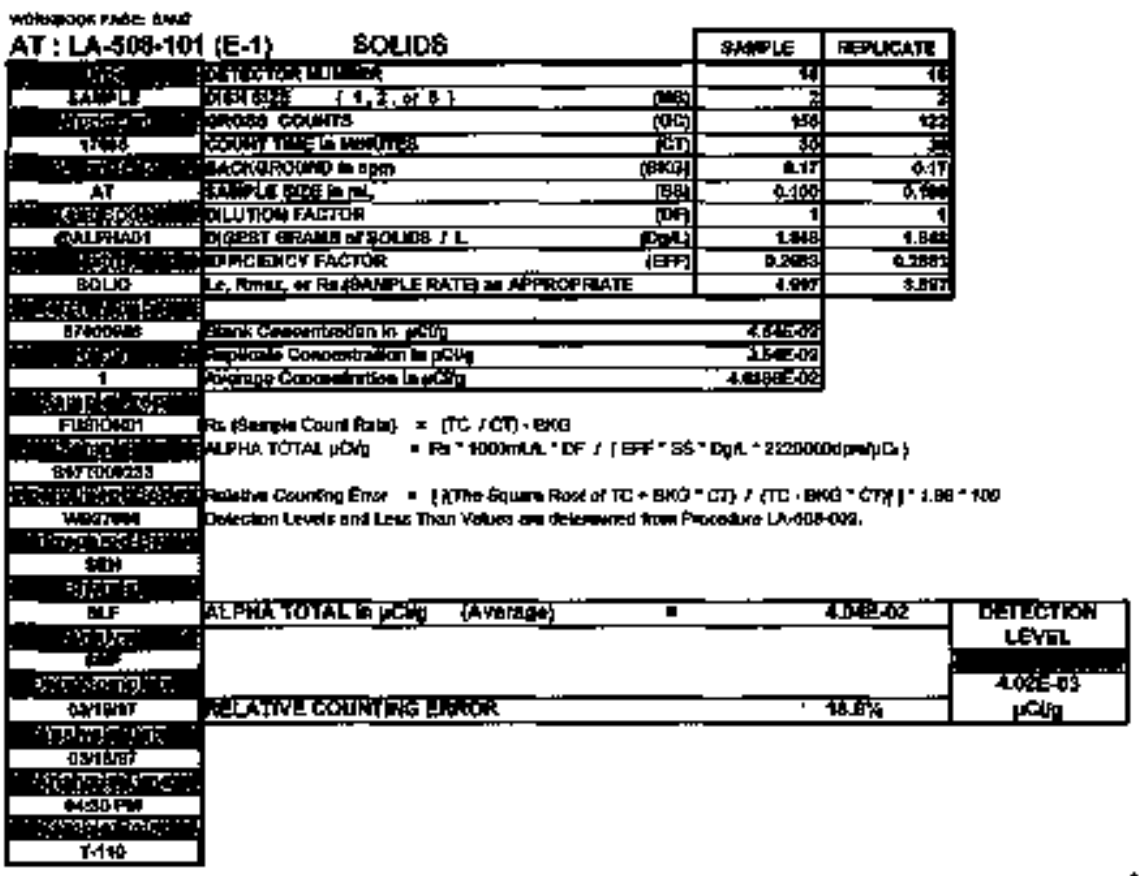

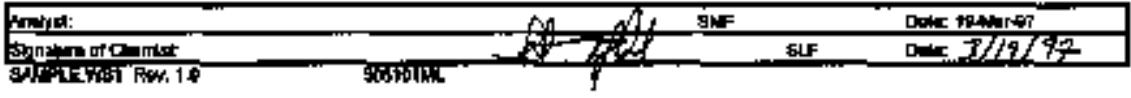


HNE.SD-WM-DP-23B, FEV. O

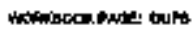

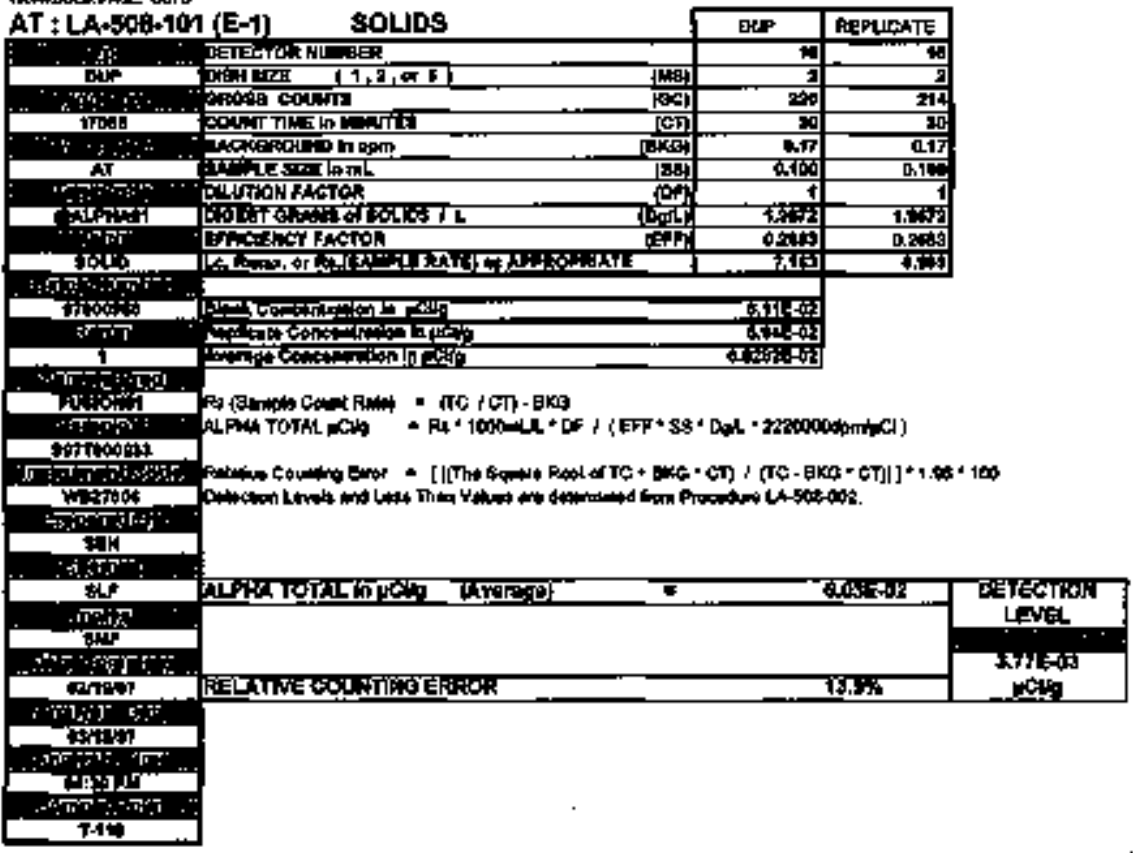

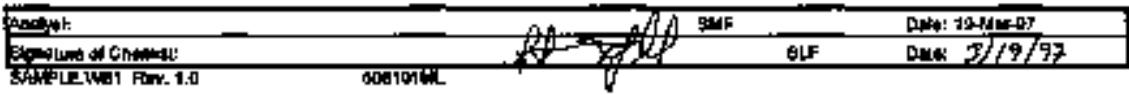




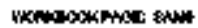

HNF-SD-WW-DP-238, REV. O

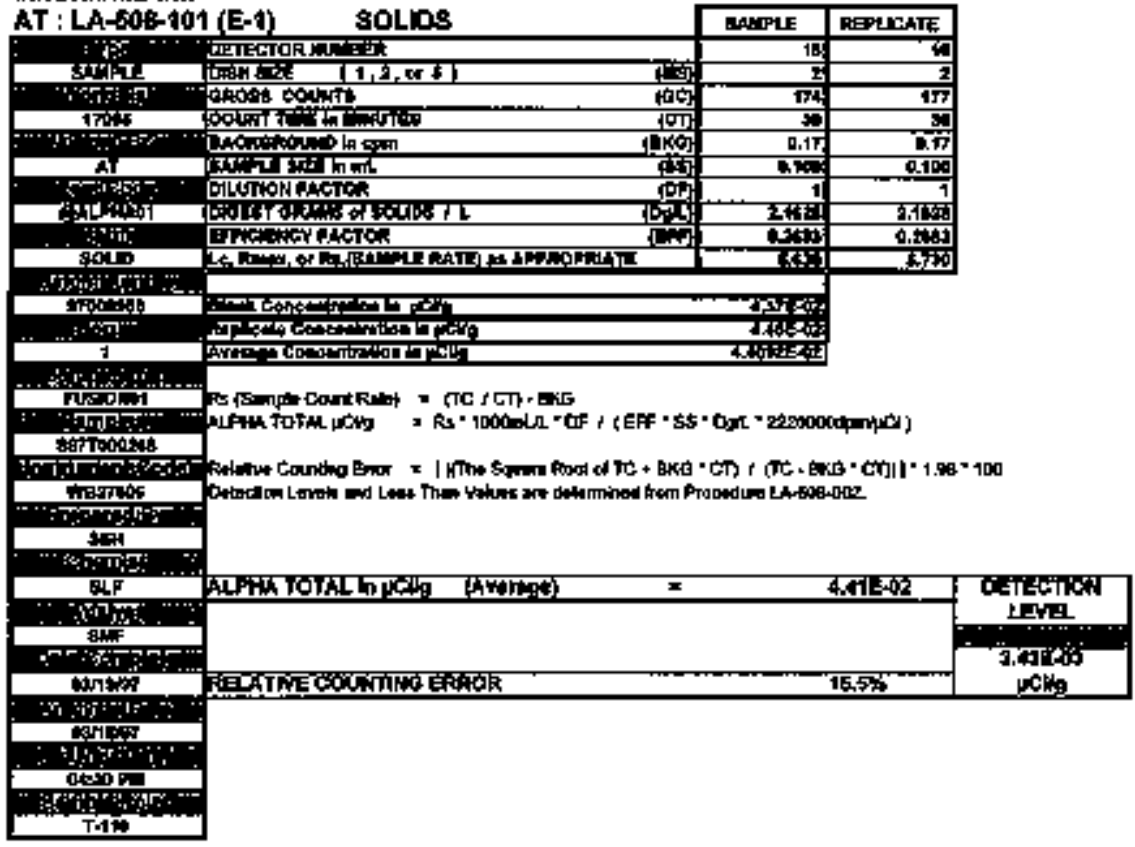


HNF-SOWW-DP-238, REV 0

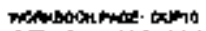

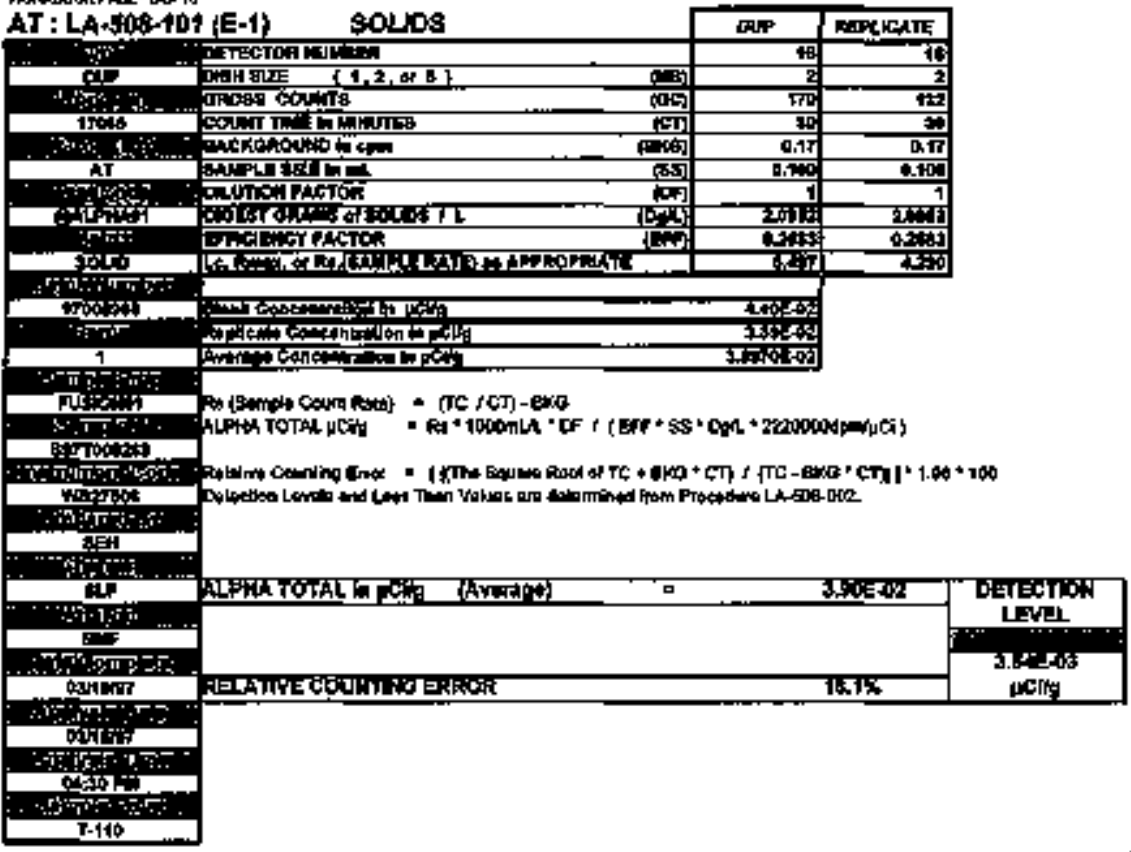

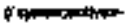

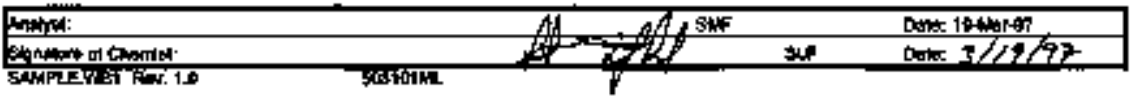




\section{LABCORE Completed RadChen Report for Worklist:f: 17066}

Anelyst snof

Instrunent: $A B 18$

Book"

Method: ReviMod

Workllst Comment: Use .100 mL smple stre. Retun H1. \$LP

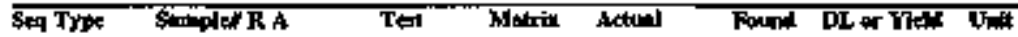

\begin{tabular}{|c|c|c|c|c|c|c|c|}
\hline 1. & - & 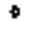 & strill. & Mngert & arch & 1.QDE-41 & 1.CSEk \\
\hline $2 \mathrm{~m}$ & & 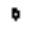 & 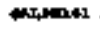 & sxapoln & 口سمा & 1.40 & $7,61 \% 000$ \\
\hline Eklas & 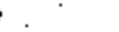 & $b$ & entat: & 1tat: & Aath & ' $\mathbf{i}$ & et \\
\hline $6+1090$ & & D & merares & & ज्ञा. & $2-40$ & s.tabetot \\
\hline Had & $\because$ & 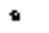 & & & 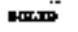 & 1. & 9,7מnatio \\
\hline & sypropenta & $0 \Rightarrow$ & encents & & HD & not & 4. astetes \\
\hline cals & Eqpoposas & I & war & whet & म्सm: & .h & $2 \operatorname{tansi}$ \\
\hline & cystsitbonara & $0 x$ & תמט & & Bष्म & 4. ass-a & 6.512-2 \\
\hline & 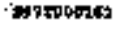 & a.t & & & & 214 & 1. $554+41$. \\
\hline & 9tprado161 & $\nabla \%$ & 11 & & ס्रा & $2.5 \mathrm{~m}=40$ & a.4T-4a \\
\hline & 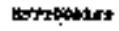 & 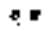 & exis & $\mathbf{s}$ & otath & س. & 4. ber-at \\
\hline & ostratald & $0 *$ & extry & we & Exht & MhL & 2. CStatid \\
\hline & 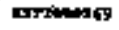 & $\boldsymbol{r}$ & & & pard & $\therefore 4,2+2-x$ & V D.dAbrt \\
\hline & otrintancs & & & & Dand & 1. . - th & $1.52=01$ \\
\hline & prisentai & 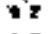 & & $\boldsymbol{I}$ & porst.. & $\underline{m} \boldsymbol{r}$ & DASEraz \\
\hline & Mraemil & 15 & exre & Ixt & monm & $\pi / 2$ & $2.412+01$ \\
\hline & 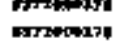 & 17 & 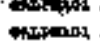 & ज्ञा & ecam & $\begin{array}{r}1.000 \\
1.09\end{array}$ & 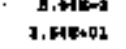 \\
\hline
\end{tabular}

\section{Final page for worklist 17066}

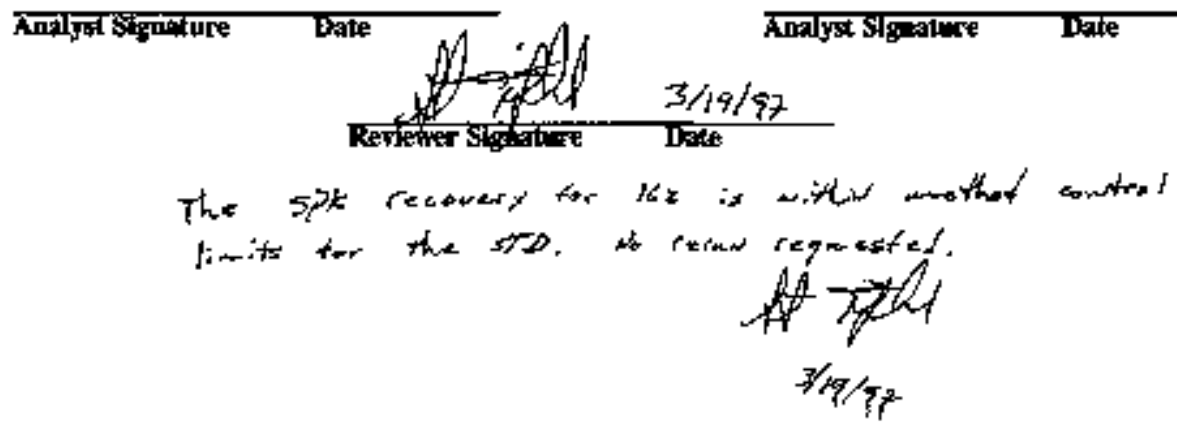

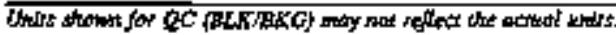




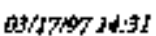

HWF-SO-WN-DP-230, FEV 0

Page: I

A.000 - I

LABCORE Data Entry Template for Worklist \# 17066

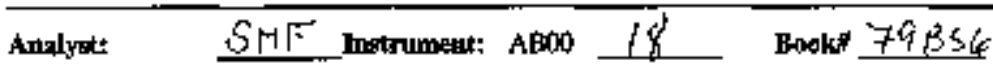

Method: LA-508-101 RewMad Fro

Forklist Cominent: Us: $100 \mathrm{~mL}$ sagple size. Rernen \#1. SLF

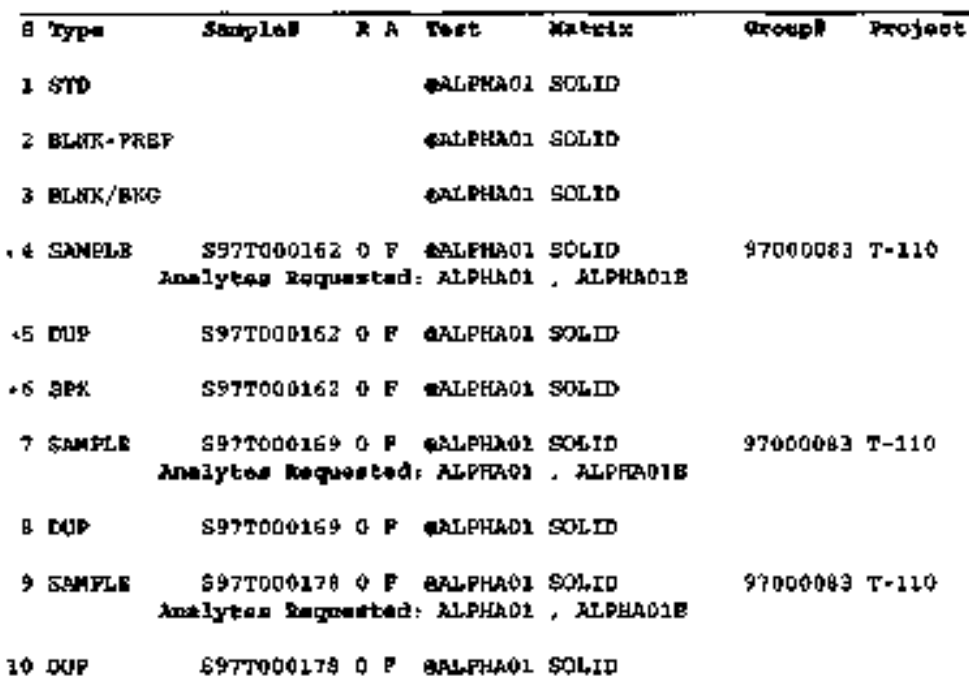

Final page for worklist \#17066

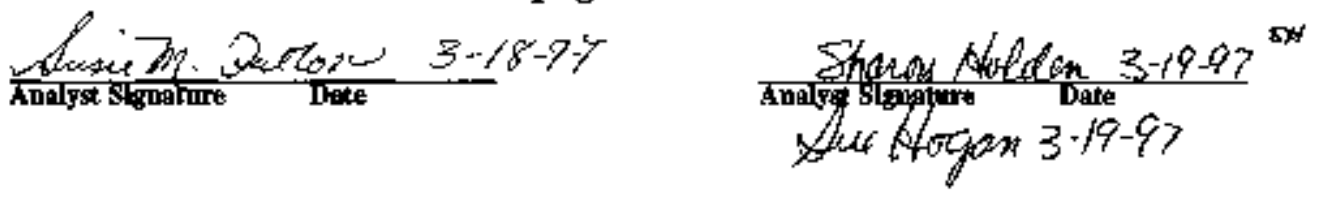

Dom Buny Cantwart:

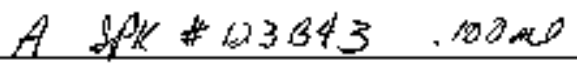

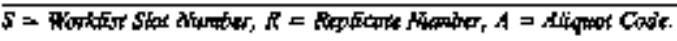

546 
HWF-SD-WW-DP-238, REV. O

WOMXOOCK PARE BTDI

AT : LA-69:109 (E-1)

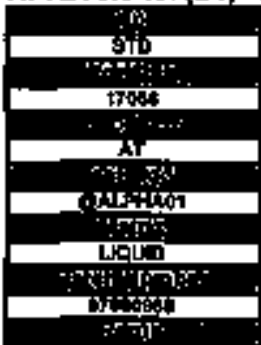

\begin{tabular}{|c|c|c|}
\hline & & ming \\
\hline
\end{tabular}

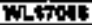

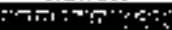

부에요

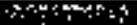

사을

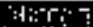

HF

$\because \cdots$

自前

:ד:"

aying

$\therefore 7: 12: 0$

Eलizm

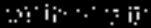

04050 Fin

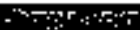

TAt
LA-GBS-113 (B-O) 8TANOARD

BEFCivial:

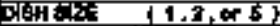

cFogB coumit:

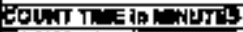

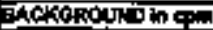

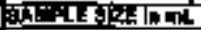

JuLITON FACTR

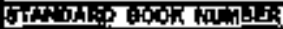

J IFG ERE FACTON

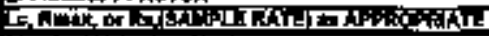

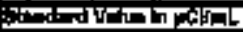

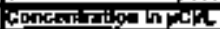

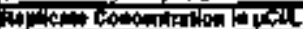

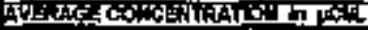

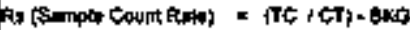

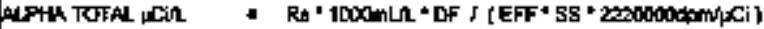

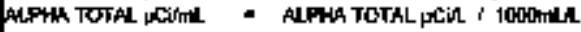

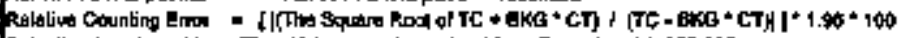

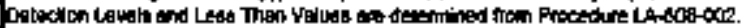

(6)

(OFr)

त्ञ

순.

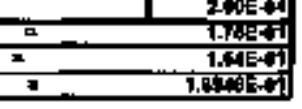

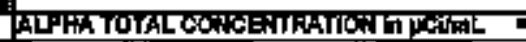

\section{(1)}

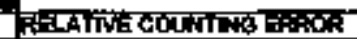

\begin{tabular}{|c|c|}
\hline $\bar{E}$ & $18=54$ \\
\hline $\bar{\theta}$ & 35 \\
\hline
\end{tabular}

1

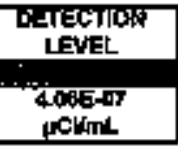

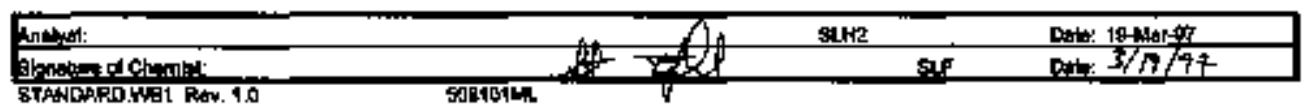




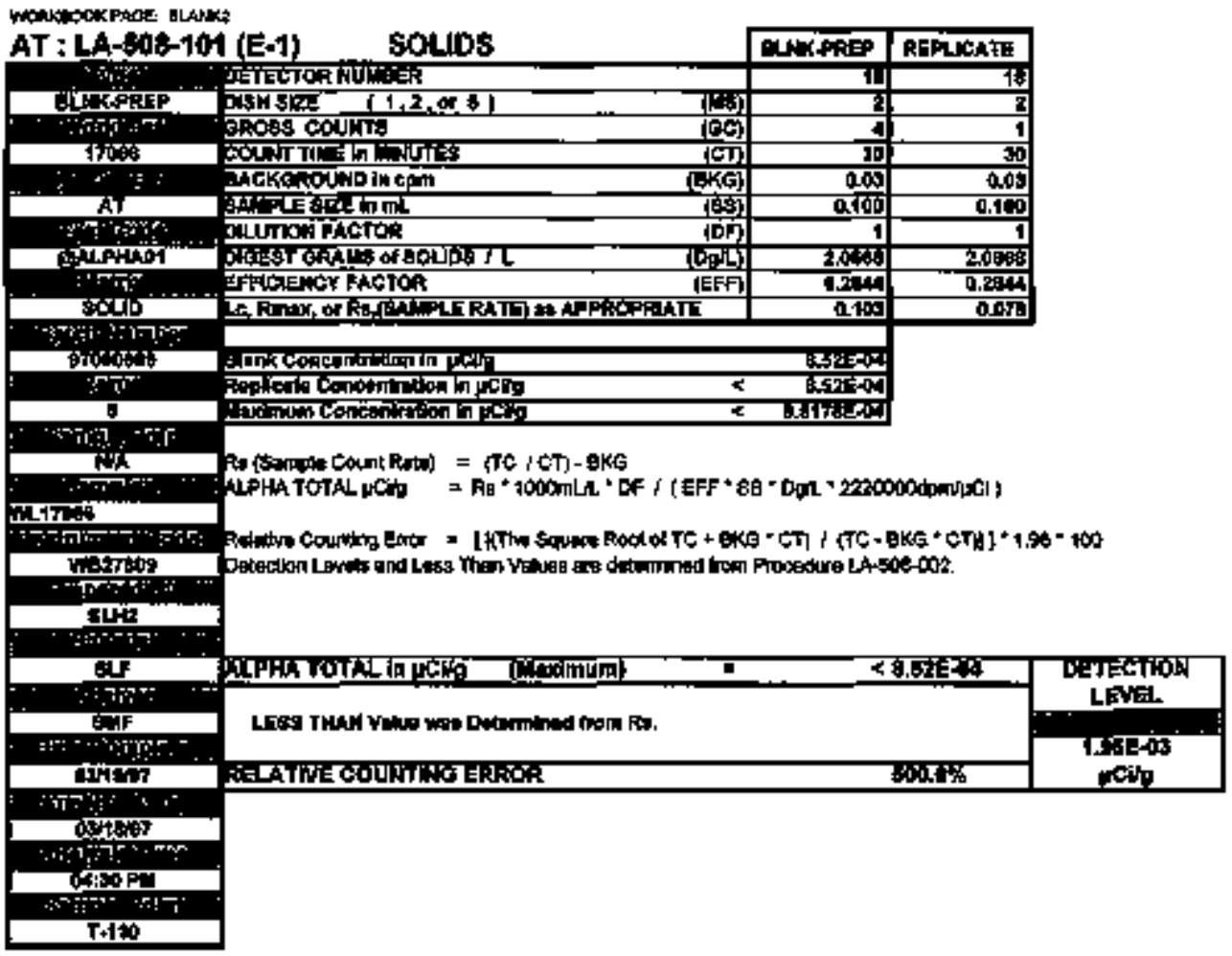

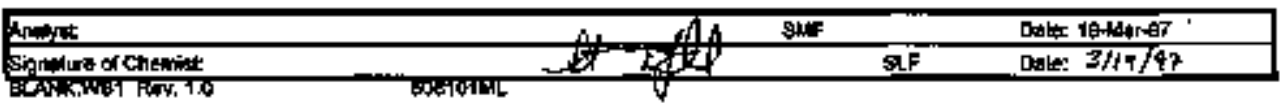




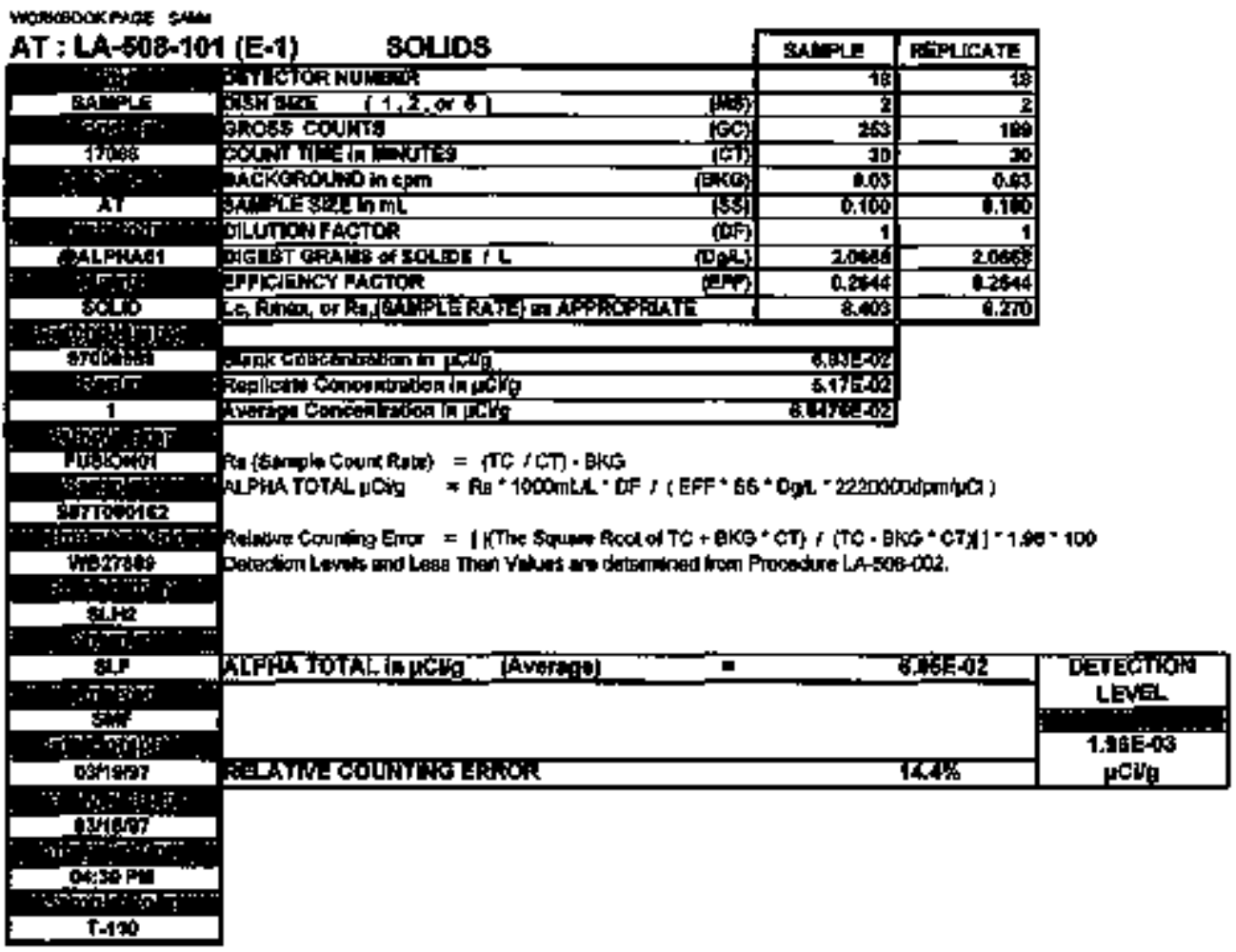

\begin{tabular}{|c|c|c|}
\hline And & 화박 & 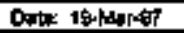 \\
\hline 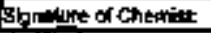 & St.F & Dotul $3 / 9 / 97$ \\
\hline
\end{tabular}


HNF-SD-WW-DP-236, REV. O

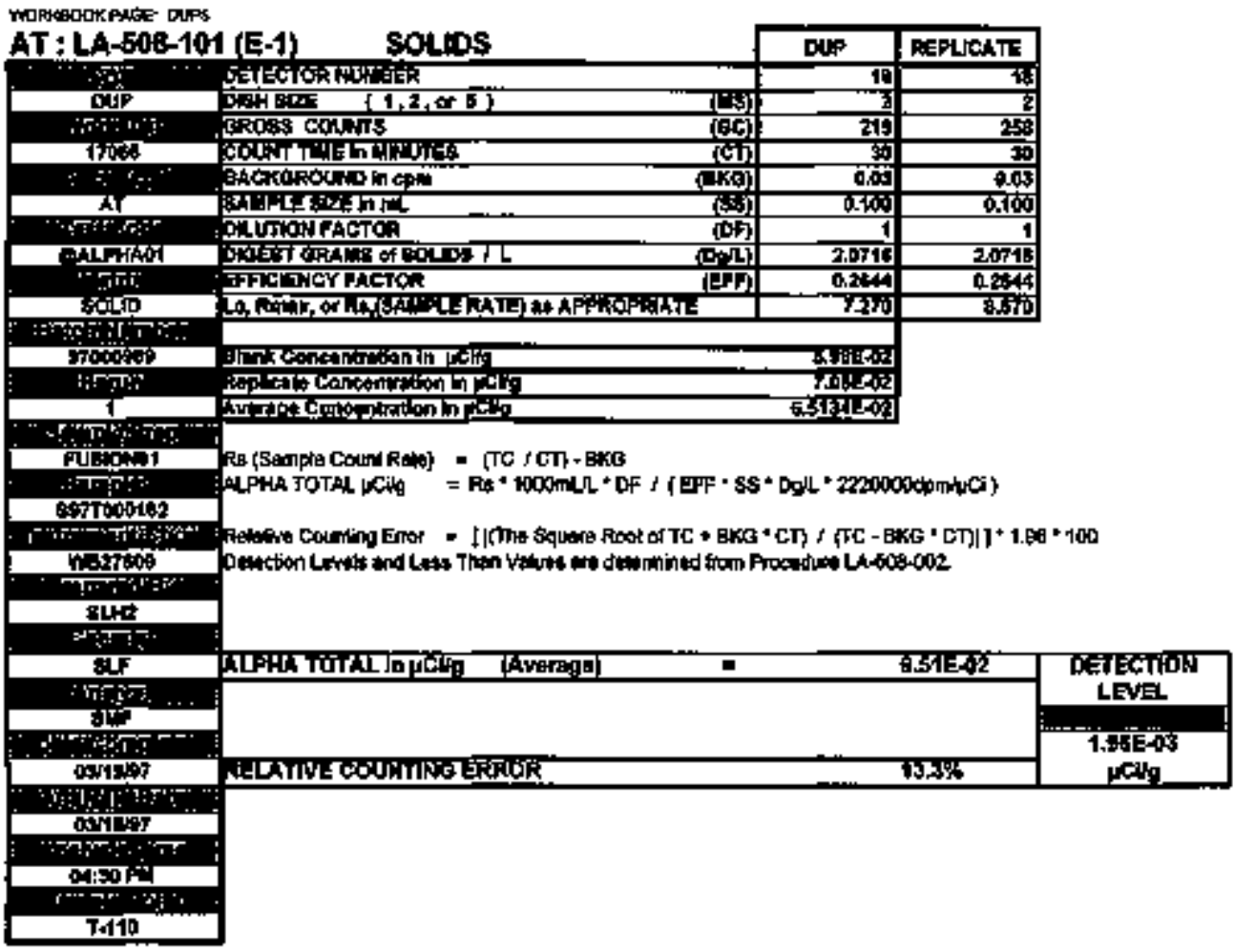

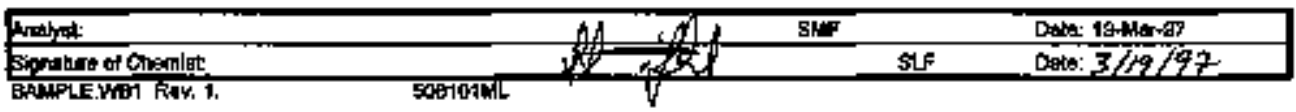


HWF-SD-WH-OP-2BS, FEV, O

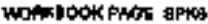

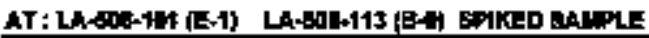

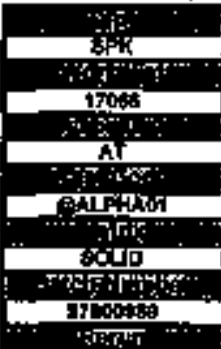

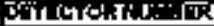

Fisस:

TOTh COUnTs

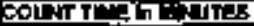

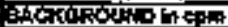

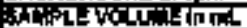

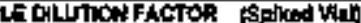

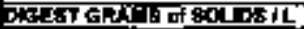

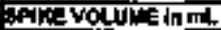

उFiri:

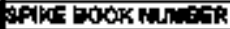

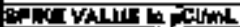

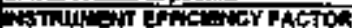

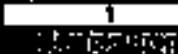

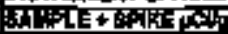

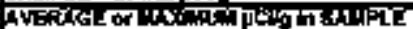

(1)

sike

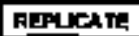

(15)

[TC]

(4)

ref?

(D)

b

[iㄷㄴㄷㅣ

torfoll

|B:F|

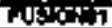

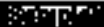

8.MTOHक

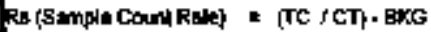

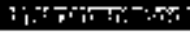
martos

: r, i, $-:-2$.

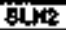

너

EंF

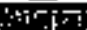

और

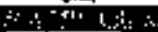

कांगिक्ष

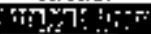

051025

$\therefore \quad 1,7: \div \quad \div: "$

at+20 Bis

MHAPLE + GPIKE vCHA

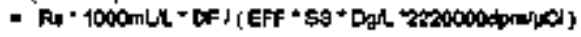

AC ACTUAL, $\%$ SVI

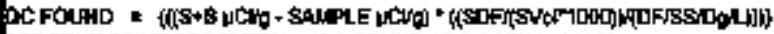

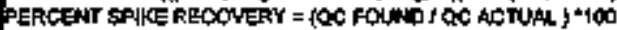

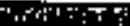

$T \times 10$

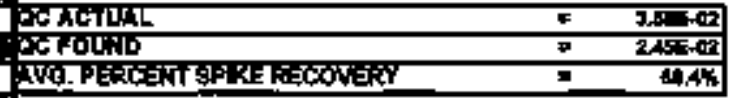

Prabst:

sojume of chmint

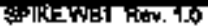

다전다는

sutz

Oniv; 19410067

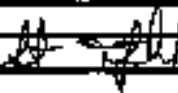

이다

Dim: $3 / 1 / 97$

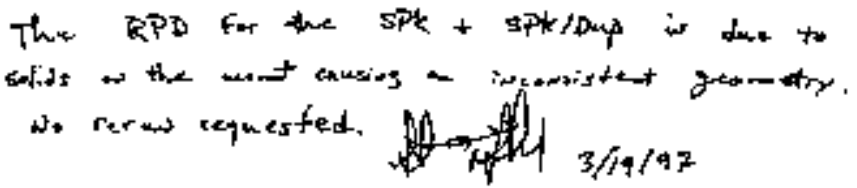


HNF-SD-WH-DP-238, REV. 0

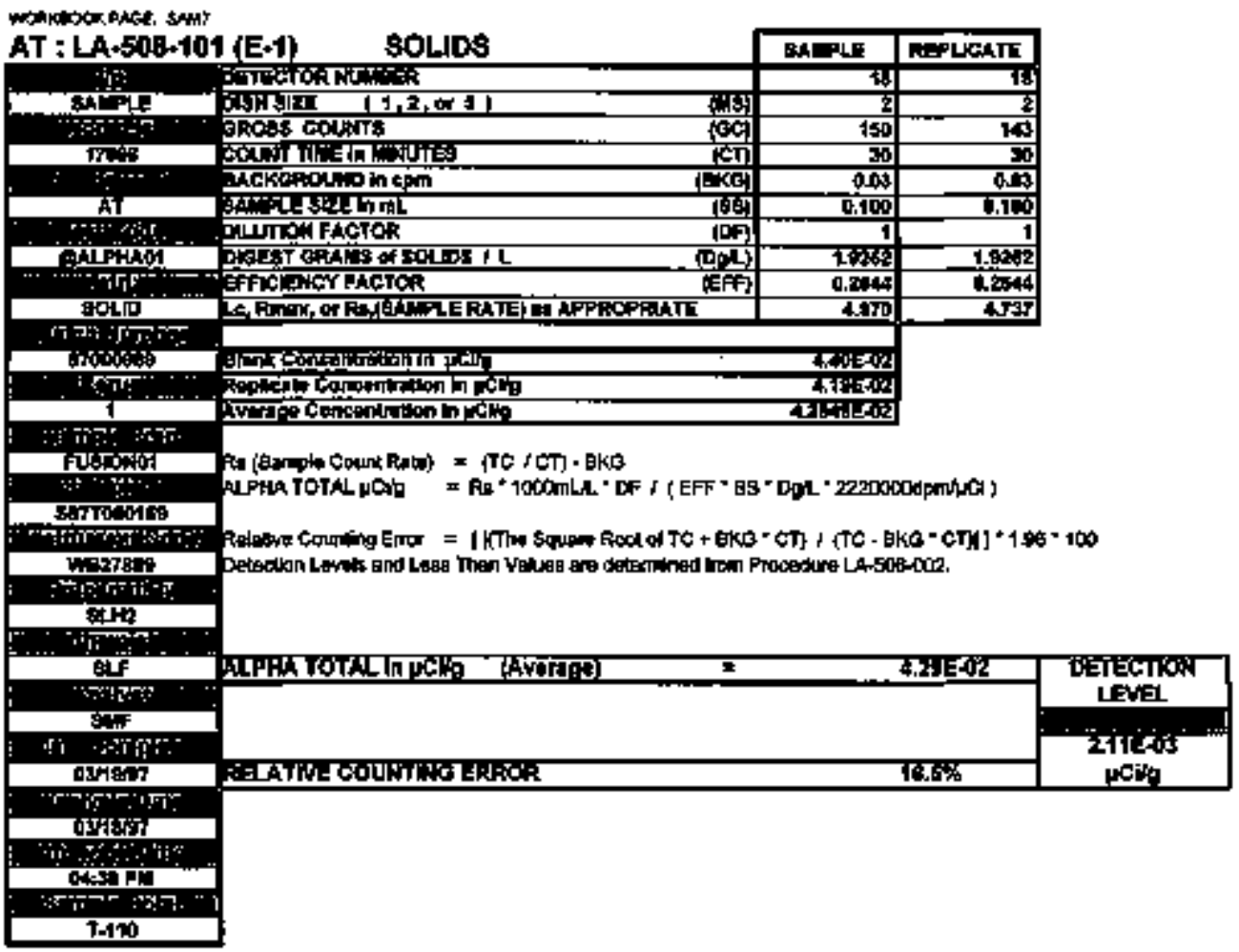

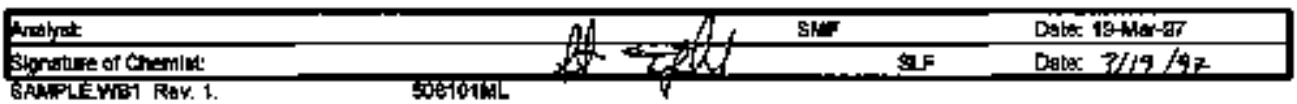

\section{2}


FNF-SD-WH-DP.Z3B, REV O

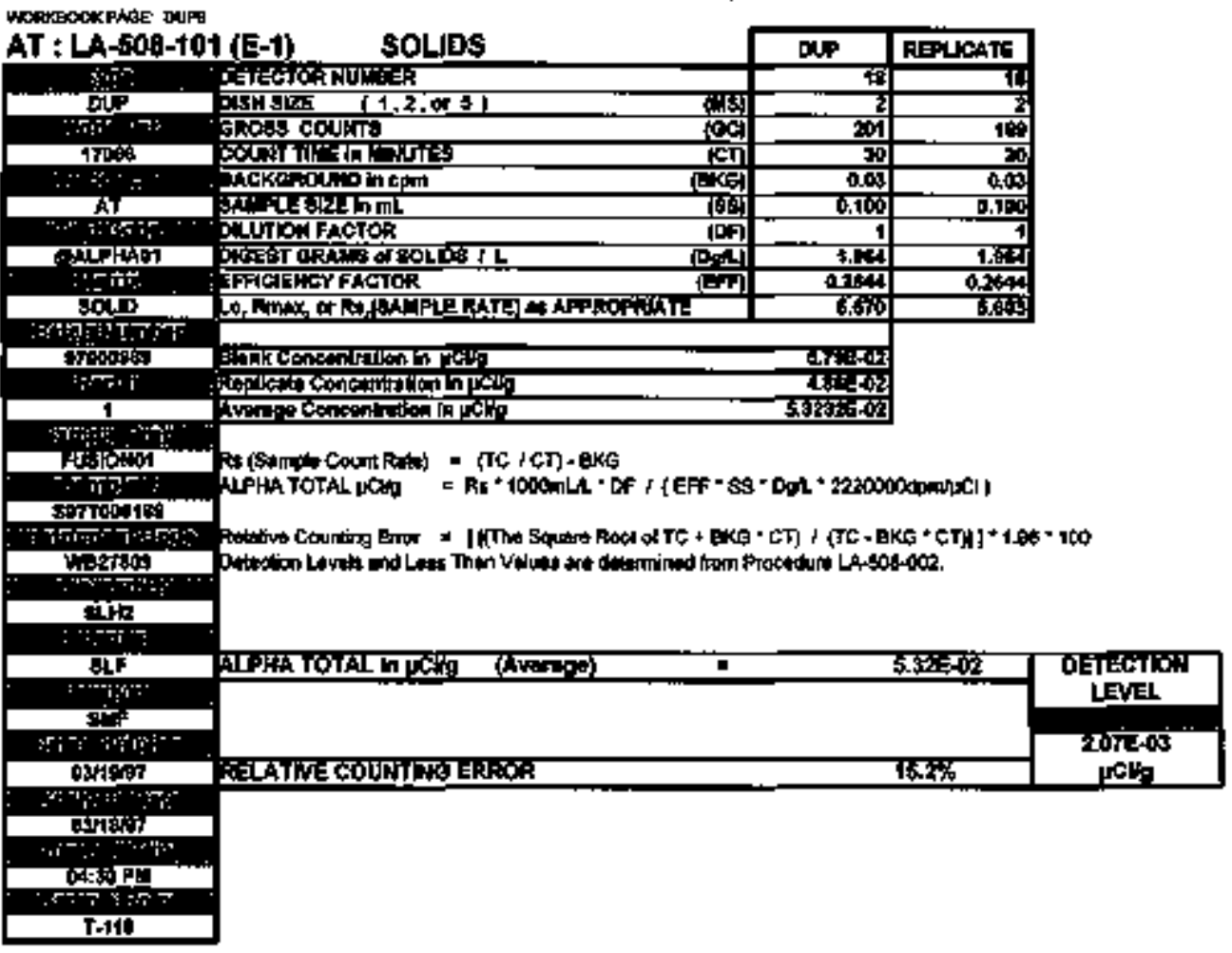

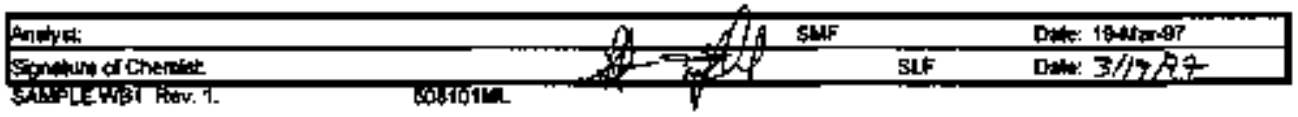


HNF-SD-WW-DP-2\%6, REV. 0

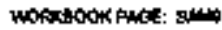

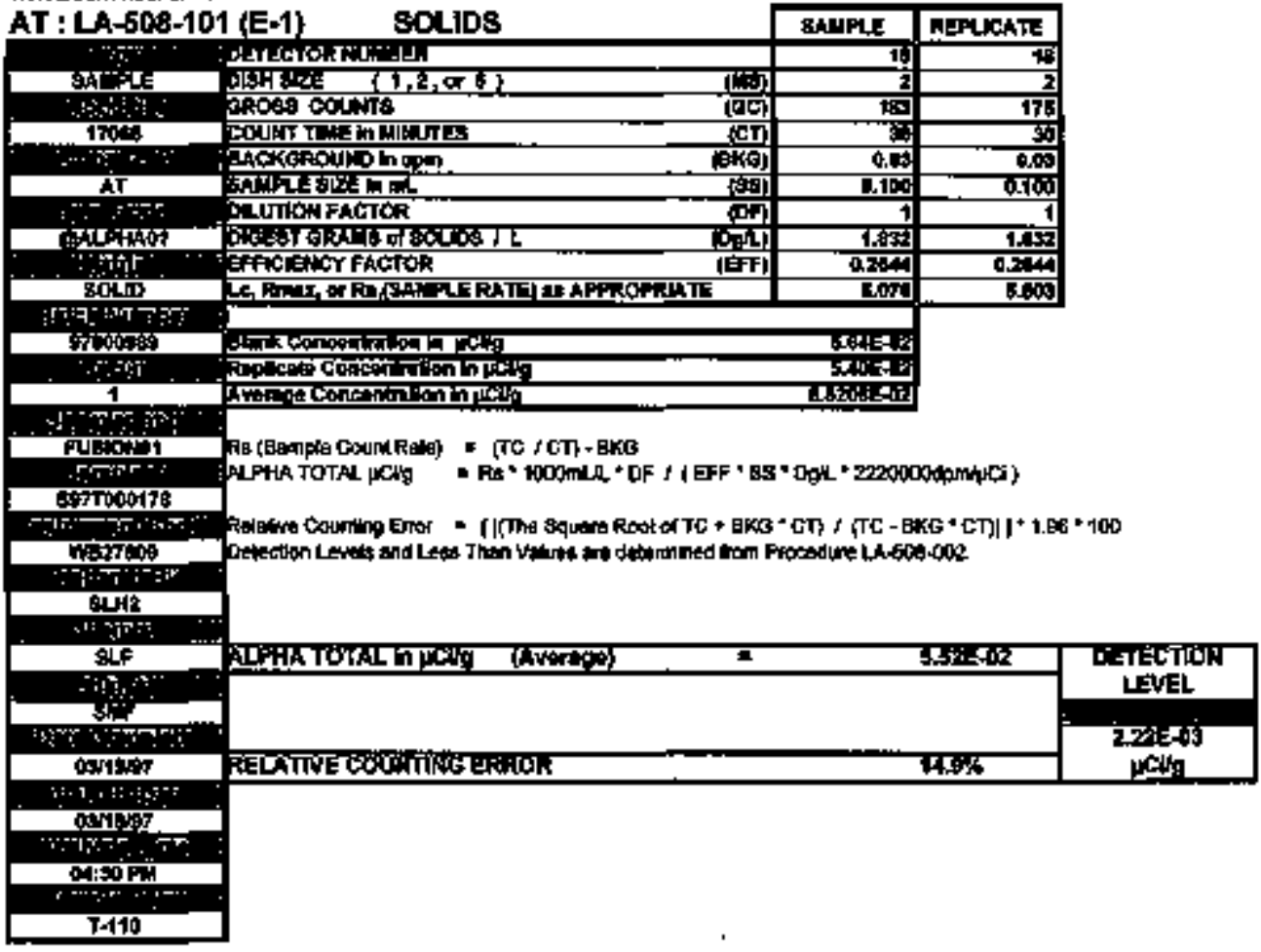

\begin{tabular}{|c|c|c|}
\hline Arabod: & 白位 & Data: 19-Mar-gl? \\
\hline
\end{tabular}




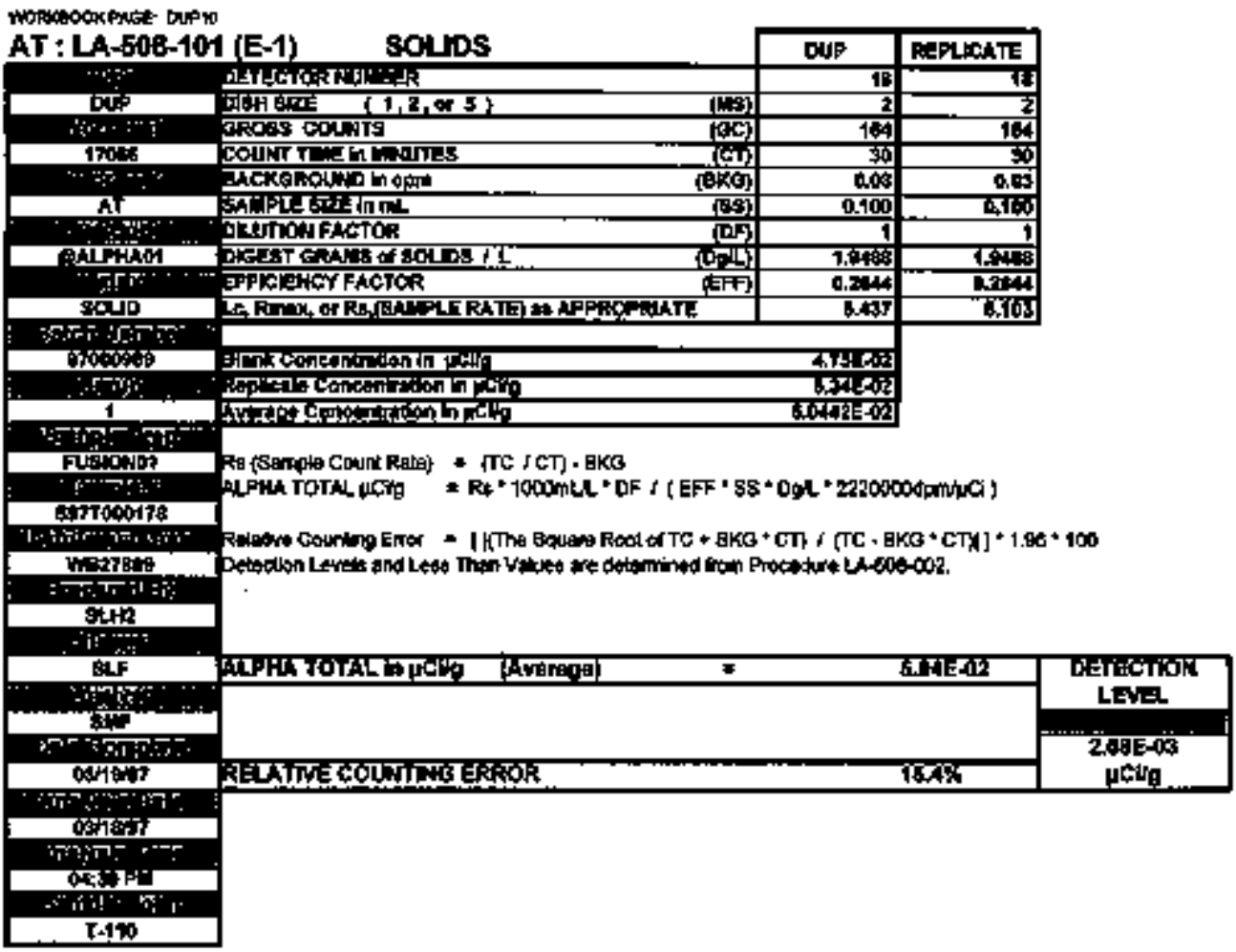

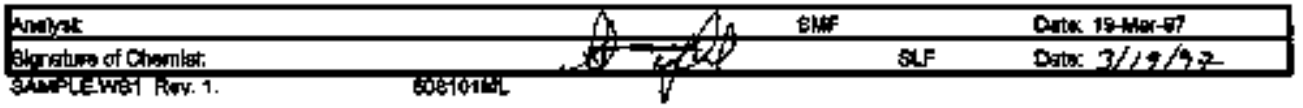




\section{LABCORE Completed RadChem Report for Worklist:; 17067}

Anshyt: sonf

Instrumen: ABI3

Book:\#

Method:

Reviluod

Torklist Comment Use 100 mL sample size. Rerun 12 SLF

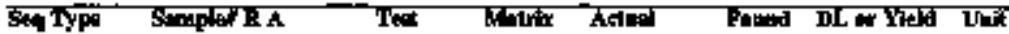

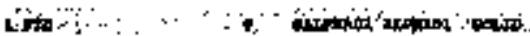

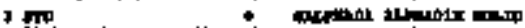

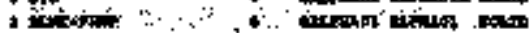

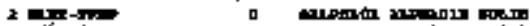

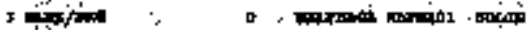

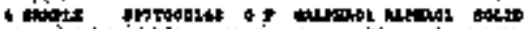

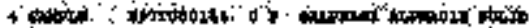

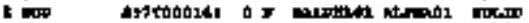

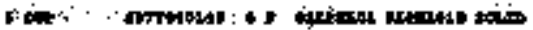

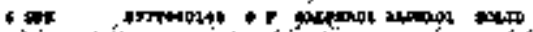

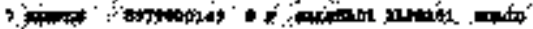

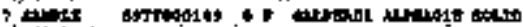

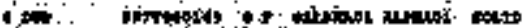

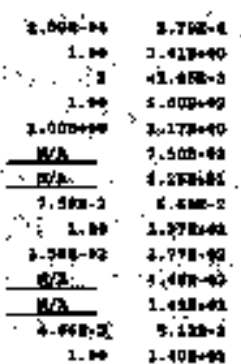

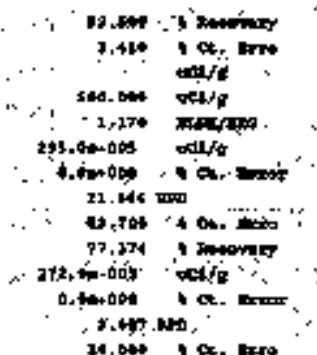

on

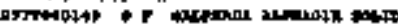

\section{Final page for worklist: 17067}

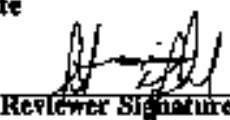




\section{LABCORE Data Entry Template for Worklist\# 17067}

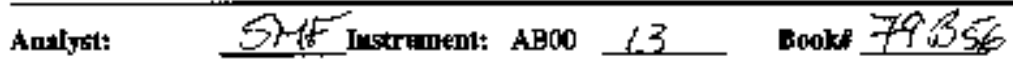

Method: LA-S008-101 RevMbod FO.

Workllst Comment: Use $.100 \mathrm{~mL}$ \$anple size. Rerun \#2. \$LP

\begin{tabular}{|c|c|c|c|c|c|c|}
\hline 1 Typd & 8anglet & $\pi x$ & Tost & Hetrix & Exoupl & Pastect \\
\hline I sTt & & & OXLPERO & 50LID & & \\
\hline 2 EESTR-PREP & & & orretadol & \$OLID & & \\
\hline 9 BLWR/BRG & & & AACPAAO1 & solID & & \\
\hline
\end{tabular}

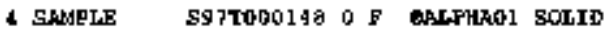

$97000083 \mathrm{~T}-110$

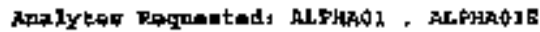

5 DOP S9TTODOLA D F EATHAOI SOLID

6 SPK S97TOHO219 O F BAPHAO SOLID

3 SANALE S97TO0O149 O F ANLFHa

$97000093 \quad T=110$

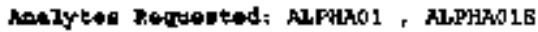

3 DurF

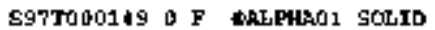

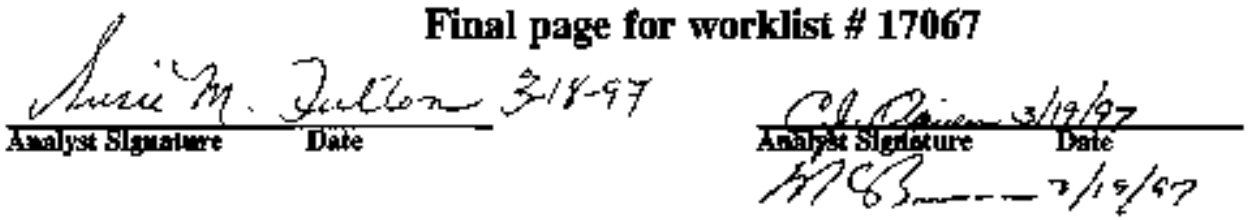

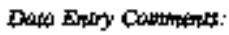

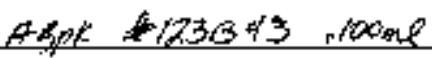


HWF-SD-WA-DP-239, FEV O

MoPKBOCX PMrE BTD

AT ; LAtho-191 [e-1]

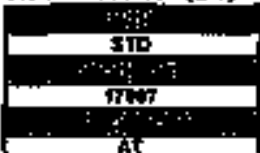

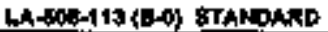

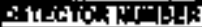

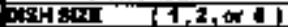

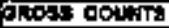

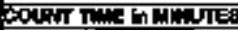

gackeriovido G epm

$\because$ T; $r_{i s ;}$

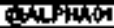

"ị:

บ1்

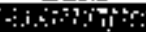

rovisis

$\therefore: 1$,

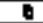

ONmin

Fution Fitcis

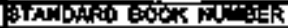

JAFC|ECY FMTOR

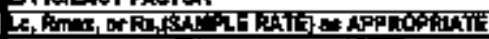

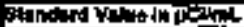

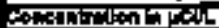

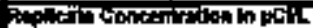

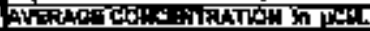

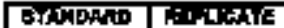

IIf

[E다.

15i

ink

[Fing)

[D.ग)

(B)A EM

IEF)

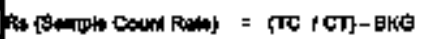

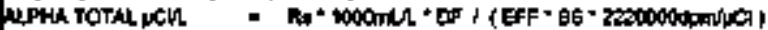

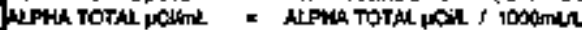

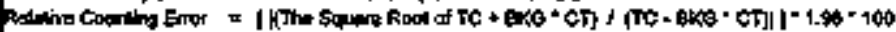

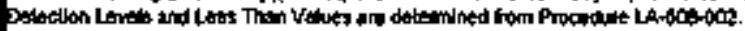

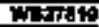

$\because \because * \because * \because \div$

960

$\therefore \cdots:$

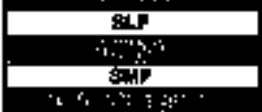

ब14

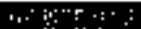

क्षिमें

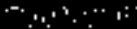

CHisfin

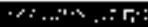

T-1T0

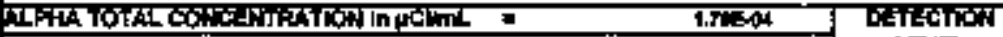
노는 rollml

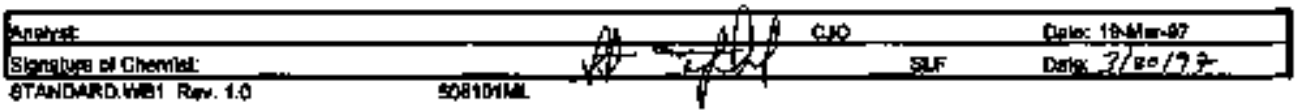




\section{HNF-SD-WH-DP-238, REV. O}

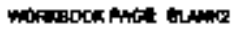

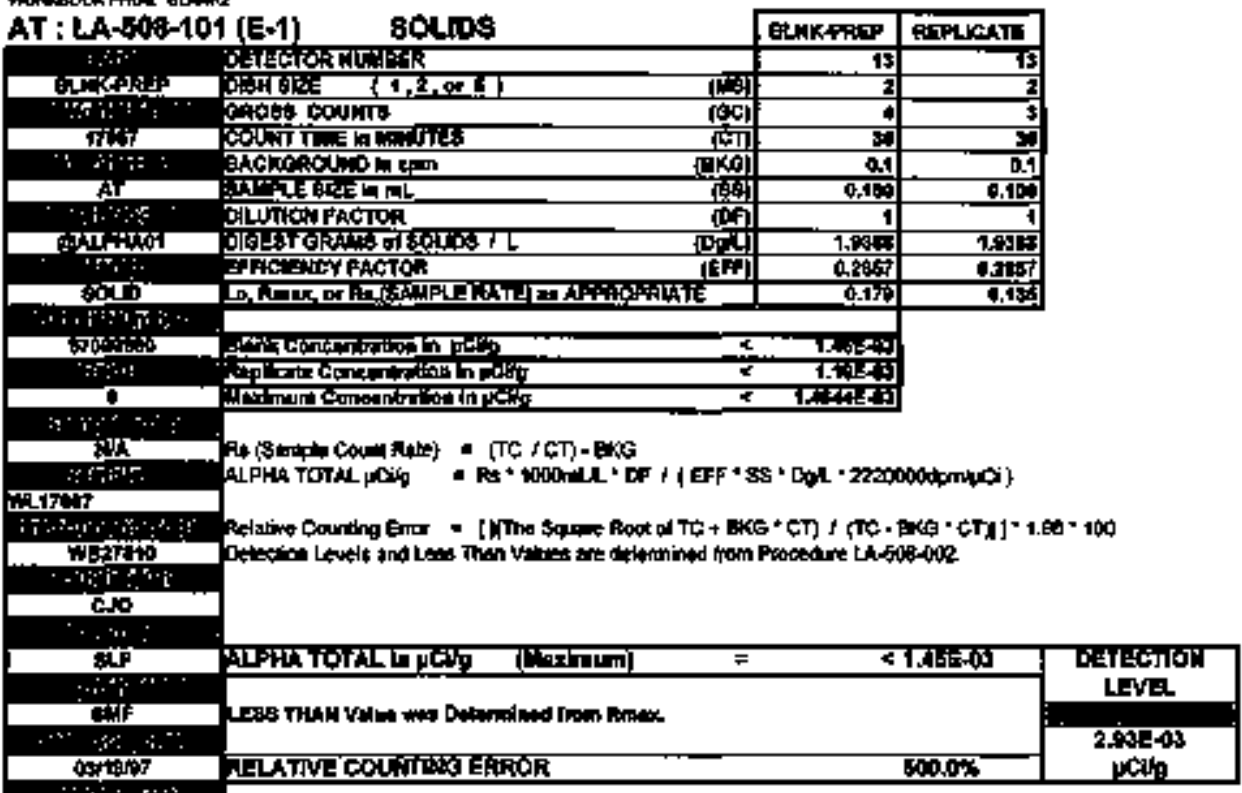

$\therefore \because \div: c: ?$

ophis

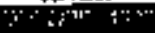

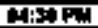

$\therefore::^{*} \times 5 \%$

F-11D

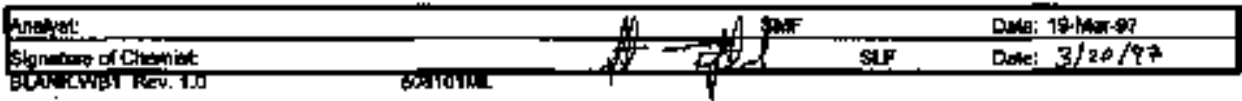


HAF-SD-WW-DP-238, REV. 0

HonkBarck DAnE BAM

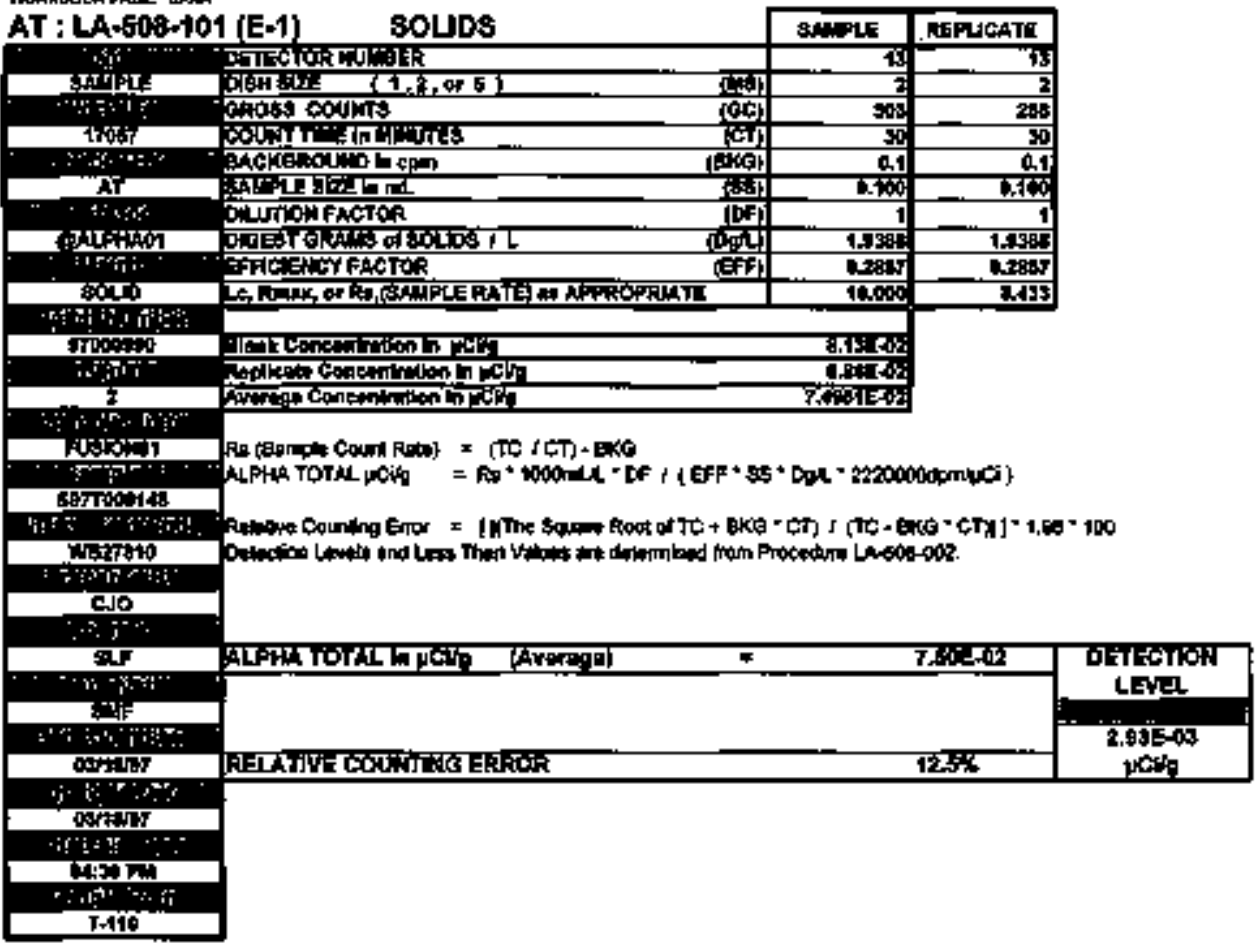

\begin{tabular}{|c|c|c|}
\hline Andred: & ENF & 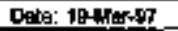 \\
\hline Slonature of Chemint: & 야 & Detre $3 / 20 \sqrt{4}$ ? \\
\hline
\end{tabular}


HNF-SD-WH-DP-238, FEV. O

Whock Ante oups

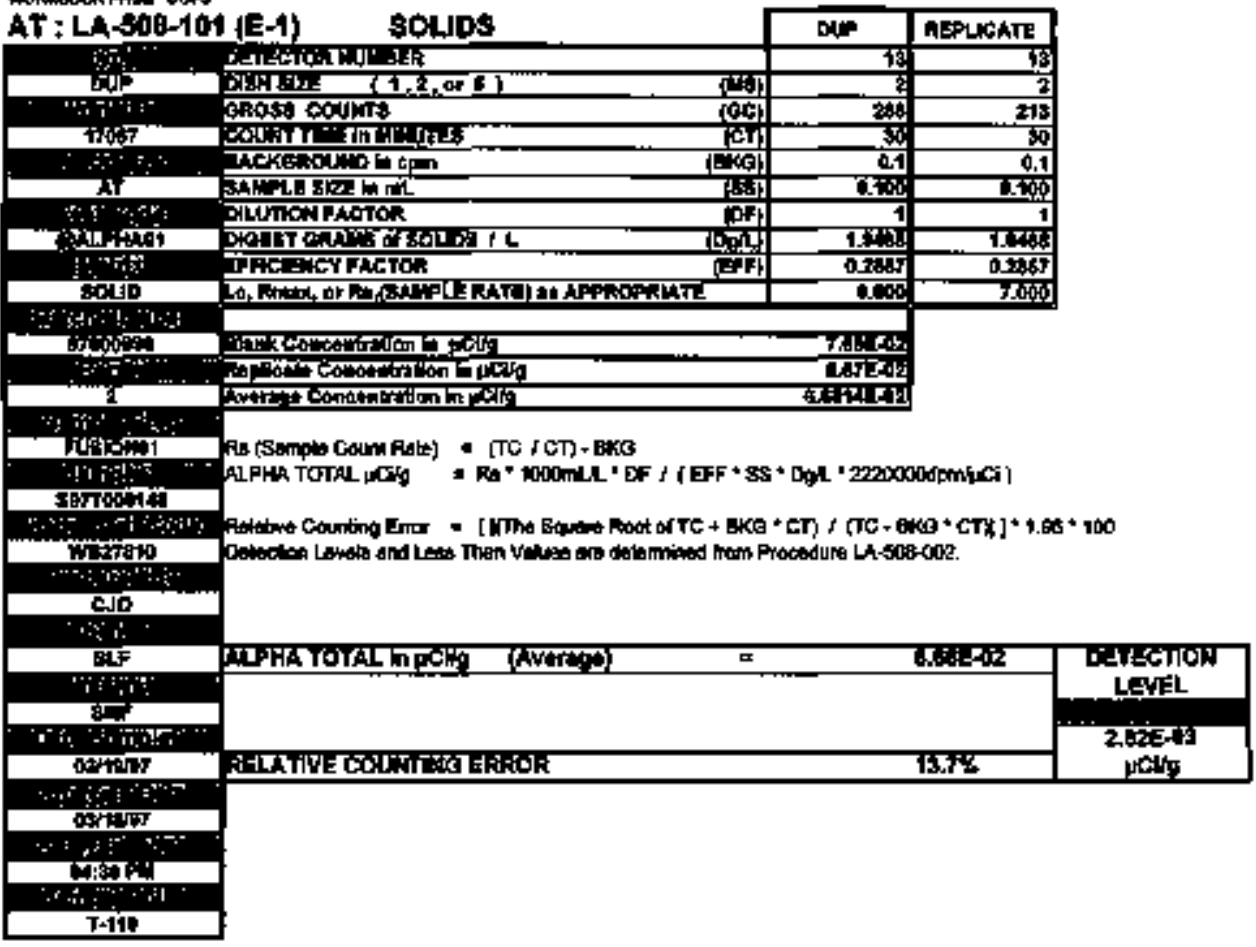

\begin{tabular}{|c|c|c|}
\hline Andret & EMF & Ow. Th- \\
\hline 3. hoture of Chamis. & SLF & Date $3 / 20 / 97$ \\
\hline
\end{tabular}


HNF-SD-WW-DF-238, REV. 0

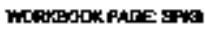

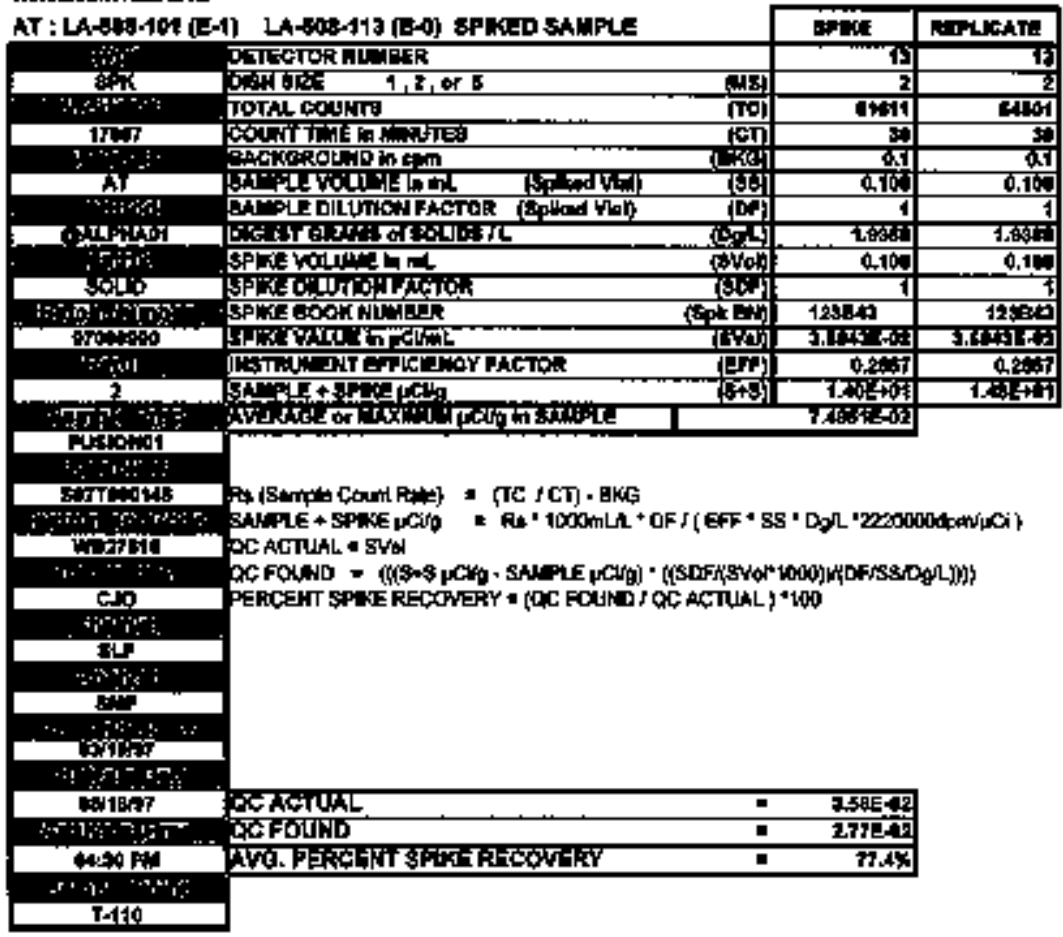

\begin{tabular}{|c|c|c|}
\hline 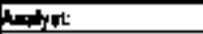 & 610 & 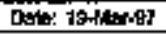 \\
\hline 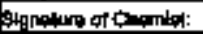 & sLf & 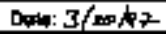 \\
\hline
\end{tabular}


HNF-SD-WM-DP-298, REV. O

monkookent: sent

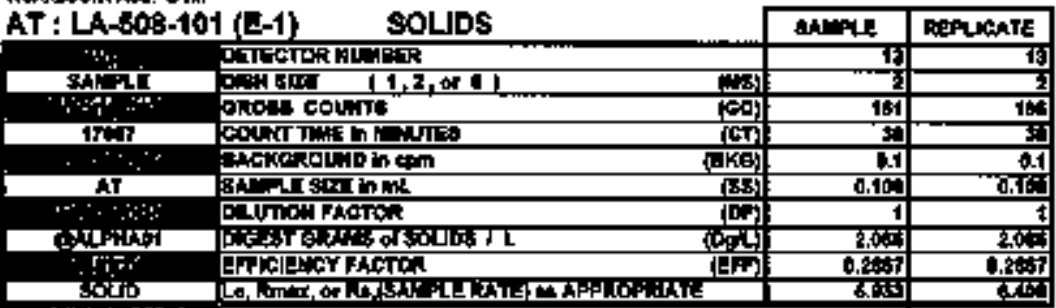

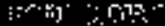

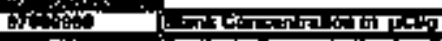

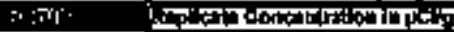

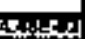

2 1

$4 \mathrm{~T} \times 1=4 \mathrm{C}$

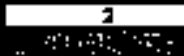

FUim

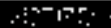

Dimpore

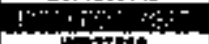

intonto

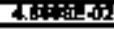

in:

ס

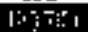

85

$\therefore \dddot{u}^{*}$ :

ind

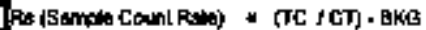

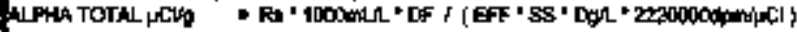

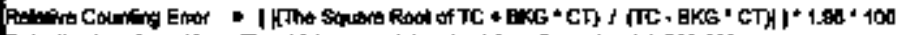

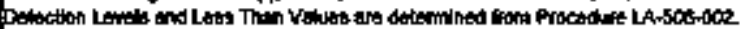

r.: : :

ETाt?

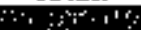

क्रांक्ष

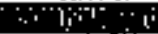

Cingin

$\because \cdots+1, \cdots ;$

$\mathbf{T}-\mathbf{4} \mathbf{6 0}$

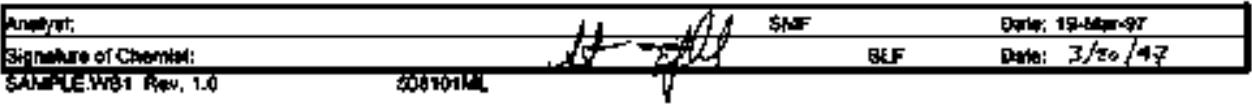


HNF-SD-WA-DP-238, REV, 0

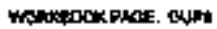

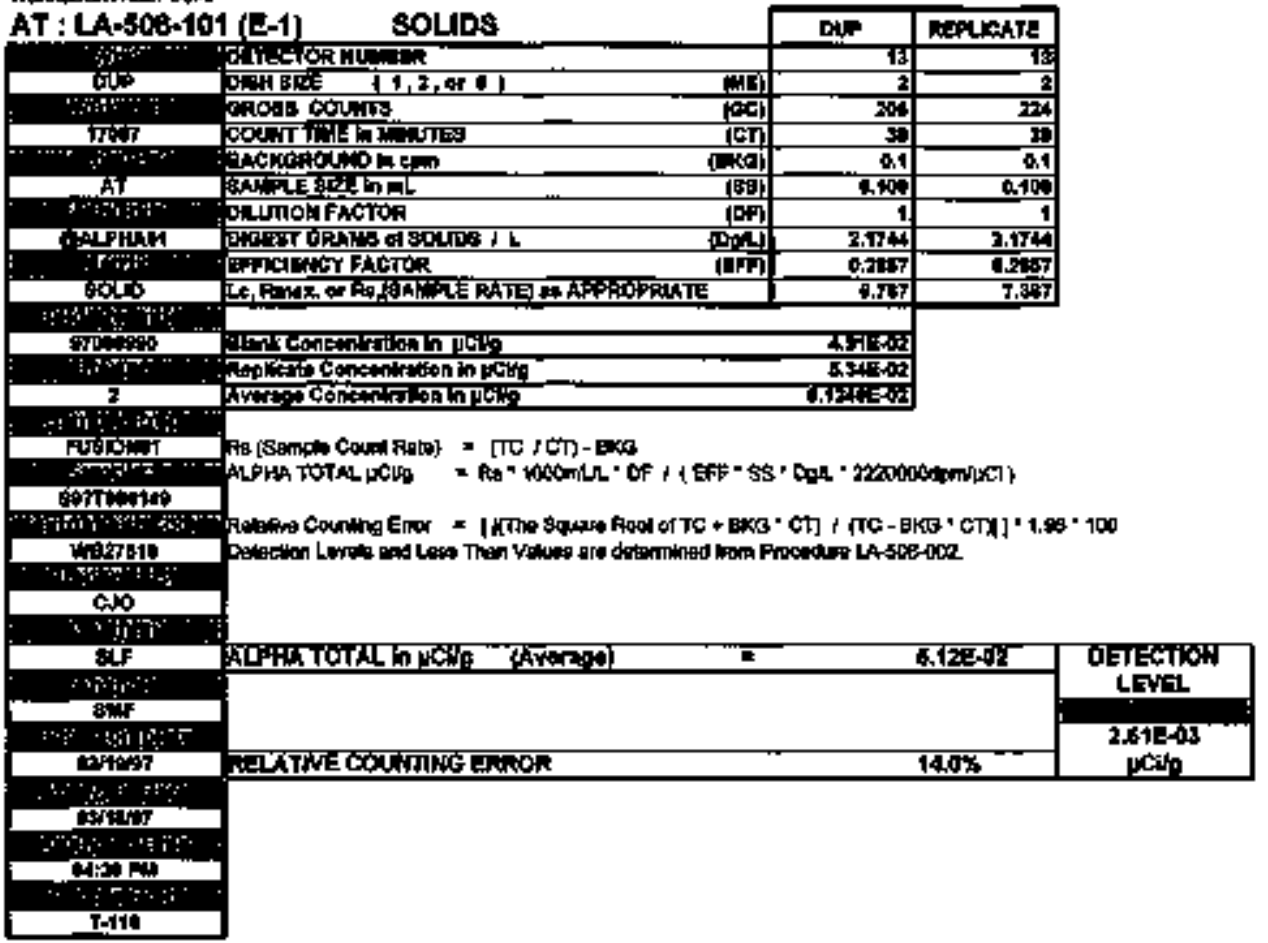

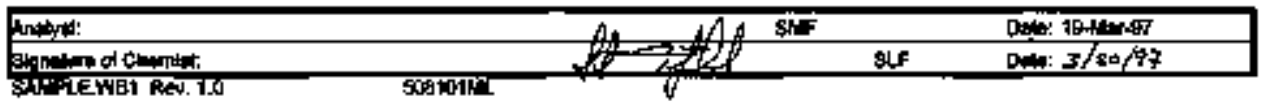




\section{LABCORE Completed RadChem Report for Worklist: 17068}

Analyst: jomv

Imstrmont: AB15

Book"

Method: Bev/Mod

Werkifst Con ment: Use , 100 mL sample size. Rerum \#1. SLF

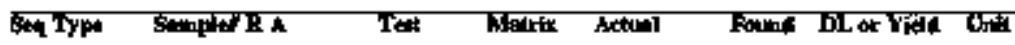

1 "

a.

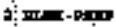

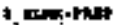

7 口का

4 enerx

+ ctivis

5 bop

i

5 atr.

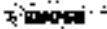

4 anore

1000

- $\mathbf{\infty}$ wrimel suarent porm

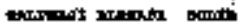

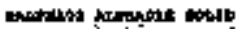

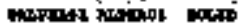

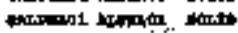

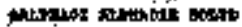

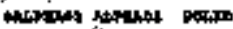

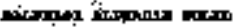

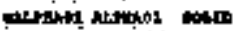

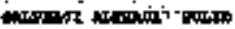

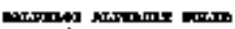

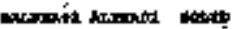
weptar akrowa expo

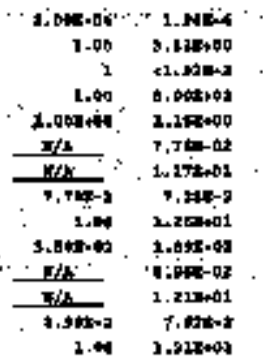

\section{Final page for worklist\# 17068}

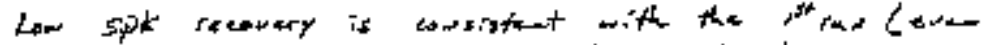

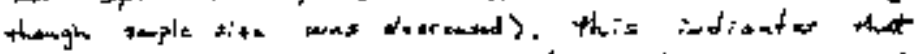
the low recovery is dut 4t matrix isterferece. No reren

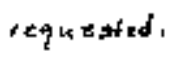<smiles>C#CC1C#CCCCCC1C#C</smiles>

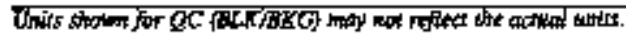




\section{LABCORE Data Entry Template for Worklist" 17068}

Amalyst: $\quad \operatorname{Sind}$ Instrument AB00

Method: LA-S0\&-101 RevMod Fo

Workllst Comment: Use , lôh nL sample size. Rerun "艹 1. SLF

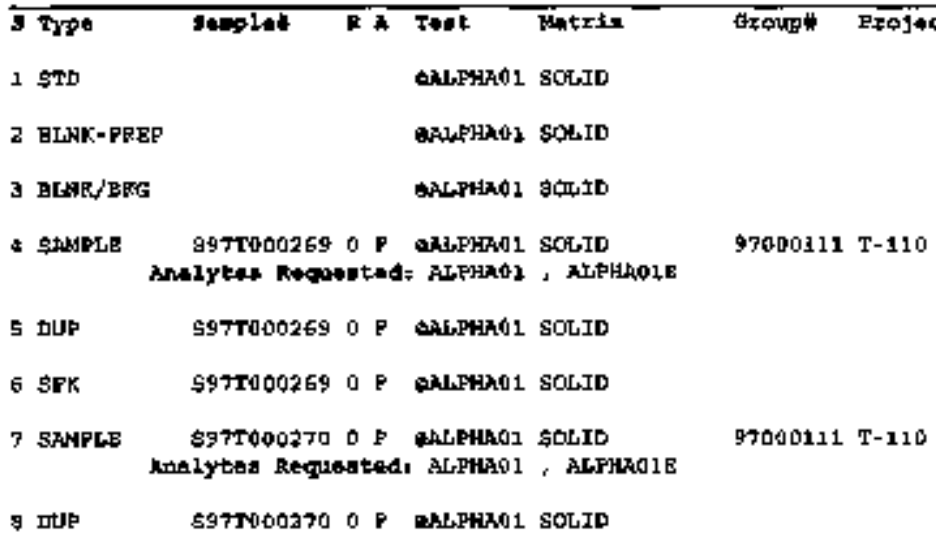

\section{Final page for worklist \# 17068}
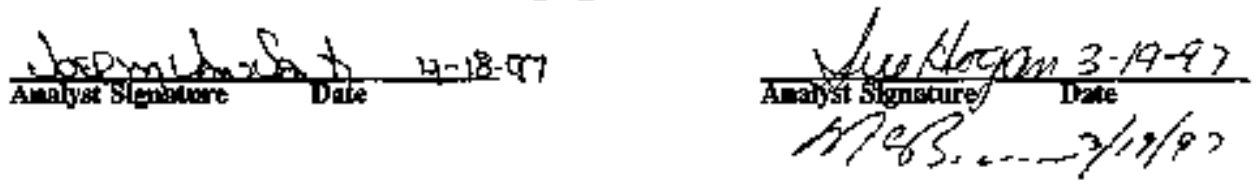

Dew Eury Cooptots:

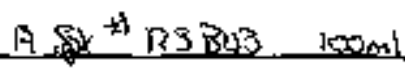

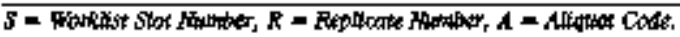


HWF-SD-WM-DP.2XB, REV. 0

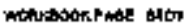

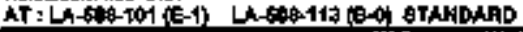

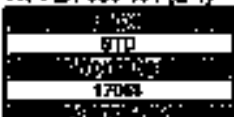

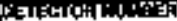

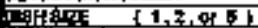

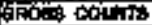

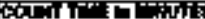

i.

T

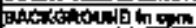

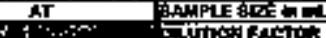

minting

$1, \because \because 3$

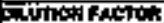

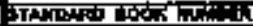

IFFEn PAGTh

पामान

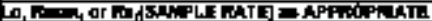

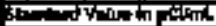

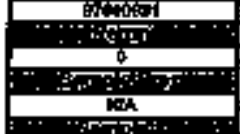

:

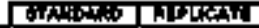

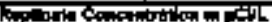

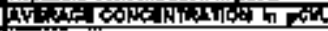

The

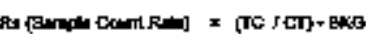

11753

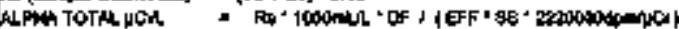

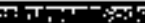

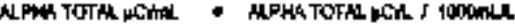

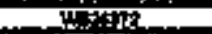

A

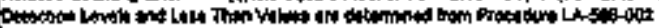

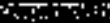

काल

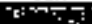

每F

$\therefore$ ता

j:

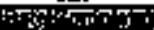

क्षाम

…7\%

Eivipr

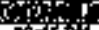

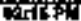

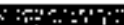

TA110

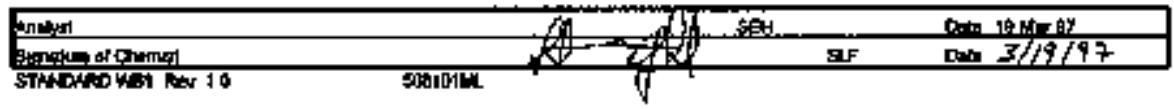


HWN-SD-WMDP-238, AEV, O

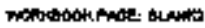

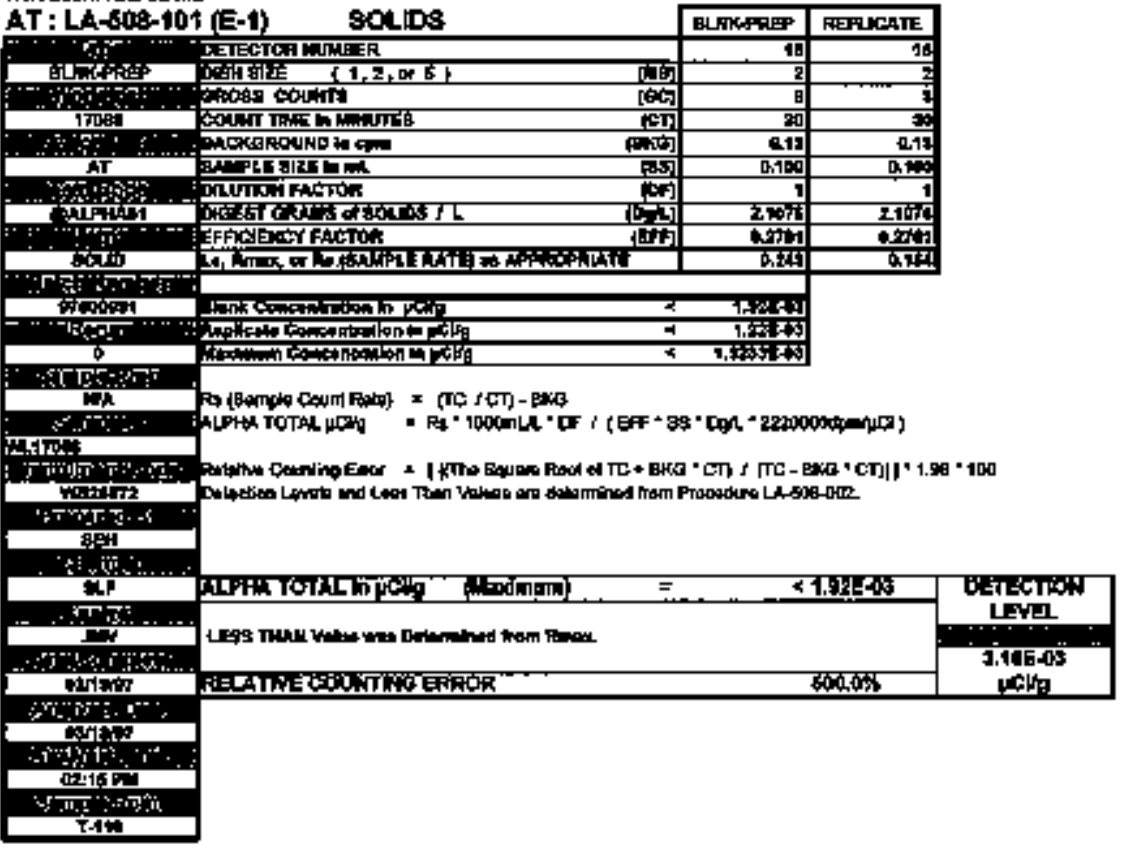

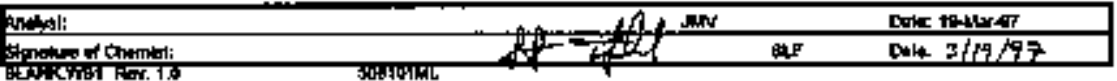


HNF-SD-WH-DP-238, REV. O

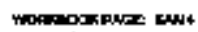

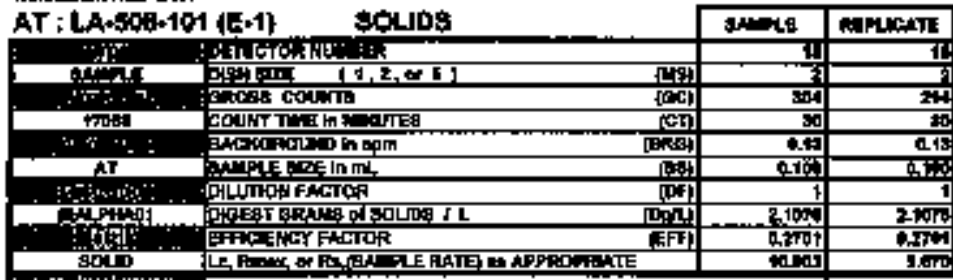

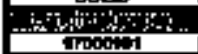
il.

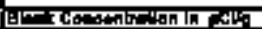

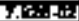

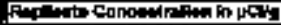

F.晒些

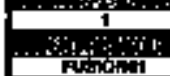

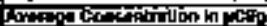

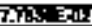

Fischen

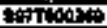

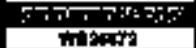

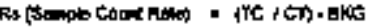

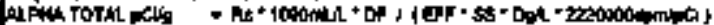

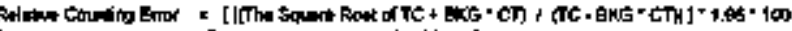

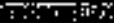

\$H

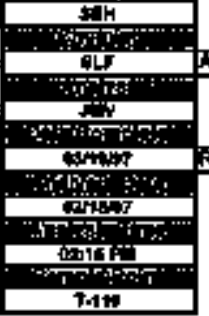

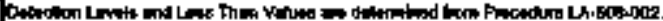

\begin{tabular}{|c|c|c|c|}
\hline 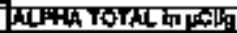 & 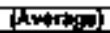 & $x$ & 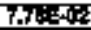 \\
\hline
\end{tabular}

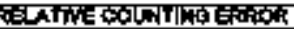

\begin{tabular}{|c|c|c|}
\hline 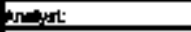 & mor & 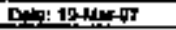 \\
\hline 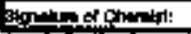 & EyF & oni: $3 / 1>197$ \\
\hline
\end{tabular}


HWF-SD-WH-DP.ZूS, FEV, O

mowhodithat: dutb

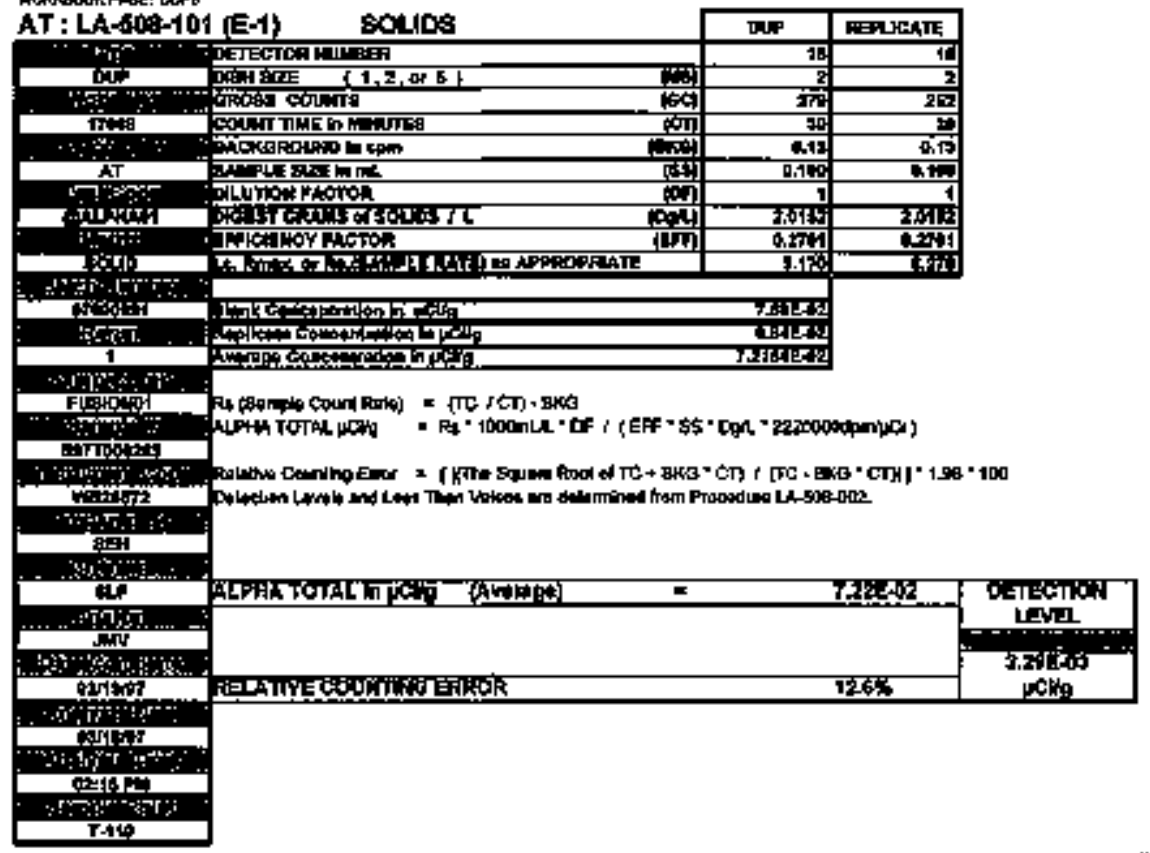

\begin{tabular}{|c|c|c|}
\hline Mtcted: & JWN & Dok: 17 lap-s7 \\
\hline shonown of Giented: & satr & Dun: $3 / 19 \mathrm{~A} 9$ \\
\hline
\end{tabular}




\section{HWF-SD-WH-DP-2SB, REV. 0}

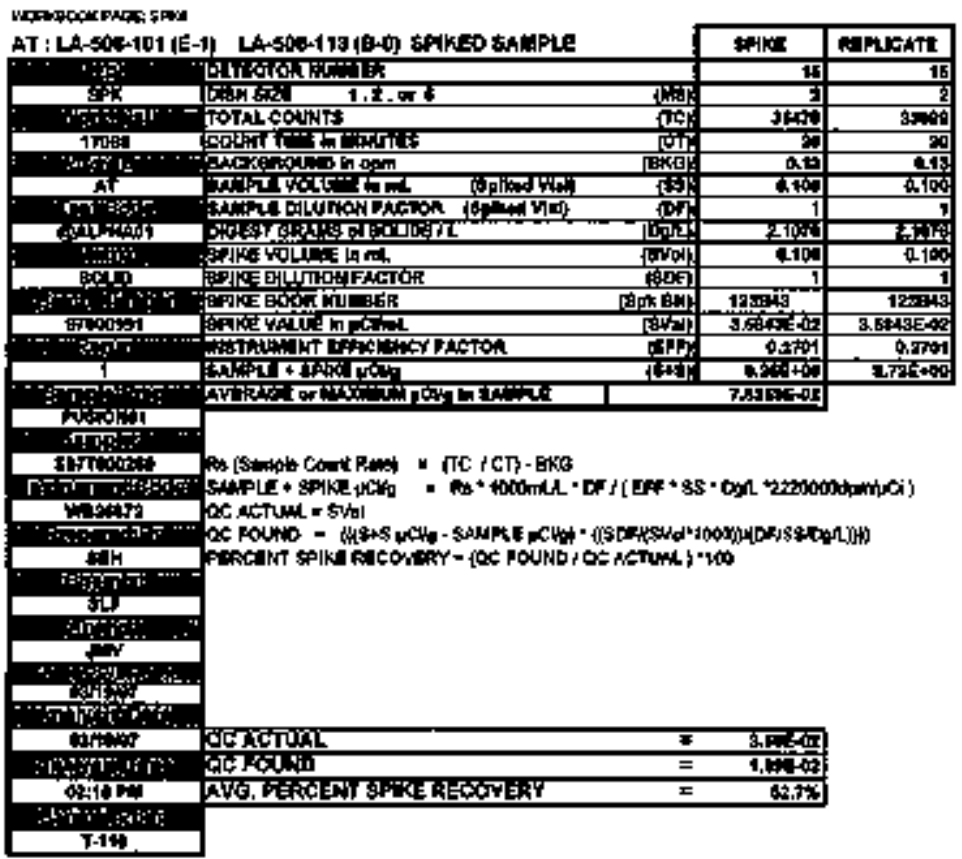

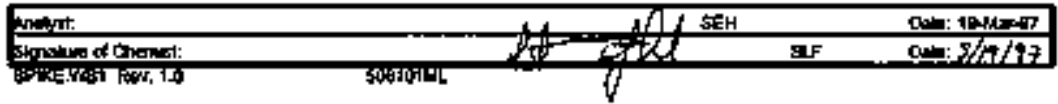




\section{HNF-SO-WH-DP-238, REV. O}

WDARXXKPASE 3wF

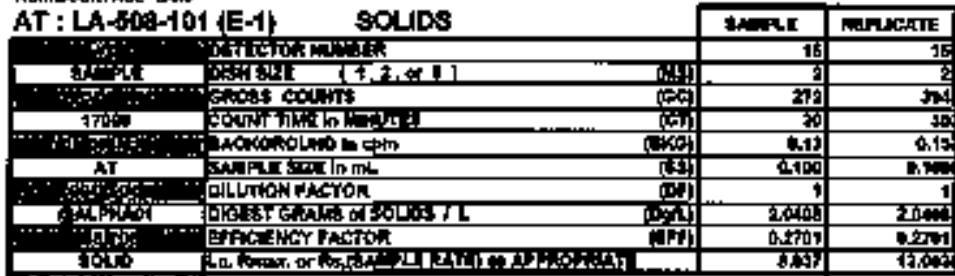

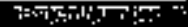

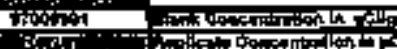

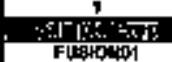

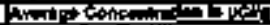

एक्षेत्र

Flespolid

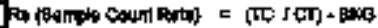

gyime

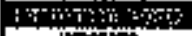

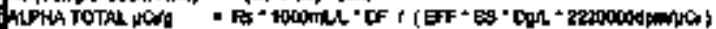

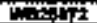

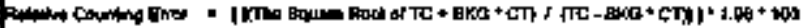

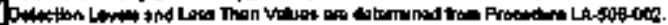

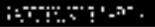

F保

政:

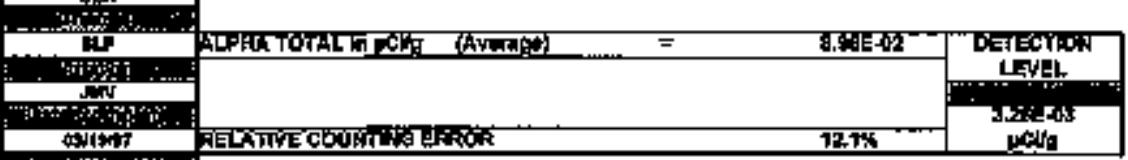

$\therefore: \cdots+\cdots: \cdots$ :

0:गाएव

:

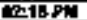

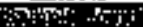

T-I4t.

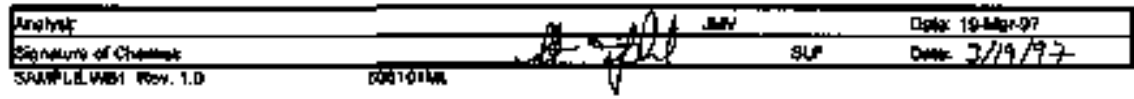




\section{HWF-SD-WHAP-23B, REV 0}

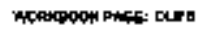

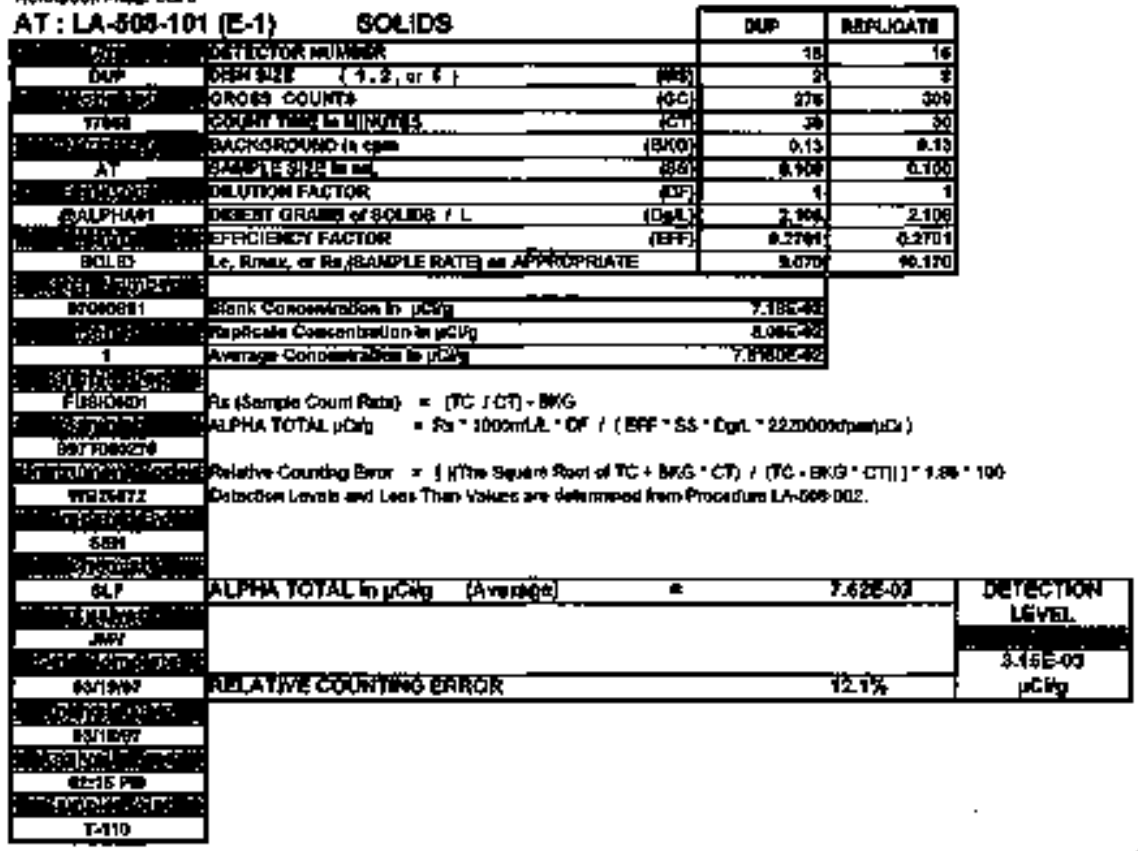

\section{느ㄴㅕㅕ:}

\%

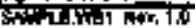

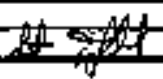

ntw

अन्नकासL

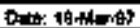

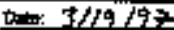




\section{LABCORE Completed RadChem Report for Worklist\#: 17072}

Analgst stb

totrument: AB16

Bookt

Method: ReyMod

Worklbst Compieat: Use $.100 \mathrm{~mL}$ sample stre, Rerun \#2. SLF

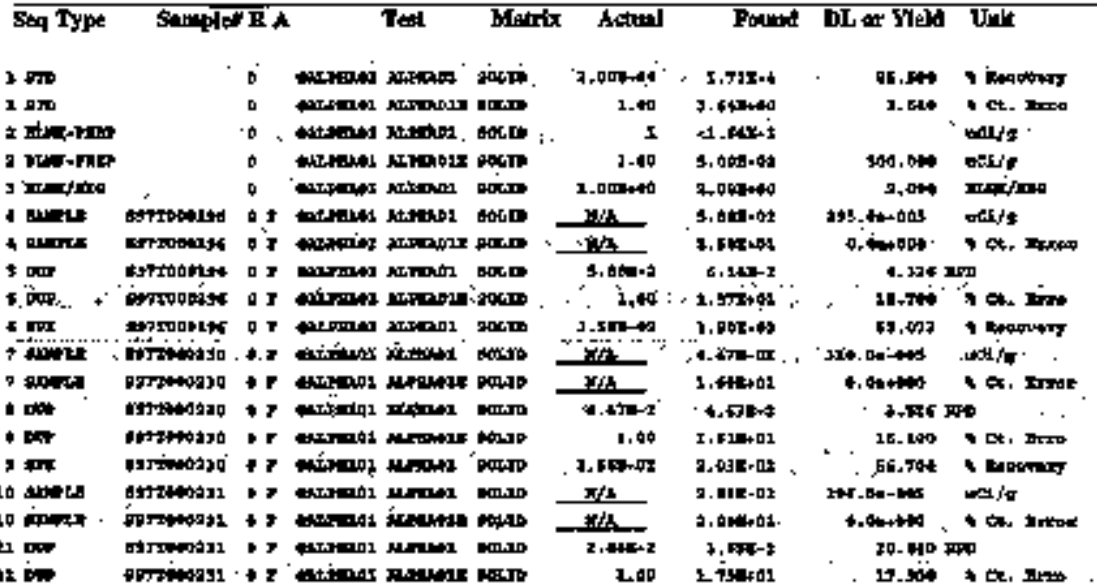

\section{Final page for worklistt 17072}

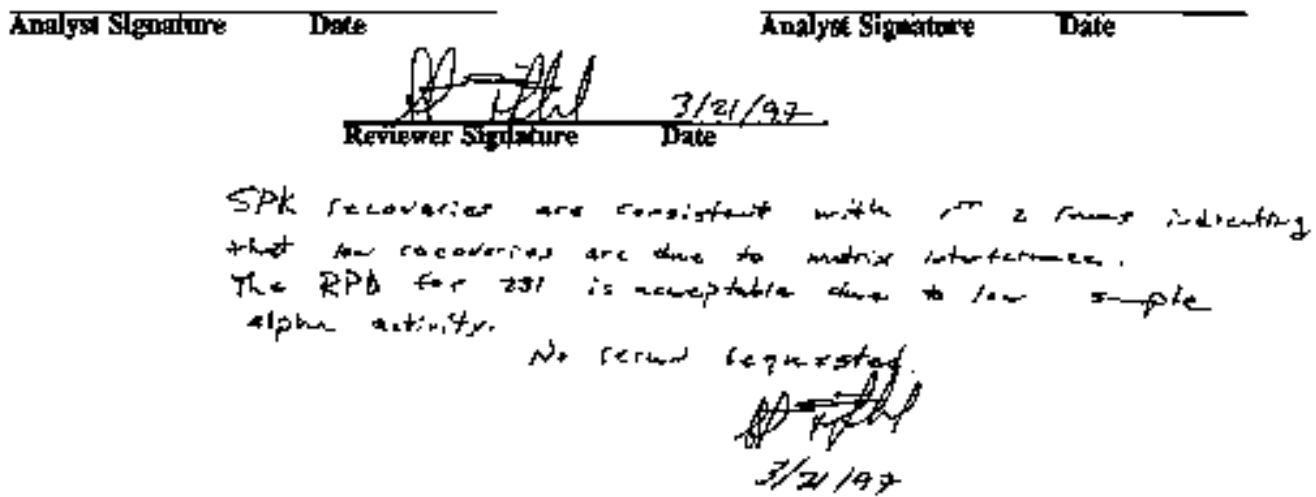

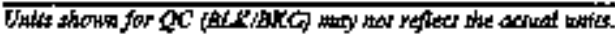




\section{LABCORE Data Entry Template for Worklist; 17072}

Anolyst:

Steflelostruments $\mathrm{ABO}$

Bontw 79856

Mellod: LA-508-101 Rev/Mad $F-0$

Workllys Comment: Use $.100 \mathrm{~mL}$ sample size. Rerun ${ }^{2}$. SLF

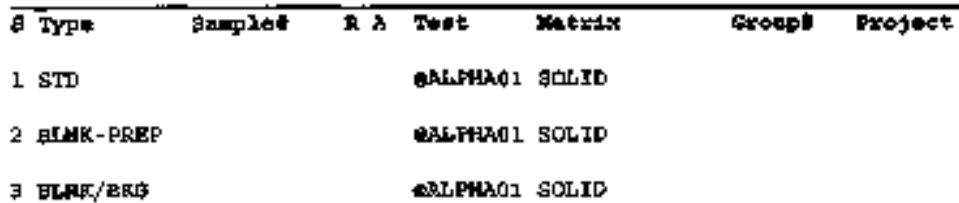

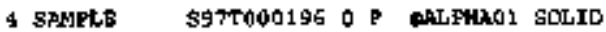

$97000093 \mathrm{~T}-110$

Amalyted Regitetod, ALFHAd , ALPHAOIE

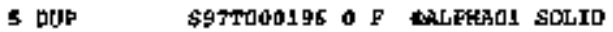

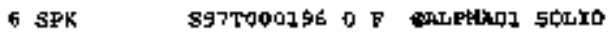

T SAMPLE

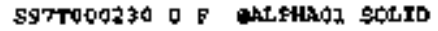

$97000171 \%-130$

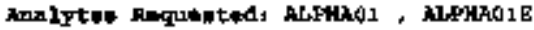

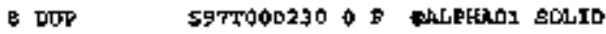

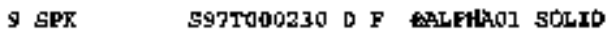

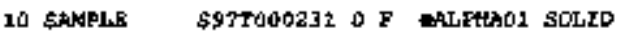

$97000111 \mathrm{~T}-210$

Ankiyted Regwested: ALPHADI . ALPHAO1E

11 DDF

\$97T000231 O F NLPEAO1 SOLI

\section{Final page for worklist \# 17072}
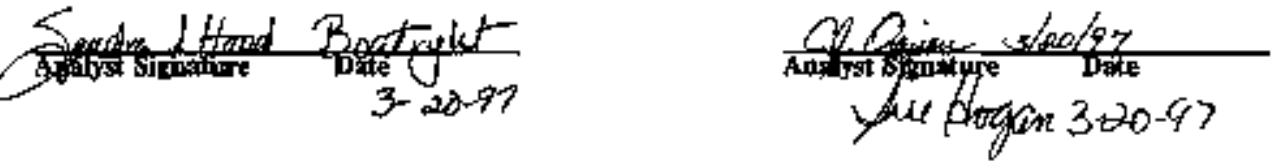

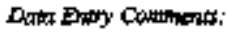

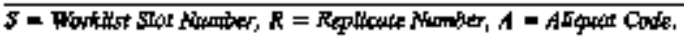




\section{HWF-SD-WMHDP.238, REV. D}

WCokxo0K PHaE sTu1

AT : LAS00-101 \{E-1\}

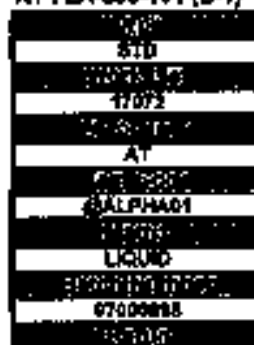

LASO9-113 (B) \$TANDARD

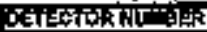

o, तf

Goos cotsing

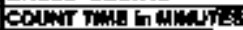

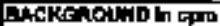

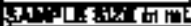

DUTOM FACTOR

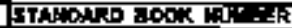

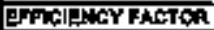

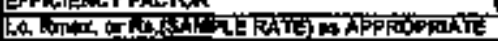

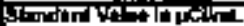

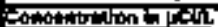

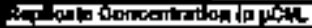

STMHDसरD

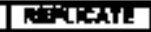

10

[G다]

[iij]

......

(1)

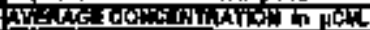

[5가]

(E.)

जitin:

IEF)

Fil::73

2005

2

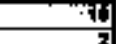

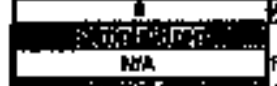

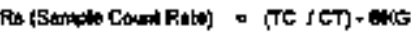

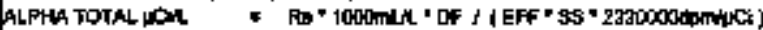

:

Whor

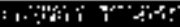
Fistist

$\because \because \cdot \cdots \cdot+3$ ebo

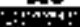

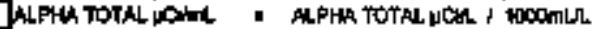

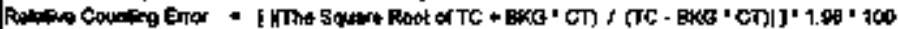

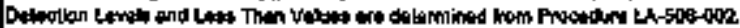

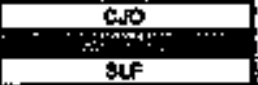

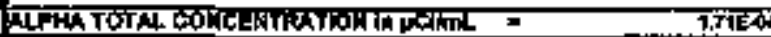

$\therefore$ and:

ज्ञस

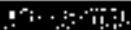
oszact

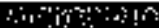
ortion

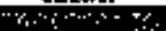

ontrin

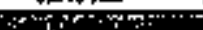

T=110

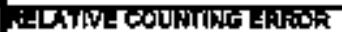

\begin{tabular}{|c|c|c|}
\hline 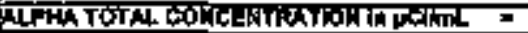 & 1.7TECOH & DETदकारकW \\
\hline & & 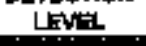 \\
\hline$-\mathrm{C}$ - & 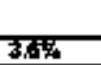 & $\begin{array}{l}\text { dostexof } \\
\text { uCthol }\end{array}$ \\
\hline
\end{tabular}

Artet:

Sisure of Chemist

GThowato.Wn' Ren 1.0

coto11

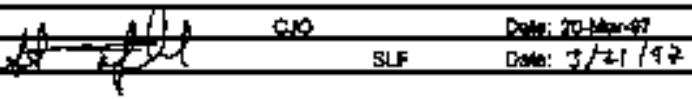

576 
IASF-SD-WHA-DP-238, REV, 0

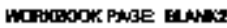

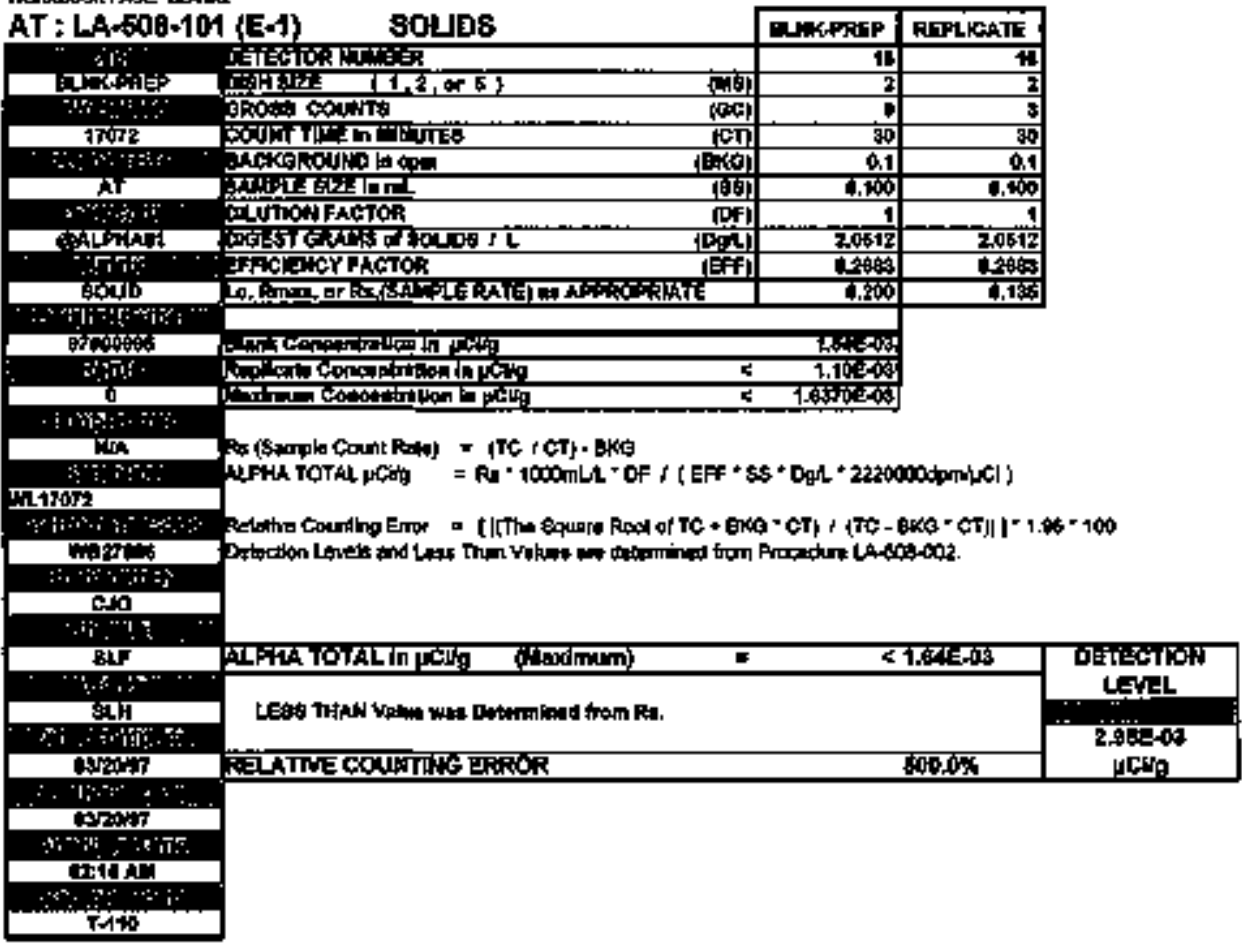

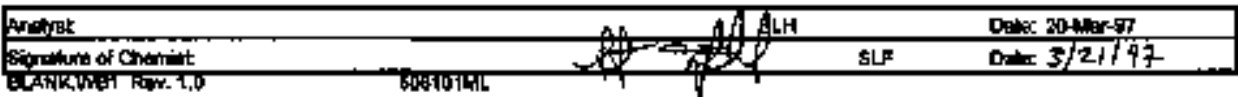




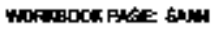

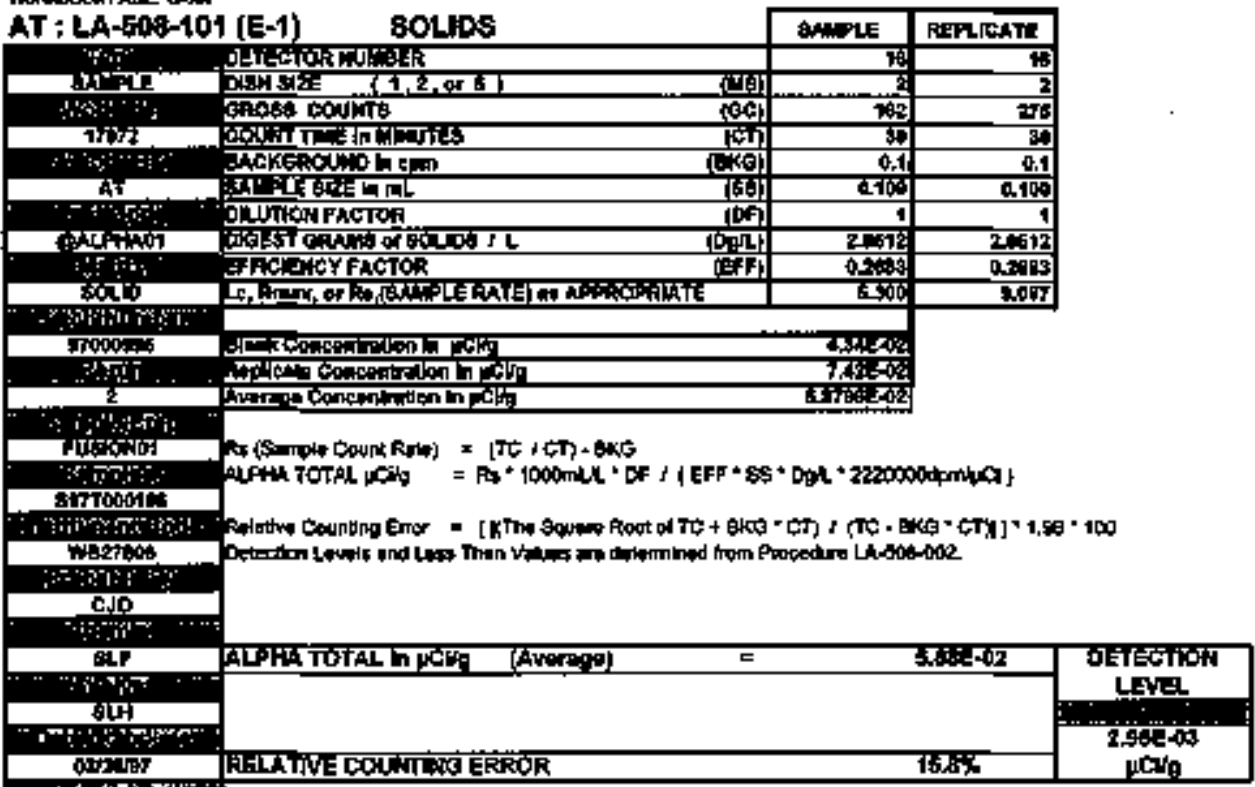

$\therefore 0^{2}$ : oring

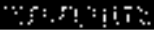

क्सात्मल

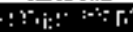

FA10

\begin{tabular}{|c|c|c|}
\hline An: & SLH & Dres: 201100-47 \\
\hline Slonture d Chemiet & S5 & Dala: $3 / 2 \sqrt{4}+2$ \\
\hline
\end{tabular}




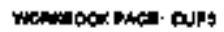

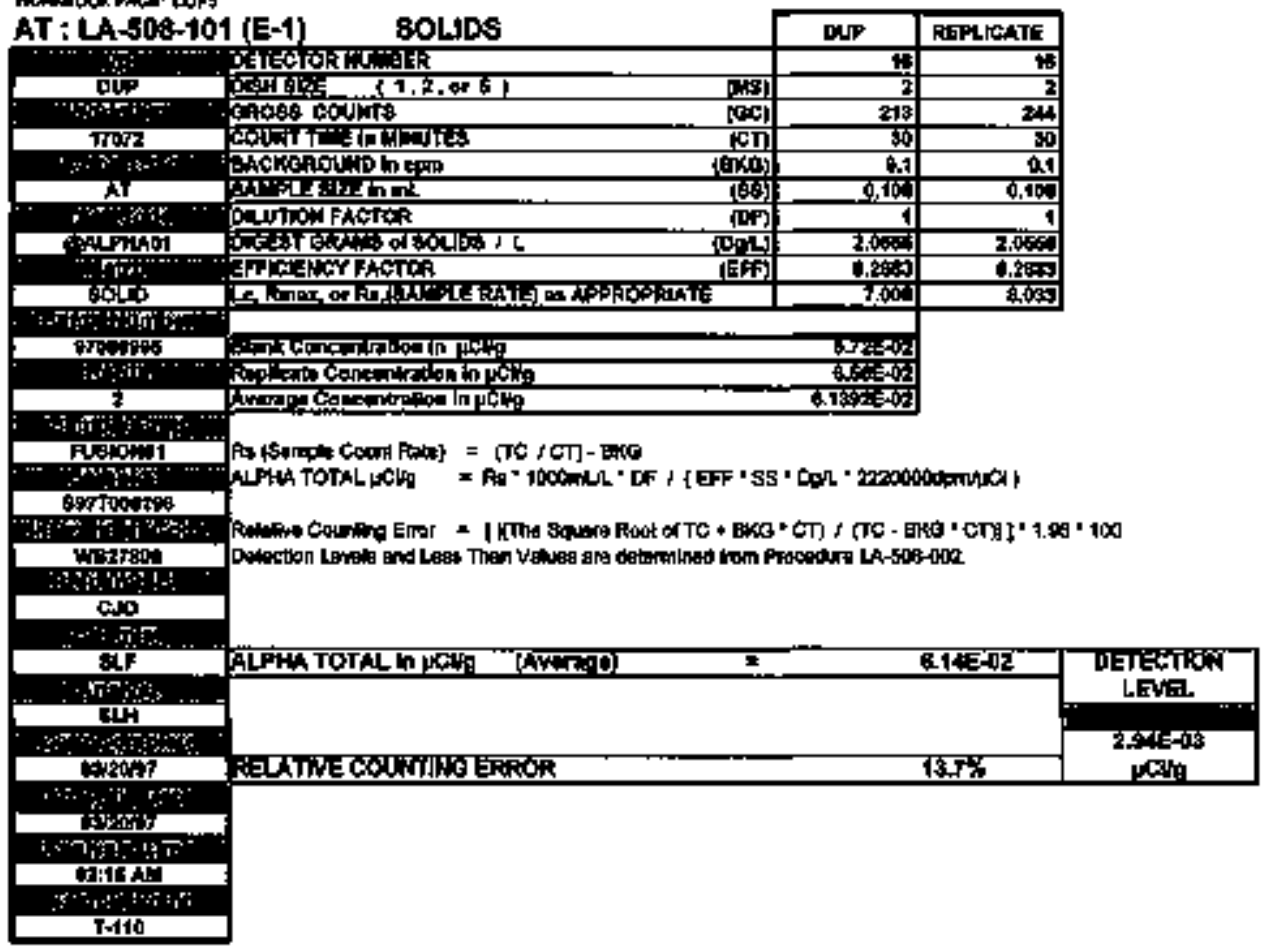

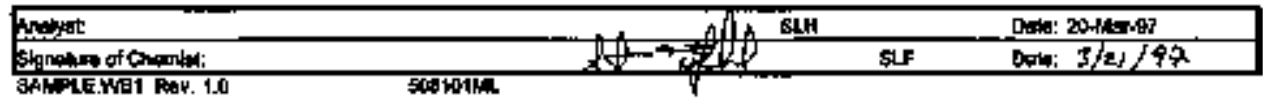


HNF-SD-WM-DP-238, REV.O

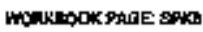

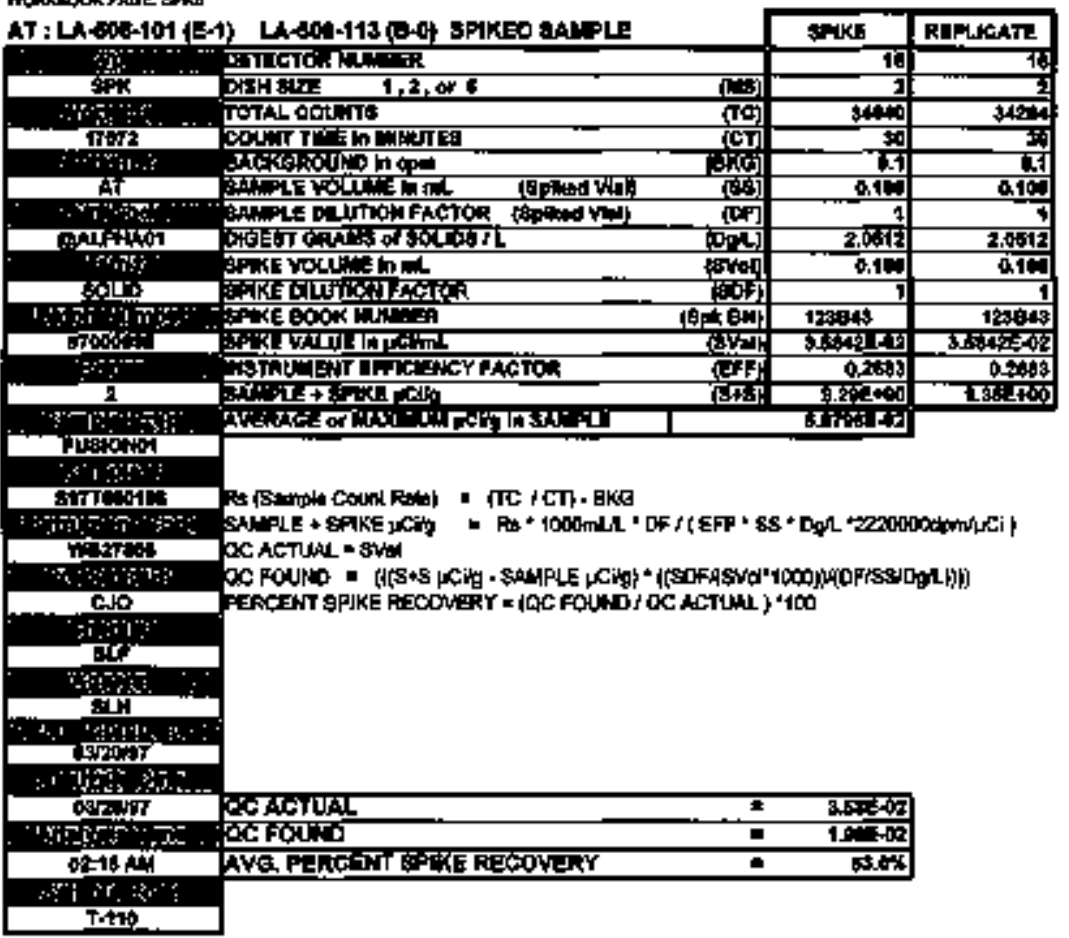

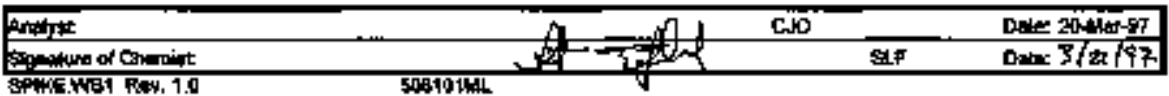

\section{0}


HWV-\$D-WW-DP-Z38, REV. 0

MLFAEOON PA3E gur

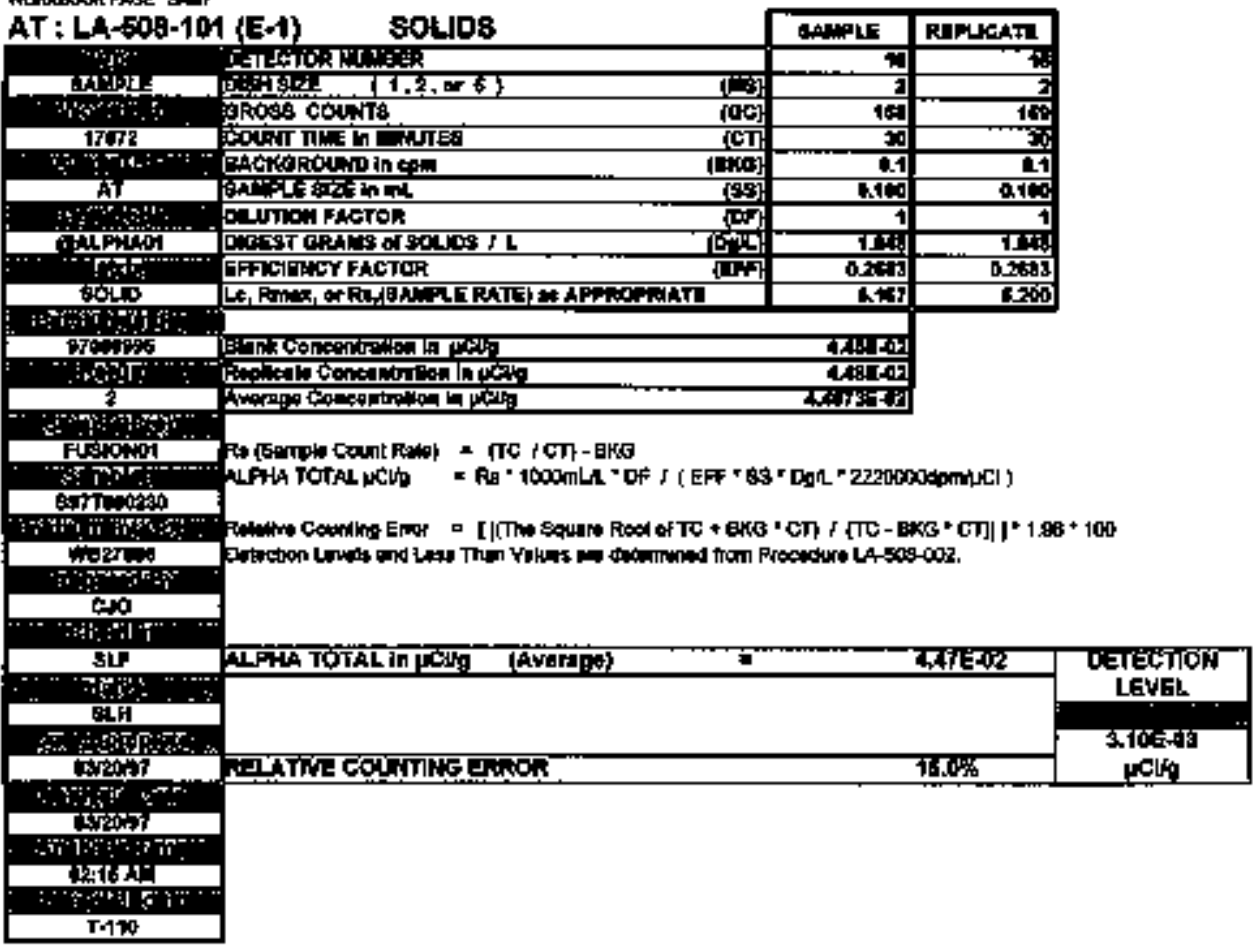

\begin{tabular}{|c|c|c|}
\hline (1) & $\Omega(A)$ SUH & 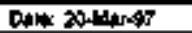 \\
\hline gipnolure of theriat. & 301 & Dolog $3 / 21 / 97$ \\
\hline
\end{tabular}


HWFSD-WM-DF-238, REV O

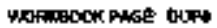

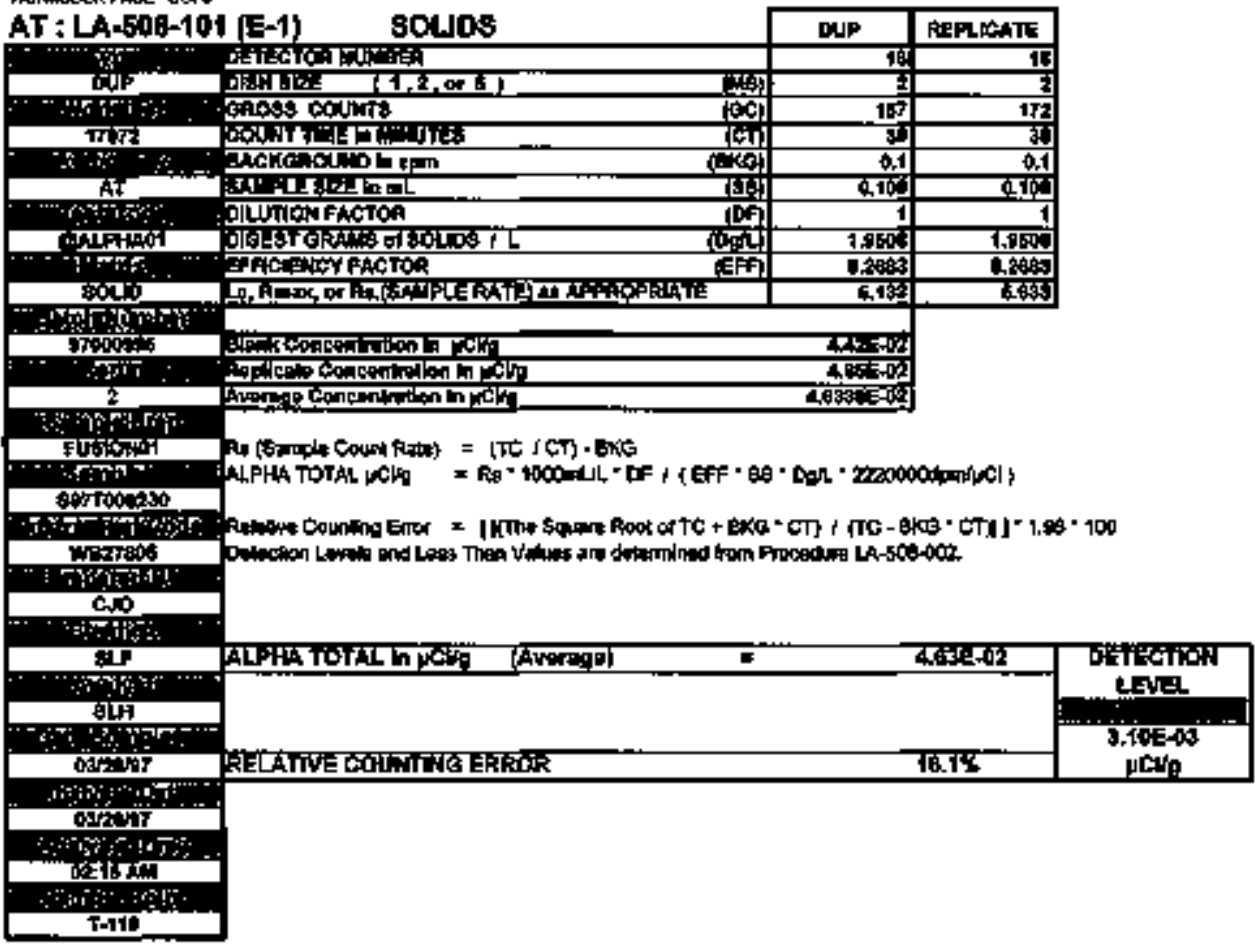

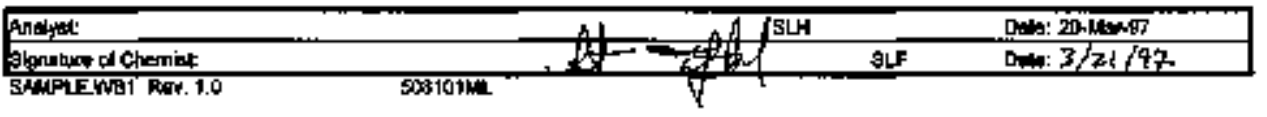




\section{HNF-SD-WW-DP-238, REV, 0}

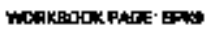

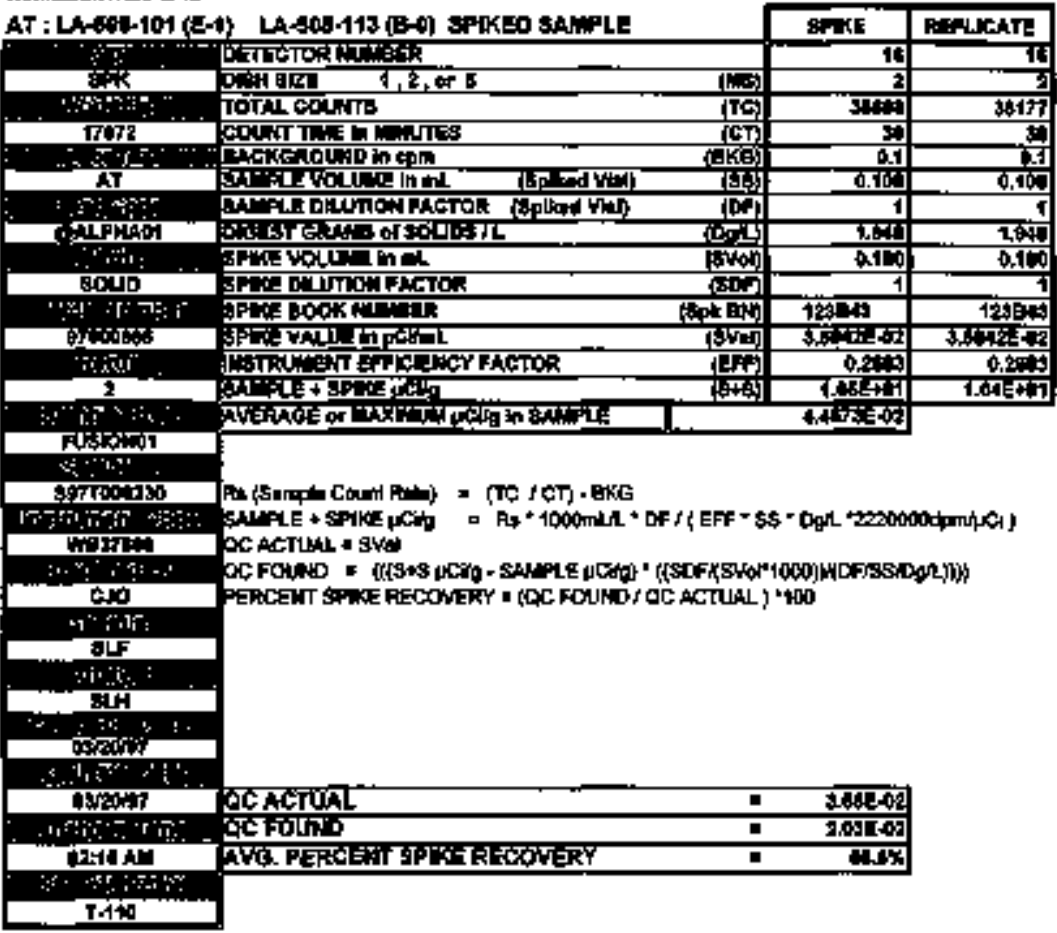

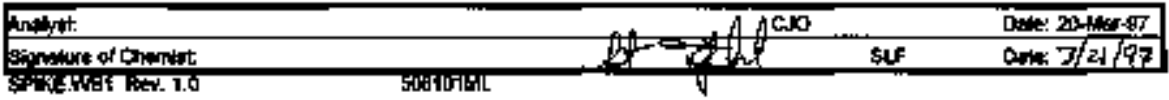


HNF-SDWW-DP-238, REY. 0

YTAKBOCX PMCE: SМню

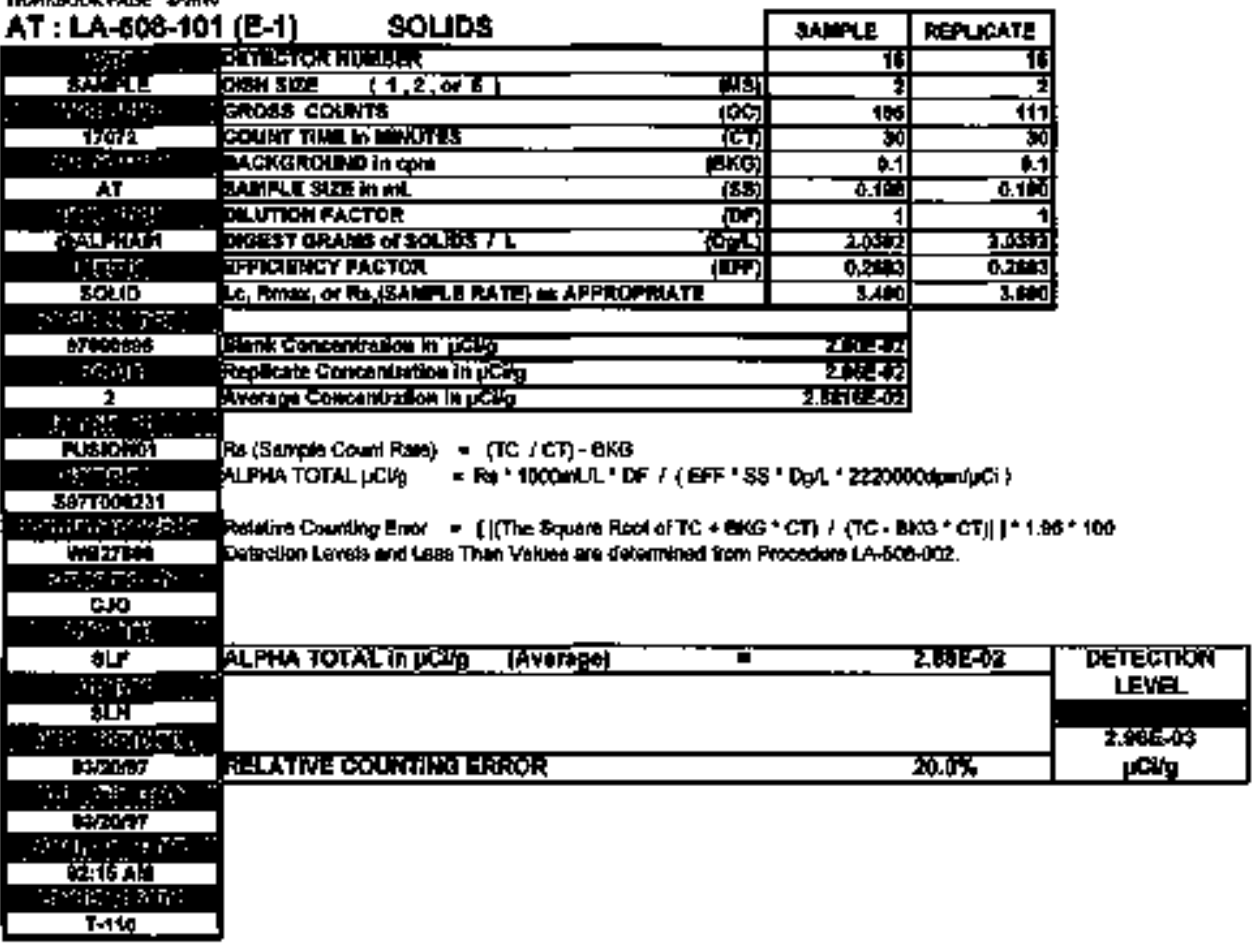

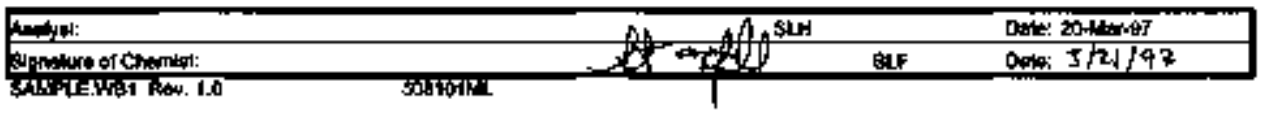

\section{4}


HNF-SD-WM-DP-239, FEV. O

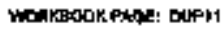

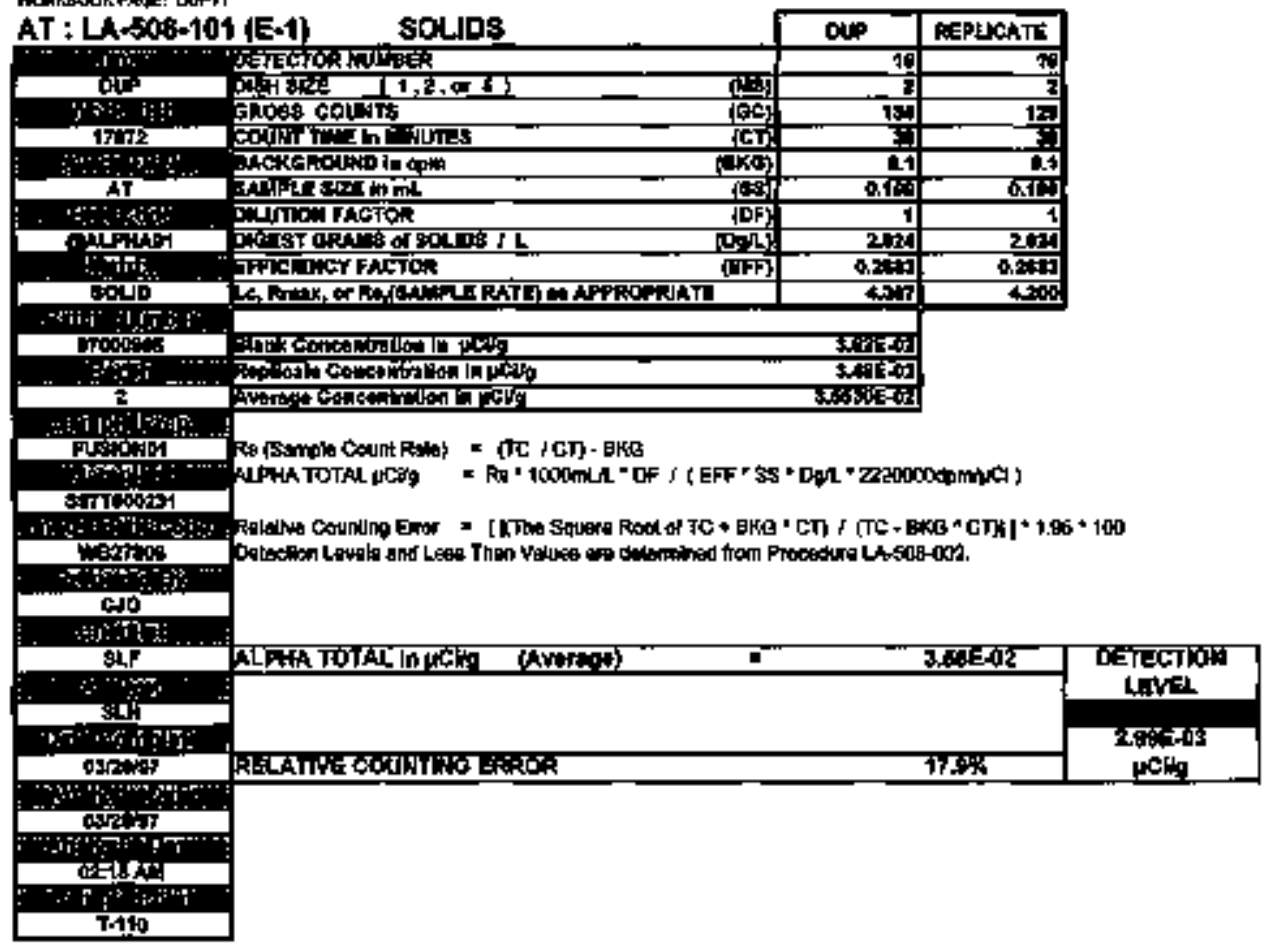

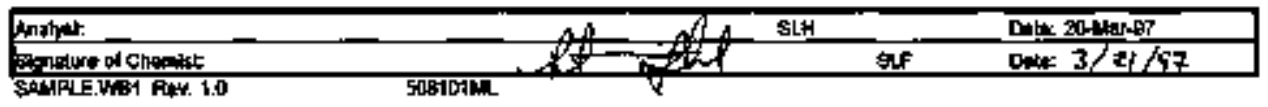




\section{LABCORE Completed RadChem Report for Worklist: 17005}

Amibyt: [wk

Instrument: AB21

Bonkf

Mothod:

Rewhod

Worklist Comment: T-110, OPL23901, Deter 9.s. by Ludlumb. Std: 1.OmL. sth

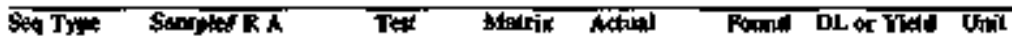

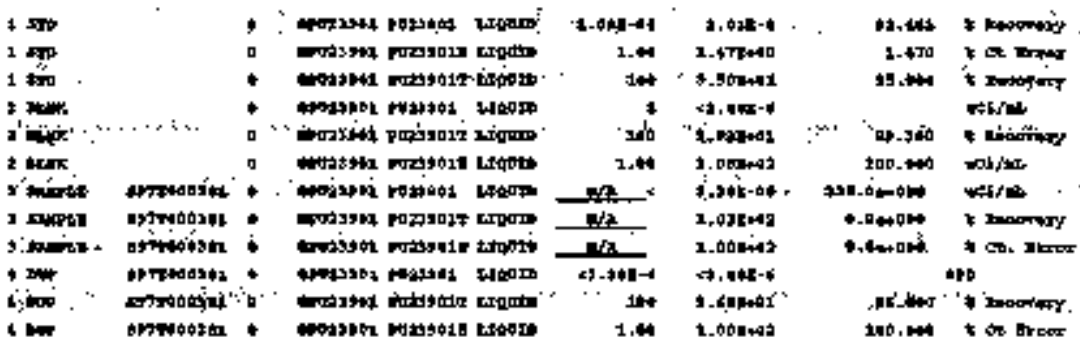

\section{Final page for worklistł 17005}

Analyat Slengiant Date

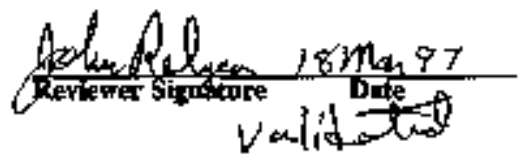




\section{LABCORE Data Entry Template for Worklist\# 17005}

Analyst:

\section{LA-943-128 Rew/Mod $6=0$}

'.t's Comment: T-110, QPU23901, Deker s.s, by Ludlum. Std: 1.0mL. skm

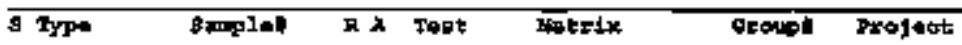

1 STD ePU23901 LIgGID

2 BLWK

-PU2J901 bIQUID

3 SAMPLE

G97T003e1 o ptus3901 Lrotith

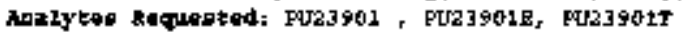

4 DUP

597T000381 o OPU23901 Liguth

Final page for worklist \# 17005
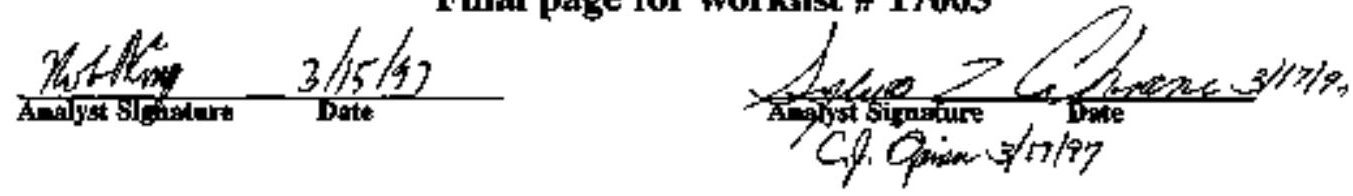

Denter Evrry Canteres:

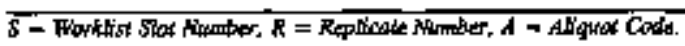


DATA COLLECTION ESHEFT FOR AMERICIUM AND PLUTONIUMA

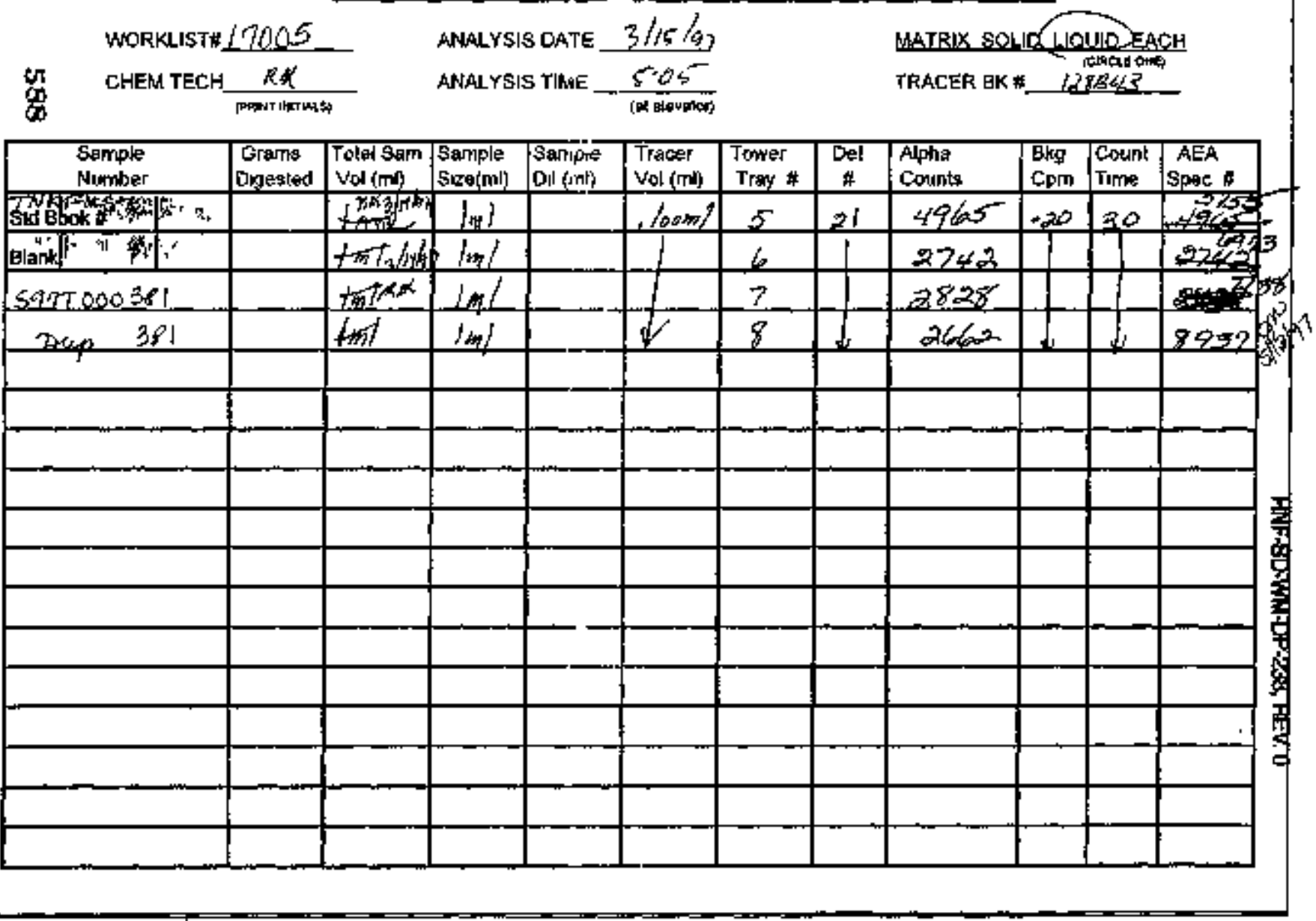


HNE-SD-WH-DP-238, AEV.O

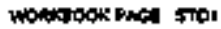

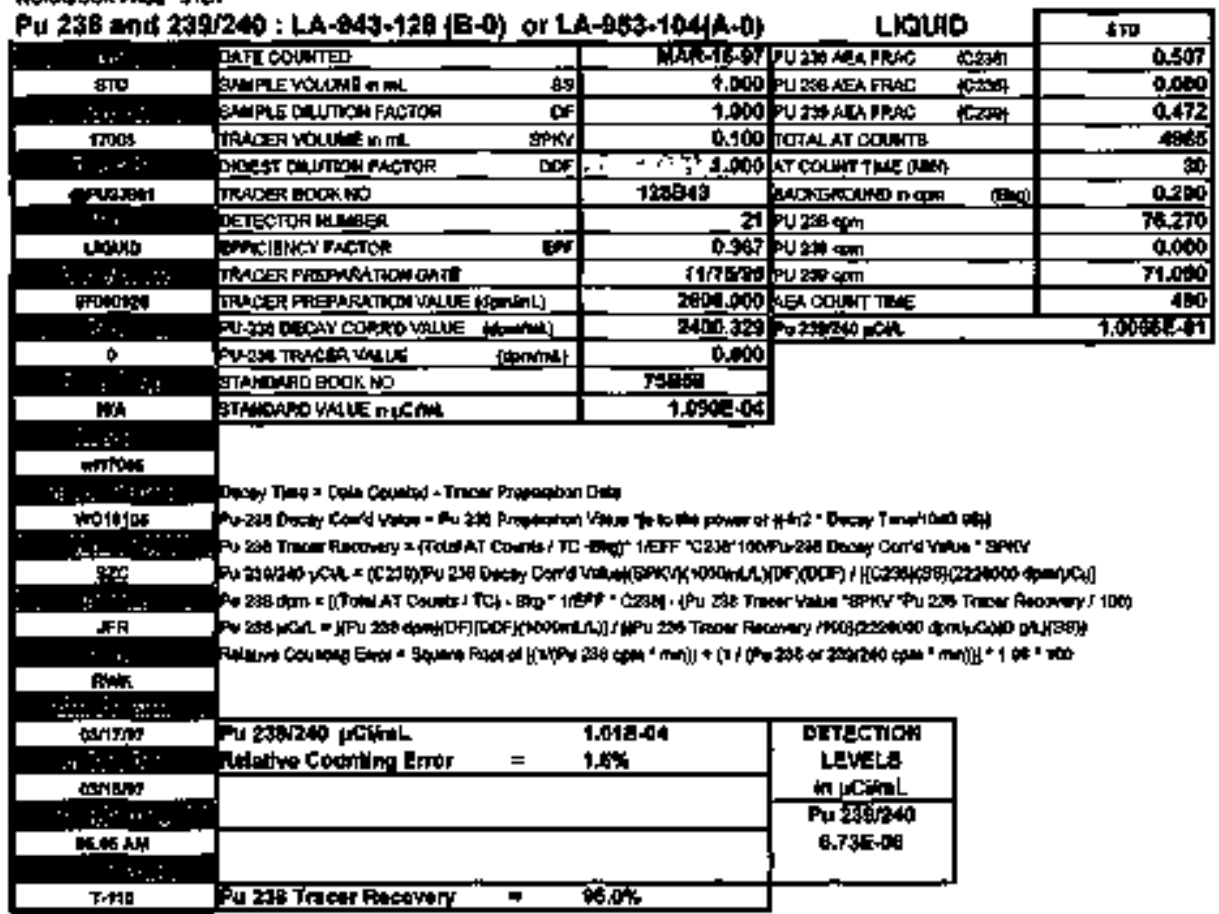

\begin{tabular}{|c|c|c|c|}
\hline Andifat & $\sin x$ & an & 17 -4tont \\
\hline 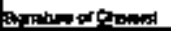 & $\boldsymbol{F}$ & 1. & $18 \mathrm{M}_{\mathrm{an}} .87$ \\
\hline
\end{tabular}




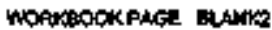

Pu 238 and 2391240 : LA-943-128 (B-0) or LA-953-104(A-0)

\begin{tabular}{|c|c|c|c|c|}
\hline \multirow{2}{*}{\multicolumn{2}{|c|}{$\mathrm{U} 238$ and $2391240:$ LA-943-128 $(\mathrm{B}-0)$}} & & LIa & BLEK \\
\hline & & CARMANA & PU Z3BAEAFRAC & 0.000 \\
\hline gutik & SAMPLE WOLUL'= In mL & 1000 & 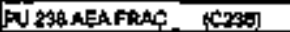 & 0,000 \\
\hline 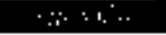 & SACHPE DUUMON FACTOR & 1,000 & PU2JTEGAFAAC & conot \\
\hline 17nets & LRACER YOUWHen & 0.000 & TOTALAT COUN'SS & 패붐 \\
\hline , $\because$ & WEEAT OULUMON FACTOR & -1.100000 & 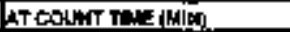 & $\$ 0$ \\
\hline 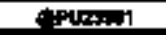 & 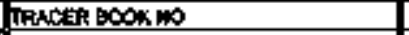 & 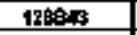 & 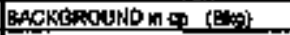 & 0.000 \\
\hline & ERTETOR NUWHA & 궉 & & TLP\$0 \\
\hline Landis & FFPKAENCYYACTOR & 0.3674 & 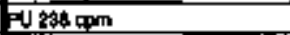 & 9,000 \\
\hline 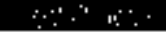 & ILRCERR PREPARATKON DATE. & 11M55 & 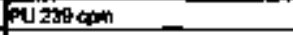 & 0.000 \\
\hline & 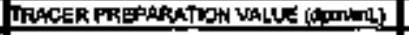 & 20,000 & AEA COCUNT TIEE & D. \\
\hline & 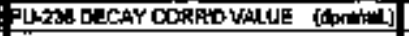 & 200.35 & 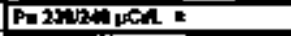 & 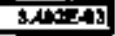 \\
\hline & FUL2SB TRACER VALUEE & 600 & & \\
\hline
\end{tabular}

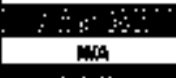

$\therefore \cdot \cdot$

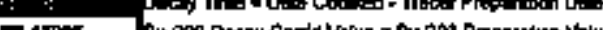

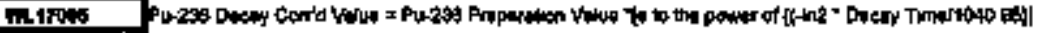

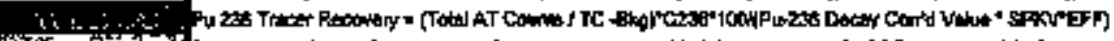

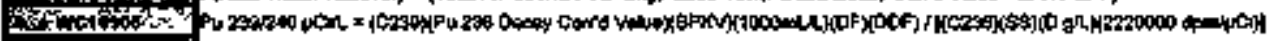

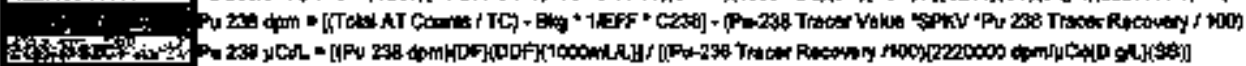

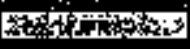

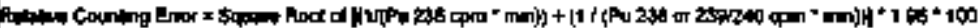

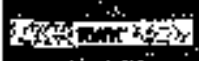

$\because \because \cdots, \cdots$

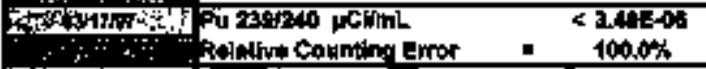

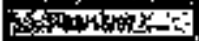

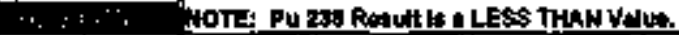

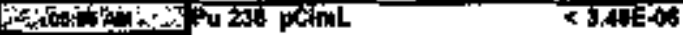

Ratutha Countine Emoe $\quad$ 100.t\%

QR

\begin{tabular}{|c|}
\hline 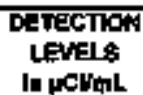 \\
\hline $\begin{array}{c}\text { PU } 25,500 \\
340 E-08\end{array}$ \\
\hline $\begin{array}{c}\text { Pu } 258 \\
340 E-08\end{array}$ \\
\hline
\end{tabular}

\begin{tabular}{|c|c|c|c|}
\hline inalitar & gurk & DE: & 1r+1:-Af \\
\hline
\end{tabular}


HWF-SD-WH-DP-23\%, REV.O

MoPABOOK PMCE Bus

Pu 238 and 2391240 : LA-943-128 (B-0) or LA-853-104(h-0)

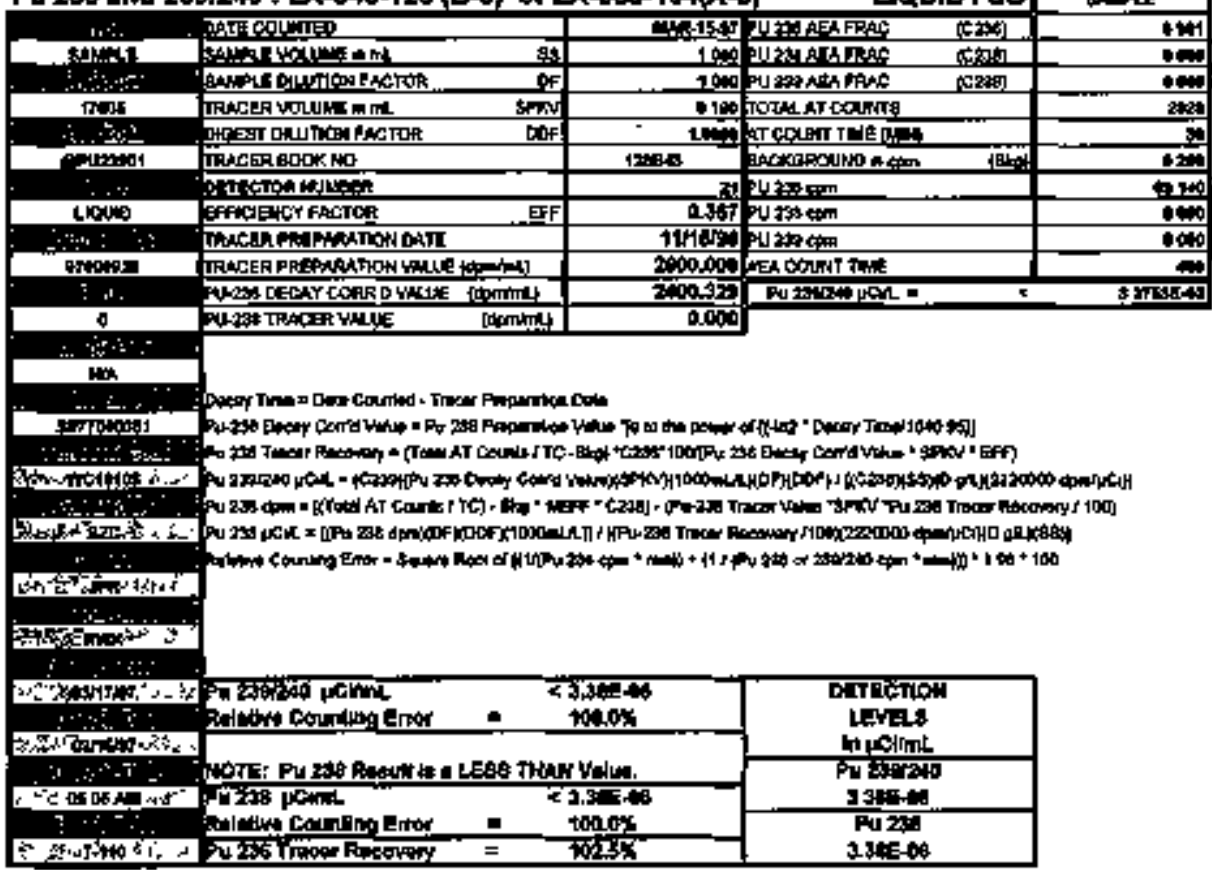

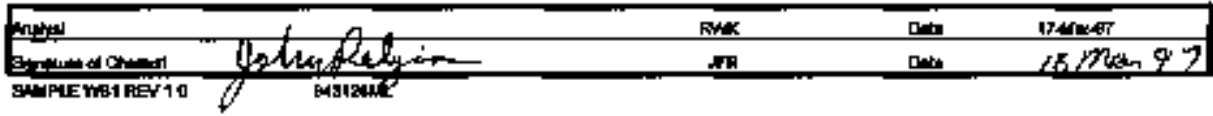




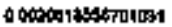

Pu 238 and 2304210 : LA-943-129 (Q-0) or LA-\$6\$-104(A-0)

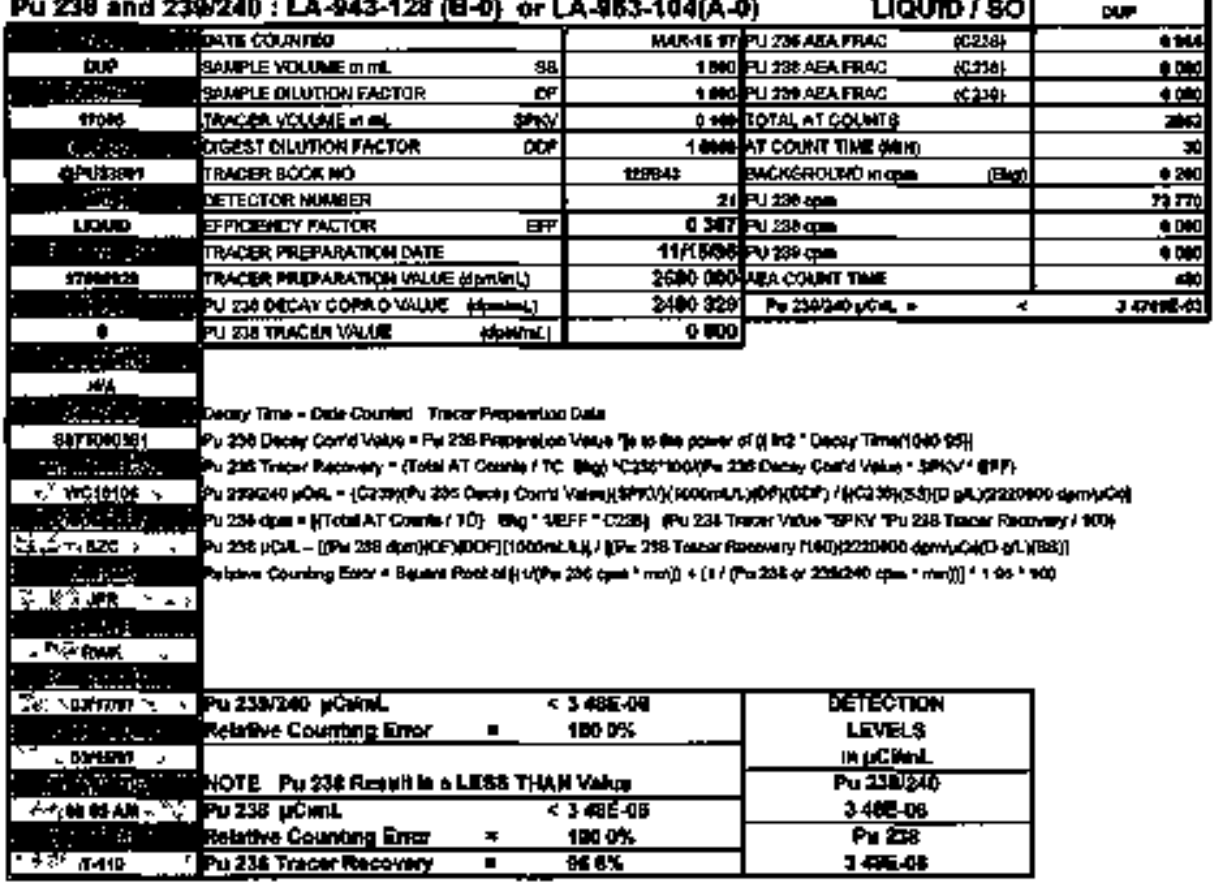

\begin{tabular}{|c|c|c|}
\hline 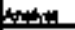 & bur & ti lint 20 \\
\hline
\end{tabular}


HNF-SD-WW-OP-238, REV 0

Restinghous: Hanford co.

G E E R A L

A L F H A E ER G T

Rev. 2.02

A $Y$ A I $S$ I $\mathbf{s}^{\prime}$

DAEA REDOCTIOA RIPORT

SALPEB

\$97T0381-DUP

File ID: 8a9937. CAF

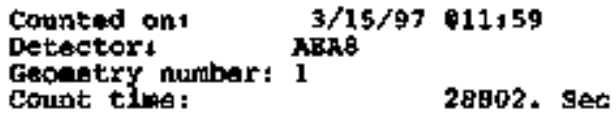

PRAK ABALYSTS

\begin{tabular}{|c|c|c|c|c|c|c|c|}
\hline $\begin{array}{l}\text { Peak } \\
\text { ID } \\
1 \\
2 \\
3 ?\end{array}$ & $\begin{array}{l}\text { Peak } h \\
\text { rnitial } \\
1777.5 \\
41.5 \\
33.2\end{array}$ & $\begin{array}{r}\text { Fight } \\
\text { Final } \\
177.5 \\
41.5 \\
13.2\end{array}$ & $\begin{array}{c}\text { Peak center } \\
\text { Inittal Final } \\
364.764 \text { 354.764 } \\
292,176291.988 \\
233.619233 .530\end{array}$ & $\begin{array}{l}\text { Initial' } \\
16.000 \\
16.000 \\
16.000\end{array}$ & $\begin{array}{l}\text { Fingl } \\
10.7 \$ 6 \\
13.486 \\
10.078\end{array}$ & $\begin{array}{l}\text { Tatt } \\
\text { Init1日1 } \\
8.000 \\
9.000 \\
8.000\end{array}$ & $\begin{array}{l}\text { F1.9a1 } \\
3.794 \\
2.944 \\
3.014\end{array}$ \\
\hline
\end{tabular}

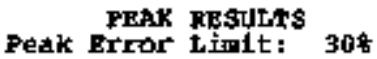

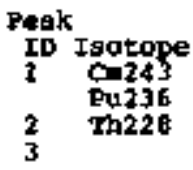

Totales
AEA

Frac

0.964

0.031

????

0.995

0.995
Peak Centrold

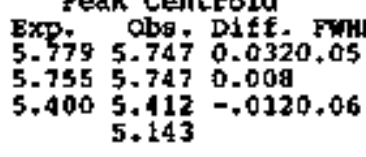

〈--valdd peaks only--〉

Count terx

Ratec/a 995

73.771 .0

$2.37 \quad 6,2$

$0.58 \quad 13.2$

$76+13$
Activity trC1/ea

$0+1268-03$

280.3

308.8

9.2

$0.941 E-04$

DETECTOR CALIBRATION
Energy (tEV) $=4.069+(0.0046)$ *Channel
Baprgy range (Hol): 4.069 To 6.424
Etficiency $=0.3605$ CPU/DFA

TOTAL COURT DATA:

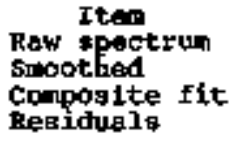

$\begin{array}{cc}\text { Total } & \text { Fecovery } \\ 36745+0 & 100+000 \\ 36745.0 & 100.000 \\ 36826.8 & 100.223 \\ -81.8 & -0.223\end{array}$

Anelyzed by: 
- " Spectrum Ba8937.ckF HNF-SD-WW-DP-228, fiEV O

1 Legend; Raw $=\ldots$ Hodeled Peaks $=1,2, \ldots$ etc Display Max.: 13425,3

,... 1

$\ldots \ldots \ldots \ldots+\ldots \ldots+\ldots 1$

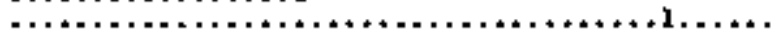

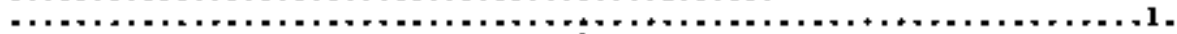

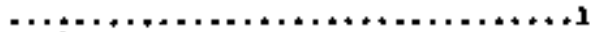

$+, 1$ 


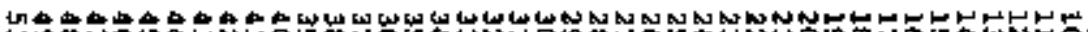

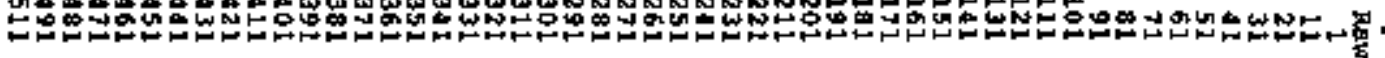

\section{a}

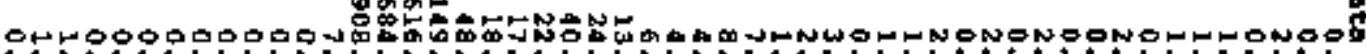

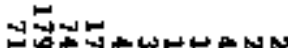

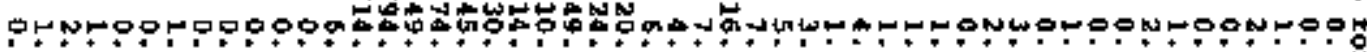

$\ln m$

Nюw

$\stackrel{\min }{\operatorname{mon}}$

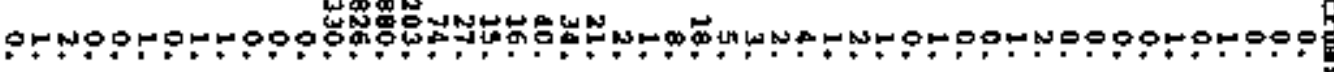

NimWN

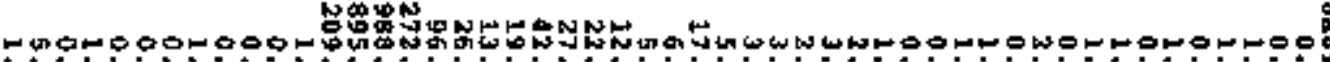

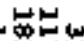

(ب)

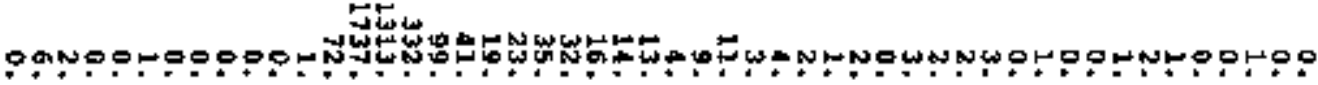

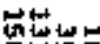

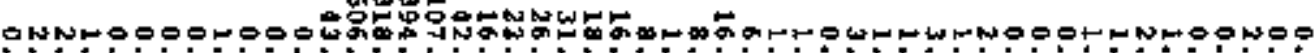

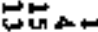

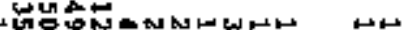

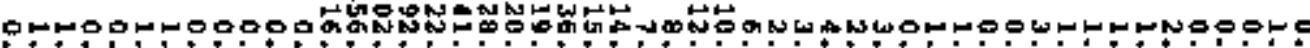

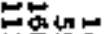

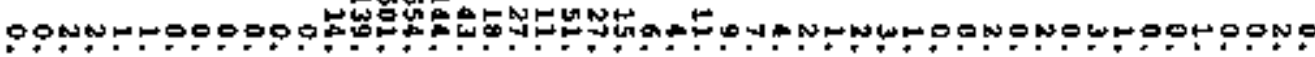


Weatinghouse Eanford Co.

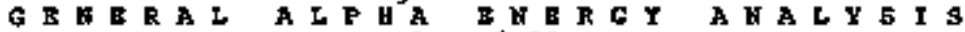

REY . 2.02

DATA RBDUCTION RRPORT

SAMPLE

FL17005-STO

Pile IDt \$a5155. CAF

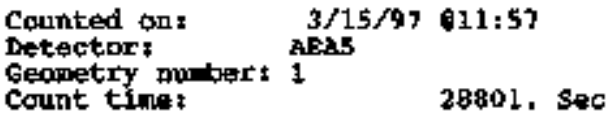

FEAR ANhLYSIS

\begin{tabular}{|c|c|c|c|c|c|c|c|c|}
\hline $\begin{array}{l}\text { Peak } \\
\text { tb } \\
1 \\
27 \\
3 \\
47 \\
5\end{array}$ & $\begin{array}{r}\text { Peak } \\
\text { Initial } \\
2145.9 \\
22.3 \\
48.3 \\
12.3 \\
2272.1\end{array}$ & $\begin{array}{r}\text { relght } \\
\text { rinal } \\
2145 \times 9 \\
22 \times 3 \\
48 \times 3 \\
21.3 \\
2272.1\end{array}$ & $\begin{array}{c}\text { Palk } \\
\text { Initial } \\
361.639 \\
304.108 \\
287.035 \\
272.240 \\
220.037\end{array}$ & $\begin{array}{r}\text { center } \\
\text { Final } \\
361.639 \\
304.109 \\
286.855 \\
770.356 \\
238.836\end{array}$ & $\begin{array}{l}\text { Initie1 } \\
12.000 \\
29.000 \\
14.000 \\
20.000 \\
12.000\end{array}$ & $\begin{array}{l}\text { Fy } \\
\text { Ftad } \\
5.740 \\
7.195 \\
3.390 \\
3.920 \\
5.470\end{array}$ & $\begin{array}{r}\text { Tal } \\
\text { Initial } \\
6.000 \\
14.000 \\
3.000 \\
5.000 \\
6.000\end{array}$ & $\begin{array}{r}\text { Final } \\
2.009 \\
14.293 \\
4.243 \\
0.598 \\
2.398\end{array}$ \\
\hline
\end{tabular}

\section{PEAK REgULTS \\ Peak srror ififtt $30 *$}

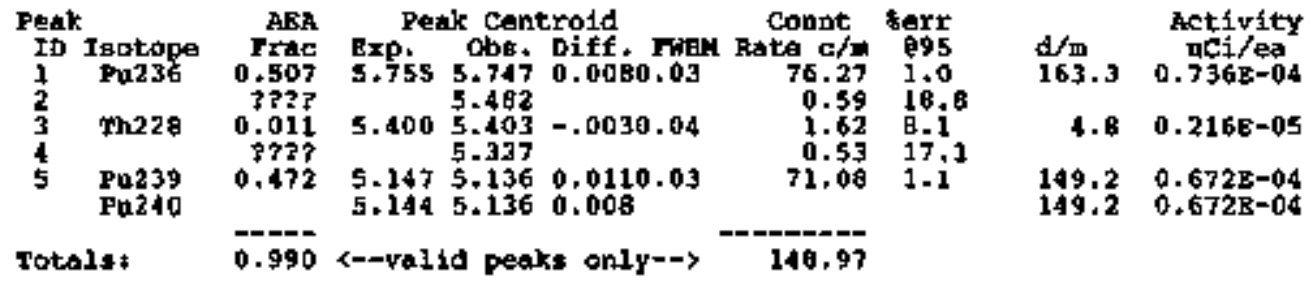

$$
\begin{aligned}
& \text { DBTBCTOR CALIBRATLOK } \\
& \text { Enargy (HBP) = } 4.083+(0.0046)+\text { Chammel } \\
& \text { Bnergy range (MeVl: 4.063 to } 6.439
\end{aligned}
$$

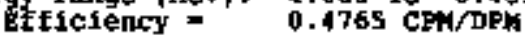

TOTAL Cotthit DATA:

Iten

Rate apectroth

Sthorathed

conposite fit

Fegiduala

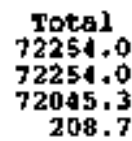

1 Recovery

100.000

100.000

99.711

0.289

Ansiyzed by 1 


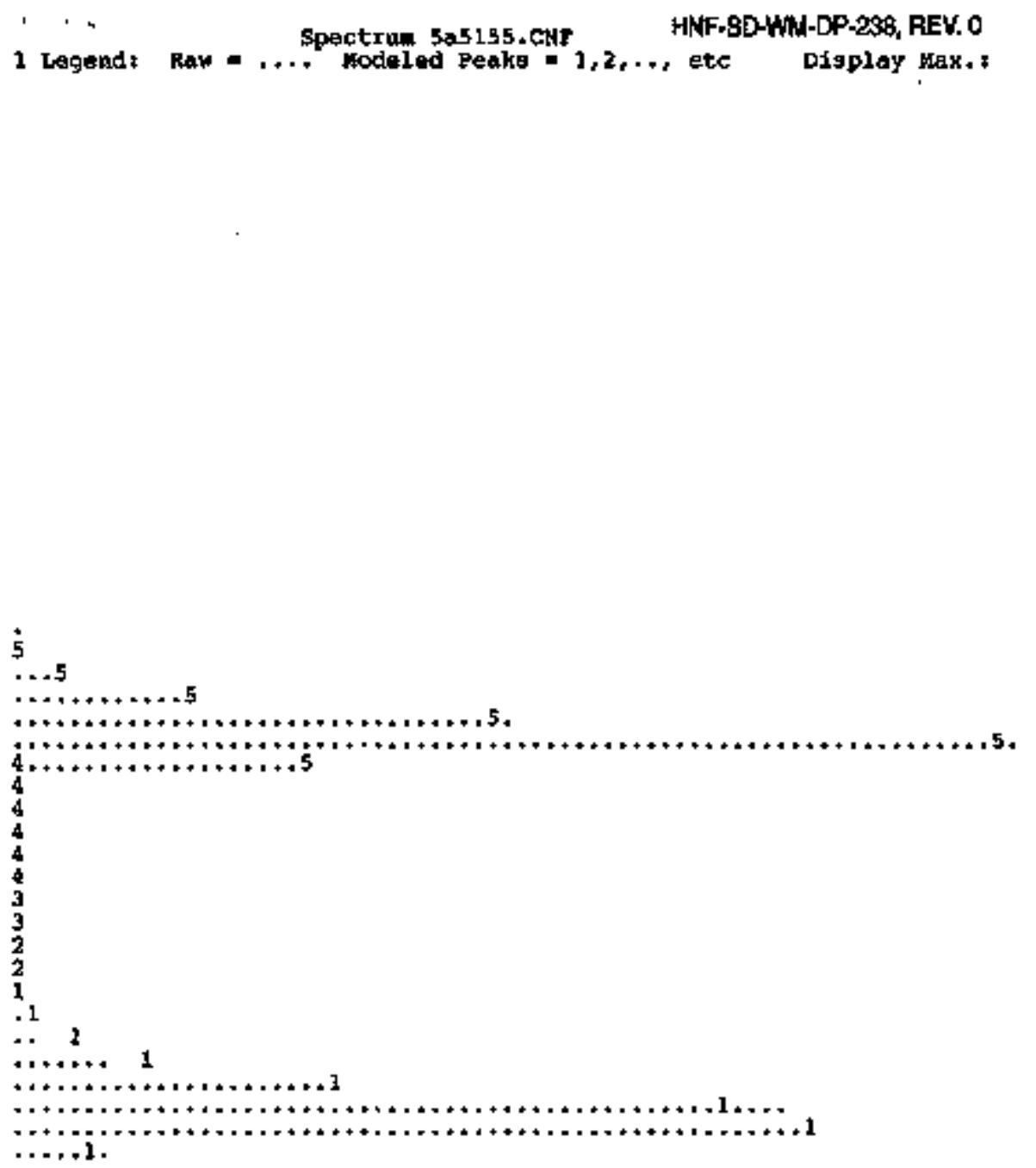


Rew Data Danp for AFA Spectrant 5a5155.CAP

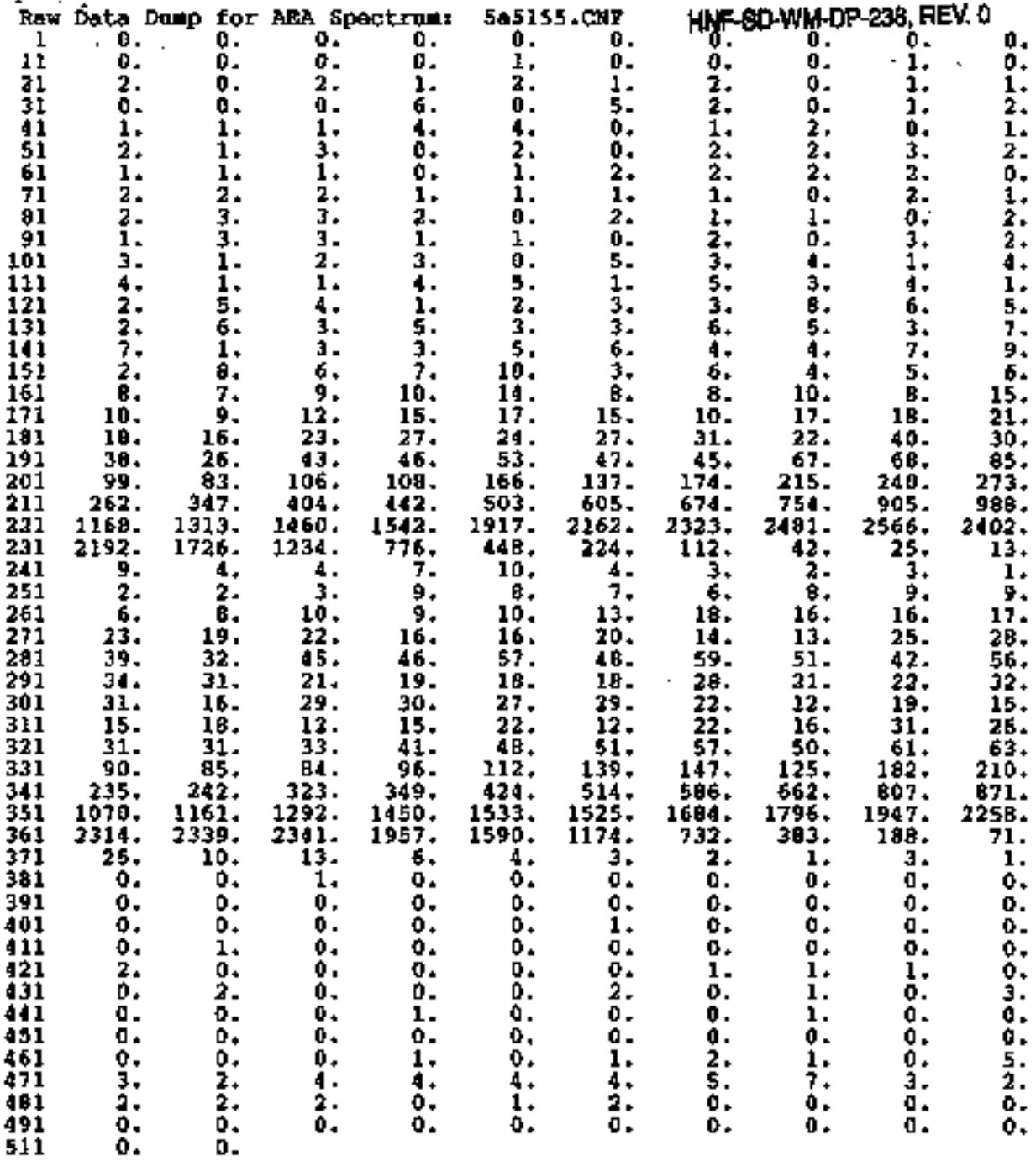


Westinghoute Hanford Ca.

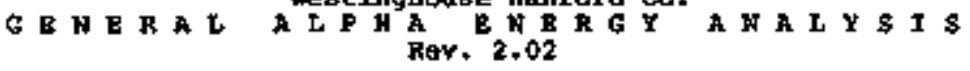

DATK REDTCTIOA REPORT

SAMPLB

File ID: 6a6973.CHF

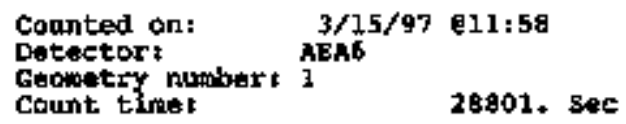

PEAK MMALYSIS

\begin{tabular}{|c|c|c|c|c|c|c|}
\hline $\begin{array}{l}\text { Penk } \\
\text { ID } \\
1 \\
27 \\
3 \\
43\end{array}$ & \begin{tabular}{rr}
\multicolumn{1}{c}{ Peak hoight } \\
In1tial & Fingal \\
1773.7 & 1771.7 \\
20.4 & 20.4 \\
43.6 & 43.6 \\
15.9 & 15.9
\end{tabular} & $\begin{array}{c}\text { Peak center } \\
\text { Initial Final } \\
360.339360 .339 \\
304.726301 .973 \\
206.297366 .192 \\
228.446228 .373\end{array}$ & $\begin{array}{l}\text { Initial } \\
16.000 \\
26.000 \\
16.000 \\
16.000\end{array}$ & $\begin{array}{l}\text { H4 } \\
\text { Findal } \\
9.430 \\
3.830 \\
9.174 \\
7.342\end{array}$ & $\begin{array}{c}\text { Tai } \\
\text { Initial } \\
8.000 \\
13.000 \\
8.000 \\
8.000\end{array}$ & $\begin{array}{r}7 \text { intal } \\
3.242 \\
13.199 \\
2.061 \\
2.220\end{array}$ \\
\hline
\end{tabular}

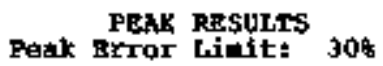

Penk

ID I gotopa

$1 \quad 0 \pi 243$

2 Th2 28

$\mathbf{3}$

Total 무:

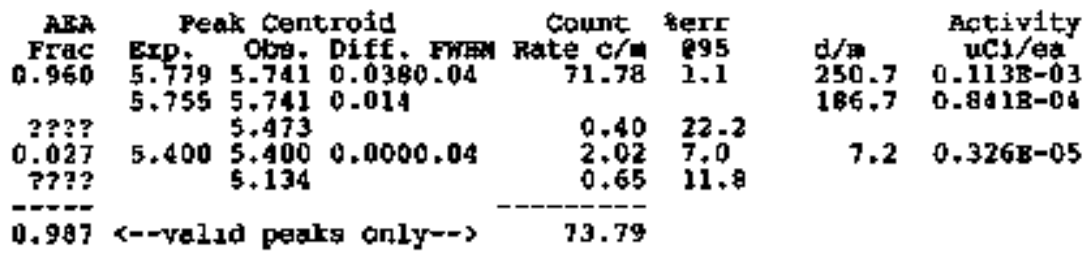

DETBCMOR CALIBRATION

$$
\begin{aligned}
& \text { Enęrgy (GBV) }=4.084+(0.0046) \text { tChanneI } \\
& \text { Buèrgy range (keV): 4.084 TO } 6.439 \\
& \text { Efficiency } 0.3922 \text { CPUDPH }
\end{aligned}
$$

TORAL COURY DATh:

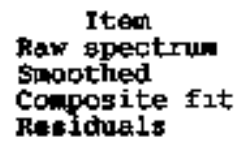

Recovery
100.000
100.020
100.139
-0.139

Analyzed by: 
- Spectrum 6a6973.chF

1 Legand: Ras - .... Modeled Feaks - 1,2, ., etc

HWF-SO-WH-DP-228, REV. 0
Display Hax,: 12124,5

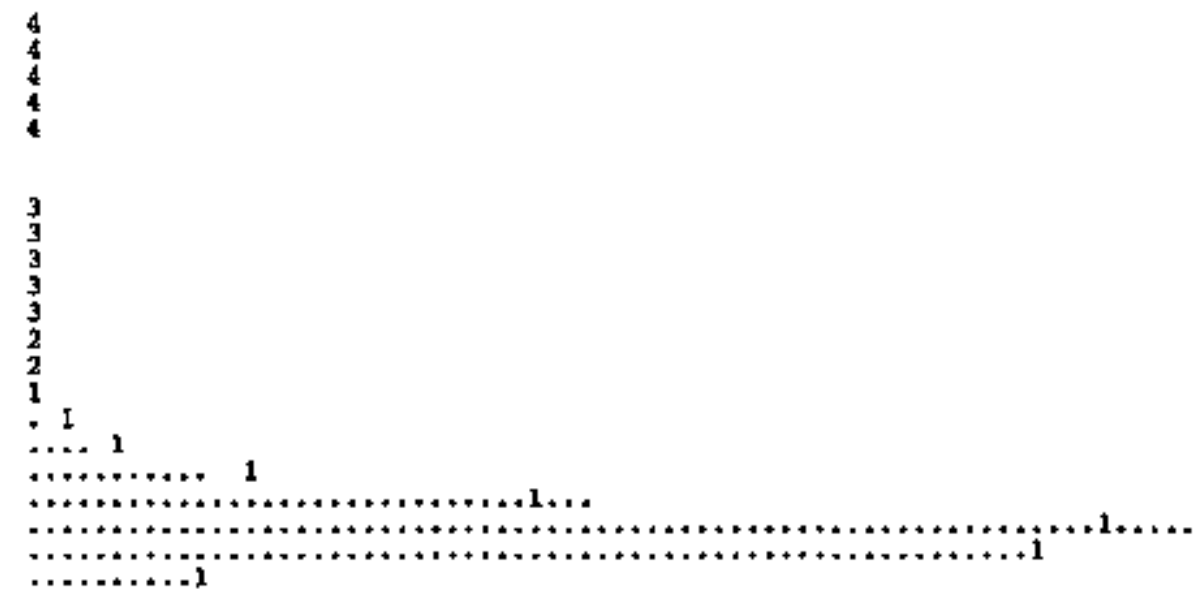




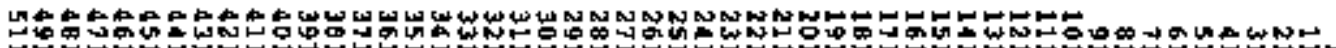

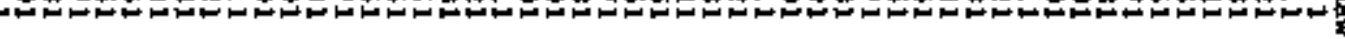

ロ姐占以

N

$$
\text { 四出 }
$$
ㅁ

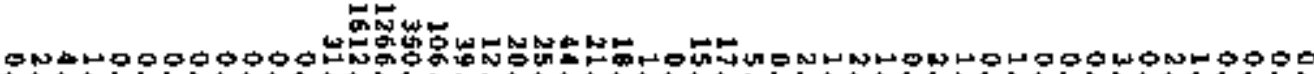

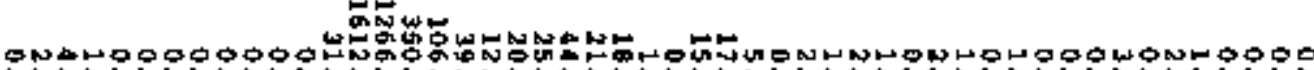
?

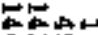

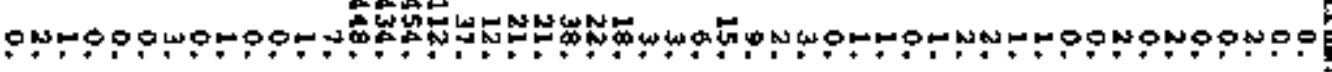

\section{UAA}

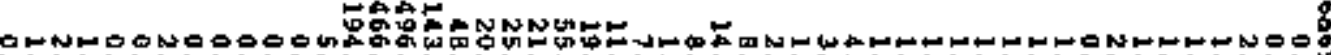

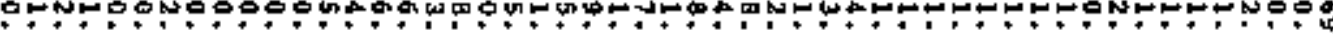

04س

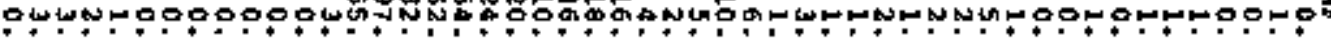

(6)

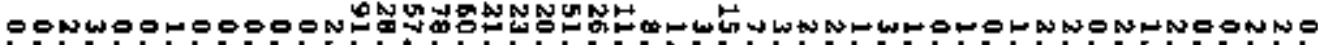

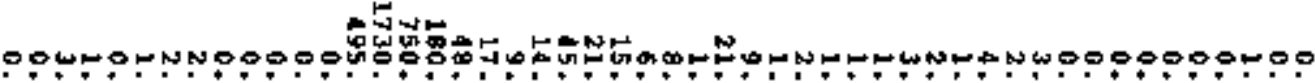

ص

-

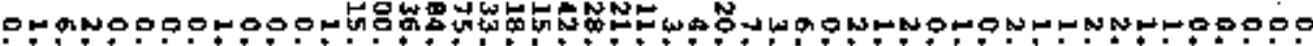

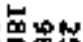

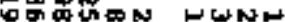


Wortinghouse Hanford Co.

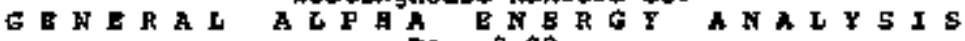
Rev. 2.02

DMEA RBDOCTIOA REPORT

SAMTtB

\$97T03B1-sus

File IDr 7a773日.CAF

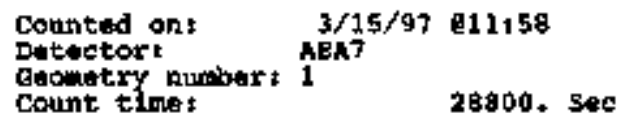

PEAR LMALYSTS

\begin{tabular}{|c|c|c|c|c|c|c|c|}
\hline $\begin{array}{l}\text { Peak } \\
\text { ID } \\
1 \\
2 \\
33\end{array}$ & $\begin{array}{c}\text { Peak b } \\
\text { Initial } \\
1488.8 \\
40.6 \\
12.0\end{array}$ & 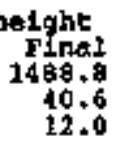 & $\begin{array}{c}\text { Peak center } \\
\text { Iritiel Final } \\
362.390 \\
2962.390 \\
220.147290 .188 \\
220.406 \quad 228.348\end{array}$ & $\begin{array}{l}\text { Tnitial } \\
22.000 \\
22,000 \\
18,000\end{array}$ & $\begin{array}{l}\text { MEN } \\
\text { Fingl } \\
17.194 \\
23.126 \\
15.558\end{array}$ & $\begin{array}{l}\text { Tritial } \\
11.000 \\
11.000 \\
9.000\end{array}$ & $\begin{array}{l}\text { Pinal } \\
6.7653 \\
6.467 \\
5.552\end{array}$ \\
\hline
\end{tabular}

\section{Feak Errox Linit: 309}

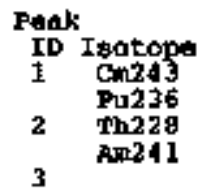

Totals;
AEA

Trac

0.961

0.032

?73?

$--193$

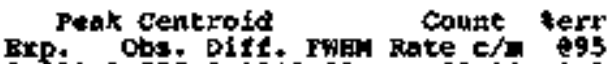

5.7795 .7550 .0240 .08

5.7555 .7550 .000

$5.4005 .416-.0160 .11$

$\$ .4795 .4160 .063$

5.125

$69,141.1$

$2.33 \quad 6.3$

$0.55 \quad 12.8$

71.47

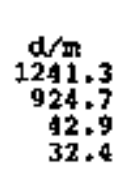

Activity uct/ea

0.5598-03

$0.417 E-03$

$0.193 E-04$

$0.146 \mathrm{~B}-04$

Analyzed by:

4 Recotrery

100.000

100.004

100.053

$-0.053$

39553.3

34570.3

$-18.3$

Channel

6.459

TOTAL COUNT DATA:

Composite fit

Residuals

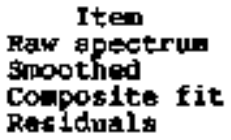


$=\quad$ spectrum 7 a7738,CNF HNF-SD-WN-DP-238, REV. 0

1 Legends Raw $=\ldots$... Modelad Peaks $=1,2, \ldots$ atc

Ditplay Max.:

11054.7

3
3
3
3
3

2
2
2
2
2
3
2

1

11

$\ldots+\ldots+1$

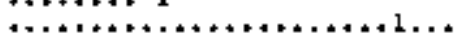

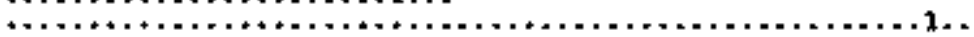

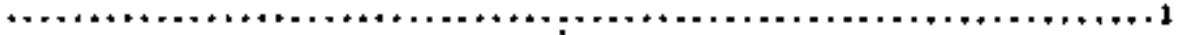

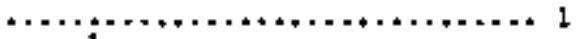

$+\ldots+1$ 


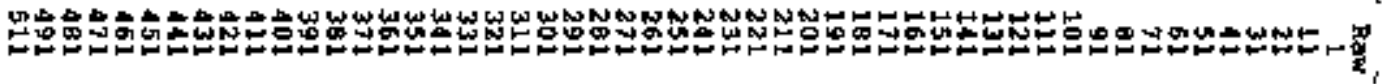

Niñ

WO

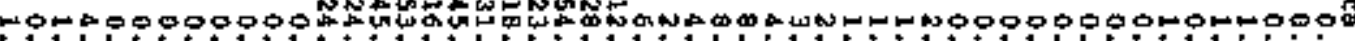

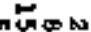

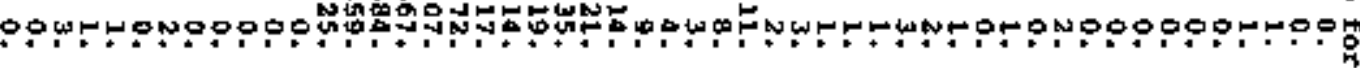

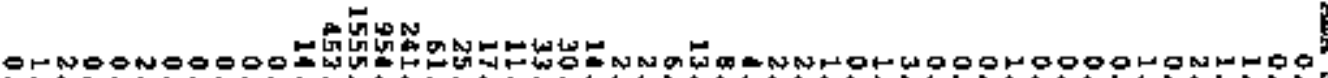

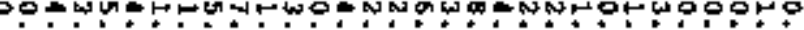

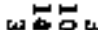

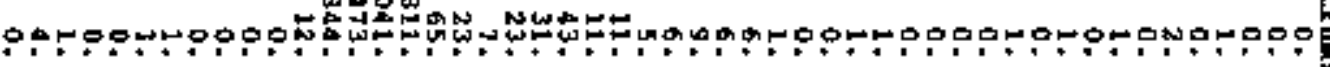

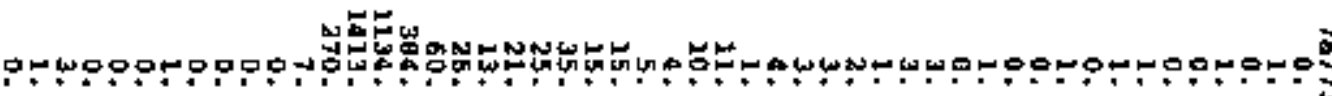

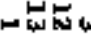

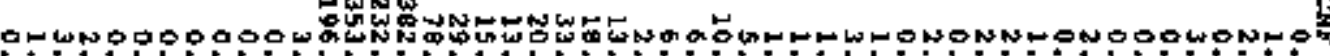

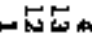

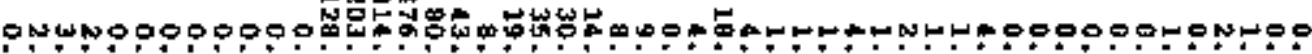

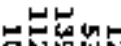

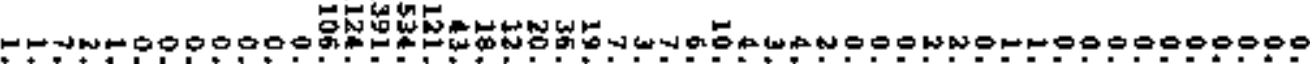
$\omega_{0}^{\infty}$

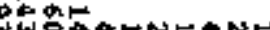

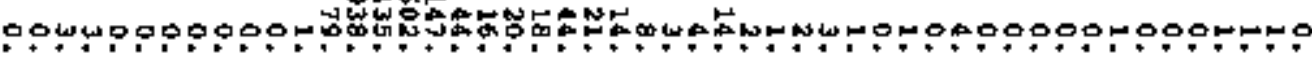

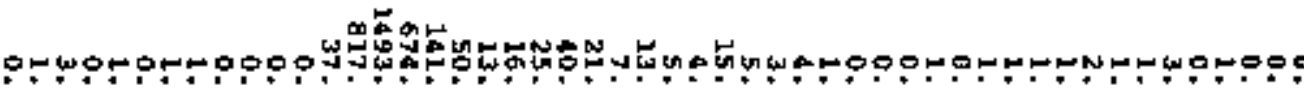


DISTMIBLTIOH SHEET

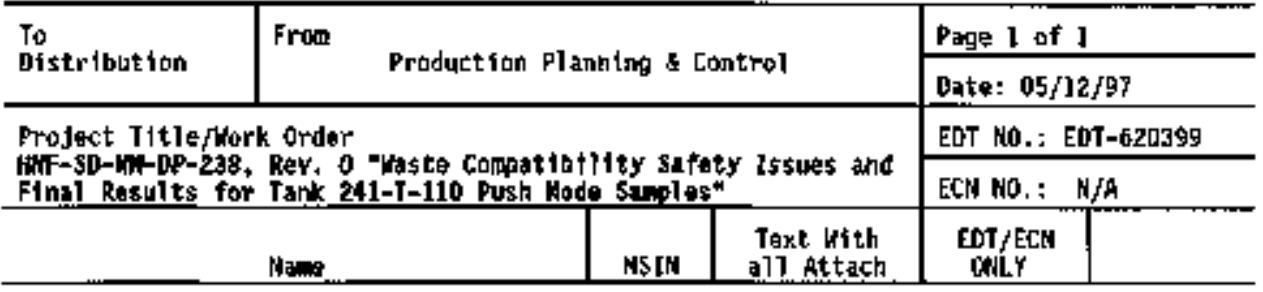

DERS Honford. Int

J. E. Meachä

ST-14 $\quad x$

U.s. Depertment of Entrgy, Rl.

C. A. Babel

57-54 $\quad x$

Loakhead Martio Han ford Core.

J. 月. Appel

T. J. Kallay

M. J. Kupfer

D. J. Micaln

J. 9. Schaffar

J. A. Yoog 4

A. E. Toung

63-21

$27-21$

H5- 49

R2-12

R2 $=12$

$\mathrm{HS}-03$

R2 $=12$

$x$

$\mathrm{X}$

$x$

Lockheed Martin Services, Ins.

Central files

A3-8B 1

Los A1 acos Technical Associates



T6-06

$x^{*}$

Pacific Horthwest Mational laboratosir

S. J. Harr's

K. L. Silvers

K $7-22$

$\mathrm{Kg}-0 \mathrm{~B}$

$x$

$x$
$x$
$x=$

Rest Federal Serrutces of Hanford. Inc.

h. W. Barnos

D. C. Hetzer

ป. L. 3tuzum

K. L. Ponell

c. M. Seidel

LTIE

T6-J6

$56-31$

T6-06

T6-0A

$T 6-04$

T6-03

SSCN Eurisys Services Carp.

B. A. Higley

$15-27$

$x$

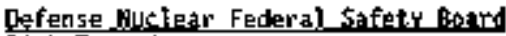

Rich Tantadonato

G25 Indiana Ave. N. Y.

$x$

Washington 0.C. 20004

- Heeds only raleasing paparwork, not a copy of the relessed documint. 